\title{
UC-NRLF
}

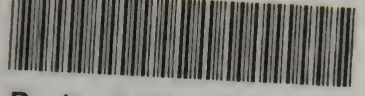

B 4 106 958 

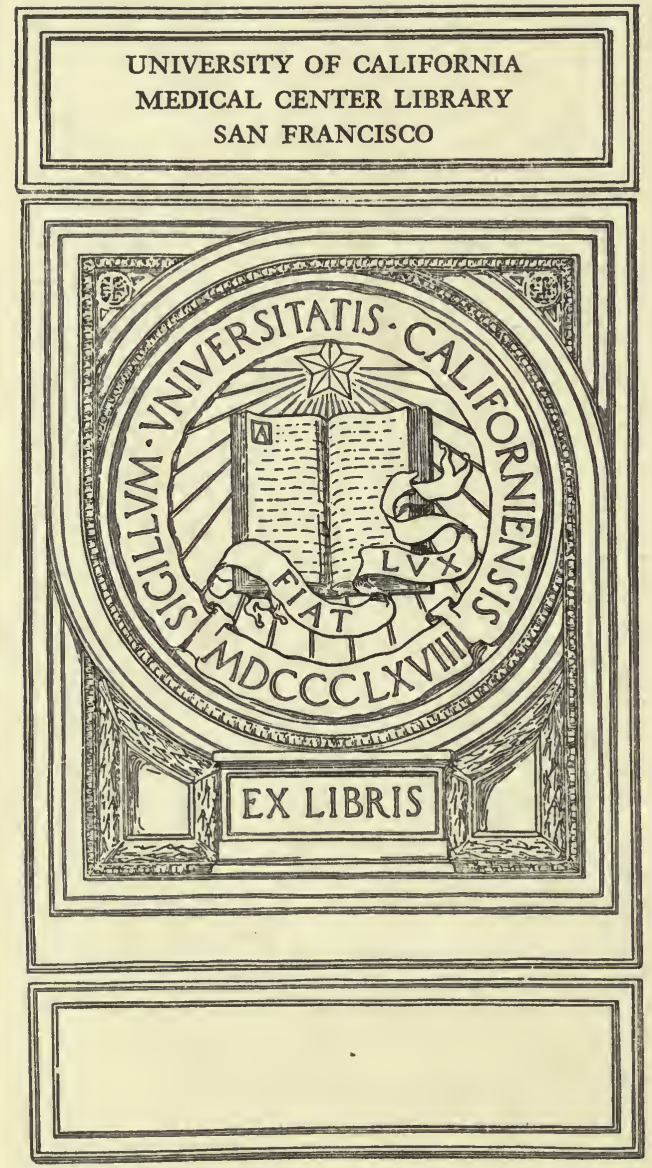


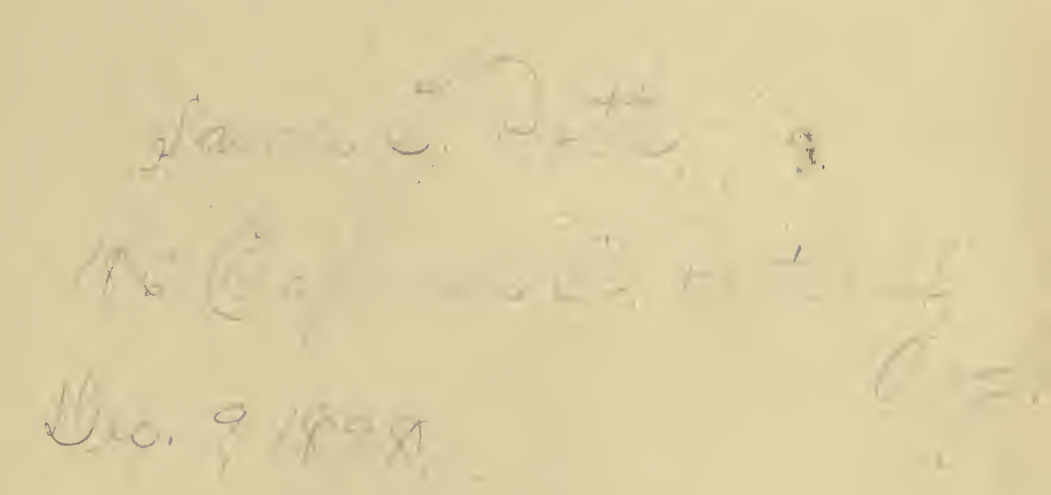


Digitized by the Internet Archive in 2007 with funding from Microsoft Corporation 




\section{A TEXT-BOOK}

oF

\section{PHYSIOLOG Y.}

BY

M. EOSTER, M. A., M.D., LL.D., F.R.S., PROFESSOR OF PHYSIOLOGY IN THE UNIVERSITY OF CAMBRIDGE, AND FELLOW OF TRINITY COLLEGE, CAMBRIDGE.

\section{SIXTH AMERICAN EDITION,}

THOROUGHLY REVISED, WITH NOTES, ADDITIONS, AND TWO HUNDRED AND FIFTY-SEVEN ILLUSTRATIONS.

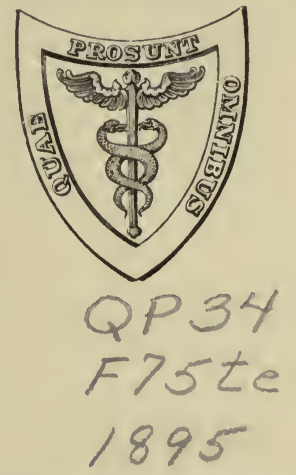

PHIL A DEL PHIA :

LEA BROTHERS \& CO. 1895 . 
Entered according to Act of Congress in the year 1895, by LEA BROTHERS \& CO.,

in the Office of the Librarian of Congress, at Washington. All rights reserved. 


\section{AMERICAN PUBLISHER'S NOTICE}

\section{TO THE SIXTH EDITION.}

Is the preparation of the Sixth American Edition every page has been subjected to careful scrutiny and considerable liberty taken with the text. Useless verbiage has been omitted, obscure sentences have been revised or entirely rewritten, a large number of typographical errors have been corrected, histological details (except of the nervous system) have been materially abridged, much that was too theoretical has been omitted, and such other alterations and additions have been made as to bring the book up to date and render it in a more advantageous form for the student. The histology of the nervous system has been retained in full, as has also the valuable Chenical Appendix - features that will be appreciated by both student and teacher. The more important additions have been distinguished by enclosure in brackets [ ].

Phila delphia, September, 1895. 



\section{CONTENTS.}

\section{BOOK I.}

BLOOD. THE TISSUES OF MOVEMENT. THE VASCULAR MECHANISM.

\section{CHAPTER I.}

BLOOD.

The Clotting of Blood . . . . . . . . . . . . . . 24

The Corpuscles of the Blood: The Red Corpuscles.. . . . . . . . . . . 37

The White or Colorless Corpuscles . . . . . . . . . . . . . 43

Blood Platelets. . . . . . . . . . . . . . . . . . . 49

The Chemical Composition of Blood . . . . . . . . . . . . 50

The Quantity of Blood, and its Distribution in the Body . . . . . . . . . 53

\section{CHAPTER II.}

\section{THE CONTRACTILE TISSUES.}

The Phenomena of Muscle and Nerve: Muscular and Nervous Irritability . . . 55

The Phenomena of a Simple Muscular Contraction . . . . . . . . . . . . 65

- Tetanic Contractions . . . . . . . . . . . . . . . . . . . . . 73

On the Changes which Take Place in a Muscle during a Contraction: The Change in Form. . . . . . . . . . . . . . . . . . 77

The Chemistry of Muscle . . . . . . . . . . . . 83

Thermal Changes . . . . . . . . . . . . . . . . 90

Electrical Changes . . . . . . . . . . . . . . 92

The Changes in a Nerve during the Passage of a Nervous Impulse . . . . . . 97

The Nature of the Changes through which an Electric Current is Able to

Generate a Nervous Impulse: Action of the Constant Current . . . . . 100

The Muscle-nerve Preparation as a Machine . . . . . . . . . . . . . 107

The Circumstances which Determine the Degree of Irritability of Muscles and Nerves . . . . . . . . . . . . . . . . . 111

The Energy of Muscle and Nerve, and the Nature of Muscular and Nervous Action . . . . . . . . . . . . . . . 115

On Some Other Forms of Contractile Tissue: Plain, Smooth or Unstriated Muscular Tissue . . . . . . . . . . . . . . . . . . 116

Ciliary Movement . . . . . . . . . . . . . . . . . . . . . 119

Amœboid Movements . . . . . . . . . . . . . . . . . . 121 
CHAPTER III.

PAGE GENERAL FEATURES OF NERVOUS TISSUES . . . . . . . . . 122

\section{CHAPTER IV.}

\section{THE VASCULAR MECHANISM.}

The Structure and Main Features of the Vascular Apparatus . . . . . . . 133

The Structure of Arteries, Capillaries, and Veins . . . . . . . . . . . . . 134

The Main Features of the Apparatus . . . . . . . . . . . . . . . . . . 134

The Main Facts of the Circulation . . . . . . . . . . . . 136

Hydraulic Principles of the Circulation . . . . . . . . . . . . . . . 144

Circumstances Determining the Rate of the Flow . . . . . . . . . . 150

The Heart . . . . . . . . . . . . . . . . . . . 157

The Phenomena of the Normal Beat . . . . . . . . . . . . . 157

Endocardiac Pressure . . . . . . . . . . . . . . 164

Summary . . . . . . . . . . . . . . . . . 174

The Work Done . . . . . . . . . . . . . . 175

The Pulse . . . . . . . . . . . . . . . . . . 176

The Regulation and Adaptation of the Vascular Mechanism: The Regulation of the Beat of the Heart . . . . . . . . . . . . . . . . . . . . . 191

The Development of the Normal Beat . . . . . . . . . . . . . . . 192

The Government of Heart-beat by the Nervous System . . . . . . . . . . 199

Other Influences Regulating or Modifying the Beat of the Heart . . . . . . . 209

Changes in the Calibre of the Minute Arteries. Vasomotor Actions . . . . . . 211

The Course of Vaso-constrictor and Vaso-dilator Fibres . . . . . . . . . . . 219

The Effects of Vasomotor Actions . . . . . . . . . . . . . . 221

Vasomotor Functions of the Central Nervous System . . . . . . . . . . . 222

The Capillary Circulation . . . . . . . . . . . . . . 231

Changes in the Quantity of Blood . . . . . . . . . . . 236

A Review of Some of the Features of the Circulation . . . . . . . . . 238

\section{BOOK II.}

\section{THE TISSUES OF CHEMICAL ACTION WITH THEIR RESPECTIVE MECHANISMS. NUTRITION.}

\section{CH A PTER I. \\ THE TISSUES AND MECHANISMS OF DIGESTION.}

The Characters and Properties of Saliva and Gastric Juice: Saliva . . . . 246

Gastric Juice . . . . . . . . . . . . . . . . . . . . . . . 251

The Act of Secretion of Saliva and Gastric Juice and the Nervous Mechanisms which Regulate It . . . . . . . . . . . . . . 260

The Changes in a Gland Constituting the Act of Secretion . . . . . . . . . 268

The Properties and Characters of Bile, Pancreatic Juice, and Succus Entericus . . 278

Bile . . . . . . . . . . . . . . . . . 278 
PAGE

Pancreatic Juice . . . . . . . . . . . . . . . . . . . . 281

Succus Entericus . . . . . . . . . . . . . . . . . . . 285

The Secretion of Pancreatic Juice and of Bile . . . . . . . . . . . 286

The Structure of the Intestines : The Small Intestine . . . . . . . . . . 292

The Muscular Mechanisms of Digestion . . . . . . . . . . . . . 293

The Changes which the Food Undergoes in the Alimentary Canal . . . . . . 306

The Changes in the Stomach . . . . . . . . . . . . 306

In the Small Intestine . . . . . . . . . . . . . . . . 308

In the Large Intestine . . . . . . . . . . . . . . . . . 312

The Feces. . . . . . . . . . . . . . . . . . . . . . . . . . . . 313

The Lacteals and the Lymphatic System . . . . . . . . . . . . . . . 313

The Nature and Movements of Lymph (including Chyle) . . . . . . . . . . 314

The Characters of Lymph . . . . . . . . . . . . . . . . 315

The Movements of $\mathrm{Lymph}$. . . . . . . . . . . . . . . . . . 317

A bsorption from the Alimentary Canal . . . . . . . . . . . . . 325

The Course Taken by the Several Products of Digestion . . . . . . . . . 325

The Mechanism of Absorption . . . . . . . . . . . . . . . 329

\section{CHAPTER II.}

\section{RESPIRATION.}

The Mechanics of Pulmonary Respiration . . . . . . . . . . . . 333

The Respiratory Movements . . . . . . . . . . . . . . . . . . . 339

Changes of the Air in Respiration . . . . . . . . . . . . . . 344

The Respiratory Changes in the Blood . . . . . . . . . . . . . . . 346

The Relations of Oxygen in the Blood . . . . . . . . . . . . . 350

Products of the Decomposition of Hæmoglobin . . . . . . . . . . . . 358

The Relations of the Carbonic Acid in the Blood . . . . . . . . . . . . 360

The Relations of the Nitrogen in the Blood . . . . . . . . . . . . . 361

The Respiratory Changes in the Lungs : The Entrance of Oxygen . . . . . . . 361

The Exit of Carbonic Acid . . . . . . . . . . . . . . . . . 364

The Respiratory Changes in the Tissues . . . . . . . . . . . . . . . 365

The Nervous Mechanism of Respiration . . . . . . . . . . . . . 369

The Effects of Changes in the Composition and Pressure of the Air Breathed . . 386

The Relations of the Respiratory System to the Vascular and Other Systems . . 390

Modified Respiratory Movements . . . . . . . . . . . . . 403

\section{CHA PTER III.}

\section{THE ELIMINATION OF WASTE PRODUCTS.}

The Composition and Characters of Urine . . . . . . . . . . . . . . . . . 404

Amounts of the Several Urinary Constituents Passed in Twenty-four Hours.

(After Parkes.) . . . . . . . . . . . . . . . . 409

The Secretion of Urine . . . . . . . . . . . . . . . 410

Secretion of the Renal Epithelium . . . . . . . . . . . . . . 417

The Discharge of Urine . . . . . . . . . . . . . . . . . 426

Micturition . . . . . . . . . . . . . . . . . . 427

The Nature and Amount of Perspiration ... . . . . . . . . 431

Cutaneous Respiration . . . . . . . . . . . . . . . . 432

The Mechanism of the Secretion of Sweat . . . . . . . . . . . 434 


\section{CHAPTER IV.}

THE METABOLIC PROCESSES OF THE BODY.

The History of Glycogen ......... PAGE

Diabetes. . . . . . . . . . . . . 437

The Formation of the Constituents of Bile . . . . . . . . . . . 453

On Urea and on Nitrogenous Metabolism in General . . . . . . . . . . 457

On Some Structures and Processes of Obscure Nature . . . . . . . . . . 465

The History of Fat. Adipose Tissue . . . . . . . . . . . . . . . 470

The Mammary Gland . . . . . . . . . . . . . . . . 477

A verage Composition of Milk in Different Animals . . . . . . . . . 480

\section{CHAPTER V.}

\section{NUTRITION.}

Statistics of Nutrition . . . . . . . . . . . . . . . 482

Comparison of Income and Output of Material . . . . . . . . . . . . 485

The Energy of the Body: The Income of Energy . . . . . . . . . . . . . 492

The Expenditure . . . . . . . . . . . . . . . . . 493

Animal Heat . . . . . . . . . . . . . . . . . . 496

On Nutrition in General . . . . . . . . . . . . 506

On Diet . . . . . . . . . . . . . . . . . . . . . . 513

\section{BOOK III.}

THE CENTRAL NERVOUS SYSTEM AND ITS INSTRUMENTS.

\section{CHA PTER I.}

THE SPINAL CORD.

On Some Features of the Spinal Nerves . . . . . . . . . . . . 525

The Structure of the Spinal Cord . . . . . . . . . . . . . . . . . 529

The Reflex Actions of the Spinal Cord . . . . . . . . . . . . 564

The Autornatic Actions of the Spinal Cord . . . . . . . . . . 578

\section{CHAPTER II.}

\section{THE BRAIN.}

On Some General Features of the Structure of the Brain . . . . . . . . . 584

The Bulb . . . . . . . . . . . . . . . . . . . . 589

The Disposition and Connections of the Gray and White Matter of the Brain:

The Gray Matter. . . . . . . . . . . . . . . . . 601

The Central Gray Matter and the Nuclei of the Cranial Nerves . . . . . . 601 
The Intermediate Gray Matter of the Crural Srstem . . . . . . . . 615

Other Collections of Gray Matter . . . . . . . . . . . . . . . . . . 625

The Arrangement of the Fibres of the Brain . . . . . . . . . . . . . . . . 627

Longitudinal Fibres of the Pedal System . . . . . . . . . . . . . . . 628

Longitudinal Fibres of the Tegmental System . . . . . . . . . . . . 631

Transverse or so-called Commissural Fibres . . . . . . . . . . . . . 634

Summary . . . . . . . . . . . . . . . . . . . . 635

On the Phenomena Exhibited by an Animal Deprived of its Cerebral Hemispheres. 637

The Machinery of Coördinated Movements . . . . . . . . . . . . . . . 643

On Some Histological Features of the Brain . . . . . . . . . . . . . . . 652

The Superficial Gray Matter of the Cerebellum . . . . . . . . . . . 653

The Cerebral Cortex . . . . . . . . . . . . . . 655

On Voluntary Movements . . . . . . . . . . . . . . . . . 661

On the Development within the Central Nervous System of Visual and of some

Other Sensations: Visual Sensations . . . . . . . . . . . . . 689

Sensations of Smell . . . . . . . . . . . . . . . 700

Sensations of Taste . . . . . . . . . . . . . . . . . . 701

Sensations of Hearing . . . . . . . . . . . . . . . 701

On the Development of Cutaneous and Some Other Sensations . . . . . . . . . 703

Some Other Aspects of the Functions of the Brain . . . . . . . . . . . . . 716

On the Time Taken up by Cerebral Operations . . . . . . . . . . 724

The Lymphatic Arrangements of the Brain and Spinal Cord . . . . . . . 728

The Vascular Arrangements of the Brain and Spinal Cord . . . . . . . . 732

\section{CHAPTER III.}

\section{SIGHT.}

The Eye . . . . . . . . . . . . . . . . . . . . 738

Dioptric Mechanisms: The Formation of the Image . . . . . . . . . . 744

Accommodation . . . . . . . . . . . . . . . . 745

Imperfections of the Dioptric A pparatus . . . . . . . . . . . . 757

Visual Sensations . . . . . . . . . . . . . . . . 759

The Origin of Visual Impulses . . . . . . . . . . . . . . . 760

Simple Sensations . . . . . . . . . . . . . . . 766

Color Sensations . . . . . . . . . . . . . . . . . . . . 769

Visual Perceptions . . . . . . . . . . . . . . . . 776

Modified Perceptions . . . . . . . . . . . . . . . . . 778

Binocular Vision : Corresponding or Identical Points . . . . . . . . . . . . . 781

Movements of the Eyeballs . . . . . . . . . . . . . . . 782

The Horupter . . . . . . . . . . . . . . . . . . 786

Visual Judgments . . . . . . . . . . . . . . . . . . . 786

The Protected Mechanisms of the Eye . . . . . . . . . . . . . 789

\section{CHA PTER IV.}

HEARING, SMELL, AND TASTE.

Hearing : The Ear . . . . . . . . . . . . . . . . 790

The Acoustic Apparatus . . . . . . . . . . . . . . . . . 798 
Auditory Sensations

\section{CHAPTER V.}

FEELING AND TOUCH.

General Sensibility and Tactile Perceptions . . . . . . . . . . . . 812

Tactile Sensations : Sensations of Pressure . . . . . . . . . . . . . 814

Sensations of Temperature . . . . . . . . . . . . . . . . 814

Tactile Perceptions and Judgments . . . . . . . . . . . . . . . 816

The Muscular Sense . . . . . . . . . . . . . . . . . . . . . . . . . . 818

CHA PTER VI.

SPECIAL MUSCULAR MECHANISMS.

The Voice: The Larynx . . . . . . . . . . . . . . . . . . 819

Speech : Vowels . . . . . . . . . . . . . . . . 826

Consonants . . . . . . . . . . . . . . . . . . . . 827

Locomotor Mechanisms . . . . . . . . . . . . . . . . . . . . . . 829

\section{BOOK IV.}

THE TISSUES AND MECHANISMS OF REPRODUCTION.

CHAPTER I.

ORGANS OF REPRODUCTION . . . . . . . . . . . 833

\section{CHAPTER II.}

MENSTRUATION

CHA PTER III.

IMPREGNATION

CH A PTER IV.

THE NUTRITION OF THE EMBRYO . . . . . . . . . . . 847

CHA PTER V. 
CHA P'TER VI.

THE PHASES OF LIFE .............

CHAPTER VII.

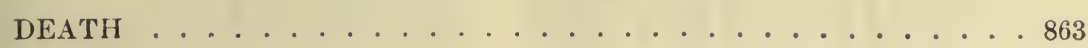

\section{A P PENDIX.}

THE CHEMICAL BASIS OF THE ANIMAL BODY $\ldots \ldots \ldots 86$ 



\section{A TEXT-BOOK OF PHYSIOLOGY.}

\section{INTRODUCTION.}

$\$ 1$. Dissection, aided by microscopical examination, teaches us that the body of man is made up of certain kinds of material, so differing from each other in optical and other physical characters and so built up together as to give the body certain structural features. Chemical examination further teaches us that these kinds of material are composed of various chemical substances, a large number of which have this characteristic, that they possess a considerable amount of potential energy capable of being set free, rendered actual, by oxidation or some other chemical change. Thus the body, as a whole, may, from a chemical point of view, be considered as a mass of various chemical substances, representing altogether a considerable capital of potential energy.

$\S 2$. This body may exist either as a living body or (for a certain time at least) as a dead body, and the living body may at any time become a dead body. At what is generally called the moment of death (but artificially so, for as we shall see the processes of death are numerous and gradual) the dead body so far as structure and chemical composition are concerned is exceedingly like the living body; indeed the differences between the two are such as can be determined only by very careful examination, and are still to a large extent estimated by drawing inferences rather than actually observed. At any rate the dead body at the moment of death resembles the living body in so far as it represents a capital of potential energy. From that moment onward, however, the capital is expended; by processes which are largely those of oxidation, the energy is gradually dissipated, leaving the body chiefly in the form of heat. While these chemical processes are going on the structural features disappear, and the body, with the loss of nearly all its energy, is at last resolved into "dust and ashes."

The characteristic of the dead body then is that, being a mass of substances of considerable potential energy, it is always more or less slowly losing energy, never gaining energy; the capital of energy present at the moment of death is more or less slowly diminished, is never increased or replaced.

\$3. When on the other hand we study a living body we are struck with the following salient facts:

1. The living body moves of itself, either moving one part of the body on another or moving the whole body from place to place. These movements are active; the body is not simply pulled or pushed by external forces, but the motive power is in the body itself, the energy of each movement is supplied by the body itself.

2. These movements are determined and influenced, indeed often seem to be started, by changes in the surroundings of the body. Sudden contact between the surface of the body and some foreign object will often call 
forth a movement. The body is sensitive to changes in its surroundings, and this sensitiveness is manifested not only by movenents but by other changes in the body.

3 . It is conţinually generating heat and giving out heat to surrounding things, the production and loss of heat, in the case of man and certain other animals, being so adjusted that the whole body is warm, that is, of a temperature higher than that of surrounding things.

4. From time to time it eats, that is to say takes into itself supplies of certain substances known as food, these substances being in the main similar to those which compose the body, and being like then chemical bodies of considerable potential energy, capable through oxidation or other chemical changes of setting free a considerable quantity of energy.

5. It is continually breathing; that is, taking in from the surrounding air supplies of oxygen.

6. It is continually, or from time to time, discharging from itself into its surroundings so-called waste matters, which waste matters may be broadly described as products of oxidation of the substances taken in as food, or of the substances composing the body.

Hence the living body may be said to be distinguished from the dead body by three main features.

The living body like the dead is continually losing energy (and losing it more rapidly than the dead body, the special breathing arrangements permitting a more rapid oxidation of its substance), but unlike the dead body is by means of food continually restoring its substance and replenishing its store of energy.

The energy set free in the dead body by the oxidation and other chemical changes of its substance leaves the body almost exclusively in the form of heat, whereas a great deal of energy leaves the living body as mechanical work, the result of various movements of the body, and as we shall see a great deal of the energy which ultimately leaves the body as heat, exists for a while within the living body in other forms than heat, though eventually transformed in to heat.

The changes in the surroundings affect the dead body at a slow rate and in a general way only, simply lessening or increasing the amount or rate of chemical change and the quantity of heat thereby set free, but never diverting the energy into some other form such as that of movement; whereas changes in the surroundings may in the case of the living body rapidly, profoundly, and in special ways affect not only the amount but also the kind of energy set free. The dead body left to itself slowly falls to pieces, slowly dissipates its store of energy, and slowly gives out heat; a higher or lower temperature, more or less moisture, a free or scanty supply of oxygen, the advent of many or few putrefactive organisms, these may quicken or slacken the rate at which energy is being dissipated but do not divert that energy from heat into motion; whereas in the living body so slight a change of surroundings as the mere touch by a hair of some particular surface, may so affect the setting free of energy as to lead to such a discharge of energy in the form of movement that the previously apparently quiescent body may be suddenly thrown into the most violent convulsions.

The differences, therefore, between living substance and dead substance though recondite are very great, and the ultimate object of physiology is to ascertain how it is that living substance can do what dead substance cannot-that is, can renew its substance and replenish the energy which it is continually losing, and can, according to the nature of its surroundings, vary not only the amount but also the kind of energy which it sets free. 
Thus there are two great divisions of physiology : one having to do with the renewal of substance and the replenishment of energy, the other having to do with the setting free of energy.

$\S 4$. Now the body of man (or one of the higher animals) is a very complicated structure, consisting of different kinds of material, which we call tissues, such as muscular, nervous, connective, and the like, variously arranged in organs such as heart, lungs, muscles, skin, etc., all built up to form the body according to certain morphological laws. But all this complication, though advantageous and indeed necessary for the fuller life of man, is not essential to the existence of life. The amoba [Fig. 1] is a

[FIG. 1.

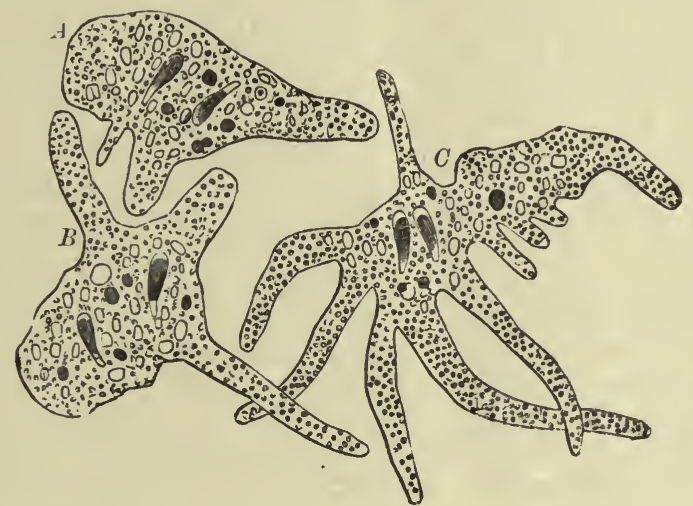

Amøba princeps, shown in different forms $(A, B, C)$ assumed by the same animal.]

living being; it renews its substance, replenishes its store of energy, and sets free energy now in one form, now in another; and yet the amoba may be said to have no tissues and no organs; at all events this is true of closely allied but not so well known simple beings. Using the more familiar amoba as a type, and, therefore, leaving on one side the nucleus, and any distinction between endosare and ectosarc, we may say that its body is homogeneous in the sense that if we divided it into small pieces, each piece would be like all the others. In another sense it is not homogeneous. For we know that the amoeba receives into its substance material as food, and that this food or part of it remains lodged in the body, until it is made use of and built up into the living substance of the body, and each piece of the living substance of the body must have in or near it some of the material which it is about to build up into itself. Further, we know that the amœa gives out waste matters such as carbonic acid and other substances, and each piece of the amœba must contain some of these waste matters about to be, but not yet, discharged from the piece. Each piece of the amœba will, therefore, contain these three things, the actual living substance, the food about to become living substance, and the waste matters which have ceased to be living substance.

Moreover, we have reasons to think that the living substance does not break down into the waste matters which leave the body at a single bound, but there are stages in the downward progress between the one and the other. Similarly, though our knowledge on this point is less sure, we have reason to think that the food is not incorporated into the living substance at a single step, but that there are stages in the upper progress from the dead food to the living substance. Each piece of the body of the amoba 
will, therefore, contain substances representing various stages of becoming living, and of ceasing to be living, as well as the living substance itself. And we may safely make this statement though we are quite unable to draw the line where the dead food on its way up becomes living, or the living substance on its way down becomes dead.

5. Nor is it necessary for our present purpose to be able to point out under the microscope, or to describe from a histological point of view, the parts which are living and the parts which are dead food or dead waste. The body of the amœba is frequently spoken of as consisting of "protoplasm." The name was originally given to the matter forming the primordial utricle of the vegetable cell as distinguished from the cell wall on the one hand, and from the fluid contents of the cell or cell sap on the other, and also we may add from the nucleus. It has since been applied very generally to such parts of animal bodies as resemble, in their general features, the primordial utricle. Thus the body of a white blood-corpuscle, or of a gland cell, or of a nerve cell, is said to consist of protoplasm. Such parts of animal bodies as do not in their general features resemble the matter of the primordial utricle are not called protoplasm, or, if they at some earlier stage did bear such resemblance, but no longer do so, are sometimes, as in the case of the substance of a muscular fibre, called "differentiated protoplasm." Protoplasm in this sense sometimes appears, as in the outer part of most anœbæ, as a mass of glassy-looking material, either continuous or interrupted by more or less spherical spaces or vacuoles filled with fluid, sometimes as in a gland cell as a more refractive, cloudy-looking, or finely granular material arranged in a more or less irregular network, or spongework, the interstices of which are occupied either by fluid or by some material different from itself. IVe shall return, however, to the features of this "protoplasm" when we come to treat of white blood-corpuscles and other "protoplasmic" structures. Meanwhile it is sufficient for our present purpose to note that lodged in the protoplasm, discontinuous with it, and forming no part of it, are in the first place collections of fluid, of watery solutions of various substances, occupying the more regular vacuoles or the more irregular spaces of the network, and in the second place discrete granules of one kind or another, also forming no part of the protoplasm itself, but lodgerl either in the bars or substance of the protoplasm or in the vacuoles or meshes.

Now, there can be little doubt that the fluids and the discrete granules are dead food or dead waste, but the present state of our knowledge will not permit us to make any very definite statement about the protoplasm itself. We may probably conclude, indeed we may be almost sure, that protoplasm in the above sense is not all living substance, that it is made up partly of the real living substance, and partly of material which is becoming living or has ceased to be living; and in the case where protoplasm is described as forming a network, it is possible that some of the material occupying the meshes of the network may be, like part of the network itself, really alive. "Protoplasm" in fact, as in the sense in which we are now using it, and shall continue to use it, is a morphological term; but it must be borne in mind that the same word "protoplasm" is also frequently used to denote what we have just now called "the real living substance." The word then embodies a physiological idea ; so used it may be applied to the living substance of all living structures, whatever the microscopical features of those structures; in this sense it cannot at present, and possibly never will be recognized by the microscope, and our knowledge of its nature must be based on inferences.

Keeping then to the phrase "living substance" we may say that each piece of the body of the amœba consists of living substance, in which are 
lodged, or with which are built up in some way or other, food and waste in various stages.

Now, an amœba may divide itself into two, each half exhibiting all the phenomena of the whole; and we can easily imagine the process to be repeated, until the amœba was divided into a multitude of exceedingly minute amœbæ, each having all the properties of the original. But it is obvious, as in the like division of a mass of a chemical substance, that the division could not be repeated indefinitely. Just as in division of the chemical mass we come to the chemical molecule, further division of which changes the properties of the substance, so in the continued division of the amœba we should come to a stage in which further division interfered with the physiological actions, we should come to a physiological unit corresponding to but greatly more complex than the chemical molecule. This unit to remain a physiological unit and to continue to live must contain not only a portion of the living substance but also the food for that living substance, in several at least of the stages, from the initial raw food up to the final "living" stages, and must similarly contain various stages of waste.

$\S 6$. Now, the great characteristic of the typical amœba (leaving out the nucleus) is that, as far as we can ascertain, all the physiological units are alike; they all do the same things. Each and every part of the body receives food more or less raw and builds it up into its own living substance; each and every part of the body may be at one time quiescent and at another in motion; each and every part is sensitive and responds by movement or otherwise to various changes in its surroundings.

The body of man, in its first stage, while it is yet an ovum, if we leave aside the nucleus and neglect differences caused by the unequal distribution of food material or yolk, may also be said to be composed of like parts or like physiological units.

By the act of segmentation, however, the ovum is divided into parts or cells which early show differences from each other; and these differences rapidly increase as development proceeds. Some cells put on certain characters and others other characters-that is to say, the cells undergo histological differentiation. And this takes place in such a way that a number of cells lying together in a group become eventually converted into a tissue, and the whole body becomes a collection of such tissues arranged together according to morphological laws, each tissue having a definite structure, its cellular nature being sometimes preserved, sometimes obscured or even lost.

This histological differentiation is accompanied by a physiological division of labor. Each tissue may be supposed to be composed of physiological units, the units of the same tissue being alike but differing from the units of other tissues; and corresponding to this difference of structure, the units of different tissues behave or act differently. Instear of all the units, as in the amœba, doing the same things equally well, the units of one tissue are told off, as it were, to do one thing especially well, or especially fully, and thus the whole labor of the body is divided among the several tissues.

$\S 7$. The several tissues may thus be classified according to the work which they have to do ; and the first great distinction is into (1) the tissues which are concerned in the setting free of energy in special ways, and (2) the tissues which are concerned in replenishing the substance and so renewing the energy of the body.

Each physiological unit of the amœeba while it is engaged in setting free energy so as to move itself, and by reasnn of its sensitiveness so directing that energy as to produce a movement suitable to the conditions of its surroundings, has at the same time to bear the labor of taking in raw food, of selecting that part of the raw food which is useful and rejecting that which 
is useless, and of working up the accepted part through a variety of stages into its own living substance-that is to say, it has at the same time that it is feeling and moving to carry on the work of digesting and assimilating. It has, moreover, at the same time to throw out the waste matters arising from the changes taking place in its own substance, having first brought these waste matters into a condition suitable for being thrown out.

$\S 8$. In the body of man, movements, as we shall see, are, broadly speaking, carried out by means of muscular tissue, and the changes in muscular tissue which lead to the setting free of energy in the form of movement are directed, governed, and adapted to the surroundings of man, by means of nervous tissue. Rays of light fall on the nervous substance of the eye called the retina, and set up in the retina changes which induce in the optic nerve other changes, which in turn are propagated to the brain as nervous impulses, both the excitation and the propagation involving an expenditure of energy. These nervous impulses reaching the brain may induce other nervous impulses which, travelling down certain nerves to certain muscles, may lead to changes in those muscles by which they suddenly grow short and pull upon the bones or other structures to which they are attached, in which case we say the man starts; or the nervous impulses reaching the brain may produce some other effects. Similarly sound falling on the ear, or contact between the skin and some foreign body, or some change in the air or other surroundings of the body, or some change within the body itself may so affect the nervous tissue of the body that nervous impulses are started and travel to this point or that, to the brain or elsewhere, and eventually may either reach some muscular tissue and so give rise to movements, or may reach other tissues and produce some other effect.

The muscular tissue then may be considered as given up to the production of movement, and the nervous tissue as given up to the generation, transformation, and propagation of nervous impulses. In each case there is an expenditure of energy, which in the case of the muscle, as we shall see, leaves the body partly as heat and partly as work done, but in the case of nervous tissue is wholly or almost wholly transformed into heat before it leaves the body; and this expenditure necessitates a replenishment of energy and a renewal of substance.

$\$ 9$. In order that these master tissues, the nervous and muscular tissues, may carry on their important works to the best advantage, they are relieved of much of the labor that falls upon each physiological unit of the amoba. They are not presented with raw food, they are not required to carry out the necessary transformations of their immediate waste matters. The whole of the rest of the body is engaged (1) in so preparing the raw food, and so bringing it to the nervous and muscular tissues that these may build it up into their own substance with the least trouble, and (2) in receiving the waste matters which arise in muscular and nervous tissues, and preparing them for rapid and easy ejection from the body.

Thus to certain tissues, which we may speak of broadly as "tissues of digestion," is allotted the duty of acting on the food and preparing it for the use of the muscular and nervous tissues; and to other tissues, which we may speak of as "tissues of excretion," is allotted the duty of clearing the body from the waste matters generated by the muscular and nervous tissues.

$\S 10$. These tissues are for the most part arranged in machines or mechanisms called organs, and the workings of these organs involves movement. The movements of these organs are carried out, like the other movements of the body, chiefly by means of muscular tissue governed by nervous_tissue. Hence we may make a distinction between the muscles which are concerned in producing an effect on the world outside man's body, the muscles by which 
man does his work in the world, and the muscles which are concerned in carrying out the movements of the internal organs. And we may similarly make a distinction between the nervous tissue concerned in carrying out the external work of the body and that concerned in regulating the movements and, as we shall see, the general conduct of the internal organs. But these two classes of muscular and nervous tissue, though distinct in work and, as we shall see, often different in structure, are not separated or isolated. On the contrary, while it is the main duty of the nervous tissue as a whole, the nervous system as we may call it, to carry out, by means of nervous impulses passing hither and thither, what may be spoken of as the work of man, and in this sense it is the master tissue, it also serves as a bond of union between itself and the muscles doing external work on the one hand, and the organs of digestion or excretion on the other, so that the activity and conduct of the latter may be adequately adapted to the needs of the former.

$\S 11$. Lastly, the food prepared and elaborated by the digestive organs is carried and presented to the muscular and nervous tissues in the form of a complex fluid known as blood, which, driven by means of a complicated mechanism known as the vascular system, circulates all over the body, visiting in turn all the tissues of the body, and by a special arrangement known as the respiratory mechanism, carrying in itself to the several tissues a supply of oxygen as well as of food more properly so called.

The motive power of this vascular system is supplied, as in the case of the digestive system, by means of muscular tissue, the activity of which is similarly governed by the nervous system, and hence the flow of blood to this part or that part is regulated according to the needs of the part.

$\S 12$. The above slight sketch will perhaps suffice to show not only how numerous but how varied are the problems. with which physiology has to deal.

In the first place, there are what may be called general problems, such as, How the food after its preparation and elaboration into blood is built up into the living substance of the several tissues? How the living substance breaks down into the dead waste? How the building up and breaking down differ in the different tissues in such a way that energy is set free in different modes, the muscular tissue contracting, the nervous tissue thrilling with a nervous impulse, the secreting tissue doing chemical work, and the like? To these general questions the answers which we can at present give can hardly be called answers at all.

In the second place, there are what may be called special problems, such as, What are the various steps by which the blood is kept replenished with food and oxygen, and kept free from an accumulation of waste, and how is the activity of the digestive, respiratory, and excretory organs, which effect this, regulated and adapted to the stress of circumstances? What are the details of the working of the vascular mechanism by which each and every tissue is forever bathed with fresh blood, and how is that working delicately adapted to all the various changes of the body? And, compared with which all other special problems are insignificant and preparatory only, How do nervous impulses so flit to and fro within the nervous system as to issue in the movements which make up what we sometimes call the life of man? It is to these special problems that we must chiefly confine our attention, and we may fitly begin with a study of the blood. 



\section{BOOK I.}

BLOOD. THE TISSUES OF IOVENENT, THE VASCULAR MECHANISII.

\section{CHA P'TER I.}

\section{BLOOD.}

$\S 13$. The several tissues are traversed by minute tubes, the capillary bloodvessels, to which blood is brought by the arteries, and from which blood is carried away by the veins. These capillaries form networks the meshes of which, differing in form and size in the different tissues, are occupied by the elements of the tissue, which consequently lie outside the capillaries.

The blood flowing through the capillaries consists, under normal conditions, of an almost colorless fluid, the plasma, in which are carried a num. ber of bodies, the red and the white corpuscles. Outside the capillary walls, filling up such spaces as exist between the capillary walls and the cells or fibres of the tissue, or between the elements of the tissue themselves, is found a colorless fluid resembling in many respects the plasma of blood, and called lymph. Thus all the elements of the tissue and the outsides of all the capillaries are bathed with lymph, which, as we shall see hereafter, is continually fiowing away from the tissue along special channels to pass into lymphatic vessels and thence into the blood.

As the blood flows through the capillaries certain constituents of the plasma (together with, at times, white.corpuscles, and under exceptional circumstances red corpuscles) pass through the capillary wall into the lymph, and certain constituents of the lymph pass through the capillary wall into the blood within the capillary. There is thus an interchange of material between the blood within the capillary and the lymph outside. A similar interchange of material is at the same time going on between the lymph and the tissue itself. Hence, by means of the lymph acting as middleman, a double interchange of material takes place between the blood within the capillary and the tissue outside the capillary. In every tissue, so long as life lasts and the blood flows through the bloodvessels, a double stream, now rapid, now slow, is passing from the blood to the tissue and from the tissue to the blood. The stream from the blood to the tissue carries to the tissue the material which the tissue needs for building itself up and for doing its work, including the all-important oxygen. The stream from the tissue to the blood carries into the blood certain of the products of the chemical changes which have been taking place in the tissue, products which may be simple waste, to be cast out of the body as soon as possible, or which may be bodies capable of being made use of by some other tissue.

A third stream, that from the lymph lying in the chinks and crannies of the tissue along the lymph channels to the larger lymph vessels, carries away from the tissue such parts of the material coming from the blood as 
are not taken up by the tissue itself and such parts of the material coming from the tissue as do not find their way into the bloodvessel.

In most tissues, as in muscle for instance, the capillary network is so close set and the muscular fibre lies so near to the bloodvessel that the lymph between the two exists only as a very thin sheet; but in some tissues, as in cartilage, the bloodvessels lie on the outside of a large mass of tissue, the interchange between the central parts of which and the nearest capillary bloodvessel is carried on through a long stretch of lymph passages. But in each case the principle is the same; the tissue, by the help of lymph, lives on the blood; and when in succeeding pages we speak of changes between the blood and the tissues, it will be understood, whether expressly stated so or not, that the changes are effected by means of the lymph. The blood may thus be regarded as an internal medium bearing the same relations to the constituent tissues that the external medium, the world, does to the whole individual. Just as the whole organism lives on the things around it, its air and its food, so the several tissues live on the complex fluid by which they are all bathed, and which is to them their inmediate air and food.

All the tissues take up oxygen from the blood and give up carbonic acid to the blood, but not always at the same rate or at the same time. Moreover, the several tissues take up from the blood and give up to the blood either different things or the same things at different rates or at different times.

From this it follows, on the one hand, that the composition and characters of the blood must be forever varying in different parts of the body and at different times; and on the other hand, that the united action of all the tissues must tend to establish and maintain an average uniform composition of the whole mass of blood. The special changes which blood is known to undergo while it passes through the several tissues will best be dealt with when the individual tissues and organs come under our consideration. At present it will be sufficient to study the main features which are presented by blood brought, so to speak, into a state of equilibrium by the common action of all the tissues.

Of all these main features of blood the most striking, if not the most important, is the property it possesses of clotting when shed.

\section{The Clotting of Blood.}

$\$ 14$. Blood, when shed from the bloodvessels of a living body, is perfectly fluid. In a short time it becomes viscid, this viscidity increasing rapidly until the whole mass of blood under observation becomes a complete jelly. The vessel into which' it has been shed can at this stage be inverted without a drop of the blood being spilt. The jelly is of the same bulk as the previously fluid blood, and if carefully shaken out will present a complete mould of the interior of the vessel. [Fig. 2.] If the blood in this jelly stage be left untouched in a glass vessel, a few drops of an almost colorless fluid soon make their appearance on the surface of the jelly. Increasing in number, and running together, the drops after a while form a superficial layer of pale, straw-colored fluid. Later on, similar layers of the same fluid are seen at the sides and finally at the bottom of the jelly, which, shrunk to a smaller size and of firmer consistency, now forms a clot or crassamentum, floating in a perfectly fluid serum. [Fig. 3.] The shrinking and condensation of the clot, and the corresponding increase of the serum, continue for some time. The upper surface of the clot is generally slightly concave. A portion of the clot examined under the microscope is seen to 
consist of a feltwork of fine granular fibrils, in the meshes of which are entangled the red and white corpuscles of the blood. In the serum nothing

[FIG. 2.

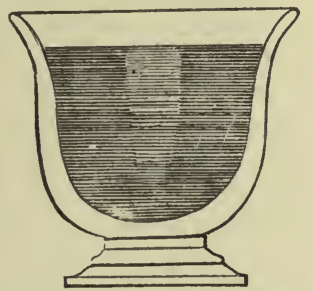

Bowl of recently coagulated blood, showing the whole mass unifurmly solidified. After Dalton.]
[FIG. 3.

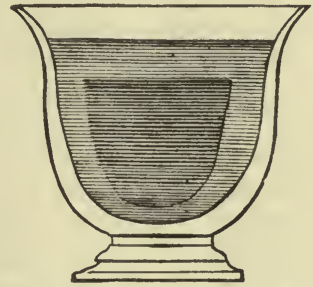

Bowl of coagulated blood, after twelve hours, showing the clot contracted and floating in the fluid serum. After Dalton.]

can be seen but a few stray corpuscles, chiefly white. The fibrils are composed of a substance called fibrin. [Fig. 4.] Hence we may speak of the clot as consisting of fibrin and corpuscles; and the act of clotting is obviously a substitution for the plasma of fibrin and serum, followed by a separation of the fibrin and corpuscles from the serum.

In man, blood when shed becomes viscid in about two or three minutes, and enters the jelly stage in about five or ten minutes. After the lapse of another few minutes the first drops of serum are seen, and clotting is generally complete in from one to several hours. The time, however, will be found to vary according to circumstances. Among animals the rapidity of clotting varies exceedingly in different species. The blood of the horse clots with remarkable slowness; so slowly, in-

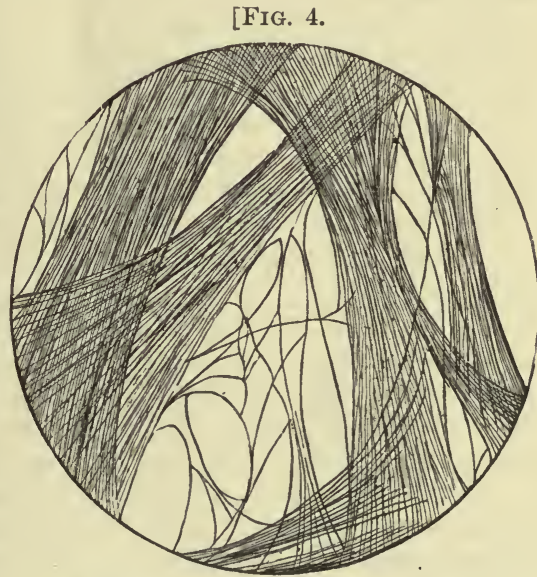

Coagulated fibrin, showing its fibrillated condition. After Dalton.] deed, that many of the red and also some of the white corpuscles (both these being specifically heavier than the plasma) have time to sink before viscidity sets in. In consequence there appears on the surface of the blood an upper layer of colorless plasma containing in its deeper portions many colorless corpuscles (which are lighter than the red). This layer clots like the other parts of the blood, forming the so-called "buffy coat." A similar buffy coat is sometimes seen in the blood of man in certain abnormal conditions of the body.

If a portion of horse's blood be surrounded by a cooling mixture of ice and salt, and thus kept about $0^{\circ} \mathrm{C}$., clotting may be almost indefinitely postponed. Under these circumstances a more complete descent of the corpuscles takes place, and a considerable quantity of colorless transparent plasma free from blood corpuscles may be obtained. A portion of this plasma removed from the freezing mixture clots in the same manner as does the entire blood. It first becomes vascid and then forms a jelly, which subsequently separates into a colorless shrunken clot and serum. This shows that the corpuscles are not an essential part of the clot. 
If a few cubic centimetres of this colorless plasma, or of a similar plasma which may be obtained from almost any blood by means which we will presently describe, be diluted with many times its bulk of a 0.6 per cent. solution of sodium chloride ${ }^{1}$ clotting is much retarded, and the various stages may be more easily watched. As the fluid is becoming viscid, fine fibrils of fibrin will be seen to be developed in it, especially at the sides of the containing vessel. As these fibrils inultiply in number, the fluid becomes more and more of the consistence of a jelly and at the same time somewhat opaque. Stirred or pulled about with a needle, the fibrils shrink up into a small opaque stringy mass; and a very considerable bulk of the jelly may by agitation be resolved into a minute fragment of shrunken fibrin floating in a quantity of what is really diluted serum. If a specimen of such diluted plasma be stirred from time to time, as soon as clotting begins, with a needle or glass rod, the fibrin may be removed piecemeal as it forms, and the jelly stage may be altogether done away with. When fresh blood which has not yet had time to clot is stirred or whipped with a bundle of rods (or anything presenting a large amount of rough surface), no jelly-like clotting takes place, but the rods become covered with a mass of shrunken fibrin. Blood thus whipped until fibrin ceases to be deposited, is found to have entirely lost its power of clotting.

Putting these facts together, it is very clear that the phenomena of the clotting of blood are caused by the appearance in the plasma of fine fibrils of fibrin. So long as these are scanty, the blood is simply viscid. When they become sufficiently numerous, they give the blood the firmness of a jelly. Soon after their formation they begin to shrink, and while shrinking enclose in their meshes the corpuscles, but squeeze out the fluid parts of the blood. Hence the appearance of the shrunken colored clot and the colorless serum.

$\$ 15$. Fibrin, whether obtained by whipping freshly shed blood, or by washing either a normal clot, or a clot obtained from colorless plasma, exhibits the same general characters. It belongs to that class of complex unstable nitrogenous bodies called proteids, which form a large portion of all living bodies and an essential part of all living structures.

Our knowledge of proteids is at present too imperfect, and probably none of them have yet been prepared in adequate purity to justify us in attempting to assign to them any definite formula; but it is important to remember their general composition. 100 parts of a proteid contain rather more than 50 parts of carbon, rather more than 15 of nitrogen, about 7 of hydrogen, and rather more than 20 of oxygen; that is to say, they contain about half their weight of carbon, and only about $\frac{1}{6}$ their weight of nitrogen ; and yet, as we shall see, they are eminently the nitrogenous substances of the body. They usually contain a small quantity ( 1 or 2 per cent.) of sulphur, and many also have some phosphorus attached to them in some way or other. IVhen burnt they leave a variable quantity of ash, consisting of inorganic salts of which the bases are chiefly sodium and potassium, and the acids chiefly hydrochloric, sulphuric, phosphoric, and carbonic.

They all give certain reactions, by which their presence may be recognized; of these the most characteristic are the following: Boiled with nitric acid they give a yellow color, which deepens into orange upon the addition of ammonia. This is called the xanthoproteic test ; the color is due to a product of decomposition. Boiled with the mixture of mercuric and mercurous nitrates known as Millon's reagent, they give a pink color. Mixed with a strong solution of sodic hydrate they give, on the addition of a drop or two of

${ }^{1}$ A solution of sodium chloride of this strength will hereafter be spoken of as " normal saline solution." 
a very weak solution of cupric sulphate, a violet or pink color which deepens on heating. These are artificial reactions, not throwing much if any light on the constitution of proteids ; but they are useful as practical tests enabling us to detect their presence.

The several members of the proteid group are at present distinguished from each other chiefly by their respective solubilities, especially in various saline solutions. Fibrin is one of the least soluble; it is insoluble in water, almost insoluble in dilute neutral saline solutions, and very sparingly soluble in more concentrated neutral saline solutions and in dilute acids and alkalies. In strong acids and alkalies it dissolves, but in the process becomes completely changed into something which is no longer fibrin. In dilute acids it swells up and becomes transparent, but when the acid is neutralized returns to its previous condition. When suspended in water and heated to $100^{\circ} \mathrm{C}$, or even to $75^{\circ} \mathrm{C}$., it becomes changed, and still less soluble than before ; it is said in this case to be coagulated by the heat, and, as we shall see, nearly all proteids have the property of being changed in nature, of undergoing coagulation and so becoming less soluble than before, by being exposed to a certain high temperature.

Fibrin, then, is a proteid distinguished from other proteids by its smaller solubility; it is further distinguished by its peculiar filamentous structure, the other proteids when obtained in a solid form appearing either in amorphous granules or, at most, in viscid masses.

$\$ 16$. We may now return to the serum.

This is perfectly fluid, and remains fluid until it decomposes. It is of a faint straw-color, due to the presence of a special pigment substance, differing from the red matter which gives redness to the red corpuscles.

Tested by the xanthoproteic and other tests it obviously contains a large quantity of proteid matter, and upon examination we find that at least two distinct proteid substances are present in it.

If crystals of magnesium sulphate be added to serum and gently stirred until they dissolve, it will be seen that the serum as it approaches saturation with the salt becomes turbid instead of remaining clear, and eventually a white amorphous granular or flocculent precipitate makes its appearance. This precipitate may be separated by decantation or filtration, washed with saturated solutions of magnesium sulphate, in which it is insoluble, until it is freed from all other constituents of the serum, and thus obtained fairly pure: It is then found to be a proteid body, distinguished by the following characters among others:

1. It is (when freed from any adherent magnesium sulphate) insoluble in distilled water; it is insoluble in concentrated solutions of neutral saline bodies, such as magnesium sulphate, sodium chloride, etc., but readily soluble in dilute (e.g., 1 per cent.) solutions of the same neutral saline bodies. Hence from its solutions in the latter it may be precipitated either by adding more neutral saline substance or by removing by dialysis the small quantity of saline substance present. When obtained in a precipitated form, and suspended in distilled water, it readily dissolves into a clear solution upon the addition of a small quantity of some neutral saline body. By these various solutions and precipitations it is not really changed in nature.

2. It readily dissolves in very dilute acids (e.g., in hydrochloric acid even when diluted to far less than 1 per cent.), and it is similarly soluble in dilute alkalies, but in being thus dissolved it is wholly changed in nature, and the solutions of it in dilute acid and dilute alkalies gives reactions quite different from those of the solution of the substance in dilute neutral saline solutions. By the acid it is converted into what is called acid-albumin, by the alkali into alkali-albumin, both of which bodies we shall have to study later on. 
3. When it is suspended in water and heated it becomes altered in character, coagulated, and all its reactions are changed. It is no longer soluble in dilute neutral saline solutions, not even in dilute acids and alkalies; it has become coagulated proteid, and is now even less soluble than fresh fibrin. When a solution of it in dilute neutral saline solution is similarly heated, a similar change takes place, a precipitate falls down which on examination is found to be coagulated proteid. 'The temperature at which this change takes place is somewhere about $75^{\circ} \mathrm{C}$., though shifting slightly according to the quantity of saline substance present in the solution.

One of the proteids present in blood-serum is paraglobulin, characterized by its solubility in dilute neutral saline solutions, its insolubility in distilled water and concentrated saline solutions, its ready solubility, and at the same time conversion into other bodies, in dilute acids and alkalies, and in its becoming converted into coagulated proteid, and so being precipitated from its solutions at $75^{\circ} \mathrm{C}$.

These reactions are given by a number of proteid bodies forming a group called globulins, the particular globulin present in blood-serum being paraglobulin.

The amount of it present in blood-serum varies in various animals, and apparently in the same animal at different times. In 100 parts by weight of serum there are generally present about 8 or 9 parts of proteids altogether, and of these some 3 or 4 , more or less, may be taken as paraglobulin.

$\S 17$. If the serum from which the paraglobulin has been precipitated by the addition of neutral salt, and removed by filtration, be subjected to dialysis, the salt added may be removed, and a clear, somewhat diluted serum free from paraglobulin may be obtained.

This still gives abundant proteid reactions, so that the serum still contains a proteid, or some proteids still more soluble than the globulins, since they will remain in solution, and are not precipitated, even when dialysis is continued until the serum is practically freed from both the neutral salt added to it and the diffusible salts previously present in the natural serum.

When this serum is heated to $75^{\circ} \mathrm{C}$. a precipitate makes its appearance; the proteids still present are coagulated at this temperature.

We have some reasons for thinking that more than one proteid is present, but they are all closely allied to each other, and we may for the present speak of them as if they were one, and call the proteid left in serum, after removal of the paraglobulin, by the name of albumin, or, to distinguish it from other albumins found elsewhere, serum-albumin. Serum-albumin is distinguished by being more soluble than the globulins, since it is soluble in distilled water, even in the absence of all neutral salts. Like the glubulins, though with much less ease, it is converted by dilute acids and dilute alkalies into acid- or into alkali-albumin. The percentage amount of serum-albumin in serum may be put down as 4 or 5 , more or less, but it varies and sometimes is less abundant than paraglobulin. In some animals (snakes) it is said to disappear during starvation.

The more important characters of the three proteids which we have just studied may be stated as follows:

Soluble in distilled water and in saline sulutions of all strengths . . serum-albumin.

Insoluble in distilled water, readily soluble in dilute saline solutions,

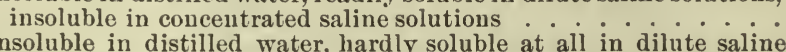

Insoluble in distilled water, hardly soluble at all in dilute saline
solutions, and very little soluble in more concentrated saline solu-

tions........................ fibrin.

Besides paraglobulin and serum-albumin, serum contains a very lärge number of substances, generally in small quantity, which, since they have 
to be extracted by special methods, are called extractives; of these some are nitrogenous, some non-nitrogeuous. Serum contains besides important inorganic saline substances; but to these we shall return.

$\S 18$. With the knowledge which we have gained of the proteids of clotted blood we may go back to the question: Clotting being due to the appearance in blood plasma of a proteid substance, fibrin, which previously did not exist in it as such, what are the causes which led to the appearance of fibrin?

We learn something by studying circumstances which affect the rapidity with which the blood of the same individual clots when shed. These are as follows :

A temperature of $40^{\circ} \mathrm{C}$, which is about or slightly above the temperature of the blood of warm-blooded animals, is perhaps the most favorable to clotting. A further rise of a few degrees is apparently also beneficial, or at least not injurious; but upon a still further rise the effect changes, and when blood is rapidly heated to $56^{\circ} \mathrm{C}$. no clotting at all may take place. At this temperature certain proteids of the blood are coagulated and precipitated before clotting can take place, and with this change the power of the blood to clot is wholly lost. If, however, the heating be not very rapid, the blood may clot before this change has time to come on. When the temperature instead of being raised is lowered below $40^{\circ} \mathrm{C}$. the clotting becomes delayed and prolonged; and at the temperature of $0^{\circ}$ or $1^{\circ} \mathrm{C}$. the blood will remain fluid, and yet capable of clotting when withdrawn from the adverse circumstances, for a very long, it might almost be said, for an indefinite time.

A small quantity of blood shed into a small vessel clots sooner than a large quantity shed into a larger one; and in general the greater the amount of foreign surface with which the blood comes in contact the more rapid the clotting. When shed blood is stirred or "whipped" the fibrin makes its appearance sooner than when the blood is left to clot in the ordinary way; so that here, too, the accelerating influence of contact with foreign bodies makes itself felt. Similarly, movement of shed blood hastens clotting, since it increases the amount of contact with foreign bodies. So also the addition of spongy platinum or of powdered charcoal, or of other inert powders, to tardily clotting blood will, by influence of surface, hasten clotting. Conversely, blood brought into contact with pure oil does not clot so rapidly as when in contact with glass or metal; and blood will continue to flow for a longer time without clotting through a tube smeared inside with oil than through a tube not so smeared. The influence of the oil in such cases is a physical not a chemical one; any pure neutral inert oil will do. As far as we know these influences affect only the rapidity with which the clotting takes place-that is, the rapidity with which the fibrin makes its appearance, not the amount of clot, not the quantity of fibrin formed, though when clotting is very much retarded by cold changes may ensue whereby the amount of clotting which eventually takes place is indirectly affected.

Mere exposure to air exerts apparently little influence on the process of clotting. Blood collected direct from a bloodvessel over mercury so as wholly to exclude the air, clots as readily as blood freely exposed to the air. It is only when blood is much laden with carbonic acid, the presence of which is antagonistic to clotting, that exclusion of air, by hindering the escape of the excess of carbonic acid, delays clotting.

These facts teach us that fibrin does not, as was once thought, make its appearance in shed blood because the blood when shed ceases to share in the movement of the circulation, or because the blood is cooled on leaving the warm body, or because the blood is then more freely exposed to the air; they further suggest the view that the fibrin is the result of some chemical change, the conversion into fibrin of something which is not fibrin, the change like 
other chemical changes being most active at an optimum temperature, and like so many other chemical changes, being assisted by the influences exerted by the presence of inert bodies.

And we have direct experimental evidence that plasma does contain an antecedent of fibrin which, by chemical change, is converted into fibrin.

$\$ 19$. If blood be received direct from the bloodvessels into one-third its bulk of a saturated solution of some neutral salt such as magnesium sulphate, and the two gently but thoroughly mixed, clotting, especially at a moderately low temperature, will be deferred for a very long time. If the mixture be allowed to stand, the corpuscles will sink, and a colorless plasma will be obtained similar to the plasma gained from horse's blood by cold, except that it contains an excess of the neutral salt. The presence of the neutral salt has acted in the same direction as cold; it has prevented the occurrence of clotting. It has not destroyed the fibrin; for if some of the plasma be diluted with from five to ten times its bulk of water, it will clot speedily in quite a normal fashion, with the production of quite normal fibrin.

The separation of the fluid plasma from the corpuscles and from other bodies heavier than the plasma is much facilitated by the use of the centrifugal machine. This consists essentially of a tireless wheel with several spokes, placed in a horizontal position and made to revolve with great velocity (1000 revolutions per minute for instance) around its axis. Tubes of metal or of very strong glass are suspended at the ends of the spokes by carefully adjusted joints. As the wheel rotates with increasing velocity, each tube gradually assumes a horizontal position, bottom outward, without spilling any of its contents. As the rapid rotation continues the corpuscles and heavier particles are driven to the bottom of the tube, and if a very rapid movement he continued for a long time will form a compact cake at the bottom of the tube. When the rotation is stopped the tubes gradually return to their upright position again without anything being spilt, and the clear plasma in each tube can then be decanted off.

If some of the colorless transparent plasma, obtained either by the action of neutral salts from any blood, or by the help of cold from horse's blood, be treated with some solid neutral salt, such as sodium chloride, to saturation, a white flaky, somewhat sticky precipitate will make its appearance. If this precipitate be removed, the fluid no longer possesses the power of clotting (or very slightly so), even though the neutral salt present be removed by dialysis, or its influence lessened by dilution. With the removal of the substance precipitated, the plasma has lost its power of clotting.

If the precipitate itself, after being washed with a saturated solution of the neutral salt (in which it is insoluble) so as to get rid of all serum and other constituents of the plasma, be treated with a small quantity of water, it readily dissolves, ${ }^{1}$ and the solution rapidly filtered gives a clear, colorless filtrate, which is at first perfectly fluid. Soon, however, the fluidity gives way to viscidity, and this in turn to a jelly condition, and finally the jelly shrinks into a clot floating in a clear field; in other words, the filtrate clots like plasma. Thus there is present in cooled plasma, and in plasma kept from clotting by the presence of neutral salts, a something precipitable by saturation with neutral salts-a something which, since it is soluble in very dilute saline solutions, cannot be fibrin itself, but which in solution speedily gives rise to the appearance of fibrin. To this substance its discoverer, Denis, gave the name of plasmine.

The substance thus precipitated is not however a single body, but a mixturo of at least two bodies. If sodium chloride be carefully added to plasma to an extent of about 13 per cent. a white flaky viscid precipitate is thrown

1 The substance itself is not soluble in distilled water, but a quantity of the neutral salt always clings to the precipitate, and thus the addition of water virtually gives rise to dilute saline solution, in which the substance is readily soluble. 
down very much like plasmine. If after the removal of the first precipitate more sodium chloride, and especially if magnesium sulphate be added, a second precipitate is thrown down, less viscid and more granular than the first.

The second precipitate when examined is found to be identical with the paraglobulin, coagulating at $75^{\circ} \mathrm{C}$., which we have already seen to be a constituent of serum.

The first precipitate is also a proteid belonging to the globulin group, but differs from paraglobulin, not only in being more readily precipitated by sodium chloride, and in being when precipitated more viscid, but also in other respects, and especially in being coagulated at a far lower temperature than paraglobulin, viz., at $56^{\circ} \mathrm{C}$. Now, while isolated paraglobulin cannot by any means known to us be converted into fibrin, and as its presence in the so-called plasmine does not seem to be essential to the formation of fibrin out of plasmine, the presence in plasmine of the body coagulating at $56^{\circ} \mathrm{C}$. does seem essential to the conversion of plasmine into fibrin, and we have reason for thinking that it is itself converted, in part at least, into fibrin. Hence it has received the name of fibrinogen.

$\$ 20$. The reasons for this view are as follows:

Besides blood, which clots naturally when shed, there are certain fluids in the body which do not clot naturally, either in the body or when shed, but which by certain artificial means may be made to clot, and in clotting to yield quite normal fibrin.

Thus the so-called serous fluid taken some hours after death ${ }^{1}$ from the pericardial, pleural, or peritoneal cavities, the fluid found in the enlarged serous sac of the testis, known as hydrocele fluid, and other similar fluids, will in the majority of cases, when obtained free from blood or other admixtures, remain fluid almost indefinitely, showing no disposition whatever to clot. $^{2}$ Yet, in most cases at all events, these fluids, when a little blood, or a piece of blood clot, or a little serum is added to them, will clot rapidly and firmly, ${ }^{3}$ giving rise to an unmistakable clot of normal fibrin, differing only from the clot of blood in that, when serum is used, it is colorless, being free from red corpuscles.

Now blood (or blood clot, or serum) contains many things, to any one of which the clotting power thus seen might be attributed. But it is found that in many cases clotting may be induced in the fluids of which we are: speaking by the mere addition, and that even in exceedingly small quantity, of a substance which can be extracted from blood, or from serum, or from blood clot, or even from washed fibrin, or indeed from other sources, a substance whose exact nature is uncertain, it being doubtful whether it is a proteid at all, and whose action is peculiar.

If serum, or whipped blood or a broken-up clot be mixed with a large quantity of alcohol and allowed to stand some days, the proteids present are in time so changed by the alcohol as to become insoluble in water. Hence if the copious precipitate caused by the alcohol, after long standing, be separated by filtration from the alcohol, dried at a low temperature, not exceeding $40^{\circ} \mathrm{C}$., and extracted with distilled water, the aqueous extract contains very little proteid matter, indeed very little organic matter at all. Nevertheless, even a small quantity of this aqueous extract added alone to certain specimens of hydrocele fluid or other of the fluids spoken of above, will bring about a speedy clotting. The same aqueous extract has also a remarkable

1 If it be removed immediately after death it generally clots readily and firmly, giving a colorless clot consisting of fibrin and white corpuscles.

${ }^{2}$ In some specimens, however, a spontaneous coagulation, generally slight, but in exceptional cases massive, may be observed.

${ }^{3}$ In a few cases no coagulation can thus be induced. 
effect in hastening the clotting of fluids which, though they will eventually clot, do so very slowly. Thus plasma may, by the careful addition of a certain quantity of neutral salt and water, be reduced to such a condition that it clots very slowly indeed, taking perhaps days to complete the process. The addition of a small quantity of the aqueous extract we are describing will, however, bring about a clotting which is at once rapid and complete.

The active substance, whatever it be, in this aqueous extract exists in small quantity only, and its clotting virtues are at once and forever lost when the solution is boiled. Further, there is no reason to think that the active substance actually enters into the formation of the fibrin to which it gives rise. It appears to belong to a class of bodies playing an important part in physiological processes, and called ferments, of which we shall have more to say hereafter. We may, therefore, speak of it as the fibrin ferment, the name given to it by its discoverer, Alexander Schnidt.

This fibrin ferment is present in and may be extracted from clotted or whipped blood, and from both the clot ${ }^{1}$ and the serum of clotted blood; and since in most, if not all, cases where blood or blood clot or serum produces clotting in hydrocele or pericardial fluid, an exactly similar clotting may be induced by the mere addition of fibrin ferment, we seem justified in concluding that the clotting virtues of the former are due to the ferment which they contain.

Now, when fibrinogen is precipitated from plasma, as above described, by sodium chloride, redissolved, and reprecipitated, more than once, it may be obtained in solution, by help of a dilute neutral saline solution, in an approximately pure condition, at all events free from other proteids. Such a solution will not clot spontaneously; it may remain fluid indefinitely; and yet on the addition of a little fibrin ferment it will clot readily and firmly, yielding quite normal fibrin.

This body fibrinogen is also present and may be separated out from the specimens of hydrocele, pericardial, and other fluids which clot on the addition of fibrin ferment, and when the fibrinogen has been wholly removed from these fluids they refuse to clot on the addition of fibrin ferment.

Paraglobulin, on the other hand, whether prepared from plasmine by separation of the fibrinogen, or from serum, or from other fluids in which it is found, cannot be converted by fibrin ferment, or indeed by any other means, into fibrin. And fibrinogen isolated, as described above, or serous fluids which contain fibrinogen, can be made, by means of fibrin ferment, to yield quite normal fibrin in the complete absence of paraglobulin. A solution of paraglobulin obtained from serum or blood clot will, it is true, clot pericardial or hydrocele fluids containing fibrinogen, or indeed a solution of fibrinogen, but this is apparently due to the fact that the paraglobulin has in these cases some fibrin ferment mixed with it; it is also possible that, under certain conditions, the presence of paraglobulin may be favorable to the action of the ferment.

When the so-called plasmine is precipitated, as directed in $\$ 19$, fibrin ferment is carried down with the fibrinogen and paraglobulin, and when the plasmine is re-dissolived the ferment is present in the solution and ready to act on the fibrinogen. Hence the re-dissolved plasmine clots spontaneously. When fibrinogen is isolated from plasma by repeated precipitation and solution, the ferment is washed away from it, and the pure ferment-free fibrinogen, ultimately obtained, does not clot spontaneously.

So far it seems clear that there does exist a proteid body, fibrinogen, which may by the action of fibrin ferment be directly, without the interven-

${ }^{1}$ A powerful solution of fibrin ferment may be readily prepared by simply extracting a washed blood clot with a 10 per cent. solution of sodium chloride. 
tion of other proteids, converted into the less soluble fibrin. Our knowledge of the constitution of proteid bodies is too imperfect to enable us to make any very definite statement as to the exact nature of the change thus effected; but we may say this much. Fibrinogen and fibrin have about the same elementary composition, fibrin containing a trifle more nitrogen. When fibrinogen is converted into fibrin by means of fibrin ferment, the weight of the fibrin produced is always less than that of the fibrinogen which is consumed, and there is always produced at the same time a certain quantity of another proteid, belonging to the globulin family. There are reasons, however, why we cannot speak of the ferment as splitting up fibrinogen into fibrin and a globulin; it seems more probable that the ferment converts the fibrinogen first into a body which we might call soluble fibrin, and then turns this body into a veritable fibrin; but further inquiries on the subject are needed:

It may be added that among the conditions necessary for the due action of fibrin ferment on fibrinogen, the presence of a certain quantity of some neutral salt seems to be one. In the total absence of all neutral salts the ferment cannot convert the fibrinogen into fibrin. There are some reasons also for thinking that the presence of a lime salt, such as calcium sulphate, though it may be in minute quantity only, is essential. If the calcium salts are taken out of fibrinogen, the fibrinogen no longer coagulates upon the addition of fibrin ferment free from lime salts. Oxalate of potassium added to freshly drawn blood also prevents coagulation, apparently because of the precipitation of the lime salts; the addition now of calcium salts is promptly followed by coagulation.

\$21. We may conclude, then, that the plasma of blood when shed, or, at all events, soon after it has been shed, contains fibrinogen; and it also seems probable that the clotting comes about because the fibrinogen is converted into fibrin by the action of fibrin ferment; but we are still far from a definite answer to the question, why blood remains fluid in the body and yet clots when shed?

We have alrearly said that blood, or blood plasma, brought up to a temperature of $56^{\circ} \mathrm{C}$. as soon as possible after its removal from the living blondvessels, gives a proteid precipitate and loses its power of clotting. This may be taken to show that blood, as it circulates in the living bloodvessels, contains fibrinogen as such, and that when the blood is heated to $56^{\circ} \mathrm{C}$, which is the coagulating point of fibrinogen, the fibrinogen present is coagulated and precipitated, and consequently no fibrin can be formed.

Further, while clotted blood undoubtedly contains an abundance of fibrin ferment, no ferment, or a minimal quantity only, is present in blood as it leaves the bloodvessels. If the blood be received directly from the bloodvessels into alcohol, the aqueous extract prepared as directed above contains no ferment, or merely a trace. Apparently the ferment makes its appearance in the blood as the result of changes taking place in the blood after it has been shed.

We might from this be inclined to conclude that blond clots when shed, but not before, because, fibrinogen being always present, the shedding brings about changes which produce fibrin ferment, not previously existing, and this acting on the fibrinogen gives rise to fibrin. But we meet with the following difficulty: A very considerable quantity of very active ferment may be injected into the blood-current of a living animal without necessarily producing any clotting at all. Obviously either blood within the bloodvessels does not contain fibrinogen as such, and the fibrinogen detected by heating the blood to $56^{\circ} \mathrm{C}$. is the result of changes which have already ensued before the temperature is reached; or in the living circulation there 
are agencies at work which prevent any ferment which may be introduced into the circulation from producing its usual effect on fibrinogen; or there are agencies at work which destroy, or do away with the fibrin, little by little, as it is formed.

$\$ 22$. And indeed, when we reflect how complex blood is and the many and great changes of which it is susceptible, we shall not wonder that the question we are putting cannot be offered off hand.

The corpuscles with which blood is crowded are living structures, and consequently are continually acting upon and being acted upon by the plasma. The red corpuscles it is true are, as we shall see, peculiar bodies, with a restricted life and a very specialized work, and possibly their influence on the plasma is not very great; but we have reason to think that the relations between the white corpuscles and the plasma are close and important.

Then again the blood is not only acting upon and being acted upon by the several tissues as it flows through the various capillaries, but along the whole of its course through the heart, arteries, capillaries, and veins, is acting upon and being acted upon by the vascular walls, which like the rest of the body are alive, and being alive are continually undergoing and promoting change.

That relations of some kind, having a direct influence on the clotting of blood, do exist between the blood and the vascular walls is shown by the following facts:

After death, when all motion of the blood has ceased, the blood remains for a long time fluid. It is not until some time afterward, at an epoch when post-mortem changes in the blood and in the bloodvessels have had time to develop themselves, that clotting begins. 'Thus some hours after death the blood in the great veins may be found still perfectly fluid. Yet such blood has not lost its power of clotting; it still clots when removed from the body, and clots too when received over mercury without exposure to air, showing that, though the blood, being highly venous, is rich in carbonic acid and contains little or no oxygen, its fluidity is not due to any excess of carbonic acid or absence of oxygen. Eventually it does clot even within the vessels, but perhaps never so firmly and completely as when shed. It clots first in the larger vessels, but remains fluid in the smaller vessels for a very long time, for many hours in fact, since in these the same bulk of blood is exposed to the influence of, and reciprocally exerts an influence on, a larger surface of the vascular walls than in the larger vessels. And if it be urged that the result is here due to influences exerted by the body at large, by the tissues as well as by the vascular walls, this objection will not hold good against the following experiment.

If the jugular vein of a large animal, such as an ox or horse, be carefully ligatured when full of blood, and the ligatured portion excised, the blood in many cases remains perfectly fluid, along the greater part of the length of the piece, for twenty-four or even forty-eight hours. The piece so ligatured may be suspended in a framework and opened at the top so as to imitate a living test-tube, and yet the blood will often remain long fluid, though a portion removed at any time into a glass or other ressel will clot in a few minutes. If two such living test-tubes be prepared, the blood may be poured from one to the other without clotting taking place.

A similar relation of the fluid to its containing living wall is seen in the case of those serous fluids which clot spontaneously. If, as soon after death as the body is cold and the fat is solidified, the pericardium be carefully removed from a sheep by an incision round the base of the heart, the pericardial fluid (which, as we have already seen, during life and some little 
time after death, possesses the power of clotting) may be kept in the pericardial bag as in a living cup for many hours without clotting, and yet a small portion removed with a pipette clots at once.

This relation between the blood and the vascular wall may be disturbed or overridden : clotting may take place or may be induced within the living bloodvessel. When the living membrane is injured, as when an artery or vein is sharply ligatured, or when it is diseased, as for instance in aneurism, a clot is apt to be formed at the injured or diseased spot; and in certain morbid conditions of the body clots are formed in various vascular tracts. Absence of motion, which in shed blood as we have seen is unfavorable to clotting, is apt within the body to lead to clotting. 'Thus, when an artery is ligatured, the blood in the tract of the artery on the cardiac side of the ligature, between the ligature and the branch last given off by the artery, ceasing to share in the circulation, remains motionless or nearly so, and along this tract a clot forms, firmest next to the ligature and ending near where the branch is given off; this perhaps may be explained by the fact that the walls of the tract suffer in their nutrition by the stagnation of the blood, and that consequently the normal relation between them and the contained blood is disturbed.

That the blood within the living bloodvessels, though not actually clotting under normal circumstances, may easily be made to clot, that the blood is always on the point of clotting, is shown by the fact that a foreign body such as a needle thrust into the interior of a bloodressel or a thread drawn through and left in a bloodvessel, is apt to become covered with fibrin. Some influence exerted by the needle or thread, whatever may be the character of that influence, is sufficient to determine a clotting, which otherwise would not have taken place.

The same instability of the blood, as regards clotting, is strikingly shown, in the case of the rabbit, by the result of injecting into the bloodvessels a small quantity of a solution of a peculiar proteid, prepared from certain structures such as the thymus body. Massive clotting of the blood in almost all the bloodvessels, small and large, takes place with great rapidity, leading to the sudden death of the animal. In contrast to this effect may be mentioned the result of injecting into the bloodvessels of a dog a quantity of a solution of a body called albumose, of which we shall hereafter have to treat as a product of the digestion of proteid substances, to the extent of 0.3 gramme per kilo of body weight. So far from producing clotting, the injected albumose has such an effect on the blood that for several hours after the injection shed blood will refuse to clot of itself and remain quite fluid, though it can be made to clot by special treatment.

$\$ 23$. All the foregoing facts tend to show that the blood as it is flowing through the healthy blondvessels is, as far as clotting is concerned, in a state of unstable equilibrium, which may at any moment be upset, even within the bloodvessels, and which is upset directly the blood is shed, with clotting as a result. Our present knowledge does not permit us to make an authoritative statement as to the exact nature of this equilibrium. There are reasons, however, for thinking that the white corpuscles play an important part in the matter. Wherever clotting occurs naturally, white corpuscles are present; and this is true not only of blood but also of such specimens of pericardial or other serous fluids as clot naturally. And many arguments which we cannot enter upon here, may be adduced all pointing to the same conclusion, that the white corpuscles play an important part in the process of clotting. But it would lead us too far into controversial matters to attempt to define what that part is, or to explain the exact nature of the equilibrium of which we have spoken. 
What we do know is that in blood soon after it has been shed the body which we have called fibrinogen is present, as also the body which we have called fibrin ferment, that the latter acting on the former will produce fibrin, and that the appearance of fibrin is undoubtedly the cause of what is called clotting. We seem justified in concluding that the clotting of shed blood is due to the conversion by ferment of fibrinogen into fibrin. The further inference that clotting within the body is the same thing as clotting outside the body, and similarly due to the transformation of fibrinogen by ferment into fibrin, though probable, is not proved. We' do not yet know the exact nature and condition of the blood within the living bloodvessels, and until we know that we cannot satisfactorily explain why blood in the living bloodvessels is usually fluid but can at times clot.

\section{The Corpuscles of the Blood.}

\section{The Red Corpuscles.}

$\S 24$. The redness of blood is due exclusively to the red corpuscles. The plasma as seen in thin layers within the living blondvessels appears colorless, as does also a thin layer of serum; but a thick layer of serum (and probably of plasma) has a faint yellowish tinge due, as we have said, to the presence of a small quantity of a special pigment.

The corpuscles appear under the microscope as fairly homogeneous, imperfectly translucent biconcave dises with a diameter of 7 to $8 \mu$ and a

[FIG. 5.

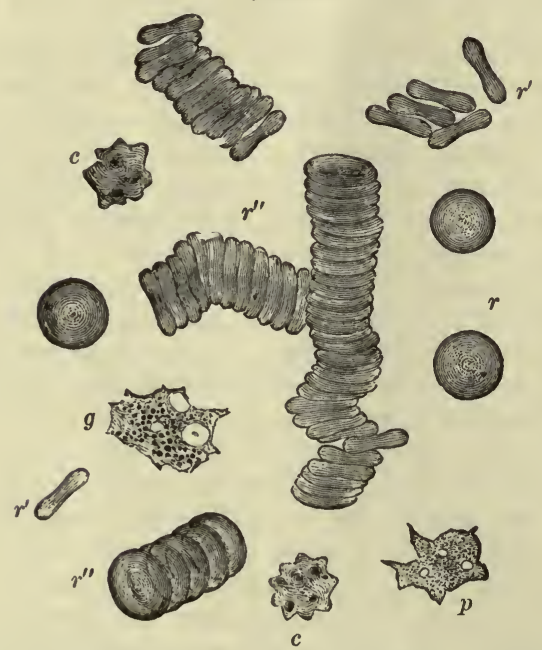

FIG. 6.
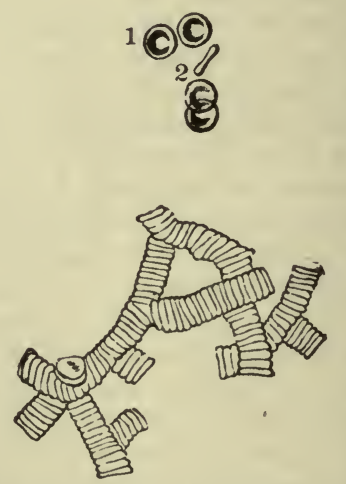

Fig. 5.-Human Blood as seen on the Warm Stage (magnified about 1200 dianieters): $r, r$, single red corpuscles seen lying flat: $r^{\prime}, r^{\prime}$, red corpuscles on their edge and viewed in profile ; $r^{\prime \prime}$, red corpuscles arranged in roùleaux; $c, c$, crenate red corpuscles; $p$, a finely granular pale corpuscle; $g$, a coarsely granular pale corpuscle. Both have two or three distinct vacuoles, and were undergoing changes of shape at the moment of observation ; in $g$ a nucleus also was visible.

Fig. 6.-Human Red Corpuscles Lying Singly and Collected into Rolls. (As seen under an ordinary high power of the microscope.)]

thickness of 1 to $2 \mu$. Being discs they are circular in outline when seen on the flat, but rod-shaped when seen in profile. [Fig. 5.] Being biconcave, with a thicker rounded rim surrounding a thinner centre, the rays of 
light in passing through them, when they are exanined by transmitted light, are more refracted at the rim than in the centre. 'The effect of this is that, when viewed at what may be considered the proper focus, the centre of a corpuscle appears clear, while a slight opacity marks out indistinctly the inner margin of the thicker rim, whereas, when the focus is shifted either up or down, the centre becomes dark and the rest of the corpuscle clear. Any body of the same shape, and composed of substances of the same refractive power, would produce the same optical effects. Otherwise the corpuscle appears homogeneous, without distinction of parts and without a nucleus. A single corpuscle seen by itself has a very faint color, looking yellow rather than red, but when several corpuscles lie one upon the top of the other the mass is distinctly red.

The red corpuscle is elastic, in the sense that it may be deformed by pressure or traction, but when the pressure or traction is removed regains its previous form. Its shape is also much influenced by the physical conditions of the plasma, serum, or fluid in which for the time being it is. If the plasma or serum be diluted with water, the disc, absorbing water, swells up into a sphere [Fig. 7], becoming a disc again on the removal of the dilution. If the serum be concentrated, the disc, giving out water, shrinks irregularly and assumes various forms; one of these forms is that of a number of blunted protuberances projecting all over the surface of the corpuscle, which is then said to be crenate; in a drop of blood examined under the microscope, crenate corpuscles are often seen at the edge of the cover-slip where evaporation is leading to concentration of the plasma, or, as it should then perhaps rather be called, serum. In blood just shed the red corpuscles are apt to adhere to each other by their

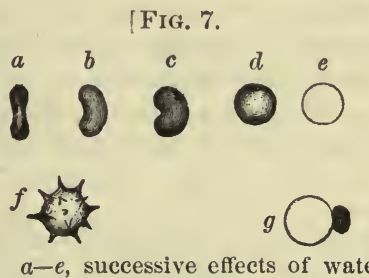

$a-e$, successive effects of water upon a red corpuscle; $f$, effect of solution of salt, crenated; $g$, effect of tannic acid.] flat surfaces, much more than to the glass or other surface with which the blood is in contact, and hence arrange themselves in rolls. 'This tendency, however, to form rolls very soon diminishes after the blood is shed.

Though a single corpuscle is somewhat translucent, a comparatively thin layer of blood is opaque; type, for instance, cannot be read through even a thin layer of blood.

When a quantity of whipped blood (or blood otherwise deprived of fibrin) is frozen and thawed several times it changes color, becoming of a darker hue, and is then found to be much more transparent, so that type can now be easily read through a moderately thin layer. It is then spoken of as laky blood. The same change may be effected by shaking the blood with ether, or by adding a small quantity of bile salts, and in other ways. Upon examination of laky blood it is found that the red corpuscles are "broken up" or at least altered, and that the redness which previously was confined to them is now diffused through the serum. Normal bloor is opaque because each corpuscle, while permitting some rays of light (chiefly red) to pass through, reflects many others, and the brightness of the hue of normal blood is due to this reflection of light from the surfaces of the several corpuscles. Laky blood is transparent because there are no longer intact corpuscles to present surfaces for the reflection of light, and the darker hue of laky blood is similarly due to the absence of reflection from the several corpuscles.

When laky blood is allowed to stand a sediment is formed (and may be separated by the centrifugal machine) which on examination is found to consist of discs, or fragments of discs, of a colorless substance exhibiting 
under high powers an obscurely spongy or reticular structure. These colorless, thin discs seen flatwise often appear as mere rings. The substance composing them stains with various reagents and may thus be made more evident.

The red corpuscle, then, consists obviously of a colorless framework, with which in normal conditions a red coloring matter is associated; but by various means the coloring matter may be driven from the framework and dissolved in the serum.

The framework is spoken of as stroma; it is a modified or differentiated protoplasm, and upon chemical analysis yields proteid substances, some of them at least belonging to the globulin group, and other matters, among which is a peculiar complex fat called lecithin, of which we shall have to speak in treating of nervous tissue.

The red coloring matter which in normal conditions is associated with this stroma is called homoglobin, and may by proper methods be split up into a proteid belonging to the globulin group, and into a colored pigment, containing iron, called hamatin. Hæmoglobin is, therefore, a very complex body. It is found to have remarkable relations to oxygen, and indeed, as we shall see, the red corpuscles by virtue of their hæmoglobin have a special work in respiration; they carry oxygen from the lungs to the several tissues. IVe shall therefore defer the further study of hæmoglobin until we have to deal with respiration.

The red corpuscle, then, consists of a disc of colorless stroma with which is associated in a peculiar way the complex colored body hremoglobin. Though the hremoglobin, as is seen in laky blood, is readily soluble in serum (and it is also soluble in plasma), in the intact normal blood it remains confined to the corpuscle; obviously there is some special connection between the stroma and the hrmoglobin ; it is not until the stroma is altered, we may perhaps say killed (as by repeated freezing and thawing), that it loses its holi on the hæmoglobin, which thus set free passes into solution in the serum. The disc of stroma when separated from the hæmoglobin has, as we have just said, an obscurely spongy texture; but we do not know accurately the exact condition of the stroma in the intact corpuscle or how it holds the hæmoglobin. There is certainly no definite membrane or envelope to the corpuscle, for by exposing blood to a high temperature, $60^{\circ} \mathrm{C}$., the corpuscle will break up into more or less spherical pieces, each still consisting of stroma and hæmoglobin.

The quantity of stroma necessary to hold a quantity of hæmoglobin is exceedingly small. Of the total solid matter of a corpuscle more than 90 per cent. is hæmoglobin. A red corpuscle in fact is a quantity of hæmoglobin held together in the form of a disc by a minimal amount of stroma. Hence whatever effect the stroma per se may have upon the plasma, this, in the case of mammals at all events, must be insignificant; the red corpuscle is practically simply a carrier of hæmoglobin.

$\$ 25$. The average number of red corpuscles in human blood may be probably put down at about 5 millions in a cubic millimetre (the range in different mammals is said to be from 3 to 18 millions), but the relation of corpuscle to plasma varies a great deal even in health, and very much in disease. Obviously the relation may be affected (1) by an increase or decrease of the plasma, (2) by an actual decrease or increase of red corpuscles. Now, the former must frequently take place. The blood, as we have already urged, is always being acted upon by changes in the tissues and, indeed, is an index of those changes; hence the plasma must be continually changing, though always striving to return to the normal condition. Thus when a large quantity of water is discharged by the kidney, the 
skin or the bowels, that water comes really from the blood, and the drain of water must tend to diminish the bulk of the plasma, and so to increase the relative number of red corpuscles, though the effect is probably soon remedied by the passage of water from the tissues into the blood. So again when a large quantity of water is drunk, this passes into the blood and tends temporarily to dilute the plasma (and so to diminish the relative number of red corpuscles), though this condition is in turn soon remedied by the passage of the superfluous fluid to the tissues and excretory organs. The greater or less number of red corpuscles, then, in a given bulk of blood may be simply due to less or more plasma, but we have reason to think that the actual number of the corpuscles in the blood does vary from time to time. This is especially seen in certain forms of disease which may be spoken of under the general term of anæmia (there being several kinds of anæmia), in which the number of red corpuseles is distinctly diminished.

The redness of blood nay, however, be influenced not only by the number of red corpuscles in each cubic millimetre of blood, but also by the amount of hæmoglobin in each corpuscle, and to a less degree by the size of the corpuscles. If we compare, with a common standard, the redness of two specimens of blood unequally red, and then determine the relative number of corpuscles in each, we may find that the less red specimen has as many corpuscles as the redder one, or at least the deficiency in redness is greater than can be accounted for by the paucity of red corpuscles. Obviously, in such a case, the red corpuscles have too little hæmoglobin. In some cases of anæmia the deficiency of hæmoglobin in each corpuscle is more striking than the scantiness of red corpuscles.

The number of corpuscles in a specimen of blood is determined by mixing a small but carefully measured quantity of the blood with a large quantity of some

[FIG. 8.

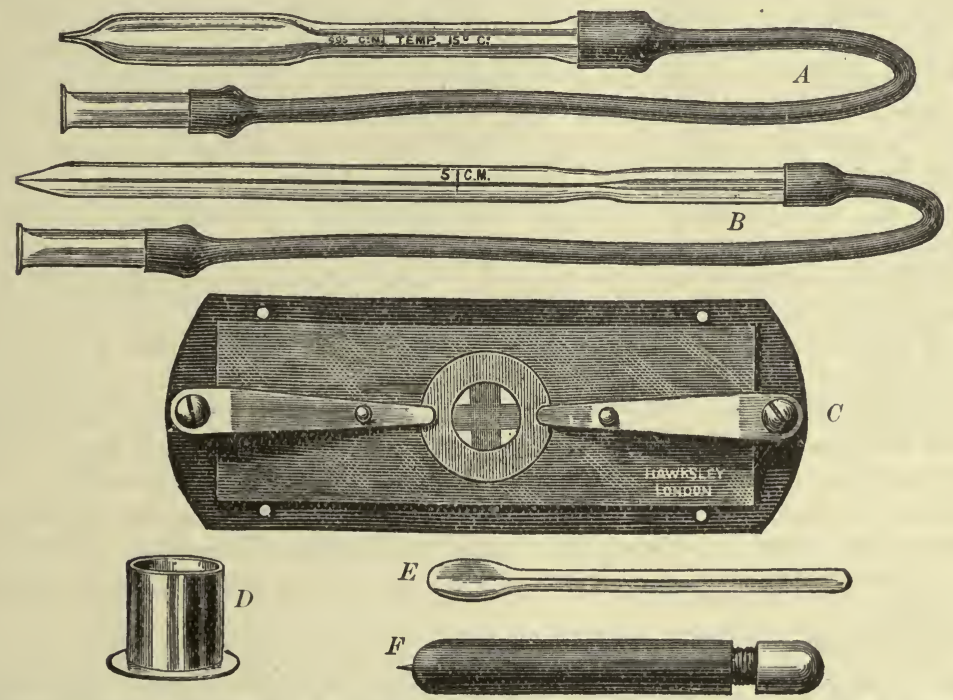

Hæmacytometer of Gowers: $A$, pipette for measuring the diluting solution ; $B$, capillary tube for measuring blood; $C$, cell with divisions on slide, corer-glass and springs; $D$, vessel to mix solutions; $E$, mixer; $F$, guarded spear-pointed needle for sticking finger.]

indifferent fluid, e. g., a 5 per cent. solution of sodium sulphate, and then actually counting the corpuscles in a known minimal bulk of the mixture. 
This, perhaps, may be most conveniently done by the method generally known as that of Gowers (Hremacytometer) [Fig. 8], improved by Malassez. A glass slide, in a metal frame, is ruled into minute rectangles, $e . g$. $\frac{1}{4} \mathrm{~mm}$. by $\frac{1}{5} \mathrm{~mm}$., so as to give a convenient area of $\frac{1}{20}$ of a square $\mathrm{mm}$. Three small screws in the frame permit a coverslip to be brought to a fixed distance, e. $g . \frac{1}{5} \mathrm{~mm}$., from the surface of the slide. The blood having been diluted, e. g. to 100 times its volume, a small quantity of the diluted (and thoroughly mixed) blood, sufficient to oceupy fully the space between the coverslip and the glass slide when the former is brought to its proper position, is placed on the slide, and the coverslip brought down. The volume of diluted blood now lying over each of the rectangles will be $\frac{1}{100}\left(\frac{1}{20} \times \frac{1}{5}\right)$ of a cubic mm.; and if, when the corpuseles have subsided, the number of corpuscles lying within a rectangle be counted, the result will give the number of corpuscles previously distributed through $\frac{1}{100}$ of a cubic $\mathrm{mm}$. of the diluted blood. This multiplied by 100 will give the number of corpuseles in 1 cubic mm. of the diluted blood, and again multiplied by 100 the number in 1 eubic $\mathrm{mm}$. of the entire blood. It is advisable to count the number of corpuscles in several of the rectangles, and to take the average. For the convenience of counting, each rectangle is subdivided into a number of very small squares, $e . g$. into 20 , each with a side of $\frac{1}{20} \mathrm{~mm}$., and so an area of $\frac{1}{400}$ of a square $\mathrm{mm}$.

Since the actual number of red corpuscles in a specinen of blood (which may be taken as a sample of the whole blood) is sometimes more, sometimes less, it is obvious that either red corpuscles may be temporarily withdrawn from and returned to the general blood current, or that certain red corpuscles are, after a while, made away with, and that new ones take their place. We have no satisfactory evidence of the former being the case in normal conditions, whereas we have evidence that old corpuscles do die and that new ones are born.

$\$ 26$. 'The red corpuscles, we have already said, are continually engaged in carrying oxygen, by means of their hæmoglobin, from the lungs to the tissues; they load themselves with oxygen at the lungs and unload at the tissues. It is extremely unlikely that this act should be repeated indefinitely without leading to changes which may be familiarly described as wear and tear, and which would ultimately lead to the death of the corpuscles.

We shall have to state later on that the liver discharges into the alimentary canal, as a constituent of bile, a considerable quantity of a pigment known as bilirubin, and that this substance has remarkable relations with, and, indeed, may be regarded as a derivative of homatin, which, as we have seen $(\$ 24)$, is a product of the decomposition of hrmoglobin. It appears probable, in fact, that the bilirubin of bile (and this as we shall see is the chief biliary pigment, and the source of the other biliary pigments) is not formed wholly anew in the body, but is manufactured in some way or other out of hæmatin derived from hæmoglobin. This must entail a daily consumption of a considerable quantity of hæmoglobin, and since we know no other source of hæmoglobin besides the red corpuscles, and have no evidence of red corpuscles continuing to exist after having lost their hæmoglobin, must, therefore, entail a daily destruction of many red corpuscles.

Even in health, then, a number of red corpuscles must be continually disappearing; and in disease the rapid and great diminution which may take place in the number of red corpuscles shows that large destruction may occur.

We cannot at present accurately trace out the steps of this disappearance of red corpuscles. In the spleen pulp, red corpuscles have been seen in various stages of disorganization, some of them lying within the substance of large colorless corpuscles, and as it were being eaten by them. There is also evidence that destruction takes place in the liver itself, and, indeed, elsewhere. But the subject has not yet been adequately worked out.

$\$ 27$. This destruction of red corpuscles necessitates the birth of new 
corpuscles, to keep up the normal supply of hremoglobin ; and, indeed, the cases in which after even great loss of blond by hemorrhage a healthy ruddiness returns, and that often rapidly, showing that the lost corpuscles have been replaced, as well as the cases of recovery from the disease anæmia, prove that red corpuscles are, even in adult life, born somewhere in the body.

In the adult, as in the embryo, the red corpuscles appear to be formed out of preceding colored nucleated cells.

In the interior of bones is a peculiar tissue called marrow, which, in most parts being very full of bloodvessels, is called red marrow. In this red marrow the capillaries and minute veins form an intricate labyrinth of relatively wide passages with very thin walls, and through this labyrinth the flow of blood is comparatively slow. In the passages of this labyrinth are found colored nucleated cells, that is to say, cells the cell substance of which has undergone more or less differentiation into hæmoglobin and stroma. And there seems to be going on in red marrow a multiplication of such colored nucleated cells, which become transformed into red non-nucleated dises, that is, into ordinary red corpuscles, and pass into the general blood current. In other words, a formation of red corpuscles, not wholly unlike that which takes place in the embryo, is in the adult continually going on in the red marrow of the bones.

A similar formation of red corpuscles has also been described, though with less evidence, as taking place in the spleen, especially under particular circumstances, such as after great loss of blood.

The formation of red corpuscles is, therefore, a special process, taking place in special regions; we have no satisfactory evidence that the ordinary white corpuscles of the blood are, as they travel in the current of the circulation, transformed into red corpuscles.

The red corpuscles, then, to sum up, are useful to the body on account of the hæmoglobin, which constitutes so nearly the whole of their solid matter. What functions the stroma may have besides the mere, so to speak, mechanical one of holding the hæmoglobin in the form of a corpuscle we do not know. The primary use of the hæmoglobin is to carry oxygen from the lungs to the tissues, and it would appear that it is advantageous to the economy that the hæmoglnbin should be as it were bottled up in corpuscles rather than simply diffused through the plasma. How long a corpuscle may live carrying oxygen we do not exactly know ; the red corpuscles of one animal, e.g., a bird, injected into the vessels of another, e. g., a mammal, disappear within a few days; but this affords no measure of the life of a corpuscle in its own home. Eventually, however, the red corpuscle dies, its place being supplied by a new one. The hæmoglobin set free from the dead corpuscles appears to have a secondary use in forming the pigment of the bile and possibly other pigments.

\section{The White or Colorless Corpuscles.}

$\S 28$. The white corpuscles are far less numerous than the red; a specimen of ordinary healthy blood will contain several hundred red corpuscles to each white corpuscle, though the proportion, even in health, varies considerably under different circumstances, ranging from 1 in 300 to 1 in 700 . But though less numerous, the white corpuscles are probably of greater importance to the blood itself than are the red corpuscles; the latter are chiefly limited to the special work of carrying oxygen from the lungs to the tissues, while the former probably exert a consilerable influence on the blood plasma itself, and help to maintain it in a proper condition. 
When seen in a normal condition, and "at rest," the white corpuscle is a small, spherical, colorless mass, varying in size, but with an average diameter of about $10 \mu$, and presenting generally a finely but sometimes a coarsely granular appearance. [Fig. 9.] The surface, even when the corpuscle is

[Fig. 9.

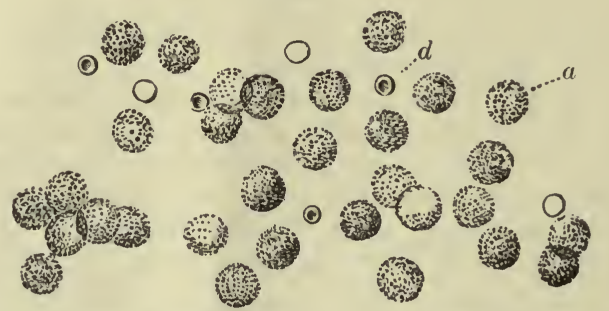

$a$, white corpuscles of human blood; $d$, red corpuscles (high power).]

perfectly at rest, is not absolutely smooth and even, but somewhat irregular, thereby contributing to the granular appearance; and at times these irregularities are exaggerated into protuberances or "pseudopodia" of varying size or form, the corpuscle in this way assuming various forms without changing its bulk, and by the assumption of a series of forms shifting its place. Of these "amoboid movements," as they are called, we shall have to speak later on.

In carrying on these amœboid movements the corpuscle may transform itself from a spherical mass into a thin, flat, irregular plate; and when this occurs there may be seen at times in the midst of the extended finely granular mass or cell body, a smaller body of different aspect and refractive power, the nucleus. The normal presence of a nucleus in the white corpuscles may also be shown by treating the corpuscle with dilute acetic acid, which swells up and renders more transparent the cell body but makes the nucleus more refractive and more sharply defined, and so more conspicuous, or by the use of staining reagents, the majority of which stain the nucleus more readily and more deeply than the cell body. In what perhaps may be considered a typical white corpuscle, the nucleus is a spherical mass about $2-3 \mu$ in diameter, but it varies in size in different corpuscles, and not unfrequently is irregular in form, at least after the action of reagents. It occasionally appears as if about to divide into fragments, and sometimes a corpuscle may contain two or even more (then generally small) nuclei. Though staining readily with staining reagents, the nucleus of an ordinary white corpuscle does not show the nuclear network which is so characteristic, as we shall see, of the nuclei of many cells, and which in these is the part of the nucleus which especially stains; in the closely allied lymph corpuscles, to which we shall have immediately to refer, a nuclear network is present.

The cell body of the white corpuscle may be taken as a good example of what we have called undifferentiated protoplasm. Optically, it consists of a uniformly transparent but somewhat refractive material or basis, in which are imbedded minute particles, generally spherical in form, and in which sometimes occur minute vacuoles filled with fluid; it is rarely, if ever, that any distinct network, like that which is sometimes observed in other cells, can be seen in the cell body of a white corpuscle whether stained or not. The imbedded particles are generally very small, and for the most part distributed uniformly over the cell body, giving it a finely granular aspect; sometimes, however, the particles are relatively large, making the corpuscles coarsely granular, the coarse granules being frequently confined to one or another 
part of the cell body. These particles or granules, whether coarse or fine, vary in nature; some of them, as shown by their greater refractive power, their staining with osmic acid, and their solution by solvents of fat, are fatty in nature; others may similarly be shown by their reactions to be proteid in nature.

The material in which these granules are imbedded, and which forms the greater part of the cell body, has no special optical features; so far as can be ascertained. it appears under the microscope to be homogeneous; no definite structure can be detected in it. It must be borne in mind that the whole corpuscle consists largely of water, the total solid matter amounting to not much more than 10 per cent. The transparent material of the cell body must, therefore, be in a condition which we may call semifluid, or semisolid, without being called upon to define what we exactly mean by these terms. This approach to fluidity appears to be connected with the great mobility of the cell body, as shown in its amoboid movements.

$\$ 29$. When we submit to chemical examination a sufficient mass of white corpuscles, separated out from the blood by special means and obtained tolerably free from red corpuscles and plasma (or apply to the white bloodcorpuscles the chemical results obtained from the more easily procured lymphcorpuscles, which, as we shall see, are very similar to, and, indeed, in many ways related to the white corpuscles of the blood), we find that this small solid matter of the corpuscle consists largely of certain proteids.

One of these proteids is a body either identical with, or closely allied to, the proteid called myosin, which we shall have to study more fully in connection with muscular tissue. At present we may simply say that myosin is a body intermediate between fibrin and globulin, being less soluble than the latter and more soluble than the former; thus while it is hardly at all soluble in a 1 per cent. solution of sodium chloride or other neutral salt, it is, unlike fibrin, speedily and wholly dissolved by a 10 per cent. solution. Myosin is further interesting because, as we shall see, just as fibrin is formed in the clotting of blood from fibrinogen, so myosin is formed out of a preceding myosinogen, during a kind of clotting which takes place in muscular fibre and which is spoken of as rigor mortis. And we have reasons for thinking that in the living white blood-corpuscle there does exist a body identical with or allied to myosinogen, which we may speak of as being in a fluid condition; and which on the death of the corpuscle is converted, by a kind of clotting, into myosin, or into an allied body which, being solid, gives the body of the corpuscle a stiffness and rigidity which it did not possess during life.

Besides this myosin or myosin-like proteid, the white corpuscles also contain either paraglobulin itself or some other member of the globulin group, as well as a body or bodies like or identical with serum-albumin.

In addition, there is present, in somewhat considerable quantity, a substance of a peculiar nature, which since it is confined to the nuclei of the corpuscles, and further seems to be present in all nuclei, has been called nuclein. This nuclein, which though a complex nitrogenous body is very different in composition and nature from proteids, is remarkable on the one hand for being a very stable inert body, and on the other for containing a large quantity (according to some observers nearly 10 per cent.) of phosphorus, which appears to enter more closely into the structure of the molecule than it does in the case of proteids.

Next in importance to the proteids, as constant constituents of the white corpuscles, come certain fats. Among these the most conspicuous is the complex fatty body lecithin.

In the case of many corpuscles at all events we have evidence of the 
presence of a member of the large group of carbohydrates, comprising starches and sugar, viz., the starch-like body glycogen, which we shall have to study more fully hereafter. This glycogen may exist in the living corpuscle as glycogen, but it is very apt, after the death of the corpuscles, to become changed by hydration into some form of sugar, such as maltose or dextrose.

Lastly, the ash of the white corpuscles is characterized by containing a relatively large quantity of potassium and of phosphates and by being relative poor in chlorides and in sodium. But in this respect the corpuscle is merely an example of what seems to be a general rule (to which, however, there may be exceptions) that while the elements of the tissues themselves are rich in potassium and phosphates, the blood plasma or lymph on which they live abounds in chlorides and sodium salts.

$\$ 30$. In the broad features above mentioned, the white blood-corpuscle may be taken as a picture and example of all living tissues. If we examine the histological elements of any tissue, whether we take an epithelium cell, or a nerve cell, or a cartilage cell, or a muscular fibre, we meet with very similar features. Studying the element morphologically, we find a nucleus ${ }^{1}$ and a cell body, the nucleus having the general characters described above with frequently other characters introduced, and the cell body consisting of at least more than one kind of material, the materials being sometimes so disposed as to produce the optical effect simply of a transparent mass in which granules are imbedded, in which case we speak of the cell body as protoplasmic, but at other times so arranged that the cell body possesses differentiated structure. Studying the element from a chemical point of view, we find proteids always present, and among these bodies identical with or more or less closely allied to myosin, we generally have evidence of the presence also of fat of some kind and of some member or members of the carbohydrate group, and the ash always contains potassium and phosphates, with sulphates, chlorides, sodium, and calcium, to which may be added magnesium and iron.

We stated in the Introduction that living matter is always undergoing chemical change; this continued chemical change we may denote by the term metabolism. We further urged that as long as living matter is alive, the chemical change or metabolism is of a double kind. On the one hand, the living substance is continually breaking down into simpler bodies, with a setting free of energy; this part of the metabolism we may speak of as made up of katabolic changes. On the other hand, the living substance is continually building itself up, embodying energy into itself and so replenishing its store of energy; this part of the metabolism we may speak of as made up of anabolic changes. IVe also urged that in every piece of living tissue there might be (1) the actual living substance itself, (2) material which is present for the purpose of becoming, and is on the way to become living substance-that is to say, food undergoing or about to undergo anabolic changes, and (3) material which has resulted from, or is resulting from, the breaking down of the living substance-that is to say, material which has undergone or is undergoing katabolic changes, and which we speak of as waste. In using the word "living substance," however, we must remember that in reality it is not a substance in the chemical sense of the word, but material undergoing a series of changes.

If, now, we ask the question, which part of the body of the white corpuscle (or of a similar element of another tissue) is the real living substance, and which part is food or waste, we ask a question which we cannot as yet definitely answer. We have at present no adequate morphological criteria

1 The existence of multinuclear structures does not affect the present argument. 
to enable us to judge, by optical characters, what is really living and what is not.

The material which appears in the cell body in the form of distinct granules, merely lodged in the more transparent material, cannot be part of the real living substance; it must be either food or waste. Many of these granules are fat, and we have at times an opportunity of observing that they have been introduced into the corpuscle from the surrounding plasma. The white corpuscle, as we have said, has the power of executing amoboid movements; it can creep around objects, envelope them with its own substance, and so put them inside itself. 'The granules of fat thus introduced may be subsequently extruded or may disappear within the corpuscle; in the latter case they are obviously changed, and apparently made use of by the corpuscle. In other words, these fatty granules are apparently food material on their way to be worked up in the living substance of the corpuscle.

But we have also evidence that similar granules of fat may make their appearance wholly within the corpuscle; they are products of the activity of the corpuscle. We have further reason to think that in some cases, at all events, they arise from the breaking down of the living substance of the corpuscle, that they are what we have called waste products.

But all the granules visible in a corpuscle are not necessarily fatty in nature; some of them may undoubtedly be proteid granules, and it is possible that some of them may at times be of carbohydrate or other nature. In all cases, however, they are either food material or waste products. And what is true of the easily distinguished granules is also true of other substances, in solution or in a solid form, but so disposed as not to be optically recognized.

Hence a part, and it may be no inconsiderable part, of the white corpuscle, may be not living substance at all, but either food or waste. Further, it does not necessarily follow that the whole of any quantity of material, fatty or otherwise, introduced into the corpuscle from without, should actually be built up into and so become part of the living substance; the changes from raw food to living substance are, as we have already said, probably many, and it may be that after a certain number of changes, few or many, part only of the material is accepted as worthy of being made alive, and the rest, being rejected, becomes at once waste matter; or the material may, even after it has undergone this or that change, never actually enter into the living substance, but all become waste matter. We say waste matter, but this does not mean useless matter. The matter so formed may without entering into the living substance be of some subsidiary use to the corpuscle, or, as probably more often happens, being discharged from the corpuscle, may be of use to some other part of the body. We do not know how the living substance builds itself up, but we seem compelled to admit that, in certain cases at all events, it is able in some way or other to produce changes on material while that material is still outside the living substance as it were, before it enters into and indeed without its ever actually entering into the composition of the living substance. On the other hand, we must equally admit that some of the waste substances are the direct products of the katabolic changes of the living substance itself-were actually once part of the living substance. Hence we ought, perhaps, to distinguish the products of the activity of living matter into waste products proper, the direct results of katabolic changes, and into by-products which are the results of changes effected by the living matter outside itself, and which cannot, therefore, be considered as necessarily either anabolic or katabolic.

Concerning the chemical characters of the living matter itself we cannot 
at present make any very definite statement. We may say that the proteid myosin, or rather the proteid antecedent or antecedents of myosin, enter in some way into its structure, but we are not justified in saying that the living substance consists only of proteid matter in a peculiar condition. And, indeed, the persistency with which some representative of fatty bodies and some representative of earbohydrates always appear in living tissue would, perhaps, rather lead us to suppose that these equally with proteid material were essential to its structure. Again, though the behavior of the nucleus, as contrasted with that of the cell body, leads us to suppose that the living substance of the former is a different kind from that of the latter, we do not know exactly in what the difference consists. The nucleus, as we have seen, contains nuclein which, perhaps, we may regard as a largely modified proteid; but being a body which is remarkable for its stability, for the difficulty with which it is changed by chemical reagents, cannot be regarded as an integral part of the essentially mobile living substance of the nucleus.

In this connection it may be worth while to again call attention to the fact that the corpuscle contains a very large quantity of water, viz., about 90 per cent. Part of this, we do not know how much, probably exists in a more or less definite combination with the protoplasm, somewhat after the manner of, to use what is a mere illustration, the water of crystallization of salts. If we imagine a whole group of different complex salts continually occupied in turn in being crystallized and being decrystallized, the water thus engaged by the salts will give us a rough image of the water which passes in and out of the substance of the corpuscle as the result of its metabolic activity. We might call this "water of metabolism." Another part of the water, carrying in this case substances in solution, probably exists in spaces or interstices too small to be seen with even the highest powers of the microscope. Still another part of the water similarly holding substances in solution exists at times in definite spaces visible under the microscope, more or less regularly spherical, and called vacuoles.

We have dwelt thus at length on the white corpuscle in the first place, because, as we have already said, what takes place in it is in a sense a picture of what takes place in all living structures, and in the second place because the facts which we have mentioned help us to understand how the white corpuscle may carry on in the blood a work of no important kind; for from what has been said it is obvious that the white corpuscle is continually acting upon and being acted upon by the plasma.

$\$ 31$. To understand, however, the work of these white corpuscles, we must learn what is known of their history.

In successive drops of blood taken at different times from the same individual, the number of colorless corpuscles will be found to vary very much, not only relatively to the red corpuscles, but also absolutely. They niust, therefore, "come and go."

In treating of the lymphatic system we shall have to point out that a very large quantity of fluid called lymph, containing a very considerable number of bodies, very similar in their general characters to the white corpuscles of the blood, is being continually poured into the vascular system at the point where the thoracic duct joins the great veins on the left side of the neck, and to a less extent where the other large lymphatics join the venous system on the right side of the neck. These corpuscles of lymph, which, as we have just said, closely resemble, and, indeed, are with difficulty distinguished from the white corpuscles of the blood, but of which, when they exist outside the vascular system, it will be convenient to speak of as leucocytes, are found along the whole length of the lymphatic system, but 
are more numerous in the lymphatic vessels after these have passed through the lymphatic glands. These lymphatic glands are partly composed of what is known as adenoid tissue, a special kind of connective tissue arranged as a delicate network. The meshes of this are crowded with colorless nucleated cells. which, though varying in size, are, for the most part, small, the nucleus being surrounded by a relatively small quantity of cell substance. Many of these cells show signs that they are undergoing cell-division, and we have reason to think that cells so formed, acquiring a larger amount of cell substance, become veritable leucocytes. In other words, leucocytes multiply in the lymphatic glands, and leaving the glands by the lymphatic vessels, niake their way to the blood. Patches and tracts of similar adenoid tissue, not arranged, however, as distinct glands, but similarly occupied by developing leucocytes and similarly connected with lymphatic vessels, are found in various parts of the body, especially in the mucous membranes. Hence we may conclude that from various parts of the body, the lymphatics are continually bringing to the blood an abundant supply of leucocytes, and that these in the blood become ordinary white corpuscles. This is probably the chief source of the white corpuscles, for though the white corpuscles have been seen dividing in the blood itself, no large increase takes place in that way.

$\S 32$. It follows that since white corpuscles are thus continually being added to the blood, white corpuscles must as continually either be destroyed, or be transformed, or escape from the interior of the bloodvessels; otherwise the blood would soon be blocked with white corpuscles.

Some do leave the bloodvessels. In treating of the circulation we shall have to point out that white corpuscles are able to pierce the walls of the capillaries and minute veins, and thus to make their way from the interiorof the bloodvessels into spaces filled with lymph, the "lymph spaces," as they are called, of the tissue lying outside the bloodvessels. This is spoken of as the "migration of the white corpuscles." In an "inflamed area" large numbers of white corpuscles are thus drained away from the blood into the lymph spaces of the tissue; and it is probable that a similar loss. takes place, more or less, under normal conditions. These migrating corpuscles may, by following the devious tracks of the lymph, find their way back into the blood; some of them, however, may remain, and undergo various changes. Thus, in inflamed areas, when suppuration follows inflammation, the white corpuscles which have migrated may become "pus corpuscles," or, where thickening and growth follow upon inflammation, may, according to many authorities, become transformed into temporary or permanent tissue, especially connective tissue; but this transformation into tissue is disputed. When an inflammation subsides without leaving any effect a few corpuscles only will be found in the tissue; those which had previously migrated must, therefore, have been disposed of in some way or another.

In speaking of the formation of red corpuscles ( $\$ 27)$ we saw that not only it is not proved that the nucleated corpuscles which give rise to red corpuscles are ordinary white corpuscles, but that in all probability the real hæmatoblasts, the parents of red corpuscles, are special corpuscles developed in the situations where the manufacture of red corpuscles takes place. So far, therefore, from assuming, as is sometimes done, that the white corpuscles of the blood are all of them on their way to become red corpuscles, it may be doubted whether any of them are. In any case, however, even making allowance for those which migrate, a very considerable number of the white corpuscles must " disappear" in some way or other from the blood stream, and we may, perhaps, speak of their disappearance as being a "destruction" 
or "dissolution." We have, as yet, no exact knowledge to guide us in the matter, but we can readily imagine, that upon the death of the corpuscle, the substances composing it, after undergoing changes, are dissolved by and become part of the plasma. If so, the corpuscles, as they die, must repeatedly influence the composition and nature of the plasma.

But if they thus affect the plasma in their death, it is even more probable that they influence it during their lif'e. Being alive, they must be continually taking in and giving out. As we have already said, they are known to ingest, after the fashion of an amøba, solid particles of various kinds, such as fat or carmine, present in the plasma, and probably digest such of these particles as are nutritious. But if they ingest these solid matters they probably also carry out the easier task of ingesting dissolved matters. If, however, they thus take in, they must also give out; and thus by the removal on the one hand of various substances from the plasma, and by the addition on the other hand of others, they must be continually influencing the plasma. We have already said that the white corpuscles in shed blood as they die are supposed to play an important part in the clotting of blood; similarly they may during their whole life be engaged in carrying out changes in the proteids of the plasma which do not lead to clotting, but which prepare them for their various uses in the body.

Pathological facts afford support to this view. The disease called leucocythæmia (or leukæmia) is characterized by an increase of the white corpuscles, both absolute and relative to the red corpuscles, the increase, due to an augmented production or possibly to a retarded destruction, being at times so great as to give the blood a pinkish-gray appearance, like that of blood mixed with pus. We accordingly find that in this disease the plasma is in many ways profoundly affected and fails to nourish the tissues. As a further illustration of the possible action of the white corpuscles we may state that, according to some observers in certain diseases in which minute organisms, such as bacteria; make their appearance in the blood, the white corpuscles "take up" these bacteria into their substance, and thus probably, by exerting an influence on them, modify the course of the disease of which these organisms are the essential cause.

If the white corpuscles are thus engaged during their life in carrying on important labors, we may expect them to differ in appearance according to their condition. Some of the corpuscles are spoken of as "faintly" or "finely" granular. Other corpuscles are spoken of as " coarsely" granular, their cell substance being loaded with conspicuously discrete granules. It may be, of course, that there are two distinct kinds of corpuscles, having different functions and possibly different origins and histories; but since intermediate forms are met with containing a few coarse granules only, it is more probable that the one form is a phase of the other; that a faintly granular corpuscle, by taking in granules from without or by producing granules within itself as products of its metabolism, may become a coarsely granular corpuscle.

Whether, however, the white corpuscles are really all of one kind, or whether they are lifferent kinds performing different functions, must at present be left an open question.

\section{Blood Platelets.}

$\S 33$. In a drop of blood examined with care immediately after removal may be seen a number of exceedingly small bodies ( $2 \mu$ to $3 \mu$ in diameter), frequently disc-shaped, but sometimes of a rounded or irregular form, homogeneous in appearance when quite fresh, but apt to assume a faintly gran- 
ular aspect. They are called blood platelets, or blood plaques. They have been supposed by some to become developed into, and, indeed, to be early stages of, the red corpuscles, and hence have been called hrmatoblasts; but this view has not been confirmed; indeed, as we have seen (\$27), the real hrematoblasts, or developing red corpuscles, are of quite a different nature.

They speedily undergo change after removal from the body, apparently dissolving in the plasma; they break up, part of their substance disappearing, while the rest becomes granular. Their granular remains are apt to run together, forming in the plasma the shapeless masses which have long been known and described as "lumps of protoplasm." By appropriate reagents, however, these platelets may be fixed and stained in the condition in which they appear after leaving the body.

The substance composing them is peculiar, and, though we may perhaps speak of them as consisting of living material, their nature is at present obscure. They may be seen within the living bloodvessels [Fig. 10], and therefore must be regarded as real parts of the blood, and not as products of the changes taking place in blood after it has been shed.

When a needle or thread or other foreign body is introduced into the interior of a bloodvessel, they are apt to collect upon, and, indeed, are the precursors of the clot which in most cases forms around the needle or thread. They are also found in the thrombi or plugs which sometimes form in the bloodvessels as the result of disease or injury. "Indeed, it has been maintained that what are called white thrombi (to distinguish them from red thrombi, which are plugs of corpuscles and fibrin) are in reality aggregations of blood platelets; and for various reasons blood platelets have been supposed to play an important part in the clotting of blood, carrying out the work which, in this respect, is by others attributed to the white corpuscles. But no very definite statement can at present be made about this ; and, indeed, the origin and whole nature of these blood platelets is at present obscure.

\section{The Chemical Composition of Blood.}

$\S 34$. We may now pass briefly in review the chief chemical characters of blood, remembering always that, as we have already urged, the chief chemical interests of blood are attached to the changes which it undergoes in the several tissues; these will be considered in connection with each tissue at the appropriate place.

The average specific gravity of human blood is 1055, varying from 1045 to 1075 within the limits of health.

The reaction of blood as it flows from the bloodvessels is found to be distinctly though feebly alkaline. If a drop be placed on a piece of faintly red, highly glazed litmus paper, and then wiped off, a blue stain will be left.

The whole blood contains, in a certain quantity, gases-viz., oxygen, carbonic acid, and nitrogen, which are held in the blood in a peculiar waywhich vary in different kinds of blood, and so serve especially to distin- 
guish arterial from venous blood, and which may be given off from blood when exposed to an atmosphere, according to the composition of that atmosphere. These gases of blood we shall study in comnection with respiration.

The normal blood consists of corpuscles and plasma.

If the corpuscles be supposed to retain the amount of water proper to them, blood may, in general terms, be considered as consisting by weight of from about one-third to somewhat less than one-half of corpuscles, the rest being plasma. As we have already seen, the number of corpuscles in a specimen of blood is found to vary considerably, not only in different animals and in different individuals, but in the same individual at different times.

The plasma is resolved by the clotting of the blood into serum and fibrin.

$\S 35$. The serum contains in 100 parts :

Proteid substances

Fats, various extractives, and saline matters .

Water .

$$
\begin{aligned}
& \text { - about } 8 \text { or } 9 \text { parts. } \\
& \text { " } 2 \text { or } 1 \text { part. }
\end{aligned}
$$

90 parts.

The proteids are paraglobulin and serum-albumin (there being probably more than one kind of serum-albumin) in varying proportion. We may, perhaps, roughly speaking, say that they occur in about equal quantities.

Conspicuous and striking as are the results of clotting, massive as appears to be the clot which is formed, it must be remembered that by far the greater part of the clot consists of corpuscles. The amount by weight of fibrin required to bind together a number of corpuscles, in order to form even a large, firm clot, is exceedingly small. Thus, the average quantity by weight of fibrin in human blood is said to be 0.2 per cent.; the amount, however, which can be obtained from a given quantity of plasma varies extremely, the variation being due not only to circumstances affecting the blood, but to the method employed.

The fats, which are scanty, except after a meal or in certain pathological conditions, consist of the neutral fats-stearin, palmitin, and olein-with a certain quantity of their respective alkaline soaps. The peculiar complex fat lecithin occurs in very small quantities only; the amount present of the peculiar alcohol cholesterin, which had so fatty an appearance is also small. Among the extractives present in serum may be put down nearly all the nitrogenous and other substances which form the extractives of the body and of food, such as urea, kreatin, sugar, lactic acid, etc. A very large number of these have been discovered in the blood under various circumstances, the consideration of which must be left for the present. The peculiar odor of blood or of serum is probably due to the presence of volatile bodies of the fatty acid series. The faint yellow color of serum is due to a special yellow pigment. The most characteristic and important chemical feature of the saline constitution of the serum is the preponderance, at least in man and most animals, of sodium salts over those of potassium. In this respect the serum offers a marked contrast to the corpuscles. Less marked, but still striking, is the abundance of chlorides and the poverty of phosphates in the serum, as compared with the corpuscles. The salts may in fact briefly be described as consisting chiefly of sodium chloride, with some amount of sodium carbonate, or more correctly sodium bicarbonate, and potassium chloride with small quantities of sodium sulphate, sodium phosphate, calcium phosphate, and magnesium phosphate. And of even the small quantity of phosphates found in the ash, part of the phosphorus exists in the serum itself, not as a phosphate, but as phosphorus in some organic body. 
$\$ 36$. The red corpuscles contain less water than the serum, the amount of solid matter being variously estimated at from 30 to 40 or more per cent. The solids are almost entirely organic matter, the inorganic salts amounting to less than 1 per cent. Of the organic matter again by far the larger part consists of hæmoglobin. In 100 parts of the dried organic matter of the corpuscles of human blood, about 90 parts are hrmoglobin, about 8 parts are proteid substances, and about 2 parts are other substances. Of the last, one of the most important, forming about a quarter of them and apparently being always present, is lecithin. Cholesterin appears also to be normally present. The proteids which form the stroma of the red corpuscles appear to belong chiefly to the glohulin family. As regards the inorganic constituents, the corpuscles are distinguished by the relative abundance of the salts of potassium and of phosphates. This at least is the case in man; the relative quantities of sodium and potassium in the corpuscles and serum respectively appear, however, to vary in different animals; in some the sodium salts are in excess even in the corpuscles.

$\$ 37$. The proteid matrix of the white corpuscles we have stated to be composed of myosin (or an allied body), paraglobulin and possibly other proteids. The nuclei contain nuclein. The white corpuscles are found to contain, in addition to proteid material, lecithin and other fats, glycogen, extractives and inorganic salts, there being in the ash, as in that of the red corpuscles, a preponderance of potassium salts and of phosphates.

The main facts of interest then in the chemical composition of the blood are as follows: The red corpuscles consist chiefly of hæmoglobin. The organic solids of serum consist partly of serum-albumin, and partly of paraglobulin. The serum or plasma contrasts, in man at least, with the corpuscles, inasmuch as the former contains chiefly chlorides and sodium salts, while the latter are richer in phosphates and potassium salts. The extractives of the blood are remarkable rather for their number and variability than for their abundance, the most constant and important being perhaps urea, kreatin, sugar, and lactic acid.

\section{The Quantity of Blood, and its Distribution in the Body.}

$\S 38$. The quantity of blood contained in the whole vascular system is a balance struck between the tissues which give to, and those which take away from, the blood. Thus the tissues of the alimentary canal largely add to the blood water and the material derived from food, while the excretory organs largely take away water and the other substances constituting the excretions. Other tissues both give and take, and the considerable drain from the blood to the lymph spaces which takes place in the capillaries is met by the flow of lymph into the great veins.

From the result of a few observations on executed criminals it has been concluded that the total quantity of blood in the human body is about $\frac{1}{13}$ th of the body weight. But in various animals, the proportion of the weight of the blood to that of the body has been found to vary very considerably in different individuals; and probably this holds good for man also, at all events within certain limits.

In the same individual the quantity probably does not vary largely. A sudden drain upon the water of the blood by great activity of the excretory organs, as by profuse sweating, or a sudden addition to the water of the blood, as by drinking large quantities of water or by injecting fluid into the bloodvessels, is rapidly compensated for by the passage of water from the tissues to the blood, or from the blood to the tissues. As we have already said, the tissues are continually striving to keep up an average composition of the 
blood, and in so doing keep up an average quantity. In starvation the quantity (and quality) of the blood is maintained for a long time at the expense of the tissues, so that after some days' privation of food and drink, while the fat, the muscles, and other tissues have been largely diminished, the quantity of blood remains nearly the same.

The total quantity of blood present in an animal body is estimated in the following way: As much blood as possible is allowed to escape from the vessels; this is measured directly. 'The vessels are then washed out with water or normal saline solution, and the washings carefully collected, mixed, and measured. A known quantity of blood is diluted with water or normal saline solution until it possesses the same tint as a measured specimen of the washings. This gives the amount of blood (or rather of hæmoglobin) in the measured specimen, from which the total quantity in the whole washings is calculated. Lastly, the whole body is carefully minced and washed free from blood. The washings are collected and filtered, and the amount of blood in them is estimated, as before, by comparison with a specimen of diluted blood. The quantity of blood, as calculated from the two washings, together with the escaped and directly measured blood, gives the total quantity of blood in the body.

The inethod is not free from objections, but other methods are even more imperfect.

The blood is in round numbers distributed as follows:

About one-fourth in the heart, lungs, large arteries and veins.

About one-fourth in the liver.

About one-fourth in the skeletal muscles.

About one-fourth in the other organs.

Since in the heart and great bloodvessels the blood is simply in transit, without undergoing any great changes (and in the lungs, as far as we know, being limited to respiratory changes), it follows that the alterations which take place in the blood passing through the liver and skeletal muscles far exceed those which occur in the rest of the body.

\section{CHA PTER II.}

\section{THE CONTRACTILE TISSUES.}

$\S 39$. In order that the blood may nourish the several tissues it is carried to and from them by the vascular mechanism; and this carriage entails active movements. In order that the blood may adequately nourish the tissues, it must be replenished by food from the alimentary canal, and purified from waste by the excretory organs; and both these processes entail movements. Hence before we proceed further we must study some of the general characters of the movements of the body.

Most of the movements of the body are carried out by means of the muscles of the trunk and limbs, which being connected with the skeleton are frequently called skeletal muscles. A skeletal muscle when subjected to certain influences suddenly shortens, bringing its two ends nearer together; and it is the shortening, acting upon various bony levers or by help of other mechanical arrangements, which produces the movement. Such a temporary shortening, called forth by certain influences, and due as we shall see to changes taking place in the muscular tissue forming the chief part of the 
muscle, is technically called a contraction of the muscle; and the muscular tissue is spoken of as a contractile tissue. The heart is chiefly composed of muscular tissue, differing in certain minor features from the muscular tissue of the skeletal muscles, and the beat of the heart is essentially a contraction of the muscular tissue composing it, a shortening of the peculiar muscular fibres of which the heart is chiefly made up. The movements of the alimentary canal and of many other organs are similarly the results of the contraction of the muscular tissue entering into the composition of those organs, of the shortening of certain muscular fibres built up into those organs. In fact, almost all the movements of the body are the result of the contraction of muscular fibres, of various nature and variously disposed.

Some few movements, however, are carried out by structures which cannot be called muscular. Thus, in the pulmonary passages and elsewhere, movement is effected by means of cilia attached to epithelium cells; and elsewhere, as in the case of the migrating white corpuscles of the blood, transference from place to place in the body is brought about by amœboid movements. But as we shall see the changes in the epithelium cell or white corpuscle which are at the bottom of ciliary or amoboid movements are, in all probability, fundamentally the same as those which take place in a muscular fibre when it contracts: they are of the nature of a contraction, and hence we may speak of all these as different forms of contractile tissue.

Of all these various forms of contractile tissue, the skeletal muscles, on account of the more complete development of their functions, will be better studied first; the others, on account of their very simplicity, are in many respects less satisfactorily understood.

All the ordinary skeletal muscles are connected with nerves. We have no reason for thinking that they are thrown into contraction, under normal conditions, otherwise than by the agency of nerves.

Muscles and nerves being thus so closely allied, and having besides so many properties in common, it will conduce to clearness and brevity if we treat them together.

\section{The Phenomena of Muscle and Nerve.}

\section{Muscular and Nervous Irritability.}

$\S 40$. The skeletal muscles of a frog, the brain and spinal cord of which have been destroyed, do not exhibit any spontaneous movements or contractions, even though the nerves be otherwise quite intact. Left undisturbed, the whole body may decompose without any contraction of any of the skeletal muscles having been witnessed. Neither the skeletal museles nor the nerves distributed to them possess any power of automatic action.

If, however, a nuscle be laid bare and be more or less violently disturbed -if, for instance, it be pinched, or touched with a hot wire, or brought into contact with certain chemical substances, or subjected to the action of galvanic currents - it will move, that is, contract, whenever it is thus disturbed. Though not exhibiting any spontaneous activity, the muscle is (and continues for some time after the general death of the animal to be) irritable. Though it remains quite quiescent when left untouched, its powers are then dormant only-not absent. These require to be roused or "stimulated " by some change or disturbance in order that they may manifest themselves. The substances or agents which are thus able to evoke the activity of an irritable muscle are spoken of as stimuli.

But to produce a contraction in a muscle the stimulus need not be applied directly to the muscle; it may be applied indirectly by means of the 
nerve. Thus, if the trunk of a nerve be pinched, or subjected to sudden heat, or dipped in certain chemical substances, or acted upon by various galvanic currents, contractions are seen in the muscles to which branches of the nerves are distributed.

The nerve, like the muscle, is irritable; it is thrown into a state of activity by a stimulus: but, unlike the muscle, it does not itself contract. The stimulus does not give rise in the nerve to any visible change of form; hut that changes of some kind or other are set up and propagated along the nerve down to the muscle is shown by the fact that the muscle contracts when a part of the nerve at some distance from itself is stimulated. Both nerve and muscle are irritable, but only the muscle is contractile-i.e., manifests its irritability by contraction. The nerve manifests its irritability by transmitting along itself, without any visible alteration of form, certain molecular changes set up by the stimulus. IVe shall call these changes thus propagated along a nerve "nervous impulses."

$\$ 41$. We have stated above that the muscle may be thrown into contraction by stimuli applied directly to itself. But it might fairly be urged that the contractions so produced are in reality due to the fact that the stimulus, although apparently applied directly to the muscle, is, after all, brought to bear on some of the many fine nerve-branches, which, as we shall see, are abundant in the muscle, passing along and between the muscular fibres, in which they finally end. The following facts, however, go far to prove that the muscular fibres themselves are capable of being directly stimulated without the intervention of any nerves: When a frog (or other animal) is poisoned with urari, the nerves may be subjected to the strongest stimuli without causing any contractions in the muscles to which they are distributed; yet even ordinary stimuli, applied directly to the muscle, readily cause contractions. If, before introducing the urari into the system, a ligature be passed underneath the sciatic nerve in one leg-for instance, the right-and drawn tightly round the whole leg to the exclusion of the nerve, it is evident that the urari, when injected into the back of the animal, will gain access to the right sciatic nerve above the ligature, but not below, while it will have free access to the rest of the body, including the whole left sciatic. If, as soon as the urari has taken effect, the two sciatic nerves be stimulated, no movement of the left leg will be produced by stimulating the left sciatic, whereas strong contractions of the muscles of the right leg below the ligature will follow stimulation of the right sciatic, whether the nerve be stimulated above or below the ligature. Now, since the upper parts of both sciatics are equally exposed to the action of the poison, it is clear that the failure of the left nerve to cause contraction is not attributable to any change having taken place in the upper portion of the nerve, else why should not the right, which has in its upper portion been equally exposed to the action of the poison, also fail? Evidently the poison acts on some parts of the nerve lower down. If a single muscle be removed from the circulation (by ligaturing its bloodvessels), previous to the poisoning with urari, that muscle will contract when any part of the nerve going to it is stimulated, though no other muscle in the body will contract when its nerve is stimulated. Here the whole nerve right down to the muscle has been exposed to the action of the poison, and yet it has lost none of its power over the muscle. On the other hand, if the muscle be allowed to remain in the body, and so be exposed to the action of the poison, but the nerve be divided high up and the part connected with the muscle gently lifted up before the urari is introduced into the system, so that no blood flows to it, and so that it is protected from tl o influence of the poison, stimulation of the nerve will be found to produce no contractions in 
the muscle, though stimuli applied directly to the muscle at once causes it to contract. From these facts it is clear that urari poisons the ends of the nerve within the muscle long before it affects the trunk; and it is exceedingly probable that it is the very extreme ends of the nerves (possibly the endplates or peculiar structures in which the nerve fibres end in the muscular fibres, for urari poisoning, at least when profound, causes a slight, but yet distinctly recognizable effect in the microscopic appearance of these structures) which are affected. The phenomena of urari poisoning go far to prove that muscles are capable of being made to contract by stimuli applied directly to the nuscular fibres themselves; and there are other facts which support this view.

$\$ 42$. When, in a recently killed frog, we stimulate by various means and in various ways the muscles and nerves, it will be observed that the movements thus produced, though very various, may be distinguished to be of two kinds. On the one hand, the result may be a mere twitch, as it were, of this or that muscle; on the other hand, one or more muscles may remain shortened or contracted for a considerable time-a limb, for instance, being raised up or stretched out, and kept raised up or stretched out for many seconds. And we find, upon examination, that a stimulus may be applied either in such a way as to produce a mere twitch, a passing rapid contraction which is over and gone in a fraction of a second, or in such a way as to keep the muscle shortened or contracted for as long a time as, up to certain limits, we may choose. The mere twitch is called a single or simple muscular contraction; the sustained contraction, which, as we shall see, is really the result of rapidly repeated simple contractions, is called a tetanic contraction.

$\S 43$. In order to study these contractions adequately, we must have recourse to the "graphic method," as it is called, and obtains a tracing or other record of the change of form of the muscle. To do this conveniently, it is best to operate with a muscle isolated from the rest of the body of a recently killed animal, and carefully prepared in such a way as to remain irritable for some time. The muscles of cold-blooded animals remain irritable after removal from the body far longer than those of warm-blooded animals, and hence those of the frog are generally made use of. We shall study presently the conditions which determine this maintenance of the irritability of muscles and nerves after removed from the body.

A muscle thus isolated, with its nerve left attached to it, is called a muscle-nerve preparation. The most convenient muscle for this purpose in the frog is, perhaps, the gastrocnemius, which should be dissected out so as to leave carefully preserved the attachment to the femur above, some portion of the tendon (tendo Achillis) below, and a considerable length of the sciatic nerve with its branches going to the muscle. (Fig. 11.)

$\S 44$. We may apply to such a muscle-nerve preparation the various kinds of stimuli (mechanical, such as pricking or pinching; thermal, such as sudden heating; chemical, such as acids or other active chemical substances; or electrical) and these we may apply either to the muscle directly or to the nerve, thus affecting the muscle indirectly. Of all these stimuli by far the most convenient for general purposes are electrical stimuli of various kinds ; and these, except for special purposes, are best applied to the nerve, and not directly to the muscle.

Of electrical stimuli, again, the currents, as they are called, generated by a voltaic cell, are most convenient, though the electricity generated by a rotating magnet, or that produced by friction may be employed. Making use of a cell or battery of cells-Daniell's, Grove's, Leclanché, or any other -we must distinguish between the current produced by the cell itself, the 
constant current, as we shall call it, and the induced current obtained from the constant current by means of an induction coil, as it is called; for

FIG. 11.

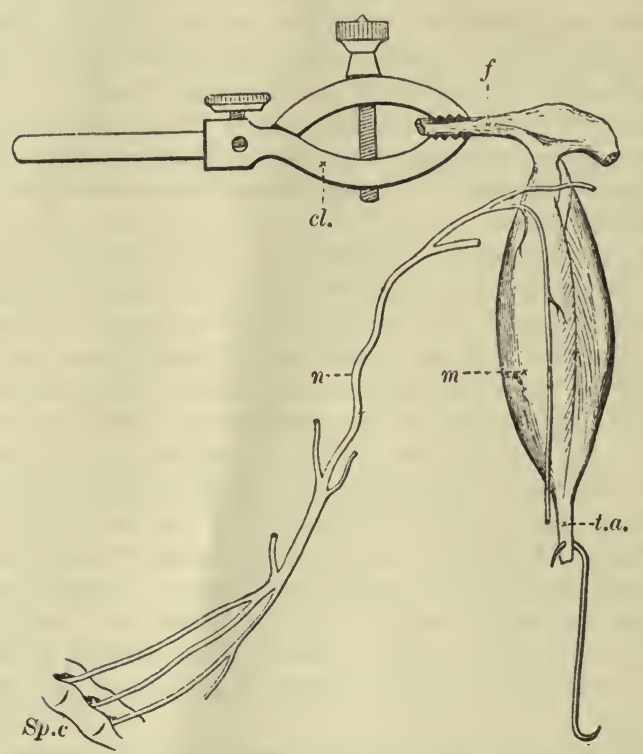

A Muscle-nerve Preparation: $m$, the muscle, gastrocnemius of frog; $n$, the sciatic nerve, all the branches being cut away except that supplying the muscle; $f$, femur; $c l$., clamp; $t$. $a .$, tendo Achillis; $s p$. c., end of spinal canal.

the physiological effects of the two kinds of current are in many ways different.

It may, perhaps, be worth while to remind the reader of the following facts :

In a galvanic battery, the substance (plate of zinc, for instance) which is acted upon and used by the liquid is called the positive element, and the substance which is not so acted upon and used up (plate, etc., of copper, platinum, or carbon, etc.) is called the negative element. A galvanic action is set up when the positive (zinc) and the negative (copper) elements are connected outside the battery by some conducting material, such as a wire, and the current is said to flow in a circuit or circle from the zinc or positive element to the copper or negative element inside the battery, and then from the copper or negative element back to the zinc or positive element through the wire outside the battery. If the conducting wire be cut through, the current ceases to flow; but if the cut ends be brought into contact, the current is re-established and continues to flow so long as the contact is good. The ends of the wires are called "poles," or when used for physiological purposes, in which case they may be fashioned in various ways, are spoken of as electrodes. When the poles are brought into contact or are connected by some conducting material, galvanic action is set up, and the current flows through the battery and wires; this is spoken of as "making the current" or "completing or closing the circuit." When the poles are drawn apart from each other, or when some non-conducting material is interposed between them, the galvanic action is arrested ; this is spoken of as "breaking the current" or "opening the circuit." The current passes from the wire conmected with the negative (copper) element in the battery to the wire connected with the positive (zinc) element in the battery; hence, the pole connected with the copper (negative) element is called the positive pole, and that connected with the zinc (positive) element is called the negative pole. When used for physiological purposes the positive pole becomes the positive elec- 
trode, and the negative pole the negative electrode. The positive electrode is often spoken of as the anode (ana, up), and the negative electrode as the kathode (kata, down).

A piece of nerve of ordinary length, though not a good conductor, is still a conductor, and when placed on the electrodes completes the circuit, permitting the current to pass through it; in order to remove the nerve from the influence of the current it must be lifted off from the electrodes. 'This is obviously inconvenient; and hence it is usual to arrange a means of opening or closing the circuit at some point along one of the two wires. This may be done in various ways -by fastening one part of the wire into a cup of mercury, and so by dipping the other part of the wire into the cup to close the circuit and nake the current, and by lifting it out of the mercury to open the circuit and break the current; or by arranging between the two parts of the wires a movable bridge of good conducting material, such as brass, which ean be put down to close the circuit or raised up to open the circuit; or in other ways. Such a means of closing and opening a circuit, and so of making or breaking a current, is called a key.

$\Lambda$ key which is frequently used by physiologists goes by the name of Du BoisReymond's key. Though undesirable in many respects, it has the advantage that it can be used in two different ways; when arranged as in A, Fig. 12, the brass bridge

FIG. 12.

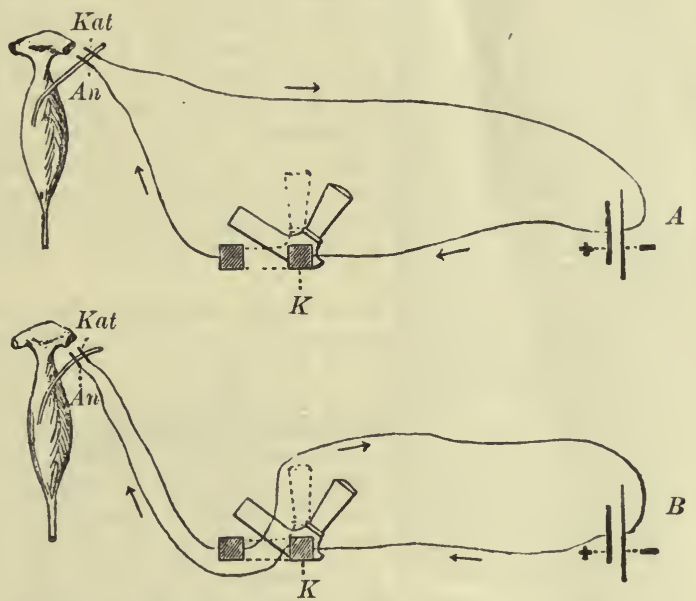

Diagram of Du Bois-Reymond Key used, A, for making and breaking ; B, for short-circuiting.

of $\mathrm{K}$, the key, being down, and forming a means of good conduction between the brass plates to which the wires are screwed, the circuit is closed and the current passes from the positive pole (end of the negative (copper) element) to the positive electrode, or anode, $A n$., through the nerve, to the negative electrode, or kathode, Kat., and thence back to the negative pole (end of the positive (zinc) element) in the battery; on raising the brass bridge, the circuit is opened, the eurrent is broken, and no current passes through the electrodes. When arranged as in B, if the brass bridge be "down," the resistance offered by it is so small, compared with the resistance offered by the nerve between the electrodes, that the whole current from the battery passes through the bridge back to the battery, and none, or only an infinitesimal portion, passes into the nerve. When, on the other hand, the bridge is raised, and so the conduction between the two sides suspended, the current is not able to pass directly from one side to the other, but can and does pass along the wire through the nerve back to the battery. Hence, in arrangement A, "putting down the key," as it is called, makes a current in the nerve, and "raising" or "opening the key" breaks the current. In arrangement B, however, putting down the key diverts the current from the nerve by sending it through the bridge, and so back to the battery; the current, instead of making the longer circuit through the electrodes, makes the shorter circuit through the 
key ; hence, this is called "short-circuiting." When the bridge is raised the current passes through the nerve on the electrodes. Thus, "putting down" and "raising " or "opening" the key have contrary effects in A and B. In B, it will be observed, the battery is always at work, the current is always flowing either through the electrodes (key up) or through the key (key down); in A, the battery is not at work until the circuit is made by putting down the key. And in many cases it is desirable to take, so to speak, a sample of the current while the battery is in full swing, rather than just as it begins to work. Moreover, in B the electrodes are, when the key is down, wholly shut off from the current; whereas, in A, when the key is up, one electrode is still in direct connection with the battery, and this connection leading to what is known as unipolar action, may give rise to stimulation of the nerve. Hence the use of the key in the form B.

Other forms of key may be used. 'Thus, in the Morse key (F, Fig. 13) contact is made by pressing down a lever handle $(h a)$; when the pressure is removed, the handle, driven up by a spring, breaks contact. In the arrangement shown in the figure, one wire from the battery being brought to the binding screw $b$, while the binding screw $a$ is connected with the other wire, putting down the handle makes connection between $a$ and $b$, and thus makes a current. By arranging the wires in the several binding screws in a different way, the making contact by depressing the handle may be used to short circuit.

In an "induction coil," Figs. 13 and 14, the wire connecting the two elements of a battery is twisted at some part of its course into a close spiral, ealled the primury coil. Thus, in Fig. 13, the wire $x^{\prime \prime \prime}$, connected with the copper or negative plate $c . p$. of the battery, $E$, joins the primary coil, $p r . c$, and then passes on as $y$ " . through the "key" $F$, to the positive (zinc) plate, z.p., of the battery. Over this primary coil, but quite unconnected with it, slides another coil, the secondary coil, s. $c$.; the ends of the wire forming this coil, $y^{\prime \prime}$ and $x^{\prime \prime}$, are continned on in the arrangement illustrated in the figure as $y^{\prime}$ and $y$, and as $x^{\prime}$ and $x$, and terminate in electrodes. If these electrodes are in contact or connected with conducting material, the circuit of the secondary coil is said to be closed; otherwise it is open.

In such an arrangement it is found that at the moment when the primary circuit is closed, $i . e$., when the primary current is " made," a secondary "induced" current is, for an exceedingly brief period of time, set up in the secondary coil. Thus, in Fig. 13, when, by moving the "key" $F, y^{\prime \prime \prime}$ and $x^{\prime \prime \prime}$, previously not in connection with each other, are put into connection, and the primary current thus made, at that instant a current appears in the wires, $v^{\prime \prime}, x^{\prime \prime}$, etc., but almost immediately disappears. A similar almost instantaneous current is also developed when the primary current is "broken," but not till then. So long as the primary current flows with uniform intensity, no current is induced in the secondary coil. It is only when the primary current is either made or broken, or suddenly varies in intensity, that a current appears in the secondary coil. In each case the current is of very brief duration, gone in an instant almost, and may therefore be spoken of as "a shock," an induction shock; being called a "making shock," when it is caused by the making, and a "breaking shock," when it is caused by the breaking of the primary circuit. The direction of the current in the making sloock is opposed to that of the primary current; thus, in the figure, while, the primary current flows from $x^{\prime \prime \prime}$ to $y^{\prime \prime \prime}$, the induced making shock flows from $y$ to $x$. The current of the breaking shock, on the other hand, flows in the same direction as the primary current from $x$ to $y$, and is therefore in direction the reverse of the making shock. Compare Fig. 14, where arrangement is shown in a diagrammatic manner.

The current from the battery, upon its first entrance into the primary coil, as it passes along each twist of that coil, gives rise in the neighboring twists of the same coil to a momentary induced current having a direction opposite to its own, and therefore tending to weaken itself. It is not until this "self-induction" has passer off that the current in the primary coil is established in its full strength. Owing to this delay in the full establishment of the current in the primary coil. the induced current in the secondary coil is developed more slowly than it would be were no such "self-induction" present. On the other hand, when the current from the battery is "broken " or "shut off" from the primary coil, no such delay is offered to its disappearance, and consequently the induced current in the second- 


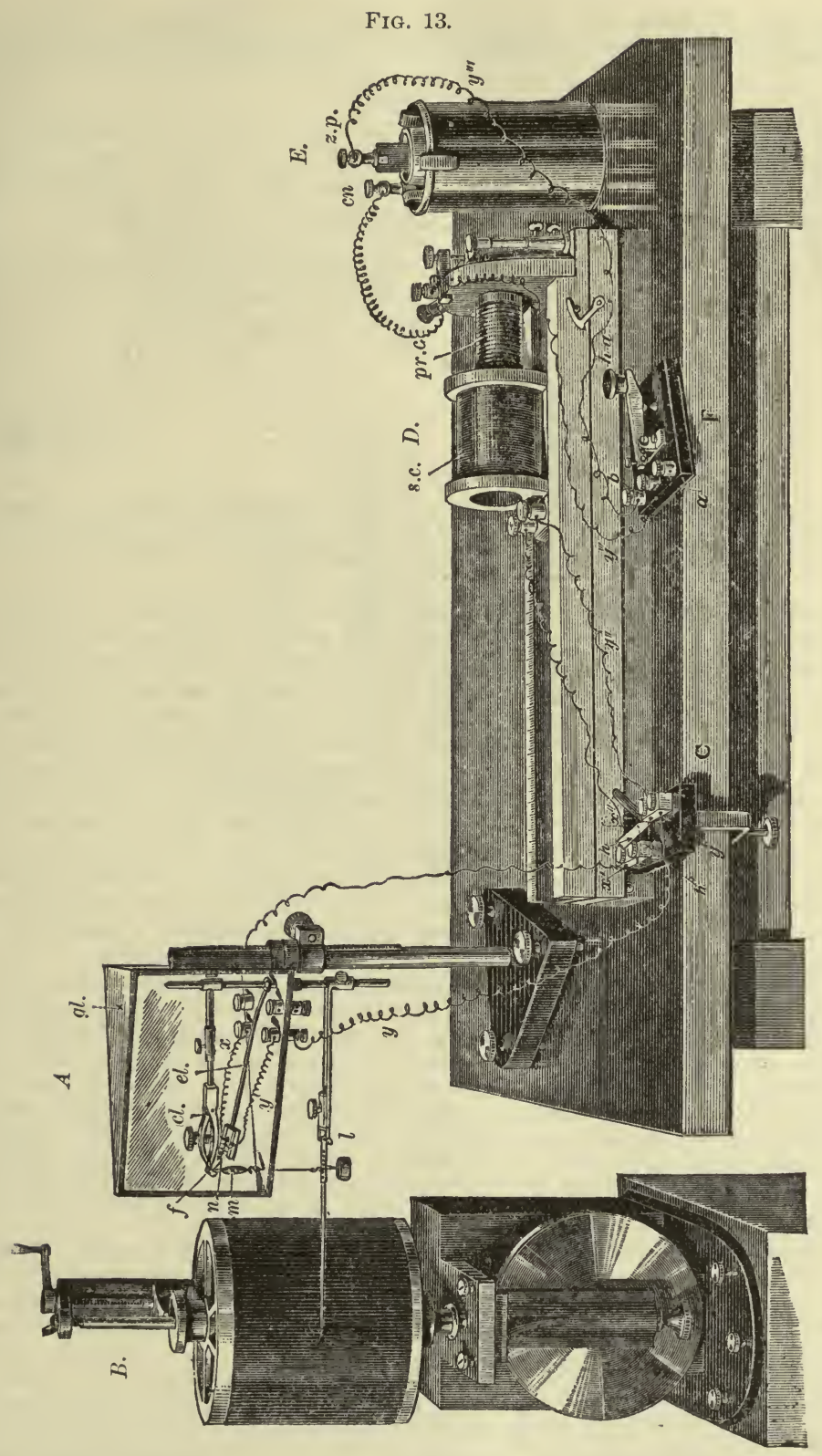

Diagram illustrating Apparatus arranged for Experiments with Muscle and Nerve: $A$. The moist chamber containing the muscle-nerve preparation. The muscle $m$, supported by the elamp $c l$., which firmly grasps the end of the femur $f$, is connected by means of the S-hook $s$ and a thread with the lever $l$, placed below the moist chamber. The nerve $n$, with a portion of the spinal column $n^{\prime}$ still attached to it, is placed on the electrode-holder $e l$, in contact with the wires $x, y$. The whole of the interior of the glass case $g l$, is kept saturated with moisture, and the electrode-holder is so constructed that a piece of moistened blotting-paper may be placed on it without coming into contact with the nerve.

$B$. The revolving cylinder bearing the smoked paper on which the lever writes.

$C$. Du Bois-Reymond's key arranged for short-circuiting. The wires $x$ and $y$ of the electrode- 
ary coil is developed with unimpeded rapidity. We shall see later on that a rapidly developed current is more effective as a stimulus than is a more slowly.

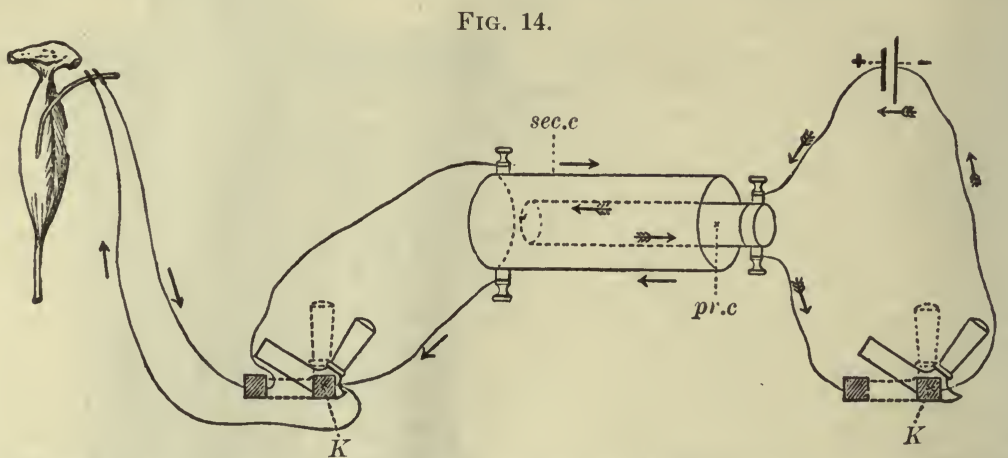

Diagram of an Induction Coil: + positive pole, end of negative element; - negative pole, end of positive element of battery; K, Du Bois-Reymond's key; $p r$. $c$. primary coil, current show 11 by feathered arrow; sc. $c$. secondary coil, current shown by unfeathered arrow.

developed current. Hence the making shock, where rapidity of production is interfered with by the self-induction of the primary coil, is less effective as a stimulus than the breaking shock whose development is not thus interfered with.

The strength of the induced current depends, on the one hand, on the strength of the current passing through the primary coil-that is, on the strength of the battery. It also depends on the relative position of the two coils. Thus a secondary coil is brought nearer and nearer to the primary coil and made to overlap it more and more; the induced current becomes stronger and stronger, though the current from the battery remains the same. With an ordinary battery, the secondary coil may be pushed to some distance away from the primary coil, and yet shocks sufficient to stimulate a muscle will be obtained. For this purpose, however, the two coils should be in the same line; when the secondary coil is placed crosswise, at right angles to the primary, no induced current is developed, and at intermediate angles the induced current has intermediate strengths.

When the primary current is repeatedly and rapidly made and broken, the secondary current being developed with each make and with each break, a rapidly recurring series of alternating currents is developed in the secondary coil and

holder are connected through binding screws in the floor of the moist chamber with the wires $x^{\prime}, y^{\prime}$, and these are secured in the key, one on either side. To the same key are attached the wires $x^{\prime \prime}, y^{\prime \prime}$, coming from the secondary coils $s$. $c$. of the induction coil $D$. This secondary coil can be made to slide up and down over the primary coil $p r . c$., with which are connected the two wires $x^{\prime \prime \prime}$ and $y^{\prime \prime \prime} ; x^{\prime \prime \prime}$ is connected directly with one pole-for instance, the copper pole $c . p$. of the battery $E ; y^{\prime \prime \prime}$ is carried to a binding screw, $a$, of the Morse key $F$, and is continued as $y$ iv from another binding screw, $b$, of the key to the zinc pole $z$. p. of the battery.

Supposing everything to be arranged, and the battery charged; on depressing the handle $h a$, of the Morse key $F$, a current will be made in the primary coil $p r . c$., passing from $c . p$. through $x^{\prime \prime \prime}$ to $p r . c$, and thence through $y^{\prime \prime}$ to $a$, thence to $b$, and so through $y^{\text {iv }}$ to $z . p$. On removing the finger from the handle of $F$, a spring thrusts up the handle, and the primary circuit is in consequence immediately broken.

At the instant that the primary current is either made or broken, an induced current is for the instant developed in the secondary coil s. $c$. If the cross-bar $h$ in the Du Bois-Reymond's key be raised (as shown in the thick line in the figure), the wires $x^{\prime \prime}, x^{\prime}, x$, the nerve between the electrodes and the wires $y, y^{\prime}, y^{\prime \prime}$, form the complete secondary circuit, and the nerve consequently experiences a making or breaking induction-shock whenever the primary current is made or broken. If the cross-bar of the Du Bois-Reymond key be shut down, as in the dotted line $h^{\prime}$ in the figure, the resistance of the cross-bar is so slight coinpared with that of the nerve and of the wires going from the key to the nerve, that the whole secondary (induced) current passes from $x^{\prime \prime}$ to $y^{\prime \prime}$ (or from $y^{\prime \prime}$ to $x^{\prime \prime}$ ) along the cross-bar, and practically none passes into the nerve. The nerve, being thus "short-circuited," is not affected by any changes in the current.

The figure is intended merely to ill ustrate the general method of studying muscular contraction ; it is not to be supposed that the details here given are universally adopted, or, indeed, the best for all purposes. 
passes through its electrodes. We shall frequently speak of this as the interrupted induction current, or more briefly the interrupted current; it is sometimes spoken of as the faradic current, and the application of it to any tissue is spoken of as faradization.

Such a repeated breaking and making of the primary current may be effected in many various ways. In the instruments commonly used for the purpose, the primary current is made and broken by means of a vibrating steel slip working against a magnet: hence the instrument is called a magnetic interruptor. See Fig. 15.

The two wires $x$ and $y$ from the battery are connected with the two brass pillars $a$ and $d$ by means of screws. Directly contact is thus made current, indicated

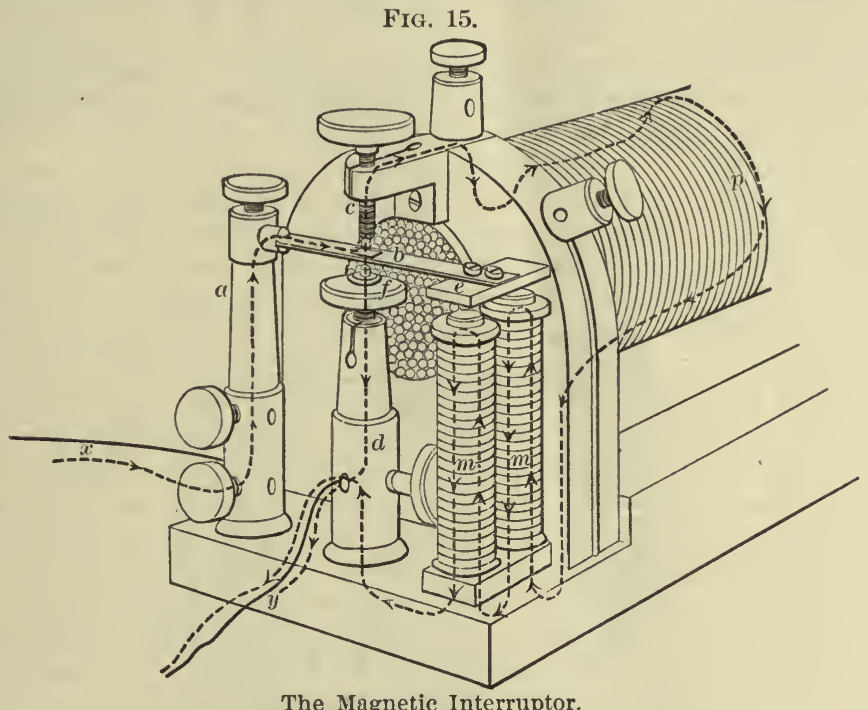

The Magnetic Interruptor.

in the figure by the thick interrupted line, passes in the direction of the arrows, up the pillar $a$, along the steel spring $b$, as far as the screw $c$, the point of which, armed with platinum, is in contact with a small platinum plate on $b$. The current passes from $b$ through $c$ and a connecting wire into the primary coil $p$. Upon its entering into the primary coil, an induced (making) current is for the instant developed in the secondary coil (not shown in the figure). From the primary coil $p$ the current passes, by a connecting wire, through the double spiral $m$, and. did nothing happen, would continue to pass from $m$ by a connecting wire to the pillar $d$, and so by the wire $y$ to the battery. The whole of this course is indieated by the thick interrupted line with its arrows.

As the current, however, passes through the spirals $m$, the iron cores of these are made magnetic. They, in consequence, draw down the iron bar $e$, fixed at the end of the spring $b$, the flexibility of the spring allowing this. But when $e$ is drawn down, the platinum plate on the upper surface of $b$ is also drawn away from the screw $c$, and thus the current is "broken" at $b$. (Sometimes the. screw $f$ is so arranged that when $e$ is drawn down a platinum plate on the under surface of $b$ is brought into contact with the platinum-armed point of the screw $f$. The current then passes from $b$, not to $c$, but to $f$, and so down the pillar $d$, in the direction indieated by the thin interrupted line. and out of the battery by the wire $y$, and is thus cut off from the primary coil. But this arrangement is unnecessary.) At the instant that the current is thus broken and so cut off from the primary coil, an induced (breaking) current is for the moment dereloped in the secondary coil. But the current is cut off not only from the primary coil, but also from the spirals $m$; in consequence, their cores cease to be magnetized, the bar $e$ ceases to be attracted by them, and the spring $b$, by virtue of its elasticity, resumes its former position 
in contact with the screw $c$. This return of the spring, however, re-establishes the current in the primary coil and in the spirals, and the spring is drawn down, to be released once more in the same manner as before. Thus, as long as the current is passing along $x$, the eontact of $b$ with $c$ is alternately being made and broken, and the current is constantly passing into and being shut off from $p$, the periods of alternation being deternined by the periods of vibration of the spring $b$. With each passage of the current into or withdrawal from the primary coil, an induced (making and, respectively, breaking) current is developed in a secondary coil.

As thus used, each "making shock," as explained above, is less powerful than the corresponding "breaking shock;" and, indeed, it sometimes happens that instead of each make, as well as each break, acting as a stimulus, giving rise to a contraction, the "breaks" only are effective, the several "makes" giving rise to no contraction.

But what is known as Helmholtz's arrangement (Fig. 16), however, the making and breaking shocks may be equalized. For this purpose the screw $c$ is raised out

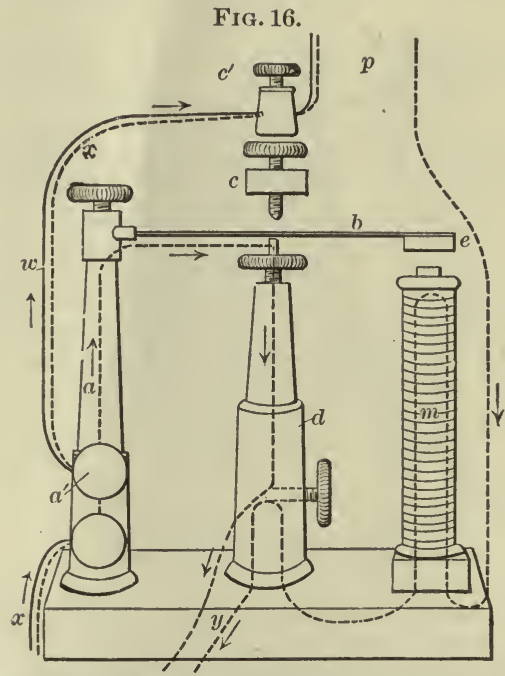

The Magnetic Interruptor with Helmholtz's Arrangement for Equalizing the Make and Break Shock.

of reach of the excursions of the spring $b$, and a moderately thick wire $w$, offering a certain amount only of resistance, is interposed between the upper binding screw $a^{\prime}$ on the pillar $a$, and the binding screw $c^{\prime}$ leading to the primary coil. Under these arrangements the current from the battery passes through $a^{\prime}$, along the interposed wire to $c^{\prime}$, through the primary coil, and thus, as before, to $m$. As before, by the magnetism of $m, e$ is drawn down and $b$ brought in contact with $f$. As the result of this contact, the current from the battery can now pass by $a, f$, and $d$ (shown by the thin interrupted line), back to the battery; but not the whole of the current, some of it can still pass along the wire $w$ to the primary coil, the relative amount being determined by the relative resistance offered by the two courses. Hence at each successive magnetization of $m$, the current in the primary coil does not entirely disappear when $l$ is brought in contact with $f$; it is only so far diminished that $m$ ceases to attract $e$, and hence by the release of $b$ from $f$ the whole current once more passes along $i v$. Since, at what corresponds to the "break" the current in the primary coil is diminished only, not absolutely done away with, self-induction makes its appearance at the "break" as well as at the "make;" thus the "breaking" and "making "induced currents or shocks in the secondary coil are equalized. They are both reduced to the lower efficiency of the "making " shock in the old arrangement; hence to produce the same strength of 
stimulus with this arrangement a stronger current must be applied or the secondary coil pushed over the primary coil to a greater extent than with the other arrangement.

\section{The Phenomena of a Simple Muscular Contraction.}

$\S 45$. If the far end of the nerve of a muscle-nerve preparation (Figs. 11 and 13) be laid on electrodes connected with the secondary coil of an induction-machine, the passage of a single induction-shock, which may be taken as a convenient form of an almost momentary stimulus, will produce no visible change in the nerve, but the muscle will give a twitch, a short, sharp contraction, $i$. e., will for an instant shorten itself, becoming thicker the while, and then return to its previous condition. If one end of the muscle be attached to a lever, while the other is fixed, the lever will by its movements indicate the extent and duration of the shortening. If the point of the lever be brought to bear on some rapidly travelling surface, on which it leaves a mark (being for this purpose armed with a pen and ink if the surface be plain paper, or with a bristle or finely pointed piece of platinum foil if the surface be smoked glass or paper), so long as the muscle remains at rest the lever will describe an even line, which we may call the base line. If, however, the muscle shortens the lever will rise above the base line and thus describe some sort of curve above the base line. Now it is found that when a single induction-shock is sent through the nerve the twitch which the muscle gives causes the lever to describe some such curve as that shown in Fig. 17 ; the lever (after a brief interval immediately succeeding the opening or shutting the key, of which we shall speak presently) rises at first rapidly but afterward more slowly, showing that the muscle is correspondingly shortening; then ceases to rise, showing that the muscle is ceasing to grow shorter; then descends, showing that the muscle is lengthening again, and finally, sooner or later, reaches and joins the base line, showing that the muscle after the shortening has regained its previous natural growth. Such a curve described by a muscle during a twitch or simple muscular contraction, caused by a single induction-shock or by any other stimulus producing the same effect, is called a curve of a simple muscular contraction, or more shortly, a "muscle-curve." It is obvious that the exact form of the curve described by identical contractions of a muscle will depend on the rapidity with which the recording surface is travelling. Thus if the surface be travelling slowly the up-stroke corresponaing to the shortening will be very abrupt
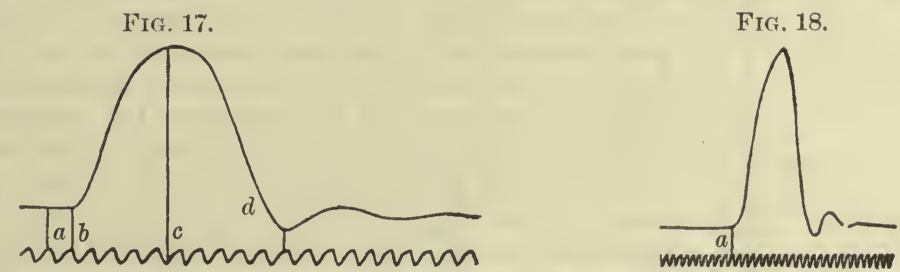

A Muscle-curve from the Gastrocnemius of the Frog: This curve, like all succeeding ones, unless otherwise indicated, is to be read from left to right-that is to say, while the lever and tuning-fork were stationary the recording surface was travelling from right to left.

$a$ indicates the moment at which the induction-shock is sent into the nerve, $b$ the commencement, $c$ the maximum, and $d$ the close of the contraction.

Below the muscle-curve is the curve drawn by a tuning-fork making 100 double vibrations a second, each complete curve representing therefore one-hundredth of a second.

and the down stroke also very steep, as in Fig. 18, which is a curve from a gastrocnemius muscle of a frog, taken with a slowly moving drum, the 
tuning-fork being the same as that used in Fig. 17 ; indeed, with a very slow movement, the two may be hardly separable from each other. On

Frg. 19.

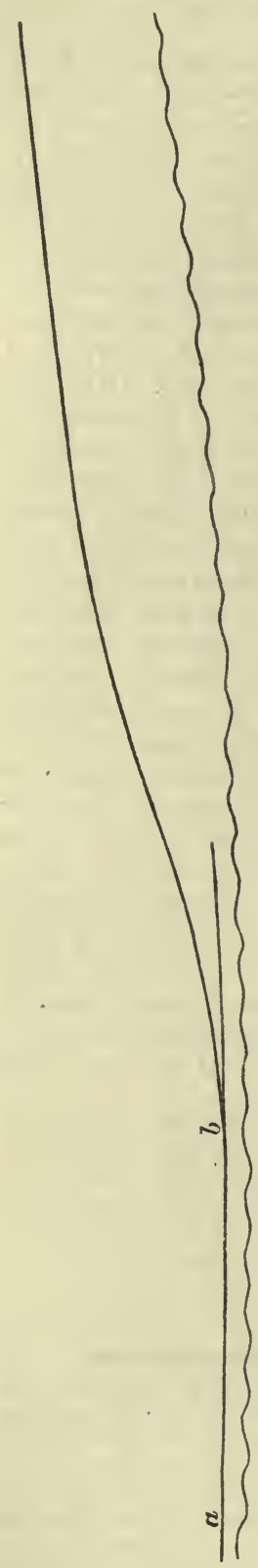
the other hand, if the surface travel very rapidly the curve may be immediately long drawn out, as in Fig. 19 , which is a curve from a gastrocnemius muscle of a frog, taken with a very rapidly moving pendulum myograph, the tuning-fork making about 500 vibrations a second. On examination, however, it will be found that both these extreme curves are fundamentally the same as the medium one, when account is taken of the different rapidities of the travelling surface in the several cases.

In order to make the "muscle-curve" complete, it is necessary to mark on the recording surface the exact time at which the induction shock is sent into the nerve, and also to note the speed at which the recording surface is travelling.

In the pendulum myograph the rate of movement can be calculated from the length of the pendulum; but even in this it is convenient, and in the case of the spring myograph and revolving cylinder is necessary, to measure the rate of movement directly by means of a vibrating tuning-fork, or of some body vibrating regularly. Indeed it is best to make such a direct measurement with each curve that is taken.

A tuning-fork, as is known, vibrates so many times a second according to its pitch. If a tuning-fork, armed with a light marker on one of its prongs and vibrating say 100 a second-i.e., executing a docible vibration, moving forward and backward, 100 times a second-be brought while vibrating to make a tracing on the recording surface immediately below the lever belonging to the muscle, we can use the curve or rather curves described by the tuning-fork to measure the duration of any part or of the whole of the muscle-curve. It is essential that at starting the point of the marker of the tuning-fork should be exactly underneath the marker of the lever, or rather, since the point of the lever as it moves up and down describes not a straight line but an arc of a circle of which its fulcrum is the centre and itself (from the fulcrum to the tip of the marker) the radius, that the point of the marker of the tuning-fork should be exactly on the arc described by the marker of the lever, either above or below it, as may prove most convenient. If, then, at starting the tuning-fork marker be thus on the arc of the lever marker, and we note on the curve of the tuning fork the place where the are of the lever cuts it at the beginning and at the end of the muscle-curve, as at Fig. 17, we can count the number of vibrations of the tuningfork which have taken place between the two marks, and so ascertain the whole time of the muscle-curve; if, for instance, there have been 10 double vibrations, each nccupying $\frac{1}{100}$ second, the whole curve has taken $\frac{1}{10}$ second to make. In the same way we 
can measure the duration of the rise of the curve or of the fall, or of any part of it.

Though the tuning-fork may, by simply striking it, be set going long enough for the purposes of an observation, it is convenient to keep it going by means of an electric current and a magnet, very much as the spring in the magnetic interruptor (Fig. 15) is kept going.

It is not necessary to use an actual tuning-fork; any rod, armed with a marker, which can be made to vibrate regularly, and whose time of vibration is known, may be used for the purpose: thus a reed, made to vibrate by a blast of air, is sometimes employed.

The exact moment at which the induction shock is thrown into the nerve may be recorded on the muscle-curve by means of a "signal," which may be applied in various ways.

A large steel lever armed with a marker is arranged over a small coil by means of a light spring in such a way that when the coil by the passage of a current through it becomes a magnet it pulls the lever down to itself; on the current being broken, and the magnetization of the coil ceasing, the lever by help of the spring flies up. The marker of such a lever is placed immediately under-i.e., at some point on the arc described by-the marker of the muscle (or other) lever. Hence by making a current in the coil and putting the signal lever down, or by breaking an already existing current, and letting the signal lever fly up, we can make at pleasure a mark corresponding to any part we please of the muscle (or other) curve.

If, in order to magnetize the coil of the signal, we use, as we may do, the primary current which generates the induction-shock, the breaking or making of the primary current, whichever we use to produce the induction-shock, will make the signal lever fly up or come down. Hence we shall have on the recording surface, under the muscle, a mark indicating the exact moment at which the primary current was broken or made. Now the time taken up by the generation of the induced current and its passage into the nerve between the electrodes is so infinitesimally small, that we may, without appreciable error, take the moment of the breaking or making of the primary current as the moment of the entrance of the inductionshock into the nerve. Thus we can mark below the muscle-curve, or by describing the arc of the muscle lever, on the muscle-curve itself, the exact moment at which the induction-shock falls into the nerve between the electrodes, as is done at $a$ in Figs. 17, 18, 19.

In the pendulum myograph a separate signal is not needed. If, having placed the muscle lever in the position in which we intend to make it record, we allow the glass plate to descend until the tooth $a^{\prime}$ just touches the rod $c$ (so that the rod is just about to be knocked down, and so break the primary circuit) and make on the base line, which is meanwhile being described by the lever marker, a mark to indicate where the point of the marker is under these circumstances, and then bring back the plate to its proper position, the mark which we have made will mark the moment of the breaking of the primary circuit, and so of the entrance of the induction-shock into the nerve. For it is just when, as the glass plate swings down, the marker of the lever comes to the mark which we have made that the rod $c$ is knocked back and the primary current is broken.

A "signal" like the above, in an improved form known as Desprez's, may be used also to record time, and thus the awkwardness of bringing a large tuningfork up to the recording surface obviated. For this purpose the signal is introduced into a circuit the current of which is continually being made and broken by a tuning-fork (Fig. 21). The tuning-fork once set vibrating continues to make and break the current at each of its vibrations, and as stated above is kept vibrating by the current. But each make or break caused by the tuning-fork affects also the small coil of the signal, causing the lever of the signal to fall down or fly up. Thus the signal describes vibration-curves synchronous with those of the tuning-fork driving it. The signal may similarly be worked by means of vibrating agents other than a tuning-fork.

Various recording surfaces may be used. The form most generally useful is a cylinder covered with smoked paper and made to revolve by clockwork or otherwise; such a cylinder driven by clockwork is shown in Fig. 13, B. By using a 


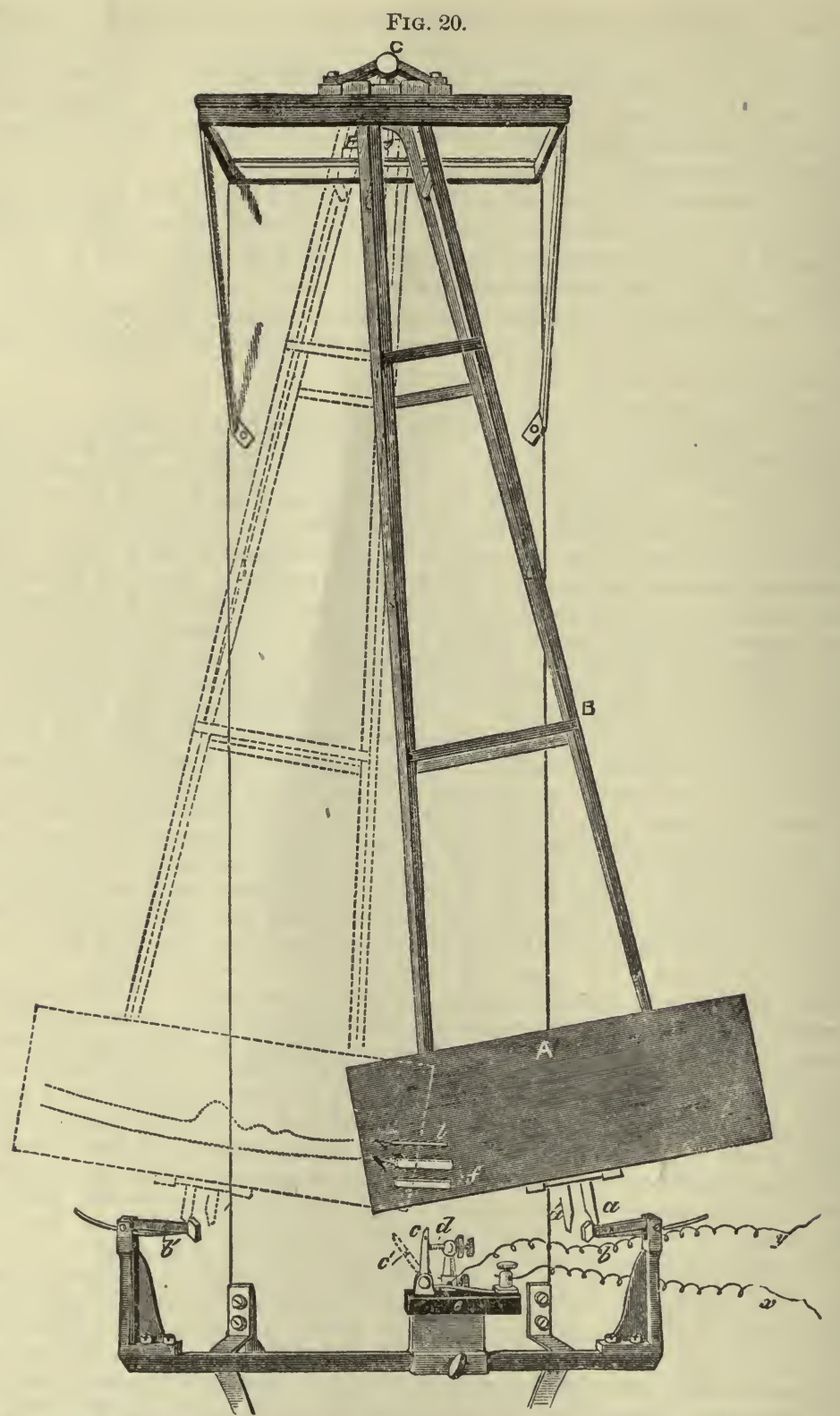

The Pendulum Myograph: The figure is diagrammatic, the essentials only of the instrument being shown. The smoker glass plate $A$ swings with the pendulum $B$ on carefully adjusted bearings at $C$. The contrivances by which the glass plate can be removed and replaced at pleasure are not shown. A second glass plate so arranged that the first glass plate may be moved up and down without altering the swing of the pendulum is also omitted. Before commeneing an experiment the pendulum is raised up (in the figure to the right), and is kept in that position by the tooth $a$ catching on the spring-catch $b$. On depressing the eateh $b$ the glass plate is set free, swings into the new position indicated by the dotted lines, and is held in that position by the tooth $a^{\prime}$ catching on the catch $b^{\prime}$. In the course of its swing the tooth $a^{\prime}$ coming in contact with the projecting steel rod $c$, knoeks it on one side into the position indieated by the 
cylinder of large radius with adequate gear, a high speed for instance, in a second, can be obtained. In the spring myograph a smoked glass plate is thrust rapidly

FI $\div$. 21.

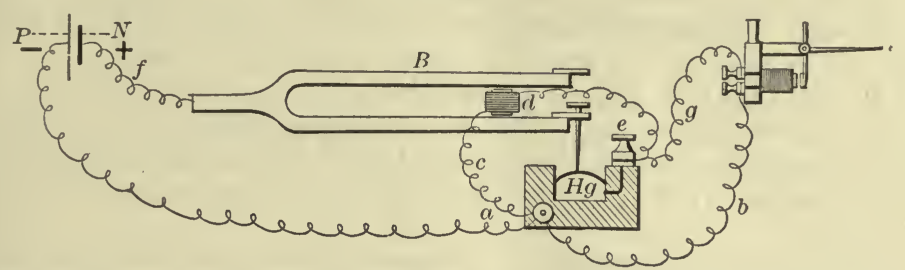

Diagram of an Arrangement of a Vibrating Tuning-fork with a Desprez Signal : The current flows along the wire $j$ cunnected with the pusitive $(+)$ pole or end of the negative plate $(N)$ of the battery, through the tuning-fork, down the pin connected with the end of the lower prong, to the mercury in the cup $H g$, and so by a wire (shown in figure) to the binding screw $e$. From this binding screw part of the current flows through the coil $d$ between the prongs of the tuningfork, and thence by the wire $c$ to the binding screw $a$, while another part flows through the wire $g$, through the coil of the Desprez signal back by the wire $b$, to the binding screw $a$. From the binding serew $a$ the current passes back to the negative $(-)$ pole or end of the positive element $(P)$ of the battery. As the current flows through the coil of the Desprez signal from $g$ to $b$, the core of coil becoming magnetized draws down the marker of the signal. As the current flows through the coil $d$, the core of that coil, also becoming magnetized, draws up the lower prong of the fork. But the pin is so adjusted that the drawing up of the prong lifts the point of the pin out of the mercury. In consequence, the current, being thus broken at $H g$, flows neither through $d$ nor through the Desprez signal. The core of the Desprez thus ceasing to be magnetized, the marker flies back, being usually assisted by a spring (not shown in the figure). But since the current ceases to flow through $d$, the core of $d$ ceases to lift up the prong, and the pin, in the descent of the prong, makes contact once more with the mercury. The re-establishment of the current, however, once more acting on the two coils, again pulls down the marker of the signal, and again by magnetizing the core of $d$ pulls up the prong and once more breaks the current. Thus the current is continually made and broken, the rapidity of the interruptions being determined by the vibration periods of the tuning-fork, and the lever of the signal rising and falling synchronously with the movements of the tuning-fork.

forward along a groove by means of a spring suddenly thrown into action. In the pendulum myograph, Fig. 20, a smoked glass plate attached to the lower end of a long frame swinging like a pendulum, is suddenly let go at a certain height, and so swings rapidly through an arc of a circle. The disadvantage of the last two methods is that the surface travels at a continually ehanging rate, whereas, in the revolving cylinder, careful construction and adjustment will secure a very uniform rate.

$\S 46$. Having thus obtained a time record, and an indication of the exact moment at which the induction-shock falls into the nerve, we may for present purposes consider the muscle-curve complete. The study of such a curve, as, for instance, that shown in Fig. 17, taken from the gastrocnemius of a frog, teaches us the following facts:

1. That although the passage of the induced current from electrode to electrode is practically instantaneous, its effect, measured from the entrance of the shock into the nerve to the return of the muscle to its natural length

dotted line $c^{\prime}$. The rod $c$ is in electric continuity with the wire $x$ of the primary coil of an induction-machine. The screw $d$ is similarly in electric continuity with the wire $y$ of the same primary coil. The screw $d$ and the rod $c$ are armed with platinum at the points at which they are in contact, and both are insulated by means of the ebonite block $e$. As long as $c$ and $d$ are in contact the circuit of the primary coil to which $x$ and $y$ belong is closed. When in its swing the tooth $a^{\prime}$ knocks $c$ away from $d$, at that instant the circuit is broken, and a "breaking " shock is sent through the electrodes connected with the secondary coil of the machine, and so through the nerve. The lever $l$, the end only of which is shown in the figure, is brought to bear on the glass plate, and when at rest describes a straight line, or more exactly an arc of a circle of large radius. The tuning-fork $f$, the ends only of the two limbs of which are shown in the figure placed immediately below the lever, serves to mark the time. 
after the shortening, takes an appreciable time. In the figure, the whole curve from $a$ to $d$ takes up about the same time as eleven double vibrations of the tuning-fork. Since each double vibration here represents $\frac{1}{10} 0$ second, the duration of the whole curve is rather more than $\frac{1}{10}$ second.

2. In the first portion of this period, from $a$ to $b$, there is no visible change, no raising of the lever, no shortening of the muscle.

3 . It is not until $b$, that is to say, after the lapse of about $\frac{1}{10} 0$ second, that the shortening begins. The shortening, as shown by the curve is at first slow, but soon becomes more rapid, and then slackens again until it reaches a maximum at $c$; the whole shortening occupying rather more than $\frac{4}{100}$ second.

4. Arrived at the maximum of shortening, the muscle at once begins to relax, the lever descending at first slowly, then more rapidly, and at last more slowly again, until at $d$ the muscle has regained its natural length; the whole return from the maximum of contraction to the natural length occupying rather more than $\frac{5}{100}$ second.

Thus a simple muscular contraction, a simple spasm or twitch, produced by a momentary stimulus, such as a single induction-shock, consists of three main phases:

1. A phase antecedent to any visible alteration in the muscle. This phase, during which invisible preparatory changes are taking place in the nerve and muscle, is called the "latent period."

2. A phase of shortening or, in the more strict meaning of the word, contraction.

3. A phase of relaxation or return to the original length.

In the case we are considering, the electrodes are supposed to be applied to the nerve at some distance from the muscle. Consequently the latent period of the curve comprises not only the preparatory actions going on in the muscle itself, but also the changes necessary to conduct the immediate effect of the induction-shock from the part of the nerve between the electrodes along a considerable length of nerve down to the muscle. It is obvious that these latter changes might be eliminated by placing the electrodes on the muscle itself or on the nerve close to the muscle. If this were done, the muscle and lever being exactly as before, and care were taken that the induction-shock entered into the nerve at the new spot, at the moment when the point of the lever had reached exactly the same point of the travelling surface as before, two curves would be gained having the relations shown in Fig. 22. The two curves resemble each other in almost all points, except that in the curve taken with the shorter piece of nerve, the latent period, the distance $a$ to $b$ as compared with the distance $a$ to $b^{\prime}$ is shortened; the contraction begins rather earlier. A study of the two curves teaches us the following two facts:

1. Shifting the electrodes from a point of the nerve at some distance from the muscle to a point of the nerve close to the muscle has only shortened the latent period a very little. Even when a very long piece of nerve is taken the difference in the two curves is very small, and, indeed, in order that it may be clearly recognized or measured, the travelling surface must be made to travel very rapidly. It is obvious, therefore, that by far the greater part of the latent period is taken up by changes in the muscle itself, changes preparatory to the actual visible shortening. Of course, even when the electrodes are placed close to the muscle, the latent. period includes the changes going on in the short piece of nerve still lying between the electrodes and the muscular fibres. To eliminate this with a view of determining the latent period in the muscle itself, the electrodes might be placed directly on the muscle poisoned with urari. If this were done, it would be 
found that the latent period remained about the same, that is to say, that in all cases the latent period is chiefly taken up by changes in the muscular as distinguished from the nervous elements.

2. Such difference as does exist between the two curves in the figure indicates the time taken up by the propagation, along the piece of nerve, of

FIG. 22.

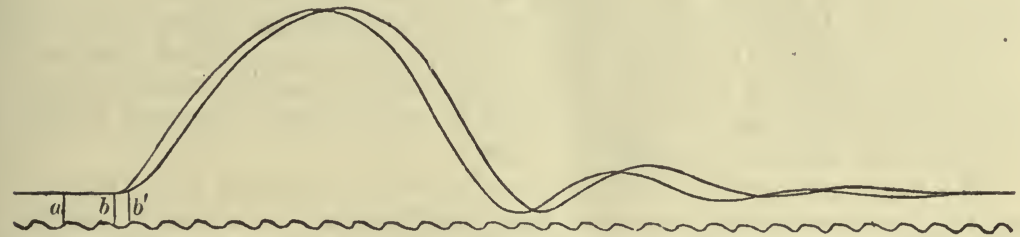

Curves Illustrating the Measurement of the Velocity of a Nervous Inpulse: The same musclenerve preparation is stimulated (1) as far as possible from the muscle, (2) as near as possible to the muscle; both contractions are registered in exactly the same way.

In (1) the stimulus enters the nerve at the time indicated by the line $a$, the contraction begins at $b^{\prime}$; the whole latent period, therefore, is indicated by the distance from $a$ to $b^{\prime}$.

In (2) the stimulus enters the nerve at exactly the same time $a$; the contraction begins at $b$; the latent period, thereforc, is indicated by the distance between $a$ and $b$.

The time taken up by the nervous impulse in passing along the length of nerve between 1 and 2 is, therefore, indicated by the distance between $b$ and $b^{\prime}$, which may be measured by the tuningfork curve below; each double vibration of the tuning-fork corresponds to $1-120$ or 0.0083 second.

the changes set up at the far end of the nerve by the induction-shock. These changes we have already spoken of as constituting a nervous impulse; and the above experiment shows that it takes a small but yet distinctly appreciable time for a nervous impulse to travel along a nerve. In the figure the difference between the two latent periods, the distance between $b$ and $b^{\prime}$, seems almost too small to measure accurately; but if a long piece of nerve be used for the experiment, and the recorling surface be made to travel very fast, the difference between the duration of the latent period when the induction-shock is sent in at a point close to the muscle, and that when it is sent in at a point as far away as possible from the muscle, may be satisfactorily measured in fractions of a second. If the length of nerve between the two points be accurately measured, the rate at which a nervous impulse travels along the nerve to a muscle can thus be easily calculated. This has been found to be in the frog about 28, and in man about 33 metres per second, but varies cónsiderably, especially in warm-blooded animals.

Thus when a momentary stimulus, such as a single induction-shock, is sent into a nerve connected with a muscle, the following events take place: a nervous impulse is started in the nerve and this travelling down to the muscle produces in the muscle, first the invisible changes which constitute the latent period, secondly the changes which bring about the shortening or contraction proper, and thirdly the changes which bring about the relaxation and return to the original length. The changes taking place in each of these three phases are changes of living matter; they vary with the condition of the living substance of the muscle, and only take place so long as the muscle is alive. Though the relaxation which brings back the muscle to its original length is assisted by the muscle being loaded with a weight or otherwise stretched, this is not essential to the actual relaxation, and with the same load the return will vary according to the condition of the muscle; the relaxation must be considered as an essential part of the whole contraction no less than the shortening itself.

$\S 47$. Not only, as we shall see later on, does the whole contraction vary 
in extent and character according to the condition of the muscle, the strength of the induction-shock, the load which the muscle is bearing, and various attendant circumstances, but the three phases may vary independently. The latent period may be longer or shorter, the shortening may take a longer or shorter time to reach the same height, and especially the relaxation may be slow or rapid, complete or imperfect. Even when the same strength of induction-shock is used the contraction may be short and sharp or very long drawn out, so that the curves described on a recording surface travelling at the same rate in the two cases appear very different; and under certain circumstances, as when a muscle is fatigued, the relaxation, more particularly the last part of it, may be so slow, that it may be several seconds before the muscle really regains its original length.

Hence, if we say that the duration of a simple muscular contraction of the gastrocnemius of a frog under ordinary circumstances is about $\frac{1}{10}$ second, of which $\frac{1}{100}$ is taken up by the latent period, $1 \frac{4}{100}$ by the contraction, and $\frac{5}{100}$ by the relaxation, these must be taken as "round numbers," stated so as to be easily remembered. The duration of each phase as well as of the whole contraction varies in different animals, in different muscles of the same animal, and in the same muscle under different conditions.

The muscle-curve which we have been discussing is a curve of changes in the length only of the muscle; but if the muscle, instead of being suspended, were laid flat on a glass plate and a lever laid over its belly, we should find, upon sending an induction-shock into the nerve, that the lever was raised, showing that the muscle during the contraction became thicker. And, if we took a graphic record of the movements of the lever, we should obtain a curve very similar to the one just discussed; after a latent period the lever would rise, showing that the muscle was getting thicker, and afterward would fall, showing that the muscle was becoming thin again. In other words, in contraction the lessening of the muscle lengthwise is accompanied by an increase crosswise; indeed, as we shall see later on, the muscle in contracting is not diminished in bulk at all (or only to an exceedingly small extent, about $\frac{1}{10000}$ of its total bulk), but makes up for its diminution in length by increasing in its other diameters.

$\$ 48$. A single induction shock is, as we have said, the most convenient form of stimulus for producing a simple muscular contraction, but this may also be obtained by other stimuli, provided that these are sufficiently sudden and short in their action, as, for instance, by a prick of, or a sharp blow on, the nerve or muscle. For the production of a single simple muscular contraction the changes in the nerve leading to the muscle must be of such a kind as to constitute what may be called a single nervous impulse, and any stimulus which will evoke a single nervous impulse only may be used to produce a simple muscular contraction.

As a rule, however, most stimuli, other than single induction-shocks, tend to produce in a nerve several nervous impulses, and, as we shall see, the nervous impulses which issue from the central nervous system, and so pass along nerves to muscles, are, as a rule, not single and simple, but complex. Hence, as a matter of fact, a simple muscular contraction is within the living body a comparatively rare event (at least as far as the skeletal muscles are concerned), and cannot easily be produced outside the body otherwise than by a single induction-shock. The ordinary form of muscular contraction is not a simple muscular contraction, but the more complex form known as a tetanic contraction, to the study of which we must now turn. 


\section{Tetanic Contraction.}

$\S 49$. If a single induction-shock be followed at a certain interval by a second shock of the same strength, the first simple contraction will be followed by a second simple contraction, both contractions being separate and distinct; and if the shocks be repeated a series of rhythmically recurring separate simple contractions may be obtained. If, however, the interval between two shocks be made short, for instance, only just long enough to allow the first contraction to have passed its maximum before the latent period of the second is over, the curves of the two contractions will bear some such relation to each other as that shown in Fig. 23 . It will be

FIG. 23.

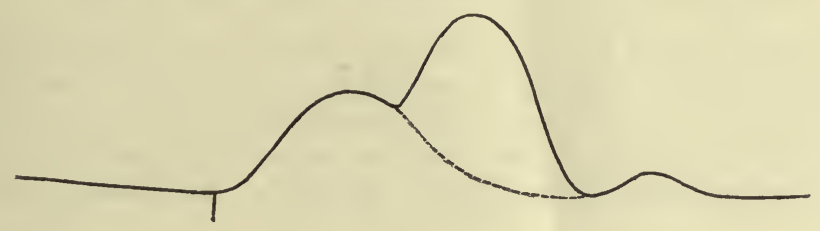

Tracing of a Double Muscle-curve. While the muscle (gastrocnemius of frog) was engaged in the first contraction (whose complete course, had nothing intervened, is indicated by the dotted line), a second induction-shock was thrown in, at such a time that the second contraction began just as the first was beginning to decline. The second curve is seen to start from the first, as does the first from the base-line.

observed that the second curve is almost in all respects like the first except that it starts, so to speak, from the first curve instead of from the base-line.

The second nervous impulse has acted on the already contracted muscle, and made it contract again just as it would have done if there had been no first impulse and the muscle had been at rest. The two contractions are added together and the lever is raised nearly double the height it would have been by either alone. If in the same way a third shock follows the second at a sufficiently short interval, a third curve is piled on top of the second; the same with a fourth, and so on. A more or less similar result would occur if the second contraction began at another phase of the first. The combined effect is, of course, greatest when the second contraction begins at the maximum of the first, being less both before and afterward.

Hence, the result of a repetition of shocks will depend largely on the rate of repetition. If, as in Fig. 24, the shocks follow each other so slowly

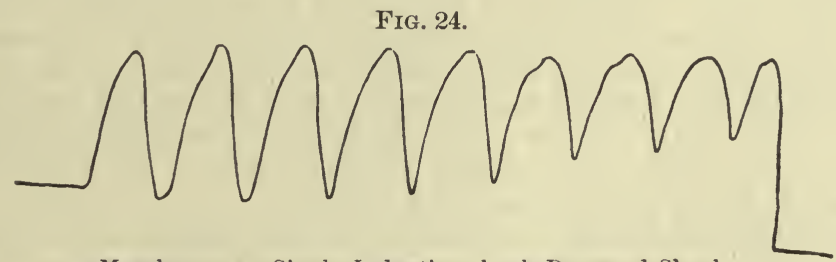

Muscle-curve. Single Induction-shock Repeated Slowly.

that one contraction is over, or almost over, before the next begins, each contraction will be distinct, or nearly distinct, and there will be little or no combined effect.

If, however, the shocks be repeated more rapidly, as in Fig. 25, each suc- 
ceeding contraction will start from some part of the preceding one, and the lever will be raised to a greater height at each contraction.

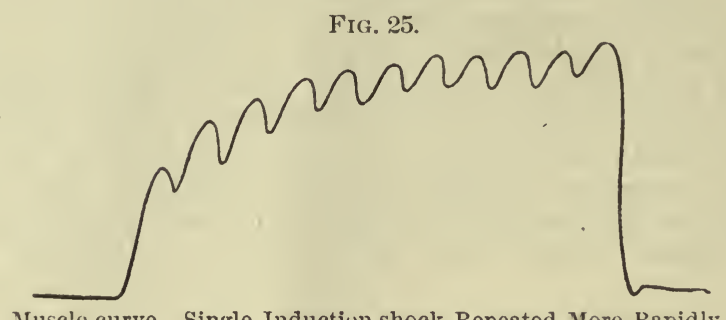

If the frequency of the shocks be still further increased, as in Fig. 26, the rise due to the combination of contraction will be still more rapid, and a smaller part of each contraction will be visible on the curve.

In each of these three curves it will be noticed that the character of the curve changes somewhat during its development. The change is the result

FIG. 26.

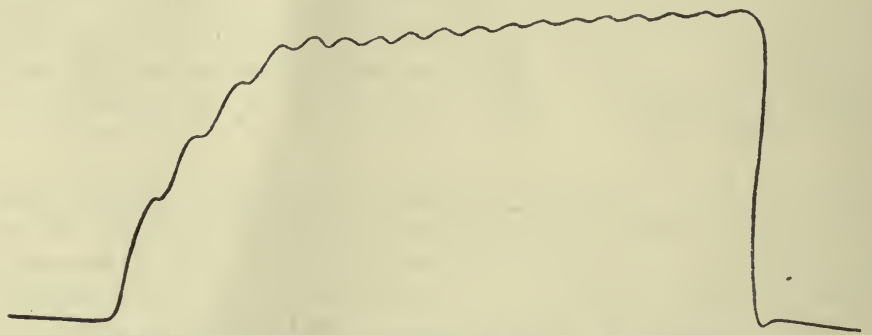

Muscle-curve. Single Induction-shock Still More Rapidly.

of commencing fatigue, caused by the repetition of the contractions, the fatigue manifesting itself by an increasing prolongation of each contraction, shown especially in a delay of relaxation, and by an increasing diminution in the height of the contraction. Thus, in Fig. 24, the contractions, quite distinct at first, become fused later ; the fifth contraction, for instance, is prolonged so that the sixth begins before the lever has reached the base line; yet the summit of the sixth is hardly higher than the summit of the fifth, since the sixth, though starting at a higher level, is a somewhat weaker contraction. See also, in Fig. 25, the lever rises rapidly at first but more slowly afterward, owing to an increasing diminution in the height of the single contractions. In Fig. 26 the increment of rise of the curve due to each contraction diminishes very rapidly, and though the lever does continue to rise during the whole series, the ascent after about the sixth contraction is very gradual indeed, and the indications of the individual contractions are much less marked than at first.

Hence, when shocks are repeated with sufficient rapidity, it results that after a certain number of shocks, the succeeding impulses do not cause any further shortening of the muscle, any further raising of the lever, but merely keep up the contraction already existing. The curve thus reaches a maximum, which it maintains, subject to the depressing effects of exhaustion, so long as the shocks are repeated. When these cease to be given, the muscle returns to its natural length. 
When the shocks succeed each other still more rapidly than in Fig. 26, the individual contractions, visible at first, may become fused together and wholly lost to view in the latter part of the curve. When the shocks succeed each other still more rapidly (the second contraction beginning in the ascending portion of the first) it becomes difficult or impossible to trace out any of the single contractions. ${ }^{1}$ The curve then described by the lever is of the kind shown in Fig. 27, where the primary current of an induction-

Fig. 27.

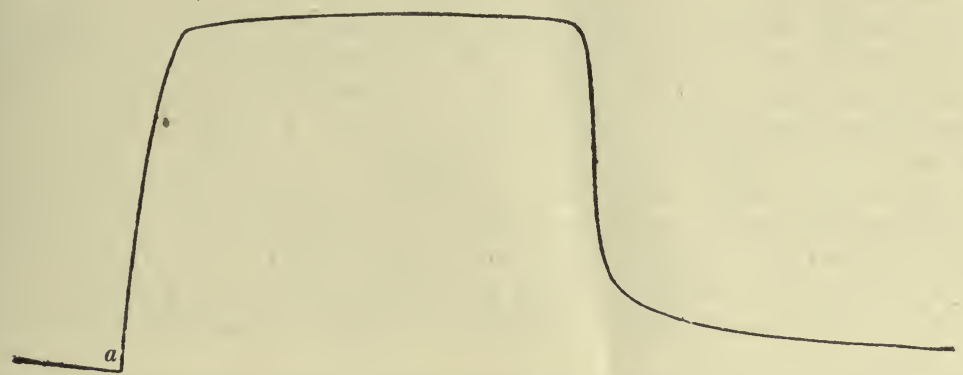

Tetanus Produced with the Ordinary Magnetic Interruptor of an Induction-machine. (Recording surface travelling slowly.) The interrupted current is thrown in at $a$.

machine was rapidly made and broken by the magnetic interruptor, Fig. 15. The lever, it will be observed, rises at $a$ (the recording surface is travelling too slowly to allow the latent period to be distinguished), at first very rapidly, in fact in an unbroken and almost a vertical line, and so very. speedily reaches the maximum, which is maintained so long as the shocks continue to be given; when these cease to be given, the curve descends at first very rapidly and then more and more gradually toward the base-line, which it reaches just at the end of the figure.

This condition of muscle, brought about by rapidly repeated shocks, this fusion of a number of simple twitches into an apparently smooth continuous effort, is known as tetanus or tetanic contraction. The above facts are most clearly shown when induction-shncks, or at least galvanic currents in some form or other, are employed. They are seen, however, whatever be the form of stimulus employed. Thus, in the case of mechanical stimuli, while a single quick blow may cause a single twitch, a pronounced tetanus may be obtained by rapidly striking successively fresh portions of a nerve. With chemical stimulation, as when a nerve is dipped in acid, it is impossible to secure a momentary application; hence tetanus, generally irregular in character, is the normal result of this mode of stimulation. In the living body, the contractions of the skeletal muscles, brought about either by the will or otherwise, are generally tetanic in character. Even very short sharp movements, such as a sudden jerk of a limb or a wink of the eyelid, are in reality examples of tetanus of short duration.

If the lever, instead of being fastened to the tendon of a muscle hung vertically, be laid across the belly of a muscle placed in a horizontal position and the muscle be thrown into tetanus by a repetition of induction-shocks, it will be seen that each shortening of the muscle is accompanied by a corresponding thickening, and that the total shortening of the tetanus is accompanied by a corresponding total thickening. And, indeed, in tetanus we can observe more easily than in a single contraction that the muscle in contract-

1 The ease with which the individual contractions can be made out depends in part, it need hardly be said, on the rapidity with which the recording surface travels. 
ing changes in form only-not in bulk. If a living muscle or group of muscles be placed in a glass jar or chamber, the closed top of which is prolonged into a narrow glass tube, and the chamber be filled with water (or preferably with a solution of sodium chloride, 0.6 per cent. in strength, usually called " normal saline solution," which is less injurious to the tissue than simple water) until the water rises into the narrow tube, it is obvious that any change in the bulk of the muscle will be easily shown by a rising or falling of the column of fluid in the narrow tube. It is found that when the muscle is made to contract, even in the most forcible manner, the change of level in the height of the column which can be observed is practically insignificant; there appears to be a fall indicating a diminution of bulk to the extent of about one ten-thousandth of the total bulk of the muscle. So that we may fairly say that in a tetanus, and hence in a simple contraction, the lessening of the length of the muscle causes a corresponding increase in the other directions; the substance of the muscle is displaced, not diminished.

$\$ 50$. So far we have spoken simply of an induction-shock or of inductionshocks without any reference to their strength, and of a living or irritable muscle without any reference to the degree or extent of its irritability. But induction-shocks may vary in strength, and the irritability of the muscle may vary.

If we slide the secondary coil a long way from the primary coil, and thus make use of extremely feeble induction-shocks, we shall probably find that these shocks, applied even to a quite fresh muscle-nerve preparation, produce no contraction. If we then gradually slide the secondary coil nearer and nearer the primary coil, and keep on trying the effects of the shocks, we shall find that after a while, in a certain position of the coils, a very feeble contraction makes its appearance. As the secondary coil comes still nearer to the primary coil, the contractions grow greater and greater. After a while, however-and that, indeed, in ordinary circumstances, very speedily increasing-the strength of the shock no longer increases the height of the contraction; the maximum contraction of which the muscle is capable with such shocks, however strong, has been reached.

If we use a tetanizing or interrupted current, we shall obtain the same general results; we may, according to the strength of the current, get no contraction at all, or contractions of various extent up to a maximum, which cannot be exceeded. Under favorable conditions the maximum contraction may be very considerable; the shortening in tetauus may amount to threefifths of the total length of the muscle.

The amount of contraction, then, depends on the strength of the stimulus, whatever be the stimulus; but this holds good within certain limits only; to this point, however, we shall return later on.

$\$ 51$. If, having ascertained in a perfectly fresh muscle-nerve preparation the amount of contraction produced by this and that strength of stimulus, we leave the preparation by itself for some time-say for a few hours -and then repeat the observations, we shall find that stronger stimulistronger shocks, for instance-are required to produce the same amount of contraction as before; that is to say, the irritability of the preparation, the power to respond to stimuli, has in the meanwhile diminished. After a further interval we should find the irritability still further diminished; even very strong shocks would be unable to evoke contractions as large as those previously caused by weak shocks. At last we should find that no shocks, no stimuli, however strong, were able to produce any visible contraction whatever. The amount of contraction, in fact, evoked by a stimulus depends not only on the strength of the stimulus, but also on the degree of irritability of the muscle-nerve preparation. 
Immediately upon removal from the body, the preparation possesses a certain amount of irritability, not differing very materially from that which the muscle and nerve possess while within and forming an integral part of the body; but after removal from the body the preparation loses irritability, the rate of loss being dependent on a variety of circumstances; and this goes on until, since no stimulus which we can apply will give rise to a contraction, we say the irritability has wholly disappeared.

We might take this disappearance of irritability as marking the death of the preparation, but it is followed sooner or later by a curious change in the muscle, which is called rigor mortis, and which we shall study presently ; and it is convenient to regard this rigor mortis as marking the death of the muscle.

The irritable muscle, then, when stimulated either directly, the stimulus being applied to itself, or indirectly, the stimulus being applied to its nerve, responds to the stimulus by a change of form which is essentially a shortening and thickening. By the shortening (and thickening) the muscle in contracting is able to do work, to move the parts to which it is attached; it thus sets free energy. We have now to study more in detail how this energy is set free, and the laws which regulate its expenditure.

\section{On the Changes which take place in a Muscle during a Contraction.}

\section{The Change in Form.}

\$2. An ordinary skeletal muscle consists of elementary muscle fibres, bound together in variously arranged bundles by connective tissue which carries bloodvessels, nerves, and lymphatics.

The contraction of a muscle is the contraction of all or some of its elementary fibres, the connective tissue being passive; hence while those fibres of muscle which end directly in the tendon, in contracting pull directly on the tendon, those which do not so end pull indirectly on the tendon by means of the connective tissue between the bundles, which connective tissue is continuous with the tendon.

Each muscle is supplied by one or more branches of nerves composed of medullated fibres, with a certain proportion of non-medullated fibres. These branches running in the connective tissue divide into smaller branches and twigs between the bundles and fibres. Some of the nerve fibres are distributed to the bloodvessels, and others end in a manner of which we shall speak later on in treating of muscular sensations; but by far the greater part of the medullated fibres and in the muscular fibres, the arrangement being such that every muscular fibre is supplied with at least one medullated nerve fibre, which joins the muscular fibre somewhere about the middle between its two ends or sometimes nearer one end, in a special nerve ending, of which we shall presently have to speak, called an endplate. The nerve fibres thus destined to end in the muscular fibres divide as they enter the muscle, so that what, as it enters the muscle, is a single nerve fibre, may, by dividing, end as several nerve fibres in several muscular fibres. Sometimes two nerve fibres join one muscular fibre, but in this case the end-plate of each nerve fibre is still at some distance from the end of the muscular fibre. It follows that when a muscular fibre is stimulated by means of a nerve fibre, the nervous impulse travelling down the nerve fibre falls into the muscular fibre not at one end but at about its middle; it is the middle of the fibre which is affected first by the nervous impulse, and the changes in the muscular substance started in the middle of the muscular fibre travel thence to the two ends of the fibre. In an ordinary skele- 
tal muscle, however, as we have said, the fibres and bundles of fibres begin and end at different distances from the ends of the muscle, and the nerve or nerves going to the muscle divide and spread out in the muscle in such a way that the end-plates, in which the individual fibres of the nerve end, are distributed widely over the muscle at very different distances from the ends of the muscle. Hence, if we suppose a single nervous impulse, such as that generated by a single induction-shock, or a series of such impulses to be started at the same time at some part of the trunk of the nerve in each of the fibres of the nerve going to the muscle, these impulses will reach very different parts of the muscle at about the same time and the contractions which they set going will begin, so to speak, nearly all over the whole muscle at the same time, and will not all start in any particular zone or area of the muscle.

$\S 53$. The wave of contraction. We have seen, however, that under the influence of urari the nerve fibre is unable to excite contractions in a muscular fibre, although the irritability of the muscular fibre itself is retained. Hence in a muscle poisoned by urari the contraction begins at that part of the muscular substance which is first affected by the stimulus, and we may start a contraction in what part of the muscle we please by properly placing the electrodes.

Some muscles, such, for instance, as the sartorius of the frog, though of some length, are composed of fibres which run parallel to each other from one end of the muscle to the other. If such a muscle be poisoned with urari so as to eliminate the action of the nerves and stimulated at one end (an induction-shock sent through a pair of electrodes placed at some little distance apart from each other at the end of the muscle may be employed, but better results are obtained if a mode of stimulation, of which we shall have to speak presently, viz. the application of the "constant current," be adopted), the contraction which ensues starts from the end stimulated, and travels thence along the muscle. If two levers be made to rest on, or be suspended from, two parts of such a muscle placed horizontally, the parts being at a known distance from each other and from the part stimulated, the progress of the contraction may be studied.

The movements of the levers indicate in this case the thickening of the fibres which is taking place at the parts on which the levers rest or to which they are attached; and if we take a graphic record of these movements, bringing the two levers to mark one immediately below the other, we shall find that the lever nearer the part stimulated begins to move earlier, reaches its maximum earlier, and returns to rest earlier than does the further lever. The contraction, started by the stimulus, in travelling along the muscle from the part stimulated reaches the nearer lever some little time before it reaches the further lever, and has passed by the nearer lever some little time before it has passed by the further lever; and the further apart the two levers are the greater will be the difference in time between their movements. In other words, the contraction travels along the muscle in the form of a wave, each part of the muscle in succession from the end stimulated swelling out and shortening as the contraction reaches it, and then returning to its original state. And what is true of the collection of parallel fibres which we call the muscle is also true of each fibre, for the swelling at any part of the muscle is only the sum of the swelling of the individual fibres; and if we were able to take a single long fibre and stimulate it at one end, we should be able, under the microscope, to see a swelling or bulging accompanied by a corresponding shortening, $i$. $e_{\text {., to }}$ see a contraction,-sweep along the fibre from end to end.

If, in the graphic record of the two levers just mentioned, we count the 
number of vibrations of the tuning-fork which intervene between the mark on the record which indicates the beginning of the rise of the near lever (that is, the arrival of the contraction wave at this lever) and the mark which indicates the beginning of the rise of the far lever, this will give us the time which it has taken the contraction wave to travel from the near to the far lever. Let us suppose this to be 0.005 second. Let us suppose the distance between the two levers to be $15 \mathrm{~mm}$. The contraction wave, then, has taken 0.005 second to travel $15 \mathrm{~mm}$., that is to say it has travelled at the rate of 3 metres per second. And, indeed, we find by this, or by other methods, that in the frog's muscles the contraction wave does travel at a rate which may be put down as from 3 to 4 metres a second, though it varies under different conditions. In the warm-blooded mammal the rate is somewhat greater, and may probably be put down at 5 metres a second in the excised muscle, rising possibly to 10 metres in a muscle within the living body.

If, again, in the graphic record of the two levers we count, in the case of either lever, the number of vibrations of the tuning-fork which intervene between the mark where the lever begins to rise and the mark where it has finished its fall and returned to the base-line, we can measure the time intervening between the contraction wave reaching the lever and leaving the lever on its way onward, that is to say we can measure the time which it has taken the contraction wave to pass over the part of the muscle on which the lever is resting. Let us suppose this time to be, say, 0.1 second. But a wave which is travelling at the rate of 3 metres a second, and takes 0.1 second to pass over any point must be $300 \mathrm{~mm}$. long. And, indeed, we find that in the frog the length of the contraction wave may be put down as varying from 200 to $400 \mathrm{~mm}$., and in the mammal it is not very different.

Now, as we have said, the very longest muscular fibre is stated to be at most only about $40 \mathrm{~mm}$. in length; hence, in an ordinary contraction, during the greater part of the duration of the contraction the whole length of the fibre will be occupied by the contraction wave. Just at the beginning of the contraction there will be a time when the front of the contraction wave has reached, for instance, only half way down the fibre (supposing the stimulus to be applied, as in the case we have been discussing, at one end only), and just at the end of contraction there will be a time, for instance, when the contraction has left the half of the fibre next to the stimulus, but has not yet cleared away from the other half. But nearly all the rest of the time every part of the fibre will be in some phase or other of contraction, though the parts nearer the stimulus will be in more advanced phases than the parts further from the stimulus.

This is true when a muscle of parallel fibres is stimulated artificially at one end of the muscles, and when, therefore, each fibre is stimulated at one end. It is, of course, all the more true when a muscle of ordinary construction is stimulated by means of its nerve. The stimulus of the nervous impulse impinges in this case on the muscle fibre at the end-plate which, as we have said, is placed toward the middle of the fibre, and the contraction wave travels from the end-plate in opposite directions toward each end, and has accordingly only about half the length of the fibre to run in. All the more, therefore, must the whole fibre be in a state of contraction at the same time.

It will be observed that the contraction wave includes not only the contraction proper and the thickening and shortening, but the relaxation and return to the natural form ; the first part of the wave up to the summit of the crest corresponds to the shortening and thickening; the decline from the summit onward corresponds to the relaxation. But we have already 
insisted that the relaxation is an essential part of the whole act; indeed, in a certain sense, as essential as the shortening itself.

\$54. Optical changes in a muscular fibre during contraction. So far we have been dealing with the muscle as a whole and as observed with the naked eye, though we have incidentally spoken of fibres. We have now, confining our attention exclusively to skeletal muscles, to consider what microscopic changes take place during a contraction, what are the relations of the histological features of the muscle fibre to the act of contraction. Unfortunately, our knowledge of the minute structure of the fibre is as yet so limited that any statements must of necessity be but speculative. When muscle contracts there is a translocation of molecules whereby there occurs not only a change of form, but other optical (polariscopical and microscopical) alterations which are due to the movement of refractive particles.

The long cylindrical sheath of sarcolemma is occupied by muscle substance. After death the muscle substance may separate from the sarcolemma, leaving the latter as a distinct sheath, but during life the muscle substance is adherent to the sarcolemma, so that no line of separation between the two can be made out; the movements of the one follow exactly all the movements of the other.

Scattered in the muscle substance, but, in the mammal, lying for the most part close under the sarcolemma, are a number of nuclei, oval in shape, with their long axes parallel to the length of the fibre. Around each nucleus is a thin layer of granular-looking substance very similar in appearance to that forming the body of a white blood-corpuscle, and like that often spoken of as undifferentiated protoplasm. A small quantity of the same granular substance is prolonged for some distance, as a narrow conical streak from each end of the nucleus, along the length of the fibre.

With the exception of these nuclei with their granular-looking bed and the end-plate or end-plates, to be presently described, all the rest of the space enclosed by the sarcolemma from one end of the fibre to the other appears to be occupied by a peculiar material, striated muscle substance.

It is called striated because it is marked out, and that along the whole length of the fibre, by transverse bands [Fig. 28], stretching right across

[FIG. 28.

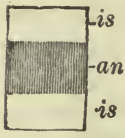

Diagrammatic Repre. sentation of a Musclecase : an, dim bands; $i s$, bright bands.] the fibre, of substance which is very transparent, bright substance, alternating with similar bands of substance which has a dim cloudy appearance, dim substance; that is to say, the fibre is marked out along its whole length by alternate bright bands and dim bands. The bright bands are on an average about $1 \mu$ or $1.5 \mu$ and the $\operatorname{dim}$ bands about $2.5 \mu$ or $3 \mu$ thick. By careful focusing, both bright bands and dim bands may be traced through the whole thickness of the fibre, so that the whole fibre appears to be composed of bright discs and dim dises placed alternately one upon the other along the whole length of the fibre, the arrangement being broken by the end-plate and here and there by the nuclei.

$\S 55$. We may now return to the question, What happens when a contraction wave sweeps over the fibre?

Muscular fibres may be examined even under high powers of the microscope while they are yet living and contractile; the contraction itself may be seen, but the rate at which the wave travels is too rapid to permit satisfactory observations to be made as to the minute changes which accompany the contraction. It frequently happens however that when living muscle has been treated with certain reagents, as for instance with osmic acid vapor, and subsequently prepared for examination, fibres are found in which a 
bulging, a thickening and shortening, over a greater or less part of the length of the fibre, has been fixed by the osmic acid or other reagent. Such a bulging obviously differs from a normal contraction in being confined to a part of the length of the fibre, whereas, as we have said, a normal wave of contraction, being very much longer than any fibre, occupies the whole length of the fibre at once. We may however regard this bulging as a very short, a very abbreviated wave of contraction, and assume that the changes visible in such a short bulging also take place in a normal contraction.

Admitting this assumption, we learn from such preparations that in the contracting region of the fibre, while both dim and bright bands become broader across the fibre, and correspondingly thinner along the length of the fibre, a remarkable change takes place between the dim bands, bright bands, and granular lines. We have seen that in the fibre at rest the intermediate line in the bright band is in most cases inconspicuous; in the contracting fibre, on the contrary, a dark line in the middle of the bright band in the position of the intermediate line becomes very distinct. As we pass along the fibre from the beginning of the contraction wave to the summit of the wave, where the thickening is greatest, this line becomes more and more striking, until at the lieight of the contraction it becomes a very marked dark line or thin dark band. Pari passu with this change, the distinction between the dim and the bright bands become less and less marked; these appear to become confused together, until at the height of the contraction, the whole space between each two now conspicuous dark lines is occupied by a substance which can be called neither dim nor bright, but which in contrast to the dark line appears more or less bright and transparent. So that in the contracting part there is, at the height of the contraction, a reversal of the state of things proper to the part at rest. The place occupied by the bright band, in the state of rest, is now largely filled by a conspicuous dark line which previously was represented by the inconspicuous intermediate line, and the place occupied by the conspicuous dim band of the fibre at rest now seems by comparison with the dark line the brighter part of the fibre. The contracting fibre is, like the fibre at rest, striated, but its striation is different in its nature from the natural striation of the resting fibre; and it is held by some that in the earlier phases of the contraction, while the old nat ural striation is being replaced by the new striation, there is a stage in which all striation is lost.

We may add that the outline of the sarcolemma, which in the fibre at rest is quite even, becomes during the contraction indented opposite the intermediate line, and bulges out in the interval between each two intermediate lines, the bulging and indentation becoming more narked the greater the contraction.

$\S 56$. We can learn something further about this remarkable change by examining the fibre under polarized light.

When ordinary light is sent through a Nicol prism (which is a rhomb of Iceland spar divided into two in a certain direction, the halves being subsequently cemented together in a special way) it undergoes a change in passing through the prism and is said to be polarized. One effect of this polarization is that a ray of light which has passed through one Nicol prism will or will not pass through a second Nicol according to the relative position of the two prisms. Thus, if the second Nicol be so placed that what is called its "optic axis" be in a line with or parallel to the optic axis of the first Nicol the light passing through the first Nicol will also pass through the second. But if the second Nicol be rotated until its optic axis is at right angles with the optic axis of the first Nicol none of the light passing through the former will pass through the latter; the prisms in this position are said to be "crossed." In intermediate positions more or less light passes through the second Nicol according to the angle between the two optic axes. 
Hence when one Nicol is placed beneath the stage of a microscope so that the light from the mirror is sent through it, and another Nicol is placed in the evepiece, the field of the microscope will appear dark when the eye-piece Nicol is rotated so that its optic axis is at right angles to the optic axis of the lower Nicol, and consequently the light passing through the lower Nicol is stopped by it. If, however, the optic axis of the eye-piece Nicol is parallel to that of the lower Nicol, the light from the latter will pass through the former and the field will be bright; and as the eye-piece is gradually rotated from one position to the other the brightness of the field will dininish or increase.

Both the Nicols are composed of doubly refractive material. If now a third doubly refractive material be placed on the stage, and therefore between the two Nicols, the light passing through the lower Nicol will (in a certain position of the doubly refractive material on the stage, that is to say, when its optic axes have a certain position) pass through it. and also through the crossed Nicol in the eyepiece. Hence the doubly refractive material on the stage (or such parts of it as are in the proper position in respect to their optic axes) will, when the eye-piece Nicol is crossed, appear illuminated and bright on a dark field. In this way the existence of doubly refractive material in a preparation may be detected.

When muscle prepared and mounted in Canada balsam is examined in the microscope between Nicol prisms, one on the stage below the object, and the other in the eye-piece, the fibres stand out as bright objects on the dark ground of the field when the axes of the prisms are crossed. On closer examination it is seen that the parts which are bright are chiefly the dim bands. This indicates that it is the dim bands which are doubly refractive, anisotropic, or are chiefly made up of anisotropic substance; there seems, however, to be some slight amount of anisotropic substance in the bright bands, though these as a whole appear singly refractive or isotropic. The fibre accordingly appears banded or striated with alternate bands of anisotropic and isotropic material. According to most authors such an alternation of anisotropic and (chiefly) isotropic bands which is obvious in a dead and prepared fibre.exists also in the living fibre; but some maintain that the living fibre is uniformly anisotropic.

Now, when a fibre contracts, in spite of the confusion previously mentioned between dim and bright bands, there is no confusion between the anisotropic and isotropic material. The anisotropic, doubly refractive bands, bright under crossed Nicols, occupying the position of the dim bands in the resting fibre, remain doubly refractive, bright under crossed Nicols, even at the very height of the contraction. The isotropic, singly refractive bands, dark under crossed Nicols, occupying the position of the bright bands in the fibre at rest, remain isotropic and dark under crossed Nicols at the very height of the contraction. All that can be seen is that the singly refractive isotropic bands become very thin indeed during the contraction, while the anistropic bands, though of course becoming thinner and broader in the contraction, do not become so thin as do the isotropic bands; in other words, while both bands become thinner and broader, the doubly refractive anisotropic band seems to increase at the expense of the singly refractive isotropic band.

$\$ 57$. The mere broadening and shortening of each section of the fibre is at bottom a translocation of the molecules of the muscle substance. If we imagine a company of 100 soldiers, ten ranks deep, with ten men in each rank, rapidly, but by a series of gradations, to extend out into a double line with 50 men in each line, we shall have a rough image of the movement of the molecules during a muscular contraction. But, from what has been said, it is obvious that the movement, in striated muscle at least, is a very complicated one; in other forms of contractile tissue it may be, as we shall. see, more simple. Why the movement is so complicated in striated muscle, what purposes it serves, why the skeletal muscles are striated, we do not at present 
know. Apparently where swift and rapid contraction is required the contractile tissue is striated muscle; but how the striation helps, so to speak, the contraction we do not know. We cannot say what share in the act of contraction is to be allotted to the several parts. Since, during a contraction, the fibre bulges out more opposite to each dim disc, and is indented opposite to each bright disc, since the dim disc is more largely composed of anisotropic material than the rest of the fibre, and since the anisotropic material in the position of the dim disc increases during a contraction, we might perhaps infer that the dim dise rather than the bright dise is the essentially active part. Assuming that the fibrillar substance is more abundant in the dim discs, while the interfibrillar substance is more abundant in the bright discs, and that the fibrillar substance is anisotropic (and hence the dim discs largely anisotropic), while the interfibrillar substance is isotropic, we might also be inclined to infer it is the fibrillar and not the interfibrillar substance which really carries out the contraction; but even this much is not yet definitely proved.

One thing must be remembered. The muscle substance, though it possesses the complicated structure, and goes through the remarkable changes which we have described, is while it is living and intact in a condition which we are driven to speak of as semi-fluid. The whole of it is essentially mobile. The very act of contraction indeed shows this; but it is mobile in the sense that no part of it, except of course the nuclei and sarcolemma, neither dim nor bright substance, neither fibrillar nor interfibrillar substance, can be regarded as a liard and fast structure. A minute nematoid worm has been seen wandering in the midst of the substance of a living contractile fibre; as it moved along, the muscle substance gave way before it, and closed up again behind it, dim bands and bright bands all falling back into their proper places. We may suppose that in this case the worm threaded its way in a fluid interfibrillar substance between and among highly extensible and elastic fibrillæ. But even on such a view, and still more on the view that the fibrillar substance also was broken and closed up again, the maintenance of such definite histological features as those which we have described in material so mobile can only be effected, even in the fibre at rest, at some considerable expenditure of energy, which energy it may be expected has a chemical source. During the contraction there is a still further expenditure of energy, some of which, as we have seen, may leave the muscle as "work done;" this energy, likewise, may be expected to have a chemical source. We must, therefore, now turn to the chemistry of muscle.

\section{The Chemistry of Muscle.}

$\S 58$. We said in the Introduction that it was difficult to make out with certainty the exact chemical differences between dead and living substance. Muscle, however, in dying undergoes a remarkable chemical change, which may be studied with comparative ease. All muscles, within a certain time after "general" death of the body, lose their irritability, which is succeeded by an event somewhat more sudden, viz., the entrance into the condition known as rigor mortis. The occurrence of rigor mortis, or cadaveric rigidity, as it is sometimes called, which may be considered as a token of the death of the muscle, is marked by the following features: The living muscle possesses a certain translucency, the rigid muscle is distinctly more opaque. The living muscle is very extensible and elastic, it stretches readily and to a considerable extent when a weight is hung upon it, or when any traction is applied to it, but speedily and, under normal circumstances, completely returns to its original length when the weight of traction is removed; as we 
shall see, however, the rapidity and completeness of the return depends on the condition of the muscle, a well-nourished active muscle regaining its normal length much more rapidly and completely than a tired and exhausted muscle. A dead rigid muscle is much less extensible, and at the same time much less elastic; the muscle now requires considerable force to stretch it, and when the force is removed, does not, as before, return to its former length. To the touch the rigid muscle has lost much of its former softness, and has become firmer and more resistant. The entrance into rigor mortis is, moreover, accompanied by a shortening or contraction, which may, under certain circumstances, be considerable. 'The energy of this contraction is not great, so that any actual shortening is easily prevented by the presence of even a slight opposing force.

Now the chemical features of the dead rigid muscle are also strikingly different from those of the living muscle.

$\S 59$. If a dead muscle, froin which all fat, tendon, fascia, and connective tissue have been as much as possible removed, and which has been freed from blood by the injection of " normal" saline solution, be minced and repeatedly washed with water, the washings will contain certain forms of albumin and certain extractive bodies of which we shall speak directly. When the washing has been continued until the wash-water gives no proteid reaction, a large portion of muscle will still remain undissolved. If this be treated with a 10 per cent. solution of a neutral salt, ammonium chloride being the best, a large portion of it will become dissolved; the solution, however, is more or less imperfect and filters with difficulty. If the filtrate be allowed to fall drop by drop into a large quantity of distilled water, a white flocculent matter will be precipitated. This flocculent precipitate is myosin. Myosin is a proteid, giving the ordinary proteid reactions, and having the same general elementary composition as other proteids. It is soluble in dilute saline solutions, especially those of ammonium chloride, and may be classed in the globulin family, though it is not so soluble as paraglobulin, requiring a stronger solution of a neutral salt to dissolve it; thus, while soluble in a 5 or 10 per cent. solution of such a salt, it is far less soluble in a 1 per cent. solution, which, as we have seen, readily dissolves paraglobulin. From its solutions in neutral saline solution it is precipitated by saturation with a neutral salt, preferably sodium chloride, and may be purified by being washed with a saturated solution, dissolved again in a weaker solution, and reprecipitated by saturation. Dissolved in saline solutions it readily coagulates when heated-i. $e_{\text {. }}$, is converted into coagulated proteid-and it is worthy of notice that it coagulates at a comparatively low temperature, viz., about $56^{\circ} \mathrm{C}$.; this, it will be remembered, is the temperature at which fibrinogen is coagulated, whereas paraglobulin, serum-albumin, and many other proteids, do not coagulate until a higher temperature $\left(75^{\circ} \mathrm{C}.\right)$ is reached. Solutions of myosin are precipitated by alcohol, and the precipitate, as in the case of other proteids, becomes, by continued action of the alcohol, altered into coagulated insoluble proteid.

We have seen that paraglobulin, and, indeed, any member of the globulin group, is very readily changed by the action of dilute acids into a body called acid-albumin, characterized by not being soluble either in water or in dilute saline solutions, but readily soluble in dilute acids and alkalies, from its solutions in either of which it is precipitated by neutralization, and by the fact that the solutions in dilute acids and alkalies are not coagulated by heat. When, therefore, a globulin is dissolved in dilute acid, what takes place is not a mere solution, but a chemical change; the globulin cannot be got back from the solution, it has been changed into acid-albu- 
min. Similarly when globulin is dissolved in dilute alkalies it is changed into alkali-albumin; and, broadly speaking, alkali-albumin precipitated by neutralization can be changed by solution with dilute acids into acid-albumin, and acid-albumin by dilute alkalies into alkali-albumin.

Now myosin is similarly, and even more readily than is globulin, converted into acid-albumin, and by treating a muscle, either washed or not, directly with dilute hydrochloric acid, the myosin may be converted into acid-albumin and dissolved out. Acid-albumin obtained by dissolving muscle in dilute acid used to be called syntonin, and it used to be said that a muscle contained syntonin; the muscle, however, contains myosin, not syntonin, but it may be useful to retain the word syntonin to denote acidalbumin obtained by the action of dilute acid on myosin. By the action of dilute alkalies, myosin may similarly be converted into alkali-albumin.

From what has been above stated it is obvious that myosin has many analogies with fibrin, and we have yet to mention some striking analogies; it is, however, much more soluble than fibrin, and, speaking generally, it may be said to be intermediate in its character between fibrin and globulin. On keeping, and especially on drying, its solubility is much diminished.

Of the substances which are left in washed muscle, from which all the nıyosin has been extracted by ammonium chloride solution, little is known. If washed muscle be treated directly with dilute hydrochloric acid, a large part of the material of the muscle passes, as we have said, at once into syntonin. The quantity of syntonin thus obtained may be taken as roughly representing the quantity of myosin previously existing in the muscle. A more prolonged action of the acid may dissolve out other proteids, besides myosin, left after the washing. The portion insoluble in dilute hydrochloric acid consists in part of the gelatin-yielding and other substances of the sarcolemma and of the connective and other tissues between the bundles, of the nuclei of these tissues and of the fibres themselves, and in part, possibly, of some portions of the muscle substance itself. We are not, however, at present in a position to make any very definite statement as to the relation of the myosin to the structural features of muscle. Since the dim bands are rendered very indistinct by the action of a 10 per cent. sodium chloride solution, we may, perhaps, infer that myosin enters largely into the composition of the dim bands, and, therefore, of the fibrillæ; but it would be hazardous to say much more than this.

$\S 60$. Living muscle may be frozen, and yet, after certain precautions, will, on being thawed, regain its irritability, or, at all events, will for a time be found to be still living in the sense that it has not yet passed into rigor mortis. We may, therefore, take living muscle which has been frozen as still living.

If living contractile muscle, freed as much as possible from blood, be frozen, and while frozen minced and rubbed up in a mortar with four times its weight of snow containing 1 per cent. of sodium chloride, a mixture is obtained which, at a temperature just below $0^{\circ} \mathrm{C}$., is sufficiently fluid to be filtered, though with difficulty. The slightly opalescent filtrate, or muscleplasma, as it is called, is at first quite fluid, but will, when exposed to the ordinary temperature, become a solid jelly, and afterward separate into a clot and serum. It will, in fact, coagulate like blood-plasma, with this difference, that the clot is not firm and fibrillar, but loose, granular, and flocculent. During the coagulation the fluid, which before was neutral or slightly alkaline, becomes distinctly acid.

The clot is myosin. It gives all the reactions of myosin obtained from dead muscle.

The serum contains an albumin very similar to, if not identical with 
serum-albumin, a globulin differing somewhat from and coagulating at a lower temperature than paraglobulin, and which to distinguish it from the globulin of blood has been called myoglobulin, some other proteids which need not be described here, and various "extractives" of which we shall speak directly. Such muscles as are red also contain a small quantity of hremoglobin, and of another allied pigment called histohomatin, to which pigments, indeed, their redness is due.

Thus, while dead muscle contains myosin, albumin, and other proteids, extractives, and certain insoluble matters, together with gelatinous and other substances not referable to the muscle substance itself, living muscle contains no myosin, but some substance or substances which bear somewhat the same relation to myosin that the antecedents of fibrin do to fibrin, and which give rise to myosin upon the death of the muscle. There are, indeed, reasons for thinking that the myosin arises from the conversion of a previously existing body which may be called myosinogen, and that the conversion takes place, or may take place, by the action of a special ferment, the conversion of myosinogen into myosin being very analngous to the conversion of fibrinogen into fibrin.

We may, in fact, speak of rigor mortis as characterized by a coagulation of the muscle-plasma, comparable to the coagulation of blood-plasma, but differing from it inasmuch as the product is not fibrin, but myosin. The rigidity, the loss of suppleness, and the diminished translucency appear to be at all events largely, though probably not wholly, due to the change from the fluid plasma to the solid myosin. We might compare a living muscle to a number of fine transparent membranous tubes containing blood-plasma. When this blood-plasma entered into the "jelly" stage of coagulation, the system of tubes would present many of the phenomena of rigor mortis. They would luse much of their suppleness and translucency, and acquire a certain amount of rigidity.

$\$ 61$. There is, however, one very marked and important difference between the rigor mortis of muscle and the coagulation of blood. Blood during its coagulation undergoes a slight change only in its reaction; but muscle during the onset of rigor mortis becomes distinctly acid.

A living muscle at rest is in reaction neutral, or, possibly from some remains of lymph adhering to it, faintly alkaline. If, on the other hand, the reaction of a thoroughly rigid muscle be tested, it will be found to be most distinctly acid. This development of an acid reaction is witnessed not only in the solid untouched fibre but also in expressed muscle-plasma; it seems to be associated in some way with the appearance of the myosin.

The exact causation of this acid reaction has not at present been clearly worked out. Since the coloration of the litmus produced is permanent, carbonic acid, which, as we shall immediately state, is set free at the same time, cannot be regarded as the active acid, for the reddening of litmus produced by carbonic acid speedily disappears on exposure. On the other hand, it is possible to extract fron rigid muscle a certain quantity of lactic acid, or rather of a variety of lactic acid known as sarcolactic acid ${ }^{1}$ and it has been thought that the appearance of the acid reaction of rigid muscle is due to a new formation or to an increased formation of this sarcolactic acid. There is much to be said in favor of this view, but it cannot at present be regarded as established heyond dispute.

Coincident with the appearance of this acid reaction, though, as we have said, not the direct cause of it, a large development of carbonic acid takes

1 There are many varieties of lactic acid, which are isomeric, having the same composition, $\mathrm{C}_{3} \mathrm{H}_{6} \mathrm{O}_{3}$, but differ in their reactions and especially in the solubility of their zinc salts. The variety present in muscle is distinguished as sarcolactic acid. 
place when muscle becomes rigid. Irritable living muscular substance, like all living substance, is continually respiring, that is to say, is continually consuming oxygen and giving out carbonic acid. In the body, the arterial blond going to the muscle gives up some of its oxygen, and gains a quantity of carbonic acid, thus becoming venous as it passes through the muscle capillaries. Even after removal from the body, the living muscle continues to take up from the surrounding atmosphere a certain quantity of oxygen and to give out a certain quantity of carbonic acid.

At the onset of rigor mortis there is a very large and sudden increase in this production of carbonic acid, in fact an outburst, as it were, of that gas. This is a phenomena deserving special attention. Knowing that the carbonic acid which is the outcome of the respiration of the whole body is the result of the oxidation of carbon-holding substances, we might very naturally suppose that the increased production of carbonic acid attendant on the development of rigor mortis is due to the fact that during that event a certain quantity of the carbon-holding constituents of the muscle are suddenly oxidized. But such a view is negatived by the following facts: In the first place, the increased production of carbonic acid during rigor mortis is not accompanied by a corresponding increase in the consumption of oxygen. In the second place, a muscle (of a frog, for instance) contains in itself no free or loosely attached oxygen; when subjected to the action of a mercurial air-pump it gives off no oxygen to a vacuum, offering in this respect a marked contrast to blood; and yet, when placed in an atmosphere free from oxygen, it will not only continue to give off carbonic acid while it remains alive, but will also exhibit at the onset of rigor mortis the same increased production of carbonic acid that is shown by a muscle placed in an atmosphere containing oxygen. It is obvious that in such a case the carbonic acid does not arise from the direct oxidation of the muscle substance, for there is no oxygen present at the time to carry on that oxidation. We are driven to suppose that during rigor mortis, some complex body, containing in itself ready-formed carbonic acid, so to speak, is split up, and thus carbonic acid is set free, the process of oxidation by which that carbonic acid was formed out of the carbon-holding constituents of the muscle having taken place at some anterior date.

Living resting muscle, then, is alkaline or neutral in reaction, and the substance of its fibres contains a coagulable plasma. Dead rigid muscle, on the other hand, is acid in reaction, and no longer contains a coagulable plasma, but is laden with the solid myosin. Further, the change from the living irritable condition to that of rigor mortis is accompanied by a large and sudden development of carbonic acid.

It is found, moreover, that there is a certain amount or parallelism between the intensity of the rigor mortis, the degree of acid reaction and the quantity of carbonic acid given ont. If we suppose, as we fairly may do, that the intensity of the rigidity is dependent on the quantity of myosin deposited in the fibres, and the acid reaction to the development, if not of lactic acid, at least of some other substance, the parallelism between the three products, myosin, acid-producing substance, and carbonic acid, would suggest the idea that all three are the results of the splitting-up of the same highly complex substance. No one has at present, however, succeeded in isolating or in otherwise definitely proving the existence of such a body, and though the idea seems tempting, it may in the end prove totally erroneous.

$\$ 62$. As to the other proteids of muscle, such as the albumin and the globulin, we know as yet nothing concerning the parts which they play and the changes which they undergo in the living muscle or in rigor mortis.

Besides the fat which is found, and that not infrequently in abundance, 
in the connective tissue between the fibres, there is also present in the muscular substance within the sarcolemma, always some and at times a great deal of fat, chiefly ordinary fat, viz., stearin, palmitin, and olein in variable proportion, but also the more complex fat lecithin. As to the function of these several fats in the life of the muscle we know little or nothing

Carbohydrates, the third of the three great classes in which we may group the energy-holding substances of which the animal body and its food are alike composed, viz., proteids, fat, and carbohydrates, are represented in muscle by a peculiar body, glycogen, which we shall have to study in detail later on. We must here merely say that glycogen is a body closely allied to starch, having a formula, which may be included under the general formula for starches, $x\left(\mathrm{C}_{6} \mathrm{H}_{10} \mathrm{O}_{5}\right)$, and may like it be converted by the action of acids, or by the action of particular ferments known as amylolytic ferments, into some form of sugar, dextrose $\left(\mathrm{C}_{6} \mathrm{H}_{12} \mathrm{O}_{6}\right)$, or some allied sugar. Many, if not all, living muscles contain a certain amount, and some, under certain circumstances, a considerable amount, of glycogen. During or after rigor mortis this glycogen is very apt to be converted into dextrose, or an allied sugar. The muscles of the embryo at an early stage contain a relatively enormous quantity of glycogen, a fact which suggests that the glycogen of muscle is carbohydrate food of the muscle about to be wrought up into the living muscular substance.

The bodies which we have called extractives are numerous and varied. They are especially interesting, since it seems probable that they are waste products of the metabolism of the muscular substance, and the study of them may be expected to throw light on the chemical change which muscular substance undergoes during life. Since, as we shall see, muscular substance forms by far the greater part of the nitrogenous, that is, proteid, portion of the borly, the nitrogenous extractives of muscle demand peculiar attention. Now the body urea, which we shall have to study in detail later on, far exceeds in importance all the other nitrogenous extractives of the body as a whole, since it is practically the one form in which nitrogenous wastes leave the body; if we include with urea the closely allied uric acid (which for present purposes may simply be regarded as a variety of urea), we may say that all the nitrogen taken in as food sooner or later leaves the body as urea; compared with this all other nitrogenous waste thrown out from the body is insignificant. Of the urea which thus leaves the body, a considerable portion must at some time or other have existed, or to speak more exactly, its nitrogen must have existed as the nitrogen of the proteids of muscular substance. Nevertheless, no urea at all is, in normal conditions, present in muscular substance either living and irritable or dead and rigid; urea does not arise in muscular substance itself as one of the immediate waste products of muscular substance.

There is, however, always present in relatively considerable amount, on an average about 0.25 per cent. of wet muscle, a remarkable body, kreatin. This is, in one sense, a compound of urea; it may be split up into urea and sareosin. This latter body is a methyl glycin, that is to say, a glycin in which methyl has been substituted for hydrogen, and glycin itself is amidoacetic acid, a compound of amidogen, that is a representative of ammonia and acetic acid. Hence kreatin contains urea, which has close relations with ammonia, together with another representative of ammonia, and a surplus of carbon and hydrogen arranged as a body belonging to the fatty acid series. We shall have to return to this kreatin and consider its relation to urea and to muscle when we come to deal with urine.

The other nitrogenous extractives, such as karnin, hypoxanthin (or 
sarkin), xanthin, taurin, etc., occur in small quantity, and need not be dwelt on here.

Among non-nitrogenous extractives the most important is the sarcolactic acid, of which we have already spoken; to this may be added sugar in some form or other, either coming from glycogen or from some other source.

The ash of muscle, like the ash of the blood corpuscles, and, indeed the ash of the tissues in general, as distinguished from the blood, or plasma, or lymph on which the tissues live, is characterized by the preponderance of potassium salts and of phosphates ; these form, in fact, nearly 80 per cent. of the whole ash.

$\S 63$. We may now pass on to the question, What are the chemical changes which take place when a living resting muscle enters into a contraction? These changes are most evident after the muscle has been subjected to a prolonged tetanus; but there can be no doubt that the chemical events of a tetanus are, like the physical events, simply the sum of the results of the constituent single contractions.

In the first place the muscle becomes acid, not so acid as in rigor mortis, but still sufficiently so after a vigorous tetanus to turn blue litmus distinctly red. The cause of the acid reaction, like that of rigor mortis, is doubtful, but is in all probability the same in both cases.

In the second place, a considerable quantity of carbonic acid is set free ; and the production of carbonic acid in muscular contraction is altogether similar to the production of carbonic acid during rigor mortis; it is not accompanied by any corresponding increase in the consumption of oxygen. This is evident even in a muscle through which the circulation of blood is still going on; for though the blood passing through a contracting muscle gives up more oxygen than the blood passing through a resting muscle, the increase in the amount of oxygen taken up falls below the increase in the carbonic acid given out. But it is still more markedly shown in a muscle removed from the body; for in such a muscle both the contraction and the increase in the production of carbonic acid will go on in the absence of oxygen. A frog's muscle suspended in an atmosphere of nitrogen will remain irritable for some considerable time, and at each vigorous tetanus an increase in the production of carbonic acid may be readily ascertained.

Moreover, there seems to be a correspondence between the energy of the contraction and the amount of carbonic acid and the degree of acid reaction produced, so that, though we are now treading on somewhat uncertain ground, we are naturally led to the view that the essential chemical process lying at the bottom of a muscular contraction as of rigor mortis is the splitting up of some highly complex substance. But here the resemblance hetween rigor mortis and contraction ends. We have no satisfactory evidence of the formation during a contraction of any body like myosin. And this difference in chemical results tallies with an important difference between rigid muscle and contracting muscle. The rigid muscle, as we have seen, becomes less extensible, less elastic, less translucent; the contracting muscle remains no less translucent, elastic, and extensible than the resting muscle, indeed, there are reasons for thinking that the muscle in contracting becomes actually more extensible for the time being.

But if during a contraction myosin is not formed, what changes of proteid or nitrogenous matter do take place? We do not know. We have no evidence that kreatin, or any other nitrogenous extractive, is increased by the contraction of muscle; we have no evidence of any nitrogen waste at all as the result of a contraction; and, indeed, as we shall see later on, the study of the waste products of the body as a whole lead us to believe that the energy of the work done by the muscles of the body comes from the poten- 
tial energy of carbon compounds, and not of nitrogen compounds at all. But to this point we shall have to return.

$\$ 64$. We may sum up the chemistry of muscle somewhat as follows:

During life the muscular substance is continually taking up from the blood, that is, from the lymph, proteid, fatty and carbohydrate material, saline matters and oxygen; these it builds up into itself, how we do not know, and so forms the peculiar complex living muscular substance. The exact nature of this living substance is unknown to us. What we do know is that it is largely composed of proteid material, and that such bodies as myosinogen, myoglobulin, and albumin have something to do with the building of it up.

During rest this muscular substance, while taking in and building itself up out of or by means of the above-mentioned materials is continually giving off carbonic acid and continually forming nitrogenous waste such as kreatin. It also probably gives off some amount of sarcolactic acid, and possibly other non-nitrogenous waste matters.

During a contraction there is a great increase in the quantity of carbonic acid given off, of lactic acid and some other substance former giving an acid reaction, a greater consumption of oxygen, though the increase is not equal to the increase of carbonic acid, but as far as we can learn, no increase of nitrogenous waste.

During rigor mortis there is a similar increased production of carbonic acid and of some other acid-producing substance, accompanied by remarkable conversion of myosinogen into myosin, by which the rigidity of the dead fibre is brought about.

\section{Thermal Changes.}

$\$ 65$. The chemical changes during a contraction set free a quantity of energy, but only a portion of this energy appears in the "work done," a considerable portion takes on the form of heat. Though we shall have hereafter to treat this subject more fully, the leading facts may be given here.

Whenever a muscle contracts its temperature rises, indicating that heat is given out. When a mercury thermometer is plunged into a mass of muscles, such as those of the thigh of the dog, a rise of the mercury is observed upon the muscles being thrown into a prolonged contraction. More exact results, however, are obtained by means of a thermopile, by the help of which the rise of temperature caused by a few repeated single contractions, or indeed by a single contraction, may be observed, and the amount of heat given out approximately measured.

The thermopile may consist either of a single junction in the form of a needle plunged into the substance of the muscle, or of sereral junctions, either in the shape of a flat surface carefully opposed to the surface of muscle (the pile being balanced so as to move with the contracting muscle, and thus to keep the contact exact), or in the shape of a thin wedge, the edge of which comprising the actual junctions, is thrust into a mass of muscles and held in position by them. In all cases the fellow-junction or junctions must be kept at a constant temperature.

Another delicate method of determining the changes of temperature of a tissne is based upon the measurement of alterations in electric resistance which a fine wire, in contact with or plunged into the tissue, undergoes as the temperature of the tissue changes.

It has been calculated that the heat given out by the muscles of the thigh of a frog in a single contraction amounts to 3.1 micro-units of heat ${ }^{1}$ for each gramme of muscle, the result being obtained by dividing by five the total amount of heat given out in five successive single contractions. It

1 The micro-unit being a milligramme of water raised one degree Centigrade. 
will, however, be safer to regard these figures as illustrative of the fact that the heat given out is considerable, rather than as data for elaborate calculations. Moreover, we have no satisfactory quantitative determinations of the heat given out by the muscles of warm-blooded animals, though there can be no doubt that it is much greater. than that given out by the muscles of the frog.

There can hardly be any doubt that the heat thus set free is the product of chemical changes within the muscle-changes which, though they cannot for the reasons given above $(\$ 63)$ be regarded as simple and direct oxidations, yet, since they are processes dependent on the antecedent entrance of oxygen into the muscle, may be spoken of in general terms as a combustion; so that the muscle may be likened to a steam-engine, in which the combustion of a certain amount of material gives rise to the development of energy in two forms, as heat and as movement, there being certain quantitative relations between the amount of energy set free as heat and that giving rise to movenent. IVe must, however, earefully guard ourselves against pressing this analogy too closely. In the steam-engine we can distinguish clearly between the fuel which, through its combustion, is the sole source of energy, and the machinery, which is not consumed to provide energy, and only suffers wear and tear. In the muscle we cannot with certainty at present make such a distinction. It may be that the chemical changes at the bottom of a contraction do not involve the real living material of the fibre, but only some substance manufactured by the living material and lodged in some way, we do not know how, in the living material. It may be that when a fibre contracts it is this substance within the fibre which explodes, and not the fibre itself. If we further suppose that this substance is some complex compound of carbon and hydrogen, into which no nitrogen enters, we shall have an explanation of the difficulty referred to above ( $\$ 63)$, namely, that nitrogenous waste is not increased by a contraction. The special contractile. carbon-hydrogen substance, may then be compared to the charge of a gun, the products of its explosion being carbonic and sarcolactic acids, while the real living material of the fibre may be compared to the gun itself, but to a gun which itself is continually undergoing change far beyond mere wear and tear, among the products of which change nitrogenous bodies like kreatin are conspicuous. This view will certainly explain why kreatin is not increased during the contraction, while the carbonic and lactic acids are. But it must be remembered that such a view is not yet proved; it may be the living material of the fibre, as a whole, which is continually breaking down in an explosive decomposition, and as continually building itself up again out of the material supplied by the blood.

In a steam-engine only a certain amount of the total potential energy of the fuel issues as work, the rest being lost as heat, the proportion varying, but the work rarely, if ever, exceeding one-tenth of the total energy, and generally heing less. In the case of the muscle we are not at present in a position to draw up an exact equation between the latent energy on the one hand, and the two forms of actual energy on the other. We have reason to think that the proportion between heat and work varies considerably under different circumstances, the work sometimes rising as high as one-fifth, sometimes possibly sinking as low as one-twenty-fourth of the total energy; and observations seem to show that the greater the resistance which the muscle has to overcome, the larger the proportion of the total energy expended which goes out as work done. The muscle, in fact, seems to be so far selfregulating that the more work it has to do the greater, within certain limits, is the economy with which it works.

Lastly, it must be remembered that the giving out of heat by the muscle 
is not confined to the occasions when it is actually contracting. When, at a later period, we treat of the heat of the body generally, evidence will be brought forward that the muscles, even when at rest, are giving rise to heat, so that the heat given out at a contraction is not some wholly new phenomenon, but a temporary exaggeration of what is continually going oll at a more feeble rate.

\section{Electrical Changes.}

$\$ 66$. Besides chemical and thermal changes, a remarkable electric change takes place whenever a muscle contracts.

Muscle-currents. - If a muscle be removed in an ordinary manner from the body, and two non-polarizable electrodes, ${ }^{1}$ connected with a delicate galvanometer and many convolutions and high resistance, be placed on two

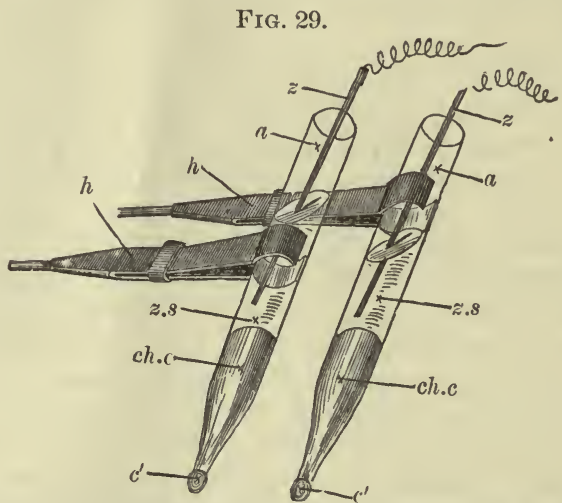

Non-polarizable Electrodes: $a$, the glass tube; $z$, the amalgamated zinc slips connected with their respective wires; $z$. s., the zinc sulphate solution; $c h . c$. , the plug of china-clay ; $c^{\prime}$, the portion of the china-clay plug projecting from the end of the tube; this can be moulded into any required form.

points of the surface of the muscle, a deflection of the galvanometer will take place, indicating the existence of a current passing through the galvanometer from the one point of the muscle to the other, the direction and amount of the deflection varying according to the position of the points. The "muscle-currents" thus revealed are seen to the best advantage when the muscle chosen is a cylindrical or prismatic one with parallel fibres, and when the two tendinous ends are cut off by clean incisions at right angles to the long axis of the muscle. The muscle then presents a transverse section (artificial) at each end and a longitudinal surface. We may speak of the latter as being divided into two equal parts by an imaginary transverse line on its surface called the "equator," containing all the points of the surface midway between the two ends. Fig. 30 is a diagrammatic representation of such a muscle, the line $a b$ being the equator. In such a muscle the development of the muscle-currents is found to be as follows:

The greatest deflection is observed when one electrode is placed at the

1 These (Fig. 29) consist essentially of a slip of thoroughly amalgamated zinc. dipping into a saturated solution of zinc sulphate, which in turn is brought into connection with the nerve or muscle by means of a plug or bridge of china-clay moistened with normal sodium chloride solution; it is important that the zinc should be thoroughly amalgamated. This form of electrode gives rise to less polarization than do simple platinum or copper electrodes. The clay affords a connection between the zinc and the tissue, which neither acts on the tissue nor is acted on by the tissue. Contact of any tissue with copper or platinum is in itself sufficient to develop a current. 
mid-point or equator of the muscle, and the other at either cut end; and the deflection is of such a kind as to show that positive currents are continually passing from the equator through the galvanometer to the cut end ; that is to say, the cut end is negative, relatively to the equator. The currents outside the muscle may be considered as completed by currents in the muscle from the cut end to the equator. In the diagram, Fig. 30, the arrows

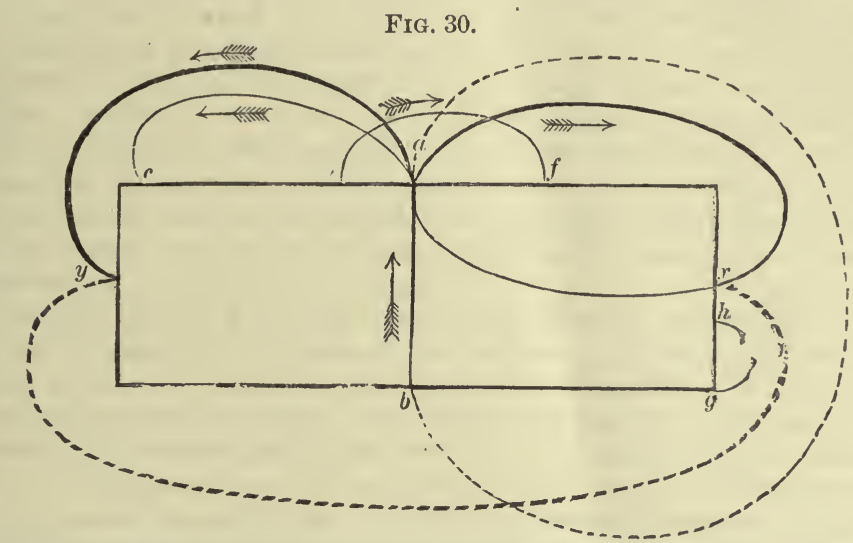

Diagram illustrating the Electric Currents of Nerve and Muscle: Being purely diagrammatic, it may serve for a piece either of nerve or of muscle, except that the currents at the transverse section cannot be shown in a nerve. The arrows show the direction of the current through the galvanometer.

$a b$, the equator. The strongest currents are those shown by the dark lines, as from $a$, at equator, to $x$ or to $y$ at the cut ends. The current from $a$ to $c$ is weaker than from $a$ to $y$, though both, as shown by the arrows, have the same direction. A current is shown from $e$, which is near the equator, to $f$, which is further from the equator. The current (in muscle) from a point in the circumference to a point nearer the centre of the transrerse section is shown at $g h$. From $a$ to $b$, or from $x$ to $y$, there is no current, as indieated by the dotted lines.

indicate the direction of the currents. If one electrode be placed at the equator $a b$, the effect is the same at whichever of the two cut ends, $x$ or $y$, the other is placed. If, one electrode remaining at the equator, the other be shifted from the cut end to a spot $(c)$ nearer to the equator, the current continues to have the same direction, but is of less intensity in proportion to the nearness of the electrodes to each other. If the two electrodes be placed at unequal distances $(e$ and $f$ ), one on either side of the equator, there will be a feeble current from the one nearer the equator to the one further off, and the current will be the feebler the more nearly they are equidistant from the equator. If they are quite equidistant-as, for instance, when one is placed on one cut end $(x)$ and the other on the other cut end $(y)$-there will be no current at all.

If one electrode be placed at the circumference of the transverse section and the other at the centre of the transverse section, there will be a current through the galvanometer from the former to the latter; there will be a current of similar direction, but of less intensity, when one electrode is at the circumference $(g)$ of the transverse section and the other at some point $(h)$ nearer the centre of the transverse section. In fact, the points which are relatively most positive and most negative to each other are points on the equator and the two centres of the transverse sections; and the intensity of the current between any two points will depend on the respective distances of those points from the equator and from the centre of the transverse section. 
Similar currents may be observed when the longitudinal surface is not the natural but an artificial one; indeed, they may be witnessed in even a piece of muscle, provided it be of cylindrical shape and composed of parallel fibres.

These " muscle-currents" are not mere transitory currents, disappearing as soon as the circuit is closed; on the contrary, they last a very considerable time. They must, therefore, be maintained by some changes going on in the muscle-by continual chemical action, in fact. They disappear as the irritability of the muscle vanishes, and are connected with those nutritive, so-called vital, changes which maintain the irritability of the muscle.

Muscle-currents, such as have just been described, may, we repeat, be observed in any cylindrical muscle suitably prepared, and similar currents, with variations which need not be discussed here, may be seen in muscles of irregular shape with obliquely or otherwise arranged fibres. And Du Bois-Reymond, to whom chiefly we are indebted for our knowledge of these currents, has been led to regard them as essential and important properties of living muscle. He has, moreover, advanced the theory that muscle may be considered as composed of electro-motive particles or molecules, each of which, like the muscle at large, has a positive equator and negative ends, the whole muscle being made up of these molecules in somewhat the same way (to use an illustration, which must not, however, be strained or considered as an exact one) as a magnet may be supposed to be made up of magnetic particles, each with its north and south pole.

There are reasons, however, for thinking that these muscle-currents have no such fundamental origin, that they are, in fact, of surface, and, indeed, of artificial origin. Without entering into the controversy on this question, the following important facts may be mentioned:

1. When a muscle is examined while it still retains uninjured its natural tendinous terminations, the currents are much weaker than when artificial transverse sections have been made; the natural tendinous end is less negative than the cut surface. But the tendinous end becomes at once negative when it is dipped in water or acid-indeed, when it is in any way injured. The less roughly, in fact, a muscle is treated the less evident are the muscle-currents; and it is maintained that if adequate care be taken to maintain a muscle in an absolutely natural condition, no such currents as those we have been describing exist at all-that natural living muscle is isoelectric, as it is called.

2. The surface of the uninjured inactive ${ }^{1}$ ventricle of the frog's heart, which is practically a mass of muscle, is isoelectric; no current is obtained when the electrodes are placed on any two points of the surface. If, however, any part of the surface be injured, or if the ventricle be cut across so as to expose a cut surface, the injured spot or the cut surface becomes at once more powerfully negative toward the uninjured surface, a strong current being developed which passes through the galvanometer from the uninjured surface to the cut surface or to the injured spot. The negativity thus developed in a cut surface passes off in the course of some hours, but may be restored by making a fresh cut and exposing a fresh surface.

The temporary duration of the negativity after injury, and its renewal upon fresh injury, in the case of the ventricle, in contrast to the more permanent negativity of injured skeletal muscle, is explained by the different structure of the two kinds of muscle. The cardiac muscle, as we shall hereafter see, is composed of short fibre-cells; when a cut is made a certain number of these fibre-cells are injured, giving rise to negativity, but the injury done to them stops with them, and is not propagated to the cells

${ }^{1}$ The necessity of its being inactive will be seen subsequently. 
with which they are in contact; hence, upon their death, the negativity and the current disappear. A fresh cut, involving new cells, produces fresh negativity and a new current. In the long fibres of the skeletal muscle, on the other hand, the effects of the injury are slowly propagated along the fibre from the spot injured.

Now, when a muscle is cut or injured, the substance of the fibres dies at the cut or injured surface. And many physiologists, among whom the most prominent is Hermann, have been led by the above and other facts to the conclusion that muscle-currents do not exist naturally in untouched, uninjured muscles, that the muscular substance is naturally, when living, isoelectric, but that whenever a portion of the muscular substance dies, it becomes, while dying, negative to the living substance, and thus gives rise to currents. They explain the typical currents (as they might be called) manifested by a muscle with a natural longitudinal surface and artificial transverse sections, by the fact that the dying cut ends are negative relatively to the rest of the inuscle.

Du Bois-Reymond and those with him offer special explanations of the above facts and of other objections which have been urged against the theory of naturally existing electro-motive molecules. Into these we cannot enter here. We must rest content with the statement that in an ordinary muscle currents such as have been described may be witnessed, but that strong arguments may be adduced in favor of the view that these currents are not "natural " phenomena, but essentially of artificial origin. It will, therefore, be best to speak of them as currents of rest.

$\$ 67$. Current of action. Negative variation of the muscle-current.-The controversy whether the "currents of rest" observable in a muscle be of natural origin or not, does not affect the truth or the importance of the fact that an electrical change takes place and a current is developed in a muscle whenever it enters into a contraction. When currents of rest are observable in a muscle, these are found to undergo a diminution upon the occurrence of a contraction, and this diminution is spoken of as "the negative variation " of the currents of rest. The negative variation may be seen when a muscle is thrown into a single contraction, but is most readily shown when the muscle is tetanized. Thus, if a pair of electrodes be placed on a muscle, one at the equator and the other at or near the transverse section, so that a considerable deflection of the galvanometer needle, indicating a considerable current of rest, be gained, the needle of the galvanometer will, when the muscle is tetanized by an interrupted current sent through its nerve (at a point too far from the muscle to allow of any escape of the current into the electrodes connected with the galvanometer), swing back toward zero; it returns to its original deflection when the tetanizing current is shut off.

Not only may this negative variation be shown by the galvanometer, but it, as well as the current of rest, may be used as a galvanic shock, and so employed to stimulate a muscle, as in the experiment known as "the rheoscopic frog." For this purpose the muscles and nerves need to be very irritable and in thoroughly good condition. Two muscle-nerve preparations, $A$ and $B$, having been made, and each placed on a glass plate for the sake of insulation, the nerve of the one, $B$, is allowed to fall on the muscle of the other, $A$, in such a way that one point of the nerve comes in contact with the equator of the muscle, and another point with one end of the muscle or with a point at some distance from the equator. At the moment the nerve is let fall and contact made, a current-viz., the "current of rest" of the muscle $A$-passes through the nerve; this acts as a stimulus to the nerve, and so causes a contraction in the muscle connected with the nerve. Thus, 
the muscle $A$ acts as a batterv, the completion of the circuit of which by means of the nerve of $B$ serves as a stimulus, causing the muscle $B$ to contract.

If, while the nerve of $B$ is still in contact with the nuscle of $A$, the nerve of the latter is tetanized with an interrupted current, not only is the muscle of $A$ thrown into tetanus, but also that of $B$, the reason being as follows: At each spasm of which the tetanus of $A$ is made up, there is a negative variation of the muscle-current of $A$. Each negative variation of the muscle-current of $A$ serves as a stimulus to the nerve of $B$, and is hence the cause of a spasm in the muscle of $B$; and the stimuli following each other rapidly, as being produced by the tetanus of $A$ they must do, the spasms in $B$ to which they give rise are also fused into a tetanus in $B$. $B$, in fact, contracts in harmony with $A$. This experiment shows that the negative variation accompanying the tetanus of a muscle, though it causes only a single swing of the galvanometer, is really made up of a series of negative variations, each single negative variation corresponding to the single spasms of which the tetanus is made up.

But an electrical change may be manifested even in cases when no currents of rest exist. We have stated $(\$ 66)$ that the surface of the uninjured inactive ventricle of the frog's heart is isoelectric, no currents being observed when the electrodes of a galvanometer are placed on two points of the surface. Nevertheless, a most distinct current is developed whenever the ventricle contracts. This may be shown either by the galvanometer or by the rheoscopic frog. If the nerve of an irritable muscle-nerve preparation be laid over a pulsating ventricle, each beat is responded to by a twitch of the muscle of the preparation. In the case of ordinary muscles, too, instances occur in which it seems impossible to regard the electrical change manifested during the contraction as the mere diminution of a pre-existing current.

Accordingly, those who deny the existence of " natural " muscle-currents speak of a muscle as developing during a contraction a "current of action," occasioned, as they believe, by the muscular substance as it is entering into the state of contraction becoming negative toward the muscular substance which is still at rest, or has returned to a state of rest. In fact, they regard the negativity of muscular substance as characteristic alike of beginning death and of a beginning contraction. So that, in muscular contraction a wave of negativity, starting from the end-plate when indirect, or from the point stimulated when direct stimulation is used, passes along the muscular substance to the ends or end of the fibre.

For instance, we will suppose two electrodes placed on two points (Fig. 31), A and $B$, of a fibre about to be stimulated by a single induction-shock at one end. Before the stimulation the fibre is isnelectric, and the needle of the galvanometer stands at zero. At a certain time after the shock has been sent through the stimulating electrodes $(x)$, as the wave of contraction is travelling down the fibre, the section of the fibre beneath $A$ will become negative toward the rest of the fibre, and so negative toward the portion of the fibre under $B$-i.e., $A$ will be negative relatively to $B$, and this will be shown by a deflection of the needle. A little later $B$ will 
be entering into contraction, and will be becoming negative toward the rest of the fibre, including the part under $A$, whose negativity by this time is passing off-that is to say, $B$ will now be negative toward $A$, and this will be shown by a deflection of the needle in a direction opposite to that of the deflection which has just previously taken place. Hence, between two electrodes placed along a fibre a single wave of contraction will give rise to two currents of different phases, to a diphasic change; and this, indeed, is found to be the case.

This being so, it is obvious that the electrical result of tetanizing a muscle when wave after wave follows along each fibre is a complex matter; but it is maintained that the apparent negative variation of tetanus can be explained as the net result of a series of currents of action due to the individual contractions, the second phase of the current in each contraction being less marked than the first phase. We cannot, however, enter more fully here into a discussion of this difficult subject.

Whichever view be taken of the nature of these phenomena, it is important to remember that the electrical changes are closely allied to the chemical events involved in the contraction of the muscle and to the change of form of the muscle. Their exact relations to each other await research.

\section{The Changes in a Nerve during the Passage of a Nervous Impulse.}

$\S 68$. The change in the form of a muscle during its contraction is a thing which can be seen and felt; but the changes in a nerve during its activity are invisible and impalpable. We stimulate one end of a nerve going to a muscle, and we see this followed by a contraction of the muscle attached to the other end; or we stimulate a nerve still connected with the central nervous system, and we see this followed by certain movements, or by other tokens which show that disturbances have been set up in the central nervous system. We know, therefore, that some changes or other, constituting what we have called a nervous impulse, have been propagated along the nerve; but the changes are such as we cannot see. It is possible, however, to learn something about them.

$\$ 69$. The chemistry of a nerve. We have spoken of the medulla as fatty, and yet it is in reality very largely composed of a substance which is not (in the strict sense of the word) a fat. When we examine chemically a quantity of nerve (or what is practically the same thing, a quantity of that part of the central nervous system which is called white matter, and which as we shall see is chiefly composed, like a nerve, of medullated nerves, and is to be preferred for chemical examination because it contains a relatively small quantity of connective tissue), we find that a very large proportion, according to some observers about half, of the dried matter consists of a peculiar body, cholesterin. Now, cholesterin is not a fat but an alcohol; like glycerin, however, which is also an alcohol, it forms compounds with fatty acids; and though we do not know definitely the chemical condition in which cholesterin exists during life in the medulla, it is more than probable that it exists in some combination with some of the really fatty bodies also present in the medulla, and not in a free isolated state. It is singular that besides being present in such large quantities in nervous tissue, and to a small extent in other tissues and in blood, cholesterin is a normal constituent of bile, and forms the greater part of gall-stones when these are present; in gall-stones it is undoubtedly present in a free state. Besides cholesterin, "white" nervous matter contains a less but still considerable quantity of a complex fat, whose nature is disputed. According to some authorities rather less than half this complex fat consists of the peculiar body lecithin, which we have already 
seen to be present also in blood corpuscles and in muscle. Lecithin contains the radicle of stearic acil (or of oleic, or of palmitic acid) associated not, as in ordinary fats, with simple glycerin, but with the more complex glycerinphosphoric acid, and further combined with a nitrngenous body, neurin, an ammonia compound of some considerable complexity; it is therefore of remarkable nature, since, though a fat, it contains both nitrogen and phosphorus. According to the same authorities the remainder of the complex fit consists of another fatty body, also apparently containing nitrogen but no phosphorus, called cerebrin. Other authorities regard both these bodies, lecithin and cerebrin, as products of decomposition of a still more complex fat, called protagon. Obviously the fat of the white matter of the central nervous system and of spinal nerves (of which fat by far the greater part must exist in the medulla, and form nearly the whole of the medulla) is a very complex body indeed, especially so if the cholesterin exists in combination with the lecithin, or cerebrin (or protagon). Being so complex it is naturally very unstable, and indeed, in its stability resembles proteid matter. Hence, probably, the reason why the medulla changes so rapidly and so profoundly after the death of the nerve.

The presence in such large quantity of this complex fatty medulla renders the chemical examination of the other constituents of a nerve very difficult, and our knowledge of the chemical nature of, and of the chemical changes going on in the axis-cylinder, is very limited. Examined under the microscope the axis-cylinder gives the xanthoproteic reaction and other indications that it is proteid in nature ; beyond this we are largely confined to inferences.

After the fats of the medulla (and the much smaller quantity of fat present in the axis-cylinder), the proteids of the axis-cylinder, and the other soluble substances present in one or the other, or gathered round the nuclei of the neurilemma, have by various means been dissolved out of a nerve fibre, certain substances still remain. One of these in small quantity is the nuclein of the nuclei; another in larger quantity is the substance neurokeratin which forms, as we have seen, a supporting framework for the medulla, and whose most marked characteristic is, perhaps, its resistance to solution.

In the ash of nerves there is a preponderance of potassium salts and phosphates, but not so marked as in the case of muscle.

$\$ 70$. The nervous impulse.-The chemical analngy between the substance of the muscle and that of the axis-cylinder would naturally lead us to suppose that the progress of a nervous impulse along a nerve fibre was accompanied by chemical changes similar to those taking place in a muscle fibre. Whatever changes, however, do or may take place are too slight to be recognized by the means at our disposal. We have no satisfactory evidence that in a nerve even repeated nervous impulses can give rise to an acid reaction, or that the death of a nerve fibre leads to such reaction. 'The gray matter of the central nervous system, it is true, is said to be slightly acid during life and to become more acid after death; but in this gray matter, nerve cells are relatively abundant; the white matter, composed chiefly of nerve fibre, is and remains, during action as well as rest, and even after death, neutral or slightly alkaline.

Nor have we satisfactory evidence that the progress of a nervous impulse is accompanied by any setting free of energy in the form of heat.

In fact, beyond the terminal results, such as a muscular contraction in the case of a nerve going to a muscle, or some affection of the central nervous system in the case of a nerve still in connection with its nervous centre, there is one event and one event only which we are able to recognize as the objective token of a nervous impulse, and that is an electric change. For a 
piece of nerve removed from the body exhibits nearly the same electric phenomena as a piece of muscle. It has an equator which is electrically positive relatively to the two cut ends. In fact, the diagram Fig. 30, and the description which was given in $\$ 66$ of the electric changes in muscle may be applied almost as well to a nerve, except that the currents are in all cases much more feeble in the case of nerves than of muscles, and the special currents from the circumference to the centre of the transverse sections cannot well be shown in a slender nerve; indeed, it is doubtful if they exist at all.

During the passage of a nervous impulse the "natural nerve current" undergoes a negative variation, just as the "natural muscle current" undergoes a negative variation during a contraction. There are, moreover, reasons in the case of the nerve, as in the case of the muscle, which lead us to doubt the pre-existence of any such "natural" currents. A nerve in an absolutely natural condition appears to be, like a muscle, isoelectric; hence we may say that in a nerve during the passage of a nervous impulse, as in a muscle during a muscular contraction, a "current of action" is developed.

This "current of action" or "negative variation" may be shown either by a galvanometer or by the rheoscopic frog. If the nerve of the "muscle nerve preparation," $B$ (see $\$ 67$ ) be placed in an appropriate manner on a thoroughly irritable nerve, $A$ (to which, of course, no muscle need be attached), touching for instance the equator and one end of the nerve, then single induction-shocks sent into the far end of $A$ will cause single spasms in the muscle of $B$, while tetanization of $A, i$. e., rapidly repeated shocks sent into $A$, will cause tetanus of the muscle of $B$.

That this current, whether it be regarded as an independent "current of action" or as a negative variation of a "pre-existing" current, is an essential feature of a nervous impulse is shown by the fact that the degree of intensity of the one varies with that of the other. They both travel, too, at the same rate. In describing the muscle-curve, and the method of measuring the muscular latent period, we have incidentally shown $(\S 46)$ how at the same time the velocity of the nervous impulse may be measured, and stated that the rate in the nerves of a frog is about 28 metres per second. By means of a special and somewhat complicated apparatus it is ascertained that the current of action travels along an isolated piece of nerve at the same rate. It also, like the molecular change in a muscle preceding the contraction, and indeed like the contraction itself, travels in the form of a wave, rising rapidly to a maximum at each point of the nerve and then more gradually declining again. The length of the wave may by special means be measured, and is found to be about $18 \mathrm{~mm}$.

When an isolated piece of nerve is stimulated in the middle, the current of action is propagated equally well in both directions, and that whether the nerve be a chiefly sensory or a chiefly motor nerve, or indeed if it be a nerveroot composed exclusively of motor or of sensory fibres. Taking the current of action as the token of a nervous impulse, we infer from this that when a nerve fibre is stimulated artificially at any part of its course, the nervous impulse set going travels in both directions.

We used just now the phrase "tetanization of a nerve," meaning the application to a nerve of rapidly repeated shocks such as would produce tetanus in the muscle to which the nerve was attached, and we shall have frequent occasion to employ the phrase. It must, however, be understood that there is in the nerve, in an ordinary way, no summation of nervous impulses comparable to the summation of museular contractions. Putting aside certain cases which we cannot discuss here, we may say that the series of shocks sent in at the far end of the nerve start a series of impulses; these 
travel down the nerve and reach the muscle as a series of distinct impulses ; and the first changes in the muscle, the molecular latent-period changes, also form a series the members of which are distinct. It is not until these molecular changes become transformed into visible changes of form that any fusion or summation takes place.

$\$ 71$. Putting together the facts contained in this and the preceding sections, the following may be taken as a brief approximate history of what takes place in a muscle and nerve when the latter is subjected to a single induction-shock. At the instant that the induced current passes into the nerve, changes occur, of whose nature we know nothing certain, except that they cause a "current of action "or " negative variation " of the "natural" nerve-current. These changes propagate themselves along the nerve in both directions as a nervous impulse in the form of a wave, having a wave-length of about $18 \mathrm{~mm}$., and a velocity (in frog's nerve) of about $28 \mathrm{~m}$. per second. Passing down the nerve fibres to the muscle, flowing along the branching and narrowing tracts, the wave at last breaks on the end-plates of the fibres of the muscle. Here it is transmitted into what we may call a muscle impulse, with a shorter, steeper wave, and a greatly diminished velocity (about $3 \mathrm{~m}$. per second). This muscle impulse, of which we know hardly more than that it is marked by a current of action, travels from each endplate in both directions to the end of the fibre, where it appears to be lost; at all events, we do not know what becomes of it. As this impulse wave, whose development takes place entirely within the latent period, leaves the end-plate, it is followed by an explosive decomposition of material, leading to a discharge of carbonic acid, to the appearance of some substance or substances with an acid reaction, and probably of other unknown things, with a considerable development of heat. This explosive decomposition gives rise to the visible contraction wave, which travels behind the invisible muscle impulse at about the same rate, but with a vastly increased wave-length. The fibre, as the wave passes over it, swells and shortens, and thus brings its two ends nearer together.

When repeated shocks are given, wave follows wave of nervous impulse, muscle impulse, and visible contraction; but the last do not keep distinct; they are fused into the continued shortening which we call tetanus.

\section{The Nature of the Changes through which an Electric Cur- rent is Able to Generate a Nervous Impulse.}

\section{Action of the Constant Current.}

$\S 72$. In the preceding account, the stimulus applied in order to give rise to a nervous impulse has always been supposed to be an induction-shock, single or repeated. This choice of stimulus has been made on account of the almost momentary duration of the induced current. Had we used a current lasting for some considerable time, the problems before us would have become more complex, in consequence of our having to distinguish between the events taking place while the current was passing through the nerve from those which occurred at the moment when the current was thrown into the nerve or at the moment when it was shut off from the nerve. These complications do arise when, instead of employing the induced current as a stimulus, we use $a$ constant current, $i . e$., when we pass through the nerve (or muscle) a current direct from the battery without the intervention of any induction-coil.

Before making the actual experiment, we might, perhaps, naturally suppose that the constant current would act as a stimulus throughout the whole 
time during which it was applied; that, so long as the current passed along the nerve, nervous impulses would be generated; and that these would throw the muscle into something, at all events, like tetanus. And, under certain conditions, this does take place; occasionally it does happen that at the moment the current is thrown into the nerve the muscle of the muscle-nerve preparation fills into a tetanus, which is continued until the current is shut off; but such a result is exceptional. In the vast majority of cases what happens is as follows: At the moment that the circuit is made, the moment that the current is thrown into the nerve, a single twitch, a simple contraction, the so-called making contraction, is witnessed; but after this has passed away the muscle remains absolutely quiescent, in spite of the current continuing to pass through the nerve, and this quiescence is maintained until the circuit is broken, until the current is shut off from the nerve, when another simple contraction, the so-called breaking contraction, is observed. The mere passage of a constant current of uniform intensity through a nerve dnes not, under ordinary circumstances, act as a stimulus generating a nervous impulse; such an impulse is only set up when the current either falls into or is shut off from the nerve. It is the entrance or the exit of the current, and not the continuance of the current, which is the stimulus. The quiescence of the nerve and muscle during the passage of the current is, however, dependent on the current remaining uniform in intensity, or, at least, not being suddenly increased or diminished. Any sufficiently sudden and large increase or diminution of the intensity of the current will act like the entrance or exit of a current, and by generating a nervous impulse give rise to a contraction. If the intensity of the current, however, be very slowly and gradually increased or diminished, a very wide range of intensity may be passed through without any contraction being seen. It is the sudden change from one condition to another, and not the condition itself, which causes the nervous impulse.

In many cases, both a "making" and a "breaking" contraction, each a simple twitch, are observed, and this is, perhaps, the commonest event; but when the current is very weak, and again when the current is very strong, either the breaking or the making contraction may be absent; $i$. e., there may be a contraction only when the current is thrown into the nerve, or only when it is shut off from the nerve.

Under ordinary circumstances the contractions witnessed with the constant current either at the make or at the break, are of the nature of a "simple" contraction; but, as has already been said, the application of the current may give rise to very pronounced tetanus. Such a tetanus is seen sometimes when the current is made, lasting during the application of the current, sometimes when the current is broken, lasting some time after the current has been wholly removed from the nerve. The former is spoken of as a "making," the latter as a "breaking" tetanus. But these exceptional results of the application of the constant current need not detain us now.

The great interest attached to the action of the constant current lies in the fact that, during the passage of the current, in spite of the absence of all nervous impulses, and therefore of all muscular contractions, the nerve is for the time both between and on each side of the electrodes profoundly modified in a most peculiar manner. This modification, important both for the light it throws on the generation of nervous impulses and for its practical applications, is known under the name of electrotonus.

$\$ 73$. Electrotonus.- The marked feature of the electrotonic condition is that the nerve, though apparently quiescent, is changed in respect to its irritability; and that in a different way in the neighborhood of the two electrodes respectively. 
Suppose that on the nerve of a muscle-nerve preparation are placed two (non-polarizable) electrodes (Fig. 32, $a, k$ ), connected with a battery and arranged with a key, so that a constant current can at pleasure be thrown into or shut off from the nerve. This constant current, whose effects we are about to study, may be called the "polarizing current." Let $a$ be the positive electrode or anode, and $k$ the negative electrode or kathode, both placed at some distance from the muscle, and also with a certain interval between each other. At the point $x$ let there be applied a pair of electrodes connected with an induction-coil. Let the muscle further be connected with a lever, so that its contractions can be recorded and their amount measured. Before the polarizing current is thrown into the nerve, let a single inductionshock of known intensity (a weak one being chosen, or, at least, not one which would cause in the muscle a maximum contraction) be thrown in at $x$. A contraction of a certain amount will follow. The contraction may

FIG. 32.
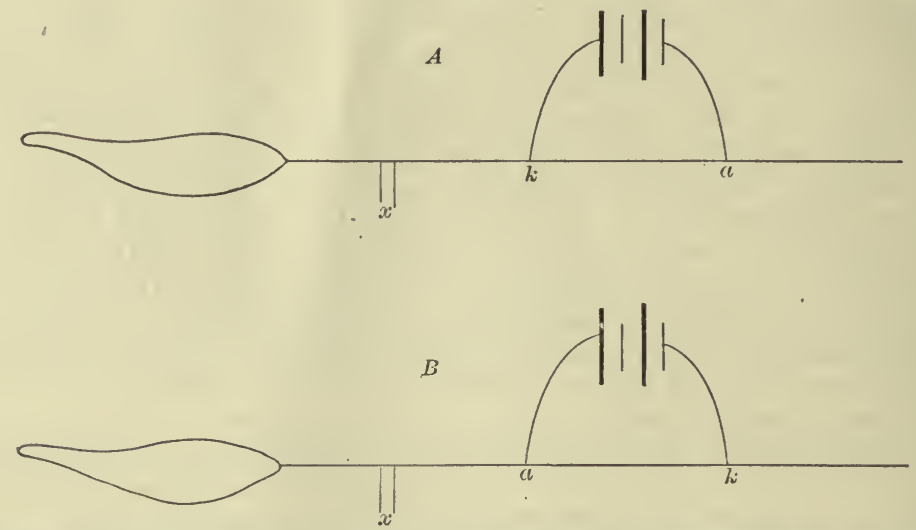

Muscle-nerve Preparations: with the nerve exposed in $A$ to a descending and in $B$ to an ascending constant current. In each, $a$ is the anode, $k$ the kathode of the constant current; $x$ represents the spot where the induction-shocks, used to test the irritability of the nerve, are sent in.

be taken as a measure of the irritability of the nerve at the point $x$. Now let the polarizing current be thrown in and let the kathode or negative pole be nearest the muscle, as in Fig. $32, A$, so that the current passes along the nerve in a direction from the central nervous system toward the muscle; such a current is spoken of as a descending one. The entrance of the polarizing current into the nerve will produce a "making" contraction; this we may neglect. If while the current is passing the same induction-shock as before be sent through $x$, the contraction which results will be found to be greater than on the former occasion. If the polarizing current be now shut off, a "breaking" contraction will probably be produced; this we also may neglect. If, now, the point $x$, after a short interval, be again tested with the same induction-shock as before, the contraction will be no longer greater, but of the same amount, or perhaps not so great as at first. During the passage of the polarizing current, therefore, the irritability of the nerve at the point $x$ has been temporarily increased, since the same shock applied to it causes a greater contraction during the presence than in the absence of the current. But this is only true so long as the polarizing current is a descending one-so long as the point $x$ lies on the side of the kathode. On the other hand, if the polarizing current had been an ascending one, with the anode or 
positive pole nearest the muscle, as in Fig. $32, B$, the irritability of the nerve at $x$ would have been found to be diminished, instead of increased, by the polarizing current; the contraction obtained during the passage of the constant current would be less than before the passage of the current, or might be absent altogether, and the contraction after the current had been shut off would be as great, or perhaps greater, than before. That is to say, when a constant current is applied to a nerve, the irritability of the nerve between the polarizing electrodes and the muscle is, during the passage of the current, increased when the kathode is nearest the muscle (and the polarizing current descending) and diminished when the anode is nearest the muscle (and the polarizing current ascending). The same result, mutatis mutandis, and with some qualifications which we need not discuss, would be gained if $x$ were placed, not between the muscle and the polarizing current, but on the far side of the latter. Hence, it may be stated generally that during the passage of a constant current through a nerve the irritability of the nerve is increased in the region of the kathode, and diminished in the region of the anode. The changes in the nerve which give rise to this increase of irritability in the region of the kathode are spoken of as katelectrotonus, and the nerve is said to be in a katelectrotonic condition. Similarly the changes in the region of the anode are spoken of as anelectrotonus, and the nerve is said to be in an anelectrotonic condition. It is also often usual to speak of the katelectrotonic increase, and anelectrotonic decrease of irritability.

This law remains true whatever be the mode adopted for determining the irritability. The result holds good not only with a single inductionshock, but also with a tetanizing interrupted current, with chemical and mechanical stimuli. It further appears to hold good not only in a dissected nerve-muscle preparation but also in the intact nerves of the living body. The increase and decrease of irritability are most marked in the immediate neighborhood of the electrodes, but spread for a considerable distance in each direction in the extrapolar regions. The same modification is not confined to the extrapolar region, but exists also in the intrapolar region. In the intrapolar region there must be, of course, a neutral or indifferent point, where the katelectrotonic increase merges into the anelectrotonic decrease,

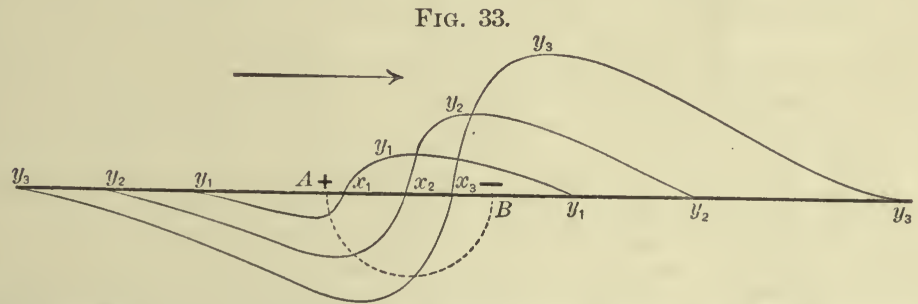

Diagram illustrating the Variations of Irritability during Electrotonus, with Polarizing Currents of Increasing Intensity. (From Pfluger) The anode is supposed to be placed at $A$, the kathode at $\mathrm{B} ; \mathrm{AB}$ is consequently the intrapolar district. In each of the three curves, the portion of the curve below the base line represents diminished irritability, that above, increased irritability. $y_{1}$ represents the effect of a weak current; the indifferent point $x_{1}$ is near the anode $\mathrm{A}$. In $y_{2}$, a stronger current, the indifferent point $x_{2}$ is nearer the kathode $\mathrm{B}$, the diminution of irritability in anelectrotonus and the increase in katelectrotonus being greater than in $y_{1}$; the effect also spreads for a greater distance along the extrapolar regions in both directions. In $y_{3}$ the same events are seen to be still more marked.

and where, therefore, the irritability is unchanged. When the polarizing current is a weak one, this indifferent point is nearer the anode than the 
kathode, but as the polarizing current increases in intensity, draws nearer and nearer the kathode (see Fig. 33).

The amount of increase and decrease is dependent: (1) On the strength of the current, the stronger current up to a certain limit producing the greater effect. (2) On the irritability of the nerve, the more irritable, better conditioned nerve being the more affected by a current of the same intensity.

In the experiments just described the increase or decrease of irritability is taken to mean that the same stimulus starts in the one case a larger or more powerful, and in the other case a smaller or less energetic impulse; but we have reason to think that the mere propagation or condition of impulses started elsewhere is also affected by the electrotonic condition. At all events anelectrotonus appears to offer an obstacle to the passage of a nervous impulse.

8 74. Electrotonic currents. During the passage of a constant current through a nerve, variations in the electric currents belonging to the nerve itself may be

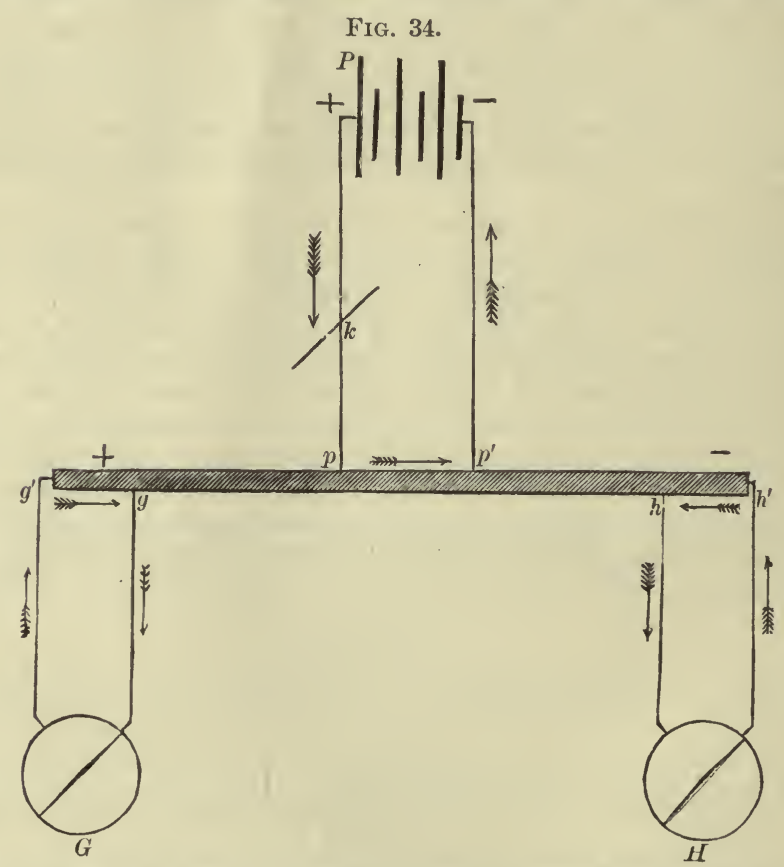

Diagram illustrating Electrotonic Currents: $P$ the polarizing battery, with $k$ a key, $p$ the anode, and $p^{\prime}$ the kathode. At the left end of the piece of nerve the natural current flows through the galvanometer $G$ from $g$ to $g^{\prime}$, in the direction of the arrows; its direction, therefore, is the same as that of the polarizing current; consequently it appears increased, as indicated by the sign +. The current at the other end of the piece of nerve, from $h$ to $h^{\prime}$ through the galvanometer $H$, flows in a contrary direction to the polarizing current; it consequently appears to be diminished, as indicated by the sign -.

N. B.-For simplicity's sake, the polarizing current is here supposed to be thrown in at the middle of a piece of nerve, and the galvanometer placed at the two ends. Of course it will be understood that the former may be thrown in anywhere, and the latter connected with any two pairs of points which will give currents.

observed ; and these variations have certain relations to the variations of the irritability of the nerve. 'Thus if a constant current supplied by the battery $P$ (Fig. 34) be applied to a piece of nerve by means of two non-polarizable electrodes 
$p, p^{\prime}$, the "currents of rest" obtainable from the various points of the ncrve will be different during the passage of the polarizing current from those which were manifest before or after the current was applied; and, moreover, the changes in the nerve-currents produced by the polarizing current will not be the same in the neighborhood of the anode $(p)$ as those in the neighborhood of the kathode $\left(p^{\prime}\right)$. Thus, let $G$ and $H$ be two galvanometers so connected with the two ends of the nerve as to afford good and clear evidence of the "currents of rest." Before the polarizing current is thrown into the nerve, the needle of $H$ will occupy a position indicating the passage of a current of a certain intensity from $h$ to $h^{\prime}$ though the galvanometer (from the positive longitudinal surface to the negative cut end of the nerve), the circuit being completed by a current in the nerve from $h^{\prime}$ to $h, i$. $e_{\text {. }}$ the current will flow in the direction of the arrow. Similarly the needle of $G$ will, by its deflection, indicate the existence of a current flowing from $g$ to $g^{\prime}$ through the galvanometer, and from $g^{\prime}$ to $g$ through the nerve, in the direction of the arrow.

At the instant that the polarizing current is thrown into the nerve at $p p^{\prime}$, the currents at $g g^{\prime}, h h^{\prime}$ will undergo a "negative variation," that is, the nerve at each point will exhibit a "current of action" corresponding to the nervous impulse, which, at the making of the polarizing current, passes in both directions along the nerve, and may cause a contraction in the attached muscle. The current of action is, as we have seen, of extremely short duration, it is over and gone in a small fraction of a second. It, therefore, must not be confounded with a permanent effect which, in the case we are dealing with, is observed in both galvanometers. This effect, which is dependent on the direction of the polarizing current, is as follows: Supposing that the polarizing current is flowing in the direction of the arrow in the figure, that is, passes in the nerve from the positive electrode or anode $p$ to the negative electrode or kathode $p^{\prime}$, it is found that the current through the galvanometer $G$ is increased, while that through $H$ is diminished. 'The polarizing current has caused the appearance, in the nerve outside the electrodes, of a current having the same direction as itself, called the "electrotonic" current; and this electrotonic current adds to, or takes away from, the natural nerve-current or "current of rest" according as it is flowing in the same direction as that or in an opposite direction.

The strength of the electrotonic current is dependent on the strength of the polarizing current, and on the length of the intrapolar region which is exposed to the polarizing current. When a strong polarizing current is used, the electromotive force of the electrotonic current may be much greater than that of the natural nerve-current.

The strength of the electrotonic current varies with the irritability, or vital condition of the nerve, being greater with the more irritable nerve; and a dead nerve will not manifest electrotonic currents. Moreover, the propagation of the current is stopped by a ligature, or by crushing the nerve.

We may speak of the conditions which give rise to this electrotonic current as a physical electrotonus analogous to that physiological electrotonus which is made known by variations in irritability. The physical electrotonic current is probably due to the escape of the polarizing current along the nerve under the peculiar conditions of the living nerve; but we must not attempt to enter here into this difficult subject or into the allied question as to the exact connection between the physical and the physiological electrotonus, though there can be little doubt that the litter is dependent on the former.

$\$ 75$. These variations of irritability at the kathode and anode respectively, thus brought about by the action of the constant current, are interesting theoretically, because we may trace a connection between them and the nervous impulse which is the result of the making or breaking of a constant current.

For we have evidence that a nervous impulse is generated when a portion of the nerve passes suddenly from a normal condition to a state of katelectrotonus or from a state of anelectrotonus back to a normal condition, but that the passage from a normal condition to anelectrotonus or from katelectrotonus back to a normal condition is unable to generate an impulse. Hence when a constant current is "made" the impulse is generated only at 
the kathode where the nerve passes suddenly into katelectrotonus; when the current on the other hand is "broken" the impulse is generated only at the anode where the nerve passes suddenly back from anelectrotonus into a normal condition. We have an indirect proof of this in the facts to which we drew attention a little while back, viz., that a contraction sometimes occurs at the "breaking" only, sometimes at the "making" only of the constant current, sometimes at both. For it is found that this depends partly on the strength of the current in relation to the irritability of the nerve, partly on the direction of the current, whether ascending or descending; and the results obtained with strong, medium and weak descending and ascending currents have been stated in the form of a "law of contraction." We need not enter into the details of this "law," but will merely say that the resulis which it formulates are best explained by the hypothesis just stated. We may add that when the constant current $\cdot$ is applied to certain structures composed of plain muscular fibres, whose rate of contraction we have seen to be slow, the making contraction may be actually seen to begin at the kathode and travel toward the anode, and the breaking contraction to begin at the anode and travel thence toward the kathode.

Since in katelectrotonus the irritability is increased, and in anelectrotonus decreased, both the entrance from the normal condition into katelectrotonus and the return from anelectrotonus to the normal condition are instances of a passage from a lower stage of irritability to a higher stage of irritability. Hence, the phenomena of electrotonus would lead us to the conception that a stimulus in provoking a nervous impulse produces its effect by, in some way or other, suddenly raising the irritability to a higher pitch. But what we are exactly to understand by raising the irritability, what molecular change is the cause of the rise, and how either electric or other stimuli can produce this change, are matters we cannot discuss here.

Besides their theoretical importance, the phenomena of electrotonus have also a practical interest. When an ascending current is passed along a nerve going to a muscle or group of muscles, the region between the electrodes and the muscle is thrown into anelectrotonus and its irritability is diminished. If the current be of adequate strength, the irritability may be so much lessened that nervous impulses cannot be generated in that part of the nerve or canuot pass along it. Hence, by this means the irregular contractions of muscles known as "cramp" may be abolished. Similarly, by bringing into a condition of anelectrotonus a portion of a sensory nerve in. which violent impulses are being generated, giving rise in the central nervous system to sensations of pain, the impulses are toned down or wholly abolished, and the pain ceases. So, on the other hand, we may at pleasure heighten the irritability of a part by throwing it into katelectrotonus. In this way the constant current, properly applied, becomes a powerful remedial means.

Lastly, though we are dealing now with nerves going to muscles-that is to say, with motor nerves only-we may add that what we have said about electrotonus and the development of nervous impulses by it appears to apply equally well to sensory nerves.

$\$ 76$. In a general way muscular fibres behave toward an electric current very much as do nerve fibres; but there are certain important differences.

In the first place, muscular fibres, devoid of nerve fibres, are much more readily thrown into contractions by the breaking and making of a constant current than by the more transient induction-shock; the muscular substance seems to be more sluggish than the nervous substance, and requires to be acted upon for a longer time. This fact may be made use of, and, indeed, is in medical practice made use of, to determine the condition of the nerves 
supplying a muscle. If the intra-muscular nerves be still in good condition, the muscle, as a whole, responds readily to single induction-shocks, because these can act upon the intra-muscular nerves. If these nerves, on the other hand, have lost their irritability, the muscle does not respond readily to single induction-shocks, or to the interrupted current, but can still easily be thrown into contraction by the constant current.

In the second place, while in a nerve no impulse is, as a rule, generated during the passage of a constant current, between the break and the make, provided that it is not too strong, and that it remains uniform in strength, in a urarized muscle, on the other hand, even with moderate and perfectly uniform currents, a kind of tetanus or apparently a series of rhythmically repeated contractions is very frequently witnessed during the passage of the current. The exact nature and cause of these phenomena in muscle, we must not, however, discuss here.

\section{The Muscle-nerve Preparation as a Machine.}

$\$ 77$. The facts described in the foregoing sections show that a muscle with its nerve may be justly regarded as a machine which, when stimulated, will do a certain amount of work. But the actual amount of work which a muscle-nerve preparation will do is found to depend on a large number of circumstances, and consequently to vary within very wide limits. These variations will be largely determined by the condition of the muscle and nerve in respect to their nutrition; in other words, by the degree of irritability manifested by the muscle or by the nerve, or by both. But quite apart from the general influences affecting its nutrition and thus its irritability, a muscle-nerve preparation is affected as regards the amount of its work by a variety of other circumstances, which we may briefly consider here, reserving to a succeeding section the study of variations in irritability.

The influence of the nature and mode of application of the stimulus. When we apply a weak stimulus-a weak induction-shock-to a nerve we get a small contraction, a slight shortening of the muscle; when we apply a stronger stimulus-a stronger induction-shock-we get a larger contraction, a greater shortening of the muscle. We take, other things being equal, the amount of contraction of the muscle as a measure of the nervous impulse, and say that in the former case a weak or slight, in the latter case a stronger or larger, nervous impulse has been generated. Now, the muscle of the muscle-nerve preparation consists of many muscular fibres, and the nerve of many nerve fibres; and we may fairly suppose that in two experiments we may in the one experiment bring the induction-shock or other stimulus to bear on a few fibres only, and in the other experiment on many or even all the fibres of the nerve. In the former case, only those muscular fibres in which the few nerve fibres stimulated end will be thrown into contraction, the others remaining quiet, and the shortening of the muscle, as a whole, since only a few fibres take part in it, will necessarily be less than when all the fibres of the nerve are stimulated and all the fibres of the muscles contract. That is to say, the amount of contraction will depend on the number of fibres stimulated. For simplicity's sake, however, we will in what follows, except when otherwise indicated, suppose that when a nerve is stimulated, all the fibres are stimulated and all the muscular fibres contract.

This being premised, we may say that, other things being equal, the magnitude of a nervous impulse, and so the magnitude of the ensuing contraction, is directly dependent on what we may call the strength of the stimulus. Thus taking a single induction-shock as the most manageable stimulus, we find that if, before we begin, we place the secondary coil (Fig. 14, sec. c.) a 
long way off the primary coil $p r . c$. no visible efiect at all follows upon the discharge of the induction-shock. The passage of the momentary weak current is either unable to produce any nervous impulse at all, or the weak nervous impulse to which it gives rise is unable to stir the sluggish muscular substance to a visible contraction. As we slide the secondary coil toward the primary, sending in an induction-shock at each new position, we find that at a certain distance between the secondary and primary coils, the muscle responds to each induction-shock ${ }^{1}$ with a contraction which makes itself visible by the slightest possible rise of the attached lever. This position of the coils, the battery remaining the same and other things being equal, marks the minimal stimulus giving rise to the minimal contraction. As the secondary coil is brought nearer to the primary, the contractions increase in height corresponding to the increase in the intensity of the stimulus. Very soon, however, an increase in the stimulus caused by further sliding the secondary coil over the primary fails to cause any increase in the contraction. This indicates that the maximal stimulus giving rise to the maximal contraction has been reached; though the shocks increase in intensity as the secondary coil is pushed further and further over the primary, the contractions remain of the same height, until fatigue lowers them.

With single induction-shocks then the muscular contraction, and by inference the nervous impulse, increases with an increase in the intensity of the stimulus, between the limits of the minimal and maximal stimuli; and this dependence of the nervous impulse, and so of the contraction, on the strength of the stimulus may be observed not only in electric but in all kinds of stimuli.

It may here be remarked that in order for a stimulus to be effective, a certain abruptness in its action is necessary. Thus as we have seen the constant current when it is passing through a nerve with uniform intensity does not give rise to a nervous impulse, and indeed it may be increased or diminished to almost any extent without generating nervous impulses, provided that the change be made gradually enough; it is only when there is a sudden change that the current becomes effective as a stimulus. And the reason why the breaking induction-shock is more potent as a stimulus than the making shock is because as we have seen ( $\$ 44)$ the current which is induced in the secondary coil of an induction-machine at the breaking of the primary circuit is more rapidly developed, and has a sharper rise, than the current which appears when the primary circuit is made. Similarly a sharp tap on a nerve will produce a contraction, when a gradually increasing pressure will fail to do so ; and in general the efficiency of a stimulus of any kind will depend in part on the suddenness or abruptness of its action.

A stimulus in order that it may be effective must have an action of a certain duration, the time necessary to produce an effect varying according to the strength of the stimulus and being different in the case of a nerve from what it is in the case of a muscle. It would appear that an electric curlent applied to a nerve must have a duration of at least about 0.0015 second to cause any contraction at all, and needs a longer time than. this to produce its full effect. A muscle fibre apart from its nerve fibre requires a still longer duration of the stimulus, and hence, as we have already stated, a muscle poisoned by urari, or which has otherwise lost the action of its nerves, will not respond as readily to induction-shocks as to the more slowly acting, breaking and making of a constant current.

In the case of electric stimuli, the same current will produce a stronger

1 In these experiments either the breaking or making shock must be used, not sometimes one and sometimes the other, for, as we have stated, the two kinds of shock differ in efficiency, the breaking being the most potent. 
contraction when it is sent along the nerve than when it is sent across the nerve; indeed it is maintained that a current which passes through a nerve in an absolutely transyerse direction is powerless to generate impulses.

It would also appear, at all events up to certain limits, that the longer the piece of nerve through which the current passes, the greater is the effect of the stimulus.

When two pairs of electrodes are placed on the nerve of a long and perfectly fresh and successful nerve-preparation, one near to the cut end, and the other nearer the muscle, it is found that the same stimulus produces a greater contraction when applied through the former pair of electrodes than through the latter. This has been interpreted as meaning that the impulse started at the further electrodes gathers strength, like an avalanche, in its progress to the muscle. It is more probable, however, that the larger contraction produced by stimulation of the part of the nerve near the cut end is due to the stimulus setting free a larger impulse, $i . e$., to this part of the nerve being more irritable. The mere section, possibly by developing nerve currents, increases for a time the irritability at the cut end. A similar greater irritability may, however, also be observed in the part of the nerve nearer the spinal cord while it is still in connection with the spinal cord; and it is possible that the irritability of a nerve may vary considerably at different points of its course.

$\S 78$. We have seen that when single stimuli are repeated with sufficient frequency, the individual contractions are fused into tetanus; as the frequency of the repetition is increased, the individual contractions are less obvious on the curve, until at last we get a curve on which they seem to be entirely lost and which we may speak of as a complete tetanus. By such a tetanus a much greater contraction, a much greater shortening of the muscle, is of course obtained than by single contractions.

The exact frequency of repetition required to produce complete tetanus will depend chiefly on the length of the individual contractions, and this varies in different animals, in different muscles of the same animal, and in the same muscle under different conditions. In a cold-blooded animal, a single contraction is as a rule more prolonged than in a warm-blooded animal, and tetanus is consequently produced in the former by a less frequent repetition of the stimulus. A tired muscle has a longer contraction than a fresh muscle, and hence in many tetanus curves the individual contractions, easily recognized at first, disappear later on, owing to the individual contractions being lengthened out by the exhaustion caused by the tetanus itself. In many animals, e.g., the rabbit, some muscles (such as the adductor magnus femoris) are pale, while others (such as the semitendinosus) are red. The red muscles are not only more richly supplied with bloodvessels, but the muscle substance of the fibres contains more hæmoglobin than the pale, and there are other structural differences. Now the single contraction of one of these red muscles is more prolonged than a single contraction of one of the pale muscles produced by the same stimulus. Hence the red muscles are thrown into complete tetanus with a repetition of much less frequency than that required for the pale muscles. Thus, ten stimuli in a second are quite sufficient to throw the red muscles of the rabbit into complete tetanus, while the pale muscles require at least twenty stimuli in a second.

So long as signs of the individual contractions are visible on the curve of tetanus it is easy to recognize that each stimulation produces one of the constituent single contractions, and that the number so to speak of the vibrations of the muscle making up the tetanus corresponds to the number of stimulations; but the question whether, when we increase the number of stimulations beyond that necessary to produce a complete tetanus, we still 
increase the number of constituent single contractions is one not so easy to answer. And connected with this question is another difficult one. What is the rate of repetition of single contractions making up those tetanic contractions which as we have said are the kind of contractions by which the voluntary, and indeed other natural, movements of the body are carried out? What is the evidence that these are really tetanic in character?

When a muscle is thrown into tetanus, a more or less musical sound is produced. This may be heard by applying a stethoscope directly over a contracting muscle, and a similar sound but of a more mixed origin and less trustworthy may be heard when the masseter muscles are forcibly contracted or when a finger is placed in the ear, and the muscles of the same arm are contracted.

When the stethoscope is placed over a muscle, the nerve of which is stimulated by induction-shocks repeated with varying frequency, the note heard will vary with the frequency of the shocks, being of higher pitch with the more frequent shocks. Now it has been thought that the vibrations of the muscle giving rise to the "muscle sound" are identical with the single contractions making up the tetanus of the muscle. And since, in the human body, when a muscle is thrown into contraction in a voluntary effort, or indeed in any of the ordinary natural movements of the body, the fundamental tone of the sound corresponds to about 19 or 20 vibrations a second, it has been concluded that the contraction taking place in such cases is a tetanus of which the individual contractions follow each other about 19 or 20 times a second. But investigations seem to show that the vibrations giving rise to the muscle sound do not really correspond to the shortenings and relaxations of the individual contractions, and that the pitch of the note cannot therefore be taken as an indication of the number of single contractions making up the tetanus; indeed, as we shall see in speaking of the sounds of the heart, a single muscular contraction may produce a sound which though differing from the sound given out during tetanus has to a certain extent musical characters. Nevertheless the special characters of the muscle sound given out by muscles in the natural movements of the body may be taken as showing at least that the contractions of the muscle in these movements are tetanic in nature, and the similarity of the note in all the voluntary efforts of the body and indeed in all movements carried out by the central nervous system is at least consonant with the view that the repetition of single contractions is of about the same frequency in all these movements. IVhat that frequency is, and whether it is exactly identical in all these movements, is not at present perhaps absolutely determined; but certain markings on the myographic tracings of these movements and other facts seem to indicate that it is about 12 a second.

$\$ 79$. The influence of the load. It might be imagined that a muscle which, when loaded with a given weight and stimulated by a current of a given intensity, had contracted to a certain extent, would only contract to half that extent when loaded with twice the weight and stimulated with the same stimulus. Such, however, is not necessarily the case; the height to which the weight is raised may be in the second instance as great, or even greater than in the first. That is to say, the resistance offered to the contraction actually augments the contraction; the tension of the muscular fibre increases the facility with which the explosive changes resulting in a contraction take place. And we have other evidence that anything which tends to stretch the muscular fibres-that any tension of the muscular fibres, whether during rest or during contraction-increases the metabolism of the muscle. There is, of course, a limit to this favorable action of the 
resistance. As the load continues to be increased, the height of the contraction is diminished, and at last a point is reached at which the muscle is unable (even when the stimulus chosen is the strongest possible) to lift the load at all.

In a muscle viewed as a machine we have to deal, not merely with the height of the contraction-that is, with the amount of shortening-but with the work done. And this is measured by multiplying the number of units of height to which the load is raised into the number of units of weight of the load. Hence, it is obvious from the foregoing observations that the work done must be largely dependent on the weight itself. Thus, there is a certain weight of load with which, in any given muscle stimulated by a given stimulus, the most work will be done, as may be seen from the following example:

$\begin{array}{lrrrrrr}\text { Load, in grammes } & 0 & 50 & 100 & 150 & 200 & 250 \\ \text { Height of contractions, in millimetres } & 14 & 9 & 7 & 5 & 2 & \text { () } \\ \text { Work done, in gram-millimetres } & 0 & 450 & 700 & 750 & 400 & 0\end{array}$

$\S 80$. The influence of the size and form of the muscle. Since all known muscular fibres are much shorter than the wave-length of a contraction, it is obvious that the longer the fibre the greater will be the shortening caused by the same contraction wave; the greater will be the height of the contraction with the same stimulus. Hence, in a muscle of parallel fibres, the height to which the load is raised as the result of a given stimulus applied to its nerve, will depend on the length of the fibres, while the maximum weight of load capable of being lifted will depend on the number of the fibres, since the load is distributed among them. Of two muscles, therefore, of equal length (and of the same quality) the most work will be done by that which has the larger number of fibres-that is to say, the fibres being of equal width, which has the greatest sectional area; and of two muscles with equal sectional areas, the most work will be done by that which is the longer. If the two nuscles are unequal both in length and sectional area, the work done will be the greater in the one which has the larger bulk, which contains the greater number of cubic units. In speaking; therefore, of the work which can be done by a muscle, we may use as a standard a cubic unit of bulk; or, the specific gravity of the muscle being the same, a unit of weight.

We learn, then, from the foregoing paragraphs that the work done by a muscle-nerve preparation will depend, not only on the activity of the nerve and muscle as determined by their own irritability, but also on the character and mode of application of the stimulus; on the kind of contraction (whether a single spasm, or a slowly repeated or a rapidly repeated tetanus) on the load itself, and on the size and form of the muscle. Taking the most favorable circumstances-viz., a well-nourished, lively preparation, a maximum stimulus causing a rapid tetanus, and an appropriate load-we may determine the maximum work done by a given weight of muscle, say one gramme. This in the case of the muscles of the frog has been estimated at about four gram-metres for one gramme of muscle.

\section{The Circumstances which Determine the Degree of Irritability of Muscles ANd Nerves.}

\$81. A muscle-nerve preparation at the time that it is removed from the body possesses a certain degree of irritability ; it responds by a contraction of a certain amount to a stimulus of a certain strength applied to the nerve or to the muscle. After a while, the exact period depending on a 
variety of circumstances, the same stimulus produces a smaller contraction, $i$. e., the irritability of the preparation has diminished. In other words, the muscle or nerve or both have become partially "exhausted," and the exhaustion subsequently increases, the same stimulus producing smaller contractions, until at last all irritability is lost, no stimulus, however strong, producing any contraction, whether applied to the nerve or directly to the muscle; and eventually the muscle, as we have seen, becomes rigid. The progress of this exhaustion is more rapid in the nerves than in the muscles; for some time after the nerve-trunk has ceased to respond to even the strongest stimulus, contractions may be obtained by applying the stimulus directly to the muscle. It is much more rapid in the warm-blooded than in the cold-blooded animals. The muscles and nerves of the former lose their irritability when removed from the body, after a period varying according to circumstances, from a few minutes to two or three hours; those of coldbloorled animals (or at least of an amphibian or a reptile) may, under favorable conditions, remain irritable for two, three, or even more days. The duration of irritability in warm-blooded animals may, however, be considerably prolonged by reducing the temperature of the body before death.

If with some thin body a sharp blow be struck across a muscle which has entered into the later stages of exhaustion a wheal lasting for several seconds is developed. This wheal appears to be a contraction wave limited to the part struck, and disappearing very slowly without extending to the neighboring muscular substance. It has been called an "idio-muscular" contraction, because it may be brought out even when ordinary stimuli have ceased to produce any effect. It may, however, be accompanied at its beginning by an ordinary contraction. It is readily produced in the living body on the pectoral and other muscles of persons suffering from phthisis and other exhausting discases.

This natural exhaustion and diminution of irritability in muscles and nerves removed from the body may be modified both in the case of the muscle and of the nerve by a variety of circumstances. Similarly, while the nerve and muscle still remain in the body, the irritability of the one or of the other may be modified either in the way of increase or of decrease by certain general influences, of which the most important are severance from the central nervous system and variations in temperature, in bloodsupply, and in functional activity.

The effects of severance from the central nervous system. When a nerve, such, for instance, as the sciatic, is divided in situ, in the living body, there is, first of all, observed a light increase of irritability, noticeable especially near the cut end, but after a while the irritability diminishes and gradually disappears. Both the slight initial increase and the subsequent decrease begin at the cut end and advance centrifugally toward the peripheral terminations. This centrifugal feature of the loss of irritability is often spoken of as the Ritter-Valli law. In a mammal it may be two or three days; in a frog, as many, or even more weeks, before irritability has disappeared from the nerve-trunk. It is maintained in the small (and especially in the intramuscular) branches for still longer periods.

In the central portion of the divided nerve similar changes may be traced as far only as the next node of Ranvier. Beyond this the nerve usually remains in a normal condition.

If the muscle thus deprived of its nervous elements be left to itself its irritability, however tested, sooner or later diminishes; but if the muscle be periodically thrown into contractions by artificial stimulation with the constant current, the decline of irritability and attendant loss of nutritive power may be postponed for some considerable time. But as far as our ex- 
perience goes at present the artificial stimulation cannot fully replace the natural one, and sooner or later the muscle like the nerve suffers degeneration, loses all irritability, and ultimately its place is taken by connective tissue.

\$2. The influence of temperature. We have already seen that sudden heat (and the same might be said of cold when sufficiently intense), applied to a limited part of a nerve or muscle, as when the nerve or muscle is touched with a hot wire, will act as a stimulus. It is, however, much more difficult to generate nervous or muscular impulses by exposing a whole nerve or muscle to a gradual rise of temperature.

A muscle may be gradually cooled to $0^{\circ} \mathrm{C}$. or below without any contraction being caused; but when it is heated to a limit, which in the case of frog's muscles is about $45^{\circ} \mathrm{C}$., of mammalian muscles about $50^{\circ} \mathrm{C}$., a sudden change takes place: the muscle falls at the limiting temperature into a rigor mortis, which is initiated by a forcible contraction or at least shortening.

Moderate warmth, e.g., in the frog an increase of temperature up to somewhat below $45^{\circ} \mathrm{C}$., favors both muscular and nervous irritability. All the molecular processes are hastened and facilitated: the contraction is for a given stimulus greater and more rapid, $i$. $e$, of shorter duration, and nervous impulses are generated more readily by slight stimuli. Owing to the quickening of the chemical changes, the supply of new material may prove insufficient; hence muscles and nerves removed from the body lose their irritability more rapidly at a high than at a low temperature.

The gradual application of cold to a nerve, especially when the temperature is thus brought near to $0^{\circ} \mathrm{C}$., slackens all the molecular processes, so that the wave of nervous impulse is lessened and prolonged, the velocity of its passage being much diminished, e. g., from 28 metres to 1 metre per second. At about $0^{\circ} \mathrm{C}$., the irritability of the nerve disappears altogether.

When a muscle is exposed to similar cold, $e . g$., to a temperature very little above zero, the contractions are remarkably prolonged; they are diminished in height at the same time, but not in proportion to the increase of their duration. Exposed to a temperature of zero or below, muscles soon lose their irritability, without, however, undergoing rigor mortis.

$\S 83$. The influence of blood-supply. When a muscle still within the body is deprived by any means of its proper blood-supply, as when the blood-vessels going to it are ligatured, the same gradual loss of irritability and final appearance of rigor mortis are observed as in muscles removed from the body. Thus, if the abdominal aorta be ligatured, the muscles of the lower limbs lose their irritability and finally become rigid. So also in systemic death, when the blood-supply to the muscles is cut off by the cessation of the circulation, loss of irritability ensues, and rigor mortis eventually follows. In a human corpse the muscles of the body enter into rigor mortis in a fixed order; first, those of the jaw and neck, then those of the trunk, next those of the arms, and lastly those of the legs. The rapidity with which rigor mortis comes on after death varies considerably, being determined both by external circumstances and by the internal conditions of the body. Thus, external warmth hastens and cold retards the onset. After great muscular exertion, as in hunted animals, and when death closes wasting diseases, rigor mortis in most cases comes on rapidly. As a general rule, it may be said that the later it is in making its appearance the more pronounced it is, and the longer it lasts; but there are many exceptions, and when the state is recognized as being fundamentally due to a clotting of myosin, it is easy to understand that the amount of rigidity, $i$. $e$., the amount of the clot, and the rapidity of the onset, $i$. e., the quickness with which 
coagulation takes place, may vary independently. The rapidity of onset after muscular exercise and wasting disease may, perhaps, be in part dependent on an increase of acid reaction, which is produced under those circumstances in the muscle, for this seems to be favorable to the coagulation of the muscle plasma. When rigor mortis has once become thoroughly established in a muscle through deprivation of blood, it cannot be removed by any subsequent supply of blood. Thus, when the abdominal aorta has remained ligatured until the lower limbs have become completely rigid, untying the ligature will not restore the muscles to an irritable condition; it simply hastens the decomposition of the dead tissues by supplying them with oxygen and, in the case of the mammal, with warmth also. A muscle, however, may acquire as a whole a certain amount of rigidity on account of some of the fibres becoming rigid, while the remainder, though they have lost their irritability, have not yet advanced into rigor mortis. At such a juncture a renewal of the blood-stream may restore the irritability of those fibres which are not yet rigid, and thus appear to do away with rigor mortis; yet, it appears that in such cases the fibres which have actually become rigid never regain their irritability, but undergo degeneration.

Mere loss of irritability, even though complete, if stopping short of the actual coagulation of the muscle substance, may be with care removed.

The influence of blood-supply cannot be so satisfactorily studied in the case of nerves as in the case of muscles; there can, however, be little doubt that the effects are analogous.

$\S 84$. The influence of functional activity. This, too, is more easily studied in the case of muscles than of nerves.

When a muscle within the body is unused, it wastes; when used it (within certain limits) grows. Both these facts show that the nutrition of a muscle is favorably affected by its functional activity. Part of this may be an indirect effect of the increased blood-supply which occurs when a muscle contracts. When a nerve going to a muscle is stimulated, the bloodvessels of the muscle dilate. Hence at the time of the contraction more blood flows through the muscle, and this increased flow continues for some little while after the contraction of the muscle has ceased. But, apart from the blood-supply, it is probable that the exhaustion caused by a contraction is immediately followed by a reaction favorable to the nutrition of the muscle; and this is a reason, possibly the chief reason, why a muscle is increased by use, that is to say, the loss of substance and energy caused by the contraction is subsequently more than made up for by increased metabolism during the following period of rest.

A muscle, even within the body, after prolonged action is fatigued, $i . e$. a stronger stimulus is required to produce the same contraction; in other words, its irritability may be lessened by functional activity. Whether functional activity, therefore, is injurious or beneficial depends on its amount in relation to the condition of the muscle. 'The muscle is sooner fatigued and exhausted than the motor nerve.

The sense of fatigue of which, after prolonged or unusual exertion, we are conscious in our own bodies, is probably of complex origin, and its nature, like that of the normal muscular sense of which we shall have to speak hereafter, is at present not thoroughly understood. It seems to be in the first place the result of changes in the muscles themselves, but it is possibly also caused by changes in the nervous apparatus concerned in muscular action, and especially in those parts of the central nervous system which are concerned in the production of voluntary impulses. In any case it cannot be taken as an adequate measure of the actual fatigue of the muscles; for a man who says he is absolutely exhausted may, under excitement, perform a 
very large amount of work with his already weary muscles. The will, in fact, rarely if ever calls forth the greatest contractions of which the muscles are capable.

Absolute (temporary) exhaustion of the muscles, so that the strongest stimuli produce no contraction, may be produced even within the body by artificial stimulation; recovery takes place on rest. Out of the body absolute exhaustion takes place readily. Here, also, recovery may take place. Whether in any given case it does occur or not, is determined by the amount of contraction causing the exhaustion, and by the previous condition of the muscle. In all cases recovery is hastened by renewal (natural or artificial) of the blood-stream.

The more rapidly the contractions follow each other, the less the interval between any two contractions, the more rapid the exhaustion. A certain number of single induction-shocks repeated rapidly, say every second or oftener, bring about exhaustive loss of irritability more rapidly than the same number of shocks repeated less rapidly, for instance every 5 or 10 seconds. Hence tetanus is a ready means of producing exhaustion.

In exhausted muscles the elasticity is much diminished; the tired muscle returns less readily to its natural length than does the fresh one.

The exhaustion due to contraction may be the result either of the consumption of the store of really contractile material present in the muscle; or of the accumulation in the tissue of the products of the act of contraction; or of both of these causes.

The restorative influence of rest, in the case of a muscle removed from the circulation, may be explained by supposing that during the repose, either the internal changes of the tissue manufacture new explosive material out of the comparatively raw material already present in the fibres, or the directly hurtful products of the act of contraction undergo changes by which they are converted into comparatively inert bodies. A stream of fresh blood may exert its restorative influence not only by quickening the above two events, but also by carrying off the immediate waste products while at the same time it brings new raw material. It is not known to what extent each of these parts is played. That the products of contraction are exhausting in their effect, is shown by the facts that the injection of a solution of the muscle-extractives into the vessels of a muscle produces exhaustion, and that exhausted muscles are recovered by the simple injection of inert saline solutions into their bloodvessels. But the matter has not yet been fully worked out.

One important element brought by fresh blood is oxygen. This, as we have seen, is not necessary for the carrying out of the actual contraction, and yet is essential to the maintenance of irritability. The oxygen absorbed by the muscle apparently enters in some peculiar way into the formation of that complex explosive material the decomposition of which in the act of contraction, though it gives rise to carbonic acid and other products of oxidation, is not in itself a process of direct oxidation.

\section{The Energy of Muscle and Nerve, and the Nature of Muscular and Nervous Action.}

$\S 85$. We may briefly recapitulate some of the chief results arrived at in the preceding pages as follows:

A muscular contraction itself is essentially a translocation of molecules, a change of form, not of bulk. We cannot say, however, anything definite as to the nature of this translocation or as to the way in which it is brought about. For instance we cannot satisfactorily explain the contraction between 
the striation of a muscular fibre and a muscular contraction. Nearly all rapidly contracting muscles are striated, and we must suppose that the striation is of some use; but it is not essential to the carrying out of a contraction, for, as we shall see, the contraction of a non-striated muscle is fundamentally the same as that of a striated muscle. But whatever be the exact way in which the translocation is effected, it is in some way or other the result of a chemical change, of an explosive decomposition of certain parts of the muscle substance. The energy which is expended in the mechanical work done by the muscle has its source in the energy latent in the muscle substance and set free by that explosion. Concerning the nature of that explosion we only know at present that it results in the production of carbonic acid and in an increase of the acid reaction, and that heat is set free as well as the specific muscular energy. There is a general parallelism between the extent of metabolism taking place and the amount of energy set free; the greater the development of carbonic acid, the larger is the contraction and the higher the temperature.

It is important to remember that, as we have already urged, relaxation, the return to the original length, is an essential part of the whole contraction no less than shortening itself. It is true that the return to the original length is assisted by the stretching exerted by the load, and in the case of muscles within the living body is secured by the action of antagonistic muscles or by various anatomical relations; but the fact that the completeness and rapidity of the return are dependent on the condition of the muscle, that is, on the complex changes within the muscle making up what we call its nutrition, the tired muscle relaxing much more slowly than the untired muscle, shows that the relaxation is due in the main to intrinsic processes going on in the muscle itself, processes which we might characterize as the reverse of those of contraction. In fact, to put the matter forcibly, adopting the illustration used in $\$ 57$, and regarding relaxation as a change of molecules from a "formation " of one hundred in two lines of fifty each to a formation of ten columns each ten deep, it would be possible to support the theory that the really active forces in muscle are those striving to maintain the latter formation in columns, and that the falling into double lines, that is to say the contraction, is the result of these forces ceasing to act; in.other words, that the contracted state of the muscular fibre is what may be called the natural state, that the relaxed condition is only brought about at the expense of changes counteracting the natural tendencies of the fibre. Without going so far as this, however, we may still recognize that both contraction and relaxation are the result of changes which, since they seem to be of a chemical nature in the one case, are probably so in the other also.

\section{On Some Other Forms of Contractile Tissue.}

\section{Plain, Smooth or Unstriated Muscular Tissue.}

$\$ 86$. This, in vertebrates at all events, rarely occurs in isolated masses or muscles, as does striated muscular tissue, but is usually found taking part in the structure of complex organs, such for instance as the intestines; hence the investigation of its properties is beset with many difficulties.

$\$ 87$. As far as we know, plain muscular tissue in its chemical features resembles striated muscular tissue. It contains albunin, some forms of globulin, and antecedents of myosin which upon the death of the fibres become myosin; for plain muscular tissue after death becomes rigid, losing its extensibility and probably becoming acid, though the acidity is not so marked as in striated muscle. Kreatin has also been found, as well as 
glycogen, and, indeed, it seems probable that the whole metabolism of plain muscular tissue is fundamentally the same as that of the striated muscles.

$\$ 88$. In their general physical features plain muscular fibres also resemble striated fibres, and like them they are irritable and contractile; when stimulated they contract. The fibres vary in natural length in different situations, those of the bloodvessels, for instance, being shorter and stouter than those of the intestine; but in the same situation the fibres may also be found in one of two different conditions. In the one case the fibres are long and thin, in the other case they are reduced in length, it may be to one-half or even to one-third, and are correspondingly thicker, broader, and less pointed at the ends, their total bulk remaining unaltered. In the former case they are relaxed or elongated, in the latter case they are contracted.

The facts of the contraction of plain muscular tissue may be studied in the intestine, the muscular coat of which consists of an outer thin sheet composed of fibres and bundles of fibres disposed longitudinally, and of an inner much thicker sheet of fibres disposed circularly ; in the ureter a similar arrangement of two coats obtains.

If a mechanical or electrical (or, indeed, any other) stimulus be brought to bear on a part of a fresh, living, still warm intestine (the small intestine is the best to work with) a circular contraction is seen to take place at the spot stimulated ; the intestine seems nipped in ringwise, as if tied round with an invisible cord, and the part so constricted, previously vascular and red, becomes pale and bloodless. The individual fibres of the circular coat in the region stimulated have each become shorter, and the total effect of the shortening of the multitude of fibres all having the same circular disposition is to constrict or narrow the lumen or tube of the intestine. The longitudinally disposed fibres of the outer longitudinal coat will at the same time similarly contract or shorten in a longitudinal direction, but this coat being relatively much thinner than the circular coat, the longitudinal contraction is altogether overshadowed by the circular contraction. A similar mode of contraction is also seen when the ureter is similarly stimulated.

The contraction thus induced is preceded by a very long latent period and lasts a very considerable time, in fact, several seconds, after which relaxation slowly takes place. We may say then that over the circularly disposed fibres of the intestine (or ureter) at the spot in question there has passed a contraction-wave remarkable for its long latent period and for the slowness of its development, the wave being propagated from fibre to fibre. From the spot so directly stimulated the contraction may pass also as a wave (with a length of $1 \mathrm{~cm}$. and a velocity of from 20 to 30 millimetres a second in the ureter), along the circular coat both upwards and downwards. The longitudinal fibres at the spot stimulated are, as we have said, also thrown into contractions of altogether similar character, and a wave of contraction may thus also travel longitudinally along the longitudinal coat both upwards and downwards. It is evident, however, that the wave of çontraction of which we are now speaking is, in one respect, different from the wave of contraction treated of in dealing with striated muscle. In the latter case the contraction-wave is a simple wave propagated along the individual fibre, and starting from the end-plate or, in the case of direct stimulation, from the part of the fibre first affected by the stimulus; we have no evidence that the contraction of one fibre can communicate contraction to neighboring fibres, or, indeed, in any way influence neighboring fibres. In the case of the intestine or ureter the wave is complex, being the sum of the contractionwaves of several fibres engaged in different phases, and is propagated from 
fibre to fibre, both in the direction of the fibres, as when the whole circumference of the intestine is engaged in the contraction, or when the wave travels longitudinally along the longitudinal coat, and also in a direction at right angles to the axes of the fibres, as when the contraction-wave travels lengthways along the circular coat of the intestine, or when it passes across a breadth of the longitudinal coat; that is to say, the changes leading to contraction are communicated not only in a direct manner across the cenent substance uniting the fibres of a bundle, but also in an indirect manner, probably by means of nerve fibres from bundle to bundle across the connective tissue between them. Moreover, it is obvious that even the contraction-wave which passes along a single unstriated fibre differs from that passing along a striated fibre, in the very great length both of its latent period and of the duration of its contraction. Hence, much more even than in the case of a striated muscle, the whole of each fibre must be occupied by the contractionwave, and, indeed, be in nearly the same phase of the contraction at the same time.

Waves of contraction thus passing along the circular and longitudinal coats of the intestine constitute what is called peristaltic action.

Like the contractions of striated muscle the contractions of plain muscles may be started by stimulation of nerves going to the part, the nerves supplying plain muscular tissue running for the most part in the so-called sympathetic system, but being, as we shall see, ultimately connected with the spinal cord or brain. Here, however, we come upon an important distinction between the striated skeletal muscles and the plain muscles of the viscera. As a general rule, the skeletal muscles are thrown into contraction only by nervous impulses reaching them along their nerves; spontaneous movements of the skeletal muscles, that is, contractions arising out of changes in the muscles themselves, are extremely rare and when they occur are abnormal ; so-called "cramps" for instance, which are prolonged tetanic contractions of skeletal muscles independent of the will, though their occurrence is largely due to the condition of the muscle itself, generally the result of overwork, are probably actually started by nervous impulses reaching them from without. On the other hand, the plain muscles of the viscera, of the intestine, uterus, and ureter, for instance, and of the bloodvessels very frequently fall into contractions and so carry out movements of the organs to which they belong quite independently of the central nervous system. These organs exhibit "spontaneous" movements quite apart from the will, quite apart from the central nervous system, and under favorable circumstances continue to do this for some time after they have been entirely isolated and removed from the body. So slight, indeed, is the connection between the movements of organs and parts supplied with plain muscular fibres and the will, that these muscular fibres have sometimes been called involuntary muscles; but this name is undesirable, since some muscles consisting entirely of plain muscular fibres (e.g., the ciliary muscles by which the eye is accommodated for viewing objects at different distances) are directly under the influence of the will, and some muscles composed of striated fibres (e.g., those of the heart) are wholly removed from the influence of the will.

We shall best study, however, the facts relating to the movements of parts provided with plain muscular fibres when we come to consider the parts themselves.

Like the skeletal muscles, whose nervous elements have been rendered functionally incapable ( $\$ 76$ ), plain muscles are much more sensitive to the making and breaking of a constant current than to induction-shocks; a current, when very brief, like that of an induction-shock, produces little or no effect. 
The contraction of plain muscular fibres is, as we said, very slow in its development and very long in its duration, even when started by a momentary stimulus, such as a single induction-shock. The contraction after a stimulation often lasts so long as to raise the question, whether what has been produced is not a single contraction but a tetanus. Tetanus, however, that is, the fusion of a series of contractions, seems to be of rare occurrence, though probably it may be induced in plain muscular tissue; but the ends of tetanus are gained by a kind of contraction which, rare or at least not prominent in skeletal muscle, becomes of great importance in plain muscular tissue by a kind of contraction called a tonic contraction. The subject is one not without difficulties, but it would appear that a plain muscular fibre may remain for a very considerable time in a state of contraction, the amount of shortening thus maintained being either small or great; it is then said to be in a state of tonic contraction. This is especially seen in the case of the plain muscular tissue of the arteries, and we shall have to return to this matter in dealing with the circulation.

The muscular tissue which enters into the construction of the heart is of a peculiar nature, being on the one hand striated and on the other in some respects similar to plain muscular tissue, but this we shall consider in dealing with the heart itself.

\section{Ciliary Movement.}

$\S 89$. Nearly all the movements of the body which are not due to physical causes, such as gravity, the diffusion of liquids, etc., are carried out by muscles, either striated or plain ; but some small and important effects in the way of movement are produced by the action. of cilia, and by those changes of protoplasm which are called amoboid.

$\$ 90$. Ciliary action, in the form in which it is most common, in mammals and, indeed, vertebrates, consists in the cilium being at one moment straight or vertical, at the next moment being bent down suddenly into a hook or sickle form, and then more slowly returning to the straight erect position. When the cilia are vigorous this double movement is repeated with very great rapidity - so rapidly that the individual movements cannot be seen; it is only when, by reason of fatigue, the action becomies slow that the movement itself can be seen; what is seen otherwise is simply the effect of the movement. The movements when slow have been counted at about eight (double movements) in a second; probably when vigorous they are repeated from twelve to twenty times a second.

The flexion takes place in one direction only, and all the cilia of each cell and, indeed, of all the cells of the same epithelium move in the same direction. Moreover, the same direction is maintained during the whole life of the epithelium; thus the cilia of the epithelium of the trachea and bronchial passages move during the whole of life in such a way as to drive the fluid lying upon them upward toward the mouth; as far as we know, in vertebrates, or at least in mammals, the direction is not and cannot by any means be reversed.

The flexion is very rapid, but the return to the erect position is much slower; hence the total effect of the blow, supposing the cilium and the cell to be fixed, is to drive the thin layer of fluid in which the cilium is working, and which always exists over the epithelium, and any particles which may be floating in that fluid, in the same direction as that in which the blow is given. If the cell be not attached, but floating free, the effect of the blow may be to drive the cell itself backward; and when perfectly fresh ciliated epithelium is teased out and examined in an inert fluid, such 
as normal saline solution, isolated cells or small groups of cells may be seen rowing themselves about, as it were, by the action of their cilia.

All the cilia of a cell move, as we have just said, in the same direction, but not quite at the same time. If we call the side of the cell toward which the cilia bend the front of the cell and the opposite side the back, the cilia at the back move a trifle before those at the front, so that the movement runs over the cell in the direction of the movement itself. Similarly, taking any one cell, the cilia of the cells behind it move slightly before and the cilia of the cells in front of it slightly after its own cilia move. Hence, in this way, along a whole stretch of epithelium, the movement or bending of the cilia sweeps over the surface in ripples or waves, very much as, when the wind blows, similar waves of bending sweep over a field of corn or tall grass. By this arrangement the efficacy of the movement is secured, and a steady stream of fluid carrying particles is driven over the surface in a uniform continued direction; if the cilia of separate cells, and still more if the separate cilia of each cell, moved independently of the others, all that would be produced would be a series of minute "wabbles," of as little use for driving the fluid definitely onward as the efforts of a boat's crew all rowing out of time are for propelling the boat.

Swift bending and slower straightening is the form of ciliary movement generally met with in the ciliated epithelium of mammals and, indeed, of vertebrates; but among the invertebrates we find other kinds of movement, such as a to-and-fro movement, equally rapid in both directions, a corkscrew movement, a simple undulatory movement, and many others. In each case the kind of movement seems adapted to secure a special end. Thus even in the mammal while the one-sided blow of the cilia of the epithelial cells secures a flow of fluid over the epithelium, the tail of the spermatozoon, which is practically a single cilium, by moving to and fro in an undulatory fashion drives the head of the spermatozoon onward in a straight line, like a boat driven by a single oar worked at the stern.

Why and exactly how the cilium of the epithelial cells bends swiftly and straightens slowly, always acting in the same direction, is a problem difficult at present to answer fully. Some have thought that the body of the cell is contractile, or contains contractile mechanisms pulling upon the cilia, which are thus simple passive puppets in the hands of the cells. But there is no satisfactory evidence for such a view. On the whole the evidence is in favor of the view that the action is carried out by the cilium itself, that the bending is a contraction of the cilium, and that the straightening corresponds to the relaxation of a muscular fibre. But even then the exact manner in which the contraction bends and the relaxation straightens the filament is not fully explained. We have no positive evidence that a longitudinal half, the inside we might say, of the filament is contractile, and the other half, the outside, elastic, a supposition which has been made to explain the bending and straightening. In fact, no adequate explanation of the matter has as yet been given, and it is really only on general grounds we conclude that the action is an effect of contractility.

In the vertebrate animal cilia are, as far as we know, wholly independent of the nervous system, and their movement is probably ceaseless. In such animals, however, as infusoria, hydrozoa, etc., the movements in a ciliary tract may often be seen to stop and to go on again, to be now fast, now slow, according to the needs of the economy, and, as it almost seems, according to the will of the creature ; indeed, in some of these animals the ciliary movements are clearly under the influence of the nervous system.

Observations with galvanic currents, constant and interrupted, have not 
led to any satisfactory results, and, as far as we know at present, ciliary action is most affected by changes of temperature and chemical media. Moderate heat quickens the movements, but a rise of temperature beyond a certain limit (about $40^{\circ} \mathrm{C}$, in the case of the pharyngeal membrane of the frog) becomes injurious; cold retards. Very dilute alkalies are favorable, acids are injurious. An excess of carbonic acid or an absence of oxygen diminishes or arrests the movements, either temporarily or permanently, according to the length of the exposure. Chloroform or ether in slight doses diminishes or suspends the action temporarily, in excess kills and disorganizes the cells.

\section{Amoeboid Movements.}

$\S 91$. The white blood-corpuscles, as we have said ( $\$ 28)$, are able of themselves to change their form and by repeated changes of form to move from place to place. Such movements of the substance of the corpuscles are called amœboid, since they closely resemble and appear to be identical in nature with the movements executed by the amœba and similar organisms. The movements of the endoplasm of the vegetable cell seems also to be of the same kind.

The amoba changes its form (and shifts its place) by throwing out projections of its substance, called pseudopodia which may be blunt and short, broad bulgings as it were, or may be so long and thin as to be mere filaments, or may be of an intermediate character. As we watch the outline of the hyaline ectosare we may see a pseudopodium beginning by a slight bulging of the outline; the bulging increases by the neighboring portions of the ectosare moving into it, the movement under the microscope reminding one of the flowing of melted glass. As the pseudopodium grows larger and engages the whole thickness of the ectosarc at the spot, the granules of the endosarc may be seen streaming into it, forming a core of endosarc in the middle of the bulging of ectosarc. The pseudopodium may continue to grow larger and larger at the expense of the rest of the body, and eventually the whole of the amoba including the nucleus may, as it were, have passed into the pseudopodium; the body of the amœba will now occupy the place of the pseudopodium instead of its old place; in other words, it will in changing its form have also changed its place.

During all these movements, and during all similar amœboid movements, the bulk of the organism will, as far as can be ascertained, have remained unchanged; the throwing out a pseudopodium in one direction is accompanied by a corresponding retraction of the body in other directions. If, as sometimes happens, the organism throws out pseudopodia in various directions at the same time, the main body from which the pseudopodia project is reduced in thickness; from being a spherical lump, for instance, it becomes a branched film. The movement is brought about not by increase or decrease of substance but by mere translocation of particles; a particle which at one moment was in one position moves into a new position, several particles thus moving toward the same point cause a bulging at that point, and several particles moving away from the same point cause a retraction at that point; but no two particles get nearer to each other so as to occupy together less space and thus lead to condensation of substance, or get further from each other so as to occupy more space and thus lead to increase of bulk.

In this respect, in that there is no change of bulk but only a shifting of particles in their relative position to each other, the amœboid movement resembles a muscular contraction; but in other respects the two kinds of movement seem different, and the question arises, have we the right to speak 
of the substance which can only execute amœboid movements as being contractile?

We may, if we admit that contractility is at bottom simply the power of shifting the relative position of particles, admit that muscular contraction is a specialized form of contraction. In a plain muscular fibre (which we may take as simpler than the striated muscle) the shifting of particles is specialized in the sense that it has always a definite relation to the long axis of the fibre; when the fibre contracts a certain number of particles assume a new position by moving at right angles to the long axis of the fibre, and the fibre in consequence becomes shorter and broader. In a white blood-corpuscle, amœba, or other organism executing amœboid movements, the shifting of the particles is not limited to any axis of the body of the organism; at the same moment one particle or one set of particles may be moving in one direction, and another particle or another set of particles in another direction. A pseudopodium short and bread, or long, thin, and filamentous, may be thrust out from any part of the surface of the body and in any direction; and a previously existing pseudopodium may be shortened, or be wholly drawn back into the substance of the body.

\section{CHA PTER III.}

\section{ON THE MORE GENERAL FEATURES OF NERVOUS TISSUES.}

$\S 92$. In the preceding chapter we have dealt with the properties of nerves going to muscles, the nerves which we called motor, and have incidentally spoken of other nerves which we called sensory. Both these kinds of nerves are connected with the brain and spinal cord, and form part of the general nervous system. We shall have to study hereafter in detail the brain and spinal cord; but the nervous system intervenes so repeatedly in the processes carried out by other tissues that it will be desirable, before proceeding further, to discuss some of its more general features.

The nervous system consists (1) of the brain and spinal cord forming together the cerebrospinal axis or central nervous system, (2) of the nerves passing from that axis to nearly all parts of the body, those which are connected with the spinal cord being called spinal and those which are connected with the brain, within the cranium, being called cranial, and (3) of ganglia distributed along the nerves in various parts of the body.

The spinal cord obviously consists of a number of segments or metameres, following in succession along its axis, each metamere giving off on each side a pair of spinal nerves; and a similar division into metameres may be traced in the brain, though less distinctly, since the cranial nerves are arranged in manner somewhat different from that of the spinal nerves. We may take a single spinal metamere, represented diagrammatically in Fig. 35, as illustrating the general features of the nervous system; and since the half on one side of the median line resembles the half on the other side we may deal with one lateral half only.

Each spinal nerve arises by two roots. The metamere of the central nervous system $C$ consists, as we shall hereafter see, of gray matter $G r$ in the interior and white matter $W$ on the outside. From the anterior part of gray matter is given off the anterior nerve root $A$ and from the posterior part the posterior nerve root $P$. The latter passes into a swelling or ganglion $G$, "the ganglion of the posterior root," or more shortly "the spinal 
FIG. 35.

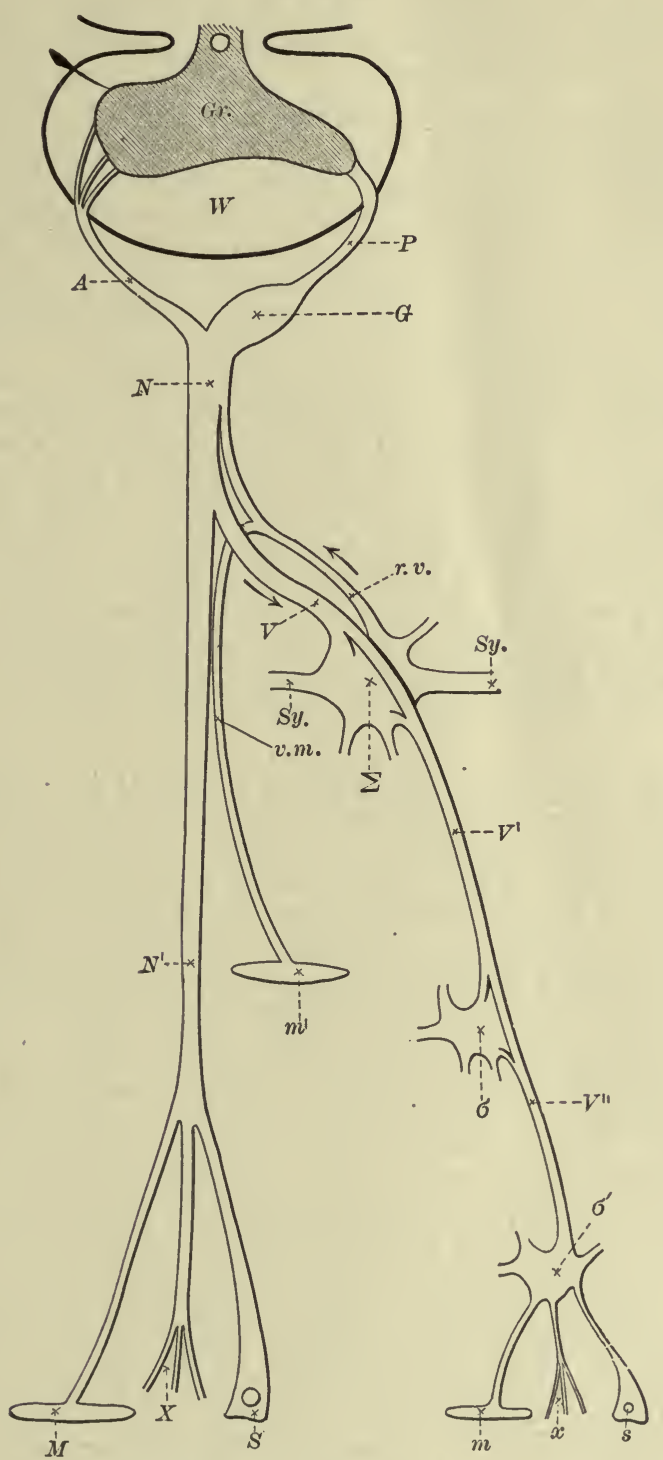

Scheme of the Nerves of a Segment of the Spinal Cord: $G r$ gray, $W$ white matter of spinal cord. $A$ anterior, $P$ posterior root $G$ ganglion on the posterior root. $N$ whole nerve, $N^{\prime}$ spinal nerve proper, ending in $\boldsymbol{M}$ skeletal or somatic muscle, $S$ somatic sensory cell or surface, $X$ in other ways. $V$ visceral nerve (white ramus communicans) passing to a ganglion of the sympathetic chain $\mathbf{\Sigma}$, and passing on as $V^{\prime}$ to supply the more distant ganglion $\sigma$, then as $V^{\prime \prime}$ to the peripheral ganglion $\sigma^{\prime}$ and ending in $m$ splanchnic muscle, $s$ splanchnic sensory cell or surface, $x$ other possible splanchnic endings. From $\mathrm{\Sigma}$ is given off the revehent nerve $r, v$ (gray ramus communicans) which partly passes backward toward the spinal cord, and partly runs as $v$. $m$, in connection with the spinal nerve, to supply vasomotor (constrictor) fibres to the muscles $\left(\mathrm{m}^{\prime}\right)$ of bloodvessels in certain parts, for example, in the limbs. Sy, the sympathetic chain uniting the ganglia of the series $\mathbf{\Sigma}$. The terminations of the other nerves arising from $\mathbf{\Sigma}, \sigma, \sigma^{\prime}$ are not shown. 
ganglion "; the anterior root does not pass into this ganglion. Beyond the ganglion the roots join to form the nerve trunk $N$. We shall later on give the evidence that the nerve fibres composing the posterior root $P$ are, as far as we know at present, exclusively occupied in carrying nervous impulses from the tissues of the body to the central nervous system, and that the fibres composing the anterior root $A$ are similarly occupied in carrying impulses from the central nervous system to the several tissues: that is to say, the former is made up of sensory fibres, or (since the impulses passing along them to the central system may give rise to effects other than sensations) afferent fibres, while the latter is made up of motor, or (since the impulses passing along them from the central nervous system may produce effects other than movements), efferent fibres. The nerve trunk $N$ is consequently a mixed nerve composed of afferent and efferent fibres.

By far the greater part of this mixed nerve, dividing into various branches, is distributed $\left(N^{\prime}\right)$ to the skin and the skeletal muscles, some of the fibres (motor) ending in muscular fibres $(\boldsymbol{M})$, others (sensory) ending in epithelial cells $(S)$ connected with the skin, which we shall consider hereafter under the name of sensory epithelial cells, while others, $X$, after dividing into minute branches and forming plexuses end, in ways not yet definitely determined, in tissues associated with the skin and skeletal muscles. Morphologists distinguish the parts which go to form the skin, skeletal muscles, etc., as somatic, from the splanchnic parts which go to form the viscera. We may, accordingly, call this main part of the spinal nerve the somatic division of the nerve.

Soon after the mixed nerve $N$ leaves the spinal canal, it gives off a small branch $V$, which under the name of (white) ramus communicans, joins one of a longitudinal series of ganglia $(\Sigma)$ conspicuous in the thorax as the main sympathetic chain. 'This branch is destined to supply the viscera, and might, therefore, be called the splanchnic division of the spinal nerve. We may say at once, without entering into details, that the whole of the sympathetic system, with its ganglia, plexuses, and nerves, is to be regarded as a development or expansion of the visceral or splanchnic divisions of certain spinal nerves. By means of this system splanchnic fibres from the central nervous system are distributed to the tissues of the viscera, some of them on their way passing through secondary ganglia $\sigma$, and, it may be, tertiary ganglia. There are, however, as we shall see, certain nerves or fibres which do not run in the sympathetic system, and yet are distributed to the viscera and are "splanchnic" in nature. We cannot, therefore, use the word sympathetic to denote all the fibres which are splanchnic in nature. On the other hand, the "splanchnic nerves" of the anatomist form a part only of the splanchnic system in the above sense, the term thus used is limited to particular nerves of the splanchnic system distributed to the abdomen; and the double use of the term splanchnic might lead to confusion. The difficulty, may, perhaps, be avoided by calling the splanchnic nerves of the anatomist "abdominal splanchnic." The majority of these splanchnic fibres seem to be efferent in nature, carrying impulses from the central nervous system to the tissues, some ending in plain muscular fibres $(m)$, others in other ways $(x)$; but some of the fibres are afferent and convey impulses from the viscera to the central nervous system, and it is probable that some of these begin or end in epithelial cells of the viscera $(s)$.

We shall have occasion in the next chapter to speak of nerves which govern the bloodvessels of the body, the so-called vasomotor nerves. A certain class of these, namely, the vaso-constrictor nerves or fibres are branches of the splanchnic divisions of the cerebro-spinal nerves, and, as we shall see, the vaso-constrictor nerves of the skeletal muscles, skin, and other parts sup- 
plied by somatic nerves, after running for some distance in the splanchnic division $(V)$, turn aside $(r . v$ and $v, m)$ and join the somatic division, the fibres of which they accompany on their way to the tissues whose bloodvessels $\left(m^{\prime}\right)$ they supply.

We have seen ( $\$ 68$ ) that a nerve going to a muscle is composed of nerve fibres, chiefly medullated, some, however, being non-medullated, bound together by connective tissues. The same description holds good for the whole somatic division of each of the spinal nerves. The splanchnic division also consists of medullated and non-medullated fibres bound together by connective tissue, but in it the non-medullated fibres preponderate, some branches appearing to contain hardly any medullated fibres at all. The non-medullated fibres which are found in the somatic division appear to be fibres which have joined that division from the splanchnic division. So prominent are non-medullated fibres in splanchnic nerves and hence in the sympathetic system that they are sonietimes called sympathetic fibres.

We have said that the axis-cylinder, whether of a medullated or nonmedullated fibre, is to be considered as a long-drawn-out process of a nerve cell. Nerve cells are found in three main situations. 1 . In the central nervous system, the brain, and spinal cord. 2. In the several ganglia placed along the course of the nerves, both the spinal ganglia, and the ganglia of the splanchnic or sympathetic system. 3. At the terminations of nerves in certain tissues. Some of these latter are to be regarded as small, more or less terminal, ganglia, and similar minute ganglia consisting of two or three cells only are found frequently along the course of splanchnic nerves and occasionally along the course of spinal nerves; such cells really, therefore, belong to the second group. But besides this, in certain situations, as, for instance, in certain organs of the skin, and in the organs of special sense, nerves, generally afferent or sensory in nature, either actually end in, or at their termination are connected with, cells which appear to be of a nervous nature; such cells form a distinct category by themselves.

Hence along its whole course a nerve consists exclusively of nerve fibres (and the connective tissue supporting them), except in the central nervous system from which it springs in the ganglia, great and small, through which it passes, or which are attached to it at one part or another of its course, in both of which situations nerve cells are found, and at its termination where its fibres may end in nerve cells.

The features of these nerve cells differ in these several situations. The characters of the terminal cells which, as we have said, are chiefly sensory, and the structure of the brain and spinal cord, we shall study in detail later on. We may here confine our attention to the nerve cells of the ganglia and to some of the broad features of the nerve cells of the spinal cord.

$\$$ 93. Spinal ganglia. When a longitudinal section of a spinal ganglion is examined under a low power, the fibres of the posterior root as they enter the ganglion are observed to spread out and pass between relatively large and conspicuously nucleated cells, which are to a large extent arranged in groups, somewhat after the fashion of a bunch of grapes. These are the nerve cells ; they have frequently a diameter of about $100 \mu$, but may be still larger or may be much smaller. In a transverse section it will be observed that a large compact mass of these cells lies on the outer side of the ganglion, and that the racemose groups on the inner side are smaller. A quantity of connective tissue carrying bloodvessels and lymphatics runs between the groups, and passing into each group runs between the cells and fibres; and a thick wrapping of connective tissue continuous with the sheath of the nerves surrounds and forms a sheath for the whole ganglion.

Each of the nerve cells-ganglionic cells, as they are called-examined 
under a higher power, either after having been isolated or in an adequately thin and prepared section, will present the following features:

The cell consists of a cell body which is, normally, pear-shaped, having a broad end in which is placed the nucleus, and a narrow end which thins out into a stalk, and is eventually continued on as a nerve fibre. The substance of the cell body is of the kind which we call finely granular protoplasm; sometimes there is an appearance of fibrillation, the fibrillæ passing in various directions in the body of the cell and being gathered together in a longitudinal direction in the stalk. Sometimes the cell body immediately around the nucleus appears of a different grain from that nearer the stalk, and not unfrequently near the nucleus is an aggregation of discrete pigment granules imbedded in the protoplasm.

The nucleus, like the nuclei of nearly all nerve cells, is large and conspicuous, and when in a normal condition is remarkably clear and refractive, though it appears to consist like other nuclei of a nuclear membrane and network and nuclear interstitial material. Even inore conspicuous, perhaps, is a very large spherical, highly refractive nucleolus; occasionally more than one nucleolus is present.

Surrounding the cell body is a distinct sheath or capsule consisting of a transparent, hyaline, or faintly fibrillated membrane, lined on the inside by one layer or by two layers of flat, polygonal, nucleated epithelioid cells or plates; that is to say, cells which resemble epithelium cells, but differ not only in being extremely flattened, but also in the cell body being transformed from ordinary granular protoplasm into a more transparent differentiated material. In stained specimens the nuclei of these plates are very conspicuous. Under normal conditions this sheath is in close contact with the whole body of the cell, but in hardened and prepared specimens the cell body is sometimes seen shrunk away from the sheath, leaving a space between them. Occasionally the cell body while remaining attached to the sheath at three or four or more points is retracted elsewhere, and accordingly assumes a more or less stellate form; but this artificial condition must not be confounded with the natural branched form which, as we shall see, other kinds of nerve cells possess.

When a section is marle through a hardened ganglion, the plane of the section passes through the stalks of few only of the cells, and that rarely for any great distance along the stalk, since in the case of many of the cells the stalk is more or less curved and consequently runs out of the plane of section; but in properly isolated cells we can see that in many cases, and we have reasons to believe that in all cases, the stalk of the cell is, as we have said, continued on into a nerve fibre. As the cell body narrows into the stalk several nuclei make their appearance, lodged on it; these are small granular nuclei, wholly unlike the nucleus of the cell body itself, and more like, though not quite like, the nuclei of the neurilemma of a nerve. They are probably of the same nature as the latter; and, indeed, as we trace the narrowing stalk downward a fine delicate sheath, which, if present, is at least not obvious over the cell body, makes its appearance, and a little further on, between this sheath, which is now clearly a neurilemma, and the stalk of the cell body, which has by this time become a cylinder of uniform width and is now obviously an axis-cylinder, a layer of medulla, very fine at first, but rapidly thickening, is established. The stalk of the nerve cell thus becomes an ordinary medullated nerve fibre. The sheath of the cell is continued also on to the nerve fibre, not as was once thought as the neurilemma, but as that special sheath of connective tissue of which we have already spoken as Henle's sheath, and which ultimately becomes fused with the connective tissue of the nerve. 
At some variable distance from the cell the nerve fibre bears the first node, and either at this or some early succeeding node the fibre divides into two; as we have seen, division of a medullated nerve fibre always takes place at a node. The two divisions thus arising run in opposite directions, forming in this way a T-piece; and while one division runs in one direction toward the posterior root, the other runs in an opposite direction toward the nerve trunk. The nerve cell is thus, as it were, a side piece attached to a fibre passing through the ganglion on its way from the posterior root to the nerve trunk. It cannot be said that in any one ganglion this connection has been traced in the case of every nerve cell of the ganglion; but the more care is taken, and the more successful the preparation, the greater is the number of cells which may be isolated with their respective T-pieces; so that we may conclude that, normally, every cell of a ganglion is connected on the one hand with a fibre of the posterior root, and on the other hand with a fibre of the nerve trunk. We have reasons further to believe that every fibre of the posterior root in passing through the ganglion on its way to the mixed nerve trunk is thus connected with a nerve cell; but this has been called in question. In certain animals-for instance, certain fishesthe cells of the spinal ganglia are not pear-shaped, but oval or fusiform, and each narrow end is prolonged into a nerve fibre, one end thus being connected with the posterior root and the other with the nerve trunk. In such a case the nerve cell is simply a direct enlargement of the axis-cylinder, with a nucleus placed in the enlargement. The nerve cells above described are similar enlargements, also bearing nuclei, placed not directly in the course of the axis-cylinder, but on nne side, and connected with the axis-cylinder by the cross-piece of the T-piece. Hence the ordinary ganglion cell is spoken of as being unipolar, those of fishes being called bipolar.

In examining spinal ganglia cells are sometimes found which bear no trace of any process connecting them with a nerve fibre. Such cells are spoken of as apolar. It is possible that such a cell may be a young cell which has not yet developed its nerve process, or an old cell which has by degeneration lost its nerve process.

$\$ 94$. The ganglia of the splanchnic system, like the spinal ganglia, consist of nerve cells and fibres imbedded in connective tissue, which, however, is of a looser and less compact nature in them than in the spinal ganglia. As far as the characters of their nuclei, the nature of their cell substance, and the possession of a sheath, are concerned, what has been said concerning the nerve cells of spinal ganglia holds, in general, good for those of splanchnic ganglia; and, indeed, in certain ganglia of the splanchnic svstem connected with the cranial nerves the nerve cells appear to be wholly like those of spinal ganglia. In most splanchnic ganglia, however, in those which are generally called sympathetic ganglia, two important differences may be observed between what we may call the characteristic nerve cell of the splanchnic ganglion and the cell of the spinal ganglion.

In the first place, while the nerve cell of the spinal ganglia has one process only, the nerve cell of the splanchnic ganglia may have, and frequently has, two, three, or even four or five processes; it is a multipolar cell.

In the second place, while these processes of the splanchnic ganglion cell are continued on as nerve fibres, as is the single process of the spinal ganglion cell, the nerve fibres so formed are, in the case of most of the processes of a cell, and sometimes in the case of all the processes, non-medullated fibres, and remain non-medullated as far as they can be traced. In some instances one process becomes at a little distance from the cell a medullated fibre, while the other processes become non-medullated fibres; 
and we are led to believe that in this case the medullated fibre is proceeding to the cell on its way from the central nervous system, and that the nonmedullated fibres are proceeding from the cell on their way to more peripherally placed parts; the nerve cell seems to serve as a centre for the division of nerve fibres, and also for the change from medullated to nonmedullated fibres.

In consequence of its thus possessing several processes, the splanchnic ganglion cell is more or less irregular and often star-like in form, in contrast to the pear shape of the spinal ganglion cell. But in certain situations in certain animals - for instance, in the frog-in many of the ganglia of the abdomen, and in the small ganglia in the heart, pear-shaped splanchnic ganglion cells are met with. In such cases the nucleated sheath is distinctly pear-shaped or balloon-shaped, and the large conspicuous nucleus is placed, as in the spinal ganglion cell, near the broad end, but the cell substance of the cell is gathered at the stalk, not into a single fibre, but into two fibres, one of which is straight and the other twisted spirally round the straight one. The two fibres run for some distance togther in the same funnel-shaped prolongation of the nucleated sheath of the cell, but eventually separate, each fibre acquiring a sheath (sheath of Henle) of its own. Generally, if not always, one fibre, usually the straight one, becomes a medullated fibre, while the other, usually the twisted or spiral one, is continued as a non-medullated fibre, while within the common nucleated sheath both fibres, especially the spiral one, bear nuclei of the same character as those seen in a corresponding situation in the spinal ganglion cell. It has been maintained that the straight and spiral fibres take origin from different parts of the nerve cell, but this has not been definitely proved.

In the walls of the intestine, in connection with splanchnic nerves, are found peculiar nerve cells forming what are known as the plexuses of Meissner and Auerbach, but we shall postpone for the present any description of these or of other peculiar splanchnic cells.

$\S 95$. In the central nervous system nerve cells are found in the so-called gray matter only; they are absent from the white matter. In the gray matter of the spinal cord, in the parts spoken of as the anterior cornua, we meet with remarkable nerve cells of the following characters. The cells are large, varying in diameter from $50 \mu$ to $140 \mu$, and each consists of a cell body surrounding a large conspicuous refractive nucleus, in which is placed an even still more conspicuous nucleolus. The nucleus resembles the nuclei of the ganglion cells already described, and the cell body, like the cell body of the ganglion cells, is composed of finely granular protoplasm, often fibrillated, though generally obscurely so; frequently a yellowish-brown pigment is deposited in a part of the cell body not far from the nucleus. The cell body is prolonged sometimes into two or three only, but generally into several processes, which appear more distinctly fibrillated than the more central parts of the cell body. These processes are of two kinds. One process, and, apparently, one only, but in the case of the cells of the anterior cornu, always one, is prolonged as a thin unbranched band, which retains a fairly uniform diameter for a considerable distance from the cell, and when successfully traced is found sooner or later to acquire a medulla and to become the axis-cylinder of a nerve fibre; the processes which thus pass out from the gray matter of the anterior cornu through the white matter form the anterior roots of the spinal nerve. Such a process is accordingly called the axis-cylinder process. The other processes of the cell rapidly branch, and so divide into very delicate filaments which are soon lost to view in the substance of the gray matter. Indeed, the gray matter is partly made up of a plexus of delicate filaments arising, on the one hand, fiom the divisions of 
processes of the nerve cells, and on the other, from the division of the axiscylinders of fibres running in the gray matter.

The cell is not surrounded like the ganglion cell by a distinct sheath. As we shall see later on, while treating in detail of the central nervous system, all the nervous elements of the spinal cord are supported by a network or spongework of delicate peculiar tissue called neuroglia, analogous to and serving much the same function as, but different in origin and nature from, connective tissue. This neuroglia forms a sheath to the nerve cell and to its processes, as well as to the nerve fibres running both in the white and the gray matter; hence within the central nervous system the fibres, whether medullated or not possess no separate neurilemma; tubular sheaths of the neuroglia give the axis-cylinder and medulla all the support they need.

All the nerve cells of the anterior cornu probably possess an axis-cylinder process, and other cells similarly provided with an axis-cylinder process are found in other parts of the gray matter. But in certain parts, as, for instance, in the posterior cornu, many of the cells appear to possess no axis-cylinder process; in such cases all the processes appear to branch out rapidly into fine filaments. Except for this absence, apparent or real, of an axis-cylinder process, such cells resemble in their general features the cells of the anterior cornu, though they are generally somewhat smaller. Speaking generally, the great feature of the nerve cells of the central nervous system as distinguished from the ganglion cells is the remarkable way in which their processes branch off into a number of delicate filaments, corresponding to the delicate filaments or fibrillæ in which at its termination in the tissues the axis-cylinder of a nerve often ends.

$\$ 96$. From the above description it is obvious that in the spinal cord (to which as representing the central nervous system we may at present confine ourselves, leaving the brain for later study) afferent fibres (fibres of the posterior root) are in some way by means of the gray matter brought into connection with efferent fibres (fibres of the anterior root) ; in other words, the spinal cord is a centre uniting afferent and efferent fibres. The spinal ganglia are not centres in this sense; the nerve cells composing the ganglia are simply relays on the afferent fibres of the posterior root, they have no connection whatever with efferent fibres, they are connected with fibres of one kind only. Concerning the ganglia of the splanchnic system we cannot in all cases make at present a positive statement, but the evidence so far at our disposal points to the conclusion that in them as in the spinal ganglia each nerve cell belongs to fibres of one function only, that where several processes of a cell are prolonged into nerve fibres these fibres have all the same function, the nerve cell being, as in the spinal ganglia, a mere relay. $\mathrm{We}$ have no satisfactory evidence that in a ganglion the fibres springing from or connected with one cell join another cell so as to convert the ganglion into a centre joining together cells whose nerve fibres have different functions.

We shall have later on to bring forward evidence that the nucleated cell body of a nerve cell in a ganglion or elsewhere is in some way or other connected with the nutrition, the growth and repair of the nerve fibres springing from it. Besides this nutritive function the multipolar cells of the splanchnic ganglia appear to serve the purpose of multiplying the tracts along which nervous impulses may pass. An impulse, for instance, reaching a multipolar cell in one of the proximal sympathetic ganglia along one fibre or process (the fibre in very many cases being a medullated fibre) can pass out of the cell in various directions along several processes or fibres, which, in the majority of cases, if not always, are non-medullated fibres. Thus these nerve cells are organs of distribution for impulses of the same kind. What further 
modifications of the impulses thus passing through them these ganglia may bring about we do not know.

It is only in some few instances that we have any indications, and those of a very doubtful character, that the ganglia of the splanchnic system can carry out either of the two great functions belonging to what is physiologically called a nerve centre, namely the function of starting nervous impulses anew from within itself, the function of an automatic centre so called, and the function of being so affected by the advent of afferent impulses as to send forth in response efferent impulses, of converting, as it were, afferent into efferent impulses, the function of a reflex centre so called.

In the central nervous system, the brain with the spinal cord, which supplies the nervous centres for automatic actions and for reflex actionsindeed all the processes taking place in the central nervous system (at least all such as come within the province of physiology) -fall into or may be considered as forming part of one or the other of these two categories.

97. Reflex actions. In a reflex action afferent impulses reaching the nervous centre give rise to the discharge of efferent impulses, the discharge following so rapidly and in such a way as to leave no doubt that it is caused by the advent at the centre of the afferent impulses. Thus a frog from which the brain has been removed while the rest of the body has been left intact will frequently remain quite motionless (as far at least as the skeletal muscles are concerned) for an almost indefinite time; but if its skin be pricked, or if in other ways afferent impulses be generated in afferent fibres by adequate stimulation, movement of the limbs or body will immediately follow. Obviously in this instance the stimulation of afferent fibres lias been the cause of the discharge of impulses along efferent fibres.

The machinery involved in a such a reflex act consists of three parts : (1) the afferent fibres, (2) the nerve centre, in this case the spinal cord, and (3)

[FIG. 36.

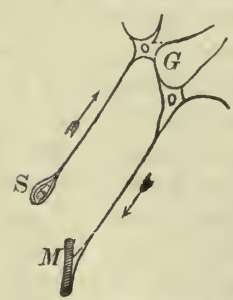
the efferent fibres. [Fig. 36.] If any one of these three parts be missing the reflex act cannot take place; if, for instance, the afferent nerves or the efferent nerves be cut across in their course, or if the centre, the spinal cord, be destroyed, the reflex action cannot take place.

Reflex actions can be carried out by means of the brain, as we shall see while studying that organ in detail, but the best and clearest examples of reflex action are manifested by the spinal cord; in fact, reflex action is one of the most important functions of the spinal cord. We shall have to study the various reflex actions

Dingram illustrating of the spinal cord in detail hereafter, but it will be Simplest Form of Re- desirable to point out here some of their general feaflex Apparatus.] tures.

When we stimulate the nerve of a muscle-nerve preparation the result, though modified in part by the condition of the muscle and nerve, whether firesh and irritable or exhausted, for instance, is directly dependent on the nature and strength of the stimulus. If we use a single induction-shock we get a simple contraction, if the interrupted current we get a tetanus, if we use a weak shock we get a slight contraction, if a strong shock a large contraction, and so on; and throughout our study of muscular contractions we assumed that the amount of contraction might be taken as a measure of the magnitude of the nervous impulses generated by the stimulus. And it need hardly be said that when we stinulate certain fibres only of a motor nerve, it is only the muscular fibres in which those nerve fibres end which are thrown into contraction. 
In a reflex action, on the other hand, the movements called forth by the same stimulus may be in one case insignificant and in another violent and excessive, the result depending on the arrangements and condition of the reflex mechanism. Thus the mere contact of a hair with the mucous membrane lining the larynx, a contact which can originate only the very slightest afferent impulses, may call forth a convulsive fit of coughing, in which a very large number of muscles are thrown into violent contractions; whereas the same contact of the hair with other surfaces of the body may produce no obvious effect at all. Similarly, while in the brainless but otherwise normal frng, a slight touch on the skin of the flank will produce nothing but a faint flicker of the underlying muscles, the same touch on the same part of a frog poisoned with strychnine will produce violent lasting tetanic contractions of nearly all the muscles of the body. Motor impulses, as we have seen, travel along motor nerves without any great expenditure of energy, and probably without increasing that expenditure as they proceed; and the same is apparently the case with afferent impulses passing along afferent nerves. When, however, in a reflex action afferent impulses reach the nerve centre, a change in the nature and magnitude of the impulses takes place. It is not that in the nerve centre the afferent impulses are simply turned aside or reflected into efferent impulses; and hence the name "reflex" action is a bad one. It is rather that the afferent impulses act afiesh, as it were, as a stimulus to the nerve centre, producing according to circumstances and conditions either a few weak efferent impulses or a multitude of strong ones. The nerve centre may be regarded as a collection of explosive charges ready to be discharged and so to start efferent impulses along certain efferent nerves, and these charges are so arranged and so related to certain afferent nerves, that afferent impulses reaching the centre along those nerves may in one case discharge a few only of the charges, and so give rise to feeble movements, and in another case discharge a very large number, and so give rise to large and violent movements. In a reflex action then the number, intensity, character, and distribution of the efferent impulses, and so the kind and amount of movement, will depend chiefly on what takes place in the centre, and this will, in turn, depend, on the one hand, on the condition of the centre, and, on the other, on the special relations of the centre of the afferent impulses. At the same time we are able to recognize in most reflex actions a certain relation between the strength of the stimulus, or the magnitude of the afferent impulses and the extent of the movement or the magnitude of the efferent impulses.

IVe may add, without going more fully into the subject here, that in most reflex actions a special relation may be observed between the part stimulated and the resulting movement. In the simplest cases of reflex action this relation is merely of such a kind that the muscles thrown into action are those governed by a motor nerve which is the fellow of the sensory nerve, the stimulation of which calls forth the movement. In the more complex reflex action of the brainless frog, and in other cases, the relation is of such a kind that the resulting movement bears an adaptation to the stimulus; the foot is withdrawn from the stimulus, or the movement is calculated to push or wipe away the stimulus. In other words, a certain purpose is evident in the reflex action.

Thus in all cases, except perhaps the very simplest, the movements called forth by a reflex action are exceedingly complex compared with those which result from the direct stimulation of a motor trunk.

$\$ 98$. Automatic actions. Efferent impulses frequently issue from the brain and spinal cord and so give rise to movements without being obviously preceded by any stimulation. Such movements are spoken of as automatic 
or spontaneous. The efferent impulses in such cases are started by changes in the nerve centre which are not the immediate result of the arrival at the nerve centre of afferent impulses from without, but which appear to arise in the nerve centre itself. Changes of this kind may recur rhythmically; thus, as we shall see, we have reason to think that in a certain part of the medulla oblongata changes of the nervous material, recurring rhythmically, lead to the rhythmic discharge along certain nerves of efferent impulses whereby muscles connected with the chest are rhythmically thrown into action and a rhythmically repeated breathing is brought about. And other similar rhythmic automatic movements may be carried out by other parts of the spinal cord.

From the brain itself a much more varied and apparently irregular discharge of efferent impulses, not the obvious result of any immediately foregoing afferent impulses, and therefore not forming part of reflex actions, is very common, constituting what we speak of as volition, efferent impulses thus arising being called volitional or voluntary impulses. The spinal cord apart from the brain does not appear capable of executing these voluntary movements; but to this subject we shall return when we come to speak of the central nervous system in detail.

We said just now that there is no satisfactory evidence that the ganglia of the splanchnic system ever act as centres of reflex action. The evidence, however, that these ganglia may serve as centres of rhythmic automatic action seems at first sight of some strength. Several organs of the body containing muscular tissue, the most notable being the heart, are during life engaged in rhythmic automatic movements, and in many cases continue these movements after removal from the body. In nearly all these cases ganglia are present in connection with the muscular tissue; and the presence and intact condition of these ganglia seem, at all events in many cases, in some way essential to the due performance of the rhythmic automatic movements. Indeed, it has been thought that the movements in question are really due to the rhythmic automatic generation in the cells of these ganglia of efferent impulses which passing down to the appropriate muscular fibres call forth the rhythmic movement. When we come to study these movements in detail, we shall find reasons for coming to the conclusion that this view is not supported by adequate evidence; and, indeed, though it is perhaps immature to make a dogmatic statement, all the evidence goes, as we have already said, to show that the great use of the ganglia of the splanchnic system, like that of the spinal ganglia, is connected with the nutrition of the nerves, and that these structures do not, like the central nervous system, act as centres either automatic or reflex.

$\$ 99$. Inhibitory nerves. We have said that the fibres of the anterior root should be called efferent rather than motor, because though they all carry impulses outward from the central nervous system to the tissues, the impulses which they carry do not in all cases lead to the contraction of muscular fibres. Some of these efferent fibres are distributed to glandular structures, for instance to the salivary glands, and impulses passing along these lead to changes in epithelial cells and their surroundings whereby, without any muscular contraction necessarily intervening, secretion is brought about ; the action of these fibres of secretion we shall study in connection with digestion.

Besides this there are efferent fibres going to muscular tissue or at all events to muscular organs, the impulses passing along which, so far from bringing about muscular contraction, diminish, hinder, or stop movements already in progress. Thus, if when the heart is beating regularly, that is to say, when the muscular fibres which make up the greater part of the 
heart are rhythmically contracting, the branches of the pneumogastric nerve going to the heart be adequately stimulated, for instance with the interrupted current, the heart will stop beating; and that not because the muscles of the heart are thrown into a continued tetanus, the rhythmic alternation of contraction and relaxation being replaced by sustained contraction, but because contraction disappears altogether, all the muscular fibres of the heart remaining for a considerable time in complete relaxation and the whole heart being quite flaccid. If a weaker stimulus be employed the beat may not be actually stopped, but slowed or weakened. And, as we shall see, there are many other cases where the stimulation of efferent fibres hinders, weakens, or altogether stops a movement already in progress. Such an effect is called an inhibition, and the fibres stimulation of which produces the effect are called "inhibitory" fibres.

The phenomena of inhibition are not, however, confined to such cases as the heart, where the efferent nerves are connected with muscular tissues. Thus the activity of a secreting gland may be inhibited, as for instance when emotion stops the secretion of saliva, and the mouth becomes dry from fear. In this instance, however, it is probable that inhibition is brought about not by inhibitory impulses passing to the gland and arresting secretion in the gland itself, but rather by an arrest, in the central nervous system, of the nervous impulses which, normally, passing down to the gland, excite it to action. And, indeed, as we shall see later on, there are many illustrations of the fact that afferent impulses reaching a nervous centre, instead of stimulating it to activity, may stop or inhibit an activity previously going on. In fact it is probable, though not actually proved in every case, that wherever in any tissue, energy is being set free, nervous impulses brought to bear on the tissue may affect the rate or amount of the energy set free in two different ways; on the one hand, they may increase or quicken the setting free of energy, and on the other hand, they may slacken, hinder, or inhibit the setting free of energy. And in at all events a large number of cases it is possible to produce the one effect by means of one set of nerve fibres and the other effect by another set of nerve fibres. We shall have occasion, however, to study the several instances of this double action in the appropriate places.

\section{CHAPTER IV.}

\section{THE VASCULAR MECHANISM.}

\section{The Structure and Main Features of the Vascular Apparatus.}

$\S 100$. The blood is the internal medium on which the tissues live; from it the tissues draw their food and oxygen, to it they give up the products of waste matters which they form. The tissues, with some few exceptions, are traversed by, and thus the elements of the tissues surrounded by, networks of minute thin-walled tubes, the capillary bloodvessels. The elementary striated muscle fibre, for instance, is surrounded by capillaries, running in the connective tissue outside but close to the sarcolemma, arranged in a network with more or less rectangular meshes. These capillaries are closed tubes with continuous walls, and the blood, which, as we shall see, is continually streaming through them, is as a whole confined to their channels and does not escape from them. The elements of the tissues lie outside the capillaries and form extra-vascular islets, of different form and size in the 
different tissues, surrounded by capillary networks. But the walls of the capillaries are so thin and of such a nature that certain of the constituents of the blood pass from the interior of the capillary, through the capillary wall to the elements of the tissue outside the capillary, and similarly certain of the constituents of the tissue, to wit, certain substances the result of the metabolism continually going on in the tissue, pass from the tissue outside the capillary through the capillary wall into the blood flowing through the capillary. Thus as we have already said, $\S 13$, there is a continual interchange of material between the blood in the capillary and the elements of the tissue outside the capillary, the lymph acting as middle-man. By this interchange the tissue lives on the blood, and the blood is affected by its passage through the tissue. In the small arteries which end in, and in the small veins which begin in the capillaries, a similar interchange takes place; but the amount of interchange diminishes as, passing in each direction from the capillaries, the walls of the arteries and veins become thicker; and indeed, in all but the minute veins and arteries, the interchange is so small that it may practically be neglected. It is in the capillaries (and minute arteries and veins) that the business of the blood is done; it is in these that the interchange takes place; and the object of the vascular mechanism is to cause the blood to flow through these in a manner best adapted for carrying on this interchange under varying circumstances. The use of the arteries is in the main simply to carry the blood in a suitable manner from the heart to the capillaries, the use of the veins is in the main simply to carry the blood from the capillaries back to the heart, and the use of the heart is in the main simply to drive the blood in a suitable manner through the arteries into the capillaries and from the capillaries back along the veins to itself again. The structure of these several parts is adapted to these several uses.

\section{Main Features of the Apparutus.}

$\S 101$. We may now pass briefly in review some of the main features of the several parts of the vascular apparatus-heart, arteries, veins, and capilliaries.

The heart is a nuscular pump-that is, a pump the force of whose strokes is supplied by the contraction of muscular fibres working intermittently, the strokes being repeated so many times (in man about 72 times) a minute. It is so constructed and furnished with valves in such a way that at each stroke it drives a certain quantity of blood with a certain force and a certain rapidity from the left ventricle into the aorta, and so into the arteries, receiving during the stroke and the interval between that stroke and the next the same quantity of blood from the veins into the right auricle. We omit for simplicity's sake the pulmonary circulation by which the same quantity of blood is driven at each stroke from the right ventricle into the lungs and received into the left auricle. The rhythm of the beat, that is the frequency of repetition of the strokes, and the characters of each beat or stroke, are determined by changes taking place in the tissues of the heart itself, though they are also influenced by causes working from without.

The arteries are tubes, with relatively stout walls, branching from the aorta all over the body. The constitution of their walls, especially of their middle coat, gives the arteries two salient properties. In the first place they are very elastic, in the sense that they will stretch readily, both lengthwise and crosswise, when pulled, and return readily to their former size and shape when the pull is taken off. If fluid be driven into one end of a piece of artery, the other end of which is tied, the artery will swell out to a very great extent, but return immediately to its former calibre when the fluid is 
let out. This elasticity is chiefly due to the elastic elements in the coats, elastic membranes, and feltworks, but the muscular fibres, being themselves also elastic, contribute to the result. By reason of their possessing such stout, elastic walls, the arteries when empty do not collapse, but remain as open tubes. In the second place the arteries, by virtue of their muscular elements, are contractile; when stimulated either directly, as by applying an electric or mechanical stimulus to the arterial walls, or indirectly, by means of the so-called vasomotor nerves, which we shall have to study presently, the arteries shrink in calibre, the circularly disposed muscular fibres contracting, and so, in proportion to the amount of their contraction, narrowing the lumen or bore of the vessel. The contraction of these arterial muscular fibres, like that of all plain, non-striated muscular fibres, is slow and long-continued, with a long latent period, as compared with the contraction of skeletal striated muscular fibres. Owing to this muscular element in the arterial walls, the calibre of an artery may be very narrow or very wide, or in an intermediate condition between the two, neither very narrow nor very wide, according as the muscular fibres are very much contracted or not contracted at all, or only moderately contracted. We have further seen that, while the relative proportion of elastic and muscular elements differs in different arteries, as a general rule the elastic elements predominate in the larger arteries and the muscular elements in the smaller arteries, so that the larger arteries may be spoken of as eminently elastic, or as especially useful on account of their elastic properties, and the smaller arteries as eminently muscular, or as especially useful on account of their muscular properties. Thus, in the minute arteries which are just passing into capillaries the muscular coat, though composed often of a single layer, and that sometimes an imperfect one, of muscular fibres, is a much more conspicuous and important part of the arterial wall than that furnished by the elastic elements.

The arteries branching out from a single aorta down to multitudinous capillaries in nearly every part of the body diminish in bore as they divide. Where an artery divides into two or gives off a branch, though the bore of each division is less than that of the artery before the division or branching, the two together are greater; that is to say, the united sectional area of the branches is greater than the sectional area of the trunk. Hence, the sectional area of the arterial bed through which the blood flows goes on increasing from the aorta to the capillaries. If all the arterial branches were thrown together into one channel, this would form a hollow cone with its apex at the aorta and its base at the capillaries. The united sectional area of the capillaries may be taken as several hundred times that of the sectional area of the aorta, so greatly does the arterial bed widen out.

The capillaries are channels of variable but exceedingly small size. The thin sheet of cemented epithelioid plates which forms the only wall of a capillary is elastic, permitting the channel offered by the same capillary to differ much in width at different times, to widen when blood and bloodcorpuscles are being pressed through it and to narrow again when the pressure is lessened or cut off. The same thin sheet permits water and substances, including gases, in solution to pass through itself from the blood to the tissue outside the capillary, and from the tissue to the blood, and thus carries on the interchange of material between the blood and the tissue. In certain circumstances, at all events, white or even red corpuscles may also pass through the wall to the tissue outside.

The minute arteries and veins with which the capillaries are continuous allow of a similar interchange of material, the more so the smaller they are. The walls of the veins are thinner, weaker and less elastic than those of 
the arteries, and possess a very variable amount of muscular tissue; they collapse when the veins are empty. Though all veins are more or less elastic and some veins are distinctly muscular, the veins as a whole cannot, like the arteries, be characterized as eminently elastic and contractile tubes; they are rather to be regarded as simple channels for conveying the blood from the capillaries to the heart, having just so much elasticity as will enable them to accommodate themselves to the quantity of blood passing through them, the same vein being at one time full and distended, and at another time empty and shrunk, and only gifted with any great amount of muscular contractility in special cases for special reasons. The united sectional area of the veins, like that of the arteries, diminishes from the capillaries to the heart; but the united sectional area of the venæ cavæ at their junction with the right auricle is greater than, nearly twice as great as, that of the aorta at its origin. The total capacity also of the veins is much greater than that of the arteries. The veins alone can hold the total mass of blood which in life is distributed over both arteries and veins. Indeed, nearly the whole blood is capable of being received by what is merely a part of the venous system, viz., the vena porta and its branches.

\section{The Main Facts of the Circulation.}

$\S 102$. Before we attempt to study in detail the working of these several parts of the mechanism, it will be well, even at the risk of some future repetition, to take a very brief survey of some of the salient points.

At each beat of the heart, which in man is repeated about 72 times a minute, the contraction or systole of the ventricles drives a certain quantity of blood, probably amounting to about 180 c.c. (4 to 6 oz.), with very great force into the aorta (and the same quantity of blood with less force into the pulmonary artery). The discharge of blood from the ventricle into the aorta is very rapid, and the time taken up by it is, as we shall see, much less than the time which intervenes between it and the next discharge of the next beat. So that the flow from the heart into the arteries is most distinctly intermittent, sudden rapid discharges alternating with relatively long intervals during which the arteries receive no blood from the heart.

At each beat of the heart just as much blood flows, as we shall see, from the veins into the right auricle as escapes from the left ventricle into the aorta; but, as we shall also see, this inflow is much slower, takes a longer time, than the discharge from the ventricle.

When the finger is placed on an artery in the living body a sense of resistance is felt, and this resistance seems to be increased at intervals, corresponding to the heart-beats, the artery at each heart-beat being felt to rise up or expand under the finger, constituting what we shall study hereafter as the pulse. In certain arteries this pulse may be seen by the eye. When the finger is similarly placed on a corresponding vein very little resistance is felt, and, under ordinary circumstances, no pulse can be perceived by the touch or by the eye.

When an artery is severed, the flow of blood from the proximal cut end, that on the heart side, is not equable, but comes in jets, corresponding to the heart-beats, though the flow does not cease between the jets. The blood is ejected with considerable force, and may in a large artery of a large animal be spurted out to the distance of some feet. The larger the artery and the nearer to the heart, the greater the force with which the blood issues, and the more marked the intermittence of the flow. The flow from the distal cut end, that away from the heart, may be very slight or may take place with 
considerable force and marked intermittence, according to the amount of collateral communication.

When a corresponding vein is severed, the flow of blood, which is chiefly from the distal cut end, that in connection with the capillaries, is not jerked but continuous; the blood comes out with comparatively little force, and "wells up" rather than "spurts out." The flow from the proximal cut end, that on the heart side, may amount to nothing at all or may be slight or may be considerable, depending on the presence or absence of valves and the amount of collateral communication.

When an artery is ligatured the vessel swells on the proximal side, toward the heart, and the throbbing of the pulse may be felt right up to the ligature. On the distal side the vessel is empty and shrunk, and no pulse can be felt in it unless there be free collateral communication.

When a vein is ligatured the vessel swells on the distal side, away from the heart, but no pulse is felt; while on the proximal side, toward the heart, it is empty and collapsed unless there be too free collateral communication.

$\S 103$. When the interior of an artery-for instance, the carotid-is placed in communication with a long glass tube of not too great a bore, held vertically, the blood, immediately upon the communication being effected, may be seen to rush into and to fill the tube for a certain distance, forming in it a column of blood of a certain height. The column rises not steadily, but by leaps, each leap corresponding to a heart-beat, and each leap being less than its predecessor; and this goes on, the increase in the height of the column at each heart-beat each time diminishing, until at last the column ceases to rise and remains for a while at a mean level, above and below which it oscillates with slight excursions at each heart-beat.

To introduce such a tube an artery-say the carotid of a rabbit-is laid bare, ligatured at a convenient spot, $l^{\prime}$ Fig. 37 , and further temporarily closed a little distance lower down nearer the heart by a small pair of "bull-dog " forceps, $b d$, or by a ligature which can be easily slipped. A longitudinal incision is now made in the artery between the forceps, $b d$, and the ligature $l^{\prime}$ (only the drop or two of blood which happens to remain inclosed between the two being lost); the end of the tube, represented by $c$ in the figure, is introduced into the artery and secured by the ligature $l$. The interior of the tube is now in free communication with the interior of the artery, but the latter is by means of the forceps at present shut off from the heart. On removing the forceps a direct communication is at once established between the tube and the artery below; in consequence the blood from the heart flows through the artery into the tube.

This experiment shows that the blood as it is flowing into the carotid is exerting a considerable pressure on the walls of the artery. At the moment when the forceps are removed there is nothing but the ordinary pressure of the atmosphere to counterbalance this pressure within the artery, and consequently a quantity of blood is pressed out into the tube; and this goes on until the column of blood in the tube reaches such a height that its weight is equal to the pressure within the artery, whereupon no more blood escapes. The whole column continues to be raised a little at each heart-beat, but sinks as much during the interval between each two beats, and thus oscillates, as we have said, above and below a mean level. In a rabbit this column of blood will generally have the height of about $90 \mathrm{~cm}$. (3 feet); that is to say, the pressure which the blood exerts on the walls of the carotid of a rabbit is equal to the pressure exerted by a column of rabbit's blood $90 \mathrm{~cm}$. high. This is equal to the pressure of a column of water about $95 \mathrm{~cm}$. high, and to the pressure of a column of mercury about $70 \mathrm{~mm}$. high.

If a like tube be similarly introduced into a corresponding vein-say the jugular vein-it will be found that the column of blood, similarly formed 
in the tube, will be a very low one, not more than a very few eentimetres high, and that while the level of the column may vary a good deal, owing, as we shall see later, to the influence of the respiratory movement, there will not, as in the artery, be oscillations corresponding to the heart-beats.

We learn, then, from this simple experiment, that in the carotid of the rabbit the blood while it flows through that vessel is exerting a considerable mean pressure on the arterial walls, equivalent to that of a column of mercury about $70 \mathrm{~mm}$. high, but that in the jugular vein the blood exerts on the venous walls a very slight mean pressure, equivalent to that of a column of mercury 3 or $4 \mathrm{~mm}$. high. We speak of this mean pressure exerted by the blood on the walls of the bloodvessels as blood-pressure, and we say that the blood-pressure in the carotid of the rabbit is very high $(70 \mathrm{~mm}$. $\mathrm{Hg})$, while that in the jugular vein is very low (only 3 or $4 \mathrm{~mm}$. $\mathrm{Hg}$.).

In the normal state of things the blood flows through the carotid to the arterial branches beyond, and through the jugular vein toward the heart; the pressure exerted by the blood on the artery or on the vein is a lateral pressure on the walls of the artery and vein, respectively. In the above experiment the pressure measured is not exactly this, but the pressure exerter at the end of the artery (or of the vein) where the tube is attached. $\mathrm{We}$ might directly measure the lateral pressure in the carotid by somewhat modifying the procedure described above. We might connect the carotid with a tube the end of which was not straight, but made in the form of a T-piece, and might introdnce the $T$-piece in such a way that the blood should flow along one limb (the vertical limb) of the T-piece from the proximal to the distal part of the earotid, and at the same time by the other (horizontal) limb of the T-piece into the main upright part of the glass tube. The column of blood in the tube would then be a measure of the pressure which the blood as it is flowing along the carotid is exerting on a portion of its walls corresponding to the mouth of the horizontal limb of the T-piece. If' we were to introduce into the aorta, at the place of origin of the carotid, a similar (larger) T-piece, and to connect the glass tube with the horizontal limb of the T-piece by a piece of elastic tubing of the same length and bore as the carotid, the column of blood rising up in the tube would be the measure of the lateral pressure exerted by the blood on the walls of the aorta at the origin of the carotid artery and transmitted to the rigid glass tube through a certain length of elastic tubing. And, indeed, what is measured in the experiment previously described is not the lateral pressure in the carotid itself at the spot where the glass tube is introduced, but the lateral pressure of the aorta at the origin of the carotid modified by the influences exerted by the length of the carotid between its origin and the spot where the tube is introduced.

$\S 104$. Such an experiment as the one described has the disadvantages that the animal is weakened by the loss of the blood which goes to form the column in the tube, and that the blood in the tube soon clots, and so brings the experiment to an end. Blood-pressure may be more conveniently studied by connecting the interior of the artery (or vein) with a mercury gauge or manometer (Fig. 37) the proximal descending limb of which, $m$, is filled above the mercury with some innocuous fluid, as is also the tube connecting the manometer with the artery. Using such an instrument, we should observe very much the same facts as in the more simple experiment.

Immediately that communication is established between the interior of the artery and the manometer, blood rushes from the former into the latter, driving some of the mereury from the descending limb, $m$, into the ascending limb, $m^{\prime}$, and thus causing the level of the mereury in the ascending limb to rise rapidly. This rise is marked by jerks corresponding with the heart- 


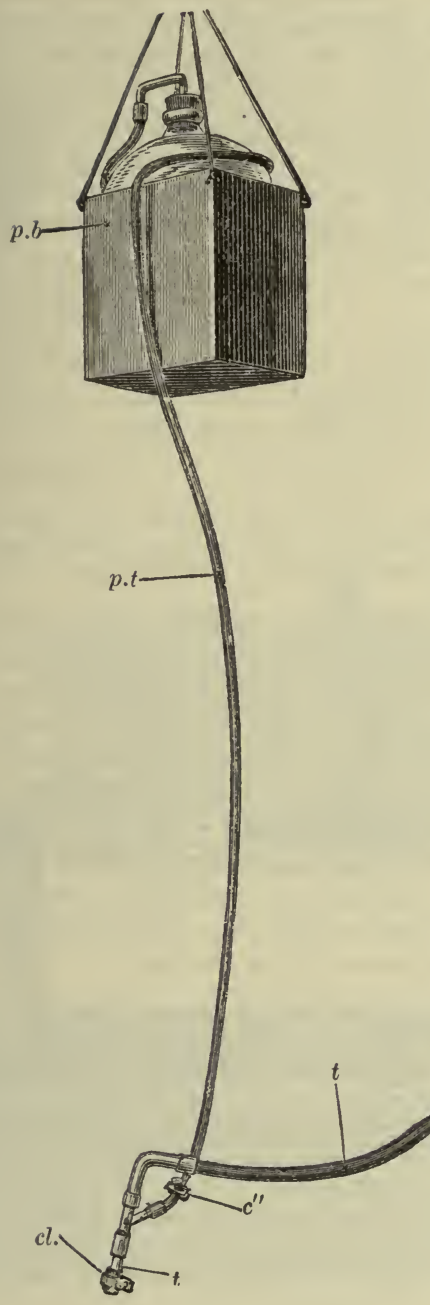

FIG. 37.

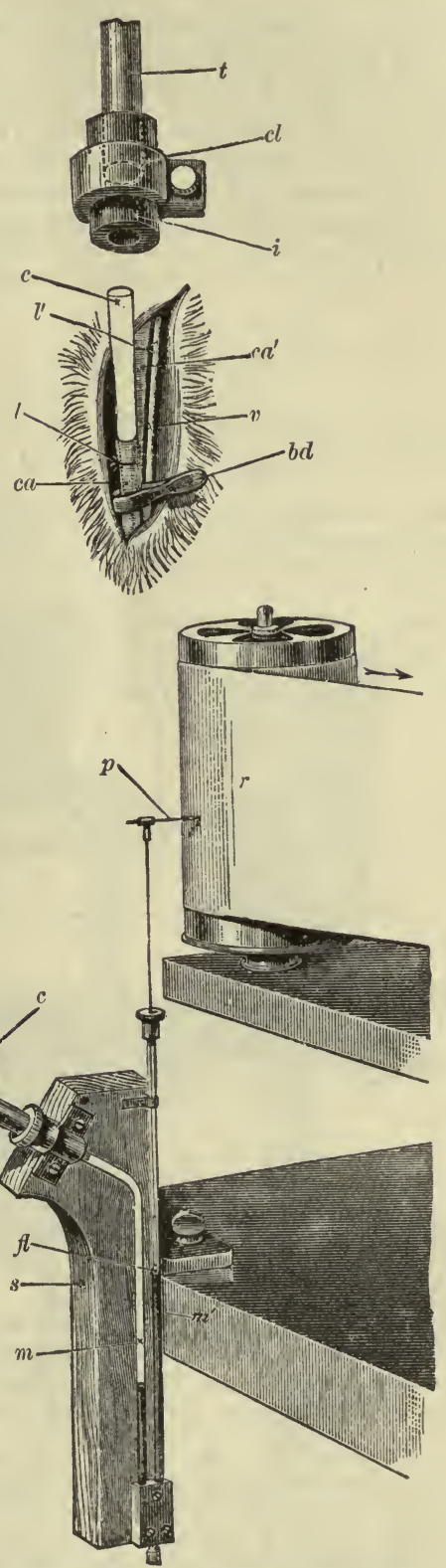

Apparatus for Investigating Blood-pressure, At the upper right-hand corner is seen, on an enlarged scale, the carotid artery, clamped by the forceps $b d$, with the vagus nerve $v$ lying by its side. The artery has been ligatured at $l^{\prime}$ and the glass eanula $c$ has been introduced into the artery between the ligature $l^{\prime}$ and the forceps $b d$, and seeured in position by the ligature $l$. The shrunken artery on the distal side of the canula is seen at $c a^{\prime}$.

$p . b$ is a box containing a bottle holding a saturated solution of sodium carbonate or a solution of sodium biearbonate of sp. gr. 1083, and capable of being raised or lowered at pleasnre. The solution flows by the tube $p . t$ regulated by the clamp $c^{\prime \prime}$ into the tube $t$. A syringe, with a stopcock, may be substituted for the bottle, and attached at $c^{\prime \prime}$. This, indeed, is in many respeets a 
beats. Having reached a certain level, the mercury ceases to rise any more. It does not, however, remain absolutely at rest, but undergoes oscillations; it keeps rising and falling. Each rise, which is very slight compared with the total height to which the mercury has risen, has the same rhythm as the systole of the ventricle. Similarly, each fall corresponds with the diastole.

If a float, swimming on the top of the mercury in the ascending limb of the manometer, and bearing a brush or other marker, be brought to bear on a travelling surface, some such tracing as that represented in Fig. 38 will be

FIG. 38.

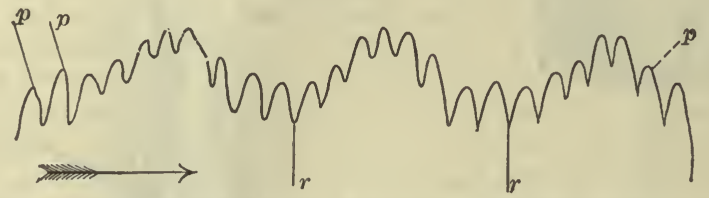

Tracing of Arterial Pressure with a Mercury Manometer: The smaller curves $p p$ are the pulse curves. The space from $r$ to $r$ embraces a respiratory undulation. The tracing is taken from a $\mathrm{dog}$, and the irregularities visible in it are those frequently met with in this animal.

described. Each of the smaller curves $(p p)$ corresponds to a heart-beat, the rise corresponding to the systole and the fall to the diastole of the ventricle. The larger undulations $(r r)$ in the tracing, which are respiratory

FIG. 39 .
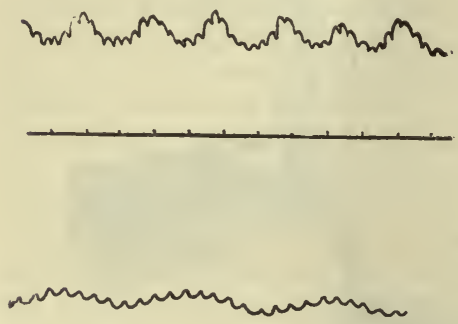

Blood-pressure Curves from the Carotid of Rabbit, the time marker in each case marking seconds.

limb of the manometer are all filled with gauge. The canula, tube, and descending ting of the blood, the one chosen being generally a strong solution (sp. gr. 1083) of sodium bicarbonate, but other fluids may be chosen. In order to avoid loss of blood, a quantity of fluid is injected into the flexible tube sufficient to raise the

more convenient plan. The tube $t$ is connected with the leaden tube $t$, and the stopcock $c$ with the manometer, of which $m$ is the descending and $m^{\prime}$ the ascending limb, and $s$ the support. The mercury in the ascending limb bears on its surface the float $f$, a long rod attached to which is fitted with the pen $p$, writing on the recording surface $r$. The clamp $c l$. at the end of the tube $t$ has an arrangement shown on a larger scale at the right hand upper corner.

The descending tube $m$ of the manometer and the tube $t$ being completely filled along its whole length with fluid to the exclusion of all air, the canula $c$ is filled with fluid, slipped into the open end of the thick-walled India-rubber tube $i$, until it meets the tube $t$ (whose position within the India-rubber tube is shown by the dotted lines), and is then securely fixed in this position by the clamp $c l$.

The stopcocks $c$ and $c^{\prime \prime}$ are now opened, and the pressure bottle raised or fluid driven in by the syringe until the mercury in the manometer is raised to the required height. The clamp $c^{\prime \prime}$ is then closed and the forceps $b d$ removed from the artery. The pressure of the blood in the carotid $c a$ is in consequence brought to bear through $t$ upon the mercury in the manometer. 
mercury in the ascending limb of the manometer to a level a very little below what may be beforehand guessed at as the probable mean pressure. When the forceps $b d$ are removed, the pressure of the blood in the carotid is transmitted through the flexible tube to the manometer, the level of the mercury in the ascending limb of which falls a little, or sinks a little at first, or may do neither, according to the success with which the probable mean pressure has been guessed, and continues to exhibit the characteristic oscillations until the experiment is brought to an end by the blood clotting or otherwise.

'Tracings of the movements of the column of mercury in the manometer may be taken either on a sinoked surface of a revolving cylinder ( Fig. 11), or by means of a brush and ink on a continuous roll of paper, as in the more complex kymograph (Fig. 40).

$\S 105$. By the help of the manometer applied to various arteries and veins we learn the following facts:

1. The mean blood-pressure is high in all the arteries, but is greater in the larger arteries nearer the heart than in the smaller arteries further from

FIG. 40.

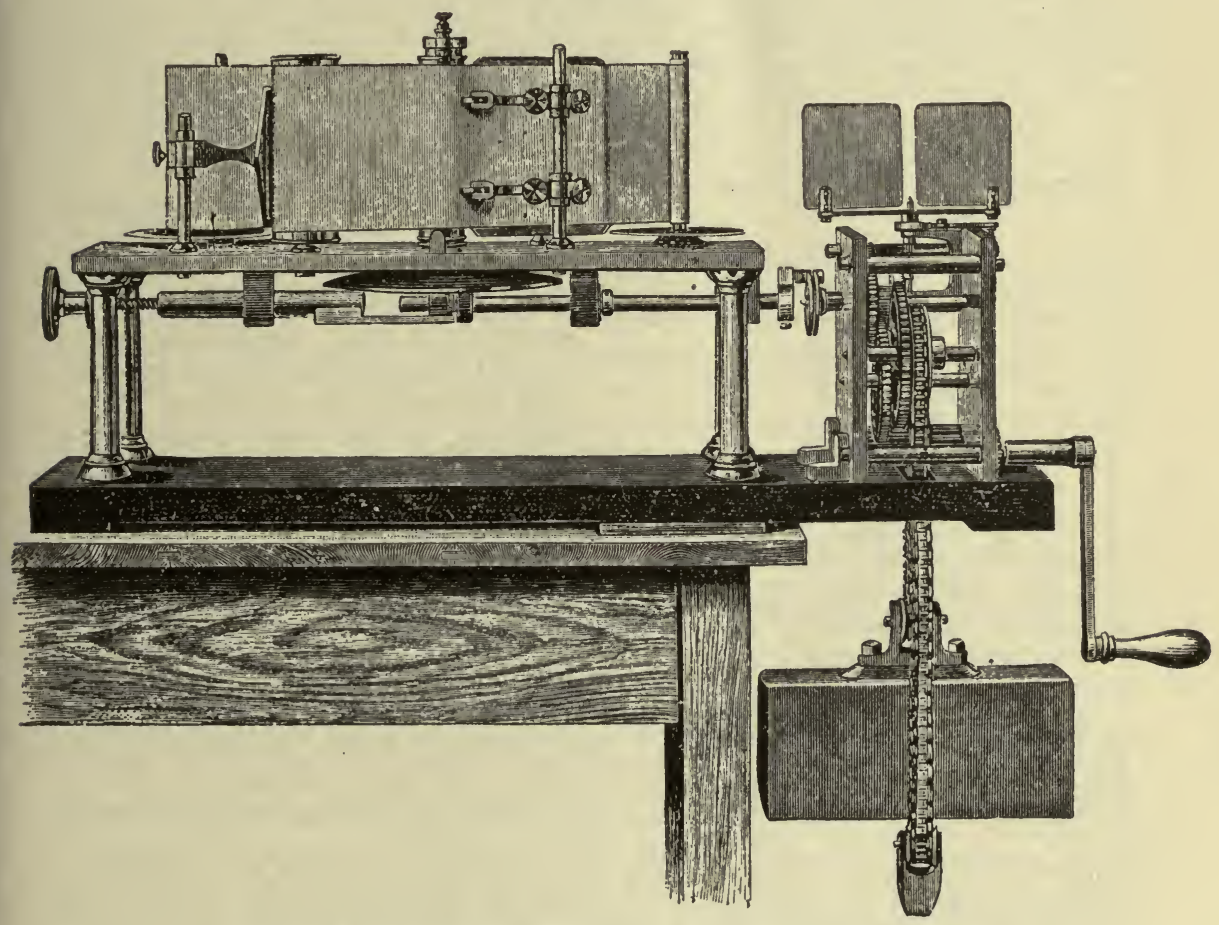

Ludwig's Kymograph for Recording on a Continuous Roll of Paper.

the heart; it diminishes, in fact, along the arterial tract from the heart toward the capillaries.

2. The mean blood-pressure is low in the veins, but is greater in the smaller veins nearer the capillaries than in the larger veins nearer the heart, diminishing, in fact, from the capillaries toward the heart. In the large veins near the heart it may be negative, that is to say, the pressure of blood in the vein bearing on the proximal descending limb of the manometer may be less than the pressure of the atmosphere on the ascending distal limb, so 
that when communication is made between the interior of the vein and the manometer, the mercury sinks in the distal and rises in the proximal limb, being sucked up toward the vein.

The manometer cannot well be applied to the capillaries, but we may measure the blood-pressure in the capillaries in an indirect way. It is well known that when any portion of the skin is pressed upon, it becomes pale and bloodless; this is due to the pressure driving the blood out of the capillaries and minute vessels and preventing any fresh blood entering into them. By carefully investigating the amount of pressure necessary to prevent the blood entering the capillaries and minute arteries of the web of the frog's foot, or of the skin beneath the nail or elsewhere in man, the internal pressure which the blood is exercising on the walls of the capillaries and minute arteries and veins may be approximately determined. In the frog's web this has been found to be equal to about 7 to $11 \mathrm{~mm}$. of mercury. In the mammal the capillary blood-pressure is naturally higher than this and may be put down at from 20 to $30 \mathrm{~mm}$. It is, therefore, considerable, being greater than that in the veins, though less than that in the arteries.

3. There is thus a continued decline of blood-pressure from the root of the aorta, through the arteries, capillaries, and veins to the right auricle. We find, however, on examination that the most marked fall of pressure

FIG. 41.

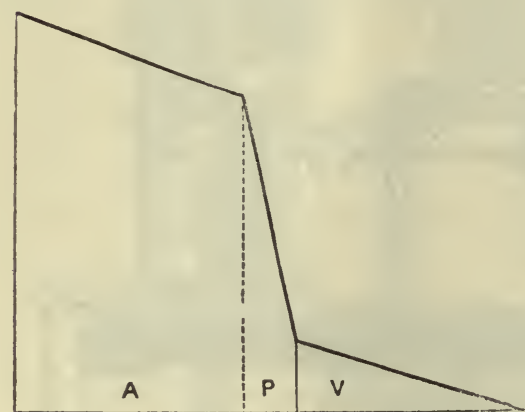

Diagram of Blood-pressure : $A$, arteries; $P$, peripheral region (minute arteries, capillaries, and veins); $r$, veins. takes place between the small arteries on the one side of the capillaries and the small veins on the other, the curve of pressure being somewhat of the form given in Fig. 41, which is simply intended to show this fact graphically and has not been constructed by exact measurements.

4. In the arteries this mean pressure is marked by oscillations corresponding to the heart beats, each oscillation consisting of a rise (increase of pressure above the mean) corresponding to the systole of the ventricle, followed by a fall (decrease of pressure below the mean) corresponding to the diastole of the ventricle.

5. These oscillations, which we may speak of as the pulse, are largest and most conspicuous in the large arteries near the heart, diminish from the heart toward the capillaries, and are, under ordinary circumstances, wholly absent from the veins along the whole extent from the capillaries to the heart.

Obviously a great change takes place in that portion of the circulation which comprises the capillaries, the minute arteries leading to and the minute veins leading away from the capillaries, and which we may speak of as the "peripheral region." It is here that a great drop of pressure takes place; it is here also that the pulse disappears.

$\S 106$. If the web of a frog's foot be examined with a microscope, the blood, as judged of by the movements of the corpuscles, is seen to be passing in a continuous stream from the small arteries through the capillaries to the veins. The velocity is greater in the arteries than in the veins, and greater in both than in the capillaries. In the arteries faint pulsations, synchronous with the heart's beat, are frequently visible; but these disappear in the capillaries, in which the flow is even, that is, not broken by pulsations, and 
this evenness of flow is continued on along the veins as far as we can trace them. Not infrequently variations in velocity and in the distribution of the blood, due to causes which will be hereafter discussed, are witnessed from time to time.

The character of the flow through the smaller capillaries is very variable. Sometimes the corpuscles are seen passing through the channel in single file with great regularity; at other times they may be few and far between. Some of the capillaries are wide enough to permit two or more corpuscles abreast. In all cases the blood as it passes through the capillary stretches and expands the walls. Sometimes a corpuscle may remain stationary at the entrance into a capillary, the channel itself being for some little distance entirely free from corpuscles. Sometimes many corpuscles will appear to remain stationary in one or more capillaries for a brief period and then to move on again. Any one of these conditions readily passes into another; and, especially with a somewhat feeble circulation, instances of all of them may be seen in the same field of the microscope. It is only when the vessels of the web are unusually full of blood that all the capillaries can be seen equally filled with corpuscles. The long, oval red corpuscle moves with its long axis parallel to the stream, occasionally rotating on its long axis, and sometinies, in the larger channels, on its short axis. The flexibility and elasticity of a corpuscle are well seen when it is being driven into a capillary narrower than itself, or when it becomes temporarily lodged at the angle between two diverging channels.

These and other phenomena, on which we shall dwell later on, nay be readily seen in the web of the frog's foot or in the stretched-out tongue or in the mesentery of the frog; and essentially similar phenomena may be observed in the mesentery or other transparent tissues of a mammal. All over the body, wherever capillaries are present, the corpuscles and the plasma are being driven in a continuous and though somewhat irregular yet on the whole steady flow through channels so minute that the passage is manifestly attended with considerable difficulties.

It is obvious that the peculiar characters of the flow through the minute arteries, capillaries, and veins affords an explanation of the great change taking place in the peripheral region between the arterial flow and the venous flow. The united sectional area of the capillaries is, as we have seen, some hundreds of times greater than the sectional area of the aorta; but this united sectional area is made up of thousands of minute passages, varying in man from 5 to $20 \mu$, some of them, therefore, being in an undistended condition, smaller than the diameter of a red corpuscle. Even were the blood a simple liquid free from all corpuscles, these extremely minute passages would occasion an enormous amount of friction, and thus present a considerable obstacle or resistance to the flow of blood through them. Still greater must be the friction and resistance occasioned by the actual blood with its red and white corpuscles. The blood in fact meets with great difficulties in its passage through the peripheral region, and sometimes, as we shall see, the friction and resistance are so great in the peripheral vessels of this or that area that no blood passes through them at all, and an arrest of the flow takes place in the area.

'The resistance to the flow of bloor thus caused by the friction generated in so many minute passages is one of the most important physical facts in the circulation. In the large arteries the friction is small; it increases gradually as they divide, but receives its chief and most important addition in the minute arteries and capillaries; it is relatively greater in the minute arteries than in the capillaries on account of the flow being more rapid in the former, 
for friction diminishes rapidly with a diminution in the rate of flow. We may speak of it as the "peripheral friction," and the resistance which it offers as the "peripheral resistance." It need perhaps hardly be said that this peripheral resistance not only opposes the flow of blood through the capillaries and minute arteries themselves where it is generated, but, working backward along the whole arterial system, has to be overcome by the heart at each systole of the ventricle.

\section{Hydraulic Principles of the Circulation.}

$\$ 107$. In the circulation, then, the following three facts of fundamental importance are met with :

1. The systole of the ventricle, driving at intervals a certain quantity of blood, with a certain force, into the aorta.

2. The peripheral resistance just described.

3. A long stretch of elastic tubing (the arteries), reaching from the ventricle to the region of peripheral resistance.

From these facts we may explain the main phenomena of the circulation, which we have previously sketched, on purely physical principles without any appeal to the special properties of living tissues, beyond the provision that the ventricle remains capable of good rhythmical contractions, that the arterial walls retain their elasticity, and that the friction between the blood and the lining of the peripheral vessels remains the same; we may thus explain the high pressure and pulsatile flow in the arteries, the steady stream through the capillaries, the low pressure and the uniform pulseless flow in the veins, and finally the continued flow of the blood from the aorta to the mouths of the venæ cavæ.

All the above phenomena in fact are the simple results of an intermittent force (like that of the systole of the ventricle) working in a closed circuit of branching tubes, so arranged that while the individual tubes first diminish in calibre (from the heart to the capillaries) and then increase (from the capillaries to the heart), the area of the bed first increases and then diminishes, the tubes together thus forming two cones placed base to base at the capillaries, with their apices converging at the heart, and presenting at their conjoined bases a conspicuous peripheral resistance, the tubing on one side, the arterial, being eminently elastic, and, on the other, the venous, affording a free and easy passage for the blood. It is the peripheral resistance (for the resistance offered by the friction in the larger vessels may, when compared with this, be practically neglected), reacting through the elastic walls of the arteries upon the intermittent force of the heart, which gives the circulation of the blood its peculiar features.

$\$ 108$. Circumstances determining the character of the flow. When fluid is driven by an intermittent force, as by a pump, through a perfectly rigid tube, such as a glass one (or a system of such tubes), there escapes at each stroke of the pump from the distal end of the tube (or system of tubes) just as much fluid as enters it at the proximal end. What happens is very like what would happen if, with a wide glass tube completely filled with billiard balls lying in a row, an additional ball were pushed in at one end: each ball would be pushed on in turn a stage further and the last ball at the further end would tumble out. The escape, moreover, takes place at the sanie time as the entrance.

This result remains the same when any resistance to the flow is introduced into the tube, as for instance when the end of the tube is narrowed. The force of the pump remaining the same, the introduction of the resistance undoubtedly lessens the quantity of fluid issuing at the distal end at. 
each stroke, but it at the same time lessens the quantity entering at the proximal end; the inflow and outflow remain equal to each other, and still occur at the same time.

In an elastic tube, such as an India-rubber one (or in a system of such tubes), whose sectional area is sufficiently great to offer but little resistance to the progress of the fluid, the flow caused by an intermittent force is also intermittent. The outflow being nearly as easy as the inflow, the elasticity of the walls of the tube is scarcely at all called into play. The tube behaves practically like a rigid tube. When, however, sufficient resistance is introduced into any part of the course, the fluid being unable to pass by the resistance as rapidly as it enters the tube from the pump, tends to accumulate on the proximal side of the resistance. 'This it is able to do by expanding the elastic walls of the tube. At each stroke of the pump a certain quantity of fluid enters the tube at the proximal end. Of this only a fraction can pass through the resistance during the stroke. At the moment when the stroke ceases, the rest still remains on the proximal side of the resistance, the elastic tube having expanded to receive it. During the interval between this and the next stroke, the distended elastic tube, striving to return to its natural undistended condition, presses on this extra quantity of fluid which it contains and tends to drive it past the resistance.

Thus, in the rigid tube (and in the elastic tube without the resistance) there issues, from the distal end of the tube at each stroke, just as much fluid as enters it at the proximal end, while between the strokes there is perfect quiet. In the elastic tube with resistance, on the contrary, the quantity which passes the resistance is only a fraction of that which enters the tube from the pump at any one stroke, the remainder or a portion of the remainder continuing to pass during the interval between the strokes. In the former case the tube is no fuller at the end of the stroke than at the beginning; in the latter case there is an accumulation of fluid between the pump and the resistance, and a corresponding distention of that part of the tube at the close of each stroke-an accumulation and distention, however, which go on diminishing during the interval between that stroke and the next. The amount of fluid thus remaining after the stroke will depend on the amount of resistance in relation to the force of the stroke and on the distensibility of the tube; and the amount which passes the resistance before the next stroke will depend on the degree of elastic reaction of which the tube is capable. Thus, if the resistance be very considerable in relation to the force of the stroke, and the tube very distensible, only a small portion of the fluid will pass the resistance, the greater part remaining lodged between the pump and the resistance. If the elastic reaction be great, a large portion of this will be passed on through the resistance before the next stroke comes. In other words, the greater the resistance (in relation to the force of the stroke), and the more the elastic force is brought into play, the less intermittent, the more nearly continuous, will be the flow on the far side of the resistance.

If the first stroke be succeeded by a second stroke before its quantity of fluid has all passed by the resistance, there will be an additional accumulation of fluid on the near side of the resistance, an additional distention of the tube, an additional strain on its elastic powers, and, in consequence, the flow between this second stroke and the third will be even more marked than that between the first and second, though all three strokes were of the same force, the addition being due to the extra amount of elastic force called into play. In fact, it is evident that, if there be a sufficient store of elastic power to fall back upon, by continually repeating the stroke a state of things will be at last arrived at in which the elastic force, called into play by the con- 
tinually increasing distention of the tube on the near side of the resistance, will be sufficient to drive through the resistance, between each two strokes, just as much fluid as enters the near end of the system as each stroke. In other words, the elastic reaction of the walls of the tube will have converted the intermittent into a continuous flow. The flow on the far side of the resistance is in this case not the direct result of the strokes of the pump. All the force of the pump is spent, first in getting up, and afterward in keeping up, the distention of the tube on the near side of the resistance; the immediate cause of the continuous flow lies in the distention of the tube which leads it to empty itself into the far side of the resistance at such a rate that it discharges through the resistance during a stroke and in the succeeding interval just as much as it receives from the pump by the stroke itself.

This is exactly what takes place in the vascular system. The friction in the minute arteries and capillaries presents a considerable resistance to the flow of blood through them into the small veins. In consequence of this resistance the force of the heart's beat is spent in maintaining the whole of the arterial system in a state of great distention ; the arterial walls are put greatly on the stretch by the pressure of the blood thrust into them by the repeated strokes of the heart; this is the pressure which we spoke of above as blood-pressure. The greatly distended arterial system is, by the elastic reaction of its elastic walls, continually tending to empty itself by overflowing through the capillaries into the venous system; and it overflows at such a rate that just as much blood passes from the arteries to the veins during each systole and its succeeding diastole as enters the aorta at each systole.

$\S 109$. Indeed, the important facts of the circulation which we have not as yet studied may be roughly but successfully imitated on an artificial model, Fig. 42 , in which an elastic syringe represents the heart, a long piece of elastic India-rubber tubing the arteries, another piece of tubing the veins, and number of smaller connecting pieces the minute arteries and capillaries. If these connecting pieces be made at first somewhat wide, so as to offer no great resistance to the flow from the artificial arteries to the artificial veins, but be so arranged that they may be made narrow by the screwing-up of clamps or otherwise, it is possible to illustrate the behavior of the vascular mechanism when the peripheral resistance is less than usual (and as we shall see later on it is possible in the living organism either to reduce or to increase what may be considered as the normal peripheral resistance), and to compare that behavior with the behavior of the mechanism when the peripheral resistance is increased.

The whole apparatus being placed flat on a table, so as to avoid differences in level in different parts of it, and filled with water, but so as not to distend the tubing, the two manometers attached, one $(A)$ to the arterial side of the tubing and the other $(V)$ to the venous side, ought to show the mercury standing at equal heights in both limbs of both instruments, since nothing but the pressure of the atmosphere is bearing on the fluid in the tubes, and that equally all over.

If, now, the connecting pieces being freely open, that is to say, the peripheral resistance being very little, we imitate a ventricular beat by the stroke of the pump, we shall observe the following: Almost immediately after the stroke the mercury in the arterial manometer will rise, but will at once fall again, and very shortly afterward the mercury in the venous tube will in a similar manner rise and fall. If we repeat the strokes with a not too rapid rhythm, each stroke having the same force, and make, as may by a simple contrivance be effected, the two manometers write on the same recording surface, we shall obtain curves like those of Fig. $43, A$ and $V$. At each stroke of the pump the mercury in the arterial manometers rises, but forthwith falls again to or 
nearly to the base line ; no mean arterial pressure, or very little, is established. The contents of the ventricle (syringe) thrown into the arterial system dis-

Fig. 42.

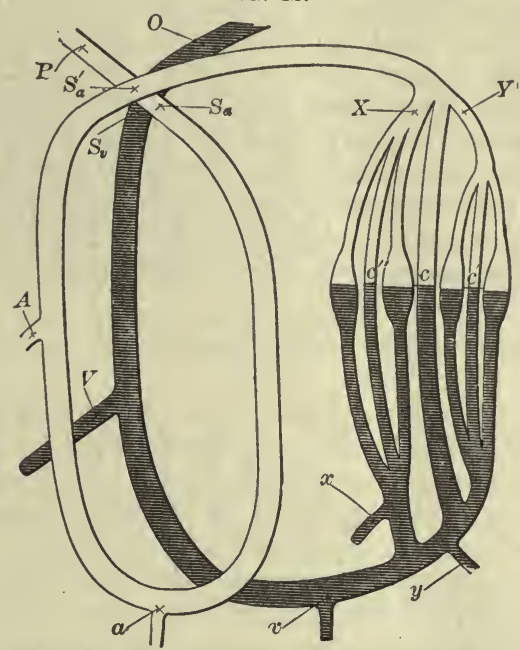

Arterial Scheme: $P$, unshaded, is an elastic tube to represent the arterial system, branching at $X$ and $Y$, and ending in the region of peripheral resistance, including the capillaries, which are imitated by filling loosely with smull pieces of sponge the parts shown as dilated in the figure. The capillaries are gathered up into the venous system, shaded, which terminates at $O$. Water is driven into the arterial system at $P$ by means of an elastic bag syringe or any other form of pump. Clamps are placed on the undilated tubes, $c, c^{\prime}, c^{\prime \prime}$. When these clamps are tightened, the only access for the water from the arterial to the venous side is through the dilated parts filled with sponge, which offers a considerable resistance to the flow of fluid through them. When the clamps are unloosed the fluid passes, with much less resistance, through the undilated tubes. Thus, by tightening or loosening the clamps the "peripheral" resistance may be increased or diminished at pleasure.

At $A$, on the arterial side, and at $V$, on the venous side, manometers can be attached. At $a$ and $v$ (and also at $x$ and $y$ ), by means of clamps, the flow of fluid from an artery and from a vein, under various conditions, may be observed. At $S a, S^{\prime} a$, and $S v$, sphygmographs may be applied.

tend it, but the passage through the peripheral region is so free that an equal quantity of fluid passes through to the veins immediately, and hence the

FIG. 43.

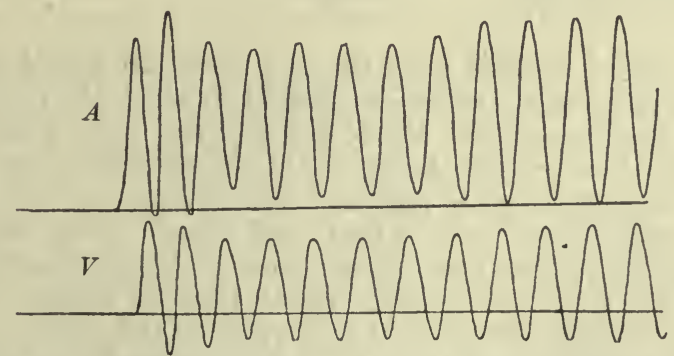

Tracings taken from an Artificial Scheme, with the Peripheral Resistance Slight: $A$, arterial; $V$, venous manometer. This figure, to save space, is on a smaller scale than the corresponding Fig. 44.

mercury at once falls. But the fluid thus passing easily into the veins distends these too, and the mercury in their manometer rises too, but only to 
fall again, as a corresponding quantity issues from the ends of the veins into the basin, which serves as an artificial auricle. Now introduce "peripheral resistance" by screwing up the clamps on the connecting tubes, and set the pump to work again as before. With the first stroke the mercury in the arterial manometer (Fig. 44, $A^{1}$ ) rises as before, but instead of falling rapidly it falls slowly, because it now takes a longer time for a quantity of fluid equal to that which has been thrust into the arterial system by the ventricular stroke to pass through the narrowed peripheral region. Before the curve has fallen to the base line, before the arterial system has had time to discharge through the narrowed peripheral region as much fluid as it received from the ventricle, a second stroke drives more fluid into the arteries, distending them this time more than it did before, and raising the mercury to

FIG. 44.

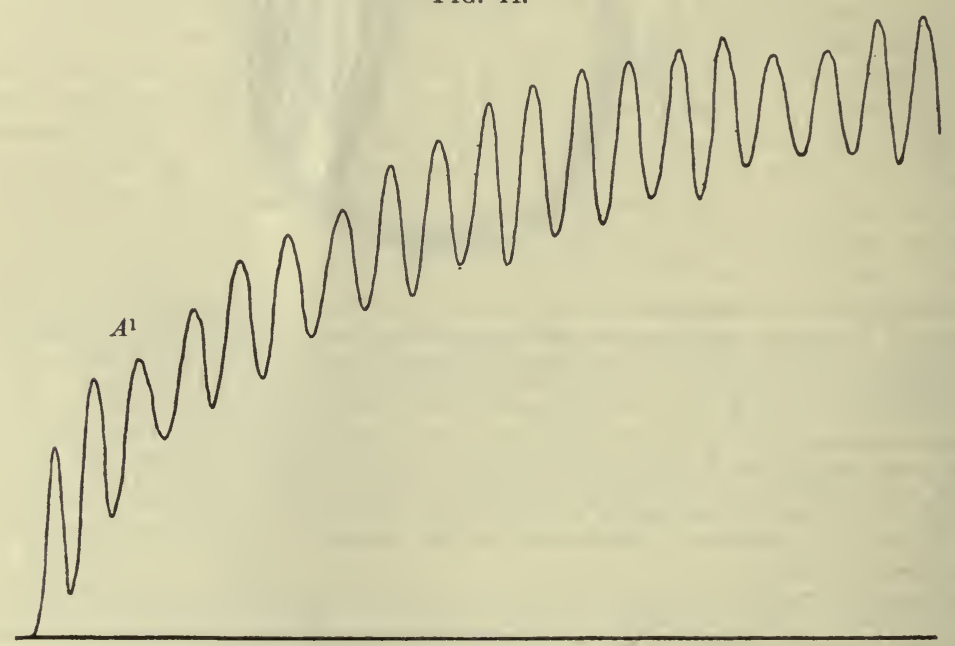

$V$

Tracings taken from an Artificial Scheme, with the Peripheral Resistance Considerable: $A^{1}$, arterial; $V^{1}$, venous manometer.

a still higher level. A third, a fourth, and succeeding strokes produce the same effect, except that the additional height to which the mercury is raised at each stroke becomes at each stroke less and less, until a state of things is reached in which the mercury, being on the fall when the stroke takes place, is by the stroke raised just as high as it was before, and then beginning to fall again is again raised just as high, and so on. With each succeeding stroke the arterial system has become more and more distended; but the more distended it is the greater is the elastic reaction brought into play ; this greater elastic reaction more and more overcomes the obstacle presented by the peripheral resistance and drives the fluid more and more rapidly through the peripheral region. At last the arterial system is so distended, and the force of the elastic reaction so great, that during the stroke and the succeeding interval just as much fiuid passes through the peripheral region as enters the arteries at the stroke. In other words, the repeated strokes have established a mean arterial pressure which, at the point where the manometer 
is affixed, is raised slightly at each ventricular stroke, and falls slightly between the strokes.

Turning now to the venous manometer, Fig. $44, V^{1}$, we observe that each stroke of the pump produces on this much less effect than it did before the introduction of the increased peripheral resistance. 'The mercury, instead of distinctly rising and falling at each stroke, now shows nothing more than very gentle undulations; it feels to a very slight degree only the direct effect of the ventricular stroke; it is simply raised slightly above the base line, and remains fairly steady at this level. The slight rise marks the mean pressure exerted by the fluid at the place of attachment of the manometer. This mean "venous" pressure is a continuation of the mean arterial pressure so obvious in the arterial manometer, but is much less than that because a large part of the arterial mean pressure has been expended in driving the fluid past the peripheral resistance. What remains is, however, sufficient to drive the fluid along the wide venous tubing right to the open end.

Thus this artificial model may be made to illustrate how it comes about that the blood flows in the arteries at a relatively high pressure, which at each ventricular systole is raised slightly above and at each diastole falls slightly below a certain mean level, and flows in the veins at a much lower pressure, which does not show the immediate effects of each heart-beat.

If two manometers, instead of one, were attached to the arterial system, one near the pump and the other further off, close to the peripheral resistance, the pressure shown by the near manometer would be found to be greater than that shown by the far one. The pressure at the far point is less because some of the pressure exerted at the near point has been used to drive the fluid from the near point to the far one. Similarly on the venous side, a manometer placed close to the peripheral region would show a higher pressure than that shown by one further off, because it is the pressure still remaining in the veins near the capillaries which, assisted, as we shall see, by other events, drives the blood onward to the larger veins. The blood-pressure is at its highest at the root of the aorta and at its lowest at the mouths of the venæ cavæ, and is falling all the way from one point to the other, because all the way it is being used up to move the blood from one point to the other. The great drop of pressure is, as we have said, in the peripheral region, because more work has to be done in driving the blood through this region than in driving the blood from the heart to this region, or from this region to the heart.

The manometer on the arterial side of the model shows, as we have seen, an oscillation of pressure, a pulse due to each heart-beat, and the same pulse may be felt by placing a finger, or rendered visible by placing a light lever, on the arterial tube. It may further be seen that this pulse is most marked nearest the pump, and becomes fainter as we pass to the periphery ; but we must reserve the features of the pulse for a special study. On the venous side of the model no pulse can be detected by the manometer or by the finger; provided that the peripheral resistance be adequate. If the peripheral resistance be diminished, as by unscrewing the clamps, then, as necessarily follows from what has gone before, the pulse passes over on to the venous side; and, as we shall have occasion to point out later on, in the living organism the peripheral resistance in particular areas may be at times so much lessened that a distinct pulsation appears in the veins.

If in the model, when the pump is in full swing, and arterial pressure well established, the arterial tube be pricked or cut, or the small side tube $\alpha$ be opened, the water will gush out in jets, as does blood from a cut artery in the living body; whereas, if the venous tube be similarly pricked or cut, or 
the small tube $v$ be opened, the water will simply ooze out or well up, as does blood from a vein in the living body. If the arterial tube be ligatured, it will swell on the pump side and shrink on the peripheral side; if the venous tube be ligatured, it will swell on the side nearest the capillaries and shrink on the other side. In short, the dead model will show all the main facts of the circulation which we have as yet described.

$\$ 110$. In the living body, however, there are certain helps to the circulation which cannot be imitated by such a model without introducing great and undesirable complications; but these chiefly affect the flow along the veins.

The veins are in many places provided with valves so constructed as to offer little or no resistance to the flow from the capillaries to the heart, but effectually to block a return toward the capillaries. Hence any external pressure brought to bear upon a vein tends to help the blood to move forward toward the heart. In the various movements carried out by the skeletal muscles, such an external pressure is brought to bear on many of the veins, and hence these movements assist the circulation. Even passive movements of the limbs have a similar effect. So, also, the movements of the alimentary canal, carried out by means of plain muscular tissue, promote the flow along the veins coming from that canal, and when we come to deal with the spleen we shall see that the plain muscular fibres which are so abundant in that organ in some animals, serve by rhythmical contractions to pump the blood regularly away from the spleen along the splenic veins.

When we come to deal with respiration, we shall see that each enlargement of the chest constituting an inspiration tends to draw the blood toward the chest, and each return or retraction of the chest walls in expiration tends to drive the blood away from the chest. 'The arrangement of the valves of the heart causes this action of the respiratory pump to promote the flow of blood in the direction of the normal circulation; and, indeed, were the heart perfectly motionless, the working of this respiratory pump alone would tend to drive the blood from the venæ cavæ through the heart into the aorta, and so to keep up the circulation; the force so exerted, however, would, without the aid of the heart, be able to overcome a very small part only of the resistance in the capillaries and small vessels of the lungs, and so would prove actually ineffectual.

There are, then, several helps to the flow along the veins, but it must be remembered that, however useful, they are helps only, and not the real cause of the circulation. The real cause of the flow is the ventricular stroke, and this is sufficient to drive the blood from the left ventricle to the right auricle, even when every muscle of the body is at rest and breathing is for a while stopped, when, therefore, all the helps we are speaking of are wanting.

\section{Circumstances Determining the Rate of the Flow.}

$\S 111$. We may now pass on to consider briefly the rate at which the blood flows through the vessels, and first the rate of flow in the arteries.

When even a small artery is severed, a considerable quantity of blood escapes from the proximal cut end in a very short space of time. That is to say, the blood moves in the arteries from the heart to the capillaries with a very considerable velocity. By various methods, this velocity of the bloodcurrent has been measured at different parts of the arterial system; the results, owing to imperfections in the methods employed, cannot be regarded as satisfactorily exact, but may be accepted as approximately true. "They show that the velocity of the arterial stream is greatest in the largest arteries near the heart, and diminishes from the heart toward the capillaries. Thus, 
in a large artery of a large animal, such as the carotid of a dog or horse, and probably in the carotid of a man, the blood flows at the rate of 300 or 500 $\mathrm{mm}$. a second. In the very small arteries the rate is probably only a few $\mathrm{mm}$. a second.

Methods. The hæmadromometer of Volkmann. [Fig. 45.] An artery-e.g., a carotid-is clamped in two places, and divided between the clamps. 'Two canulæ,

[FIG. 45 .
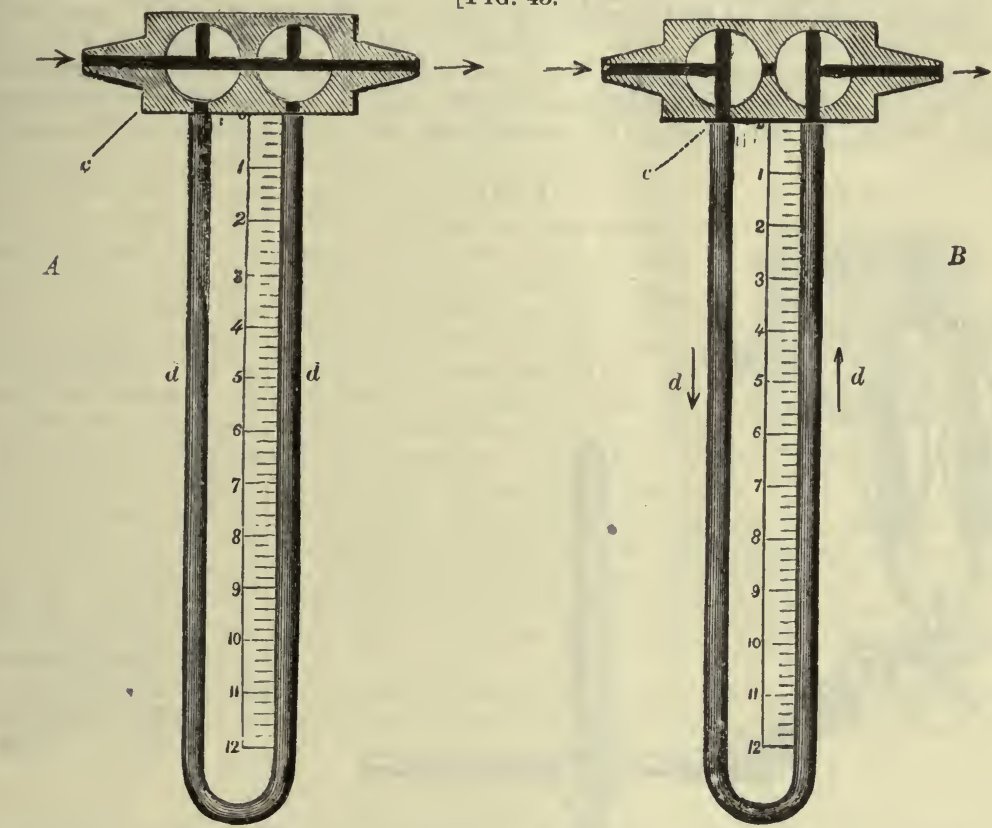

Volkmann's Hæmadromomeier: The conical portions of the instrument are inserted in the cut ends of a vein or artery. By a simple arrangement of a double stopcock the blood-current can be made to pass immediately through the transverse arm, as in $A$, or to pass through the graduated $\mathrm{U}$-shaped tube, as in $B$.]

of a bore as nearly equal as possible to that of the artery, or of a known bore, are inserted in the two ends. The two canulæ are connected by means of two stopcocks, which work together, with the two ends of a long glass tube, bent in the shape of a $U$, and filled with normal saline solution, or with a colored innocuous fluid. The clamps on the artery being released, a turn of the stopcocks permits the blood to enter the proximal end of the long $U$-tube, along which it courses, driving the fluid out into the artery through the distal end. Attached to the tube is a graduated scale, by means of which the velocity with which the blood flows along the tube may be read off. Even supposing the canulæ to be of the same bore as the artery, it is evident that the conditions of the flow through the tube are such as will only admit of the result thus gained being considered as an approximative estimation of the real velocity in the artery itself.

The rheometer (Stromuhr) of Ludwig. This consists of two glass bulbs, $A$ and $B$, Fig. 46, communicating above with each other and with the common tube $C$, by which they can be filled. Their lower ends are fixed in the metal disc $D$, which can be made to rotate, through two right angles, round the lower disc $E$. In the upper disc are two holes, $a$ and $b$, continuous with $A$ and $B$ respectively, and in the lower disc are two similar holes $a^{\prime}$ and $b^{\prime}$, similarly continuous with the tubes $G$ and $I$. Hence, in the position of the dises shown in the figure, the tube $G$ is continuous through the two dises with the bulb $A$, and the tube $H$ with the bulb $B$. On turning the disc $D$ through two right angles, the tube $G$ 
becomes continuous with $B$ instead of $A$, and the tube $H$ with $A$ instead of $B$. There is a further arrangement, omitted from the figure for the sake of simplicity, by which when the dise $D$ is turned through one instead of two right angles from either of the above positions, $G$ becomes directly continuous with $H$, both being completely shut off fiom the bulbs.

The ends of the tubes $H$ and $G$ are made to fit exactly into two canula inserted into the two cut ends of the artery about to be experimented upon, and having a bore as nearly equal as possible to that of the artery.

The method of experimenting is as follows: The dise $D$, being placed in the intermediate position, so that $a$ and $b$ are both cut off from $a^{\prime}$ and $b^{\prime}$, the bulb $A$ is filled with pure olive oil up to the mark $x$, and the bulb $B$, the rest of $A$, and the junction $C$, with defibrinated blood; and $C$ is then clamped. The tubes $H$ and $G$ are also filled with defibrinated blood, and $G$ is inserted into the canula of the central, $H$ into that of the peripheral, end of the artery. On removing

FIG. 46.

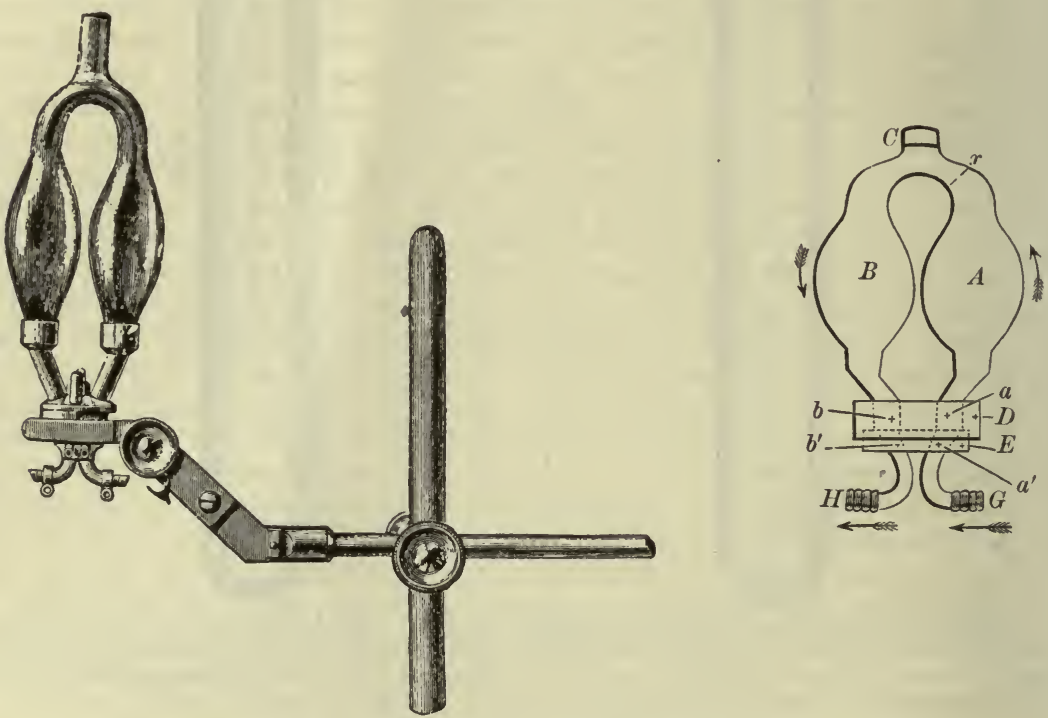

Ludwig's Stromuhr and a Diagrammatic Representation of the Same.

the clamps from the artery the blood flows through $G$ to $H$, and so back into the artery. The observation now begins by turning the disc $D$ into the position shown in the figure; the blood then flows into $A$, driving the oil there contained out before it into the bulb $B$, in the direction of the arrow, the defibrinated blood previously present in $B$ passing by $H$ into the artery, and so into the system. At the moment that the blood is seen to rise to the mark $x$, the disc $D$ is with all possible rapidity turned through two right angles; and thus the bulb $B$, now largely filled with oil, placed in communication with $G$. The blood-stream now drives the oil back into $A$, and the new blood in $A$ through $H$ into the artery. As soon as the oil has wholly returned to its original position, the dise is again turned round, and $A$ once more placed in communication with $G$, and the oil once more driven from $A$ to $B$. And this is repeated several times. indeed generally until the clotting of the blood or the admixture of the oil with the blood puts an end to the experiment. Thus the flow of blood is used to fill alternately with blood or oil the space of the bulb $A$, whose cavity as far as the mark $x$ has been exactly measured; hence if the number of times in any given time the disc $D$ has to be turned round be known, the number of times $A$ has been filled is also known, and thus the quantity of blood which has passed in that time through the canula connected with the tube $G$ is directly measured. For instance, sup- 
posing that the quantity held by the bulb $A$ when filled up to the mark $x$ is 5 c.c., and supposing that from the moment of allowing the first 5 c.c. of blood to begin to enter the tube to the moment when the escape of the last 5 c.c. from the artery into the tube was complete, 100 seconds had elapsed, during which time 5 c.c. has been received ten times into the tube from the artery (all but the last 5 c.c. being returned into the distal portion of the artery), obviously 0.5 c.c. of blood had flowed fiom the proximal section of the artery in one second. Hence, supposing that the diameter of the canula (and of the artery, they being the same) were $2 \mathrm{~mm}$., with area therefore of 3.14 square mm., an outflow through the section of 0.5 c.c. or $500 \mathrm{~mm}$. in a second would give $\left(\frac{500}{3.14}\right)$ a velocity of about 159 $\mathrm{mm}$. in a second.

The hæmatachometer of Vierordt [Fig. 47] is constructed on the principle of measuring the velocity of the current by observing the amount of deviation undergone by a pendulum, the free end of which hangs loosely in the stream. A square or rectangular chamber, one side of which is of glass and marked with a graduated scale in the form of an arc of a circle, is connected by means of two short tubes with the two cut ends of an artery; the blood consequently flows from the proximal (central) portion of the artery through the chamber into the distal portion of the artery. Within the chamber and suspended from its roof is a short pendulum, which when the blood-stream is cut off from the chamber hangs motionless in a vertical position, but when the blood is allowed to flow through the chamber, is driven by the force of the current out of its position of rest. The

[FIG. 47.

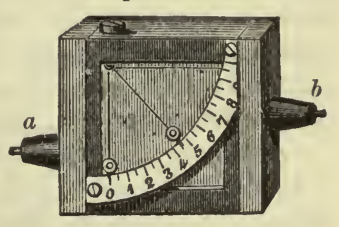

Hæmatachometer of vierordt : $a, b$, mouthpieces.] pendulum is so placed that a marker attached to its free end travels close to the inner surface of the glass side along the arc of the graduated side. Hence the amount of deviation from a vertical position may easily be read off on the scale from the outside. The graduation of the scale having been carried out by experimenting with streams of known velocity, the velocity can at once be calculated from the amount of deviation.

An instrument based on the same principle has been invented by Chauveau and improved by Lortet, Fig. 48 . In this the part which corresponds to the pendulum

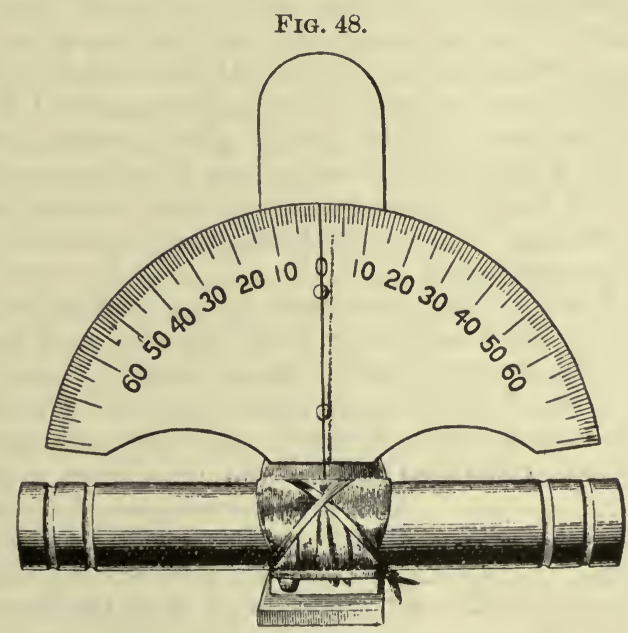

Hæmatachometer of Chauveau and Lortet.

in Vierordt's instrument is prolonged outside the chamber, and thus the portion within the chamber is made to form the short arm of a lever, the fulcrum of which is at the point where the wall of the chamber is traversed, and the long arm of which projects outside. A somewhat wide tube, the wall of which is at one point 
composed of an India-rubber membrane, is introduced between the two cut ends of an artery. A long light lever pierces the India-rubber membrane. The short expanded arm of this lever projecting within the tube is moved on its finlerum in the India-rubber ring by the current of blood passing through the tube, the greater the velocity of the current the larger being the excursion of the lever. The movements of the short arm give rise to corresponding movements in the opposite direction of the long arm outside the tube, and these, by means of a marker attached to the end of the long arm, may be directly inscribed on a recording surfice. This instrument is very well adapted for observing changes in the velocity of the flow. In determining actual velocities, for which purpose it has to be experimentally graduated, it is not so useful.

In the capillaries, the rate is slowest of all. In the web of the frog the flow as judged by the movement of the red corpuscles may be directly measured under the microscope by means of a micrometer, and is found to be about half a millimeter in a second; but this is probably a low estimate, since it is only when the circulation is somewhat slow, slower perhaps than what ought to be considered the normal rate, that the red corpuscles can be distinctly seen. In the mammal the rate has been estimated at about 0.75 millimetres a second, but is probably quicker than even this.

As regards the veins, the flow is very slow in the small veins emerging from the capillaries, but increases as these join into larger trunks, until in a large vein, such as the jugular of the dog, the rate is about $200 \mathrm{~mm}$. a second.

$\S 112$. It will be seen, then, that the velocity of the flow is in inverse proportion to the width of the bed, to the united sectional areas of the vessels. It is greatest at the aorta, it diminishes along the arterial system to the capillaries, to the united bases of the cones spoken of in $\$ 101$, where it is least, and from thence increases again along the venous system.

And, indeed, it is this width of the bed, and this alone, which determines the general velocity of the flow at various parts of the system. The slowness of the flow in the capillaries is not due to there being so much more friction in their narrow channels than in the wider canals of the larger arteries. For the peripheral resistance caused by the friction in the capillaries and small arteries is an obstacle not only to the flow of blood through these small vessels where the resistance is actually generated, but also to the escape of the blood from the large into the small arteries, and indeed from the heart into the large arteries. It exerts its influence along the whole arterial tract. And it is obvious that if it were this peripheral resistance which checked the flow in the capillaries, there could be no recovery of velocity along the venous tract.

The blood is flowing through a closed system of tubes, the bloodvessels, under the influence of one propelling force, the systole of the ventricle, for this is the force which drives the blood from ventricle to auricle, though, as we have seen, its action is modified in the several parts of the system. In such a system the same quantity of fluid must pass each section of the system at the same time, otherwise there would be a block at one place and a

FIG. 49.

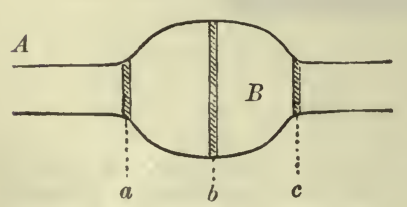
deficiency at another. If, for instance, a fluid is made to flow by some one force, pressure or gravity, through a tube $A$ (Fig. 49) with an enlargement $B$, it is obvious that the same quantity of fluid must pass through the section $b$ as passes through the section $a$ in the same time-for instance, a second. Otherwise, if less passes through $b$ than $a$, the fluid would accumulate in $B$, or if more, $B$ would be emptied. In the same way just as much must pass in the 
same time through the section $c$ as passes through $a$ or $b$. But if just as many particles of water have to get through the narrow section $a$ in the same time as they have to get through the broader section $c$, they must move quicker through $a$ than through $c$, or more slowly through $c$ than through $a$. For the same reason water flowing along a river impelled by one force-viz., that of gravity-rushes rapidly through a "narrow" and flows sluggishly when the river widens out into a "broad." The flow through $B$ will be similarly slackened if $B$, instead of being simply a single enlargement of the tube $A$, consists of a number of small tubes branching out from $A$, with a united sectional area greater than the sectional area of $A$. In each of such small tubes, at the line $c$, for instance, the flow will be slower than at $a$, where the small tubes branch out from $A$, or at $b$, where they join again to form a single tube. Hence it is that the blood rushes swiftly through the arteries, tarries slowly through the capillaries, but quickens its pace again in the veins.

An apparent contradiction to this principle that the rate of flow is dependent on the width of the bed is seen in the case where, the fluid having alternative routes, one of the routes is temporarily widened. Suppose a tube $A$ dividing into two branches of equal length $x$ and $y$ which unite again to form the tube $V$. Suppose, to start with, $x$ and $y$ are of equal diameter; then the resistance offered by each being equal, the flow will be equally rapid through the two, being just so rapid that as much fluid passes in a given time through $x$ and $y$ together as passes through $A$ or through $V$. But now suppose $y$ to be widened; the widening will diminish the resistance offered by $y$, and in consequence, supposing that no material change takes place in the pressure or force which is driving the fluid along, more fluid will now pass along $y$ in a given time than did before; that is to say, the rapidity of the flow in $y$ will be increased. It will be increased at the expense of the flow through $x$, since it will still hold good that the flow through $x$ and $y$ together is equal to the flow through $A$ and through $V$. We shall have occasion later on to point out that a small artery, or a set of small arteries, may be more or less suddenly widened without materially affecting the general blood-pressure which is driving the blood through the artery or set of arteries. In such cases the flow of blood through the widened artery or arteries is for the time being increased in rapidity, not only in spite of, but actually in consequence of, the artery being widened.

It must be understood in fact that this dependence of the rapidity of the flow on the width of the bed applies to the general rate of flow of the whole circulation, and that, besides the above instance, other special and temporary variations occur due to particular circumstances. Thus changes of pressure may alter the rapidity of flow. The cause of the flow through the whole system is the pressure of the ventricular systole manifested as what we have called blood-pressure. At each point along the system nearer the left ventricle, and therefore further from the right auricle, the pressure is greater than at a point further from the left ventricle and so nearer the right auricle; it is this difference of pressure which is the real cause of the flow from the one point to the other; and other things being equal the rapidity of the flow will depend on the amount of the difference of pressure. Hence, temporary or local variations in rapidity of flow may be caused by the establishment of temporary or local differences of pressure. For example, at any point along the arterial system the flow is increased in rapidity during the temporary increase of pressure due to the ventricular systole, $i . e$, the pulse, and diminished during the subsequent teniporary decrease, the increase and decrease being the more marked the nearer the point to the heart. And we shall probably meet later on with other instances. 
\$113. Time of the entire circuit. It is obvious from the foregoing that a red corpuscle in performing the whole circuit, in travelling from the left ventricle back to the left ventricle, would spend a large portion of its time in the capillaries, minute arteries, and veins. The entire time taken up in the whole circuit has been approximately estimated by measuring the time it takes for an easily recognized chemical substance after injection into the jugular vein of one side to appear in the blood of the jugular vein of the other side.

While small quantities of blood are being drawn at frequently repeated intervals from the jugular vein of one side, or while the blood from the vein is being allowed to fall in a minute stream on an absorbent paper covering some travelling surface, an iron salt such as potassium ferrocyanide (or preferably sodium ferrocyanide as being more innocuous) is injected into the jugular vein of the other side. If the time of the injection be noted, and the time after the injection into one side at which evidence of the presence of the iron salt can be detected in the sample of blood from the vein of the other side be noted, this gives the time it has taken the salt to perform the circuit; and on the supposition that mere diffusion does not materially affect the result, the time which it takes the blood to perform the same circuit is thereby given.

In the horse this time has been experimentally determined at about 30 seconds and in the dog at about 15 seconds. In man it is probably from 20 to 25 seconds.

Taking the rate of flow through the capillaries at about $1 \mathrm{~mm}$. a second it would take a corpuscle as long a time to get through about $20 \mathrm{~mm}$. of capillaries as to perform the whole circuit. Hence, if any corpuscle had in its circuit to pass through $10 \mathrm{~mm}$. of capillaries, half the whole time of its journey would be spent in the narrow channels of the capillaries. Inasmuch as the purposes served by the blood are chiefly carried out in the capillaries, it is obviously of advantage that its stay in them should be prolonged. Since, however, the average length of a capillary is about $0.5 \mathrm{~mm}$., about half a second is spent in the capillaries of the tissues and another half second in the capillaries of the lungs.

$\S 114$. We may now briefly summarize the broad features of the circulation, which we have seen may be explained on purely physical principles, it being assumed that the ventricle delivers a certain quantity of blood with a certain force into the aorta at regular intervals, and that the physical properties of the bloodvessels remain the same.

We have seen that owing to the peripheral resistance offered by the capillaries and small vessels the direct effect of the ventricular stroke is to establish in the arteries a mean arterial pressure which is greatest at the root of the aorta and diminishes toward the small arteries, some of it being used up to drive the blood from the aorta to the small arteries, but which retains at the region of the small arteries sufficient power to drive through the small arteries, capillaries, and veins just as much blood as is being thrown into the aorta by the ventricular stroke. We have seen, further, that in the large arteries at each stroke the pressure rises and falls a little above and below the mean, thus constituting the pulse, but that this extra distention with its subsequent recoil diminishes along the arterial tract and finally vanishes; it diminishes and vanishes because it ton, like the whole force of the ventricular stroke, of a fraction of which it is the expression, is used up in establishing the mean pressure; we shall, however, consider again later on the special features of this pulse. We have seen, further, that the task of driving the blood through the peripheral resistance of the small arteries and capillaries consumes much of this mean pressure, which consequently is much less in the small veins than in the corresponding small arteries, but 
that sufficient remains to drive the blood, even without the help of the auxiliary agents which are generally in action, from the small veins right back to the auricle. Lastly we have seen that while the above is the cause of the flow from ventricle to auricle, the changing rate of the flow, the diminishing swiftness in the arteries, the sluggish crawl through the capillaries, the increasing quickness through the veins are determined by the changing width of the vascular "bed."

Before we proceed to consider any further details as to the phenomena of the flow through the vessels, we must turn aside to study the heart.

\section{The Heart.}

$\S 115$. The heart is a valvular pump which works on mechanical principles, but the motive power of which is supplied by the contraction of its muscular fibres. Its action consequently presents problems which are partly mechanical and partly vital. Regarded as a pump, its effects are determined by the frequency of the beats, by the force of each beat, by the character of each beat-whether, for instance, slow and lingering, or sudden and sharpand by the quantity of fluid ejected at each beat. Hence, with a given frequency, force, and character of beat, and a given quantity ejected at each beat, the problems which have to be dealt with are for the most part mechanical. The vital problems are chiefly connected with the causes which determine the frequency, force, and character of the beat. The quantity ejected at each beat is governed more by the state of the rest of the body than by that of the heart itself.

\section{The Phenomena of the Normal Beat.}

The visible movements. When the chest of a mammal is opened and artificial respiration kept up the heart may be watched beating. Owing to the removal of the chest-wall, what is seen is not absolutely identical with what takes place within the intact chest, but the main events are the same in both cases. A complete beat of the whole heart or cardiac cycle may be observed to take place as follows:

The great veins, inferior and superior venæ cavæ, and pulmonary veins are seen, while full of blood, to contract in the neighborhood of the heart; the contraction runs in a peristaltic wave toward the auricles, increasing in intensity as it goes. Arrived at the auricles, which are then full of blood, the wave suddenly spreads, at a rate too rapid to be fairly judged by the eye, over the whole of those organs, which accordingly contract with a sudden sharp systole. In the systole, the walls of the auricles press toward the auriculo-ventricular orifices, and the auricular appendages are drawn inward, becoming smaller and paler. During the auricular systole, the ventricles may be seen to become turgid. Then follows, as it were immediately, the ventricular systole, during which the ventricles become more conical. Held between the fingers they are felt to become tense and hard. As the systole progresses, the aorta and pulmonary arteries expand and elongate, the apex is tilted slightly upward, and the heart twists somewhat on its long axis, moving from the left and behind toward the front and right so that more of the left ventricle becomes displayed. As the systole gives way to the succeeding diastole, the ventricles resume their previous form and position, the aorta and pulmonary artery shrink and shorten, the heart turns back toward the left, and thus the cycle is completed.

In the normal beat, the two ventricles are perfectly synchronous in action, they contract at the same time and relax at the same time, and the 
two auricles are similarly synchronous in action. It has been maintained, however, that the synchronism may at times not be perfect.

Before we attempt to study in detail the several parts of this complicated series of events, it will be convenient to take a rapid survey of what is taking place within the heart during such a cycle.

$\S 116$. The cardiac cycle. IVe may take as the end of the cycle the moment at which the ventricles having emptied their contents have relaxed and returned to the diastolic or resting position and form. At this moment the blood is flowing freely with a fair rapidity, but as we have seen at a very low pressure, through the venæ cavæ into the right auricle (we may confine ourselves at first to the right side), and since there is now nothing to keep the tricuspid valve shut, some of this blood probably finds its way into the ventricle also. This goes on for some little time, and then comes the sharp, short systole of the auricle, which, since it begins, as we have seen, as a wave of contraction running forward along the ends of the venæ cavæ, drives the blood not backward into the veins but forward into the ventricle; this end is further secured by the fact that the systole has behind it on the venous side the pressure of the blood in the veins, increasing, as we have seen, backward toward the capillaries, and before it the relatively empty cavity of the ventricle, in which the pressure is at first very low. By the complete contraction of the auricular walls the complete or nearly complete emptying of the cavity is insured. No valves are present in the mouth of the superior vena cava, for they are not needed; and the imperfect Eustachian valve at the mouth of the inferior vena cava cannot be of any great use in the adult, though in its more developed state in the fotus it had an important function in directing the blood of the inferior vena cava through the foramen ovale into the left auricle. The valves in the coronary vein are, however, probably of some use in preventing a reflux into that vessel.

As the blood is being driven by the auricular systole into the ventricle, a reflux current is probably set up, by which the blood, passing along the sides of the ventricle, gets between them and the flaps of the tricuspid valve and so tends to float these up. [Figs. 50,51.] It is further probable that
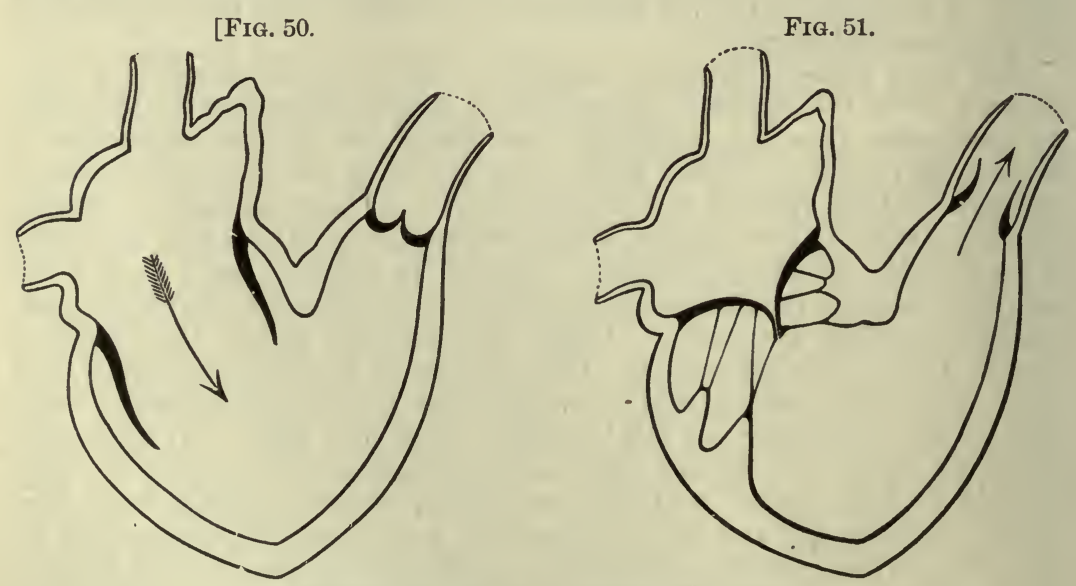

Diagrams of Valves of the Heart. After Dalton.]

the same reflux current, continuing somewhat later than the flow into the ventricle, is sufficient to bring the flaps into apposition, withnut any regurgitation into the auricle, at the close of the auricular systole, before the ven- 
tricular systole has begun. According to some authors, however, the closure of the valve is effected, at the very beginning of the ventricular systole, by the contraction of the papillary muscles; the chordæ tendinex of a papillary muscle are attached to the adjacent edges of two flaps, so that the shortening of the muscle tends to bring these edges into apposition.

The auricular systole is, as we have said, immediately followed by that of the ventricle. Whether the contraction of the ventricular walls (which as we shall see is a simple though prolonged contraction and not a tetanus) begins at one point and swiftly travels over the rest of the fibres, or begins all over the ventricle at once, is a question not at present definitely settled; but in any case the walls exert on the contents a pressure which is soon brought to bear on the whole contents and very rapidly rises to a maximum. The only effect upon the valve of this increasing intra-ventricular pressure is to render the valve more and more tense, and in consequence more secure, the chordæ tendineæ (the slackening of which through the change of form of the ventricle is probably obviated by a regulative contraction of the papillary muscles) at the same time preventing the valve from being inverted or even bulging largely into the auricle, and indeed, according to some observers, keeping the valvular sheet actually convex to the ventricular cavity, by which means the complete emptying of the ventricle is more fully effected. [Figs. 50,51.] The connection, to which we have just referred, of the chordæ of the same papillary muscle with the adjacent edges of two flaps, also assists in keeping the flaps in more complete apposition. Morever the extreme borders of the valves, outside the attachments of the chordæ, are excessively thin, so that when the valve is closed, these thin portions are pressed flat together back to back; hence, while the tougher central parts of the valves bear the force of the ventricular systole, the opposed thin membranous edges, pressed together by the blood, more completely secure the closure of the orifice.

At the commencement of the ventricular systole the semilunar valves of the pulmonary artery are closed, and are kept closed by the high pressure of the blood in the artery. As, however, the ventricle continues to press with greater and greater force on its contents, making the ventricle hard and tense to the touch, the pressure within the ventricle becomes greater than that in the pulmonary artery, and this greater pressure forces open the semilunar valves and allows the escape of the contents into the artery. The ventricular systole may be seen and felt in the exposed heart to be of some duration; it is strong enough and long enough to empty the ventricle completely ; indeed, as we shall see, it probably lasts longer than the discharge of blood, so that there is a brief period during which the ventricle is empty but yet contracted.

During the ventricular systole the semilunar valves are pressed outward toward but not close to the arterial walls, reflux currents probably keeping them in an intermediate position, so that their orifice forms an equilateral triangle with curved sides; thus they offer little obstacle to the escape of blood from the cavity of the ventricle. The ventricle, as we have seen, propels the blood with great force and rapidity into the pulmonary artery, and the whole contents are speedily ejected. Now, when a force which is driving a fluid with great rapidity along a closed channel suddenly ceases to act, the fluid, by its momentum, continues to move onward after the force has ceased; in consequence of this a negative pressure makes its appearance in the rear of the fluid, and, sucking the fluid back again, sets up a reflux current. So when the last portions of blood leave the ventricle a negative pressure makes its appearance behind them, and leads to a reflux current from the artery toward the ventricle. This alone would be sufficient to bring 
the valves together; and, in the opinion of some, is the real cause of the closure of the valves; others, however, as we shall see later on, maintain that subsequent to this reflux due to mere negative pressure a somewhat later reflux, in which the elastic reaction of the arterial walls is concerned, more completely fills and renders tense the pockets, causing their free margins to come into close and firm contact, and thus entirely blocks the way. The corpora Arantii meet in the centre, and the thin membranous festoons or lunulæ are brought into exact apposition. As in the tricuspid valves, so here, while the pressure of the blood is borne by the tougher bodies of the several valves, each two thin adjacent lunulæ, pressed together by the blood acting on both sides of them, are kept in complete contact, without any strain being put upon them; in this way the orifice is closed in a most efficient manner.

The ventricular systole now passes off, the muscular walls relax, the ventricle returns to its previous form and position, and the cycle is once more ended.

What thus takes place in the right side takes place in the left side also. There is the same sudden sharp auricular systole beginning at the roots of the pulmonary veins, the same systole of the ventricle, but, as we shall see, one much more powerful and exerting much more force; the mitral valve with its two flaps acts exactly like the tricuspid valve, and the action of the semilunar valves of the aorta simply repeats that of the valves of the pulmonary artery.

We may now proceed to study some of the cardiac events in detail.

$\S 117$. The change of form. The exact determination of the changes in form and position of the heart, especially of the ventricles, during a cardiac cycle is attended with difficulties.

The ventricles, for instance, are continually changing their form : they change while their cavities are being filled from the auricles, they change while the contraction of their walls is getting up the pressure on their contents, they change while under the influence of that pressure, their contents being discharged into the arteries, and they change when, their cavities having been emptied, their muscular walls relax.

We may take it for granted that the internal cavities are obliterated by the systole, for it is probable that practically the whole contents are driven out at each stroke, and probably also each cavity is emptied from its apex toward the mouth of the artery.

With regard to changes in external form, there seems no doubt that the side-to-side diameter is much lessened. It seems also clear that the front-to back diameter is greater during the whole time of the systole than during the diastole, the increase taking place during the first part of the systole. If a light lever be placed on the surface of the heart of a mammal, the chest having been opened and artificial respiration being kept up, some such curve as that represented in Fig. 52 is obtained. The rise of the lever in describing such a curve is due to the elevation of the part of the front surface of the heart on which the lever is resting. Such an elevation might be caused, especially if the lever were placed near the apex, by the heart being "tilted" upward during the systole, but only a small portion at most of the rise can be attributed to this cause; the rise is perhaps best seen when the lever is placed in the middle portion of the ventricle, and must be chiefly due to an increase in the front-to-back diameter of the ventricle during the beat. We shall discuss this curve later on in connection with other curves and may here simply say that the part of the curve from $b^{\prime}$ to $d$ probably corresponds to the actual systole of the ventricle, that is, to the time during which the fibres of the ventricle are undergoing contraction, the sudden fall from $d$ 
onward representing the relaxation which forms the first part of the diastole. If this interpretation of the curve be correct, it is obvious that the front-to-

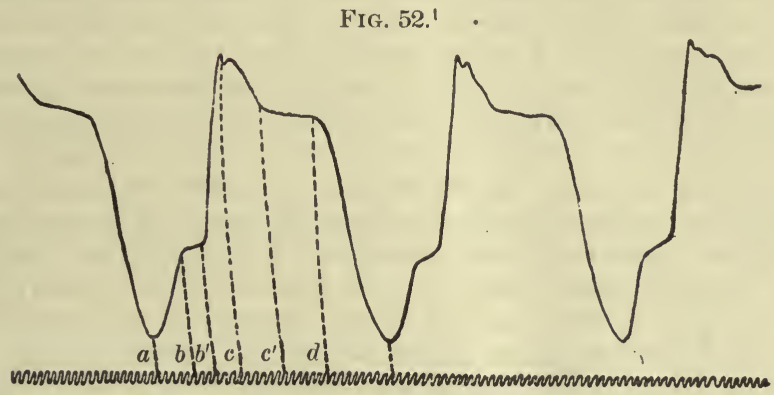

Tracing from Heart of Cat, obtained by placing a Light Lever on the Ventricle, the Chest having been Opened. The tuning-fork eurve marks 50 vibrations per second.

back diameter is greater during the whole of the systole than it is during diastole, since the lever is raised up all this time.

This increase of the front-to-back diameter combined with a decrease of the side-to-side diameter has for a result a change in the form of the section of the base of the ventricles. During the diastole this has somewhat the form of an ellipse with the long axis from side to side, but with the front part of the ellipse much more convex than the back, since the back surface of the ventricle is somewhat flattened. During the systole this ellipse is by the shortening of the side-to-side diameter and the increase of the front-toback diameter converted into a figure much more nearly resembling a circle. It is urged, moreover, that the whole of the base is constricted, and that the greater efficiency of the auriculo-ventricular valves is thereby secured.

As to the behavior of the long diameter from base to apex observers are not agreed. Some maintain that it is shortened, and others that it is practically unchanged. If any shortening does take place, it must be largely compensated by the elongation of the great vessels, which, as stated above,

1 The vertical or rather curved lines (segments of circles) introduced into this and many other curves are of use for the purpose of measuring parts of the curve. A complete curve should exhibit an "abscissa" line. This may be drawn by allowing the lever, arranged for the experiment but remaining at rest, to mark with its point on the recording surface set in motion; a straight line, the abscissa line, is thus described, and may be drawn before or after the curve itself is made, and may be placed above or preferably below the curve. When a tuning-fork or other time-marker is used, the line of the timemarker or a line drawn through the curves of the tuning-fork will serve as an abscissa line. After a tracing has been made, the recording surface should be brought back to such a position that the point of the lever coincides with some point of the curve which it is desired to mark ; if the lever be then gently moved up and down, the point of the lever will describe a segment of a circle (the centre of which lies at the axis of the lever), which segment should be made long enough to cut both the curve and the abscissa line (the tuning-fork curves or other time-marking line) where this is drawn. By moving the recording surface backward and forward similar segments of circles may be drawn through other points of the curve. The lines $a, b, c$ in Fig. 52 were thus drawn. The distance between any two of these points may thus be measured on the tuning-fork curve or other time curve, or on the abscissa line. Similar lines may be drawn on the tracing after its removal from the recording instrument in the following way: Take a pair of compasses, the two points of which are fixed just as far apart as the length of the lever used in the experiment, measured from its axis to its writing point. By means of the compasses find the position on the tracing of the centre of the circle of which any one of the previously drawn curved lines forms a segment. Through this centre draw a line parallel to the abscissa. By keeping one point of the compass on this line but moving it along the line backward or forward a segment of a circle may be drawn so as to cut any point of the curve that may be desired, and also the abscissa line or the time line. Such a segment of a circle may be used for the same purposes as the original one, and any number of such segments may be drawn. 
may be seen in an inspection of the beating heart. For there is evidence that the apex, though, as we have seen, it is during the systole somewhat twisted round and at the same time brought closer to the chest-wall, does not change its position up or down-i. e., in the long axis of the body. If in a rabbit or dog a needle be thrust through the chest-wall so that its point plunges into the apex of the heart, though the needle quivers its head moves neither up nor down, as it would do if its point in the apex moved down or up.

$\$ 118$. Cardiac impulse. If the hand be placed on the chest, a shock or impulse will be felt at each beat, and on examination this impulse, "cardiac impulse," will be found to be synchronous with the systole of the ventricle. In man, the cardiac impulse may be most distinctly felt in the fifth costal interspace, about an inch below and a little to the median side of the left nipple. In an animal the same impulse may also be felt in another wayviz., by making an incision through the diaphragm from the abdomen, and placing the finger between the chest-will and the apex. It then can be distinctly recognized as the result of the hardening of the ventricle during the systole. And the impulse which is felt on the outside of the chest is chiefly the effect of the same hardening of the stationary portion of the ventricle in contact with the chest-wall, transmitted through the chest-wall to the finger. In its flaccid state, during diastole, the apex is (in a standing position, at least) at this point in contact with the chest-wall, lying between it and the tolerably resistant diaphragm. During the systole, while being brought even closer to the chest-wall by the tilting of the ventricle and by the movement to the front and to the right, of which we have already spoken, it suddenly grows tense and hard. The ventricles, in executing their systole, have to contract against resistance. They have to produce within their cavities pressures greater than those in the aorta and pulmonary arteries, respectively. 'This is, in fact, the object of the systole. Hence, during the swift systole, the ventricular portion of the heart becomes suddenly tense, somewhat in the same way as a bladder full of fluid would become tense and hard when forcibly squeezed. The sudden pressure exerted by the ventricle, thus become suddenly tense and hard, aided by the closer contact of the apex with the chest-wall (which, however, by itself without the hardening of contraction would be insufficient to produce the effect), gives an insulse of shock both to the chest-wall and to the diaphragm, which may be felt readily both on the chest-wall, and also through the diaphragm when the abdomen is opened and the finger inserted. If the modification of the sphygmograph (of which we shall speak in dealing, later on, with the pulse), called the cardiograph, be placed on the spot where the impulse is felt most strongly, the lever is seen to be raised during the systole of the ventricles, and to fall again as the systole passes away, very much as if it were placed on the heart directly. A tracing may thus be obtained (see Fig. 58), of which we shall have to speak more fully immediately (see $\$ 122$ ). If the button of the lever be placed, not on the exact spot of the impulse, but at a little distance from it, the lever will be depressed during the systole. While at the spot of impulse itself the contact of the ventricle is increased during systole, away from the spot the ventricle retires from the chest-wall (by the diminution of its right-to-left diameter), and hence, by the mediastinal attachments of the pericardium, draws the chest-wall after it.

$\S 119$. The sounds of the heart. When the ear is applied to the chest, either directly or by means of a stethoscope, two sounds are heard, the first a comparatively long, dull, booming sound, the second a short, sharp, sudden one. Between the first and second sounds the interval of time is very 
short-too short to be measurable-but between the second and the succeeding first sound there is a distinct pause. The sounds have been likened to the pronunciation of the syllables lūbb, dŭp, so that the cardiac cycle, as far as the sounds are concerned, might be represented by : lübb, dŭp, pause.

The second sound, which is short and sharp, presents no difficulties. It is coincident in point of time with the closure of the semilunar valves, and is heard to the best advantage over the second right costal cartilage close to its junction with the sternum-i. $e$., at the point where the aortic arch comes nearest to the surface, and to which sounds generated at the aortic orifice would be best conducted. Its characters are such as would belong to a sound generated by membranes like the semilunar valves being suddenly made tense and so thrown into vibrations. It is obscured and altered or replaced by a " murmur" when the semilunar valves are affected by disease, and may be artificially obliterated, a murmur taking its place, by passing a wire down the arteries and hooking up the aortic valves. There can be no doubt, in fact, that the second sound is due to the semilunar valves being thrown into vibrations at their sudden closure. The sound heard at the second right costal cartilage is chiefly that generated by the aortic valves, and murmurs or other alterations in the sound caused by changes in the aortic valves are heard most clearly at this spot. But even here the sound is not exclusively of aortic origin, for in certain cases in which the semilunar valves on the two sides of the heart are not wholly synchronous in action the sound heard here is double ("reduplicated second sound"), one being due to the aorta and one to the pulmonary artery. While the sound is listened to on the left side of the sternum at the same level, the pulmonary artery is supposed to have the chief share in producing what is heard, and changes in the sound heard more clearly here than on the right side are taken as indications of mischief in the julmonary valves.

The first sound, longer, duller, and of a more "booming" character than the second, heard with greatest distinctness at the spot where the cardiac impulse is felt, presents many difficulties in the way of a complete explanation. It is heard distinctly when the chest-walls are removed. 'The cardiac impulse, therefore, can have little or nothing to do with it. In point of time it is coincident with the systole of the ventricles, and may be heard to the greatest advantage at the spot of the cardiac impulse-that is to say, at the place where the ventricles come nearest to the surface, and to which sounds generated in the ventricle would be best conducted.

It is more closely coincident with the closure and consequent vibrations of the auriculo-ventricular valves than with the entire systole; for, on the one hand, it dies away before the second sound begins, whereas, as we shall see, the actual systole lasts up to, if not beyond, the closure of the semilunar valves; and, on the other hand, the auriculo-rentricular valve ceases to be tense and to vibrate as soon as the contents of the ventricle are driven out. This suggests that the sound is caused by the sudden tension of the auriculo-ventricular valves, and this view is supported by the facts that the sound is obscured, altered, or replaced by murmurs when the tricuspid or mitral valves are diseased, and that the sound is also altered, or, according to some observers, wholly done away with, when blood is prevented from entering the ventricles by ligature of the venæ cavæ. On the other hand, the sound has not the sharp character which one would expect in a sound generated by the vibration of membranes such as the valves in question, but in its booming qualities rather suggests a muscular sound. Further, according to some observers, the sound, though somewhat modified, may still be heard when 
the large veins are clamped so that no blood enters the ventricle, and, indeed, may be recognized in the few beats given by a mammalian ventricle rapidly cut out of the living body by an incision carried below the auriculo-ventricular ring. Hence the view has been adopted that this first sound is a muscular sound. In discussing the muscular sound of skeletal muscle (see $\S 78$ ), we saw reasons to distrust the view that this sound was generated by the repeated individual simple contractions which made up the tetanus, and hence correspond in tone to the number of those simple contractions repeated in a second, and to adopt the view that the sound was really due to a repetition of unequal tensions occurring in a muscle during the contraction. Now, the ventricular systole is undoubtedly a simple contraction, a prolonged simple contraction, not a tetanus, and therefore under the old view of the nature of a muscular sound, could not produce such a sound; but, accepting the other view, and reflecting how complex must be the course of the systolic wave of contraction over the twisted fibres of the ventricle, we shall not find great difficulty in supposing that that wave is capable in its progress of producing such repetitions of unequal tensions as might give rise to a " muscular sound," and consequently in regarding the first sound as mainly so caused. Accepting such a view of the origin of the sound, we should expect to find the tension of the muscular fibres, and so the nature of sound dependent on the quantity of fluid present in the ventricular cavities, and hence modified by ligature of the great veins, and still more by the total removal of the auricles with the auriculo-ventricular valves. We may add that we should expect to find it modified by the escape of blood from the ventricles into the arteries during the systole itself, and might regard this as explaining why it dies away before the ventricle has ceased to contract.

Moreover, seeing that the auriculo-ventricular valves must be thrown into sudden tension at the onset of the ventricular systole, which, as we have seen, is developed with considerable rapidity, not far removed at all events from the rapidity with which the semilunar valves are closed, a rapidity, therefore, capable of giving rise to vibrations of the valves adequate to produce a sound, it is difficult to escape the conclusion that the closure of these valves must also generate a sound which in a normally beating heart is mingled in some way with the sound of muscular origin, although the ear cannot detect the mixture.

If we accept this view, that the sound is of double origin, partly " muscular," partly "valvular," both causes being dependent on the tension of the ventricular cavities, we can perhaps more easily understand how it is that the normal first sound is at times so largely, indeed we may say so completely, altered and obscured in diseases of the auriculo-ventricular valves.

Since the left ventricle forms the entire left apex of the heart; the murmurs or other changes of the first sound heard most distinctly at the spot of cardiac impulse belong to the mitral valve of the left ventricle. Murmurs generated in the tricuspid valve of the right ventricle are heard more distinctly in the median line below the end of the sternum.

\section{Endocardiac Pressure.}

$\S 120$. Since the heart exists for the purpose of exerting pressure on the blood within its cavities, by which pressure the circulation of the blood is effected, the study of the characters of this endocardiac pressure possesses great interest. Unfortunately, the observation of this pressure is attended with great difficulties. The ordinary mercury manometer which is so useful in studying the pressure in the arteries fails us when applied to the heart. It is true that a long canula, or tube open at the end, filled with sodium 
carbonate solution, may be introduced into the jugular vein and so slipped down into either the right auricle or the right ventricle, or may be similarly introduced into the carotid artery and with care slipped down through the aorta, past the semilunar valves, into the left ventricle, and having been thus introduced may, like the ordinary canula used in studying arterial pressure ( $\$ 104)$, be brought into connection with a mercury manometer. In this way, as in the case of an artery, a graphic record may be obtained of the changes of pressure taking place in either of the above three cavities. But the changes in the ventricular cavities are so great and rapid, that the inertia of the mercury, an evil in the case of an artery, comes so largely into play that the curve described by the float on the mercury is far from being an accurate record of the changes of pressure in the cavity.

The mercury manometer may, however, be made to yield valuable results by adopting the ingenious contrivance of converting the ordinary manometer into a maximum or a minimum instrument.

The principle of the maximum manometer, Fig. 53, consists in the introduction into the tube leading from the heart to the mercury column of a (modified cup-

[FIG. 53.

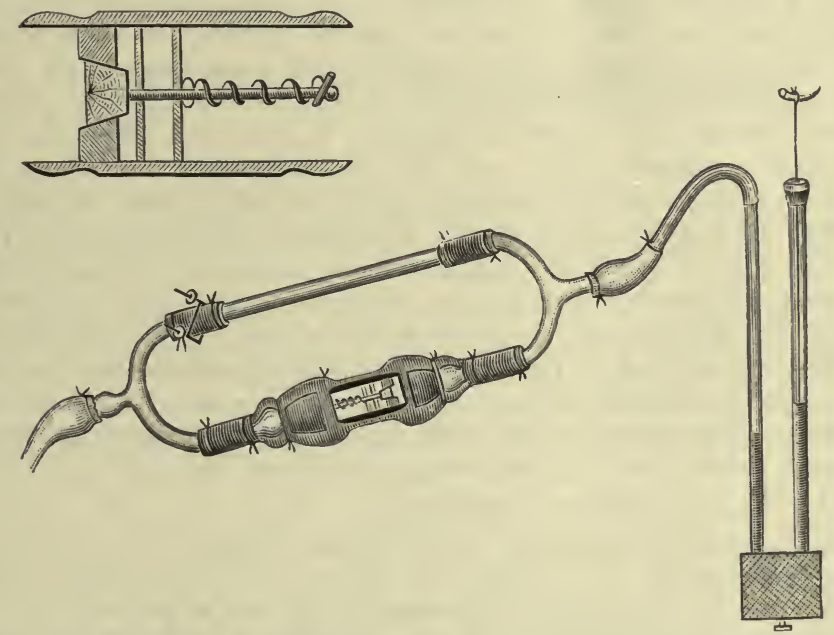

The Maximum Manometer of Goltz and Gaule. At $e$ a connection is made with the tube leading to the heart. When the screw-clamp $k$ is closed, the valve $v$ comes into action, and the instrument, in the position of the valve shown in the figure, is a maximum manometer. By reversing the direction of $v$ it is converted into a minimum manometer. When $k$ is opened, the variations of pressure are conveyed along $a$, and the instrument then acts like an ordinary manometer.

and-ball) valve, opening, like the aortic semilunar valves, easily from the heart, but closing firmly when fluid attempts to return to the heart. The highest pressure is that which drives the longest column of fluid past the valve, raising the mercury column to a corresponding height. Since this column, once past the valve, cannot return, the mercury remains at the height to which it was raised by it and thus records the maximum pressure. By reversing the direction of the valve, the manometer is converted from a maximum into a minimum instrument.

The maximum manometer applied to the cavity of either ventricle or of the right auricle, gives a record of the highest pressure reached within that cavity, and the minimum manometer similarly shows the lowest pressure reached, during the time that the instrument is applied.

The maximum manometer thus employed shows that the maximum pres- 
sure in the left ventricle is distinctly greater than the mean pressure in the aorta (the ordinary mercury manometer having previously given the paradoxical result, due to the inertia of the mercury, that the mean pressure in the left ventricle might be less than in the aorta), that the maximum pressure in the right ventricle is less than in the left, and in the right auricle is still less. In the dog, for example, the pressure in the left ventricle reaches a maximum of about $140 \mathrm{~mm}$. (mercury), in the right ventricle of about 60 $\mathrm{mm}$., and in the right auricle of about $20 \mathrm{~mm}$.

But the chief interest attaches to the minimum pressure observed; for the minimum manometer records a negative pressure in the cavities of the heart$i$. $e$, shows that the pressure in them may fall below that of the atmosphere. Thus in the left ventricle (of the $\mathrm{dog}$ ) a minimum pressure varying from -52 to $-20 \mathrm{~mm}$. may be reached, the minimum of the right ventricle being from -17 to $-16 \mathrm{~mm}$., and of the right auricle from -12 to $-17 \mathrm{~mm}$. Part of this diminution of pressure in the cardiac cavities may be due, as will be explained in a later part of this work, to the aspiration of the thorax in the respiratory movements. But even when the thorax is opened, and artificial respiration kept up, under which circumstances no such aspiration takes place, a negative pressure is still observed, the pressure in the left ventricle still sinking as low as $-24 \mathrm{~mm}$. Now, what the instrument actually shows is that at some time or other during the number of beats which took place while the instrument was applied (and these may have been very few) the pressure in the ventricle sank so many $\mathrm{mm}$. below that of the atmosphere. Since the negative pressure is observed when the heart is beating quite regularly, each beat being exactly like the others, we may infer that a negative pressure occurs at some period or other of each cardiac cycle. But the instrument obviously gives us no information as to the exact phase of the beat in which the negative pressure occurs; to this point as well as to the importance of this negative pressure we shall return presently.

$\S 121$. The difficulties due to the inertia of the mercury may be obviated by adopting the method of Chauveau and Marey, which consists in introducing in a large animal, such as a horse, through a bloodvessel into a cavity of the heart a tube ending in an elastic bag (Fig. 54, A) fashioned something like a sound, both tube and bag being filled with air, and the tube being connected with a recording "tambour."

A tube of appropriate curvature, $A, b$, Fig. 54, is furnished at its end with an elastic bag or "ampulla," $a$. When it is desired to explore simultaneously both auricle and ventricle, the sound is furnished with two ampulla with two small elastic bags, one at the extreme end and the other at such a distance that when the former is within the cavity of the ventricle the latter is in the cavity of the auricle. Such an instrument is spoken of as a "cardiac sound." Fach "ampulla" communicates by a separate air-tight tube with an air-tight tambour (Fig. 54, B) on which a lever rests, so that any pressure on the ampulla is communicated to the cavity of its respective tambour, the lever of which is raised in proportion. When two ampulla are used the writing points of both levers are brought to bear on the same recording surface exactly underneath each other. The tube is carefully introduced through the right jugular vein into the right side of the heart until the lower (ventricular) ampulla is fairly in the cavity of the right ventricle, and consequently the upper (auricular) ampulla in the cavity of the right auricle. Changes of pressure on either ampulla then cause movements of the corresponding lever. When the pressure, for instance, on the ampulla in the auricle is increased, the auricular lever is raised and describes on the recording surface an ascending curve; when the pressure is taken off the curve descends; and so also with the ventricle.

The "sound" may in a similar manner be readily introduced through the

1 These numbers are to be considered merely as instances which have been observed, and not as averages drawn from a large number of cases. 
carotid artery into the left ventricle and the changes taking place in that chamber also explored.

When this instrument is applied to the right auricle and ventricle some such record is obtained as that shown in Fig. 55, where the upper curve is a

FIG. 54.

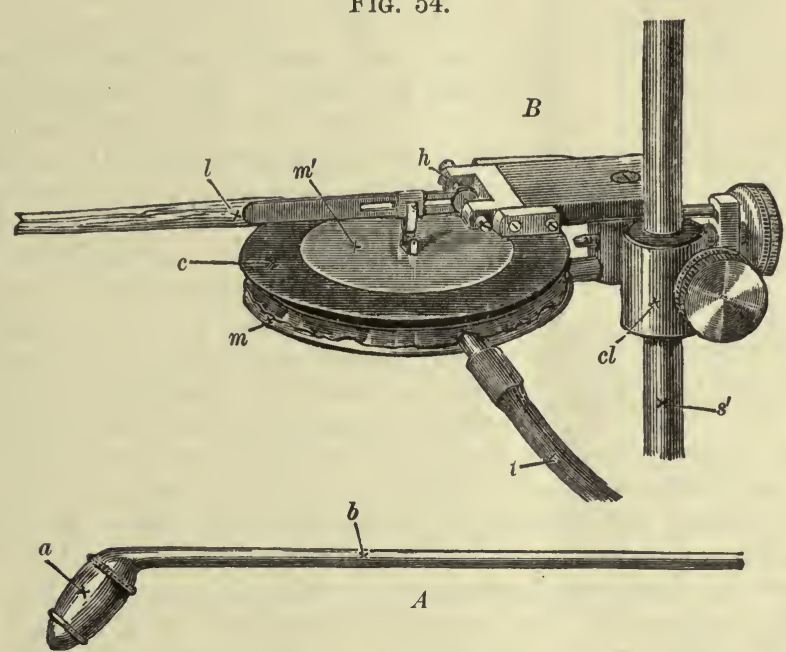

Marey's Tambour, with Cardiac Sound: $A$. A simple cardiac sound such as may be used for exploration of the left ventricle. The portion $a$ of the ampulla at the end is of thin India-rubber, stretched over an open framework with metallic supports above and below. The long tube $b$ serves to introduce it into the cavity which it is desired to explore.

$B$. The tambour. The metal chamber $m$ is covered in an air-tight manner with the Indiarubber $c$, bearing a thin metal plate $m^{\prime}$ to which is attached the lever $l$ moving on the hinge $h$. The whole tambour can be placed by means of the clamp $c l$ at any height on the upright $s^{\prime}$. The India-rubber tube $t$ serves to connect the interior of the tambour either with the cavity of the ampulla of $A$ or with any other cavity. Supposing that the tube $t$ were connected with $b$, any pressure exerted on $a$ would cause the roof of the tambour to rise and the point of the lever would be proportionately raised.

tracing taken from the right auricle and the lower curve from the right ventricle of the horse, both curves being taken simultaneously on the same recording surface.

In these curves the rise of the lever indicates pressure exerted upon the corresponding ampulla, and the upper curve from the right auricle shows the sudden brief pressure $(b)$ exerted by the sudden and brief auricular systole. The lower curve from the right ventricle shows that the pressure exerted by the ventricular systole begins almost immediately after the auricular systole, increases very rapidly indeed, so that the lever rises in almost a straight line up to $c^{\prime}$, is continued for some considerable time, and then falls very rapidly to reach the base line. But it may be doubted whether the instrument can be trusted to tell much more than this. The pressure recorded by each lever is the pressure exerted on the ampulla, and this may continue to be exerted after all blood has been discharged from the cavity, the walls of the emptied cavity closing round and pressing on the ampulla. But, as we shall presently see, it is of great interest to determine, not only the force and duration of the pressure exerted by the ventricular systcle, but also whether or no the fibres continue contracted and exerting pressure for an appreciable time after the blond has been forced out of the cavity. The figure, moreover, it need hardly be said, does not by 
itself give any information as to the relative amounts of pressure ex-

FIG. 55 .

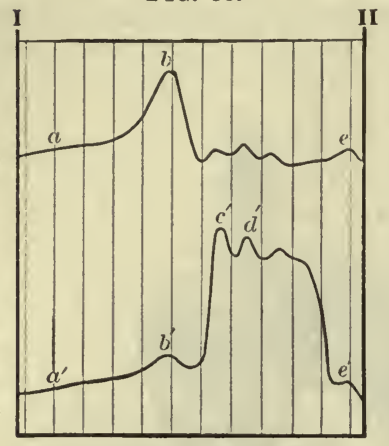

Simultaneous Tracings from the Right Auricle and Ventricle of the Horse. (After Chauveau and Marey.)

erted by the auricle and ventricle respectively. In the curve the auricular lever rises about half as high as the ventricular lever; but we must not infer from this that the auricular stroke is half as strong as the ventricular stroke; the former is arranged so as to move much more readily, to be much more sensitive than the latter. The instrument, it is true, may be experimentally graduated, and may then be used to determine the actual amount of pressure; but for this purpose is not wholly satisfactory. We may add that the irregularities seen on the ventricular curve during the ventricular systole, and on the auricular curve at the same time, have given rise to much debate, and need not be discussed here. On the whole, the method, though useful for giving a graphic view of the series of events within the cardiac cavities during a cardiac cycle, the short auricular pressure, the long-continued ventricular pressure, lasting nearly half the whole period, and the subsequent pause when both parts are at rest or in diastole, cannot with safety be used for drawing more detailed conclusions.

Perhaps the least untrustworthy method of recording the changes of endocardiac pressure is that recently introduced by Roy and Rolleston, though difficulties present themselves in the interpretation of the curves obtained by it.

By means of a short canula introduced through a large vessel, or directly, as a trocar, through the walls of the ventricle (or auricle), the blood in the cavity is brought to bear on an easily moving piston. The movements of the piston are recorded by a lever, and the evils of inertia are met by making the piston and lever work against the torsion of a steel ribbon, the length of which, and consequently the resistance offered by which, and hence the excursions of the piston, can be varied at pleasure.

The curves obtained by this method vary according to circumstances. We may take as fair examples two curves from the left ventricle, one (Fig. $56, A$ ) of a rapidly beating, and the other (Fig. $56, B$ ) of a slowly beating heart.

$\S 122$. In attempting to interpret these curves with the view of learning • the changes of pressure taking place in the heart, it is desirable to study them in connection with the tracing of which we have already spoken (Fig. 57), taken.by means of a light lever placed on the exposed ventricle, and which, as we have seen, is a curve of the changes taking place in the front-to-back diameter of the ventricle; or we may use what is very nearly the same thing, viz., a cardiographic tracing (Fig. 58); that is to say, a tracing of the cardiac impulse which is a curve of changes in the pressure exerted by the apex of the heart on the chest-wall.

Various forms of cardiograph have been used to record the cardiac impulse. In some the pressure of the impulse, as in the sphygmograph, is transmitted direetly to a lever which writes upon a travelling surface. In others the impulse is, by means of an ivory button, brought to bear on an air-chamber. connected by a tube with a tambour, as in Fig. 54 ; the pressure of the cardiac impulse compresses the air in the air-chamber, and through this the air in the chamber of the tambour by which the lever is raised. In such delicate and complicated movements as those of the heart, however, the use of long tubes filled with air is liable to introduce various errors. 
We may begin our study of these curves at any point in the cycle; let it be the point $b^{\prime}$ in Fig. 56 . From this point the curve rises very abruptly,

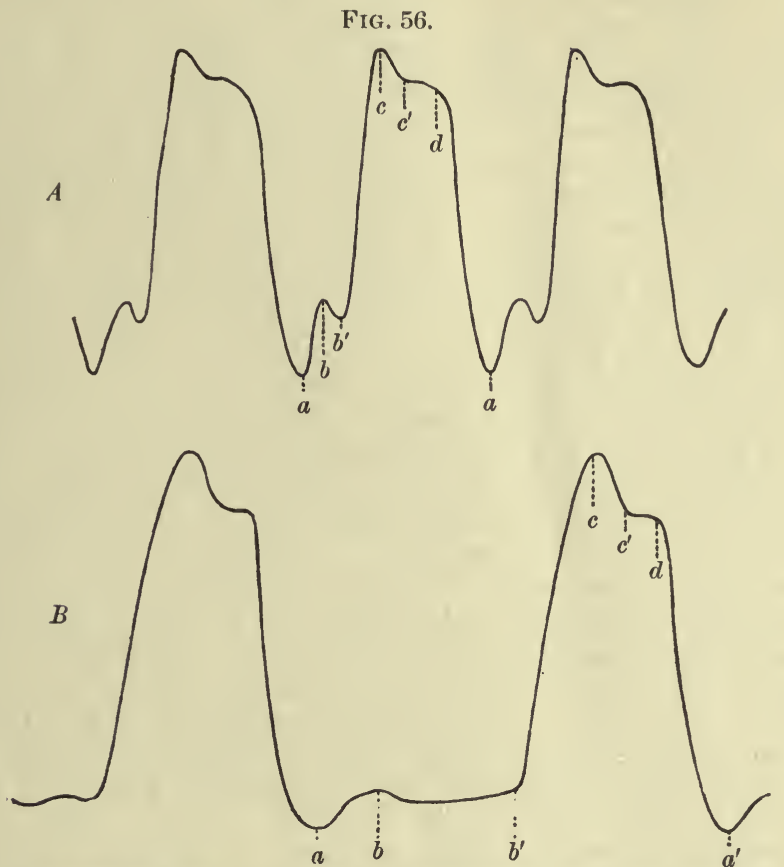

Curves of Endocardiac Pressure-from Left Ventricle of Dog: A, a quickly beating, $B$, a more slowly beating, heart. The letters in this and the succeeding Figs. 57, 58, are explained in the text.

almost in the vertical line, to a maximum at $c$, and the same sudden large rise to a maximum occurs in the front-to-back diameter of the ventricles (Fig.

FIG. 57.

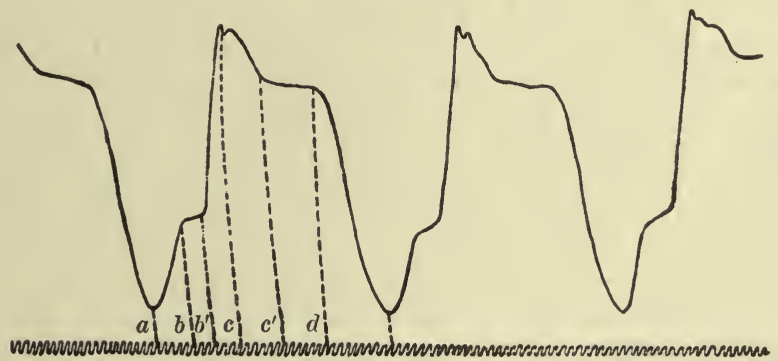

(See Fig. 52.)

57 ) and in the pressure of the apex against the chest wall (Fig. 58). There can be no doubt that this corresponds to the first part of the systole of the ventricles. By the sudden onset of the contraction of the ventricular fibres pressure is brought to bear on the contents of the ventricle, and there being as yet no escape for the blood, by the increasing contraction of the fibres the pressure becomes greater and greater. At the point $c$ a 
change take place in all three curves; the rise is converted into a fall, which, however, is very gradual as far as $d$. In the case of the front-to-

FIG. 58.

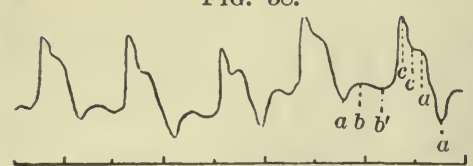

Cardograin from Man back diameter curve (Fig. 57) we may interpret this as meaning that while the continued contraction of the muscular fibres still maintains that change in the form of the ventricle by which the frontto-back diameter is increased, that same diameter is somewhat lessened by a diminution of the volume of the ventricles due to the escape of blood into the great arteries; and the cardiographic tracing admits of a similar interpretation-the apex relaxes its pressure on the chest-wall. We may extend the same interpretation to the pressure curve (Fig. 56). Somewhere about $c$ the pressure in the (left) ventricle has become higher than the pressure in the aorta, and in consequence blood escapes from the former into the latter. Whether the exact moment of the opening of the valves is absolutely identical with the turn of the curve at $c$, the curve beginning to fall at the moment when the area of high pressure in the ventricle is made continuous with the area of lower pressure in the aorta, or whether it occurs a little before $c$, the still increasing contraction of the ventricular fibres still increasing the pressure on the column of blood as it begins to move from the cavity of the ventricle into the aorta, may be left for the present undecided. The sudden fall from $d$ to $a$ admits of only one interpretation, and that in all the curves; this can only be due to the sudden relaxation of the muscular fibres of the ventricle, whereby the front-to-back diameter suddenly diminishes, the apex suddenly ceases to press on the chest-wall, and the pressure which the ventricular walls were previously exerting on the fluid in the canula introduced into its cavity also suddenly ceases. From $b^{\prime}$ to $d$, then, the ventricular walls are still contracting; during the whole of this time the real systole is being continued, but gives place at $d$ to a rapid relaxation which ushers in or forms the first part of the sequent diastole. Some little time after the beginning of this systole, somewhere about $c$, as we have seen, blood begins to escape from the ventricle into the aorta; this escape is certainly completed by the time $d$ is reached, and we have reason to think that it is really completed some little time before. The entrance into the aorta of the column of blood ejected by the ventricle distends that vessel, and the distention passes on, as we have seen, along the arterial track as the pulse. If, now, we measure the time during which the aorta, even near the heart, is being distended by the injection of the ventricular contents, we find this to be appreciably less than the time from $c$ to $d$, during which the systole of the ventricle is still going on, though the contents have already begun to escape at about $c$. This means that the ventricle, though empty, remains contracted for some little time after its contents have left the cavity. It is possible that the point $c^{\prime}$ in the three figures under discussion, where the descent of the lever changes in rate, becoming less rapid, corresponds to the end of the outflow from the ventricle; but this is not certain, and, indeed, the exact interpretation of this part of the curve is especially difficult.

The escape from the ventricle is rapid and forcible; the flow ceases suddenly. Hence, as we have already stated, $\S 116$, owing to the column of blood tending to move on by virtue of its inertia after the propelling force has ceased to act, a negative pressure makes its appearance behind the column of blood discharged from the ventricle, and as soon as the column is lodged in the aorta leads to a reflux toward the ventricle. This reflux 
would of itself have the effect of closing the valves even were the aorta a rigid tube. But the aorta is extensible and elastic and the effects of the movement of the column of fluid are combined with the effects of the movement of the arterial walls; the elastic action of the arterial walls, in a manner which we shall discuss later on in dealing with the pulse, also leads to a reflux. It has been urged that the reflux due to the negative pressure of the mere movement of the column of blood being more rapid, occurs independently of and earlier than the reflux due to the elastic recoil, the former closing the valves, the latter securing their complete closure. Be this so or no the valves are probably closed almost immediately after the escape of the ventricular contents, though observers are not agreed upon this point, some urging that the valves are not closed until so late a period as the point $d$, just as relaxation is about to begin. In the curves we are now considering, a notch, followed by a rise, or at least a more or less abrupt change in the course of the curve at $c^{\prime}$, is sometimes observed in that part of the curve which intervenes between the first large rise and the final sudden fall; and this secondary rise has been taken to indicate the closure of the semilunar valves. Sometimes two such notches and peaks are seen, and the occurrence of the two has been attributed to a want of synchronism in the closure of the pulmonary and aortic semilunar valves, the latter closing some little time before the former. But it is by no means clear that these notches and peaks are thus due to the closure of the valves; they may possibly have another origin, they are not always present, and, indeed, it does not seem certain that the closing of the valves should necessarily make an impress on the ventricular curve.

$\S 123$. In the performance of the ventricle, then (and what has been said of the left ventricle applies also to the right ventricle), there appear to be four stages:

1. A rapid "getting up" of pressure within the ventricle, all the valves being as yet closed; this continues until the pressure within the ventricle, becoming greater than that in the aorta, throws open the aortic valves.

2. The escape of the contents of the ventricle into the aorta, the contraction of the ventricular walls still continuing.

3. Further maintenance of the contraction for some little time after the main body, at all events, of the contents have passed the aortic valves; by this the complete emptying of the ventricle seems assured.

4. Sudden and rapid relaxation of the ventricular walls.

These four events together make up a large portion, and in a quickly beating heart the greater portion, of the whole cardiac cycle.

Meanwhile, that is, during the time from $b^{\prime}$ to $a$, blood has been flowing from the great veins into the auricle; during the interval from $b^{\prime}$ to $d$ none of this can pass into the ventricle since this is still contracted, but with the commencement of relaxation from $d$ onward there is no longer any obstacle ; on the contrary, as we shall see, an inducement for the blood to pass from the auricle into the ventricle.

For a brief time, as we have seen, there is probably an unbroken flow from the great veins (pulmonary or venæ cavæ) through the auricle into the ventricle, leading to a steady but slight increase of the front-to-back diameter, to a slight pressure of the apex on the chest-wall, and to a slight increase of intra-ventricular pressure, especially shown in the curve of the slowly beating heart of the horse (Fig. 55). In Fig. 57 the sudden rise due to the ventricular systole is preceded by a rise $b$ followed by a fall, forming thus, as it were, a shoulder on the curve. This has been interpreted as indicating the sharp transient auricular systole; the sudden injection of the 
auricular contents into the ventricle increases the front-to-back diameter of the ventricle, and the momentum of the rapid stroke being considerable, the lever is in each case carried too far forward, so that the rise is followed by a fall, producing a notch. A similar though somewhat different shoulder is also seen in the cardiogram, Fig. 58. In the curve of ventricular pressure taken by means of the cardiac sound (Fig. 55) there is a similar temporary increase $b^{\prime}$ in the ventricular pressure coincident with the auricular stroke $b$, and in the "piston" pressure-curve of the rapidly beating heart (Fig. 56, A) there is a similar shoulder $b$ just preceding the rise of the ventricular systole. The meaning of the last curve is, however, doubtful, for in the similar curve of the more slowly beating heart (Fig. $56, B$ ) it occurs inmediately after the relaxation of the ventricle, some time before the occurrence of the auricular systole, and in many curves taken by the same method is absent altogether. The exact meaning, therefore, of the shoulder $b$ in the other curves must be left at present undecided.

$\S 124$. We have still to consider the negative pressure shown by the minimum manometer. This instrument, as we have said, merely shows that the pressure in the ventricle (or auricle) becomes negative at some phase or other of the cardiac cycle, but does not tell us in which phase it occurs.

Now there are two ways in which such a negative pressure might originate. In the first place, as we have just seen, a negative pressure makes its appearance in the rear of the column of blood driven from the ventricle into the aorta with great suddenness and rapidity. But this negative pressure, as we have also seen, follows the column into the aorta past the semilunar valves, and in part, at all events, determines the closure of the semilunar valves. Hence if this is the negative pressure which the minimum manometer records, it ought to be shown not only when the end of the tube connected with the manometer is in the cavity of the ventricle, but also when the tube is slipped out of the ventricle just past the semilunar valves. When the tube, however, is in the latter situation the manometer does not show the same marked negative pressure that it does when the tube is in the ventricle; the negative pressure which occurs in the aorta at each beat is sufficient to produce such an effect on the minimum manometer as is produced when the instrument is in the ventricle. Hence we infer that the negative pressure shown by the minimum manometer is not produced in this way. We may, moreover, conclude that the semilunar valves are closed before this negative pressure makes its appearance in the ventricle; otherwise, however produced, it would be transmitted from the interior of the ventricle through the open valves to the root of the aorta beyond.

But there is another event which might give rise to a negative pressure. The relaxation of the ventricular walls is, as the curves (Figs. 56, 57, 58) show, a rapid process, something quite distinct from the mere filling of the ventricular cavities with blood from the auricles; and, though some have objected to the view, it may be urged that this return of the ventricle from its contracted (and emptied) condition to its normal form would develop a negative pressure. This return is probably simply the total result of the return of each fibre or fibre cell to its natural condition, though some have urged that the extra quantity of blood thrown into the coronary arteries at the systole helps to unfold the ventricles somewhat in the way that fluid driven between the two walls of a double-walled collapsed ball or cup will unfold it.

Accepting the return of the ventricles to their normal form as the cause of the negative pressure (and it may be remarked that the return of the thick-walled left ventricle naturally exerts a greater negative pressure than the thin-walled right ventricle), it is obvious that the negative pressure will 
assist the circulation by sucking the blood which meanwhile has been accumulated in the auricle, from that cavity into the ventricle, the auriculo-ventricular valves easily giving way. At the same time this very flow from the auricle will at once put an end to the negative pressure, which obviously can be of brief duration only. It may be further urged in support of this view, that even when the thorax is opened, so that the respiratory movements can no longer act toward producing a negative pressure in the auricle and great veins, a minimum manometer placed in the right auricle shows frequently no pressure at all (that is, a pressure equal to that of the atmosphere) and sometimes a decidedly negative pressure. Seeing that the blood under these circumstances is being driven along the great veins by a pressure which though low is always above that of the atmosphere, we may conclude that the negative pressure produced in the ventricle is the cause of this lowering of the pressure in the auricle, though it is unable to make itself felt along the great veins.

$\S 125$. The duration of the several phases. We may, first of all, distinguish certain main phases: (1) The systole of the auricles. (2) The systole, proper, of the ventricles, during which their fibres are in a state of contraction, lasting to $d$ in Figs. $56,57,58$. (3) The diastole of the ventriclesthat is to say, the time intervening between their fibres ceasing to contract and commencing to contract again. To these we may perhaps add (4) The pause or rest of the whole heart, comprising the period from the end of the relaxation of the ventricles to the beginning of the systole of the auricles; during this time the walls are undergoing no active changes, neither contracting nor relaxing, their cavities being simply passively filled by the influx of blood.

The mere inspection of almost any series of cardiac curves, however taken-those, for instance, which we have just discussed-will show, apart from any accurate measurements, that the systole of the auricles is always very brief, that the systole of the ventricles is always very prolonged-always occupying a considerable portion of the whole cycle-and that the diastole of the whole heart, reckoned from the end either of the systole or of the relaxation of the ventricle, is very various, being in quickly beating hearts very short and in slowly beating hearts decidedly longer.

When we desire to arrive at more complete measurements, we are obliged to make use of calculations based on various data; and these give only approximate results. Naturally, the most interest is attached to the duration of events in the human heart.

The datum which perhaps has been most largely used is the interval between the beginning of the first and the occurrence of the second sound. This may be determined with approximate correctness, and is found to vary from 0.301 to 0.327 second, occupying from 40 to 46 per cent. of the whole period, and being fairly constant for different rates of heart-beat. That is to say, in a rapidly beating heart it is the pauses which are shortened, and not the duration of the actual beats.

The observer, listening to the sounds of the heart, makes a signal at each event on a recording surface, the difference in time between the marks being measured by means of the vibrations of a tuning-fork recorded on the same surface. $\mathrm{By}$ practice it is found possible to reduce the errors of observation within very small limits.

Now, whatever be the exact causation of the first sound, it is undoubtedly coincident with the systole of the ventricles, though possibly the actual commencement of its becoming audible may be slightly behind the actual beginning of the muscular contractions. Similarly the occurrence of the second 
sound, which, as we have seen, is certainly due to the closure of the semilunar valves, has been taken to mark the close of the ventricular systole. And on this supposition the interval between the beginning of the first and the occurrence of the second sound has been regarded as indicating approximately the duration of the ventricular systole- $i$. e., the period during which the ventricular fibres are contracting.

The determination of the separate duration of each of the three periods of the ventricular systole-viz., the getting up of the pressure, the discharge of the contents, and the remaining emptied but contracted-is subjected to so much uncertainty that it neer not be insisted on here; it may, however, be said that, roughly speaking, each phase occupies probably about 0.1 second.

In a heart beating 72 times a minute, which may be taken as the normal rate, each entire cardiac cycle would last about 0.8 second, and taking 0.3 second as the duration of the ventricular systole, the deduction of this would leave 0.5 second for the whole diastole of the ventricle, including its relaxation, the latter occupying about or somewhat less than 0.1 second. In the latter part of this period there occurs the systole of the auricles, the exact duration of which it is difficult to determine, it being hard to say when it really begins, but which, if the contraction of the great veins be included, may perhaps be taken as lasting, on an average, 0.1 second. The "passive interval," therefore, during which neither auricle nor ventricle is undergoing contractions, lasts about 0.4 second, and the absolute pause or rest during which neither auricle nor ventricle is contracting or relaxing, about 0.3 second; if, however, a longer period be allotted to the ventricular systole, these periods must be proportionately shortened. The systole of the ventricle follows so immediately upon that of the auricles, that practically no interval exists between the two events.

The duration of the several phases may, for convenience sake, be arranged in a tabular form as follows:

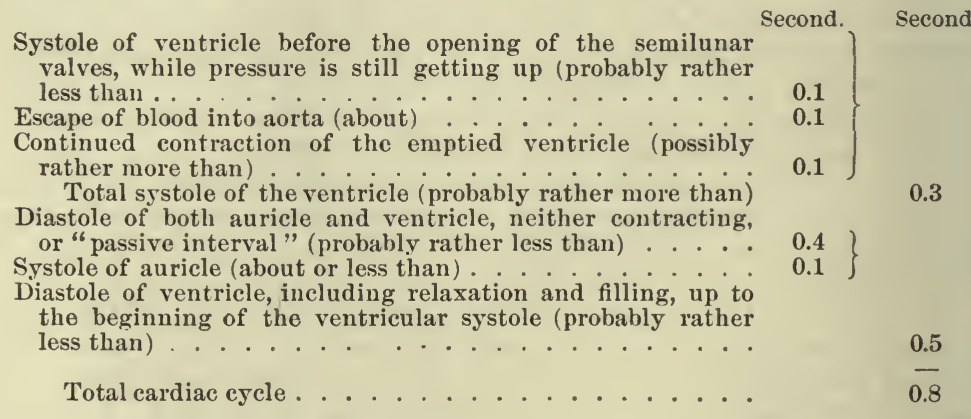

\section{Summary.}

$\S 126$. We may now briefly recapitulate the main facts connected with the passage of blood through the heart. The right auricle during its diastole, by the relaxation of its muscular fibres, and by the fact that all backward pressure from the ventricle is removed by the closing of the tricuspid valves, offers but little resistance to the ingress of blood from the veins. On the other hand, the blood in the trunks of both the superior and inferior vena cava is under a pressure, which, though diminishing toward the heart, remains higher than the pressure obtaining in the interior of the auricle; the blood in consequence flows into the empty auricle, its progress in the case of the superior vena cava heing assisted by gravity. At each inspiration this flow (as we shall see in speaking of respiration) is favored by the diminution 
of pressure in the heart and great vessels caused by the respiratory movements. Before this flow has gone on very long, the diastole of the ventricle begins, its cavity dilates, the flaps of the tricuspid valve fall back, and blood for some little time flows in an unbroken stream from the venæ cavæ into the ventricle. In a short time, however, probably before very much blood has had time to enter the ventricle, the auricle is full; and forthwith its sharp sudden systole takes place. Partly by reason of the backward pressure in the veins, which increases rapidly from the heart toward the capillaries, and which, at some distance from the heart, is assisted by the presence of valves in the venous trunks, but still more from the fact that the systole begins at the great veins themselves and spreads thence over the auricle, the force of the auricular contraction is spent in driving the blood, not back into the veins, but into the ventricle, where the pressure is still exceedingly low. Whether there is any backward flow at all into the great veins, or whether by the progressive character of the systole the flow of blood continues, so to speak, to follow up the systole without break, so that the stream from the veins into the auricle is really continuous, is at present doubtful; though a slight positive wave of pressure synchronous with the auricular systole, travelling backward along the great veins, has been observed, at least in cases where the heart is beating vigorously.

The ventricle thus being filled by the auricular systole, the play of the tricuspid valves described above comes into action, the auricular systole is followed by that of the ventricle, and the pressure within the ventricle, cut off from the auricle by the tricuspid valves, is brought to bear on the pulmonary semilunar valves and the column of blood on the other side of those valves. As soon as by the rapidly increasing shortening of the ventricular fibres the pressure within the ventricle becomes greater than that in the pulmonary artery, the semilunar valves open and the still continuing systole discharges the contents of the ventricle into that vessel.

As the ventricle thus rapidly and forcibly empties itself, either the transient negative pressure which makes its appearance in the rear of the ejected column of blood, or the elastic action of the aortic walls, leads to a reflux of blood toward the ventricle, the effect of which, however, is to close the semilunar valves and thus to shut off the blood in the distended arteries from the emptied ventricle. Either immediately at or more probably some little time after this closing of the valves the ventricular systole ends and relaxation begins; then once more the cavity of the ventricle becomes unfolded and finally distended by the influx of blood, a negative pressure developed by the relaxation probably aiding the flow from the auricle and great veins.

During the whole of this time the left side has with still greater energy been executing the same manœuvre. At the same time that the venæ cavæ are filling the right auricle the pulmonary veins are filling the left auricle. At the same time that the right auricle is contracting tle left auricle is contracting, too. The systole of the left ventricle is synchronous with that of the right ventricle, but executed with greater force; and the flow of blood is guided on the left side by the mitral and aortic valves in the same way that it is on the right by the tricuspid valves and the valves of the pulmonary artery.

\section{The Work Done.}

$\$ 127$. The most important factor of all in the determination of the work of the vascular mechanism is the quantity of blood ejected from the ventricle into the aorta at each systole. The general result of some of these calculations gives about 180 grammes (6 ozs.) as the quantity of blood which is 
driven from each ventricle at each systole in a full-grown man of average size and weight, but this estimate is probably too high.

In the dog the quantity has been experimentally determined, by allowing the heart to deliver its contents through one branch of the aorta, all others being ligatured or blocked, into a receiver, the contents of which are at intervals, by an ingenious contrivance, returned to the right auricle. The time taken to fill the receiver and the number of beats executed during that time being noted, the average quantity ejected at a beat is thus given. It is found to vary widely.

$V$ arious methods have been adopted for calculating the average amount of blood ejected at each ventricular systole. The simplest method is to measure the capacity of the recently removed and as yet not rigid ventricle, filled with blood under a pressure equal to the calculated average pressure in the ventricle. On the supposition that the whole contents of the ventricle are ejected at each systole this would give the quantity driven into the aorta at each stroke. The other methods are very indirect.

It is evident that exactly the same quantity must issue at a beat from each ventricle; for if the right ventricle at each beat gave out rather less than the left, after a certain number of beats the whole of the blood would be gathered in the systemic circulation. Similarly, if the left ventricle gave out less than the right, all the blood would soon be crowded into the lungs. The fact that the pressure in the right ventricle is so much less than that in the left (probably 30 or $40 \mathrm{~mm}$. as compared with $200 \mathrm{~mm}$. of mercury), is due, not to differences in the quantity of blood in the cavities, but to the fact that the peripheral resistance which has to be overcome in the lungs is so nuuch less than that in the rest of the body.

It must be remembered that though it is of advantage to speak of an average quantity ejected at each stroke, it is more than probable that that quantity may vary within very wide limits. Taking, however, 180 grammes as the quantity, in man, ejected at each stroke at a pressure of $250 \mathrm{~mm} .^{1}$ of mercury, which is equivalent to 3.21 metres of blood, this means that the left ventricle is capable at its systole of lifting 180 grammes 3.21 metres high, $i$. $e_{\text {. }}$, it does 578 gramme-metres of work at each beat. Supposing the heart to beat 72 times a minute, this would give for the day's work of the left ventricle nearly 60,000 kilogramme-metres. Calculating the work of the right ventricle at one-fourth that of the left, the work of the whole heart would amount to 75,000 kilogramme-metres, which is just about the amount of work done in the ascent of Snowdon by a tolerably heavy man.

A calculation of more practical value is the following: Taking the quantity of blood as $\frac{1}{13}$ of the body-weight, the blood of a man weighing 75 kilos would be about 5760 grammes. If 180 grammes left the ventricle at each beat, a quantity equivalent to the whole blood would pass through the heart in 32 beats, $i$. e., in less than half a minute.

\section{The Pulse.}

$\S 128$. We have seen that the arteries, though always distended, undergo at each systole of the ventricle a temporary additional distention, so that when a finger is placed on an artery, such as the radial, an intermittent pressure on the finger, coming and going with the beat of the heart, is felt, and when a light lever is placed on the artery, the lever is raised at each beat, falling between.

This intermittent expansion which we call the pulse, corresponding to the jerking outflow of blood from a severed artery, is present in the arteries only, being, except under particular circumstances, absent from the veins and capillaries. The expansion is frequently visible to the eye, and in some.

${ }^{1} \mathrm{~A}$ high estimate is purposely taken here. 
cases, as where an artery has a bend, may cause a certain amount of locomotion of the vessel.

The temporary increase of pressure which is the cause of the temporary increase of expansion makes itself felt, as we have seen, in the curve of arterial pressure taken by the mercury manometer; but the inertia of the mercury prevents the special characters of each increase becoming visible. In Fick's spring manometer (Fig. 59), in which the increase of pressure unfolds a curved spring and so moves a lever, the inertia is much less, and satisfactory tracings may be taken by this instrument. Other instruments have also been devised for recording the special characters of each increase

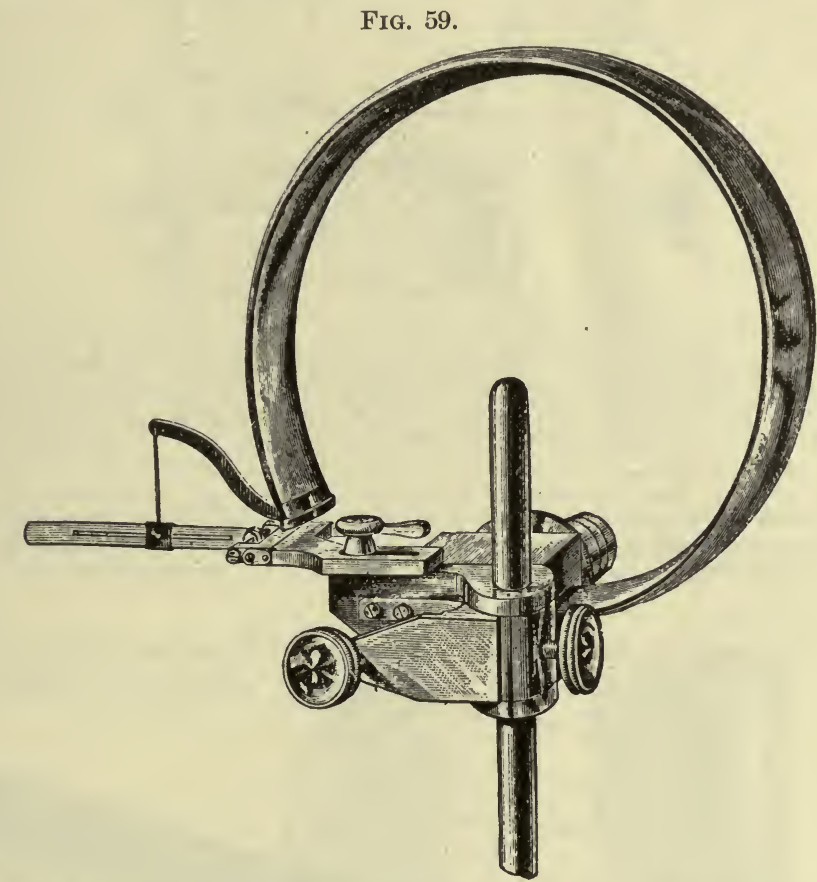

Fick's Spring Manometer. The flattened tube in the form of a hoop is firmly fixed at one end, while the other free end is attached to a lever. The interior of the tube, filled with spirit, is brought, by means of a tube containing sodium carbonate solution, into connection with an artery, in much the same way as in the case of the mercury manometer. The increase of pressure in the artery being transmitted to the hollow hoop, tends to straighten it, and correspondingly moves the attached lever.

of pressure or of the expansion of the artery which is the result of that increase. The easiest and most common method of registering the expansion of an artery is that of simply bringing a light lever to bear on the outside of the artery.

A lever specially adapted to record a pulse tracing is called a sphygmograph, the instrument generally comprising a small travelling recording surface on which the lever writes. There are many different forms of sphygmograph, but the general plan of structure is the same. Fig. 60 represents in a diagrammatic form the essential parts of the sphygmograph, known as Dudgeon's [and Fig. 61, Marey's, which is in more common use]. The instrument is generally applied to the radial artery because the arm affords a convenient support to the fulcrum of the lever, and because the position of 
the artery, near to the surface and with the support of the radius below so that adequate pressure can be brought to bear by the lever on the artery, is

FIG. 60.

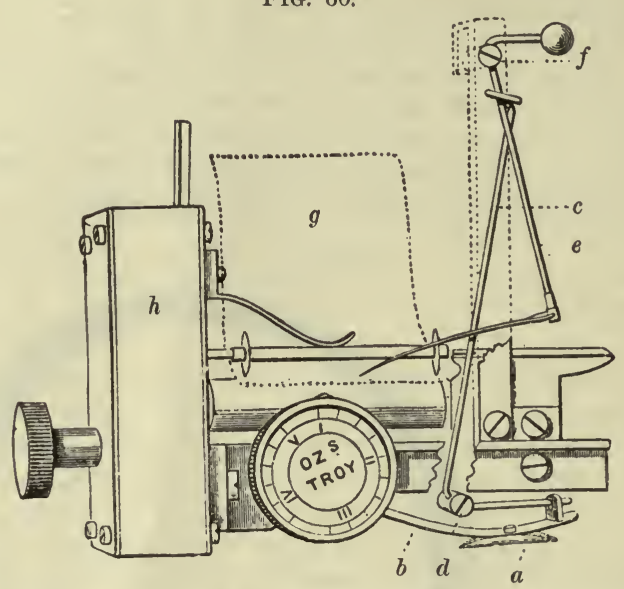

Diagram of a Sphygmograph (Dudgeon's). Certain supporting parts are omitted so that the multiplying levers may be displayed. $a$ is a small metal plate which is kept pressed on the artery by the spring $b$. The vertical movements of $a$ cause to-and-fro morements of the lever $c$ about the fixed point $d$. These are communicated to and magnified by the lever $e$, which moves round the fixed point $f$. The free end of this lever carries a light steel marker whieh rests on a strip of smoked paper $g$. The paper is placed beneath two small wheels and rests on a roller which can be rotated by means of eloek-work contained in the box $h$. The paper is thus caused to travel at a uniform rate. The screw graduated in ounces (Troy) is brought to bear on the spring $b$ by means of a cam and by this the pressure put on the artery can be regulated. 'The levers magnify the pulse-movements fifty times.

favorable for making observations. It can, of course, be applied to other arteries. When applied to the radial artery some such tracing as that

[FIG. 61.

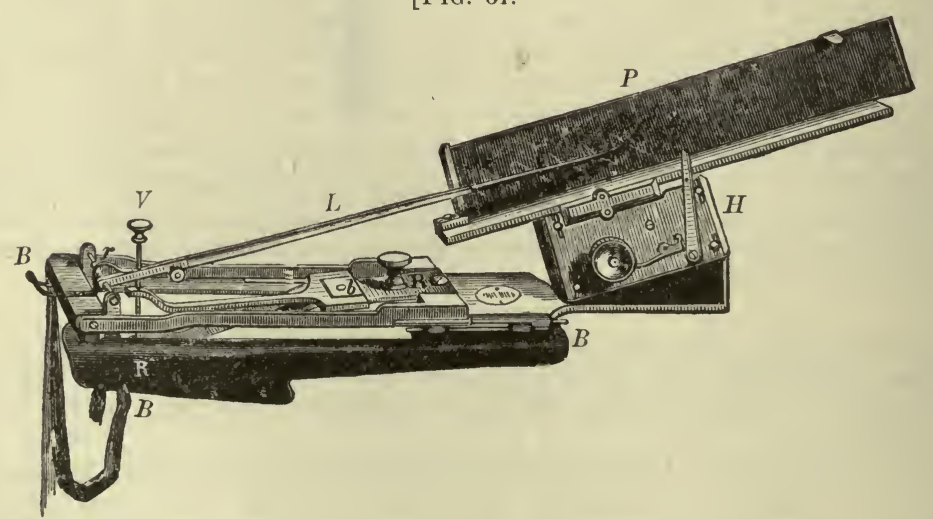

Marey's Sphygmograph: $B, B$ is where the sphygmograph is applied to the arm; $R$, spring which rests upon radial artery ; $V$, screw for adjusting marking lever $L ; H$, clock-work $: P$, smoked paper upon which traeing is made; $r$, small spring for causing descent of lever after raising.]

shown in Fig. 62 is obtained. At each heart beat the lever rises rapidly and then falls more gradually in a line which is more or less uneven. 
$\S 129$. We have now to study the nature and characters of the pulse in greater detail.

We may say at once, and indeed have already incidentally seen, that the pulse is essentially due to the action of physical causes; it is the

FIG. 62.

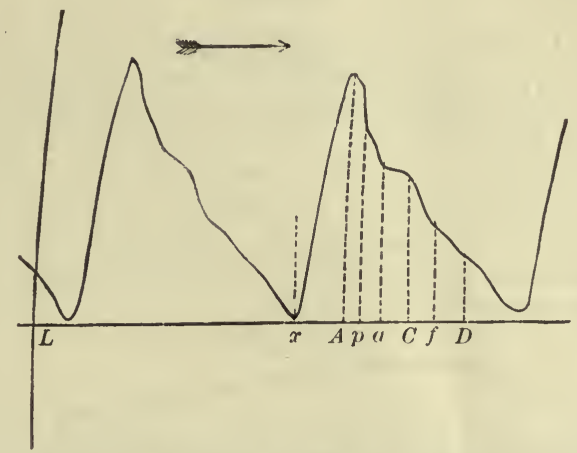

Pulse Tracing from the Radial Artery of Man The vertical curved line $L$, gives the tracing which the recording lever made when the blackened paper was motionless. The curved interrupted lines show the distance from one another in time of the chief phases of the pulse-wave, viz., $x=$ commencement and $A$ end of expansion of artery; $p$, pre-dicrotic notch. $d$, dicrotic notch. $C$, dicrotic crest. $D$, Post-dicrotic crest. $f$, the post-dicrotic notch. These are explained in the text later on.

physical result of the sudden injection of the contents of the ventricle into the elastic tubes called arteries; its more important features may be ex-

[FIG. 63.

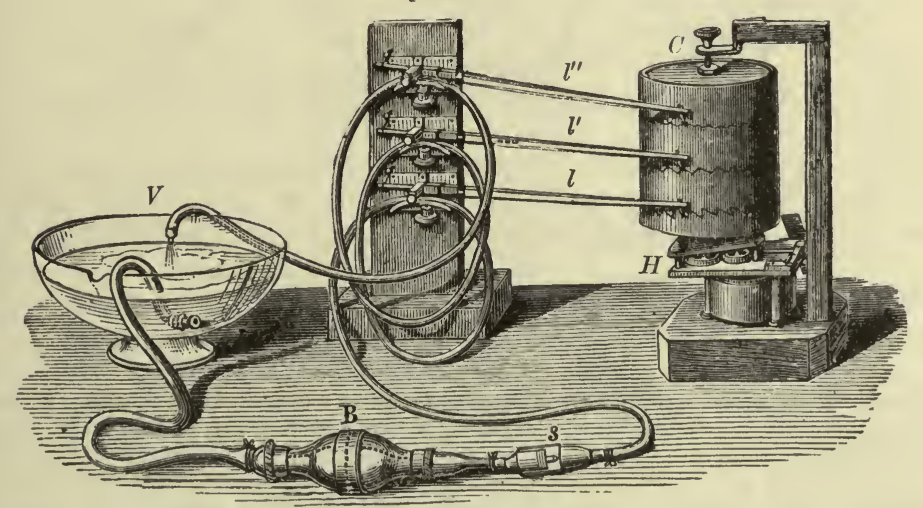

Apparatus of Marey for showing Mode in which Pulse is Propagated in the Arteries: $B$ is a rubber pump, with valve attachment, to prevent a regurgitant current; $l, l^{\prime}, l^{\prime \prime}$, are levers resting on a gum tube, at intervals of $20 \mathrm{~cm}$. of tubing; $C$, drum upon which tracing is made; $H$, clockwork to revolve drum.]

plained on physical principles and may be illustrated by means of an artificial model [Fig. 63].

If two or more levers be placed on the arterial tubes of an artificial model, Fig. 63, one near to the pump, and the others equal distances apart, with a considerable length of tubing between the proximal and distal levers, and the levers be made to write, on a vertical line, on a recording surface, 
so that their curves can be more easily compared, the following facts may be ubserved when the pump is set to work regularly.

At each stroke of the pump, each lever rises until it reaches a maximum (Fig. 64, 1a, 2a, etc.) and then falls again, thus describing a curve.

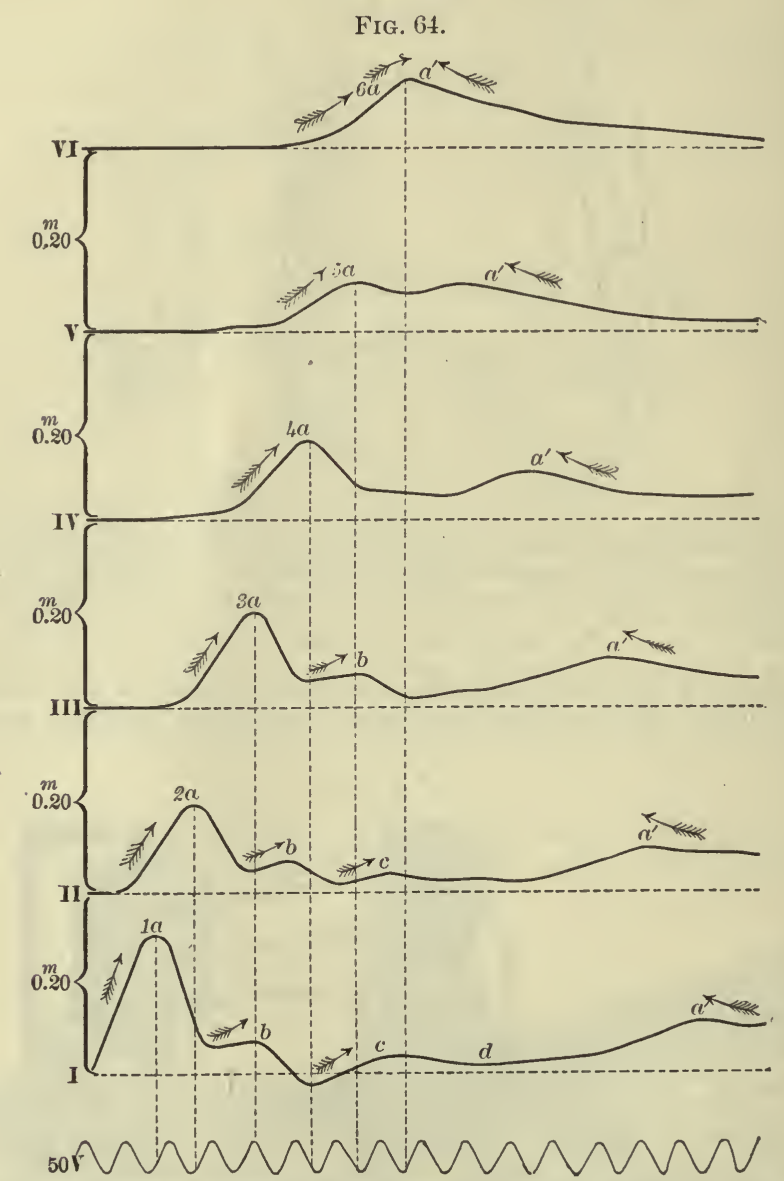

Pulse-curve described by a series of Sphygmographic Levers. placed at intervals of $20 \mathrm{~cm}$. from each other along an elastic tube into which fluid is forced by the sudden stroke of a pump. The pulse-wave is travelling from left to right, as indicated by the arrows over the primary $(a)$ and secondary $(b, c)$ pulse-waves. The dotted vertical lines drawn from the summit of the sereral primary waves to the tuning-fork curve below, each complete vibration of which occupies $1-50$ second, allow the time to be measured which is taken up by the wave in passing along $20 \mathrm{~cm}$. of the tubing. The waves $a^{\prime}$ are waves reflected from the closed distal end of the tubing; this is indicated by the direction of the arrows. It will be observed that in the more distant lever VI, the reflected wave, having but a slight distance to travel, becomes fused with the primary wave. (From Marey.)

The rise is due to the expansion of the part of the tube under the lever, and the fall is due to that part of the tube running after the expansion to its previous calibre. The curve is, therefore, the curve of the expansion (and return) of the tube at the point at which the lever rests. We may call it the pulse-curve. It is obvious that the expansion passes by the lever 
in the form of a wave. At one moment the lever is at rest; the tube beneath it is simply distended to the normal amount indicative of the mean pressure which at the time obtains in the arterial tubes of the model; at the next moment the pulse expansion reaches the lever, and the lever begins to rise; it continues to rise until the top of the wave reaches it, after which it falls again until finally it comes to rest, the wave having completely passed by.

It may perhaps be as well at once to warn the reader that the figure which we call the pulse-curve is not a representation of the pulse-wave itself; it is simply a representation of the movements, up and down, of the piece of the wall of the tubing at the spot on which the lever rests during the time that the wave is passing over that spot. We may roughly represent the wave in the diagram Fig. 65 in which the wave shown by the dotted line is passing over the tube (shown in a condition of rest by the thick double line) in the direction from $H$ to $C$. It must, however, be remembered

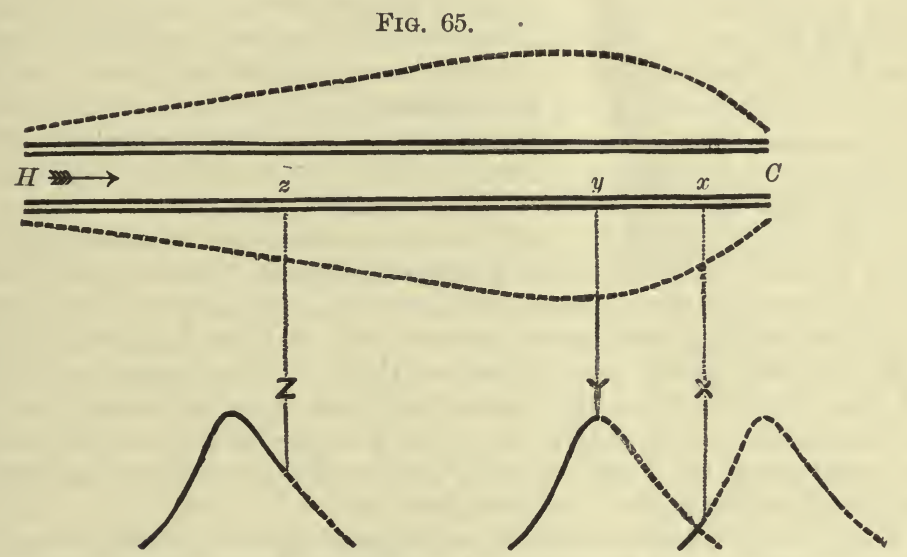

A rough Diagrammatic Representation of a Pulse-wave passing over an Artery.

that the wave thus figured is a much shorter wave than is the pulse-wave in reality (that being, as we shall see, about 6 metres long), $i$. $e$., occupies a smaller length of the arterial system from the heart $H$ toward the capillaries $C$.

The curves below, $X, Y, Z$, represent, in a similarly diagrammatic fashion, the curves described, during the passage of the wave, by levers placed on the points $x, y, z$. At $Z$ the greater part of the wave has already passed under the lever, which during its passage has already described the greater part of its curve, shown by the thick line, and has only now to describe the small part, shown by the dotted line, corresponding to the remainder of the wave from $Z$ to $H$. At $Y$ the lever is at the summit of the wave. At $X$ the lever has only described a small part of the beginning of the wave, viz., from $C$ to $x$, the rest of the curve, as shown by the dotted line, having yet to be described.

But to return to the consideration of Fig. 64 .

$\$ 130$. The rise of each lever is somewhat sudden, but the fall is more gradual, and is generally marked with some irregularities which we shall study presently. The rise is sudden because the sharp stroke of the pump suddenly drives a quantity of fluid into the tubing and so suddenly expands the tube; the fall is more gradual because the elastic reaction of the walls of the tube, which brings about the return of the tube to its former calibre 
after the expanding power of the pump has ceased, is more gradual in its action.

These features, the suddenness of the rise or up-stroke, and the more gradual slope of the fall or down-stroke, are seen also in natural pulse-curves
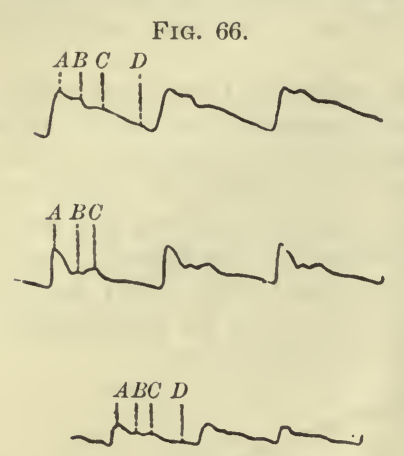

Pulse-tracings from the same Radial Artery under Different Pressures of the Lever. (The letters are explained in a later part of the text.)

taken from living arteries (Figs. 62, 66, etc.). Indeed, the difference between the up-stroke and the down-stroke is even more marked in the latter than in the former, the delivery of blood from the ventricle being more rapid than the issue of water from a pump as ordinarily worked.

It may here be noted that the actual size of the curve, that is, the amount of excursion of the lever, depends in part (as does also to a great extent the form of the curve) on the amount of pressure exerted by the lever on the tube. If the lever only just touches the tube in its expanded state, the rise will be insignificant. If, on the other hand, the lever be pressed down too firmly, the tube beneath will not be able to expand as it otherwise would, and the rise of the lever will be proportionately diminished. There is a certain pressure which must be exerted by the lever on the tube, the exact amount depending on the expansive power of the tubing and on the pressure exerted by the fluid in the tube, in order that the tracing may be best marked. This is shown in Fig. 66, in which are given three tracings taken from the same radial artery with the same instrument; in the lower curve the pressure of the lever is too great, in the upper curve too small, to bring out the characters seen most distinctly in the middle curve with a medium pressure.

$\$ 131$. It will be observed that in Fig. 64 , curve I., which is nearer the pump, rises higher, and rises more rapidly than curve II., which is further away from the pump; that is to say, at the lever further away from the pump the expansion is less and takes place more slowly than at the lever nearer the pump. Similarly in curve IV. the rise is still less, and takes place still less rapidly than in II., and the same change is seen still more marked in V. as compared with IV. In fact, if a number of levers were placed at equal distances along the arterial tubing of the model and the model were working properly, with an adequate peripheral resistance, we might trace out step by step how the expansion, as it travelled along the tube, got less and less in amount and at the same time became more gradual in its development, the curre becoming lower and more flattened out, until in the neighborhood of the artificial capillaries there was hardly any trace of it left. In other words, we might trace out step by step the gradual disappearance of the pulse.

The same changes, the same gradual lowering and flattening of the curve, may be seen in natural pulse-tracings, as for instance in Fig. 67, which is a tracing from the dorsalis pedis artery, compared with the tracing from the ra-

FIG. 67.

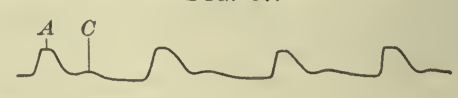

Pulse-tracing from Dorsalis Pedis, taken from the same individual as Fig. 66 .

dial artery, Fig. 66, taken from the same individual with the same instrument on the same occasion. This feature is, of course, not obvious in all 
pulse-curves taken from different individuals with different instruments and under varied circumstances; but if a series of curves from different arteries were carefully taken under the same conditions it would be found that the aortic tracing is higher and more sudden than the carotid tracing, which, again, is higher and more sudden than the radial tracing-the tibial tracing being in turn still lower and more flattened. The pulse-curve dies out by becoming lower and lower and more and more flattened out.

And a little consideration will show us that this must be so. The systole of the ventricle drives a quantity of blood into the already full aorta. The sudden injection of this quantity of blood expands the portion of the aorta next to the heart, the part immediately adjacent to the semilunar valves beginning to expand first, and the expansion travelling thence on to the end of this portion. In the same way the expansion travels on from this portion through all the succeeding portions of the arterial system. For the total expansion required to make room for the new quantity of blood is not provided by that portion alone of the aorta into which the blood is actually received; it is supplied by the whole arterial system; the old quantity of blood which is replaced by the new in this first portion has to find room for itself in the rest of the arterial space. As the expansion travels onward, however, the increase of pressure which each portion transmits to the succeeding portion will be less than that which it received from the preceding portion. For the whole increase of pressure due to the systole of the ventricle has to be distributed over the whole of the arterial system; the general mean arterial pressure is, as we have seen, maintained by repeated systoles, and any one systole has to make its contribution to that mean pressure; the increase of pressure which starts from the ventricle must, therefore, leave behind at each stage of its progress a fraction of itself; that is to say, the expansion is continually growing less, as the pulse travels from the heart to the capillaries. Moreover, while the expansion of the aorta next to the heart is, so to speak, the direct effect of the systole of the ventricle, the expansion of the more distant artery is the effect of the systole transmitted by the help of the elastic reaction of the arterial tract between the heart and the distant artery; and since this elastic reaction is slower in development than the actual systole, the expansion of the more distant artery is slower than that of the aorta, the up-stroke of the pulse-curve is less sudden, and the whole pulse-curve is more flattened.

The object of the systole is to supply a contribution to the mean pressure, and the pulse is an oscillation above and below that mean pressure-an oscillation which diminishes from the heart on wards, being damped by the elastic walls of the arteries, and so, little by little, converted into mean pressure until in the capillaries the mean pressure alone remains-the oscillations having disappeared.

$\S 132$. If in the model the points of the two levers at different distances from the pump be placed exactly one under the other on the recording surface, it is obvious that, the levers being alike except for their position on the tube, any difference in time between the movements of the two levers will be shown by an interval between the beginnings of the curves they describe, the recording surface being made to travel sufficiently rapidly.

If the movemients of the two levers be thus compared, it will be seen that the far lever (Fig. 64, II.) commences later than the near one (Fig. 64, I.); the further apart the two levers are, the greater is the interval in time between their curves. Compare the series I. to VI. (Fig. 64). This means that the wave of expansion, the pulse-wave, takes some time to travel along the tube. In the same way it would be found that the rise of the near lever 
began some fraction of a second after the stroke of the pump, meaning that time is required for the transmission of the wave.

The velocity with which the pulse-wave travels depends chiefly on the amount of rigidity possessed by the tubing. The more extensible (with corresponding elastic reaction) the tube, the slower is the wave; the more rigid the tube becomes, the faster the wave travels; in a perfectly rigid tube, what in the elastic tube would be the pulse, becomes a mere shock travelling with very great rapidity. The width of the tube is of much less influence. though according to some observers the wave travels more slowly in the wider tubes.

The rate at which the normal pulse-wave travels in the human body has been variously estimated at from 10 to 5 metres per second. In all probability the lower estimate is the more correct one; but it must be remembered that the rate may vary very considerably under different conditions. According to all observers the velocity of the wave in passing from the groin to the foot is greater than in passing from the axilla to the wrist (6 metres against 5 metres). This is probably due to the fact that the femoral artery with its branches is more rigid than the axillary and its branches. So also in the arteries of children, the wave travels more slowly than in the more rigid arteries of the adult. The velocity is also increased by circumstances which heighten and decreased by those which lessen the mean arterial pressure, since with increasing pressure the arterial walls become more and with diminishing pressure less rigid. Probably, also, the velocity of the pulsewave depends on conditions of the arterial walls which we cannot adequately describe as mere differences in rigidity. In experimenting with artificial tubes it is found that different qualities of India-rubber give rise to very different results.

Care must be taken not to confound the progress of the pulse-wave$i$. $e$, of the expansion of the arterial wall-with the actual onward movement of the blood itself. The pulse-wave travels over the moving blood somewhat as a rapidly moving natural wave travels along a sluggishly flowing river. Thus while the velocity of the pulse-wave is 6 or possibly even 10 metres per second, that of the current of the blood is not more than half a metre per second even in the large arteries, and is still less in the smaller ones.

$\S 133$. Referring again to the caution given above not to regard the pulsecurve as a picture of the pulse-wave, we may now add that the pulse-wave is of very considerable length. If we know how long it takes for the pulsewave to pass over any point in the arteries and how fast it is travelling, we can easily calculate the length of the wave. In an ordinary pulse-curve the artery, owing to the slow return, is.seen not to regain the ealibre which it had before the expansion, until just as the next expansion begins-that is to say, the pulse-wave takes the whole time of a cardiac cycle, viz., $\frac{8}{10}$ ths second to pass by the lever. Taking the velocity of the pulse-wave as 6 metres per second the length of the wave will be $\frac{8}{10}$ ths of 6 metres-or nearly 5 metres. And even if we took a smaller estimate, by supposing that the real expansion and return of the artery at any point took much less time, say $\frac{4}{10}$ ths second, the length of the pulse-wave would still be more than 2 metres. But even in the tallest man the capillaries furthest from the heart, those in the tips of the toes, are not 2 metres distant from the heart. In other words, the length of the pulse-wave is much greater than the whole length of the arterial system, so that the beginning of each wave has become lost in the small arteries and capillaries some time before the end of it has finally passed away from the beginning of the aorta.

We must now return to the consideration of certain special features in 
the pulse, which from the indications they give or suggest. of the condition of the vascular system are often of great interest.

$\$ 134$. Dicrotism. In nearly all pulse-tracings, the curve of the expansion and recoil of the artery is broken by two, three, or several smaller elevations and depressions; secondary waves are imposed upon the fundamental or primary wave. In the sphygmographic tracing from the carotid, Fig. 68,

FIG. 68.

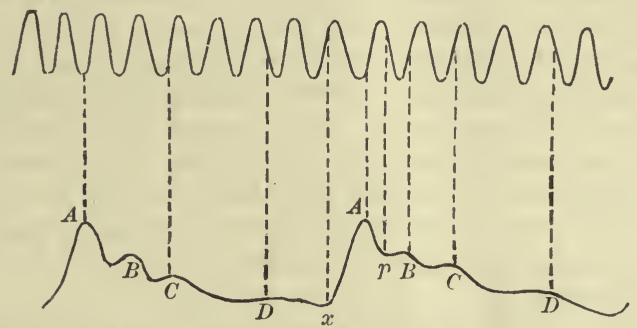

Pulse-tracing from Carotid Artery of Healthy Man (from Moens) : $x$, commencement of expansion of artery. $A$, summit of the first rise. $C$, dicrotic secondary wave. $B$, pre-dicrotic secondary wave; $p$, notch preceding this. $D$, succeding secondary wave. The curve above is that of a tuning-fork with ten double vibrations in a second.

and in many of the other tracings given, these secondary elevations are marked, as $\dot{B}, C, D$. When one such secondary elevation only is conspicuous, so that the pulse-curve presents two notable crests only, the primary crest and a secondary one, the pulse is said to be "dicrotic"; when two secondary crests are prominent, the pulse is often called "tricrotic"; where several, "polycrotic." As a general rule, the secondary elevations appear only ou the descending limb of the primary wave, as in most of the curves given, and the curve is then spoken of as "katacrotic." Sometimes, however, the first elevation or crest is not the highest, but appears on the ascending portion of the main curve; such a curve is spoken of as " anacrotic," Fig. 69 .

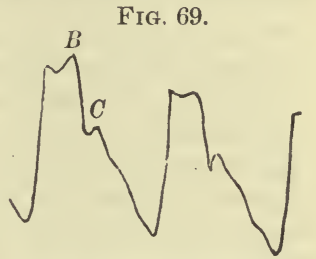

Anacrotic Sphygmographtracing from the Ascending Aorta. (Aneurism.)

Of these secondary elevations the most frequent, conspicuous, and important is the one which appears some way down on the descending limb, and is marked $C$ on Fig. 68 and on most of the curves here given. It is more or less distinctly visible on all sphygmograms, and may be seen in those of the aorta as well as of other arteries. Sometimes it is so slight as to be hardly discernible; at other times it may be so marked as to give rise to a really double pulse (Fig. 70), $i$. e., a pulse which can be felt as double by the

Fig. 70.

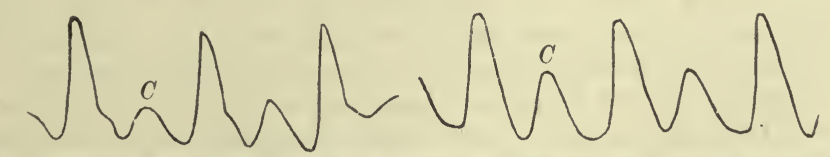

Two Grades of Marked Dicrotism in Radial Pulse of Man. (Typhoid fever.)

finger; hence it has been called the dicrotic elevation or the dicrotic wave, the notch preceding the elevation being spoken of as the "dicrotic notch." Neither it nor any other secondary elevations can be recognized in the tracings of blood-pressure taken with a manometer. This may be explained, 
as we have said ( $\$ 128)$ by the fact that the movements of the mercury column are too sluggish to reproduce these finer variations; but dicrotism is also conspicuous by its absence in the tracings given by more delicately responsive instruments. Moreover, when the normal pulse is felt by the finger, most persons find themselves unable to detect any dicrotism. But that it does really exist in the normal pulse is shown by the fact that it appears in a most unmistakable manner in the tracing obtained by allowing the blood to spurt directly from an opened small artery, such as the dorsalis pedis, upon a recording surface.

Less constant and conspicuous than the dicrotic wave, but yet appearing in most sphygmograms, is an elevation which appears higher up on the descending limb of the main wave; it is marked $B$ in Fig. 68 , and on several of the other curves, and is frequently called the pre-dicrotic wave; it may become very prominent. Sometimes other secondary waves, often called "post-dicrotic," are seen following the dicrotic wave, as at $D$ in Fig. 68, and some other curves; but these are not often present, and usually, even when present, inconspicuous.

When tracings are taken from several arteries, or from the same artery under different conditions of the body, these secondary waves are found to vary very considerably, giving rise to many characteristic forms of pulsecurve. Were we able with certainty to trace back the several features of the curves to their respective causes, an adequate examination of sphygmographic tracings would undoubtedly disclose much valuable information concerning the condition of the body presenting them. Unfortunately, the problem of the origin of these secondary waves is a most difficult and complex one ; so much so, that the detailed interpretation of a sphygmographic tracing is still in most cases extremely uncertain.

$\$ 135$. The chief interest attaches to the nature and meaning of the dicrotic wave. In general, the main conditions favoring dicrotism are (1) a highly extensible and elastic arterial wall, (2) a comparatively low mean pressure, leaving the extensible and elastic reaction of the arterial wall free scope to act, and (3) a sufficiently vigorous and sufficiently rapid stroke of the ventricle, and the discharge of a large quantity of blood into the aorta. The development of the dicrotic wave may probably be explained as follows:

At each beat the time during which the contents of the left ventricle are injected into the aorta is, as we have seen ( $\$ 125)$, very brief. The expansion of the aorta is very sudden, and the cessation of that expansion is also very sudden.

Now, when fluid is being driven with even a steady pressure through an elastic tube or a system of elastic tubes, levers placed on the tube will describe curves indicating variations in the diameter of the tube, if the inflow into the tube be suddenly stopped, as by sharply turning a stop-cock; and a comparison of levers placed at different distances from the stop-cock will show that these variations of diameter travel down the tube from the stopcock in the form of waves. The lever near the stop-cock will first of all fall, but speedily begin to rise again, and this subsequent rise will be followed by another fall, after which there may be one or more succeeding rises and fallsthat is, oscillations-with decreasing amplitudes, until the fluid comes to rest. The levers further from the stop-cock will describe curves similar to the above in form but of less amplitude, and it will be found that these occur somewhat later in time, the more so the further the lever is from the stopcock. Obviously these waves are generated at or near the stop-cock, and travel thence along the tubing.

We may infer that at each beat of the heart similar waves would be generated at the foot of the aorta upon the sudden cessation of the flow from 
the ventricle, and would travel thence along the elastic arteries. The facts that each beat is rapidly succeeded by another, and that the flow which suddenly ceases is also, by the nature of the ventricular stroke, suddenly generated, may render the waves more complicated, but will not change their essential nature.

The exact interpretation of the generation of these waves is perhaps not without difficulty, but two factors seem of especial importance. In the first place, as we have already more than once said, when a rapid flow is suddenly stopped a negative pressure makes its appearance behind the column of fluid. In a rigid tube this simply tends to a reflux of fluid. In an elastic tube its effects are complicated by the second factor, the elastic action and inertia of the walls of the tube. Upon the sudden cessation of the flow, the expansion of the tube, or as we may at once say, of the aorta, ceases, the vessel begins to shrink, and the lever placed on its walls falls, as from $A$ onward in the pulsecurve. This shrinking is in part due to the elastic reaction of the walls of the aorta, but is increased by the "suction" action of the negative pressure spoken of above. In thus shrinking, however, under these combined causes, the aorta, through the inertia of its walls, overshoots the mark, it is carried beyond its natural calibre-i.e., the diameter it would possess if left to itself with the pressure inside and outside equal ; it shrinks too much and consequently begins again to expand. This secondary expansion (taking for simplicity sake a pulse-curve in which the so-called pre-dicrotic wave, $B$, is absent or inconspicuous) causes the secondary rise of the lever up to $C$ - that is, the dicrotic rise. In thus expanding again the aorta tends to draw back toward the heart the column of blood which by loss of momentum had come to rest, or, indeed, under the influence of the negative pressure spoken of above, was already undergoing a reflux. In this secondary expansion, moreover, the aorta is by the inertia of its walls, aided by that of the blood, again carried, so to speak, beyond its mark, so that no sooner has it become expanded and filled with fluid to a certain extent than it again begins to shrink as from $C$ onward. And this shrinking may in a similar manner to the first be followed by a further expansion and shrinking, giving rise to a postdicrotic wave, or it may be to post-dicrotic waves. And the successive changes thus inaugurated at the root of the aorta travel as so many waves along the arterial system, diminishing as they go. It will be observed that for the development of these waves a certain quality in the walls of the tubing is necessary. The tube must be such as possesses when at rest an open lumen; the walls must be of such a kind that the tube remains open when empty$i$. e., when the atmospheric pressure is equal inside and outside-so that when it shrinks too much it expands again in striving to retain its natural calibre.

This we have seen to be a characteristic of the arteries. A collapsible tube of thin membrane will not show the phenomena; such a tube when the stop-cock is turned collapses and empties itself, continuing to be collapsed without any effort to expand again.

In the above explanation no mention has been made of the closing of the semilunar valves; we shall have to speak of these a little later on in referring to the pre-dicrotic wave, and shall see that, under the view we have just given, the closing of the semilunar valves is to be regarded rather as the effect than the cause of the dicrotic wave. Many authors, however, give an interpretation of the dicrotic wave different from that detailed above. Thus, it is held that the primary shrinking from $A$ onward, being brought to bear on the column of blood already come to rest, in face of the great pressure in front, drives the blood back against the semilunar valves, thus closing them, and that the impact of the column of blood against the valves starts a new wave of expansion, which reinforcing the natural tendency of 
the elastic walls to expand again after their primary shrinking, produces tho dicrotic wave $C$. On this view, it is the blood driven back from the valves which expands the artery; on the view given above, it is the expanding artery which draws the blood back toward the valves.

Moreover, quite other views have been or are held concerning this dicrotic wave. According to many authors, it is what is called a "reflected" wave. Thus, when the tube of the artificial model bearing two levers is blocked just beyond the far lever, the primary wave is seen to be accompanied by a second wave, which at the far lever is seen close to, and often fused into, the primary wave (Fig. 64, VI. $\alpha^{\prime}$ ), but at the near lever is at some distance from it (Fig. 64, I. $a^{\prime}$ ), being the further from it the longer the interval between the lever and the block in the tube. The second wave is evidently the primary wave reflected at the block and travelling backward toward the pump. It thus, of course, passes the far lever before the near one. And it has been argued that the dicrotic wave of the pulse is really such a reflected wave, started either at the minute arteries and capillaries, or at the points of bifurcation of the larger arteries, and travelling backward to the aorta. But if this were the case, the distance between the primary crest and the dicrotic crest ought to be less in arteries more distant from than in those nearer to the heart, just as in the artificial scheme the reflected wave is fused with a primary wave near the block (Fig. 64 , VI. $6 a \cdot a^{\prime}$ ), but becomes more and more separated from it the further back toward the pump we trace it (Fig. 64, I. $1 a . a^{\prime}$ ). Now this is not the case with the dicrotic wave. Careful measurements show that the distance between the primary and dicrotic crests is either greater, or certainly not less, in the smaller or more distant arteries than in the larger or nearer ones. This feature indeed proves that the dicrotic wave cannot be due to reflection at the periphery, or, indeed, in any way a retrograde wave. Besides, the multitudinous peripheral division would render one large peripherally reflected wave impossible. Again, the more rapidly the primary wave.is obliterated, or at least diminished, on its way to the periphery, the less conspicuous should be the dicrotic wave. Hence increased extensibility and increased elastic reaction of the arterial walls which tend to use up rapidly the primary wave, should also lessen the dicrotic wave. But as a matter of fact these conditions, as we have said, are favorable to the prominence of the dicrotic wave.

On the other hand, these and the other conditions which favor dicrotism in the pulse are exactly those which would favor such a development of secondary waves as has been described above, and their absence would be unfavorable to the occurrence of such waves. Thus dicrotism is less marked in rigid arteries (such as those of old people) than in healthy elastic ones; the rigid wall neither expands so readily nor shrinks so readily, and hence does not so readily give rise to such secondary waves. Again, dicrotism is more marked when the mean arterial pressure is low than when it is high; indeed, dicrotism may be induced when absent, or increased when slightly marked by diminishing, in one way or another, the mean pressure. Now, when the pressure is high, the arteries are kept continually much expanded, and are therefore the less capable of further expansion; that is to say, are, so far, more rigid. Hence the additional expansion due to the systole is not very great; there is a less tendency for the arterial walls to swing backward and forward, so to speak, and hence a less tendency to the development of secondary waves. When the mean pressure is low, the opposite state of things exists ; supposing, of course, that the ventricular stroke is adequately vigorous (the low pressure being due, not to diminished cardiac force, but to diminished peripheral resistance), the relatively empty but highly distensible 
artery is rapidly expanded, and, falling rapidly back, enters upon a secondary (dicrotic) expansion, and even a third.

Moreover, the same principles may be applied to explain why sometimes dicrotism will appear marked in a particular artery while it remains little marked in the rest of the system. In experimenting with an artificial tubing such as the arterial model, the physical characters of which remain the same throughout, both the primary and the secondary waves retain the same characters as they travel along the tubing, save only that both gradually diminish toward the periphery; and in the natural circulation, when the vascular conditions are fairly uniform throughout, the pulse-curve, as a rule, possesses the same general characters throughout, save that it is gradually "damped off." But suppose we were to substitute for the first section of the tubing a piece of perfectly rigid tubing; this at the stroke of the pump, on account of its being rigid, would show neither primary nor secondary expansion, but the expanding force of the pump's stroke would be transmitted through it to the second elastic section, and here the primary and secondary waves would at once become evident. This is an extreme case, but the same thing would be seen to a less degree in passing from a more rigid, that is, less extensible and elastic section, to a less rigid, more extensible and elastic section ; the primary and secondary expansions, in spite of the general damping effect, would suddenly increase. Similarly in the living body a pulsecurve which, so long as it is travelling along arteries in which the mean pressure is high, and which are therefore practically somewhat rigid, is not markedly dicrotic, may become very markedly dicrotic when it comes to a particular artery in which the mean pressure is low (and we shall see presently that such a case may occur), and the walls of which are therefore for the time being relatively more distensible than the rest.

Lastly, we may recall the observation made above $(\$ 130)$, that the curve of expansion of an elastic tube is modified by the pressure exerted by the lever employed to record it, and that hence, in the same artery and with the same instrument, the size, form, and even the special features of the curve vary according to the amount of pressure with which the lever is pressed upon the artery. Accordingly the amount of dicrotism apparent in a pulse may be modified by the pressure exerted by the lever. In Fig. 64, for instance, the dicrotic wave is more evident in the middle than in the upper tracing.

$\S 136$. The pre-dicrotic wave (marked $B$ on Fig. 68, and on several other of the pulse-curves), which precedes the dicrotic wave and is still more variable than that wave, being sometimes slight or even invisible and sometimes conspicuous, has given rise to much controversy. In the interpretation of the dicrotic wave given in the preceding paragraph it was stated that the negative pressure developed on the cessation of the flow in the rear of the column of blood, led by itself to a reflux toward the ventricle; and it has been suggested that this reflux meeting and closing the semilunar valves starts a small wave of expansion before the larger dicrotic wave has had time to develop itself. On this view the semilunar valves would be actually closed before the occurrence of the secondary dicrotic expansion of the arterial walls, though the larger, more powerful reflux of this later event must render the closure more complete, and in doing so possibly gives rise to the second sound. According, however, to the second view given in the same paragraph, which regards the reflux due to the shrinking of the artery in face of the great pressure in front as firmly closing the semilunar valves, and as thus starting the secondary dicrotic wave of expansion, the firm closing of the semilunar valves must take place before the beginning, not during the development of the dicrotic wave; it 
is still possible, however, even on this view, as on the other, to suppose that an antecedent reflux, due to the negative pressure succeeding the cessation of flow from the ventricle, closes the valves and starts the pre-dicrotic wave. But the matter is one not yet beyond the stage of controversy.

$\$ 137$. In an anacrotic pulse the first rise is not the highest, but a second rise ( $B$, Fig. 69) which follows and is separated from it by a notch is higher than, or at least as high as, itself. Such an anacrotic wave, though it may sometimes be produced temporarily in healthy persons, is generally associated with diseased conditions, usually such in which the arteries are abnormally rigid. In describing the ventricular systole, we spoke of the pressure within the ventricle as reaching its maximum just before the opening of the semilunar valves; and this is apparently the normal event; but there are curves which seem to show that after the first sudden rise of pressure which opens the valves, followed by a brief lessening of pressure, which appears on the curve as a notch, the pressure may again rise, and that to a point higher than before. And a similar curve is sometimes described by the front-to-back diameter of the ventricle. The systole opens the valve as it were with a burst; this is followed by a slight relapse, and then the systole, strengthening again, discharges the whole of the ventricular contents into the aorta and so brings about a tardy maximum expansion. And what is thus started in the aorta travels onward over the arterial system. It is difficult to see how these anacrotic events can be produced, except by a certain irregularity in the ventricular systole; and, indeed, the anacrotic pulse is frequently associated with some disease or defect of the ventricle.

$\$ 138$. Venous pulse. Under certain circumstances the pulse may be carried on from the arteries through the capillaries into the veins. Thus, as we shall see later on, when the salivary gland is actively secreting, the blood may issue from the gland through the veins in a rapid pulsating stream. The nervous events which give rise to the secretion of saliva, lead at the same time, by the agency of vasomotor nerves, of which we will presently speak, to a dilatation of the small arteries of the gland. When the gland is at rest the minute arteries are, as we shall see, somewhat constricted and narrowed, and thus contribute largely to the peripheral resistance in the part; this peripheral resistance throws into action the elastic properties of the small arteries leading to the gland, and the remnant of the pulse reaching these arteries is, as we before explained, finally destroyed. When the minute arteries are dilated, their widened channels allow the blood to flow more easily through them and with less friction; the peripheral resistance which they normally offer is thus lessened. In consequence of this the elasticity of the walls of the small arteries is brought into play to a less extent than before, and these small arteries cease to do their share in destroying the pulse which comes down to them from the larger arteries. As in the case of the artificial model, where the "peripheral" tubing is kept open, not enough elasticity is brought into play to convert the intermittent arterial flow into a continuous one, and the pulse which reaches the arteries of the gland passes on through them and through the capillaries, and is continued on into the veins. A similar venous pulse is also sometimes seen in other organs.

Careful tracings of the great veins in the neighborhood of the heart show elevations and depressions, which appear due to the variations of intracardiac (auricular) pressure, and which may, perhaps, be spoken of as constituting a "venous pulse," though they have a quite different origin from the venous pulse just described in the salivary gland; but at present they need further elucidation. In cases, however, of insufficiency of the 
tricuspid valves, the systole of the ventricle makes itself distinctly felt in the great veins; and a distention travelling backward from the heart becomes very visible in the veins of the neck. This is sometimes spoken of as a venous pulse.

Variations of pressure in the great veins, due to the respiratory movements, are also sometimes spoken of as a venous pulse; the nature of these variations will be explained in treating of respiration.

\section{The Regulation and Adaptation of the Vascular Mechanism.}

\section{The Regulation of the Beat of the Heart.}

$\S 139$. So far the facts with which we have had to deal, with the exception of the heart's beat itself, have been simply physical facts. All the essential phenomena which we have studied may be reproduced on a dead model. Such an unvarying mechanical vascular system would, however, be useless to a living body whose actions were at all complicated. The prominent feature of a living mechanism is the power of adapting itself to changes in its internal and external circumstances.

The vascular mechanism in all animals in which it is present is capable of local and general modifications, adapting it to local and general changes of circumstance. These modifications fall into two great classes:

1. Changes in the heart's beat. These, being central, have, of course, a general effect; they influence or may influence the whole body.

2. Changes in the peripheral resistance, due to variations in the calibre of the minute arteries, brought about by the agency of their contractile muscular coats. These changes may be either local, affecting a particular vascular area only, or general, affecting all or nearly all the bloodvessels of the body.

These two classes of events are chiefly governed by the nervous system. It is by means of the nervous system that the heart's beat and the calibre of the minute arteries are brought into relation with each other, and with almost every part of the body. It is by means of the nervous system acting either on the heart or on the small arteries, or on both, that a change of circumstances affecting either the whole or a part of the body is met by compensating or regulative changes in the flow of blood. It is by means of the wervous system that an organ has a more full supply of blood when at work than when at rest, that the tide of blood through the skin rises and ebbs with the rise and fall of the temperature of the air, that the work of the heart is tempered to meet the strain of overfull arteries, and that the arterial gates open and shut as the force of the central pump waxes and wanes. The study of these changes becomes, therefore, to a large extent a study of nervous actions.

The circulation may also be modified by events not. belonging to either of the above two classes. Thus, in this or that peripheral area, changes in the capillary walls and the walls of the minute arteries and veins may lead to an increase of the tendency of the blood corpuscles to arlhere to the vascular walls, and so, quite apart from any change in the calibre of the bloodvessels, may lead to increase of the peripheral resistance. This is seen in an extreme case in inflammation, but may possibly intervene to a less extent in the ordinary condition of the circulation, and may also be under the influence of the nervous system. Further, any decided change in the quantity of blood actually in circulation must also influence the working of the vascular mechanism. But both these changes are unimportant compared with the other two kinds of changes. Hence, the two most important problems for us to study are, 1 , how the nervous system regulates the beat of the heart, 
and 2, how the nervous system regulates the calibre of the bloodvessels. IVe will first consider the former problem.

\section{The Development of the Normal Beat.}

$\$ 140$. The heart of a mammal, or of a warm-blooded animal, generally ceases to beat within a few minutes after being removed from the body in the ordinary way, the hearts of newly-born animals continuing, however, to beat for a longer time than those of adults. Hence, though by special precaution and by means of an artificial circulation of blood, an isolated mammalian heart may be preserved in a pulsating condition for a much longer time, our knowledge of the exact nature and of the causes of the cardiac beat is as yet very largely based on the study of the hearts of coldblooded animals, which will continue to beat for hours, or under favorable circumstances even for days, after they have been removed from the body with only ordinary care. We have reason to think that the mechanism by which the beat is carried on varies in some of its secondary features in different kinds of animals; that the heart, for instance, of the eel, the snake, the tortoise, and the frog, differ in some minor details of behavior, both from each other and from the bird and the mammal ; but we may, at first at all events, take the heart of the frog as illustrating the main and important truths concerning the causes and mechanism of the beat.

In studying elosely the phenomena of the beat of the heart it becomes necessary to obtain a graphic record of various movements.

1. In the frog or other cold-blooded animal, a light lever may be placed direetly on the ventricle (or on an auricle, etc.), and changes of form, due either to distention by the influx of blood or to the systole, will cause movements of the lever, which may be recorded on a travelling surface. The same methods, as we have seen, may be applied to the mammalian heart.

2. Or, as in Gaskell's method, the heart may be fixed by a clamp carefully adjusted around the auriculo-ventricular groove, while the apex of the ventricle and some portion of one auricle are attached by threads to horizontal levers placed respectively above and below the heart. The auricle and the ventricle each in its systole pulls at the lever attached to it, and the times and extent of the contractions may thus be recorded.

3. A record of endocardiac pressure may be taken in the frog or tortoise, as in the mammal, by means of an appropriate manometer. And in these animals, at all events, it is easy to keep up an artificial circulation. A canula is introduced into the sinus venosus, and another into the ventricle through the aorta. Serum or dilute blood (or any other fluid which it may be desired to employ) is driven by moderate pressure through the former; to the latter is attached a tube connected by means of a side piece with a small mereury manometer. So long as the exit-tube is open at the end fluid flows freely through the heart and apparatus. Upon closing the exit-tube at its far end the force of the ventricular systole is brought to bear on the manometer, the index of which registers in the usual way the movements of the mercury column. Newell Martin has sueceeded in applying a modification of this method to the mammalian heart.

4. The movements of the ventricle may be registered by introducing into it through the auriculo-ventricular orifice a so-called "perfusion" canula, Figs. 71 and $72, \mathrm{I}$., with a double tube, one inside the other, and tying the rentricle on to the canula at the auriculo-ventricular groove, or at any level below that which may be desired. 'The blood or other fluid is driven at an adequate pressure through the tube $a$, enters the ventricle, and returns by the tube $b$. If $b$ be connected with a manometer as in method 3 , the movements of the ventricle may be registered.

5. In the apparatus of Roy, Fig. 72, II., the exit-tube is free, but the ventricle (the same method may be adopted for the whole heart) is placed in an air-tight chamber filled with oil, or partly with normal saline solution and partly with oil. By means of the tube $b$ the interior of the chamber $a$ is continuous with that of a small cylinder $c$ in which a piston $d$, secured by a thin flexible animal membrane 
works up and down. 'The piston again bears on a lever $e$ by means of which its movements may be registered. When the ventricle contracts, and by contracting diminishes in volume, there is a lessening of pressure in the interior of the chamber; this is transmitted to the cylinder, and the piston correspondingly rises, earrying with it the lever. As the ventricle subsequently becomes distended the pressure in the chamber is increased, and the piston and lever sink. In this way variations in the volume of the ventricle may be recorded, without any great interference with the flow of blood or fluid through it.

The heart of the frog, as we have just said, will continue to beat for hours after removal from the body, even after the cavities have been cleared of blood, and, indeed, when they are almost empty of all fluid. The beats thus carried out are in all important respects identical with the beats executed by the heart in its normal condition within the living body. Hence we may infer that the beat of the heart is an automatic action; the muscular

FIG. 71.

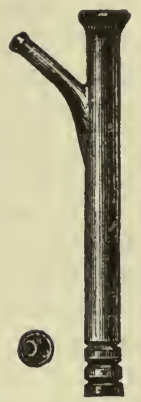

A Perfusion Canula. contractions which constitute the beat are due to causes which arise spontaneously in the heart itself.

In the frog's heart, as in that of the mammal, $\$ 115$, there is a distinct sequence of events which is the same whether the heart be removed from, or

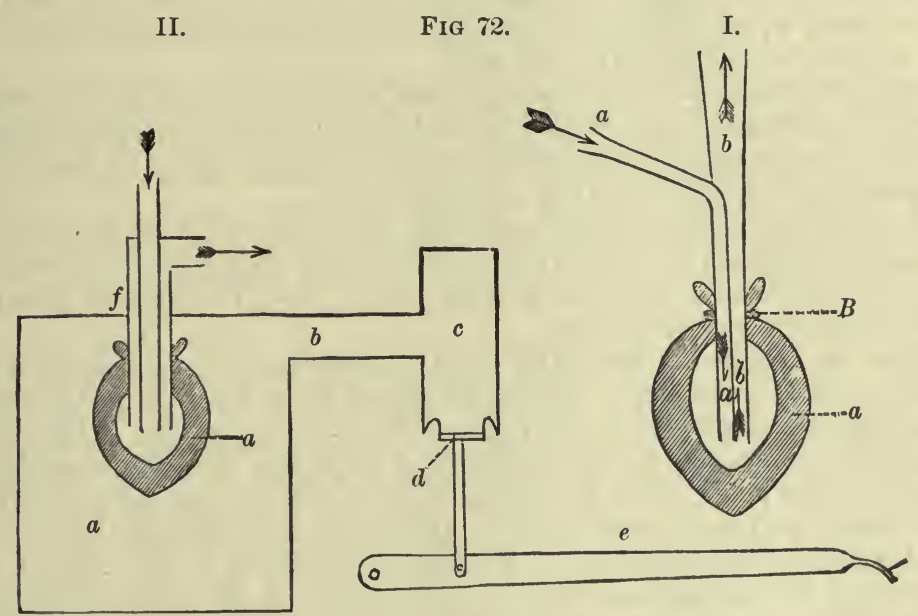

Purely Diagrammatic Figures of-I. Perfusion canula tied into frog's ventricle: $a$, entrance: $b$, exit-tube ; $A$, wall of ventricle; $B$, ligature.

II. Roy's apparatus modified by Gaskill, $a$, chamber filled with saline solution and oil, containing the ventricle $A$ tied on to perfusion canula $f ; b$, tube leading to cylinder $c$, in which moves piston $d$, working the lever $e$.

be still in its normal condition within, the body. First comes the beat of the sinus venosus, preceded by a more or less peristaltic contraction of the large veins leading into it, next follows the sharp beat of the two auricles together, then comes the longer beat of the ventricle, and lastly, the cycle is completed by the beat of the bulbus arteriosus, which does not, like the mammalian aorta, simply recoil by elastic reaction after distention by the ventricular stroke, but carries out a distinct muscular contraction passing in a wave from the ventricle outward.

When the heart in dying ceases to heat, the several movements cease, as a rule, in an order the inverse of the above. Omitting the bulbus arte- 
riosus, which sometimes exhibits great rhythmical power, we may say that first the ventricle fails, then the auricles fail, and lastly the sinus venosus fails.

The heart after it has ceased to beat spontaneously remains for some time irritable-that is, capable of executing a beat, or a short series of beats, when stimulated either mechanically, as by touching it with a blunt needle or electrically by an induction shock or in other ways. 'The artificial beat so called forth may be in its main features identical with the natural beat, all the divisions of the heart taking part in the beat, and the sequence of events being the same as in the natural beat. Thus when the sinus is pricked the beat of the sinus may be followed by a beat of the auricles and of the ventricle; and even when the ventricle is stimulated, the directly following beat of the ventricle may be succeeded by a complete beat of the whole heart.

Under certain circumstances, however, the division directly stimulated is the only one to beat; when the ventricle is pricked for instance it alone beats, or when the sinus is pricked it alone beats. The results of stimulation, moreover, may differ according to the condition of the heart and according to the particular spot to which the stimulus is applied.

With an increasing loss of irritability, the response to stimulation ceases in the several divisions in the same order as that of the failure of the natural beat-the ventricle ceases to respond first, then the auricles, and lastly the sinus venosus, which frequently responds to stimulation long after the other divisions have ceased to make any sign.

It would appear as if the sinus venosus, auricles, and ventricle formed a descending series in respect to their irritability and to the power they possess of carrying on spontaneous rhythmic beats, the sinus being the most potent. This is also seen in the following experiments:

In order that the frog's heart may beat after removal from the body with the nearest approach in rapidity, regularity, and endurance to the normal condition, the removal must be carried out so that the excised heart still retains the sinus venosus intact.

When the incision is carried through the auricles so as to leave the sinus venosus behind in the body, the sinus venosus beats forcibly and regularly, having suffered hardly any interruption from the operation. The excised heart, however, remains in the majority of cases for some time motionless. Stimulated by a prick or an induction-shock, it will perhaps give one, two, or several beats, and then come to rest. In the majority of cases, however, the animal having previously been in a vigorous condition, it will after a while recommence its spontaneous beating, the systole of the ventricle following that of the auricles; but the rhythm of beat will not be the same as that of the sinus venosus left in the body, but will be slower, and the beats will not continue to go on for so long a time as will those of a heart still retaining the sinus venosus.

When the incision is carried through the auriculo-ventricular groove, so as to leave the auricles and sinus venosus within the body, and to isolate the ventricle only, the results are similar but more marked. The sinus and auricles beat regularly and vigorously, with their proper sequence, but the ventricle, after a few rapid contractions due to the incision acting as a stimulus, generally remains for a long time quiescent. When stimulated, however, the ventricle will give one, two, or several beats, and after a while, in many cases at least, will eventually set up a spontaneous pulsation with an independent rhythm; and this may last for some considerable time, but the beats are not so regular and will not go on for so long a time as will those of a ventricle to which the auricles are still attached. 
If a transverse incision be carried through the ventricle at about its upper third, leaving the base of the ventricle still attached to the auricles, the portion of the heart left in the body will go on pulsating regularly, with the ordinary sequence of sinus, auricles, ventricle, but the isolated lower twothirds of the ventricle will not beat spontaneously at all, however long it be left. Moreover, in response to a single stimulus such as an induction-shock or a gentle prick it gives, not, as in the case of the entire ventricle when stimulated at the base or of the ventricle to which the auricles are attached, a series of beats, but a single beat.

Lastly, to complete the story, we may add that when the heart is bisected longitudinally, each half continues to beat spontaneously, with an independent rhythm, so that the beats of the.two halves are not necessarily synchronous, and this continuance of spontaneous pulsations after longitudinal bisection may be seen in the conjoined auricle and ventricle, or in the isolated auricles, or in the isolated but entire ventricle. Moreover, the auricles may be divided in many ways and yet many of the segments will continue beating; small pieces even may be seen under the microscope pulsating, feebly, it is true, but distinctly and rhythmically.

In these experiments, then, the various parts of the frog's heart also form, as regards the power of spontaneous pulsation, a descending series: sinus venosus, auricles, entire ventricle, lower portions of ventricle, the last exhibiting under ordinary circumstances no spontaneous pulsations at all.

$\S 141$. Now we have seen $(\S 139)$ that these parts form to a certain extent a similar descending series as regards the presence of ganglia; at least so far that the ganglia are very numerous in the sinus venosus, that they occur in the auricles, and that while Bidder's ganglia are present at the junction of the ventricle with the auricles, ganglia are wholly absent from the rest of the ventricle. Hence, on the assumption (which we have already, $\S 96$, seen reason to doubt) that the nerve cells of ganglia are similar in general functions to the nerve cells of the central nervous system, the view very naturally presents itself that the rhythmic spontaneous beat of the heart of the frog is due to the spontaneous generation in the ganglionic nerve cells of rhythmic motor impulses, which, passing down to the muscular. fibres of the several parts, cause rhythmic contractions of these fibres, the sequence and coördination of the beating of the several divisions of the heart being the result of a coördination between the several ganglia in regard to the generation of impulses. Under this view the cardiac muscular fibre simply responds to the motor impulses reaching it along its motor nerve fibre in the same way as the skeletal muscular fibre responds to the motor impulses reaching it along its motor nerve fibre; in both cases the muscular fibre is, as it were, a passive instrument in the hands of the motor nerve, or rather of the nervous centre (ganglion or spinal cord) from which the motor nerve proceeds. And the view, thus based on the fact of the frog's heart, has been extended to the hearts of (vertebrate) animals generally.

There are reasons, however, which show that this view is not tenable.

For instance, the lower two-thirds, or lower third, or even the mere tip of the frog's ventricle-that is to say, parts which are admitted not to contain nerve cells - may, by special means, be induced to carry on for a considerable time a rhythmic beat, which in its main features is identical with the spontaneous beat of the ventricle of the intact heart. If such a part of the frog's ventricle be tied on to the end of a perfusion canula (Fig. 71), the portion of the ventricular cavity belonging to the part may be adequately distended and at the same time be "fed" with a suitable fluid, such as blood, made to flow through the canula; it will then be found that the portion of ventricle so treated will, after a preliminary period of qui- 
escence, commence to beat, apparently spontaneously, and will continue so beating for a long period of time. It may be said that in this case the distention of the cavity and the supply of blood or other fluid acts as a stimulus; but if so the stimulus is a continuous one, or at least not a rhythmic one, and yet the beat is most regularly rhythmic.

Then, again, the reluctance of the ventricle to execute spontaneous rhythmic beats is to a certain extent peculiar to the frog. The ventricle of the tortoise, for instance, the greater part of the substance of which is as free from nerve cells as is that of the frog, will beat spontaneously when isolated from the auricles with great ease and for a long time. Further, a mere strip of this ventricular muscle tissue, if kept gently extended and continually moistened with blood or other suitable fluid, will continue to beat spontaneously with very great regularity for hours, or even days, especially if the series be started by the preliminary application of induction-shocks rhythmically repeated.

In connection with this question we may call attention to the fact that the cardiac muscular fibre is not wholly like the skeletal muscular fibre; in many respects the contraction or beat of the former is in its very nature different from the contraction of the latter ; the former cannot be considered, like the latter, a mere instrument in the hands of the motor nerve fibre. The features of the beat or contraction of cardiac muscle may be studied on the isolated and quiescent ventricle, or part of the ventricle, of the frog. When such a ventricle is stimulated by a single stimulus, such as a single induction-shock or a single touch with a blunt needle, a beat may or may not result. If it follows it resembles, in all its general features at least, a spontaneous beat. Between the application of the stimulus and the first appearance of any contraction is a very long latent period, varying according to circumstances, but in a vigorous fresh frog's ventricle being about 0.3 second. The beat itself lasts a variable but considerable time, rising slowly to a maximum and declining slowly again. Of course, when the beat is recorded by means of a light lever placed on the ventricle, what the tracing shows is really the increase in the front-to-back diameter of the ventricle during the beat-that is to say, one of the results of the contraction of the cardiac fibres-and gives, in an indirect manner only, the extent of the contraction of the fibres themselves; and the same is the case with the other methods of recording the movements of the whole ventricle. We may, however, study in a more direct way the contraction of a few fibres by taking a slip of the ventricle (and for this purpose the tortoise is preferable to the frog) and suspending it to a lever after the fashion of a musclenerve preparation. We then get a curve of contraction, characterized by a long latent period, a slow long-continued rise, and a slow long-continued fall-a contraction, in fact, more like that of a plain muscular fibre than of a skeletal muscular fibre. In the tortoise the contraction is particularly long, the contraction of even the skeletal muscles being long in that animal; it is less long, but still long, in the frog; shorter still, but yet long as compared with the skeletal muscles, in the mammal.

The beat of the ventricle, then, is a single but relatively slow, prolonged contraction wave sweeping over the peculiar cardiac muscle-cell, passing through the cement substance from cell to cell along the fibre, from fibre to fibre along the bundle, and from bundle to bundle over the labyrinth of the ventricular walls.

Like the case of the skeletal muscle, this single contraction is accompanied by an electric change, a current of action. The intact ventricle at rest is, as we have already said $(\$ 66)$, isoelectric, but each part just as it is about to enter into a state of contraction becomes negative toward the rest. 
Hence, when the electrodes of a galvanometer are placed on two points, $A, B$, of the surface of the ventricle, a diphasic variation of the galvanometer needle is seen just as a beat, natural or excited, is about to occur. Supposing that the wave of contraction reaches $A$ first, this will become negative toward the rest of the ventricle, including $B$, but when the wave some time afterward reaches $B, B$ will become negative toward the rest of the ventricle, including $A$. Compare $\$ 67$.

The beat of the auricles, that of the sinus venosus, and that of the bulbus arteriosus are similar in their main features to that of the ventricle, so that the whole beat may be considered to be a wave of contraction sweeping through the heart from sinus to bulbus; but the arrangement of fibres is such that this beat is cut up into sections in such a way that the sinus, the auricles, the ventricle, and the bulbus have each a beat, so to speak, to themselves. In a normal state of things these several parts of the whole beat follow each other in the sequence we have described, but under abnormal conditions the sequence may be reversed, or one section may beat while the others are at rest, or the several sections may beat out of time with each other.

So far the description of the contraction which is the foundation of the beat differs from that of a skeletal muscle in degree only; but now comes an important difference. When we stimulate a skeletal muscle with a strong stimulus we get a large contraction; when we apply a weak stimulus we get a small contraction; within certaiu limits (see $\$ 77$ ) the contraction is proportional to the stimulus. This is not the ease with the quiescent ventricle or heart. When we apply a strong induction-shock we get a beat of a certain strength; if we now apply a weak shock, we get either no beat at all or quite as strong a beat as with the stronger stimulus. That is to say, the magnitude of the beat depends on the condition of the ventricle (or heart), and not on the magnitude of the stimulus. If the stimulus can stir the ventricle up to beat at all, the beat is the best which the ventricle at the time can accomplish; the stimulus either produces its maximum effect or none at all. It would seem as if the stimulus does not produce a contraction in the same way that it does when it is brought to bear on a skeletal muscle, but rather stirs up the heart in such a way as to enable it to execute a spontaneous beat, which, without the extra stimulus, it could not bring about. And this is further illustrated by the fact that when a ventricle is beating rhythmically, either spontaneously or as the result of rhythmic stimulation, the kind of effect produced by a new stimulus thrown in will depend upon the exact phase of the cycle of the beat at which it is thrown in. If it is thrown in just as a relaxation is taking place, a beat follows prematurely, before the next beat would naturally follow, this premature beat being obviously produced by the stimulus. But if it he thrown in just as a contraction is beginning, no premature beat follows; the ventricle does not seem to feel the stimulus at all. There is a period during which the ventricle is insensible to stimuli, and that however strong; this period is called the "refractory" period. (There is, it may be mentioned, a similar refractory period in skeletal muscle, but it is of exceedingly short duration.) From this it results that, when a succession of stimuli repeated at a certain rate are sent into the ventricle, the number of beats does not correspond to the number of stimuli; some of the stimuli falling in refractory periods are ineffective and produce no beat. Hence, also, it is difficult if not impossible to produce a real tetanus of the ventricle, to fuse a number of beats into one. And there are other facts tending to show that the contraction of a cardiac muscular fibre, even when induced by artificial stimulation, is of a peculiar nature, and that the analogy with the contraction of a skeletal muscular fibre, 
induced by motor impulses reaching it along its nerve, does not hold good.

These and other considerations, taken together with the facts already mentioned, that portions of cardiac muscular tissue in which ganglionic cells are certainly not present, can in various animals be induced, either easily or with difficulty, to execute rhythmic beats which have all the appearance of being spontaneous in nature, lead us to conclude that the beat of the heart is not the result of rhythmic impulses proceeding from the cells of the ganglia to passive muscular fibres, but is mainly the result of changes taking place in the muscular tissue itself. And here we may call attention to the peculiar histological features of cardiac muscular tissue; though so far differentiated as to be striated, its cellular constitution and its "protoplasmic" features, including the obscurity of the striation, show that the differentiation is incomplete. Now, one attribute of undifferentiated primordial protoplasm is the power of spontaneous movement.

$\$ 142$. We have, moreover, evidence that it is the muscular tissue, and not the arrangement of ganglia and nerves, which is primarily concerned in maintaining the remarkable sequence of sinus beat, auricle beat, and ventricle beat. This is perhaps better seen in the heart of the tortoise than in that of the frog.

In this animal the nerves passing from the sinus to the ventricle may be divided, or the several ganglia may be respectively removed, and yet the normal sequence is maintained. On the other hand, we find that interference with the muscular substance of the auricle, when carried to a certain extent, prevents the beat of the auricle passing over to the ventricle, so that the sequence is broken after the auricle beat. If, for instance, the auricle be cut through until only a narrow bridge of muscle be left connecting the part of the auricle adjoining the sinus with the part adjoining the auriculo-ventricular ring, or if this part be compressed with a clamp, a state of things may be brought about in which every second beat only, or every third beat only, of the sinus and auricle is followed by a beat of the ventricle; and then, if the bridge be still further narrowed or the clamp screwed tighter, the ventricle does not at all follow in its beat the sequence of sinus and auricle, though it may after a while set up an independent rhythm of its own. This experiment suggests, and other facts support, the view that the normal sequence is maintained as follows: The beat begins in the sinus (including the ends of the veins); the contraction wave, beginning at the ends of the veins, travels over the muscular tissue of the sinus, and reaching the auricle starts a contraction in that segment of the heart; similarly the contraction wave of the auricular beat reaching the ventricle starts a ventricular beat, which in turn in like fashion starts the beat of the bulbus. And in hearts in a certain condition it is possible by stimulation to reverse this sequence, or to produce, by alternate stimulation, an alternation of a normal and a reversed sequence; thus in the heart of the skate, in a certain condition, mechanical stimulation of the bulbus by indicating a heat of the bulbus will start a sequence of the bulbus, ventricle, auricle, and sinus, and similar stimulation of the sinus will produce a normal sequence of sinus, auricle, ventricle, and bulbus.

It would, perhaps, be premature to insist that the nervous elements do not intervene in any way in the maintenance of this sequence; but the evidence shows that they are not the main factors, and we have at present no satisfactory indications of the way in which they do or may intervene.

Two questions naturally suggest themselves here. The first is, Why does the cardiac cycle begin with the sinus beat? We have previously (\$ 141) given the evidence that the sinus has a greater potentiality of beating than 
the other parts; in and by itself it beats more readily and with a quicker rhythm than the other parts. When we ask the further question, Why has it this greater potentiality? the only answer we can at present give is that it is inborn in the substance of the sinus. The problem is somewhat of the same kind as why the heart of one animal beats so much quicker than that of another. All we can say at present is that the rate is the outcome of the molecular constitution of tissue, without being able to define that molecular constitution.

The second question is, Why does not the contraction wave starting at the sinus spread as a continuous wave over the whole heart? why is it broken up into sinus beat, auricle beat, ventricle beat? We may here call to mind the fact mentioned in $\$ 140$ of the existence, more or less marked in all hearts and well seen in the heart of the tortoise, of a muscular ring or collar between the sinus and the auricle, and of a similar ring between the auricle and ventricle. The muscular tissue in these rings seems to be of a somewhat different nature from the muscular tissue forming the body of the sinus, or of the auricle, or of the ventricle. If we suppose that this tissue has a low conducting power, it may offer sufficient resistance to the progress of the contraction to permit the sinus, for example, to carry out or to be far on in the development of its beat before the auricle begins its beat (and thus bisect, so to speak, the beat which would otherwise be common to the two), and yet not offer so much resistance as to prevent the contraction wave passing ultimately on from the sinus to the auricle. We may in the tortoise by careful clamping or section of the auricle in its middle, by which an obstacle to the contraction wave is introduced, bisect the single auricular beat into two beats, one of the part between the sinus and the obstacle and another between the obstacle and the ventricular. We may thus consider the breaking up the primitive unbroken peristaltic wave of contraction from sinus to bulbus to be due to the introduction of tissue of lower conducting power at the junctions of the several parts.

We do not say that this is the complete solution of the problem, but it at least offers an approximate solution; and here as elsewhere we have no satisfactory evidence of nervous elements being main factors in the matter.

In the above we have dealt chiefly with the heart of the cold-blooded animal, but as far as we know the same conclusions hold good for the mammalian heart also.

The question now arises, If the ganglia are not the prime cause of the heart's rhythmic beat, or of the maintenance of the normal sequence, what purposes do they serve? But before we even attempt to answer this question we must deal with the nervous mechanisms by which the beat of the heart, thus arising spontaneously within the tissues of the heart itself, is modified and regulated to meet the requirements of the rest of the body.

\section{The Government of the Heart-beat by the Nervous System.}

$\S 143$. It will be convenient to begin with the heart of the frog, which, as we have seen, is connected with the central nervous system through and therefore governed by the two vagi nerves, each of which though apparently a single nerve contains, as we shall see, fibres of different origin and nature.

If while the beats of the heart of a frog are being carefully registered an interrupted current of moderate strength be sent through one of the vagi, the heart is seen to stop beating. It remains for a time in diastole, perfectly 
motionless and flaccid; all the muscular fibres of the several chambers are for the time being in a state of relaxation. The heart has been inhibited by the impulses descending down the vagus from the part of the nerve stimulated.

If the duration of the stimulation be short and the strength of the current great, the standstill may continue after the current has been shut off; the beats when they reappear are generally at first feeble and infrequent, but soon reach or even go beyond their previous vigor and frequency. If the duration of the current be very long, the heart may recommence beating while the stimulation is still going on, but the beats are feeble and infrequent though gradually increasing in strength and frequency. The effect of the stimulation is at its maximum at or soon after the commencenent of the application of the stimulus, gradually declining afterward; but even at the end of a very prolonged stimulation the beats may still be less in force or in frequency, or in both, than they were before the nerve was stimulated, and on the removal of the current may show signs of recovery by an increase in force and frequency. The effect is not produced instantaneously ; if on the curve the point be exactly marked when the current is thrown in, as at on, Fig. 73, it will frequently be found that one beat at least occurs after the

FIG 73.

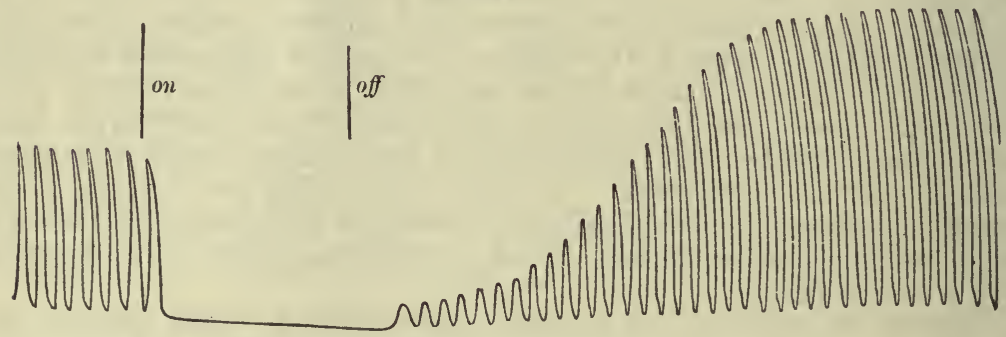

Inhibition of Frog's Heart by Stimulation of Vagus Nerve: on marks the time at which the interrupted current was thrown into the vagus, off when it was shut off. The time-marker below marks seconds. The beats were registered by suspending the ventricle from a clamp attached to the aorta and attaching a light lever to the tip of the ventricle.

current has passed into the nerve; the development of that beat has taken place before the impulses descending the vagus have had time to affect the heart.

The stimulus need not necessarily be the interrupted current ; mechanical, chemical, or thermal stimulation of the vagus will also produce inhibition; but in order to get a marked effect it is desirable to make use of not a single nervous impulse but a series of nervous impulses; thus it is difficult to obtain any recognizable result by employing a single induction-shock of moderate intensity only. As we shall see later on, "natural" nervous impulses descending the vagus from the central nervous system, and started there, by afferent impulses or otherwise, as parts of a reflex act, may produce inhibition.

The stimulus may be applied to any part of the course of the vagus from high up in the neck right down to the sinus ; indeed, very marked results are obtained by applying the electrodes directly to the sinus, where, as we have seen, the two nerves plunge into the substance of the heart. The stimulus may also be applied to either vagus, though in the frog and some other animals, one vagus is sometimes more powerful than the other. Thus, it not infrequently happens that even strong stimulation of the vagus on one 
sile produces no change of the rhythm, while even moderate stimulation of the nerve on the other side of the neck brings the heart to a standstill at once.

If during the inhibition the ventricle or other part of the heart be stimulated directly-for instance, mechanically by the prick of a needle-a beat may follow; that is to say, the impulses descending the vagus, while inhibiting the spontaneous beats, have not wholly abolished the actual irritability of the cardiac tissues.

With a current of even moderate intensity, such a current, for instance, as would produce a marked tetanus of a muscle-nerve preparation, the standstill is complete, that is to say, a certain number of beats are entirely dropped; but with a weak current the inhibition is partial only, the heart does not stand absolutely still but the beats are slowed, the intervals between them being prolonged, or weakened only without much slowing, or both slowed and weakened. Sometines the slowing and sometimes the weakening is the more conspicuous result.

It sometimes happens that, when in the frog the vagus is stimulated in the neck, the effect is very different from that just described; for the beats are increased in frequency, though they may be at first diminished in force. And, occasionally, the beats are increased both in force and in frequency; the result is augmentation, not inhibition. But this is due to the fact that in the frog the vagus along the greater part of its course is a mixed nerve and contains fibres other than those of the vagus proper.

$\S 144$. If we examine the vagus nerve closely, tracing it up to the brain, we find that just as the nerve has pierced the cranium, just where it passes through the ganglion ( $G$. V., Fig. 74), certain fibres pass into it from the sympathetic nerve of the neck, $S y$., of the further connections of which we shall speak presently.

This being the case, we may expect that we should get different results according as we stimulated (1) the vagus in the cranium before it was joined by the sympathetic, (2) the sympathetic fibres before they join the vagus, and (3) the vagus trunk containing the real vagus and the sympathetic fibres added. What we have previously described are the ordinary results of stimulating the mixed trunk, and these, as we have said, are not wholly constant, though usually and in the main most distinct inhibitory results follow.

If we stimulate the sympathetic in the neck, as at $S y$., Fig. 74, cutting the nerve below, so as to block all impulses from passing downward, and only allow impulses to pass up to the vagus and thence down the mixed vagus trunk to the heart, we get very remarkable results. The beat of the heart, instead of being inhibited, is augmented; the beats are increased either in frequency or in force, or most generally both in frequency and in force. The effect is, perhaps, best seen when the heart before stimulation is beating slowly and feebly; upon stimulation of the cervical sympathetic the beats at once improve in vigor and frequency; indeed, a heart which, for one reason or another, has almost ceased to beat may, by proper stimulation of the sympathetic, be called back into vigorous activity.

If, on the other hand, we stimulate the vagus before it has been joined by the sympathetic fibres (and to insure the result not being marred by any escape of the stimulating current on to the sympathetic fibres it is necessary to stimulate the vagus within the cranium), we get pure and constant inhibitory results - the beats are for a time wholly abolished, or are slowed, or are weakened, or are both slowed and weakened.

Obviously, then, the heart of the frog is supplied through the vagus by two sets of fibres coming from the central nervous system, the one by the 
vagus proper and the other by the cervical sympathetic nerve, and these two sets have opposite and antagonistic effects upon the heart. We find upon examination that we can make the following statements concerning them :

The one set, those belonging to the vagus proper, are inhibitory; they weaken the systole and prolong the diastole, the effect with a strong stimula-

Fig. 74.

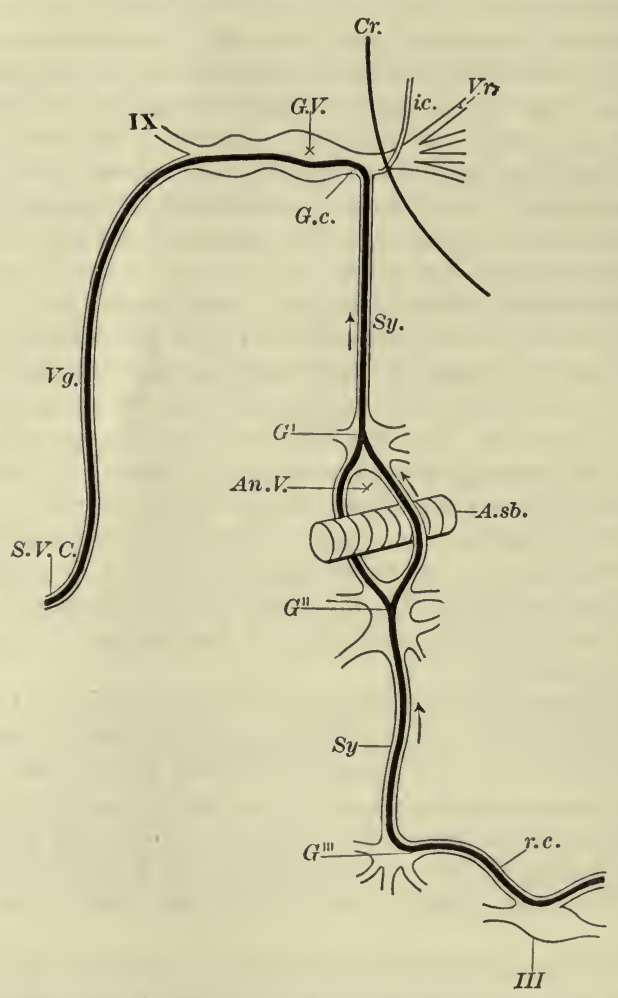

Diagrammatic Representation of the Course of Cardiac Augmentor Fibres in the Frog: V.r., roots of vagus (and IXth) nerve. G.V., ganglion of same. Cr., line of cranial wall. Vg.. vagus trunk. IX., ninth, glosso-pharyngeal nerve. S. V.C., superior vena cava. Sy., sympathetic nerve in neck. G. c., junction of sympathetic ganglion with vagus ganglion sending $i . c$. intra-cranial fibres passing to Gasserian ganglion. The rest of the fibres pass along the vagus trunk. $G^{1}$, splanchnic ganglion connected with the first spinal nerve. $G^{11}$, splanchnic ganglion of the second spinal nerve. $A n . V$, annulus of Vieussens. A.sb., subclavian artery. $G^{\prime \prime \prime}$, splanchnic ganglion of the third spinal nerve. III., third spinal nerve. r.c., ramus communicans.

The course of the augmentor fibres is shown by the thick black line. They may be traced from the spinal cord by the anterior root of the third spinal nerve, through the ramus communicans to the corresponding splanchnic ganglion $G^{111}$ and thence by the second ganglion $G^{11}$ the annulus of Vieussens, and the first ganglion $G^{1}$ to the cervical sympathetic Sy. and so by the vagus trunk to the superior vena cava S.V.C.

tion being complete, so that the heart is for a time brought to a standstill. Sometimes the slowing, sometimes the weakening, is the more prominent. When the nerve and the heart are in good condition it needs only a slight stimulus, a weak current, to produce a marked effect; and it may be mentioned that the more vigorous the heart, the more rapidly it is beating, the easier it is to bring about inhibition. Although, as we have said, the effect 
is at its maximum soon after the beginning of stimulation, a very prolonged inhibition may be produced by prolonged stimulation; indeed by rhythmical stimulation of the vagus the heart may be kept perfectly quiescent for a very long time and yet beat vigorously upon the cessation of the stimulus. In other words, the mechanism of inhibition - that is, the fibres of the vagus and the part or substance of the heart upon which these act to produce inhibition, whatever that part or substance may be-is not readily exhausted. Further, the inhibition when it ceases is, frequently at all events, followed by a period of reaction, during which the heart for a while beats nore vigorously and rapidly than before. Indeed, the total effect of stimulating the vagus fibres is not to exhaust the heart, but rather to strengthen it; and by repeated inhibitions carefully administered, a feebly beating heart may be nursed into vigorous activity.

The other set, those joining the vagus from the sympathetic, are "augmentor" or " accelerating" fibres; the latter name is the more common, but the former is more accurate, since the effect of stimulating these fibres is to increase not only the rapidity but the force of the beat; not only is the diastole shortened, but the systole is strengthened, sometimes the one result and sometimes the other being the more prominent. In contrast with the case of the vagus fibres, a somewhat strong stimulation is required to produce an effect; the time required for the maximum effect to be produced is also remarkably long. Moreover, at all events, in the case of a heart in which the circulation is not maintained, and which is therefore cut off from its normal nutritive supply, the augmentor fibres are far less easily exhausted than are the inhibitory fibres. Hence, when in such a heart both sets of fibres are stimulated together, as when the vagus trunk in the neck is stimulated, the first effects produced are those of inhibition; but these on continued stimulation may become mixed with those of augmentation, and finally the latter alone remain. Lastly, the contrast is completed by the fact that the augmentation resulting from the stimulation of the sympathetic is followed by a period of reaction in which the beats are feebler; in other words, augmentation is followed by exhaustion; and, indeed, by repeated stimulation of these sympathetic fibres a fairly vigorous bloodless heart may be reduced to a very feeble condition.

By watching the effects of stimulating the sympathetic nerve at various points of its course we may trace these augmentor fibres from their junction with the vagus down the short sympathetic of the neck through the first splanchnic or sympathetic ganglion connected with the first spinal nerve, $G^{1}$ (Fig. 74), through one or both the loops of the annulus of Vieussens, $A n$. V, through the second ganglion connected with the second spinal nerve, $G^{11}$, to the third ganglion connected with the third spinal nerve, $G^{111}$, and thence through the ramus communicans or visceral branch of that ganglion, $r . c$., to the third spinal nerve, $I I I$., by the anterior root of which they reach the spinal cord.

$\S 145$. Both sets of fibres may then be traced to the central nervous system; and we find accordingly that the heart may be inhibited or augmented by nervous impulses which are started in the nervous system either by afferent impulses as part of a reflex act or otherwise, and which pass to the heart by the inhibitory or by the augmenting tract.

Thus, if the medulla oblongata, or a particular part of the medulla oblongata which is specially connected with the vagus nerve, be stimulated, the heart is inhibited; if, for instance, a needle be thrust into this part, the heart stands still. This region in question may be stirred into action in a "reflex" manner by afferent impulses reaching it from various parts of the body. Thus, if the abdomen of a frog be laid bare, and the intestines be struck sharply 
with the handle of a scalpel, the heart will stand still in diastole with all the phenomena of vagus inhibition. If the nervi mesenterici, or the connections of these nerves with the spinal cord, be stimulated with the interrupted current, cardiac inhibition is similarly produced. If in these two experiments both vagi are divided, or the medulla oblongata is destroyed, inhibition is not produced, however much either the intestine or the mesenteric nerves be stimulated. This shows that the phenomena are caused by impulses ascending along the mesenteric nerves to the medulla, and so affecting a portion of that organ as to give rise by reflex action to impulses which descend the vagi as inhibitory impulses. The portion of the medulla thus mediating between the afferent and efferent impulses may be spoken of as the cardioinhibitory centre.

Reflex inhibition through one vagus may be brought about by stimulation of the central end of the other. In general the alimentary tract seems in closer connection with the cardio-inhibitory centre than other parts of the body; and if the peritoneal surface of the intestine be inflamed, very gentle stimulation of the inflamed surface will produce marked inhibition. But apparently stimuli, if sufficiently powerful, will through reflex action produce inhibition, whatever be the part of the body to which they are applied. Thus, crushing a frog's foot will stop the heart, and adequate stimulation of most afferent nerves will produce some amount of inhibition.

The details of the reflex chain and the portion of the centre concerned in the development of augmenting impulses have not been worked out so fully as in the case of inhibitory impulses, but there can be little doubt that the former; like the latter, are governed by the central nervous system.

$\$ 146$. So far we have been dealing with the heart of the frog, but the main facts which we have stated regarding inhibition and augmentation of the heart beat apply also to other vertebrate animals, including mammals ; and, indeed, we meet similar phenomena in the hearts of invertebrate animals.

If in a mammal the heart be exposed to view by opening the thorax, and the vagus nerve be stimulated in the neck, the heart may be seen to stand still in diastole, with all the parts flaccid and at rest. If the current employed be too weak, the result as in the frog is not an actual arrest, but a slowing or weakening of the beats. If a light lever be placed on the heart a graphic record of the standstill or of the slowing, of the complete or incomplete inhibition, may be obtained. The result of stimulating the vagus is also well shown on the blood-pressure curve, the effect of complete cardiac inhibition on blood-pressure being most striking. If, while a tracing of arterial pressure is being taken, the beat of the heart be suddenly arrested, some such curve as that represented in Fig. 75 will be obtained. It will be observed that two beats follow the application of the current marked by the point $a$, which corresponds to the signal $x$ on the line below. Then for a space of time no beats at all are seen, the next beat $b$ taking place almost immediately after the shutting off the current at $y$. Immediately after the last beat following $a$ there is a sudden fall of the blood-pressure. At the pulse due to the last systole the arterial systen is at its maximum of distention ; forthwith the elastic reaction of the arterial walls propels the blood forward into the veins, and, there being no fresh fluid injected from the heart, the fall of the mercury is unbroken, being rapid at first, but slower afterward, as the elastic force of the arterial walls is more and more used up. With the returning beats the pressure correspondingly rises in successive leaps until the normal mean pressure is regained. The size of these returning leaps of the mercury may seem disproportionately large, but it must be remembered that by far the greater part of the force of the first few strokes of the heart 
is expended in distending the arterial system, a small portion only of the blood which is ejected into the arteries passing on into the veins. As the arterial pressure rises, more and more blood passes at each beat through the capillaries, and the rise of the pressure at each beat becomes less and less, until at last the whole contents of the ventricle pass at each stroke into the veins, and the mean arterial pressure is established. To this it may be added that, as we have seen, the force of the individual beats may be somewhat greater after than before inhibition. Besides, when the mercury

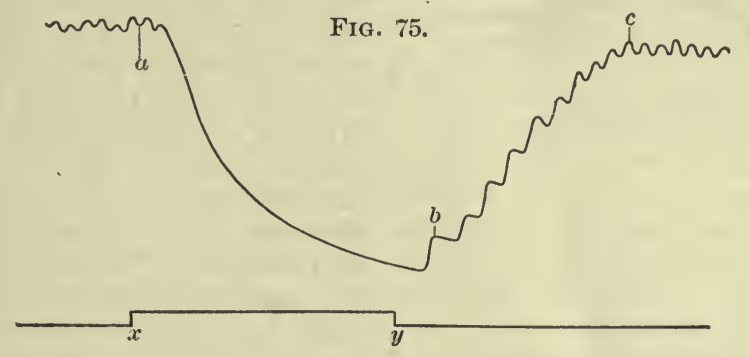

Tracing showing the Influence of Cardiac Inhibition on Blood-pressure. From a Rabbit. $x$, the marks on the signal line when the current is thrown into, and $y$, shut off from the vagus. The time-marker below marks seconds, the heart, as is frequently the case in the rabbit, beating very rapidly.

monometer is used, the inertia of the mercury tends to magnify the effects of the initial beats.

In the mammal inhibition may be brought about by impulses passing along fibres which, starting in the medulla oblongata, run down over the vagus nerve and reach the heart by the cardiac nerves. It would appear, however, that the inhibitory fibres do not belong to the vagus proper, but leave the central nervous system by the spinal accessory nerve. Thus if the roots of the spinal accessory be divided, those of the vagus proper being left intact, the spinal accessory fibres in the vagus trunk degenerate, and when this takes place stimulation of the vagus trunk fails to produce the ordinary inhibitory effects. In the mammal, as in the frog, inhibition may be brought about not only by artificial stimulation of the vagus trunk, but by stimulation in a reflex manner or otherwise of the cardio-inhibitory centre. Thus the fainting which often follows upon a blow on the stomach is a repetition of the result just mentioned as obtained on the frog by striking the stomach or stimulating the nervi mesenterici. So also the fainting,. complete or partial, which accompanies severe pain or mental emotion, is an illustration of cardiac inhibition by the vagus. In fact, cardiac inhibition so far from being a mere laboratory experiment enters repeatedly into the every-day working of our own organism as well as that of other living beings.

Indeed there is some reason for thinking that the central nervous system by means of the cardiac inhibitory fibres keeps as it were a continual rein on the heart, for, in the dog at least, section of both vagi causes a quickening of the heart's beat.

In the dog the augmentor fibres (Fig. 76) leave the spinal cord by the anterior roots of the second and third dorsal nerves, possibly also to some extent by the fourth and fifth, pass along the rami communicantes of those nerves to the ganglion stellatum, first thoracic ganglion, or respectively to one or other of the ganglia forming part of the thoracic, splanchnic, or sympathetic chain immediately below, and thence upward through the 
annulus of Vieussens, passing along one or other or both loops, to the inferior cervical ganglion. Their further course to the heart is along the nerves springing either from the inferior cer-

FIG. 76.

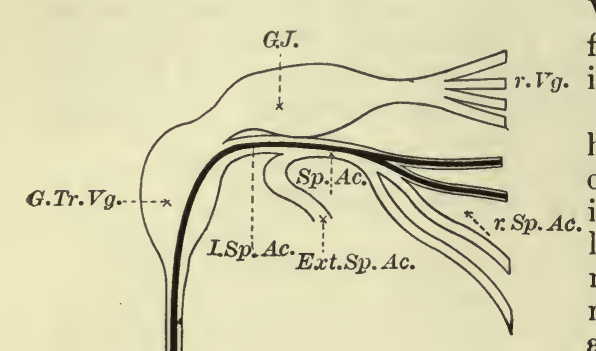

Vieussens directly. Their exact path from the ganglia in fact seems to vary in different individuals.

The path of the augmentor fibres has not been worked out so fully in other mammals as in the dog, but it is most probable that in all cases they leave the spinal cord by the anterior roots of the second and third dorsal nerves (possibly also by the fourth and fifth) and, passing up the sympathetic chain to the ganglion stellatum and annulus of Vieussens, proceed to the heart by nerves branching off from some part or other of the annulus or from the lower and middle. cervical ganglia.

Diagrammatic Representation of the Cardial Inhibitory and Augmentor Fibres in the Dog. The upper portion of the figure represents the inhibitory, the lower the augmentor fibres. $r . V g$., roots of the ragus: r.Sp.Ac., roots of the spinal accessory; both drawn very diagrammatically. G.J., ganglion jugulare; G.Tr. Vg., ganglion trunci vagi; Sp.Ac., spinal accessory trunk; ext.Sp.Ac., external spinal accessory; i.Sp. $A c$, internal spinal accessory; $V g$., trunk of vagus nerve: n.c., branches going to heart; C.Sy., cervical sympathetic; G.C., lower cervical ganglion: $A . s b .$, subclavian artery: $A n . V$. , annulus of Vieussens: G.St.(Th.1), ganglion stellatum or first thoracic ganglion: G.Th.2, G.Th ${ }^{3}$, G.Th. ${ }^{4}$, second, third, and fourth thoracic ganglia: D.II., D.III., D.IV., D.V., second third, fourth, and fifth thoracic spinal nerves; r.c., ramus communicans; n.c., nerves (cardiac) passing to heart (superior vena cava) from cervical ganglion and from the annulus of Vieussens.

The inhibitory fibres, shown by black line, run in the upper (medullary roots) of the spinal accessory, by the internal branch of the spinal accessory, past the ganglion trunci vagi, along the trunk of the vagus, and so by branches to the superior vena cava and the heart.

The augmentor fibres, also shown by black line, pass from the spinal cord by the anterior roots of the second and third thoracic nerves (possibly also from fourth and fifth as indicated by broken black line), pass the second and first (stellate) thoracic ganglia by the annulus of Vieussens to the lower cervical ganglion, from whence, as also from the annulus itself, they pass along the cardiac nerves to the superior vena cava.

The effects of stimulating these augmentor fibres in the mammal are, in general, the same as those witnessed in the frog. In the mammal, as in the frog, impulses along these augmentor fibres may be originated in the central 
nervous system, and that probably in various ways. That palpitation of the heart which is so conspicuous an effect of certain emotions is probably due to the sudden positive action of augmenting impulses, though it may possibly be due, in part at least, to sudden withdrawal of normal, continuous, tonic, and inhibitory impulses.

In the mammal, then, as in the frog, the heart is governed by two sets of nerves, the one antagonistic to the other. In the dog the roots of the spinal accessory nerve, by which inhibitory fibres leave the central nervous system, consists entirely of medullated fibres. Among these are fibres of fine calibre, $2 \mu-3 \mu$ in diameter, which may be traced down the trunk of the vagus, along the branches going to the heart, right down to the heart itself. There can be little doubt that these medullated fibres of fine calibre are the inhibitory fibres of the vagus, and indeed there is evidence which renders it probable that the inhibitory fibres of the heart are always medullated fibres of fine calibre, which continue as medullated fibres right down to the heart, but eventually lose their medulla in the heart itself.

The anterior roots of the second and third dorsal nerves, and the (white) rami communicantes belonging to them, which, as we have just seen, contain in the dog augmentor fibres, also consist exclusively of medullated fibres. But the nerves which convey the augmenting impulses from the lower cervical ganglion, or from the annulus of Vieussens to the heart, consist of non-medullated fibres. Hence, the augmentor fibres must have lost their medulla, and become continuous with non-medullated fibres somewhere in their course along the sympathetic chain. It is probable that the change occurs in the ganglion stellatum and lower cervical ganglion, and it is further probable that the change is effected by the medullated fibre passing into one of the ganglion cells, and so losing its medulla, the impulses which it conveys passing out of the nerve cell by one or more of the other processes of the cell which are continued on as non-medullated fibres. Cf. $\$ 94$.

In the $d o g$ then these two sets of nerve fibres, antagonistic to each other in function, differ in structure, the augmentor fibres early losing their medulla, and hence being over a large part of their course non-medullated fibres, whereas the inhibitory fibres are medullated fibres which, though they may pass by or through ganglia (as the ganglion jugulare and ganglion trunci vagi), do not lose their medulla in these ganglia, but remain as medullated fibres right down to the heart. And this difference in structure appears to hold good for all mammals, and is possibly true for vertebrates generally.

$\$ 147$. The question, What is the exact nature of the change brought about by the inhibitory and augmenting impulses respectively on their arrival at the heart? or, in other words, by virtue of what events produced in the heart itself do the impulses of one kind bring about inhibition, of the other kind augmentation? is a very difficult one, which we cannot attempt to discuss fully here. We may, if we please, speak of an " inhibitory mechanism" placed in the heart itself, but we have no exact knowledge of the nature of such a mechanism. Still less do we possess any satisfactory information as to an augmenting mechanism. It has been suggested that some of the ganglia in the heart serve as such an inhibitory (or augmenting) mechanism; but there is evidence that the inhibitory impulses produce their effect by acting directly on the muscular fibres, or at all events do not produce their effect by acting exclusively on any ganglia. One evidence of this kind is supplied by the action of the drug atropine.

If, either in a frog or a ninammal, or other animal, after the vagus fibres 
have been proved by trial to produce upon stimulation the usual inhibitory effects, a small quantity of atropine be introduced into the circulation (when the experiment is conducted on a living animal, or be applied in a weak solution to the heart itself' when the experiment is conducted, as in the case of a frog, on an excised heart, or after the circulation has ceased), it will after a short time be found not only that the stimulation, the application of a current, for instance, which previously when applied to the vagus produced marked inhibition, now produces no inhibition, but even that the strongest stimulus, the strongest current applied to the vagus will wholly fail to affect the heart, provided that there be no escape of current on to the cardiac tissues themselves; under the influence of even a small dose of atropine, the strongest stimulation of the vagus will not produce standstill or appreciable slowing or weakening of the beat.

Now it might be supposed that the atropine produces this remarkable effect by acting on some ganglionic or other mechanism intervening between the vagus fibres and the cardiac muscular tissue; but we have evidence that the atropine acts either on the muscular tissue itself or on the very endings of the nerves in the muscular fibres. We have said $(\S 140)$ that a properly prepared strip of the ventricle of the tortoise will execute for a long time spontaneous rhythmic contractions, it will go on "beating" for a long time. A strip of the auricle will exhibit the same phenomena even still more readily. If now, while such a strip from the auricle is satisfactorily beating, a gentle interrupted current be passed through it, it will stop beating; the current inhibits the spontaneous beats; a very gentle interrupted current must be used, otherwise the effect is obscured by the more direct stimulating action of the current. If now the strip be gently bathed with a weak solution of atropine no such inhibitory effect is produced by the interrupted current; the beats go on regardless of the action of the current. The interruption of this experiment is that in the first case the interrupter current stimulated the fine termination of the inhibitory fibres in the muscular strip, and that in the second case the atropine produced some effect either on these fine fibres, or on their connections with the muscular substance or on the actual muscular substance itself, by virtue of which they ceased to act. But if this be so, if the same inhibitory effects are produced alike by stimulating the vagus trunk and stimulating the very endings of the nerves in the muscles of the heart, if not the actual muscular tissue itself, then there is no need to suppose the existence of any special inhibitory mechanism placed between the fibres in the vagus branches and the cardiac muscular tissue.

The action of atropine on the heart is, so to speak, complemented by the action of muscarine, the active principle of many poisonous mushrooms. If a small quantity of muscarine be introduced into the circulation, or applied directly to the heart, the beats become slow and feeble, and if the dose be adequate the heart is brought to a complete standstill. The effect is in some respects like that of powerful stimulation of the vagus, but the standstill is much more complete, the effect is much more profound. Now if, in a frog, the heart be brought to a standstill by a dose of muscarine, the application of an adequate quantity of atropine will bring back the beats to quite their normal strength. The one drug is, as far as the heart is concerned (and, indeed, in many other respects), the antidote of the other. And, as in the case of atropine, so in the case of muscarine, there is evidence that the drug acts not on any ganglionic mechanisms, but on the cardiac tissue itself.

The conclusion that inhibition is the result of changes in the cardiac tissue itself may serve to explain why in inhibition sometimes the slowing, 
sometimes the weakening is the more prominent. When the inhibitory impulses, by reason of particular fibres being affected or otherwise, are brought to bear chiefly on those parts of the heart, such as the sinus, which possessing higher rhythmic potentiality (see $\$ 142$ ) determine the sequence and set the rate of rhythm, it is the rate which is most markedly affected. When, on the other hand, the inhibitory impulses fall chiefly on the parts possessing lower rhythmic potentiality, the most marked effect is a diminution in the force of the contractions.

There is no adequate evidence then that the cardiac ganglia act as an inhibitory mechanism in the sense that they produce important changes in the nature of the impulses reaching them along vagus inhibitory fibres before those impulses pass on to the muscular tissue. We may add that there is similarly no adequate evidence that any of the ganglia act as an "augmenting" mechanism. We have previously seen (\$\$ 141,142) reasons for thinking the ganglia are not centres for the origination or regulation of the spontaneous beats. The question then arises, What are their functions? To this question we cannot at present give a wholly satisfactory answer.

The inhibitory fibres remain, as we have seen, medullated fibres until they reach the heart, but it would appear that they lose their medulla somewhere in the heart before they actually reach the muscular tissue, and it is probable that the loss takes place in connection with some of the cardiac ganglia much in the same way that the augmenting fibres lose their medulla in the ganglia of the sympathetic chain; but we do not know what is the physiological effect or the purpose of this loss of the medulla, and we cannot suppose that this is the sole or even chief use of the ganglia. Coincident with the loss of the medulla an increase of fibres frequently takes place, more than one non-medullated fibre leaving a nerve cell into which one medullated fibre enters; and we may suppose that this mode of branching has purposes not fulfilled by the mere division of a fibre. Then again, bearing in mind the nutritive or "trophic" function of the spinal ganglia alluded to in $\$ 96$, we may suppose that the cardiac ganglia are in some way concerned in the nutrition of the cardiac nerve fibres. But our knowledge is not yet sufficiently ripe to allow exact statements to be made.

\section{Other Influences Regulating or Modifying the Beat of the Heart.}

$\S 148$. Important as is the regulation of the heart by the nervous system, it must be borne in mind that other influences are or may be at work. The beat of the heart may, for instance, be modified by influences bearing directly on the nutrition of the heart. The tissues of the heart, like all other tissues, need an adequate supply of blood of a proper quality; if the blood vary in quality or quantity the beat of the heart is correspondingly affected. The excised frog's heart, as we have seen, continues to beat for some considerable time, though apparently empty of blood. After a while, however, the beats diminish and disappear; and their disappearance is greatly hastened by washing out the heart with a normal saline solution, which when allowed to flow through the cavities of the heart readily permeates the tissues on account of the peculiar construction of the ventricular walls. If such a "washed out" quiescent heart be fed with a perfusion canula, in the manner described ( $\$ 141)$, with diluted blood (of the rabbit, sheep, etc.), it may be restored to functional activity. A similar but less complete restoration may be witnessed if serum be used instead of blood; and a heart fed regularly with fresh supplies of blood or even of serum may be kept beating for a 
very great length of time. In treating of the skeletal muscles we saw that in their case the exhaustion following upon withdrawal of the blood-stream might be attributed either to an inadequate supply of new nutritive material and oxygen, or to an accumulation in the muscular substance of the products of muscular metabolism, or to both causes combined. And the same considerations hold good for the nervous and muscular structures of the heart, though the subject has not yet been sufficiently well worked out to permit any very definite statements to be made. It seems probable, however, that an important factor in the matter is the accumulation in the muscular fibres and in the surrounding lymph of carbonic acid, and especially of the substances which give rise to the acid reaction.

When the frog's heart is thus "fed" with various substances the interesting fact is brought to light that some substances, such, for instance, as very dilute lactic acid, lear to increased expansion, and others, such, for instance, as very dilute solutions of sodium hydrate, to diminished expansion, that is to continued contraction, of the quiescent ventricle. It would appear that the muscular fibres of the ventricle over and above their rhythmic contractions are capable of varying in length, so that at one time they are longer, and the ventricle when pressure is applied to it internally dilates beyond the normal, while at another time they are shorter, and the ventricle, with the same internal pressure, is contracted beyond the normal. Further, in the frog at least, when the pause between two beats is lengthened the relaxation of the ventricle goes on increasing, so that apparently the ventricle when beating normally is already somewhat contracted when a new beat begins. In other words, the ventricle possesses what we shall speak of in reference to arteries as tonicity or tonic contraction, and the amount of this tonic contraction, and in consequence the capacity of the ventricle, varies according to circumstances. We have, moreover, evidence that inhibitory impulses diminish and augmenting impulses increase this tonic contraction.

When the frog's ventricle is thus artificially fed with serum or even with blood, the beats, whether spontaneous or provoked by stimulation, are apt to become intermittent and to arrange themselves into groups. This intermittence is possibly due to the serum or blood being unable to carry on nutrition in a completely normal manner, and to the consequent production of abnormal chemical substances; and it is probable that cardiac intermittences seen during life have often a similar causation. Various chemical substances in the blood, natural or morbid, may thus affect the heart's beat by acting on its muscular fibres or its nervous elements, or both, and that probably in various ways, modifying in different directions the rhythm or the individual contractions, or both.

The physical or mechanical circumstances of the heart also affect its beat; of these perhaps the most important is the amount of the distention of its cavities. The contractions of cardiac muscle, like those of ordinary muscle (see $\$ 79$ ), are increased up to a certain limit by the resistance which they have to overcome; a full ventricle will, other things being equal, contract more vigorously than one less full; though, as in ordinary muscle, the limit at which resistance is beneficial may be passed, and an overfull ventricle will fail to beat at all.

Under normal conditions the ventricle probably empties itself completely at each systole. Hence an increase in the quantity of blood in the ventricle would augment the work done in two ways: the quantity thrown out would be greater, and the increased quantity would be ejected with greater force. Further, since the distention of the ventricle is (at the commencement of the systole at all events) dependent on the auricular systole, the work of the 
ventricle (and so of the heart as a whole) is in a measure governed by the auricle.

An interesting combination of direct mechanical effects and indirect nervous effects is seen in the relation of the heart's beat to blood-pressure. When the blood-pressure is high, not only is the resistance to the ventricular systole increased, but other things being equal, more blood flows (in the mammalian heart) through the coronary artery. Both these events would increase the activity of the heart, and we might expect that the increase would be manifest in the rate of the rhythm as well as in the force of the individual beats. As a matter of fact, however, we do not find this. On the contrary the relation of heart-beat to pressure may be put almost in the form of a law, that the "rate of the beat is in inverse ratio to the arterial pressure," a rise of pressure being accompanied by a diminution, and fall of pressure with an increase, of the pulse-rate. This, however, only holds good if the vagi be intact. If these be previously divided, then in whatever way the blood-pressure be raised-whether by injecting blood or clamping the aorta or increasing the peripheral resistance, through that action of the vasomotor nerves which we shall have to describe directly-or in whatever way it be lowered, no such clear and decided inverse relation between blood-pressure and pulse-rate is observed. It is inferred, therefore, that increased bloodpressure causes a slowing of the pulse, when the vagi are intact, because the cardio-inhibitory centre in the medulla is stimulated by the high pressure, either directly by the pressure obtaining in the bloodvessels of the medulla, or in some indirect manner, and the heart in consequence to a certain extent inhibited.

\section{Changes in the Calibre of the Minute Arteries. Vasomotor}

\section{Actions.}

$\S 149$. All arteries contain plain muscular fibres, for the most part circularly disposed, and most abundant in, or sometimes almost entirely confined to, the middle coat. Moreover, as the arteries become smaller the muscular element, as a rule, becomes more and more prominent as compared with the other elements, until, in the minute arteries, the middle coat consists almost entirely of a series of plain muscular fibres wrapped around the internal coat. Nerve fibres, of whose nature and course we shall presently speak, are distributed largely to the arteries and appear to end chiefly in fine plexuses around the muscular fibre, but their exact terminations have not as yet been clearly made out. By mechanical, electrical, or other stimulation, this muscular coat may, in the living artery, be made to contract. During this contraction, which has the slow character belonging to the contractions of all plain muscles, the calibre of the vessel is diminished. The veins also, as we have seen, possess nuscular elements, but these vary in amount and distribution very much more in the veins than in the arteries. Most veins, however, are contractile, and may vary in calibre according to the condition of their muscular elements. Veins are also supplied with nerves. It will be of advantage, however, to consider separately the little we know concerning the changes in the veins, and to confine ourselves at present to the changes in the arteries.

If the web of a frog's foot be watched under the microscope, any individual small artery will be found to vary considerably in calibre from time to time, being sometimes narrowed and sometimes dilated; and these changes may take place without any obvious changes either in the heart-beat or in the general circulation; they are clearly changes of the artery itself. During the narrowing, which is obviously due to a contraction of the muscular 
coat of the artery, the capillaries fed by the artery and the veins into which these lead become less filled with blood and paler. During the widening, which corresponds to the relaxation of the muscular coat, the same parts are fuller of blood and redder. It is obvious that, the pressure at the entrance into any given artery remaining the same, more blood will enter the artery when relaxation takes place, and consequently the resistance offered by the artery is diminished, and less when contraction occurs and the resistance is consequently increased; the blood flows in the direction of least resistance.

The extent and intensity of the narrowing or widening, the constriction or dilatation which may thus be observed in the frog's web, vary very largely. Variations of slight extent, either more or less regular and rhythmic or irregular, occur even when the animal is apparently subjected to no disturbing causes, and may be spoken of as spontaneous ; larger changes may follow events occurring in various parts of the body ; while as the result of experimental interference the arteries may become either constricted, in some cases almost to obliteration, or dilated until they acquire double or more than double their normal diameter. This constriction or dilation may be brought about not only by treatment applied directly to the web, but also by changes affecting the nerve of the leg or other parts of the body. Thus, section of the sciatic nerve is generally followed by a widening which may be slight or which may be very marked, and which is sometimes preceded by a passing constriction; while stimulation of the peripheral stump of the divided nerve by an interrupted current of moderate intensity generally gives rise to constriction, often so great as almost to obliterate some of the minute arteries.

Obviously, then, the contractile muscular elements of the minute arteries of the web of the frog's foot are capable by contraction or relaxation of causing decrease or increase of the calibre of the arteries; and this condition of constriction or dilation may be brought about through the agency of the nerves. Indeed, not only in the frog, but also, and still more so, in warmblooded animals, have we evidence that in the case of nearly all, if not all, the arteries of the body, the condition of the muscular coat, and so the calibre of the artery, is governed by means of nerves; these nerves have received the general name of vasomotor nerves.

$\$ 150$. If the ear of a rabbit, preferably a light-colored one, be held up before the light, a fairly conspicuous artery will be seen running up the middle line of the ear accompanied by its broader and more obvious veins. If this artery be carefully watched it will be found, in most instances, to be undergoing rhythmic changes of calibre, constriction alternating with dilatation. At one moment the artery appears as a delicate, hardly visible, pale streak, the whole ear being at the same time pallid. After a while the artery slowly widens out, becomes broad and red, the whole ear blushing, and many small vessels previously invisible coming into view. Again the artery narrows and the blush fades away; and this may be repeated at. somewhat irregular intervals of a minute, more or less. The extent and regularity of the rhythm are usually markedly increased if the rabbit be held up by the ears for a short time previous to the observation. Similarly rhythmic variations in the calibre of the arteries have been observed in several places, e.g., in the vessels of the mesentery and elsewhere; probably they are widely spread.

Sometimes no such variations are seen; the artery remains constant in a condition intermediate between the more extreme widening and extreme narrowing just described. In fact, we may speak of an artery as being at any given time in one of three phases. It may be very constricted, in which case its muscular fibres are very much contracted; or it may be dilated, in 
which case its muscular fibres are relaxed; or it may be moderately constricted, the muscular fibres being contracted to a certain extent, and remaining in such a condition that they may, on the one hand, pass into stronger contraction, leading to marked constriction, or, on the other hand, into distinct relaxation, leading to dilatation. We have reason to think, as we shall see, that many arteries of the body are kept habitually, or at least for long periods together, in this intermediate condition, which is frequently spoken of as tonic contraction, or tonus, or arterial tone.

$\S 151$. If, now, in a vigorous rabbit, in which the heart is beating with adequate strength and the whole circulation is in a satisfactory condition, the cervical sympathetic nerve be divided on one side of the neck, remarkable changes may be observed in the bloodvessels of the ear of the same side. The arteries and veins widen, they together with the small veins and the capillaries become full of blood, many vessels previously invisible come into view, the whole ear blushes, and if the rhythmic changes described above were previously going on, these now cease; and, in consequence of the extra supply of warm blood, the whole ear becomes distinctly warmer. Now these changes take place, or may take place, without any alteration in the heartbeat or in the general circulation. Obviously the arteries of the ear have, in consequence of the section of the nerve, lost the tonic contraction which previously existed; their muscular coats, previously somewhat contracted, have become quite relaxed, and whatever rhythmic contractions were previously going on have ceased. The more marked the previous tonic contraction, and the more vigorous the heart-beats, so that there is an adequate supply of blood to fill the widened channels, the more striking the results. Sometimes, as when the heart is feeble, or the pre-existing tonic contraction is slight, the section of the nerve produces no very obvious change.

If, now, the upper segment of the divided cervical sympathetic nervethat is, the portion of the nerve passing upward to the head and ear-be laid upon the electrodes of an induction machine and a gentle interrupted current be sent through the nerve, new changes take place in the bloodvessels of the ear. A short time after the application of the current, for in this effect there is a latent period of very appreciable duration, the ear grows paler and cooler, many small vessels previously conspicuous become again invisible, the main artery shrinks to the thinnest thread, and the main veins become correspondingly small. When the current is shut off from the nerve these effects still last some time, but eventually pass off; the ear reddens, blushes once more, and indeed may become even redder and hotter, with the vessels more filled with blood than before. Obviously the current has generated in the cervical sympathetic nerve impulses which, passing upward to the ear and finding their way to the muscular coats of the arteries of the ear, have thrown the muscles of those coats into forcible contractions, and have thus brought about a forcible narrowing of the calibre of the arteries - a forcible constriction. Through the narrowed constricted arteries less blood finds its way, and hence the paleness and coldness of the ear. If the impulses thus generated be very strong, the constriction of the arteries may be so great that the smallest quantity only of blood can make its way through them, and the ear may become almost bloodless. If the impulses be weak, the constriction induced may be slight only; and, indeed, by careful manipulation the nerve may be induced to send up to the ear impulses only just sufficiently strong to restore the moderate tonic constriction which existed before the nerve was divided.

We infer from these experiments that among the various nerve fibres making up the cervical sympathetic, there are certain fibres which, passing 
upward to the head, become connected with the arteries of the ear, and that these fibres are of such a kind that impulses generated in them and passing upward to the ear lead to marked contraction of the muscular fibres of the arteries, and thus produce constriction. These fibres are vasomotor fibres for the bloodvessels of the ear. From the loss of tone, so frequently following section of the cervical sympathetic, we may further infer that, normally during life, impulses of a gentle kind are continually passing along these fibres upward through the cervical sympathetic, which impulses, reaching the arteries of the ear, maintain the normal tone of those arteries. But, as we said, the existence of this tone is not so constant, and these tonic impulses

Fig. 77.

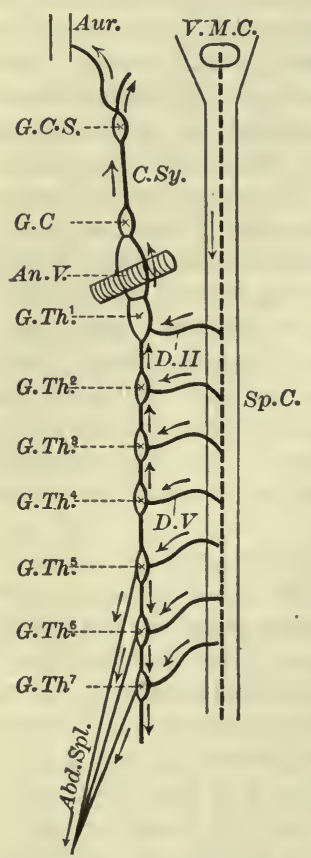

Diagram illustrating the Paths of Vaso-constrictor Fibres along the Cervical Sympathetic and (part of) the $\mathrm{Ab}$ dominal Splanchnic: Aur., artery of ear; G.C.s., superior cervical ganglion: $A b d$. Spl., upper roots of and part of abdominal splanchnic nerve; $\boldsymbol{V}$. $M$. C., vasomotor centre in medulla. The other references are the same as in Fig. 76,8 146. The paths of the constrictor fibres are shown by the arrows. The dotted line in the spinal cord, Sp. C., is to indicate the passage of constrictor impulses down the cord from the vasomotor centre in the medulla. bloodvessels of the ear of that side; and section

are not so conspicuous as the artificial constrictor impulses generated by stimulation of the nerve.

$\S 151$. The above results are obtained whatever be the region of the cervical sympathetic which we divide or stimulate from the upper cervical ganglion to the lower. We may, therefore, describe these vasomotor impulses as passing upward from the lower cervical ganglion along the cervical sympathetic to the upper cervical ganglion, from which they issue by branches which ultimately find their way to the ear. But these impulses do not start from the lower cervical ganglion; on the contrary, by repeating the experiments of division and stimulation in a series of animals, we may trace the path of these impulses from the lower cervical ganglion (Fig. 77) through the annulus of Vieussens to the ganglion stellatum or first thoracic ganglion, and thence either along the ramus communicans (visceral branch) to the anterior root of the second dorsal nerve, and thus to the spinal cord, or lower down along the thoracic sympathetic chain, and thence by other rami communicantes to some other of the upper dorsal nerves, and thus to the spinal cord. The path taken by these vasomotor impulses for the ear is in fact very similar to that of the augmentor fibres for the heart (cf. Fig. 76), from the spinal cord up to the annulus of Vieussens and to the lower cervical ganglion; but there they part company. We can thus trace these impulses along the cervical sympathetic to the anterior roots of certain dorsal nerves, and through these to a particular part of the spinal cord, where we will for the present leave them. We may accordingly speak of vasomotor fibres for the ear as passing from the dorsal spinal cord to the ear along the track just marked out; stimulation of these fibres at their origin in the spinal cord or at any part of their course (along the anterior roots of the second, third, or other upper dorsal nerves, visceral branches of those nerves, ganglion stellatum or upper part of thoracic sympathetic chain, annulus of Vieussens, etc.) leads to constriction in the 
existing tonic constriction of the bloodvessels of the ear, though this effect is not so constant or striking as that of stimulation.

$\S 153$. We must now turn to another case. In dealing with digestion we shall have to study the submaxillary salivary gland. We may for the present simply say that this is a glandular mass well supplied with bloodvessels, and possessing a double nervous supply. On the one hand it receives fibres from the cervical sympathetic, Fig. 78, v. sym. (in the dog, in which the effects which we are about to describe are best seen, the vagus and cervical

Fig. 78.

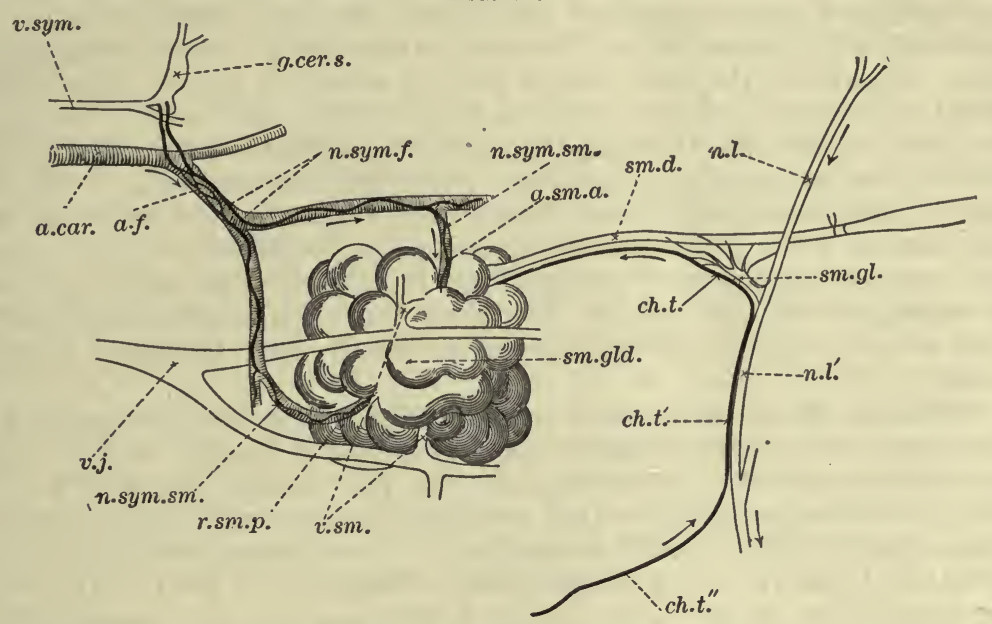

Diagrammatic Representation of the Submaxillary Gland of the Dog, with its Nerves and Bloodvessels. The dissection has been on an animal lying on its back, but since all the parts shown in the figure cannot be seen fronı any one point of view, the figure does not give the exact anatomical relations of the several structures.

$s m$. gld. The submaxillary gland, into the duct $(s m . d$.) of which a canula has been tied. The sublingual gland and ductare notshown. n.l., n.l'. The lingual branch of the fifth nerve, the part n.l. is going to the tongue. ch.t., ch. $t^{\prime}$., ch. $t^{\prime \prime}$. The chorda tympani. The part ch. $t^{\prime \prime}$. is proceeding from the facial nerve; at $c h . t^{\prime}$. it becomes conjoined with the lingual $n . l^{\prime}$., and afterward diverging passes as ch.t. to the gland along the duct; the continuation of the nerve in company with the lingual n.l. is not shown. $s m$. gl. The submaxillary ganglion with its several roots. $a$. car. The carotid artery, two small branches of which, $a . s m$. $a$. and $r . s m$. p., pass to the anterior and posterior parts of the gland. $v .8 m$. The anterior and posterior veins from the gland, falling into $v . j$., the jugular vein. $v$. $8 y m$. The conjoined vagus and sympathetic trunks. g.cer. 8 . The upper cervical ganglion, two branches of which, forming a plexus (a.f.) over the facial artery, are distributed $(n . s y m . s m$.) along the two glandular arteries to the anterior and posterior portions of the gland.

The arrows indicate the direction taken by the nervous impulses during reflex stimulation of the gland. They ascend to the brain by the lingual and descend by the chorda tympani.

sympathetic are enclosed in a common sheath so as to form what appears to be a single trunk), which reach the gland in company with the arteries supplying the gland $(n . s y m$. sm.). On the other hand, it receives fibres from a small nerve called the chorda tympani $(c h . t$.), which, springing from the seventh cranial (facial) nerve, crosses the tympanum of the ear (hence the name) and, joining the lingual branch of the fifth nerve, runs for some distance in company with that nerve, and then ends partly on the tongue, and partly in a small nerve which, leaving the lingual nerve before reaching the tongue, runs along the duct of the submaxillary gland, and is lost in the substance of the gland; a small branch is also given off to the sublingual gland. 
Now when the chorda tympani is simply divided no very remarkable changes take place in the bloodvessels of the gland, but if the peripheral segment of the divided nerve, that still in connection with the gland, be stimulated, very marked results follow. The small arteries of the gland become very much dilated and the whole gland becomes flushed. (As we shall see later on, the gland at the same time secretes saliva copiously, but this does not concern us just now.) Changes in the calibre of the bloodvessels are of course not so readily seen in a compact gland as in a thin, extended ear; but if a fine tube be placed in one of the small veins by which the blood returns from the gland, the effects on the bloodvessels of stimulating the chorda tympani become very obvious. Before stimulation the blood trickles out in a thin slow stream of a dark venous color; during stimulation the blood rushes out in a rapid full stream, often with a distinct pulsation and frequently of a color which is still scarlet and arterial in spite of the blood having traversed the capillaries of the gland; the blood rushes so rapidly through the widened bloodvessels that it has not time to undergo completely that change from arterial to venous which normally occurs while the blood is traversing the capillaries of the gland. This state of things may continue for some time after the stimulation has ceased, but before long the flow from the veins slackens, the issuing blood becomes darker and venous, and eventually the circulation becomes normal.

Obviously the chorda tympani contains fibres which we may speak of as "vasomotor," since stimulation of them produces a change in, and brings about a movement in the bloodvessels; but the change produced is of a character the very opposite to that produced in the bloodvessels of the ear by stimulation of the cervical sympathetic. There stimulation of the nerve caused contraction of the muscular fibres, constriction of the small arteries; here stimulation of the nerve causes a widening of the arteries, which widening is undoubtedly due to relaxation of the muscular fibres. Hence we must distinguish between two kinds of vasomotor fibres, fibres the stimulation of which produces constriction, vaso-constrictor fibres, and fibres the stimulation of which causes the arteries to dilate, vaso-dilator fibres, the one kind being the antagonist of the other.

The reader can hardly fail to be struck with the analogy between these two kinds of vasomotor fibres on the one hand, and the inhibitory and augmentor fibres of the heart on the other hand. The augmentor cardiac fibres increase the rhythm and the force of the heart-beats; the vaso-constrictor fibres increase the contractions of the muscular fibres of the arteries; the one works upon a rhythmically active tissue, the other upon a tissue whose work is more or less continuous, but the effect is in each case similar-an increase of the work. 'The inhibitory cardiac fibres slacken or stop the rhythm of the heart and diminish the beats; the vaso-dilator fibres diminish the previously existing contraction of the muscular fibres of the arteries so that these expand under the pressure of the blood.

$\$ 154$. But we must return to the vasomotor nerves. The cervical sympathetic contains vaso-constrictor fibres for the ear, and we may now add for other regions, also of the head and face. Thus the branches of the cervical sympathetic, going to the submaxillary gland of which we just spoke (Fig. $78, n . s y m . s m$.$) , contain vaso-constrictor fibres for the vessels of the gland;$ stimulation of these fibres produces on the vessels of the gland an effect exactly the opposite of that produced by stimulation of the chorda tympani. But to this particular point we shall have to return when we deal with the gland in connection with digestion. A more important fact for our present purpose is that the cervical sympathetic appears to contain only vaso-con- 
strictor fibres; if we put aside as exceptional and doubtful the result of certain observers who obtained vaso-dilator effects in the mouth and face, we may say that in no region to which the fibres of the cervical sympathetic are distributed can any vaso-dilator action be observed as the result of stimulation of the nerve at any part of its course. In the chorda tympani, on the other hand, the vasonotor fibres are exclusively vaso-dilator fibres, and this is true both of the part of the nerve ending in the submaxillary and sublingual glands and the rest of the ending of the nerve in the tongue. Stimulation of the chorda tympani (as far as the vasomotor functions of the nerve are concerned, for it has, as we shall see, other functions) at any part of its course, from its leaving the facial nerve to its endings in the tongue or gland, produces only vaso-dilator effects, never vaso-constrictor effects.

With many other nerves of the body the case is different. In the frog, division of the sciatic nerve leads to a widening of the arteries of the web of the foot of the same side, and stimulation of the peripheral end of the nerve causes a constriction of the vessels, which, if the stimulation be strong, may be so great that the web appears for the time being to be devoid of blood. Also, in a mammal, division of the sciatic nerve causes a similar widening of the small arteries of the skin of the leg. Where the condition of the circulation can be readily examined, as, for instance, in the hairless balls of the toes, especially when these are not pigmented, the vessels are seen to be dilated and injected, and a thermometer placed between the toes shows a rise of temperature amounting, it may be, to several degrees. If, moreover, the peripheral end of the divided nerve be stimulated, the vessels of the skin liecome constricted, the skin grows pale, and the temperature of the foot falls. And very similar results are obtained in the forelimb by division and subsequent stimulation of the nerves of the brachial plexus.

The quantity of blood present in the bloodvessels of the mammal, though it may sometimes be observed directly, has frequently to be determined indireetly. The temperature of passive struetures subject to cooling influences, such as the skin, is largely dependent on the supply of blood; the more abundant the supply the warmer the part. Hence, in these parts variations in the quantity of blood may be inferred from variations of temperature; but in dealing with more active structures there are obviously sources of error in the possibility of the treatment adopted, such as the stimulation of a nerve giving rise to an increase of temperature due to increased metabolism, independent of variations in blood-supply.

The quantity of blood may also be determined by the plethysmograph. In this instrument a part of the body, such as the arm, is introduced into a closed chamber filled with fluid, ex. gr., a large glass tube, the opening by which the arm is introduced being secured with a stout eaoutchouc membrane. An increase or decrease of blood sent into the arm will lead to an increase or decrease of the volume of the arm, and this will make itself felt by an increase or diminution of pressure in the fluid of the closed chamber, which may be registered and measured in the usual way. We shall have to speak again of a modifieation of this instrument when we are dealing with the kidney.

So far the results are quite like those obtained by division and stimulation of the cervical sympathetic, and we might infer that the sciatic nerve and brachial plexus contain vaso-constrictor fibres for the vessels of the skin of the hind-limb and fore-limb, vaso-dilator fibres being absent. But sometimes a different result is obtained: on stimulating the divided sciatic nerve the vessels of the foot are not constricted, but dilated-perhaps widely dilated. And this vaso-dilator action is almost sure to be manifested when the nerve is divided, and the peripheral stump stimulated some days after division, by which time commencing degeneration has begun to interfere with the irrita- 
bility of the nerve. For example, if the sciatic be divided, and some days afterward, by which time the flushing and increased temperature of the foot following upon the section has wholly or largely passed away, the peripheral stump be stimulated with an interrupted current, a renewed flushing and rise of temperature is the result. We are led to conclude that the sciatic nerve (and the same holds good for the brachial plexus) contains both vasoconstrictor and vaso-dilator fibres, and to interpret the varying results as due to variations in the relative irritability of the two sets of fibres. The constrictor fibres appear to predominate in these nerves, and hence constriction is the more common result of stimulation ; the constrictor fibres also appear to be more readily affected by a tetanizing current than the dilator fibres. When the nerve after division commences to degenerate, the constrictor fibres lose their irritability earlier than the dilator fibres, so that at a certain stage a stimulus, such as the interrupted current, while it fails to affect the constrictor fibres, readily throws into action the dilator fibres. The latter, indeed, in contrast to ordinary motor nerves ( $\S 81$ ), retain their irritability after section of the nerve for very many days. The result is, perhaps, even still more striking if a mechanical stimulus, such as that of "crimping" the nerve by repeated snips with the scissors, be employed. Exposure to a low temperature again seems to depress the constrictors more than the dilators; hence, when the leg is placed in ice-cold water stimulation of the sciatic, even when the nerve has been but recently divided, throws the dilator only into action and produces flushing of the skin with blood. Rhythmical stimulation, moreover, of even a freshly divided nerve produces dilatation. And there are other facts which support the same view that the sciatic nerve (and brachial plexus) contains both vaso-constrictor and vaso-dilator fibres which are differently affected by different circumstances. We may point out that the case of the vagus of the frog is a very analogous one; in it are both cardiac inhibitory (true vagus) and cardiac augmentor (sympathetic) fibres, but the former, like the vaso-constrictor fibres in the sciatic, are predominant, and special means are required to show the presence of the latter.

In the splanchnic nerve (abdominal splanchnic), which supplies fibres to the bloodvessels of so large a part of the abdominal viscera, there is abundant evidence of the presence of vaso-constrictor fibres, but the presence of vaso-dilator fibres has not clearly been shown. Division of this nerve leads to a widening of the bloodvessels of the abdominal viscera, stimulation of the nerve to a constriction; and, as we shall see, since the amount of bloodvessels thus governed by this nerve is very large indeed, interference either in the one direction or the other with its vasomotor functions produces very marked results, not only on the circulation in the abdomen, but on the whole vascular system.

In nerves going to muscles vaso-dilator fibres predominate; indeed, in these the presence of any vaso-constrictor fibres at all has not at present been satisfactorily established. When a muscle contracts there is always an increased flow of blood through the muscle; this may be in part a mere mechanical result of the change of form, the shortening and thickening of the fibres opening out the minute bloodvessels, but is not wholly, and probably not even largely, thus produced. A notable feature of vasomotor fibres is that, in very many cases at all events, their action is not affected by small or moderate doses of urari such as render the motor nerves of striated muscle powerless. Thus, in a frog placed under the influence of a moderate amount of urari, stimulation of a nerve going to a muscle will produce vasomotor effects unaccompanied and unobscured by any contraction of the striated fibres. By placing a thin muscle of a frog, such as the mylo-hyoid, 
under the microscope, and watching the calibre of the sinall arteries and the circulation of the blood through them while the nerve is being stimulated, the widening of the bloodvessels as the result of the stimulation may be actually observed. This experiment appears not to succeed in a mammal; and it has been suggested that when a muscle contracts some of the chemical products of the metabolism of the muscle may, by direct action on the minute bloodvessels apart from any nervous agency, lead to a widening of those bloodvessels; this, however, is doubtful. With regard to the vaso-constrictor fibres, the only evidence that they exist in muscles is that when the nerve of a muscle is divided the bloodvessels of the muscle widen, somewhat like bloodvessels of the ear after division of the cervical sympathetic. This suggests the presence of vaso-constrictor fibres carrying the kind of influence which we called tonic, leading to an habitual moderate constriction; it cannot, however, be regarded by itself as conclusive evidence; but we must not discuss the matter here.

Speaking generally, then, most if not all the arteries of the body are supplied with vasomotor fibres running in this or that nerve, the fibres being either vaso-constrictor or vaso-dilator, and some nerves containing one kind of fibres only, some both in varying proportion. Almost every nerve in the body, therefore, may be looked upon as influencing a certain set of bloodvessels, as governing a vascular area, the area being large or small, and the government being exclusively constrictor or exclusively dilator, or mixed.

\section{The Course of Vaso-constrictor and Vaso-dilator Fibres.}

$\S 155$. Both the vaso-constrictor and the vaso-dilator fibres have their origin in the central nervous system, the spinal cord, or the brain, but the course of the two sets appears to be very different.

In the mammal, as far as we know at present, all the vaso-constrictor fibres for the whole body take their origin in the middle region of the spinal cord, or rather leave the spinal cord by the nerves belonging to this middle region. Thus in the dog the vaso-constrictor fibres, not only for the trunk but for the limbs, head, face, and tail, leave the spinal cord by the anterior roots of the spinal nerves reaching from about the second dorsal to the fourth lumbar nerve, both inclusive. Running in the case of each nerve root to the mixed nerve trunk they pass along the visceral branch, white ramus communicans, to the chain of splanchnic ganglia lying in the thorax and abdomen-the so-called thoracic and abdominal sympathetic chain (Fig. 77). From these ganglia they reach their destination in various ways. Thus those going to the head and neck pass chiefly through the second and third dorsal and partly through the fourth, fifth and first dorsal nerves, thence upward through the annulus of Vieussens to the lower cervical ganglion, and thence, as we have seen, up the cervical sympathetic. Those for the abdominal viscera pass off in a similar way to the abdominal splanchnic nerves (Fig. 77, $a b d . s p l$.). Those destined for the fore limbs pass along in the fourth to the ninth dorsal nerves, both inclusive, chiefly in the seventh, and sometimes a few in the tenth, and so reach the brachial plexus; while those for the leg pass through the eleventh dorsal to the third lumbar, both inclusive, a few passing through the tenth dorsal and the fourth lumbar, and finally to the sciatic plexus. Those for the tail pass through in the first to third lumbar inclusive. The constrictor fibres of the skin of the trunk probably reach the spinal nerves in which they ultimately run in a similar manner. All the vaso-constrictor fibres, whatever their destination, leave the spinal cord by the anterior roots of spinal nerves, and then, passing through the appropriate visceral branches, join the thoracic or abdominal 
chain of splanchnic ganglia. In these ganglia the fibres undergo a remarkable change. Along the anterior root and along the visceral branch they are medullated fibres, but long before they reach the bloodvessels for which they are destined they become non-medullated fibres; they appear to lose their medulla in the system of splanchnic ganglia. We may add that in the anterior roots and along the visceral branches, white rami communicantes, these fibres are invariably of small diameter, not more than $1.8 \mu$ to $3.6 \mu$.

$\$ 156$. The course of the vaso-dilator fibres appears to be a somewhat different one, some apparently accompanying the vaso-constrictor fibres, and others ruming an independent course, though the details have as yet been fully worked out in the case of only a few of the fibres. It is chiefly in the nerves belonging to the cranial and sacral regions of the central nervous system, whence, as we have seen, no vaso-constrictor fibres are known to issue, that the course of the vaso-dilator fibres has been successfully traced. Thus the vaso-dilator fibres for the submaxillary gland running in the chorda tympani may be traced, as we have seen, back to the facial or seventh nerve; and the continuation of the chorda tympani along the lingual nerve to the tongue contains vaso-dilator fibres for that organ; when the lingual is stimulated, the bloodvessels of the tongue dilate owing to the stimulation of the conjoined corda tympani fibres. The ramus tympanicus of the glosso-pharyngeal nerve contains vaso-dilator fibres for the parotid gland, and it appears probable that the trigeminal nerve contains vaso-dilator fibres for the eye and nose and possibly for other parts. In the anterior roots of the sacral nerves run vaso-dilator fibres which pass into the so-called nervi erigentes, the nerves stimulation of which by leading to a widening of the arteries of the penis, brings about the erection of that organ, the effect being assisted by a simultaneous hindrance to the venous outflow. Though vaso-dilator fibres are, as we have seen, present in the nerves of the limbs, and probably also in those of the trunk, the investigation of their several paths is rendered very difficult by the concomitant presence of vaso-constrictor fibres. There are some reasons for thinking that the vaso-dilator fibres in these nerves pursue a direct course from the spinal cord through the anterior spinal roots, and thus afford a contrast with the constrictor fibres of the same nerves, which, as we have seen, take a roundabout course, passing into the splanchnic system before they join the nerve trunk. Our information, however, is too imperfect to allow any very positive statement to be made. Accepting this view, however, we may say that while all the vaso-constrictor fibres, as far as we know, come from a particular, though considerable, part of the spinal cord and pass into the splanchnic system on their way to their several destinations, the vaso-dilator fibres arise from all parts of the spinal cord as well as from the medulla oblongata, and pursue a more or less direct course to their destination.

Further, while the vaso-dilator fibres, as they leave the central nervous system, are, like the vaso-constrictor fibres, fine medullated fibres, unlike the vaso-constrictors they retain their medulla for the greater part of their course, and only lose it near their termination in the tissue whose bloodvessels they supply.

Lastly, while the vaso-constrictor fibres, as in the case of the cervical sympathetic, of the abdominal splanchnic, and of the nerves of the skin, and probably in all cases, are normally in a state of moderate activity (so long as they remain in connection with the central nervous system), the moderate activity maintaining that moderate constriction which we spoke of above as "tone," the vaso-dilators appear to possess no such continued activity. Section of vaso-constrictor fibres leads to loss of tone, diminution of constriction, lasting, as we shall see, for some considerable time; but 
section of vaso-dilators, according, at all events, to most observers, does not lead to analogous constriction or diminution of dilatation; all that is observed is a transient increase of dilatation due probably to the section acting as a transient stimulus to the nerve at the place of section. But before we study the use made by the central nervous system of vasomotor nerves, it will be best to consider briefly some features of

\section{The Effects of Vasomotor Actions.}

$\S 157$. A very little consideration will show that vasomotor action is a most important factor in the circulation. In the first place the whole flow of blood in the body is adapted to and governed by what we may call the general tone of the arteries of the body at large. In a normal condition of the body a very large number of the minute arteries of the body are in a state of tonic, i.e., of moderate, contraction, and it is the narrowing due to this contraction which forms a large item of that peripheral resistance which we have seen to be one of the great factors of blood-pressure. The normal general blood-pressure, and, therefore, the normal flow of blood, is in fact dependent on the "general tone" of the minute arteries.

In the second place local vasomotor changes in the condition of the

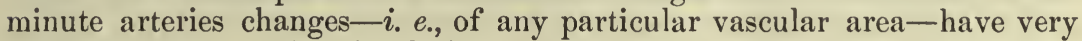
decided effects on the circulation. The changes, though local themselves, may have effects which are both local and general, as the following considerations will show:

Let us suppose that the artery $A$ is in a condition of normal tone, is midway between extreme constriction and dilatation. The flow through $A$ is determined by the resistance in $A$, and in the vascular tract which it supplies in relation to the mean arterial pressure, which is again dependent on the way in which the heart is beating and on the peripheral resistance of all the small arteries and capillaries, $A$ included. If, while the heart and the rest of the arteries remain unchanged, $A$ be constricted, the peripheral resistance in $A$ will increase, and this increase of resistance will lead to an increase of the general arterial pressure. Since, as we have seen ( $\$ 108)$, it is arterial pressure which is the immediate cause of the flow from the arteries to the veins, this increase of arterial pressure will tend to drive more blood from the arteries into the veins. The constriction of $A$, however, by increasing the resistance, opposes any increase of the flow through $A$ itself, in fact will make the flow through $A$ less than before. The whole increase of discharge from the arterial into the venous system will take place through the arteries in which the resistance remains unchanged, that is, through channels other than $A$. Thus, as the result of the constriction of an artery there occur (1) diminished flow through the artery itself, (2) increased general arterial pressure, leading to (3) increased flow through the other arteries. If, on the other hand, $A$ be dilated, while the heart and other arteries remain unchanged, the peripheral resistance in $A$ is diminished. This leads to a lowering of the general arterial pressure, which in turn tends to drive less blood from the arteries into the veins. The dilatation of $A$, however, by diminishing the resistance, permits, even with the lowered pressure, more blood to pass through $A$ itself than before. Hence the diminished flow tells all the more on the rest of the arteries in which the resistance remains unchanged. Thus, as the result of the dilatation of any artery, there occur (1) increased flow of blood through the artery itself, (2) diminished general pressure, and (3) diminished flow through the other arteries. Where the artery thus constricted or dilated is small, the local effect, the diminution or increase of flow through itself, is much more marked than the general effects, 
the change in blood-pressure and the flow through other arteries. When, however, the area, the arteries of which are affected, is large, the general effects are very striking. Thus if while a tracing of the blood-pressure is being taken by means of a manometer connected with the carotid artery, the abdominanl splanchnic nerves be divided, a conspicunus but steady fall of pressure is observed, very similar to but more marked than that which is seen in Fig. 99. The section of the abdominal splanchnic nerves causes the mesenteric and other abdominal arteries to dilate, and these being very numerous, a large amount of peripheral resistance is taken away, and the blood-pressure falls accordingly; a large increase of flow into the portal veins takes place, and the supply of blood to the face, arms, and legs is proportionately diminished. It will be observed that the dilatation of the arteries is not instantaneous but somewhat gradual, as shown by the pressure sinking not abruptly but with a gentle curve.

The general effects on blood-pressure by vasomotor changes are so marked that the manometer may be used to detect vasomotor actions. Thus, if the stimulation of a particular nerve or any other operation leads to a marked rise of the mean blood-pressure, unaccompanied by any changes in the heart-beat, we may infer that constriction has taken place in the arteries of some considerable vascular area; and similarly, if the effect be a fall of blood-pressure, we may infer that constriction has given way to dilatation.

\section{Vasomotor Functions of the Central Nervous System.}

$\S 158$. The central nervous system, to which we have traced the vasomotor nerves, makes use of these nerves to regulate the flow of blood through the various organs and parts of the body ; by the local effects thus produced it assists or otherwise influences the functional activity of this or that tissue; by the general effects it secures the well-being of the body.

The use of the vaso-rlilator nerves, which is more simple than that of the vaso-constrictors since it appears not to be complicated by the presence of habitual tonic influences, is frequently conspicuous as part of a reflex act. Thus, when food is placed in the mouth, afferent impulses, generated in the nerves of taste, give rise in the central nervous system to efferent impulses, which descend the chorda tympani and other nerves to the salivary glands, and, by dilating the bloodvessels, secure a copious flow of blood through the glands, while, as we shall see later on, they excite them to secrete. The centre of this reflex action appears to lie in the medulla oblongata, and may be thrown into activity not only by impulses reaching it along the specific nerves of taste, but also by impulses passing along other channels; thus, emotions started in the brain by the sight of food or otherwise may give rise to impulses passing down along the central nervous system itself to the medulla oblongata, or even in the stomach may send impulses up the vagus nerve, or stimulation of one kind or another may send impulses up almost any sentient nerve, and these various impulses reaching the medulla may, by reflex action, throw into activity the vaso-dilator fibres of the chorda tympani and other analogous nerves, and bring about a flushing of the salivary glands, while at the same time they cause the glands to secrete.

The vaso-dilator fibres of the nervi erigentes may be thrown into activity in a similar reflex way, the centre in this case being placed in the sacral or lumbar portion of the spinal cord, though it is easily thrown into activity by impulses descending down the spinal cord from the brain that such a centre does exist, is shown by the fact that when in a dog the spinal cord is completely divided in the dorsal region, erection of the penis may readily be 
brought about by stimulation of the sentient surfaces. And other instances might be quoted in which vaso-dilator fibres appear to be connected with a "centre" soon after their entrance into the nervous system.

If, as seems probable ( $\$ 153$ ), the bloodvessels of a muscle dilate by vasomotor action whenever the muscle is thrown into contraction, either in a reflex or voluntary movement, the vaso-dilator fibres of the muscle would seem to be thrown into action by impulses arising in the spinal cord not far from the origin of the ordinary motor impulses and accompanying those motor impulses along the motor nerve.

$\$ 159$. The case of the vaso-constrictor fibres is somewhat more complicated on account of the existence of tonic influences; since the same fibres may, on the one hand, by an increase in the impulses passing along them, be the means of constriction, and, on the other hand, by the removal or diminution of the tonic influences passing along them, be the means of dilatation. We have already traced all the vaso-constrictor fibres from the middle region of the spinal cord to the splanchnic system in the thorax and abdomen, from whence they pass (1) by the abdominal splanchnic and by the hypogastric nerves to the viscera of the abdomen and pelvis (concerning the vasomotor nerves of the thoracic viscera we know at present very little); (2) by the cervical sympathetic or cervical splanchnic, as it might be called, to the skin of the head and neck, the salivary glands and mouth, the eyes and other parts, and probably the brain, including its membranes; (3) by the brachial and sciatic plexuses to the skin of the fore and hind limbs, and by various other nerves to the skin of the trunk. The chief parts of the body supplied by vaso-constrictor fibres appear to be the skin, with its appendages, and the alimentary canal, with its appendages, glandular and other; the great mass of skeletal muscles appears to receive an insignificant supply of vaso-constrictor fibres.

If, now, in an animal, the spinal cord be divided in the lower dorsal region, the skin of the legs becomes flushed, their temperature frequently rises, and there is a certain amount of fall in the general blood-pressure as measured, for instance, in the carotid; and this state of things may last for some considerable time. Obviously, the section of the spinal cord has cut off the usual tonic influences descending to the lower limbs; in consequence the bloodvessels have become dilated, thus causing the general peripheral resistance to become proportionately diminished, and the general bloodpressure to fall. The tonic vaso-constrictor impulses for the lower limbs, therefore, have their origin in the central nervous system higher up than the lower dorsal region of the spinal cord.

If the spinal cord be divided between the roots of the fifth and sixth dorsal nerves (that is to say, at the level where the path of the splanchnic fibres from the cord seems to divide (see Fig. 77) those issuing above passing upward to the fore limbs and head, and those issuing below passing to the abrlomen and lower limbs), the cutaneous bloodvessels of the lower limbs dilate, as in the former case, and on examination it will be found that the bloodvessels of the abdomen are also largely dilated; at the same time the blood-pressure undergoes a very marked fall ; it may, indeed, be reduced to a very few millimetres of mercury. Obviously, the tonic vasn-constrictor impulses passing to the abdomen and to the lower limbs take origin in the central nervous system higher up than the level of the fifth dorsal nerve.

If the section of the spinal cord be made above the level of the second dorsal nerve, in addition to the above-mentioned results the vessels of the head and face also become dilated ; but, in consequence of the fall of general blood-pressure just mentioned, these vessels never become so full of blood, the loss of tone is not so obvious in them as after simple division of the 
cervical sympathetic, since the latter operation produces little or no effect on the general blood-pressure.

Obviously, then, the tonic vaso-constrictor impulses, which passing to the skin and viscera of the body maintain that tonic narrowing of so many small arteries by which the general peripheral resistance, and so the general bloodpressure, is maintained, proceed from some part of the central nervous system higher up than the upper dorsal region of the spinal cord. And, since exactly the same results follow upon section of the spinal cord in the cervical region right up to the lower limit of the medulla oblongata, we infer that these tonic impulses proceed from the medulla oblongata.

On the other hand, we may remove the whole of the brain right down to the upper parts of the medulla, and yet produce no flushing, or only a slight transient flushing, of any part of the body, and no fall at all, or only a slight transient fall, of the general blood-pressure. We, therefore, seem justified in assuming the existence in the medulla oblongata of a nervous centre, which we may speak of as a vasomotor centre, or the medullary vasomotor centre, from which proceed tonic vaso-constrictor impulses, or which regulates the emission and distribution of such tonic vaso-constrictor impulses or influences over various parts of the body.

$\$ 160$. The existence of this vasomotor centre may, moreover, be shown in another way. The extent or amount of the tonic constrictor impulses proceeding from it may be increased or diminished, the activity of the centre may be augmented or inhibited by impulses reaching it along various afferent nerves; and provided no marked changes in the heart-beat take place at the same time, a rise or fall of general blood-pressure may be taken as a token of an increase or decrease of the activity of the centre.

In the rabbit there is found in the neck, lying side by side with the cervical sympathetic nerve and running for some distance in company with it, a slender nerve which may be ultimately traced down to the heart, and which if traced upward is found to eome off somewhat high up from the vagus, by two or more roots, one of which is generally a branch of the superior laryngeal nerve. This nerve (the fibres constituting which are, in the $d o g$, bound up with the vagus, and do not form an independent nerve) appears to be exclusively an afferent nerve; when, after division of the nerve the peripheral end, the end still in connection with the heart, is stimulated, no marked results follow. The beginnings of the nerve in the heart are therefore quite different from the endings of the inhibitory fibres of the vagus, or of the augmentor fibres of the splanchnic (sympathetic) system; the nerve has nothing to do with the nervous regulation of the heart (see $\$ \$ 139$ et seq.). If, now, while the pressure in an artery such as the carotid is

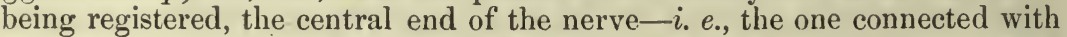
the brain-be stimulated with the interrupted current, a gradual but marked fall of pressure (Fig. 79) in the carotid is observed, lasting, when the period of stimulation is short, some time after the removal of the stimulus. Since the beat of the heart is not markedly changed, the fall of pressure must be due to the diminution of peripheral resistance occasioned by the dilatation of some arteries. And it is probable that the arteries thus dilated are chiefly, if not exclusively, those arteries of the abdominal viscera which are governed by the abdominal splanchnic nerves; for if these nerves are divided on both sides previous to the experiment, the fall of pressure when the nerve is stimulated is very small-in fact, almost insignificant. The inference we draw is as follows : The afferent impulses, passing upward along the nerve in question, have so affected some part of the central nervous system that the influences. which, in a normal condition of things, passing. along the abdominal splanchnic nerves keep the minute arteries of the 
abdominal viscera in a state of moderate tonic constriction, fail altogether, and those arteries in consequence dilate just as they do when the abdominal

Fig. 79.

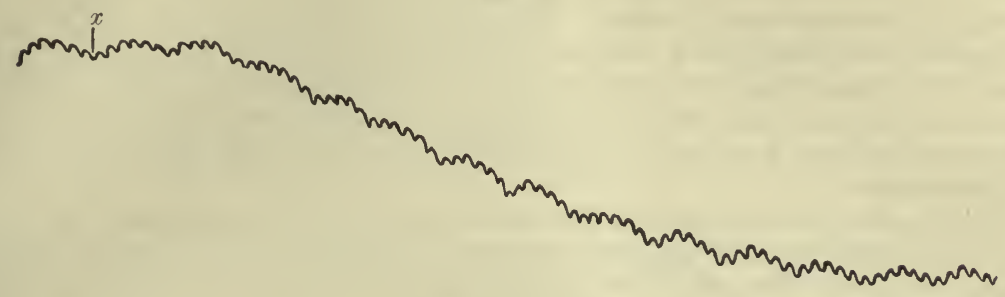

Tracing showing the Effect on Blood-pressure of Stimulating the Central End of the Depressor Nerve in the Rabbit. On the time-marker below the intervals correspond to seconds. At $x$ an interrupted current was thrown into the nerve.

splanchnic nerves are divided, the effect being possibly increased by the similar dilatation of other vascular areas. Since stimulation of the nerve of which we are speaking always produces a fall, never a rise, of blood-pressure-the amount of fall, of course, being dependent on circumstances, such as the condition of the nervous system, state of blond-pressure, etc.-the nerve is known by the name of the depressor nerve. As we shall point out later on, by means of this afferent nerve from the heart the peripheral resistance is, in the living body, lowered to suit the weakened powers of a laboring heart.

This gradual lowering of blood-pressure by diminution of peripheral resistance affords a marked contrast to the sudden lowering of blood-pressure by cardiac inhibition. (Compare Fig. 79 with Fig. 75.)

$\S 161$. But the general blood-pressure may be modified by afferent impulses passing along other nerves than the depressor, the modification taking on, according to circumstances, the form either of decrease or of increase.

Thus, if, in an animal placed under the influence of urari (some anæsthetic other than chloral, etc., being used), the central stump of the divided sciatic nerve be stimulated, an increase of blood-pressure (Fig. 80) almost

\section{Fig. 80.}

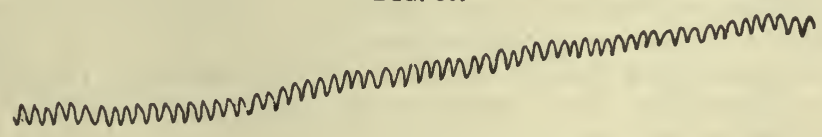

$x$

Effect on Blood-pressure Curve of Stimulating Sciatic Nerve under Urari. (Cat.) $x$ marks the moment in which the current was thrown into the nerve. Artificial respiration was carried on, and the usual respiratory undulations are absent.

exactly the reverse of the decrease brought about by stimulating the depressor, is observed. The curve of the blood-pressure, after a latent period during which no changes are visible, rises steadily without any corresponding change in the heart's beat, reaches a maximum, and after a 
while slowly falls again, the fall sometimes beginning to appear before the stimulus has been removed. There can be no doubt that the rise of pressure is due to the constriction of certain arteries; the arteries in question being those of the abdominal splanchuic area certainly, and possibly those of other vascular areas as well. The effect is not confined to the sciatic; stimulation of any nerve containing afferent fibres may produce the same rise of pressure, and so constant is the result that the experiment has been made use of as a method of determining the existence of afferent fibres in any given nerve and even the paths of centripetal impulses through the spinal cord.

If, on the other hand, the animal be under the influence not, of urari, but of a large dose of chloral, instead of a rise of blood-pressure, a fall quite similar to that caused by stimulating the depressor is observed when an afferent nerve is stimulated. The condition of the central nervous system seems to determine whether the effect of afferent impulses on the central nervous system is one leading to an augmentation of vaso-constrictor impulses and so to a rise, or one leading to a diminution of vaso-constrictor impulses and so to a fall of blood-pressure.

$\S 162$. We have used the words " central nervous system " in speaking of the above; we have evidence, however, that the part of the central nervous system acted on by the afferent impulses is the vasomotor centre in the medulla oblongata, and that the effects in the way of diminution (depressor) or of augmentation (pressor) are the results of afferent impulses inhibiting or augmenting the tonic activity of this centre or of a part of this centre especially connected with abdominal splanchnic nerves. The whole brain may be removed right down to the medulla oblongata, and yet the effects of stimulation in the direction either of diminution or of augmentation may still be brought about. If the medulla oblongata be removed, these effects vanish too, though all the rest of the nervous system be left intact. Nay, more, by partially interfering with the medulla oblongata, we may partially diminish these effects and thus mark out, so to speak, the limits of the centre in question within the medulla itself. Thus, in an intact animal under urari, stimulation of the sciatic nerve with a stimulus of a certain strength will produce a rise of blood-pressure up to a certain extent. After removal of the whole brain right down to the medulla oblongata, the same stimulation will produce the same rise as before; the vasomotor centre has not been interfered with. Directly, however, in proceeding downward, the region of the centre in question is reached, stimulation of the sciatic produces less and less rise, until at last when the lower limit of the centre is arrived at no effect at all on bloodpressure can be produced by even strong stimulation of the sciatic or other afferent nerve. In this way the lower limit of the medullary vasomotor centre has been determined in the rabbit at a horizontal line drawn about 4 or $5 \mathrm{~mm}$. above the point of the calamus scriptorius, and the upper limit at about $4 \mathrm{~mm}$. higher up-i. e., about 1 or $2 \mathrm{~mm}$. below the corpora quadrigemina. When transverse sections of the brain are carried successively lower and lower down, an effect on blood-pressure in the way of lowering it and also of diminishing the rise of blood-pressure resulting from stimulation of the sciatic, is first observer when the upper limit is reached. On carrying the sections still lower, the effects of stimulating the sciatic become less and less, until when the lower limit is reached no effects at all are observed. The centre appears to be bilateral, the halves being placed not in the middle line, but more sideways and rather nearer the anterior than the posterior surface. It may perhaps be more closely defined as a small prismatic space in the forward prolongation of the lateral columns after they have given off their fibres to the decussating pyramids. This space is largely occupied by a mass 
of gray matter, called by Clarke the antero-lateral nucleus, and containing large multipolar cells ; but it is by no means certain that this group of nerve cells really acts as the centre in question.

$\$ 163$. The above experiments appear to afford adequate evidence that, in a normal state of the body, the integrity of the medullary vasomotor centre is essential to the production and distribution of those continued constrictor impulses by which the general arterial tone of the body is maintained, and that an increase or decrease of vaso-constrictor action in particular arteries, or in the arteries generally, is brought about by means of the same medullary vasomotor centre. But we must not, therefore, conclude that this small portion of the medulla oblongata is the only part of the central nervous system which can act as a centre for vaso-constrictor fibres; and, as we have seen, there is no evidence at present that the vaso-dilator fibres are connected with either this or any other one centre. In the frog reflex vasomotor effects may be obtained by stimulating various afferent nerves after the whole medulla has been removed, and, indeed, even when only a comparatively small portion of the spinal cord has been left intact and connected, on the one hand, with the afferent nerve which is being stimulated, and, on the other, with the efferent nerves in which run the vasomotor fibres whose action is being studied. In the mammal such effects do not so readily appear, but may with care and under special conditions be obtained. Thus in the dog, when the spinal cord is divided in the dorsal region, the arteries of the hind limbs and hinder part of the body, as we have already said ( $\$ 158$ ), become dilated. This one would naturally expect as the result of their severance from the medullary vasomotor centre. But if the animal be kept in good condition for some time, a normal or nearly normal arterial tone is after a while re-established; and the tone thus regained may, by afferent impulses reaching the cord below the section, be modified in the direction certainly of diminution-i. e., dilatation-and possibly, but this is by no means so certain, of increase-i.e., constriction. Dilatation of various cutaneous vessels of the limbs may be readily produced by stimulation of the central stump of one or another nerve.

These remarkable results, which, though they are most striking in connection with the lower part of the spinal cord, hold good apparently for other parts also of the spinal core, naturally suggest a doubt whether the explanation just given above of the effects of section of the medulla oblongata is a valid one. When we come to study the central nervous system, we shall again and again see that the immediate effect of operative interference with these delicate structures is a temporary suspension of nearly all their functions. This is often spoken of as "shock" and may be regarded as an extreme form of inhibition. An example of it occurs in the above experiment of section of the dorsal cord. For some time after the operation the vaso-dilator nervi erigentes (which, as far as we know, have no special connection with the medullary vasomotor centre) cannot be thrown into activity as part of a reflex action; their centre remains for some time inactive. After a while, however, it recovers, and erection of the penis through the nervi erigentes may then still be brought about by suitable stimulation of sensory surfaces. Hence the question may fairly be put whether the effects of cutting and injuring the structures which we have spoken of as the medullary vasomotor centre, are not in reality simply those of shock, whether the vascular dilatation which follows upon sections of the so-called medullary vasomotor centre, does not come about because section of or injury to this region exercises a strong inhibitory influence on all the vasomotor centres situated in the spinal cord below. Owing to the special function of the medulla oblongata in carrying on the all-important work of respiration, a mammal 
whose medulla has been divided cannot be kept alive for any length of time. We cannot, therefore, put the matter to the simple experimental test of extirpating the supposed medullary vasomotor centre and seeing what happens when the animal has completely recovered from the effects of the operation; we have to be guided in our decision by more or less indirect arguments. And against the argument that the effects are those of shock, we may put the argument, evidence for which we shall meet with in dealing with the central nervous system, that when one part of the central nervous system is removed or in any way placed hors de combat, another part may vicariously take on its function; in the absence of the medullary vasomotor centre, its function may be performed by other parts of the spinal cord which in its presence do no such work.

And we may, in connection with this, call attention to the fact that the dilatation or loss of tone which follows upon section of the cervical sympathetic (and the same is true of the abdominal splanchnic) is not always, though it may be sometimes, permanent; in a certain number of cases it has been found that after a while, it may not be until after several days, the dilatation disappears and the arteries regain their usual calibre; on the other hand, in some cases no such return has been observed after months or even years. This recovery, when it occurs, cannot always be attributed to any regeneration of vasomotor fibres in the sympathetic, for it is stated to have been observed when the whole length of the nerve including the superior cervical ganglion has been removed. When recovery of tone has thus taken place, dilatation or increased constriction may be occasioned by local treatment ; the ear may be made to blush or pale by the application of heat or cold, by geutle stroking or rough handling and the like; but neither the one nor the other condition can be brought about by the intervention of the central nervous system. So also the spontaneous rhythmic variations in the calibre of the arteries of the ear of which we speak, though they cease for a time after division of the cervical sympathetic, may in some cases eventually reappear and that even if the superior cervical ganglion be removed; in other cases they do not. And the analogous rhythmic variations of the veins of the bat's wing have been proved experimentally to go on vigorously when all connection with the central nervous system has been severed; they may continue, in fact, in isolated pieces of the wing provided that the vessels are adequately filled and distended with blood or fluid. From these and other facts, even after making allowance for the negative cases, we may conclude that what we have spoken of as the tone of the vessels of the face, though influenced by and in a measure dependent on the central nervous system, is not simply the result of an effort of that system. The muscular walls of the arteries are not mere passive instruments worked by the central nervous system through the vasomotor fibres; they appear to have an intrinsic tone of their own, and it seems natural to suppose that when the central nervous system causes dilatation or constriction of the vessels of the face, it makes use, in so doing, of this intrinsic local tone. It has been supposed that this intrinsic tone is dependent on some local nervous mechanism; in the ear at least no such mechanism has yet been found; and, indeed, as we have said above ( $\$ 176)$, no such peripheral nervous mechanism is really necessary. In the case both of a vessel governed by vaso-dilator fibres and one governed by vaso-constrictor fibres, we may suppose a certain natural condition of the muscular fibres which we may call a condition of equilibrium. In a vessel governed only by vaso-dilator fibres, if there be such, this condition of equilibrium is the permanent condition of the muscular fibre, from which it is disturbed by vaso-dilator impulses, but to which it speedily returns. In a vessel governed by vaso-constrictor fibres, and subject to tone, the muscu- 
lar fibre is habitually kept on the constrictor side of this equilibrium, and, as in the cases quoted above, may strive of itself toward some amount of active constriction even when separated from the central nervous system.

But to return to the medullary vasomotor centre. Without attempting to discuss the matter fully, we may say that, after all due weight has been attached to the play of inhibitory impulses or "shock" as a result of operative interference, there still remains a balance of evidence in favor of the view that the region of the medulla of which we are speaking does really act as a general vasomotor centre in the manner previously explained, and plays an important part in the vasomotor regulation of the living body.

It is not, however, to be regarded as the single vasomotor centre, whence alone can issue tonic-constrictor impulses or whither afferent impulses from all parts of the body must always travel before they can affect the vasomotor impulses passing along this or that nerve. We are rather to suppose that the spinal cord along its whole length contains, interlaced with the reflex and other mechanisms by which the skeletal muscles are governed, vasomotor centres and mechanisms of varied complexity, the details of whose functions and topography have yet largely to be worked out; and though, as we have seen, the medullary centre is essentially a centre of impulses issuing along vaso-constrictor fibres, it is possible that there are ties between it and vaso-dilator fibres also. As in the absence of the sinus venosus, the auricles and ventricle of the frog's heart may still continue to beat, so in the absence of the medulla oblongata these spinal vasomotor centres provide for the vascular emergencies which arise. As, however, in the normal entire frog's heart, the sinus, so to speak, gives the word and governs the work of the whole organ, so the medullary vasomotor centre rules and co-ordinates the lesser centres of the cord, and through them presides over the chief vascular areas of the body. By means of these vasomotor central mechanisms, by means of the head centre in the medulla, and the subsidiary centres in the spinal cord, the delicate machinery of the circulation which determines the blood-supply, and so the activity of each tissue and organ, is able to respond by narrowing or widening arteries to the evervarying demands and to meet by compensating changes the shocks and strains of daily life.

$\S 164$. We may sum up the history of vasomotor actions somewhat as follows:

All, or nearly all—or, as far as we know, all - the arteries of the body are connected with the central nervous system by nerve fibres, called vasomotor fibres, the action of which varies the amount of contraction of the muscular coats of the arteries, and so leads to changes in calibre. The action of these vasomotor fibres is more manifest and probably more important in the case of small and minute arteries than in the case of larger ones.

These vasomotor fibres are of two kinds: the one kind, vaso-constrictor fibres, are of such a nature or have such connections at their central origin or peripheral endings that stimulation of them produces narrowing, constriction of the arteries; and during life these fibres appear to be the means by which the central nervous system exerts a continued tonic influence on the arteries and maintains an arterial "tone." The other kind, the vasodilator fibres, are of such a kind or have such connections that stimulation of them produces widening, dilation of the arteries. There is no adequate evidence that these vaso-dilator fibres serve as channels for tonic dilating impulses or influences.

The vaso-constrictor fibres leave the spinal cord by the anterior roots of the nerves coming from middle regions only of the spinal cord (in the dog, 
and probably in other mammals from about the first dorsal to the fifth lumbar nerve), pass into the splanchnic ganglia connected with those nerves (thoracic and abdominal chain of sympathetic ganglia), where the fibres lose their medulla, and proceed to their destination as non-medullated fibres, either still in so-called sympathetic nerves, such as splanchnic, cervical sympathetic, hypogastric, etc., or along recurrent branches of the splanchnic system, to join the spinal nerves of the arm, leg, and trunk.

In the intact organism the emission and distribution along these vasoconstrictor fibres of tonic-constrictor impulses, by which general and local arterial tone is maintained and regulated, is governed by a limited portion of the medulla oblongata known as the medullary vasomotor centre; and when some change of conditions or other natural stimulus brings about a change in the activity of the vaso-constrictor fibres of one or more vascular areas, or of all the arteries supplied with vaso-constrictor fibres, this same medullary vasomotor centre appears in such cases to play the part of a centre of reflex action. Nevertheless, in cases where the nervous connections of this medullary vasomotor centre with a vascular area are cut off by an operation, as by section of the cord, other parts of the spinal cord may act as centres for the vaso-constrictor fibres of the area, and possibly these subordinate centres may be to a certain extent in action in the intact organism.

The vaso-dilator fibres appear to take origin in various parts of the central nervous system and to proceed in a direct course to their destination along the (anterior) roots and as part of the trunks and branches of various cerebro-spinal nerves; they do not lose their medulla until they approach their termination. They do not appear to serve as channels of tonic dilating influences; they are thrown into action generally as part of a reflex action, and their centre in the reflex act appears in each case to lie in the central nervous system not far from the centre of the ordinary motor fibres which they accompany.

The effects of the activity of the vaso-dilator fibres appear to be essentially local in nature; when any set of them come into action the vascular area which these govern is dilated. And the vascular areas so governed are relatively so small that changes in them produce little or no effect on the vascular system in general.

The effects of changes in the activity of the vaso-constrictor fibres are both local and general, and may be also double in nature. By an inhibition of tonic-constrictor impulses a certain amount of dilation may be effected; by an augmentation of constrictor impulses, constriction, it may be of considerable extent, may be brought about. When the vascular area so affected is small, the effects are local, more or less blood is distributed through the area; when the vascular area affected is large, the inhibition of constriction may lead to a marked fall, and an augmentation of constriction to a marked rise of general blood-pressure.

$\$ 165$. We shall have occasion later on again and again to point out instances of the effects of vasomotor action, both local and general, but we may here quote one or two characteristic ones. "Blushing" is one. Nervous impulses started in some parts of the brain by an emotion produce a powerful inhibition of that part of the medullary vasomotor centre which governs the vascular areas of the head supplied by the cervical sympathetic, and hence has an effect on the vasomotor fibres of the cervical sympathetic almost exactly the same as that produced by section of the nerve. In consequence the muscular walls of the arteries of the head and face relax, the arteries dilate, and the whole region becomes suffused. Sometimes an emotion gives rise not to blushing, but to the opposite effect, viz., to pallor. 
In a great number of cases this has quite a different cause, being due to a sudden diminution or even temporary arrest of the heart's beats; but in some cases it may occur without any change in the beat of the heart, and is then due to a condition the very converse of that of blushing, that is, to an increased arterial constriction ; and this increased constriction, like the dilatation of blushing, is effected through the agency of the central nervous system and the cervical sympathetic.

The vascular condition of the skin at large affords another instance. When the temperature of the air is low the vessels of the skin are constricted and the skin is pale; when the temperature of the air is high the vessels of the skin are dilated and the skin is red and flushed. In both these cases the effect is mainly a reflex one, it being the central nervous system which brings about augmentation of constriction in the one case and inhibition in the other, though possibly some slight effect is reproduced by the direct action of the cold or heat on the vessels of the skin simply. Moreover, the vascular changes in the skin are accompanied by corresponding vascular changes in the viscera (chiefly abdominal) of a reverse kind. When the vessels of the skin are dilated those of the viscera are constricted, and vice versa, so that a considerable portion of the whole blood ebbs and flows, so to spzak, according to circumstances from skin to viscera and from viscera to skin. By these changes, as we shall see later on, the maintenance of the normal temperature of the body is in large measure secured.

When food is placed in the mouth the bloodvessels of the salivary glands, as we have seen, are flushed with blood as an adjuvant to the secretion of digestive fluid; and as the food passes along the alimentary canal, each section in turn, with the glandular appendages belonging to it, welcomes its advent by flushing with blood, the dilatation being sometimes, as in the case of the salivary gland, the result of the activity chiefly of vaso-dilator fibres, but sometimes the result of the cessation of constrictor impulses and sometimes the result of the two combined. So also when the kidney secretes urine, its vessels become dilated, and in general, wherever functional activity comes into play, the metabolism of tissue which is the basis of that activity is assisted by a more generous flow of blood through the tissue.

$\S 166$. Vasomotor nerves of the veins. Although the veins are provided with muscular fibres and are distinctly contractile, and although rhythmic variations of calibre due to contractions may be seen in the great veins opening into the heart, in the veins of the bat's wing, and elsewhere, and similar rhythmic variations, also possibly due to active rhythmic contractions, but possibly also of an entirely passive nature, have been observed in the portal veins, very little is known of any nervous arrangements governing the veins. When in the frog the brain and spinal cord are destroyed, very little blood comes back to the heart as compared with the normal supply, and the heart in consequence appears almost bloodless and beats feebly. This has been, by some, regarded as more than can be accounted for by mere loss of arterial tone, and accordingly interpreted as indicating the existence of a normal tone in the veins dependent on the central nervous system. When the latter is destroyed, the veins become abnormally distended and a large quantity of blood becomes lodged and hidden, as it were, in them.

\section{The Capillary Circulation.}

$\S 167$. We have already some time back ( $\$ 106)$ mentioned some of the salient features of the circulation through the capillaries, viz., the difficult passage of the corpuscles (generally in single file, though sometimes in the larger channels two or more abreast) and plasma through the narrow chan- 
nels, in a stream which though more or less irregular is steady and even, not broken by pulsations, and slower than that in either the arteries or the veins. We have further seen that the capillaries vary very much in width from time to time; and there can be no doubt that the changes in their calibre are chiefly of a passive nature. They are expanded when a large supply of blood reaches them through the supplying arteries, and, by virtue of their elasticity, shrink again when the supply is lessened or withdrawn; they may also become expanded by an obstacle to the venous outflow.

On the other hand, as we have also stated, there is a certain amount of evidence that, in young animals at all events, the calibre of a capillary canal may vary, quite independently of the arterial supply or the venous outflow, in consequence of changes in the form of the epithelioid cells, allied to the changes which in a muscle-fibre or muscle-cell constitute a contraction; and though the matter requires further investigation, it is possible that these active changes play an important part in determining the quantity of blood passing through a capillary area; but there is as yet no satisfactory evidence that they, like the corresponding changes in the arteries, are governed by the nervous system.

Over and above these changes of form, the capillaries and minute vessels are subject to changes and exert influences by virtue of which they play an important part in the work of the circulation. Their condition determines the amount of resistance offered by their channels to the flow of blood through those channels, and determines the amount and character of that interchange between the blood and the tissues which is the main fact of the circulation.

If the web of the frog's foot, or, better still, if some transparent tissue of a mammal be watched under the microscope, it will be observed that, while in the small capillaries the corpuscles are pressed through the channel in single file, one after the other, each corpuscle as it passes occupying the whole bore of the capillary, in the larger capillaries (of the mammal), and especially in the small arteries and veins which permit the passage of more than one corpuscle abreast, the red corpuscles run in the middle of the channel, forming a colored core, between which and the sides of the vessels all around is a colorless layer, containing no red corpuscles, called the "plasmatic layer " or "peripheral zone." This division into a peripheral zone and an axial stream is due to the fact that in any stream passing through a closed channel the friction is greatest at the sides, and diminishes toward the axis. The corpuscles pass where the friction is least, in the axis. A quite similar axial core is seen when any fine particles are driven with a sufficient velocity in a stream of fluid through a narrow tube. As the velocity is diminished the axial core becomes less marked and disappears.

In the peripheral zone, especially in that of the veins, are frequently seen white corpuscles, sometimes clinging to the sides of the vessel, sometimes rolling slowly along, and in general moving irregularly, stopping for a while and then suddenly moving on. The greater the velocity of the flow of blood, the fewer the white corpuscles in the peripheral zone, and with a very rapid flow they, as well as the red corpuscles, may be all confined to the axial stream. The presence of the white corpuscles in the peripheral zone has been attributed to their being specially lighter than the red corpuscles, since when fine particles of two kinds, one lighter than the other, are driven through a narrow tube, the heavier particles flow in the axis and the lighter in the more peripheral portions of the stream. But, besides this, the white corpuscles have a greater tendency to adhere to surfaces than have the red, as is seen by the manner in which the former become fixed to the glass slide and cover-slip when a drop of blood is mounted for microscopical 
examination. They probably thus adhere by virtue of the amœboid movements of their protoplasm, so that the adhesion is to be considered not so much a mere physical as a physiological process, and hence may be expected to vary with the varying nutritive conditions of the corpuscles and of the bloodvessels. Thus while the appearance of the white corpuscles in the peripheral zone may be due to their lightness, their temporary attachment to the sides of the vessels and characteristic progression is the result of their power to adhere; and as we shall presently see, their amœboid movements may carry them on beyond mere adhesion.

$\S 168$. These are the phenomena of the normal circulation, and may be regarded as indicating a state of equilibrium between the blond on the one hand and the bloodvessels with the tissues on the other; but a different state of things sets in when that equilibrium is overthrown by causes leading to what is called inflammation or to allied conditions.

If an irritant, such as a drop of chloroform or a little diluted oil of mustard, be applied to a small portion of a frog's web, tongue, mesentery, or some other transparent tissue, the following changes may be observed under the microscope; they may also be seen in the mesentery or other transparent tissue of a mammal. The first effect that is noticed is a dilatation of the arteries, accompanied by a quickening of the stream. The irritant, probably by a direct action on the muscular fibres of the arteries, has led to a relaxation of the muscular coat and hence to a widening; and we have already ( $\$ 112)$, explained how such a widening in a small artery may lead to a temporary thickening of the stream. In consequence of the greater flow through the arteries, the capillaries become filled with corpuscles, and many passages, previously invisible or nearly so on account of their containing no corpuscles, now come into view. The veins at the same time appear enlarged and full. If the stimulus be very slight, this may all pass away, the arteries gaining their normal constriction and the capillaries and veins returning to their normal condition; in other words, the effect of the stimulus in such a case is simply a temporary blush. Unless, however, the chloroform or mustard be applied with especial care the effects are much more profound, and a series of remarkable changes sets in.

In the normal circulation, as we have just said, white corpuscles may be seen in the peripheral, plasmatic zone, but they are scanty in number, and each one after staying for a little time in one spot suddenly gets free, sometimes almost by a jerk, as it were, and then rolls on for a greater or less distance. In the area now under consideration a large number of white corpuscles soon gather in the peripheral zones, especially of the veins and venous capillaries (that is, of the larger capillaries which are joining to form veins), but also, to a less extent, of the arteries; and this takes place although the vessels still remain dilated and the stream still continues rapid though not so rapid as at first. Each white corpuscle appears to exhibit a greater tendency to stick to the sides of the vessels, and though driven away from the arteries by the stronger arterial stream, beconses lodged, so to speak, in the veins. Since new white corpuscles are continually being brought by the blood-stream on to the scene, the number of them in the peripheral zones of the veins increases more and more, and this may go on until the inner surface of the veins and venous capillaries appears to be lined with a layer of white corpuscles. The small capillaries, too, contain more white corpuscles than usual, and even in the arteries these are abundant, though not forming the distinct layer seen in the veins. The white corpuscles, however, are not the only bodies present in the peripheral zone. Though in the normal circulation blood-platelets (see $\S 33$ ) cannot 
be seen in the peripheral zone, and hence must be confined (on the view, which has the greater support, that these bodies are really present in quite normal blood) to the axial stream, they make their appearance in that zone with the changes which we are now describing. Indeed, in many cases they are far more abundant than the white corpuscles, the latter appearing imbedded at intervals in masses of the former. Soon after their appearance the individual platelets lose their outline and run together into formless masses.

$\$ 169$. This much, the appearance of numerous white corpuscles and platelets in the peripheral zones may take place while the stream, though less rapid than at the very first, still remains rapid; so rapid at all events that, owing to the increased width of the passages, in spite of the obstruction offered by the adherent white corpuscles, the total quantity of blood flowing in a given time through the inflamed area is greater than normal. But soon, though the vessels still remain dilated, the stream is observed most distinctly to slacken and then a remarkable phenomenon makes its appearance. The white corpuscles lying in contact with the walls of the veins or of the capillaries are seen to thrust processes through the walls; and, the process of a corpuscle increasing at the expense of the rest of the body of the corpuscle, the whole corpuscle, by what appears to be an example of amœboid movement, makes its way through the wall of the vessel into the lymph space outside; the perforation appears to take place in the cement substance joining the epithelioid plates together. This is the migration of the white corpuscles to which we alluded in $\S 32$, and takes place chiefly in the veins and capillaries, not at all or to a very slight extent in the arteries. Through this migration the lymph spaces around the vessels in the inflamed area become crowded with white corpuscles. At the same time the lymph in the same spaces not only increases in amount but changes somewhat in its chemical characters; it becomes more distinctly and readily coagulable, and is sometimes spoken of as "exudation fluid," or by the older writers as " coagulable lymph." This turgescence of the lymph spaces, together with the dilated, crowded condition of the bloodvessels, gives rise to the swelling which is one of the features of inflammation.

If the inflammation now passes off the white corpuscles cease to emigrate, cease to stick for any length of time to the sides of the vessels, the stream of blood through the vessells quickens again, and the vessels themselves, though they may remain for a long time dilated, eventually regain their calibre, and a normal circulation is re-established. The migrated corpuscles move away from the region, along the labyrinth of lymph spaces, and the surplus lymph also passes away along the lymph spaces and lymphatic vessels.

$\$ 170$. 'The condition of things, however, instead of passing off may go on to a further stage. More and more white corpuscles, arrested in their passage, crowd the channels and block the way, so that though the vessels remain dilated the stream becomes slower and slower, until at last it stops altogether and "stagnation" or "stasis" sets in. The red corpuscles are driven in, often in masses, among the white corpuscles and platelets, the distinction between axial stream and peripheral zone becoming lost; and arteries, veins, and capillaries, all distended, sometimes enormously so, are filled with a mass of mingled red and white corpuscles and platelets. When actual stagnation occurs the red corpuscles run together so that their outlines are no longer distinguishable; they appear to become fused into a homogeneous red mass. And it may now be observed that, not only white corpuscles but also red corpuscles make their way through 
the distended and altered walls of the capillaries, chiefly, at all events, at the junctions of the epithelioid plates, into the lymph spaces beyond. This is spoken of as the diapedesis of the red corpuscles.

This latter "stagnation" stage of inflammation may be the prelude to further mischief and indeed to the death of the inflamed tissue, but it, too, like the earlier stages, may pass away. As it passes away the outlines of the corpuscles become once more distinct, those on the venous side of the block gradually drop away into the neighboring currents-little by little the whole obstruction is removed, and the current through the area is re-established.

The slowing and final arrest of the blood current described above is not due to any lessening of the heart's beat; the arterial pulsations, or at least the arterial flow, may be seen to be continued in full force down to the affected area, and there to cease very suddenly. It is not due to any constriction of the small arteries increasing the peripheral resistance, for these continue dilated, sometimes exceedingly so. It must, therefore, be due to some new and unusual resistance occurring in the area itself, and there can be no doubt that this is to be found in an increased tendency of the corpuscles, especially of the white corpuscles, to stick to the sides of the vessels. The increase of adhesiveness is not caused by any change confined to the corpuscles themselves; for if, after a temporary delay, one set of corpuscles has managed to pass away from the affected area, the next set of corpuscles brought to the area in the blood-stream is subjected to the same delay and the same apparent fusion. The cause of the increased adhesiveness must, therefore, lie in the walls of the bloodvessels or in the tissue of which these form a part. That the increased adhesion is due to the vascular walls and not primarily to the corpuscles themselves is further shown by the fact that if in the frog, an artificial blood of normal saline solution to which milk has been added be substituted for normal blood, a stasis may by irritants be induced in which oil-globules play the part of corpuscles, and by their aggregation bring about an arrest of the flow.

We are driven to conclude that there exist in health certain relations between the blood, on the one hand, and the walls of the vessel on the other, by which the tendency of the corpuscles to adhere to the bloodvessels is kept within certain limits; these relations consequently determine the normal flow, with its axial stream and peripheral zone, and the normal amount of peripheral resistance; in inflammations these relations, in a manner we cannot as yet fully explain, are disturbed so that the tendency of the corpuscles to adhere to the sides of the vessel is largely and progressively increased. Hence the tarrying of the corpuscles in spite of the widening of their path, and finally their agglomeration and fusion in the distended channels.

We may add that the changes occurring in the vascular walls also at least facilitate the migration of the corpuscles, and modify the passage from the blood to the tissue of the fluid parts of the blood, the lymph of inflamed areas being richer in proteids than normal lymph.

We must not, however, pursue this subject of inflammation any further. We have said enough to show that the peripheral resistance (and consequently all that depends on that peripheral resistance) is not wholly determined by the varying width of the minute passages, but is also dependent on the vital condition of the tissue of which the walls of the passage form a part. When the tissue is in health, a certain resistance is offered to the passage of blood through the capillaries and other minute vessels, and the whole vascular mechanism is adapted to overcome this resistance to such an extent that a 
normal circulation can take place. When the tissue becomes affected, the disturbance of the relations between the tissue and the blood may, as in the later stages of inflammation, so augment the resistance that the passage of the blood becomes at first difficult and ultimately impossible. And it is quite open to us to suppose that under certain circumstances the reverse of the above may occur in this or that area, conditions in which the resistance may be lowered below the normal and the circulation in the area quickened. Thus the vital condition of the tissue becomes a factor in the maintenance of the circulation; and it is possible, though not yet proved, that these vital conditions are directly under the dominion of the nervous system.

$\$ 171$. Changes in the peripheral resistance may also be brought about by changes in the character of the blood, especially by changes in the relative amount of gases present. When a stream of defibrinated blood is artificially driven through a perfectly fresh excised organ such as the kidney, it is found that the resistance to the flow of blood through the organ, measured for instance by the amount of outflow in relation to the pressure exerted, varies considerably owing to changes taking place in the organ, and may be increased by increasing the venous character and diminished by increasing the arterial character of the blood. Remarkable changes in the resistance are also brought about by the addition of small quantities of certain drugs such as chloral, atropine, etc., to the blood.

These changes have been attributed to the altered blood acting on the walls of the vessels, inducing, for instance, constriction or widening of the small arteries, or it may be affecting the capillaries, for it has been asserted that the epithelioid plates of the capillaries vary in form according to the relative quantities of carbonic acid and oxygen present in the blood. But this is not the whole explanation of the matter, since similar variations in resistance are met with when blood is driven through fine capillary tubes of inert matter. In such experiments it is found that the resistance to the flow increases with a diminution of the oxygen carried by the red corpuscles, and is modified by the addition to the blood of even small quantities of certain drugs.

It is obvious then that in the living body the peripheral resistance, being the outcome of complex conditions, may be modified in many ways. Experience teaches us that, even in dealing with non-living inert matter, the flow of fluid through capillary tubes may be modified on the one hand by changes in the substance of which the tubes are composed, and on the other hand by changes in the chemical nature (even independent of the specific gravity) of the fluid which is used. In the living body both the fluid, the blood, and the walls of the minute vessels being both alive, are incessantly subject to change; the changes in the one, moreover, are capable of reacting upon and inducing changes in the other; and, lastly, the changes both of the one and of the other may be primarily set going by events taking place in some part of the body far away from the region in which these changes are modifying the resistance to the flow.

\section{Changes in the Quantity of Blood.}

$\$ 172$. In an artificial scheme changes in the total quantity of fluid in circulation will have an immediate and direct effect on the arterial pressure, increase of the quantity heightening and decrease diminishing it. This effect will be produced partly by the pump being more or less filled at each stroke, and partly by the peripheral resistance being increased or diminished by the greater or less fulness of the small peripheral channels. The venous 
pressure will under all circumstances be raised with the increase of fluid, but the arterial pressure will be raised in proportion only so long as the elastic walls of the arterial tubes are able to exert their elasticity.

In the natural circulation the direct results of change of quantity are modified by compensatory arrangements. Thus experiment shows that when an animal with normal blood-pressure is bled from one carotid, the pressure in the other carotid sinks so long as the bleeding is going on, ${ }^{1}$ and remains depressed for a brief period after the bleeding has ceased. In a short time, however, it regains or nearly regains, the normal height. This recovery of blood-pressure, after hemorrhage, is witnessed so long as the loss of blood does not amount to more than about 3 per cent. of the bodyweight. Beyond that a large and frequently a sudden dangerous permanent depression is observed.

The restoration of the pressure after the cessation of the bleeding is too rapid to permit us to suppose that the quantity of fluid in the bloodvessels is repaired by the withdrawal of lymph from the extra-vascular elements of the tissues. In all probability the result is gained by an increased action of the vasomotor nerves, increasing the peripheral resistance, the vasomotor centres being thrown into increased action by the diminution of their bloodsupply. When the loss of blood has gone beyond a certain limit, this vasomotor action is insufficient to compensate the diminished quantity (possibly the vasomotor centres in part become exhausted), and a considerable depression takes place; but at this epoch the loss of blood frequently causes anæmic convulsions.

Similarly when an additional quantity of blood is injected into the vessels, no marked increase of blood-pressure is observed so long as the vasomotor centre in the medulla oblongata is intact. If, however, the cervical spinal cord be divided previous to the injection, the pressure, which on account of the removal of the medullary vasomotor centre is very low, is permanently raised by the injection of blood. At each injection the pressure rises, falls somewhat afterward, but eventually remains at a higher level than before. This rise is stated to continue until the amount of blood in the vessels above the normal quantity reaches from 2 to 3 per cent. of the body-weight, beyond which point it is said no further rise of pressure occurs.

These facts seem to show, in the first place, that when the volume of the blood is increased, compensation is effected by a lessening of the peripheral resistance by means of a vaso-dilator action of the vasomotor centres, so that the normal blood-pressure remains constant. They further show that a much greater quantity of blood can be lodged in the bloodvessels than is normally present in them. That the additional quantity injected does remain in the vessels is proved by the absence of extravasations and of any considerable increase of the extra-vascular lymphatic fluids. It has already been insisted that, in health, the veins and capillaries must be regarded as being far from filled, for were they to receive all the blood which they can, even at a low pressure, hold, the whole quantity of blood in the body would be lodged in them alone. In these cases of large addition of blood the extra quantity appears to be lodged in the small veins and capillaries (especially of the internal organs), which are abnormally distended to contain the surplus.

We learn from these facts the two practical lessons, first, that bloodpressure cannot be lowered directly by bleeding, unless the quantity removed be dangerously large, and secondly, that there is no necessary connection

${ }^{1}$ Chiefly in consequence of the free opening in the vessel from which the bleeding is going on cutting off a great deal of the peripheral resistance and so leading to a general lowering of the blood-pressure. 
between a high blood-pressure and fulness of blood or plethora, since an enormous quantity of blood may be driven into the vessels without any marked rise of pressure.

\section{A Review of Some of the Features of the Circulation.}

$\$ 173$. The facts dwelt on in the foregoing sections have shown us that the factors of the vascular mechanism may be regarded as of two kinds: one constant or approximately constant, the other variable.

The constant factors are supplied by the length, natural bore, and distribution of the bloodvessels, by the extensibility and elastic reaction of their walls, and by such mechanical contrivances as the valves. By the natural bore of the various bloodvessels is meant the diameter which each would assume if the muscular fibres were wholly at rest, and the pressure of fluid within the vessel were equal to the pressure outside. It is obvious, however, that even these factors are only approximately constant for the life of an individual. The length and distribution of the vessels change with the growth of the whole body or parts of the body, and the physical qualities of the walls, especially of the arterial walls, their extensibility, and elastic reaction change continually with the age of the individual. As the body grows older the once supple and elastic arteries become more and more stiff and rigid, and often in midlle life, or it may be earlier, a lessening of arterial resilience which proportionately impairs the value of the vascular mechanism as an agent of nutrition, marks a step toward the grave. The valvular mechanisms, too, also show signs of age as years advance, and more or less marked and increasing imperfections diminish the usefulness of the machine.

The chief variable factors are, on the one hand, the beat of the heart, and, on the other, the peripheral resistance, the variations in the latter being chiefly brought about by muscular contraction or relaxation in the minute arteries, but also, though to what extent has not yet been accurately determined, by the condition of the walls of the minute vessels according to which the blood can pass through them with less or with greater ease, as well as by the character of the circulating blood.

$\S 174$. These two chief variables, the beat of the heart and the width of the minute arteries, are known to be governed and regulated by the central nervous system, which adapts each to the circumstance of the moment, and at the same time brings the two into mutual interdependence; but the central nervous system is not the only means of government; there are other modes of regulation, and so other safeguards.

Thus while undoubtedly the two prominent governors of the heart are the inhibitory fibres of the vagus and the augmentor fibres from the splanchnic system, the one slowing the rhythm and weakening the stroke, the other quickening the rhythm and strengthening the stroke, other causes may vary the beat in the absence of any action of either of these two nerves. Mere distention of the ventricle, by increasing the tension of the ventricular fibres, and so increasing the force of the contraction of each fibre (see $\$$ 148), brings about a more forcible beat. As we shall see in dealing with respiration, a powerful inspiration leads to a larger flow of blood into the heart, and forthwith the ventricle, out of its very fulness, gives stronger beats for the time. So also when by valvular disease or otherwise an unusual obstacle is presented to the outflow from the ventricle, increased vigor in the strokes of the distended organ strives to compensate the mischief. As, however, in the case of the skeletal muscle, the tension, if too great, may be injurious. In a similar manner the auricle, by a stronger or a weaker con- 
traction, may distend the ventricle to a greater or to a less extent, and so produce a stronger or weaker ventricular systole.

$\S 175$. Still more efficient, perhaps, as a direct governor of the heart's beat is the quality and quantity of blood passing in the mammal through the coronary arteries and regulating the nutrition of the cardiac substance. In the absence of all interference by inhibitory and augmentor fibres the heart will continue beating with a certain rhythm and force, determined by the metabolism going on certainly in its muscular, and possibly to a certain extent also in its nervous elements. We have seen that the energy set free in an ordinary skeletal muscle, in response to a stinulus, may vary from nothing to a maximum according to the metabolism going on-according to the nutritive vigor of the muscular fibres. The spontaneous rhythmic beat of the cardiac substance may be regarded as the outcome of a metabolism more highly pitched, more elaborate, of a higher order than that which simply furnishes the ordinary skeletal fibre with mere irritability toward stimuli. All the more, therefore, may the beat be expected to be influenced by any change in the metabolism of the cardiac substance, and so by any change in the blood which furnishes the basis for that metabolism. Hence the beat of the heart, quite apart from extrinsic nervous influences, may vary largely in consequence of changes in its own metabolism, which in turn may result from alterations in the blood-supply, or may have a deeper origin, and be due to the fact that the cardiac substance, owing to failure in its molecular organization (a failure which may be temporary or permanent), is unable to avail itself properly of the nutritive opportunities afforded by a normal quantity of normal blood.

$\$ 176$. As is well known, the beat of the heart may become temporarily or permanently irregular; that many hearts go on beating day after day, vear after year, without any such irregularity is a striking proof of the complete balance which usually obtains between the several factors of which we are speaking. Sometimes such cardiac irregularities, those of a transient nature and brief duration, are the results of extrinsic nervous influences. Some events taking place in the stomach, for instance, give rise to afferent impulses which ascending from the mucous membrane of the stomach along certain afferent fibres of the vagus to the medulla oblongata, so augment the action of the cardio-inhibitory centre as to stop the heart for a beat or two, the stoppage being frequently followed by a temporary increase in the rapidity and force of the beat. Such a passing failure of the heart-beat, in its sudden onset, in its brief duration, and in the reaction which follows, very closely resembles the temporary inhibition brought about by artificial stimulation of the vagus. But these characters are not essential to cardiac inlibition. For it must be remembered that the central nervous system possesses, in the form of natural nervous impulses of various origin, a means of stimulation far finer, more delicate and more varied than anything we can effect by our rough means of induction coils and electrodes. Thus in many cases of fainting, the hesrt-beats, instead of stopping abruptly, gradually die away or fade away it may be to an absolute brief arrest, but more frequently merely down to a feebleness which is insufficient to supply the brain with a quantity of blood arlequate to maintain consciousness, and then in many cases, at all events, are resumed, or recover strength gradually and quietly without any boisterous reaction. In all probability all cases of simple fainting from emotion, pain, digestive troubles, etc., as distinguished from the syncope of actual heart disease, are instances of vagus inhibition, and though we cannot accurately reproduce their varied phases by direct stimulation of the vagus trunk, we may approach them more nearly by producing reflex inhibition, as by mechanical irritation of the abdomen. (See $\$ 145$. .) 
Whether definite temporary irregularity is ever brought about by means of the augmentor fibres we have at present no clear evidence; but cases do occur of palpitation without previous stoppage, cases in which a few hurried strong beats come on, pass off, and are followed by feebler beats; and these may possibly be due to some transient influence of augmentor fibres thrown into activity as part of a reflex act or otherwise. And though we have no direct experimental evidence, it is very probable that the acceleration or augmentation of the beat, or a combination of the two, which so often follows emotion, is carried out by augmentor fibres.

In all probability, however, irregularity in the heart-beat is much more frequently the result of intrinsic events, or the product of a disordered nutrition of the cardiac substance. The normal nutrition sets the pace of the normal rhythm. We cannot explain how this is affected; nor can we explain why in one individual the normal pace is set so low as 50 or even 30 beats a minute, and in another as high as 90 a minute or even more, while in most persons it is about 70 a minute. The slower or the quicker the pace, though not normal to the species, must be considered as normal to the individual, for it may be kept up through long years in an organism capable of carrying on a normal man's duties and work. So long as we cannot explain these differences we cannot hope to explain how it is that a disordered nutrition brings about an irregular heart-beat, either the more regular irregularity of a "dropping" pulse, that is, a failure of sequence rather than an irregularity, or a more actively irregular rhythm, such as that accompanying a dilated ventricle. We may, however, distinguish two kinds of irregularity : one in which, in spite of all favorable nutritive conditions, the cardiac substance cannot secure, even perhaps for a minute, a steady rhythm; and another in which the rhythm, though normal under ordinary circumstances, is, so to speak, in a condition of unstable equilibrium, so that a very slight change in conditions, too much or too little blood, or some small alteration in the composition of the blood, or the advent of some, it may be slight, nervous impulse, augmentor or inhibitory, develops a temporary irregularity.

$\$ 177$. No one thing, perhaps, concerning the heart is more striking than the fact that a heart which has gone on beating for many years, with only temporary irregularities, and those few and far between, a heart which must, therefore, have executed with long-continued regularity, many millions of beats, should suddenly, apparently without warning, after a brief, flickering struggle, cease to beat any more. But we must remember that each beat is an effort-an effort, moreover, which, as we have seen ( $\$ 141)$, is the best that the heart can make at the moment; the accomplishment of each beat is, so to speak, a hurdle which has to be leaped -one of the long series of hurdles which make up the steeple-chase of life. At any one leap failure may occur; so long as failure does not occur, so long as the beat is made, and a fair proportion of the ventricular contents are discharged into the great vessels, the chief end is gained, and whether the leap is made clumsily or well is, relatively considered, of secondary importance. But if the beat be not made, everything almost (provided that the miss be due, not to vagus inhibition, but to intrinsic events) is unfavorable for a succeeding beat; the mysterious molecular changes, by which the actual occurrence of one beat prepares the way for the next, are missing, the favorable influences of the extra rush of blood through the coronary arteries due to a preceding beat are missing also, and even the distention of the cardiac cavities, at first favorable, speedily passes the limit and becomes unfavorable. And these untoward influences accumulate rapidly as the first miss is followed by a second and by a third. In this way a heart, which has been brought into a state of unstable equilibrium 
by disordered nutrition (as, for instance, by imperfect coronary circulation, such as seems to accompany diseases of the aortic valves leading to regurgitation from the aorta into the ventricle, in which cases sudden death is not uncommon), which is able just to accomplish each beat, but no more, which has but a scanty saving store of energy, under some strain or other untoward influence, misses a leap, falls, and is no more able to rise. Doubtless in such cases could adequate artificial aid be promptly applied in time, could the fallen heart be stirred even to a single good beat, the favorable reaction of that beat might bring a successor, and so once more start the series; but such a period of grace, of potential recovery, is a brief one. Even a coarse skeletal muscle, when cut off from the circulation, soon loses its irritability beyond all recovery, and the heart cut off from its own influence on itself runs down so rapidly that the period of possible recovery is measured chiefly by seconds.

$\S 178$. Turning now to the minute arteries and the peripheral resistance which they regulate, we may call to mind the existence of the two kinds of mechanism, the vaso-constrictor mechanism, which, owing to the maintenance by the central nervous system of a tonic influence, can be worked both in a positive constrictor and in a negative dilator direction, and the vaso-dilator mechanism, which, as far as we know, exerts its influence in one direction only, viz., to dilate the bloodvessels. The latter dilator mechanism seems, as we have seen, to be used in special instances only, as seen in the cases of the chorda tympani and nervi erigentes; the use of the former constrictor mechanism appears to be more general. Thus: the relaxation of the cutaneous arteries of the head and neck, which is the essential feature in blushing, seems due to mere loss of tone, to the removal of constrictor influences previously exerted through the vaso-constrictor fibres of the cervical sympathetic. Though probably dilator fibres pass directly along the roots of the cervical and of certain cranial nerves to the nerves of the head and neck, we have no evidence that these come into play in blushing; as we have seen, blushing may be imitated by mere section of the cervical sympathetic. So also the "glow" and redness of the skin of the whole body-i.e., dilatation generally of the cutaneous arteries - which is produced by external warmth, is probably another instance of diminished activity of tonic constrictor influences; though the result, that the dilatation produced by warming an animal in an oven is greater than that produced by section of nerves, seems to point to the dilator fibres for the cutaneous vessels which, as we have seen, probably exist in the sciatic and brachial plexuses and possibly in all the spinal nerves, also taking part in the action. A similar loss of constrictor action in the cutaneous vessels may be the result of certain emotions, whether going so far as actual blushing of the body, or merely producing a "glow." The effect of cold, on the other hand, and of certain emotions, or of emotions under certain conditions, is to increase the constrictor action on the cutaneous vessels, and the skin grows pale. It may be worth while to point out that in both the above cases, while both the cold and warmth produce their effect chiefly at all events through the central nervous system, and very slightly, if at all, by direct action on the skin, their action on the central nervous system is not simply a general augmentation or inhibition of the whole vasomotor centre. On the contrary, the cold, while it constricts the cutaneous vessels, so acts on the vasomotor centre as to inhibit that portion of the vasomotor centre which governs the abdominal splanchnic area ; while less blood is carried to the colder skin, by the opening up of the splanchnic area more blood is turned on to the warmer regions of the body, and the rise of blood-pressure which the constriction of the cutaneous vessels 
tended to produce, and which might be undesirable, is thereby prevented. Conversely when warmth dilates the cutaneous vessels, it at the same time constricts the abdominal splanchnic area, and prevents an undesirable fall of pressure.

The warm and flushed condition of the skin, which follows the drinking of alcoholic fluids, is probably in a similar manner the result of an inhibition of that part of the vasomotor centre which governs the cutaneous arteries; and it is probable also that except for the local effect of the fluid on the gastric mucous membrane, whereby some anount of blushing of the gastric bloodvessels takes place as a reflex act, this effect on the vessels of the skin is accompanied by an inverse constrictor action in the splanchnic area. This last point, however, has not been proved experimentally and may not occur, since the influence of the alcohol is at the same time to increase the heart's action, and thus to obviate the fall of pressure which would certainly occur were the cutaneous and splanchnic vascular areas to be dilated at the same time. This effect of the alcohol on the heart may be a direct action of the alcohol on the cardiac substance, being carried thither by the blood; but the effect, in being an augmentation of the force, and acceleration of the pace of the heart-beat of a temporary character, followed by a reaction in the direction of feebleness and slowness, so strikingly resemble the effects of artificially stimulating the cardiac augmentor fibres, that it is at least probable that the alcohol does act upon the cardiac augmentor mechanism.

$\S 179$. The influence on the body of exercise illustrates both the manner in which the two vascular factors, the heart-beat and the peripheral resistance, are modified by circumstances, and the mutual action of these on each other.

When the body passes from a condition of comparative rest and quiet to one of exertion and movement, the metabolism of the skeletal muscles (and of the nervous system) is increased and more heat is generated in them. We know for certain that the increased metabolism throws into the blood of the veins coming from the muscles an increased amount of carbonic acid, and it is probable, but not so certain, that it also loads the blood with lactic acid and other metabolic products; at the same time, there is an increased consumption of oxygen; the blood of the body tends to become less arterial and more venous. In dealing with respiration, we shall see that the influence thus exerted on the blood leads to an increase in the respiratory movements, and we shall further see that the more vigorous working of the respiratory pump, since it promotes the flow of blood to and through the heart and lungs, quickens and strengthens the heart-beats. Possibly this mere mechanical effect of the more vigorous breathing is sufficient by itself to account for the increase in the frequency and vigor of the heart's action, but it is more than probable that it is the changed condition of the blood, which, while it hurries on the respiratory pump, also stimulates the vascular pump, either by a direct action on the cardiac substance, or through the medium of the central nervous system and the augmentor fibres. If, as experiments seem to show, the increased vigor of the respiratory movements compensates, or even over-compensates, the tendency of the whole blood to become more venous, so that during exercise the blood, which is distributed by the aorta, actually does not contain more carbonic acid and less oxygen than the rest, but even the reverse, then these efforts must be due to some of the products of muscular metabolism other than carbonic acid.

The same changed condition of blood, while it thus excites the heart, dilates the cutaneous vessels, as is clearly shown by the warm flushed skin, and at the same time throws into activity the perspiratory mechanism with which 
we shall hereafter have to deal. There can be no doubt, as we shall see later on, that the perspiration which accompanies muscular exercise is brought about by means of the central nervous system, and we may almost with certainty conclude that the dilatation of the cutaneous arteries is also brought about by means of the central nervous system, and most probably by means of an inhibition of that part of the vasomotor centre which maintains under ordinary circumstances a greater or less tonic constriction of the cutanerus arteries; how far this may be assisted by the special action of vaso-dilator fibres we do not know.

This widening of the cutaneous arteries diminishes largely the peripheral resistance, and so tends to lower the blood-pressure. Moreover, with each effort of each skeletal muscle the minute arteries of that muscle are dilated, so that during exercise, and especially during vigorous exercise calling into action many skeletal muscles, there must be in the body at large a very considerable widening of the minute arteries distributed to the various muscles, and in consequence a very considerable diminution of the peripheral resistance. These two diminutions of peripheral resistance, cutaneous and muscular, would tend to lower the blood-pressure--a result which would be most injurious, since the increased metabolism of the muscles demands a more rapid circulation in order to get rid of the products of metabolism, and for a rapid circulation a high blood-pressure is in most cases necessary, and in all cases advantageous. The evil is, in part at all events, met by the increased force and frequency of the heart's beats, for, as we have said again and again, the mean blood-pressure is the product of the heart-beat working against the peripheral resistance, and may remain constant when one factor is increased or diminished, provided that the other factor be proportionately diminished or increased. It is possible, then, that the mere increase in the heart's beats are, during exercise, sufficient to neutralize the diminution of peripheral resistance, or even to raise the blood-pressure above the normal ; and, indeed, we find, as a matter of fact, that during exercise there is such an increase of the mean blood-pressure. But it is more than probable that much valuable labor of the heart is economized by neutralizing the imminent fall of blood-pressure in another manner. It would appear that while that part of the vasomotor centre which governs the cutaneous vascular area is being inhibited, that part which governs the abdominal splanchnic area is, on the contrary, being augmented. And in this way a double end is gained. On the one hand, the mean blood-pressure is maintained or increased in a more economical manner than by increasing the heart-beats, and, on the other hand, the blood during the exercise is turned away from the digestive organs, which at the time are, or ought to be, at rest, and therefore requiring comparatively little blood. These organs certainly, at all events, ought not during exercise to be engaged in the task of digesting and absorbing food, and the old saying, "after dinner sit a while," may serve as an illustration of the working of the vascular mechanism with which we are dealing. The duty which some of the digestive organs have to carry out in the way of excretion of metabolic waste products is during exercise probably taken on by the flushed and perspiring skin. It is true that at the beginning of a period of exercise, before the skin, so to speak, has settled down to its work, an increased flow of urine, dependent on or accompanied by an increased flow of blood through the kidney, may make its appearance; but in this case, as we shall see later on in dealing with the kidney, the flow of blood through the kidney may be increased in spite of constriction of the rest of the splanchnic area, and, besides, such an initial increase of urine speedily gives way to a decrease.

$\S 180$. The effect of food on the vascular mechanism affords a marked 
contrast to the effect of bodily labor. The most marked result is a widening of the whole abdominal splanchnic area, accompanied by so much constriction of the cutaneous vascular area, and so much increase of the heart's beat, as is sufficient to neutralize the tendency of the widening of the splanchnic area to lower the mean pressure, or perhaps even sufficient to raise slightly the mean pressure.

Any large widening of the cutaneous area, especially if accompanied by muscular labor and the incident widening of the arteries of the muscles, would tend so to lower the general blood-pressure (unless met by a wasteful use of cardiac energy) as injuriously to lessen the flow through the active digesting viscera. A moderate constriction of the cutaneous vessels, on the other hand, by throwing more blood on the abdominal splanchnic area without tasking the heart, is favorable to digestion, and is probably the physiological explanation of the old saying, "If you eat till you're cold, you'll live to be old."

In fact, during life there seems to be a continual give-and-take between the bloodvessels of the somatic and those of the splanchnic divisions of the body ; to fill the one, the other is proportionately emptied, and vice versa.

$\S 181$. We have seen $(\$ 160)$ that certain afferent fibres of the vagus forming in the rabbit a separate nerve, the depressor nerve, are associated with the vaso-constrictor nerves and the vasomotor centre in such a way that impulses passing centripetally along them from the heart lower the bloodpressure by diminishing the peripheral resistance, probably inhibiting the tonic constrictor influences exerted along the abdominal splanchnic nerves, and so, as it were, opening the splanchnic flood-gates. We do not possess much exact information about the use of these afferent depressor fibres in the living body, but probably when the heart is laboring against the bloodpressure which is too high for its powers, the condition of the heart starts impulses which, passing along the depressor fibres up to the medulla oblongata, temper down, so to speak, the blood-pressure to suit the cardiac strength.

We have, moreover, reason to think that not only does the heart thus regulate the blood-pressure by means of the depressor fibres, but also that the blood-pressure, acting, as it were, in the reverse direction, regulates the heart-beat; a too high pressure, by acting directly on the cardio-inhibitory centre in the medulla oblongata (either directly - that is, as the result of the vascular condition of the medulla itself-or indirectly, by impulses reaching the medulla along afferent nerves from various parts of the body) may send inhibitory impulses down the vagus, or so slacken or tone down the heart-beats.

In the following sections of this work we shall see repeated instances, similar to or even more striking than the above, of the management of the vascular mechanism by means of the nervous system, and we, therefore, need dwell no longer on the subject.

We may simply repeat that at the centre lies the cardiac muscular fibre, and at the periphery the plain muscular fibre of the minute artery. On these two elements the central nervous system, directed by this or that impulse reaching it along afferent nerve fibres, or affected directly by this or that influence, is during life continually playing, now augmenting, now inhibiting, now the one, now the other, and so, by help of the elasticity of the arteries and the mechanism of the valves, directing the blood-flow according to the needs of the body. 


\section{BOOK II.}

\section{THE TISSUES OF CHEMICAL ACTION. THEIR RESPECTIVE MECHANISMS, NUTRITION.}

\section{CH A P T E R I. \\ THE TISSUES AND MECHANISMS OF DIGESTION.}

$\S 182$. THE food in passing along the alimentary canal is subjected to the action of certain juices supplied by the secretory activity of the epithelial cells which line the canal itself or which form part of its glandular appendages. These juices, viz., saliva, gastric juice, bile, pancreatic juice, and the secretions of the small and large intestines, poured upon and mingling with the food produce in it such changes that, from being largely insoluble, it becomes largely soluble, or otherwise modify it in such a way that the larger part of what is eaten passes into the blood, either directly by means of the capillaries of the alimentary canal, or indirectly by means of the lacteal system, while the smaller part is discharged as excrement.

Those parts of the food which are thus digested, absorbed, and made use of by the body, are spoken of as food-stuffs (they have also been called alimentary principles), and may be conveniently divided into four great classes:

1. Proteids. We have previously $(\$ 15)$ spoken of the chief characters of this class, and have dealt with several members in treating of blood and muscle. We may here repeat that in general composition they contain in 100 parts by weight "in round numbers" rather more than 15 parts of nitrogen, rather more than 50 parts of carbon, about 7 parts of hydrogen, and rather more than 20 parts of oxygen; though essentially the nitrogenous bodies of food and of the body, they are made up of carbon to the extent of more than half their weight.

The nitrogenous body gelatin, which occurs largely in animal food, and some other bodies of less importance, while more closely allied to proteid bodies than to any other class of organic substances, differ considerably from proteids in composition and especially in their behavior in the body; they are not of sufficient importance to form a class by themselves.

2. Fats, frequently but erroneously called hydrocarbons. These vary very widely in chemical composition, ranging from such a comparatively simple fat as butyrin to the highly complex lecithin ( $\$ 69)$; they all possess, in view of the oxidation of both their carbon and their hydrogen, a large amount of potential energy.

3. Carbohydrates, or sugars and starches. These possess, weight for weight, relatively less potential energy than do fats; they already contain in themselves a large amount of combined oxygen, and when completely oxidized give out, weight for weight, less heat than do fats. 
4. Saline or mineral bodies, and water. These salts are for the most part inorganic salts, and this class differs from the three preceding classes inasmuch as the usefulness of its members to the body lies not so much in the amount of energy which may be given out by their oxidation as in the various influences which, by their presence, they exercise on the metabolic events of the body.

These several food-stuffs are variously acted upon in the several parts of the alimentary canal, and we may distinguish, as the food passes along the digestive tract, three main stages: digestion in the mouth and stomach, digestion in the small intestine, and digestion in the large intestine. In many animals the first stage is, to a large extent, preparatory only to the second, which in all the animals is the stage in which the food undergoes the greatest change; in the third stage the changes begun in the previous stages are completed, and this stage is especially characterized by the absorption of fluid from the interior of the alimentary canal.

It will be convenient to study these stages more or less apart, though not wholly so, and it will also be convenient to consider the whole subject of digestion under the following heads :

First, the characters and properties of the various juices and the changes which they bring about in the food eaten.

Secondly, the nature of the processes by means of which the epithelium cells of the various glands and various tracts of the canal are able to manufacture so many various juices out of the common source, the blood, and the manner in which the secretory activity of the cells is regulated and subjected to the needs of the economy.

Thirdly, the mechanisms, here as elsewhere, chiefly of a muscular nature, by which the food is passed along the canal and most efficiently brought into contact with the several juices.

Fourthly and lastly, the means by which the nutritious digested material is separated from the undigested or excremental material and absorbed into the blood.

\section{The Characters and Properties of Saliva and Gastric Juice.}

\section{Saliva.}

$\$ 183$. Mixed saliva, as it appears in the mouth, is a thick, glairy, generally frothy and turbid fluid. Under the microscope it is seen to contain, beside the molecular débris of food, bacteria and other organisms (frequently cryptogamic spores), epithelium scales, mucous corpuscles and granules, and the so-called salivary corpuscles. Its reaction in a healthy subject is alkaline, especially when the secretion is abundant. When the saliva is scanty, or when the subject suffers from dyspepsia, the reaction of the mouth may be acid. Saliva contains but little solid matter, on an average probably about 5 per cent., the specific gravity varying from 1002 to 1006. Of these solids, rather less than half, about 2 per cent, are salts (including at times a minute quantity of potassium sulphocyanate). The organic bodies which can be recognized in it are globulin and serum albumin (see $§ \S 16,17$ ), found in small quantities only-other obscure bodies occurring in minute quantity, and mucin; the latter is by far the most conspicuous organic constituent, the glairiness or ropiness of mixed and other kinds of saliva being due to its presence.

Mucin. If acetic acid be cautiously added to mixed saliva, the viscidity of the saliva is increased, and on further addition of the acid a semi-opaque ropy mass separates out, leaving the rest of the saliva limpid. This ropy 
mass, which is mucin, if stirred carefully with a glass rod, shrinks, becoming opaque, clings to the glass rod, and may be thus removed from the fluid. If the quantity of mucin be small and the saliva be violently shaken or stirred while the acid is being added, the mucin is apt to be precipitated in flakes, and may then be separated by filtration. It may be added that the precipitation of mucin by acid is greatly influenced by the presence of sodium chloride and other salts; thus, after the addition of sodium chloride, acetic acid, even in considerable excess, will not cause a precipitate of mucin.

Mucin, thus prepared and purified by washing with acetic acid, swells out in water without actually dissolving; it will, however, dissolve into a viscid fluid readily in dilute ( 0.1 per cent.) solutions of potassium hydrate, more slowly in solutions of alkaline salts. In order to filter a mucin solution, great dilution with water is necessary.

Mucin is precipitated by strong alcohol and by various metallic salts ; it may also be precipitated by dilute mineral acids, but the precipitate is then soluble in excess of the acid.

Mucin gives the three proteid reactions mentioned in $\S 15$, but it is a very complex borly, more complex even than proteids, for by treatment with dilute mineral acids and in other ways, it may be converted into some form of proteid (acid-albumin when dilute mineral acid is used), while at the same time there is formed a body which appears to be carbohydrate and resembles a sugar in having the power of reducing cupric sulphate solutions. Solutions of mucin, moreover, on mere keeping are apt to lose their viscidity and to become converted into a proteid not unlike the body peptone, which, as we shall see, is the result of gastric digestion, and into a reducing body. Several kinds of mucin appear to exist in various animal bodies, but they seem all to agree in the character that they can by appropriate treatment be split up into a proteid of some kind and into a carbohydrate or allied body.

184. The chief purpose served by the saliva in digestion is to moisten and soften the food and to assist in mastication and deglutition. In some animals this is its only function. In other animals and in man it has a specific solvent action on some of the food-stuffs. Such minerals as are soluble in slightly alkaline fluids are dissolved by it. On fats it has no effect save that of producing a very feeble emulsion. On proteids it has also no specific action, though pieces of meat, cooked or uncooked, appear greatly altered after they have been masticated for some time; the chief alteration, however, which thus takes place is a change in the hæmoglobin and a general softening of the muscular fibres by aid of the alkalinity of the saliva. Of course, when particles of food are retained for a long time in the mouth, as in the interstices or in cavities of the teeth, the bacteria or other organisms which are always present in the mouth may produce much more profound changes, but these are not the legitimate products of the action of saliva. The characteristic property of saliva is that of converting starch into some form of sugar.

Action of saliva on starch. If to a quantity of boiled starch, which is always more or less viscid and somewhat opaque or turbid, a small quantity of saliva be added, it will be found after a short time that an important change has taken place, inasmuch as the mixture has lost its previous viscidity and become thinner and more transparent. In order to understand this change the reader must bear in mind the existence of the following bodies, all belonging to the class of carbohydrates:

1. Starch, which forms with water not a true solution but a more or less viscid mixture, and gives a characteristic blue color with iodine. The formula is $\mathrm{C}_{6} \mathrm{H}_{10} \mathrm{O}_{5}$, or more correctly $\left(\mathrm{C}_{6} \mathrm{H}_{10} \mathrm{O}_{5}\right)^{n}$ since the molecule of starch 
is some multiple ( $n$ being not less than 5 ) of the simpler formula. A kind of starch known as soluble starch, while giving a blue color with iodine, forms, unlike ordinary starch, a clear solution.

2. Dextrins, differing from starch in forming a clear solution. Of these there are at least two ; one, erythrodextrin, often spoken of simply as dextrin, giving a port-wine red color with iodine, and second, achroodextrin, which gives no color at all with iodine. 'The formula for dextrin is the same as that for starch, but has a smaller molecule and might be represented by $\left(\mathrm{C}_{6} \mathrm{H}_{10} \mathrm{O}_{5}\right)^{n \prime}$.

3. Dextrose, also called glucose or grape-sugar, giving no coloration with iodine, but characterized by the power of reducing cupric and other metallic salts; thus, when dextrose is boiled with a fluid known as Fehling's fluid, which is a solution of hydrated cupric oxide in an excess of caustic alkali and double tartrate of sodium and potassium, the cupric oxide is reduced and a red or yellow deposit of cuprous oxide is thrown down. This reaction serves with others as a convenient test for dextrose. Neither starch nor that commonest form of sugar known as cane-sugar gives the reaction; whether the dextrins do is doubtful. The formula for dextrose is $\mathrm{C}_{6} \mathrm{H}_{12} \mathrm{O}_{6}$; it is more simple than that of starch or dextrin and contains an additional $\mathrm{H}_{2} \mathrm{O}$ for every $\mathrm{C}_{6}$. Unlike starch and dextrin it can be obtained in a crystalline form, either from aqueous solutions (it being readily soluble in water), in which case the crystals contain water of crystallization, or from its solutions in alcohol (in which it is sparingly soluble), in which case the crystals have no water of crystallization. Solutions of dextrose have a marked dextrorotatory power with rays of light.

4. Maltose, very similar to dextrose, and like it capable of reducing cupric salts. The formula is somewhat different, being $\mathrm{C}_{12} \mathrm{H}_{22} \mathrm{O}_{11}$. Besides this, it differs from dextrose chiefly in its smaller power-i.e., a given weight will not convert so much cupric oxide into cuprous oxide as will the same weight of dextrose - and in having a stronger rotatory action on rays of light. Like dextrose it can be crystallized, the crystals fron aqueous solutions containing water of crystallization.

Now, when a quantity of starch is boiled with water we may recognize in the viscid imperfect solution, on the one hand, the presence of the starch by the blue color which the addition of iodine gives rise to; and on the other hand, the absence of sugar (maltose, dextrose), by the fact that when boiled with Fehling's fluid no reduction takes place and no. cuprous oxide is precipitated.

If, however, the boiled starch be submitted for a while to the action of saliva, especially at a somewhat high temperature such as $35^{\circ}$ or $40^{\circ} \mathrm{C}$., it is found that the subsequent addition of iodine gives no blue color at all or very much less color, showing that the starch has disappeared or diminished; on the other hand, the mixture readily gives a precipitate of cuprous oxide when boiled with Fehling's fluid, showing that maltose or dextrose is present. That is to say, the saliva has converted the starch into maltose or dextrose. The presence of the previously absent sugar may also be shown by fermentation and by the other tests for sugar. Moreover, if an adequately large quantity of starch be subjected to the charge, the sugar formed may be isclated, and its characters determined. When this is done it is found that while some dextrose is formed the greater part of the sugar which appears is in the form of maltose. As is well known, starch may, by the action of dilute acid, be converted into dextrin, and by further action into sugar; but the sugar thus formed is always wholly dextrose, and not maltose at all. The action of saliva in this respect differs from the action of dilute acid. 
While the conversion of the starch by the saliva is going on the addition of iodine. frequently gives rise to a red or violet color instead of a pure blue, but when the conversion is complete no coloration at all is observed. The appearance of this red color indicates the presence of dextrin (erythrodextrin); the violet color is due to the red being mixed with the blue of still unchanged starch.

The appearance of dextrin shows that the action of the saliva on the starch is somewhat complex; and this is still further proved by the fact that even when the saliva has completed its work the whole of the starch does not reappear as maltose or dextrose. A considerable quantity of the other dextrin (achroodextrin) always appears and remains unchanged to the end; and there are probably several other bodies also formed out of the starch, the relative proportions varying according to circumstances. The change, therefore, though perhaps we may speak of it in a general way as one of hydration, cannot be exhibited under a simple formula, and we may rest content for the present with the statement that starch when subjected to the action of saliva is converted chiefly into the sugar known as maltose with a comparatively small quantity of dextrose and to some extent into achroodextrin (erythrodextrin appearing temporarily only in the process), other bodies on which we need not dwell being formed at the same time.

Raw unboiled starch undergoes a similar change but at a much slower rate. This is due to the fact that in the curiously formed starch grain the true starch, or granulose, is invested with coats of cellulose. This latter material, which requires previous treatment with sulphuric acid before it will give the blue reaction on the addition of iodine, is apparently not acted upon by saliva. Hence the saliva can only get at the granulose by traversing the coats of cellulose, and the conversion of the former is thereby much hindered and delayed.

$\S 185$. The conversion of starch into sugar, and this we may speak of as the amylolytic action of saliva, will go on at the ordinary temperature of the atmosphere. The lower the temperature the slower the change, and at about $0^{\circ} \mathrm{C}$. the conversion is indefinitely prolonged. After exposure to this cold for even a considerable time the action recommences when the temperature is again raised. Increase of temperature up to about $35^{\circ}-40^{\circ}$, or even a little higher, favors the change, the greatest activity being said to be manifested at about $40^{\circ}$. Much beyond this point, however, increase of temperature becomes injurious, markedly so at $60^{\circ}$ or $70^{\circ}$; and saliva which has been boiled for a few minutes not only has no action on starch while at that temperature, but does not regain its powers on cooling. By being boiled, the amylolytic activity of saliva is permanently destroyed.

The action of saliva on starch is most rapid when the reaction of the mixture is neutral or nearly so; it is hindered or arrested by a distinctly acid reaction. Indeed, the presence of even a very small quantity of free acid, at all events of hydrochloric acid, at the temperature of the body, not only suspends the action but speedily leads to permanent abolition of the activity of the juice. The bearing of this will be seen later on.

The action of saliva is hampered by the presence in a concentrated state of the product of its own action-that is, of sugar If a small quantity of saliva be added to a thick mass of boiled starch, the action will after a while slacken, and eventually come to almost a standstill, long before all the starch has been converted. On diluting the mixture with water, the action will recommence. If the products of action be removed as soon as they are formed, dialysis for example, a small quantity of saliva will, if sufficient time 
be allowed, convert into sugar a very large, one might almost say an indefinite, quantity of starch. Whether the particular constituent on which the activity of saliva depends is at all consumed in its action has not at present been definitely settled.

On what constituents do the amylolytic virtues of saliva depend?

If saliva, filtered and thus freed from much of its mucin and from other formed constituents, be treated with ten or fifteen times its bulk of alcohol, a precipitate is formed containing, besides other substances, all the proteid matters. Upon standing under the alcohol for some time (several days), the proteids thus precipitated become coagulated and insoluble in water. Hence, an aqueous extract of the precipitate, made after this interval, contains very little proteid material; yet it is exceedingly active. Moreover, by other more elaborate methods there may be obtained from saliva solutions which appear to be almost entirely free from proteids and yet are intensely amylolytic. But even these probably contain other bodies beside the really active constituent. Whatever the active substance be in itself, it exists in such extremely small quantities that it has never yet been satisfactorily isolated; and indeed the only clear evidence we have of its existence is the manifestation of its peculiar powers.

The salient features of this body, this amylolytic agent, which we may call ptyalin, are then: 1 st, its presence in minute and almost inappreciable quantity. $2 d$, the close dependence of its activity on temperature. $3 d$, its permanent and total destruction by a high temperature and by various chemical reagents. 4th, the want of any clear proof that it itself undergoes any change during the manifestations of its power-that is to say, the energy necessary for the transformation which it effects does not come out of itself; if it is at all used up in its action, the loss is rather that of simple wear and tear of a machine than that of a substance expended to do work. 5th, the action which it induces is probably of such a kind (splitting up of a molecule with assumption of water) as is effected by that particular class of agents called " hydrolytic."

These features mark out the amylolytic active body of saliva as belonging to the classs of ferments $;^{1}$ and we may henceforward speak of the amylolytic ferment of saliva. The fibrin-ferment (\$20) is so called because its action in many ways resembles that of the ferment of which we are now speaking.

$\S 186$. Mixed saliva, whose properties we have just discussed, is the result of the mingling in various proportions of saliva from the parotid, submaxillary, and sublingual glands with the secretion from the buccal glands. These constituent juices have their own special characters, and these are not the same in all animals. Moreover, in the same individual the secretion differs in composition and properties according to circumstances; thus, as we shall see in detail hereafter, the saliva from the submaxillary gland secreted under the influence of the chorda tympani nerve is different from that which is obtained from the same gland by stimulating the sympathetic nerve.

\footnotetext{
1 Ferments may, for the present at least, be divided into two elasses, commonly called organized and unorganized. Of the former, yeast may be taken as a well-known example. The fermentative activity of yeast which leads to the conversion of sugar into aleohol, is dependent on the life of the yeast-cell. Unless the yeast-cell be living and functional, fermentation does not take place; when the yeast-cell dies fermentation eeases; and no substance obtained from the fluid parts of yeast, by preeipitation with alcohol or otherwise, will give rise to alcoholic fermentation. The salivary ferment belongs to the latter class; it is a substanee, not a living organism like veast. It may be added, however, that possibly the organized ferment, the yeast for instance, produces its effect by means of an ordinary unorganized ferment which it generates, but which is immediately made away with.
} 
In man pure parotid saliva may easily be obtained by introducing a fine canula into the opening of the Stenonian duct, and submaxillary saliva, or rather a mixture of submaxillary and sublingual saliva, by similar catheterization of the Whartonian duct. In animals the duct may be dissected out and a canula introduced.

Parotid saliva in man is clear and limpid, not viscid; the reaction of the first drops secreted is often acid, the succeeding portions, at all events when the flow is at all copious, are alkaline; that is to say, the natural secretion is alkaline, but this may be obscured by acid changes taking place in the fluid which has been retained in the duct, possibly by the formation of an excess of carbonic acid. On standing the clear fluid becomes turbid from a precipitate of calcic carbonate, due to an escape of carbonic acid. It contains globulin and some other forms of albumin, with little or no mucin. Potassium sulphocyanate may also sometimes be detected, but structural elements are absent.

Submaxillary saliva, in man and in most animals, differs from parotid saliva in being more alkaline, and from the presence of mucin more viscid; it contains salivary corpuscles, that is bodies closely resembling if not identical with leucocytes, and, often in abundance, amorphous masses. The socalled chorda saliva in the dog, that is to say, saliva obtained by stimulating the chorda tympani nerve (of which we shall presently speak), is under ordinary circumstances thimmer and less viscid, contains less mucin and fewer structural elements than the so-called synpathetic saliva, which is remarkable for its viscidity, its structural elements, and for its larger total of solids.

Sublingual saliva is more viseid and contains more salts (in the dog about 1 per cent.) than the submaxillary saliva.

The action of saliva varies in intensity in different animals. Thus in man, the pig, the guinea-pig, and the rat, both parotid and submaxillary and mixed saliva are amylolytic; the submaxillary saliva being in most cases more active than the parotid. In the rabbit, while the submaxillary saliva has scarcely any action, that of the parotid is energetic. The saliva of the cat is much less active than the above; that of the dog is still less active, indeed is almost inert. In the horse, sheep, and ox, the amolytic powers of either mixed saliva or of any one of the constituent juices are extremely feeble.

Where the saliva of any gland is active, an aqueous infusion of the same gland is also active. The importance and bearing of this statement will be seen later on. From the aqueous infusion of the gland, as from saliva itself, the ferment may be approximately isolated. In some cases at least some ferment may be extracted from the gland even when the secretion is itself inactive. In fact, a ready method of preparing a highly amylolytic liquid tolerably free from proteid and other impurities is to mince finely a gland known to have an active secretion, such, for instance, as that of a rat, to dehydrate it by allowing it to stand under absolute alcohol for some days, and then, having poured off most of the alcohol and removed the remainder by evaporation at a low temperature, to cover the pieces of gland with strong glycerin. Though some of the ferment appears to be destroyed by the alcohol, a mere drop of such a glycerin extract rapidly converts starch into sugar.

\section{Gastric Juice.}

$\S 187$. There is no difficulty in obtaining what may be fairly considered as a normal saliva; but there are many obstacles in the way of determining the normal characters of the secretion of the stomach. IVhen no food is 
taken the stomach is at rest and no secretion takes place. When food is taken, the characters of the gastric juice secreted are obscured by the food with which it is mingled. The gastric membrane may, it is true, be artificially stimulated, by touch, for instance, and a secretion obtained. This we may speak of as gastric juice, but it may be doubted whether it ought to be considered as normal gastric juice. And indeed, as we shall see, even the juice which is poured into the stomach during a meal, varies in composition as digestion is going on. Hence the characters which we shall give of gastric juice must be considered as having a general value only.

Gastric juice, obtained in as normal a condition as possible from the healthy stomach of a fasting dog by means of a gastric fistula, is a thin, almost colorless fluid with a sour taste and odor.

In the operation for gastric fistula an incision is made through the abdominal walls, along the linea alba, the stomach is opened, and the lips of the gastric wound securely sewn to those of the incision in the abdominal walls. Union soon takes place, so that a permanent opening from the exterior into the inside of the stomach is established. A tube of proper construction, introduced at the time of the operation, becomes firmly secured in place by the contraction of healing. Through the tube the contents of the stomach can be received, and the mucous membrane stimulated at pleasure.

When obtained from a natural fistula in man, its specific gravity has been found to differ little from that of water, varying from 1.001 to 1.010 , and the amount of solids present to be correspondingly small. In animals pure gastric juice seems to be equally poor in solids, the higher estimates which some observers have obtained being probably due to admixture with food, etc.

Of the solid matters present about half are inorganic salts, chiefly alkaline (sodium) chlorides, with small quantities of phosphates. The organic material consists of pepsin, a body to be described immediately, mixed with other substances of undetermined nature. In a healthy stomach gastric juice contains a very small quantity only of mucin, unless some submaxillary saliva has been swallowed.

The reaction is distinctly acid, and the acidity is normally due to free hydrochloric acid. This is shown by various proofs, among which we may mention the conclusive fact that the amount of chlorine present in gastric juice is more than would suffice to form chlorides with all the bases present, and that the excess, if regarded as existing in the form of hydrochloric acid, corresponds exactly to the quantity of free acid present. Lactic and butyric and other acids when present are secondary products, arising either by their respective fermentations from articles of food, or from the decomposition of their alkaline or other salts. In man the amount of free hydrochloric acid in healthy juice may be stated to be about 0.2 per cent., but in some animals it is probably higher.

$\$ 188$. On starch gastric juice has no amylolytic action; on the contrary, when saliva is mixed with gastric juice any amylolytic ferment which may be present in the former is at once prevented from acting by the acidity of the mixture. Moreover, in a very short time, especially at the temperature of the body, the amylolytic ferment is destroyed by the acid, so that even on neutralization the mixture is unable to convert starch into sugar.

On dextrose healthy gastric juice has no effect. And its power of converting cane-sugar seems to be less than that of hydrochloric acid diluted to the same degree of acidity as itself. In an unhealthy stomach, however, containing much mucus, the gastric juice is very active in converting canesugar into dextrose. This power seems to be due to the presence in the mucus of a special ferment, analogous to, but quite distinct from, the 
ptyalin of saliva. An excessive quantity of cane-sugar introduced into the stomach causes a secretion of mucus, and hence provides for its own conversion.

On fats gastric juice has at most a limited action. When adipose tissue is eaten, the chief change which takes place in the stomach is that the proteid and gelatiniferous envelopes of the fat-cells are dissolved, and the fats set free. Though there is experimental evidence that emulsion of fats to a certain extent does take place in the stomach, the great mass of the fat of a meal is not so changed.

Such minerals as are soluble in free hydrochloric acid are for the most part dissolved; though there is a difference in this and in some other respects between gastric juice and simple free hydrochloric acid diluted with water to the same degree of acidity as the juice, the presence either of the pepsin or of other bodies apparently modifying the solvent action of the acid.

The essential property of gastric juice is the power of dissolving proteid matters and of converting them into a substance called peptone.

Action of gastric juice on proteids. The results are essentially the same, whether natural juice obtained by means of a fistula, or artificial juice, $i . e$. an acid infusion of the mucous membrane of the stomach, be used.

Artificial gastric juice may be prepared in any of the following ways:

1. 'The mucous membrane of a pig's or dog's stomach is removed from the muscular coat, finely minced, rubbed in a mortar with pounded glass and extracted with water. The aqueous extract filtered and acidulated (it is in itself somewhat acid), until it has a free acidity corresponding to 0.2 per cent. of hydrochloric acid, contains but little of the products of digestion, such as peptone, but is fairly potent.

2. The mucous membrane similarly prepared and minced is allowed to digest at $35^{\circ} \mathrm{C}$. in a large quantity of hydrochloric acid diluted to 0.2 per cent. The greater part of the membrane disappears, shreds only being left, and the somewhat opalescent liquid can be decanted and filtered. The filtrate has powerful digestive (peptic) properties, but contains a considerable amount of the products of digestion (peptone, etc.) arising from the digestion of the mucous membrane itself. $^{1}$

3. The mucous membrane, similarly prepared and minced, is thrown into a comparatively large quantity of concentrated glycerin, and allowed to stand. The membrane may be previously dehydrated by being allowed to stand under alcohol, but this is not necessary, and a too prolonged action of the alcohol injures or even destroys the activity of the product. The decanted clear glycerin, in which a comparatively small quantity of the ordinary proteids of the mucous membrane are dissolved, if added to hydrochloric acid of 0.2 per cent. (about 1 c.c. of the glycerin to 100 c.c. of the dilute acid are sufficient), makes an artificial juice tolerably free from ordinary proteids and peptone, and of remarkable potency, the presence of the glycerin not interfering with the results.

Before proceeding to study the action of gastric juice on proteids, it will be useful to review very briefly the chief characters of the more important members of the group.

The more important proteids which we have thus far studied are: 1. Fibrin, insoluble in water and not really soluble (i.e., without change) in saline solutions. 2. Myosin, insoluble in water, but soluble in saline solutions, provided these are not too dilute or too concentrated. 3. Globulin (including paraglobulin, fibrinogen, etc.), insoluble in water, but readily soluble in even very dilute saline solutions. 4. Albumin, serum-albumin, soluble in water in the absence of all salts. 5. Acid-albumin, into which globulins and myosin are rapidly converted by the action of dilute acids,

1 These, however, may be removed by concentration at $40^{\circ} \mathrm{C}$. and subsequent dialysis. 
the particular acid-albumin into which the myosin of muscle is changed, being sometimes called syntonin. If the reagent used be not dilute acid, but dilute alkali, the product is called alkali-albumin. The two bodies, acid-albumin and alkali-albumin, are very parallel in their characters, and may readily be converted, the one into the other, by the use of dilute alkali or dilute acid respectively. Their most important common characters are insolubility in water and in saline solutions and ready solubility in dilute acids and alkalies. 6. Coagulated proteids. As we have seen, when fibrin suspended in water, serum-albumin in solution, acid-albumin or alkalialbumin suspended in water, or paraglobulin suspended in water or dissolved in a dilute saline solution, are heated to a temperature which for the whole group may be put down at about $75^{\circ}$ to $80^{\circ} \mathrm{C}$., each of them becomes coagulated, and after the change is insoluble in water, saline solutions, dilute acids, etc., in fact in everything but very strong acids. Myosin and fibrinogen undergo a similar change at a lower temperature, viz., about $56^{\circ} \mathrm{C}$. We may, for present purposes, speak of all these proteids thus changed under the one term of coagulated proteids.

To the above list we may now add two other proteids, viz.: 7. A kind of albumin which forms the great bulk of the proteid matter present in raw "white of egg," and which, since it differs in minor characters from the albumin of blood and of the tissues, is called egg-albumin. 8. The peculiar proteid casein, an important constituent of milk. This may perhaps be regarded as a naturally occurring alkali-albumin, since it has many resemblances to the artificial alkali-albumin; but for several reasons it is desirable to consider it as an independent body.

Egg-albumin, like serum-albumin, becomes coagulated at a temperature of about $75^{\circ}$ to $80^{\circ} \mathrm{C}$, and though casein as it naturally exists in milk is not coagulated on boiling, when separated out in a special way, and suspended in water in which it is insoluble, it becomes coagulated at about $75^{\circ}$ to $80^{\circ} \mathrm{C}$.

It will be observed that all these proteids form, as regards their solubilities, a descending series in the following order: coagulated proteids; fibrin ; acid-albumin with alkali-albumin, and casein; myosin, globulin ; serumalbumin with egg-albumin.

We must now return to the action of gastric juice.

If a few shreds of fibrin, obtained by whipping blood, after being thoroughly washed and boiled and thus by the boiling coagulated, be thrown into a quantity of gastric juice, and the mixture be exposed to a temperature of from $35^{\circ}$ to $40^{\circ} \mathrm{C}$., the fibrin will speedily, in some cases in a few minutes, be dissolved. The shreds first swell up and become transparent, then gradually dissolve, and finally disappear with the exception of some granular débris, the amount of which, though generally small, varies according to circumstances. If raw, that is, unboiled, uncoagulated fibrin be employed the same changes may be observed, but they take place much more rapidly.

If small morsels of coagulated albumin, such as white of egg, be treated in the same way, the same solution is observed. The pieces become transparent at their surfaces ; this is especially seen at the edges, which gradually become rounded down; and solution steadily progresses from the outside of the piece inward.

If any other form of coagulated albumin (e.g., precipitated acid-or alkali-albumin, suspended in water and boiled) be treated in the same way, a similar solution takes place. The readiness with which the solution is effected, will depend, cceteris paribus, on the smallness of the pieces, or rather on the amount of surface as compared with bulk, which is presented to the action of the juice. 
Gastric juice then readily dissolves coagulated proteids which otherwise are insoluble, or soluble only, and that with difficulty, in very strong acids.

When proteids which are soluble in water, or in dilute acid, are treated with gastric juice, no visible change takes place; but nevertheless, it is found on examination that the solutions have undergone a remarkable change, the nature of which is easily seen by contrasting it with the change effected by dilute acid alone. If raw white of egg, largely diluted with water and strained, be treated with a sufficient quantity of dilute hydrochloric acid, the opalescence or turbidity which appeared in the white of egg on dilution (and which is due to the precipitation of various forms of globulin accompanying the egg-albumin in the raw white) disappears, and a clear mixture results. If a portion of the mixture be at once boiled, a large deposit of coagulated albumin occurs. If, however, the mixture be exposed to $50^{\circ}$ to $55^{\circ} \mathrm{C}$. for some time, the amount of coagulation which is produced by boiling a specimen becomes less, and, finally, boiling produces no coagulation whatever. By neutralization, however, the whole of the albumin (with such restrictions as the presence of certain neutral salts may cause) may be obtained in the form of acid-albumin, the filtrate after neutralization containing no proteids at all (or a very small quantity). Thus the whole of the albumin present in the white of egg may be, in time, converted, by the simple action of dilute hydrochloric acid, into acid-albumin. Serum-albumin similarly treated undergoes in course of time a similar conversion into acid-albumin, and we have already seen ( $\$ 59)$ that solutions of myosin or of any of the globulins are with remarkable rapidity converted into acid-albumin. Thus simple dilute hydrochloric acid of the same degree of acidity as gastric juice, merely converts these proteids into acid-albumin, the rapidity of the change differing with the different proteids, being in some cases very slow, and requiring a relatively high temperature.

If the same white of egg or serum-albumin be treated with gastric juice instead of simple dilute hydrochloric acid, the events for some time seem the same. Thus after a while boiling causes no coagulation, while neutralization gives a considerable precipitate of a proteid body, which being insoluble in water and in sodium chloride solutions and soluble in dilute alkali and acids, at least closely resembles acid-albumin. But it is found that only a portion of the proteid originally present in the white of egg or serum-albumin can thus be regained by precipitation. Though the neutralization be carried out with the greatest care it will be found, on filtering off the neutralization precipitate, that is, the acid-albumin, that the filtrate, as shown on employing the various tests for proteid (see $\$ 15$ ) or on adding an adequate quantity of strong alcohol, still contains a very considerable quantity of proteid matter ; and, on the whole, the longer the digestion is carried on, the greater is the proportion borne by the proteid remaining in solution to the precipitate thrown down on neutralization; indeed, in some cases at all events, all the proteid matter originally present remains in solution, and there is 110 neutralization precipitation at all, or at most a wholly insignificant one.

$\S 189$. The proteid matter, thus remaining in solution after neutralization differs from all the proteids which we have hitherto studied, inasmuch as, though existing in a neutral solution, it is not coagulated by heat, like the egg-albumin or serum-albumin from which it has been produced; the solution, after the neutralization precipitate has been filtered off, remains quite clear when boiled. The only other solutions of proteids which do not coagulate on boiling are solutions of acid- or alkali-albumin ; but these solutions must be acid or alkali respectively; the acid-albumin or alkali-albumin is insoluble in a neutral solution, and when simply suspended in water is 
readily coagulated at a temperature of $75^{\circ} \mathrm{C}$. This new proteid matter of which we are speaking is soluble in neutral solutions, indeed in distilled water, and can under no circumstances be coagulated by heat.

Upon examination we find that the new proteid matter thus left in solution consists of at least two distinct proteid bodies. If to the solution ammonium sulphate be added, part of the proteid matter is precipitated while part is still left in solution. The proteid body thus thrown down is called albumose (there are several varieties of albumose, but these need not now detain us). It approaches albumin in nature by reason of the fact that it will not diffuse through membranes ; that it differs, however, widely from that proteid is shown by its solutions not coagulating on boiling. The body which is not thrown down by ammonium sulphate is called peptone; it differs from albumose in being diffusible, for it will pass through membranes. The diffusion is not nearly so rapid as that of salts, sugar, and other similar substances; indeed solutions of peptones may be freed from salts by dialysis. But it is very marked as compared with that of other proteids; these pass through membranes with the greatest difficulty, if at all. Peptone is insoluble in alcohol, and may be precipitated from its solutions by the addition of an adequate quantity of this reagent; but for this purpose a very large excess of alcohol is needed, otherwise much of the peptone remains in solution. It may be kept under alcohol for a long time without undergoing change, whereas other proteids are more or less slowly coagulated by alcohol. A useful test for peptone is furnished by the fact that a solution of peptone, mixed with a strong solution of caustic potash, gives on addition of a mere trace of cupric sulphate in the cold a pink color, whereas other proteids give a violet color. In applying this test, however, care must be taken not to add too much cupric sulphate, since in that case a violet color, deepening on boiling, that is, the ordinary proteid reaction (see $\$ 15$ ), is obtained.

There are reasons for thinking that there are several kinds, or at least more than one kind, of peptone ; but we may for the present regard the substance as one. For a long time albumose was confounded with peptone, and many of the commercial forms of "peptone" consist largely of albumose; indeed, the two are closely allied and have many reactions in common, the most striking differences being that peptone is diffusible, while albumose is not, or hardly at all, and that peptone is not, like albumose, precipitated by ammonium sulphate. The amount of albumose appearing in a digestion experiment, relative to the amount of true peptone, depends on the activity of the juice, and other circumstances. We may regard albumose as a less complete product of digestion than peptone.

The precipitate thrown down by neutralization after the action of gastric juice on egg- or serum-albumin resembles, in its general characters, acidalbumin. Since, however, it probably is distinguishable from the body or bodies produced by the action of simple acid on muscle or white of egg, it is best to reserve for it the name of parapeptone, which was originally applied to it.

Thus the digestion by gastric juice of solutions of egg-albumin or serumalbumin results in the conversion of all the proteids present into peptone, albumose, and parapeptone, of which the first may be considered as the final and chief product, and the other two as intermediate products, occurring in varying quantity, possibly not always formed, and probably of secondary importance. When fibrin, either raw or boiled, or any form of coagulated proteid is dissolved and seems to disappear under the influence of gastric juice, the same products, peptone, albumose, and parapeptone make their appearance. The same bodies result when myosin or any of the globulins are subjected to the action of the juice; and acid-albumin or alkali-albumin is similarly converted into albumose and peptone. 
It is obvious that the effect of the action of the gastric juice is to change the less soluble proteid into a more soluble form, the change being either completed up to the stage of peptone, the most soluble of all proteids, or being left in part incomplete. This will be seen from the following tabular arrangement of proteids according to their solubilities:

\section{Soluble in distilled water.}

Aqueous solutions not coagulated on boiling:

Diffusible ... . . . . . . Peptone.

Not diffusible . . . . . . . . . Albumose.

Aqueous solutions coagulated on boiling . . . . Albumin.

\section{Insoluble in distilled water.}

Readily soluble in dilute saline solutions $(\mathrm{NaCl} 1$ per cent.) . . . . . .

Soluble only in stronger saline solutions ( $\mathrm{NaCl} 5$ to 10

per cent.) . ........................ Myosin.

\section{Insoluble in dilute saline solutions.}

Readily soluble in dilute acid ( $\mathrm{HCl} 0.1$ per cent.) in the cold .

Soluble with difficulty in dilute acid, that is at high temperature $\left(60^{\circ} \mathrm{C}\right.$. $)$ and after prolonged treatment only .

Insoluble in dilute acids, soluble only in strong acids . Coagulated proteid.

Globulins.

Milk when treated with gastric juice is first of all "curdled." This is the result partially of the action of the free acid, but chiefly of the special action of a particular constituent of gastric juice, of which we shall speak hereafter. The curd onnsists of a particular proteid matter mixed with fat; and this proteid matter is subsequently dissolved with the same appearance of peptone, albumose, and parapeptone as in the case of other proteids. In fact, the digestion by gastric juice of all the varieties of proteids consists in the conversion of the proteid into peptone, with the concomitant appearance of a certain variable amount of albumose and parapeptone.

$\$ 190$. Circumstances affecting gastric digestion. The solvent action of gastric juice on proteids is modified by a variety of circumstances. The nature of the proteid itself makes a difference, though this is determined probably by physical rather than by chemical characters. Hence in making a series of comparative trials the same proteid should be used, and the form of proteid most convenient for the purpose is fibrin. If it be desired simply to ascertain whether any given specimen has any digestive powers at all, it is best to use boiled fibrin, since raw fibrin is eventually dissolved by dilute hydrochloric acid alone, probably on account of some pepsin previously present in the blood becoming entangled with the fibrin during clotting. But in estimating quantitatively the peptic power of two specimens of gastric juice under different conditions, raw fibrin prepared by Grützner's method is most convenient.

Portions of well-washed fibrin are stained with carmine and again washed to remove the superfluous coloring matter. A fragment of this colored fibrin thrown into an active juice on becoming dissolved, gives up its color to the fluid. Hence if the same stock of colored fibrin be used in a series of experiments. and the same bulks of fibrin and of fluid be used in each case, the amount of fibrin dissolved may be fairly estimated by the depth of tint given to the fluid. Fibrin thus colored with carmine may be preserved in ether. 
Since, if sufficient time be allowed, even a small quantity of gastric juice will dissolve at least a very large if not an indefinite quantity of fibrin, we are led to take, as a measure of the activity of a specimen of gastric juice, not the quantity of fibrin which it will ultimately dissolve, but the rapidity with which it dissolves a given quantity.

The greater the surface presented to the action of the juice, the more rapid the solution ; hence minute division and constant movement favor digestion. And this is probably, in part at least, the reason why a fragment of spongy filamentous fibrin is more readily dissolved than a solid clump of boiled white of egg of the same size. Neutralization of the juice wholly arrests digestion; fibrin may be submitted for an almost indefinite time to the action of neutralizerl gastric juice without being digested. If the neutralized juice be properly acidified, it may again become active; when gastric juice, however, has been made alkaline, and kept for some time at a temperature of $35^{\circ} \mathrm{C}$., its solvent powers are not only suspended but actually destroyed. Digestion is most rapid with dilute hydrochloric acid of 0.2 per cent. (the acidity of natural gastric juice). If the juice contains much more or much less free acid than this, its activity is distinctly impaired. Other acids, lactic, phosphoric, etc., may be substituted for hydrochloric; but they are not so effectual, and the degree of acidity most useful varies with the different acids. The presence of neutral salts, such as sodium chloride, in excess is injurious. 'The action of mammalian gastric juice is most rapid at $35^{\circ}-40^{\circ} \mathrm{C}$.; at the ordinary temperature it is much slower, and at about $0^{\circ} \mathrm{C}$. ceases altogether. The juice may be kept, however, at $0^{\circ} \mathrm{C}$. for an indefinite period without injury to its powers. The gastric juice of coldblooded vertebrates is relatively more active at low temperatures than that of warm-blooded mammals or birds.

At temperatures much above $40^{\circ}$ or $45^{\circ}$ the action of the juice is impaired. By boiling for a few minutes the activity of the most powerful juice is irrevocably destroyed. The presence in a concentrated form of the products of digestion hinders the process of solution. If a large quantity of fibrin be placed in a small quantity of juice, digestion is soon arrested; on dilution with the normal hydrochloric acid ( 0.2 per cent.), or if the mixture be submitted to dialysis to remove the peptones formed, and its acidity be kept up to the normal, the action recommences. By removing the products of digestion as fast as they are formed, and by keeping the acidity up to the normal, a given amount of gastric juice may be made to digest a very large quantity of proteid material. Whether the quantity is really unlimited is disputed; but in any case the energies of the juice are not rapidly exhausted by the act of digestion.

$\S 191$. Nature of the action. All these facts go to show that the digestive action of gastric juice on proteids, like that of saliva on starch, is a fermentaction; in other words, that the solvent action of gastric juice is essentially due to the presence in it of a ferment-body. To this ferment-body, which as yet has been only approximately isolated, the name of pepsin has been given. It is present not only in gastric juice, but also in the glands of the gastric mucous membrane, especially in certain parts and under certain conditions which we shall study presently. The glycerin extract of gastric mucous membrane, at any rate of that which has been dehydrated, contains a minimal quantity of proteid matter, and yet is intensely peptic. Other methods, such as the elaborate one of Briicke, give us a material which, though containing nitrogen, exhibits none of the ordinary proteid reactions, and yet in concert with normal dilute hydrochloric acid is peptic in a very high degree. IVe seem, therefore, justified in asserting that pepsin is not a proteid, but it would be hazardous to make any dogmatic statement con- 
cerning a substance obtained in so small a quantity at a time that its exact chemical characters have not yet been ascertained. At present the manifestation of peptic powers is our only safe test of the presence of pepsin.

In one important respect pepsin, the ferment of gastric juice, differs from .ptyalin, the ferment of saliva. Saliva is active in a perfectly neutral medium, and there seems to be no special connection between the ferment and any alkali or acid. In gastric juice, however, there is a strong tie between the acid and the ferment, so strong that some writers speak of pepsin and hydrochloric acid as forming together a compound, peptohydrochloric acid.

In the absence of exact knowledge of the constitution of proteids, we cannot state distinctly what is the precise nature of the change into peptone; the various proteids differ from each other in elementary composition quite as widely as does peptone from any of them. Judging from the analogy with the action of saliva on starch, we may fairly suppose that the process is at bottom one of hydration; and this view is further suggested by the fact that peptone closely resembling, if not identical with, that obtained by gastric digestion, may be obtained by the action of the strong acids, by the prolonged action of dilute acids especially at a high temperature, or simply by digestion with superheated water in a Papin's digester, that is to say, by means of agents which, in other cases produce their effects by bringing about hydrolytic changes; beyond this we cannot at present go.

$\S 192$. All proteids, as far as we know, are converted by pepsin into peptone. Concerning the action of gastric juice on other nitrogenous substances more or less allied to proteids, but not truly proteid in nature, our knowledge is at present imperfect. Mucin, nuclein, and the chemical basis of horny tissues are wholly unaffected by gastric juice. The gelatiniferous tissues are dissolved by it; and the bundles and membranes of connective tissue are very speedily so far affected by it that at a very early stage of digestion the bundles and elementary fibres of muscles which are bound together by connective tissue fall asunder; moreover, both prepared gelatin and the gelatiniferous basis of connective tissue in its natural condition, that is without being previously heated with water, are by it changed into a substance so far analogous with peptone that the characteristic property of gelatinization is entirely lost. Chondrin and the elastic tissues undergo a similar change.

$\$ 193$. Action of gastric juice on milk. It has long been known that an infusion of calves' stomach, called rennet, has a remarkable effect in rapidly curdling milk, and this property is made use of in the manufacture of cheese. Gastric juice has a similar effect: milk when subjected to the action of gastric juice is first curdled and then digested. If a few drops of gastric juice be added to a little milk in a test-tube, and the mixture exposed to a temperature of $40^{\circ} \mathrm{C}$., the milk will curdle into a complete clot in a rery short time. If the action be continued the curd or clot will be ultimately dissolved and digested. Milk contains, besides a peculiar form, or peculiar forms of albumin, fats, milk-sugar, and various salines, the peculiar proteid casein. In natural milk casein is present in solution, and "curdling" consists essentially in the soluble casein being converted (or more probably as we shall see presently, split up) into an insoluble modification of casein, which as it is being precipitated carries down with it a great deal of the fat and so forms the "curd." Now casein is readily precipitated from milk upon the addition of a small quantity of acid, and it might be supposed that the curdling effect of gastric juice was due to its acid reaction. 
But this is not the case, for neutralized gastric juice, or neutral rennet, is equally efficacious.

The curdling action of rennet is closely dependent on temperature, being like the peptic action of gastric juice favored by a rise of temperature up to about $40^{\circ} \mathrm{C}$. Moreover the curdling action is destroyed by previous boiling of the juice or rennet. These facts suggest that a ferment is at the bottom of the matter; and, indeed, all the features of the action support this view. Moreover, as a matter of fact, a curdling ferment may be extracted by glycerin and by the other methods used for preparing ferments. The ferment, however, is not pepsin, but some other body; and the two may be separated from each other.

It might be thought that the rennet-ferment, rennin we may call it, acted by inducing a fermentation in the sugar of milk, giving rise to lactic acid which precipitated the casein by virtue of its being an acid. But this view is disproved by the following facts which show that the ferment produces its curdling effect by acting directly on the natural casein itself. Casein may be precipitated unchanged, that is, capable of redissolving in water (the presence of calcic phosphate being assumed) by saturating milk with neutral saline bodies (such as sodium chloride or magnesium sulphate); and by being precipitated and redissolved more than once may be obtained largely free from fat and wholly free from milk-sugar. Such solutions of isolated casein freed from milk-sugar may be made to curdle like natural milk by the addition of rennin, showing that the milk-sugar has nothing to do with the matter. Moreover, the precipitate thrown down from milk by dilute acids, lactic acid included, is itself unaltered or very slightly altered casein, not curd, and with care may be so prepared as to be redissolved into solutions which curdle with rennin, like solutions of casein prepared by means of neutral salts.

When isolated casein is curdled by means of reunin, two proteids, it is stated, make their appearance, one which is soluble and allied to albumin, and another which is insoluble and forms the curd. Curdling, therefore, according to this result appears to be the splitting up by a ferment of a more complex body; and it is interesting to observe, as perhaps throwing light on the somewhat analogous formation of the fibrin, that this curdling action will not take place if calcic phosphate be wholly absent from the mixture. The calcic phosphate appears to play a peculiar part in determining the insolubility of the curd, for there is evidence that in the absence of calcic phosphate the ferment has power to attack the casein and split it up, but that both products remain in solution; if calcic phosphate be present, the one, viz., the curd, ${ }^{1}$ becomes insoluble.

Rennin is abundant in the gastric juice and in the gastric mucous membrane of ruminants, but is also found in the gastric juice of other animals, and either it, or what we shall presently have occasion to speak of as the antecedent of the ferment or zymogen, is present also in the mucous membrane of the stomach of most animals. A very similar if not identical ferment has also been found in many plants.

\section{The Act of Secretion of Saliva and Gastric Juice and the Nervous Mechanisms which Regulate it.}

$\S 194$. The saliva and gastric juice whose properties we have studied, though so different from each other, are both drawn ultimately from one

\footnotetext{
${ }^{1}$ It might be useful, in order to distinguish the curd from the natural soluble casein, to call the former tyrein (rvpós, cheese), and so reserve the name of casein for the latter.
} 
common source, the blood, and they are poured into the alimentary canal, not in a continuous flow, but intermittently as occasion may demand. The epithelial cells which supply them have their periods of rest and of activity, and the amount and quality of the fluids which the cells secrete are determined by the needs of the economy as the food passes along the canal. We have now to consider how the epithelial cell manufactures its special secretion out of the materials supplied to it by the blood, and how the cell is called into activity by the presence of food, it may be as in the case of saliva at some distance from itself, or by circumstances which do not bear directly on itself. In dealing with these matters in connection with the digestive juices, we shall have to enter at some length into the physiology of secretion in general.

The question which presents itself first is: By what mechanism is the activity of the secreting cells brought into play?

While fasting, a small quantity only of saliva is poured into the mouth; the buccal cavity is just moist and nothing more. When food is taken, or when any sapid or stimulating substance, or indeed a body of any kind, is introduced into the mouth, a flow is induced which may be very copious. Indeed the quantity secreted in ordinary life during 24 hours has been roughly calculated at as much as from 1 to 2 litres. An abundant secretion in the absence of food in the mouth may be called forth by an emotion, as when the mouth waters at the sight of food, or by a smell, or by events occurring in the stomach, as in some cases of nausea. Evidently in these instances some nervous mechanism is at work. In studying the action of this nervous mechanism, it will be of advantage to confine our attention at first to the submaxillary gland.

$\S 195$. The submaxillary gland is supplied with two sets of nerves. These are represented in Fig. 81 , which is a very diagrammatic rendering of the appearances presented when the submaxillary gland is prepared for an experiment in a dog, the animal being placed on its back and the gland exposed from the neck. The one set, and that the more important, belongs to the chorda tympani nerve (ch.t." ). This is a small nerve, which branches off from the facial or seventh cranial nerve in the Fallopian canal before the nerve issues from the skull. Whether it really belongs to the facial proper has been doubted; in man the fibres which form it are either fibres coming not from the roots of the facial proper, but from the portio intermedia Wrisbergi, or, according to some, fibres which, though joining the facial in the Fallopian canal, are ultimately derived from another (the fifth) cranial nerve. Leaving the facial nerve, the chorda tympani passes through the tympanic cavity or drum of the ear (hence the name) and joins or rather runs in company (ch.t.') with the lingual or gustatory branch of the fifth nerve. Some of the fibres run on with the lingual right down to the tongue (these are not shown in the figure), but many leave the lingual as a slender nerve (ch.t.), which, reaching Wharton's duct or duct of the submaxillary gland $(s m . d$.$) runs along the duct to the gland. As$ the nerve courses along the duct nerve-cells make their appearance among the fibres, and these are especially abundant just after the duct enters the hilus of the gland. The fibres may be traced into the gland for some distance, but, as we have said, their ultimate ending has not yet been definitely made out. Along its whole course up to the gland, the fibres of the chorda are very fine medullated fibres, but they lose their medulla in the gland.

The other set of nerve-fibres reaches the gland along the small arteries of the gland. These are non-medullated fibres mixed with a few medullated fibres, and may be traced back to the superior cervical ganglion. From thence they may be traced still further back down the cervical sympathetic 
to the spinal cord, following apparently the same tract as the vaso-constrictor fibres, treated of in $\S 152$.

$\$ 196$. If a tube be placed in the duct, it is seen that when sapid substances are placed on the tongue, or the tongue is stimulated in any nther way, or the lingual nerve is laid bare and stimulated with an interrupted current, a copious flow of saliva takes place. If the sympathetic be divided, stimulation of the tongue or lingual nerve still produces a flow. But if the small chorda nerve be divided, stimulation of the tongue or lingual nerve produces no flow.

Evidently the flow of saliva is a nervous reflex action, the lingual nerve serving as the chamnel for the afferent and the small chorda nerve for the efferent impulses. If the trunk of the lingual be divided above the point where the chorda leaves it, as at $n . l^{\prime}$, Fig. 81 , stimulation of the (front part

Fig. 81.

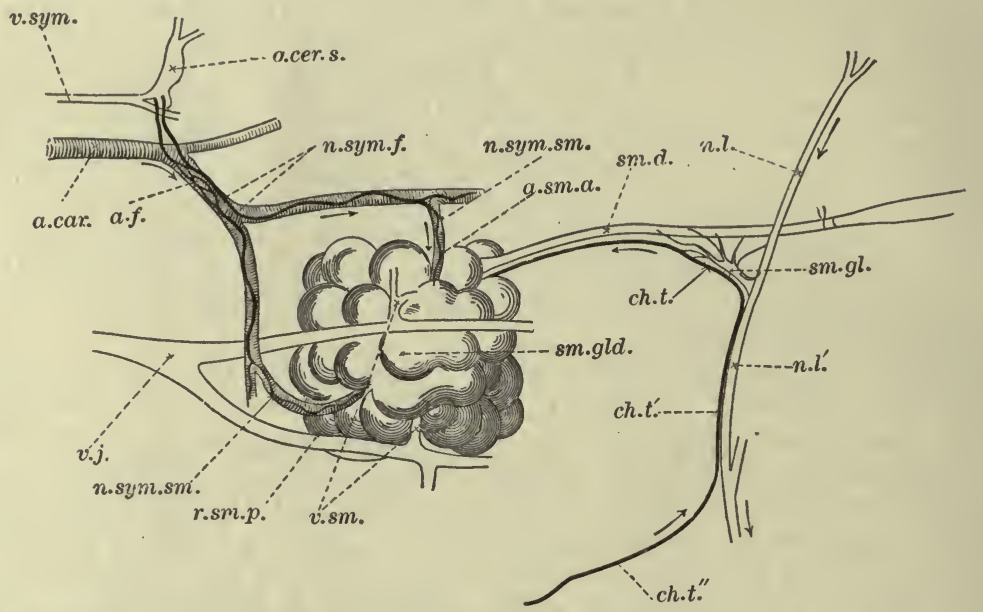

Diagrammatic Representation of the Submaxillary Gland of the Dog, with its Nerves and Bloodvessels. The dissection has been on an animal lying on its back, but since all the parts shown in the figure cannot be seen from any nue point of view, the figure does not give the exact anatomical relations of the several structures.

$s m$. gld. The submaxillary gland, into the duct $(s m . d$.) of which a callula has been tied. The sublingual gland and duet are not shown. n.l.,n.l'. The lingual branch of the fifth nerve, the part n.l. is going to the tongue. ch.t., ch.t'., ch.t". The chorda tympani. The part $c h . t^{\prime \prime}$. is proceeding from the facial nerve; at $c h . t^{\prime}$. it becomes conjoined with the lingual $n . l^{\prime}$., and afterward diverging passes as ch.t. to the gland along the duct; the continuation of the nerve in company with the lingual $n . l$., is not shown. $8 m . g l$. The submaxillary ganglion with its several roots. $a . c a r$. The carotid artery, two small branches of which, $a . s m$. $a$. and $r$. sm.p. pass to the anterior and posterior parts of the gland. $v . s m$. The anterior and posterior veins from the gland, falling into $v . j$., the jugular vein. $v$. sym. The conjoined vagus and sympathetic trunks. g.cer. s. The upper cervical ganglion, two branches of which, forming a plexus $(a, f)$ over the facial artery, are distributed $(n .8 y m . s m$.) along the two glandular arteries to the anterior and posterior portions of the gland.

The arrows indicate the direction taken by the nervous impulses during reflex stimulation of the gland. They ascend to the brain by the lingual and descend by the chorda tympani.

of ) tongue produces, under ordinary circumstances, no flow. This shows that the centre of the reflex action is higher up than the point of section; it lies in fact in the brain.

In the angle between the lingual and the chorda, where the latter leaves the former to pass to the gland, lies the small submaxillary ganglion (represented diagrammatically in Fig. 81, sm. gl.). This consists of small masses of nerve-cells 
lying on the small bundles of nerve-fibres which spread out like a fan from the lingual and chorda tympani nerves $(c h . t$.) toward the ducts of the submaxillary and sublingual glands. It has been much debated whether this ganglion can act as a centre of reflex action in connection with the submaxillary gland, but no conclusive evidence that it does so act has as yet been shown; it probably belongs in reality to the sublingual gland.

Stimulation of the glosso-pharyngeal is even more effectual than that of the lingual. Probably this indeed is the chief afferent nerve in ordinary secretion. Stimulation of the mucous membrane of the stomach (as by food introduced through a gastric fistula) or of the vagus may also produce a flow of saliva, as indeed may stimulation of the sciatic, and probably of many other afferent nerves. All these cases are instances of reflex action, the cerebro-spinal system acting as a centre. We may further define the centre as a part of the medulla oblongata, apparently not far removed from the vasomotor centre. When the brain is removed down to the medulla oblongata, that organ being left intact, a flow of saliva mav still be obtained by adequate stimulation of various afferent nerves; when the medulla is destroyed no such action is possible. And a flow of saliva may be produced by direct stimulation of the medulla itself. When a flow of saliva is excited by ideas, or by emotions, the nervous processes begin in the higher parts of the brain, and descend thence to the medulla before they give rise to distinctly efferent impulses; and it would appear that these higher parts of the brain are called into action when a flow of saliva is excited by distinct sensations of taste.

Considering, then, the flow of saliva as a reflex act, the centre of which lies in the medulla oblongata, we may imagine the efferent impulses passing from that centre to the gland either by the chorda tympani or by the sympathetic nerve. Although it would perhaps be rash to say that in this relation the sympathetic nerve never acts as an efferent channel, as a matter of fact we have no satisfactory experimental evidence that it does so; and we may, therefore, state that, practically, the chorda tympani is the sole efferent nerve. Section of that nerve, either where the fibres pass from the lingual nerve and the submaxillary ganglion to the gland, or where it runs in the same sheath as the lingual, or in any part of its course from the main facial trunk to the lingual, puts an end, as far as we know, to the possibility of any flow being excited by stimuli applied to the sensory nerves, or to the sentient surfaces of the mouth or of other parts of the body.

The natural reflex act of secretion may be inhibited, like the reflex action of the vasomotor nerves, at its centre. Thus when, as in the old rice ordeal, fear parches the mouth, it is probable that the afferent impulses caused by the presence of food in the mouth cease, through emotional inhibition of their reflex centre, to give rise to efferent impulses.

$\$ 197$. In life, then, the flow of saliva is brought about by the advent to the gland along the chorda tympani of efferent impulses, started chiefly by reflex actions. The inquiry thus narrows itself to the question: In what manner do these efferent impulses cause the increase of flow?

If in a dog a tube be introduced into Wharton's duct, and the chorda be divided, the flow, if any be going on, is from the lack of efferent impulses arrested. On passing an interrupted current through the peripheral portion of the chorda, a copious secretion at once takes place, and the saliva begins to rise rapidly in the tube; a very short time after the application of the current the flow reaches a maximum which is maintained for some time, and then, if the current be long continued, gradually lessens. If the current be applied for a short time only, the secretion may last for some time after the current has been shut off. The saliva thus obtained is but slightly viscid, 
and under the microscope a very few salivary corpuscles, and occasionally only, amorphous lumps of peculiar material, probably mucous in nature, are seen. If the gland itself be watched, while its activity is thus roused, it will be seen (as we have already said, $§ 153$ ) that its arteries are dilated and its capillaries filled, and that the blood flows rapidly through the veins in a full stream and of bright arterial hue, frequently with pulsating movements. If a vein of the gland be opened, this large increase of flow, and the lessening of the ordinary deoxygenation of the blood consequent upon the rapid stream will be still more evident. It is clear that excitation of the chorda largely dilates the arteries; the nerve acts energetically as a vaso-dilator nerve.

Thus stimulation of the chorda brings about two events: a dilatation of the bloodvessels of the gland, and a flow of saliva. This question at once arises, Is the latter simply the result of the former or is the flow caused by some direct action on the secreting cells, apart from the increased bloodsupply? In support of the former view we might argue that the activity of the epithelial secreting cell, like that of any other form of protoplasm, is dependent on blood-supply. When the small arteries of the gland dilate, while the pressure in the arteries on the side toward the heart is (as we have previously seen when treating generally of blood-pressure, $\S 109$ ) correspondingly diminished, the pressure on the far side in the capillaries and veins is increased; hence the capillaries become fuller, and more blood passes through them in a given time. From this we might infer that a larger amount of nutritive material would pass away from the capillaries into the surrounding lymph-spaces, and so into the epithelial cells, the result of which would naturally be to quicken the processes going on in the cells, and to stir these up to greater activity. But even admitting all this it does not necessarily follow that the activity thus excited should take on the form of secretion. It is quite possible to conceive that the increased blood-supply should lead only to the accumulation in the cell of the constituents of the saliva, or of the raw materials for their construction, and not to a discharge of the secretion. A man works better for being fed, but feeding does not make him work in the absence of any stimulus. The increased blood-supply, therefore, while favorable to active secretion, need not necessarily bring it about. Moreover, the following facts distinctly show that it need not. When a canula is tied into the duct and the chorda is energetically stimulated, the pressure acquired by the saliva accumulated in the canula and in the duct may exceed for the time being the arterial blood-pressure, even that of the carotid artery ; that is to say, the pressure of fluid in the gland outside the bloodvessels is greater than that of the blood inside the bloodvessels. This must, whatever be the exact mode of transit of nutritive material through the vascular walls, tend to check that transit. Again, if the head of an animal be rapidly cut off, and the chorda immediately stimulated, a flow of saliva takes place far too copious to be accounted for by the emptying of the salivary channels through any supposed contraction of their walls. In this case secretion is excited in the gland though the blood-supply is limited to the small quantity still remaining in the bloodvessels. Lastly, if a small quantity of atropine be injected into the veins, stinulation of the chorda produces no secretion of saliva at all, though the dilatation of the bloodvessels takes place as usual; in spite of the greatly increased bloodsupply no secretion at all takes place. These facts prove that the secretory activity is not simply the result of vascular changes, but may be called forth independently; they further lead us to suppose that the chorda contains two sets of fibres, one which we may call secretory filores, acting directly on the secreting structures only, and the other vaso-dilator fibres, acting on the 
bloodvessels only, and further that atropine, while it has no effect on the latter, paralyzes the former just as it paralyzes the inhibitory fibres of the vagus. Hence when the chorda is stimulated there pass down the nerve, in addition to impulses affecting the blood-supply, impulses affecting directly the protoplasm of the secreting cells, and calling it into action, just as similar impulses call into action the contractility of the substance of a muscular fibre. Indeed, the two things, secreting activity and contracting activity, are very parallel.

Since the chorda acts thus directly on the secreting cells, we should expect to find an anatomical connection between the cells and the nerve; and some authors have maintained that the nerve-fibres may be traced into the cells. But, save perhaps in the case of certain glands of invertebrates (so-called salivary glands of Blatta), the evidence, as we have said, is as yet not convincing.

$\S 198$. When the cervical sympathetic is stimulated, the vascular effects, as we have already said ( $\S 154)$, are the exact contrary of those seen when the chorda is stimulated. The small arteries are constricted, and a small quantity of dark venous blood escapes by the veins. Sometimes, indeed, the flow through the gland is almost arrested. The sympathetic, therefore, acts as a vaso-constrictor nerve, and in this sense is antagonistic to the chorda.

As concerns the flow of saliva brought about by stimulation of the sympathetic, in the case of the submaxillary gland of the dog the effects are very peculiar. A slight flow results, and the saliva so secreted is remarkably viscid, of higher specific gravity, and richer in corpuscles and in the above-mentioned amorphous lumps than is the chorda saliva. This action of the sympathetic is little or not at all affected by atropine.

In the submaxillary gland of the dog, then, the contrast between the effects of chorda stimulation and those of sympathetic stimulation are very marked; the former gives rise to vascular dilatation with a copious flow of fairly limpid saliva poor in solids, the latter to vascular constriction with a scanty flow of viscid saliva richer in solids. And in other animals a similar contrast prevails, though with minor differences. Thus, in the rabbit both chorda saliva and sympathetic saliva are limpid and free from mucus, though the latter contains more proteids; in the cat, chorda saliva is more viscid than sympathetic saliva; but in both these cases, as in the dog, stimulation of the chorda causes a copious flow with dilated bloodvessels, and stimulation of the sympathetic a scanty flow with vascular constriction. We shall return again presently to these different actions of the two nerves; meanwhile we have seen enough of the history of the submaxillary gland to learn that secretion in this instance is a reflex action, the efferent impulses of which directly affect the secreting cells, and that the vascular phenomena may assist, but are not the direct cause of the flow.

$\S 199$. We have dwelt long on this gland because it has been more fruitfully studied than any other. But the nervous mechanisms of the other salivary glands are in their main features similar. Thus the secretion of the parotid gland, like that of the submaxillary, is governed by two sets of fibres; one of cerebro-spinal origin, running along the auriculo-temporal branch of the fifth nerve, but originating possibly in the glosso-pharyngeal, and the other of sympathetic origin coming from the cervical sympathetic. Stimulation of the cerebro-spinal fibres produces a copious flow of limpid saliva, free from mucus; stimulation of the cervical sympathetic gives rise in the rabbit to a secretion also free from mucus, but rich in proteids and of greater amylolytic power than the cerebro-spinal secretion; in the dog little or no secretion is produced, though, as we shall see later on, certain changes 
are brought about in the gland itself. In both animals the cerebro-spinal fibres are vaso-dilator, and the sympathetic fibres vaso-constrictor in action.

$\S 200$. The secretion of gastric juice. Though a certain amount of gastric juice may sometimes be found in the stomachs of fasting animals, it may be stated generally that the stomach, like the salivary glands, remains inactive, yielding no secretion, so long as it is not stimulated by food or otherwise. The advent of food into the stomach, however, at once causes a copious flow of gastric juice; and the quantity secreted in the twenty-four hours is probably very considerable, but we have no trustworthy data for calculating the exact amount. So also when the gastric mucous membrane is stimulated mechanically, as with a feather, secretion is excited; but to a very small amount even when the whole interior surface of the stomach is thus repeatedly stimulated. The most efficient stimulus is the natural stimulus, viz., food; though dilute alkalies seem to have unusually powerful stimulating effects; thus the swallowing of saliva at once provokes a flow of gastric juice.

During fasting the gastric membrane is of a pale gray color, somewhat dry, covered with a thin layer of mucus, and thrown into folds; during digestion it becomes red, flushed, and tumid, the folds disappear, and minute drops of fluid appearing at the mouths of the glands, speedily run together into small streams. When the secretion is very active, the blood flows from the capillaries into the veins in a rapid stream without losing its bright arterial hue. The secretion of gastric juice is, in fact, accompanied by vascular dilatation in the same way as is the secretion of saliva.

$\$ 201$. Seeing that, unlike the case of the salivary secretion, food is brought into the immediate neighborhood of the secreting cells, it is exceedingly probable that a great deal of the secretion is the result of the working of a local mechanism; and this view is supported by the fact that when a mechanical stimulus is applied to one spot of the gastric membrane the secretion is limited to the neighborhood of that spot and is not excited in distant parts. This local mechanism may be nervous in nature or the effect of the stimulus may perhaps be conveyed directly from cell to cell, from the mouth of the gland to its extreme base, without the intervention of any nervous elements; but the vascular changes at least would seem to imply the presence of a nervous mechanism.

The stomach is supplied with nerve fibres from the two vagi nerves and from the solar plexus of the splanchnic system. The two vagi after forming the osophageal plexus on the osophagus are gathered together again as two main trunks which run along the osophagus-the left in the front, the right at the back-to the stomach. The left or anterior nerve is distributed to the smaller curvature and the front surface of the stomach, forming a plexus in which nerve-cells are present; and branches pass on to the liver and probably to the duodenum. The right or posterior nerve is distributed to the hinder surface of the stomach, but only to the extent of about one-third of its fibres; about two-thirds of the fibres pass on to the solar plexus. The fibres of the vagus nerves thus distributed to the stomach are for the most part non-medullated fibres; by the time the vagus reaches the abdomen it consists almost exclusively of non-medullated fibres, medullated fibres being very few ; the large number of medullated fibres which the nerve contains in the upper part of the neck pass off into the laryngeal, cardiac, and other branches.

From the solar plexus nerves, arranged largely in plexuses, pass in company with the divisions of the cœliac artery, coronary artery of the stomach and branches of the hepatic artery, to the stomach. Though the two abdo- 
minal splanchnic nerves which join the solar plexus (semilunar ganglia) are chiefly composed of medullated fibres, the nerves which pass from the plexus to the stomach are to a large extent composed of non-medullated fibres. All these nerves, both branches of the vagi and those from the solar plexus, lie at first in company with the arteries on the surface of the stomach beneath the peritoneum. From thence they pass inward, still in company with arteries, and form, on the one hand, a plexus containing nerve-cells between the longitudinal and circular muscular coats corresponding to what in the intestine we shall have to speak of as the plexus of Auerbach, whence fibres are distributed to the two muscular coats; and, on the other hand, a plexus in the submucous coat, also containing nerve-cells, corresponding to what is known in the intestine as Meissner's plexus. From this latter plexus fibres pass to the mucous membrane; some of these end in the mascularis mucosæ; whether any are connected with the gastric glands, and if so how, is not at present known.

There are no facts which afford satisfactory evidence that any part of this arrangement of nerves supplies such a local nervous mechanism as was suggested above. The importance, however, of such a local mechanism whatever its nature, and the subordinate value of any connection between the gastric membrane and the central nervous system, is further shown by the fact that a secretion of quite normal gastric juice will go on after both vagi, or the nerves from the solar plexus going to the stomach have been divided, and, indeed, when all the nervous connections of the stomach are so far as possible severed. And all attempts to provoke or modify gastric secretion by the stimulation of the nerves going to the stomach have hitherto failed. On the other hand, in cases of gastric fistula, where by complete occlusion of the œsophagus stimulation by the descent of saliva has been avoided, the mere sight or smell of food has been seen to provoke a lively secretion of gastric juice. This must have been due to some nervous action; and the same may be said of the cases where emotions of grief or anger suddenly arrest the secretion going on or prevent the secretion which would otherwise have taken place as the result of the presence of food in the stomach. So that much has yet to be learned in this matter.

$\$ 202$. The contrast presented between the scanty secretion resulting from mechanical stimulation and the copious flow which actual food induces is interesting because it seems to show that the secretory activity of the cells is heightened by the absorption of certain products derived from the portions of food first digested. This is well illustrated by the following experiment of Heidenhain. This observer, adopting the method employed for the intestine, of which we shall speak later on, succeeded in isolating a portion of the fundus from the rest of the stomach ; that is to say, he cut out a portion of the fundus, sewed together the cut edges of the main stomach, so as to form a smaller but otherwise complete organ, while by sutures he converted the excised piece of fundus into a small independent stomach opening on to the exterior by a fistulous orifice. When food was introduced into the main stomach secretion also took place in the isolated fundus. This at first sight might seem the result of a nervous reflex act; but it was observed that the secondary secretion in the fundus was dependent on actual digestion taking place in the main stomach. If the material introduced into the main stomach were indigestible or digested with difficulty, so that little or no products of digestion were formed and absorbed into the blood, such ex. gr. as pieces of ligamentum nuchæ, very little secretion took place in the isolated fundus. We quote this now as bearing on the question of a possible nervous mechanism of gastric secretion, but we shall have to return to it under another aspect. 


\section{The Changes in a Gland constituting the Act of Secretion.}

$\S 203$. We have now to consider what are the changes in the glandular cells and their surroundings which cause this flow of fluid possessing specific characters into the lumen of an alveolus, and so into a duct. It will be convenient to begin with the pancreas.

The thin extended pancreas of a rabbit may, by means of special precautions, be spread out on the stage of a microscope and examined with even high powers, while the animal is not only alive but under such conditions that the gland remains in a nearly normal state, capable of secreting vigorously. It is possible under these circumstances to observe eren minutely the appearances presented by the gland when at rest and loaded, and to watch the changes which take place during secretion.

When the animal has not been digesting for some little time, and the gland is therefore "loaded," the outlines of the individual cells are very indistinct, the lumen of the alveolus is invisible or very inconspicuous, and each cell is crowded with small refractive spherical granules, forming an irregular granular mass which hides the nucleus and leaves only a very narrow clear outer zone next to the basement membrane, or it may be hardly any such zone at all. (Fig. 82, A.)

The blood-supply, moreover, is scanty, the small arteries being constricted and the capillaries imperfectly filled with corpuscles.

If, however, the same pancreas be examined while it is in a state of activity, either from the presence of food in the stomach or from the injection of some stimulating drug, such as pilocarpine, a very different state of things is seen. The individual cells (Fig. $82, B$ ) have become smaller and much more dis-

FIG. 82.
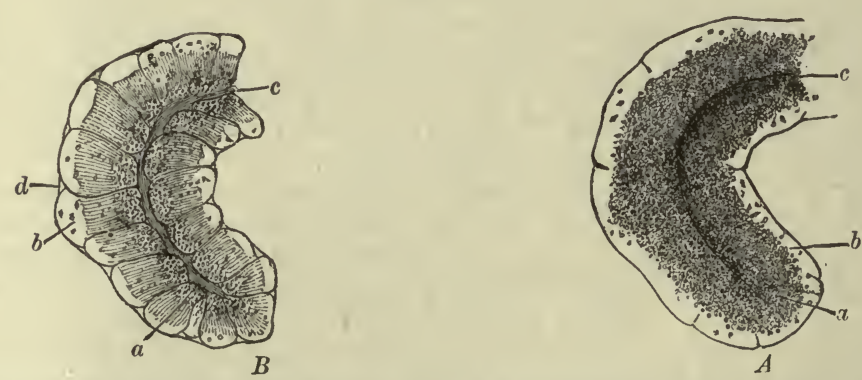

A Portion of the Pancreas of the Rabbit. (Kuhne and Sheridan Lea.) $A$ at rest, $B$ in a state of activity. $a$ the inner granular zone, in which $A$ is larger, and more elosely studded with fine granules, than in $B$, in which the granules are fewer and coarser. $b$ the outer transparent zone, small in $A$, larger in $B$, and in the latter marked with faint striæ. $c$ the lumen, very obvious in $B$, but indistinct in $A$. $d$ an indentation at the junction of two cells, seen in $B$, but not oceurring in $A$.

tinct in outline and the contour of the alveolus, which previously was even, is now wavy, the basement membrane being indented at the junction of the cells, also the lumen of the alveolus is now wider and more conspicuous. In each cell the granules have become much fewer in number and, as it were, have retreated to the inner margin, so that the inner granular zone is much narrower and the outer transparent zone much broader than before; the latter, too, is frequently marked at its inner part by delicate striæ running into the inner zone. At the same time the bloodvessels are largely dilated and the stream of blood through the capillaries is full and rapid.

With care the change from the one state of things to the other may be 
watched under the microscope. The vascular changes can, of course, be easily appreciated, but the granules may also be seen to diminish in number. Those at the inner margin seem to be discharged into the lumen, and those nearer the outer margin to travel inward through the cell-substance toward the lumen, the faint striæ spoken of above, apparently, at all events, being the marks of their paths. Obviously, during secretion, the granules with which the cell-substance was "loaded" are "discharged" from the cell into the lumen of the alveolus. What changes these granules may undergo during the discharge we shall consider presently.

Sections of the prepared and hardened pancreas of any animal tell nearly the same tale as that thus told by the living pancreas of the rabit. In sections, for instance, of the pancreas of a dog which has not been fed, and therefore has not been digesting, for some hours (twenty-four or thirty), the cells are seen to be crowded with granules (which, however, are usually shrunken and irregular owing to the influence of the hardening agent), leaving a very narrow outer zone. In similar sections of the pancreas of a dog which has been recently fed, six hours before for example, and in which, therefore, the gland has been for some time actively secreting, the granules are far less numerous, and the clear outer zone accordingly much broader and more conspicuous. With osmic acid these granules stain well, and are preserved in their spherical form, so that the cell thus stained maintains much of the appearance of a living cell. But with carmine, hæmatoxylin, etc., the granules do not stain nearly so readily as does the cell-substance of the cells, so that a discharged cell stains more deeply than does a loaded cell because the staining of the "protoplasmic" cell-substance is not so much obscured by the unstained granules; besides which, however, the actual cellsubstance stains probably somewhat more deeply in the discharged cell. It may he added that in the discharged cell the nucleus is conspicuous and well formed; in the loaded cell it is generally in prepared sections, more or less irregular, possibly because in these it is less dense and more watery than in the discharged cell, and so shrinks under the influence of the reagents employed.

These several observations suggest the conclusion that in a gland at rest the cell is occupied in forming by means of the metabolism of its cell-substance and lodging in itself ( $\$ 30$ ) certain granules of peculiar substance intended to be a part and probably an important part of the secretion. This goes on until the cell is more or less completely "loaded." In such a cell the amount of actual living cell-substance is relatively small, its place is largely occupied by granules, and in itself has been partly consumed in forming the granules. During the act of secretion the granules are discharged to form part of the secretion, other matters, including water, as we shall see, making up the whole secretion; and the cell would be proportionately reduced in size were it not that the act of the discharge seems to stimulate the cell-substance to a new activity of growth, so that the new cell-substance is formed; this, however, is in turn soon in part consumed in order to form new granules. And what is thus seen with considerable distinctness and ease in the pancreas, is seen with more or less distinctness in other glands.

$\S 204$. When we study an albuminous gland, the parotid gland, for instance, in a living state, we find that the changes which take place during activity are quite comparable to those of the pancreas. During rest (Fig. $83, A)$, the cells are large, their outlines very indistinct, in fact almost iuvisible, and the cell-substance is studded with granules. During activity (Fig. 83, $B$ ) the cells become smaller, their outlines more distinct, and the granules disappear, especially from the outer portions of each cell. After 
prolonged activity, as in Fig. 83, $C$, the cells are still smaller, with their outlines still more distinct, and the granules have disappeared almost entirely, a few only being left at the extreme inner margin of each cell, abutting upon the conspicuous, almost gaping lumen of the alveolus. And

FIG. 83.
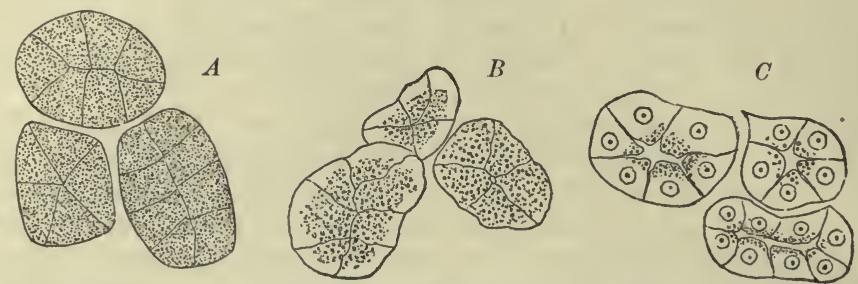

Changes in the Parotid during Secretion. (Langley.) The figure, which is somewhat diagrammatic, represents the microscopic changes which may be observed in the living gland. $A$. During rest. The obscure outlines of the cells are introduced to show the relative size of the cells; they could not be readily seen in the specimen itself. $B$. After moderate stimulation. $C$. After prolonged stimulation. The nuclei are diagrammatic, and introduced to show their appearance and position.

upon special examination it is found that the nuclei are large and round. In fact, we might almost take the parotid, as thus studied, to be more truly typical of secretory changes than even the pancreas. For the demarcation of an inner and outer zone is not a necessary feature of a secreting cell at rest. What is essential is that the cell-substance manufactures material, which for a while, that is during rest, is deposited in the cell, generally in the form of granules but not necessarily so, and that during activity this material is used up, the disappearance of the granules, when these are visible, being naturally earliest and most marked at the outer portions of each cell, and progressing inward toward the lumen, the whole cell becoming smaller and, as it were, shrunken.

In the cells of the parotid gland and other albuminous cells the granules seen in the living or fresh cell differ from the granules seen in the pancreatic cell, inasmuch as they are easily dissolved or broken up by the action of alcohol, chromic acid, and the other usual hardening reagents, and hence in hardened specimens have disappeared. In consequence, in sections of hardened and prepared albuminous glands the difference between resting or loaded and active or discharged cells may appear not very conspicuous; and this is especially the case in the parotid gland of the rabbit when the

FIG. 84.

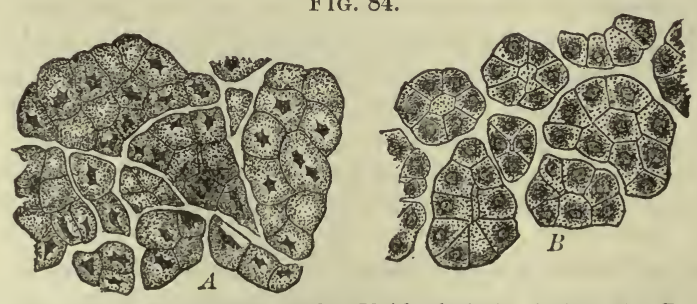

Sections of the Parotid of the Rabbit. (After Heidenhain.) A. At rest. B. After stimulation of the cervical sympathetic. Both sections are from hardened gland.

activity has been called into play by stimulation of the auriculo-temporal nerve. When, however, either in the rabbit or the dog the cervical sympathetic is stimulated, though the stimulation gives rise in the rabbit to 
little secretion of saliva and in the dog to none at all, a marked effect on the gland is produced, and changes in the same direction as those already described may be observed. During rest the cells of the parotid as seen in sections of the gland hardened in alcohol (Fig. 84, A) are pale, transparent, staining with difficulty, and the nuclei possess irregular outlines as if shrunken by the reagents employed. After stimulation of the sympathetic the protoplasm of the cells becomes turbid (Fig. 84, $B$ ), and stains much more readily, while the nuclei are no longer irregular in outline, but round and large, with conspicuous nucleoli, the whole cell at the same time, at least after prolonged stimulation, becoming distinctly smaller.

$\$ 205$. In a mucous gland the changes which take place are of a like kind, though apparently somewhat more complicated, owing probably to the peculiar characters of the mucin which is so conspicuous a constituent of the secretion.

If a piece of resting loaded submaxillary gland be teased out, while fresh and warm from the borly, in normal saline solution, the cell-substance of the mucous cells (Fig. 85,a) is seen to be crowded with granules or spherules, which may fairly be compared with the granules of the pancreas, though perhaps less dense and solid than these.

If a piece of a gland which has been secreting for some time, and is therefore a discharged gland, be examined in the same way (Fig. 85, $b$ ), the granules are far less numerous and largely confined to the part of the cell nearer the lumen, the outer part of the cell around the nucleus consisting of ordinary "protoplasmic" cell-substance. The distinction, however, between an inner "granular zone" next to the lumen and an outer "clear zone" next to the basement membrane is less distinct than in the pancreas, partly because the granules do not disappear in so regular a manner as in the pancreas; and partly because the outer zone of the mucous cell, as it forms, is less homogeneous than that of the pancreatic cell.

The "granules" or "spherules" of the mucous cell are moreover of a peculiar nature. If the fresh cell, showing granules (either many as in the case of a loaded or few as in the case of a discharged cell), be irrigated with water or with dilute acids or dilute alkalies, the granules swell up (Fig. 85, $a^{\prime}, b^{\prime}$, into a transparent mass, giving the reactions of mucin, traversed by a network of "protoplasmic" cell-substance. In this way is produced an appearance very similar to that shown in sections of mucous glands hardened and stained in the ordinary way.

In the loaded mucous cell in hardened

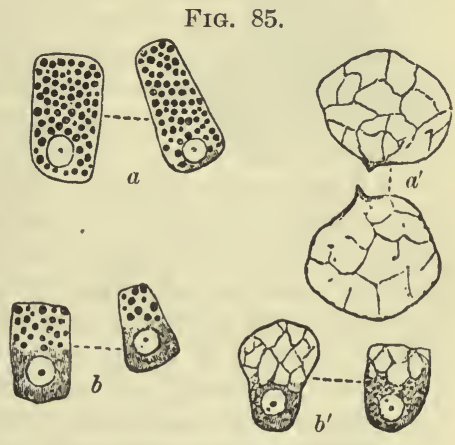

Mucous Cells from a fresh Submaxil lary Gland of Dog. (Langley.) $a$ and $b$ isolated in 2 per cent. salt solution: $a$, from loaded gland; $b$, from discharged gland (the nuclei are usually more obscured by granules than is here represented). On teasing out a fresh fragment in 2 to 5 per cent. salt solution, the cells usually become broken up so that isolated cells are rarely obtained entire ; isolated cells are common if the gland be left in the body for a day after death; $a^{\prime}$, $b^{\prime}$, treated with dilute acid: $a^{\prime}$, from loaded; $b^{\prime}$, from discharged gland. and stained preparations (Fig. 86, a) there is seen a small quantity of protoplasmic cell-substance gathered round the nucleus at the outer part of the cell next to the basement membrane; the rest of the cell consists of a network of cell-substance, the interstices being filled with transparent material, which, unlike the network itself and the mass of cell-substance round the nucleus, does not stain with 
carmine or with certain other dyes. The discharged cell in similar preparations (Fig. 86, b) differs from the loaded cell in the amount of transparent

Fig. 86 .
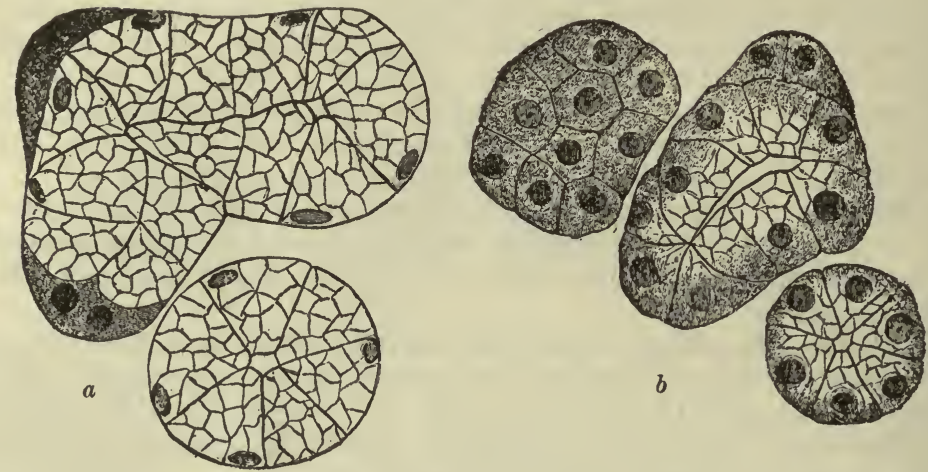

Alveoli of Dog's Submaxillary Gland Hardened in Alcohol and Stained with Carmine. (Langley.) The network is diagrammatic. $a$, from a loaded gland. $b$, from a discharged gland; the chorda tympani having been stimulated at short intervals during five hours.

non-staining material being much less and chiefly confined to the inner part of the cell, while the protoplasmic cell-substance around the now large and well-formed nucleus is not only, both relatively and absolutely, greater in amount, but stains still more deeply than in the loaded cell.

It would appear, therefore, that in the mucous cell, as in the pancreatic cell, the cell-substance forms and deposits in itself certain material in the form of granules. During secretion these granules disappear and presumably form part of the secretion.

$\S 206$. The "central" or "chief" cells of the gastric gland also exhibit similar changes. In such an animal as the newt these cells may, though with difficulty, be examined in the living state. They are then found to be studded with granules when the stomach is at rest. During digestion these granules become much less numerous and are chiefly gathered near the lumen, leaving in each cell a clear outer zone. And in many mammals the same abundance of granules in the loaded cell, the same paucity of granules for the most part restricted to an inner zone in the discharged cell, may be demonstrated by the use of osmic acid (Fig. 87).

When the stomach is hardened by alcohol these changes, like the similar changes in an albuminous cell, are obscured by the shrinking of the "granules," or by their swelling up and becoming diffused through the rest of the cell-substance; so that, though in sections so prepared very striking differences are seen between loaded and discharged cells, these are unlike those seen in living glands. In specimens taken from an animal which has not been fed for some time, the central cells of the gastric glands are pale, finely granular, and do not stain readily with carmine and other dyes. During the early stages of gastric digestion, the same cells are found somewhat swollen, but turbid and more coarsely granular; they stain much more readily. At a later stage they become smaller and shrunken, but are even more turbid and granular than before, and stain still more deeply. This is true not only of the central cells in the cardiac glands, but also of the cells of which the pyloric glands are built up. In a loaded cell very little staining takes place, because the amount of living staining cell-substance is small relatively to the amount of material with which it is loaded and 
which does not stain readily. In the cell which after great activity has discharged itself', the cell is smaller, but what remains is largely living cell-substance, some of it new, and all staining readily. It would appear also, that during the activity of the cell some substances, capable of being precipitated by alcohol, make their appearance, and the presence of this material adds to the turbid and granular aspect of the cell ; possibly, also, this material contributes to the staining. A similar material seems to make its appearance in the cells of albuminous glands.

In the ovoid or border cells no very characteristic changes make their appearance. During digestion they become larger, more swollen, as it were, and in consequence bulge out the basement membrane, but no characteristic disappearance of granules can be observed. In the living state, the cell-substance of these ovoid cells appears finely granular, but in hardened and prepared sections has a coarsely granular, "reticulate" look, which is perhaps less marked in the swollen active cells than in the resting cells.

$\S 207$. All these various secreting cells, then-pancreatic cell, mucous cell, albuminous cell, and central gastric cell-exhibit the same series of events, modified to a certain extent in the several cases. In each case the "protoplasmic" cell-substance manufactures and lodges in itself material destined to form part of the juice secreted. In the fresh cell this material may generally be recognized under the microscope by its optical characters as granules; these, however, are apt to become altered by reagents. But we must guard ourselves against the assumption that the material which can thus be recognized is the only material thus stored up; we may in future, by chemical or other means, be able to differ-

FIG. 87.

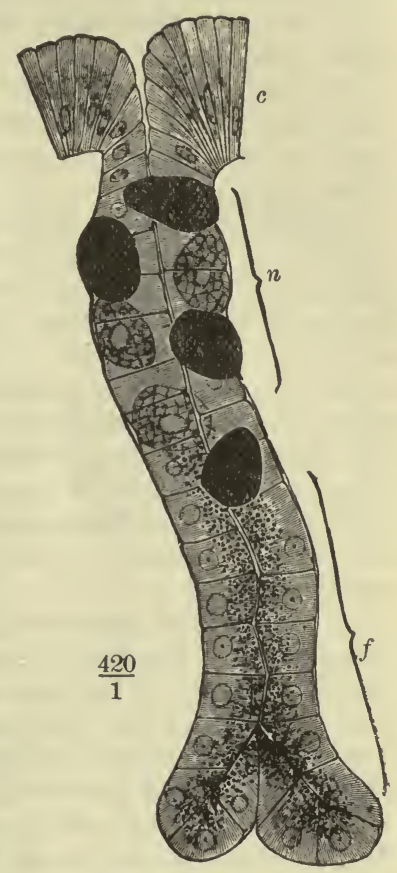

Gastric Gland of Mammal (Bat) during Activity. (Langley.) $c$, the mouth of the gland with its cylindrical cells. $n$, the neck, containing conspicuous ovoid cells, with their coarse protoplasmic network. $f$, the body of the gland. The granules are seen in the central cells to be limited to the inner portions of each cell, the round nucleus of which is conspicuous. entiate other parts of the cell-body as being also material similarly stored up.

During activity, while the gland is secreting, this material, either unchanged or after undergoing change, is wholly or partially discharged from the cell. The cell, in consequence of having thus got rid of more or less of its load, consists to a larger extent of actual living cell-substance, this being in many cases increased by rapid new-growth, though the bulk of the discharged cell may be less than that of the loaded cell.

This activity of growth continues after the act of secretion, but the discharged cell soon begins again the task of loading itself with new secretion material for the next act of secretion.

Thus in most cases there is, corresponding to the intermittence of secretion, an alteration of discharge and loading; but it must be borne in mind that such an alteration is not absolutely necessary, even in the case of intermittent secretion. We can easily imagine that the discharge, say of 
"granules" during secretion, should stir up the cell to an increased activity in forming granules, and that the formative activity should cease when the secretory activity ceased. In such a case the number of new granules formed might always be equal to the number of old granules used up, and the active cell, in spite of its discharge, would possess as many granules-that is to say, as large a load-as the cell at rest. And in the central gastric cells of some animals it would appear that such a continued balancing of load and discharge does actually take place, so that no distinction in granules can be observed between resting and active cells.

$\$ 208$. We spoke just now of the material stored up in the cell and destined to form part of the secretion as undergoing change before it was discharged. In the mucous cell we have seen that the material deposited in the living cell has at first the form of granules. These granules, however, are easily converted into a transparent material lodged in the spaces of the cell-substance, which material, even if not exactly identical with, at least closely resembles, the mucin found in the secretion; and apparently in the act of secretion the granules do undergo some such change. In the case of some other glands, moreover, we have chemical as well as optical evidence that the material stored up in the cells is, in part at least, not the actual substance appearing in the secretion, but an antecedent of that substance.

An important constituent of pancreatic juice is, as we shall see later on, a body called trypsin, a ferment very similar to pepsin, acting on proteid bodies and converting them into peptone and other substances. Though in many respects alike, pepsin and trypsin are quite distinct bodies, and differ markedly in this, that while an acid medium is necessary for the action of pepsin, an alkaline medium is necessary for the action of trypsin; and accordingly the pancreatic juice is alkaline in contrast to the acidity of gastric juice. Trypsin can, like pepsin ( $\$ 191$ ), be extracted with glycerin from substances in which it occurs; glycerin extracts of trypsin, however, need for the manifestation of their powers the presence of a weak alkali, such as a 1 per cent. solution of sodium carbonate.

Now, trypsin is present in abundance in normal pancreatic juice; but a loaded pancreas, one which is ripe for secretion, and which if excited to secrete would immediately pour out a juice rich in trypsin, contains no trypsin or a mere trace of it; nay, even a pancreas which is engaged in the act of secreting contains in its actual cells an insignificant quantity only of trypsin, as is shown by the following experiment:

If the pancreas of an animal, even of one in full digestion, be treated, while still warm from the body, with glycerin, the glycerin extract, as judged of by its action on fibrin in the presence of sodium carbonate, is inert or nearly so as regards proteid hodies. If, however, the same pancreas be kept for twenty-four hours before being treated with glycerin, the glycerin extract readily digests fibrin and other proteids in the presence of an alkali. If the pancreas, while still warm, be rubbed up in a mortar for a few minutes with dilute acetic acid, and then treated with glycerin, the glycerin extract is strongly proteolytic. If the glycerin extract obtained without acid from the warm pancreas, and therefore inert, be diluted largely with water and kept at $35^{\circ} \mathrm{C}$. for some time, it becomes active. If treated with acidulated instead of distilled water, its activity is much sooner developed. If the inert glycerin extract of warm pancreas be precipitated with alcohol in excess, the precipitate, inert as a proteolytic ferment when fresh, becomes active when exposed for some time in an aqueous solution, rapidly so when treated with acidulated water. These facts show that a pancreas taken fresh from the body, even 
during full digestion, contains but little ready-made ferment, though there is present in it a body which, by some kind of decomposition, gives birth to the ferment. We may remark incidentally, that though the presence of an alkali is essential to the proteolytic action of the actual ferment, the formation of the ferment out of its forerunner is favored by the presence of a small quantity of acid; the acid must be used with care, since the trypsin, once formed, is destroyed by acids. To this body, this mother of the ferment, which has not at present been satisfactorily isolated, but which appears to be a complex body, splitting up iuto the ferment, which, as we have seen, is at all events not certainly a proteid body, and into an undeniably proteid body, the name of zymogen has been applied. But it is better to reserve the term zymogen as a generic name for all such bodies as, not being themselves actual ferments, may by internal changes give rise to ferments-for all " mothers of ferment," in fact-and to give to the particular mother of the pancreatic p:oteolytic ferment the name trypsinogen.

Evidence of a similar kind shows that the gastric glands, both the cardiac and the pyloric glands, while they contain comparatively little actual pepsin, contain a considerable quantity of zymogen of pepsin, or pepsinogen; and there can be little doubt but that this pepsinogen is lodged in the central cells of the cardiac glands and in the somewhat similar cells which line the whole of the pyloric glands.

$\S 209$. The act of secretion itself. The above discussion prepares us at once for the statement that the old view of secretion, according to which the gland picks out, separates, secretes (hence the name secretion), and so filters, as it were, frum the common store of the blood the several constituents of the juice, is untenable. According to that view the specific activity of any one gland was confined to the task of letting certain constituents of the blood pass from the capillaries surrounding the alveolus through the cells to the channels of the ducts, while refusing a passage to others. We now know that certain important constituents of each juice, the pepsin of gastric juice, the mucin of saliva and the like are formed in the cell, and not obtained ready made from the blood. A minute quantity of pepsin does exist, it is true, in the blood, but there are reasons for thinking that this has made its way back into the blood, either being absorbed from the interior of the stomach or, as seems more probable, picked up directly from the gastric glands; and so with some of the other constituents of other juices. The chief or specific constituents of each juice are formed in the cell itself.

But the juice secreted by any gland consists not only of the specific substances such as mucin, pepsin, or other ferment, or other bodies, found in it alone, but also of a large quantity of water, and of various other substances, chiefly salines, common to it, to other juices, and to the blood. And the question arises, Is the water, are the salts and other common substances, furnished by the same act as that which supplies the specific constituents?

Certain facts suggest that they are not. For instance, as mentioned some time ago, in the submaxillary gland of the dog stimulation of the chorda tympani produces a copious flow of saliva, which is usually thin and limpid, whilst stimulation of the cervical sympathetic produces a scanty flow of thick viscid saliva. That is to say, stimulation of the chorda has a marked effect in promoting the discharge of water, while stimulation of the sympathetic has a marked effect in promoting the discharge of mucin. To this we may add the case of the parotid of the dog. In this gland stimulation of a cerebro-spinal nerve, the auriculo-temporal, produces a copious flow of limpid saliva, while stimulation of the sympathetic produces itself little or no secretion at all; but when the sympathetic and the cerebro-spinal nerves are stimulated at the same time, the saliva which flows is much richer in solid 
and especially in organic matter than when the cerebro-spinal nerve is stimulated alone. And we have already seen that in this gland the microscopic changes following upon sympathetic stimulation are more conspicuous than those which follow upon cerebro-spinal stimulation.

These and other facts have led to the conception that the act of secretion consists of two parts, which in one case may coincide, in another may take place apart or in different proportions. On the one hand, there is the discharge of water carrying with it common soluble substances, chiefly salines, derived from the blood; on the other haud, a metabolic activity of the cellsubstance gives rise to the specific constituents of the juice. To put the matter broadly, the latter process produces the specific constituents, the former washes these and other matters into the duct. It has been further supposed that two kinds of nerve fibres exist: one governing the former process and, in the case of the submaxillary gland for instance, preponderating, though not to the total exclusion of the other kind, in the chorda tympani ; the other governing the latter process and preponderating in the branches of the cervical sympathetic. These have been called respectively "secretory" and "trophic" fibres; but these terms are not desirable. It may here be remarked that even the former process is a distinct activity of the gland and not a mere infiltration. For, as we have seen in the case of the salivary glands, when atropine is given, not only do the specific constituents cease to be ejected as a consequence of stimulation of the chorda, but the discharge of water, in spite of the bloodvessels becoming dilated, is also arrested : no saliva at all leaves the gland. And what is true of the salivary glands as regards the dependence of the flow of water on something else besides the mere pressure of the bloor in the bloodvessels appears to hold good with other glands also. Indeed, it has been suggested that the very discharge of water is due to an activity of the cell; the hypothesis has been put forward that changes in the cell give rise to the formation in the cell of substances which absorb water from the blood or lymph on the one side and give it up on the other side into the lumen of the alveolus. Such an hypothesis cannot be regarded as proved; but the mere putting it forward raises a doubt as to the validity of the distinction on which we have been dwelling; and other considerations point in the same direction. For instance, if the common soluble salts present in a juice, as distinguished from the specific constituents, were merely carried into the juice by the rush, so to speak, of water, we should expect to find the percentage of these salts either remaining the same or perhaps decreasing when the juice was secreted more rapidly and in fuller volume. But under these circumstances the percentage very frequently increases; and in general we find that under various circumstances the proportion of salts secreted to the quantity of water secreted may vary considerably. Obviously, while something determines the quantity of water passing into the alveolus, something else determines how much of common soluble salts that water contains, and still something else determines to what extent that water is also laden with specific constituents and other organic bodies. The whole action is too complicated to be described as consisting merely of the two processes mentioned above, but the time has not yet come for clear and definite statements. Everything, however, tends to show that the cell is the prime agent in the whole business, though we cannot at present define the nature of the several changes in the cell, nor can we say how those changes are exactly related to each other, to changes of the blood-pressure in the blondvessels, or, we may add, to changes taking place in the lymph-spaces which lie between the blood and the cell.

We may perhaps add that since in certain cutaneous secreting glands 
the alveolus, or what corresponds to the alveolus, is wrapped round with plain muscular fibres, the contraction of which appears to force the secretion outward, the idea has been suggested that in glands, such as we are now considering, the cell-substance, making use of "protoplasmic" contraction instead of actual muscular contraction, may force part of the cell contents into the lumen of the alveolus. Such a mode of secretion would be comparable to the ejection of undigested material, or "excretion," by an amœba. But we have no satisfactory evidence in favor of this view.

$\$ 210$. Throughout the above we have spoken as if the secretion were furnished exclusively by the cells of the alveoli or secreting portion of the gland, as if the epithelial cells lining the ducts, or conducting portion of the gland, contributed nothing to the act. In the gastric glands the slender cells lining the mouths of the glands (which correspond to ducts) and covering the ridges between, are mucous cells secreting into the stomach generally a small, but under abnormal conditions a large amount of mucus, which has its uses but is not an essential part of the gastric juice. In the salivary glands we can hardly suppose that the long stretch of characteristic columnar epithelium which reaches from the alveoli to the mouth of the long main duct serves simply to furnish a smooth lining to the conducting passages; but we have as yet no clear indications of what the function of this epithelium can be.

$\$ 211$. Before we leave the mechanism of secretion there are one or more accessory points which deserve attention.

In treating just now of the gastric glands we spoke as if pepsin were the only important constituent of gastric juice, whereas, as we have previously seen, the acid is equally essential. The formation of the free acid of the gastric juice is very obscure, and many ingenious but unsatisfactory views have been put forward to explain it. It seems natural to suppose that it arises in some way from the decomposition of sodium chloride drawn from the blood; and this is supported by the fact that when the secretion of gastric juice is actively going on, the amount of chlorides leaving the blood by the kidney is proportionately diminished; but nothing definite can at present be stated as to the mechanism of that decomposition.

In the frog, while pepsin free from acid is secreted by the glands of the lower portion of the oesophagus, an acid juice is afforded by glands in the stomach itself, which have accordingly been called oxyntic ( $\delta \xi \dot{v} \nu \varepsilon i \nu$, to sharpen, acidulate) glands; but these oxyntic glands appear also to secrete pepsin. In the mainmal the isolated pylorus secretes an alkaline juice; in fact, the appearance of an acid juice is limited to those portions of the stomach in which the glands contain both "chief" or "central" and "ovoid" or "border" cells. Now, from what has been previously said, there can be no doubt that the chief cells do secrete pepsin. On the other hand, there is no evidence whatever of the formation of pepsin by the "border" or "ovoid" cells, though this was once supposed to be the case, and these cells were unfortunately formerly' called "peptic" cells. Hence it has been inferred that the border cells secrete acid; but the argument is at present one of exclusion only, there being no direct proof that these cells actually manufacture the acid.

The rennin appears to be formed by the same cells which manufacture the pepsin, that is, by the chief cells of the fundus generally, and to some extent by the cells of the pyloric glands. We may add that we have evidence of the existence of a zymogen of rennin analogous to the zymogen of pepsin or of trypsin.

$\$ 212$. Seeing the great solvent power of both gastric and pancreatic juice the question is naturally suggested, Why does not the stomach digest itself? 
After death, the stomach is frequently found partly digested, viz., in cases when death has taken place suddenly on a full stomach. In an ordinary death, the membrane ceases to secrete before the circulation is at an end. That there is no special virtue in living things which prevents their being digested is shown by the fact that the leg of a living frog or the ear of a living rabbit introduced into the stomach of a dog, through a gastric fistula, is readily digested. It has been suggested that the blood-current keeps up an alkalinity sufficient to neutralize the acidity of the juice in the region of the glands themselves; but will not explain why the pancreatic juice, which is active in an alkaline medium, does not digest the proteids of the pancreas itself, or why the digestive cells of the bloodless actinozoon or hydrozoon do not digest themselves. We might add, it does not explain why the amoba, while dissolving the protoplasm of the swallowed diatom, does not dissolve its own protoplasm. We cannot answer this question at all at present, any more than the similar one, why the delicate protoplasm of the amøba resists during life the entrance into itself by osmosis of more water than it requires to carry on its work, while a few moments after it is dead water enters freely by osmosis, and the effects of that entrance become abundantly evident by the formation of bullæ and the breaking up of the protoplasm.

\section{The Properties and Characters of Bile, Pancreatic Juice, and Succus Entericus.}

$\$ 213$. In the living body the food, subjected to the action first of the saliva and then of the gastric juice, undergoes in the stomach changes which we shall presently consider in detail, and the food so changed is passed on into the small intestine, where it is further subjected to the action of the bile secreted by the liver, of pancreatic juice secreted by the pancreas, and possibly to some extent, though this is by no means certain, of a juice secreted by the intestine itself and called succus entericus. It will be convenient to study the minute structure of the liver in connection with other functions of the liver more important, perhaps, than that of the secretion of bile, namely, the formation of glycogen, and other metabolic events occurring in the hepatic cells; we have already studied the structure of the pancreas; and the structure of the intestine will best be considered by itself. We, therefore, turn at once to the properties and characters of the abovenamed juices.

\section{Bile.}

Though bile, after secretion in the lobules of the liver, is passed on along the hepatic duct, it is in the case of most animals not poured at once into the duodenum, but taken by the cystic duct to the reservoir of the gallbladder. Here it remains until such time as it is needed, when a quantity is poured along the common bile duct into the intestine.

The quality of bile varies much, not only in different animals, but in the same aninial at different times. It is, moreover, affected by the length of the sojourn in the gall-bladder; bile taken direct from the hepatic duct, especially when secreted rapidly, contains little or no mucus; that taken from the gall-bladder, as of slaughtered oxen or sheep, is loaded with mucus. The color of the bile of carnivorous and omnivorous animals, and of man, is generally a bright golden red; of herbivorous animals, a yellowish green or a bright green or a dirty green, according to circumstances, being much modified by retention in the gall-bladder. The reaction is neutral or alkaline. The following may be taken as the average composition of human 
bile taken from the gall-bladder, and therefore containing much more mucus as well as, relatively to the solids, more water than bile from the hepatic duct.

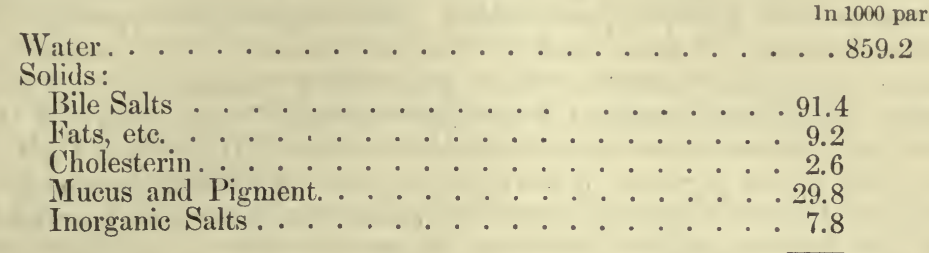

The entire absence of proteids is a marked feature of bile : pancreatic juice, as we shall see, contains a considerable quantity; saliva, as we have seen, a small quantity; normal gastric juice probably still less, and bile none at all. Even the bile which has been retained some time in the gall-bladder, though rich in mucus, contains no proteids.

The constituents which form, apart from the mucus, the great bulk of the solids of bile, and which deserve chief attention, are the pigments and the bile-salts; of these we shall speak immediately.

With regard to the inorganic salts actually present as such sodium salts are conspicuous, sodium chloride, amounting to 0.2 or more per cent., sodium phosphate to nearly as much, the rest being earthy phosphates and other matters in small quantity. The presence of iron, to the extent of about 0.006 per cent., is interesting, since, as we shall see, there are reasons for thinking that the pigment of bile, itself free from iron, is derived from iron-holding hæmoglobin; some, at least, of the iron set free during the conversion of hæmoglobin into bile pigment, which probably takes place in the liver, finds its way into the bile. Bile also appears to contain a small quantity, at all events occasionally, of other metals, such as manganese and copper; metals introduced into the body are apt to be retained in the liver and eventually leave it by the bile.

The small quantity of fat present consists in part of the complex body, lecithin:

The peculiar body, cholesterin, which though fatty-looking (hence the name "bile fat") is really an alcohol with the composition $\mathrm{C}_{26} \mathrm{H}_{44} \mathrm{O}$, is conspicuous by its quantity and constancy. It forms the greater part of most gall-stones, though some are composed chiefly of pigment. Insoluble in water and cold alcohol, though soluble in hot alcohol and readily soluble in ether, chloroform, etc., it is dissolved by the bile-salts in aqueous solution and hence is present in solution in bile Its physiological functions are obscure.

The ash of bile consists largely of soda, derived partly from the sudium chloride and partly from the bile-salts, of sulphates derived chiefly if not wholly from the latter, and of phosphates partly ready formed and in part derived from the lecithin.

$\$ 214$. Pigments of bile. The natural golden-red color of normal human or carnivorous bile is due to the presence of bilirubin. This, which is also the chief pigmentary constituent of gall-stones, and occurs largely in the urine of jaundice, may be obtained in the form either of an orange-colored amorphous powder or of well-formed rhombic tablets and prisms. Insoluble in water, and but little soluble in ether and alcohol, it is readily soluble in chloroform and in alkaline fluids. Its composition is $\mathrm{C}_{16} \mathrm{H}_{18} \mathrm{~N}_{2} \mathrm{O}_{3}$. Treated 
with oxidizing agents, such as nitric acid yellow with nitrous acid, it displays a succession of colors in the order of the spectrum. The yellowish goldenred becomes green, this a greenish-blue, then blue, next violet, afterward a dirty red, and finally a pale yellow. This characteristic reaction of bilirubin is the basis of the so-called Gmelin's test for bile-pigments. Each of these stages represents a distinct pigmentary substance. An alkaline solution of bilirubin, exposed in a shallow vessel to the action of the air, turns green, becoming converted into biliverdin $\left(\mathrm{C}_{16} \mathrm{H}_{20} \mathrm{~N}_{2} \mathrm{O}_{5}\right.$ or $\mathrm{C}_{16} \mathrm{H}_{18} \mathrm{~N}_{2} \mathrm{O}_{4}$, Maly), the green pigment of herbivorous bile. Biliverdin is also found at times in the urine of jaundice, and is probably the body which gives to bile which has been exposed to the action of gastric juice, as in biliary vomits, its characteristic green hue. It is the first stage of the oxidation of bilirubin in Gmelin's test. Treated with oxidizing agents biliverdin runs through the same series of colors as bilirubin, with the exception of the initial golden-red.

$\$ 215$. The bile-salts. These consist, in man and many animals, of sodium glycocholate and taurocholate, the proportion of the two varying in different animals. In man both the total quantity of bile-salts and the proportion of the one bile-salt to the other seem to vary a good deal, but the glycocholate is said to be always the more abundant. In ox-gall sodium glycocholate is abundant and taurncholate scanty. 'The bile-salts of the dog, cat, bear, and other carnivora consist exclusively of the latter.

Insoluble in ether, but soluble in alcohol and in water, the aqueous solutions having a decided alkaline reaction, both salts may be obtained by crystallization in fine acicular needles. They are exceedingly deliquescent. The solutions of both acids have a dextro-rotary action on polarized light.

Preparation. Bile, mixed with animal charcoal, is evaporated to dryness and extracted with alcohol. If not colorless, the alcoholic filtrate must be further decolorized with animal eharcoal, and the alcohol distilled off. The dry residue is treated with absolute alcohol, and to the alcoholic filtrate anhydrous ether is added as long as any precipitate is formed. On standing the eloudy precipitate becomes transformed into a crystalline mass at the bottom of the vessel. If the alcohol be not absolute, the erystals are very apt to be changed into a thick syrupy fluid. This mass of erystals has been often spoken of as bilin. Both salts are thus precipitated, so that in such a bile as that of the ox or man bilin eonsists both of sodium glycocholate and sodium taurocholate. The two may be separated by precipitation from their aqueous solutions with sugar of lead, which throws down the former much more readily than the latter. The acids may be separated from their respective salts by dilute sulphuric action, or by the action of lead acetate and sulphydric acid.

On boiling with dilute acids (sulphuric, hydrochloric), or caustic potash or baryta water, glycocholic acid is split up into cholalic (cholic) acid and glycin. Taurocholic acid may similarly be split up into cholalic acid and taurin. Thus :

$$
\begin{aligned}
& \text { Glycocholic acid. Cholalic acid. Glycin. } \\
& \mathrm{C}_{26} \mathrm{H}_{43} \mathrm{NO}_{6}+\mathrm{H}_{2} \mathrm{O}=\mathrm{C}_{24} \mathrm{H}_{40} \mathrm{O}_{5}+\mathrm{CH}_{2} \cdot \mathrm{NH}_{2}(\mathrm{CO} . \mathrm{OH}) \text {. } \\
& \text { Taurocholic acid. Cholalic acid. Taurin. } \\
& \mathrm{C}_{26} \mathrm{H}_{45} \mathrm{NSO}_{7}+\mathrm{H}_{2} \mathrm{O}=\mathrm{C}_{24} \mathrm{H}_{40} \mathrm{O}_{5}+\mathrm{C}_{2} \mathrm{H}_{4} \cdot \mathrm{NH}_{2} \cdot \mathrm{SO}_{3} \mathrm{H} \text {. }
\end{aligned}
$$

Both acids contain the same non-nitrogenous acid, cholalic acid; but this acid is in the first case associated or conjugated with the important nitrogenous body glycin, or amido-acetic acid, which is a compound formed from ammonia and one of the "fatty acid" series, viz., acetic; and in the second case with taurin, or amido-jsethionic acid, that is a compound into which representatives of ammonia, of the ethyl group, and of sulphuric acid enter. 
The decomposition of the bile acids into cholalic acid and taurin or glycin respectively takes place naturally in the intestine, the glycin and taurin being probably absorbed, so that from the two acids, after they have served their purpose in digestion, the two ammonia compounds are returned into the blood. Each of the two acids, or cholalic acid alone, when treated with sulphuric acid and cane-sugar, gives a magnificent purple color (Pettenkofer's test), with a characteristic spectrum. A similar color may, however, often be produced by the action of the same bodies on albumin, amyl alcohol, and some other organic bodies.

$\$ 216$. Action of bile on food. In some animals at least bile contains a ferment capable of converting starch into sugar ; but its action in this respect is wholly subordinate.

On proteids bile has no direct digestive action whatever, but being, generally at least, alkaline, and often strongly so, tends to neutralize the acid contents of the stomach as they pass into the duodenum, and, as we shall see, so prepares the way for the action of the pancreatic juice. To peptic action it is distinctly antagonistic; the presence of a sufficient quantity of bile renders gastric juice inert toward proteids. Moreover, when bile, or a solution of bile-salts, is added to a fluid containing the products of gastric digestion, a precipitate takes place, consisting of parapeptone (when present), peptone, pepsin, and bile-salts. The precipitate is redissolved in an excess of bile or solution of bile-salts; but the pepsin, though redissolved, remains inert toward proteids. This precipitation actually does take place in the duodenum, and we shall speak of it again later on.

With regard to the action of bile on fats, the following statements may be made:

Bile has a slight solvent action on fats, as seen in its use by painters. It has by itself a slight but only slight emulsifying power; a mixture of oil and bile separates after shaking rather less rapidly than a mixture of oil and water. With fatty acids bile forms soap. It is, moreover, a solvent of solid soaps, and it would appear that the emulsion of fats is under certain circumstances at all events facilitated by the presence of soaps in solution. Hence bile is probably of much greater use as an emulsion agent when mixed with pancreatic juice than when acting by itself alone. To this point we shall return. Lastly, the passage of fats through membranes is assisted by wetting the membranes with bile or with a solution of bile-salts. Oil will pass to a certain extent through a filter-paper kept wet with a solution of bile-salts, whereas it will not pass or passes with extreme difficulty through one kept constantly wet with distilled water.

Bile possesses some antiseptic qualities. Out of the body its presence hinders various putrefactive processes; and when it is prevented from flowing into the alimentary canal, the contents of the intestine undergo changes different from those which take place under normal conditions, and leading to the appearance of various products, especially of ill-smelling gases.

These various actions of bile seem to be dependent on the bile-salts and not on the pigmentary or other constituents.

\section{Pancreatic Juice.}

$\S 217$. Natural healthy pancreatic juice obtained by means of a temporary pancreatic fistula differs from the digestive juices of which we have already spoken in the comparatively large quantity of proteids which it contains. Its composition varies according to the rate of secretion, for, with the more rapid flow, the increase of total solids does not keep pace with that of the water, though the ash remains remarkably constant. 
By an incision through the linea alba the pancreatic duct (or ducts) can easily be found either in the rabbit or in the dog, and a canula secured in it. There is no difficulty about a temporary fistula; but with permanent fistula the secretion is apt to become altered in nature, and to lose niany of its characteristic properties. Some, however, have succeeded in obtaining permanent fistulae without any impairment of the secretion.

Healthy pancreatic juice is a clear, somewhat viscid fluid, frothing when shaken. It has a very decided alkaline reaction, and contains few or no structural constituents.

The average amount of solids in the pancreatic juice (of the dog) obtained from a temporary fistula is about 8 to 10 per cent.; but in even thoroughly active juice obtained from a permanent fistula is not more than about 2 to 5 per cent., 0.8 being inorganic matter; and this is probably the normal amount. The important constituents of quite fresh juice are albumin, a peculiar form of proteid allied to myosin, giving rise to a sort of clotting, a small amount of fats and soaps, and a comparatively large quantity of sodium carbonate, to which the alkaline reaction of the juice is due, and which seems to be peculiarly associated with the proteids.

Since, as we shall presently see, pancreatic juice contains a ferment acting energetically on proteid matters in an alkaline medium, it rapidly digests its own proteid constituents, and, when kept, speedily changes in character. The myosin-like clot is dissolved, and the juice soon contains a peculiar form of alkali-albumin (precipitable by saturation with magnesium sulphate), as well as small quantities of leucin, tyrosin, and peptone, which seem to be the products of self-digestion and are entirely absent from the perfectly fresh juice.

§ 218. Action on food-stuffs. On starch pancreatic juice acts with great energy, rapidly converting it into sugar (chiefly maltose). All that has been said in this respect concerning saliva might be repeated in the case of pancreatic juice, except that the activity of the latter is far greater than that of the former. Pancreatic juice and the aqueous infusion of the gland are always capable of converting starch into sugar, whether the animal from which they were taken be starving or well fed. From the juice, or, by the glycerin method from the gland itself, an amylolytic ferment may be approximately isolated.

On proteids pancreatic juice also exercises a solvent action, so far similar to that of gastric juice that by it proteids are converted into peptone. If a few shreds of fibrin are thrown into a small quantity of pancreatic juice, they speedily disappear, especially at a temperature of $35^{\circ} \mathrm{C}$, and the mixture is found to contain peptone. The activity of the juice in thus converting proteids into peptone is favored by increase of temperature up to $40^{\circ}$ or thereabouts, and hindered by low temperatures; it is permanently destroyed by boiling. The digestive powers of the juice in fact depend, like those of gastric juice, on the presence of a ferment which, as we have already said, may be isolated much in the same way as pepsin is isolated, and to which the name trypsin has been given.

The appearance of fibrin undergoing pancreatic digestion is, however, different from that undergoing peptic digestion. In the former case the fibrin does not swell up, but remains as opaque as before, and appears to suffer corrosion rather than solution. But there is a still more important distinction between pancreatic and peptic digestion of proteids. Peptic digestion is essentially an acid digestion; we have seen that the action only takes place in the presence of an acid, and is arrested by neutralization. Pancreatic digestion, on the other hand, may be regarded as an alkaline digestion; the action is most energetic when some alkali is present, and the 
activity of an alkaline juice is hindered or delayed by neutralization and arrested by acidification, at least with nineral acids. The glycerin extract of pancreas is under all circumstances as inert in the presence of free mineral acid as that of the stomach in the presence of alkalies. If the digestive mixture be supplied with sodium carbonate to the extent of 1 per cent., digestion proceeds rapidly, just as does a peptic mixture when acidulated with hydrocholoric acid to the extent of 0.2 per cent. Sodium carbonate of 1 per cent. seems in fact to play in tryptic digestion a part altogether comparable to that of hydrochloric acid of 0.2 per cent. in gastric digestion. And just as pepsin is rapidly destroyed by being heated to about $40^{\circ}$ with a 1 per cent. solution of sodium carbonate, so trypsin is rapidly destroyed by being similarly heated with dilute hydrochloric acid of 0.2 per cent. Alkaline bile, which arrests peptic digestion, seems, if anything, favorable to tryptic digestion.

Corresponding to this difference in the helpmate of the ferment, there is in the two cases a difference in the nature of the products. In both cases peptone is produced, and such differences as can be detected between pancreatic and gastric peptones are relatively small; but in pancreatic digestion the by-product is not, as in gastric digestion, a kind of acid-albumin, but, as might be expected, a body having more analogy with alkali-albumin. Moreover, before the alkali-albumin is actually formed, the fibrin becomes altered and takes on characters intermediate between those of alkali-albumin and of ordinary albumin; and when fresh raw, $i . e$. , unboiled, fibrin is acted upon by pancreatic juice, one or more globulins appear as initial products.

Further, there are evidences that differences of even a more profound nature than the above exist between pancreatic and gastric digestion. One of these is the appearance in the pancreatic digestion of proteids of two remarkable nitrogenous crystalline bodies, leucin and tyrosin. When fibrin (or other proteid) is submitted to the action of pancreatic juice, the amount of peptone which can be recovered from the mixture falls far short of the original amount of proteids, much more so than in the case of gastric juice; and the longer the digestive action, the greater is this apparent loss. If a pancreatic digestive mixture be freed from the alkali-albumin by neutralization and filtration, the filtrate yields, when concentrated by evaporation, a crop of crystals of tyrosin. If these be removed the peptone may be precipitated from the concentrated filtrate by the addition of a large excess of alcohol and separated by filtration. The second filtrate, upon being concentrated by evaporation, yields abundant crystals of leucin and traces of tyrosin. Thus, by the action of the pancreatic juice, a considerable amount of the proteid which is being digested is so broken up as to give rise to products which are no longer proteid in nature. From this breaking up of the proteid there arise leucin, tyrosin, and probably several other bodies, such as fatty acids and volatile substances.

As is well known, leucin and tyrosin are the bodies which make their appearance when proteids or gelatin are acted on by dilute acids, alkalies, or various oxidizing agents. Leucin is a body which, in an impure state, crystallizes in minute round lumps with an obscure radiate striation, but when pure forms thin glittering flat crystals. It has the formula $\mathrm{C}_{6} \mathrm{H}_{13} \mathrm{NO}_{2}$ or $\mathrm{C}_{5} \mathrm{H}_{10} \cdot \mathrm{NH}_{2}(\mathrm{CO} . \mathrm{OH})$, and is amido-caproic acid. Now, caproic acid is one of the "fatty acid" series, so that leucin may be regarded as a compound of ammonia with a fatty acid. Tyrosin, $\mathrm{C}_{9} \mathrm{H}_{11} \mathrm{NO}_{3}$, on the other hand, belongs to the "aromatic" series; it is a phenyl compound, and hence allied to benzoic acid and hippuric acid. So that in pancreatic digestion the large complex proteid molecule is split up into fatty acid and aromatic molecules, some other bodies of less importance making their appearance at the same 
time. We infer that the proteid molecules are in some way built up out of "fatty acid" and "aromatic" molecules, together with other components, and we shall later on see additional reasons for this view.

Among the supplementary products of pancreatic digestion may be mentioned the body indol $\left(\mathrm{C}_{8} \mathrm{H}_{7} \mathrm{~N}\right)$, to which apparently the strong and peculiarly fecal odor which sometimes makes its appearance during pancreatic digestion is due. Indol, however, unlike the leucin and tyrosin, is not a product of pure pancreatic digestion, but of an accompanying decomposition due to the action of organized ferments. A pancreatic digestive mixture soon becomes swarming with bacteria, in spite of ordinary precautions, when natural juice or an infusion of the gland is used. When isolated ferment is used and atmospheric germs are excluded, or when pancreatic digestion is carried on in the presence of salicylic acid or thymol, which prevent the development of bacteria and like organisms but permit the action of the trypsin, no odor is perceived and no indol is produced.

After long-continued digestion, especially when accompanied by putrefactive decomposition, the amount of proteids which are carried beyond the peptone stage and broken up may be very great.

On the gelatiniferous elements of the tissues as they actually exist in the tissue previous to any treatment, pancreatic juice appears to have no solvent action. The fibrillæ and bundles of fibrillæ of ordinary untouched connective tissue are not digested by pancreatic juice, which in this respect affords a striking contrast to gastric juice. But when they have been previously treated with acid or boiled, so as to become converted into actual gelatin, trypsin is able to dissolve them, apparently changing them much in the same way as does pepsin. Trypsin, unlike pepsin, will dissolve mucin. Like pepsin, it is inert toward nuclein, horny tissues, and the so-called amyloid matter.

On fats pancreatic juice has a twofold action. In the first place it emulsifies fats. If hog's lard be gently heated until it melts and be then mixed with pancreatic juice before it solidifies on cooling, a creamy emulsion lasting for almost an indefinite time is formed. So also when olive oil is shaken up with pancreatic juice, the separation of the two fluids takes place very slowly, and a drop of the mixture under the microscope shows that the division of the fat is very minute. An alkaline aqueous infusion of the gland has similar emulsifying powers. In the second place pancreatic juice splits up neutral fats into their respective acids and glycerin. Thus, palmitin (or tripalmitin) $\left(\mathrm{C}_{15} \mathrm{H}_{31} \text {. CO.O }\right)_{3} \cdot \mathrm{C}_{3} \mathrm{H}_{5}$ is with the assumption of $3 \mathrm{H}_{2} \mathrm{O}$ split up into three molecules of palmitic acid $3\left(\mathrm{C}_{15} \mathrm{H}_{31} . \mathrm{CO} . \mathrm{OH}\right)$ and one of glycerin $\left(\mathrm{C}_{3} \mathrm{H}_{5}\right)\left(\mathrm{OH}_{3}\right)$; and so with the other neutral fats. If perfectly neutral fat be treated with pancreatic juice, especially at the body-temperature, the emulsion which is formed speedily takes on an acid reaction, and by appropriate means not only the corresponding fatty acids, but glycerin may be obtained from the mixture. When alkali is present, the fatty acids thus set free form their corresponding soaps. Pancreatic juice contains fat and is consequently apt after collection to have its alkalinity reduced, and an aqueous infusion of a pancreatic gland (which always contains a considerable amount of fat) very speedily becomes acid.

Thus pancreatic juice is remarkable for the power it possesses of acting on all the food-stuffs, on starch, fats, and proteids.

The action on starch, the action on proteids, and the splitting up of neutral fats appear to be due to the presence of three distinct ferments, and methods have been suggested for isolating them. The emulsifying power, on the other hand, is connected with the general composition of the juice (or of the aqueous infusion of the gland), being probably in large measure depend- 
ent on the alkali and the alkali-albumin present. The proteolytic ferment, trypsin, as ordinarily prepared seems to be proteid in nature and capable of giving rise by digestion to peptone; but it may be doubted, as in the case of pepsin and other ferments, whether the pure ferment has yet been isolated. There are no means of distinguishing the amylolytic ferment of the pancreas from ptyalin. The term pancreatin has been variously applied to many different preparations from the gland, and its use had, perhaps, better be avoided.

The action of pancreatic juice or of the infusion or extract of the gland, on starch, is seen under all circumstances, whether the animal be fasting or not. The same may probably be said of the action on fats. On proteids the natural juice, when secreted in a normal state, is always active. The glycerin extract or aqueous infusion of the gland, on the contrary, as we have already explained ( $\$ 208$ ), is active in proportion as the trypsinogen has been converted into trypsin.

\section{Succus Entericus.}

$\$ 219$. When in a living animal a portion of the small intestine is ligatured, so that the secretions coming down from above cannot enter its canal, while yet the blood-supply is maintained as usual, a small amount of secretion collects in its interior. This is spoken of as the succus entericus, and is supposed to be furnished by the glands of Lieberkühn, of which we shall presently speak.

Succus entericus may be obtained by the following method, known as that of Thiry modified by Vella. The small intestine is divided in two places at some distance $(30$ to $50 \mathrm{~cm}$.) part. By fine sutures the lower end of the upper section is carefully united with the upper end of the lower section, thus, as it were, cutting out a whole piece of the small intestine from the alimentary tract. In successful cases union between the cut surfaces takes place, and a shortened but otherwise satisfactory canal is re-established. Of the isolated piece, the two ends are separately brought through incisions in the abdominal wall, and their mouths carefully fastened in such a manner that each mouth of the piece opens on to the exterior. During the process of healing two fistula are thus established, one leading to the beginning of and the other to the end of a short piece of intestine quite isolated from the rest of the alimentary canal; by means of these openings a small quantity of fluid can be obtained.

The quantity secreted is said to be considerably increased by the administration of pilocarpine.

Succus entericus obtained fiom the dog by the above method is a clear yellowish fluid having a faintly alkaline reaction and containing a certain quantity of mucus. It is said to convert starch into sugar and proteids into peptone (the action being very similar to that of pancreatic juice), to split up neutral fats, to emulsify fats, and to curdle milk. It is also said to convert rapidly cane-sugar into grape-sugar, and by a fermentative action to convert cane-sugar into lactic acid, and this again into butyric acid, with the evolution of carbonic acid and free hydrogen.

According to the above results succus entericus is to be regarded as an important secretion acting on all kinds of food. But even at its best its actious are slow and feeble. Moreover, many observers have obtained negative results, so that the various statements are conflicting. Besides, we have no exact knowledge as to the amount to which such a secretion takes place under normal circumstances in the living body. We may, therefore, conclude that, at present at all events, we have no satisfactory reasons for supposing that the actual digestion of food in the intestine is, to any great extent, aided by such a juice. 
Of the possible action of other secretions of the alimentary canal, as of the cæcum and large intestine, we shall speak when we come to consider the changes in the alimentary canal.

$\$ 220$. Gall-stones. Concretions, often of considerable size, known as gallstones, are not unfrequently formed in the gall-bladder, and smaller concretions are sometimes formed in the bile passages. In man two kinds of gall-stones are common. One kind consists almost entirely of cholesterin, sometimes nearly free from any admixture with pigment, sometimes more or less discolored with pigment. Gall-stones of this kind have a crystalline structure, and when broken or cut show frequently radiate and concentric markings. The other kind consists chiefly of bilirubin in combination with calcium. Gall-stones of this kind are dark-colored and amorphous. Less common than the above are small, dark-colored stones, having often a mulberry shape, consisting not of bilirubin itself, but of one or other derivative of bilirubin. Gall-stones consisting almost entirely of inorganic salts, calcic carbonates and phosphates, are also occasionally met with. In the lower animals, in oxen for instance, bilirubin gall-stones are not uncommon, but cholesterin gall-stones are rare.

A gall-stone appears always to contain a more or less obvious "nucleus," around which the material of the stone has been deposited, and which may be regarded as the origin of the stone; the real cause of the formation of the stone lies, however, in certain changes in the bile, by which the cholesterin, or bilirubin, or other constituent ceases to remain dissolved in the bile. But we cannot discuss this matter here.

\section{The Secretion of Pancreatic Juice and of Bile.}

$\S 221$. The secretion of pancreatic juice. Although in some cases, as that of the parotid of the sheep, the flow of saliva is continuous or nearly so, in most animals, as in man, the intermittence of the secretion is very nearly absolute. While food is in the mouth saliva flows freely, but between meals only just sufficient is secreted to keep the mouth moist, and probably the greater part of this is supplied not by the larger salivary but by the small buccal glands. The flow of pancreatic juice, on the other hand, is much more prolonged, being in the rabbit continuous, and in the dog lasting for twenty hours after food. But this contrast between the secretion of saliva and that of pancreatic juice is natural, since the stay of food in the mouth, even during a protracted feast, is relatively short, whereas the time during which the material of a meal is able in some way or other to affect the pancreas is very prolonged.

The flow, though continuous or nearly so, is not uniform. In the dog the flow of pancreatic juice begins immediately after food has been taken, and rises to a maximum which may be reached within the first or, as in the case furnishing the diagram given in Fig. 88, the second hour, but which more commonly is not reached until the third or fourth hour. This rise is then followed by a fall, after which there is a secondary rise, reaching a second maximum at a very variable time, but generally between the fifth and seventh hours. 'This second maximum, however, is never so high as the first.

The second rise may be due to material absorbed from the intestines being carried in the circulation to the pancreas, and so directly exciting the gland to activity, much in the same way as, in the case of the stomach, the absorption of digested material promotes the flow of gastric juice (see $\$ 202$ ); and a similar absorption may contribute to the first rise also, but it is more probable that so marked and sudden a rise as this is carried out by some 
nervous mechanism. The details of this mechanism have, however, not as yet been satisfactorily worked out.

The pancreas derives its nerves, which reach it along its bloodvessels, from the solar plexus of the splanchnic system, but the ultimate origin of the fibres have not been traced out; some of them, however, certainly come through the plexus from the right vagus.

Stimulation of the medulla oblongata, or of the spinal cord, will call forth secretion in a quiescent gland, or increase a secretion already going on. From this we may infer the existence of a reflex mechanism, though we cannot as yet trace out satisfactorily the exact path of either the afferent or the efferent impulses; all we can say is, that the latter do not reach the

FIG. 88.

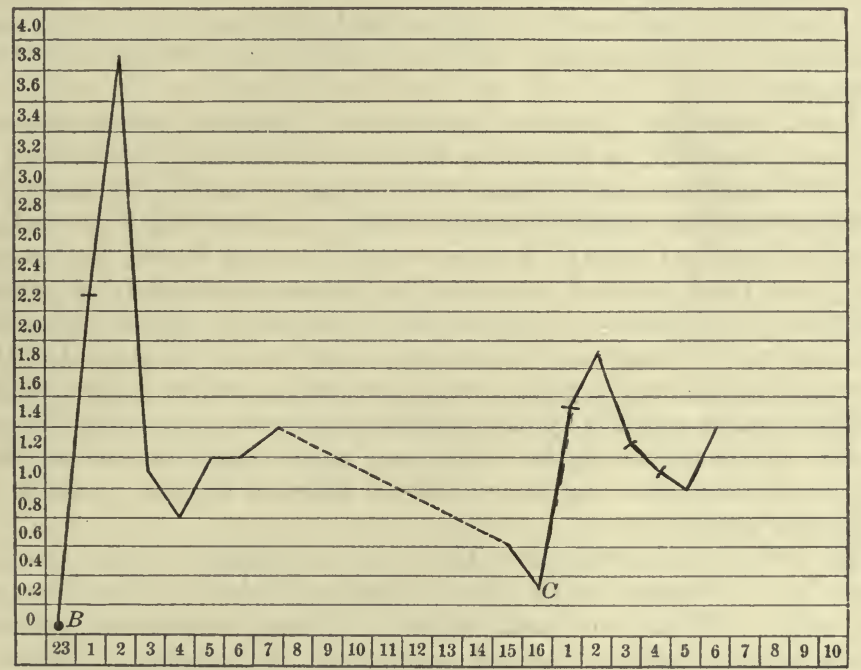

Diagram illustrating the Influence of Food on the Secretion of Pancreatic Juice. (N. O. Bernstein.) The abscissæ represent hours after taking food; the ordinates represent in c.c. the secretion in ten minutes. A marked rise is seen at $B$ immediately after food was taken, with a secondary rise between the fourth and fifth hours afterward. Where the line is dotted the observation was interrupted. On food being again given at $C$, another rise is seen, followed in turn by a depression and a secondary rise at the fifth hour. A very similar curve would represent the secretion of bile.

pancreas by the vagus, since stimulation of the medulla is effective after section of both vagi.

A secretion already going on may be arrested by stimulation of the central end of the vagus, and the stoppage of the secretion, which has been observed as occurring during and after vomiting, is probably brought about in this way. This effect, which, however, is not confined to the vagus, stimulation of other afferent nerves, such as the sciatic, producing the same effect, may be regarded (in the absence of any proof that the result is due to reflex constriction of the pancreatic bloodvessels unduly checking the blood-supply) as an inhibition of a reflex mechanism at its centre in the medulla, or in some other part of the central nervous system, much in the same way as fear inhibits at the central nervous system the secretion of saliva following food in the mouth ( $\$ 196)$. But if so, then we must regard the secretion of pancreatic juice as closely resembling that of saliva, inasmuch as it is called 
forth by a reflex act. Yet it is stated that, unlike the case of saliva, the secretion of pancreatic juice continues after all the nerves going to the gland have been divided, an operation which would do away with the possibility of reflex action. Such an experiment, however, cannot be regarded as decisive, since it is almost impossible to be sure of dividing all the nerves.

No evidence has yet been brought forward to prove the existence of any double nervous mechanism similar to that of chorda fibres and sympathetic fibres in the salivary gland. All that can be said is that, when the gland is stimulated to secrete, the bloodvessels are dilated as in the salivary gland; and we have already ( $\$ 203)$ dwelt on the histological changes which accompany secretion. We may add that when the gland is stimulated to increased secretion, the increase is not merely an increase of water; the discharge of solids is increased even more than the discharge of water, so that the percentage of solids in the juice increases.

The quantity of pancreatic juice secreted, in the case of man, in twentyfour hours has been calculated at 300 c.c., but such a calculation is of very uncertain value.

We have seen (\$197) that in the salivary glands the pressure which may be exerted by the fluid in the ducts is very considerable, exceeding it may be even the blood-pressure in the carotid artery. In this respect the pancreas differs from the salivary glands. When, in a rabbit, a canula connected with a vertical tube or a manometer is placed in the pancreatic duct, the column of fluid does not rise above a height corresponding to a pressure of about $17 \mathrm{~mm}$. of mercury. But at this pressure the gland becomes oedematous on account of the juice secreted passing back through the walls of the ducts and alveoli into the connective tissue; a much higher pressure is needed to render a salivary gland oedematous; and whether the low pressure observed in the pancreas is due to the ease with which odema takes place, or to the actual secretion not being able to reach a higher pressure, cannot be stated with certainty.

$\$ 222$. The secretion of bile. The act of secretion of bile by the liver must not be confounded with the discharge of bile from the bile-duct into the duodenum. When the acid contents of the stomach are poured over the orifice of the biliary duct, a gush of bile takes place. Indeed, stimulation of this region of the duodenum with a dilute acid at once calls forth a flow, though alkaline fluids so applied have little or no effect. When no such acid fluid is passing into the duodenum no bile is, under normal circumstances, discharged into the intestine. The discharge is due to a contraction of the muscular walls of the gall-bladder and ducts, accompanied by a relaxation of the sphincter of the orifice; both acts are probably of a reflex nature, but the details of the mechanism have not been worked out.

The secretion of bile, on the other hand, as shown by the result of biliary fistulæ, is continuous; it appears never to cease. When no food is taken the bile passes from the liver along the hepatic and then back along the cystic duct (the flow being aided probably by peristaltic contractions of the muscular fibres of the duct) to the gall-bladder, where it is temporarily stored; hence in starving animals, when no discharge is excited by food, the gall-bladder becomes greatly distended with bile. But the secretion, though continuous, is not uniform. The rate of secretion varies, and is especially influenced by food; it is seen to rise rapidly after meals, reaching its maximum, in dogs, in from four to eight hours. There seems to be an immediate, sudden rise when food is taken, then a fall, followed subsequently by a more gradual rise up to the maximum, and ending in a final fall to the lowest point. The curve of secretion, in fact, resembles that of 
the secretion of pancreatic juice in having a double rise; and as in that case so in this, it is very probable that the first rise is in part the result of nervous action, and it is also possible that nervous influences intervene in the second, more lasting rise; but, as we shall see presently, even nervous influences may affect the liver in a very indirect manner, and our knowledge as to any direct action of the nervous system on the liver is at present very imperfect.

The liver receives its chief nervous supply from the solar plexus, and to a great extent through that part of the solar plexus called the hepatic plexus, which embraces the portal vein, hepatic artery, and bile-duct, as these plunge into the liver at the porta. The solar plexus is fed by the two abdominal splanchnic nerves, major and minor, by other smaller nerves from the lower parts of the splanchnic (sympathetic) chain, and by the terminal portion of the right vagus nerve. Small branches from the left vagus, rami hepatici, also pass directly to the liver from the termination of that nerve on the stomach, finding their way also through the porta. The fibres thus entering the liver from the several sources are, for the most part, non-medullated fibres; with these, however, are mixed a certain number of medullated fibres.

As to the functions of these nerves in reference to the secretion of bile, we may say at once that no satisfactory or exact statement can at present be marle.

$\$ 223$. It must be remembered, however, that the liver is so peculiarly related to the other organs of digestion, and its vascular arrangements so special that, with regard to it, as compared with many other organs, an intrinsic nervous mechanism must occupy a more or less subordinate position. The blood-supply of the pancreas for instance is dependent chiefly on the width for the time being of the pancreatic arteries; it will be affected, of course, by the general arterial pressure and by any circumstances which affect the outflow by the pancreatic veins, and therefore by the condition of the portal venous system of which those veins form a part; but in the main, the amount of blood bathing the alveoli of the pancreas will depend on whether the pancreatic arteries are constricted or dilated. The quality of the blood reaching the pancreas, being arterial blood drawn direct from the arterial foundation, will be modified only by such circumstances as modify the general mass of the blood.

Very different is the case of the liver. The supply of arterial blood coming direct through the hepatic artery is small compared with the mass pouring through the vena portx; it moreover, as we shall see, is distributed in capillaries among the small interlobular branches of the vena portæand has become venous, indeed merged with the portal blood, before it reaches the actual lobules. The supply of blood 'for the liver is mainly that through the vena portæ; and this supply is not, like an arterial supply, a fairly uniform one, modified chiefly by the vasomotor events of the organ itself, but is dependent on what happens to be taking place in the alimentary canal and in abdominal organs other than the liver itself. When no food is being digested and the alimentary canal is at rest, the vessels of that canal, as we have already said in speaking of the stomach, are like those of the pancreas and salivary glands, in a state of tonic constriction; a relatively small quantity of blood passes through them; hence the flow through the vena portæ is relatively inconsiderable, and the pressure in that vessel is low. When digestion is going on all the minute arteries of the stomach, intestine, spleen and pancreas are dilated, and general arterial pressure being by some means or other maintained (see $\$ 180$ ), a relatively large quantity of blood rushes into the vena portæ and the pressure in that 
vessel becomes much increased, though, of course, remaining lower than the general arterial pressure. Moreover, during digestion, peristaltic movements of the muscular coats of the alimentary canal are, as we have seen, active; and these movements, serving as aids to the circulation (see $\$ 110$ ), help to increase the portal flow. Further, the spleen, as we shall see in speaking of that organ, is in many animals richly provided with plain muscular fibres, and in such cases seems, especially during digestion, to act as a muscular pump driving the blood onward, with increased vigor, along the splenic veins to the liver. So that even were the liver not connected with the central nervous system by a single nervous tie, the tide of blood through the liver would ebb and flow according to the absence or presence of food in the alimentary canal.

An increase of blood-supply does not, of course, necessarily mean an increase of secretory activity. As we have seen, $\$ 197$, in the presence of atropine the secretion of saliva may stand still in spite of dilated bloodvessels and the consequent rush of blood; but we may safely assert that, other things being equal, a fuller blood-supply is favorable to activity. Apparently a mere change in the quantity of blood bathing an alveolus will not start in the cells the changes which constitute the act of secretion, any more than an increase in the blood bathing a muscular fibre will necessarily set going a contraction; but unless there be some counteracting influence at work, a fuller and richer lymph around a cell will naturally lead to the cell taking up more material from the lymph, and so will increase the cell's store of energy. Hence, especially in the hepatic cell, which appears to be always at work, always undergoing metabolism of such a kind as to give rise to bile, we might fairly expect the greater flow through the portal vein to quicken the flow through the bile-duct.

And, as a matter of fact, we do find vaso-constrictor action dominant over the secretion. In the various experiments which have been made to ascertain the action of the nervous system on the secretion of bile, it has always been found that stimulation of the medulla oblongata, or of the spinal cord, or of the abdominal splanchnic nerves, stops, or at least checks the flow of bile. Now the effect of these stimulations is, as we have already seen more than once, a powerful constricting action on the abdominal bloodvessels; by such stimulation the blood-supply of the liver is materially diminished, and in consequence the secretory activity is slackened or arrested.

But there is something besides the mere quantity of blood to be considered in this relation. The blond which passes from the alimentary canal at rest is ordinary venous blood, laden simply with earbonic acid and the ordinary products of the metabolism of the muscular and mucous coats of the canal. When digestion is going on the portal blood is laden, as we shall see, with some, at all events, of the products of digestion, with sugar probably and with various proteil bodies. And it is quite possible or even probable that some of these bodies in the portal blood reaching the hepatic cells, stir them up to secretory activity; indeed, this view may be regarded as supported by the fact that proteid food increases the quantity of bile secreted, whereas fatty food, which as we shall see passes chiefly, if not wholly, not by the portal vein but by the lymphaties, and which is probably largely disposed of in some way or other before it can reach the liver, has no such effect.

Hence we may infer that at all events the second increase of the flow of bile which occurs during the later stages of digestion, may be to a large extent the direct effect of blood, laden with digestive products, passing from the stomach and intestines, especially the latter, to the liver 
by the portal vein, quite independent of any direct nervous action on the liver itself; and, indeed, it is possible that the first rise also may be partly due to the increased flow of blood from the stomach, aided by the absorption from that organ of a certain amount of digested material. Since, however, there is no evidence of any decrease in blood-supply, or in the rate of absorption, corresponding to the fall between the two rises, some influences other than those which we are discussing must be at work in the matter.

$\$ 224$. The blood-supply of the liver being thus, quite apart from any nervous supply of its own, so closely dependent on what is going on in the alimentary canal, it will be convenient to say a few words nore concerning the vasomotor nerves of that canal. As we have already said, in speaking of the vascular system, $\S 155$, the vaso-constrictor fibres for the stomach and intestines, large and small, issuing from what we may call the vaso-constrictor region of the cord, pass for the most part through the two abdominal splanchnic nerves, major and minor, a small number only passing out below the roots of those nerves. When these splanchnic nerves are divided the vessels of the canal are dilated, when they are centrifugally stimulated the vessels are constricted. Whether there be any distinct vaso-dilator fibres for all or any part of the canal, and if so what course they take, is not known. When no food has for some time been taken, the mucous membrane of the stomach as seen through a gastric fistula is pale; the bloodvessels are constricted. And, as far as we know, a similar condition obtains throughout the small and large intestines. When food is taken the mucous membrane of the stomach becomes flushed; its vessels become dilated. This appears to be the result of an inhibition of the previously existing tonic constriction; at least we have no evidence supporting any other explanation. Apparently the presence of food in the stomach starts in the mucous membrane influences which, ascending to the central nervous system, inhibit the vasomotor centre for the abdominal splanchnic nerves or such part of that centre as governs the vaso-constrictor fibres of the stomach. By what path such afferent impulses reach the central nervous system is not as yet definitely settled; but possibly by the vagus nerve, if it be true, as stated, that centripetal stimulation of that nerve, while it raises the general blood-pressure by increasing, in a reflex manner, vaso-constriction in other regions, leads to a dilatation of the gastric vessels. So also it is probable that as the food reaches succeeding sections of the alimentary canal, those in turn in a similar manner become flushed with blood. In the frog there is some evidence that vaso-constrictors leaving the spinal cord by consecutive spinal nerves, govern the bloodvessels of consecutive sections of the alimentary canal.

All this flushing of the canal with blood leads, we repeat, to an increased flow of blood at a higher pressure through the portal vein. Whether there be any additional mechanism set to work, such as, for instance, which some observations suggest, a rhythmical peristaltic contraction of the portal vein, by which the blood is still more rapidly hurried to the liver, and whether the increased venous supply through the portal vein is accompanied by a corresponding increase of the lesser supply of arterial blood through the hepatic artery, is not known. It may, perhaps, be here remarked that there is no need for any increase of arterial blood, since the blood from the alimentary canal, owing to its more rapid passage through the minute vessels, is probably like the corresponding blood in the veins of an active salivary gland (though probably also not to the same extent) less venous than usual during digestion, in spite of the extra quantity of carbonic acid thrown into it by the increased metabolism of the muscular coat during the peristaltic movements. 
$\S 225$. It is interesting to observe that the pressure under which the bile is secreted is relatively low, like that of the pancreatic juice, not high like that of the saliva; it is much lower than the arterial pressure in the same animal, whereas in the case of saliva $(\$ 197)$ the pressure is greater than the blood-pressure in the carotid artery. But, in the case of bile, since the blood which flows through the hepatic lobules is, mainly, venous portal blood, we have to compare the pressure of the secretion, not with arterial pressure but with the venous pressure in the portal system; and in the dog it has been found that while the pressure of the bile secreted stood at about $200 \mathrm{~mm}$. of a solution of sodium carbonate - that is, about $15 \mathrm{~mm}$. mercury-the bloodpressure in a branch of the superior mesenteric vein stood only at about 90 $\mathrm{mm}$. of the same solution-that is, about $7 \mathrm{~mm}$. mercury. Now, the venous pressure in the mesenteric veins is higher, though only slightly higher, than that in the portal vein into which these pour their blood (the difference of pressure being the main cause why the blood flows from the one into the other), and is, therefore, certainly higher than the pressure in the portal capillaries of the hepatic lobules. So that what is true of the salivary gland is also true, on a different scale, of the liver, viz., that the pressure exerted by the secretion is higher than the pressure of the blood in the vessels feeding the secreting cells.

$\$ 226$. If the pressure in the bile-duct be artificially increased, as by pouring fluid into the glass tube or manometer with which the canula in the duct is connected, a resorption of the secreted bile takes place; and resorption will also take place within the body, when the pressure generated by the act of secretion itself reaches and is maintained at a sufficiently high level. Thus, when in the living body the bile-duct is ligatured, or becomes obstructed by gall-stones or otherwise, fluid is accumulated on the near side of the ligature at a pressure which goes on increasing until resorption of the bile takes place, bile salts and biliary pigments are thrown back upon the system, and "jaundice" results. It would appear that in these cases resorption takes place through the interlobular bile-ducts and not through the hepatic cells or other structures within the lobules. The high pressure in the ducts does not lead to a reversal of the current in the hepatic cells (at most it slackens or possibly stops the current), but the bile secreted into the interlobular ducts escapes from these. It further appears that the escape is not into the bloodvessels but into the lymphatics; the bile salts, pigments, and other constituents are carried into the thoracic duct, and in an indirect manner only find their way into the blood stream.

To complete the history of the secretion of bile we ought now to turn to the manufacture of the biliary constituents within the cells. But since the hepatic cells are also engaged in labors other and more important, perhaps, than that of secreting bile, it will be convenient to defer what we have to say on this point until we come to speak of the formation of glycogen and of the general metabolic events taking place in the liver.

\section{The Muscular Mechanisis of Digestion.}

$\$ 227$. From its entrance into the mouth until such remnant of it as is undigested leaves the body, the food is continually subjected to movements having for their object the trituration of the food as in mastication, or its more complete mixture with the digestive juices, or its forward progress through the alimentary canal. 'These various movements may briefly be considered in detail :

\$228. Peristaltic movements. Putting aside the somewhat complicated pharyngeal part of deglutition, and taking the osophageal movements by 
themselves, we find that these, together with the movements of the stomach and of the small and large intestines right down to the anus are more or less alike, and may be described under the general name of "peristaltic" movements. We have already in $\$ 88$ spoken of these, but it may be well to consider them briefly again under a general aspect, before dwelling on the special movements of the several parts of the alimentary canal.

The muscular coat of the alimentary canal consists, as we have seen, of two layers, separated more or less distinctly by a sheet of connective tissue, an outer thimner longitudinal layer, and an inner thicker circular layer; and a similar arrangement obtains in nearly all the muscular hollow tubes of the body, except the arteries, in which the muscular elements are present not so much for the purpose of driving the blood onward as for the sake of regulating the irrigation.

The action of the circular coat is fairly simple. A contraction starting at any part, travels onward in the same direction, generally downward, that is to say, from a part nearer the mouth to a part nearer the rectum, for a greater or less distance, the circularly disposed bundles contracting in sequence. The result is a narrowing or constriction of the tube which, travelling more or less slowly along the tube, drives the contents onward; when a butcher empties the intestine of a slaughtered animal by squeezing it high up with his hand or with his thumb and finger, and carrying the squeezing action downward along the length of the intestine, he makes the passive intestine do very much what the circular coat does actively, by contraction, in the living animal.

The action of the longitudinal coat is perhaps not so clear; but a contraction of the longitudinal coat taking place in any segment of the tube would tend to draw the tube over the contents lying immediately above, or below, the segment, very much as a glove is drawn over a finger. And a succession of such contractions travelling along the tube would lead to a movement of the contents in the same direction. Were the circular coat absent a longitudinal coat might by itself possibly suffice to propel the contents along the tube. In the presence of the circular coat, the action of the longitudinal coat in any segment of the tube, if taking place immediately before the circular contraction, would, by filling the segment with contents, render the squeezing action of the circular coat more efficient; if taking place immediately after the circular contraction, it would help in quickening the return of the tube to its normal calibre, for the contraction of the longitudinal coat tends to shorten and widen the segment, and thus would prepare it for new contents. We can hardly imagine that the two coats would contract at the same time, since they would tend to neutralize each other's action. Indeed, we may probably go farther and assume that in each segment of the canal first the longitudinal coat contracts while the circular coat is relaxed, and that then the circular coat contracts while the longitudinal relaxes. When we come to deal with respiration we shall meet with a similar double antagonistic and successive action between inspiratory and expiratory muscles; we shall further see reason to think that the processes which start the expiratory act tend to check or inhibit the inspiratory act and vice versa; and very possibly a like see-saw of stimulation and inhibition obtains in the muscles of the alimentary canal.

It must be remembered that the circular coat is always much thicker than the longitudinal coat; and we may infer that while the chief work of driving the contents onward falls on the former, the latter assists the work, either in the way which we have suggested or in some other. way.

In the small intestine the tube is hung loosely and much twisted, so that many loops are formed; the contents, moreover, are largely fluid. Hence, 
the steady onward movement, such as is seen when more solid contents pass along the straight and somewhat firmly attached osophagus, is complicated by movements due to a loop being projected forward by the entrance of fluid from above, or being dragged down by the weight of its new contents, or, on the other hand, due to a loop being retracted by the driving on ward of its contents and the emptying of itself, and the like. In this way a peculiar writhing movement of the bowel is brought about, and the phrase "peristaltic movements" is generally used to denote this total effect of the contraction of the muscular coats ; it will, however, be best to restrict the meaning to the progressive contraction of the circular coat assisted, in most cases, by a similar progressive contraction of the longitudinal coat.

$\$ 229$. Mastication. This in man consists chiefly of an up-and-down movement of the lower jaw, combined in the grinding action of the molar teeth, with a certain amount of lateral fore-and-aft movement. The lower jaw is raised by means of the temporal, masseter, and internal pterygoid muscles. The slighter effort at depression brings into action chiefly the digastric muscle, though the mylo-hyoid and genio-hyoid probably share in the matter. Contraction of the external pterygoids pulls forward the condyles and thrusts the lower teeth in front of the upper. Contraction of the pterygoids on one side will also throw the teeth on to the opposite side. The lower horizontally placed fibres of the temporal serve to retract the jaw.

During mastication the food is moved to and fro and rolled about by the movements of the tongue. These are affected by the muscles of that organ governed by the hypoglossal nerve.

The act of mastication is a voluntary one, guided as are so many voluntary acts, not only by muscular sense, but also by contact sensations. The motor fibres of the fifth cranial nerve convey motor impulses from the brain to the above-mentioned muscles; but paralysis of the sensory fibres of the same nerve renders mastication difficult by depriving the will of the aid of the usual sensations.

\$230. Deglutition. The food when sufficiently masticated is, by the movements of the tongue, gathered up into a bolus on the middle of the upper surface of that organ. The front of the tongue being raised-partly by its intrinsic muscles and partly by the stylo-glossus - the bolus is thrust back between the tongue and the palate through the anterior pillars of the fauces or isthmus faucium. Immediately before it arrives there the soft palate is raised by the levator palati, and so brought to touch the posterior wall of the pharynx, which, by the contraction of the upper margin of the superior constrictor of the pharynx, bulges somewhat forward. The elevation of the soft palate causes a distinct rise of pressure in the nasal chambers? this can be shown by introducing a water manometer into one nostril and closing the other just previous to swallowing. By the contraction of the palato-pharyngeal muscles which lie in the posterior pillars of the fauces the curved edges of those pillars are made straight, and thus tend to meet in the middle line, the small gap between them being filled up by the uvula. Through these manœuvres the entrance into the posterior nares is blocked, while the soft palate is formed into a sloping roof, guiding the bolus down the pharynx. By the contraction of the stylo-pharyngeus and palatopharyngeus, the funnel-shaped bag of the pharynx is brought up to meet the descending morsel, very much as a glove may be drawn up over the finger.

Meanwhile in the larynx, as shown by the laryngoscope, the arytenoid cartilages and vocal cords are approximated, the latter being also raised so that they come very near to the false vocal cords; and the cushion at the base of the epiglottis covers the rima glottidis, while the epiglottis itself is 
depressed over the larynx. The thyroid cartilage is now, by the action of the laryngeal muscles, suddenly raised up behind the hyoid bone, and thus assists the epiglottis to cover the glottis. This movement of the thyroid can easily be felt on the outside. Thus, both the entrance into the posterior nares and that into the larynx being closed, the impulse given to the bolus by the tongue can have no other effect than to propel it beneath the sloping soft palate over the incline formed by the root of the tongue and the epiglottis. The palato-glossi or constrictores isthmi faucium, which lie in the anterior pillars of the fauces, by contracting close the door behind the food which has passed them.

When the bolus of food is large it is received by the middle and lower constrictors of the pharynx, which, contracting in sequence from above downward, thrust it into the œesophagus, along which it is driven by a similar series of successive contractions which we shall speak of immediately as peristaltic action. This comparatively slow descent of the food from the pharynx into the stomach may be readily seen if animals with long necks, such as horses and dogs, be watched while swallowing. When, however, the morsel is not large, or when the substance swallowed is liquid, the movement of the back part of the tongue may be sufficient not merely to introduce the food into the grasp of the constrictors of the pharynx, but even to propel it rapidly, to shoot it, in fact, along the lax oesophagus before the muscles of that organ have time to contract. In such a mode of swallowing the middle and lower constrictors take little or no part in driving the food onward, though they and the osophagus appear to contract from above downward after the food has passed by them, as if to complete the act and to insure that nothing has been left behind. Deglutition in this fashion still remains possible after these constrictors have become paralyzed by section of their motor nerves.

When a second act of deglutition succeeds the first with sufficient rapidity, the nervous changes which start the pharyngeal movements of the second act appear to inhibit the osophageal movements of the first act; and when swallowing is repeated rapidly several times in succession, the oesophagus remains quiet and lax during the whole time until immediately after the last swallow, when a peristaltic movement closes the series.

When the stethoscope is applied over the œsophagus, at different regions, a sound is heard during deglutition; sometimes two sounds are heard. 'The first and most constant is coincident with the passage of the bolus, and is due to this and to the muscular sound of the contracting muscles. The later and less constant sound appears to be caused by a quantity of air-bubbles with which the bolus was entangled, lodged at the cardiac end of the oesophagus, being forced into the stomach by the sequent peristaltic contraction of the osophagus.

It will be seen from what has been said that deglutition, though a continuous act, may be regarded as divided into three stages: 'The first stage is the thrusting of the food through the isthmus faucium; this may be either of long or short duration. The second stage is the passage through the upper part of the pharynx. Here the food traverses a region common both to the food and to respiration, and in consequence the movement is as rapid as possible. The third stage is the descent through the grasp of the constrictors. Here the food has passed the respiratory orifice, and in consequence its passage again becomes comparatively slow, except in case of fluids and small morsels, when, as we have seen, it may continue to be rapid. The passage along the osophagus may, perhaps, be regarded as constituting a fourth stage; but it will be more convenient to consider the osophageal movements by themselves. 
The first stage in this complicated process is undoubtedly a voluntary act. The raising of the soft palate and the approximation of the posterior pillars may also be at times voluntary, since they have been seen, in a case where the pharynx was laid bare by an operation, to take place before the food had touched these parts; but the movement may take place without any exercise of the will and in the absence of consciousness. Indeed, the second stage, taken as a whole, though some of the earlier component movements are, as it were, on the borderland between the voluntary and involuntary kingdoms, must be regarded as a reflex act. The third and last stage, whatever be the exact form which it takes, is undoubtedly reflex; the will has no power whatever over it, and can neither originate, stop, nor modifiy it.

Deglutition in fact as a whole is a reflex act; it cannot take place unless sone stimulus be applied to the mucous membrane of the fauces. When we voluntarily bring about swallowing movements with the mouth empty, we supply the necessary stimulus by forcing with the tongue a small quantity of saliva into the fauces, or by touching the fauces with the tongue itself.

In the reflex act of deglutition, caused in the ordinary way by the food coming in contact with the fauces, the afferent impulses originated in the fauces are carried up to the nervous centre by the glosso-pharyngeal nerve, by branches of the fifth, and by the pharyngeal branches of the superior laryngeal division of the vagus. The latter seem of special importance, since the act of swallowing, quite apart from the presence of food in the mouth, may be brought about by centripetal stimulation of the superior laryngeal nerve. The efferent impulses descend the hypoglossal to the muscles of the tongue, and pass down the glosso-pharyngeal, the vagus through the pharyngeal plexus, the fifth, and the spinal accessory, to the muscles of the fauces and pharynx; their exact paths being as yet not fully known, and probably varying in different animals. The laryngeal muscles are governed by the laryngeal branches of the vagus.

The centre of the reflex act lies in the medulla oblongata. Deglutition can be excited, by tickling the fauces, in an animal rendered unconscious by removal of the brain, provided the medulla be left. If the medulla be destroyed, deglutition is impossible. The centre for deglutition lies higher up than that of respiration, so that in the diseases or injuries involving the upper part of the medulla oblongata, the former act may be impaired or rendered impossible while the latter remains untouched. It has been said to form part of the superior olivary bodies, but this view is based on anatomical grounds only. We shall have to deal with this and similar matters in treating of the central nervous system. It is probable that, as is.the case in so many other reflex acts, the whole movement can be called forth by stimuli affecting the centre directly, and not acting on the usual afferent nerves.

$\$ 231$. Movements of the oesophagus. These, as we have just said, are fairly simple. The circular contraction begun by the constrictors of the pharynx is continued along the circular coat of the cesophagus, and assisted by an accompanying contraction of the longitudinal coat, the direction being always, save in the abnormal action of vomiting, from above downward.

It will be remembered that the muscular bundles of the œesophagus are composed of striated fibres in the upper part, and of plain unstriated fibre cells in the lower part, the transition occupying a different level in different animals. Nevertheless, as far as the peristaltic movement is concerned, the two kinds of fibres behave in the same way, except that the peristaltic wave, if we may so call it, travels more rapidly in the striated region. 
These peristaltic movements of the œsophagus may, like those of the intestine, be seen after removal of the organ from the body ; and, indeed, may continue to appear, upon stimulation, for an unusual length of time. They may, therefore, be carried out by the muscular elements, with or without the help of the nervous elements imbedded in them, apart from any action of the central nervous system. Nevertheless, in the living body, the movements of the osophagus seem to be in a special way dependent on the central nervous system; the contractions are not started and carried out by the walls of the tube alone, and so transmitted from section to section in the walls of the tube itself; but afferent impulses started in the pharynx and passing to the medulla oblongata, give rise to reflex efferent impulses which descend along nervous tracts to successive portions of the organ. If the nesophagus be cut across some way down, or if a portion of the middle region be excised, stimulation of the pharynx will produce a peristaltic contraction, which travelling downward will not stop at the cut or excision, but will be continued on into the lower disconnected portion by means of the central nervous system. And it is stated that ordinary peristaltic contractions of the lower part of the œesophagus can be readily excited by stimulation of the pharynx, but not by stimuli applied to its own mucous membrane. In the reflex act which thus brings about the peristaltic contraction of the cesophagus the afferent nerves are those of the pharynx, viz., the superior laryngeal nerve and pharyngeal branches of the vagus, branches of the fifth, and in some animals, at least, branches of the glosso-pharyngeal, but chiefly the first; and œesophageal movements can easily be excited by centripetal stimulation of the superior laryngeal. The centre lies in the medulla oblongata, being a part of the general deglutition centre; and efferent impulses pass along fibres of the vagus, reaching the upper part of the oesophagus by the recurrent laryngeal nerves, and the lower part through the esophageal plexus of the vagus. Section of the trunk of the vagus renders difficult the passage of food along the osophagus, and stimulation of the peripheral stump causes œesophageal contractions.

The force of this movement in the œosophagus is considerable; thus in the dog a ball, pulling by means of a pulley against a weight of 250 grammes, has been found to be readily carried down from the pharynx to the stomach.

At the junction of the œesophagus with the stomach the circular fibres usually remain in a more or less permanent condition of tonic or obscurely rhythmic contraction, more particularly when the stomach is full of food, and thus serve as a sphincter to prevent the return of food from the stomach into the esophagus. Upon the arrival of the bolus of food at the end of the osophagus, the centre for this sphincter is inhibited and the orifice is thus opened up. Possibly the patency of the orifice is still further secured by a contraction of the longitudinal muscular fibres which radiate from the end of the osophagus over the stomach.

$\$ 232$. Movements of the stomach. While the object of the osophageal movement is simply to carry the swallowed bolus with all due speed to the stomach, and while the intestinal movement has, in like manner, simply to carry the intestinal contents onward, the twisted course of the looped path insuring all the mixing of the constituents of the contents that may be necessary, the movements of the stomach have a double object: on the one hand to provide an adequate exposure of the contents of the dilated chamber to the influence of the gastric juice, and on the other to propel the partially digested food, when ready, into the duodenum. We may, accordingly, distinguish between what we may call the "churning" and the "propulsive" movements of the stomach. 
When the stomach is empty all the muscular fibres, as we have said, longitudinal, circular, and oblique, fall into a condition which we may perhaps speak of as an obscure tonic contraction. The whole stomach is small and contracted, its cavity is nearly obliterated, and the mucous membrane, owing to the predominance of the circular coat, is, like the lining membrane of an empty artery, thrown into longitudinal folds. As more and more food enters the stomach all the coats become relaxed, with the exception of the pyloric sphincter, which remains at first permanently closed, and the less marked cardiac sphincter, which merely relaxes from time to time at each act of swallowing. No sooner, however, do the coats thus become relaxed than they set up obscure rhythmical peristaltic contractions, giving rise to the "churning" movements. These movements have been described as of such a kind that the contents flow in a main current from the cardia along the greater curvature to the pylorus, and back to the cardia along the lesser curvature, subsidiary currents mixing the peripheral portions of the contents with the more central; it may be doubted, however, whether any such regularity of flow is marked or constant, and it is not easy to see by what combination and sequence of contractions in the three coats, longitudinal, circular, and oblique, such a regular flow can be produced. But in any case, by such rhythmical contractions the food and gastric juice are rolled about and mixed together. These churning movements are feeble at first, even though the stomach be filled and distended by a large meal rapidly eaten; they become more and more pronounced as digestion proceeds.

Before digestion has proceeded very far the "propulsive" movements begin. These occur at intervals, and are repeated at first slowly but afterward more rapidly. Each movement consists in a contraction of the circular muscular fibres more powerful than any taking part in the churning movements, and leading to a circular constriction which, beginning apparently at about the obscurely defined groove which marks the beginning of the antrum pylori, travels down toward the pylorus, propelling the food onward. This movement is accompanied or rather preceded by a relaxation of, that is to say, in all probability an inhibition of the permanent contraction of, the sphincter pylori itself, in order that the gastric contents may pass into the duodenum. But the occurrence of this relaxation is determined by the nature of the gastric contents; for if the propulsive movement drives large uncligested pieces toward the pylorus, the sphincter is apt to close again, the result of which is that the undigested morsels are carried back into the main body of the stomach.

The combined effect, then, of the churning and of the propulsive movements is, after a certain part of the meal has been reduced to a thick fluid condition somewhat resembling pea soup and often called chyme, to strain off this more fluid part into the duodenum, and to submit the remaining still solid pieces to the further action of the gastric juice.

As digestion proceeds, more and more material leaves the stomach, which is thus gradually emptied, the last portions which are carried through heing those parts of the food which are least digestible, and any wholly indigestible foreign bodies which happen to have been swallowed; the latter may perhaps never leave the stomach at all. The presence of food leards to the development of the movements; but evidently it is not the mere mechanical repletion of the organ which is the cause of the movements, since the stomach is fullest at the beginning when the movements are slight, and becomes emptier as they grow more forcible. The one thing which does increase pari passu with the movements is the acidity, which is at a minimum when the (generally alkaline) food has been swallowed, and increases steadily on- 
ward. It has not, however, been definitely shown that the increasing acidity is the efficient stimulus giving rise to the movements.

The movements of even a full stomach are said to cease during sleep. The nervous mechanism of the gastric movements had better be considered in connection with that of the intestinal movements.

$\S 233$. Vomiting. In a conscious individual this act is preceded by feelings of nausea, during which a copious flow of saliva into the mouth takes place. This being swallowed carries down with it a certain quantity of air, the presence of which in the stomach, by assisting in the opening of the cardiac sphincter, subsequently facilitates the discharge of the gastric contents. The nausea is generally succeeded at first by ineffectual retching in which a deep inspiratory effort is made, so that the diaphragm is thrust down as low as possible against the stomach, the lower ribs being at the same time forcibly drawn in; since during this inspiratory effort the glottis is kept closed, no air can enter into the lungs ; but some is drawn into the pharynx, and thence probably descends by a swallowing action into the stomach. When retching passes on to actual vomiting this inspiratory effort is succeeded by a sudden violent expiratory contraction of the abdominal walls, the glottis still being closed, so that the whole force of the effort is spent, as we shall see it is in defecation, in pressure on the abdominal contents. 'The stomach is, therefore, forcibly compressed from without. At the same time, or rather immediately before the expiratory effort, by a contraction of its longitudinal fibres the osophagus is shortened and the cardiac orifice of the stomach brought close under the diaphragm, while apparently by an inhibition of the circular sphincter, aided perhaps by a contraction of the fibres which radiate from the end of the oesophagus over the stomach, the cardiac orifice, which is normally closed, is somewhat suddenly dilated. This dilatation opens the way for the contents of the stomach, which, pressed upon by the contraction of the abdomen, and to a certain but probably only to a slight extent by the contraction of the gastric walls, are driven forcibly up the œosphagus. The mouth being widely open, and the neck stretched to afford as straight a course as possible, the vomit is ejected from the body. At this moment there is an additional expiratory effort which serves to prevent the vomit passing into the larynx. In most cases, too, the posterior pillars of the fauces are approximated, in order to close the nasal passage against the ascending stream. This, however, in severe vomiting is frequently ineffectual.

Thus in vomiting there are two distinct acts: the dilatation of the cardiac orifice and the extrinsic pressure of the abdominal walls in an expiratory effort. Without the former the latter, even when distressingly vigorous, is ineffectual. Without the latter, as in urari poisoning, the intrinsic movements of the stomach itself are rarely efficient to do more than eject gas, and, it may be, a very small quantity of food or fluid. Pyrosis or waterbrash is, however, probably brought about by this intrinsic action of the stomach.

During vomiting the pylorus is generally closed, so that but little material escapes into the duodenum. When the gall-bladder is full, a copious flow of bile into the duodenum accompanies the act of vomiting. Part of this may find its way into the stomach, as in bilious vomiting, the pylorus then having evidently been opened.

The nervous mechanism of vomiting is complicated and in many aspects obscure. The efferent impulses which cause the expiratory effort must come from the respiratory centre in the medulla; with these we shall deal in speaking of respiration. The dilatation of the cardiac orifice is caused, in part at least, by impulses descending the vagi, since when these are cut real vomiting with discharge of the gastric contents, if it takes place at all, be- 
comes difficult through want of readiness in the dilatation. Such intrinsic movements of the stomach as do take place and the movements of the œsophagus appear to be carried out by the usual nerves. The efferent impulses which cause the flow of saliva in the introductory nausea also descend along the usual nerves such as the chorda tympani. 'These various impulses may best be considered as starting from a vomiting centre in the medulla, having close relations with the respiratory centre. This centre may be excited, may be thrown into action, in a reflex manner, by stimuli applied to peripheral nerves, as when vomiting is induced by tickling the fauces, or by irritation of the gastric membrawe, or by obstruction of the intestine due to ligature, hernia, etc. That the vomiting in the last instance is due to nervous action, and not to any regurgitation of the intestinal contents, is shown by the fict that it will take place when the intestine is perfectly empty and may be prevented by section of the mesenteric nerves. The vomiting attending renal and biliary calculi is apparently also reflex in origin. Vomiting in fact, as a rule, is a reflex action, the afferent impulses passing along one or other nerves, but most frequently along those connected with the alimentary canal, that is, along afferent fibres running in the vagus or in the splanchnic nerves. The centre, however, may be affected directly, as probably in the cases of some poisons, and in some instances of vomiting from disease of the medulla oblongata. Lastly, it may be thrown into action by impulses reaching it from parts of the brain higher up than itself, as in cases of vomiting produced by smells, tastes, or emotions, or by the recollection of past events, and in some cases of vomiting due to cerebral disease.

Many emetics, such as tartar emetic, appear to act directly on the centre, since, introduced into the blood, they will produce vomiting after a bladder has been substituted for the whole stomach. Others again, such as mustard and water, act in a reflex manner by irritation of the gastric mucous membrane. With others, again, which cause vomiting by developing a nauseous taste, the action involves parts of the brain higher than the centre itself.

$\S 234$. Movements of the small intestine. These, as we have already said, are the typical peristaltic movements, simple except in so far as they are complicated by the existence of the pendent lonps, the peculiar oscillating movements of which appear to be produced chiefly by the longitudinal fibres.

The peristaltic movements, as a rule, take place from above downward, and a wave beginning at the pylorus may be traced a long way down. But contractions may, and in all probability occasionally do, begin at various points along the length of the intestine. A movement started by artificial stimulation some way down the intestine, may travel not only downward but also upward; it has been disputed, however, whether in the living body any natural backward peristaltic movement really takes place. In the living body the intestines have periods of rest, alternating with periods of activity, the occurrence of the periods depending on various circumstances; the intensity of the movements also varies very considerably.

$\$ 235$. Movements of the large intestine. They are fundamentally the same as those of the small intestine, but distinct in so far as the latter cease at the ileo-creal valve, at which spot the former normally begin; they are simpler, inasmuch as the pendent loops are absent, and not so vigorous, since relatively to the diameter of the tube the amount of muscular fibre is less. Along the colon where the sacculi are well developed the movement may perhaps be described as almost intermittent from sacculus to sacculus, the contents of one sacculus being driven by the peristaltic contractions of its circular fibres into the next sacculus, which prepares to receive them by a relaxation of its circular and a contraction of its longitudinal fibres.

Since the lips of the ileo-crcal valve are placed transversely across the 
cæcum, not only does distention of the cæcum, by stretching the valve along the line of the lips, bring them into apposition, but the pressure exerted by the peristaltic movement has the same effect. In this way any return of the contents from the large to the small intestine is prevented.

Arrived at the sigmoid flexure, the contents, now more or less solid feces, are supported by the bladder and the sacrum, so that they do not press on the sphincter ani.

$\$ 236$. Defecation. This is a mixed act, being superficially the result of an effort of the will, and yet carried out by means of an involuntary mechanism. Part of the voluntary effort consists in producing a pressure-effect, by means of the abdominal muscles. These are contracted forcibly as in expiration, but the glottis being closed and the escape of air from the lungs prevented, the whole force of the pressure is brought to bear on the abdomen itself, and so drives the contents of the descending colon onward toward the rectum. The sigmoid flexure is by its position sheltered from this pressure; a body introduced per anum into the empty rectum is not affected by even forcible contractions of the abdominal walls.

The anus is guarded by the sphincter ani, which is habitually in a state of normal tonic contraction, capable of being increased or diminished by a stimulus applied, either internally or externally, to the anus. The tonic contraction is in part at least due to the action of a nervous centre situated in the lumbar spinal cord. If the nervous connection of the sphincter with the spinal cord be broken, relaxation takes place. If the spinal cord be divided somewhat higher up, for instance in the dorsal region, the sphincter, after the depressing effect of the operation, which may last several days, has passed off; regains and subsequently maintains its tonicity, showing that the centre is not placed higher up than the lumbar region of the cord. The increased or diminished contraction following on local stimulation is probably due to reflex augmentation or inhibition of the action of this centre. The centre is also subject to influences proceeding from higher regions of the cord, and from the brain. By the action of the will, by emotions, or by other nervous events, the lumbar sphincter centre may be inhibited, and thus the sphincter itself relaxed; or augmented, and thus the sphincter tightened. A second item, therefore, of the voluntary process in defecation is the inhibition of the lumbar sphincter centre, and consequent relaxation of the sphincter muscle. Since the lumbar centre may remain wholly efficient when separated from the brain, the paralysis of the sphincter which occurs in certain cerebral diseases is probably due to inhibition of this lumbar centre, and not to paralysis of any cerebral centre.

Thus a voluntary contraction of the abdominal.walls, accompanied by a relaxation of the sphincter, might press the contents of the descending colon into the rectum and out at the anus. Since, however, as we have seen, the pressure of the abdominal walls is warded off the sigmoid flexure, such a mode of defecation would always end in leaving the sigmoid flexure full. Hence the necessity for these more or less voluntary acts being accompanied by an involuntary augmentation of the peristaltic action of the large intestine, sigmoid flexure, and rectum.

In the movements of the rectum we can trace out more distinctly than in other regions of the alimentary canal the separate actions of the longitudinal and circular fibres. The former, by means of contractions travelling from above downward, shorten the rectum, and, since the anus affords a more or less fixed support, pull the rectum and its contents down; the latter, by means of contractions travelling from above downward but taking place somewhat later, narrow the rectum and so squeeze the contents onward and outward. 
Defecation then appears to take place in the following manner: The large intestine and signoid flexure becoming more and more full, stronger and stronger peristaltic action is excited in their walls. By this means the feces are driven into the rectum, and so, by a continuance of the movements, increasing in vigor, against the sphincter. Through a voluntary act, or sometimes at least by a simple reflex action, the lumbar sphincter centre is inhibited and the sphincter relaxed. At the same time the contraction of the abdominal muscles presses firmly on the descending colon, and thus, contractions of the levator ani assisting, the contents of the rectum are ejected.

It must, however, be remembered that, while in appealing to our own consciousness, the contraction of the abdominal walls and the relaxation of the sphincter seem purely voluntary efforts, the whole act of defecation, including both of these seemingly so voluntary components, may take place in the absence of consciousness, and indeed, in the case of the dog at least, after the complete severance of the lumbar from the dorsal cord. In such cases the whole act must be purely reflex, excited by the presence of feces in the rectum.

$\S 237$. The nervous mechanisms of gastric and intestinal movements. Both the stomach and intestines when removed from the body and thus wholly separated from the central nervous system may, by direct stimulation, be readily excited to movements; and indeed in the absence of all obvious stimuli movements which seem to be spontaneous may at times be observed. The movements of which we are speaking are orderly movements of a peristaltic nature, not mere local contractions of a few bundles of plain muscular fibres. The alimentary canal, therefore, like the heart, though to a less degree, possesses within itself such mechanisms as are requisite for carrying out its own movements; and, as in the case of the heart, there is no adequate evidence that the ganglia scattered in its muscular wallsnamely, those forming the plexus of Auerbach-play any prime part in developing these movements.

On the other hand, powerful movements of a peristaltic kind may be induced, not only as we have already seen in the osophagus but also in the stomach, in the small intestines, and even in the large intestines by stimulation of the vagus nerve.

The chief and usual cause of the movements of the stomach and intestines is the presence of food in their interior. But we do not know definitely the exact manner in which the food produces the movement. It may be that the food, by stimulating the mucous membrane, sends up afferent impulses, and that these give rise by reflex action to efferent impulses which descend the vagus fibres to successive portions of the canal, in a manner similar to that already described in reference to the osophagus. If this be so the efferent impulses reach the stomach and upper part of the duodenum by the terminal portions of the two vagi, Fig. $89, R . V ., L . V$., and reach the intestines by the portion of the right or posterior vagus, Fig. $89, R^{\prime} . V^{\prime}$., which passes into the solar plexus and thence by the mesenteric nerves. The afferent impulses from the stomach travel also apparently by the vagus; the paths of those from the intestines have not yet been determined.

But that such a reflex action through vagus fibres is not the only means by which the presence of food brings about the movements in question, is shown by the fact that these continue to be developed after section of both vagus nerves. Probably the whole action is a mixed one which we may picture to ourselves somewhat as follows: The alimentary canal possesses a power of spontaneous movement, feeble it is true, very inferior to that of the heart and very apt to be latent, but still existing. 'The presence of food in 
some way or other, by some direct action quite apart from the central nervous system, is able to increase this power so that, without any aid from the central nervous system, as after section of the vagi, adequate peristaltic movements can, under favorable circumstances, be carried out. Nevertheless, in

FIG. 89.

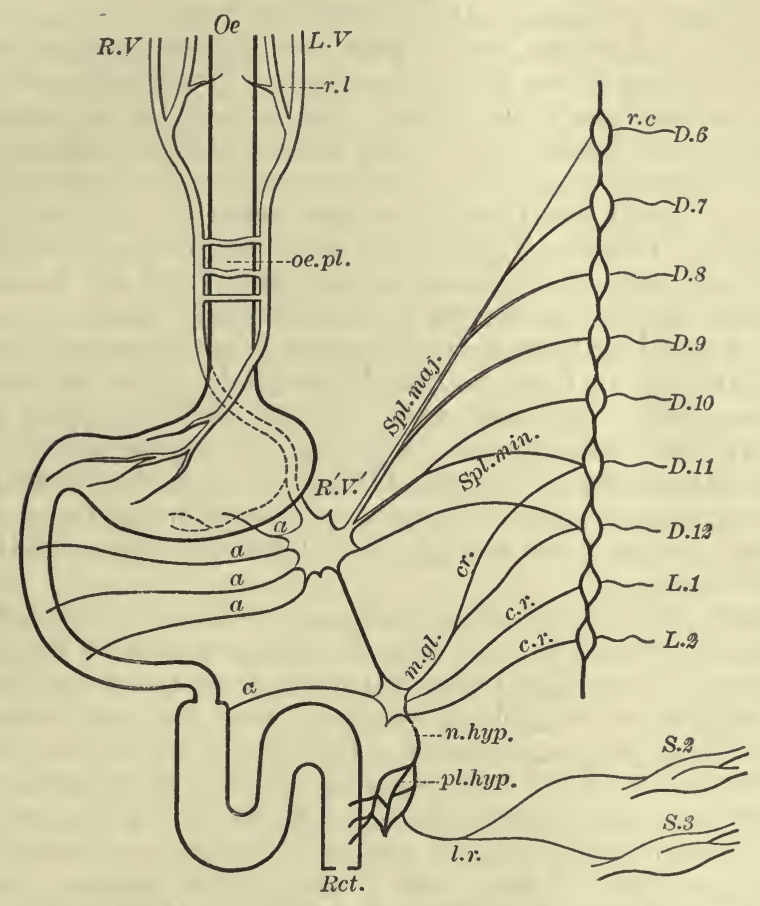

Diagram to illustrate the Nerves of the Alimentary Canal in the Dog. ${ }^{1}$ The figure is, for the sake of simplicity, made as diagrammatic as possible, and does not represent the anatomical relations. $O e$. to Ret. The alimentary canal, œsophagus, stomach, small intestine, large intestine, rectum. $L . V$. Left vagus nerve, ending on front of stomach. r.l. Recurrent laryngeal nerve supplying upper part of cesophagus. $R$. V. Right ragus, joining left vagus in œsophageal plexus, oe. pl., supplying posterior part of stomach and entinued as $R^{\prime}$. $I^{\prime \prime}$. to join the solar plexus, here represented by a single ganglion and connected with the inferior mesenterie ganglion (or plexus) m.gl. a Branches from the solar plexus to stomach and small intestine and from the mesenteric ganglion to the large intestine. Spl. maj. Large splanehnic nerve arising from the thoracic ganglia and rami communicantes, r.c., belonging to dorsal nerves from the 6 th to the 9 th (or 10 th). Spl. min. Small splanchnic nerve similarly arising from 10th and 11 th dorsal nerves. These both join the solar plexus and thence make their way to the alimentary canal. c.r. Nerves from the ganglia, ete., belonging to 11th and 12th dorsal and 1st and $2 \mathrm{~d}$ lumbar nerves, proceeding to the inferior mesenterie ganglia (or plexus), $m$. gl., and thence by the hypogastric nerve, $n$. hyp., and the hypo gastric plexus, $p l$. hyp., to the circular muscles of the rectum. l.r. Nerves from the $2 d$ and $\mathrm{d} d$ sacral nerves, S.2, S.3 (nervi erigentes), proceeding by the hypogastric plexus to the longitudinal muscles of the rectum.

the normal course of events, satisfactory movements are still further secured by the reflex action through vagus fibres just described. Thus, in the dog, the act of swallowing food or even the mere smell of food has been observed to increase the movements of a piece of intestine isolated from the rest of

1 It was not observed until too late that in the diagram of the nerves of the alimentary canal in the dog, twelve dorsal nerves had been represented. The figure, as stated, makes no pretence to anatomical exactuess; but it would have been better to represent either thirteen or fifteen dorsal nerves. 
the alimentary canal but retaining its connections with the central nervous system. . Under this view the peristaltic movements produced by centrifugal stimulation of the vagus in the neck are comparable not so much with the contraction of a skeletal muscle when its motor nerve is stimulated as with the beats which may be called forth in an inhibited or otherwise quiescent heart by stimulation of the cardiac augmentor fibres.

Indeed, we may, perhaps, call the vagus fibres which pass to the stomach and intestines (and these, we may remark, are, like the cardiac augmentor fibres, non-medullated fibres along the greater part of their course) augmentor fibres rather than motor fibres. IVe have all the more reason to do so since there exist companion, but antagonistic, inhibitory fibres. If while lively peristaltic action is going on in the bowels the splanchnic nerves be stimulated, the bowels are brought to rest, often in a very abrupt and marked manner. Inhibitory fibres, therefore, run in the splanchnic nerves (Fig. 89, Spl. maj. and min.), passing along them from the spinal cord to the abdominal plexuses, and thence to the alimentary canal.

It will be noticed that the splanchnic nerves, while containing vaso-constrictor, $i . e$., augmentor, fibres for the bloodvessels of the intestines, carry inhibitory fibres for the muscular coat; and probably the vagus, while containing augmentor fibres for the muscular coat, carries inhibitory dilator fibres for the bloodvessels. It may further be remarked that the vagus, while supplying augmentor fibres for the muscular mechanisms of the alimentary canal, carries, as we so well know, inhibitory fibres for the cardiac muscular mechanism.

In the above statement we have purposely used the general term, peristaltic movement; but, as we have seen, in the movements of the alimentary canal, two sets of muscles are concerned-the circular and the longitudinal. Now, in the rectum we are able to recognize that the two sets of muscles have quite distinct nervous supplies. The longitudinal coat is governed by nerve-fibres which, in the dog, leave the spinal cord in the anterior roots of the second and third sacral nerves (Fig. 89, S.2, S. 3), pass along the branches of those nerves frequently spoken of as the nervi erigentes, $l . r$, to the hypogastric plexus ( $p l$. hyp.), and thence to the rectum. Stimulation of these nerves causes contractions of the rectum, which are confined to the longitudinal coat and, as we have said, pull the rectum down. The circular coat is governed by fibres which leave the spinal cord by the anterior roots of the lower dorsal and first two lumbar nerves, Fig. 89 (coming from the lower part of that spinal region from which, as we have seen $(\$ 155)$ the vaso-constrictor fibres take origin), and, early losing their medulla, pass to the rectum by the inferior mesenteric ganglia, the hypogastric nerves, and hypogastric plexus (Fig. 89, m. gl., n. hyp., pl. hyp.). Stimulation of these fibres gives rise to contractions which are confined to the circular coat and squeeze out the contents of the rectum. A similar double nervous supply probably governs the longitudinal and circular coats along the whole alimentary canal; but the details of such a supply are at present unknown.

Our knowledge, moreover, concerning the details of any special nervous mechanisms, by means of which the more complicated movements of the stomach, including the closing and opening of the sphincters, are carried out, is at present very imperfect. We cannot add to what we have incidentally said in speaking of vomiting.

The movements of the rectum, including the sigmoid flexure, appear to be much more closely dependent on the central nervous system than are those of the rest of the alimentary canal. As we have said. the movements of both large and small intestine are rather assisted and aug- 
mented than primarily called forth by impulses descending from the central nervous system along the vagus fibres. As the large intestine, however, passes into the rectum, government by the vagus is replaced by government through the lumbar cord and the nerves just previously nientioned; and this government appears to be not so much mere augmentation as the actual carrying out of the movements through reflex action. Hence, this is the part of intestinal movement which fails in diseases of the central nervous system, the failure leading to obstinate constipation, if not to actual difficulty of defecation. The presence of feces in the sigmoid flexure no longer stirs up the reflex mechanism for their discharge; meanwhile the more independent movements of the higher parts of the canal continue to drive the contents onward, and hence the feces accumulate in the sigmoid flexure and colon awaiting the delayed action of the imperfect reflex mechanism. With regard to the exact manner in which the presence of food acts as a stimulus, it may be worth while to remark that, although in the stomach, as we have seen, mere fulness is not the efficient cause of the movements, since these become more active as digestion proceeds and the bulk of the contents diminishes, yet in the intestine distention of the bowel up to certain limits most distinctly increases the vigor of the movements just as distention of the cardiac cavities within certain limits improves the cardiac stroke. This is well seen in obstruction of the bowels, in which case the bowel distended above the obstruction is frequently thrown into violent peristaltic movements. This effect is in part at least due to the distention extending the muscular fibres, and so in a direct manner promoting their contraction (see $\$ 77$ ), but may be in part due to augmentor impulses excited in a reflex manner. Probably in an intestine isolated from the central nervous system food provokes peristaltic movements much more by causing distention and so stretching the muscular coats than by acting as a stimulus to the mucous membrane, either through chemical action or in any similar way.

$\S 238$. Next to the presence of food in the interior of the alimentary canal a deficient oxygenation of the blood supplied to the walls of the canal or the sudden cutting off of the supply of blood may be regarded as the most powerful provocatives of peristaltic action. When the aorta is clamped, or when the respiration is seriously interfered with, peristaltic movements become very pronounced. Thus, in death by asphyxia or suffocation, an involuntary discharge of feces, which is in part at least the result of increased peristaltic action, is not an unfrequent result; and the marked peristaltic movements which are so frequently seen in an animal when the abdomen is laid open immediately after death appear to be due to the cessation of the circulation and the consequent failure in the supply of blood to the walls of the alimentary canal, and not, as has been suggested, to the contact with air of the peritoneal surface. Since it is blood which brings oxygen to the tissues, failure in the supply of blood is tantamount to failure in the supply of oxygen; but the blood current brings other things besides oxygen and also takes things away; and the failure of this action also, probably, as well as failure in the supply of oxygen, provokes the movements in question.

The movements thus produced are to some extent the result of the deficient supply of blood acting directly on the walls of the canal, though in asphyxia at all events this effect may be increased by the too venous blood stimulating the central nervous system, and thus sending augmentor impulses down the vagus.

With regard to the mode of action of the drugs which promote peristaltic action, it will be sufficient here to say that while some, such as 
nicotin, appear to act directly on the walls of the canal, others, such as strychnia, produce their effect chiefly by acting through the central nervous system.

\section{The Changes which the Food Uxdergoes in the Alimentary}

\section{CANal.}

$\S 239$. Having studied the properties of the digestive juices as exhibited outside the body, and the various mechanisms by means of which the food introduced into the body is brought under the influence of those juices, we have now to consider what, as matters of fact, are the actual changes which the food does undergo in passing along the alimentary canal; what are the steps by which the contents of the canal are gradually converted into feces. The events which lead to this conversion are twofold. On the one hand the digestive juices do bring about, inside the alimentary canal, changes which in the main are the same as those observed in laboratory experiments outside the body and described in previous sections, though the results are somewhat modified by the special conditions which obtain within the body. On the other hand, absorption, that is to say, the passage from the interior of the canal into the bloodvessels and lymphatics, of digested material in company with water is going on along the whole length of the canal, and especially in the small and large intestines. It will be convenient to confine ourselves at present to the study of the first class of events, the changes effected in the canal, merely noting the disappearance of this or that product, and deferring the difficult problem of how absorption takes place to a subsequent and separate discussion.

In the mouth the presence of the food, assisted by the movements of the jaw, causes, as we have seen, a flow of saliva. By mastication, and by the addition of mucous saliva, the food is broken into small pieces, moistened, and gathered into a convenient bolus for degiutition. In man some of the starch is, even during the short stay of the food in the mouth, converted into sugar; for if boiled starch free from sugar be even momentarily held in the mouth and then ejected into water (kept boiling to destroy the ferment), it will be found to contain a decided amount of sugar. In many animals no such change takes place. The viscid saliva of the dog serves almost solely to assist in deglutition; and even the longer stay which food makes in the mouth of the horse is insufficient to produce any marked conversion of the starch it may contain. During the rapid transit through the cesophagus no appreciable change takes place.

The amount of absorption of digested material, or even of simple water, from the mouth or cosophagus must always be insignificant.

\section{The Changes in the Stomach.}

$\$ 240$. The arrival of the food, the reaction of which is either naturally alkaline, or is made alkaline, or at least is reduced in acidity, by the addition of saliva, causes a flow of gastric juice. This, already commencing while the food is yet in the mouth, increases as the food accumulates in the stomach, and as, by the churning gastric movements, one part after another of the food is brought into contact with the mucous membrane.

The characters of the juice appear to change somewhat as the act of digestion proceeds. The amount of pepsin in the gastric contents increases for some time after food is taken, and probably the actual secretion increases also. The acidity of the gastric contents is at first very feeble; indeed in man, in some cases at least, for some little time after the beginning of a meal no free acid is present, and during this period the conversion of starch into 
sugar may continue. This condition, however, is temporary only; very soon the contents become acid, arresting the action of and ultimately destroying the amylolytic ferment; and, since the rate of secretion of acid appears to be fairly constant, the contents of the stomach, unless fresh alkaline food be taken, become more acid as digestion goes on.

The gross effect of gastric digestion is to break up and partly to dissolve the larger lumps of masticated food into a thick grayish soup-like liquid called chyme, with which are still mixed in variable quantity larger and smaller masses of less changed food. This is the result, partly of the solution of proteid matters, partly of the solution of the gelatiniferous connective tissue holding the proteid elements together. In a fragment of meat, for instance, the muscular fibres, through the solution of the connective tissue binding them together, fall asunder, the sarcolemma is dissolved, and the fibres themselves split up sometimes longitudinally but most frequently by transverse cleavage into discs, and are ultimately more or less reduced partly into a granular mass, partly to actual solution. In a piece of tissue containing fat, the connective tissue binding the fat cells together and the envelopes of the fat cells are dissolved, so that the fat, fluid at the temperature of the body, is set free from the individual cells and runs together into larger and smaller masses. In vegetable tissue the proteid elements are in part dissolved and, though there is no evidence that in man cellulose is dissolved in the stomach, the whole tissue is softened and to a certain extent disintegrated. Milk is curdled and the curd subsequently more or less dissolved.

The thick soup-like acid chyme consists accordingly partly of substances which have entered into actual solution, partly of mere particles or droplets of proteid, fatty or other nature, and partly of masses small or great which may be recognized under the microscope as more or less changed portions of animal or vegetable tissue. The amount of material actually dissolved is in most specimens of chyme exceedingly small. When the solid parts are removed by filtration the clear filtrate contains beside salts, pepsin and free hydrochloric acid (the constituents of the gastric juice), a small amount of sugar, of parapeptone and of peptone. The sugar is often absent, the parapeptone is not always present, and the amount of peptone (or albumose) is always small.

During gastric digestion the chyme thus formed is from time to time ejected through the pylorus, accompanied by even large morsels of solid less-digested matter. This may occur immediately when water alone is taken and within a few minutes of food having been taken; but the larger escape from the stomach probably does not in man begin until from one to two, and lasts from four to five, hours after the meal, becoming more rapid toward the end, and such pieces as are the least broken up by the gastric juice and movements being the last to leave the stomach.

The time taken up in gastric digestion probably varies in the same animal not only with different articles of food, but also with varying conditions of the stomach and of the body at large. In different animals it varies very considerably, being from twelve to twenty-four hours in the dog after a full meal, while the stomachs of rabbits are never empty but always remain largely filled with food, even during starvation. In man the stomach probably becomes empty between the usual meals.

The total amount of change which the food undergoes in the stomach, that is the share taken by the stomach in the whole work of digestion, seems to vary largely in different animals, and in the same animal differs according to the nature of the meal. In a dog fed on an exclusively meat diet, a very large part of the digestion is said to be carried out by the 
stomach, very little work apparently being left for the intestines; that is to say, the larger part of the meal is reduced in the stomach to actual solution and a considerable quantity is probably absorbed directly from the stomach. In such cases the amount of peptone found in the stomach during the digestion of the meal is found to be fairly constant, from which it may be inferred that the peptone is absorbed as soon as it is formed. There is also evidence that fat may to a certain extent undergo in the stomach changes leading to emulsion, similar to those which, as we shall see, are carried out in the small intestine.

But such cases as these cannot be regarded as typical cases of gastric digestion, and in man, at all events, living on a mixed diet the work of the stomach appears to be to a large extent preparatory only to the subsequent labors of the intestine. It is true that our information on this matter is imperfect, being chiefly drawn from the study of cases of gastric or duodenal. fistula, in which probably the order of things is not normal, or being in large measure deductions from experiments on animals, whose economy in this respect must be largely different from our own; but we are probably safe in concluding that, in ourselves, the chief effect of gastric digestion is by means of the disintegration spoken of above to reduce the lumps of food to the more uniform chyme and so to facilitate the changes which take place in the small intestine. During the disintegration some of the proteid in the meal is converted into peptone; and the peptone so formed is probably absorbed at once; but much proteid remains unchanged or at least is not converted into peptone, and the fats and starches undergo in themselves very little change indeed.

In the act of swallowing, no inconsiderable quantity of air is carried down into the stomach, entangled in the saliva or in the food. This may be returned in eructations. When the gas of eructation or that obtained directly from the stomach is examined, it is found to consist chiefly of nitrogen and carbonic acid, the oxygen of the atmospheric air having been largely absorbed. In most cases the carbonic acid is derived by simple diffusion from the blood, or from the tissues of the stomach, which similarly take up the oxygen. In many cases of flatulency, however, it may arise from a fermentative decomposition of the sugar which has been taken as such in food or which has been produced from the starch, the gas being either formed in the stomach or passing upward from the intestine through the pylorus.

The enormous quantity of gas which is discharged through the mouth in cases of hysterical flatulency, even on a perfectly empty stomach, and which seems to consist largely of carbonic acid, presents difficulties in the way of explanation; it is possible that it may be simply diffused from the blood, but it is also possible that in many cases it is derived from air which the patient has hysterically swallowed, the oxygen having been removed, in the stomach, by absorption and replaced by carbonic acid.

\section{In the Small Intestine.}

$\S 241$. The semi-digested acid food, or chyme, as it passes over the biliary orifice, causes, as we have seen ( $\$ 223)$, gushes of bile, and at the same time the pancreatic juice flows into the intestine freely. These two alkaline fluids, especially the more strongly and constantly alkaline pancreatic juice, tend to neutralize the acidity of the chyme, but the contents of the duodenum do not become distinctly alkaline until some distance from the pylorus is reached. The rapidity with which the change in the reaction is completed is not the same in all animals, and in the same animal 
appears to vary according to the nature of the food and various circumstances. In man, living on a mixed diet, the contents have probably become distinctly alkaline before they have passed far down the duodenum. On the other hand, in dogs the contents of the small intestine have been observed to be acid throughout, and that not only when fed on starch and fat, which might, by an acid fermentation of which we shall presently speak, give rise to an acid reaction, but even when fed on meat.

The conversion of starch into sugar, which, as we have seen, is sooner or later arrested in the stomach, is resumed with great activity and indeed completed by the pancreatic juice, possibly assisted by the succus entericus, the presence of bile being said to increase the activity of the pancreatic amylolytic ferment. The conversion begins as soon as the acidity of the chyme is sufficiently reduced and continues along the intestine; portions, howerer, of still undigested starch may be found in the large intestine, and even at times in the feces.

The pancreatic juice, as we have seen, emulsifies fats, and also splits them into their respective fatty acids and glycerin. The fatty acids thus set free become converted by means of the alkaline contents of the intestine into soaps; but to what extent saponification thus takes place is not exactly known. Undoubtedly soaps have to a small extent been found both in portal blood and in the thoracic duct after a meal; but there is no proof that any large quantity of fat is introduced in this form into the circulation. On the other hand, the presence of neutral fats in the lacteals, and to a slight extent in portal blood, is a conspicuous result of the digestion of fatty matters; and in all probability saponification in the intestine is a subsidiary process, the effect of which is rather to facilitate the emulsion of neutral fats than to introduce soaps as such into the blood, for the presence of soluble soaps favors the emulsion of neutral fats. Hence a rancid fat, $i$. e., a fat containing a certain amount of free fatty acid, forms an emulsion with an alkaline fluid more readily than does a quite neutral fat. A drop of rancid oil let fall on the surface of an alkaline fluid, such as a solution of sodium carbonate of suitable strength, rapidly forms a broad ring of emulsion, and that even without the least agitation. As saponification takes place at the junction of the oil and alkaline fluid, currents are set up by which globules of oil are detached from the main drop and driven out in a centrifugal direction; the intensity of the currents and the consequent amount of emulsion depend on the concentration of the alkaline medium and on the solubility of the soaps which are formed. Now the bile and pancreatic juice supply just such conditions as the above for emulsionizing fats; they both together afford an alkaline medium, the pancreatic juice gives rise to an adequate amount of free fatty acid, and the bile in addition brings into solution the soaps as they are formed. So that we may speak of the emulsion of fats in the small intestine as being carried on by the bile and pancreatic juice acting in conjunction; and as a matter of fact the bile and pancreatic juice do largely emulsify the contents of the small intestine, so that the grayish turbid chyme is changed into a creamy-looking fluid, which has been sometimes called chyle. It is advisable, however, to reserve this name for the contents of the lacteals. Many of the fats present in food-for instance, butter-already contain some fatty acids when eaten; for these fats the initial action of the pancreatic juice is less necessary.

This mutual help of bile and pancreatic juice in producing an emulsion explains to a certain extent the controversy which long existed between those who maintained that the bile and those who maintained that the pancreatic 
juice was necessary for the digestion and absorption of fatty food. That the pancreatic juice does produce in the intestine such a change as favors the transference of neutral fats from the intestine into the lacteals is shown by the fact that in diseases affecting the pancreas much fatty food frequently passes through the intestine undigested and great wasting ensues; but it cannot be maintained that the pancreatic juice is the sole agent in this matter, since in animals in which the pancreatic ducts have been successfully ligatured chyle is still found in the lacteals. On the other hand, that the bile is of use in the digestion of fat is shown by the prevalence of fatty stools in case of obstruction of the bile-ducts; and though the operation of ligaturing the bile-ducts and leading all the bile externally through a fistula of the gall-bladder is open to objection, since it in some way or other so exhausts the animal as indirectly to affect digestion, still the results of experiments in which the resorption of fat was distinctly lessened (the quantity of fat in the lacteals falling from 3.2 to 0.2 per cent.) by the ligature and fistula obviously point to the same conclusion. That in man the succus entericus possesses a wholly insufficient emulsifying power is shown by the observation of a case in which the duodenum opened on the surface by a fistula in such a way that the lower part of the intestine could be kept free from the contents of the upper part containing the bile and pancreatic juice and matters proceeding from the stomach. Fats introduced into the lower part, where they could not be acted upon either by the bile or by the pancreatic juice, were but slightly digested. IVithout denying the possible assistance of the succus entericus, or even of gastric juice, we may conclude that the digestion of fat is in the main carried out by the conjoint action of bile and pancreatic juice.

$\S 242$. We have seen $(\$ 216)$ that the addition of bile to a digesting mixture gives rise to a precipitate. This is partly a coarse, flocculent precipitate, consisting of parapeptone with some amount of bile acids, and partly of a finer, more granular precipitate, which is longer in falling down and consists chiefly of bile acids with a variable amount of peptone; the latter is redissolved on the further addition of bile, even though the reaction of the mixture remain acid. In the upper part of the duodenum the inner surface, if examined while digestion is going on, is found to be lined by a colored flocculent and granular material, which is probably a precipitate thus formed; the purpose of this precipitation is probably to delay the passage of the undigested parapeptone along the duodenum. Moreover, apart from this precipitation, bile arrests the action of pepsin, even while the reaction of the mixture still remains acid; and as soon as an alkaline reaction is established the pepsin is apparently destroyed by the trypsin, so that with the flow of bile and pancreatic juice into the duodenum the processes which have been going on in the stomach come to an end. In fact, it would seem that the juices of the various districts of the alimentary canal are mutually destructive; thus, while pepsin in an acid solution destroys the active constituents of saliva and of pancreatic juice (probably also those of the succus entericus), it is in its turn antagonized or destroyed by the bile and the other alkaline juices of the intestine. Hence, pancreatic juice introduced through the mouth must lose its powers in the stomach, and can only be of use as an alkaline medium containing certain proteid matters. On the other hand, if, as we have reason to believe, the contents of the stomach as they issue from the pylorus still contain a large quantity of undigested proteids, these must be digested by the pancreatic juice (with or without the assistance of the succus entericus), the action of which seems to be assisted, or at least not hindered, by bile. And in dogs fed through a duodenal fistula, so that all gastric digestion is excluded, proteids are completely digested 
and give rise to quite normal feces. To what stage the pancreatic digestion is carried, whether peptone is practically the only product, or whether the pancreatic juice in the body, as out of the body, carries on its work in the more destructive form, whereby the proteid material subjected to it is so broken down as to give rise to appreciable quantities of leucin and tyrosin, is at present not exactly known. Leucin and tyrosin have been found in the intestinal contents, and may therefore be formed during normal digestion, but whether an insignificant quantity or a considerable quantity of the proteid material of food is thus hurried into a crystalline form cannot be definitely stated. The extent to which the action is carried is probably different in different animals, and probably varies also according to the nature of the meal and the condition of the body. Possibly when a large and unnecessary quantity of proteid material is taken at a meal, together with other substances, no inconsiderable amount of the proteids undergo this profound change, and, as we shall see, rapidly leave the body as urea without having been used by the tissues, their contribution to the energy of the body being limited to the heat given out during the changes by which they are converted into urea. To this apparently wasteful use of proteids we shall return in speaking of what is called the "luxus consumption" of food.

$\$ 243$. In dealing with the action of pancreatic juice we drew attention ( $\$ 218$ ) to the difference between the results of pure tryptic digestion and those obtained when bacteria or other microörganisms were allowed to be present. We saw that indol, for example, was the product of the action of these organisms, not of trypsin. Now indol is formed in varying quantity during the digestion which actually takes place in the intestine, some of it at times appearing in the urine as indigo-yielding substance (indican). Moreover, bacteria and other microörganisms are present in the intestinal contents. Hence, we must regard the changes taking place in the intestine not as the pure results of the action of the several digestive juices, but as those results modified by or mixed with the results of the action of microörganisms. We spoke above ( $\$ 216$ ) of bile as being antiseptic, but this must be understood as meaning not that the presence of bile arrests the action of all microorganisms within the intestine, but that it modifies their action, keeping it within certain limits and along certain lines.

Concerning the exact nature and extent of the changes thus due to microorganisms, our knowledge is at present very imperfect. The proteids and the carbohydrates seem to be the food-stuff's on which these organisms produce their chief effect. Out of the proteids they give rise not only to indol, but to several other compounds, among which may be mentioned phenol $\left(\mathrm{C}_{6} \mathrm{H}_{6} \mathrm{O}\right)$, of which a small quantity may be recognized in the feces, the rest being absorbed and appearing in the urine in the form of certain phenolcompounds, such as phenyl-sulphuric acid. Out of proteids they may also form the peculiar poisonous bodies called ptomaines, which appear in the ordinary putrefaction of proteids. But their most conspicuous effects are those on the carbohydrates. As the food descends the intestine, the presence of lactic acid becomes more and more obvious; indeed, in some cases the naturally alkaline reaction of the intestinal contents may in the lower part of the intestine be changed into an acid one by the presence of lactic acid. Now, lactic acid may be formed out of sugar by means of a special organism inducing what is spoken of as the lactic acid fermentation. And we have every reason to believe that in even normal digestion a certain quantity of sugar, either eaten as such or arising from the amylolytic conversion of starch, does not pass away from the intestine into the blood as sugar, but undergoes this fermentation into lactic acid. To what extent this change 
takes place we do not know ; the amount probably varies according to the amount of carbohydrates eaten, the condition of the alimentary canal, and other circumstances. It may be under certain circumstances simply a part of normal digestion; under other circumstances it may be excessive and give rise to troubles.

That fermentative changes may occur in the small intestine is further indicated by the facts that the gas there present may contain free hydrogen, and that chyme, after removal from the intestine, continues at the temperature of the body to produce carbonic acid and hydrogen in equal volumes. This suggests the possibility of the sugar of the intestinal contents undergoing the butyric acid fermentation, during which, as is well known, carbonic anhydride and hydrogen are evolved. By this change the sugar is removed from the carbohydrate group into the fatty acid group; it is thus, so to speak, put on its way to become fat. We shall see hereafter that sugar may be somewhere in the body converted into fat; this conversion, however, takes place chiefly if not wholly in the tissues, and such change as may take place in the alimentary canal is to be regarded as suggestive rather than as important.

The hydrogen thus occurring in the intestine may also arise from the proteid decompositions spoken of above. However arising it may act as a reducing agent-reducing sulphates, for instance-and thus giving rise to sulphides and to sulphuretted hydrogen; as a reducing agent it assists in the formation of the fecal and urinary pigments.

Thus, during the transit of the food through the small intestine, by the action of the bile and pancreatic juice, and possibly to some extent of the succus entericus, assisted by various microörganisms, the proteids are largely dissolved and converted into peptone and other products, the starch is changed into sugar, the sugar possibly being in part further converted into lactic and other acids, and the fats are largely emulsified and to some extent saponified. These products as they are formed pass into either the lacteals or the portal bloodvessels, so that the contents of the small intestine, by the time they reach the ileo-crcal valve, are largely-but by no means wholly deprived of their nutritious constituents. So far as water is concerned, the secretion of water into the small intestine maintains such a relation to the absorption from it that the intestinal contents at the end of the ileum, though much changed, are about as fluid as in the duodenum.

\section{In the Large Intestine.}

$\S 244$. The contents, whether alkaline or not, in the ileum now become once more distinctly acid. This, however, is not caused by any acid secretion from the mucous membrane; the reaction of the intestinal walls in the large as in the small intestine is alkaline. It must, therefore, arise from acid fermentations going on in the contents themselves; and that fermentations do go on is shown by the appearance of marsh gas as well as hydrogen in this portion of the alimentary canal. The character and amount of fermentation probably depend largely on the nature of the food, and probably also vary in different animals.

Of the particular changes which take place in the large intestine we have no very definite knowledge; but it is exceedingly probable that in the voluminous cæcum of the herbivora a large amount of digestion of a peculiar kind goes on. We know that in herbivora a considerable quantity of cellulose disappears in passing through the alimentary canal, and even in man some is digested. It seems probable that this cellulose digestion takes place in the large intestine, and is the result of fermentative changes 
carried out by means of microörganisms, marsh gas being one of the products formed at the same time.

Be this as it may, whether digestion, properly so called, is all but complete at the ileo-creal valve, or whether important changes still await the chyme in the large intestine, one great characteristic of the work done in the colon is absorption. By the abstraction of all the soluble constituents, and especially by the withdrawal of water, the liquid chyme becomes as it approaches the rectum converted into the firm solid feces, and the color shifts from the bright orange, which the gray chyme gradually assumes after admixture with bile, into a darker and dirtier brown.

\section{The Feces.}

$\S 245$. These consist in the first place of the indigestible and undigested constituents of the meal; shreds of elastic tissue, hairs and other horny elements, much cellulose and chlorophyll from vegetable, and some connective tissue from animal food, fragments of disintegrated muscular fibre, fat-cells, and not unfrequently undigested starch-corpuscles. The amount of each must of course vary very largely according to the nature of the food, and the digestive powers, temporary or permanent, of the individual. In the second place, to these must be added substances not distinctly recognizable as parts of the food, but derived from the secretions of the alimentary canal. The feces contain mucus in variable amount, sometimes albumin, cholesterin, butyric and other fatty acids, lime and magnesia soaps, coloring matters, and inorganic salts, especially earthy phosphates, crystals of ammonio-magnesia phosphates being very conspicuous. The reaction is generally but not always acid. They also contain a ferment similar in its action to pepsin, and an amylolytic ferment similar to that of saliva or of pancreatic juice. The bile salts are represented by a small quantity of cholalic acid, or some product of that body, and sometimes a very small quantity of taurin. The glycin and most or all of the taurin have been absorbed from the intestine, and the cholalic acid has been partly absorbed and partly decomposed. The fact that the feces become "clay-colored" when the bile is cut off from the intestine shows that the bile-pigment is at least the mother of the fecal pigment; and a special pigment, which has been isolated and called stercobilin, is said to be identical with the substance called urobilin, which may be formed from bilirubin. As other special constituents of the feces may be mentioned excretin, a somewhat complex nitrogenous body, whose exact chemical nature is at present uncertain, and skatol $\left(\mathrm{C}_{9} \mathrm{H}_{9} \mathrm{~N}\right)$, a nitrogenous body which like indol is derived from the decomposition of proteids by means of microörganisms, and which is the chief cause of the fecal odor, since only a small quantity of indol remains in the feces. These odoriferous bodies are derived directly from the food; at the same time it is quite possible that other specific odoriferous substances may be secreted directly from the intestinal wall, especially from that of the large intestine.

\section{The Lacteals and the Lymphatic System.}

\$246. We have seen that absorption does, or at least may, take place from the stomach. We have also stated that a large absorption, especially of water, occurs along the whole large intestine. Nevertheless, it is during the transit of food along the small intestine that the largest and most important part of the digested material passes away from the canal, partly into the lacteals, partly into the portal vessels. The portal vessels are simply parts of the general vascular system; the lacteals, into which, we may at 
once say, the greater part of the fat passes, are similarly parts of the general lymphatic system, being in fact the lymphatic vessels of the alimentary canal, and especially of the small intestine. The only reason for the special name of lacteals is that, unlike the lymphatic vessels of other parts of the body, the lymphatics of the intestine contain at times a fluid of a milky white appearance. Hence for the better understanding of absorption by the lacteals it will be desirable to study at some length the whole subject of the lymphatic system.

The lymphatic vessels may be said to begin in minute passages, possessing special characters, known as lymph-capillaries. Broadly speaking, these lymph-capillaries are found, in the mammal, in all parts of the body in which connective tissue is found; and they have special connections with those minute spaces in connective tissue which we have already more than once spoken of as lymph-spaces.

These lymph-capillaries, which, as we shall see, are frequently arranged in plexuses, are continuous with other passages also minute but of a different and more regular structure, the lymphatic vessels proper, which are gathered into larger and larger vessels, all running like the bloodvessels in a bed of connective tissue, until at last all the lymphatic vessels of the body join either the great thoracic duct which opens by a valvular orifice into the venous system at the junction of the left jugular and subclavian veins, or the small right lymphatic trunk which similarly opens into the junction of the right jugular and subclavian veins. Serous cavities may be regarded as large lymph-spaces connected with lymphatic vessels.

\section{The Nature and Movements of Lymph (including Chyle).}

$\S 247$. We are led to regard the multitudinous spaces, both small and great, of connective tissue all over the body, including among these the "serous cavities," as forming the beginning or roots of the lymphatic system. Into these spaces certain parts of the plasma of the blood transude and so become lymph (to what extent the epithelioid lining of the large serous cavities plays in regulating the transudation of serous fluid, $i$. e., of lymph, into those cavities we do not know); from these spaces the lymph is continually flowing through the lymph-capillaries into the lymphatic vessels, and so by the thoracic duct and right lymphatic trunk back into the blood system.

The amount of lymph occupying the lymph-spaces, lymph-capillaries, and minute lymphatic vessels of any region varies from time to time according to circumstances. A hand, for instance, which has been kept hanging down for some time becomes swollen and the skin tense; if it be raised the swelling lessens and the skin becomes loose; and a similar temporary swelling of the skin of the limbs, and of the skin generally, is frequently the result of active exercise. Such a swelling is partly due to the bloodvessels being dilated, or to the return flow along the veins being retarded, so that the blood capillaries become distended with blood, but is much more largely owing to the lymph-spaces and lymphatic vessels of the skin and underlying structures being unusually filled with lymph. On the other hand the skin may become shrivelled and dry from a deficiency of lymph in the lymph-spaces and vessels. Under even normal circumstances the quantity of lymph in the tissues may vary considerably, and under abnormal circumstances a very large amount of lymph may greatly distend the spaces of the connective tissue of the skin and other structures, giving rise to oedema or dropsy. Obviously there are agencies at work in the body by which the appearance of lymph in the spaces or its removal 
thence along the lymph-channels, or both, may be either increased or diminished.

\section{The Characters of Lymph.}

$\$ 248$. As it slowly flows from its origin in the tissues to the mouth of the thoracic duct (we may for simplicity's sake onit the right lymphatic trunk) the lymph is subjected to the influence of the lymphatic glands, and is possibly affected by the walls of the lymph-vessels. Moreover, the lymph coming from one tissue differs more or less in certain characters from the lymph arising in another tissue, just as the venous blood of one organ differs from the venous blond of another organ ; and these differences may be exaggerated by the activity of theone or other tissue. Of these differences by far the most striking is that between the lymph coming from the alimentary canal during active digestion and known as chyle, and the lymph coming from other parts of the body. When digestion is not going on, and when, consequently, no considerable absorption of material from the alimentary canal into the lacteals is taking place, the fluid flowing along the lacteals is lymph, not differing from the lymph of other regions to any marked degree.

The food, accordingly, which flows along the thoracic duct in an animal which has not been fed for some considerable time may be taken as illustrating the general characters of lymph. The contents of the thoracic duct may be obtained by laying bare the junction of the subclavian and jugular (in the dog the junction of the axillary and jugular) veins, and introducing a canula into the duct as it enters into the venous system at that point. The operation is not unattended with difficulties.

Lymph, so obtained, is a clear transparent or slightly opalescent fluid which left to itself soon clots. The clotting is not so pronounced as that of blood, but clotting is caused as in blood by the appearance of fibrin. The fibrin which is formed, though scanty ( 0.5 per cent.), is identical apparently with that of blood, and, as far as we know, all that has been said previously, (\$ 14-23), concerning the nature of clotting blood applies equally well to lymph.

Examined with the microscope lymph contains a number of corpuscles, lymph-corpuscles, which in all their characters as far as is at present known are identical with white blood-corpuscles; they vary in size from $5 \mu$ to $15 \mu$, and the smaller corpuscles are much more abundant in lymph than in blood. Like the white corpuscles of blood they exhibit amoboid movements. Their number varies in different animals, and, apparently, in the same animal, according to circumstances; on the whole perhaps it may be said that lymph-corpuscles are about as numerous in lymph as white corpuscles in blood. Even when every care is taken to avoid admixture with blood, lymph, and especially chyle, not unfrequently contains a certain number of red blood-corpuscles; sometimes these are sufficient to give the lymph (or chyle) a reddish tinge. They have been observed within the living lymphatic vessels, even within small ones, and have probably in some manner or other made their way from the blood into the lymph channels.

$\S 249$. The chemical composition of lymph, even when taken in each case from the thoracic duct, varies a good deal. The total solids are much less than in blood, amounting in general to not more than 5 or 6 per cent. Hence the venous blood of a vascular area contains rather more solids than the arterial blood of the same area, since the blood in giving rise to the lymph during its passage through the capillaries from the arteries to the veins has parted with relatively more water than solid matter. 
The proteids amount on the average to about 3 or 4 per cent., that is to say, to about half as much as in blood, the particular proteids present being the same as in blood, viz., albumin, paraglobulin and antecedents of fibrin. In lymph, as distinguished from chyle, the quantity of fat is small, and consists of the usual neutral fats and the soaps of their fatty acids, together with lecithin; cholesterin may also be present. A certain amount of sugar (dextrose) appears to be always present, and several observers have found an appreciable quantity of urea. The ash of lymph like that of blood-serum contains a considerable quantity of sodium chloride, while phosphates and potash are scanty ; it also contains iron, apparently in too great a quantity to be accounted for by the few red corpuscles which may be present. From lymph a certain amount of gas can be extracted, consisting chiefly or almost exclusively of carbonic acid, with a small quantity of nitrogen, the amount of oxygen present being exceedingly small. The importance of this we shall see when we come to study respiration.

Broadly speaking, we may say that all the substances present in bloodplasma are present also in lymph, but are accompanied by a larger quantity of water.

$\$ 250$. Lymph may also be obtained from separate regions of the body, as from the lower or upper limbs, for instance, by introducing a fine canula into a lymphatic vessel. In its general features the lymph so obtained resembles that taken from the thoracic duct. Analyses of the lymph distending the subcutaneous connective tissue in cases of dropsy show that this contains much less solid matter than normal lymph taken from the thoracic duct or larger lymphatic vessels. From this it has been inferred that the lymph normally existing in the lymph-spaces, lymph-capillaries and minute vessels contains an excess of water; and, indeed, it has been asserted that the percentage of solids increases in passing from the smaller to the larger vessels; but this cannot be regarded as distinctly proved. The number of corpuscles, however, as we have already said, appears to be increased in passing through the lymphatic glands. It has also been stated that the lymph in the finer lymph-vessels clots even less firmly than that in the thoracic duct. From this we may infer that some of the leucocytes in the adenoid tissue of the follicles of a lymphatic gland find their way into the lymph-sinus, and so into the efferent lymphatics, and that some of the fibrin factors are added to the lymph, or, at least, that some changes favorable to clotting are brought about.

$\$ 251$. The large serous cavities of the peritoneum, pericardium, etc., are parts of the lymphatic system ; indeed, pericardial or other serous fluid has all the general characters of lymph. We have already said $(\$ 20)$, that these fluids, when taken fresh from the body, clot (this is, at least, the case in most animals) ; the clot, when examined microscopically, is found to consist of colorless corpuscles like those of lymph or of blood entangled in the meshes of fibrin. Both in their proteid and other chemical constituents these serous fluids resemble lymph. Analyses of the accumulations of fluid occasionally occurring in these cavities show that they contain sometimes less and sometimes more solid matter than ordinary lymph. The aqueous humor of the eye contains very little solid matter; and the cerebro-spinal fluid is so peculiar that it had better be considered by itself in connection with the nervous system.

\$252. Chyle. In fasting animals the fluid flowing along the lacteals, as may be seen by inspection of the mesentery, is clear and transparent; it is lymph, differing, as we have said, in no essential respects from the lymph flowing along other lymphatic vessels. Shortly after a meal containing fat (and every meal does contain some fat), the lymph becomes white and 
opaque like milk, the more so the richer the meal is in fat; it is then called chyle. Owing to the relatively large quantity of this milky fluid which for some time after a meal continues to be poured into the thoracic duct, the contents of that duct also become milky, and are also called chyle. In the thoracic duct the chyle of the lacteals is more or less mixed with lymph from other lymphatic vessels, but the former is so preponderating that the contents of the duct may be taken as illustrating the nature of chyle.

Chyle differs from lymph in one important respect, and one only : whereas lymph ordinarily contains a small quantity only of fat, chyle contains a very large amount. The actual amount of fat present in the chyle of the thoracic duct varies, as may be expected, very considerably, according to the nature of the meal, the stage of digestion, and various circumstances. Five per cent. is a very common amount; in the dog it has been found to vary from 2 to 15 per cent. The increase in fat is chiefly if not exclusively due to an increase in the neutral fats; though whether the small quantity of soaps and of lecithin present is greater than in lymph has not been distinctly ascertained. Cholesterin is probably present in greater amount than in lymph, since it probably comes from the hile poured into the intestines during digestion; but this is not certain. How far the nature of the fat, that is, the proportion of the various kinds of fiat, of stearin, etc., varies with the fats present in the meal has not been definitely ascertained.

The condition of the fat in chyle is peculiar. Some of it exists, like the fat in milk, in the form of fat-globules of various sizes, but all small. A very considerable quantity, however, is present in the form of exceedingly minute spherules or granules, far smaller than any globules to be seen in milk ; these exhibit active "Brownian movements." "The fat present in this form is spoken of as the "molecular basis" of chyle, and is very distinctive of chyle. In the emulsified contents of the intestine, often called chyle, the fat is finely divided, and to a large extent into small globules, but there is nothing corresponding to this molecular basis; the fat does not assume this condition until it has passed out of the intestine into the lacteals. Lymph examined with the microseope shows besides the white corpuscles only very few oil-globules and nothing of this molecular basis. Just as in fact lymph is, broadly speaking, blood minus its red corpuscles, so chyle is lymph plus a very large quantity of minutely divided neutral fat.

The total amount of lymph or of chyle which enters the blood system through the thoracic duct, though it probably varies considerably, is probably also always very large. It has been calculated that in a well-fed animal a quantity equal at least to that of the whole blood may pass through the thoracic duct in twenty-four hours, and of this it is supposed that about half comes through the lacteals from the alimentary canal, and therefore to a large extent from food, and the remainder from the body at large. These calculations are based on uncertain data, and cannot, therefore, be taken as of exact value, but we may use them for the sake of an illustration. Thus, in a man of average weight, that is, about 154 kilos., the quantity of blood ( $\$ 38$ ) being $\frac{1}{13}$ of the body weight is about 12 kilos. The quantity of lymph or chyle, therefore, discharged into the blood in an hour would be according to this calculation half a kilo., or something less than half a litre; and since the flow must vary considerably in the twenty-four hours would be sometimes less, and therefore sometimes even more, than this.

\section{The Movements of Lymph.}

$\S 253$. Making every allowance for the uncertainty of the calculations detailed in the preceding paragraph, it is obvious that the lymph must flow 
with a not inconsiderable rapidity (if we take about half the above estimate, the rate will be about 5 c.c. per minute) through the thoracic duct, and therefore must also be continually streaming into that duct along the various lymphatic channels from the manifold lymph-spaces of the body. This onward progress of the lymph is determined by a variety of circumstances. In the first place, the remarkably widespread presence of valves in the lymphatic vessels causes every pressure exerted on the tissues in which they lie to assist in the propulsion forward of the lymph. Hence all muscular movements increase the flow. If a canula be inserted in one of the larger lymphatic trunks of the limb of a dog, the discharge of lymph from the canula will be more distinctly increased by movements, even passive movements, of the limb than by anything else. When we come to speak of the entrance of chyle into the lacteal radicles of the villi, we shall see that, at all events according to one view, the muscular fibres of the villus act as a kind of muscular pump, driving the chyle past the valved end of the lacteal radicle into the lymphatic canals below. In addition to the presence of valves along the course of the vessels, the opening of the thoracic duct into the venous system is guarded by a valve, so that every escape of lymph or chyle from the duct into the veins becomes itself a help to the flow. In the second place, we have already seen that the blood-pressure in the capillaries and minute vessels is considerably greater than that in the large veins, such as the jugular; in fact, this difference of pressure is the cause of the flow of blood from the capillaries to the heart. Now the lymph in the lymphatic spaces outside the capillaries and minute vessels undoubtedly stands at a lower pressure than the blood inside the capillaries; otherwise the transudation from the blood into the tissues would be checked; but the difference is probably much less than the difference between the pressure in the capillaries and that in the large venous trunks. So that the lymph in the lymph-spaces of the tissues may be considered as standing at a higher pressure than the blood in the venous trunks, for instance, in the jugular vein. That is to say, the lymphatic vessels as a whole form a system of channels leading from a region of higher pressure, viz., the lymph-spaces of the tissues, to a region of lower pressure, viz., the interior of the jugular and subclavian veins. This difference of pressure will, as in the case of the bloodvessels, cause the lymph to flow onward in a continuous stream. Further, this flow, caused by the lowness of the mean venous pressure at the subclavian vein, will be assisted at every respiratory movement, since at every inspiration the pressure in the venous trunks becomes, as we shall see in dealing with respiration, negative, and thus lymph will be sucked in from the thoracic duct, while the increase of pressure in the great veins during expiration is warded off from the duct by the valve at its opening. In the third place, the flow may be increased by rhythmical contractions of the walls of the lymphatics themselves, which, as we have seen, are remarkably muscular; and the peculiar interlacing of the muscular fibres above each valve suggests that the walls here act after the fashion of a tiny heart and by a rhythmical systole drive on the fluid, which by the action of the valve below collects at the spot. IVe have, however, no experimental proof of this; for, though rhythmic variations have been observed in the lacteals of the mesentery, it is maintained that these are simply passive, $i$. e., caused by the rhythmic peristaltic action of the intestine, each contraction of the intestine filling the lymph-channels more fully, and are not due to contractions of the walls of the lacteal vessels themselves. In some of the lower animals, for instance in the frog, the muscular walls of the vessels are developed at places into distinctly contractile propulsive organs, spoken of as lymph-hearts. Lastly, it is at least open for us, on the strength of the analogy that osmosis may give rise to increased 
pressure on one side of a diffusion septum, to suppose that the very processes which give rise to the appearance of lymph in the lymph-spaces of the tissues, tend themselves to promote the flow of lymph. We have at least, under all circumstances, one or other of these causes at work, promoting a continual flow from the lymphatic roots to the great veins. They are together sufficient to drive, in man, the lymph from the lower limbs and trunk, against the effects of gravity, into the veins of the neck. In the upper limb the influences of gravity, owing to the varied movements of the limb, are as often favorable to as opposed to the natural flow of the lymph; but, as we have already said, a long-continued unfavorable action of gravity, especially in the absence of the aid of movements in the skeletal muscles, as when the arm hangs down motionless for some time, leads to accumulation of lymph at its origin in the lymph-spaces. The strength of the causes combining to drive on the lymph is strikingly shown in animals when the thoracic duct is ligatured; in such cases a very great distention of the lymphatic vessels below the ligature is observed.

$\S 254$. Although the phenomena of disease and, perhaps, general considerations render it probable that the nervous system governs in some way the stream of lymph, regulating, it may be, not only the flow along the definite lymph-canals, but also the transit of plasma into the lymph-spaces and the escape of lymph thence into the definite canals, our knowledge on these points is very imperfect. We have no proof that the muscular fibres in the walls of the lymphatic vessels are governed by nerves, or that the lymph-spaces are influenced directly by nervous action; and most of the attempts to demonstrate any direct action of the nervous system on the lymphatics have hitherto failed.

$\$ 255$. The passage of material, namely, of water containing certain substances in solution, fiom the interiur of the blondvessel, where they form part of the plasma, into the lymph-capillary where they are called lymph, consists of two steps: the passage from the bloodvessel into the lymph-space, and the passage from the lymph-space into the lymph-capillary ; for, as we have seen, it is only in particular places that the lymph-capillary immediately surrounds the bloodvessel. Once arrived in the lymph-capillary the lymph finds an open path along the rest of the lymphatic system, but the connection between the lymph-space and the lynuph-capillary is, as we have seen, peculiar and at least not a free and open one.

The passage of material from the bloodvessel into the lymph-space we speak of as transudation. What can we say as to the nature of this process? There are two known physical processes with which we may compare it: diffusion through a membranous or other porous partition, and filtration through a similar partition. Diffusion, though influenced by fluid pressure, is not the direct result of fluid pressure, but may, on the contrary, be the cause of differences of pressure on the two sides of the partition, and may work against fluid pressure. When a strong solution and a weak solution of salt are separated by a diffusion septum, diffusion takes place whether the columns of fluid be at the same level on the two sides of the septum or at different levels; and if the columns be at the same level to start with, that of the stronger solution soon comes to exceed the other in height, on account of the osmotic flow of water from the weaker into the stronger solution. Filtration, on the other hand, is the direct result of pressure; without difference of pressure filtration does not take place; and, the filter remaining of the same nature and in the same condition, the amount of filtrate is dependent on the amount of pressure. May we speak of the process of transudation as a simple process of diffusion or a simple process of filtration? that is to say, can all the phenomena of transudation be explained as simply 
the results of one or other of these physical processes? Diffusion by itself will not account for the results; for the proteids of the blood-plasma are indiffusible or very nearly so, and yet the lymph contains a considerable quantity of these proteids. We have no satisfactory knowledge of the exact composition of lymph as it exists in the lymph-spaces. In the lymph of the larger lymph-trunks the diffusible saline substances are present in about the same proportion, and the indiffusible proteids to about or less than half as much as in blood-serum; and we may perhaps assume that the lymph in the lymph-spaces contains relatively less proteids, but has otherwise the same composition as blood-plasma. Mere diffusion would not give rise to a fluid of such a nature. Can we speak of transudation, then, as a filtration? The blood is undoubtedly flowing through the capillaries and other small vessels under a certain pressure; we have seen $(\$ 105)$ that the pressure is roughly speaking about $30 \mathrm{~mm}$. $\mathrm{Hg}$.; and it would be possible to select such a filter or porous partition as would at about this pressure permit the passage of a certain quantity of the inorganic and crystalline constituents of blood-plasma to pass through in company with a relatively smaller quantity of the proteids and a large quantity of the water, the red and white corpuscles being excluded. Such a filtrate would be more or less of the nature of lymph; and so far we might be justified in speaking of the transudation of lymph as a process of filtration. But the transit through the living wall of the bloodvessel is affected by circumstances in a manner so different from the manner in which the same circumstances affect a transit through an ordinary lifeless filter, that we gain but little and may be led into error by speaking of the process as a filtration. Substances in solution or otherwise, pass through a filter when the pressure is sufficient to drive them through the passages furnished by the interstices existing in the substance of the filter. In the case of an ordinary filter the substance of the filter is within limits permanent, and the passages correspondingly constant. The living wall of a capillary, however, is not a constant unchanging thing. The epithelioid plates and other elements which constitute it are alive, and being alive are continually undergoing change and are especially subject to change ; moreover, as we have seen ( $\$ 22,23)$, the vascular walls appear to be continually acting upon and being acted upon by the blood. Hence a change in the blood tends to cause changes in them; and these changes may materially affect in one direction or another their action as filters. In an ordinary filter increase of pressure necessarily entails increase of filtration; in a living filter it may or may not, and the same increase of pressure may according to circumstances produce very different results as regards the transudation of lymph.

Thus it seems reasonable to suppose, as we have suggested ( $\$ 197)$, that, other things being the same, an increase of blood-pressure should necessarily increase the transudation of lymph. Hence when a small artery dilates, since the pressure in the still smaller branches and capillaries of that artery is, as we have more than once pointed out, increased, more lymph appears in the lymph-spaces; indeed it is one of the main purposes of the widening of small arteries to supply the elements of the tissue with more lymph, that is, with more food. But it does not therefore follow that under all circumstances widening of the artery should increase the passage of lymph; something may occur to counteract the natural effect of the increased pressure in the bloodvessels. An instance of this seems to be afforded by the case of the submaxillary gland, when the chorda nerve is stimulated while the gland is under the influence of atropine. As we have seen, though the arteries dilate, no secretion takes place; and we cannot explain the ahsence of a flow into the alveoli by supposing that the extra amount of lymph which would 
in normal circumstances form part of the secretion, and in the case of a fairly copious secretion would be considerable, now passes away by the lymphatics without reaching the cells of the alveoli, for in such cases no extra flow in the lymphatics leading from the gland has been observed, and there is no accumulation of lymph in the connective tissue of the gland. Apparently, for some reason or other, in spite of the increased pressure in the bloodvessels, more lymph than usual does not pass into the lymph-spaces.

Then again, as we shall presently have occasion to point out, an increase of pressure in the bloodvessels produced by obstruction to the venous outflow is much more efficient in promoting an increase of transudation, at all events an abnormal increase, than is an increase of arterial pressure; and the difference between the two cases appears to be too great to be accounted for on the ground that an obstruction to the venous outflow raises the pressure within the capillaries and small vessels more readily and to a higher degree than does the widening of the arteries. Moreover, that obstruction to venous outflow does not produce its effects in the way of transudation simply and merely by raising the capillary pressure is shown by the fact that the same amount of obstruction may or may not give rise to excessive transudation, according to the condition of the blood or other circumstances. For instance, though the obstruction produced by ligaturing a vein frequently causes excessive transudation, it does not always cause it, and the femoral vein of a dog may be ligatured without any excessive transudation taking place; yet if, after the ligature, certain changes be induced in the blood, excessive transudation occurs in the leg the vein of which has been ligatured, but not elsewhere. Pointing toward the same conclusion is the fact that excessive transudation more readily occurs when a vein is plugged by a thrombus arising from abnormal conditions of the vascular system than when a vein is simply ligatured. And in general we may say, and this is a point to which we shall return, that two things chiefly determine the amount of transudation: the pressure of the blood in the bloodvessels, and the condition of the vascular walls in relation to the blood, the latter being at least as important as the former.

Another aspect of the matter, moreover, deserves attention. In filtration the movement takes place through the filter in one direction only, whereas in the living body, the passage of material through the capillary wall takes place in two opposite directions. In all the tissues, though more perhaps in certain tissues than in others, the passage from the bloodvessel into the lymph-space is accompanied by a passage from the lymph-space into the blood; while food for the tissue passes in one direction, waste products pass in the other. In a secreting gland the greater part of the lymph coming from the bloodvessels, the water and other matters, pass away into the lumen of the alveolus after undergoing changes in the cell; but even in such a case there is some return from the cells into the bloodvessels, carbonic acid, for instance, if nothing else, is given up by the cells to the blood; and in such organs as a muscle or the liver, the backward stream of material from the tissue to the blood is extensive and important. Moreover, this backward stream works against pressure; indeed, as may be seen in a muscle, it is when the bloodvessels are dilated and the pressure in the capillaries and small vessels highest, as during and after the contraction of the muscle, that the passage from the tissue into the blood is most energetic. Many of the waste products of the tissue are, it is true, diffusible, and we might be tempted to say that while the lymph which feeds the tissue traverses the vascular wall by filtration in the direction of pressure, the waste products return to the blood against pressure by diffusion; but such a view cannot at present be regarded as proved; and if it be true, as is maintained by some, that 
lymph, including the proteids, may at times be reabsorbed from the tissue in to the bloodvessels, it is distinctly contradicted. Finally, the introduction of certain substances (termed lymphagogues) into the blond, is followed by an increase in the quantity of lymph without any related change in the bloodpressure. We shall have to return to this question when we come to deal with the secretion of urine; but meanwhile we may adopt the conclusion, which is especially supported by the phenomena of disease, that while diffusion and filtration play their respective parts, diffusible substances passing in and out of the blood more readily than indiffusible substances and an increase of pressure tending to promote transudation, the condition of the vascular wall so profoundly influences the transit of material as to render the process very complex. We may probably regard it as too complex to be compared even with filtration through a filter capable of widely changing in texture from time to time, and as more nearly resembling the process of secretion.

Concerning the passage of the lymph from the confined lymph-spaces into the open gangways of the lymph-capillaries we know very little. If, as some think, the cavity of the lymph-capillary is shut off on all sides and completely by a continuous lining of sinuous epithelioid plates, then the passage from the lymph-space into it must be regarded as a sort of repetition of the passage from the blood-capillary into the lymph-space, as a second transudation. But if, as others think, and as on the whole seems more probable, the lymph-spaces open at places directly into the lymph-capillaries, the passage is a simply mechanical affair determined by the freedom of these openings.

In either case the flow from the lymph-spaces will be facilitated by all events which promote, and checked by those which hinder the flow of lymph along the lymph-capillaries and the other lymphatic channels.

We may here remark as influencing the quantity of lymph in the lymphspaces and vessels that the quantity of lymph taken up from the lymphspaces by the actual elements of the tissue may vary considerably. We remarked in $\$ 30$ on the peculiar relations of living tissue to water, and there are reasons for thinking that the very substance of a cell or a fibre (a muscular fibre, for instance) may hold in itself a larger quantity of water at one time than at another. The water thus taken up or given out, and the substances which may be carried in solution by that water, come from and go to the lymph. The condition of the tissue determines by itself the amount of lymph in the lymph-spaces.

$\S 256$. Under the influence of all these several actions the lymph in the various lymph-spaces of the body varies in amount from time to time, but under normal circunistances never exceeds certain limits. Under pathological conditions those limits may be exceeded, and the result is known as oedema or dropsy. Similar excessive accumulations of lymph may occur not in the ordinary lymph-spaces, but in those larger lymph-spaces, the serous cavities, any large excess of fluid in the peritoneal cavity being known as ascites.

The possible causes of œedema are, on the one hand, an obstruction to the flow of lymph from the lymph-spaces, and on the other hand an excessive transudation, the lymph gathering in the lymph-spaces faster than it can be carried away by a normal flow ; with the former the lymphatic system itself, with the latter chiefly the vascular system, is concerned. As a matter of fact, however, odema is almost always, if not always, due to abnormal conditions of the vascular system, and is the result not of hindered outflow, but of excessive transudation.

Owing to the numerous anastomoses of the lymph-vessels and the conse- 
quent establishment of collateral streams, obstruction in the lymph-passages themselves rarely if ever gives rise to odema ; and it may be here remarked that owing to the same free collateral communication between the lymphvessels the labyrinthine passages of the lymphatic glands do not offer the serious obstacle to the onward flow of the general lymph-stream, as might at first sight be supposed. Nor have we at present any knowledge which would lead us to suppose that any physiological changes in the walls of the lymphatic vessels or of the lymph-capillaries, or in the lymph-spaces, by giving rise in some way to obstacles to the flow of lymph, ever lead to an accumulation of lymph in the latter.

One kind of œedema we have already touched upon in speaking of the capillary circulation ( $\$ 169)$, viz., the "inflammatory" œdema. In this kind of nedema, owing to changes in the vascular walls, a larger amount of transudation passes into the lymph-spaces, and that transudation is richer in proteid matters, and contains a larger amount of the fibrin factors, or at all events, is much more distinctly coagulable than ordinary lymph, as well as crowded with migrating corpuscles. Allied to this inflammatory œedema is the increase of Iymph, also apparently changed somewhat in character, which appears as "effusion" in the serous cavities when these are inflamed, as in pleurisy and peritonitis.

One of the most common forms of œdema is an œdema of primarily, though not wholly, mechanical origin-odema arising from obstruction to the venous flow; under these circumstances more lymph passes into the lymph-spaces than the lymph-vessels are able to carry away. If the femoral vein be tied the leg may become odematous, and, as we have said, odema is a common result of the plugging or obstruction of veins through disease; the odema which is so common an accompaniment of heart disease, involving obstruction to the return of venous blood to the right side of the heart, and the ascites which follows upon hindrance to the portal flow, are instances of œedema of this kind. We have already remarked on the relation of transudation to blood-pressure, and in venous obstruction the rise of pressure within the small bloodvessels is distinguished from that due to arterial dilatation by being accompanied with a want of adequate renewal of the blood; this probably affects the epithelioid lining of the bloodvessels in such a way as to increase the transudation. And, indeed, as is seen in case of heart disease with prolonged or repeated venous obstruction, the œdema, as time goes on and the tissues become impaired, is more easily excited and with greater difficulty removed, though the actual amount of obstruction, the actual increase of pressure in the small vessels, remains the same, or at least is not proportionately increased.

Still another kind of æedema is one due to changes taking place in the blood, quite apart from variations of blood-pressure. This kind of oedema is seen in some diseases of the kidney, in "Bright's disease," for instance. In such cases the blood contains less proteids, and indeed less solids, is more watery and of lower specific gravity than is normal. But the ordema is not in these cases to be explained on the view that the more watery blood passes more readily through the capillary walls, for it may be shown experimentally that the mere thinning of the blood, as by the injection of normal saline solution into the bloodvessels, will not at once lead to odema, at least in the limbs and trunk, and it is these which in Bright's disease especially become œdematous. In all probability the œedema of Bright's disease, if it be really due to the abnormal character of the blood, is produced by the abnormal blood so acting on the bloodvessels that these allow a transudation greater than the normal. Finally, œdema may be due to abnormal conditions of the tissues themselves. 
But these are pathological questions into which we must not enter here. We have touched upon them because they illustrate the important processes taking place in the lymph-spaces, and, as we have more than once insisted, the lymph in the lymph-spaces is the middleman of all the tissues, and hence facts illustrating the laws which govern the flow of lymph into and out of the lymph-spaces are of fundamental physiological importance.

$\$ 257$. Lymph-hearts. In the frog and other amphibia and in reptiles the flow of lymph into the venous system is assisted by rhythmically pulsating muscular lymph-hearts, which present many curious analogies with the blood-heart. 'The frog possesses four lymph-hearts. Of these, two belonging to the hind limbs are placed one on each side of the coccyx near its end, and, being covered only by aponeurosis and the skin, may without dissection be seen beating. Two anterior ones are placed on the transverse processes of the third vertebra, and are covered from view by the shoulder girdle. Each lymph-heart is a more or less oval sac lying in one of those lymph sacs or cavities lined with sinuous epithelioid plates, which, as we have said, are present in the frog. It is continued at one end, by an orifice guarded with valves, into a small vein which opens, in the case of the posterior heart, into a crural vein, and in the case of the anterior hearts into a jugular vein. The wall consists of muscular fibres arranged in a plexiform manner and supported by a considerable amount of connective tissue. These fibres are striated and branched and are intermediate in character between cardiac and skeletal muscular fibres. Nerve-fibres terminate in these muscular fibres, and the muscular wall, unlike that of the blood-heart, is supplied with capillary bloodvessels. The interior is lined with epithelioid plates of sinuous outline, and this lymphatic lining is continued along a number of openings or pores, by which the cavity of the heart opens into the surrounding lymph-space. When the heart contracts the contents are driven into the vein, the lymphatic pores being closed by the approximation of the contracting muscular fibres; when the heart dilates the fluid in the vein is prevented from returning by the valves at its mouth, while the lymph enters readily from the surrounding space through the now open pores. In the frog regular lymphatic vessels are scanty; hence these lymph-hearts become of considerable importance in promoting the flow of lymph. The lymph-hearts of reptilia are similar in structure and function. In the frog, in which they have been chiefly studied, the action of the lymph-hearts is in a measure dependent on the spinal cord. The posterior lymph-hearts belonging to the hind limbs are connected by means of the delicate tenth pair of spinal nerves with a region of the cord opposite the sixth or seventh vertebra in such a way that section of the nerve or destruction of the particular region of the cord suspends or destroys their activity. The anterior pair are similarly connected with a region of the spinal cord opposite the third vertebra. Each pair therefore seems to have a "centre" in the spinal cord; but it is probable, though observers are not wholly agreed, that the hearts, after destruction of their spinal centre, ultimately resume their rhythmic beats, so that the dependence of their activity on the spinal centre is not an absolute one. Like the heart of the blood-system, the lymph-hearts may be inhibited, and that in a reflex manner, the inhibition centre being moreover in the medulla oblongata. If a frog be carefully observed, the activity of the lymph-hearts will be found to vary largely, and these variations appear to be in part due to nervous influences, so that in this way the movement of lymph, and hence the processes of absorption, are in this animal directly dependent on the nervous system. 


\section{Absorption from the Alimentary Canal.}

$\$ 258$. We may now return to consider the absorption of the products of digestion, that is to say, the passage of these bodies from the interior of the alimentary canal, where they are really outside the body proper, into the body itself. For simplicity's sake we may consider digestion in a broad way as the conversion of practically non-diffusible proteids and starch into more diffusible peptone and highly diffusible sugar, and as the emulsifying, or division into minute particles of fats. We have reason to believe that some of the sugar may be changed into lactic acid, or even into butyric or other acids, that some of the proteids are carried beyond the peptone condition into leucin and other bodies, and that some of the fat may be saponified; and it may be that some of the proteid material of the food passes into the body as albumose or even as parapeptone, or in some other little changed condition. But we may probably with safety, for present purposes, assume that the greater part of the proteid is absorbed as peptone, that carbohydrates are mainly absorbed as sugar, and that the greater part of the fat passes into the body as emulsified but otherwise unchanged neutral fat; and we may neglect the other conditions of digested food as subsidiary, and as far as absorption is concerned, unimportant.

We have seen that two paths are open for these products of digestion, one by the capillaries of the portal system, the other by the lacteals. It cannot be a matter of indifference which course is taken. For if the products pass by the lacteals they fall into the general blood-current after having undergone only such changes as they may experience in the lymphatic system ; while if they pass into the portal vein they are subjected to certain powerful influences of the liver (which we shall study in a future chapter) before they find their way to the right side of the heart, and if the substances be prevented from reaching the liver, as when in the dog the portal vein is connected with the inferior vena cava and they are thus at once transmitted to the general circulation, serious results ensue. We may, therefore, consider first which of the two paths is, as a matter of fact, taken by the several products, and subsequently study the mechanism of absorption in the two cases.

\section{The Course taken by the Several Products of Digestion.}

$\S 259$. From what has already been said we have been led to regard the villi as the most active organs of absorption, and the structure of a villus leads us further to conclude that the diffusible peptones and sugar pass, together with the water in which they are dissolved, into the superficially placed capillary network of the villus and so into the portal system, while the merely emulsified fat, unable to traverse the wall of the capillary, passes on to the deep-seated lacteal radicle, and so finds its way into the lymphatic system. And the results of observation and experiment, as far as they go, support this view.

Fats. After a meal containing fat the lymph of the lacteals contains fat, and is now called chyle; and the richer the meal in fat the more conspicuous is the fat in the lymph-vessels. We cannot, however, prove that all the fat of a meal absorbed from the alimentary canal is poured by the thoracic duct into the venous system. If a meal containing a known quantity of fat be given to a dog and the small quantity of fat present in the feces corresponding to the meal be subtracted from that amount, we can determine the amount of fat absorbed, for we have no evidence whatever that any appreciable amount of fat undergoes a destructive decomposition in the alimentary canal. Collecting by means of a canula inserted into the thoracic duct the whole of 
the chyle during and after the meal, so long as it remains milky, showing that fat is being absorbed, we can ascertain the quantity of absorbed fat, which would, but for the operation, have passed into the venous system. When this has been done, a very remarkable deficit, amounting it may be to 40 or 50 per cent., has been observed ; that is to say, of every 100 parts of fat which disappear from the alimentary canal only about 60 parts find their way through the thoracic duct into the venous system.

Are we then to conclude that the missing quantity finds its way into the portal system? Now the portal blood does, during digestion, contain a certain quantity of fat; indeed, the serum is said at times to appear milky from the presence of fat. But the whole circulating blood during the digestion of a fatty meal contains, for a while, the fat poured into it by the thoracic duct; and it has been ascertained in the dog that the blood of the portal vein during digestion contains not more but less fat than the blood of the carotid artery, so that the fat which appears in the portal blood during digestion is, for the most part at least, not fat absorbed by the capillaries of the alimentary canal, but fat absorbed by the lacteals. Moreover, when the chyle of the thoracic duct is diverted through a canula, and not allowed to flow into the blood, the quantity of fat in the portal blood, as in the blood at large, is very small indeed. Lastly, when a villus of an intestine in full digestion of fat is treated with osmic acid, fat cannot be recognized by the microscope within the capillaries or other bloodvessels, though it abounds outside them in the substance of the villus and in the lacteal radicle.

We may probably, therefore, infer with safety that all or at least very nearly all the fat absorbed from the intestine takes the path of the lacteals. As to the deficit mentioned above, that is as yet without explanation. It may be that in some way, on its course, in the lymphatic glands, for instance, the fat is taken away from the chyle, hidden so to speak somewhere away from both chyle and blood; but on this point we have no exact information.

$\$ 260$. Water and salts. If, in an animal, the rate of flow of lymph or chyle through a canula placed in the thoracic duct be watched, and water or, to avoid the injurious effect of simple water on the mucous membrane, normal saline solution, be then injected in not too great quantity into the intestine, no marked increase in the flow of chyle through the canula is observed. From this we may infer that the water of the intestinal contents is absorbed not into the lacteals but into the portal system. If, however, a very large quantity of the normal saline solution be injected so as to distend the intestine, then the flow of chyle is increased to some extent. It would appear, therefore, that while under normal conditions the water passes from the intestine mainly into the portal blood, some of it may under some circumstances pass into the lacteals.

With regard to the course taken by ordinary saline matters we possess no detailed information. When special salts, such as potassium iodide and others, easily recognized by appropriate tests, are introduced into the intestine, they may be speedily detected both in the blood and in the contents of the thoracic duct; but whether, in such cases, these salts find their way into the thoracic duct by the lacteal radicle of the villi, or pass into the lymph stream at some later part of its course, we do not know. Nor can we with regard to such a salt as sodium chloride, state absolutely that it passes mainly with the water into the portal blood, though we may fairly suppose this to be the case.

$\S 261$. Sugar. Both blood and chyle contain, normally, a certain small amount of sugar; and careful inquiries show that the percentage of sugar in chyle and in general blood is fairly constant, neither being to any marked extent increased by even amylaceous meals; on the other hand, a ineal con- 
taining sugar or starch does temporarily increase the quantity of sugar in the portal blood. From this we may infer that such portions of the sugar of the intestinal contents as are absorbed as sugar pass exclusively by the portal vein. We may, however, here call attention to the difficulties attending an argument of this kind. In the first place the quantitative determination of a small amount of sugar in so complex a fluid as blood is attended with great difficulties and uncertainties. In the second place a very large quantity of blood is at any one moment streaming through the capillaries of the alimentary canal; and we may perhaps speak of the quantity which passes through them during the whole period of digestion as being enormous. Hence though each 100 c.c. in passing through the capillaries might take up a quantity of sugar so small as to fall almost within the limit of errors of observation, yet the whole quantity absorbed during the hours of digestion might be considerable; or to put it in another way, an error of observation, unavoidable with our present means of analysis, on a sample of blood taken from the portal vessels might lead to a wholly unwarranted conclusion that sugar was or was not being absorbed. Making every allowance, however, for these difficulties, the increase of sugar which has been observed in the portal blood during digestion seems too great to permit of any other conclusion than that sugar is really absorbed from the alimentary canal by the bloodvessels.

When, however, a large quantity of sugar dissolved in a large quantity of water is present in the intestine, the sugar in the chyle is said to be increased. In such a case the excess of water, as stated above, passes into the lacteals, and in so doing appears to carry some of the sugar with it.

In this connection it should be remembered that the sugar resulting from digestion is for the most part maltose, while that in the portal blood is dextrose, the former being changed into the latter probably while passing through the intestinal epithelium.

$\$ 262$. Proteids. The difficulties attending the experimental determination of the path taken by proteids are greater even than in the case of sugar, for the exact quantitative estimation of peptone in blood (and we are assuming that proteids are mainly absorbed as peptone) is a task of the greatest difficulty-one compared with which that of estimating sugar appears almost easy. Bearing this in mind we may state that all observers are agreed that peptone is absent from chyle or at least that its presence cannot be satisfactorily proved. On the other hand, while some observers have succeeded in finding peptone in the portal blood after food, but not during fasting, many have failed to demonstrate the presence of peptone in the blood either of the portal vein or of the vessels at large even after a meal containing large quantities of proteids. Of course, as we argued in speaking of the absorption of sugar, the quantity of peptone passing into the portal blood at any moment might be small, and yet a considerable quantity might so pass during the hours of digestion. We may suppose, moreover, that that which does pass is immediately converted, possibly by some ferment action, into one or other of the natural proteids of the blood, or otherwise disposed of; and, indeed, peptone injected carefully and slowly into a vein disappears from the blood, though little or even none passes out by the kidney. And the view that peptone is so changed, possibly in the very act of absorption, is supported not only by the statement that peptone may be found in the practically bloodless wall, that is, mucous membrane, of the intestine removed from a dead animal even when it appears to be absent from the blood, but also and especially by the following observation. If an artificial circulation of blood be kept up in the mesenteric arteries supplying a loop of intestine removed from the body, the loop may be kept alive for some considerable time. During this survival a considerable quantity of peptone placed in the cavity 
of the loop will disappear, $i . e$, will be absorbed, but cannot be recovered from the blood which is being used for the artificial circulation, and which escapes from the veins after traversing the intestinal capillaries. The disappearance is not due to any action of the blood itself, for peptone introduced into the blood before it is driven through the mesenteric arteries in the experinent may be recovered from the blood as it escapes from the mesenteric veins. It would seem as if the peptone were changed before it actually gets from the interior of the intestine into the interior of the capillaries.

But the argument that the absence of peptone from the blood is no proof that the peptone is not absorbed into the blood may also be applied to the chyle, and thus leaves us unable to draw a conclusion as to the path of the proteids. The following indirect proof that peptone does not pass into the chyle has been offered, but it too is open to objection. We shall see hereafter that the absorption of proteid material leads to an increase in the elimination of urea by the kidneys. So marked is this increase, that unless there be clearly some other causes at work leading to an increase of urea, such as fever for instance, an increase of urea in the urine following upon the administration of proteid food may be taken as a proof that the proteid food has been digested and absorbed. Now if in a dog the thoracic duct be successfully ligatured so that the chyle cannot pass as usual into the blood, and the dog be fed on proteid food, as free as possible from fat, so as not unnecessarily to load the obstructed lacteals, an increase in the urea of the urine is observed as usual. Obviously in such a case the proteid food is absorbed, and obviously also does not pass into the blood through the thoracic duct (the success of the ligature having been proved by post-mortem examination). But the experiment, though as far as it goes supporting, does not rigorously prove, the view that the proteids are absorbed by the capillaries of the alimentary canal; for the thoracic duct and lymphatics below the ligature were found largely distended, and lymph and chyle appear to have escaped from the vessels; hence it is possible that some at least of the proteids were absorbed by the lacteals of the intestine, but finding their usual path blocked made their way into the blood stream.

We may, therefore, say that the results of experiment, while they do not definitely prove, give some support to, and at least do not contradict, the view which we a little while ago put forward as probable, namely, that proteids, transformed into diffusible peptones, pass into the bloodvessels and not into the lacteals.

But, if this view be provisionally accepted, it must be on the understanding that it is probable only; and it may be that proteids do not take the same paths and are not absorbed in the same condition in all animals. The experiments just related were performed on dogs, that is to say, on carnivorous animals whose (natural) food contains a considerable quantity of fat, and whose lacteals might, therefore, be considered as preoccupied in the absorption of fat. The food of herbivora on the other hand contains a relatively small amount of fat; and if in these animals all the proteids and carbohydrates are absorbed by the blondvessels, there is comparatively little left for the lacteals to do. Yet in these animals the lacteals and lymphatics are well developed. In the villus of a herbivorous guinea-pig or rabbit, though the reticular tissue is very scanty as compared with that present in the villus of a dog, the lacteal chamber is, relatively to the diameter of the villus, not merely as large as, but much larger than, in the dog. It is difficult to suppose that this wide chamber is intended solely for the absorption of the relatively small amount of fat present in vegetable food. The question which we are discussing is clearly at present to be regarded as by no means settled. 


\section{The Mechanism of Absorption.}

$\$ 263$. The absorption of fats. We have now to consider the manner in which these several substances pass into either the lacteal radicle or the capillary bloodvessels. It will be convenient to begin with the absorption of the fats.

We have seen reason $(\$ 241)$, to think that the fats, remaining chiefly as neutral fats, are emulsified in the intestine by means of the bile and pancreatic juice, the small quantity of soap which is formed probably serving simply the purpose of facilitating the emulsification.

The neutral fats so emulsified pass in the first instance into the bodies of the columnar cells of the villi. It has, it is true, been maintained by some that they pass between the cells and not into them; but the evidence is distinctly against this view. Since no such collections of fat globules are seen in the cubical cells of the glands of Lieberkuhn we infer that these have nothing to do with the absorption of fat.

How the fat enters into the substance of the cell we do not know. We may presume that the striated border plays some part, but what part we do not know. Though, as we have seen, the rods making up the border appear able to move, to change their form, we have no evidence that the fat is introduced into the cells by means of any movements of these rods. We may imagine that the globules pass into the cell-substance by help in some way of these rods through amœboid movements comparable with the ingestive movements of the body of an amoba; but we have no positive evidence to support this view. IVe said that bile promotes the passage of fat through membranes, possibly by in some way promoting a closer contact between the particles of fat and the substance of the membrane; but even if bile has this effect on the surface of the cells, its action in this respect can be subsidiary only.

When fatty acids are ingested neutral fats appear in the chyle, indicating a synthesis of fatty acids into neutral fats in the epithelium of the villi.

Within the columnar cell the fat may be seen, both in osmic acid preparations and in fresh living cells, to be disposed in globules of various sizes, some large and some small, each globule placed in a space of the protoplasmic cell-substance. It does not follow that the fat actually entered the cell exactly in the form of these globules; it may be that the fat passes the striated border in very minute spherules which, reaching the body of the cell, run together into larger globules; but whether this is so or not we do not know.

From the columnar cell the fat passes into the spaces of the reticular tissue of the villus. It has, it is true, been contended that it passes along the substance of the bars of the reticulum; but in carefully prepared osmic acid specimens of a villus in active digestion of fatty food, the fat may be distinctly recognized as largely filling up, still in the form of globules of various sizes, the spaces in the meshes of the reticulum which are not occupied by the leucocytes or allied wandering cells. The bases of the columnar cells, through the gaps in the basement membrane, directly abut upon the labyrinth of spaces; and the fat once out of the base of the cell is free in the spaces of the labyrinth. How it issues from the cell we do not exactly know; possibly by a process analogous to the excretion of solid matters by an amœba.

From the labyrinth of spaces of the reticulum of the villus the fat passes into the cavity of the lacteal radicle; and it is worthy of note that in the passage it undergoes a change. In the interior of the intestine, in the substance of the columnar cell, and apparently in the labyrinth of the reticu- 
lum, it is simply emulsified fat consisting of globules small and large; within the lacteal radicle it consists partly of the same easily recognized globules but partly of the extremely divided " molecular basis" ( $\$ 252)$; it is now no longer emulsified fat but chyle. How and by what meaus this extremely minute division of the globular fat into the " nolecular basis" takes place we do not know ; nor do we know the exact manner in which the fat passes from the spaces of the reticulum into the interior of the radicle.

IVe may here, perhaps, remark that the contents of the lacteal radicle consist not exclusively of fat, but of fat accompanied by the proteid and other substances which go to make up the chyle. Proteid and other substances besides fat are also present in the lymph which occupies in part the labyrinth of the body of the villus, and are derived, like the lymph elsewhere, from the blood of adjacent capillaries; at least, they are in part so derived, though it may be not wholly, for, as we have just seen, the passage of proteid material from the intestine into the substance of the villus past the capillaries, though not proved, must still be considered as possible.

The spaces of the reticulum of the villus are more or less occupied by wandering cells of which we spoke under the general term of leucocytes. These do not all present the same appearances and most probably are not all of the same kind.

Some of these leucocytes wander not only through the labyrinth of the reticulum but pass into the epithelium between the cells, and may project processes into or even make their way eventually into the interior of the intestine; or following the reverse course may wander from between the epithelial cells into the body of the villus; some of them, moreover, undoubtedly contain fat. Hence the view has been suggested that these leucocytes are important agents, indeed the chief agents in the absorption of fat. It has been supposed that they, receiving the globules of fat into their cellsubstance, in fact eating the fat exactly after the manner of an amoba, either while projecting between the columnar cells, in which case they carry their burden of fat through the epithelium into the villus, or while wandering in the labyrinth of the villus bear it away bodily into the lymphatic system. But the number of leucocytes really containing any appreciable quantity of fat is too small to account for the amount of fat absorbed; Nor is the abundance of leucocytes in the mucous membrane during the period of digestion a sure proof that they are concerned in absorption, but rather an indication only that active changes of some kind are going on, since after the administration of a saline such as magnesium sulphate, which produces effects the very reverse of absorption, these leucocytes are present in unusual numbers. Moreover, under some circumstances, as in the villi of a new-born puppy after a meal of milk, they are absent even when digestion of fat is rapidly going on and the lacteals are filling with fat. In fact, what we stated above concerning the presence of fat in the bodies of the columnar cells shows that leucocytes can have little to do in transferring fat from the interior of the intestine into the body of the villus; and there are no adequate reasons for attributing to them any real share in the transference of fat from the body of the villus into the lacteal chamber.

$\$ 264$. The lacteal chamber opens at the base of the villus into the valved lymphatic vessels lying below, and in these the flow of lymph (chyle) is being promoted by the various causes detailed in $\$ 253$. The pressure, for instance, exerted by the peristaltic contractions of the intestine helps to empty the lymphatic vessel into which a lacteal chamber opens and so promotes the emptying of the latter. In addition to this the plain muscular fibres of the villus supply a special muscular pump for the emptying and filling of the lacteal chamber. These fibres and small bundles of fibres, 
though running in various directions and varying in number and arrangement in different animals, take on the whole a longitudinal direction parallel to the long axis of the villus. It has been supposed that in contracting and shortening the villus they compress the lacteal and thus empty it, and that when they relax and the villus elongates again, the emptied chamber fills once more. But a different interpretation of their action has been offered somewhat as follows. When the muscular fibres contract they shorten the villus. In thus becoming shorter the body of the villus becomes proportionately broader, since probably no great change of bulk in the reticulum takes place; in this broadening the part to give way will be the lacteal chamber, which thus becomes broader and larger. When the muscular fibres relax, the reticulum, the bars of which have been put on the stretch in a lateral direction, by elastic reaction brings back the villus to its former length, and the lacteal chamber elongates and narrows. On this view the muscular contraction expands and so fills, while the relaxation narrows and so empties, the lacteal chamber. Whichever view we adopt, we may at least conclude that contractions and relaxations of the muscular fibres in some way or other alternately fill and empty the lacteal chamber, and in all probability, at all events during digestion, rhythmical contractions of these fibres are continually going on. When the villus is shortened by the contraction of the muscular fibres, the columnar cells are compressed, becoming longer and narrower; when the muscular fibres relax and the villus elongates, the columnar cells return to their previous form. The alternating changes of form to which the columnar cells are thus subjected, and the alternating changes of pressure taking place in the reticulum, may also serve to promote the passage of material through the one and through the other.

$\S 265$. The absorption of diffusible substances and of water. On the provisional assumption which we have made that the proteids are converted into peptone, we may consider, for the present at all events, peptone, sugar, and soluble salts as together forming a class distinguished from fats by their being diffusible, some more so than others. And we have made the further provisional assumption that these pass into the bloodvessels and not into the lacteals.

The network of capillary bloodvessels is spread immediately beneath the basement membrane, and all the material which enters the lacteal chamber has to run the gauntlet of the meshes of this network. During digestion the capillaries of the intestine are filled and distended, so that at a time when absorption is taking place these meshes between the capillaries are unusually narrow. From the interior of these capillaires, as elsewhere, transudation is taking place; these capillaries supply the lymph which helps to fill up the labyrinth of the reticulum and the lacteal chamber. But to a much greater extent than elsewhere (cf. $\$ 255$ ) this current of transudation from within the capillary to without is accompanied by a reverse current from without to within. The diffusible substances in question pass from the intestine through the layer of epithelial cells, through the attenuated reticular lymph-space between the basement membrane and the capillary wall, and through the capillary wall into the blood current. 'Their passage consists of two stages: that through the epithelial cells from the intestine to the lymph-space, and that from the lymph-space into the bloodvessels. These two stages may be expected to differ, seeing that the structures concerned are different; but we may at first consider them as one, and speak of the passage from the intestine into the blood as a single event.

In speaking of these substances as diffusible, we are using the term in 
reference to the well-known passage of such substances through thin membranes or porous partitions. When a strong solution of sugar or of common salt is separated by a thin membrane (vegetable parchment, dead urinary bladder, dead intestine, etc.) from a weak solution of sugar or of salt, the sugar or salt passes with a certain rapidity from the stronger to the weaker solution, and water passes from the weaker solution to the stronger; if, to begin with, simple water be substituted for the weaker solution, the effect is at first still more striking. Peptone passes in the same manner, but as we have seen, much more slowly. The process is spoken of as a physical one, since it is not accompanied necessarily by any chemical change in the diffusing substance, nor is there any necessary change in the membrane or partition. The rate at which a substance diffuses, and the total amount of diffusion which can take place, are determined by certain qualities of the substance (which we may call physical, though they depend on the chemical nature of the substance) in relation to certain qualities of the membrane; thus two salts may diffuse through the same membrane at different rates, with different rates in the associated current of water, the osmotic current as it is called, from the weaker to the stronger solution; and the same substance may pass at different rates through different membranes. By a number of observations, in which various substances in solution and several known membranes or partitions have been employed, a certain number of "laws of diffusion" have been established.

Now if, by the statement that diffusible substances pass by diffusion into the blood-capillaries of the intestine, we are led to expect that the passage takes place exactly according to the laws established by observations on ordinary membranes, we should be led into error; for the disappearance of these substances from the interior of the intestine does not take place according to the laws which regulate their disappearance from one side of an ordinary diffusion septum. This can be ascertained by introducing solutions of the substances, of various strength, into a loop of intestine, isolated in the living animal by the method described in $\$ 219$, and watching their disappearance by analysis of the contents of the loop. For instance, sodium sulphate passes through an ordinary diffusion septum with a rapidity rather greater than that of dextrose, whereas dextrose disappears from the intestine distinctly more rapidly than sodium sulphate; peptone, which diffuses very slowly indeed through an ordinary diffusion septum, disappears rapidly (though not so rapidly as dextrose) from the intestine; and when the details of the disappearance from the intestine of weak solutions of two salts which diffuse through an ordinary membrane at differents rates, which have, as it is said, different osmotic equivalents, are studied, these details are quite different from those of ordinary diffusion. The more the matter is studied, the more decidedly apparent becomes the difference between ordinary diffusion and the absorption of diffusible substances from the intestine.

\section{CHAPTER II.}

\section{RESPIRATION.}

\section{The Structure of the Iuungs and Bronchial Passages.}

$\$ 266$. Ore particular item of the body's income, viz., oxygen, is peculiarly associated with one particular item of the body's waste, viz., carbonic acid, inasmuch as the means which are applied for the introduction of the 
former are also used for the getting rid of the latter. Both are gases, and the ingress of the one as well as the egress of the other is far more dependent on the simple physical process of diffusion than on any active vital processes carried on by means of tissues. Oxygen passes from the air into the blood mainly by diffusion, and mainly by diffusion also from the blood into the tissues; in the same way carbonic acid passes mainly by diffusion from the tissues into the blood and from the blood into the air. IVhereas, as we have seen in the secretion of the digestive juices the epithelial cell plays an all-important part, in respiration the entrance of oxygen from the lungs into the blood, and from the blood into the tissue, and the passage of carbonic acid in the contrary direction, are affected, if at all, in a wholly subordinate manner, by the behavior of the pulmonary, or of the capillary epithelium. What we have to deal with in respiration, then, is not so much the vital activities of any particular tissue, as the various mechanisms by which a rapid interchange between the air and the blood is effected, the means by which the blood is enabled to carry oxygen and carbonic acid to and from the tissues, and the manner in which the several tissues take oxygen from and give carbonic acid up to the blood. We have reasons for thinking that oxygen can be taken into the blood, not only from the lungs but also to a certain small extent from the skin, and, as we have seen, from the alimentary canal also ; and carbonic acid certainly passes away from the skin, and through the various secretions, as well as by the lungs. Still the lungs are so eminently the channel of the interchange of gases between the body and the air, that in dealing at present with respiration, we shall confine ourselves entirely to pulmonary respiration, leaving the consideration of the subsidiary respiratory processes till we come to study the secretions of which they respectively form part.

\section{The Mechanics of Pulmonary Respiration.}

$\$ 267$. The lungs are placed, in a state which is always one of distention, sometimes greater, sometimes less, in the air-tight thorax, the cavity of which they, together with the heart, great bloodvessels, and other organs, completely fill. By the contraction of certain muscles the cavity of the thorax is enlarged. The lungs must follow this enlargement and be themselves enlarged, otherwise the pleural cavities would be enlarged; but this is impossible so long as the walls are intact. The enlargement of the lung consists chiefly in an enlargement or expansion of the pulmonary alveoli, the air in which becomes, by the expansion, rarefied. That is to say, the pressure of the air within the lungs becomes less than that of the air outside the body, and this difference of pressure causes a rush of air through the trachea into the lungs until an equilibrium of pressure is established between the air inside the lungs and that outside. This constitutes inspiration. On relaxation of the respiratory muscles (the muscles whose contractions have brought about the thoracic expansion), the elasticity of the lungs and chest-walls, aided, perhaps, to some extent by the contraction of certain muscles, causes the chest to return to its original size; in consequence of this the pressure within the lungs becomes greater than that outside, and thus air rushes out of the trachea until equilibrium is once more established. This constitutes expiration; the inspiratory and expiratory act together forming a respiration. The fresh air introduced into the upper part of the pulmonary passages by the inspiratory movement contains more oxygen and less carbonic acid than the old air previously present in the lungs. By diffusion the new or tidal air, as it is frequently called, gives up its oxygen to, and takes carbonic acid from, the old or stationary air, as it has been called, 
and thus when it leaves the chest in expiration has been the means of both introducing oxygen into the chest and of removing carbonic acid from it. In this way, by the ebb and flow of the tidal air, and by diffusion between it and the stationary air, the whole air in the lungs is being constantly renewed through the alternate expansions and contractions of the chest.

$\S 268$. In ordinary respiration the expansion of the chest never reaches its maximum; by more forcible muscular contraction, by what is called labored inspiration, an additional thoracic expansion can be brought about, leading to an inrush of a certain additional quantity of air before equilibrium is established. This additional quantity is often spoken of as complemental air. In the same way in ordinary respiration the contraction of the chest never reaches its maximum. By calling into use additional muscles, by a labored expiration an additional quantity of air, the so-called reserve or supplemental air, may be driven out. But even after the most forcible expiration, a considerable quantity of air, the residual air, still remains in the lungs. The natural condition of the lungs in the chest is, in fact, one of partial distention. The elastic pulmonary tissue is always to a certain extent on the stretch; it is always, so to speak, striving to pull asunder the pulmonary from the parietal pleura; but this it cannot do, because the air can have no access to the pleural cavity. When, however, the chest ceases to be air-tight, when by a puncture of the chest-wall or diaphragm air is freely introduced into the pleural chamber, the elasticity of the lungs pulls the pulmonary away from the parietal pleura and the lungs collapse, driving out by the windpipe a considerable quantity of the residual air. Even then, however, the lungs are not completely emptied, some air still remaining in them; this is probably air imprisoned in the infundibula by collapse of the bronchioles, which, as we have seen, have flaccid and not rigid walls. If, in a living animal, the pressure of the atmosphere continue to have access to the outside of a lung, the air thus imprisoned is gradually absorbed and the lung becomes solid. The same result may occur from the pressure of fluid accumulated in the pleural eavity.

It need hardly be added that when the pleura is punctured and air can gain free admittance from the exterior in the pleural chamber, since the resistance to the entrance of the air into the pleural chamber is far less than the resistance to the entrance into the lungs, the effect of the respiratory movements is simply to drive air in and out of that chamber, instead of in and out of the lung. There is, in consequence, no renewal of the air within the lungs under those circumstances. If there be a sufficient obstacle to the entrance of air into the pleural chamber, such as a fold of tissue blocking up the opening, the expansion of the chest may still lead to a distention of the lungs, and in this way, in some cases, puncture of the chest-walls has not seriously interfered with respiration. The parietal and pulmonary pleura are, in normal circumstances, separated by a very thin layer only of fluid, so that we may, perhaps, speak of them as being in a state of " adhesion," such as obtains between two wet membranes superimposed. And it has been suggested that this adhesion, having to be overeome before the two surfaces can separate, assists in preventing the entrance of air into the pleural cavity after puncture of the thorax; but it has not been clearly shown that this is really of importance in the matter.

$\$ 269$. Before birth the lungs contain no air ; they are in the condition called atelectatic. The walls of the alveoli, the epithelial lining of which is, at that time, well developed, consisting of distinctly nucleated cells with granular cell-substance, are in contact, the cavity of the alveolus not having 
as yet come into existence; the walls of the bronchioles are similarly in a collapsed condition, with their walls touching; the more rigid bronchia, like the trachea, possess some amount of lumen, which, however, is occupied by fluid. When the chest expands with the first breath taken, the pressure of the inspired air has to overcome the "adhesion " obtaining between the walls of the alveoli, thus in contact with each other and also those of the bronchioles. The force spent in thus opening out and unfolding, so to speak, the alveoli and bronchioles is considerable, and in the expiration succeeding the first inspiration most of the air thus introduced remains, the force exerted by the chest in returning to its previous dimensions after the breathing in and the elastic action of the alveoli being insufficient to bring the walls of the alveoli again into contact. Succeeding breaths unfold the lungs more and more, until all the alveoli and bronchioles are opened up, and then the whole force of the expiratory act is directed to driving out the previously inspired air.

It is not, however, until some time after birth that the lungs pass into that further distended state of which we spoke above. In a newly-born animal there is no negative pressure obtaining in the pleural cavities; the lungs, when at rest, are not on the stretch, and opening the thorax does not lead to collapse of the lungs. The state of things obtaining later on is established, not at once, but gradually, and is apparently brought about by the thorax growing more rapidly, and so becoming relatively more capacious than the lungs. The distention of the lungs in the adult may be familiarly described as being due to the chest being too large for the lungs.

$\$ 270$. In man the pressure exerted by the elasticity of the lungs alone amounts to about 5 or $7 \mathrm{~mm}$. of mercury. This is estimated by tying a manometer into the windpipe of a dead subject and observing the rise of mercury which takes place when the chest-walls are punctured. If we took $7.6 \mathrm{~mm}$. as the pressure this would be just $-\frac{1}{0} 0$ of the pressure of the atmosphere. If the chest be forcibly distended beforehand, a much larger rise of the mercury is observed, amounting, in the case of a distention corresponding to a very forcible inspiration, to $30 \mathrm{~mm}$. In the living body this mechanical elastic force of the lungs may be assisted by the contraction of the plain muscular fibres of the bronchi; the pressure, however, which can be exerted by these probably does not exceed 1 or $2 \mathrm{~mm}$.

When a manometer is introduced into a lateral opening of the windpipe of an animal, the mercury will fall, indicating a negative pressure, as it is called, during inspiration, and rise, indicating a positive pressure, during expiration, both fall and rise being slight and varying according to the freerlom with which the air passes in and out of the chest. When a manometer is fitted with air-tight closure into the mouth, or better, in order to avoid the suction-action of the mouth, into one nostril, the other nostril and the mouth being closed, and efforts of inspiration and expiration are made, the mercury falls or undergoes negative pressure with inspiration, and rises or undergoes positive pressure during expiration. It has been found in this way that the negative pressure of a strong inspiratory effort may vary from 30 to $74 \mathrm{~mm}$., and the positive pressure of a strong expiration from 62 to $100 \mathrm{~mm}$.

The total amount of air which can be given out by the most forcible expiration following upon the most forcible inspiration, that is, the sum of the complemental, tidal, and reserve airs, has been called the "vital capacity ;" "extreme differential capacity" is a better phrase. It may be measured by a modification of a gas-meter called a spirometer; and though it 
varies largely, the average may be put down at $3000-4000$ c.c. (200 to 250 cubic inches).

Of the whole measure of vital capacity, about 500 c.c. (30 cubic inches) may be put down as the average amount of tidal air, the remainder being nearly equally divided between the complemental and reserve airs. The quantity left in the lungs after the deepest expiration amounts to about 1400 to 2000 c.c.

Since the respiratory movements are so easily affected by various circumstances, the simple fact of attention being directed to the breathing being sufficient to cause modifications both of the rate and depth of the respiration, it becomes very difficult to fix the volume of an average breath. Thus various authors have given figures varying from 53 c.c. to 792 c.c. The statement made above is the mean of observations varying from 177 to 699 c.c.

$\$ 271$. Graphic records of respiratory movements. These may be obtained in various ways.

The simplest, readiest, and perhaps the most generally useful method is that of recording the movements of the column of air. This may be effected by introducing a T-piece into the trachea, one cross-piece being left open and the other connected with a Marey's tambour or with a receiver, which in turn is connected with a tambour (see Fig. 54 and Fig. 90). The movements of the column of air in the trachea are transmitted to the tambour, the consequent expansions and contractions of which are transmitted to the recording drum by means of a lever resting on it.

If, a receiver being used, the open $\in$ nd of the $\vdash$ be closed, the animal breathes into and out of the receiver, and the movements of the tambour are greatly increased. This has the disadvantage that the air in the receiver soon becomes unfit for further respiration. A similar increase of the movements of the lever of the tambour may be obtained by connecting a piece of India-rubber tubing to the open end of the $\vdash$. By increasing the length of this tube, or slightly contracting it, the movements of the lever may be increased without very seriously interfering with the breathing of the animal.

In another method the movements of the chest are recorded. When a small animal, such as a rabbit, is used, the whole animal may be placed in an air-tight box, breathing being carried on by means of a tube inserted into the trachea and carried through an air-tight orifice in the wall of the box. By another orifice and tube the air in the box is brought into connection with a tambour, which accordingly registers the changes of pressure in the air of the box produced by the movements of the chest (and body), and thus indirectly the movements of the chest. In man and larger animals the changes in the girth of the chest may be conveniently recorded by means of Marey's pneumograph. This consists of a hollow elastic cylinder, or a cylinder with elastic ends, the interior of which is connected with a tambour. By means of a strap attached to each end of the cylinder the instrument can be buckled round the chest like a girdle. When the chest expands, the ends of the cylinder are pulled out, and the air within the chamber rarefied; in consequence the lever of the tambour connected with its interior is depressed; conversely, when the chest contracts, the lever is elevated. The pneumatograph of Fick is somewhat similar. Or changes in one or other diameter of the chest may be recorded by what may be called the "calipers" method, as in the recording stethometer of Burdon-Sanderson. This consists of a rectangular framework constructed of two rigid parallel bars joined at right angles to a cross-piece. The free ends of the bars, the distance between which can be regulated at pleasure, are armed, the one with a tambour, the other simply with an ivory button. The tambour bears on the metal plate of its membrane $\left(m^{\prime}\right.$, Fig. 54) a small ivory button in place of the lever. When it is desired to record the changes occurring in any diameter of the chest, e. $q$. , an antero-posterior diameter from a point in the sternum to a point in the back, the instrument is made to encircle the chest somewhat after the fashion of a pair of calipers, the ivory button at one free end being placed on the spine of a vertebra behind and the tambour at the other on the sternum in front in the line of the diameter which 
FIG. 90.

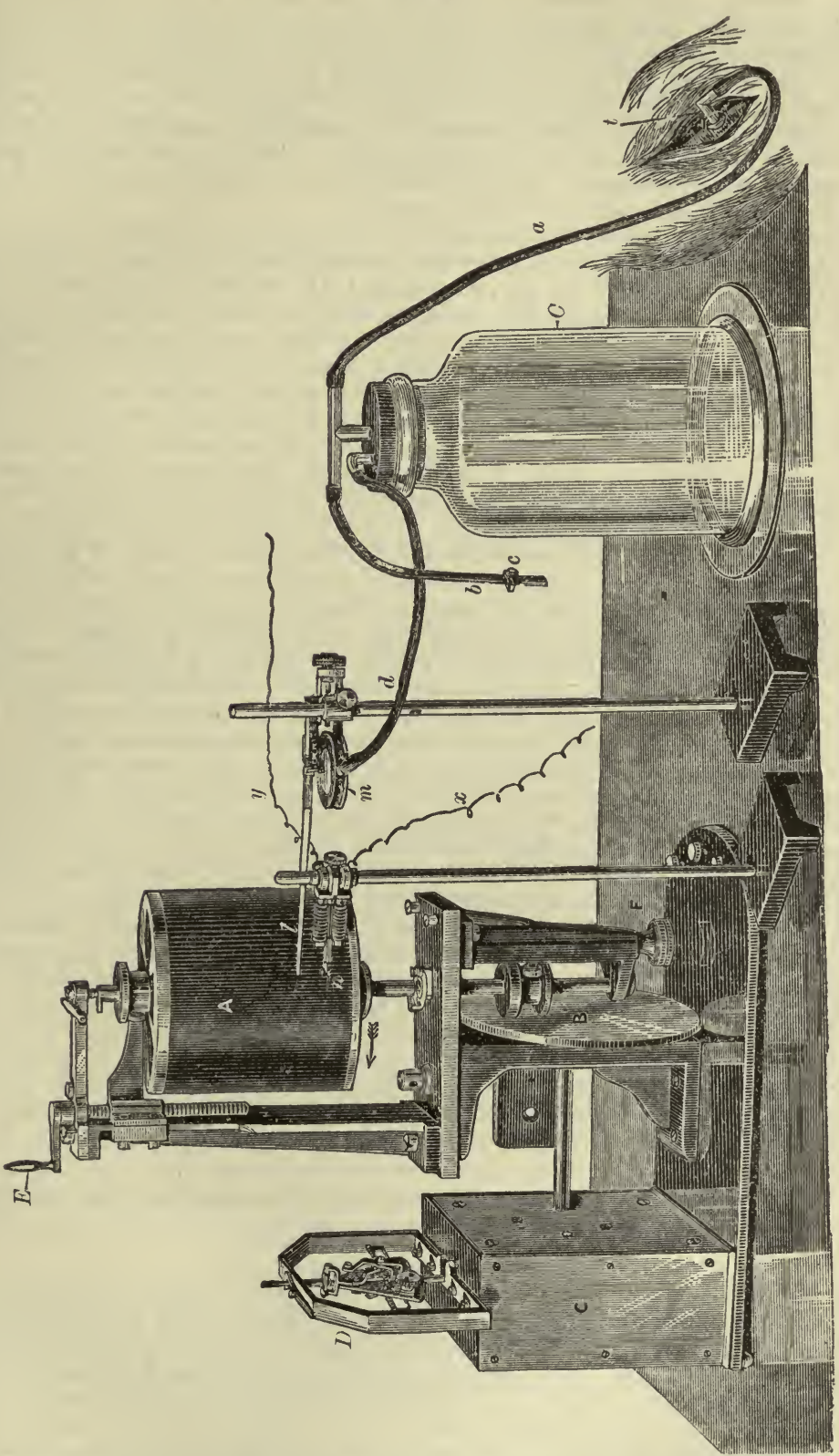

Apparatus for taking Tracings of the Movements of the Column of Air in Respiration. The recording apparatus shown is the ordinary cylinder recording apparatus. The cylinder $A$, covered with smoked paper, is by means of the friction-plate $B$ put into revolution by the spring elockwork in $C$, regulated by Foucault's regulator $D$. By means of the screw $E$, the cylinder can be raised or lowered, and by means of the screw $F$ its speed may be increased or diminished.

The tracheotomy tube $t$ fixed in the trachea of an animal is connected by India-rubber tubing $a$ with a glass $T$-piece inserted into the large jar $G$. From the other end of the T-piece proceeds a second piece of tubing $b$, the end of which can be either closed or partially obstructed at pleas- 
is being studied. The distance between the free ends of the instrument being carefully adjusted so that the button of the tambour presses lightly on the sternum, any variations in the length of the diameter in question will, since the framework of the tambour is immobile, give rise to variations of pressure within the tambour. These variations of the "receiving" tambour, as it is called, are conveyed by a flexible tube containing air to a second or "recording" tambour, the lever of which records the variations on a travelling surface. For the purpose of measuring the extent of the movements the instrument must be experimentally graduated. Other forms of ealipers may, of course. be used.

By still another method the variations in intra-thoracic pressure, by means of which the movements of the chest walls produce the movement of air in the lungs, may be recorded. This may be effected by introducing carefully, to the total exclusion of air, into a pleural cavity or into the pericardial cavity, a canula connected by a rigid tube with a manometer. With each inspiration a negative pressure, or rather an increase of the existing negative pressure, is produced, the mercury, or fluid, in the manometer returning at each expiration. An easier method of recording this intra-thoracic pressure is to introduce into the oesophagus an el istic sound (similar to the cardiac sound, Fig. 54) connected with a tambour. The oesophagus within the thorax, like the heart ant great vessels, as we shall see, is affected as well as the lungs by the variations of intra-thoracic pressure brought about by the respiratory movements.

In yet another method the movements of the diaphragm, which, as we shall see, serve as the prime agent in bringing aloout the enlargement of the thoracic cavity, are recorded. 'This may be done by inserting, through an incision in the abdominal wall, a flat elastic bag between the diaphragm and abdominal organs. When in inspiration the diaphragm dessends, it exerts on the lag a pressure which, by means of a tube, may be communicated to a tambour. Or a needle may be thrust through the chest wall so as to rest upon or transfix the diaphragm, and the head of the needle outside the body connected by a thread or otherwise with a lever; each upward and downward movement of the head of the needle, corresponding to the downward and upward movements of the diaphragm, is registered by the lever.

Various modifications of these several methouls have been adopted by various observers. They all, however, leave much to be desired. A very ingenious method of registering the contractions of the diaphragm has recently been introduced. In the rabbit two slips of muscular fibres forming part of the diaphragm, one on each side of the ensiform cartilage, are so disposed and possess such attachments that one or both of them may be isolated without injury to either nerves or bloodvessels, and arranged so that while one end of the slip is securely fixed to the chest-wall as a fixed point, the other end can by a thread be brought to bear on a lever. The slip, even when thus arranged, appears to contract rhythmically in complete unison with the contractions of the whole of the rest of the diaphragm: it serves, so to speak, as a sample of the diaphragm; and hence its contractions, like those of the whole diaphragm, may be taken as a record of respiratory movements. The record has to be corrected for variations in the position of the fixed point.

$\S 272$. In these various ways curves are obtained, which, while differing in detail, exhibit the same general features, and more or less resemble the curve shown in Fig. 91.

As the figure shows, inspiration begins somewhat suddenly and arlvances rapidly, being followed immediately by expiration, which is carried out at first rapidly, but afterwarl more and more slowly. Such pauses as are seen usually occur between the end of expiration and the beginning of inspira-

ure by means of the screw clamp $c$. From the jar proceeds a third piece of tubing $d$, connected with a Marey's tambour $m$ (see Fig. 54), the lever of which $l$ writes on the recording surface. When the tube $b$ is open the animal breathes freely through this, and the movements in the air of $G$ and consequently in the tambour are slight. On closing the clamp $c$, the animal breathes only the air contained in the jar, and the movements of the lever of the tambour become consequently much more marked.

Below the lever is seen a small time-narker $n$ connected with an electro-magnet, the current through which, coming from a battery by the wires $x$ and $y$, is made and broken by a clock-work or metronome. 
tion. In normal breathing hardly any such pause exists, but in cases where the respiration becomies infrequent, pauses of considerable length may be observed. As we shall see in detail hereafter, the several parts of the whole act vary much, under various circumstances, in relation to each other. Sometimes expiration, sometimes inspiration is prolonged; and either inspi-

[FIG. 91.

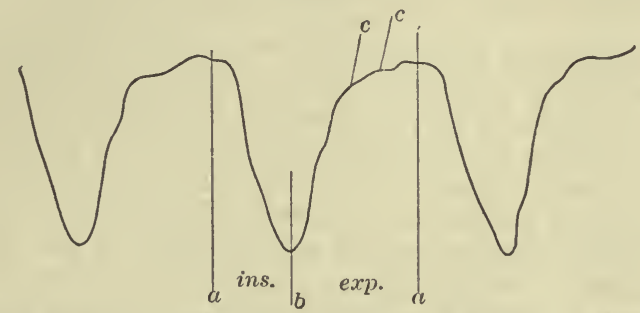

Tracing of Thoracic Respiratory Movements obtained by means of Marey's Pneumograph. A whole respiratory phase is comprised between $a$ and $a$; inspiration, during which the lever descends, extending from $a$ to $b$, and expiration from $b$ to $a$. The undulations at $c$ are caused by the heart's beat.]

ration or expiration may be slow or rapid in its development. At times the chest may remain for a while at the height of inspiration, thus making a pause between inspiration and expiration.

In what may be considered as normal breathing the respiratory act is repeated about seventeen times a minute, the duration of the inspiration as compared with that of the expiration (and such pause as may exist) being about as ten to twelve; but the rate varies very largely, and in this, as in the volume of each breath, it is very difficult to fix a satisfactory average, the figures given varying from twenty to thirteen a minute. It varies according to age and sex. It is influenced by the position of the body, being quicker in standing than in lying, and in lying than in sitting. Muscular exertion and emotional conditions affect it deeply. In fact, almost every event which occurs in the body may influence it. We shall have to consider in detail hereafter the manner in which these influences are brought to bear.

When the ordinary respiratory movements prove insufficient to effect the necessary changes in the blood, their rhythm and character become changed. Normal respiration gives place to labored respiration, and this in turn to dyspnoca, which, unless some restorative event occurs, terminates in asphyxia. These abnormal conditions we shall study more fully hereafter.

\section{The Respiratory Movements.}

$\S 273$. When the movements of the chest during normal breathing are watched, or when a graphic record is taken by one or other of the methods just described, it is seen that during inspiration an enlargement takes place in the antero-posterior diameter, the sternum being thrown forward, and at the same time moving upward. The lateral width of the chest is also increased. The vertical increase of the cavity is not so obvious from the outside, though when the movements of the diaphragm are watched by means of an inserted needle or otherwise, it is clear that the upper surface of that organ descends at each inspiration, the anterior walls of the abdomen bulging out at the same time. In the female human subject, the movement of the upper part of the chest is very conspicuous, the breast rising and falling with every respiration; in the male, however, the movements are almost en- 
tirely confined to the lower part of the chest. In labored respiration all parts of the chest are alternately expanded and contracted, the breast rising and falling as well in the male as in the female. We have now to consider these several movements in greater detail, and to study the means by which they are carried out.

$\$ 274$. Inspiration. There are two chief means by which the chest is enlarged in normal inspiration, viz., the descent of the diaphragm and the elevation of the ribs. The former causes that movement in the lower part of the chest and abdomen so characteristic of male breathing, which is hence called diaphragmatic; the latter causes the movement of the upper chest characteristic of female breathing, which is called costal. These two main factors are assisted by less important and subsidiary events.

Even in the female human subject, the share taken in respiration by the diaphragm is an important one ; in the male the diaphragm must be regarded as the chief respiratory agent, and in some animals its use, for this purpose, is so prominent that the movements of the ribs may in normal breathing be almost neglected. In the rabbit for instance, in normal breathing, almost all the respiratory work is done by the contractions of the diaphragm.

The descent of the diaphragm is effected by means of the contraction of its muscular fibres. When at rest the diaphragm presents a convex surface to the thorax: when contracted it becomes much flatter, and in consequence the level of the chest-floor is lowered, the vertical diameter of the chest being proportionately enlarged. In descending, the diaphragm presses on the ahdominal viscera, and so causes a projection of the flaccid abdominal walls. From its attachments to the sternum and the false ribs, the diaphragm, while contracting, naturally tends to pull the sternum and the upper false ribs downward and inward, and the lower false ribs upward and inward, toward the lumbar spine. In normal breathing, this teudency produces little effect, being counteracted by the accompanying general costal elevation, and by certain special muscles to be mentioned presently. In forced inspiration, however, and especially where there is any obstruction to the entrance of air into the lungs, the lower ribs may be so much drawn in by the contraction of the diaphragm that the girth of the trunk at this point is obviously diminished.

$\$ 275$. The elevation of the ribs is a much more complex matter than the descent of the diaphragm. If we examine any one rib, such as the fifth, we find that while it moves freely on its vertebral articulation, it inclines when in the position of rest in an oblique direction from the spine to the sternum; hence it is obvious that when the rib is raised, its sternal attachment must not only be carried upward but also thrown forward. The rib may in fact be regarded as a radius, moving on the vertebral articulation as a centre, and causing the sternal attachment to describe an arc of a circle in the vertical plane of the body; as the rib is carried upward from an oblique to a more horizontal position, the sternal attachment must of necessity be carried further away in front of the spine. Since all the ribs have a downward slanting direction, they must all tend, when raised toward the horizontal position, to thrust the sternum forward, some more than others, according to their slope and length. The elasticity of the sternum and costal cartilages, assisted by the articulation of the sternum to the clavicle above, permits the front surface of the chest to be thus thrust forward as well as upward, when the ribs are raised. By this action, the antero-posterior diameter of the chest is enlarged.

Since the ribs form arches which increase in their sweep as one proceeds from the first downward as far at least as the seventh, it is evident that when a lower rib such as the fifth is elevated so as to occupy or to approach 
toward the position of the one above it, the chest at that level will become wider from side to side, in proportion as the fifth arch is wider than the fourth. Thus the elevation of the rib increases not only the antero-posterior but also the transverse diameter of the chest. Further, on account of the resistance of the sternum, the angles between the ribs and their cartilages are, in the elevation of the ribs, somewhat opened out, and thus also the transverse as well as the antero-posterior diameter, somewhat increased. In more than one way, then, the elevation of the ribs enlarges the dimensions of the chest.

$\S 276$. The ribs are raised by the contraction of certain muscles. Of these the external intercostals are perhaps the most important. Even in the case where two ribs, such as the fifth and sixth, are isolated from the rest of the thoracic cage, by section of the structures occupying the intercostal spaces above and below, the contraction of the external intercostal muscle of the intervening space raises the two ribs, thus bringing them toward the position in which the fibres of the muscle have the shortest length, viz., the horizontal one. This elevating action is, in the entire chest, further favored by the fact that the first rib is less movable than the second, and so affords a comparatively fixed base for the action of the muscles between the two, the second in turn supporting the third, and so on, while the scaleni muscles in addition serve to render fixed, or to raise, the first two ribs. So that in normal respiration, the act may probably be described as beginning by a contraction of the scaleni. The first two ribs being thus raised or at least fixed, the contraction of the series of external intercostal muscles acts at a great disadvantage.

While the elevating, $i . e$., inspiratory, action of the external intercostals is admitted by nearly all authors, the function of the internal intercostals has been much disputed. Some regard their action as wholly inspiratory ; others maintain, what is perhaps the more commonly adopted view, that while those parts of them which lie between the sternal cartilages act like the external intercostals as elevators, $i . e$, as inspiratory in function, those parts which lie between the osseous ribs act as depressors, $i$. e., as expiratory in function.

In the well-known model consisting of two rigid bars representing the ribs, moving vertically by means of their articulations within an upright representing the spine, and connected at their free ends by a piece representing the sternum, it is undoubtedly true that stretched elastic bands attached to the bars in such a way as to represent respectively the external and internal intercostals, viz., sloping in the one case downward and forward, and in the other downward and backward, do, on being left free to contract, in the former case elevate and in the latter depress the ribs. Such a model, however, does not fairly represent the natural conditions of the ribs, which are not straight and rigid, but peculiarly curved and of varying elasticity, capable moreover of rotation on their own axes, and having their movements determined by the characters of their vertebral articulations. The mechanical conditions in fact of these muscles are so complex, that a deduction of their actions from simple mechanical principles, or from the direction of the fibres, must be exceedingly difficult and dangerous. Actual experiments on the cat and dog tend to show that in these animals the contraction of the internal intercostals, along their whole length, takes place, in point of time, alternately with that of the diaphragm, and thus offer an argument in favor of these muscles being expiratory in function.

Next in importance to the external intercostals come the levatores costarum, which, though small muscles, are able, from the nearness of their costal insertions to the fulcrum, to produce considerable movement of the 
sternal ends of the ribs. The external intercostals and the levatores costarum with the scaleni may fairly be said to be the elevators of the ribs, $i$. $e$, the chief muscles of costal inspiration in normal breathing.

It must be added, however, that some observers deny that either set of intercostal muscles take any important part in raising the ribs. They hold that the chief if not the only use of these muscles is by their contraction to render the intercostal spaces firm and the whole thoracic cage rigid, so that the thorax is moved as a whole by the other muscles mentioned, and the intercostal spaces do not give way during the respiratory movements.

Additional space in the transverse diameter is afforded probably by the rotation of the ribs on an antero-posterior axis; but this movement is quite subsidiary and unimportant. When the chest is at rest, the ribs are somewhat inclined with their lower borders directed inward as well as downward. When they are drawn up by the action of the intercostal muscles, their lower borders are everted. Thus their flat sides are presented to the thoracic cavity, which is thereby slightly increased in width.

$\$ 277$. Labored inspiration. When respiration becomes labored, other muscles are brought into play. The scaleni are strongly contracted, so as distinctly to raise or at least give a very fixed support to the first and second ribs. In the same way the serratus posticus superior, which descends from the fixed spine in the lower cervical and upper dorsal regions to the second, third, fourth, and fifth ribs, by its contractions raises those ribs. In labored breathing a function of the lower false ribs, not very noticeable in easy breathing, comes into play. They are depressed, retracted, and fixed, thereby giving increased support to the diaphragm, and directing the whole energies of that muscle to the vertical enlargement of the chest. In this way the serratus posticus inferior, which passes upward from the lumbar aponeurosis to the last four ribs, by depressing and fixing those ribs becomes an adjuvant inspiratory muscle. The quadratus lumborum and lower portions of the sacro-lumbalis may have a similar function.

All these muscles may come into action even in breathing which, though deeper than usual, can hardly perhaps be called labored. When, however, the need for greater inspiratory efforts becomes urgent, all the muscles which can, from any fixed point, act in enlarging the chest, come into play. Thus the arms and shoulder being fixed, the serratus magnus passing from the scapula to the middle of the first eight or nine ribs, the pectoralis minor passing from the coracoid to the front parts of the third, fourth, and fifth ribs, the pectoralis major passing from the humerus to the costal cartilages, from the second to the sixth, and that portion of the latissimus dorsi which passes from the humerus to the last three ribs, all serve to elevate the ribs and thus to enlarge the chest. The sterno-mastoid and other muscles passing from the neck to the sternum, are also called into action. In fact, every muscle which by its contraction can either elevate the ribs or contribute to the fixed support of muscles which do elevate the ribs, such as the trapezius, levator anguli scapule, and rhomboidei by fixing the scapula, may, in the inspiratory efforts which accompany dyspnoa, be brought into play.

\$278. Expiration. In normal easy breathing, expiration is in the main a simple effect of elastic reaction. By the inspiratory effort the elastic tissue of the lungs is put on the stretch; so long as the inspiratory muscles continue contracting, the tissue remains stretched; but directly those muscles relax, the elasticity of the lungs comes into play and drives out a portion of the air contained in them. Similarly the elastic: sternum and costal cartilages are by the elevation of the ribs put on the stretch; they are driven into a position which is unnatural to them. When the intercostal and other elevator muscles cease to contract, the elasticity of the sternum and costal car- 
tilages cause them to return to their previous position, thus depressing the ribs and diminishing the dimensions of the chest. When the diaphragm descends, in pushing down the abdominal viscera, it puts the abdominal walls on the stretch; and hence, when at the end of inspiration the diaphragm relaxes, the abdominal walls return to their place, and by pressing on the abdominal viscera, push the diaphragm up again into its position of rest. Expiration, then, during easy breathing is, in the main, simple elastic reaction; but there is probably some, though possibly in most cases a very slight, expenditure of muscular energy to bring the chest more rapidly to its former condition. This is, as we have seen, supposed by many to be afforded by the internal intercostals acting as depressors of the ribs. If these do not act in this way, we may suppose that the elastic return of the abdominal walls is accompanied and assisted by a contraction of the abdominal muscles. The triangularis sterni, the effect of whose contraction is to pull down the costal cartilages, may also be regarded as an expiratory muscle.

When expiration becomes labored, the abdominal muscles become important expiratory agents. By pressing on the contents of the abdomen, they thrust them and therefore the diaphragm also, up toward the chest, the vertical diameter of which is thereby lessened, while by pulling down the sternum and the middle and lower ribs they lessen also the activity of the chest in its antero-posterior and transverse diameters. They are, in fact, the chief expiratory muscles, though they are doubtless assisted by the serratus posticus inferior and portions of the sacro-lumbalis, since when the diaphragm is not contracting, the depression of the lower ribs which the contraction of these muscles causes, serves only to narrow the chest. As expiration becomes more and more forced, every muscle in the body which can either by contracting depress the ribs or press on the abdominal viscera, or afford fixed support to muscles having those actions, is called into play.

$\$ 279$. Facial and laryngeal respiration. The thoracic respiratory movements are accompanied by associated respiratory movements of other parts of the body, more particularly of the face and of the glottis.

In normal healthy respiration the current of air which passes in and out of the lungs, travels, not through the mouth but through the nose, chiefly through the lower nasal meatus. The ingoing air, by exposure to the vascular mucous membrane of the narrow and winding nasal passages, is more efficiently warmed than it would be if it passed through the mouth; and at the same time the mouth is thereby protected from the desiccating effect of the continual inroad of comparatively dry air.

During each inspiratory effort the nostrils are expanded, probably lyy the action of the dilatores naris, and thus the entrance of air facilitated. The return to their previous condition during expiration is effected by the elasticity of the nasal cartilages, assisted perhaps by the compressores naris. This movement of the nostrils, perceptible in many people even during tranquil breathing, becomes very obvious in labored respiration.

When the mouth is closed, the soft palate, which is held somewhat tense, is swayed by the respiratory current, but entirely in a passive manner, and it is not until the larynx is reached by the ingoing air that any active movements are met with. When the larynx (the details of which we shall have to deal with at a later part of this work) is examined with the laryngoscope, it is frequently seen that, while during inspiration the glottis is widely open, with each expiration the arytenoid cartilages approach each other so as to narrow the glottis, the cartilages of Santorini projecting inward at the same time. Thus, synchronous with the respiratory expansion and contraction of the chest, and the respiratory elevation and depression of the alæ nasi, there is a rhythmic widening and narrowing of the glottis. Like the movements 
of the nostril, this respiratory action of the glottis is much more evident in labored than in tranquil breathing. Indeed, in the latter case it is frequently absent. The manner in which this rhythmic opening and narrowing is effected will be described when we come to study the production of the voice. Whether there exists a rhythmic contraction and expansion of the trachea and bronchial passages, especially the smaller and more exclusively muscular ones, effected by means of the plain muscular tissue of those organs and synchronous with the respiratory movements of the chest, is uncertain.

\section{Changes of the Air in Respiration.}

$\S 280$. During its stay in the lungs, or rather during its stay in the bronchial passages, the tidal air (by means of diffusion chiefly) effects exchanges with the stationary air ; in consequence the expired air differs from inspired air in several important particulars.

The temperature of expired air is variable, but under ordinary circumstances is higher than that of the inspired air. At an average temperature of the atmosphere, for instance at about $20^{\circ} \mathrm{C}$., the temperature of expired air is in the mouth $33.9^{\circ}$, in the nose $35.3^{\circ}$. When the external temperature is low, that of the expired air sinks somewhat, but not to any great extent, thus at $-6.3^{\circ} \mathrm{C}$. it is $29.8^{\circ} \mathrm{C}$. When the external temperature is high, the expired air may become cooler than the inspired, thus at $41.9^{\circ}$ it has been found to be $38.1^{\circ}$. The expired air takes its temperature from that of the body, that is, of the blood, and this as we shall see later on, while generally higher may, at times, be lower than that of the atmosphere. The exact temperature of the expired air in fact depends on the relative temperatures of the blood and inspired air, and on the depth and rate of breathing. The change in temperature takes place not in the lungs but in the upper passages, and chiefly in the nose and pharynx.

$\$ 281$. The expired air is loaded with aqueous vapor. The point of saturation of any gas, that is, the utmost quantity of water which any given volume of gas can take up as aqueous vapor, varies with its temperature, being higher with the higher temperature. For its own temperature expired air is, according to most observers, saturated with aqueous vapor. The moisture, like the warmth, is imparted not in the depths of the lung but in the upper passages. The inspired air as it passes into the bronchia is already saturated with moisture.

$\$ 282$. The expired air contains about 4 or 5 per cent. less oxygen, and about 4 per cent. more carbonic acid than the inspired air, the quantity of nitrogen suffering but little change. Thus

\begin{tabular}{|c|c|c|c|}
\hline ed air & $\begin{array}{l}\text { Oxygen. } \\
20.81\end{array}$ & $\begin{array}{c}\text { Nitrogen. } \\
79.15\end{array}$ & $\begin{array}{c}\text { Carbonic acid } \\
0.04\end{array}$ \\
\hline 8 & 16.033 & 79.587 & 4.38 \\
\hline
\end{tabular}

The quantity of nitrogen in the expired air is sometimes found to be slightly greater than as in the above table, but sometimes equal to, and sometimes less than, that of the inspired air.

In a single breath the air is richer in carbonic acid (and poorer in oxygen) at the end than at the beginning of the breath. Hence, the longer the breath is held, the greater the (artificial) pause between inspiration and expiration, the higher the percentage of carbonic acid in the expired air. Thus, by increasing the interval between two expirations to 100 seconds, the percentage may be raised to 7.5. When the rate of breathing remains the 
same, by increasing the depth of the breathing the percentage of carbonic acid in each breath is lowered, but the total quantity of carbonic acid expired in a given time is increased. Similarly, when the depth of breathing remains the same, by quickening the rate the percentage of carbonic acid in each breath is lowered, but the quantity expired in a given time is increased.

Taking, as we have done, the amount of tidal air passing in and nut of the chest of an average man at 500 c.c., such a person will expire about 22 c.c. of carbonic acid at each breath; this, reckoning the rate of breathing at 17 a minute, would give over 500 litres of carbonic acid for the day's production. Actual determinations, however, give a rather smaller total than this; thus, in a series of experiments of which we shall have to speak hereafter, the total daily excretion of carbonic acid in an average man was found to be 800 grms., $i$. e., rather more than 400 litres (406), containing 218.1 grms. carbon and 581.9 grms. oxygen, the oxygen which actually disappeared from the inspired air at the same time being about 700 grms. This amount, it should be said, represents, owing to the manner in which the experiment was conducted, the gases given nut and taken in, not by the lungs only, but by the whole body; but the amount of carbonic acid given out by other channels than the lungs is, as we shall see, very slight (10 grms. or even less), so that 800 grms. may be taken as the average production of carbonic acid by an average man. The quantity, however, both of oxygen consumed and of carbonic acid given out, is subject to very wide variations ; thus, in the observations of which we are speaking, the daily quantity of carbonic acid varied from 686 to 1285 grms., and that of the oxygen from 594 to 1072 grms. These variations and their causes will be discussed when we come to deal with the problems of nutrition.

$\$ 283$. When the total quantity of tidal air given out at any expiration is compared with that taken in at the corresponding inspiration, it is found that, both being dried and measured at the same temperature and pressure, the expired air is less in volume than the inspired air, the difference amounting to about $\frac{1}{40}$ th to $\frac{1}{50}$ th of the volume of the latter. Hence, when an animal is made to breathe in a confined space, the air is absolutely diminished in volume. The approximate equivalence in volume between inspired and expired air arises from the fact that the volume of any given quantity of carbonic acid is equal to the volume of the oxygen consumed to produce it; the slight falling short of the expired air is due to the circumstances that all the oxygen inspired does not reappear in the carbonic acid expired, some having formed within the body other combinations.

$\$ 284$. Besides carbonic acid, expired air contains various substances which may be spoken of as impurities, many of an unknown nature, and all in small amounts. Traces of ammonia have been detected in expired air, even in that taken directly from the trachea, in which case its presence could not be due to decomposing food lingering in the mouth. When the expired air is condensed by being conveyed into a cooled receiver, the aqueous product is found to contain organic matter, which from the presence of microörganisms introduced in the inspired air, is very apt rapidly to putrefy. The organic substances thus shown to be present in the expired air are the cause in part of the odor of breath. It is probable that some of them are of a poisonous nature, either poisonous in themselves as coming direct from and produced in some way or other in the pulmonary apparatus, or poisonous as being the products of putrefactive decomposition; for various animal substances and fluids give rise by decomposition to distinct poisonous products known as ptomaines, and it is possible that some of the constituents of expired air are of an allied nature. In any case the 
substances present have a deleterious action, for an atmosphere containing simply 1 per cent. of carbonic acid (with a corresponding diminution of oxygen) has very little effect on the animal economy, whereas an atmosphere in which the carbonic acid has been raised to 1 per cent. by breathing is highly injurious. In fact, air rendered so far impure by breathing that the carbonic acid amounts to 0.08 per cent. is distinctly unwholesome, not so much on account of the carbonic acid as of the accompanying impurities. Since these impurities are of unknown nature and cannot be estimated, the easily determined carbonic acid is usually taken as an indirect measure of their presence. IVe have seen that the average man loads at each breath 500 c.c. of air with carbonic acid to the extent of 4 per cent. He will accordingly at each breath load 2 litres to the extent of 1 per cent.; and in one hour, if he breathe 17 times a minute, will load rather more than 2000 litres to the same extent. At the very least, then, a man ought to be supplied with this quantity of air hourly, and if the air is to be kept fairly wholesome, that is with the carbonic acid reduced below 0.01 per cent., he should have even more than ten times as much.

\section{The Respiratory Changes in the Blood.}

$\S 285$. While the air in passing in and out of the lungs is thus robbed of a portion of its oxygen and loaded with a certain quantity of carbonic acid, the blood as it streams along the pulmonary capillaries undergoes important correlative changes. As it leaves the right ventricle it is venous blood of a dark purple or maroon color; when it falls into the left auricle it is arterial blood of a bright scarlet hue. In passing through the capillaries of the body from the left to the right side of the heart it is again changed from the arterial to the venous condition. We have to inquire, What are the essential differences between arterial and venous blood, by what means is the venous blood changed into arterial in the lungs, and the arterial into venous in the rest of the body, and what relations do these changes in the blood bear to the changes in the air which we have already studied?

The facts that venous blood at once becomes arterial in appearance on being exposed to or shaken up with air or oxygen, and that arterial blood becomes venous in appearance when kept for some little time in a closed vessel, or when submitted to a current of some indifferent gas such as nitrogen or hydrogen, prepare us for the statement that the fundamental difference between venous and arterial blood is in the relative proportion of the oxygen and carbonic acid gases contained in each. From both a certain quantity of gas can be extracted by means which do not otherwise materially alter the constitution of the blood; and this gas when obtained from arterial blood is found to contain more oxygen and less carbonic acid than that obtained from venous blood. This is the real differential character in the two bloods; all other differences are either, as we shall see to be the case with the color, dependent on this, or are unimportant and fluctuating.

If the quantity of gas which can be extracted by the mercurial air-pump from 100 volumes of blood be measured at $0^{\circ} \mathrm{C}$. and a pressure of $760 \mathrm{~mm}$., it is found to amount in round numbers to 60 volumes.

The vacuum produced by the ordinary mechanical air-pump is insufficient to extract all the gas from blood. Hence it becomes necessary to use a mercury pump capable of producing a large Torricellian vacuum. In the form of mercurial pump which bears Ludwig's name (Fig. 92), two large globes of glass, one fixed and the other movable, are connected by a flexible tube: the fixed globe is made to com- 
municate by means of air-tight stopcocks alternately with a receiver containing the blood and with a recciver to collect the gas. When the movable globe filled with mercury is raised above the fixed one, the mercury from the former runs

FIG. 92.

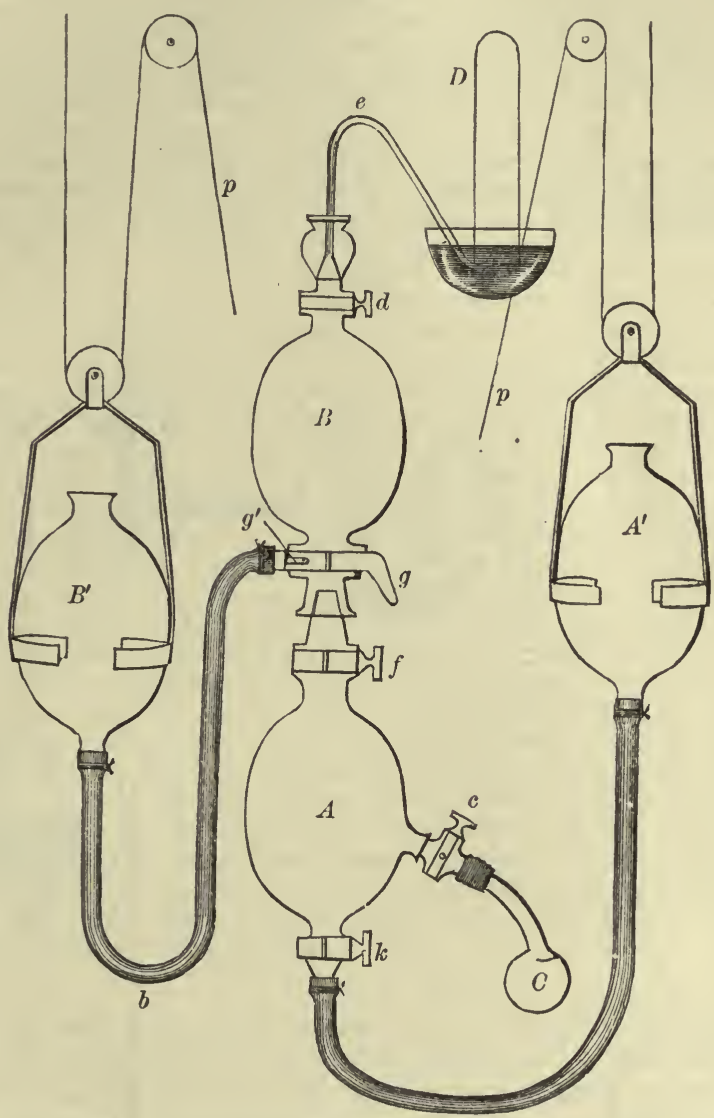

Diagrammatic illustration of Ludwig's Mercurial Gas-pump: $A$ and $B$ are two glass globes connected by strong India-rubber tubes, $a$ and $b$, with two similar glass globes, $A^{\prime}$ and $B^{\prime} . A$ is further connected by means of the stopcock $c$ with the receiver $C$ containing the blood (or other fluid) to be analyzed, and $B$ by means of the stopcock $d$ and the tube $e$ with the receiver $D$ for receiving the gases. $A$ and $B$ are also connected with each other by means of the stopcocks $f$ and $g$, the latter being so arranged that $B$ also communicates with $B^{\prime}$ by the passage $g^{\prime} . A^{\prime}$ and $B^{\prime}$ being full of mercury, and the cocks $k, f, g$, and $d$ being open, but $c$ and $g^{\prime}$ closed, on raising $A^{\prime}$ by means of the pulley $p$ the mercury of $A^{\prime}$ fills $A$, driving ont the air contained in it into $B$, and so out through $e$. When the mercury has risen above $g, f$ is closed, and $g^{\prime}$ being opened, $B^{\prime}$ is in turn raised until $B$ is completely filled with mercury, all the air previously in it being driven out through $e$. Upon closing $d$ and lowering $B^{\prime}$ the whole of the mercury in $B$ falls in $B^{\prime}$, and a vacuum consequently is established in $B$. On closing $g^{\prime}$, but opening $g, f$, and $k$, and lowering $A^{\prime}$, a vacuum is similarly established in $A$ and in the junction between $A$ and $B$. If the cock $c$ be now opened, the gases of the blood in $C$ escape into the vacuum of $A$ and $B$. By raising $A^{\prime}$ after the closure of $c$ and opening of $d$, the gases so set free are driven from $A$ into $B$, and by the raising of $B^{\prime}$ from $B$, through $e$ into the receiver $D$, standing over mercury.

into and completely fills the latter, the air previously present being driven out. After adjusting the cocks, the movable globe is then depressed thirty inches below the fixed one, in which the consequent fall of the mercury produces an almost complete vacuum. By turning the proper cock this vacuum is put into 
connection with the receiver containing the blood, which thereupon becomes proportionately exhausted. By again adjusting the cocks and once more elevating the movable globe, the gas thus extracted is driven out of the fixed globe into a receiver. The vacuum is then once more established and the operation repeated as long as gas continues to be given off from the blood.

A modified form of pump working on the same principles as that of Ludwig, but involving the use of only one globe to be made vacuous and one movable reservoir for mercury, has been constructed by Pflüger. It presents several advantages over the one just described, the chief being that (1) non-defibrinated blood may be used for the extraction of gases, (2) the vacuum into which the gases are evolved is large, (3) this vacuum is kept dry by being connected laterally with a vacuous chamber containing sulphuric acid. The details of this construction are, however, complicated, and the greatest care is required in its use to avoid break-

FIG. 93.

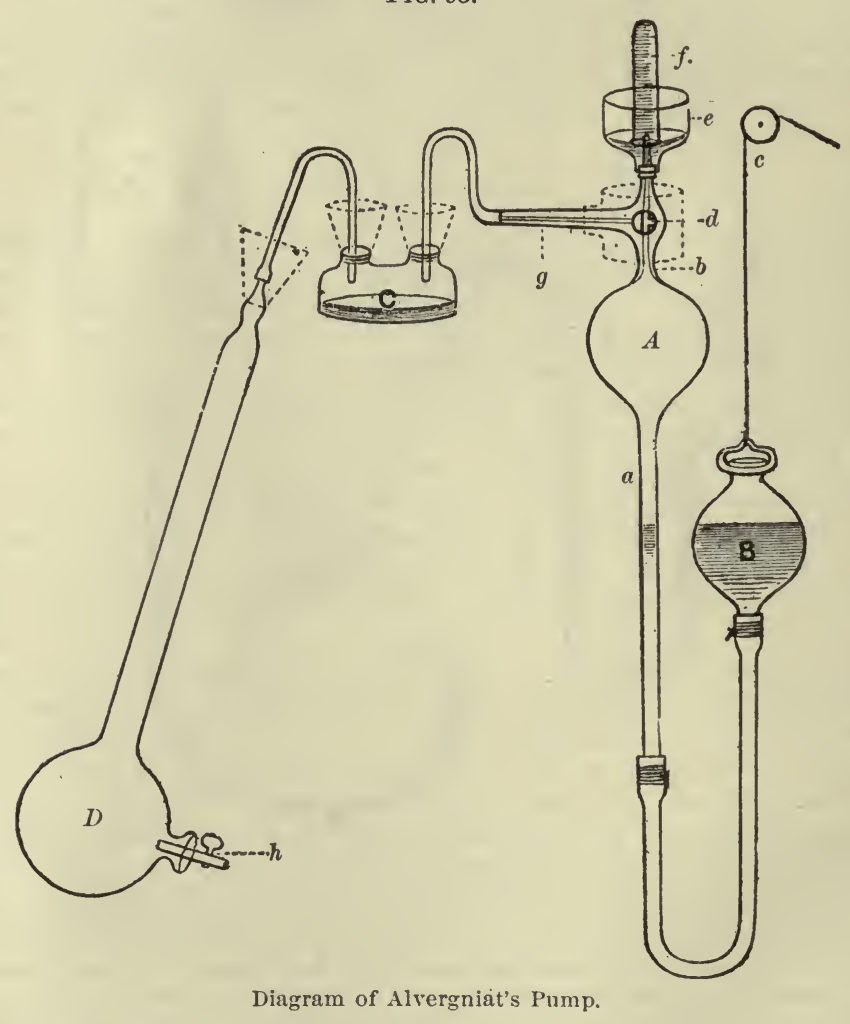

age. Of later years a simplified form of pump has been introduced for laboratory work. It was first used by Gréhant and Paul Bert, and is now frequently called an Alvergniat's pump, from the name of its present maker. Fig. 93 gives a diagrammatic representation of its construction.

$A$ is a glass bulb some five inches in diameter, blown on to a glass tube $a$ below and on to a vertical tube $b$ above. The lower end of $a$ is connected by a thickwalled India-rubber tube with a reservoir for mercury $B$, which can be raised and lowered by means of a string passing over a pulley $c$. The vertical tube $b$ is thickened at one place, and into this thickened portion a three-way tap $d$ is ground. The upper end of $b$ is prolonged (above the three-way tap) into a fine point. This point passes by a tight joint through the bottom of a vessel $e$, which can be partly filled with mercury, and over which a receiver $f$, filled with mercury 
for the collection of the gases, can be inverted. A tube $g$ fused on laterally to one opening of the three-way tap $d$ places the latter in connection with a thickwalled Woulff's bottle $C$ containing a layer of strong sulphuric acid. The second tubulure of this bottle is similarly connected by an elastic tube with the vessel I), into which blood or other fluid may be introduced by means of the tap $h$. All the movable joints of the apparatus are protected by India-rubber tubes into which water can be poured, and a metal easing around the tap $d$, which may also be filled with water, similarly prevents the possibility of any leakage here.

The pump is used as follows: By placing the tap $d$ in the position shown in the figure and raising $B$, the bulb $A$ may be filled with mereury up to the top, the contained air being expelled through the upper end of $b$. By a slight turn of the tap all connection between $A$ and either the tube $g$ or the upper part of $b$ may be cut off, and on lowering $B$ a vacuum is established in the bulb $A$ and part of the tube a. A may now be connected by the tap $d$ with the tube $g$, and hence with $C$ and $D$, and, $h$ being elosed, a partial vacuum is established in $C$ and $D$. By means of the tap $d$ the air in $A$ may be cut off from $g$, and on raising $B$ and placing the plug of $d$ as shown in the figure this air may be expelled through the upper end of $b$. By slightly turning $d$ and lowering $B$ a vacuum is again established in $A$, and, as before, a further portion of air in $C$ and $D$ may be allowed to pass over into $A$ and the vacuum in $D$ and $C$ increased. In this way all the air in $D$ can be extracted, the final stages being facilitated by the admission of a little water into $D$, the last traces of air being driven over into $A$ by the rush of vapor from the water. A known rolume of blood having been collected over mercury in a small tube is now allowed to enter $D$ through the tap $h$ and yields up its gases to the vacuum. A repetition of the processes by which the air in $D$ was originally extracted will now remove the gases which have been given off from the known volume of blood, the only difference being that now the tube $f$ filled with mercury is inverted in the trough $e$ over the upper end of the tube $b$. In this way the gases originally in $D$ are nut allowed to escape into the air, as was the case when the apparatus was being originally made vacuous, but are collected in $f$ for subsequent analysis. During the extraction of the gases from the blood the bulb $D$ is immersed in a vessel of warm water, to facilitate the exit of the gases and, by causing the formation of large quantities of aqueous vapor, to sweep the gases rapidly over into $A$. The sulphuric acid chamber $C$ dries the vacuum before the admission of the blood into $D$, and hence makes it more perfect and causes the most complete and rapid evolution of gases from the blood.

The average composition of the gas thus obtained from each of the two kinds of blood (the arterial blood being taken from a large artery, and the venous blood from the right side of the heart) is, stated in round numbers, as follows:

From 100 vols. may be obtained-

$\begin{array}{cccc} & \text { of oxygen. } & \text { Of carbonic acid. } & \text { Of nitrogen. } \\ \text { Of arterial blood, } & 20 \text { vols. } & 40 \text { vols. } & 1 \text { to } 2 \text { vols. } \\ \text { Of venous blood, } & 8 \text { to } 12 \text { vols. } & 46 \text { vols. } & 1 \text { to } 2 \text { vols. }\end{array}$
all measured at $760 \mathrm{~mm}$. and $0^{\circ} \mathrm{C}$.

That is to say, venous blood, as compared with arterial blood, contains 8 to 12 per cent. less oxygen and 6 per cent. more carbonic acid. It must be remembered, however, that while arterial blood from whatever artery taken has always nearly the same proportion of gases, or at all events the same amount of oxygen, the amount of oxygen in venous blood, even when taken from the same vein, may vary a good deal, still more so when it is taken from different veins. 'The reason of this we shall see hereafter.

It will be convenient to consider the relations of each of these gases separately. 


\section{The Relations of Oxygen in the Blood.}

$\S 286$. When a liquid such as water is exposed to an atmosphere containing a gas such as oxygen, some of the oxygen will be dissolved in the water, that is to say, will be absorbed from the atmosphere. The quantity which is so absorbed will depend on the pressure of the oxygen in the atmosphere above; the greater the pressure of the oxygen, the larger the amount which will be absorbed. If the pressure of the whole atmosphere remain the same, at $760 \mathrm{~mm}$. of mercury for instance (the ordinary atmospheric pressure), the pressure of the oxygen may be increased or diminished by increasing or diminishing the proportion of oxygen in the atmosphere. So that with an atmosphere remaining at any given pressure the quantity of oxygen absorbed will depend on the quantity present in that atmosphere. If, on the other hand, water, already containing a good deal of oxygen dissolved in it, be exposed to an atmosphere containing little or no oxygen, the oxygen will escape from the water into the atmosphere. The oxygen, in fact, which is dissolved in the water, like the oxygen in the atmosphere above, stands at a certain pressure, the amount of pressure depending on the quantity dissolved; and when water containing oxygen dissolved in it is exposed to any atmosphere, the result, that is, whether the oxygen escapes from the water into the atmosphere or passes from the atmosphere into the water, depends on whether the pressure of the oxygen in the water is greater or less than the pressure of the oxygen in the atmosphere. Hence, when water is exposed to oxygen, the oxygen either escapes or is absorbed until equilibrium is established between the pressure of the oxygen in the atmosphere above and the pressure of the oxygen in the water below. This result is, as far as mere absorption and escape are concerned, quite independent of what other gases are present in the water or in the atmosphere. Suppose a half-litre of water was lying at the bottom of a two-litre flask, and that the atmosphere in the flask above the water was one-third oxygen; it would make no difference, as far as the absorption of oxygen by the water was concerned, whether the remaining two-thirds of the atmosphere was carbonic acid or nitrogen or hydrogen, or whether the space above the water was a vacuum filled to one-third with pure oxygen. Hence, it is said that the absorption of any gas depends on the partial pressure of that gas in the atmosphere to which the liquid is exposed. This is true not only of oxygen and water, but of all gases and liquids which do not enter into chemical combination with each other. Different liquids will, of course, absorb different gases with differing readiness; but with the same gas and the same liquid, the amount absorbed will depend directly on the partial pressure of the gas in the overlying space. It should be added that the process is much influenced by temperature. Hence, to state the matter generally, the absorption of any gas by any liquid will depend on the nature of the gas, the nature of the liquid, the pressure of the gas, and the temperature at which both stand.

Now it might be supposed, and indeed was once supposed, that the oxygen in the blood was simply dissolved by the blood. If this were so, then the amount of oxygen present in any given quantity of blood exposed to any given atmosphere ought to rise and fall steadily and regularly as the partial pressure of oxygen in that atmosphere is increased or diminished; the ahsorption (or escape) of oxygen ought to follow what is known as the Henry-Dalton law of pressures. But this is found not to be the case. If we expose blood containing little or no oxygen to a succession of atmospheres containing increasing quantities of oxygen, we find that at first there is a very rapid absorption of the available oxygen, and then this somewhat suddenly ceases or becomes very small ; and if, on the other hand, we submit 
arterial blood to successively diminishing pressures, we find that for a long time very little oxygen is given off, and then suddenly the escape becomes very rapid. The absorption of oxygen by blood does not follow the general law of absorption according to pressure. The phenomena, on the other hand, suggest the idea that the oxygen in the blood is in some particular combination with a substance or some substances present in the blood, the combination being of such a kind that it holds good during a lowering of pressure down to a certain limit, and that then dissociation readily occurs; we may add that this limit is very closely dependent on temperature. It is, however, not to be supposed that as the pressure is lowered, no oxygen whatever is given off from the substance until a certain point is reached, and that at that point the whole store is in an instant dissociated, no more remaining to be given off. The case is rather that while pressure is being lowered down to a certain point, no appreciable dissociation takes place, and that then having begun it iucreases rapidly with each further lowering of pressure until the whole of the oxygen is given off. During the narrow range, between the first beginning to give off oxygen and the completion of the giving off, the compound of the oxygen with the substance or substances may be spoken of as partly, that is more or less, dissociated. What is the substance or what are the substances with which the oxygen is thus peculiarly combined?

If serum free from red corpuscles be used in such absorption experiments, it is found that, as compared with the entire blood, very little oxygen is absorbed, about as much as would be absorbed by the same quantity of water; and such as is absorbed does follow the law of pressure. In natural arterial blood the quantity of oxygen which can be obtained from serum is exceedingly small; it does not amount to half a volume in one hundred volumes of the entire blood to which the serum belonged. It is evident that the oxygen which is present in blood is in some way or other peculiarly connected with the red corpuscles. Now, the distinguishing feature of the red corpuscles is the presence of hæmoglobin. We have already seen (\$24) that this constitutes 90 per cent. of the dried red corpuscles. There can be i priori little doubt that this must be the substance with which the oxygen is associated, and to the properties of this body we must therefore direct our attention.

$\S 287$. Homoglobin. When separated from the other constituents of the serum, hæmoglobin appears as a substance, either amorphous or crystalline, readily soluble in water (especially in warm water) and in serum.

Since hæmoglobin is soluble in serum, and since the identity of the crystals observed occasionally within the corpuscles with those obtained in other ways shows that the hæmoglobin as it exists in the corpuscle is the same thing as that which is artificially prepared from blood, it is evident that some peculiar relationship between the stroma and the hæmoglobin must, in natural blood, keep the latter from being dissolved by the serum. Hence, in preparing hæmoglobin it is necessary first of all to break up this connection and to set the hæmoglobin free from the corpuscles. This may be done by the addition of water, of ether, of chloroform, and of bile-salts, or by repeatedly freezirig and thawing: blood so treated becomes "laky" (cf. \& 24). It is also of advantage previously to remove the alkaline serum as much as possible, so as to operate only on the red corpuscles. The stroma and hæmoglobin being thus separated, a solution of hremoglobin is the result. The alkalinity of the solution, when present, being reduced by the cautious addition of dilute acetic acid, and the solvent power of the aqueous medium being diminished by the addition of onefourth its bulk of alcohol, the mixture, set aside in a temperature of $0^{\circ} \mathrm{C}$. in order still further to reduce the solubility of the hæmoglobin, readily crystallizes, when the blood used is that of the dog, cat, horse, rat, guinea-pig, etc. In the case of the dog, indeed, it is simply sufficient to add ether carefully to the blood until it just becomes "laky," and then to let it stand in a cool place; the mixture soon becomes 
a mass of crystals. The crystals may be separated by filtration, redissolved in water, and recrystallized.

Hæmoglobin from the blood of the rat, guinea-pig, squirrel, hedgehog, horse, cat, dog, goose, and some other animals, crystallizes readily, the crystals being generally slender, four-sided prisms belonging to the rhombic

[FIG. 94.

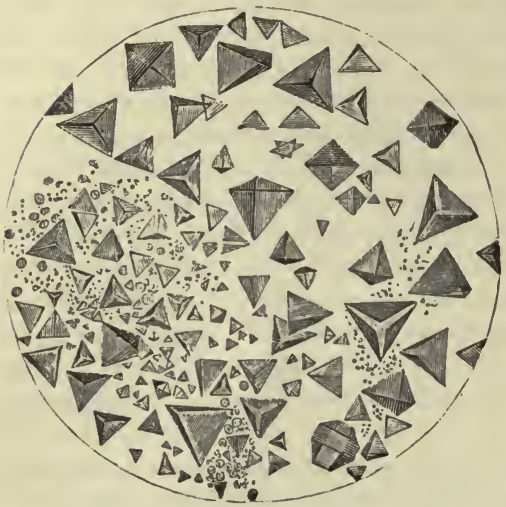

Tetrahedral from Blood of the Pig.] system and often appearing quite acicular. [Figs. 94, 95, 96.] The crystals from the blood of the guineapig are octahedral, but also belong to the rhombic system; those of the squirrel are six-sided plates. The blood of the $n x$, sheep, rabbit, pig, and man erystallizes with difficulty. Why these differences exist is not known; but the composition and the amount of water of crystallization vary somewhat in the crystals obtained from different animals. In the dog the percentage composition of the crystals has been determined as C. $53.85, \mathrm{H} .7 .32$, N. 16.17, O. 21.84 , S. 0.39 , Fe. 0.43 , with 3 to 4 per cent. of water of crystallization. It will thus be seen that hæmoglobin contains, in addition to the other elements usually present in proteid substances, a certain amount of iron ; that is to say, the element iron is a distinct part of the hæmoglobin

[FIG, 95.

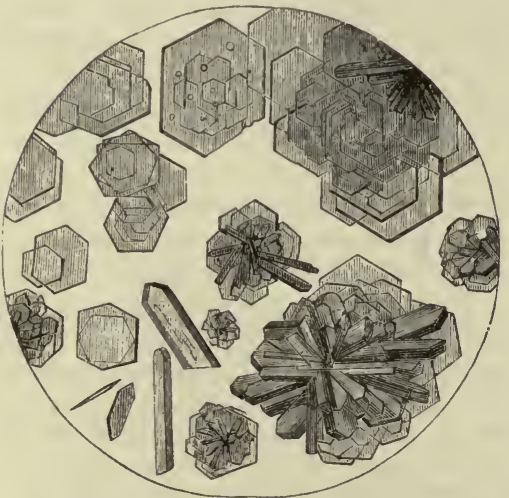

Hexagonal Crystals from Blood of Squirrel. On these six-sided plates, prismatic crystals, grouped in a stellate manner, not infrequently occur. (After Funke)].
[FIG. 96.

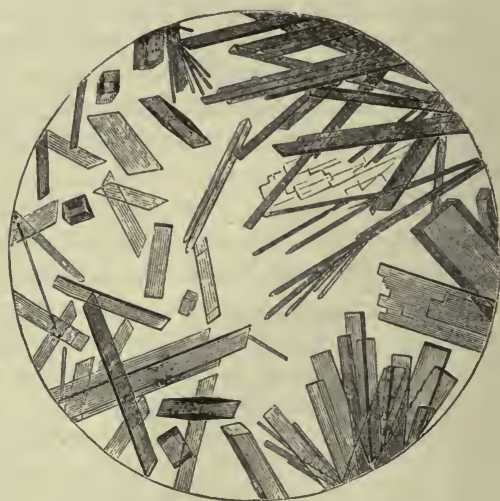

Prismatic, from Human Blood.]

molecule, a fact which of itself renders hæmoglobin remarkable among the chemical substances present in the animal body.

$\$ 288$. The crystals when seen in a sufficiently thick layer under the microscope have the same bright scarlet color as arterial blood has to the naked eye; when seen in a mass they naturally appear darker. An aqueous solution of hæmoglobin, obtained by dissolving purified crystals in distilled 
water, has also the same bright arterial color. A tolerably dilute solution placed before the spectroscope is found to absorb certain rays of light in a peculiar and characteristic manner. A portion of the red end of the spectrum is absorbed, as is also a much larger portion of the blue end; but what is most striking is the presence of two strongly marked absorption bands, lying between the solar lines $D$ and $E$. (See Fig. 97.) Of these the one toward the red side, sometimes spoken of as the band $(a)$, is the thinnest, but the most intense, and in extremely dilute solutions (Fig. 97, 1) is the only one visible; its middle lies at some little distance to the blue side of $D$. Its position may be more exactly defined by expressing it in wave-lengths. As is well known, the rays of light which make up the spectrum differ in the length of their ways, diminishing from the red end, where the waves are longest, to the blue end, where they are shortest. Thus, Frauenhofer's line $D$ corresponds to rays having a wave-length of 589.4 millionths of a millimetre. Using the same unit, the centre of this absorption band, $a$, of hæmoglobin corresponds to the wave-length 578; as may be seen in Fig. 97, where, however, the numbers of the divisions of the scale indicate only 100,000 ths of a millimetre. The other, sometimes called $b$, much broader, lies a little to the red side of $E$, its blueward edge, even in moderately dilute solutions (Fig. 97, 2), coming close up to that line; its centre corresponds to about wave-length 539. Each band is thickest in the middle and gradually thins away at the edges. These two absorption bands are extremely characteristic of a solution of hæmoglobin. Even in very dilute solutions both bands are visible (they may be seen in a thickness of $1 \mathrm{~cm}$. in a solution containing 1 grm. of hæmoglobin in 10 litres of water), and that when scarcely any of the extreme red end and very little of the blue end is cut off. They then appear not only faint but narrow. As the strength of the solution is increased the bands broaden and become more intense; at the same time both the red end, and still more the blue end, of the whole spectrum are encroached upon (Fig. $97,3)$. This may go on until the two absorption bands become fused together into one broad band (Fig. 97, 4). The only rays of light which pass through the hæmoglobin solution are those in the green between the blueward edge of the united bands and the general absorption, which is now rapidly advancing from the blue end, and those in the red between the united bands and the general absorption at the red end. If the solution be still further increased in strength, the interval on the blue side of the united bands becomes absorbed also, so that the only rays which pass through are the red rays lying to the red side of $D$; these are the last to disappear, and hence the natural red color of the solution as seen by transmitted light. Exactly the same appearances are seen when crystals of hæmoglobin are examined with a micro-spectroscope. They are also seen when arterial blood itself (diluted with saline solutions, so that the corpuscles remain in as natural a condition as possible) is examined with the spectroscope, as well as when a drop of blood, which from the necessary exposure to air is always arterial, is examined with the micro-spectroscope. In fact, the spectrum of hæmoglobin is the spectrum of normal arterial blood.

$\$ 289$. When crystals of hæmoglobin, prepared in the way described above, are subjected to the vacuum of the mercurial air-pump, they give off a certain quantity of oxygen, and at the same time they change in color. The quantity of oxygen given off is definite, $1 \mathrm{grm}$. of the crystals giving off 1.59 c.c. of oxygen measured at $760 \mathrm{~mm}$. $\mathrm{Hg}_{\text {. and }} 0^{\circ} \mathrm{C}$. In other words, the crystals of hæmoglobin, over and above the oxygen which enters intimately into the composition of the molecule (and which alone is given in the elementary composition previously stated), contain another quantity of oxygen, which is in loose combination only, and which may be dissociated from 
them by subjecting them to a sufficiently low pressure. The change of color which ensues when this loosely combined oxygen is removed, is character-

FIG. 9\%.

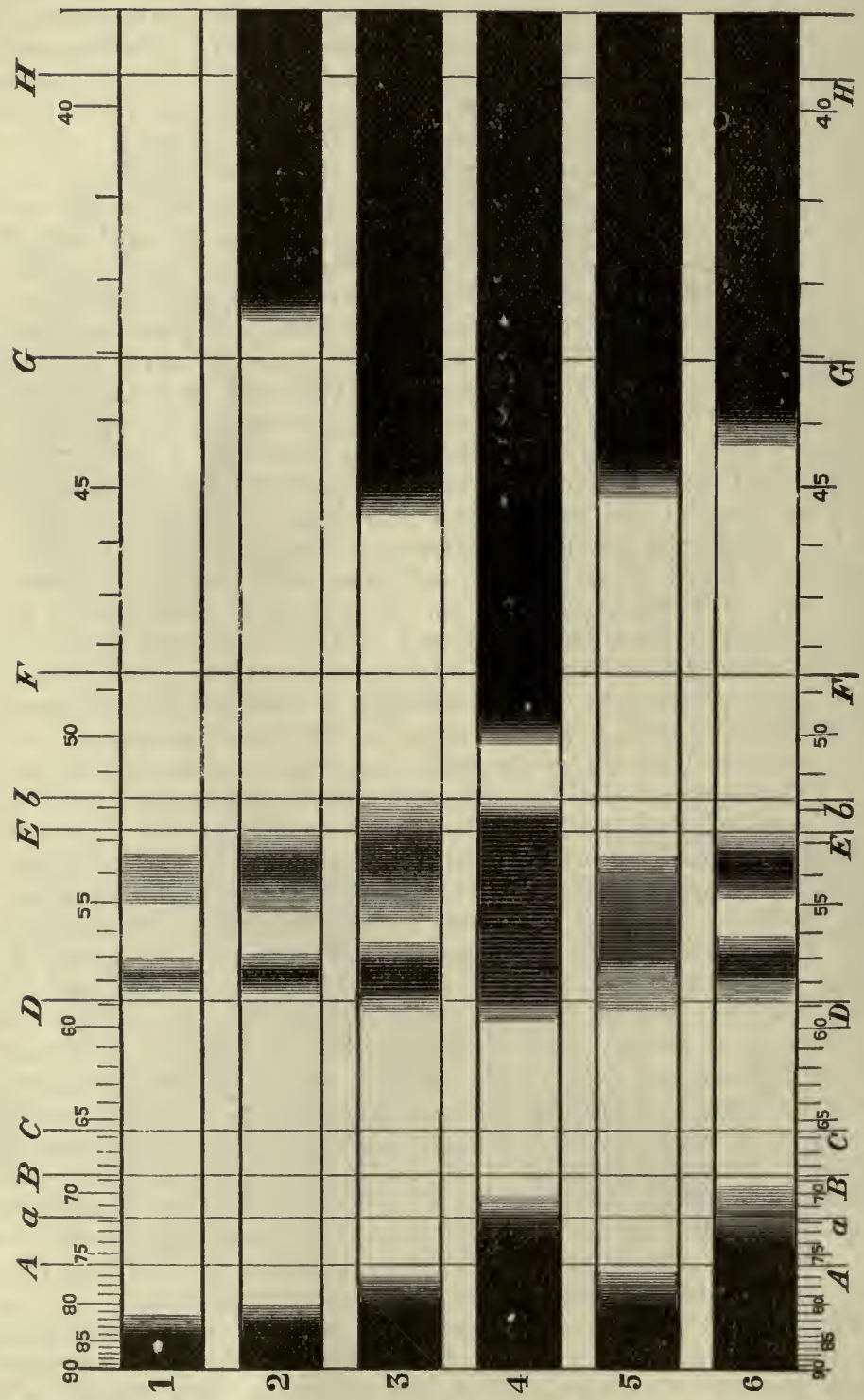

The Spectra of Oxy-hæmoglobin in Different Grades of Concentration, of (reduced) Hæmoglobin, and of Carbonic-oxide-hæmoglobin. (After P'reyer and Gamgee) : 1 to 4 , solution of oxyhæmoglobin containing-(1) less than 0.01 per cent., (2) 0.09 per cent., (3) 0.37 per cent., (4) 0.8 per cent. 5, solution of (reduced) hæmoglobin containing about 0.2 per cent. 6 , solution of carbonicoxide-hæmoglobin. In each of the six cases the layer brought before the spectroscope was $1 \mathrm{~cm}$. in thickness. The letters $(A, a$, etc.) indicate Frauenhofer's lines, and the figures wave-lengths expressed in 100,000 th s of a millimetre. 
istic ; the crystals become darker and more of a purple hue, and at the same time dichroic, so that while the thicker parts are purple, the thin edges appear greenish.

An ordinary solution of hæmoglobin, like the crystals from which it is formed, contains a definite quantity of oxygen in a similarly peculiar loose combination ; this oxygen it also gives up when subjected in the air-pump to sufficiently low pressure, becoming at the same time of a purplish hue. This loosely combined oxygen may also be removed by passing a stream of hydrogen or other indifferent gas through the solution; the stream of hydrogen acts like an oxygen-vacuum to the hæmoglobin and thus disassociation is effected. Carbonic acid gas is unsuitable for this purpose, since, as we shall see, being an acid, it acts in another way on the hæmoglobin. The oxygen may also be removed from the hæmoglobin not only by physical but also by chemical means, as by the use of reducing agents. Thus if a few drops of ammonium sulphide or of an alkaline solution of ferrous sulphate kept from precipitation by the presence of tartaric acid, be added to a solution of hæmoglobin, or even to an unpurified solution of blood corpuscles such as is afforded by the washings from a blood-clot, the oxygen in loose combination with the hrmoglobin is immediately seized upon by the reducing agent. This may be recognized at once by the characteristic change of color; from a bright scarlet the solution becomes of a purplishclaret color, when seen in any thickness, but greenish when sufficiently thin; the color of the reduced solution is exactly like that of the crystals from which the loose oxygen has been removed by the air-pump.

Examined by the spectroscope, this reduced solution, or solution of reduced homoglobin, as we may now call it, offers a spectrum (Fig. 97, 5) very different from that of the unreduced solution. The two absorption bands have disappeared, and in their place there is seen a single, much broader, but at the same time much fainter band, whose middle occupies a position about midway between the two absorption bands of the unreduced solution, though the redward edge of the band shades away rather further toward the red than does the other edge toward the blue; its centre corresponds to about wave-length 555. At the same time the general absorption of the spectrum is different from that of the unreduced solution; less of the blue end is absorbed. Even when the solutions become tolerably concentrated, many of the bluish-green rays to the blue side of the single band still pass through. Hence the difference in color between hæmoglobin which retains the loosely combined oxygen, ${ }^{1}$ and hæmoglobin which has lost its oxygen and become reduced. In tolerably concentrated solutions, or tolerably thick layers, the former lets through the red and orange-yellow rays, the latter the red and the bluish-green rays. Accordingly, the one appears scarlet, the other purple. In dilute solutions, or in a thin layer, the reduced hæmoglobin lets through so much of the green rays that they preponderate over the red, and the resulting impression is one of green. In the unreduced hæmoglobin or oxy-hæmoglobin, the potent yellow which is blocked out in the reduced hæmoglobin makes itself felt, so that a very thin layer of oxyhæmoglobin, as in a single corpuscle seen under the microscope, appears yellow rather than red.

It must be remembered that when we speak of reduced hæmoglobin (or more briefly hæmoglobin) with a purple color and a characteristic onebanded spectrum, we mean hæmoglobin which has lost all its loosely associated oxygen. If a quantity of $0 x y$-hæmoglobin be exposed to an insuffi-

1 For brevity's sake we may call the hæmoglobin containing oxygen in loose combination, oxy-hæmoglobin. and the hæmoglobin from which this loosely combined oxygen has been removed, reduced hæmoglobin or sinply hæmoglobin. 
ciently low pressure, or to the action of an insufficient quantity of the reducing action, it gives up a part only of its oxygen; it is only partly reduced. Such a partly reduced solution still shows the two bands of oxyhæmoglobin.

$\S 290$. When the hæmoglobin solution (or crystal), which has lost its oxygen by the action either of the air-pump or of a reducing agent or by the passage of an indifferent gas, is exposed to air containing oxygen, an absorption of oxygen at once takes place. If sufficient oxygen be present, the hæmoglobin seizes upon sufficient oxygen to obtain its full complement, each gramme taking up in combination 1.59 c.c. of oxygen; if there be an insufficient quantity of oxygen the hæmoglobin still remains partly reduced; or perhaps we may say that a part only of the hremoglobin gets its allowance while the remainder continues reduced. If the amount of oxygen be sufficient, the solution (or crystal), as it takes up the oxygen, regains its bright scarlet color and its characteristic absorption spectrum, the single band being replaced by the two. Thus if a solution of oxy-hæmoglobin in a test-tube, after being reduced by the action of a drop or two of ammonium sulphide solution and thus showing the purple color and the single band, be shaken up with air, the bright scarlet color at once returns, and when the fluid is placed before the spectroscope, it is seen that the single faint broad band of the reduced hrmoglobin has wholly disappeared, and that in its place are the two sharp thinner bands of the oxy-hæmoglobin. If left to stand in the test-tube the quantity of reducing agent still present is generally sufficient again to rob the hæmoglobin of the oxygen thus newly acquired, and soon the scarlet hue fades back again into the purple, the two bands giving place to the one. Another shake and exposure to air will, however, again bring back the scarlet hue and the two bands; and once more these may disappear. In fact, a few drops of the reducing fluid will allow this game of hrmoglobin taking oxygen from the air and giving it up to the reducer to be played over and over again; at each turn of the game the color shifts from scarlet to purple and from purple to scarlet, while the two bands exchange for the one and the one for the two.

$\$ 291$. Color of venous and arterial blood. Evidently we have in these properties of hæmoglobin an explanation of at least one-half of the great respiratory process, and they teach us the meaning of the change of color which takes place when venous blood becomes arterial or arterial venous.

In venous blood, as it issues from the right ventricle, the oxygen present is insufficient to satisfy wholly the hæmoglobin of the red corpuscles; the hæmoglobin is, to a large extent, reduced, hence the purple color of venous blood. When ordinary venous blood, diluted without access of oxygen, is brought before the spectroscope, the two bands of oxy-hæmoglobin are seen. This is explained by the fact that in partly reduced hæmoglobin, which we may conveniently regard as a mixture of oxy-hæmoglobin and (reduced) hæmoglobin, the two sharp bands of the former are always much more readily seen than the much fainter band of the latter. Now in ordinary venous blood there is always some loose oxygen, removable by diminished pressure or otherwise; the hæmoglobin is only partly reduced, there is always some, indeed a considerable quantity, of oxy-hæmoglobin as well as (reduced) hæmoglobin. It is only under special circumstances, as, for instance, after death by what we shall presently speak of as asphyxia, that all the loose oxygen of the blood disappears; and then the two bands of oxy-hrmoglobin vanish too. If even only a small quantity of oxygen be present, so distinct are the two bands that a solution of completely reduced hæmoglobin may be used as a test for the presence of oxygen; if oxygen be present in any fluid 
to which the reduced hæmoglobin is added, the single band immediately gives way to the two bands of oxy-hæmoglobin.

As the venous blood passes through the capillaries of the lungs, this reduced hæmoglobin takes from the pulmonary air its complement of oxygen, all or nearly all the hæmoglobin of the red corpuscles becomes oxy-hæmoglobin, and the purple color forthwith shifts into scarlet. For careful observations show that the hrmoglobin of arterial blood is saturated or nearly saturated with oxygen; it probably falls short of complete saturation by about 1 vol. of oxygen in $100 \mathrm{vols}$. of blood. By increasing the pressure of the oxygen, an additional quantity may be driven into the blood, but this, after the hæmoglobin has become completely saturated, is effected by simple absorption. The quantity so added is extremely small compared with the total quantity combined with the hæmoglobin.

Passing from the left ventricle to the capillaries of the tissues the oxyhæmoglobin gives up some of its oxygen to the tissues, becoming, in part, reduced hæmoglobin, and the blood in consequence becomes once more venous, with a purple hue. Thus the red corpuscles by virtue of their hæmoglobin are emphatically oxygen-carriers. Undergoing no intrinsic change in itself, the hæmoglobin combines in the lungs with oxygen, which it carries to the tissues; these, more greedy of oxygen than itself, rob it of its charge, and the reduced hæmoglobin hurries back to the lungs in the venous blood for another portion. The change from venous to arterial blood is then in part (for as we shall see there are other events as well) a peculiar combination of lıæmoglobin with oxygen, while the change from arterial to venous is, in part also, a reduction of oxy-hæmoglobin; and the difference of color between venous and arterial blood depends almost entirely on the fact that the reduced hæmoglobin of the former is of purple color, while the oxyhæmoglobin of the latter is of a scarlet color.

There may be other causes of the change of color, but these are wholly subsidiary and unimportant. When a corpuscle swells, its refractive power is diminished, and in consequence the number of rays which pass into and are absorbed by it are increased at the expense of those reflected from its surface; anything therefore which swells the corpuscles, such as the addition of water, tends to darken blood, and anything, such as a concentrated saline solution, which causes the corpuscles to shrink, tends to brighten blood. Carbonic acid has apparently some influence in swelling the corpuscles, and therefore may aid in darkening the venous blood.

$\S 292$. We have spoken of the combination of hæmoglobin with oxygen as being a peculiar one. The peculiarity consists in the facts that the oxygen may be associated and dissociated, without any general disturbance of the molecule of hæmoglobin, and that dissociation may be brought about very readily. Hæmoglobin combines in a wholly similar manner with other gases. If carbonic oxide (monoxide) be passed through a solution of hæmoglobin, a change of color takes place, a peculiar bluish tinge making its appearance. At the same time the spectrum is altered; two bands are still visible, but on accurate measurement it is seen that they are placed more toward the blue end than are the otherwise similar bands of oxy-hrmoglobin (see Fig. 97,6); their centres corresponding respectively to about wavelengths 572 and 533 , while those of oxy-hæmoglobin, as we have seen, correspond to 578 and 539 . When a known quantity of carbonic oxide gas is sent through a hæmoglobin solution, it will be found on examination that a certain amount of the gas has been retained, an equal volume of oxygen appearing in its place in the gas which issues from the solution. If the solution so treated be crystallized, the crystals will have the same characteristic color and give the same absorption spectrum as the solution; when subjected to 
the action of the mercurial pump, they will give off a definite quantity of carbonic oxide, 1 gramme of the erystals yielding 1.59 c.c. of the gas. In fact hæmoglobin combines lnosely with earbonic oxide just as it does with oxygen; but its affinity with the former is greater than with the latter. While carbonic oxide readily turns out oxygen, oxygen cannot so readily turn out carbonic oxide. Indeed, carbonic oxide has been used as a means of driving out and measuring the quantity of oxygen present in any given blood. This property of carbonic oxide explains its poisonous nature. When the gas is breathed, the reduced and the unreduced hæmoglobin of the venous blood unite with the carbonic oxide, and hence the peculiar, bright cherry-red color observable in the blood and tissues in cases of poisoning by this gas. The carbonic-oxide hæmoglobin, however, is of no use in respiration; it is not an oxygen-carrier, nay more, it will not readily, though it does so slowly and eventually, give up its carbonic oxide for oxygen, when the poisonous gas ceases to enter the chest and is replaced by pure air. The organism is killed by suffocation, by want of oxygen, in spite of the blood not assuming any dark venous color; to adopt a phrase which has been used, the corpuscles are paralyzed.

Hæmoglobin smilarly forms a compound, having a characteristic spectrum, with nitric oxide, more stable even than that with carbonic oxide.

It has been supposed by some that the oxygen thus associated with hæmoglobin is in the condition known as ozone; but the arguments urged in support of this view are inconclusive.

\section{Products of the Decomposition of Homoglobin.}

$\S 293$. Although a crystalline body, hæmoglobin diffuses with great difficulty. This arises from the fact that it is in part a proteid body; it consists of a colorless proteid, associated with a colored substance, which may be separated out from the hæmoglobin, though not in the exact condition in which it naturally exists in the compound; this substance when separated out appears as a brownish-red body known as homatin. All the iron belonging to the hæmoglobin is in reality attached to the hæmatin. A solution of hæmoglobin, when heated, coagulates, the exact degree at which the coagulation takes place depending on the amount of dilution; at the same time it turns brown from the setting free of the hæmatin. If a strong solution of hæmoglobin be treated with acetic (or other) acid, the same brown color, from the appearance of hæmatin, is observed. The proteid constituent, however, is not coagulated, but by the action of the acid passes into the state of acid-albumin. On adding ether to the mixture, and shaking, the hæmatin is dissolved in the supernatant acid ether, which it colors a dark red, and which, examined with the spectroscope, is found to possess a well-marked spectrum, the spectrum of the so-called acid hæmatin of Stokes (Fig. 98,6). The proteid in the water below the ether appears in a coagulated form owing to the action of the ether. In a somewhat similar manner alkalies split up hæmoglobin into a proteid constituent and hæmatin.

The exact nature of the proteid constituent of hæmoglobin has not as yet been clearly determined. It was supposed to be globulin (hence the name hæmatoglobulin, contracted into hæmoglobin), but though belonging to the globulin family, has characters of its own; it is possibly a mixture of two or more distinct proteids. It has been provisionally named globin and is said to be free from ash.

§ 294. Hæmatin when separated from its proteid fellow, and purified, appears as a dark-brown amorphous powder, or as a scaly mass with a 
RESPIRATORY CHANGES IN THE BLOOD.

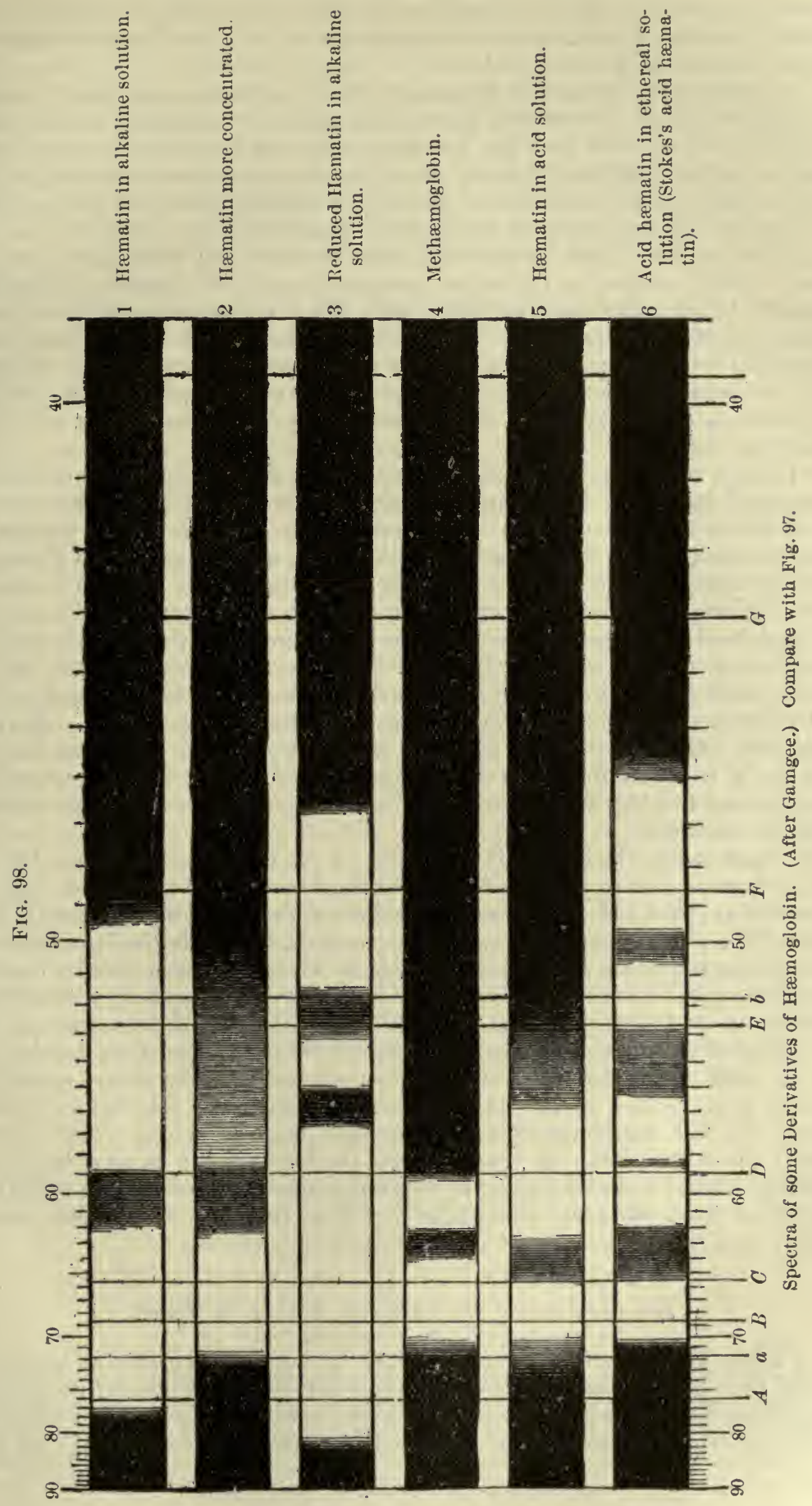


metallic lustre, having the probable composition of $\mathrm{C}_{32}, \mathrm{H}_{34}, \mathrm{~N}_{4}, \mathrm{Fe}, \mathrm{O}_{5 .}{ }^{1}$ It is fairly soluble in dilute acid or alkaline solutions, and then gives characteristic spectra (Fig. 98, 1, 2, 5).

An interesting feature in hæmatin is that its alkaline solution is capable of being reduced by reducing agents, the spectrum changing at the same time (Fig. 98, 3), and that the reduced solution will, like the hæmoglobin, take up oxygen again on being brought into contact with air or oxygen. This would seem to indicate that the oxygen-holding power of hæmoglobin is connected exclusively with its hæmatin constituent.

By the action of strong sulphuric acid hæmatin may be rolbbed of all its iron. It still retains the feature of possessing color, the solution of iron-free hæmatin being a dark rich brownish-red ; but is no longer capable of combining loosely with oxygen. This indicates that the iron is in some way associated with the peculiar respiratory functions of hæmoglobin; though it is obviously an error to suppose, as was once supposed, that the change from venous to arterial blood consists essentially in a change from a ferrous to a ferric salt.

Though not crystallizable itself, hæmatin forms with hydrochloric acid a compound, occurring in minute rhombic crystals, known as homin crystals.

When blood is left until it decomposes, the hæmoglobin is very apt to become changed into a peculiar borly known as methomoglobin, in the spectrum of which a very conspicuous band is seen in the red between $C$ and $D$ (see Fig. 98,4). The same change may be brought about by the action of weak acids, such as carbonic acid, by ozone, and by other agents such as nitrites and potassium permanganate. When a strean of carbonic acid is driven through blood or through a solution of hæmoglobin the band in the red characteristic of methæmoglobin so(n) makes its appearance. Methæmoglobin differs but little, if at all, in elementary composition from hæmoglobin; it is maintainer that it contains the same quantity of oxygen as oxy-hæmoglobin but in a more stable condition, more intimately associated with the molecule.

In conclusion, the condition of oxygen in the blood is as follows: Of the whole quantity of oxygen in the blood, only a minute fraction is simply absorbed or dissolved according to the law of pressure (the Henry-Dalton law). The great mass is in a state of combination with the hæmoglobin, the connection being of such a kind that while the hrmoglobin readily combines with the oxygen of the air to which it is exposed, dissociation readily occurs at low pressures, or in the presence of indifferent gases, or by the action of substances having a greater affinity for oxygen than has hæmoglobin itself. 'The difference between venous and arterial blood, as far as oxygen is concerned, is that while in arterial blood the hrmoglobin holds nearly its full complement of oxygen and may be spoken of as nearly wholly oxy-hæmoglobin, in venous blood the hæmoglobin is to a large but variable extent reduced; and the characteristic colors of venous and arterial blood are in the main due to the fact that the color of reduced hæmoglobin is purple, while that of oxy-hrmoglobin is scarlet.

\section{The Relations of the Carbonic Acid in the Blood.}

$\$ 295$. The presence of carbonic acid in the blood appears to be determined by conditions more complex in their nature and at present not so well understood as those which determine the presence of oxygen. The carbonic acid is not simply dissolved in the blood; its absorption by blood does not

1 This formula is the old one of Hoppe-Seyler. 
follow the law of pressures. It exists in association with some substance or substances in the blood, and its escape from the blood is a process of dissociation. We cimnot, however, speak of it as being associated, to the same extent as is the oxygen, with the hrmoglobin of the red corpuscles. So fur from the red corpuscles containing the great mass of the carbonic acid, the quantity of this gas which is present in a volume of serum is according to some observers actually greater than that which is present in an equal volume of blood, $i$. e., an equal volume of mixed corpuscles and serum; that is to say, the carbonic acid is much more largely associated with the serum (or, in the living blood, with the plasma) than with the red corpuscles.

When serum is subjected to the action of the mercurial pump, by far the greater part of the carbonic acid is given off'; but a small additional quantity ( 2 to 5 vols. per cent.) may be extracted by the subsequent addition of an acid. This latter portion may be spoken of as "fixed" carbonic acid in distinction to the larger "loose" portion which is given off to the vacuum. When, however, the whole blood is subjected to the vacuum until the carbouic acid ceases to be given off, the subsequent addition of acid is said not to set free any further quantity; so that when serum is mixed with corpuscles all the carbonic acid may be spoken of as "loose"; and it is stated that the excess of carbonic acid in a quantity of serum over that present in the same bulk of entire blood, corresponds to the fixed portion in serum which has to be driven off by an acid. Moreover, even those who maintain that the quantity of carbonic acid in entire blood is less than that in an equal volume of serum, admit that the carbonic acid exists in some way or other at a higher pressure in and is more readily given off from entire blood than from serum. It seems probable that the carbonic acid exists associated with some substance or substances in the serum, or rather plasma, but that the conditions of its association (and those of its dissociation) are determined by the action of some substance or substances present in the corpuscles. It has been suggested that the association of the carbonic acid in the plasma is with one or other of the proteids of the plasma; but it has also been suggested that the association is one with sodium as sodium bicarbonate, and further than the hæmoglobin of the corpuscles plays a part in promoting the dissociation of the sodium bicarbonate or even the carbonate, and thus keeping up the carbonic acid of the entire blood. Other observers, however, maintain that the plasma does not hold this exclusive possession of the carbonic acid, but that a considerable quantity of this gas is in some way associated with the red corpuscles. Indeed, further investigations are necessary before the matter can be said to have been placed on a satisfactory footing.

\section{The Relations of the Nitrogen in the Blood.}

$\$ 296$. The small quantity of this gas which is present in both arterial and venous blood seems to exist in a state of simple solution.

\section{The Respiratory Changes in the Lungs.}

\section{The Entrance of Oxygen.}

$\S 297$. We have already seen that the blood in passing through the lungs takes up a certain variable quantity (from 8 to 12 vols. per cent.) of oxygen. We have further seen that the quantity so taken up, putting aside the insignificant fraction simply absorbed, enters into direct but loose combination with the hrmoglobin. In drawing a distinction between the oxy- 
gen simply absorbed and that entering into combination with the hæmoglobin, it must not be understood that the latter is wholly independent of pressure. On the contrary, all chemical compounds are in various degrees subject to dissociation at certain pressures and temperatures; and the existence of the somewhat loose compound of oxygen and hæmoglobin is dependent on the partial pressure of oxygen in the atmosphere to which the hæmoglobin is exposed. Not only will a solution of hæmoglobin or a quantity of blood either absorb oxygen, and thus undergo association or undergo dissociation and give off oxygen according as the partial pressure of oxygen in the atmosphere to which it is exposed is high or low, but also the amount taken up or given off will depend on the degree of the partial pressure; the hæmoglobin as we have seen may be partially as well as wholly reduced. The law, however, according to which absorption or escape thus takes place is quite different from that observed in the simple absorption of oxygen by liquids. The association or dissociation is further especially dependent on temperature, a high temperature favoring dissociation, so that at a high temperature less oxygen is taken up than would be taken up (or, as the case may be, more given off than would be given off') at a lower temperature, the partial pressure of the oxygen in the atmosphere remaining the same.

Moreover, in the blood we have to deal not with hæmoglobin in simple solution, in which the molecules are dispersed uniformly through the solvent, but with the hæmoglobin segregated into minute isolated masses, bottled up as it were in the individual corpuscles. The hæmoglobin of each corpuscle is separated from its fellows by a layer, thin it may be but still a distinct layer, of colorless, hæmoglobinless plasma. As the corpuscle makes its way through the narrow capillary paths of a pulmonary alveolus, it is separated from the air of the alveolus by a thin layer of plasma as well as by the film of the conjoined capillary and alveolar walls; and a like layer of plasma separates it from its fellows as it journeys in company with them through the wider passages of the arteries and veins. Through this layer of plasma, which containing no hæmoglobin can hold oxygen in simple solution only, the oxygen has to pass on its way to and from the corpuscle; and every corpuscle may be considered as governing, as far as oxygen is concerned, a zone of plasma immediately surrounding itself. The corpuscle takes its oxygen directly from this zone and gives up its oxygen directly to this zone; and the pressure at which at any moment the oxygen exists in this zone will depend on the pressure of oxygen outside the zone, in the air of the pulmonary alveolus, for instance, and on the smaller or greater amount of oxygen associated with the hæmoglobin of the corpuscle.

The evidence, so far as it goes, seems to show that blood absorbs oxygen in the same way as an aqueous solution of hremoglobin of the same concentration; the zone of plasma spoken of above as surrounding each corpuscle behaves as far as regards the passage of oxygen to and from the corpuscles in no essentially different respect from the way the molecules of water belonging to a molecule of dissolved hæmoglobin behave in regard to the absorption or the giving off of oxygen by an aqueous solution of hrmoglobin.

The film of the conjoined capillary and alveolar wall is a thin membrane soaked with lymph and wet; we cannot speak of it as actually secreting a liquid secretion into the alveolus, for the cavity of the alveolus is filled with air which, though saturated with moisture, is air, not a liquid; still enough passes through the film to keep it continually moist. Through this film the oxygen has to make its way in order to gain access to the plasma and so to the corpuscle; it makes its way dissolved in the fluid, that is the lymph, 
which keeps the film moist. This film, moreover, is composed of living matter, and the considerations which a little while back (\$265) we urged concerning the diffusion through a living membrane of solid substances in solution, hold good also for the diffusion of gases in solution.

IVe have now to consider the question, Are the conditions in which hæmoglobin and oxygen exist in ordinary venous blood as it flows to the lungs, of such a kind that the venous blood in passing through the pulmonary capillaries will find the partial pressure of the oxygen in the pulmonary alveoli sufficient to bring about the association of the additional quantity of oxygen whereby the venous is converted into arterial blood?

$\$ 298$. In man, as we have seen, expired air contains about 16 per cent. of oxygen. The air in the pulmonary alveoli must contain less than this, since the expired air consists of a tidal air mixed by diffusion with the stationary air. How much less it contains we do not exactly know, but probably the difference is not very great. At the ordinary atmospheric pressure of $760 \mathrm{~mm} .16$ per cent. is equivalent to a partial pressure of 122 $\mathrm{mm}$. The question, therefore, stands thus, Will venous blood, exposed at the temperature of the body to a partial pressure of less than $122 \mathrm{~mm}$. (less than 16 per cent.) of oxygen take up sufficient oxygen (from 8 to 12 vols. per cent.) to convert it into arterial blood? Numerous experiments have been made (chiefly but not exclusively on the dog) to determine on the one hand the oxygen-pressure of both arterial and venous blood $\left(i . e_{\text {., the partial }}\right.$ pressure of oxygen in an atmosphere exposed to which the arterial blood neither gives up nor takes in oxygen, and the same for venous blood), and on the other hand the behavior at the temperature of the body or at ordinary temperatures of blood or of solutions of hæmoglobin (for the two, as we have just said, behave in this respect very much alike) toward an atmosphere in which the partial pressure of oxygen is made to vary. Without going into detail, we may state that these experiments show that the partial pressure of oxygen in the lungs is amply sufficient to bring about, at the temperature of the body, the association of that additional amount of oxygen by which venous blood becomes arterial. When a solution of hæmoglobin or when blnod is successively exposed to increasing oxygen pressures, as the partial pressure of oxygen is gradually increased, the curve of absorption rises at first very rapidly but afterward more slowly; that is to say, the later additions of oxygen at the higher pressures are proportionately less than the earlier ones at the lower pressures. And this is consonant with what appears to be the fact, that the hæmoglobin of arterial blood, though nearly saturated with oxygen, $i$. e., associated with almost its full complement of oxygen, is not quite saturated. When arterial blood is thoroughly exposed to air it takes up rather more than 1 vol. per cent. of oxygen; and that appears to represent the difference between exposing blood to pure air, such as enters or ought to enter the mouth in inspiration, and exposing blood to the air as it exists in the pulmonary alveoli. The greater relative absorption at the lower pressures has a beneficial effect, inasmuch as it still permits a considerable quantity of oxygen to be absorbed even when the partial pressure of oxygen in the air in the lungs is largely reduced, as in ascending to great heights.

Observations made both with dog's blood and ox's blood seem to show that arterial blood ceases to take up oxygen and begins to give off oxygen; in other words, that dissociation begins to take place when the partial pressure of the oxygen in the atmosphere to which it is exposed sinks to about $60 \mathrm{~mm}$. of mercury; that is to say, when the whole atmospheric pressure is reduced from $760 \mathrm{~mm}$. to about $300 \mathrm{~mm}$., or when the percentage of oxygen in the atmosphere is reduced by decidedly more than half. And 
this accords with the observation that in man, when the oxygen of inspired air is gradually diminished without any other change in the air, synuptoms of dyspnœa do not make their appearance until the oxygen sinks to 10 per cent. in the inspired air, and must therefore be less than this in the pulmonary alveoli. We may remark that at ordinary altitudes, even taking into account the diminution the oxygen undergoes before it reaches the pulmonary alveoli, the partial pressure of the oxygen in the atmosphere leaves a wide margin of safety. But at an altitude of 5500 metres (17,000 feet), at which the pressure of the whole atmosphere stands at about the limit given above of $300 \mathrm{~mm}$., the partial pressure of the oxygen will be such that the venous blood cannot take up the quantity of oxygen proper to convert it into arterial blood, since at this limit arterial blood begins to give off oxygen. We may add that it is at this altitude that breathing becomes especially difficult; but to this we shall return.

$\$ 299$. The statements made so far refer to ordinary breathing, but the question may be asked, What happens when the renewal of the air in the pulmonary alveoli ceases, as when the trachea is obstructed? In such a case the oxygen in the alveoli is found to diminish rapidly, so that the partial pressure of oxygen in them soon falls below the oxygen-pressure of ordinary venous blood. But in such a case the blood is no longer ordinary venous blood; instead of being moderately it is largely and increasingly reduced; instead of containing a comparatively small amount, it contains a large and gradually increasing amount of reduced hæmoglobin. And as the reduction continues to increase, the oxygen-pressure of the venous blood also continues to decrease; it thus keeps below that of the air in the lungs. Hence, apparently, even the last traces of oxygen in the lungs may be taken up by the blood and carried away to the tissues. Whether or not the pulmonary tissue and the capillary walls, because of their being living structures, have any influence upon the transmission of oxygen must still be considered an unsettled question.

\section{The Exit of Carbonic Acid.}

$\$ 300$. It seems natural to suppose that the carbonic acid would escape by diffusion from the blood of the alveolar capillaries into the air of the alveoli. But in order that diffusion should thus take place, the carbonic acid pressure of the air in the pulmonary alveoli must always be less than that of the venous blood of the pulmonary artery, and ought not to exceed that of the blood of the pulmonary vein. There are, however, many practical difficulties in the way of an exact determination of the carbonic acid pressure of the pulmonary alveoli (for, though it must be greater than that of the expired air, it is difficult to say how much greater), and of the carbonic acid pressure of the blood at the same time, so as to be in a position to compare the one with the other. In the case of oxygen there is always present in the lungs a surplus of the gas, a portion only being absorbed at each breath; in the case of carbonic acid the whole quantity comes direct from the blood, and any modifications in breathing seriously affect the amount given out. Thus, when the breath is held for some time the percentage of carbonic acid in the expired air reaches 7 or 8 per cent., but we cannot take this as a measure of the normal percentage of carbonic acid in the pulmonary alveoli, since by the mere holding of the breath the carbonic acid in the blood, and hence in the pulmonary alveoli, is increased beyond the normal.

The difficulties of the problem seem, however, to have been overcome by an ingenious experiment in which there is introduced into the bronchus of 
the lung of a dog a catheter, round which is arranged a small bag; by the inflation of this bag, the bronchus, whenever desired, can be completely blocked up. Thus, without any marked disturbance of the general breathing, and therefore without any marked change in the normal proportions of the gases of the blood, the experimenter is able to stop the ingress of fresh air into a limited portion of the lung. At the same time he is enabled by means of the catheter to withdraw a sample of the air of the same limited portion and by analysis to determine the amount of carbonic acid which it contains, or in other words, the partial pressure of the carbonic acid. The blood passing through the alveolar capillaries of this limited portion of the lung naturally possesses the same carbonic acid pressure as the rest of the venous blood flowing through the pulmonary artery - a pressure which, though varying slightly from moment to moment, will maintain a normal average. On the supposition that carbonic acid passes simply by diffusion from the pulmonary blood into the air of the alveoli, because the carbonic acid pressure of the latter is normally lower than that of the former, one would expect to find that the air in the occluded portion of the lung would continue to take up carbonic acid until an equilibrium was established between it and the carbonic acid pressure of the venous blood. Consequently, if after an occlusion, say of some minutes (by which time the equilibrium might fairly be assumed to have been established), the carbonic acid pressure of the air of the occluded portion were determined, it ought to be found to be equal to, and not more than equal to, the carbonic acid pressure of the venous blood of the pulmonary artery. And this is the result which has been arrived at: it has been found that the pressures of the carbonic acid of the occluded air and of the venous blood of the right side of the heart are just about equal. Hence the evidence, so far as it goes, is distinctly in favor of the view that the escape of carbonic acid from the blood into the pulmonary alveoli is simply due to diffusion, and that there is no need to seek for any further explanation.

As far then as can be seen at present, both the entrance of oxygen and the exit of carbonic acid by which venous blood is converted into arterial are the simple physical results of the exposure of the blood in the pulmonary capillary to the air of the pulmonary alveoli.

\section{The Respiratory Changes in the Tissues.}

$\S 301$. In passing through the several tissues the arterial blood becomes once more venous. The oxy-hæmoglobin becomes considerably reduced, and a quantity of carbonic acid passes from the tissues into the blood. The amount of change varies in the various tissues, and in the same tissue may vary at different times. Thus, in a gland at rest, as we have seen, the venous blood is dark, showing that the hæmoglobin is to a large extent in the reduced condition; when the gland is active, the venous blood in its color, and in the extent to which the hæmoglobin is in the condition of oxy-hæmoglobin, resembles closely arterial blood. The blood therefore which issues from a gland at rest is more "venous" than that from an active gland; though owing to the more rapid flow of blood which, as we saw in an earlier section, accompanies the activity of the gland, the total quantity of oxygen taken up from and of carbonic acid discharged into the blood from the gland in a given time may be greater than the latter. The blood, on the other hand, which comes from an active, i.e., a contracting muscle, is, in spite of the more rapid flow, not only richer in carbonic acid, but also, though not to a corresponding amount, poorer in oxygen than the blood which flows from a muscle at rest. 
In all these cases the great question which comes up for our consideration is this: Does the oxygen pass from the blood into the tissues, and does the oxidation take place in the tissues, giving rise to carbonic acid, which passes in turn away from the tissues into the blood? or do certain oxidizable reducing substances pass from the tissues into the blood, and there become oxidized into carbonic acid and other products, so that the chief oxidation takes place in the blood itself?

There are, it is true, reducing oxidizable substances in the blood, but these are small in amount, and the quantity of carbonic acid to which they give rise when the blood containing them is agitated with air or oxygen, is so small as scarcely to exceed the errors of observation.

We may add that the oxidative power which the blood itself removed from the body is able to exert on substances which are undoubtedly oxidized in the body is so small that it may be neglected in the present considerations. If grape-sugar be added to blood or to a solution of hæmoglobin, the mixture may be kept for a long time at the temperature of the body without undergoing oxidation. Even within the body a slight excess of sugar in the blood over a certain percentage wholly escapes oxidation, and is discharged unchanged. Many easily oxidized substances, such as pyrogallic acid, pass largely through the blood of a living body and are discharged in the urine without being oxidized; though perhaps in some of these cases, what appears to be an absence of oxidation is really an oxidation followed by a subsequent equivalent reduction taking place in the urine or elsewhere. The organic acids, such as citric, even in combination with alkaline bases, are only partially oxidized; when administered as acids, and not as salts, they are hardly oxidized at all. It is, of course, quite possible that the changes which the blood undergoes when shed might interfere with its oxidative action, and hence the fact that shed blood has little or no oxidizing power, is not a satisfactory proof that the unchanged blood within the living vessels may not have such a power. But did oxidation take place largely in the blood itself, one would expect even highly diffusible substances to be oxidized in their transit; whereas if we suppose the oxidation to take place in the tissues, it becomes intelligible why such diffusible substances as those which the tissues in general refuse to take up largely should readily pass unchanged from the blood through the excreting organs.

On the other hand, it will be remembered in speaking of muscle, we drew attention ( $\$ 61)$ to the fact that a frog's muscle removed from the body (and the same is true of the muscles of other animals) contains no free oxygen whatever; none can be obtained from it by the mercurial air-pump. Yet such a muscle will not only when at rest go on producing and discharging a certain quantity, but also when it contracts evolve a very considerable quantity, of carbonic acid. Moreover, this discharge of carbonic acid will go on for a certain time in muscles under circumstances in which it is impossible for them to obtain oxygen from without. Oxygen, it is true, is necessary for the life of the muscle; when venous instead of arterial hlood is sent through the bloodvessels of a muscle, the irritability speedily disappears, and unless fresh oxygen be administered the muscle soon dies. The muscle may, however, during the interval in which irritability is still retained after the supply of oxygen has been cut off, continue to contract vigorously. The supply of oxygen, though necessary for the maintenance of irritability, is not necessary for the manifestation of that irritability, is not necessary for that explosive decomposition which develops a contraction. A frog's muscle will continue to contract and to produce carbonic acid in an atmosphere of hydrogen or nitrogen, that is, in the total absence of free hydrogen, both from itself and from the medium in which it is placed. 
Thus, on the one hand, the muscle seems to have the property of taking up and fixing in some way or other the oxygen to which it is exposed, of storing it up in its own substance in such a condition that it cannot be removed by simple diminished pressure (so that the pressure of oxygen in the muscular substance may be considered as always nil) and yet has not entered into any distinct combination which we can speak of as an oxidation, but is still available for such a purpose. On the other hand. the muscular substance is always undergoing a decomposition of such a kind that carbonic acid is set free, sometimes, as when the muscle is at rest, in small, sometimes, as during a contraction, in large quantities. The oxygen present in this carbonic acid, as an oxidation product, comes from the previously existing store of which we have just spoken. The oxygen taken in by the muscle, whatever be its exact condition immediately upon its entrance into the muscular substance, in the phase which has been called "intra-molecular," sooner or later enters into a combination, or perhaps we should rather say, enters into a series of combinations. We have previously urged $(\S 30)$ that all living substance may be regarded as incessantly undergoing changes of a double kind, changes of building up and changes of breaking down. In the endproducts of the breaking down, in the carbonic acid given out by muscle, for instance, we can recognize an oxidation product; but we do not know exactly at what stage or exactly in what way the oxygen is combined with the carbon. We may imagine that the oxygen as it comes from the blood is caught up, so to speak, by and disappears in the building-up process, and that through those processes it is made part of complex decomposable substances whose decomposition ultimately gives rise to the carbonic acid; but, as far as actual knowledge goes, we cannot as yet trace out the steps taken by the oxygen from the moment it slips from the blood into the muscular substance to the moment when it issues united with carbon as carbonic acid.

But if the oxygen-pressure of the muscular tissue be thus always nil, oxygen will be always passing over from the blood corpuscles, in which it is at a comparatively high pressure, through the plasma, through the capillary walls, the lymph-spaces, and the sarcolemma, into the muscular substance, and as soon as it arrives there will be in some manner or other hidden away, leaving the oxygen-pressure of the muscular substance once more nil. Conversely, the carbonic acid produced by the decomposition of the muscular substance will tend to raise the carbonic acid pressure of the muscle until it exceeds that of the blood; whereupon carbonic acid will pass from the muscle into the blood, its place in the muscular substance being supplied by gas freshly generated. There will always in fact be a stream of oxygen from the blood to the muscle and of carbonic acid from the muscle to the blood. The respiration of the muscle, then, does not consist in throwing into the blood oxidizable substances, there to be oxidized into carbonic acid and other matters; but it does consist in the assumption and storing up of oxygen somehow or other in its substance, in the building up by help of that oxygen of explosive decomposable substances, and in the carrying out of decompositions whereby carbonic acid and other matters are discharged first into the substance of the muscle and subsequently into the blood.

$\S 302$. Our knowledge of the respiratory changes in muscle is more complete than in the case of any other tissue; but we have no reason to suppose that the phenomena of muscle are exceptional. On the contrary, all the available evidence goes to show that in all tissues the oxidation takes place in the tissue, and not in the adjoining blood. It is a remarkable fact that lymph, serous fluids, bile, urine, and milk contain a mere trace of free or loosely combined oxygen, but a very considerable quantity of carbonic acid. And we may probably assert with safety with regard to all the tissues, that 
in the tissues themselves, in the lymph which bathes their lymph-spaces, and in the secretions which some of them pour forth, free oxygen is either wholly absent or so scanty that their oxygen-pressure may be regarded as nil, while carbonic acid is so abundant that the pressure of carbonic acid in them may be regarded as exceeding that of venous blood. An exception seems to be presented by the case of the lymph flowing along the larger lymphatic vessels, for in this the amount of carbonic acid, while usually higher than that of arterial blood, is lower than that of the general venous blood; but this probably is due to the fact that the lymph in its passage onward is largely exposed to arterial blood in the connective tissues and in the lymphatic glands, where the production of carbonic acid is slight as compared to that going on in muscles. All the facts point to the conclusion that it is the tissues, and not the blood, which become primarily loaded with carbonic acid, the latter simply receiving the gas from the former by diffusion, except the (probably) small quantity which results from the metabolism of the blood corpuscles; and that the oxygen which passes from the blood into the tissues is at once taken up and placed under such conditions that it is no longer removable by diminished pressure.

We have seen that in muscle the production of carbonic acid is not directly dependent on the consumption of oxygen. The muscle produces carbonic acid in an atmosphere of hydrogen. What is true of muscle is true also of other tissues and of the body at large. It was shown long ago that animals might continue to breathe out carbonic acid in an atmosphere of nitrogen or hydrogen; and this has more recently been illustrated by the remarkable experiment that a frog kept at a low temperature will live for several hours, and continue to produce carbonic acid, in an atmosphere absolutely free from oxygen. The carbonic acid produced during this period was made by help of the oxygen inspired in the hours anterior to the commencement of the experiment. The oxygen then absorbed was stowed away from the hrmoglobin into the tissues, it was made use of to build up the explosive compounds, whose explosions later on gave rise to the carbonic acid. Or, to adopt a simile which has been suggested, the oxygen helps to wind up the vital clock; but once wound up the clock will go on for a period without further winding. The frog will continue to live, to move, to produce carbonic acid for a while without any fresh oxygen, as we know of old it will without any fresh food; it will continue to do so till the explosive compounds which the oxygen built up are exhausted; it will go on until the vital clock has run down.

$\S 303$. To sum up, then, the results of respiration in its chemical aspects. As the blood passes through the lungs, the low oxygen pressure of the venous blood permits the entrance of oxygen from the air of the pulmonary alveolus, through the thin alveolar walls, through the thin capillary sheath, through the thin layer of blood-plasma to the red corpuscle, and the reduced hrmoglobin of the venous blood becomes wholly, or all but wholly, oxyhæmoglobin. Hurried to the tissues, the oxygen, at comparatively high pressure in the arterial blood, passes largely into them. In the tissues the oxygen-pressure is always kept at an exceedingly low pitch by the fact that they, in some way at present unknown to us, pack away at every moment into some stable combination each molecule of oxygen which they receive from the blood. With its oxy-hæmoglobin largely but not wholly reduced, the blood passes on as venous blood. To what extent the hæmoglobin is reduced will depend on the activity of the tissue itself. The quantity of hæmoglobin in the blood is the measure of limit of the oxidizing power of the body at large; but within that limit the amount of oxidation is determined by the tissue, and by the tissue alone. 
We cannot trace the oxygen through its sojourn in the tissue. We only know that sooner or later it comes back combined in carbonic acid (and other matters not now under consideration). Owing to the continual production of carbonic acid, the pressure of that gas in the extra-vascular elements of the tissue is always higher than that in the blood; the gas accordingly passes from the tissue into the blood, and the venous blood passes on not only with its hamoglobin more or less reduced, $i . e$, with its oxygen-pressure decreased, but also with its carbonic acid pressure increased. Arrived at the lungs, the blood finds the pulmonary air at a lower carbonic acid pressure than itself. The gas accordingly streams through the thin vascular and alveolar walls until the pressure without the bloodvessel is equal to the pressure within. At the same time the blood finds in the air of the pulmonary alveoli a supply of oxygen, more than adequate to convert, not entirely but nearly so, the reduced hæmoglobin back again to oxy-hæmoglobin. Thus the air of the pulmonary alveoli, having given up oxygen to the blood and taken up carbonic acid from the blood, having in consequence a higher carbonic acid pressure and a lower oxygen pressure than the tidal air in the bronchial passages, mixes rapidly with this by diffusion. The mixture is further assisted by ascending and descending currents ; and the tidal air issues from the chest at the breathing out poorer in oxygen and richer in carbonic acid than the tidal air which entered at the breathing in.

\section{The Nervous Mechanism of Respiration.}

$\S 304$. Breathing is an involuntary act. Though the diaphragm and all the other muscles employed in respiration are voluntary muscles, $i . e$., muscles which can be called into action by a direct effort of the will, and though respiration may be modified within very wide limits by the will, yet we habitually breathe without the intervention of the will; the normal breathing may continue, not only in the absence of consciousness, but even after the removal of all the parts of the brain above the medulla oblongata.

We have already seen how complicated is even a simple respiratory act. A very large number of muscles are called into play. Many of these are very far apart from each other, such as the diaphragm and the nasal muscles; yet they act in harmonious sequence in point of time. If the lower intercostal muscles contracted before the scaleni, or if the diaphragm contracted alternately with the other chest-muscles, the satisfactory entrance and exit of air would be impossible. These muscles, moreover, are coördinated also in respect of the amount of their several contractions; a gentle and ordinary contraction of the diaphragm is accompanied by gentle and ordinary contractions of the intercostals, and these are preceded by gentle and ordinary contractions of the scaleni. A forcible contraction of the scaleni, followed by simply a gentle contraction of the intercostals, would perhaps hinder rather than assist inspiration, and at all events would be waste of power. Further, the whole complex inspiratory effort is often followed by a less marked but still complex expiratory action. It is impossible that all these so carefully coördinated muscular contractions should be brought about in any other way than by coördinate nervous impulses descending along efferent nerves from a coördinating nervous centre. By experiment we find this to be the case.

When in a rabbit the trunk of a phrenic nerve is cut, the diaphragm on that side remains motionless, and respiration goes on without it. When both nerves are cut, the whole diaphragm remains quiescent, though the costal respiration becomes excessively labored.

When an intercustal nerve is cut, no active respiratory movements are 
seen in the intercostal muscles of the corresponding space, and when the spinal cord is divided below the origin of the seventh cervical spinal nerve, that is, below the exits of the roots of the phrenic nerves, costal respiration ceases, though the diaphragm continues to act, and that with increased vigor. When the cord is divided just below the medulla, all thoracic movements cease, but the respiratory actions of the nostrils and glottis still continue. These, however, disappear when the facial and recurrent laryngeal nerves are divided. We have already stated that after removal of the brain above the medulla, respiration still continues very much as usual, the modifications which ensue from the loss of the brain being unessential. Hence, putting all these facts together, it is clear that the respiratory movements are, as we suggested, brought about by coördinated impulses which, developed in the central nervous system and starting in the first instance in the medulla, find their way along the several efferent nerves. The proof is completed by the fact that the removal of', or extensive injury to, the medulla alone is, save in exceptional cases which we will discuss presently, at once followed by the cessation of all respiratory movements, even though the rest of the nervous system including every muscle and every nerve concerned be left intact. Nay more, if only a small portion of the medulla, a tract whose limits have not been clearly defined, but which may be described as lying below the vasomotor centre in the immediate neighborhood of the nuclei of the vagus nerves, be removed or injured, respiration ceases, and death at once ensues. Hence this portion of the nervous system was called by Flourens the vital knot, or ganglion of life, nœud vital. We shall speak of it as the respiratory centre.

$\S 305$. The nature of this centre must be exceedingly complex; for while even in ordinary respiration it gives rise to a whole group of coördinate nervous impulses of inspiration followed in due sequence by a smaller but still coördinate group of expiratory impulses of an antagonistic nature, in labored respiration fresh and larger impulses are generated, though still in coördination with the normal ones, the expiratory events being especiallv augmented; and in the cases of more extreme dyspnœa and asphyxia impulses overfow, so to speak, from it in all directions, though only gradually losing their coördination, until almost every muscle in the body is thrown into contractions.

We must not, however, conceive of this centre as one of such a kind that the impulses leave it fully coördinated and equipped so that nothing remains for them but to travel, unchanged, along the several efferent nerve-fibres to their several muscular destinations. On the contrary, we have reason to think that the respiratory motor nerves, like other motor nerves, are connected, just as they are about to issue from the spinal cord, with a nervous machinery in which nerve-cells play a part-a point which we shall consider more fully in treating of the spinal cord; we have reason to think that the respiratory impulses starting from the respiratory centre pass into and are modified by secondary spinal nervous mechanisms before they issue along the motor nerve roots. Indeed, observations show that under particular conditions, and especially in young animals, respiratory movements may he carried out in the entire absence of the medulla oblongati. Thus if, in a kitten or puppy or young rabbit, after division of the spinal cord below the medulla artificial respiration be kept up, and then pauses be made in the artificial respiration, during these pauses not only may what appear to be respiratory movements be induced in a reflex manner, by pinching or by blowing on the skin, but, especially if the excitability of the spinal cord be heightened by small doses of strychnine, even spontaneous efforts of breathing may occasionally be observed. These are the exceptional instances mentioned above. 
Since in such cases the rhythmically repeated movements of the respiratory muscles are sometimes accompanied by rhythmic movements of the fore and hind limbs not respiratory in nature, it may be doubted whether these experiments really prove the existence of distinct respiratory centres in the spinal cord; and at most they merely show that the respiratory nervous niechanism is not entirely confined, as was once thought, to the centre in the medulla, but also embraces other subsidiary mechanisms, which may perhaps he spoken of as centres, in the spinal cord below. It has, indeed, been maintained by some that these lower spinal centres are the chief centres, and that the medullary centre acts merely in the way of regulating these; but it is difficult to reconcile this view with the experience that interference with the medulla, limited entirely to the medulla, so often leads to the entire abolition of the respiratory movements. This matter is not at present thoroughly worked out, but we shall probably not greatly err in regarding the respiratory nervous system as in many ways analogous to the vasomotor nervous system, with its head centre in the medulla and secondary centres elsewhere, and in continuing to speak of the centre in the medulla as being "the respiration centre," while admitting that it works through other nervous machinery placed lower down in the spinal cord, and that this subordinate machinery may in exceptional cases carry out, though inadequately, the work of the chief centre.

$\S 306$. Admitting then the existence of this medullary respiratory centre the question naturally arises, Are we to regard its rhythmic action as due essentially to changes taking place in itself, or as due to afferent nervous impulses or other stimuli which affect it in a rhythmic manner from without? In other words, Is the action of the centre automatic or purely reflex? We know that the centre may be influenced by impulses proceeding from without, and that the breathing may be affected by the action of the will, or by an emotion, or by a dash of cold water on the skin, or in a hundred other ways; but the fact that the action of the centre may be thus modified from without, is no proof that the continuance of its activity is dependent on extrinsic causes.

In attempting to decide this question we naturally turn to the pneumogastric as being the nerve most likely to serve as the channel of afferent impulses setting in action the respiratory centre. If both vagus nerves be divided, respiration still continues, thongh in a modified form. This proves distinctly that afferent impulses ascending those nerves are not the efficient cause of the respiratory movements. We have seen that when the spinal cord is divided below the medulla, the facial and laryngeal movements still continue. This proves that the respiratory centre is still in action, though its activity is unable to manifest itself in any thoracic movement. But when the cord is thus divided, the respiratory centre is cut off from all sensory impulses, save those which may pass into it from the cranial nerves of sensory function; and that these sensory cranial nerves are not specially concerned in developing the activity of the respiratory centre is shown by the fact that the division of these cranial nerves by themselves, when the medulla and spinal cord are left intact, does not do away with the continuance of respiration. One cranial nerve, as we shall see, is especially concerned in respiration, viz., the ragus nerve; but if, after removal of the brain above the medulla, both vagus nerves be divided, respiration still goes on ; indeed, the respiratory impulses proceeding from the centre are, though in a peculiar way, exaggerated. Hence, though we cannot put the matter to an experimental test by dividing every sensory nerve in the body, while leaving the motor nerves of respiration intact, such an operation being practically impossible, we may infer that the respiratory impulses proceed- 
ing from the respiratory centre are not simply afferent impulses reaching the centre along afferent nerves and transformed by reflex action in that centre. They evidently start de novo from the centre itself, however mucil their characters may be affected by afferent impulses, reaching that centre at the time of their being generated. The action of the centre is automatic, not simply reflex.

$\$ 307$. We find, on inquiry, that the activity of the centre is profoundly influenced by two classes of events. These, as we might expect, are, on the one hand, events producing changes in the quality of the blood distributed to the medulla by the left ventricle, especially as regards its gases, that is to say, events modifying the interchange taking place in the lungs; and, on the other hand, nervous impulses started in various ways and reaching the centre along various nerves or nervous tracts. It will be convenient to consider the latter first.

Afferent nervous impulses may affect the centre in many various ways. The whole act of breathing or of taking a breath is a double act, consisting of an inspiration and an expiration, and nervous impulses may especially affect the one or the other. One mode of ureathing may differ from another in the depth of the individual breath, in the volume of air taken in and given out; and nervous impulses may increase or may diminish the depth of a breath, the volume of air respired. One mode of breathing again differs from another in the rapidity with which one breath succeeds another, that is, in the rate of rhythm; and nervous impulses may slow or may quicken the rate of rhythm. Then, again, combination of affects so numerous and varied as almost to baffle description, may result from the influence of various nervous impulses. Emotions may affect a single breath or a long scries of breaths, may quicken the rhythm while making each breath

Fig. 99.

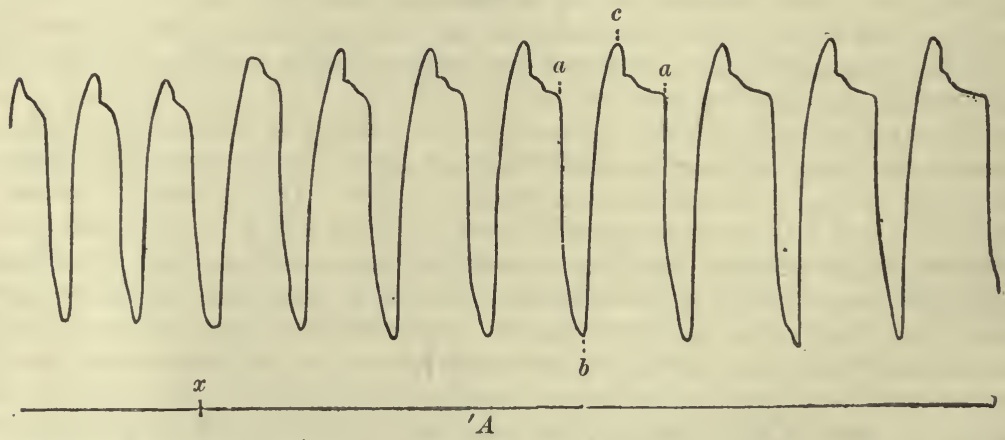

Effect on Respiration of Section of One Vagus. The vagus was divided at the point marked $x$. The curve was obtained by means of a tambour connected with a receiver into which the animal (rabbit) breathed, as shown in Fig. 90, the lever falling in inspiration as air is sucked out of the tambour and rising in expiration as the air returns. Inspiration begins at $a$ and ends at $b$. Expiration begins at $b$ and ends at $c$. The lever gradually falls between $c$ and $a$, owing to the escape of air from the apparatus.

more shallow, or may, at the same time, make each breath deeper, or may slow the rhythm in either the one or the other manner, and may bear chiefly on inspiration or on expiration. Moreover, there is not an afferent nerve in the body which by means of afferent impulses passing along it may not be the instrument of influencing the respiratory centre. Of all the automatic centres in the body the respiratory centre is the one whose independence is most obscured by the repeated effects of afferent nervous impulses. 
Certain afferent nerves, however, appear to be more closely connected with it than others; and of these the most conspicuous and important are the two vagus nerves, which we have already mentioned in this connection. Their importance is well illustrated by the following experiments: If one vagus be divided in an ordinary way, without any special precautions, the respiration is either not materially changed, or if affected becomes slower (Fig. 99). If both be divided (Fig. 100) it becomes very slow, the pauses between expiration and inspiration being markedly prolonged. The character of the respiratory movement, too, is markedly changed; each respiration is fuller and deeper, so much so, indeed, that, according to some observers, what is lost in rate is gained in extent, the amount of carbonic acid pro-

FIG. 100.

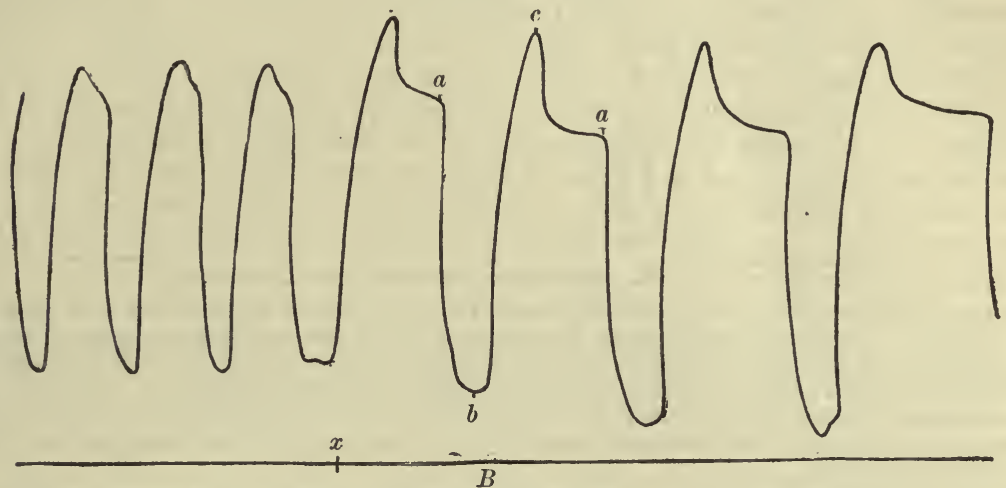

Effect on Respiration of Section of Both Vagus Nerves. The curve was obtained in the same way as Fig. 99. The second vagus nerve was divided at $x$.

duced and oxygen consumed in a given period remaining after division of the nerves about the same as when these were intact; but it is undesirable to insist ton much on the exactness of this compensation.

Fig. 101.

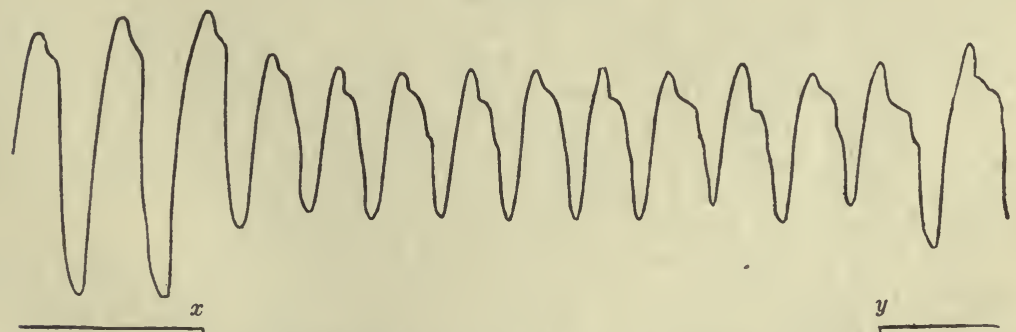

Quickening of Respiration by Gentle Stimulation of the Central End of the Vagus Trunk. The curve was obtained in the same way as Figs. 99, 100. Stimulation of the vagus began at $x$ and ended at $y$.

When after division of both vagus nerves in the neck, the medulla being intact, the central stump, that connected with the central nervous system, of one of them is stimulated with a gentle interrupted current, the effects are not always the same; one of two results may follow, and that whichever of the two nerves be used. In a certain number of cases, and these may, perhaps, be regarded as the more typical ones, the respiration, which from the 
division of the nerves had become slow, is quickened again (Fig. 101), and with care, by a proper application of the stimulus, the normal respiratory rhythm may for a time be restored. Upon the cessation of the stimulus the slower rhythm returns. If the current be increased in strength, the rhythm may in some cases be so accelerated that inspiration begins before the expiration of the preceding breath is completed (Fig. 102), and this may go on

Fig. 102.

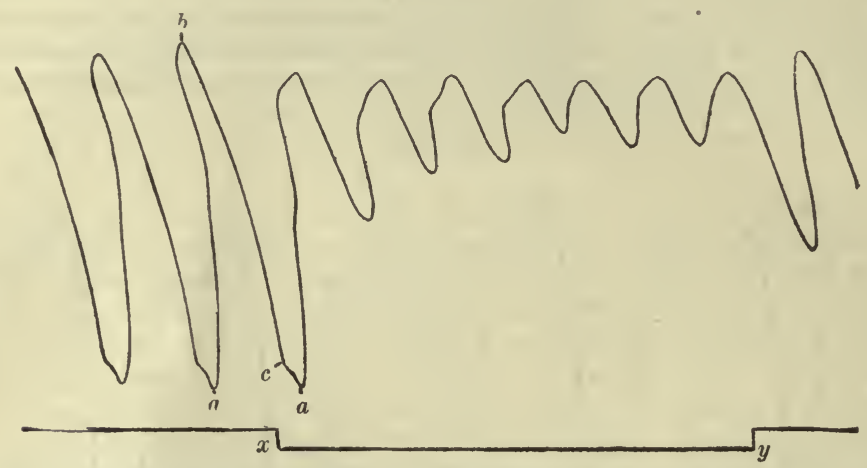

Stimulation of Vagus Leading to Inspiratory Increase: This curve, unlike the preceding, was obtained by inserting a needle through the body wall, so as to rest on the diaphragm, and attaching a lever to the needle. (See $\& 271$.) The lever rises with each contraction of the diaphragm, so that inspiration begins at $a$ and ends at $b$, expiration begins at $b$ and ends at $c$, the interval between $c$ and $a$ corresponding to the pause. Stimulation of the vagus begins at $x$. It will be seen that upon stimulation the inspiratory rises of the lever begin long before the preceding expirations are complete.

until at last the diaphragm is brought into a condition of prolonged tetanus, and a standstill of respiration in an extreme inspiratory phase is the result. On the other hand, in a certain number of cases the result is of an opposite character. Even though the respiration be already slowed by division of the nerves, stimulation produces a still further slowing, the pauses between each expiration and the succeeding inspiration are prolonged (cf. Fig. 103), and in a certain number of cases actual standstill is brought about, but a standstill of a kind the opposite of the one just described, since the diaphragm which in that case was in prolonged tetanus is in this case completely relaxed and remains for some time in the condition in which it is at the close of an ordinary breath. In a certain number of cases, and these are not uncommon, the result is intermediate between the two above extremes; the diaphragm stands still in a prolonged contraction in a position which is intermediate between the height of inspiration and expiration.

These results suggest the conclusion that the vagus nerve (we are dealing now with the main trunk of the nerve) contains afferent fibres of two kinds connected with the respiratory centre; one kind augmenting the action of the centre somewhat in the same way as the augmentor cardiac fibres augment the beat of the heart, and the other kind having an inhibitory effect. Apparently sometimes the one and sometimes the other kind is, according to circumstances, most provoked by the stimulation, much in the same way as stimulation of the vagus in the frog, which, as we have seen, $\S 144$, is the channel for both inhibitory and augmentor cardiac impulses, produces sometimes inhibition, sometimes augmentation of the heart-beat. To affect the heart of course the stimulation of the vagus must be centrifugal, directed toward the periphery, whereas to affect the respiration it must be centripetal, applied to the part of the nerve connected with the brain; and 
while the usual effect on the heart of ordinary stimulation of the vagus is inhibition, augmentation only occurring in special cases, the most common effect on respiration is augmentation, though inhibition is not unfrequently seen. When the experiment is conducted on an animal under the full influence of chloral, stimulation of the vagus generally produces inhibition of respiration, probably because the chloral renders the respiratory centre more susceptible to inhibitory influences.

$\$ 308$. We said just now "the action of the centre" ; but the respiratory centre is a double one; it gives rise to inspiratory and to expiratory afferent impulses, and these are antagonistic the one to the other. If inspiratory and expiratory impulses issued from the centre at the same time and in equal potency, there could be no breathing at all, they would neutralize each other's effects; and, indeed, any amount of inspiratory impulse is antagonistic to a simultaneous expiratory impulse, and vice versa. Hence, for the adequate services of the respiratory centre we might expect to find that each kind of afferent impulse ascending the vagus affected the centre in a double and opposite way, inhibiting expiration while augmenting inspiration, or inhibiting inspiration while augmenting expiration. If we allow ourselves to speak of the whole respiratory centre as consisting of two parts, one the inspiratory part, or inspiratory centre concerned in the issue of inspiratory impulses, and the other the expiratory part, or expiratory centre concerned in the issue of expiratory impulses, we may suppose that these centres are so related to each other that afferent impulses, reaching the medulla, which augment or inhibit the one, necessarily inhibit or augment the other. We need perhaps hardly add that of these two centres we should expect to find the inspiratory centre the dominant and the most responsive one; in normal breathing it comes almost alone into obvious use, since, as we have seen, the expiratory muscles have then a very slight task only, the chest being emptied chiefly by elastic reaction; and, speaking generally, breathing in is the first consideration-we breathe out mostly because we have already breathed in.

There are many facts which support this view of the double antagonistic action of afferent respiratory impulses. If the central end of the superior laryngeal branch of the vagus be stimulated the effects are much more constant than those of stimulating the main vagus trunk. Whether the main trunk of the nerve be previnusly severed or not, the result of centripetal stimulation of the superior laryngeal branch is always in the direction of a slowing of the respiration (Fig. 103); and this may by proper stimulation be carried so far that a complete standstill of respiration in the phase of rest is brought about. While the main trunk of the vagus contains fibres of two kinds, both augmentory and inhibitory of inspiration, the superior laryngeal branch appears to contain one kind only, those which inhibit inspiration. If now while this experiment is being conducted on a rabbit the abdomen be watched, it will be seen that the inhibition of inspiration is accompanied by a contraction of the abdominal nuscles, that is by an effort at expiration; the stimulation of the nerve while inhibiting respiration provokes, to a certain extent, expiration.

$\$ 309$. That the trunk of the vagus is the channel of these two kinds of impulses, of a mutually antagonistic character, is further shown by applying what may be considered as natural stimuli to the endings of the nerve in the lungs; and the results so obtained have an especial value since the artificial stimulation of a nerve-fibre, at a part of its course by means of an electric current is at best a rough process, by which we cannot hope to do more than approximate to the results actually taking place in the living body when the nerve is stimulater at its endings by natural stimuli; and 
the approximation is perhaps less in the case of the exquisitely sensitive respiratory centre than in many other cases.

FIG. 103.

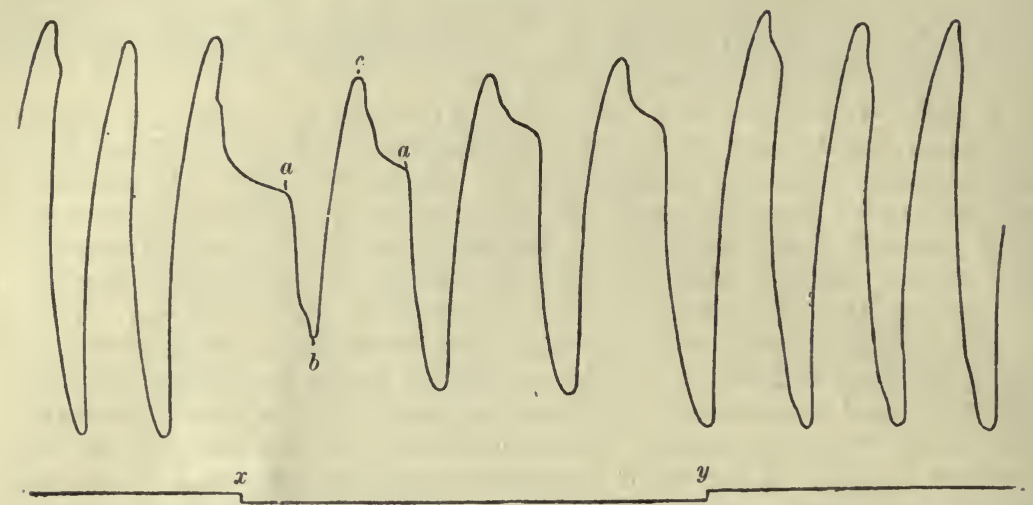

Slowing of Respiration by Stimulation of Superior Laryngeal Nerve. This curve was obtained in the same way as Figs. 99, 100, 101, and the letters have the same meaning as in those figures. Stimulation begins at $x$ and ends at $y$.

If in an animal in which a careful graphic record of the respiratory movements is being taken, the trachea be suddenly closed at the summit of

FIG. 104.
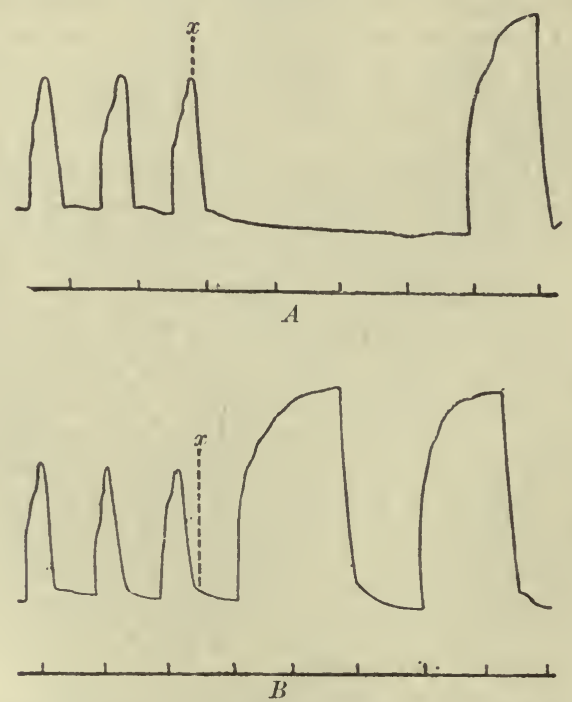

Effects of Distention and Collapse of Lung. (Hear.) Both curves are described by a lever attrehed, as stated in 3271 , to a slip of the diaphragm of a rabbit. A contraction of the diaphragm (inspiration) raises the lever; during relaxation of the diaphragm the lever fails. In $A$, the trachea is elosed at $x$, the height of inspiration : a pause follows during which the lever gradually sinks until an inspiration (a very powerful one) sets in. In $B$, the trachea is closed at the end of expiration, $x$; there follow powerful inspirations.

an inspiration, the result is a pause before the succeeding inspiration follows, that is to say, a partial or temporary inhibition of inspiration ; and if during 
such an experiment on a rabbit a curve be taken by means of the isolated slip of the diapliragm, $\S 271$, it will be seen (Fig. 104, A) that the slip elongates somewhat; that is to say, previously in a state of slight tonic contraction, it changes in the direction of expiration. If, on the other hand, the trachea be suddenly closed at the end of an expiration (Fig. 104, B), when the lungs have returned to their emptied condition, the result is an increase of the sequent inspirations, that is to say, an augmentation of inspiratory impulses. If the chest or if the lung only be gently inflated, a temporary cessation of all inspiration may be produced, accompanied sometimes by an attempt at expiration. If, on the other hand, air be sucked out of the

Fig. 105.

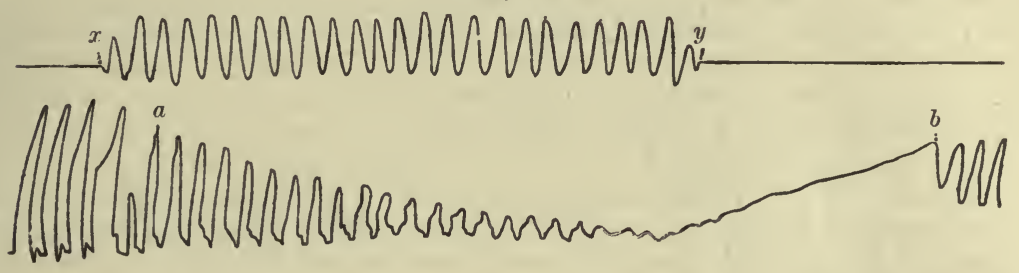

Effects of Repeated Inflations. Positive Ventilation. (Head.) The lower curve is described, as in Fig. 104, by a lever attached to a slip of the diaphragm. The upper curve shows the inflations from $x$ to $y$, which were made without any attempt to draw the air out at each inflation: each rise on this curve denotes an inflation. It will be observed that as the inflations are continued the respiratory movements of the diaphragm are gradually "knocked down."

chest, or if one lung be made to collapse by puncture of one pleural chamber, a prolonged inspiration is the frequent result, the diaphragm being thrown into a prolonged inspiratory tetanus. If the lungs are repeatedly inflated, without any means being taken to draw out the air after each inflation (Fig. 105), a procedure which we may speak of as positive ventilation, the result is that the inspiratory efforts are diminished, and if the ventilation is continued may cease altogether. If, on the other hand, air is repeatedly sucked out of the lungs without any corresponding inflations (negative ventilation), the inspiratory efforts are increased (Fig. 106), and the increase may be such as to bring the diaphragm to a state of tetanus. And in general, though several complications occur which we cannot discuss here, the results of inflation of the lungs on the one hand and of suction or collapse of the lungs on the other hand, show that the mere inflation or perhaps rather the mere distention of the lung tends to inhibit inspiratory and usher in expiratory impulses, while collapse of the lung tends to inhibit expiratory and to develop inspiratory impulses, the effect on the inspiratory impulses, as might be expected from the dominance of the inspiratory portion of the centre, being more marked than the effect on the expiratory impulses. That the instrument by which these effects are produced is the vagus nerve is shown by the fact that they are no longer distinctly recognizable when both vagus nerves are divided. And that the results are due to the mere mechanical expansion and collapse of the lung in insufflation and collapse, and not to any chemical influences exerted by the larger amount or smaller amount of air present in the lung in the two cases increasing or diminishing the absorption of oxygen and escape of carbonic acid, is shown by the fact that the results remain in their main features the same when some indifferent gas, such as hydrogen, is used for inflation instead of air or oxygen. We infer therefore that the expansion of the pulmonary alveoli in some way or other so stimulates the endings in the 
lung of the pulmonary branches of the vagus, that impulses are generated which ascending the vagus trunk inhibit the inspiratory processes in the respiratory centre; and that conversely collapse of the lung similarly generates impulses which are augnentative of inspiratory impulses. And, assuming on the strength of analogy the existence in the vagus of two sets of fibres we may say that expansion stimulates the endings of the fibres which inhibit inspiration and concurreutly tend to augment expiration, while collapse stimulates the fibres which inhibit expiration and augment inspiration. The respiratory pump may thus be looked upon as a self-regu-

FIG. 106.

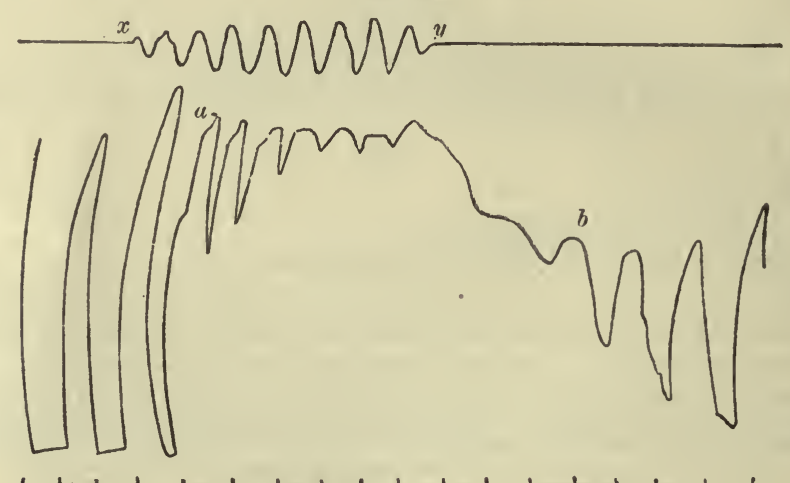

Effects of liepeated Suctions of the Lungs. Negative Ventilation. (Head.) The curve corresponds exactly to Fig. 105, except that the lungs are subjected to repeated suctions without corresponding inflations. The result is that the inspirations are repeated in such a way as to lead almost to an inspiratory tetanus of the diaphragm.

lating mechanism; the expansion of the lungs which is the result of the efferent inspiratory impulses tends to check the issue of these impulses and to inaugurate the sequent expiration; and the return of the lungs in expiration tends to set going the succeeding inspiration.

The regulative influence exerted by impulses normally ascending the vagus nerves is further shown by the following striking experiment: As we have already seen, the brain above the medulla may be removed without any extraordinary change in the respiration taking place. We have also seen that when both vagus nerves are divided the respiration is slower and deeper, but is otherwise regular. If, however, after the removal of the brain above the medulla both vagus nerves are divided, if the respiratory centre be cut off at one and the same time from impulses passing down from the higher parts of the brain and from impulses ascending the vagus nerves, the result is that the respirations take on the form of a series of long continued inspiratory spasms. It would seem as if there were a tendency in the respiratory centre to go off into tetanic inspiratory explosions, that this tendency is held in check by impulses from the brain when the vagus nerves are divided, and by impulses along the vagus nerves when the brain is removed, but meets with no arlequate checks when impulses from both sources are cut off at the same time.

$\$ 310$. Hypotheses have been put forward to explain the changes in the respiratory centre which lead to the rhythmic discharge of inspiratory and expiratory impulses and the further changes which result from the advent of augmenting and inhibitory impulses; but these as yet remain mere hypotheses, and it would not be profitable to discuss them here. We may add 
that, though the analogy of the cardiac nervous mechanism, in which we can anatomically distinguish between augmentor and inhibitory fibres, justifies us in speaking of augmentor and inhibitory and respiratory fibres as existing in the vagus nerve, we are not as yet able to distinguish them by anatomical methods. We may further add that, so exquisitely sensitive is the respiratory centre to these afferent impulses stimuli too slight to produce any appreciable effect when applied to afferent nerves connected with an ordinary centre, such as a spinal reflex centre, may produce marked effects on the respiratory centre. For instance, the feeble electric current which is developed when the cut end of a divided vagus is replaced in the wound, the circuit between the cut end and the longitudinal surface of the nerve being closed through the blood or lymph of the wound, is often sufficient to develop inhibitory impulses. Again, when the connection of the respiratory centre with the lungs through the vagus nerves is abolished, not by section of the nerves, but by freezing both nerves at some part of the course of each nerve (an operation which, while completely blocking the passage of impulses along the nerve-fibres, does not itself act as a stimulus), the effect on the respiratory movements is much more in the direction of increasing and prolonging the inspiratory act than that of slowing the rhythm. Hence it would appear that what-we have previously described as the result of dividing both vagus nerves is partly due to the blocking of natural impulses and partly to the section of the nerves, and possibly to electric currents developed as suggested above, acting as stimuli and thus giving rise to artificial impulses.

$\S 311$. The double or alternate respiratory action of the vagus nerves, on which we have dwelt above, may be taken as, in a general way, illustrative of the manner in which other afferent nerves and various parts of the cerebrum are enabled to influence respiration. As we have already said, and, indeed, know from daily experience, of all the apsychical nervous centres the respiratory centre is the one which is most frequently and most deeply affected by nervous impulses from various quarters. Besides the changes brought about by the will (and when we breathe voluntarily we probably make use, to some extent, of the normal nervous machinery of respiration, working through this, rather than sending independent volitional impulses direct to the diaphragm and other respiratory muscles), we find that emotions and painful sensations alter profoundly the character of the respiratory movements. And though these effects may be partly indirect (the emotion modifying the heart-beat or the tonus arteries, and so influencing the flow of blood through the respiratory centre), they are chiefly due to the direct action of nervous impulses reaching that centre from higher parts of the brain. So, also, impulses from almost every sentient surface or passing along almost every sensory nerve may modify respiration in one direction or another. The influence in this way of stimuli applied to the skin is well known to all; but, perhaps, next to the vagus the nerve most closely connected with the respiratory centre is the fifth nerve, branches of which guard the nasal respiratory channels; the slightest stimulation of the nostrils at once affects the breathing and most frequently arrests it. The effects of stimuli of various strengths brought to bear on various nerves are very varied. Sometimes the result is an increase of inspiration, and that either by a quickening of the rhythm or by an increase of the individual breaths or by a combination of the two. Sometimes the result is inhibition of inspiration, accompanied or not by an increase of expiration, and sometimes, as when the stimulation causes a cough, the expiratory results may be out of all proportion to the modifications of inspiration. While in the case of some nerves, for instance, 
as we have seen, the superior laryngeal, and, it is said, the splanchnic nerves, the effects are exclusively, or, at least, chiefly inhibitory of inspiration and augmentative of expiration, that is, expiratory, and in others, perhaps, chiefly augmentative of inspiration, or inspiratory, in the case of most nerves the effect may be, according to circumstances, either in the one direction or the other. Perhaps, as a rule, weak stimuli tend to augment, and strong to inhibit inspiration; but the effects of artificially stimulating sensory nerves are complicated and often confused, because powerful afferent impulses by giving rise to pain may, through impulses generated by the pain itself and descending to the medulla from the brain, act in an indirect as well as in a direct manner; and the prominence of the indirect painful impulses will, in any experiment, depend on the anæsthetic used. We may say, however, that in all cases the effect is very largely determined by the condition at the time being of the respiratory centre itself; and that, in turn, is determined not only by things which affect its nutrition, such as the character of the blood circulating in it, but also by the nature and amount of the other afferent impulses which are playing upon it at the same time. Thus, as we shall presently see, the effect of a stimulus applied to the vagus, when the respiratory centre is inadequately supplied with arterial blood, is not the same when the centre has its normal supply of normal blood. So also a stimulus which, applied to the vagus or to another nerve in an intact animal, simply quickens inspiration, applied in an animal whose cerebral hemispheres have been removed will call forth a prolonged tetanic inspiratory gasp. The respiratory centre responds, in fact, in the most intricate and varied manner to nervous impulses proceeding from all parts of the body, and thus delicately adjusts the working of the respiratory pump to the needs of the economy.

$\S 312$. The complicated nature of the respiratory centre is further shown by the fact that it appears to consist of two lateral halves which normally work in unison and yet may be made to work independently. If the medulla oblongata be carefully divided in the middle line respiration may continue to go on in quite a normal fashion. If, however, one vagus be then divided, the respiratory movements, both costal and diaphragmatic, on the side of the body on which division of the vagus has taken place, become slower than those on the other side, so that the two sides are no longer synchronous; and a stimulus confined to one vagus affects the respiratory movements of that side of the body only. So, also, a section of a lateral half of the cord below the medulla stops the respiratory movements on that side alone.

$\$ 313$. Besides these nervous influences, however, there is another circumstance which, perhaps, above all others affects the respiratory centre, and that is the condition of the blood in respect to its respiratory changes; the more venous (less arterial) the blood, the greater is the activity of the respiratory centre. When by reason either of any hindrance to the entrance of air into the chest or other interference with the due interchange between the blood and the pulmonary air or of a greater respiratory activity of the tissues, as during muscular exertion, the blood becomes less arterial, more venous, $i$. $e$, with a smaller charge of oxygen and more heavily laden with carbonic acid, the respiration from being normal becomes labored. We may speak of normal breathing as eupnoea, and say that this, when the blood is insufficiently arterialized, passes into dyspnoea, an intermediate stage in which the respiratory movements are simply exaggerated being known as hyperpnoa. The modifications of breathing thus caused by deficient arterialization of blood are especially characterized by an increase in the total energy of the respiratory impulses generated, and in this respect differ from the modifications 
resulting from interference with the nervous arrangements such as those $f(0)$ lowing upon section of the vagus nerves, in which case, as we have seen, the rhythm is much more profoundly affected than the amount. In dyspnoa the breathing is frequently quicker as well as deeper, there is an increase in the sum of efferent respiratory impulses, and the expiratory impulses, which in normal respiration are very slight, acquire a pronounced importance. As the blood becomes in cases of obstruction less and less arterial, more and more venous, the discharge from the respiratory centre becomes more and more vehement, and instead of confining itself to the usual tracts and passing down to the ordinary respiratory muscles, overflows into other tracts and puts into action other muscles, until there is, perhaps, hardly a muscle in the body which is not made to feel its effects. The muscles which are thus more and more thrown into action are especially those tending to carry out or to assist expiration; and at last, if no relief is afforded, the violent but still definite respiratory movements give way to general convulsions of the whole body, which, however, have to a certain extent an expiratory character. With the onset of these convulsions dyspnoa is said to have passed into asphyxia. By the violence of these convulsions the whole nervous system becomes exhausted, the convulsions cease, and death is ushered in through a few infrequent and long-drawn breaths; but to this matter we shall return. The effect of venous blood, then, is to augment all those natural explosive decompositions of the substance of the central nervous system which give rise to respiratory impulses; it increases their amount and also quickens their rhythm. The latter change, however, is much less marked than the former, the respiration being much more deepened than hurried, and the several respiratory acts are never so much hastened as to catch each other up, and so to produce an inspiratory tetanus like that resulting from stimulation of the vagus. On the contrary, especially as exhaustion begins to set in, the rhythm becomes slower than proportionate to the weakening of the individual movements.

$\S 314$. The question naturally arises, Does this condition of the blood affect the substance of the central nervous system, that is to say, the respiratory centre in the medulla (and the subsidiary spinal nervous mechanisms) directly, or does it produce its effect by stimulating the peripheral ends of afferent nerves in various parts of the body, and, by the generation there of afferent impulses, indirectly modify the action of the central nervous system? IVithout denying the possibility that the latter mode of action may help in the matter, as regards not only the vagus, but all afferent nerves, the following facts seem to show that the main effect is produced by the direct action of the blood on the central nervous system, and, indeed, on the medullary respiratory centre itself. If the spinal cord be divided below the medulla oblongata, and both vagi be cut, want of proper aëration of the blood still produces an increased activity of the respiratory centre, as shown by the increased vigor of the facial respiratory movements. If the supply of blood be cut off from the medulla by ligature of the carotid and intervertebral arteries dyspnoen is produced, though the operation produces at first no change in the blood generally, but simply affects the respiratory condition of the medulla itself by cutting off its blood-supply, the immediate result of which is an accumulation of carbonic acid and a paucity of available oxygen in the nervous substance of that region. If the blood in the carotid artery in an animal be warmed above the normal, a dyspnoa is produced which, though apparently not quite identical with the dyspnoa caused by imperfect arterialization of the blood, shows that the too high temperature of the blood directly affects the activity of the respiratory centre. IVe may conclude, therefore, that the condition of the blood affects respiration by 
acting directly on the respiratory centre. Moreover, it is the medullary centre which, at all events in adult animals, is affected by the too venous blood, since after the division of the spinal cord below the medulla, dyspnœic thoracic respiratory movements and convulsions do not follow upon exclusion of air. They are, however, stated to occur in new-born animals, indicating that the subsidiary mechanisms in the upper spinal cord, of which we spoke in $\$ 306$, may be also affected by the too venous blood; but the doubts which we previously urged hold good in these cases also.

While the respiratory centre is thus being affected by the too venous blood, it is, until exhaustion begins to set in, more irritable, more easily and largely affected by afferent impulses than in its normal condition. During dyspnoa a stimulus which applied to the vagus or to some other sensory nerve under normal conditions would produce little or no effect, may start very powerful respiratory movements.

$\S 315$. Deficient aëration produces two effects in blood : it diminishes the oxygen and increases the carbonic acid. Do both of these changes affect the respiratory centre, or only one, and if so, which? When an animal is made to breathe an atmosphere containing nitrogen only, the exit of carbonic acid by diffusion is not affected, and the blood, as is proved by actual analysis, contains no excess of carbonic acid. Yet all the phenomena of dyspnoa are present, and if the experiment be continued, convulsions ensue and the animal dies in asphyxia. In this case the result can only be attributed to the deficiency of oxygen. On the other hand, if an animal be made to breathe an atmosphere rich in carbonic acid, but at the same time containing abundance of oxygen, though the breathing becomes markedly deeper and also somewhat more frequent, there is no culmination in a convulsive asphyxia, even when the quantity of carbonic acid in the blood, as shown by direct analysis, is very largely increased. On the contrary, the increase in the respiratory movements may after a while pass off; the animal becoming unconscious, and appearing to be suffering rather from a narcotic poison than from simple dyspnoea ; the excess of carbonic acid in the blood appears to affect other parts of the central nervous system, and especially portions of the brain, more profoundly than it does the respiratory centre. It has been maintained by some that while a deficiency of oxygen promotes inspiratory movements, an excess of carbonic acid stimulates the expiratory movements, the nervous mechanisms being so arranged that a lack of oxygen leads to an effort to get more of it, and a too great load of carbonic acid to an effort to get rid of it; but the facts are opposed to the existence of any such teleological adaptation. It is obvious, however, that a lack of oxygen and an excess of carbonic acid affect the respiratory centre in very different ways, and that in ordinary cases of interference with the interchange in the lungs, as in deficient aëration, it is the lack of oxygen which plays the principal part in developing the abnormal respiratory movements. We may infer that it too is chiefly concerned in regulating the more normal respiration, but cannot as yet say what is the exact share so be attributed to the carbonic acid.

We may here point out that it is not to be supposed that each breath is determined by the condition of the blood flowing through the capillaries of the medulla at the moment preceding that breath; it is not to be imagined that each breath is the result of the lack of oxygen felt immediately before. On the contrary, as we have previously urged, the respiratory centre, like the cardiac substance, is an automatic centre; the respiratory impulses issue from it in rhythmic series as a result of the molecular changes of the metabolism going on in its substance; and whatever affects that rhythm, whether few or many beats be influenced, produces its result by modifying 
that metabolism. A lack of oxygen in the blood, or a nervous impulse along an afferent fibre, both affect the centre by modifying its metabolism; but each probably affects it in a different way. It is beyond our present knowledge to explain how either the one or the other acts. We may imagine that a lack of oxygen on the other hand has a more profound effect in modifying the whole complex series of metabolic changes, the whole chain of building up and breaking down processes, thus in some way or other rendering the whole edifice, so to speak, more unstable; and that an afferent augmenting impulse (and possibly an excess of carbonic acid) acts rather after the fashion of what we are accustomed to call a stimulus, and fires oft a larger amount of the already stored up explosive compounds. And we may further imagine that the special feature of the substance of the respiratory centre is that the metabolism is so arranged as to be thus, unlike that of other living substances, rendered unstable and more explosive, not simply diminished or deadened by a lack of oxygen. But these as yet are matters of speculation.

We may, perhaps, add that, under various nutritive conditions, the sensitiveness of the metabolism of the respiratory centre to lack of oxygen may vary widely. Thus, while undoubtedly under the normal nutritive conditions afforded by the ordinary supply of normal blood to the medulla, lack of oxygen in that blood at once provokes increased respiratory movements, it need not do so under other nutritive conditions of the medulla. By transfusion a large proportion of the hæmoglobin-holding blood may in an animal be gradually replaced by hæmoglobinless normal saline solution. In such a. case the amount of oxygen brought to the medulla by the diluted blood must be greatly diminisher, and yet if the change be made sufficiently slowly, no conspicuous dyspnoea is produced; under the new strange nutritrive conditions of the diluted blood the medulla is not affected in the same way as before by lack of oxygen.

$\S 316$. There are reasons for thinking that conditions of the blood, other than variations in the amount of oxygen and carbonic acid, may also materially affect the working of the respiratory centre. It is a matter of common experience that muscular exertion, especially if at all excessive, increases the respiratory movements; violent exercise soon puts a man "out of breath." This increased activity of the respiratory centre is in large measure at all events caused by the character of the blood which during and for some little time after the movements is carried to the medulla, and not by any nervous impulses sent up to the medulla from the contracting muscles. This is shown by the fact that if in an animal the spinal cord be divided in the dorsal or lumbar region and the hind limbs be powerfully tetanized, the respiratory movements are increased; the animal pants as it would do if it had been rumning. In such a case the only connection between the hind limbs and the respiratory centre is through the blood; it must be some change in the blood caused by the muscular contractions which affects the medulla when the blood passes from the hind limbs to be distributed by the heart to the medulla. Now when a nuscle contracts, its consumption of oxygen and production of carbonic acid, especially the latter $(\$ 60)$, are increaserl; the blood leaving the muscle is more venous than usual. Hence, when many muscles are contracting powerfully, the blood carried to the right side of the heart is more venous than usual; and we might expect that it is this unusually vemous blood failing to be adequately arterialized in the lungs, and hence reaching the merlulla from the left side of the heart in a more venous, less completely arterialized, condition than usual, which stirs up the respiratory centre to increaser activity.

On examination, however, it is found that the blood leaving the left side 
of the heart in such cases is not less arterialized, but, if anything, more arterialized than usual. The increased respiratory movements induced by the changed b!ood soon prove sufficient, or even more than sufficient, to give the blood the extra quantity of oxysen and to remove the extra quantity of carbonic acid. Obviously the blood coming from the tetanized muscles affects the respiratory centre by virtue of some quality which, unlike that due to the deficiency of oxygen or excess of carbonic acid, is not immediately affected by the passage through the lungs. Whether the quality in question be dependent on an excess of sarcolactic acid, or on some other product or products of muscular metabolism, we do not as yet know. But the fact that substances in the blood may so affect the respiratory centre is interesting, since it shows by how many safeguards the working of the respiratory centre is carefully adapted to the needs of the economy.

Thus a change in the circumstances surrounding an animal body, or a change in the body itself, may, in one or more of several ways-by acting as a stimulus to some afferent nerves and so sending up afferent nervous impulses to the respiratory centre, or by interfering with the interchange of gases in the lungs, or by otherwise altering the proportion of the gases present in the blood reaching the respiratory centre, or by generating or increasing in that blood some substance or substances tending to affect the nutrition of the respiratory centre-affect the working of the all-important breathing mechanism. And the affection so wrought has generally an adaptative character; it generally tends to protect the organism against the evil effects of the change.

\$317. Apnoea. When we attempt to hold our breath, we find that we can do this fur a limited time only ; sooner or later a breath must come; but, as is well known, the time during which we can remain without breathing may on occasion be much prolonged, if we first of all take a series of deep breaths. It is probable, though perhaps not distinctly proved, that when we breathe voluntarily, or when by an act of the will we hold the respiratory apparatus in any one respiratory phase, the nervous impulses, generated by the will, do not pass down by a direct and independent course to the respiratory muscles, but that the will makes use or modifies the activity of the medullary and spinal nervous respiratory mechanisms. The breath sooner or later inevitably follows because at last the natural impulses proceeding from the respiratory centre become too imperious to be any longer held in check by the impulses of volition passing down to the centre from the brain. The fact that a series of deep breaths, a thorough ventilation of the lungs, postpones the victory of the unconscious centre, shows that such a ventilation in some way delays the development of the natural respiratory impulses. A similar but still more marked delay may often be seen in an animal under artificial respiration. If in a rabbit artificial respiration is carried on very vigorously for a while, and then suddenly stopped, the animal does not immediately begin to breathe. For a variable period no respiratory movements at all take place, and breathing when it does begin occurs gently and normally, only passing into dyspnoa if the animal is unable to breathe of itself; and even then the transition is quite gradual. Evidently during this period the respiratory centre is in a state of complete rest, no explosions are taking place, no respiratory impulses are being generated, and the quiet transition from this condition to that of normal respiration shows that the subsequent generation of impulses is attended by no great disturbance. Not only is the centre at rest, but it is less irritable than the normal; impulses along the vagus or other nerves which otherwise would produce respiratory explosions are now ineffectual. This state of things is known as that of apnoe, the converse of dyspnœa ; and the longer pause in breathing men- 
tioned above as possible after unusual ventilation of the lungs may be regarded as a brief apnœa.

Now it seemed natural to suppose that such a state of rest of the respiratory centre was brought about by the more than necessarily ample supply of oxygen afforded by the previous increased inspiratory movements; and, indeed, it was maintained that apnœa was the result of too great, just as dyspnoed is the result of too little, arterialization of the blood reaching the respiratory centre. It was argued that owing to the increased vigor of the artificial respiratory movements the hæmoglobin of the arterial blood, which in normal breathing is not quite saturated with oxygen, became almost completely so, and that at the same time the quantity of oxygen simply dissolved in the blood became largely increased and its tension largely augmented, But there are reasons which render such a view untenable. In the first place there is no direct and satisfactory proof that in apnoa the arterial blood is overloaded with oxygen as supposed; indeed, during the course of apnoa before it has come to an end the blood becomes distinctly less arterial, more venous than usual. In the second place apnoea if not entirely impossible, is much nore difficult to bring about when both vagus nerves are divided, and if it does occur after section of the vagus nerves has not the same characters as ordinary apnoea. Now, when artificial respiration is being carried on, section of the vagus nerves can have no effect on the quantity of oxygen taken up by the blood in the lungs. But the vagus nerves are the channel of impulses affecting the respiratory centre, and this relation of the apnœa to the vagus nerves suggests another and different interpretation of apnœa. As we have seen, expansion of the lung by acting in some way or other on the pulmonary terminations of the vagus nerve sends up along that nerve impulses which inhibit inspiration. And it is argued that repeated forcible inflations of the lungs produce apnœa by generating potent inhibitory impulses, which by a kind of summation of their effects in the medulla stop for a while the generation of respiratory impulses in the respiratory centre. This conclusion, moreover, is strongly supported by the fact that an apnoa may be produced, so long as the vagus nerves are intact, by forcible artificial respiration with hydrogen instead of atmospheric air; in other words, the inhibitory impulses generated in the vagus nerves by the inflation are sufficient wholly to neutralize the development of respiratory impulses which the deficient arterialization of the blood would otherwise have produced. The exact nature and development of such a summation of inhibitory impulses, especially in the presence of correlative augmentative impulses called forth by the corresponding successive collapses of the lungs, is too complex a matter to be dwelt on here. Moreover, an apnoa may be produced, though, as we have said, with difficulty after section of both vagus nerves; but in this case air and not hydrogen must be used for inflation, the use of the latter, in contrast to the result when the nerves are intact, leading to dyspnœa. The subject cannot as yet be considered as fully cleared up. That apnoa as ordinarily produced is in some way the result of inhibitory impulses generated by the inflations can, however, hardly be doubted.

$\$ 318$. Secondary respiratory rhythm-Cheyne-Stokes respiration. A remarkable abnormal rhythm of respiration, first observed by Cheyne but afterward more fully studied by Stokes, and hence called by their combined names, occurs in certain pathological cases. The respiratory movements gradually decrease both in extent and rapidity until they cease altogether, and a condition of apnœa, lasting it may be for several seconds, ensues. This is followed by a feeble respiration, succeeded in turn by a somewhat stronger one, and thus the respiration returns gradually to the normal, or may even rise to hyperpnœa or slight dyspnœa, after which it again declines 
in a similar manner. A secondary rhythm of respiration is thus developed, periods of normal or slight dyspnœic respiration alternating by gradual transitions with periods of apnœa. The cause of the phenomena is not thoroughly understood. Whether the waning and waxing of the respiratory movements be due to corresponding rhythmic changes in the nutrition of the respiratory centre itself, or to a rhythmic increase and decrease of inhibitory impulses playing upon that centre from other parts of the body, for instance from higher regions of the brain, has not yet been settled. It frequently appears in connection with a fatty condition of the heart, but has been met with in various maladies. Closely similar phenomena have been observed during sleep, under perfectly normal conditions; and this fact is rather in favor of the latter of the two explanations just given. The phenomena present a striking analogy with the "groups" of heart-beats so frequently seen in the frog's ventricle placed under abnormal circumstances.

\section{The Effects of Changes in the Composition and Pressure} of the Air Breathed.

$\$ 319$. The preceding sections have shown us that the respiratory mechanism is arranged to work satisfactorily when the lungs are adequately supplied with air of the ordinary composition of, and at the ordinary pressure of, the atmosphere. We have further seen that the mechanism can adapt itself within certain limits to changes in the composition and pressure of the air supplied. We may now consider briefly what takes place when those limits are overstepped. The most striking effects are seen when, on account of occlusion of the trachea, or by breathing in a confined space, or for other reasons, a due supply of air not being obtained, normal respiration gives place, through an intermediate phase of dyspnoea, to the condition known as asphyxia ; this, unless remedial measures be taken, rapidly proves fatal.

Asphyxia. As soon as the blood becomes less arterial, more venous than normal, the respiratory movements become deeper and at the same time more frequent; both the inspiratory and expiratory phases are exaggerated, the supplementary muscles spoken of $(\$ 276)$ are brought into play, and the rate of the rhythm is hurried. These effects, as we have seen, are chiefly to be ascribed to the deficiency of oxygen in the blood.

As the blood continues to become more and more venous the respiratory movements continue to increase both in force and frequency, a larger number of muscles being called into action and that to an increasing extent. Very soon, however, it may be observed that the expiratory movements are becoming more marked than the inspiratory. Every muscle which can in any way assist in expiration is in turn brought into play; and at last almost all the muscles of the body are involved in the struggle. The orderly expiratory movements culminate in expiratory convulsions, the order and sequence of which are obscured by their violence and extent. That these convulsions, through which dyspnoa merges into asphyxia, are due to a stimulation (by the venous blood) of the medulla oblongata, is proved by the fact that they fail to make their appearance when the spinal cord has been previously divided below the medulla, though they still occur after those portions of the brain which lie above the medulla have been removed. It is usual to speak of a "convulsive centre" in the medulla, the stimulation of which gives rise to these convulsions; but if we accept the existence of such a centre we must at the same time admit that it is connected by the closest ties with the normal expiratory division of the respiratory centre, since every intervening step may be observed between a simple slight ex- 
piratory movement of normal respiration and the most violent convulsion of asphyxia. An additional proof that these convulsions are carried out by the agency of the medulla is afforded by the fact that convulsions of a wholly similar character are witnessed when the supply of blood to the medulla is suddenly cut off by ligaturing the blondvessels of the head. In this case the nervous centres, being no longer furnished with fresh blood, become rapidly asphyxiated through lack of oxygen, and expiratory convulsions quite similar to those of ordinary asphyxia, and preceded like them by a passing phase of dyspnœea, make their appearance. Similar "anæmic" convulsions are seen after a sudden and large loss of blood from the body at large, the medulla being similarly stimulated by the lack of arterial blood. In ordinary fainting, which is loss of consciousness due to an insufficient supply of blood to the brain, the diminution of blood-supply is not great enough to produce these convulsions.

Such violent efforts speedily exhaust the nervous system; and the convulsions after being maintained for a brief period suddenly cease and are followed by a period of calm. The calm is one of exhaustion; the pupils, dilated to the utmost, are unaffected by light; touching the cornea calls forth no movement of the eyelids, and, indeed, no reflex actions can anywhere be produced by the stimulation of sentient surfaces. All expiratory active movements have ceased; the muscles of the body are flaccid and quiet; and though from time to time the respiratory centre gathers sufficient energy to develop respiratory movements, these resemble those of quiet normal breathing, in being, as far as muscular actions are concerned, almost entirely inspiratory. They occur at long intervals, like those after section of the vagi; and like them are deep and slow. The exhausted respiratory centre takes some time to develop an inspiratory explosion; but the impulse when it is generated is proportionately strong. It seems as if the resistance which had in each case to be overcome was considerable, and the effort in consequence, when successful, productive of a large effect.

Very soon these inspiratory efforts become less frequent; their rhythm becomes irregular; long pauses, each one of which seems a final one, are succeeded by several somewhat rapidly repeated inspirations. The pauses become longer, and the inspiratory movements shallower. Each inspiration is accompanied by the contraction of accessory muscles, especially of the face, so that each breath becomes more and more a prolonged gasp. The inspiratory gasps spread into a convulsive stretching of the whole body ; and with extended limbs, and a straightened trunk, with the head thrown back, the mouth widely open, the face drawn, and the nostrils dilated, the last breath is taken in.

Thus we are able to distinguish three stages in the phenomena which result from a continued deficiency of air: 1. A stage of dyspnœa, characterized by an increase of the respiratory movements both of inspiration and expiration. 2. A convulsive stage, characterized by the dominance of the expiratory efforts, and culminating in general convulsions. 3. A stage of exhaustion, in which lingering and long-drawn inspirations gradually die out. When brought about by sudden occlusion of the trachea these events run through their course in about four or five minutes in the dog and in about three or four minutes in the rabbit. The first stage passes gradually into the second, convulsions appearing at the end of the first minute. The transition from the second stage to the third is somewhat abrupt, the convulsions suddenly ceasing early in the second minute. The remaining time is occupied in the third stage.

The duration of asphyxia varies not only in different animals, but in the same animal under different circumstances. Newly born and young animals 
need much longer immersion in water before death by asphyxia occurs than do adults. Thus while in a full-grown dog recovery from drowning is unusual after one and a half minutes, a newborn puppy has been known to bear an immersion of as much as fifty minutes. The cause of the difference lies in the fact that in the quite young or rather just-born animal the respiratory changes of the tissues are much less active. These consume less oxygen, and the general store of oxygen in the blood has a less rapid demand made upon it. The respiratory activity of the tissues may also be lessened by a deficiency in the circulation; hence bodies in a state of syncope at the time when the deprivation of oxygen begins can endure the loss for a much longer period than can bodies in which the circulation is in full swing. There being the same store of oxygen in the blood in each case, the quicker circulation must of necessity bring about the speedier exhaustion of the store. So also anæsthetics may diminish the effects and delav the final results: large doses of anæsthetics may prevent the exaggerated and convulsive movements. In many cases of drowning, death is hastened by the entrance of water into the lungs.

By training, the respiratory centre may be accustomed to bear a scanty supply of oxygen for a much longer time than usual before dyspnœea sets in, as is seen in the case of divers.

The phenomena of slow asphyxia, where the supply of air is gradually diminished, are fundamentally the same as those resulting from a sudden and total deprivation. The same stages are seen, but their development takes place more slowly.

$\$ 320$. Deficiency of air results not only in a diminution of the oxygen but also in an increase of the carbonic acid of the blood. We have seen, however ( $\$ 315)$, that the phenomena of asphyxia are in the main due to the former, and that the accumulation of carbonic acid in the blood has subsidiary effects only.

If the percentage of oxygen in the inspired air be increased instead of diminished, the total pressure of the atmosphere reniaining the same, the partial pressure of the oxygen alone being changed, no marked results follow. We have already seen $(\$ 297)$ that the percentage of oxygen in the ordinary atmosphere leaves a wide margin of safety, and that ( $\$ 317$ ) the phenomena of apnœa are in the main at least to be explained as the result not of an increase in the oxygen of the blood but of nervous impulses ascending the vagus nerves. We have no satisfactory evidence that, provided the respiratory mechanism is in good working order, an increase of oxygen in the inspired air even to a whole atmosphere seriously modifies the respiratory act; and it may be doubted whether any effect is produced even when the mechanism is impaired.

$\$ 321$. The composition of the atmosphere, the pressure remaining the same, may be modified by the introduction of foreign gases. To some of these the respiratory mechanism is indifferent; for instance, hydrogen may be substituted for nitrogen without any change in the respiration, provided, of course, that the oxygen is not diminished. Other gases may produce poisonous effects, either by interfering with some of the respiratory processes or in other ways. Thus carbon monoxide, by combining with the hæmoglobin of the red corpuscles, and so preventing the corpuscles from acting as oxygen-carriers, produces asphyxia through deficiency of oxygen. Sulphuretted hydrogen interferes with the oxygenation of the blood by acting as a reducing agent. Some gases while allowing the ordinary respiratory changes of the blood to go on as usual produce toxic effects by acting on one or other of the tissues. Thus, as we have seen, an excess of carbonic acid in the blood seems to have a special effect on the central nervous system and 
so acts as a narcotic poison. The peculiar effects of nitrous oxide (laughing gas) are similarly due to the direct action of the gas in the blood on the central nervous system. Some gases are irrespirable and may interfere with respiration, even causing suffocation, on account of their causing spasm of the glottis, and this is said to be, to a certain extent, the case with an atmosphere which is wholly or largely composed of carbonic acid.

$\$ 322$. The effects of changes in atmospheric pressure. Diminution of pressure. The partial pressure of the oxygen in the inspired air may be changed, not only by altering the composition of the air entering at the ordinary atmospheric pressure, but also by altering the total pressure of the atmosphere without changing its composition. The results of the latter are, however, complicated; we have then to deal not merely with the effects on the interchange of gases in the lungs but with the effects on the whole organism. All the complicated machinery of the body is adapted and arranged to work under what we may call ordinary atmospheric pressure, that is to say, within the limits of $760 \mathrm{~mm}$. mercury at the sea level and about $500 \mathrm{~mm}$., corresponding to an altitude of 6000 feet, this being the range of ordinary human dwellings. Any great increase or decrease of pressure beyond these limits will affect not only the exit of carbonic acid from and the entrance of oxygen into the blood, but, in varying degree, all the physical and chemical processes of the body. A gross instance of this is seen when an animal is suddenly subjected to a great diminution of pressure, as when it is placed in the receiver of an air-pump and the receiver rapidly exhausted. The animal is soon thrown into fatal convulsions, which are in part, but only in part, due to the liberation of gas from the blood within the bloodvessels; the gas so set free mechanically interferes with the circulation, as by obstructing the play of the cardiac valves, or by plugging the smaller bloodvessels, and thus helps to bring the machine to a standstill. The free gas found in the vessels upon examination after death is said to be composed chiefly of nitrogen, the carbonic acid and the oxygen, which probably were also set free, having been reabsorbed before the examination was made.

But, quite apart from gross effects of this kind, it is very obvious that the organism must in many ways suffer from a diminution of pressure. The complex and delicately balanced vascular system is constructed to work at the ordinary atmospheric pressure. The force of the heart-beat and the tonic contraction of the small arteries are, so to speak, pitched to meet the influence exerted on the outside of the bloodvessels by the ordinary pressure of the atmosphere; and any great diminution of that pressure must produce a greater or less disarrangement of the vascular mechanism until it is counterbalanced by some compensating changes. And a little reflection will supply many other instances.

We have already called attention $(\$ 297)$ to the fact that, the total pressure of the atmosphere remaining the same, the partial pressure of the oxygen in the inspired air may be reduced as low as about $76 \mathrm{~mm}$. (10 per cent.) without seriously modifying the respiration. In order to attain this diminution of the partial pressure of the oxygen without changing the composition of the atmosphere, the total pressure of the atmosphere must be reduced to the limit of $300 \mathrm{~mm}$., corresponding to an altitude of 17,000 feet. Now it is a matter of common experience that in ascending a mountain "distress" is felt long before such an altitude is reached. The distress felt on such occasions is probably due not so much, if indeed at all directly, to the diminution of oxygen as to a general disarrangement of the organism and perhaps more particularly of the vascular system. The nose-bleeding which is so frequent an occurrence under the circumstances shows that the minute 
bloodvessels more directly exposed to the diminution of pressure are profoundly affected by it; and what is true of them is, probably, in various ways and to different degrees, true of the whole vascular system. The breathlessness which is so marked a feature on these occasions seems due not so much to the fact that the blood which reaches the respiratory nervous centres is deficient in oxygen, as to the fact that the troubled vascular system fails to deliver to those centres their blood in an adequate fashion.

It is a feature of the vascular system, and indeed of the other mechanisms of the body in which nervous factors intervene, that they possess the power of adapting themselves to changed conditions; and as is well known, the human organism somewhat rapidly becomes accustomed to these moderate altitudes. Practice and custom have far less effect, though they have some, on the more fundamental processes depending on the actual supply of oxygen; and it is at the extreme altitudes, where in addition to the other troubles a deficiency of oxygen definitely makes itself felt, that the body seems to fail in adapting itself to the new circumstances.

The addition of these troubles not directly respiratory in nature, when the supply of oxygen is diminished by a diminution of the total pressure, perhaps explains why though an adequate lowering of pressure will produce asphyxia, that asphyxia is somewhat different from the ordinary asphyxia due to deprivation of air or oxygen. Convulsions which are essential to ordinary asphyxia are at times wholly absent; the nervous system under the peculiar conditions does not respond to the stimulus of the lack of oxygen; and other nervous symptoms, such as a rapid onset of feebleness amounting almost to paralysis, are apt to make their appearance.

$\$ 323$. The effects of increase of atmospheric pressure. These are in many ways remarkable. Up to a pressure of several atmospheres of air, the only symptoms which present themselves are those somewhat resembling narcotic poisoning. The animal becomes sleepy and stupid, the result probably not so much of respiratory changes, as of the effects of the increased pressure on the whole organism to which we have just alluded. At a pressure, however, of 15 atmospheres of air, or what amounts to the same thing, of 3 atmospheres of oxygen, and upward, a very remarkable phenomenon presents itself. The animals die of asphyxia and convulsions, exactly in the same way as when oxygen is deficient. Corresponding with this it is found that the production of carbonic acid is diminished. That is to say, when the pressure of the oxygen is increased beyond a certain limit, the oxidations of the body are diminished, and with a still further increase of oxygen are arrested altogether. The oxidation of phosphorus is perhaps analogous; at a high pressure of oxygen phosphorus will not burn. Not only animals, but plants, bacteria, and organized ferments, are similarly killed by too great a pressure of oxygen.

\section{The Relations of the Respiratory System to the Vascular and} OTHER Systems.

$\S 324$. Many events in the body show the influence which the respiratory movements exert on the circulation. When the brain of a living mammal is exposed by the removal of the skull, a rhythmic rise and fall of the cerebral mass, a pulsation of the brain, quite distinct from the movements caused by the pulse in the arteries of the brain, is observed; and upon examination it will be found that these movements are synchronous with the respiratory movements, the brain rising up during expiration and sinking during inspiration. They disappear when the arteries going to the brain are ligatured, or when the venous sinuses of the dura mater are laid open so as to admit of a 
free escape of the venous blood. They evidently arise from the expiratory movements in some way hindering and the inspiratory movements assisting the return of blood from the brain. We have already (\$105) stated that during inspiration the pressure of blood in the great veins may become negative, $i$. e., may sink below the pressure of the atmosphere; and a puncture of one of these veins may cause death by air being actually drawn into the vein and thus into the heart during an inspiratory movement. When the veins of an animal are laid bare in the neck and watched, the so-called pulsus venosus may be observed in them, that is, they swell up during expiration and diminish again during inspiration. And indeed a little consideration will show that the expansion and contraction of the chest must have a decided effect on the flow of blood through the thoracic portion of, and thus indirectly on that through the whole of, the vascular system.

This is well illustrated by the effects of respiration on arterial bloodpressure. We have seen, while treating of the circulation, that the arterial blood-pressure curves are marked by undulations, which, since their rhythm is synchronous with that of the respiratory movements, are evidently in some way connected with respiration. Similar undulations may be observed in the pulse-tracings taken from man.

Fig. 107.

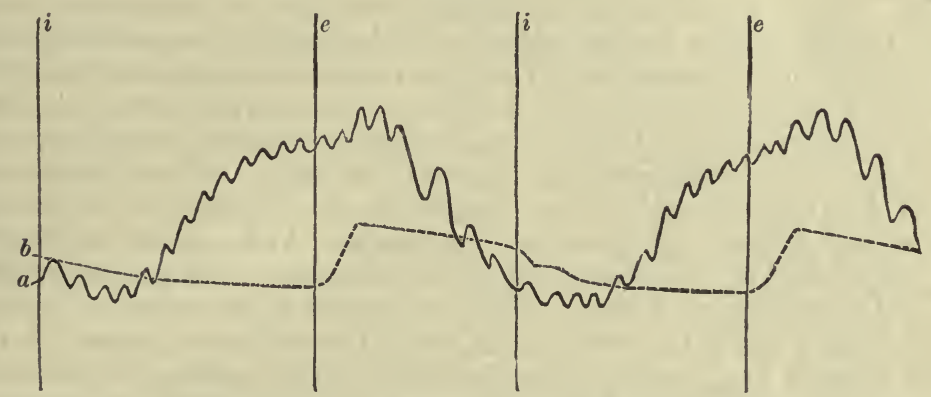

Comparison of Blood-pressure Curve with Curve of Intra-thoracic Pressure. (Dog.) $a$ is the blood-pressure curve taken by means of a mercury manometer; it shows the respiratory undulation, the slower beats on the descent being very marked. $b$ is the curve of intra-thoracic pressure obtained by connecting one limb of a manometer with the pleural cavity. Inspiration begins at $i$, expiration at $e$. With the beginning of inspiration (i) the expansion of the chest causes a marked fall of the mereury in the intra-thoracic manometer : but the effect soon diminishes, since the lessening of intra-thoracic pressure does not bear on the manometer alone, but on the lungs also; and as the lungs expand more and more the fall in the mercury becomes less and less until toward the end of inspiration the curve becomes very nearly a straight line. Conversely, the return of the chest at the beginning of expiration ie) produces at first a marked rise of the mercury in the manometer; but this soon ceases as the air leaves the chest and the lungs shrink, whereupon the mercury falls slowly.

When these undulations of the blood-pressure curve are compared carefully with the respiratory movements or with the variations of intra-thoracic pressure, what is most commonly observed is that while the blood-pressure, on the whole, rises during inspiration and falls during expiration, neither the rise nor the fall is exactly synchronous with either inspiration or expiration. Fig. 107 shows two tracings from a dog taken at the same time, one, $a$, being the ordinary blood-pressure curve from the carotid, and the other, $b$, representing the condition of the intra-thoracic pressure as obtained by carefully bringing a manometer into connection with the pleural cavity. On comparing the two curves it is evident that neither the rise nor the fall of arterial pressure coincides exactly either with inspiration or with expiration. At 
the beginning of inspiration (i) the arterial pressure is seen to be falling; it soon, however, begins to rise, but does not reach the maximum until some time after expiration $(e)$ has begun; the fall continues during the remainder of expiration, and passes on into the succeeding inspiration. This suggests the idea that, while inspiration tends to increase and expiration to diminish the blood-pressure, there are causes at work which in each case delay the effect.

Extended observations, however, show that such a relation as that shown in the figure, though frequent, is not constant. In fact, the effects of the respiratory movements on blood-pressure are found to vary very widely according as the respiration is quick or slow, easy and shallow, or labored and deep, and especially as the air enters into the chest readily or with difficulty. Moreover, respiratory undulations of blood-pressure are seen not only with natural but also with artificial respiration; in the latter the mechanical conditions are to a large extent the reverse of those of the former, and might fairly be expected to affect the circulation in a different way. The causation of these respiratory undulations is, in fact, complex. The respiratory act affects the vascular system in several different ways, and the general effect varies according as one or other influence is predominant. These several actions are sufficiently interesting and important to deserve discussion.

\$ 325. The heart and great bloodvessels are, like the lungs, placed in the air-tight thoracic cavity, and are subject like the lungs to the pumping action of the respiratory movements. Were there no lungs present in the chest, the whole force of the expansion of the thorax in inspiration would be directed to drawing blood from the extra-thoracic vessels toward the heart, and conversely in expiration the effect of the return of the thorax to its previous dimensions would be to drive the blood thus drawn in back again from the heart toward the extra-thoracic vessels. And, even in the presence of the lungs, some of this effect is still felt. The main purpose and the main result of the expansion of the chest in inspiration is, of course, to draw air into the lungs; by that expansion the air in the pulmonary alveoli is rarefied and brought to a lower pressure than that of the atmosphere outside the chest; and the difference of pressure thus set up leads to an inrush of inspired air until an equilibrium of pressure is established between the air in the lungs and that outside the chest. Before, however, the inspired air can fill a pulmonary alveolus the elastic walls of the alveolus have to be distended, and that distention is effected by means of the pressure which causes the inspired air to enter. Part of the atmospheric pressure, in fact, which causes the entrance of the air into the lung is spent in overcoming the elasticity of the pulmonary passages and cells. So that while by the inrush of inspired air the difference of pressure between the air inside the pulmonary alveoli and that outside the chest, brought about by the thoracic expansion, is completely neutralized, the difference between the pressure to which the parts lying within the thorax, but outside the lungs, are exposed and that outside the chest is not so completely neutralized. The pressure on these parts always falls short of the pressure of the atmosphere by the amount of pressure necessary to counterbalance the elasticity of the pulmonary passages and alveoli. Consequently, any structure lying within the thorax, but outside the lungs, is never, even at the conclusion of an inspiration, when the lungs are filled with air, subject to a pressure as great as that of the atmosphere. And, since the fraction of the atmospheric pressure which is thus spent in distending the lungs increases as the lungs become more and more stretched, it follows that the fuller the inspiration the greater is the difference between the pressure on structures within the thorax, but outside the 
lungs, and the ordinary pressure of the atmosphere. Now, we have seen that the pressure necessary to counterbalance the elasticity of the lungs, when they are completely at rest (in the pause between expiration and inspiration), is in man about 5 to $7 \mathrm{~mm}$. of mercury, and that when the lungs are fully distended, as at the end of a forcible inspiration, the pressure rises to as much as $30 \mathrm{~mm}$. of mercury. Hence, at the height of a forcible inspiration the pressure exerted on the heart and great vessels within the thorax is $30 \mathrm{~mm}$. less than the ordinary atmospheric pressure of $760 \mathrm{~mm}$., and even when the chest is completely at rest, at the end of an inspiration, the pressure on the heart and great vessels is slightly (by about $5 \mathrm{~mm}$. of mercury) below that of the atmosphere. We may add that any obstacle to the free ingress of the inspired air, any difficulty in the full expansion of the pulmonary alveoli, of course increases the negative pressure to which the thoracic structures outside the lungs are subjected by the expansion of the chest. Hence, when the trachea is closed a very large part of the thoracic expansion is directed to increasing the negative pressure around the heart and great bloodvessels.

During an inspiration, then, the pressure around the heart and great bloodvessels becomes considerably less than that of the atmosphere on the vessels outside the thorax. During expiration this pressure returns toward that of the atmosphere, but in ordinary breathing never quite reaches it. It is only in forcible expiration that the pressure on the thoracic vascular organs reaches or exceeds that of the atmosphere. But.if during inspiration the pressure bearing on the right auricle and the venæ cavæ become less than the pressure which is bearing on the jugular, subclavian, and other veins outside the thorax, this nust result in an increased flow from the latter into the former. Hence, during each inspiration a larger quantity of blood enters the right side of the heart. This probably leads to a stronger stroke of the heart, and at all events causes a larger quantity to be ejected by the right ventricle; this causes a larger quantity to escape from the left ventricle, and thus more blood is thrown into the aorta, and the arterial pressure proportionately increased. During expiration the converse takes place. The pressure on the intra-thoracic bloodvessels returns to the normal, the flow of blood from the veins outside the thorax into the venæ cava and right auricle is no longer assisted, and in consequence less blood passes through the heart into the aorta, and arterial pressure falls again. During forced expiration the intra-thoracic pressure may be so great as to afford a distinct obstacle to the flow from the veins into the heart.

The effect of the respiratory movements on the arteries is naturally different from that on the veins. During inspiration the diminution of pressure in the thorax around the aortic arch tends to expand the aortic arch and thus to check the onward flow of blood and to diminish the pressure of blood within the aorta. During expiration the increase of pressure outside the aortic arch of course tends to increase also the blood-pressure within the aorta, acting in fact just in the same way as if the coats of the aorta themselves contracted. Thus, as far as arterial blood-pressure is concerned, the effects of the respiratory movements on the great veins and great arteries respectively are antagonistic to each other; the effect on the veins being to increase arterial pressure during inspiration and to diminish it during expiration, while the effect on the arteries is to diminish arterial pressure during inspiration and to increase it during expiration. But we should naturally expect the effect on the thin-walled veins to be greater than that on the stout, thick-walled arteries, so much so that the direct effect on the arteries may be neglected. That is to say, we should expect the blood-pressure to rise during inspiration and to fall during expiration. This, as we have seen, 
is frequently the case, and, indeed, when the breathing is deep and labored, and especially during violent and sudden respiratory movements, the influence in this direction on the blood-pressure curve of the pumping action of the chest is unmistakable.

In attempting, however, to estimate the effect of the respiratory movements on blood-pressure we must bear in mind what is taking place in the abdomen. In inspiration the descent of the diaphragm compresses the abdominal viscera, and so, while at the very first it drives a quantity of blood onward along the inferior vena cava, subsequently hinders the upward flow from the abdomen and lower limbs; at the same time by compressing the abdominal aorta, it tends to raise the pressure in the thoracic aorta and its branches, while lowering that of the abdominal aorta and its branches. The effect of easy expiration would be the converse of this; but in forced expiration the pressure of the contracting abdominal muscles would, as in inspiration, first tend to drive the blood onward along the vena cava, but subsequently to hinder the flow both along the vena cava and the aorta. The effect of the abdominal movements therefore is mixed and variable, and their influence on the blood-pressure in the femoral artery must be different from that on the radial artery or other branch of the thoracic aorta. It is difficult to predict what in all cases the effect would be; and the matter cannot be settled by eliminating the movements of the diaphragm through section of the phrenic nerves, since in such a case the whole working of the respiratory pump is materially affected.

$\$ 326$. In addition to the influence thus exerted by the thoracic movements on the great veins leading to and the great arteries leading from the heart, we have to consider the behavior of the pulmonary vessels themselves under the varying thoracic pressure. These, like the venæ cavæ and aorta, tend to expand under the influence of the inspiratory expansion of the chest, and thus to become fuller of blood, very much as they would if the whole lung were placed under a large cupping-glass. The first effect of this increased filling of the pulmonary vessels would be to retain for a while a certain quantity of blood in the lungs and thus to lessen the amount falling into the left auricle. But this would be temporary only, and the widening of the pulmonary vessels would speedily produce an exactly contrary effect, namely, an increased flow through the lungs due to the diminished resistance offered by the widened passages. Conversely, the first effect of expiration would be an increased flow into the left auricle due to the additional quantity of blood driven onward by the partial collapse of the pulmonary vessels, followed by a more significant diminished flow caused by the greater resistance now offered by the narrower vascular channels. Thus the effect of inspiration in this way would be first to diminish the flow into the left auricle and so into the left ventricle, but afterward, for the rest of the inspiration until the beginning of expiration, to increase the flow into the ventricle; while conversely the effect of expiration would be first, for a brief period, to increase, and afterward, during the rest of the movement, to diminish the flow of blood into the left ventricle. Further, while this may be considered as the effect on the pulmonary vessels, large and small taken altogether, the influence both of the thoracic negative pressure during inspiration, and the return in a positive direction during expiration, will bear more on the thin-walled pulmonary veins than on the stouter pulmonary artery; that is to say, as inspiration becomes established, there will be a diminution of pressure in the pulmonary veins greater than that in the pulmonary artery, and this will be an additional influence favoring the flow into the left ventricle; during expiration a similar difference of effect will be felt in the contrary direction. During the increase of flow into the rentricle 
the quantity of blood ejected at each stroke will increase, and each stroke will $(\$ 148)$ be increased in vigor, in consequence of which the arterial pressure will rise. Conversely, during the decrease of flow into the ventricle the arterial pressure will fall. Hence the general effect of the movements of the chest on the pulmonary vessels will be during the beginning of inspiration to continue the lowering of arterial pressure which was taking place during expiration, but subsequently to raise the arterial pressure; and conversely, at the beginning of expiration to continue the rise of arterial pressure which was taking place during inspiration, but subsequently to lower the arterial pressure. In ordinary breathing, as we have seen, what may be considered as the norinal relations of blood-pressure to the respiratory movements are precisely of this kind.

$\$ 327$. Effects of the respiratory movements, however, are seen not only in natural but also in artificial respiration. When, for instance, in an animal under urari, artificial is substituted for natural respiration, undulations of the blood-pressure curve, synchronous with the respiratory movements, are still observed (Fig. 108), though generally less in extent than those seen under natural conditions.

Now in artificial respiration, the mechanical conditions under which the thoracic viscera are placed as regards pressure, are the exact opposite of those existing during natural respiration, for when air is blown into the trachea to distend the lungs, the pressure within the chest is increased instead of diminished. Under these circumstances, applying the considerations laid down in the preceding paragraph with regard to natural respiration, we should expect to find that while the first effect of an artificial inspiration would be to drive an additional quantity of blood out of the lungs. into the left ventricle, and thus to raise arterial pressure, this would be in turn followed by a fall of arterial pressure due to the increased resistance offered both to the passage of blood through the lungs and to the entrance of blood through the venæ cavæ into the right auricle. Conversely, the effect of the succeeding expiration would be an initial continuance of the fall of arterial pressure succeeded by a rise. In other words, we should expect to find in artificial respiration effects exactly the reverse of those which we find in normal respiration; and, indeed, in many curves of blood-pressure taken during artificial respiration this is the case.

Both in natural and in artificial respiration, however, the features of the blood-pressure curve vary according as the breathing is hurried or slow, shallow or deep, and according to the facility with which air enters the chest, so much so that at times the blood-pressure curves of natural and artificial respiration may closely resemble each other. And a little consideration would lead us to expect this.

We have seen that the rise in arterial pressure which marks the respiratory undulation is, in the main, due to a temporary greater amount of blood thrown into the aorta by the left ventricle, and that, correspondingly, the fall of pressure completing the undulation is in the main due to a temporary lessening of the amount so thrown. Though the causes discussed in $\$ 325$ undoubtedly make themselves prominent in labored and violent respiratory movements, we may conclude that in ordinary respiration, both natural and artificial, the main events producing the respiratory undulations are those discussed in $\$ 326$. We may restate the conclusions of that discussion by saying that the respiratory movements affect the amount of flow of blood into the left ventricle, and so the discharge of blood from the left ventricle into the aorta, in two main ways. In the first place, through the widening or narrowing of the pulmonary vessels they alter 
the capacity of the vessels to hold blood for the time being. In the second place, in consequence of the difference of resistance, occasioned by the widening or narrowing, they alter the rate of flow through the pulmonary vessels. The first factor is a brief and passing one; the extra room due to widening is soon filled up, the narrowed ressels soon discharge the quantity which they can no longer hold. But the second factor is a more lasting one; so long as in the respiratory movement the vessels remain widened or narrowed so long is the rate of flow increased or diminished. These two factors produce opposite effects, and hence the total result of any particular kind of respiration will depend on their relative prominence. With quickly repeated respiratory movements the first factor comes to the front; when the respiratory movements are more slowly repeated and more slowly carried out the second factor is the more potent. Hence it comes about that in quickly repeated artificial respiration where the first factor is predominant, and the prominent effect of each inflation is to diminish the capacity of, and so to empty the pulmonary vessels and to increase the flow into the ventricle whereby the pressure rises in inflation, that is in inspiration, the blood-pressure curve stimulates that of a slowly repeated natural respiration, where the pressure also rises in inspiration, but where, the second factor being predominant, the rise of pressure brought "about by each inspiration is due mainly to the more rapid flow through the widened pulmonary vessels. And other illustrations of a like kind could be given.

$\S 328$. Besides the mechanical effects of the respiratory movements the vascular system is influenced by respiration through the changes in the gases of the blood.

Changes in the blood may affect, on the one hand, the vasomotor system and, on the other hand, the heart. They may further affect the heart either directly by acting on the cardiac tissues, or indirectly by means of the inhibitory and augmentor cardiac nerves. They may also, probably, affect the peripheral vessels, not only through vasomotor nerves, but by acting directly on the walls of the smaller vessels. We have indications of an action of respiration on the cardio-inhibitory system, even in normal quiet respiration. One striking feature of the respiratory undulation in the bloodpressure curve of the $\operatorname{dog}^{1}$ is the fact that the pulse-rate is quickened during the rise of the undulation and becomes slower during the fall. (See Fig. 107.) A similar influence may be seen in pulse-tracings taken from man. The quickening of the beat might be considered as itself partly accounting for the rise of pressure, or, on the other hand, it might be urged that the increased flow of blood which causes the rise of pressure, at the same time leads to the quickening of the beat, were it not for one fact, viz., that the difference is at once done away with, without any other essential change in the undulations, by section of both vagus nerves. Evidently the slower pulse during the fall is caused by a coincident stimulation of the cardio-inhibitory centre in the medulla ublongata, the quicker pulse during the rise being due to the fact that, during that interval, the centre is comparatively at rest. We have here indications that, while the respiratory centre in the medulla oblongata is at work, sending out rhythmic impulses of inspiration and expiration, the neighboring cardio-inhibitory centre is, as it were, ly sympathy, thrown into an activity of such a kind that its influence over the heart waxes with each expiration and wanes with each inspiration. We cannot as yet explain exactly the manner in which the activity of the one centre influences that of the other; it may be that during the expiratory phase the

${ }^{1}$ In the rabbit, the respiratory undulations, though well marked, present a very small difference of pulse-rate in the rise and fall. 
blood reaching the medulla is not quite so well arterialized, especially as far as the escape of carbonic acid is concerned, as during the inspiratory phase, and that the cardio-inhibitory centre is sufficiently sensitive to appreciate the slight difference; but of this we cannot be sure.

$\S 329$. When through interference with the pulmonary interchange the blood sent out from the left ventricle becomes and continues to be less arterialized than usual, the effects on both the heart and the vasomotor system become conspicuous. The rhythm of the heart-beats is most distinctly slowed. This, under ordinary circumstances, when the vagus nerves are intact, is probably in part the result of vagus inhibition, the venous blood, as suggested above, stimulating the cardio-inhibitory centre in the medulla. But the slowing is not wholly caused in this way, for it is still conspicuous in an animal placed under urari and with both vagus nerves divided. Compare curves 3 and 4 with 1 and 2 in Fig. 108. How this slowing is brought about is not very clear. When venous blood is sent through an excised heart, the beat is, it is true, slowed, but it is also and still more conspicuously weakened. Now when the blood becomes too venous, as is shown in Fig. 108, even after the action of the vagus nerves has been eliminated by section and also by urari, the slowing is out of proportion to the weakening, since, as we shall presently see, the blood-pressure rises; and though that rise is chiefly due to vasomotor constriction, still it could not take place if the cardiac stroke were very notably weakened. It may be that the venous blood stimulates the cardiac augmentor mechanism in such a way as to bring about an augmentation of the cardiac stroke rather than a quickening of the rhythm; but this has not been definitely proved. In any case a slow beat, with such a maintenance of the strength of the cardiac strokes as permits the continuance for some considerable time of a high blood-pressure, is met when the arterialization of the blood is interfered with. Sooner or later, however, the deficiency of oxygen in the blood dininishes the store of explosive compounds in the cardiac muscular substance, the beats lessen in force, often showing a temporary increase in frequency, and soon become irregular.

$\$ 330$. The effects of deficient arterialization on the vasomotor system are well shown when in an animal placed under a moderate dose of urari so as to eliminate the complications due to contractions of the skeletal muscles, with both vagi divided so as to insure the elimination of inhibitory impulses from the medulla, artificial respiration is suspended. Soon after the respiration is stopped, a very large but steady rise of pressure is observed. (See Fig. 108.) The rise so witnessed is very similar to that brought about by powerfully stimulating a number of vaso-constrictor nerves; and there can be no doubt that it is due to the venous blood stimulating the vasomotor centre in the medulla, and thus causing constriction of the small arteries of the body, especially those of the splanchnic area, since, as we shall see, in speaking of the skin, a too venous blood leads to a widening of the cutaneous arteries. We say "stimulating the medullary vasomotor centre" because, though we must admit that, since a rise of pressure follows upon dyspnœa when the spinal cord has been previously divided below the medulla, the venous blood may stimulate other vasomotor centres in the spinal cord and possibly even act directly on local peripheral mechanisms, yet the fact that the rise of pressure is much less under these circumstances shows that the medullary centre plays the chief part. As we have just said, the effect of this vasoconstriction in raising the pressure, if not assisted by an increase, at all events is not neutralized by an adequate decrease of the cardiac stroke. Upon the cessation of the artificial respiration, the respiratory undulations of course cease also, so that the blood-pressure curve rises at first steadily in almost a straight line broken only by the heart-beats; yet after a while new 
undulations, the so-called Traube or Traube-Hering curves, make their appearance (Fig. 108, 2, 3), very similar to the previous ones, except that their curves are larger and of a more sweeping character. These new undulations, since they appear in the absence of all thoracic or pulmonary movements, passive or active, and are witnessed even when both vagi are cut, must be of vasomotor origin; the rhythmic rise must be due to a rhythmic constriction of the small arteries, and this probably is caused by a rhythmic discharge from vasomotor centres, and especially from the medullary vasomotor centre. The undulations are maintained as long as the bloodpressure continues to rise. With the increasing venosity of the blood, however, both the vasomotor centres and the heart become enfeebled; the undulations disappear, and the blood-pressure rapidly sinks.

FIG. 108.

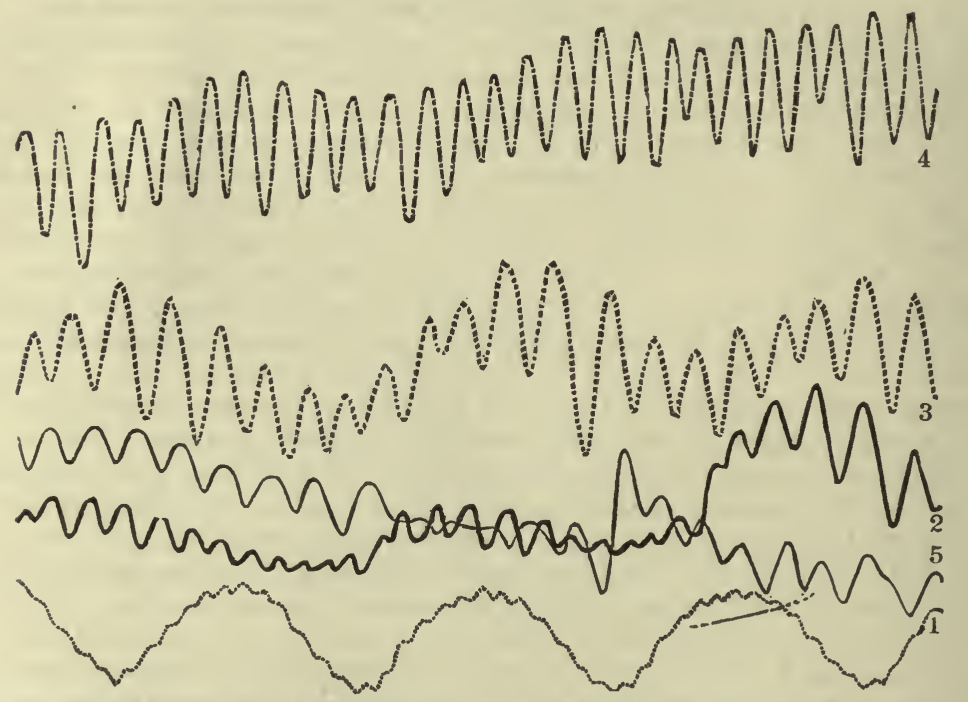

Blood-pressure Curves during a Suspension of Breathing. (Traube-Hering Curves.) The curves, 1, 2, 3, 4, 5, are portions selected from one long continuous tracing forming the record of a prolonged observation, so that the several curves represent successive stages of the same experiment. Each curve is placed in its proper position relative to the base line, which, to save space, is omitted ; and it is obvious that, starting from the stage represented by 1 , the blood-pressure rises in stages 2,3 , and 4 , but falls again in stage 5. Curve 1 is taken from a period when artificial respiration was being kept up, and the undulations visible are those the nature of which have been discussed; the vagus nerves having been cut the pulsations on the ascent and descent of the undulations do not differ. When the artificial respiration was suspended these undulations disappeared, and the blood-pressure rose steadily, while the heart-beats became slower. Soon, as shown in curve 2, new undulations appeared. A little later the blood-pressure was still rising, the heart-beats still slower, but the undulations still more obvious (curve 3). Still later (curve 4) the pressure was still higher, but the heart-beats were quicker and the undulations flatter. The pressure then began to fall rapidly (curve 5), and continued to fall until some time later artificial respiration was resumed.

We may here incidentally remark that the occurrence of long, slow undulations is not dependent on the cessation of the respiratory movements, and on an abnormally venous condition of the blood. They are sometimes (Fig. 109) seen in animals whose breathing is fairly normal. We need not discuss them any further now, and have introduced them chiefly to illustrate the fact that the vasomotor nervous system is apt to fall into a condition of rhythmic activity. It has been suggested that the normal 
respiratory undulations may be due to a rhythmic rise and fall of the activity of the vasomotor centre, synchronous, like that of the cardioinhibitory centre, with the respiratory movements. There can, however, be no doubt that the respiratory variations in blood-pressure are due to the mechanical conditions discussed above, and that vasomotor influences intervene but little if at all.

Fig. 109.

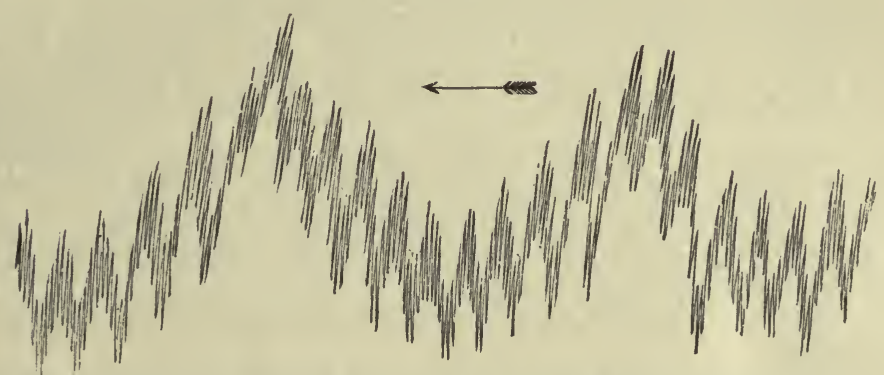

Blood-pressure Curve of a Rabbit, Recorded on a Slowly Moving Surface, to show TraubeHering Curves. (The curve was described not by means of a mercury manometer, but by an instrument similar to but not identical with Fick's spring kymograph.) In each heart-beat the upward and downward strokes are very close together, but may be easily distinguished by the help of a lens. The undulations of the next order are those of respiration. The wider sweeps are the Traube-Hering curves, of which two complete curves and portions of two others are shown. Each Traube-Hering curve comprises about nine respiratory curves, and each respiratory curve about the same number of heart-beats.

$\S 331$. The further general effects, similar to the above, on the vascular system of deficient arterialization of the blood may be studied by taking a blood-pressure tracing from the carotid or other artery of an animal while the interference with respiration is pushed on to a fatal asphyxia. During the first and second stages of the asphyxia the blood-pressure rises rapidly, attaining a height far above the normal. During the third stage it falls even more rapidly, repassing the normal and becoming nil as death ensues. If the animal, no urari having been given, is breathing of itself, and if, as usually is the case, the asphyxia is brought about by occlusion of the trachea, so that the mechanical effects of the respiratory movements are exaggerated by the air being unable to enter the chest, the respiratory undulations of the pressure-curve due to the mechanical causes discussed above are, especially during the first stage, extensive, abrupt, and irregular, the inspiratory movements being accompanied by a conspicuous fall of pressure. When the animal has been previously placed under urari, so that the respiratory impulses cannot manifest themselves by any muscular movements, the rise of the j,ressure-curve, as we have already said, is at first steady and unbroken, but after a variable period Traube's curves make their appearance. As during the third stage the pressure sinks, these undulations pass away.

The heart-beats are at first somewhat quickened, but speedily become slow, at the same time, as we have seen, not notably losing force, so that the pulse-curves on the tracing are exceedingly bold and striking. But the boldness of the curve of the mercury manometer is, it must be remembered, partly the mere result of the slowness of the rhythm; the mercury has time to fall largely between each two beats. (Fig. 108, 3 and 4.) Even while the blood-pressure is sinking, and when the cardiac stroke is now certainly lessening in vigor, the slowness of the cardiac rhythm is still sufficient to maintain somewhat these characters of the curve. The strokes at last, how- 
ever, rapidly fail in strength and become irregular, though the heart continues to beat for some seconds after the respiratory movements have ceased.

If the chest of an animal be opened under artificial respiration, and asphyxia brought on by cessation of the respiration, it will be seen that the heart during the second and third stages becomes completely gorged with venous blood, all the cavities as well as the large veins being distended to the utmost. If the heart be watched to the close of the events, it will be seen that the feebler strokes which come on toward the end of the third stage are quite unable to empty its cavities; and when the last beat has passed away its parts are still choked with blood. The veins spurt out when pricked; and it may frequently be observed that the beats recommence when the over-distention of the heart's cavities is relieved by puncture of the great vessels. When rigor mortis sets in after death by asphyxia, the left side of the heart is more or less emptied of its contents; but not so the right side. Hence, in an ordinary post-morten examination in cases of death by asphyxia, while the left side is found comparatively empty, the right appears gorged.

The various phenomena of asphyxia are probably brought about in the following way :

The increasingly venous character of the blood augments the action of the vasomotor centres, both the medullary centre and the subsidiary centres in the spinal cord, and thus leads to a constriction of the small arteries, especially of the splanchnic area. This is the chief cause of the markedly increased blood-pressure; though the venous blood may possibly also act directly on peripheral vasomotor mechanisms, or what is more likely, may increase the peripheral resistance in the capillaries themselves, since there are reasons for thinking ( $\$ 171)$ that venous blood rich in carbonic acid meets with more friction, and passes less easily through the capillaries than does blood less venous in character.

This increased peripheral resistance and the high blood-pressure to which it gives rise, while tending to increase the distention of the left ventricle and so indirectly helping to augment the force of the heart's beat, soon becomes a direct obstacle to the heart emptying itself of its contents. On the other hand, the labored respiratory movements favor the flow of venous blood toward the heart, which in consequence becomes more and more full. This repletion is, moreover, assisted by the marked infrequency of the beats which is soon developed. This in turn depends in part on the cardio-inhibitory centre in the medulla being stimulated by the venous blood, but, as we have previously seen, cannot be wholly accounted for in this way. The increased resistance in front, the augmented supply from behind, and the long pauses between the strokes, all concur in distending the heart more and more.

When the large veins have become full of blood, the inspiratory movements can no longer have their usual effect in facilitating the venous flow into the right auricle. The chief effect of the chest movement, as far as the circulation is concerned, is to widen and so to increase the capacity of the pulmonary vessels, and at the same time to diminish the pressure around the large arteries; hence the marked sinking of the blood-pressure during each inspiratory movement.

The distention of the cardiac cavities, at first favorable to the heart-beat, as it increases becomes injurious; and the cardiac tissues after a while become enfeebled by the action of the venous blood, so that the strokes of the heart become weaker and irregular.

On account of this increasing feebleness of the heart's beat, accompanied by more or less irregularity, the blood-pressure, in spite of the continued 
arterial constriction, begins to fall, since less and less blood is pumped into the arterial system; the boldness of the pulse-curves at this stage is chiefly due to the infrequency of the strokes. As the quantity which passes from the heart into the arteries becomes less second by second, the pressure gets lower and lower, the descent being assisted by the exhaustion of the vasomotor centre, until almost before the last beats it has sunk to zero. Thus at the close of asphyxia, while the heart and venous system are distended with blood, the arterial system is less than normally full.

$\$ 332$. While changes occurring primarily in the respiratory system thus affect the vascular system, conversely changes occurring primarily in the vascular system affect the respiratory system. Two kinds of change in the vascular system bearing on two parts of the respiratory system deserve special attention.

In the first place the respiratory mechanism may be affected by changes in the blood-supply to the respiratory centre in the medulla. We have already seen ( $\$ 314$ ) that the sudden cutting off of the supply of blood to the medulla gives rise to dyspnoic respiratory movements and may lead to expiratory convulsions. That is an extreme case; but, short of that, the activity of the respiratory centre, the extent and character of the respiratory explosions which take place in it, may be varied according as the constricted or dilated condition of the small arteries branching off from the basilar artery or of the basilar artery itself allows a scanty or a full flow of blood through the medulla. And it is possible that some forms of dyspnoea may be brought about in this way.

Much more common and important, however, is the second kind of change, that affecting the circulation through the lungs. In the normal organism an adequate supply of arterial blood to the tissues is secured by an adequate renewal of the air in the pulmonary alveoli, and an adequately rapid flow of blood through the pulmonary capillaries. When, as by obstruction in the pulmonary arteries, or by failure of the cardiac valves, or, and perhaps especially, by an insufficient cardiac stroke, the stream of blood from the lungs into the left ventricle is lessened either in amount or in rapidity, less oxygen is carried to the tissues, including the nervous tissue of the medulla, and dyspnœa or "want of breath" follows. When the circulation through the lungs is in full healthy swing, the hæmoglobin of the red corpuscles is as we have seen saturated or nearly saturated with oxygen. If owing to a slower stream the red corpuscles tarry longer in their passage along the walls of the pulmonary alveoli they cannot thereby take up a compensating addition of oxygen, indeed, it is doubtful if they can take up any additional oxygen at all. The blood falling under these circumstances into the left ventricle and sent thence over the body is not more arterial than usual; at the same time the amount of blood sent out at each heartstroke is less, often much less, than the normal; and the medulla as well as other tissues suffer in consequence from a deficiency of oxygen. The deficient supply to the medulla manifests itself in dyspnoic or at least in labored breathing, which sometimes, through the mechanical influences discussed above, has the happy result of improving the pulmonary circulation and so produces compensating effects. When the pulmonary artery is suddenly plugged with a clot the primary and urgent symptom is "want of breath," though air enters freely into the chest; and "cardiac dyspnoa" is a common symptom of cardiac disease.

$\S 333$. Other systems of the body are also related to the respiratory system, though by ties less striking than those which bind to it the vascular system. We have seen that deficient arterialization of the blood stirs up the muscles of the alimentary canal to increased activity, and we shall presently 
see that the same condition has a notable effect in promoting the perspiration; it probably has a similar influence over other secretions. On the other hand, as we have seen ( $\$ 316)$, there are reasons for thinking that the activity of the respiratory centre and so the energy of the whole respiratory act is influenced by chemical changes, other than the decrease of oxygen and increase of carbonic acid, brought about in the blood by the activity of the skeletal muscles.

The closeness and the intricacy of the ties which thus connect the respiratory system with almost all parts of the body may be illustrated by considering the effects of muscular work on the body, and the conditions which, apart from the capacity of the muscles themselves and of the motor nervous apparatus which puts them to work, determine the power of the body to do work. During work, especially arduous work, the muscular contractions rob the blood of much oxygen and load it with much carbonic acid. This change in the blood would itself increase the activity of the respiratory centre and the energy of the respiratory movements, and might be sufficient to secure such an increase of these movements that the deficiency of oxygen and increase of carbonic acid should never overstep certain limits. But, as we have said, apparently other products of muscular metabolism act so potently in stimulating the respiratory centre, that the respiratory movements are more than sufficient to compensate the changes in the gases of the blood. The efficacy of the augmented respiratory movements is much increased by a concomitant increase in cardiac activity and a swifter or fuller strean of blood through the lungs; indeed, unless backed up by the cardiac increase, the mere increase of the pulmonary ventilation might prove inadequate.

Hence the capacity for arduous muscular labor is determined not by the respiratory mechanism alone, nor by the vascular system alone, but by both, and especially by both working together in harmony and concert. The increased ventilation would be idle unless it were accompanied by a quicker circulation, and the quicker circulation would similarly be of comparatively little use unless accompanied by increased ventilation. To a bystander the working of the respiratory pump is much more obvious than that of the vascular system, and indeed the subject himself is much more directly conscious of changes in the former than of changes in the latter. Hence when the organism ceases to be able to meet the demands which the labor is making upon it, the subject is said to be "out of breath," though in a large number of cases the failure lies much more at the door of the vascular than of the respiratory system. And, as a rule, it may perhaps be said that when two men differ in their capacity for strenuous work, such as running a race, the difference, though it is often familiarly spoken of as one of "wind" or power of breathing, is in reality not a difference in ventilating capacity but a difference in the power of the heart to keep up to and work in harmony with the increased respiratory movements.

Thus there are two main factors in respiration, the respiratory mechanism proper, and the circulation, the one bringing the air to the blood and the other the blood to the air. We may remind the reader that there is also a third factor, and that one of great moment, the amount of hæmogiobin, that is, the number of red corpuscles, in the blood. The amount of oxygen taken up from the lungs depends not only on the strokes of the respiratory and the vascular pumps, but also on the richness of the blood in red corpuscles. A body which from loss of blood or from disease is anæmic is thrown out of breath by very slight exertion, not so much because the respiratory or the vascular pump is weak, but because, through lack of oxygen-carriers, with their best efforts the combined pumps can only deliver to the tissues, including the medulla, an inadequate supply of oxygen. And fat persons, 
whose store of hæmoglobin in proportion to their body weight is always below par, are proverbially "scant of breath."

\section{Modified Respiratory Movements.}

$\S 334$. The respiratory mechanism with its adjuncts, in addition to its respiratory function, becomes of service, especially in the case of man, as a means of expressing emotions. The respiratory column of air, moreover, in its exit from the chest, is frequently made use of in a mechanical way to expel bodies from the upper air-passages. Hence arise a number of peculiarly modified and more or less complicated respiratory movements, sighing, coughing, laughter, etc., adapted to secure special ends which are not distinctly respiratory. They are all essentially reflex in character, the stimulus determining each movement, sometimes affecting a peripheral afferent nerve as in the case of coughing, sometimes working through the higher parts of the brain as in laughter and crying, sometimes possibly as in yawning and sighing, acting on the respiratory centre itself. Like the simple respiratory act, they may with more or less success be carried out by a direct effort of the will.

Sighing is a deep and long-drawn inspiration, chiefly through the nose, followed by a somewhat shorter, but correspondingly large expiration.

Yawning is similarly a deep inspiration, deeper and longer continued than a sigh, drawn through the widely open mouth, and accompanied by a peculiar depression of the lower jaw and frequently by an elevation of the shoulders.

Hiccough consists in a sudden inspiratory contraction of the diaphragm, in the course of which the glottis suddenly closes, so that the further entrance of air into the chest is prevented, while the impulse of the column of air just entering, as it strikes upon the closed glottis, gives rise to a well-known accompanying sound. The afferent impulses of the reflex act are conveyed by the gastric branches of the vagus. The closure of the glottis is carried out by means of the inferior laryngeal nerve. See Voice.

In sobbing a series of similar convulsive inspirations follow each other slowly, the glottis being closed earlier than in the case of hiccough, so that little or no air enters into the chest.

Coughing consists in the first place of a deep and long-drawn inspiration by which the lungs are well filled with air. 'This is followed by a complete closure of the glottis, and then comes a sudden and forcible expiration, in the midst of which the glottis suddenly opens, and thus a blast of air is driven through the upper respiratory passages. The afferent impulses of this reflex act are, in most cases, as when a foreign body is lodged in the larynx or by the side of the epiglottis, conveyed by the superior laryngeal nerve; but the movement may arise from stimuli applied to other afferent branches of the vagus, such as those supplying the bronchial passages and stomach and the auricular branch distributed to the meatus externus. Stimulation of other nerves also, such as those of the skin by a draught of cold air, may develop a cough.

In sneezing the general movement is essentially the same, except that the opening from the pharynx into the mouth is closed by the contraction of the anterior pillars of the fauces and the descent of the soft palate, so that the force of the blast is driven entirely through the nose. The afferent impulses here usually come from the nasal branches of the fifth. When sneezing, however, is produced by a bright light, the optic nerve would seem to be the afferent nerve.

Laughing consists essentially in an inspiration succeeded, not by one, but 
by a whole series, often long continued, of short spasmodic expirations, the glottis being freely open during the whole time, and the vocal cords being thrown into characteristic vibrations.

In crying, the respiratory movements are modified in the same way as in laughing; the rhythm and the accompanying facial expressions are, however, different, though laughing and crying frequently become indistinguishable.

\section{CHA PTER III.}

\section{THE ELIMINATION OF WASTE PRODUCTS.}

$\S 335$. We have traced the food from the alimentary canal into the blood, and, did the state of our knowledge permit, the natural course of our study would be to trace the food from the blood into the tissues, and then to follow the products of the activity of the tissues back into the blood and so out of the body. This, however, we cannot as yet satisfactorily do ; and it will be more convenient to study first the final products of the metabolism of the body, and the manner in which they are eliminated, and afterward to return to the discussion of the intervening steps.

Our food consists of certain food-stuffs, viz., proteids, fats, and carbohydrates, of various salts, and of water. In their passage through the blood and tissues of the body, the proteids, fats, and carbohydrates are converted into urea (or some closely allied body), carbonic acid, and water, the nitrogen of the urea being furnished by the proteids alone. Many of the proteids contain sulphur, and also have phosphorus attached to them in some combination or other, and some of the fats taken as food contain phosphorus; these elements ultimately undergo oxidation into phosphates and sulphates, and leave the body in that form in company with the other salts.

Broadly speaking, then, the waste products of the animal economy are urea, carbonic acid, salts, and water. These leave the body by one or other of three main channels, the lungs, the skin, and the kidney. Some part, it is true, leaves the body by the bowels, for, as we have seen, the feces contain, besides undigested portions of food, substances which have been secreted into the bowel, and are, therefore, waste products; but the amount of these is so small that they may be neglected.

The lungs serve as the channel for the discharge of the greater part of the carbonic acid, and a considerable quantity of water; this discharge we have just studied. Through the skin there leave the body a comparatively small quantity of salts, a little carbonic acid, and a variable but on the whole large quantity of water.

The kidneys discharge all or nearly all the urea and allied bodies, the greater portion of the salts, and a large amount of water, with an insignificant quantity of carbonic acid. They are especially important, since by them practically all the nitrogenous waste leaves the body; and to them we will turn first.

\section{The Composition and Characters of Urine.}

$\S 336$. These are so fully dwelt upon in special works that we may confine ourselves here to salient points. The healthy urine of man is a clear yellowish 
slightly fluorescent fluid of a peculiar odor, saline taste, and acid reaction, having a mean specific gravity of 1020 , and generally holding in suspension a little mucus. The mucus, when present, comes from the urinary passages, as do also the occasional epithelial cells. All the rest of the urine may be considered as the secretion of the kidney.

The urine, as we have said, is the chief channel by which solid matters leave the body, a small quantity only passing by the skin and practically none by the lungs. Hence, neglecting for the present the skin, we may say that all the substances taken into the body sooner or later leave the body by the urine, save the few substances which may be retained permanently within the body and the substances which make up the body at the moment of its death. We accordingly find that the urine contains a large number of substances, the exact amount of each substance present in a given quantity of urine varying, in the case of every substance somewhat, and in the cases of many substances very largely, from time to time. The composition of urine is not only complex but extremely variable.

Moreover, a little consideration will show that the several substances present in urine must have very different histories. Some of the constituents of urine appear in it in the exact form in which they were introduced into the mouth; they have been simply absorbed from the alimentary canal into the blood and excreted by the kidney without undergoing change ; they are derived directly and without change from the food.

Others again are the products of changes which the food has undergone in the body; and these changes may be slight or may be extensive, and may take place on the one hand in the alimentary canal, or during a brief transit of the substance in the blood-stream, or even in the urine itself, may, so to speak, be superficial; or on the other hand may take place in the very depths of the tissues and be closely associated with the very life of the tissues. We shall, however, have to return to these matters later on, and may here briefly consider what substances are, normally and abnormally, present in urine, and the chief features of the fluid itself.

$\$ 337$. Besides water, the constituents of urine are:

Nitrogenous crystalline bodies. Neglecting the small proportion of these bodies which, especially in the case of flesh-eaters, are introduced into the economy with the food, as kreatin and the like, and so pass into the urine with no or with comparatively little change, we may on the whole regard the substances of this class as the products of the changes which the proteid matters (and allied substances such as gelatin and the like) present in food have undergone either while the food was simply food, still in the alimentary canal, for instance, or after the food had been built up into the tissues of the body.

Of these by far the most important, in the urine of man and mammalia, is the body urea $\left(\mathrm{N}_{2} \mathrm{H}_{4} \mathrm{CO}\right)$. It is the chief form in which, in these animals, nitrogen leaves the body. We shall have to discuss the relations and formation of urea later on, but meanwhile we will simply state that it has remarkable double connections with two great groups. On the one hand, it is related to the ammonia group, and by hydration is readily converted into ammonium carbonate $\left(\mathrm{N}_{2} \mathrm{H}_{4} \mathrm{CO}+2 \mathrm{H}_{2} \mathrm{O}=\left(\mathrm{NH}_{4}\right)_{2} \mathrm{CO}_{3}\right)$. On the other hand, it is related to the great cyanogen group, ammonium cyanate and urea being isomeric, and the former by simple heating being converted into the latter $\left(\mathrm{NH}_{4} \cdot \mathrm{CNO}=\mathrm{N}_{2} \mathrm{H}_{4} \mathrm{CO}\right)$.

Though a base, forming salts with acids, such as nitrates, oxalates, etc., urea occurs in urine in a free and independent condition.

Closely allied to urea, occurring apparently as a by-product of the same line of metabolism, is uric acid $\left(\mathrm{C}_{5} \mathrm{H}_{4} \mathrm{~N}_{4} \mathrm{O}_{3}\right)$ which is found always in 
the urine of man, occurring in small but variable quantity. In the urine of some animals, such as birds and reptiles, it occurs in abundance, and indeed in these replaces urea as the chief nitrogenous excretion. Uric acid is a more complex body than urea, one molecule of uric acid splitting up, under the influence of certain reagents, into two molecules of urea and a compound of oxalic acid. Its decomposition products, however, under different reagents are very numerous and complex, though urea occurs among them frequently and characteristically. Uric acid may be synthetically produced out of urea and glycin (glycocol).

It is a weak dibasic acid, and occurs in normal human urine, not as a free acid but as an acid salt, being combined with potassium and sodium, and to a less extent with calcium and ammonium. In quite normal urine these salts are soluble in the urine, even after the fluid has cooled down to the ordinary temperature of the air; but not infrequently the urates, soluble in the urine at the temperature at which it leaves the body, are precipitated when the fluid cools, forming the well-known "deposit of urates." On further standing the salts are apt to be decomposed and thus to give rise to crystals of uric acid.

Besides urea and uric acid the urine contains small but variable quantities of more or less nearly allied bodies, such as kreatinin, xanthin, hypoxanthin, and guanin. Concerning these we will at present only say that kreatinin is a hydrated form of the body kreatin which we spoke of $(\S 62)$ as a constituent of muscles. Kreatin by hydration is readily converted into kreatinin, and kreatinin by dehydration into kreatin; kreatin introduced into the alimentary canal or into the blood appears in the urine as kreatinin; and in flesh-eaters some at least of the kreatinin of the urine is derived directly from the kreatin present in the meat eaten as food; but we shall discuss the subject of kreatin later on.

Besides the above, such bodies as leucin, taurin, cystin, allantoin, and ammonium oxalurate are occasionally found in urine, but cannot be regarded as constituents of normal urine.

In the urine of man hippuric acid appears to be always present in small quantities, and in the urine of herbivora occurs in large quantities. In these latter it is derived more or less directly, by changes of which we shall have to speak in a succeeding chapter, from constituents of the food-containing bodies belonging to the aromatic group (benzoic acid series); but the small quantity present in man and other carnivora appears to come from the metabolism of proteid matter which, as we have already seen, contains an aromatic constituent. Another member of the aromatic group, tyrosin, is occasionally present in urine; and as more regular constituents of normal urine may be mentioned certain phenol compounds, such as phenylsulphuric acid, the phenol constituents of which are derived from the action of microorganisms in the alimentary canal (see $\$ 243$ ); these substances, though they no longer contain nitrogen, take origin from bodies of the aromatic series. Similar changes are also the source of indigo compounds (indican) in the urine, derived from indol (see $\$ 219$ ).

$\$ 338$. Inorganic salts. These for the most part exist in urine in natural sulution, the composition of the ash almost exactly corresponding with the results of the direct analysis of the fluid; in this respect, urine contrasts forcibly with blood, the ash of which is largely composed of inorganic substances, which previous to the incineration existed in peculiar combination with proteid and other complex bodies. In the ash of urine there is rather more sulphur than corresponds to the sulphuric acid directly determined; this indicates the existence in urine of some sulphur-holding complex body. And there are traces of iron, pointing to some similar iron-holding sub- 
stance. But otherwise, all the substances found in the ash exist as salts in the natural fluid.

The chief bases are sodium, potassium, calcium and magnesium in the form of chlorides, phosphates and sulphates. The exact way in which the several bases and acids are combined is to some extent a matter of uncertainty ; but sodium chloride is certainly present and in considerable quantity; it is the most abundant and important inorganic constituent. A large portion of the phosphoric acid seems to exist as acid sodium phosphate, the rest as soluble calcium and magnesium phosphates. The remaining chief salts, occurring, however, in smaller quantity, are potassium and sodium sulphate and calcium chloride.

Ammonia occurs in small quantity, alkaline carbonates are frequently found, traces of nitrates are at all events occasionally present, as also indications of salicylates and of sulpho-cyanates.

The phosphates are derived partly from the phosphates taken as such in food, partly from the phosphorus or phosphates peculiarly associated with the proteids, and partly from the phosphorus of certain complex fats such as lecithin. When urine becomes alkaline (and, as we shall presently see, it may do so by changes taking place in itself) the calcic and magnesic phosphates are converted into basic salts which, being insoluble, are precipitated, the sodium phosphate remaining in solution. When the alkalinity, as is frequently the case, is due to ammonia, ammonio-magnesium phosphate is formed and is apt to appear in crystals. The sulphates are derived partly from the sulphates taken as such in food and partly from the sulphur of the proteids. The carbonates, when occurring in large quantity, generally have their origin in the oxidation of such salts as citrates, tartrates, etc. The bases present depend largely on the nature of the food taken. Thus with a vegetable diet, the excess of the alkalies in the food reappears in the urine; with an animal diet, the earthy bases in a similar way come to the front.

$\$ 339$. Non-nitrogenous bodies. These exist in very small quantities, and many of them are probably of uncertain occurrence. Some of these are organic acids, the most constant perhaps being oxalic acid; to this may be added glycerin-phosphoric, lactic, formic, acetic, butyric and possibly succinic acids. Inosit has also been said to occur normally. It has been maintained that minute quantities of sugar (dextrose) are invariably present in even healthy urine; this, however, has not as yet been placed beyond all doubt. The nature of the substances which give to urine its characteristic odor has not been made out; probably there are more such bodies than one.

\$ 340. Pigments. Urine is always colored, the tint varying from a light to a dark yellow with an admixture of brown. In the course of twentyfour hours, a not inconsiderable quantity of pigment must leave the body by the urine; but the nature of the normal pigment or pigments of urine is at present obscure and the subject of much controversy. The matter is apparently further complicated by the presence in urine of what have been called "chromogens," that is to say, bodies which are not colored themselves but which readily give rise to pigments upon oxidation; and it is probable that some of these "chromogens" of the urine are reduction products of the respective pigments, the reduction taking place in the urine after secretion, or during or even before secretion. There is frequently present in urine, especially in cases of fever, a pignent which has been isolated and determined, which has a characteristic spectrum, and which, being maintained by some to be a derivative of bilirubin, has been called urobilin. It is not this urobilin, however, which gives to urine its ordinary 
color. Some observers, on the other hand, maintain that normal urine does contain and, in part at least, owes its normal color to a somewhat similar but different body, which in consequence they have called "normal" urobilin. It is in fact not possible, at the present moment, to make definite and satisfactory statements as to whether urine contains one or more than one normal pigment, as to its or their nature, as to whether they are derived from bile pigment or directly from the hæmatin or hremoglobin or in other ways, or as to the several steps by which they are produced. There are also abnormal coloring matters present on occasion, such for instance as the peculiar red coloring matter occurring sometimes in the urine of acute rheumatism, which has been called uroerythrin; but our knowledge concerning these is very imperfect.

$\S 341$. Ferments and other bodies. Even normal urine has frequently been found to contain a small quantity, hardly amounting to more than a trace, of proteid material, apparently an albumin; but the normal presence of even this small quantity has been disputed. Urine, however, certainly contains ferment bodies.

When urine is treated with many times its volume of alcohol, a granular or flocculent precipitate is thrown down, consisting chiefly of phosphates, together with some other substances or probably several other substances, in very small quantities. An aqueous solution of the precipitate, which may be freed from the phosphates, is both amylolytic and proteolytic. Ferments may also and more readily be extracted from urine by allowing shreds of fibrin to soak in the urine for a few hours, and then removing and washing them. The ferments become entangled in the fibrin in such a way as not to be easily removed by washing. The washed shreds will convert starch into sugar; and when treated with dilute hydrochloric acid digest themselves, showing the presence of pepsin. By this method it has been ascertained that an amylolytic ferment and pepsin are present in quantities which. vary in the twenty-four hours according to the meals. Rennin has also been found, and, at times at least, trypsin. From this it appears that some of the ferments of the alimentary canal escape from the body by the urine, being probably reabsorbed directly from the respective gland; the quantity which thus escapes is insignificant.

A small quantity of gas, about 15 vols. per cent., can be extracted by the mercurial pump from urine received direct from the body without exposure to air. The gas so obtained consists chiefly of carbonic acid, nitrogen being very scanty, and oxygen occurring in very small quantities or being wholly absent. The meaning of this we have already touched upon in speaking of respiration (see $\$ 302$ ).

$\$ 342$. 'The quantities in which these multifarious bodies, all of which, as we have seen, we may perhaps regard as constituents of normal urine, are present in different specimens of urine, vary within very wide limits, being dependent on the nature of the food taken and on the conditions of the body. 'The amount not of water only, but of many of the other several constituents, varies widely and indeed rapidly, so that the percentage composition of urine will vary from hour to hour if not from minute to minute. The causes which determine these variations in the nature and amount of urine we shall study later on. Meanwhile what may be called the average composition of human urine is shown in the following table in which the acids and bases are put down separately. 
Amounts of the Several Urinary Constituents Passed in Twenty-Four Hours. (After Parkes.)

\begin{tabular}{|c|c|c|c|c|c|c|c|c|c|c|c|c|c|c|}
\hline & & & & & & & & & & & & $\begin{array}{l}\text { By an average } \\
\text { man of } 66 \text { kilos. }\end{array}$ & & $\begin{array}{l}\text { Per } 1 \text { kilo } \\
\text { f body-weight. }\end{array}$ \\
\hline Water . . & & & - & & & & • & - & & • & & 1500.000 & grms. & 23.0000 grms. \\
\hline Total solids. & . & . & ${ }^{-}$ & ${ }^{\circ}$ & . & . & . & . & . & . & . & tec & & 1.1000 \\
\hline Urea . & . & . & - & . & • & • & . & . & • & . & & .33 .180 & & 0.5000 \\
\hline Uric acid . & & & . & . & . & . & . & . & . & . & • & . 0.555 & & 0.0084 \\
\hline Hippuric ac & aid & . & - & . & . & • & . & . & • & - & & . 0.400 & & 0.0060 \\
\hline Kreatinin. & . & . & 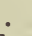 & - & & • & . & & & • & & . 0.910 & & 0.0140 \\
\hline Pigment, an & ad & otl & he & & & bst & $\tan$ & ces & & . & & .10 .000 & & 0.1510 \\
\hline Sulphuric a & cid & & . & - & - & . & . & . & . & - & • & . $\quad 2.012$ & & 0.0305 \\
\hline Phosphoric & aci & & . & . & . & • & . & . & 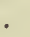 & 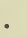 & & .3 .164 & & 0.0480 \\
\hline Chlorine . & . & & - & . & . & - & . & & -5 & . & & $\begin{array}{r}7.000 \\
(8.21)\end{array}$ & & 0.1260 \\
\hline Ammonia . & - & • & • & . & 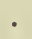 & & - & ${ }^{\circ}$ & & & & . 0.770 & & \\
\hline Potassium & . & • & . & . & . & • & . & . & . & • & & . 2.500 & & \\
\hline Sodium . . & - & - & - & - & 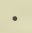 & - & - & - & • & • & & .11 .090 & & \\
\hline Calcium . & . & - & - & $\cdot$ & • & 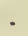 & - & . & • & - & & . 0.260 & & \\
\hline Magnesium & & . & & . & . & - & . & . & . & - & & $\begin{array}{r}0.207 \\
72.000\end{array}$ & & \\
\hline
\end{tabular}

$\$ 343$. The acidity of urine. The healthy urine of man is acid, owing to the presence of acid sodium phosphate, the absence of free acid being shown by the fact that sodium hyposulphite gives no precipitate. The amount of acidity is about equivalent to 2 grammes of oxalic acid in twenty-four hours, but the degree of acidity at any one time varies much during the day, being in an inverse ratio to the amount of acid secreted by the stomach; thus it decreases after food is taken, and increases again as gastric digestion comes to an end. It varies with the nature of the food; with a vegetable diet the excess of alkalies in the food, being secreted by the urine, lears to alkalinity, or at least to diminished acidity, whereas this effect is wanting with an animal diet, in which the alkalies are less abundant, earthy bases preponderating. Hence the urine of carnivora is generally very acid, while that of herbivora is alkaline. The latter, when fasting, are for the time being carnivorous, living entirely on their own bodies, and hence their urine becomes under these circumstances acid.

The natural acidity increases for some time after the urine has been discharged, owing to the formation of fresh acid, apparently by some kind of fermentation. This increase of acid frequently causes a precipitation of urates, which the previous acidity, even after the cooling of the urine, had been insufficient to throw down. After a while, however, the acid reaction gives way to alkalinity. This is caused by a conversion of the urea into ammonium carbonate through the agency of a specific " organized" ferment. This ferment as a general rule does not make its appearance except in urine exposed to the air ; it is only in unhealthy conditions that the fermentation takes place within the bladder, and in such cases is due either to microorganisms introduced into the bladder from without, during the use of instruments for instance, or to the action of an unorganized ferment, secreted, apparently by the walls of the bladder.

$\$ 344$. Abnormal constituents of urine. The structural elements found in the urine under various circumstances are blood, pus and mucous corpuscles, epithelium from the bladder and kidney, and spermatozoa. To these may be arlded the so-called "casts" which are either " epithelial casts," that is to say, eylinders of more or less altered epithelial cells shed from the tubules, or structureless, "fibrinous" casts, which are cylinders of peculiar material moulded in the lumina of the tubules; the exact nature of this material is 
at present a matter of doubt; it is not always the same, but appears not to be fibrin.

The most common and important abnormal constituents of urine are albumin, giving rise to albuminuria, and sugar, giving rise to glycosuria or diabetes. The soluble proteids, generally spoken of as "albumin," in the urine differ in different cases. The exact determination of their nature is a matter of some difficulty, since, as we have seen, we have in differentiating the various proteids to trust largely to their behavior as regards precipitation upon the addition of certain saline bodies; and the presence of saline bodies in the natural urine introduces complications. It would appear, however, that the proteids usually present are serum-albumin and globulin; these are not, however, as a rule, if ever, present in the same relative proportions as in blood-plasma; and either the one or the other may be present by itself. A form of albumose ( $\$ 189$ ), called hemi-albumose, is sometimes found, and, indeer, probably very many distinct kinds of proteids are from time to time present. If egg-albumin be injected into the blood it appears in the urine as egg-albumin, and peptone similarly injected appears as peptone.

The sugar which is found in the urine of diabetes is undistinguishable from ordinary dextrose; but whether it is absolutely identical with that body, or whether the sugar in all cases of diabetic urine is exactly the same in character, cannot, perhaps, as yet, be regarded as definitely settled.

When blood is mingled with urine in the kidney and in the urinary passages the constituents of the former are, of course, added to those of the latter; and when, as sometimes happens, chyle from the lacteals makes its way into the kidneys, the urine contains the fats and other constituents of chyle. Fats, however, may be present without the urine being distinctly "chylous."

Cholesterin, bile-acids, bile-pigments, and one or other of a large number of bodies arising from a disordered metabolism of the body, such as leucin, tyrosin, acetone (in cases of diabetes), oxalic acid, taurin, eystin, and many others are also found more or less frequently; some of these, indeed, have been regarded as normal constituents. Besides these the urine serves as the chief channel of elimination for various bodies, not proper constituents of food, which may happen to have been taken into the system. Thus various minerals, alkaloids, salts, pigmentary and odoriferous matters, may be passed unchanged. Many substances thus occasionally taken undergo, however, changes in passing through the body: the most important of these, since the changes which they undergo throw light on the metabolic processes of the body, will be considered in a succeeding chapter.

\section{The Secretion of Urine.}

$\S 345$. The kidney consists of two parts, so distinct in structure that it seems impossible to resist the conclusion that their functions are different, and that the mechanism by which the urine secreted is of a double kind. On the one hand, the tubuli uriniferi, with their characteristic epithelium, seem obviously to be actively secreting structures comparable to the secreting alveoli of the salivary and other glands. On the other hand, the Malpighian capsules, with their glomeruli, are organs of a peculiar nature, with an almost insignificant epithelium, and their structure irresistibly suggests that they act rather as what may be called, in a general way, a filtering than as a truly secreting mechanism. Hence has arisen the view 
which frequently bears the name of Bowman, since he was the first to put it forward, that certain constituents only of the urine are secreted after the fashion of other secreting glands by the tubuli uriniferi, and that the rest of the constituents, including a great deal of the water, with such highly soluble and diffusible salts as pre-exist in adequate quantity in the blood, are, as it were, filtered off by the glomeruli of the Malpighian capsules. We shall see, later on, reason to doubt whether we are justified in applying the term "filtration," which has a definite physical meaning, to the process by which water and other substances pass from the bloodvessels of the glomerulus into the lumen of the tubule; for that process is, as we shall find, peculiar and complex. But such a doubt need not prevent us from recognizing that the whole act of secretion of urine consists of two parts, one of which is much more closely dependent on the flow of blood through the kidney than is the ordinary process of secretion, such as has hitherto come before us, and another part which seems to bear the same relation to the flow of blood as does ordinary secretion.

That the work of the kidney is, to an unusual degree, dependent on the flow of blood throngh it, seems suggested by the vascular arrangements; for these are extremely favorable to a full and rapid stream of blood through the organ. The short and relatively broad renal artery comes off direct from the abdominal aorta, where the blood-pressure is extremely high; the renal vein opens directly into the vena cava, where the blood-pressure is extremely low. Between the mouth of the renal artery and the mouth of the renal vein the difference of pressure is very great, indeed; and, as we have seen in treating of the vascular system, it is the difference of pressure between two points of the vascular tract which is the actual cause of the flow of blood from the one point to the other. The difference of pressure, indeed, which drives the blood through the limited area of the kidney is the same difference of pressure which drives the blood along the abdominal aorta down both legs back again to the vena cava.

This free and abundant supply of blood is regulated, is either increased or diminished, according to the needs of the moment, by the vasomotor system ; this is shown by experimental aud other results, which it will be profitable to study in some detail. Before entering into these details, however, it will be well to call attention to the fact that when vasomotor events modify the flow of blood through an organ, they produce their effects in one direction or another by working on arterial blood-pressure. Thus, as we shall see, when stimulation or section of a nerve increases the flow of blood through the kidney, it does so by increasing the pressure in the small vessels of the kidney, including the capillary loops of the glomeruli. In such a case the walls of the glomerular loops, through which the passage of materials to form (part of) the urine takes place, are subjected to two influences-on the one hand, to a fuller, more rapid flow of blood past them, and, on the other, to an increase of the pressure which that blood, as it passes along, exerts on them. We shall have, subsequently, to discuss the share taken by these two influences in determining and modifying the passage of material through the walls of the glomerular loops; and this will bear on the question of filtration, to which we have above alluded; but, for the present, it will be convenient to deal with the effects of variation in blood-pressure apart from this secondary question.

$\$ 346$. The vasomotor mechanisms of the kidrey. It may be shown experimentally that the kidney is supplied with a vasomotor mechanism as well developed, perhaps, as that of any part of the body. By means of a modification of the plethysmograph (Figs. 110,111), we can readily observe the variations which take place in the volume of the kidney. 
The instrument consists of two parts, one of which (Fig. 110), called the oncometer, ${ }^{1}$ is applied to the organ about to be studied, while the other (Fig. 111), called the oncograph, is the recording part of the apparatus. Any diminution in the volume of the organ (Fig. 110, $K$ ), kidney, spleen, etc., as the case may be, diminishes the pressure on the fluid in the chamber $\alpha$; some of the fluid in the chamber $M$ (Fig. 111) accordingly passes through the tube $K$ (Fig. 111) and the tube $T$ (Fig. 110) to the chamber $a$; the piston $D$ accordingly falls and with it the lever $H$. Similarly an increase in the volume of the organ causes the lever to rise.

FIG. 110.

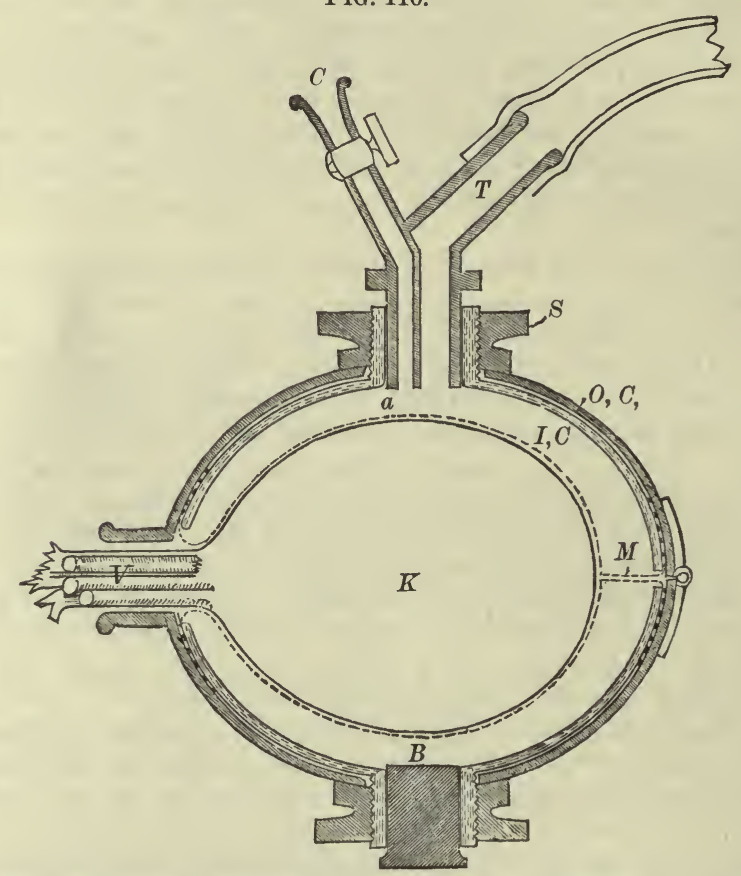

Renal Oncometer. (Seen in section, semi-diagrammatic.) $K$, kidney ; $V$, vessels and nerves imbedded in fat, etc., entering hilus of organ; $O, C$, and $I, C$, outer and inner metal capsules screwed together by the screw $S$, and holding between them the edge of the membrane $M$, which applies itself to the surface of the kidney and forms with the metal capsule two chambers, $a$ and $B$, one of which $(B)$ is closed by a plug filling the opening $B$, while the other $(a)$ communicates by a tube $T$ with the recording instrument. The other opening, $C$ (which is closed by a small tap), is for the purpose of filling the chamber $a$ with warm oil after the kidney has been placed in the box, the other chamber $B$ having been previously partly filled, the quantity introduced into it depending upon the size of the kidney.

The volume of the kidney may be increased by a swelling of its constituent cells and other structural elements, by an accumulation of lymph in its lymph-spaces, and by a distention of its blnodvessels. Compared with the third, the two former causes are in health so insignificant and problematical that they may be disregarded. Further, the distention of the bloodvessels will in general depend on the constriction or dilatation of the renal arteries and their ramifications, for distention due to venous obstruction will only occur in special cases. Hence variations in the volume of the kidney may be taken as a measure of variations in its vascular supply-increase of volume indicating dilated renal vessels, and decrease of volume indicating constriction of the renal vessels.

I From oncos, bulk. 
When by means of the instrument just described a tracing is taken of the volume of a kidney in what may be considered a normal condition, some such result as that shown in Fig. 112 is obtained.

FIG. 111.

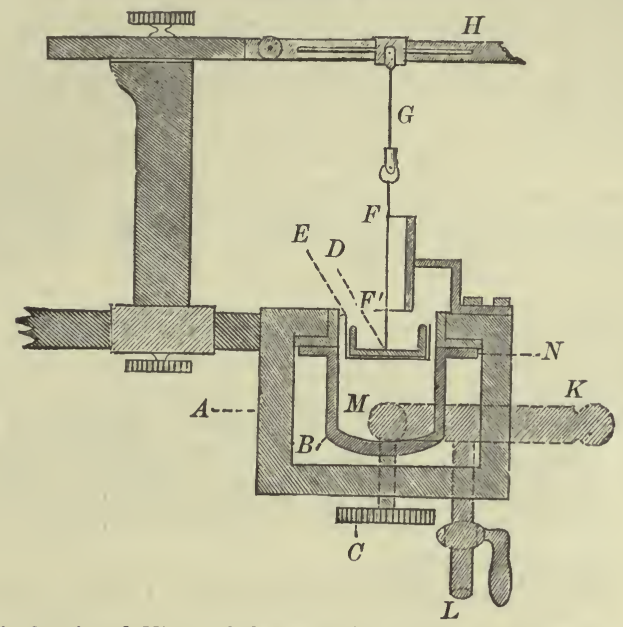

Semi-diagrammatic Sectional View of Oncograph. (Half natural size.) $K$, tube connecting instrument with oncometer; $D$, piston floating on oil contained in the cavity $M$; the oil is prevented from escaping by the side of the piston by the delicate flexible membrane $E$, which does not interfere with the movements of the piston; $H$, recording lever connected with the piston by a needle $G$ passing through the guides $F, F^{\prime}$. The screw $C$ is for the purpose of clamping the edge of the membrane between the two ring-shaped surfaces at $N$, while the side tube $L$ is for the purpose of filling the instrument.

The volume of the kidney is seen to be so delicately responsive to changes in the mean arterial pressure that the curve reproduces almost exactly a blood-pressure curve, showing not only the respiratory undulations

FIG. 112.

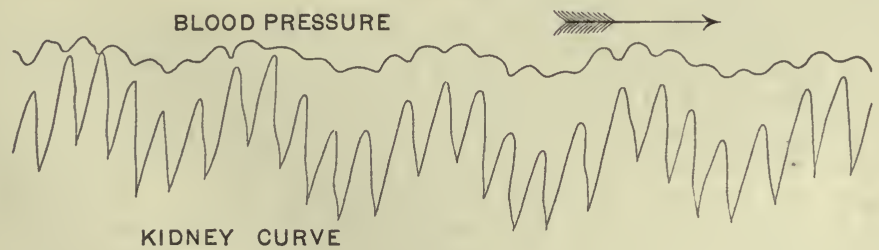

KIDNEY CURVE

Blood-pressure Tracing, and Curve from Renal Oncometer. (Natural size.) The blood-pressure abscissa line has been raised $2.75 \mathrm{~cm}$. (the actual medium blood-pressure having been 115 $\mathrm{mm}$. Hg.). The time-curve gives interruptions recurring every three seconds.

but even the rise and fall due to the individual heart-beats. With each rise of mean arterial pressure more blood is driven into the renal vessels and the kidney swells; with each fall of pressure less blood enters and the kidney shrinks. On other tracings taken in the same way may often be seen (not 
shown in Fig. 112) the wider variations corresponding to the Traube-Hering curves; but it will be observed that in these the kidney shrinks with the rise of pressure and swells with the fall. For as we have seen ( $\$ 331$ ) the rise in the Traube-Hering undulation is due to an augmentation of peripheral resistance caused by the constriction of minute arteries; and this constriction occurs in the kidney as elsewhere; the renal arterioles take their share in producing the result, and in consequence of their constriction the kidney shrinks. Similarly the relaxation of the renal vessels contributes to bring about the sequent fall.

$\$ 347$. In the course of a discussion in an earlier part of this work $(\S 157)$ on the local and general effects of arterial constriction and dilatation, we saw that the local blood-pressure in and flow of blood through the capillaries and other minute vessels of this or that vascular area may be increased-

1. By an increase of the general blood-pressure brought about- $(a)$ by an increased force, frequency, etc., of the heart's beat, $(b)$ by the constriction of the small arteries supplying areas other than the area in question.

2. By a relaxation of the artery (or arteries) supplying the area itself, which, while diminishing the pressure in the artery itself, increases the pressure in the capillaries and small veins which the artery supplies. It need hardly be added that this local relaxation must not be accompanied by a too great dilatation elsewhere.

The same local blood-pressure and flow of blood may similarly be diminished-

1. By a constriction of the artery of the area itself (and its branches), which, while increasing the pressure on the cardiac side of the artery, diminishes the pressure in the capillaries and veins which are supplied by the artery. This again must not be accompanied by a too great constriction elsewhere.

2. By a lowering of the general blood-pressure, brought about- $(a)$ by diminished force, etc., of the heart's beat, $(b)$ by a general dilatation of the small arteries of the body at large, or by a dilatation of vascular areas other than the area in question.

Applying these considerations to the bloodvessels of the kidney, we should expect to find the following :

A rise in general blood-pressure, and that means a rise of pressure in the abdominal aorta at the mouth of the renal artery, will cause a greater flow of blood through, and so an expansion of the kidney, provided that the renal arteries themselves are not unduly constricted at the same time. This is well shown, as we have seen, in the curve given above, where the increase of pressure due to each heart-beat, as well as that due to each respiratory movement, being of central origin and not due to arterial constriction and being unaccompanied by any compensating constriction of the renal artery, leads to expansion of the kidney, that is, to a greater flow of blood through the kidney.

If, however, the rise of general blood-pressure be due to events which at the same time cause a constriction of the renal arteries, the flow through the kidney may not only not be increased, but even-be diminished; the kidney may shrink instead of expanding. Thus if dyspnœa be brought about, as by stopping artificial respiration during an experiment, the kidney at once shrinks; the too venous blood stimulates the vasomotor centre, and probably also by direct action on the bloodvessels leads to a general arterial constriction and so to a rise of blood-pressure; but the renal vessels are involved in this constriction, so much so that their constricted condition more than counterbalances the general rise of blood-pressure, and less blood flows through 
the renal vessels. So also when the medulla or spinal cord is directly stimulated by induction shocks (the animal being under urari so as to eliminate the complications due to contractions of the skeletal muscles) the renal vessels share so fully in the arterial constriction which results that, in spite of the great rise of mean pressure which is induced, less blood than normal passes through the renal vessels, and the kidney shrinks. Or if the abdominal splanchnic nerves be stimulated, since, as we shall see, these carry vasoconstrictor fibres for the kidney, in spite of the rise of blood-pressure which follows, the kidney shriiks on account of the great constriction of the renal vessels.

On the other hand, if a rise of blood-pressure be for any reason not accompanied by a compensating constriction of the renal arteries, that rise, whether it be brought about by general constriction of arteries other than the renal or by an increase of the cardiac delivery, causes the kidney to swell, showing a greater flow of blood. Such a condition of things may be induced by section of the nerves of the renal plexus, whereby the paths of all vaso-constrictor impulses to the kidney are blocked. After this has been done, a rise of general pressure, whether by dyspnœa, or by direct stimulation of the spinal cord, or by stimulation of the abdominal splanchnic nerves, leads to a greater flow through the renal vessels and an increased expansion of the kidney.

A rise of general blood-pressure, then, may be accompanied by either a shrinking or a swelling of the kidney, by either a greater or lesser flow of blood through the kidney, according to the concomitant condition of the renal vessels; or, indeed, may under certain circumstances be accompanied by no cliange at all in the renal circulation, the local effects exactly counterbalancing the general ones.

Conversely, in a similar way, a fall of blood-pressure leads to a lesser flow through the renal vessels and a shrinkage of the kidney unless it be accompanied by a dilatation of the renal vessels out of proportion to the general fall. Thus when the spinal cord is divided below the medulla the fall of general blood-pressure is, as we have seen ( $\$ 159)$, very marked, being due to an abolition for the time being of wonted constrictor impulses. The pressure in the aorta falls rapidly, and at the same time, owing to the more open pathway through the region of peripheral resistance in the body generally, the pressure in the vena cava is increased; the difference of pressure between the mouth of the renal artery in the aorta and the mouth of the renal vein in the vena cava is so largely reduced that in spite of the concomitant relaxed condition of the renal vessels themselves the flow of blood through the kidney is largely diminished.

It will of course be understood that, the general blood-pressure remaining the same, the flow through the kidney will at once be, on the one hand, increased by dilatation and, on the other, decreased by constriction of the renal vessels themselves. The constricted or dilated condition of the renal vessels can by themselves produce but little effect on the pressure either in the aorta or in the vena cava; and the difference between the pressure at the mouth of the renal artery and that at the mouth of the renal vein remaining the same, the more open passages of the dilated renal vessels must lead to a fuller, and the narrower passages of the constricted renal vessels to a scantier, flow through the kidney.

$\$ 348$. By means of the oncometer, watching the shrinking and swelling of the kidney and thus judging of the flow of blood through it, the results being always interpreted with reference to the general blood-pressure on the lines of the above discussion, the paths of vasomotor impulses to the kidney have been approximately made out. Vaso-constrictor fibres for the kidney 
are supplied from what we have previously ( $\$ 155$ and elsewhere) spoken of as the vaso-constrictor region of the spinal cord. They issue from the spinal cord by the anterior roots of a large number of the spinal nerves taking origin from this region, and may be traced (in the dog) as high up as the sixth dorsal, a few perhaps even to the fourth dorsal, and as low down as the second lumbar (fourth lumbar if only thirteen nerves be counted as dorsal); but most seem to pass by the eleventh, twelfth, and thirteenth dorsal nerves. Passing through the corresponding ganglia of the splanchnic (sympathetic) chain, these fibres reach the solar plexus and thus the renal plexus by the abdominal splanchnic nerve ; those, however, coming from some of the lower nerves apparently do not contribute to the splanchnic nerve, but take a separate course. Centrifugal stimulation of these anterior roots produces shrinking of the kidney, all the more marked and distinct in the case of the eleventh, twelfth, and thirteenth dorsal roots because the effect on the kidney is then not so much masked by vasomotor effects on other organs. Stimulation of the higher roots also produces shrinking of the kidney, but less marked, since in these cases the stimulation bears at the same time largely on vaso-constrictor fibres for other abdomiual organs, and so by raising the general blond-pressure tends to neutralize the local effect on the kidney. And even the very decided shrinking of the kidney which results from the stimulation of the splanchnic trunk itself is less than would take place if the stimulation affected the vessels of the kidney only.

$\S 349$. We stated in $\S 154$ that by the method of slowly repeated rhythmical stimulation the presence of vaso-dilator fibres in the sciatic nerve might be detected, though these are largely mixed with vaso-constrictor fibres; and slow rhythmical stimulation of the anterior roots of the abovementioned lower dorsal nerves leads, not, as does ordinary rapidly interrupted stimulation, to shrinking, but to swelling of the kidney, showing that these ruots contain vaso-dilator fibres as well as vaso-constrictor fibres. The higher (anterior) roots also appear to contain some renal vaso-dilator fibres; but the effect of stimulating them by the slow rhythmic method is more masked by a concomitant dilatation of the vessels of the other abdominal organs, the roots in question containing vaso-dilator as well as vaso-constrictor fibres for those organs; this leads to a fall of general blood-pressure whereby the tendency of the kidney to swell is counteracted.

$\$ 350$. It is obvious then that by means of this vasomotor mechanism the How of blood through the kidney is governed by the central nervous system in such a way that afferent impulses, started in this or that region or surface, and passing up to the central nervous system, may lead either to constriction or to dilatation of the renal vessels; and to such actions of this kind we shall presently return. Meanwhile we wish to call attention to the fact that the volume of the kidney is remarkably sensitive to chemical changes taking place in the blood. The injection into the blood of even a small quantity of water causes a transient shrinking of the kidney followed by a more lasting expansion. The injection of urea and some other diuretics produces the same effect to a more marked degree, leading especially to a swelling which lasts for some considerable time, while the injection of normal saline solution, and especially of such diuretics as sodium acetate, causes an expansion from the very first, the primary shrinking being absent. It is, moreover, worthy of note that these effects of diuretics and of chemical changes in the blood are observed even after all the renal nerves have apparently been completely severed. Hence the changes in volume caused by the presence of these substances in the blood must be due to the substances acting either upon some peripheral vasomotor mechanism, or, even more directly, on the bloodvessels themselves. It may be added that they 
will produce considerable effects in the kidney itself without appreciably modifying the general blood-pressure.

$\$ 351$. If, while the kidney is in the oncometer, and the various experiments on section and stimulation of nerves and the like are being carried on, a canula be tied in the ureter, the secretion of urine may be watched at the same time. It will then be seen that the flow of urine through the end of the canula is not equable, and does not either increase or decrease in an even manner. On the contrary, it will frequently be found that a sort of gush of urine takes place, several drops following each other in rapid succession, followed by a cessation of flow; and if the ureter be watched it will be seen that the gushes of urine are synchronous with waves of peristaltic contraction sweeping down the ureter. Obviously the urine collects, to a certain extent, in the pelvis of the kidney, and is driven thence by muscular action from time to time; to this point we shall return later on.

Making every allowance, however, for these irregularities of flow, we may take the rate of flow from the end of the canula as a measure of the rate of secretion; and it is found that as a general rule increased flow of urine is coincident with swelling of the kidney, that is with a greater flow of blood through it, and diminished or arrested flow of urine is coincident with shrinking of the kidney, that is, with a diminished flow of blood through it.

A striking instance of this is afforded by the expriment of dividing in the dog the spinal cord below the medulla. 'The blood-pressure then, as we know, falls rapidly, owing to the removal of constrictor impulses from the small arteries and the general diminution of peripheral resistance which follows upon so many small arteries becoming dilated; and though the renal arteries probably share in the general relaxation, yet, owing to the fall of pressure in the aorta conjoined as this is by a corresponding rise of pressure in the vena cava, the flow of blood through the kidney is largely diminished. We find that after the operation the secretion of urine is greatly diminished; indeed, in most cases the flow from the end of a canula is almost arrested. In fact, we may almost make the general assertion that, when in the dog the blood-pressure falls to about $30 \mathrm{~mm}$. Hg. or less, the secretion of urine is for the time stopped. These and other results support the view stated above that the secretion of urine is in quite a special way dependent on the flow of blood through the kidney; and we may further conclude that the secretion which is so particularly influenced by the flow of blood is that special kind of secretion, allied to filtration, which takes place through the glomeruli, and not the more ordinary kind of secretion by means of the epithelium of the tubuli uriniferi. But before we proceed to discuss how the increased flow of blood increases the glomerular flow of urine, we must turn to consider the functions of the epithelium of the tubuli.

\section{Secretion by the Renal Epithelium.}

$\S 352$. The glomerular mechanism is, after all, a small portion only of the whole kidney, and the epithelium over a large part of the course of the tubuli uriniferi bears most distinctly the characters of an active secreting epithelium. These facts would lead us a priori to suppose that the flow of urine is in part the result of an active secretion comparable to that of the salivary or other glands which we have already studied. And we have experimental and other evidence that such is the case.

In the first place, a flow of urine may be artificially excited even when the natural flow has been arrested by diminution of blood-pressure. Thus 
if, when the urine has ceased to flow in consequence of a section of the medulla oblongata, certain substances, such as urea, urates, sodium acetate, and the like, be injected into the blood, a more or less copious secretion is at once set up. This secretion is, or at least may be, unaccompanied by any rise of general blood-pressure sufficient to account for the increased secretion as the mere result of an increased flow of blood. It is true (as we have seen, $§ 350)$ that the injection of these substances leads to an expansion of the kidney, an expansion which is probably due to a local dilatation of the small renal arteries; but the flow of urine which is observed in these cases is too great to be accounted for by any increase of flow of blood which the local dilatation may bring about; and hence we conclude that the increase of secretion is of a different kind from that which follows upon mere increase of blood-flow. It seems much more reasonable to suppose that the presence of the above substances in the blood excites the renal epithelial cells to an unwonted activity, causing them to pour into the interior of the tubules a copious secretion, just as the presence of pilocarpine in the blood will cause the salivary cells to pour forth their secretion into the lumen of their ducts; and that this activity of the epithelial cells is accompanied, also as in the case of the submaxillary and other glands, by a vascular dilatation, which, though adjuvant and beneficial, is not the distinct cause of the activity. This view is further supported by the following remarkable experiment, which goes far to show that of the various substances which, having found their way into the blood, are thrown out by the kidney, some pass into the urine through the glomeruli, while others are distinctly secreted by the tubuli uriniferi, the discharge of the latter being accompanied by a general activity of the secreting cells, as shown by the flow of water taking place at the same time.

In the amphibia the kidney has a double vascular supply; it receives arterial blood from the renal artery, but there is also poured into it venous blood from another source. The femoral vein divides at the top of the thigh into two branches, one of which runs along the front of the abdomen to meet its fellow in the middle line and form the anterior abdominal vein, while the other passes to the outer border of the kidney and branches in the substance of that organ, forming the so-called reual portal system. Now the glomeruli, in some species at least of these animals, are supplied exclusively by the branches of the renal artery, the renal vena portæ only serving to form the capillary plexus around the tubuli uriniferi, which is also supplied by the efferent vessels of the glomeruli. From this it is obvious that if the renal artery be tied, the blood is shut off entirely from the glomeruli; and actual observation of the kidney has, in the animals in question, shown that under these circumstance there is no reflux from the capillary network surrounding the tubules back to the glomeruli ; thus the kidney by this simple operation is transformed into an ordinary secreting gland devoid of any special filtering mechanism. Such a kidney may be used to ascertain what substances are excreted by the glomeruli, and what by the tubules in some other part of their course. It is found that urea injected into the blood gives rise to a secretion of urine when the renal arteries are tied; this substance, therefore, is secreted by the epithelium of the tubules, and in being so secreted gives rise at the same time to a flow of water through the cells into the interior of the tubules. Sugar and peptones, on the other hand, which injected into the blood readily pass through the untouched kidney and appear in the urine, do not pass through a kidney the renal arteries of which have been tied, even when a diuretic such as urea is given at the same time in order to secure a flow of urine. These substances, therefore, are excreted by the glomeruli. 
The validity of this experiment, which may be accepted as indicating a marked difference between glomerular secretion on the one hand and epithelial or tubular secretion on the other, depends on the absence of any collateral circulation whereby the glomeruli may be supplied with blood after ligature of the renal artery. In these animals anastonıses occur between the renal arteries and the arteries of the generative organs; and unless the renal artery be so tied as to aroid these collateral communications the results of the experiment are different.

Additional evidence in favor of the secretory activity of the epithelial cells is afforded by the following observation. Into the veins of animals in which the urinary flow had been arrested by section of the spinal cord below the medulla a quantity of the blue coloring material known as sodium sulphindigotate ${ }^{1}$ is injected. This substance is rapidly excreted on the one hand by the liver in the bile, and on the other hand by the kidney. By varying the quantity injected, killing the animals at appropriate times after the injection of the material, and examining the kidneys microscopically and otherwise, it may be ascertained that the pigment so injected passes from the blood into the renal epithelium, and from thence into the channels of the tubules. There being no stream of fluid through the tubules, owing to the arrest of urinary flow by means of the preliminary operation, the pigment travels very little way down the interior of tubules, and remains very much where it was cast out by the epithelial cells. There are no traces whatever of the pigment having passed by the glomeruli; and the cells which appear most distinctly to take up and eject it are those lining such portions of the tubules (viz., the first and second convoluted tubules, zigzag tubules, and ascending limbs of the loops of Henle) as from their microscopic features have been supposed to be the actively secreting portions of the entire tubules. The following observation which has been made is of a peculiarly interesting character. After injecting a certain quantity of pigment, and allowing such a time to elapse as might be judged from previous experiments would suffice for the passage of the material through the epithelium to be pretty well completed, a second quantity was injected. It was found that the excretion of this second quantity was most incomplete and imperfect. It seems as if the cells were exhausted by their previous efforts, just as a muscle which has been severely tetanized will not respond to a renewed stimulation.

The above observation may he objected to on the ground that this coloring matter does not occur as a constituent of the blood either in lealth or disease, and especially that the absence of any concomitant discharge of fluid from the cells excites suspicion that the process observed was not really one of secretion; for the injection of such substances as urea or urates into the blood does cause a copious flow of fluid, and indeed thus prevents the microscopic tracking out of their passage, which in the case of urates might otherwise be done much in the same way as with the sodium sulphindigotate. Moreover, other observers have naintained that the sodium sulphindigotate does like ordinary carmine pass through the glomeruli. But their results may probably be explained by the glomeruli having been damaged by a too rapid or too abundant injection ; and in the case of the amphibian kidney, when sodium sulphindigotate is injected after ligature of renal arteries, no urine is found in the bladder, but the pigment can be traced through the epithelium of the secreting portions of the tubules. Without insisting too much on the value of the sodium sulphindigotate experiments, they may be taken as fairly supporting the view which we are considering. We may add

\footnotetext{
1 Sometimes called indigo-carmine, though this name is more properly applied to a crude, impure preparation of potassium sulphiudigotate.
} 
that in birds, the urine of which contains little water, urates may be detected in the epithelium of the tubules, but not in the capsules.

Though much remains to be cleared up, we may, for the present, conclude that the secretion of urine does consist of two separate and distinct acts; secretion by the glomeruli, which we may for brevity's sake speak of as glomerular secretion, and secretion by the epithelium of the tubuli, which we may speak of similarly as tubular secretion. But these forms of secretion, especially the former, but to a certain extent the latter also, differ from the secretion of such a gland as the salivary, and both deserve some special consideration.

$\$ 353$. The nature of glomeiular secretion. We have seen that the expansion of the kidney which has for its accompaniment an increased flow of urine is one brought about by the renal artery and its various branches becoming dilated, under such circumstances that the difference between the blood-pressure in the aorta at the mouth of the renal artery and the bloodpressure at the vena cava at the mouth of the renal vein is at the same time increased, or at all events is not diminished.

In dealing with the vascular system we saw that relaxation of a small artery, taking place without any marked change in the general blood-pressure and in neighboring arteries, leads to a fuller and more rapid stream of blood through the capillaries supplied by the artery, and that at the same time the pressure in the capillaries themselves is increased; owing to the decrease of peripheral resistance through the widening of the artery, the great fall of pressure (see $\$ 105$ ) so characteristic of the peripheral region is shifted from the arterial side of the capillaries toward the venous side and to the capillaries themselves.

Hence, as we have already said, when the renal artery dilates two things happen in the loops of the glomeruli: a fuller, more rapid stream of blood passes through them, and that blood as it flows through them is exerting a greater pressure than before on their walls. How does each of the events stand toward the secretion of urine?

We have not at present the means of inducing a fuller and more rapid flow without increasing the pressure; but we may easily obtain increase of pressure without the fuller and more rapid flow. If we hinder or obstruct the outflow through the renal vein we at once increase the pressure in the glomerular loops as in the other capillaries of the kidney. Now, when the blood-pressure in the glomeruli is thus raised by partial obstruction to the venous outflow, the flow of urine so far from being increased is diminished. Obviously, then, the passage of water and material through the walls of the glomerular loops, to go to form the urine, is not the result of mere pressure, and cannot, therefore, be spoken of properly as a process of filtration. (Cf. § 255.) And we may here draw a comparison between the passage of water and material through the wall of a capillary in an ordinary situation to form lymph and the passage through the wall of the glomerular loop to form urine or part of urine. The former, as we have seen ( $\$ 255)$, appears to be directly dependent on pressure, though influenced as we have also seen in a very material way by the condition of the vascular wall; and hindrance to venous outflow, so inefficient in promoting a flow of urine, is as we have seen especially favorable to the transudation of lymph. In the former case the substances which pass through the capillary wall may be described as the constituents of the blood generally, proteids as well as salts and other soluble and diffusible matters. Through the wall of the glomerular loop there pass, so long as that wall is sound and intact, neither albumin nor globulin nor fibrin factor, but only water accompanier by some, and apparently a selection of some, of the soluble diffusible constitu- 
ents of the blood; for, as we have said, the presence of proteids in normal urine is contested, and, at most, there is present a very small quantity only (which, moreover, may come from the tubular epithelium). This difference in the material which passes through may be referred to the differences in the nature of the partition. The transudation of lymph takes place through the capillary wall; between the blood on one side and the lymph in the lymph-space on the other is only the thin film of conjoined epithelioid plates. But the corresponding wall of the glomerular loop is covered over and wrapped around, so to speak, by an adherent layer of cells, which though reduced and thin are still epithelial cells; the materials which go to form urine have to pass through these cells as well as through the film of epithelioid plates. It seems to be this layer of cells which determines what shall pass and what shall not.

Obviously the passage through this epithelium is of a peculiar nature. The necessary condition for the due accomplishment of the passage is as we have seen a full and rapid stream of (arterial) blood; the high pressure which accompanies that full and rapid stream, though probably under normal circumstances an adjuvant, is by itself helpless. Thus when the pressure is raised by venous obstruction, in which case the high pressure is accompanied by a slow stream or by actual arrest of the flow, even the passage of mere water is retarded. Seeing that many of the constituents of urine are diffusible substances certainly preëxisting in the blood, inorganic salines for instance, and seeing that diffusible abnormal constituents of blood, such as peptone and sugar, pass into the urine not by the tubular epithelium but by the glomeruli, we might expect that diffusion, in contrast to filtration (see $\$ 265$ ), played an important part in the passage; and a full rapid stream would undoubtedly favor diffusion. But diffusion by itself will not explain matters. Egg-albumin differs very slightly as regards diffusibility from serum-albumin, and yet while at the most a minute quantity only of the latter passes into the urine in normal circumstances, the former when injected into the blood at once makes its way into the urine, presumably by the glomeruli. On the other hand urea is an eminently diffusible body, and yet if we can trust the experiments on the amphibian kidney, the main mass at all events of the urea of the urine passes by the epithelium of the tubules.

The important part played by the epithelium is shown when the epithelium is deranged. If the renal artery be temporarily ligatured or otherwise obstructed, so that the glomeruli are shut off from the blood-supply for some little time, the secretion of urine is stopped; on reëstablishment of the circulation the secretion of urine slowly returns, and the urine is then found to be albuminous, remaining so for some little time. The serum-albumin and globulin which could not pass through the intact epithelium, can pass through when the epithelium is damaged by interference with its nutrition. The appearance of albumin in the urine (albuminuria) is a not infrequent symptom of kidney disease, and its presence in other than minute quantities indicates imperfections in the glomerular epithelium. But even under unhealthy conditions that epithelium still governs to a certain extent the passage of material; for the proteids of the blood-plasma do not pass through bodily or in a proportion which corresponds either to the relative proportion in which they exist in the plasma or to the relative ease (or difficulty) with which they pass through membranes. Though the "albumin" of albuminous urine frequently consists of both serum-albumin and globulin, these do not necessarily occur in the same proportion as in blood; they vary in urine much more than they do in blood; and indeed the one or the other may be absent; moreover, fibrin factors are very rarely found. 
Hæmoglobinuria, or the presence of hæmoglobin in urine, may be brought about by injecting into the bloodvessels laky blood, or some substance such as pyrogallic acid, which will "break up" the corpuscles of the blood. Now, in such cases there is evidence that the hromoglobin passes through the glomeruli ; minute disc-like masses of hæmoglobin, the so-called "menisci," are, by appropriate methods of preparation, found in situ in the capsules. Such a passage is very far removed from being a process of diffusion.

We may conclude, then, that the passage of material through the glomeruli, like the transudation of lymph, and even to a more marked extent, is a complex affair in which the ordinary physical processes of diffusion and filtration may play their part, but are not masters of the situation.

$\$ 354$. The work of the epithelium of the tubules. As we have said, the structural features of the epithelial cells of the tubules seem to justify the conclusion that they exercise a secretory, activity comparable with that of a salivary or a gastric gland. But their work is in many ways peculiar. In the case of the salivary, gastric, and pancreatic glands there can be no doubt that the specific constituents of the several secretions, mucin, pepsin, trypsin, and the like, are manufactured in the alveolar cells out of antecedents of some nature or other. The evidence, as we have seen, is all against the view that these glands merely withdraw, secrete in the old sense of the word, from the blood these substances preëxisting in the blood. When the salivary glands are extirpated, or the pancreas or the stomach removed, there is no accumulation in the blood of the specific constituents of the corresponding secretions. So also when the liver is extirpated there is no accumulation in the blood of either bile acids or bile pigment. With regard to the kidney and the most important constituent of urine, namely, urea, the case is different. If the kidneys in a mammal be extirpated, or if the kidneys by disease or by ligature of the ureters be so damaged as to be unable to carry on their work, an accuınulation takes place in blood, not as was once thought, of some antecedent of urea, such as kreatin, but of urea itself. In the case of birds and reptiles which excrete not urea, but chiefly uric acid, the accumulation is one of uric acid. Obviously in secreting urea the work of the epithelium of the tubules is largely, if not exclusively, confined to simply picking the urea out of the blood and pushing it, so to speak, into the lumina of the tubules. IVe might, perhaps, say exclusively, for there is no evidence that any urea at all is actually manufactured in the kidney.

But even this mere picking up the urea is after all not a simple process; the epithelial cell of the tubule is not a mere passive sieve of peculiar structure, especially adapted to strain off the urea from the blood. As we have already seen, when urea or uric acid is injected into the blood the result is not a mere increase in the proportions of urea (or uric acid) present in the urine which is being secreted. The injection leads to an increased flow of urine, the whole activity of the cell is stirred up, and other constituents, not at the moment like the urea existing in excess in the blood, are discharged into the lumina of the tubules together with the urea.

How the urea, which is in this peculiar manner taken out of the blood, comes to make its appearance in the blood is a problem in which the kidney is not concerned, and with which we shall deal in treating of the metabolic events of the body generally.

$\$ 355$. In the case of some other constituents of the urine we have evidence that the cells do something more than simply pick the constituent out of the blood. Hippuric acid, as we have seen, occurs in small quantity in the urine of man, and in larger amount in the urine of herbivora. Now, hippuric acid may be formed by the combination, with dehydration, of benzoic acid and glycin $\left(\mathrm{C}_{7} \mathrm{H}_{6} \mathrm{O}_{2}+\mathrm{C}_{2} \mathrm{H}_{5} \mathrm{NO}_{2}-\mathrm{H}_{2} \mathrm{O}=\mathrm{C}_{9} \mathrm{H}_{9} \mathrm{NO}_{3}\right)$; and benzoic 
acid introduced into the alimentary canal or injected into the blood reappears in large measure in the urine as hippuric acid. Somewhere in the body the benzoic acid meets with and combines with glycin. And we have experimental proof that the combination may and probably does take place in the kidney.

If a circulation of blood be kept up through the bloodvessels of the kidney freshly removed from a living animal, and benzoic acid and glycin be added to the blood as it is about to enter into the kidney, hippuric acid will be found in the blood issuing from the kidney, especially if the same blood be passed through the kidney several times; the blood used must be blood containing oxy-hæmoglobin, carbonic-oxide hæmoglobin not producing the effect. The mere mixing with the blood itself is insufficient; and if the blood be sent not through a kidney just removed from the living body, but through one taken from a dead body or one which has been left to itself for some time after removal from a living body, the synthesis will not be effected. To carry out the combination by means of the kidney which has been removed from the body, the kidney must retain for a while its own life, it must be a "surviving" kidney. Nor is it absolutely necessary to bring the benzoic acid and glycin to the kidney by means of a blood-stream. If a "surviving" kidney be divided rapidly into small pieces and the benzoic acid rapidly mixed with the pieces, hippuric acid is formed. Nor is it necessary to furnish the glycin. If benzoic acid alone be used, hippuric acid is formed all the same. Glycin, as we have previously said, cannot be recognized as a normal constituent of any of the tissues; nevertheless, as we have seen in speaking of glycocholic acid in the bile, and as we shall see later on, glycin must make a momentary appearance in various metabolic processes of the body, being immediately on its appearance converted into something else, so that it never remains as glycin. It apparently is formed in the kidney, and is thus momentarily available for the conversion of benzoic into hippuric acid.

It seems probable, therefore, that, with regard to this particular constituent of urine, hippuric acid, the cells of the tubules have the power of effecting a combination between the benzoic acid brought to them by the blood and the glycin which they furnish by means of their own metabolism, and in this way produce hippuric acid.

Not only benzoic acid, but many other bodies taken into the system, reappear in the urine combined with glycin, and in their cases also the combination probably takes place through the activity of the cells of the tubules of the kidney. Moreover, other changes than the assumption of glycin, the various changes which many chemical substances taken into the system undergo before reappearing in the urine, probably also take place to a large extent in the kidney, and are also carried out by means of the epithelium of the tubules.

What other constituents of normal urine are produced in this or a similar manner we do not as yet definitely know. The pigment urobilin, which, as we have seen, is supposed to be a derivative from bilirubin, may be brought ready-formed from the liver or may have the finishing touches given to it in the kidney itself; and the other normal or abnormal urinary pigments possibly arise either directly from hæmoglobin or indirectly from that body through the biliary pigment by a transformation taking place in the cells of the tubules. There is also evidence in frogs that acid sodium phosphate is furnished by the cells of the tubules.

In conclusion, then, we may say that the activity of the epithelium of the kidney appears especially modified, as compared with other secreting glands, to meet the special object which the kidney has to secure. The purpose of 
the kidney is not to provide a fluid, urine, which can be made use of for the needs of the body, but to cast out waste matter's from the body. Hence its secretory activity is limited largely to the mere discharge of matters which reach it preëxistent in the blood, though in several cases it gives the final shape to the excreted substance before it passes into the ureter.

$\$ 356$. We may illustrate the preceding discussions by briefly passing in review sonse of the more usual ways in which the secretion of urine is in ordinary life modified.

In the preceding section the composition of urine was illustrated by the daily output of the several constituents, rather than by a percentage account of any specimen of urine, for the reason that the composition of urine varies within extremely wide limits. This is especially the case as regards the proportion of water to solids. One urine may be of high specific gravity with a small amount of water relatively to the solids, while another may have so little color and such a low specific gravity as to appear hardly more than water. The reason of these extreme differences lies in the fact that the kidney is not only the channel by which waste solids leave the body, but also an important outlet for the discharge of the stream of water which, in order that the various processes of the body may be duly carried on, is continually passing through the system. It is frequently of advantage to the body to discharge through the kidney a large amount of water, more or less irrespective of the solid matters which are, so to speak, washed away with it; and hence the advantage of the glomerular mechanism so specially adapted for the discharge of water.

As we shall see presently, to the skin also falls the duty of discharging large quantities of water. The respiratory organs also, as we have seen, serve for the discharge of water; but the amount which the latter put out can only be varied by the inconvenient method of increasing or diminishing the whole act of breathing. Hence we find special relations between the skin and the kidneys, correlating the work of the one to that of the other as regards this particular work of the discharge of water.

When the body is exposed to cold the discharge of water from the skin in the form of sweat is checked, and the cutaneous vessels are constricted. At the same time the bloodvessels of the abdominal viscera, including the kidneys are dilated, but not out of proportion to the constriction of the cutaneous vessels, for the general blood-pressure does not fall, but if anything rises somewhat. Thus there is established just the state of things which is favorable to a full and rapid stream of blood through the renal glomeruli, and an increased flow of urine results. It is possible, we may, perhaps, say probable, that the nervous system affords a special tie between the skin and the kidney, so that under the circumstances in question the renal arteries are dilated even more than those of the other abdominal viscera; but this has not been proved experimentally. It is also possible that by another reflex mechanism of the central nervous system the skin may work upon the kidney, not by the vasomotor nerves alone, but also by nerves governing the secretory activity of the tubules; but we have no satisfactory indications of any such mechanisms, and it seems more probable that the connections should be with the glomerular mechanism, since the chief object at all events is to get rid of water.

Conversely, when the body is exposed to warmth the skin perspires freely and the cutaneous vessels are widely dilated; and conversely also the renal and other abdominal vessels are constricted, so that a slow and small stream of blood trickles through the glomeruli, and the urine which is secreted is scanty.

$\$ 357$. Even more important than its relations to the skin are the rela- 
tions of the kidney to the water absorbed by the alimentary canal; this is especially seen when large quantities of fluid are drunk. The whole of the water thus introduced into the alimentary canal passes into the blood, for in a healthy organism no anount of fluid drunk, unless it throws the economy out of order, can affect the amount of water present in the feces. But the addition to the blood of even a very large quantity of fluid does not, as we have seen, by its mere quantity ( $\$ 172)$, increase the general blood-pressure, and therefore cannot in this way produce what it undoubtedly does produce, an increased flow of urine.

The fluid so absorbed may act on the kidney in two ways. On the one hand, as we have seen $(\$ 350)$, the injection of water into the blood produces a local dilatation of the renal vessels, as indicated by the swelling of the kidney. Thus the absorption of mere water from the alimentary canal may stir up to greater activity the glomerular mechanism, and in so) doing may be assisted by the presence of various substances absorbed from the alimentary canal with the water, for some of these also may similarly lead to dilatation of the renal vessels.

On the other hand, some or other of the chemical bodies thus passing into the blood with the water drunk may excite the secretory activity of the tubules, and that either by acting directly on the epithelium as they are carried through the kidney in the blond of the renal arteries, or indirectly through some intervention of the central nervous system.

Our knowledge is at present too scanty to enable us to decide which of these two methods is the one usually emplnyed by the organism; but the inordinate flow of urine, so poor in solids as to be little more than water, which may be directed through the kidney by means of an adequate "drinking bout," would lead us to conclude that in such cases the organism, striving, though too often in vain, to free itself fron the evils to which it is being subjected, has recourse rather to the simpler glomerular mechanism than to the more expensive tissue-wasting activity of the tubules; and the urine in such cases is probably discharged chiefly by the method of dilating the renal vessels and thus throwing the poisoned blood into the glomeruli.

When, however, fluid is taken simply as a proper accompaniment of solid food, the increase of urine which results has probably another origin. As we have already said, and as we shall point out more fully later on, the absorption of proteid material, which is a constituent and generally a conspicunus constituent of every meal, leads to a formation of urea; and urea, as we have seen reason to believe, directly stimulates the epithelium of the tubules to secretory activity. And what seems prominently true of urea is probably true of many other products of digestion; so that the increased flow of urine which follows an ordinary meal accompanied by not more than the ordinary amount of fluid, is the result of the labors of the epithelium of the tubules as well as of the fuller stream of blood through the glomeruli.

$\$ 358$. What has just been said concerning the influence on the kidney of foorl and water may be applied also to the action of substances which being especially efficacious in promoting a flow of urine when taken into the body are called "diuretics." The several actions of various diuretics are very varied, and it would be out of place to discuss them fully. We may, however, say that while the action of some appears simple that of others is complex.

Such agents as sodium acetate and potassium nitrate probably produce their effect chiefly by acting directly on the kidney, including, as we have seen $(\$ 350)$, local vascular dilatation and so working on the glomeruli, but probably at the same time also stirring up, after the fashion of urea, the 
epithelium of the tubules to secretory activity, the accompanying fuller stream of blood through the whole kidney being, as in the case of the salivary and other glands, a useful adjuvant.

The diuretic effect of such an agent as digitalis is probably more complex. By increasing the cardiac stroke, and at the same time constricting many small vessels, digitalis raises the general blood-pressure; but the tendency of the increased blood-pressure to increase the flow of urine may be counterbalanced by the constriction of the renal vessels themselves. Ancl while it is a matter of common experience that digitalis is very effective as a diuretic in cardiac disease, there is great doubt whether it really acts as a diuretic in health; in cardiac disease it probably raises the blood-pressure bv improving the cardiac stroke and not by constriction of the bloodvessels. But even in the absence of cardiac disease, digitalis has been found in certain cases to act as a powerful diuretic, and in these cases either it must act directly on the tubular epithelium or its effects in constricting the renal arteries must be less than its effects on other small arteries or must pass off before the influence of the heightened blood-pressure has disappeared.

$\$ 359$. Quite removed from the intervention of chemical substances in the blood and yet most striking is the influence on the kidney of the central nervous system. The potent influence of emotions in promoting the secretion of urine is proverbial, and the general features of "nervous" urine, the water increased out of proportion to the solid constituents, especially seen in the "urina hysterica," which is hardly more than simple water, often discharged in enormous quantity, at once suggests the view that impulses originating in the brain and passing down to the kidney along the vaso-dilator fibres, of whose existence evidence was given in $\$ 349$, lead to dilated bloodressels and great play of glomerular activity, without perhaps producing. any other direct effect on the economy; though possibly the same emotions by constricting the cutaneous and, it may be, other vessels may raise the general blood-pressure and so help the dilated renal vessels.

\section{The Discharge of Urine.}

$\$ 360$. The urine, like the bile, is secreted continuously; the flow may rise and fall, but in health never absolutely ceases for any length of time. The cessation of renal activity, the so-called suppression of urine, entails speedy death. The minute streams passing continuously-now more rapidly, now more slowly-along the collecting and discharging tubules, are gathered into the renal pelvis, whence the fluid is carried along the ureters into the bladder partly by pressure and gravity, and from time to time partly, as we have already said ( $\$ 351)$, by the peristaltic contractions of the muscular walls of the ureter.

If in a living animal a ureter be laid bare and stimulated, mechanically or otherwise, at a part of its course, waves of peristaltic contraction may be seen to pass in both directions from the spot stimulated-up toward the kidney and down toward the bladder. In the absence of artificial stimulation spontaneous waves of contraction make their appearance, sometimes repeated with tolerable regularity (about every twenty seconds in the rabbit), sometimes occurring in groups with longer pauses between. These spontaneous contractions invariably pass in one direction, from the kidney to the bladder, and their frequency and vigor seem to be determined by the activity of the secretion of urine. But they are not directly called forth by the urine, either mechanically distending the tube or chemically stimulating the inner surface, for regularly recurring contractions may be observed in a 
kidney and ureter removed from the body, or even in an isolated exeised piece of the ureter.

The rhythmically repeated contractions arise spontaneously in the muscular coat of the ureter much in the same way as the similar cardiac contractions arise in the muscular substance of the heart; and it may here be mentioned in support of what was urged in $\S 141$ with regard to the heartbeats not being started by nerve-cells, that rhythmically repeated spontaneous peristaltic contractions have been observed in isolated pieces of ureter taken from the middle of its course, in which no nerve-cells and indeed no distinct nerve-fibres could be observed.

In the living body these spontaneous movements, beats they might be called, are subordinated to the flow of urine into the pelvis; the more active the secretion of urine, the more frequent and vigorous are the beats of the pelvis and ureter ; but the exact mechanism by which the secretion and the movements are maintained in harmony has not yet been cleared up.

\section{Micturition.}

$\$ 361$. In the urinary bladder the urine is collected, its return into the ureters being prevented by the oblique entrance into the bladder and valvular nature of the orifices of those tubes, and its discharge from thence in considerable quantity is effected from time to time by a somewhat complex muscular mechanism, of the nature and working of which the following is a brief account :

The involuntary muscular fibres forming the greater part of the vesical walls are arranged, partly in a more or less longitudinal direction and partly in a circular manner. After it has been emptied the bladder is contracted and thrown into folds; as the urine gradually collects, the bladder becomes mere and more distended. The escape of the fluid is in part prevented by the resistance offered by the elastic fibres in the walls of the urethra, which help to keep the urethral channel closed. But this is not all, for observation shows that fluid is retained within the bladder up to a pressure of twenty inches of water, so long as the bladder is governed by an intact spinal cord, but gives way to a pressure of six inches only when the lumbar spinal cord is destroyed or the vesical nerves are severed. This affords very strong evidence that the obstruction at the neck of the bladder to the exit of urine depends on some tonic muscular contraction maintained by a reflex or automatic action of the lumbar spinal cord. And it has been maintained that it is the circularly disposed fibres specially developed around the neck of the bladder which are the subjects of this tonic contraction and thus the chief cause of retention; hence the name sphincter vesicr. The continuity of these fibres, however, with the rest of the circular fibres of the bladder suggests that they probably do not act as a sphincter, but that their use lies in their contracting after the rest of the vesical fibres, and thus finishing the evacuation of the bladder. The resistance in question is supplied by a tonic contraction not of the circular fibres of the bladder itself, but of the muscular fibres-partly plain, partly striated-surrounding the prostatic portion of the urethra, and constituting the splincter vesicce externus or prostaticus, or sphincter of Henle. It is stated that artificially excited contractions of these fibres will resist a pressure of fluid in the bladder.

When the bladder has become full, we feel the need of making water, the sensation being heightened, if not caused, by the trickling of a few drops of urine from the full bladder into the urethra. We are then conscious of an effort; during this effort the bladder is thrown into a long-continued con- 
traction of an obscurely peristaltic nature, the force of which is more than. sufficient to overcome the resistance offered by the urethra, and the urine issues in a stream, the sphincter vesicæ externus being at the same time either relaxed after the fashion of the sphincter ani, or at least overcome. In its passage along the urethra, the exit of the urine, at all events of the last portions, is forwarded by irregularly rhythmic contractions of the bulbo-cavernosus or ejaculator urinæ muscle, the contractions of which compress the urethra; and the whole act is further assisted by pressure on the bladder exerted by means of the abdominal muscles, very much the same as in defecation.

In the case of the rectum we were able ( $\$ 237)$ to distinguish between the actions of the longitudinal and of the circular coats, and we said that the two coats had distinct nervous supplies (Fig. 89). The bladder has, as we have said, a similar nerve supply, and it is very probable, but not yet distinctly proven, that this like double supply has a like double action. Stimulation of the branches coming from the sacral nerves, at the same time that it throws the longitudinal coat of the rectum into contractions, brings about in the dog, in which the longitudinal fibres of the bladder are much more pronounced than the circular, powerful vesical contractions. Moreover, stimulation of the sacral nerves on one side produces unilateral contraction of the bladder. From this we may infer that the sacral nerves govern the longitudinal coat. Stimulation of the hypogastric nerves carrying fibres from the dorsal and upper lumbar cord (see Fig. 109), while throwing the circular coat of the rectum into strong contractions, gives rise to vesical contractions, but these are by no means so marked as those which appear when the sacral nerves are stimulated. We may probably conclude that the more important fibres in the fundus of the bladder, which are for the most part longitudinal, are to be regarded as governed chiefly by the sacral nervefibres, while the circular muscular fibres around the neck of the bladder, whose contraction completes, as it were, the emptying of the bladder, are those on which the hypogastric nerve-fibres have the chief influence.

$\S 362$. We said just now "when the bladder has become full," but this must not be understood to mean "when the bladder has received a certain quantity of fluid." On the contrary, it is a matter of common experience that we feel the desire to make water sometimes when a large quantity and sometimes when a small quantity of urine has accumulated in the bladder. We have evidence that the bladder possesses to a very high degree that obscure continuous contraction which we speak of as " tone " and, further, that the amount of its tone is exceedingly variable, the organ, quite independently of distinct efforts at micturition, being at one time contracted and at another flaccid and distended. When it is in a contracted state, a small quantity of fluid may exert the same effect on the vesical walls as a larger quantity when the bladder is flaccid. Hence, while the determining cause of the desire to make water is the pressure of the urine upon the vesical walls, the quantity needed to produce the necessary fulness is dependent on the amount of tonic contraction of the muscular fibres existing at the time. And we have evidence that this tone is regulated by the nervous system.

$\$ 363$. Micturition as sketched above seems at first sight, and especially when we appeal to our own consciousness, a purely voluntary act. A voluntary effort throws the muscular fibres of the bladder into contractions, an accompanying voluntary effort lessens the tone of the sphincter externus, probably by inhibiting its centre in the spinal cord, while other voluntary efforts throw the ejaculator and abdominal muscles into contractions, and the resistance of the urethra being thereby overcome, the exit of the urine naturally follows. 
There are facts, however, which prevent the acceptance of so simple a view. In the first place, in cases of urethral obstruction, where the bladder cannot be emptied when it reaches its accustomed fulness, the increasing distention sets up fruitless but powerful contractions of the vesical walls, contractions which are clearly involuntary in nature, which wane or disappear, and return again and again in a rhythmic manner, and which may be so strong and powerful as to cause great suffering. It seems that the fibres of the bladder, like all other muscular fibres, have their contractions augmented in proportion as they are subjected to tension. Just as a previously quiescent ventricle of a frog's heart may be excited to a rhythmic beat by distending its cavity with blood, so the quiescent bladder may, quite independently of the will, be excited, by the distention of its cavity, to a peristaltic action which in normal cases is never carried beyond a first effort, since witl that the bladder is emptied and the stimulus is removed, but which in cases of obstruction is enabled clearly to manifest its rhythmic nature.

In the second place it has been shown that quite normal micturition may take place in a dog in which the lumbar region of the spinal cord has been completely and permanently separated by section from the upper dorsal region. In such a case there can be no exercise of volition, and the whole process appears as a reflex action. When under these circumstances the bladder becomes full (and otherwise apparently the act fails) any slight stimulus, such as sponging the anus or slight pressure on the abdominal walls, causes a complete act of micturition; the bladder is entirely emptied, and the stream of urine toward the end of the act undergoes rhythmical augmentations due to contractions of the ejaculator urinæ. These facts can only be interpreted on the view that there exists in the lower spinal cord (of the dog) what we may speak of as a micturition centre capable of being thrown into action by appropriate afferent impulses, the action of the centre being such as to cause a contraction of the walls of the bladder and of the ejaculator urinæ, and at the same time to suspend the tone of the sphincter vesica externus. Clinical experience also goes to show the existence of a similar micturition centre in man, placed higher up in the cord than the corresponding "genital" centre governing the genital organs.

Moreover, we have, in the case both of man and of other animals, experimental and other evidence that contraction of the bladder is frequently brought about by reflex action. Thus the pressure within the bladder when observed for any length of time is found to be subject to considerable and manifold variations. Over and above passive changes in pressure due to the respiratory movements, through which the bladder is pressed upon at each descent of the diaphragm, active contractions, of a strength inadequate to bring about micturition, are from time to time observed. These in some instances appear to be spontaneous, or to be the result of emotions, but they may be readily induced in a reflex manner by stimulating various sentient surfaces or sensory nerves. And common experience affords many instances where vesical contractions thus brought about in a reflex manner acquire strength adequate to empty the bladder.

Observation of vesical pressure may be most conveniently carried out by introducing into the bladder a catheter connected with a water manometer and a registering apparatus, and so arranged as to allow fluid to be driven into or received from the bladder at pleasure.

$\$ 364$. Involuntary micturition obviously of reflex nature has frequently been observed in cases of paralysis from disease of or injury to the spinal cord; and the involuntary micturition which is common in children, as the result of irritation of the penis and genital organs, and which some- 
times occurs in the adult as the result of emotions, or at least sensory impressions, appears to be the result of reflex action. In these several cases we may fairly suppose that the centre in the spinal cord is affected by afferent impulses reaching it along various sensory nerves or descending from the brain. Hence we are led to the conception that when we make water by a conscious effort of the will, what occurs is not a direct action of the will on the muscular walls of the bladder, but that impulses started by the will descend from the brain after the fashion of afferent impulses and thus in a reflex manner throw into action the micturition centre in the spinal cord. We may draw an analogy between the micturition apparatus and the respiratory mechanism. We saw reasons in the latter case to think that when the will interfered with the respiratory movements, it did so by acting upon the nervous mechanism in the central nervous system and not by acting directly on the muscular fibres of the diaphragm and other respiratory muscles. And the case of the plain muscular fibres of the bladder seems even stronger than that of respiratory muscles so largely skeletal in nature. We might also draw an analogy with the heart. We are not able to throw into action, by any direct effort of the will, the cardiac augmentor mechanism. Were we able to do so powerfully and suddenly, we might throw into violent action a weakly beating heart much in the same way that we empty an obscurely contracting bladder. Nor is this view negatived by the fact that paralysis of the bladder, or rather inability to make water either voluntarily or in a reflex manner, is a common symptom of cerebral or spinal disease or injury. Putting aside the cases in which the reflex act is not called forth because the appropriate stimulus has not been applied, the failure in micturition under these circumstances may be explained by supposing that the shock of the spinal injury or some extension of the disease has rendered the spinal centre unable to act.

The so-called incontinence of urine in children is simply an easily excited and frequently repeated reflex micturition. In cases of cerebral or spinal disease a form of incontinence is frequently met with which seems to be of a different nature. The bladder becoming full, but, owing to a failure in the mechanism of voluntary or reflex micturition, being unable to empty itself by a complete contraction, a continued dribbling of urine takes place through the urethra, the fulness of the bladder being sufficient to overcome the resistance at the neck of the urethra. It is probable, however, that even in these cases the flow is partly caused by obscure, unfelt, intrinsic contractions of the bladder.

$\$ 365$. Whether, under normal conditions, the urine undergoes any notable change during its stay in the bladder has been much debated. Experiments show that poisonous substances injected into the bladder with all due care to avoid any abrasion of the epithelium are absorbed and produce their usual effects. It has also been stated that if a solution of urea be injected into the bladder after ligature of both ureters, and allowed to stay for some hours, part of the urea disappears. But at present there is no very decided proof that under ordinary conditions either the water or other constituents of urine are to any appreciable extent absorbed by the bladder.

Under abnormal conditions, as in inflammation or irritation of the bladder, the urine may have undergone marked changes during its stay in the bladder, one of the most common being a change of some of the urea into ammonium carbonate, by which the urine also becomes alkaline. Under abnormal conditions also, the mucus of the urine, which in a healthy man is insignificant, though in some animals, for instance the horse, it occurs in considerable quantity, is largely increased during the stay in the bladder. 
Since there are in man no goblet cells in the vesical epithelium (in the frog they are present) or mucous glands in the walls of the bladder, this mucus must be supplied by an abnormal metabolism of the ordinary epithelial cells.

\section{The Nature and Amount of Perspiration.}

$\S 366$. The quantity of matter which leaves the human body by way of the skin is very considerable. Thus, it has been estimated that while 0.5 gramme passes away through the lungs per minute, as much as 0.8 gramme passes through the skin. The amount, however, varies extremely; it has been calculated, from data gained by enclosing the arm in a caoutchouc bag, that the total amount of perspiration from the whole body in twenty-four hours might range from 2 to 20 kilos; but such a mode of calculation is obviously open to many sources of error.

Of the whole amount thus discharged, part passes away at once as watery vapor mixed with volatile matters, while part may remain for a time as a fluid on the skin; the former is frequently spoken of as insensible, the latter as sensible, perspiration or sweat. The proportion of the insensible to the sensible perspiration will depend on the rapidity of the secretion in reference to the dryness, temperature, and amount of movement of the surrounding atmosphere. Thus, supposing the rate of secretion to remain constant, the drier and hotter the air, and more rapidly the strata of air in contact with the body are renewed, the greater is the amount of sensible perspiration, which is, by evaporation, converted into the insensible condition ; and, conversely, when the air is cool, moist, and stagnant, a large amount of the total perspiration may remain on the skin as sensible sweat. Since, as the name implies, we are ourselves aware of the sensible perspiration only, it may, and frequently does, happen that we seem to ourselves to be perspiring freely, when, in reality, it is not so much the total perspiration which is being increased as the relative proportion of the sensible perspiration. The rate of secretion may, however, be so much increased that no amount of dryness or heat, or movement of the atmosphere, is sufficient to carry out the necessary evaporation, and thus the sensible perspiration may become abundant in a hot, dry air. And, practically, this is the usual occurrence, since, certainly, a high temperature conduces, as we shall point out presently, to an increase of the secretion, and it is possible that mere dryness of the air has a similar effect.

The amount of perspiration given off is affected not only by the condition of the atmosphere, but also by the circumstances of the body. 'Thus it is influenced by the nature and quantity of food eaten, by the amount of fluid drunk, by the character of exercise taken, by the relative activity of the other excreting organs, more particularly of the kidney, by mental conditions, and the like. Variations may also be induced by drugs and by diseased conditions. How these various influences produce their effects we shall study immediately.

The fluid peispiration, or sweat, when collected, is found to be a clear, colorless fluid of a distinctly salt taste, with a strong and distinctive odor, varying according to the part of the body from which it is taken. Besides accidental epidermic scales, it contains no structural elements.

Sweat, as a whole, is furnished partly by the sweat-glands and partly by the sebaceous glands, for, as we shall see, the small amount which simply transudes through the epidermis, apart from the glands, may be neglected. Now, the secretions firom these two kinds of glands differ widely in nature, and the characters of the sweat, as a whole, will vary according to the rela- 
tive proportion of the two kinds of secretion. The secretion of the sebacenus glands appears to be fairly constant, the larger variations of the total sweat depending chiefly on the varying activity of the sweat-glands. Hence, when sweat is scanty, the constituents of the sebum influence largely the characters of the sweat; when, on the contrary, the sweat is very abundant, these may be disregarded, and the sweat may be considered as the product of the sweatglands.

We are not able at present to make a complete statement as to what bodies occur exclusively in the sebum and what in the secretion of the sweat-glainds. The former consists, very largely, of fats and fatty acids, and appears to contain some form or forms of proteids; but we have reason to think that the sweat-glands secrete, in some quantity, some forms of fat, and especially volatile fatty acids.

When sweat is scanty, the reaction is generally acid, but when abundant, is alkaline; and when a portion of the skin is well washed the sweat which is collected immediately afterward is usually alkaline. From this we may infer that the secretion of the sweat-glands is naturally alkaline, but that when mixed, sweat is acid ; the acidity being due to fatty (or other) acids of the sebum. In the horse, which is singular in haircovered animals for its frequent profuse sweating, the sweat is said to be always acid and to contain a considerable quantity of some form of proteid. These features are probaby due to the large admixture of sebum from the numerous sebaceous glands connected with the hairs.

Taking ordinary sweat, such as may be obtained by enclosing the arm in a bag, we may say that in man the average amount of solids is from 1 to 2 per cent., of which about two-thirds consist of organic substances. The chief normal constituents are: (1) Sodium chloride, with small quantities of other inorganic salts. (2) Various acids of the fatty series, such as formic; acetic, butyric, with probably propionic, caproic, and caprylic. The presence of these latter is inferred from the odor; it is probable that many various volatile acids are present in small quantities. Lactic acid, which has been reckoned as a normal constituent, is stated not to be present in health. (3) Neutral fats and cholesterin; these have been detected even in places, such as the palm of the hand, where sebaceous glands are not present. (4) The evidence goes to show that neither urea nor any ammonia compound exists in the normal secretion to any extent, though some observers have found a considerable quantity of urea (calculated at $10 \mathrm{grms}$. in the twenty-four hours for the whole body). Apparently a small amount of nitrogen leaves the body through the skin, but this is probably supplied by the sebum or by the epidermis.

In various forms of disease the sweat has been found to contain, sometimes in considerable quantities, blood, albumin, urea (particularly in cholera), uric acid, calcium oxalate, sugar (in diabetic patients), lactic acid, indigo (or indigo-yielding bodies, giving rise to "blue" sweat), bile, and other pigments. Iodine and potassium iodide, succinic, tartaric, and benzoic (partly as hippuric) acids have been found in sweat when taken internally as medicines.

\section{Cutaneous Respiration.}

$\$ 367$. A frog whose lungs have been removed will continue to live for some time; and during that period will continue, not only to produce carbonic acid, but also to consume oxygen. In other words, the frog is able to breathe without lungs, respiration being carried on efficiently by means of 
the skin. In mammals and in man this cutaneous respiration is, by reason of the thickness of the epidermis, restricted within very narrow limits; and, indeed, it has been questioned whether it can be spoken of at all as a true respiration. When the body remains for some time in a closed chamber to which the air passing in and out of the lungs has no access (as when the body is enclosed in a large air-tight bag fitting tightly round the neck, or where a tube in the trachea carries air to and from the lungs of an animal placed in an air-tight box), it is found that the air in the chamber loses oxygen and gains carbonic acid. The amount of carbonic acid which is thus thrown off by the skin of an average man in twenty-four hours amounts to about 10 grnis., or according to some observers to (no more than) about 4 grms., increasing with a rise of temperature and being very markedly augmented by bodily exercise. It is stated that the amount of oxygen consumed is about equal in volume to that of the carbonic acid given off, but some observers make it rather less. It may be doubted, however, whether the carbonic acid comes direct from the blood; it may come from decomposition taking place in the sweat-of carbonates, for instance. Similarly the oxygen which disappears may be simply used in oxidizing some of the constituents of the sweat. It is evident that the loss which the body suffers through the skin consists, besides a small quantity of sodium chloride, chiefly of water.

When an animal, a rabbit for instance, is covered over with an impermeable varnish, such as gelatin, so that all exit or entrance of gases or liquids by the skin is prevented, death shortly ensues. This result cannot be due, as once thought, to arrest of cutaneous respiration, seeing how insignificant and doubtful is the gaseous interchange by the skin as compared with that by the lungs. Nor are the symptoms at all those of asphyxia, but rather of some kind of poisoning, marked by a very great fall of temperature, which, however, seems to be the result not of diminished production of heat, but of an increase of the discharge of heat from the surface. The animal may be restored, or at all events its life may be prolonged with the abatement of the symptoms, if the great loss of heat which is evidently taking place be prevented by covering the body thickly with cotton-wool or keeping it in a warm atmosphere. The symptoms have not as yet been clearly analyzed, but they seem to be due in part to a pyrexia or fever possibly caused by the retention within or reabsorption into the blood of some of the constituents of the sweat, or by the products of some abnormal metabolism, and in part to a dilatation of the cutaneous vessels caused by the application of varnish; owing to the dilated condition of the cutaneous vessels the loss of heat through the skin is abnormally large, even though the varnish may not be a good conductor.

$\$ 368$. Absorption by the skin. Although under normal circumstances the skin serves only as a channel of loss to the body, it has been maintained that it may, under particular circumstances, be a means of gain, and the little which we have to say on this matter may perhaps be said here. Cases are on record where bodies are said to have gained in weight by immersion in a bath, or by exposure to a moist atmosphere during a given period, in which no fond or drink was taken, or to have gained more than the weight of the food or drink taken; the gain in such cases must have been due to the absorption of water by the skin. Direct experiments, however, throw doubt on these statements, for they show that under ordinary circumstances such a gain by the skin is slight, being apparently due to mere imbibition of water by the upper layers of the epidermis.

Absorption of various substances takes place very readily by abraded surfaces where the dermis is laid bare or covered only by the lowest layers 
of epidermis, but it has been debated whether substances in aqueous solution can be absorbed by the skin when the epidermis is intact, the evidence on this point being contradictory. In the case of the skin of the frog an absorption of water and of various soluble substances certainly takes place. In the case of the sound human skin there are no à priori reasons why water carrying substances dissolved in it should not pass inward through the corneous as well as the other layers of the epidermis, the amount so passing depending, among other things, upon the condition of the skin; and common experience seems to show that it does. Nevertheless, the results of actual experiment are conflicting. Some observers maintain that soluble non-volatile substances are not absorbed, and that volatile substances, such as iodine, which may be detected in the system after a bath containing them, are absorbed not by the skin, but by the mucous membrane of the respiratory organs, the substance making its way to the latter by volatilization from the surface of the bath. Others, again, have found evidence of absorption, especia!ly with volatile substances, even when care has been taken to avoid all error's; and the greater weight may perhaps be given to these, since they accord with common experience. The conflict of experimental results, however, at least shows that we do not fully understand the conditions under which such absorption takes place.

There is, moreover, evidence that even solid particles can pass through an intact skin. The lymphatics in the skin of a newborn infant have been found crowded with the particles of the peculiar fatty secretion which covers the skin at birth; and solid particles rubbed into even the sound skin may, especially when applied in a fatty vehicle, as $e . g$., in the well-known mercury ointment, find their way into the underlying lymphatics. The wandering leucocytes which are at times found among the epidermic cells may perhaps take part in this transport.

\section{The Mechanism of the Secretion of Sweat.}

$\$ 369$. In dealing with the manner in which various circumstances affect the amount of sweat secreted we may, as we have already said, consider the sweat as a whole to be supplied by the sweat-glands alone. For though it seems evident that some amount of fluid must pass by simple transudation through the ordinary epidermis of the portions of skin intervening between the mouths of the glands, yet on the whole it is probable that the portion which so passes is a small fraction only of the total quantity secreted by the skin; and direct experiment shows that even the simple evaporation of water is much greater from those parts of the skin in which the glands are abundant, than from those in which they are scanty. We have as yet no evidence that the sebaceous glands vary in activity; their very peculiar form of secretion, if we may speak of it as a secretion, is not adapted to sudden changes, and at all events we have as yet no evidence that circumstances rapidly and largely modify the amount of sebum discharced by healthy sebaceous glands.

The secreting activity of the skin, like that of the other glands, is usually accompanied and aided by vascular dilatation. In one of the early experiments on division of the cervical sympathetic it was observed that. in the case of the horse, the vascular dilatation of the face on the side operated on was accompanied by increased perspiration. Indeed, the comnection between the state of the cutaneous bloodvessels and the amount of perspiration is a matter of daily observation. When the vessels of the skin are constricted, the secretion of the skin is diminished; when they are dilated, it becomes abundant. In this way, as we shall later on point out, the temperature of 
the body is largely regulated. When the surrounding atmosphere is warm, the cutaneous vessels are dilated, the amount of sweat secreted is increased, and the consequently augmented evaporation tends to cool down the body. On the other hand, when the atmosphere is cold, the cutaneous vessels are constricted, perspiration is scanty, and less heat is lost to the body by evaporation.

The analogy with the other secreting organs which we have already studied leads us, however, to infer that there are special nerves directly governing the activity of the sudoriparous glands, independent of variations in the vascular supply. And not only is this view suggested by many facts, such as the profuse perspiration of the death-agony, of various crises of disease, and of certain mental emotions, and the cold sweats occurring in phthisis and other maladies, in all of which the skin is anæmic rather than hyperæmic, but we have direct experimental evidence of a nervous mechanism of perspiration as complete as the vasomotor mechanism.

If in the cat ${ }^{1}$ the peripheral stump of the divided sciatic nerve be stimulated with the interrupted current, drops of sweat may readily be observed to gather on the hairless sole of the foot of that side. The sweating is not due to any increase of blood-supply, for it may be observed when the cutaneous vessels are thrown into a state of constriction by the stimulus, or even when the aorta or crural artery is clamped previous to the stimulation, and indeed may be obtained by stimulating the sciatic nerve of a recently amputated leg. Moreover, when atropine has been injected, the stimulation produces no sweat, though vasomotor effects follow as usual. The analogy between the sweat-glands of the foot and such a gland as the submaxillary is in fact very close, and we are justified in speaking of the sciatic nerve as containing secretory fibres distributed to the sudoriparous glands of the foot. Similar results may be obtained with the nerves of the fore limb. And in ourselves a copious secretion of sweat may be induced by tetanizing through the skin the nerves of the limbs or the face.

If a cat in which the sciatic nerve has been divided on one side be exposed to a high temperature in a heated chamber, the limb the nerve of which has been divided remains dry, while the feet or the other limbs sweat freely. This result shows that the sweating which is caused by exposure of the body to high temperatures is brought about by the agency of the central nervous system, and not by a local action on the sweat-glands; for the foot of the limb whose nerve has been divided is equally exposed to the high temperature. A high temperature it is true up to a certain limit increases the irritability of the epithelium of the sweat-glands and predisposes it to secrete, just as it promotes action in the case of a muscle or nerve or other forms of living substance. Thus stimulation of the sciatic in the cat produces a much more abundant secretion in a limb exposed to a temperature of $35^{\circ}$ or somewhat above, than in one which has been exposed to a distinctly lower temperature, and in a limb which has been placed in ice-cold water hardly any secretion at all can be gained ; but apparently mere rise of temperature without nervestimulation will not give rise to a secretory activity of the glands. The sweating caused by a dyspnœic condition of blood, and such appears to be the sweat of the death-agony, is similarly brought about by the agency of the central nervous system. When an animal with the sciatic nerve divided on one side is made dyspnœic, no sweat appears in the hind limb of that side though abundance is seen in the other feet.

\footnotetext{
1 The cat sweats freely in the hairless soles of the feet but not on any part of the body covered with hairs. The dog also sweats in the same regions but not so freely as the cat; indeed, sweating is often absent, the ducts being stopped by growth of the corneous epidermis. Rabbits and other rodents appear not to sweat at all. The snout of the pig sweats freely ; and the often profuse sweating of the horse, a singular event among haircovered animals, is known to all.
} 
Sweating may be brought about as a reflex act. Thus when the central stump of the divided sciatic is stimulated sweating is induced in the other limbs, and in ourselves the introduction of pungent substances into the mouth will frequently give rise to a copious perspiration over the side of the face. We are thus lead to speak of sweat centres, analogous to the vasomotor centres, as existing in the central nervous system; and as in the case of vasomotor centres, a dispute has arisen as to whether there is a dominant sweat in the medulla oblongata or whether such centres are more generally distributed over the whole of the spinal cord.

It does not at present appear certain whether the sweating caused by heat is carried out by direct action of the heated blood on the sweat centres, or by the higher temperature stimulating the skin and so sending up afferent impulses which produce the effect in a reflex manner; but in the case of dyspnoea at least we may fairly suppose that the action of the venous blood is chiefly if not exclusively on the nerve centres. Some drugs, such as pilocarpine, which cause sweating, appear to produce their effect chiefly by a local action on the glands, since the action continues after the division of the nerves (though pilocarpine apparently has as well some slight action on the nerve centres) and the antagonistic action of atropine is similarly local. Picrotoxine and strychnine appear to produce their sweating action chiefly if not exclusively by acting on the central nervous system, while nicotine seems to act both centrally and peripherally.

$\$ 370$. The sweat-fibres for the hind foot (in the cat) appear to leave the spinal cord by the roots of the twelfth thoracic to the third lumbar nerve inclusive, pass along the rami communicantes to the abdominal sympathetic, and thus reach the sciatic nerve. They thus follow very much the course of the vaso-constrictor fibres of the lower limb. The sweat-nerves for the fore-foot leave the spinal cord by the roots of the fourth to the ninth or tenth, chiefly in the sixth, seventh, and eighth dorsal nerve, inclusive, pass into the thoracic sympathetic, thence into the ganglion stellatum, and so join the brachial plexus by the fine branches passing from the ganglion to the spinal nerves. The course to the fore-foot is finally along the median and ulnar nerves respectively. In the horse the sweat-fibres for the side of the face and in the pig those for the snout appear to run in branches of the fifth nerve and not in the facial; in the latter animal at least some of these fibres reach the fifth nerve from the cervical sympathetic, but apparently not all.

$\$ 371$. The fact mentioned above that in the horse, after section of the cervical sympathetic nerve on one side of the neck, profuse sweating is apt to break out on that side of the face, has suggested the idea that this nerve conveys inhibitory impulses to the sweat-glands of the head and face, and that when it is divided the sweat fibres running in the fifth nerve, having nothing to counteract them, set up sweating. But it is probably sufficient in this case to suppose that the glands predisposed to activity by the higher temperature brought about by the section of the sympathetic dilating the bloodvessels, are more easily excited by any stimulus working upon them through the fifth nerve. And though the idea of a double nervous mechanism, augmenting and inhibitory, governing the activity of the sweat-glands, is a tempting one, there are at present no satisfactory reasons for adopting it. 


\section{CHA PTER I V.}

\section{THE METABOLIC PROCESSES OF THE BODY.}

$\S 372$. WE have followed the food through its changes in the alimentary canal, and have seen it enter into the blood, either directly or by the intermediate channel of the lacteals, in the form of peptone (or otherwise modified albumin), sugar, lactic acid, and fats, accompanied by various salts and water. We have further seen that the waste products which leave the body are urea, carbonic acid, salts and water. We have now to attempt to connect together the food and the waste products; to trace out as far as we are able the various steps by which the one is transformed into the other. There remains the further task to inquire into the manner in which the energy set free in this transformation is distributed and made use of.

The master tissues of the body are the muscular and nervous tissues; all the other tissues may be regarded as the servants of these. And we may fairly presume that, besides the digestive and excretory tissues which we have already studied, many parts of the body are engaged either in further elaborating the comparatively raw food which enters the blood, in order that it may be assimilated with the least possible labor by the master tissues, or in so modifying the waste products which arise from the activity of the master tissues that they may be removed from the body as speedily as possible. There can be no doubt that manifold intermediate changes of this kind do take place in the body ; but our knowledge of the matter is at present very imperfect. In a few instances only can we localize these metabolic actions and speak of distinct metabolic tissues. In the majority of cases we can only trace out or infer chemical changes, without being able to say more than that they do take place somewhere; and in consequence, perhaps somewhat loosely, speak of them as taking place in the blood.

How little we know concerning the metabolism of the master tissues themselves was shown when we were dealing with these tissues in an earlier part of this work; but success in the study of these can hardly be expected until our knowledge is increased as regards the changes which the blood undergoes before it reaches and after it leaves the muscle or the nerve. The fact that a large part of the absorbed food is carried through the liver before it is thrown on the general circulation leads us to suppose that in this large organ important metabolic processes are carried on; and observation with experiment confirms this view. Important as the secretion of bile may be, the other metabolic functions of the liver are of still greater importance.

\section{The History of Glycogen.}

$\S 373$. If the liver of a well-fed animal be removed immediately after death, rapidly divided into small pieces, thrown into boiling water, rubbed up and boiled, a decoction may be obtained which after careful neutralization and filtration will be tolerably free from proteid matter. Such a decoction is remarkably opalescent, milky in fact in appearance, much more so than a similar decoction from muscle or other tissue, and remains opalescent even after repeated filtration. Treated with iodine, the solution turns a brownish-red, port-wine red color, not unlike that given by dextrin 
when iodine is added; the color disappears on warming, but reappears on cooling provided that not too much proteid matter has been left in the solution. Treated with Fehling's fluid or other tests for sugar, the solution is found to contain a small and variable, but only a small, quantity of sugar.

If the solution be exposed, preferably in a warm room, to the action of saliva or of some other amylolytic ferment, or be boiled with dilute acid, the opalescence disappears; and the now clear transparent solution gives no longer the port-wine reaction with iodine. Tested, moreover, with Fehling's fluid or by other means it is now found to contain a considerable quantity of sugar.

If alcohol be added to the opalescent solution until the mixture contains 60 per cent. of the alcohol (previous concentration by evaporation being desirable) a white amorphous precipitate is thrown down. This precipitate, removed by filtration, boiled with an alcoholic solution of potash in which it is insoluble, but which dissolves and destroys any proteids which may be present, treated with ether to remove fatty impurities, and washed with alcohol, may be obtained in a pure condition. It then appears as a white amorphous powder, fairly soluble in water, but always giving rise to a milky opalescent solution unless an excess of alkali be present, in which case the opalescence may be slight or absent.

The opalescent solution of this purified material gives a port-wine reaction with iodine, but no reaction whatever with Fehling's fluid or the other sugar tests. Treated with an amylolytic ferment or boiled with dilute acid, the solution, like the raw decoction of liver, loses its opalescence and its port-wine reaction with iodine, but now gives an abundant evidence of the presence of sugar, dextrose if boiling with acid has been employed, maltose chiefly if an amylolytic ferment has been used. If quantitative determination be employed it will be found that the amount of sugar obtained is proportionate to the amount of the white powder acted upon; in other words the substance forming an opalescent solution is converted into sugar, the solution of which is clear. Obviously the substance is a body allied to the starch; and this is confirmed by the elementary composition, which is found to be $\mathrm{C}_{6} \mathrm{H}_{10} \mathrm{O}_{5}$ or some multiple of this.

Hence this body is called glycogen. And it is obvious from what has been stated above that the liver of a well-fed animal at the moment of death contains a considerable quantity of glycogen either in a free state or in such a condition that it is set free by subjecting the liver to the action of boiling water. We may add that it occurs in the liver in the hepatic cells, for the reaction of a port-wine color given under certain conditions by the hepatic cells is due to the presence of glycogen in them.

$\$ 374$. If the liver, instead of being treated immediately upon the death of the animal, is allowed to remain in the body of the dead animal for several hours, especially in a warm place, before a decoction is made of it, the decoction will be found to have little or no opalescence, to be quite clear, to give little or no port-wine reaction with iodine, but to contain a very considerable quantity of sugar. As we have said above, the decoction even of a liver taken immediately after death generally contains some little sugar, and the quantity of sugar in the liver appears, as a rule, to increase steadily after death, the amount of glycogen diminishing at the same time. The glycogen then present in the liver at the moment of death is gradually after death by some action or other converted into sugar.

The action is that of some agency whose activity is destroyed by the temperature of boiling water; hence the directions repeatedly given above to throw the liver into boiling water. This naturally suggests the presence in 
the liver of an amylolytic ferment. But not only have attempts to isolate from the liver an amylolytic ferment failed, in the hands of most observers at least, but the exact nature of the sugar which appears shows that the change is not effected by an ordinary amylolytic ferment. In the case of the amylolytic ferment of saliva, pancreatic juice, intestinal juice, and indeed of all other amylolytic animal fluids, the sugar into which starch or glycogeu is converted is maltose. Now, the sugar which appears in the liver after death is dextrose, identical, as far at least as can at present be made out, with ordinary dextrose. We are led, therefore, to infer that the change of glycogen into sugar which appears to go on after death is carried out by some action of the liver, probably of the hepatic cell itself, which is done away with by a temperature of $100^{\circ} \mathrm{C}$., but which is not the action of a ferment calpable of being isolated.

$\$ 375$. We have used above the phrase "well-fed" animal because the amount of glycogen present in the liver of an animal at any one time is very variable, and especially dependent on the amount and nature of the food previously taken. When all food is withheld from an animal the glycogen in the liver diminishes, rapidly at first, but more slowly afterward. Even after some days' starvation a small quantity is frequently still found; but in rabbits, at all events, the whole may eventually disappear.

If an animal, after having been starved until its liver may be assumed to be free, or almost free, from glycogen, be fed on a diet rich in carbohydrates or on one consisting exclusively of carbohydrates, the liver will in a short time be found to contain a very large quantity of glycogen. Obviously the presence of carbohydrates in food leads to an accumulation of glycogen in the liver; and this is true both of starch and of dextrin and of the various forms of sugar-cane, grape, and milk sugar. The effect may be quite a rapid one, for glycogen has been found in the liver in considerable quantity within a few hours after the introduction of sugar into the alimentary canal of a starving animal.

If an animal similarly starved be fed on an exclusively meat diet a certain amount of glycogen is found in the liver. This appears to be especially the case with dogs (probably with other carnivorous animals also), and in earlier works on the subject the constant presence of glycogen in the livers of dogs fed on meat was regarded as an important indication of the formation within the body of non-nitrogenous from nitrogenous material. But in the first place, the quantity of glycogen thus stored up in the liver as the result of a meat diet is much less than that which follows upon a carbohydrate diet; and in the second place, ordinary meat, especially horseflesh on which dogs in such experiments are usually fed, contains in itself $(\$ 62)$ a certain amount either of glycogen or some form of sugar. Moreover, when animals are fed not on meat, but on purified proteid, such as fibrin, casein, or albumin, the quantity of glycogen in the liver becomes still smaller, though, according to most observers, remaining greater than during starvation. We may infer, therefore, that part of the glycogen which appears in the liver after a meat diet is really due to carbohydrate materials present in the meat. Part, however, would appear to be the result of the actual proteid food; and we have similar evidence that gelatin taken as food leads to the formation of some glycogen in the liver. But in this respect these nitrogenous substances fall far short of carbohydrate material.

With regard to fats, all observers are agreed that these lead to no accumulation of glycogen in the liver; an animal fed on an exclusively fatty diet has no more glycogen in its liver than a starving animal.

Hence of the three great classes of fond-stuffs the carbohydrates stand out prominently as the substances which taken as food lead to an accumu- 
lation of glycogen in the liver. We may remark that the greatest accumulation of glycogen is effected not by a pure carbohydrate diet, but by a mixed diet rich in carbohydrates. A quantity of carbohydrate mixed with a certain proportion of proteid gives rise to a larger amount of glycogen in the liver than the same quantity of carbohydrate given by itself; and it is possible that the presence of an appropriate quantity of fat still further assists the accumulation. But this result probably depends, in part at least, on the fact that, though differences may be met with in different animals, a mixture of the several classes of food-stuffs is more readily digested, resulting in more nutritive material being thrown upon the blood, than is a meal consisting exclusively of one kind of food-stuff alone.

As far as we know at present the glycogen which thus appears in the liver as the result of feeding either with any of the various forms of carbohydrates or with proteids, or with other substances, is of the same kind and presents the same characters; at least we have no evidence to the contrary.

The storing-up of glycogen in the liver is also influenced by other circumstances than the taking of food. For instance, in the frog an increase of glycogen takes place during the winter months. In the summer months the liver of a frog will be found to contain very little glycogen (Fig. 114, C),

FIG. 113.

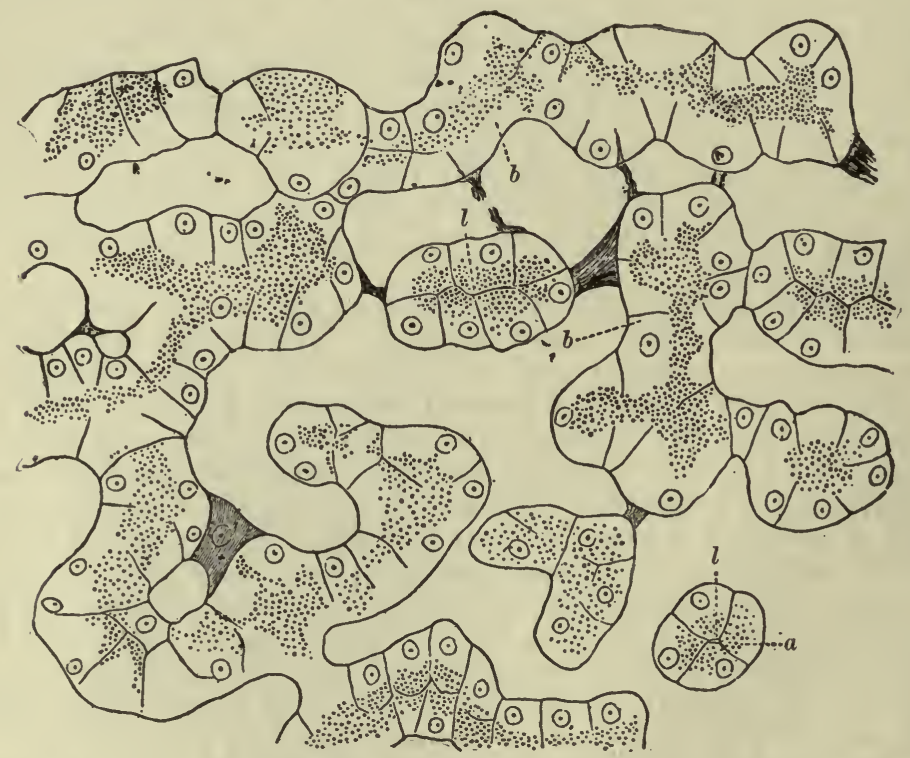

Section of Liver of Frog. (Langley.) The figure shows the tubular structure of the liver. At $a$, a tubule is seen in transverse, at $b$ in longitudinal section; $l$, lumen of tubule. The liver was that of a winter frog, and the cells show an inner zone of proteid granules; the outer zone was chiefly occupied by glycogen.

unless the animal has been unusually well fed; whereas a liver examined in midwinter (Figs. 113, 114, A) will be found to contain a considerable quantity, even though no food has been taken for months. In such a rase the material for the formation of the glycogen in the liver must have been furnished by some part of the body of the frog, and could not, as may be the case when a meal leads immediately to an increase of glycogen, be supplied 
directly from the food. It seems as if in the summer the frog lives up to its capital of hepatic glycogen, spending it as fast almost as it is made, but that during the winter a quantity is funded to provide for the demands of the late winter and early spring.

This winter storage of hepatic glycogen in the frog seems closely dependent on temperature. If a winter frog, whose liver is presumably more or less loaded with glycogen, be exposed for some time to a temperature of $20^{\circ}$ or a little higher, the liver will afterward be found to contain little or no glycogen (Fig. 114, B); and conversely, if a summer frog be exposed to untimely cold, glycogen, though not in any great quantity, begins to be stored up in the liver.

$\S 376$. Before we attempt to discuss further how food and other circum-

FIG. 114.
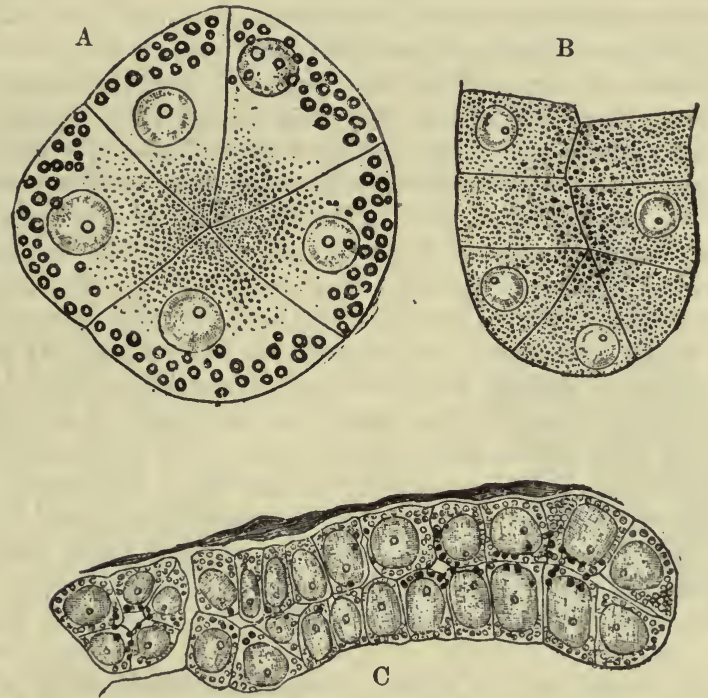

Three Phases of the Hepatic Cells of the Frog. (Langley.) A, cells rich in glycogen. Taken from a frog during winter. The cells are large and proteid granules are massed around the lumen, the homogeneous outer zones of the cells are largely composed of glycogen, which is present in considerable abundance. The outer zones contain numerous fat globules, shown as dark spots; but, as stated in the text, these fat globules vary much. $B$, cells poor in glycogen. Taken from a winter frog which had been kept at $22^{\circ} \mathrm{C}$. for ten days. The cells contain very little glycogen, and the proteid granules are dispersed throughout the cell. In a summer frog well fed on proteids the cells would present a very similar appearance. $C$, starved cells. Taken from a summer frog after a long fast. The cells are small and almost free from glycogen. The proteid granules are dispersed throughout the cell. All the specimens were hardened in 1 per cent. osmic acid, and are drawn to the same, or nearly to the same scale.

stances thus affect the glycogen in the liver, it will be desirable to take up the matter which we left on one side, viz., the consideration of the histological changes occurring in the hepatic cells under various conditions. It will be convenient to begin with the cells of the more distinctly tubular gland of the frog.

In a frog which has not been subjected to any special treatment the cell substance of the hepatic cell (cf. Fig. 114, A) will generally be found to contain lodged in itself three kinds of material, the presence of which, if not directly recognizable in the fresh cell, may be demonstrated by the use of various reagents. In the first place, oil globules of variable size 
and in variable amount are scattered throughout the cell; sometimes, as we have already said, these are extremely abundant; but there is otherwise nothing very special about these fat globules in the hepatic cell to demand any discussion concerning them apart from the general discussion on the formation of fat, into which we shall euter later on.

In the second place, a number of small discrete granules may be seen lodged in the cell substance. These appear to be of a proteid nature and are generally most abundant on the inner side of the cell near the lumen of the bile passage. The presence of these granules is closely dependent on the activity of the digestive processes. They diminish when digestion is going on and accumulate again afterward. Putting aside certain details, we may say that these granules behave very much like the granules in an albuminous salivary cell, a pancreatic cell, or a chief gastric cell ; and we may probably safely conclude that they, like the granules in these cells, are in some way concerned in the formation of the secretion; that is, in their case, bile.

In the third place, the cell contains, more especially in its outer parts nearer the bloodvessel, away from the lumen of the bile passage, a variable quantity of material which differs from the ordinary cell substance in being hyaline and refractive, and hence glassy-looking, and in staining port-wine red with iodine, instead of brownish-yellow, as does ordinary cell substance. This material is, though with some little difficulty, soluble in water, and by this means may be dissolved out from the cell. When this is done the places which it occupied appear as vacuoles or gaps of various sizes limited by bars of the cell substance, which thus take on the form of a network, the meshes of which are wider and more conspicuous in the outer part of the cell, in which the hyaline material was previously most abundant. In the inner part of the cell where the hyaline material was scanty the cell substance is more dense, and even in the outer part a shell of more dense, less reticulate cell substance affords a definite outline to the cell. There can be no doubt that this hyaline material is either actual glycogen, such as may be extracted from the liver, or, as seems more probable from its definite solubility, glycogen in some more or less loose combination with some other body, a combination, however, of such a kind that the iodine reaction makes itself felt.

$\$ 377$. The above may be taken as a general description of a cell in an ordinary condition. The question now comes before us, What changes are brought about by various foods or by the absence of food?

If a frog be largely fed on a diet containing large quantities of carbohydrates, the liver will be found rich in glycogen, and the cells will present the following characters: The cell is relatively large ( $c f$. Fig. 114, A), and, as it were, swollen; the cell substance is largely occupied by the hyaline material just spoken of, especially in its outer parts, so that in sections prepared and mounted in the ordinary way in which the glycogen has been dissolved out, the greater part of the cell consists of a loose open network of bars of stained cell substance with wide meshes; a certain quantity of more solid, generally granular-looking cell substance occupies the part of the cell nearest the lumen, and a thin shell of cell substance forms an envelope for the rest of the celi. The nucleus is large and distinct, but though changes in the nucleus accompanying changes in the cell substance have been described, they are not sufficiently important to detain us now. When such a cell is seen in a perfectly fresh state, the hyaline refractive material (which, we need hardly say, gives a marked reaction with iodine) often hides the nucleus and the greater part of the cell substance proper.

If, on the other hand, the frog be fed on a proteid diet free from carbohydrates-for instance, on fibrin - the liver contains little or no glycogen, 
and the hepatic cells are not only much smaller, but present an appearance very different from the above ( $c \dot{f}$. Fig. $114, \mathrm{~B})$. Little or no hyaline material is visible, the cells give little or no port-wine reaction with iodine, but only the usual brown-yellow proteid reaction, and in specimens prepared and mounted in the ordinary way the cell substance appears densely granular throughout.

Lastly, if the frog be starved, and if to the effects of starvation there be added those of exposure to a high temperature $\left(25^{\circ} \mathrm{C}\right.$.), by which, as we have seen, the hepatic cells are markedly affected, the liver is found to be free from glycogen and the hepatic cells to be extremely small (cf. Fig. 114, C), only half the size or even less of those of the well-fed frog, but otherwise much like the cells in a frog fed on proteid material.

$\S 378$. In the mammal changes in the hepatic cells similar to those just described as occurring in the frog have also been observed. When the animal is fed on a diet rich in carbohydrates, and when, therefore, as we have seen, the liver abounds in glycogen, the hepatic cells (Fig. 115) are larger (so large that they have by some authors been described as compressing the lobular capillaries) and loaded with the same refractive hyaline material staining port-wine red with iodine. When this material is dissolved out a coarse open network of cell substance is displayed. The

FIG. 115.

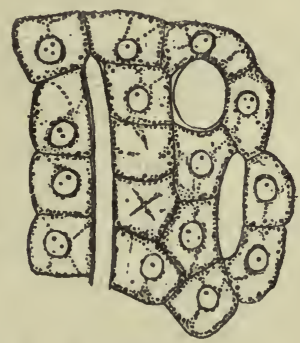

Section of Mammalian Liver rich in Glycogen. (Langley.) Osmic acid specimen, glycogen not dissolved out.

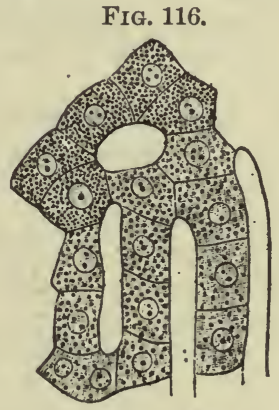

Section of Mammalian Liver containing little or no Glycogen. (Langley.) Osmic acid specimen. The granules are not well preserved in some of the cells.

most marked point of difference between the mammalian and frog's hepatic cell under these conditions is that in the former, the hyaline glycogenic substance is gathered at first centrally around the nucleus (not more on the outer side, as is the case in the frog) and spreads from the centre toward the periphery, always leaving on the extreme outside a somewhat thick shell of cell substance, which in hardened and prepared specimens may strikingly simulate a thickened cell-wall. We may add that in an animal thus fed the whole liver is very large, and, as it were, swollen ; it is also soft and tears easily.

In an animal fed on proteids alone, for instance on fibrin, the liver frequently contains some glycogen and the hepatic cells contain a small quantity of hyaline glycogenic material. As in the corresponding case in the frog, the cells are comparatively small, and the cell substance appears finely and uniformly granular.

In a starved mammal the liver is small, dense to the touch, and tough; it contains a trace only of glycogen or none at all; the cells (Fig. 116) are small, as it were shrunken, and the cell substance, which gives no port-wine reaction, or a mere trace only, with iodine, is still more finely granular.

$\S 379$. The microscopic appearances just described show, and indeed 
general considerations lead us to the same conclusion, that the processes taking place in an hepatic cell are very complex. In the first place, the constituents of bile are being formed and discharged into the bile passages after the fashion of ordinary secreting glands. In the second place, a formation of glycogen is also taking place, and we shall have presently to consider briefly the relations of the one process to the other. In the third place, as is especially indicated by the somewhat peculiar effects on the hepatic cell of food exclusively proteid in nature, other processes, similar perhaps to the formation of glycogen, but not resulting in the storage of any carbohydrate material, and dealing possibly with proteid substances, also take place. Hence the exact interpretation of all the changes which may be observed becomes exceedingly difficult.

Leaving the processes of the first and third kind wholly on one side for the present, and confining our attention entirely to the glycogen, it is obvious that the hepatic cell manufactures the glycogen in some way or other and lodges it in its own substance for the time very much in the same way that a secreting cell manufactures and lodges in itself for a time material for the secretion which it is about to pour forth. There is this difference, that in the one case the material of the secretion, after undergoing, as we have seen, more or less change, is cast out into the lumen of the alveolus, whereas in the other case the glycogen, which must undergo change, since it may be made to disappear rapidly from the hepatic cell, is not when changed cast out into the bile passages; it must therefore be sent back again to the blood.

$\S 380$. We say " manufacture the glycogen in some way or other," and we have now to inquire what we know concerning the nature and the several steps of this manufacture.

We have already seen that the presence of glycogen in the liver is especially favored by a carbohydrate diet; and in our studies on digestion we have seen reason to think that a very large part at all events of the carbohydrate material of a meal is absorbed as sugar by the capillaries of the intestiue and carried as sugar to the liver in the portal blood. Hence, it seems only reasomable to conclude that the glycogen which makes its appearance in the liver after an amylaceous meal arises from a direct conversion of the sugar carried to the liver by the portal vein, the sugar becoming through some action of the hepatic cell substance dehydrated into glycogen, or animal starch, as it has been called, the process being a reverse of that by which in the alimentary canal starch is hydrated into sugar through the action of the salivary and pancreatic ferments. Vegetable cells can undoubtedly convert both starch into sugar and sugar into starch; and there are no à priori arguments or positive facts which would lead us to suppose that the activity of animal living substance cannot accomplish the latter as well as the former of these changes. We are quite ignorant, it is true, of the exact way in which either the hydration or the dehydration is effected by living substances; but we are equally ignorant of the exact way in which an amylolytic ferment effects the hydration of starch into sugar, which it carries out with so much apparent ease. It is not a great assumption to suppose that the continually changing living substance, which in its changes is continually giving out energy, has the power of acting on molecules of starch or of sugar in contact with or even only near to itself, and so of hydrating starch into sugar or of dehydrating sugar into starch. The latter process may be a more difficult one than the former, but not one beyond the power of the living substance. We may fairly suppose that a quantity of sugar in solution present in a vacuole, for instance, of the hepatic cell substance can be, by some action of the cell substance, converted into 
glycogen in a solid form, filling up the vacuole. Again, as we have incidentally mentioned, sugar injected into the jugular vein readily gives rise to sugar in the urine; but a very considerable quantity can be slowly injected into the portal vein without any appearing in the urine. This suggests the idea that the liver, so to speak, catches the sugar as it is passing through the hepatic capillaries and at once dehydrates it into glycogen.

Similar consideration may also be applied to the case mentioned above of the appearance of glycogen in the hepatic cells of winter (fasting) frogs. We have reason to think that sugar makes its appearance as a product of the metabolism of various tissues. The sugar thus arising finding its way into blood may be made use of at once elsewhere, converted speedily, for instance, into carbonic acid and so got rid of. But we can readily imagine that under certain circumstances, as for instance when the activities of the animal were lessened by a low temperature, it was not so made use of and remained in the blood. If so, it would in the course of the circulation be carried to the liver, and might be at once taken up by the hepatic cells and converted into glycogen; and these might be so active that the blood was never at any time allowed to remain loaded with sugar to such an extent as to permit a loss through the urine.

$\$ 381$. Upon such a view, the carbohydrate taken as food would be converted into glycogen by the agency of the hepatic cell, without at any time becoming an integral part of the living substance of the cell. Such a view may be the true one; but it is open for us to look at the matter in another light. We may push still further the allalogy between the glycogen of the hepatic cell and the material with which a secreting cell is loaded. In dealing with secretions we saw reasons for regarding such a body as mucin to be a product of the metabolism of the cell substance of the mucous cell; and we may similarly regard glycogen, or sugar readily convertible into glycogen, or at least some or other carbohydrate material, as a normal product of the metabolism of the hepatic cell. We may thus conceive of the hepatic cells as being continually engaged in giving rise to carbohydrate material in the form either of sugar or of some other body; and we may suppose that under certain circumstances, as in the absence of adequate food, the carbohydrate material thus formed is at once discharged into the blood of the hepatic vein for the general use of the body, but that under other circumstances, as when an amylaceous meal has been taken, the immediate wants of the economy being covered by the carbohydrates of the meal, the carbohydrate products of the hepatic metabolism are stored up as glycogen. Under such a view the sugar of the meal is used up somewhere in the body, and the glycogen to the storage of which in the liver it gives rise comes direct from the hepatic substance. And a similar explanation may be given of the storing-up of the glycogen in the liver under such circumstances as those of the winter frog previously mentioned.

We do not possess at present experimental or other evidence of so clear a kind as to enable us to decide dogmatically between these two views. We have seen that proteid food, though in this respect falling far below carbohydrated food, does or may give rise to a certain amount of glycogen in the liver; and gelatin seems to have the same effect. Further, in certain cases of the disease diabetes, of which we shall have to speak presently, and which is characterized by the presence of a large amount of sugar in the blood, sugar continues to be formed in large quantity, even when the diet is restricted to proteid and fatty matters, all carbohydrates being excluded. Now in diabetes we have reason to believe that the large quantity of sugar in the blood is accompanied by a large deposition of glycogen in the liver, and indeed in other tissues; for in the few cases which have been examined 
sufficiently soon after death, and in which owing to the suddenness of death there was no opportunity for stored-up glycogen to disappear, a very large quantity of glycogen has been found in the liver or some other organs. Hence the phenomena of diabetes may be taken as showing, in a much more striking manner than do any experiments, that proteid material taken as food may give rise to hepatic glycogen.

$\S 382$. We may now turn to another question, the answer of which is in a measure dependent on the one which we have just discussed. What is the use and purpose of this hepatic glycogen? What ultimately becomes of the glycogen thus for a while stored up in the liver?

One view which has been put forward is as follows: We have evidence, as we shall presently learn, that a great deal of the fat of the body is not taken as such in the food, but is constructed anew in the body out of other substances. Both carbohydrates and proteids, taken in excess or under certain circumstances, lead to an accumulation of fat; and we have reason to believe that carbohydrates, on the one hand, and the carbon-holding portions of various proteids, on the other, may by some process or other be converted into fat. And it has been suggested that the glycogen in the liver is a phase of a constructive fatty metabolism, that it is material on its way to become fat.

Another view, one which has already been suggested while we were dealing with the manner of formation of glycogen, makes use of the formation of fat for the purposes of analogy only. Seeing that adipose tissue serves as a storehouse of fat which is not wanted by the body at the moment, but may be wanted presently, the question readily presents itself, May not the hepatic glycogen have an analogous function? May we not regard the presence of glycogen in the liver as in large measure due to the fact that it is deposited there simply as a store of carbohydrate material, being accunulated whenever amylaceous material is abundant in the alimentary canal, and being converted into sugar and so drawn upon by the body at large to meet the general demands for carbohydrate material during the intervals when food is not being taken? And we can accept this view without being able to say definitely what becomes of the sugar thus thrown into the hepatic blood. It was formally believed that this sugar underwent an immediate and direct oxidation as it was circulating in the blood, but we have already dwelt ( $\$ 360)$ on the objections to such a view. It is sufficient for us at the present to admit that the sugar is made use of in some way or other.

Now, many considerations lead us to believe that a certain average composition is necessary for that great internal medium the blood, in order that the several tissues may thrive upon it to the best advantage, one element of that composition being a certain percentage of sugar. It would appear that some at least if not all of the tissues are continually drawing upon the blood for sugar, and that hence a certain supply must be kept up to meet this demand. On the other hand an excess of sugar in the blood itself would be injurious to the tissues. And as a matter of fact we find that the quantity of sugar in the blood is small but constant; it remains about the same when food is being taken as in the intervals between meals. If sugar be injected into the jugular vein in too large quantities or to rapidly, a certain quantity appears in the urine, indicating an effort of the system to throw off the excess and so bring back the blood to its average condition. The maintenance of such a constant percentage of sugar would obviously be provided for, or at least largely assisted by the liver acting as a structure where the sugar might at once and without much labor be packed away in the form of the less soluble glycogen, at those times when, as during an amylaceous meal, sugar is rapidly passing into the blood, and there is danger of 
the blood becoming loaded with far more sugar than is needed for the time being; and it may be incidently noted that a larger quantity of sugar may be injected into the portal than into the jugular vein without any reappearing in the urine, apparently because a large portion of it is in such a case retained in the liver as glycogen. At those times, on the other hand, when we may suppose that sugar ceases to pass into the blood from the alimentary canal, the average percentage in the blood is maintained by the glycogen previously stored up becoming reconverted into sugar, and being slowly discharged into the hepatic blood.

Moreover, this view, that the glycogen of the liver is a reserve fund of carbohydrate material, is strongly supported by the analogy of the migration of starch in the vegetable kingdom. We know that the starch of the leaves of a plant, whether itself having previously passed through a glucose stage or not, is normally converted into sugar, and carried down to the roots or other parts, where it frequently becomes once more changed back again into starch.

$\$ 383$. Glycogen is found in other parts of the body than the liver, and a study of the facts relating to the presence of glycogen in other tissues will help us to a true conception of the purposes of the hepatic glycogen. Next to the liver, the skeletal muscles are perhaps the most conspicuous glycogenholders. So frequently is glycogen found in muscle that it may be regarded as an ordinary though not an invariable constituent of that tissue; indeed it may almost be considered as a constituent of all contractile tissues. The quantity varies very largely both in the different muscles of the same animal and corresponding muscles of different animals. It disappears, according to some observers, readily upon starvation, even before the hepatic glycogen is exhausted; but all observers are not agreed on this point, and in some muscles, at least, it appears to be retained for a very long time. It is said to be increased in quantity when the nerve of the muscle is divided, and the muscle thus brought into a state of quiescence. On the other hand it diminishes or even disappears, being apparently converted into dextrose, when the muscle enters into rigor mortis. Some observers have found that it diminishes during tetanus, and maintain that it, after conversion into dextrose, is used up in the act of contraction, forming through its oxidation the immediate supply of the energy set free in the contraction. But even granting that the glycogen in a muscle may be diminished during prolonged labor, it cannot be admitted that the oxidation or other chemical change of glycogen is a necessary part of the ordinary metabolism of a muscular contraction, since many muscles wholly free from glycogen are perfectly well able to carry on long-continued contractions.

Another view of the use of glycogen in muscle is suggested by the fact that undeveloped embryonic muscles are peculiarly rich in glycogen. In a young embryo, at the time when the muscular substance, though undergoing striation, is still largely "protoplasmic" in nature, the quantity of glycogen present is enormous; it frequently amounts to 40 per cent. of the dry material. At this period the hepatic cells are immature and very little glycogen is present in them. Later on, as the muscles become more wholly striated, the glycogen largely disappears from the muscle, and very soon afterward begins to be stored up in the liver.

The meaning of this can hardly be mistaken. The glycogen in the immature muscle is a store of carbohydrate material, laid down on the spot, and ready at once to be used in what we may probably call the fierce metabolic struggle by which the simple protoplasmic cell substance of the rudiment of the muscular fibre is transformed into the highly differentiated striated contractile substance. And we shall probably not err in considering the 
glycogen of the mature muscle to hold a similar position ; it is carbohydrate material stored up on the spot, a local branch, so to speak, of the great carbohydrate bank. It is destined to become part of the contractile substance, and as such will contribute to the energy set free in a muscular contraction; but its energy is only available in this way after it has undergone the necessary metabolism and become part of muscular substance; it cannot be fired off in a contraction while it lies as raw glycogen, or even as dextrose, in the interstices of the muscular fibre.

$\$ 384$. Glycogen may also be found in considerable quantity in the placenta. Here, as we shall see in the latter part of this work, it is laid down in epithelial cells which lie on the boundary between the maternal and the foetal tissues. And here, too, there can be little doubt that it is a store of carbohydrate material for the nourishment of the foetus.

It has also been found in leucocytes, in cartilage corpuscles, especially in those large rapidly growing and rapidly multiplying cartilage corpuscles which lie in the outer zone of endochondral ossification, and in other situations. In cases of diabetes, where the body is overloaded with carbohydrate material, it has been found in considerable quantity in the testis, in the brain, and elsewhere. Its occurrence in these situations, and under these circumstances, may be regarded as additional evidence of the truth of the view which we have expounded above, that the main purpose of the deposition of glycogen is to afford a store, either general or local, of carbohydrate material, which can be packed away without much trouble so long as it remains glycogen, but which can be drawn upon as a source of soluble circulating sugar whenever the needs of this or that tissue demand it. It thus forms a very complete analogue to the vegetable starch, and fitly earns the name of animal starch.

We have some reasons for thinking that there are several varieties of glycogen, and that the glycogen which exists in muscle is not quite identical with that which occurs in the liver. Indeed, there seem to be intermediate stages between glycogen and starch, or dextrin. The physiological value of these differences has not yet, however, been elearly determined, and, with this caution, we may continue to speak of glycogen as a single substance.

\section{Diabetes.}

$\S 385$. Natural diabetes is a disease characterized by the appearance of a large quantity of sugar in the urine, due, as we have already said, to the presence of an abnormal quantity of sugar in the blood. Into the pathology of the various forms of this disease it is impossible to enter here; but a temporary diabetes, the appearance for a while of a large quantity of sugar in the urine, may be artificially produced in animals in several ways.

If the medulla oblongata of a well-fed rabbit be punctured in the region which we have previously described $(\$ 162)$ as that of the vasomotor centre (the area marked out as the "diabetic area," agreeing very closely with that defined as the vasomotor area), though the animal need not necessarily be in any other way obviously affected hy the operation, its urine will be found, in an hour or two, or even less, to be increased in amount and to contain a considerable quantity of sugar. A little later the quantity of sugar will have reached a maximum, after which it declines, and in a day or two, or even less, the urine will again be perfectly normal. The better fed the animal, or, more exactly, the richer in glycogen the liver, at the time of the operation, the greater the amount of sugar. If the animal be pre- 
viously starved so that the liver contains little or $n 0$ glycogen, the urine will, after the operation, contain little or no sugar. It is clear that the urinary sugar of this form of artificial diabetes comes from the glycogen of the liver. The puncture of the medulla causes such a change in the liver that the previously stored-up glycogen disappears, and the blood becomes loaded with sugar, much, if not all, of which passes away by the urine. In the absence of any proof to the contrary, we may assume that in this form of artificial diabetes, the glycogen previously present in the liver becomes converted into sugar, just as we know that it does become so converted by post-mortem changes. The glycogenic function of the liver is, therefore, subject to the influences of the nervous system, and, in particular, to the influence of a region of the cerebro-spinal centre, which we already know as the vasomotor centre, or at least of a part of that region.

Before we attempt to discuss this nervous influence we must say a few words on the nerves of the liver.

$\S 386$. The liver is supplied with nerves from the hepatic plexus, which passes into the liver at the porta and, running in the portal canal with the hepatic artery and portal vein, is distributed to various parts of the organ. This plexus, which is the only nerve supplying the liver, consists partly of medullated and partly of non-medullated fibres, and is an extension of the great solar plexus, already often mentioned. Into that plexus, as we have already seen, the right (posterior) vagus sends the greater part of its fibres, and in that plexus both the abdominal splanchnic nerves, major and minor, end, on both sides of the body. The left (anterior) vagus forms slight connections only with the solar plexus, but sends off a very distinct branch directly to the hepatic plexus. The liver, therefore, has nervous connection with the central nervous system by both vagus nerves and by the (abdominal) splanchnic nerves. Besides this, other nerve-fibres find their way through the splanchnic sympathetic chain, or possibly otherwise, to the solar plexus from the spinal cord without taking part in either of the splanchnic nerves; and these may, perhaps, join the hepatic plexus.

Concerning the destination of the fibres of the hepatic plexus within the liver we know little or nothing definitely. Some, undoubtedly, supply the hepatic artery and its branches; but we cannot at present say what proportion of the whole number of fibres end in this way. Some, again, are destined for the bile-ducts, and before the plexus passes into the liver it sends fibres to the gall-bladder; these probably end in the muscular coats of these organs. Whether any of the nerve-fibres end in the remarkably muscular coats of the portal vein, or whether, as theoretical reasons would, perhaps, lead us to suppose, some are connected with the hepatic cells, we do not for certain know, though some observers have claimed to have traced nerve-fibres directly into the hepatic cells.

$\S 387$. With regard to the exact nature of the influence started by the puncture of the medulla, and the path by which that influence reaches the liver, our information is at present very imperfect. One thing seems clear, viz., that the influence in question is not carried down by the main vagus trunks; for not only has the section of both these nerves in the neck no marked effect in the way of producing diabetes, but the "diabetic puncture" of the medulla oblongata is as efficient after division of both vagus nerves as before. Seeing how close to, or almost indentical with, the vasomotor centre is the diabetic centre, if we may use the phrase, it seems natural to suppose that the undue conversion of glycogen into sugar which follows the puncture is the result of some vasomotor disturbance in the liver, for instance, dilatation of the hepatic artery. But we have no clear proof that this is the true explanation. 
$\S 388$. A temporary diabetes may be brought about by the administration of the substance phloridzin. This, however, is a glucoside, and part of the sugar which appears in the urine, after a dose of it, may come direct from the drug itself; but the quantity of sugar discharged is too great to be accounted for in this way, and similar diabetic effects are produced by the administration of phloretin, a derivate of phloridzin, not a glucoside, and not giving rise to sugar by its own decomposition. The sugar which appears in the urine after a dose of this substance seems to conie in part at least from the hepatic store of glycogen when that is present; but the drug will give rise to sugar in the urine of starving animals, from whose livers (and other tissues) glycogen is presumably absent.

Artificial diabetes is also a prominent symptom of urari poisoning. This is not due to the artificial respiration, which is had recourse to in order to keep the urarized animals alive ; because, though disturbance of the respiratory functions sufficient to interfere with the hepatic circulation may produce sugar in the urine, artificial respiration may with care be carried on without any sugar making its appearance. Moreover, urari causes diabetes in frogs, although in these aninals respiration can be satisfactorily carried on without any pulmonary respiratory movements. The exact way in which this form of diabetes is brought about has not yet been clearly made out.

A very similar diabetes is seen in carbonic-oxide poisoning; and is one of the results of a sufficient dose of morphia, of amyl nitrite and of some other drugs.

There can be no doubt that in diabetes, arising from whatever cause, the sugar appears in the urine because the blood contains more sugar than usual. The system can only dispose (either by oxidation, or as seems more probable in other ways) of a certain quantity of sugar in a certain time. Sugar injected into the jugular vein reappears in the urine whenever the injection becomes so rapid that the percentage of sugar in the blood reaches a certain (low) limit. Sugar in the urine means an excess of sugar in the blood. How in natural diabetes that excess arises has not at present been clearly made out. It may be that some forms of diabetes resemble the artificial diabetes just described as resulting from puncture of the medulla, and arise from a too rapicl conversion of the hepatic glycogen, or from carbohydrate material failing to be stored up as glycogen, or from an excessive manufacture of carbohydrate material by the hepatic cells. All forms of diabetes, however, cannot be satisfactorily explained in this way; and it has been suggested, though adequate proof has not yet been supplied, that the sugar of diabetes is of a peculiar nature and accumulates in the blood because it is unable to undergo those changes, whatever they be, which befall the normal sugar of the blood. We cannot here discuss the subject in detail; but there is much to be said in favor of the view that the sources of the excess of sugar in the blood may be various, and hence that several distinct varieties of diabetes may exist. In severe cases of diabetes the aberrant nature of the metabolism which is going on in some or other of the tissues of the body is shown by the appearance of abnormal substances in the urine. Thus acetone is frequently present, and the fatal issue of certain cases has been attributed to poisoning by that substance; oxybutyric acid and other various organic, chiefly volatile, acids are also sometimes present. But in respect to these and other abnormal bodies we are not at present clear whether they are, like the sugar itself, the products of an abnormal metabolism which is the root of the disease, or whether they are secondary products, that is to say, products of the general disordered metabolism induced by the constant presence in the blood of an excess of 
sugar. We have already, in discussing the formation of glycogen, called attention to the fact that in severe cases of diabetes the sugar must have a non-amylaceous source; and the fact that the urea is increased (and that too in some cases in ratio with the sugar) in diabetes, suggests that the sugar may arise from proteids which have been split up into a nitrogenous (urea) and a non-nitrogenous moiety, and so points out the way in which proteids may be a source of glycogen.

As a sort of converse to diabetes we may mention that the administration of arsenic in sufficient doses or for an adequate time prevents an accumulation of glycogen in the liver and apparently in the body generally, whatever be the diet used. The presence of the netal in the hepatic cell seems to prevent the cell substance from manufacturing glycogen either fiom carbohydrate material brought to it, or out of its own substance. As another kind of converse we may also state that the administration of grlycerin, especially through the alimentary canal, diminishes the effect of the diabetic puncture, or of morphia or of other poisoning, in hurrying on the hepatic store of glycogen into sugar, and thus diminishes the sugar in the urine; the presence of the glycerin in the hepatic cell appears to be in some way a hindrance to the conversion of the glycogen into sugar. Now glycerin injected into the alimentary canal of a normal animal leads to an increase of glycogen in the liver; and the view very naturally suggests itself that this increase arising from the glycerin is to be explained by the glycerin inhibiting in some way a normal conversion of the glycogen store into sugar which is continually going on, and thus increasing for the time that store.

\section{SPLEEN,}

$\S 389$. The movements of the spleen. After a meal the spleen increases in size, reaching its maximum about five hours after the taking of food; it remains swollen for some time, and then returns to its normal bulk. In certain diseases, such as the pyrexia attendant on certain fevers or inflammations, and more especially in ague, a somewhat similar temporary enlargement takes place. In prolonged ague a permanent hypertrophy of the spleen, the so-called ague-cake, occurs.

The turgescence of the spleen seems to be due to a relaxation both of the small arteries and of the muscular tissue of the capsule and of the trabeculæ; to be, in fact, a vascular dilatation accompanied by a local inhibition of the tonic contraction of the other plain muscular fibres entering into the structure of the organ, the latter, at all events in some animals, being probably the more important of the two. And the condition of the spleen, like that of other vascular areas, appears to be regulated by the central nervous system, the digestive turgescence being fairly comparable to the flushed condition of the pancreas and of the gastric membrane during their phases of activity.

The application of the plethysmographic method to the spleen, carried out in the way which we described in speaking of the kidney ( $\$ 346$ ), enables us to study more exactly the variations in volume which the organ undergoes.

A "spleen curve" (Fig. 117) taken in the same way as a "kidney curve" does not, in the dog at all events, show variations in the volume of the spleen corresponding with the pulse waves. The kidney curve, as we have seen, ( $\$ 346)$, gives clear indications of each heart-beat, but the spleen curve shows, besides the larger waves of which we shall speak directly, only undulations due to the respiratory n:ovements; and these, always very slight, are 
sometimes not visible. In other words, the spleen does not expand with the increase of blood-pressure occurring in the splenic arteries after each heartbeat; this may be due to the muscular coat resisting expansion. Moreover, when the supply of blood to the spleen is wholly and suddenly cut off; as by clamping the aorta, the spleen curve sinks very slowly, showing that the spleen is diminishing in volume not suddenly but very slowly. The pathway of the blood through the splenic reticulum is peculiar; and increase or decrease in the volume of the spleen means more or less blood held in the spleen-pulp, not necessarily a greater or less flow of blood through the organ.

Of special interest are the large slow variations of volume which, besides the respiratory undulations, the spleen curve usually shows, as seen in the figure. Rhythmic contractions and expansions, though not always present, frequently make their appearance, each contraction with its fellow expansion lasting in the cat and dog about a minute, and recurring with great regu-

FIG. 117.

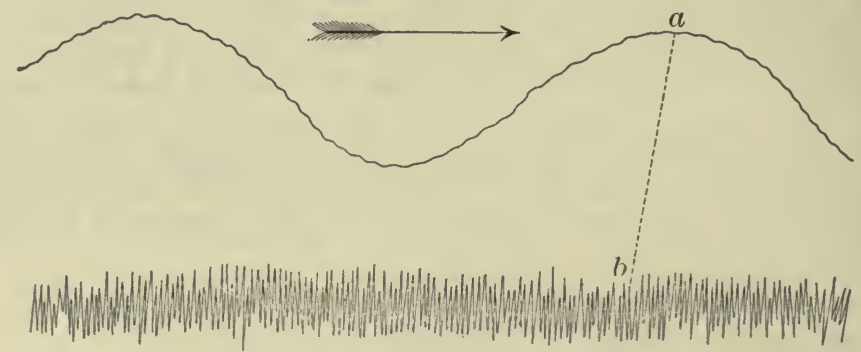

Normal Spleen Curve from Dog. (Roy.) The upper curve is the spleen curve showing the rhythmic contractions and expansions; the smaller waves are due to the respiratory movements. The lower curve is the blood-pressure curve, and the point $a$ of the spleen curve corresponds in time to the point $b$ of the blood-pressure curve. The marks on the time-curve below indicate seconds.

larity for a long time; and besides these the volume varies widely from time to time. There can be little doubt but that the rhythmic variations in volume are due in these animals to rhythmic contractions, with intervening relaxations, of the muscular trabeculæ and capsule; the slower variations are also probably due to the same cause. In many animals the contractility of the splenic tissue is shown by the white lines of constriction which appear when the electrodes of an induction machine in action are drawn over its surface; and similiar lines may be produced by mechanical stimulation with the point of a needle. So that the spleen in these animals may be considered as a muscular organ, now expanding to receive a larger quantity of blood and now contracting to drive the blood on to the liver. When the muscular elements are scanty in or absent from the capsule and trabeculæ, the expansion and contraction of the whole organ must depend alone or chiefly on variations in the width of the supplying arteries. We have evidence, moreover, that the muscular activity of the spleen, whether of the muscular capsule and trabeculæ and arteries combined, or of the latter alone, is under the dominion of the nervous system. A rapid contraction of the spleen may be brought about in a direct inanner by stimulation of the splanchnic or vagus nerves, or in a reflex manner by stimulation of the central end of a sensory nerve; it may also be caused by stimulation of the medulla oblongata with 
a galvanic current or by means of asphyxia. Though the matter has not yet been fully worked out, we have already sufficiently clear indications that the flow of blood through the spleen is, through the agency of the nervous system, varied to meet changing needs. At one time a small quantity of blood is passing through or is being held by the organ, and the metabolic changes which it undergoes in the transit are comparatively slight. At another time a larger quantity of blood enters the organ, and is let loose, so to speak, into the splenic pulp, there to undergo more profound changes, and afterward to be ejected by the rhythmic contractions of the muscular trabeculæ.

It is further obvious that these changes going on in the spleen must have an important influence on the changes going on in the liver; it cannot be of indifference to the latter organ, whether a relatively small quantity of blood, relatively little changed, reaches it from the spleen, or whether it receives a relatively large quantity of blood, profoundly altered by the changes which it has undergone in the spleen-pulp.

$\$ 390$. The chemical constituents of the spleen. Besides the chemical bodies which one would expect to find in a vascular, muscular organ full of blood, the spleen contains bodies, lodged apparently in the spleen-pulp, which give it special chemical characters. One of the most important of these is a special proteid of the nature of alkali-albumin, holding iron in some way peculiarly associated with it. The occurrence of this ferruginous proteid, accompanied as it is by several peculiar but at present little-understood pigments, rich in carbon, which are partly present in the cells spoken of above and partly deposited in the branched cells of the reticulum, appears to be connected with the changes undergone by the hæmoglobin which we shall presently discuss. The inorganic salts of the spleen, or at least those of its ash, are remarkable for the large amount of both soda and phosphates and the small amount of potash and chlorides which they contain, thus differing from those of blood corpuscles on the one hand, and from those of bloodserum on the other. But perhaps the most striking feature of the spleenpulp is its richness in the so-called extractives. Of these the most common and plentiful are succinic, formic, acetic, butyric, and lactic acids, inosit, leucin, xanthin, hypoxanthin, and uric acid. Tyrosin apparently is not present in the perfectly fresh spleen, though leucin is ; both are found when decomposition has set in. The constant presence of uric acid is remarkable, especially since it has been found even in the spleen of animals, such as the herbivora, whose urine contains none.

The richness of the spleen in these extractives is an indication of the importance of the metabolic events with which the organ has to do; but it will be more profitable to discuss what goes on in the spleen in connection with the metabolic changes in the other parts of the body, in the liver for instance, than to attempt to lay down any so-called "functions" of the spleen. When we confine our attention to the spleen itself we learn very little; thus the whole organ may be successfully removed without any very obvious changes in the economy resulting. We may return, therefore, to the discussion of the formation of the bilirubin of bile, and of the changes undergone by hæmoglobin, with which, as we shall see, the spleen is connected, and which, moreover, has to do with the formation of other pigments.

\section{The Formation of the Constituents of Bile.}

§391. Bile pigments. After extirpation of the liver no accumulation of bile pigment or bile salts takes place in the blood. This is well shown in 
frogs, which survive the operation for some considerable time; but the same results have been obtained in birds (geese and ducks). There can be no doubt, therefore, that these substances are formed in the liver, and not simply withdrawn from the blood by the liver in some such way as we have seen reason to think urea is withdrawn from the blood by the kidney.

When the plasma of circulating blood is made to contain hæmoglobin detached from the corpuscles, bile pigment frequently makes its appearance in the urine. The presence of free hæmoglobin may be obtained by injecting into the veins a solution of hæmoglobin or blood made "laky" by freezing and thawing or by the addition of a small quantity of bile salts, or by simply injecting into the veins a quantity of distilled water or a small quantity of ether or chloroform or of bile salts, all of which tend to "break up" red corpuscles and set free hæmoglobin. A similar result occurs in poisoning by certain drugs, such as toluylendiamine. Under these circumstances not only does bile pigment, bilirubin, make its appearance in the urine, but the quantity of bilirubin secreted by the liver is increased. Obviously the presence of dissolved hæmoglobin in the plasma of the blood, and, presumably more especially of the blood reaching the liver by the portal vein, leads to an increased formation of bilirubin, which take place in such a manner that the whole of the bilirubin so formed does not pass into the bile but part is retained in or thrown back into the circulation and appears in the urine.

We have already mentioned the chemical connection between hæmoglobin and bilirubin. Hæmoglobin after the detachment of its proteids component becomes hæmatin $\left(\mathrm{C}_{32} \mathrm{H}_{32} \mathrm{~N}_{4} \mathrm{FeO}_{4}\right)$. By treatment with sulphuric acid or otherwise ( $\$ 294$ ), hrematin may be deprived of its iron; and this iron-free hæmatin (sometimes called hæmatoporphyrin) is said to have the composition $\mathrm{C}_{32} \mathrm{H}_{32} \mathrm{~N}_{4} \mathrm{O}_{5}$, differing from bilirubin only in its oxygen and hydrogen $\left(\mathrm{C}_{32} \mathrm{H}_{32} \mathrm{~N}_{4} \mathrm{O}_{5}+2 \mathrm{H}_{2} \mathrm{O}-\mathrm{O}=\mathrm{C}_{32} \mathrm{H}_{36} \mathrm{~N}_{4} \mathrm{O}_{6}\right){ }^{1} \quad$ Moreover, in old blood-clots in the body the hrmoglobin of the clot becomes in time transformed into an iron-free body which has been called hrematoidin, but which both in composition and reactions appears to be identical with bilirubin.

These several facts lead us to the conclusion that the bilirubin of the bile is simply some of the hrmoglobin of the blood transformed by the throwing off of its proteid and its iron components. It is natural to suppose that the transformation takes place in, and is effected by, the agency of the hepatic cells; and this view is supported by the fact that the hepatic cells are characterized by containing certain peculiar iron compounds. When all the blood is carefully washed out of the liver by injection through the bloodvessels, by which means the remaining bile is got rid of at the same time, the hepatic substance is found to contain a small quantity of iron, sufficient to give the cells a diffused dark color when treated with ammonium sulphide; the exact amount appears to vary largely, but the causes of the variation have not been determined. That this iron is in organic combination is indicated by the fact that with potassium ferrocyanide and sulphocyanide the blue or red action is not observed until after treatment with hydrochloric acid. Apparently there are several such compounds, of a proteid or of a nuclein ( $\$ 29)$ nature, from some of which the iron is more easily removed than others, and these compounds appear to be present in both the cell substance and the nucleus. It will be remembered ( $\$ 213$ ) that bile contains a distinct quantity of iron, which probably has its origin in the iron thus set free from hrmoglobin and retained in the hepatic cell; but it does not follow that all the iron thus set free makes its way into the bile; and, indeed, the quantity of iron discharged in the bile in twenty-four hours

1 Doubling the formula for bilirubin given in ?, 214 . 
is much smaller than the quantity calculated to be set free in the formation out of the hrmoglobin of the quantity of bilirubin discharged during the same period. Apparently the iron compounds of the hepatic cell have some other work than the simple discharge of iron into the bile.

The fiact mentioned above, that the presence of free hæmoglobin in the blood leads not only to an increase of bilirubin in the bile, but also to its presence in the urine, offers some difficulties ; for if the bilirubin be formed out of hrmoglobin by and in the hepatic cell, one would expect to find that the whole of it passed into the bile, and that it could not appear in the blood and so in the urine unless reabsorption from the bile passages, due to obstruction took place; and there is no evidence of any sufficient obstruction occurring in these cases. Indeed the presence of bilirubin in the urine in these cases has been urged by some as an argument that bilirubin is formed in the blood or at least elsewhere than in the liver, and is simply excreted by the liver. Not only, however, as stated above, is there no accumulation of bile in the blood after extirpation of the liver, but that operation prevents the appearance of bilirubin in the urine as a consequence of the presence of free hæmoglobin in the blood. The phenomena in question, therefore, do not disprove that the bilirubin is formed in the liver; they may be taken, however, to show that that formation, viewed as a secretory act, is peculiar, since the hepatic cell appears under certain circumstances to discharge its product of secretion into the blood or lymph as well as into the bile passages.

$\$ 392$. We may assume then that the hepatic cell has the power of splitting up the hæmoglobin brought to it, and of discharging part as bilirubin while it retains for a time the iron component in some organic combination; and, if we further assume that it works upon the entire hæmoglobin we may presume that makes some subsequent use of the proteid component. But are we justified in assuming that the whole work is done by the hepatic cells? Are we to conclude that bilirubin is manufactured by some act of the hepatic cells which includes not only the conversion of hæmoglobin into bilirubin, but also the extraction of the hæmoglobin from the red corpuscles as these are streaming slowly through the lobular hepatic capillaries in close contact with the hepatic cells? Now, as far as we know at present, hæmoglobin can only be set free by means of a disintegration of the corpuscles; we have no instances of a corpuscle parting with some of its hæmoglobin and proceeding on its way otherwise unchanged; and we have no histological evidence of any disintegration of red corpuscles in the liver corresponding to the formation of bile. Nor can we draw any conclusion from the result of a comparative enumeration of red corpuscles in the portal and hepatic blood, for these are too insecure to rest any conclusion upon. On the other hand, as we have just seen, the presence in the plasma of the blood of hæmoglobin in a free condition is peculiarly potent in exciting the formation of bilirubin. The evillence, therefore, is very strong for the view that as far as the formation of the greater part at least of the bilirubin is concerned, the action of the hepatic cell is limited to converting into bilirubin the free hæmoglobin offered to it by the portal blood.

By what means, under normal conditions, is the presence of that free hrmoglobin secured? We have seen reason to conclude from histological appearances that a certain number of red corpuscles undergo change in the spleen-pulp; and it seems natural to infer that one duty of the spleen is to set free hæmoglobin from the corpuscles and thus, through the splenic veins and so the portal vein, to supply the liver with material for bilirubin. But this cannot be the only source, since the secretion of bile continues after extirpation of the spleen. There must, therefore, be other regions of the 
body in which a similar change of red corpuscles is going on; it has been suggested that the red marrow of bones is one of these; but further information on these points is needed.

We may then go so far as to say that the bilirubin of the bile is derived from the hrmoglobin of the blood, and that the later stages of the transformation, including the discharge of the iron of the hæmatin component, take place in and by means of the hepatic cell; but much beyond this is at present uncertain. It must be remembered too that, though after extirpation of the liver no accumulation of bilirubin takes place, showing that the bilirubin is formed by the liver, yet the whole change from red corpuscles to bilirubin may occasionally take place quite apart from the liver, as shown by the presence of hæmatoidin in old blood-clots.

$\$$ 393. The formation of the bile-acids. About this we know still less. Taking glycocholic and taurocholic acids as the typical bile-acids, recognizing ( $\$ 215$ ) that these arise from the union of cholalic acid with glycin and taurin respectively, and remembering that taurin is found in several tissues, and that glycin (see $\$ 355$ ) though not an actual constituent of any of the tissues, must certainly arise in tissue metabolism, we may conclude that the chief work in this respect of the hepatic cell is to provide cholalic acid, and to effect the combination with glycin and taurin, though possibly some amount of either one or the other of these bodies may be furnished by the hepatic substance itself. As to how cholalic acid arises out of the metabolism of the hepatic cell, we know no more than we do about the formation of kreatin in muscle $\mathrm{rr}^{\circ}$ of pepsin in a gastric cell. We are equally ignorant about the origin of glycin and taurin, and cannot explain why in one animal glycocholic and in another taurocholic acid is prominent in the bile, though the two bodies, as shown especially by the presence of sulphur in the taurin, are widely different. It has been observed that the presence of bile in the intestines seems to excite the liver to increased biliary action; since the bileacids are rapidly changed in the intestine and the cholalic acid speedily altered, it seems probable that the increased biliary activity is due to the absorption of the glycin and taurin respectively. From which we may conclude that the presence of these bodies stirs up the hepatic cell to an increased formation of cholalic acid.

$\$ 394$. As a general rule, the formation of bile-acids runs parallel with the formation of bile-pigment, an increase or decrease of bile meaning an increase or decrease of both constituents. But there are some facts which seem to show that the two actions may be dissociated. The condition or symptom known as "jaundice" is essentially an excess of bilirubin in the blood, whereby the tissues such as the skin and the fluids such as the urine are colored with the yellow pigment. In most of the maladies in which jaundice is a symptom, there is evidence of an obstruction to the flow of bile through the bile passages; and the presence of bile in the blood, and hence in the tissues at large, is in such cases due to the fact that the bile after secretion by the hepatic cells is reabsorbed from the bile-ducts (see $\S 226)$.

But in certain cases where jaundice is a prominent symptom, no evidence of any obstruction whatever to the flow of bile can be obtained. This is the case in the jaundice of yellow fever and of a peculiar allied malady known as " acute yellow atrophy of the liver." Now in these cases there is no evidence of an accumulation in the blood or elsewhere of bile-acids as there is of bile pigment. And in the obscure malady known as simple or idiopathic jamdice, in which though the anatomical conditions are unknown there is it least no sign of obstruction, the urine though loaded with bile pigment is said to contain no bile-acids. 
$\S 395$. The question may be asked, Is the secretion of bile independent of or in some way or other connected with the glycogenic activity of the cells? To this we cannot at present give a definite answer. In some of the invertebrata the cells in the organ, called a liver, which manufacture glycogen, are distinct from those which secrete bile or other digestive juices; and it might be inferred that in the vertebrate the two actions, though taking place, as they certainly do, in the same cell, take place apart and distinct. There are facts which seem to indicate that the two are intimately connected; but we have as yet no exact knowledge concerning the matter. It has been urged that the portal blood is chiefly concerned with the formation of glycogen, and the blood of the hepatic artery with the secretion of bile; but there is no adequate support of this view. It must be remembered, moreover, that, in addition to the formation of glycogen and the secretion of bile, other metabolic events, especially affecting proteid or at least nitrogenous constituents of the body, are also taking place; and to these we must now turn.

\section{On Urea and on Nitrogenous Metabolism in General.}

$\S 396$. We have seen that nitrogenous proteid material in some form or other enters into the composition of all the tissues of the body, and we have further seen that it is so conspicuously and constantly present wherever living subtances are manifesting vital energies as to justify the conclusion that the changes which it undergoes are in some way essential to the manifestation of those energies. We have seen, it is true, reason to think that in some tissues at least, in muscle for instance, a large part of the energy set free during activity preëxisted as latent energy and had its immediate source not in proteid (nitrogenous) but in some other constituents of muscle; and indeed, as we shall see later on, the greater part of the whole energy of the hody must be regarded as the energy of carbon compounds and not of nitrogen compounds; but this is quite consistent with the view that proteid material in some way or other essentially intervenes in, we may perhaps go so far as to say directs, the changes by which in the body energy is set free in the peculiar way which we speak of as living.

IVe have seen that at all events the greater part of the proteid material of the food enters the blood as proteid material either as peptone or in some other form, and is carried as proteid material to the tissues.

We have seen that the nitrogen of proteid material leaves the body so largely in the form of urea, that the other nitrogenous excretions may for the time be left out of consideration.

And lastly we have seen reason to think that this urea which leaves the body in urine is brought to the kidney as urea in the blood, the kidneys themselves apparently having no special power of forming urea out of something which is not urea, but only contributing to the general stock of urea by virtue of their own proteid metabolism. We have now to study the little we know concerning the steps by which the proteid material of the food and of the body is converted into this urea of the blood, which is the source of the urea of the urine.

$\$ 397$. In the first place we may take it for granted that the urea carried to the kidney in the blood had an antecedent in something which was not urea. We can hardly suppose that the proteid constituent of living substance, when in the course of its metabolism it ceases to be proteid, breaks up at once into urea and into non-nitrogenous bodies. All we have learned goes to show that what we call metabolism is not a single abrupt change, but consists essentially in a series of changes; and we may safely conclude 
that proteid material in becoming urea passes through phases in which the nitrogen exists in chemical combinations distinct from proteid material on the one hand and urea on the other.

In the second place it is extremely probable that the series of changes by which proteid material becomes urea is not the same in all the tissues and on all occasions. We should naturally expect to find the proteid material following different lines of metabolism in different places or under different circumstances, the different lines all converging to the same body, urea, because for some reasons or other urea appears to be, in the main, the most convenient form in which the nitrogen can leave the blood and the body.

We should accordingly expect to find, on the one hand, various nitrogenous bodies resulting from proteid metabolism in various parts of the body, and, on the other hand, arrangements by means of which these various bodies were reduced to the common form urea, preparatory to their discharge from the body by the kidney. And actual observation as far as it goes supports this view, though our knowledge of the whole matter is very imperfect.

$\S 398$. We may turn our attention first to the metabolism of the skeletal muscles, since these represent, as far as mere quantity is concerned, by far the greater part of the proteid capital of the body. "We may safely infer that they furnish a large part of the urea of the urine; though undoubtedly a small mass of tissue might by reason of its more rapid metabolism work over a greater quantity of proteid material than a much larger mass with a slower metabolism; yet we have no reason to think that the proteid metabolism of skeletal muscle, obscure though it is in its nature, is so slow as to neutralize the probable effect of the great bulk of muscle existing in the body.

In dealing with the chemistry of muscle ( $\$ 62)$ we saw that urea, save in the exceptional instances of certain cartilaginous fishes, was conspicuous by its absence from the extract of muscle, whereas, a very appreciable quantity of kreatin was invariably present, and, indeed, was the prominent nitrogenous crystalline constituent of that extract. It seems difficult to resist the conclusion that kreatin is the main normal nitrogenous product of the metabolism of skeletal muscles. If we accept this view, then, upon the fact of the presence of kreatin in, and the absence of urea from, the muscle itself, we may base the conclusion that while the muscle produces kreatin as an antecedent of urea, the kreatin so produced is converted into urea in some part of the body other than the muscle itself. Kreatin, as we have already seen, may be easily split up, and we may probably with safety assume is split up, somewhere in the hody, into urea and sarcosin. But sarcosin does not appear in the urine as such; hence, the conversion of kreatin into (part of) the urea of the urine entails as well the further conversion of sarcosin into urea. Now sarcosin, as we have seen, is methyl-glycin; we may regard it for our present purposes as simple glycin, and hence the total conversion of kreatin into urea entails the conversion of glycin into urea. This, however, does not offer any additional difficulty, since we know from direct observation that glycin introduced into the alimentary canal does not reappear as such in the urine, but produces a corresponding increase in the urea of the urine; from which we infer that glycin absorbed from the alimentary canal is, somewhere in the body, converted into urea. We shall speak of this conversion later on, and shall then see that, as far as urea is concerned, glycin (amido-acetic acid) and sarcosin (methyl glycin, methyl-anido-acetic acid) undergo the same change, the amide moiety in each case being converted into urea, while the non-nitrogenous moiety is oxidized and thrown off: 
Meanwhile, we may state the conclusion at which we have provisionally arrived, namely, that the nitrogenous metabolism of muscle probably gives rise to kreatin, which, in some part of the body other than muscle, is probably split up into urea, ready for excretion, and into sarcosin which also, somewhere in the body, is further converted into urea. And bearing in mind the large mass of the skeletal muscles, we may further conclude that a large portion of the urea leaving the body by the urine is formed in this way.

$\$ 399$. We must not, however, leave this statement without referring to a difficulty. Kreatinin, as we have seen, is so frequently found in urine as to be regarded as a normal constituent, at all events, of human urine; and kreatinin is, as we have seen, the urinary form, so to speak, of kreatin; the one body easily changes into the other by the assumption or removal of $\mathrm{H}_{2} \mathrm{O}$. This suggests the question, Is not the kreatinin of urine the representative of the kreatin of the muscles, which is thus exerted directly without undergoing the change into urea just discussed? In answer to this we may say, in the first place, that the quantity of kreatinin in the urine, though variable, is small; we may put the average at about $1 \mathrm{grm}$. in twenty-four hours. Now, muscle contains from 0.2 to 0.4 per cent. of kreatin; and this, taking the total muscle of the body (to say nothing of other sources of kreatin, which we shall mention presently) at about 30 kilos. would give 60 to $120 \mathrm{grms}$. of kreatin as present in the muscles of the body at any one moment. We can hardly suppose that the metabolism of muscle is so slow as out of this stock only to provide the $1 \mathrm{grm}$. of kreatinin in twenty-four hours. Moreover, the kreatin in urine vanishes during starvation, is very markedly increased by a diet of flesh which contains kreatin, and is not increased either by muscular exercise (which, however, would only indirectly affect nitrogenous metabolism of muscle), or by such conditions, fever, for instance, as notably increase the urea of urine by increasing the nitrogenous metabolism of muscle. We infer, therefore, that the normal presence of kreatinin in urine is due to the direct administration of kreatin present in a (normal) flesh diet, and has nothing to do with the muscular metabolism of the individual who is secreting the kreatinin in his urine.

The fact, however, that the kreatin present in the muscle of the food and absorbed from the alimentary canal does not undergo a change into urea, but is excreted as kreatinin, that is, virtually as kreatin, warns us to be careful in adopting the conclusion arrived at above, that the kreatin produced by muscular metabolism in the living body is a conspicuous antecedent of the urea of the urine. It is difficult to see why kreatin passing into the blood of the capillaries of the muscle should be changed into urea, while that which passes into the capillaries of the portal system is not; for reasons which will be apparent presently, we should rather expect that the latter being more directly exposed to the influence of the liver would be more readily and more completely converted than the former. Indeed, the question forces itself upon us, Is kreatin, after all, the natural main product of the nitrogenous metabolism of muscle? It is possible that in the normal metabolism of the livin muscle the nitrogen leaves the muscular substance and passes into the blood in another form, as some substance not kreatin, and that it is as the muscle dies that kreatin is formed, just as the solid myosin is unknown to living fibre but makes its appearance in a dying one? We have no positive evidence, however, that this is so, and, meanwhile, may continue to suppose that kreatin is formed, and that, in consequence, kreatin is a conspicuous antecedent of the urea of the urine; but we must not regard this as proved. 
$\$ 400$. Our knowledge of the metabolism of the nervous tissues is, as we have seen, very imperfect $(\$ 70)$, but the presence of the kreatin in the central nervous system leads us to infer that the nitrogenous metabolism of the living substance of nerve-cells and of the axis-cylinder of nerve-fibres is, in its broad features, identical with that of muscle substance. The mass, however, of the nerve-cells and axis-cylinder of the body, all put together, is small, compared with the mass of skeletal muscle; moreover, the energy set free by the metabolism of a mass of nervous matter though "higher" in quality is less in quantity than that set free by the metabolism of an equal mass of muscle, or, in other words, its metabolism is less rapid. Hence, we may probably consider the metabolism of the nervous system as a mere addition to that of the muscular system, at least as regards the point on which we are now dwelling. The amount of nitrogenous metabolism taking place in connective tissue, cartilage, bone, and the skin is probably still less, and, for our present purposes, needs no special discussion.

$\S 401$. The nitrogenous metabolism of the glands, however, more particularly that of the liver, does deserve special consideration; and we may at once turn to a quite different aspect of the question in hand.

When the rate of discharge of urea from the body is observed during a period of some length, especially under varied circumstances, the direct effect of nitrogenous food becomes more striking. We have already said, and shall again return to the point, that muscular contraction does not directly increase the output of urea; the discharge of urea, for instance, is not necessarily increased by even great bodily labor. The introduction, however, of even a small quantity of proteid material into the alimentary canal at once increases the urea of the urine; and in the curve of the discharge of urea in the twenty-four hours each meal is followed by a conspicuous rise. The absorption of proteid material from the alimentary canal is followed by an immediate proportionate increase in the quantity of urea which is secreted by the kidneys, and that, as we have seen, means an increase in the urea brought to the kidney by the renal artery. What is the origin of this additional urea?

Two views present themselves. On the one hand, since some portion of the proteid material of every meal, at all events of every necessary meal, goes to repair the proteid waste continually going on in the parts of the body where proteid metabolism is taking place, we may suppose that the presence of an extra quantity of proteid material thrown upon the blood from the food acts as a stimulus to the tissues, to the muscles, for instance, as well as others, stirs them up to increased nitrogenous metabolism and thus produces an increase of energy, chiefly if not exclusively in the form of heat, accompanied by an increase of the antecedents of urea and so of urea. In other words, the increase of urea in question is the result of an increase in the general nitrogenous metabolism of the body.

On the other hand, we may suppose that in order to prevent the whole body being encumbered with it, this excess of proteid food material is, in some special part of the body, split up into a nitrogenous and a non-nitrogenous moiety, and that, while the latter is stored up as fat or glycogen, the former is at once converted into urea and got rid of. We have already ( $\$ 218$ ) seen that a step in this direction may take place while the food is as yet in the alimentary canal; we have seen that pancreatic juice may carry part of the proteids on which it acts beyond the stage of albumose and peptone, and reduce that part into leucin, tyrosin, and other bodies. We do not know, as we have already said, to what extent this more profound digestion by pancreatic juice does actually take place in the living body ; it may take 
place to a very slight extent and it may under certain circumstances take place to a considerable extent. But in any case it illustrates the way in which a somewhat similar disruption of proteid material, a disruption which may be broadly described as a splitting up of the proteid into a nitrogenous and a non-nitrogenous moiety, may take place somewhere in the body and so lead to the sudden formation of some antecedent of urea. The antecedent may be leucin or may be some other body or bodies.

In support of this view may be urged the fact that such bodies as leucin, glycin, asparagin, and many others when introduced into the alimentary canal are transformed into urea. When these bodies are administered in not too great quantities they do not reappear in the urine, but the urea is proportionately increased.

$\$ 402$. We have seen reason to think that the proteids of a meal are absorbed not by the lacteals but by the portal bloodvessels, and such bodies as leucin probably take the same course. This being so, all these bodies pass through the liver and are subjected to such influences as may be exerted by the hepatic cells. Now, we have no positive evidence that the liver does or can exert such an action on proteid material itself as to separate a relatively simple nitrogen compound from the remaining constituents, leaving these to form a body rich in carbon; we have no positive proof that the increase of proteid metabolism just spoken of as leading to an increase of urea takes place in the liver rather than in the tissues at large; we may go so far perhaps as to suspect that it is largely or wholly confined to the liver, but we have no convincing demonstration. We have, however, a convergence of evidence that the last stage of the process, namely, the conversion into urea of some product of proteid metabolism, which though allied to is not exactly urea, does occur in the liver. In the first place, a large quantity of urea seems to be present in the liver of mammals; in this respect the liver presents a strong contrast to the muscles; in the liver of birds the urea is represented by urates. Moreover, when a stream of fresh blood is passed several times through the liver of an animal recently killed, the percentage of urea in the blood so used is found to be decidedly increased. This, however, does not prove that urea is formed in the liver, since the increased quantity of urea in the blood which had been circulated might have been simply urea which had been washed out from the liver, where it had previously been staying. Still as far as it goes it is suggestive. In the second place, in certain cases of a form of disease of the liver known as acute yellow atrophy in which the hepatic cells are so changed that their functional activity is largely diminished, the urea of the urine not only undergoes a very marked decrease but appears to be replaced to a very large extent by leucin. This fact suggests that leucin (and not for instance kreatin) is the chief immediate product of the nitrogenous metabolism of the body, and that the leucin thus produced is in a normal state of things converted into urea by the liver. And in this connection it may be remarked that not only is leucin found in nearly all the tissues after death, especially in the glandular tissues, but also appears with striking readiness in almost all decompositions of proteids, and is, moreover, a product of decomposition of gelatiniferous substances. Without going, however, so far as to conclude that leucin is the chief antecedent of urea, we may take the above observation as indicating that the normal liver has, in some way or other, the power of converting leucin into urea. If this be so we may also venture to suppose that when such bodies as leucin, glycin, etc., introduced into the alimentary canal appear in the urine as urea the transformation has taken place in the liver. The body tyrosin which so often accompanies leucin, belonging as it does to the aromatic series, stands on a different footing from leucin and the like. 
$\$ 403$. The transformation, however, of leucin into urea raises a new point of view. Leucin, as we know, is amido-caproic acid; and, with our present chemical knowledge, we can conceive of no other way in which leucin can be converted into urea than by the complete reduction of the former to the ammonia condition (the caproic acid residue being either elaborated into a fat or oxidized into carbonic acid) and by a reconstruction of the latter out of the ammonia so formed. We have a somewhat parallel case in glycin, which is amido-acetic acid; here, too, a reconstruction of urea out of an ammonia phase must take place. Moreover, when ammonium chloride is given to a dog a very large portion reappears as urea, $i . e$, there is an increase in the urea of the urine corresponding to a large portion of the nitrogen contained in the ammonium chloride. And in the case of other animals also, indeed of man himself, there is evidence that somewhere in the body ammonia may be converted into urea. Hence in all these cases where ammonia or ammonia compounds are changed into urea, the last step at all events is one of synthesis; and this suggests the possibility that in the ordinary proteid metabolism also, the downward katabolic series of changes may finish off with a synthetic effort, the last stage of the former being the appearance of an ammonia compound which is subsequently reconstructed into urea.

This synthesis, like the transformation of leucin and other bodies, probably takes place in the liver; and in support of this view we have a certain amount of experimental evidence. Birds may be kept alive after total extirpation of the liver for a longer time than can mammals; and when in geese the liver is removed the uric acid (representing in these animals the urea of the manmal) is largely decreased, while the ammonia of the urine is largely increased. After the removal of the liver also, leucin, glycin, and other amides or amido-acids administered by the alimentary canal no longer increase the uric acid of the urine, as they do in the intact animal. In these animals, the synthesis of ammonia compounds into uric acid, which is parallel to the synthesis into urea occurring in the mammal, seems to take place in the liver, and we may infer is in some way or other effected by the hepatic cells.

As to the exact way in which ammonia, either as such or in form of an amide or amido-acid changes into urea, we have no certain knowledge. Ammonium carbonate, we know, is readily formed out of urea by simple hydration, and we may imagine that the living organism can carry out the reverse process and dehydrate ammonium carbonate into urea. There is, however, a certain amount of evidence that not ammonium carbonate but ammonium carbamate is the immediate antecedent of urea; and, indeed, out of the body, by electrolyzing a solution of ammonium carbamate with alternating currents, a certain amount of urea may be artificially produced. But this is a matter too obscure to be discussed here.

$\$ 404$. Uric acid. This, like urea, is a normal constituent of human urine, and, like urea, has been found in the blood, in the liver, and in the spleen; it is a conspicuous constituent of an extract of the latter organ. In some animals, such as birds and most reptiles, it takes the place of urea. In various diseases the quantity in the urine is increased; and at times, as in gout, uric acid accumulates in the blood, and a deposit of urates takes place in the tissues. Since by oxidation a molecule of uric acid can be split up into two molecules of urea, and a molecule of some carbon acid, uric acid is commonly spoken of as a less oxidized product of proteid metabolism than urea. But there is no evidence whatever to show that the former is a necessary antecedent of the latter; on the contrary, all the facts known go to show that the appearance of uric acid is the result of a metab- 
olism slightly diverging from that leading to urea; indeerl, it is probable that the divergence occurs toward the end of the series of changes, for urea given by the mouth to birds appears in the urine as uric acid, and, conversely, uric acid given to mammals appears in the urine as area. We have no evidence to prove that the cause of the divergence lies in an insufficient supply of oxygen to the organism at large; on the contrary, uric acid occurs in the rapidly breathing birds as well as in the more torpid reptiles. Nor can the fact that in the frog, again, urea replaces uric acid be explained by reference to that animal having so large a cutaneous in addition to its pulmonary respiration. The final causes of the divergence are to be sought rather in the fact that urea is the form adapted to a fluid, and uric acid to a more solid excrement. Nor is there in man or the mammal any satisfactory physiological or clinical evidence that an increase of uric acid is the result of deficient oxidation. The absolute amount of uric acid discharged by man and its proportion to the urea passed at the same time varies a good deal. There is no positive evidence that the quantity excreted is necessarily increased by nitrogenous diet, unless some disorder supervenes; indeed, it is asserted that both absolutely and relatively to the urea the quantity excreted is greater upon a mixed diet than upon a highly proteid one. Alkalies in the food seem undoubtedly to diminish it, and alcohol, at least in excess, to increase it.

So far from considering uric acid as a less oxidized antecedent of urea, we ought, perhaps, rather to regard its appearance as a result of a synthesis in which urea or some allied body takes part. As we have said, uric acid may be formed synthetically by heating together urea and glycin; and it has more recently been similarly prepared from various allied bodies. As to where or how such a synthesis is effected in the living body, we know little or nothing for certain, and can only make conjectures. The constant presence of uric acid in the spleen, however, and the frequently noted connection between a rise and fall of uric acid in the urine and variations in the volume and therefore presumably in the activity of the spleen, suggest that the change may be brought about in that organ; but it must be remembered that in birds and reptiles the formation of uric acid seems to be effected in the same organs as that of urea and in an analogous manner; and the arguments which we have used concerning the formation of urea in the liver of nammals, may be applied to the formation of uric acid in the livers of birds and reptiles. It is more probable, therefore, that in the mammal the turn to uric acid rather than urea is given in the liver, the spleen, however, possibly playing its part also in the matter.

$\$ 405$. Of the meaning of the appearance in the tissues of such bodies as xanthin, hypoxanthin, guanin, and the like, and of the exact nature of the metabolism which gives rise to them or which they themselves undergo, we know little or nothing. The presence of these several bodies may be taken as illustrating the complex and varied nature of proteid metabolism to which we referred above. Urea is the chief end-product of proteir metabolism, but that end is probably reached in several ways; so that probably a very large number of nitrogenous chemical substances make a momentary appearance in the body. Some of these fail to become urea, and either without or after further change make their appearance in the urine. But we do not know whether their appearance is accidental, the result of imperfect chemical machinery, or whether they, though small in quantity, serve some special ends in the economy. Perhaps sometimes or with some of them it is the one case, at other times or with others it is the other case.

When proteid material undergoes outside the body, either by the action of trypsin or as the result of decomposition or under the influence of chem- 
ical agents, that change by which it is converted into leucin, the leucin which appears in some considerable quantities is accompanied by tyrosin, which appears in smaller quantities, as well as by other bodies. The almost constant appearance of tyrosin as a result of the decomposition of proteid material leads one, as we have previously said, to the conception that some representative of the aromatic series enters into the constitution of proteid substance; and it is possible that the hippuric acid of flesh-eating animals derives its benzoic acid constituent from this aromatic radicle of proteid matter. Tyrosin itself does not appear in the body as a normal product of proteid metabolism, and we are therefore led to infer that in proteid metabolism the aromatic radicle takes on some other form. Whether, as in tyrosin, the aromatic (phenyl) nucleus is associated with an ammonia representative or no, we do not know. But if it is, then, since neither tyrosin nor any similar body is a constituent of normal urine, the ammonia constituent is somewhere dissociated from the phenyl one; and while the former contributes to the stock of urea, the latter is either discharged by the urine as hippuric acid, having as we have seen effected in the kidney a new association with the ammonia representative, glycin, or leaves the body as one or other of the urinary phenyl compounds, or possibly may be oxidized somewhere into carbonic acid and water. Our knowledge on this point is limited, but we have ventured to refer to the point since it further illustrates the complexity of proteid metabolism.

$\S 406$. In speaking of urea ( $\$ 337)$ we alluded to its relations to the cyanogen compounds. Bearing in mind the peculiarly large amount of energy set free as heat during the isomeric transformation of many cyanogen compounds, as well as the large store of potential energy existing in cyanogen itself, the heat of combustion of which is very large, and contrasting these properties with those of ammonia and the ammonia compounds, we cannot help being tempted toward the view that in the actual living structure the nitrogen exists in the form of cyanogen compounds, and that in the passage to dead nitrogenous waste, during which energy is set free, the cyanogen compound changes to the amide or other ammonia representative. And there are several facts which lend support to such a view, such as the presence of sulphocyanates in saliva and urine, which we may look upon as a sort of leakage of cyanogen factors, the artificial production of kreatinin out of cyamide and sarcosin, and other facts. But the matter, though it deserves to be borne in mind, is too obscure to be dwelt on here.

$\S 407$. We may now briefly sum up the varied discussions which have occupied us in the present section.

Urea is the main end-product of proteid metabolism. Unlike hippuric acid and some other constituents of urine, urea is simply excreted by the kidneys, being brought to them in the blood, they apparently, beyond the simple act of excretion, doing no more than merely contributing to the stock of urea in so far as they are masses of proteid material undergoing proteid metabolism as part of their general life. What are the immediate antecedents of urea we do not clearly know ; but it is probable that they are not one but several and indeed possibly many. We have reason to think that urea may be formed out of amides or amido-acids, or out of ammonia itself by a synthetic process; and we have indications that this synthesis is effected in the liver by the agency of the hepatic cells. But we do not know whether this synthesis bears only on particular nitrogen-holding substances of food or of the body, or whether it comes into play in the normal metabolism of proteid material. If the kreatin which is so conspicuous a constituent of muscular and nervous structures is a stage in the direct line to urea, then the synthesis would affect only the sarcosin which 
the kreatin in becoming urea sets free. But it is by no means clear that kreatin is such a stage.

The evidence as far as it goes tends to show that the metabolism of proteid is very complex and varied, that a large number of nitrogen-holding substances make a momentary appearance in the body, taking origin at this or that step in the downward stairs of katabolic metabolism and changing into something else at the next step, and that the presence in various parts of the body and even in the urine, in small quantities, of so many varied nitrogenous crystalline substances, forming a large part of what are known as extractives, has to do with this varied metabolism. Possibly the transformations by which nitrogen thus passes downward take place to a certain extent in such organs as the liver and the spleen, which are remarkably rich in these extractives.

\section{On Some Structures and Processes of Obscure Nature.}

$\$ 408$. The thyroid body. Certain structures of obscure nature, but probably connected in some way or other with some of the metabolic processes in the body, are often spoken of under the undesirable name of "ductless glands." Such are the thyroid body or gland, the pituitary body, the thymus, and the suprarenal capsules. These differ from each other so essentially that the only plea which can be urged in favor of considering them together is convenience and our ignorance of their respective functions.

The thyroid body is the one of the group most deserving to be called a gland, since it, like the lungs, arises as a two-lobed diverticulum from the ventral surface of the anterior part of the alimentary canal, and at first, like the lungs also, behaves as if it were about to become a double racemose gland. The connection with the throat, however, which should have become a duct, is soon obliterated, and the two lobes, united with each other by an isthmus acruss the trachea, lose all traces of any branching ducts within them, and become transformed into masses of isolated ductless alveoli bound together with connective tissue.

Hence, when a section is taken through a hardened and prepared lobe of an adult thyroid, what is seen is a limiting capsule of connective tissue sending into the interior numerous septa, which surround and separate from each other round or oval spaces, the sections of the isolated alveoli. These are of variable size, some being visible to the naked eye, and each is lined by a single layer of low columnar or cubical nucleated cells resting on a basement membrane, leaving a large cavity, which in fresh specimens is filled with a glairy fluid. The cells present no special characters.

The septa of connective tissue, fairly rich in elastic elements, but remarkably free from adipose tissue, contain numerous bloodvessels derived from the superior and inferior thyroid arteries, the branches of which, relatively large and frequently anastomosing, end for the most part in capillary networks round the alveoli; from these capillaries and those of the septa the blood is gathered into veins also relatively large, which, forming plexuses on the surface of the organ, end in the superior middle and inferior thyroid veins. 'The thyroid body is thus furnished with an abundant supply of blood.

The serta also contain a very large number of lymphatic vessels, which, both on the surface of the organ and along the septa, are arranged in plexuses of anastomosing trunks of considerable size. Small nodules of adenoid tissue are also found in the septa.

The nerves of the thyroid body are also abundant. They are, in man, derived chiefly from the cervical sympathetic nerve, passing off from the 
middle and lower cervical ganglia; their exact terminations within the organ is not known. Fine filaments are also said to be given off to it from the external branch of the superior laryngeal nerve.

The "accessory" thyroid bodies often found are of the same nature as the main body.

Very frequently, so frequently in the adult as to be of almost normal occurrence, the alveoli contain not simple glairy fluid but a more solid clear material called "colloid;" this generally appears in the centre of an alveolus and may fill up the whole lumen; occasionally more or less changed epithelial cells may be seen lying between it and the layer of cells resting on the basement membrane. Extravasations of blood into the alveoli are also not uncommon.

The thyroid body is very apt to become enlarged, sometimes enormously so, and is then spoken of as goitre. The enlargement may be due simply to an increase in the number of otherwise fairly normal alveoli and septa. But very often a number of alveoli become more or less confluent, forming a cyst; and at times the whole gland appears to be composed of a number of cysts of varying size, frequently loaded with "colloid" material. There is also a form of goitre in which the enlargement is chiefly or even exclusively due to an increase in the vascular supply, the bloodvessels being abnormally distended; and this apparently may occur without any structural changes in the walls of the bloodvessels. Sometimes, however, the arteries undergo aneurismal enlargements, with changes in their coats.

'The glairiness of the fluid contents of the alveoli has generally been attributed to the presence of mucin, and this body has also been said to have been found within the lymphatic vessels running in the septa; but some observers have urged that the material in question is not true mucin, but a peculiar form (or forms) of proteid substance. "The "colloid" material so frequently appearing has also been regarded as allied to mucin, but its exact nature has not as yet been satisfactorily determined. Besides these special substances the alveoli or cysts also contain serum-albumin and globulin. The "extractives" of the thyroid appear to contain kreatin or kreatinin in not inconsiderable quantities, xanthin, and lactic (paralactic) acid; guanin is said to be absent. In large and old eysts cholesterin is sometimes present; and when, as often happens, extravasations of blood into the cysts have taken place, hæmoglobin, or at a later stage hæmatoidin (bilirubin) has been found.

$\S 409$. The large supply of blood to the thyroid suggests the idea that the organ is the seat of some of the subsidiary metabolic processes to which we referred in the last section, and this view is supported by the presence of the extractives just mentioned; but we have no detailed knowledge of what actually goes on.

The presence of the peculiar mucin-like body in the alveoli, and the tendency to "colloid formation" further suggests some relation of the organ to the formation or distribution of mucin; and this view has derived a certain support from some experimental results, but these, though numerous, have proved neither uniform nor accordant. When in certain animals (monkeys, dogs, and other carnivora, and the same has been observed in man) the gland is extirpated, even with the greatest care, the operation is frequently followed by the occurrence of peculiar nervous symptoms, such as muscular twitchings and tremors, spasms, and even tetanic convulsions (more especially observed in young animals), accompanied or succeeded by irregularity or failure of voluntary movements; subsequently there may ensue varied symptoms which may be described under the general term of disordered nutrition, ending eventually in death. In a certain number of cases, however, in the 
above kinds of animal, no serious symptoms follow, even the total extirpation of the organ producing no marked effect; and in rabbits and other herbivorous animals removal is said never to be followed by any of the above results. It has been urged that the symptoms when seen are the effects not of the mere absence of the organ, but of mischief set up by the operation in adjoining structures, more especially in the laryngeal nerves and vagus trunks; but this does not seem a valid explanation. If, as suggested above, certain metabolic processes are normally going on in the organ, we may fairly suppose that, in the absence of the organ, the interruption of the normal sequence of chemical change would throw upon the circulation certain strange substances which, acting like a poison, might produce the nervous symptoms, throw into disorder the nutrition of various tissues, and finally bring about death. We may further explain the cases where symptoms are absent by supposing that, for some reason or other, "things have taken a different turn," the particular poisonous substance have not made their appearance, but innocuous ones have taken their place; and we know how slight a change in chemical composition may turn a poison into an inert body. This, of course, remains a mere supposition until we can state what the exact metabolic processes are, and name the substances which work the mischief; but it seems more reasonable to accept such a provisional supposition, than to conclude that the thyroid may be removed without producing any effect whatever on the organism. An animal without a thyroid may appear perfectly well, because the circumstances to which it is exposed do not happen to test the imperfection from which it is really suffering, just as a man's inability to swim may not be apparent until he happens to fall into the water. The animals which do succumb to the operation of removal of the organ are, for some reason or other, put to the test, and are found wanting. The very discordance of the experimental results points the physiological moral that the phenomena which we are as yet able to observe form, as it were, a mere surface covering intricate processes at present wholly, or nearly wholly, hidden from us.

The above experimental results receive additional interest and at the same time support from clinical experience. The connection between goitre and cretinism-the latter disease being, broadly speaking, a result of disordered nutrition telling largely on the nervous system-has long been recognized; and attention has also been called to some tie between disease of the thyroid and a morbid condition, known as myxœedema, in a certain number of cases of which mucin or a mucin-like body has been found in great excess in the skin and in other tissues. In monkeys the removal of the thyroid has, in some cases, been followed, besides the symptoms mentioned above, some of which resemble those of myxœdema, by an accumulation of mucin or a mucin-like body in the skin and various tissues. It is very difficult not to connect this with the formation in the thyroid of colloid material in the contents of the alveoli. But we know so little about the nature of mucin and its allies, about their real relations to more ordinary proteid substances, and about the part which they play in physiological processes, that any views as to the exact connection between the presence of mucin in the tissues at large and changes taking place in the thyroid must be at present to a large extent speculation.

The large vascular supply of the thyroid, and the phenomena of a disease known as exophthalmic goitre, in which vascular enlargement of the thyroid is associated with cardiac symptoms, and other vascular disturbances, especially of the head, have suggested that, apart from metabolic processes, the circulation in the thyroid may, perhaps in a more or less mechanical way, be connected with and influence the cir- 
culation in the brain. But the exact nature of this influence has not been made clear.

$\$ 410$. The pituitary body. The lower, posterior, lobe of this organ resembles the thyroid body (the upper, anterior, lobe is of quite distinct nature, being really a part of the central nervous system).

Concerning the processes which take place in these alveoli, and the purposes of the organ as a whole, we know absolutely nothing.

$\$ 411$. The supra-renal bodies. A (mammalian) supra-renal body, when cut across, is seen to consist of two distinct parts, an outer thicker cortical part, of yellowish color, striated radially, and an inner thicker medullary part of dark color. At the depression on the anterior surface called the hilus, whence issues the comparatively large supra-renal vein, the cortex thins away so that the merlulla comes to the surface. These two parts, cortex and medulla, are not, like the cortex and medulla of a lymphatic gland, different arrangements of the same material, but are of essentially different nature, and, indeed, are of different origin. The medulla is derived from, is a modification of, sympathetic ganglia, while the cortex is derived from masses of mesoblastic cells surrounding the great bloodvessels; and in some animals the two form wholly separate bodies. The so-called accessory suprarenals are composed of cortex alone.

The whole organ is surrounded by a capsule of connective tissue, free from muscular fibres, and not very rich in elastic elements. From the capsule septa pass inward and form a framework, the cavities of which are filled by cells or groups of cells differing in nature and differently arranged in the cortex and in the medulla. The middle larger part of the cortex is composed of somewhat long solid columns of polyhedral cells, lodged in corresponding meshes of the framework. The columns, which are three or four cells thick and several cells in length, though somewhat irregular and varying in size, do not anastomose, being wholly separated from each other by the bars of connective tissue, and possess no central cavity or lumen. The bloodvessels, which are abundant in these bars of connective tissue, do not penetrate the columus. The cell substance of the cells is of a yellowish color, often containing yellowish oil globules, and possesses a clear round nucleus.

In the outer part of the cortex immediately underneath the capsule is a thin zone in which the groups of cells are not columnar, but rounded and irregular; and again in the inner part of the cortex abutting on the medulla is another thin zone, in which the columnar arrangement is lost, the cells being here disposed in a network of thin cords, and the individual cells to a large extent separated from each other by delicate continuations of the coarser connective-tissue septa. Hence, the main median part of the cortex, which from the prominent columnar arrangement appears striated radially, is often called the zona fasciculata, the thin outer part the zona glomerulosa, and the thin inner part the zona reticularis; but as far as the essential characters of the cells are concerned, all the three zones are alike.

The medulla also consists of cells or groups of cells lying in the meshes of a connective-tissue framework, but the cells are of a different nature from those of the cortex. They are irregular and often branched, and their cell substance, though it sometimes contains pigment, is generally clear and transparent. The medulla, noreover, is further distinguished from the cortex by the abundant supply of bloodvessels and of nerves.

The cells of the medulla and of the inner zone (zona reticularis) of the cortex are very apt to undergo change after death, and to become diffluent.

The arteries which come from the aorta and from the renal and phrenic arteries pass into the organ on the surface, and traversing the cortex, supply- 
ing as they go both capsule and cortex with a moderate number of vessels, end in the medulla the connective-tissue bars of which bear numerous large venous sinuses, into which the capillaries pour their blood, and from which the blood is gathered up into the supra-renal vein.

A large number of nerves, consisting chiefly of medullated fibres from the solar plexus, the renal plexus, the phrenic nerve, and the vagus, pass in to the supra-renal body at the hilus and on the under surface, and forming numerous plexuses, coarse and fine, some carrying small groups of nerve cells, end chiefly in the medulla, though some pass on to the cortex. The ultimate endings are not yet known.

The lymphatics are fairly numerous, and form plexuses in the capsule and in the connective tissue of the framework; it is stated that the lymphatic vessels surrounding the groups of cells in the cortex communicate with spaces between the cells.

$\S 412$. Besides the ordinary proteid and other chemical constituents, the supra-renal body contains some substance or substances possessing striking color reactions, giving a dark-blue or dark-green color with ferric chloride, and a carmine-red tint with various oxidizing agents. This substance (whose nature is not exactly known, and which is confined to or most abundant in the medulla) is not soluble in the ordinary solvents of pigments, such as alcohol, ether, chloroform, etc., but is readily soluble in dilute acids.

Among the extractives, hippuric and benzoic acid, and taurocholic acid or taurin have been found, but it is not certain that these are normal constituents.

$\$ 413$. Some of the histulogical features of the supra-renal bodies, namely, the groups of cells and their abundant blood-supply, suggest, on the one hand, that important metabolic processes take place in them, some of which are probably connected with the history of the pigments of the body at large. On the other hand, the unusually large nerve-supply, and the derivation of part of the body from the sympathetic ganglia, suggest peculiar nervous connections. And the organ has often served as a startingpoint for speculations in these two directions; but our exact knowledge concerning them is very limited. The results of experiment have taught us little; extirpation, for example, has been often followed by the death of the animal operated upon, but the cause of the death in such cases is by no means clear.

One fact, gained by clinical experience, is the only real item of knowledge which we possess. Disease of the supra-renal bodies, apparently tubercular in nature and beginning in the medulla, is so often associated with a change in the color or with an increase of the pigment of the skin"bronzed skin," "Addison's disease" - that some connection between the two must exist; but the several links of the chain are as yet unknown. It is tempting to associate the increase of pigment in the bronzed skin with the chromogen or color-yielding substance spoken of above; but we have no warrant for doing so, such, for instance, as any indication of ties between the supra-renal bodies and changes either in hæmoglobin itself or in bilirubin, which two bodies we have reason to regard more particularly as mothers of pigment. Moreover the bronzed skin is only one of the symptoms of Addison's disease, failure of nutrition and nervous symptoms being also present.

$\$ 414$. The thymus. This, though it arises in the embryo as a paired outgrowth from the epithelial walls of a pair of visceral clefts, and thus begins as an epithelial structure into which mesoblastic elements subsequently intrude, soon puts on such characters as to appear essentially a lymphatic structure, and, indeed, might be regarded as a part of the lymphatic system. 
It consists of a capsule of connective tissue, plain muscular fibres being absent, and septa or trabeculie of the same nature which divide the organ into a number of irregular more or less cylindrical anastomosing follicles or lobules, and send finer radiating. septa into the interior of each lobule. These lobules present the same characters throughout the whole mass of the organ, there not being, as in a lymphatic gland, any distinction between a cortex and a medulla of the whole body. The words are, however, applied to each lobule, to distinguish the central from the peripheral part of the lobule itself. Both the central medulla and the peripheral cortex of each lobule consist of a framework of reticular connective tissue, which in the cortex is identical with or closely allied to adenoid tissue, but in the medulla is coarser and more open and to a larger extent composed of branched anastomosing epithelioid cells. The meshes of the cortex are crowded with leucocytes, but these are much less abundant in and more easily fall out of the medulla, so that in sections the medulla appears more transparent than the cortex. It will be observed that this arrangement is almost the reverse of that obtaining in the alveolus of a lymphatic gland, in which the finer gland substance with its adenoid tissue crowded with leucocytes is placed in the centre, suirounded by the more open network of the lymph sinus.

The bloodvessels of the thymus rumning along the septa form capillary networks which, though closer and more abundant in the cortical than in the medullary portions of the lobules, have no such special arrangements as obtains in lymphatic glands.

Lymphatic vessels, abundant in the capsule and septa, are undoubtedly in connection with the substance of the lobules.

The medullary substance frequently contains bodies, known as "concentric capsules," nests of concentrically disposed nucleated flattened epithelial or epithelioid cells. They appear to arise from a proliferation of the epithelioid cells lining small bloodvessels, and have been supposed to be connected with the degenerative changes by which, with obliteration of the vessels, the whole organ dwindles away soon after birth.

$\S 415$. From the thymus there mav be extracted by means of saline solution a form of globulin or a proteid allied to globulin which, like the corresponding bodies from lymphatic glands or from leucocytes, seems to have some special relations to the formation of fibrin. Thus, as has already been said ( $\$ 22$ ), a solution of this globulin-like body from the thymus, injected into the veins will give rise to extensive intra-vascular clotting.

The thymus, like the other bodies on which we are now dwelling, is also rich in extractives. Thus xanthin, hypoxanthin, leucin, lactic, succinic and other acids have been found in it.

But of what really takes place in the body we have no exact knowledge. Since the thymus is best developed before birth, disappearing after birth at a rate which varies much in different individuals and still more in different kinds of animals, and being eventually replaced by fat and connective tissue, it is obvious that its chief functions are in some way associated with events taking place before birth or in early life.

\section{The History of Fat. Adipose Tissue.}

$\$ 416$. Globules of fat of various sizes make their appearance in the very elements of most of the tissues, in muscular fibres, in epithelial cells, in nerve cells, in leucocytes, and so on; and the medulla of medullated nerves consists largely of a peculiar fatty material. Besides this certain cells of connective tissue at various times, and in various places, become so 
loaded with fat that groups of the cells become practically masses of fat. Connective tissue thus loaded with fat is called adipose tissue; and masses of adipose tissue of all manner of sizes and of shapes adapted to the several situations are found in various parts of the body. Many of the internal organs, more especially the kidneys, are wrapped in adipose tissue ; but the largest deposit is one lying in the subcutaneous tissue, sometimes called the "panniculus adiposus"; and a "fat" body is distinguished from a "lean" body chiefly, though by no means exclusively, by the amount of subcutaneous adipose tissue.

Of all the tissues of the body adipose tissue is the most fluctuating in bulk; within a very short space of time a large amount of 'adipose tissue may disappear, and within an almost equally short time the quantity present in a body may be several times multiplied. When too much or too little food is given it is the subsequent adipose tissue which first and most rapidly increases or decreases in bulk.

$\$ 417$. A small piece of adipose tissue, examined under a low power, appears to be made up almost entirely of rounded masses of highly refractive material, closely packed together. These rounded masses, which stain an intense black with osmic acid and give other reactions of fat, are arranged in irregular lobules; between the lobules, and between the individual rounded masses, may be seen a small amount of fibrillated connective tissue carrying bloodvessels.

When the tissue has been hardened and stained, and the fat has been removed by solvents, what was previously only visible as a rounded mass of fat is now seen, under higher powers, to be a cell, but a cell nearly the whole of the cell substance of which has become transformed into a single large vacuole. Over the greater part of the circumference of the cell the cell substance is reduced to a mere thin shell or envelope, or cell membrane, but at one part a thicker disc-like remnant is seen, and in this is placed a rounded or oval, often flattened nucleus. Between these fat-cells may be seen a few bundles of connective tissue forming a scanty loose network, the rounded meshes of which are occupied by the fat-cells, the matrix of the bundles appearing at places continuous with, or adherent to, the envelopes of the cells; ordinary connective-tissue corpuscles are also here and there present, though rarely visible between the larger, $50 \mu$ to $130 \%$, fat-cells. In injected specimens it is further seen that the connective-tissue meshwork carries small bloodvessels, which form capillary networks around the groups of fat-cells and even around individual cells. After death, upon cooling, the fat in the fat-cells may solidify in crystals.

It is obvious that a fat-cell is a cell belonging to connective tissue, in the cell substance of which fat has been collected to such an extent that the cell, which increases largely in bulk during the process, is almost wholly transformed into a large vacuole filled with fat, the cell substance being reduced to a thin envelope of the vacuole, thickened at one part where the nucleus, thrust on one side by the gathering fat, is placed. Adipose tissue is a collection of such fat-cells held together by a meagre quantity of vascular connective tissue.

By studying the development of adipose tissue in the embryo or elsewhere, we may trace out the steps of the formation of the fat-cells. In the embryo, in a situation where adipose tissue is about to be formed, the connective tissue is seen to contain a number of small nucleated cells, rounded or somewhat irregular in form, the cell substance of which at first presents no special characters, and contains not more than what may be called the ordinary amount of fat globules or spherules. Very soon, however, these minute drops or specks increase in number, the cell substance at the same 
time increasing in bulk while remaining round or becoming more distinctly so, and the smaller drops run together into larger ones [Fig. 118]. This goes on: the fat increasing in quantity coalesces more and more, and the cell, as a whole, becomes larger and larger, the cell substance at first keeping up in bulk with the increasing fat, but subsequently ceasing to increase, being apparently used up in the formation of the fat. 'Thus the original small "protoplasmic" cell is at last transformed into the larger fat-cell, all the fat having run together into a vesicle the envelope of which, thickened on one side to carry the nucleus, is furnished by the remnant of the cell substance. In some cases, the nucleus instead of being pushed early on one side, remains central though the collection of fat has become considerable; it is, however, eventually displaced. The whole process appears very similar to the deposition of mucin in the cells of a mucous gland $(\$ 205)$; and we

[FIG. 118.

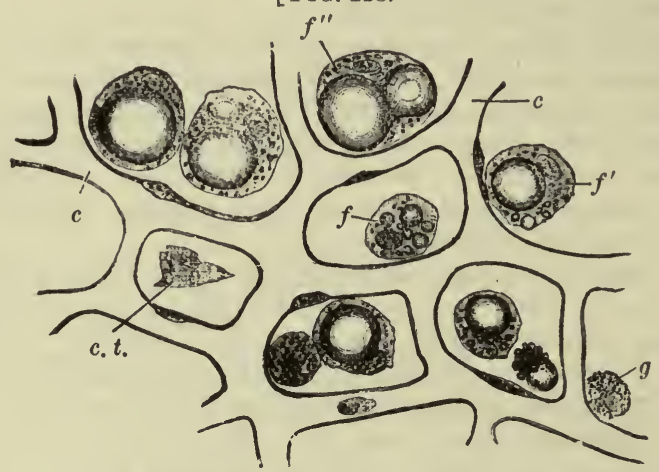

Deposition of Fat in Connective-tissue Cells : $f$, a ccll with a few isolated fat-droplets in its protoplasm; $f^{\prime}$, a cell with a single large and several minute drops ; $f^{\prime \prime}$, fusion of two large drops: $g$, granular or plasma cell, not yet exhibiting any fat deposition ; c.t., flat connective-tissue corpuscles; c. c., network of capillaries.]

may by analogy infer that the fat-cell becomes a fat-cell by the cell manufacturing fat in some way or other, and depositing the fat so formed in the interstices of its substance. The most striking superficial distinctions seem to be that in the mucous cell the granules or spherules remain discrete within the cell, being separated by bars of cell substance, whereas in the fatcell the globules, as they form, run together until at last they unite into a single mass; and further that while in the mucous cell, even when most heavily loaded, a relatively large amount of active cell-substance still remains, in the fat-cell a mere remnant is left and that chiefly surrounding the displaced nucleus.

Some observers are of opinion that the cells belonging to connective tissue which thus become fat-cells of adipose tissue belong exclusively to the kind which we spoke of as plasma cells, but this is doubtful. Others again, while admitting that the cells which become fat-cells resemble in appearance ordinary connective-tissue corpuscles and may like them be branched, believe them nevertheless to constitute a special kind of connective-tissue corpuscle, being led to this view by the fact, that though adipose tissue is very generally distributed throughout the comnective tissue of the body, it is apt to appear in particular situations, rather than in others, and in some tracts of connective tissue never under normal circumstances makes its appearance. Others again maintain that, under favorable circumstances, any connective tissue corpuscle may become a fat-cell. 
The fat in the interior of bones forming the yellow marrow appears to have the same general structure and to be formed in the same way as the rest of the adipose tissue.

$\$ 418$. The fat thus deposited in a fat-cell sooner or later disappears. It is nat injected bodily into the surrounding lymph-spaces of the connective tissue, but passes away either into the blood stream or into the lymphatics by some processes not as yet fully understood. The shell of cell substance which forms the envelope of the fat-cell is probably of a differentiated nature, and may have properties which assist the escape of the fat; but on this point we have no exact knowledge. The disappearance of the fat appears to take place in two different ways. On the one hand, and this perhaps is the more ordinary method, the fat gradually disappears, little by little, and the rounded distended vesicle gradually assumes the characters of a connective-tissue corpuscle, even of a branched one. On the other hand, especially when the disappearance is rapid and total, the space previously occupied by fat becomes filled with a clear fluid resembling lymph, the fat vesicle being transformed into a lymph vesicle. This condition, however, is temporary only, the lymph is subsequently absorbed and the vesicle shrinks. At times the emptying of the cell, whether by the one method or the other, is followed by a rejuvenescence of the cell, the nucleus by division gives rise to several nuclei, and the cell divides into new cells, each of which may, under appropriate conditions, develop again into a fat-cell.

$\$ 419$. The fat thus lodged in adipose tissue varies somewhat in composition in various animals, but is chiefly composed of olein, palmitin, and stearin in varying proportions, with small quantities of the glycerin compounds of such fatty acids as butyric, capronic, caprylic, etc., together with a little lecithin and cholesterin. "The "fat" of one animal, that is, the fat thus contained in adipose tissue, differs from the fat of another animal partly by the presence of more or less of one or more of these less abundant fats, but chiefly by the proportion in which the three main fats, olein, palnitin, and stearin, are respectively present in the mixed fat. The melting-points of these three fats being different, the melting-point of the fat of the body will differ according to the relative proportions in which the three are present. Thus the subcutaneous fat of man melts at from $15^{\circ}$ to $22^{\circ}$ or higher, the fat round the kidney being firmer and not melting until $25^{\circ}$; the fat of the dog melts at about $22^{\circ}$, that of the goose at about $25^{\circ}$, of the ox at about $40^{\circ}$, and of the sheep at $50^{\circ}$, the less resistant fat of the man and dog containing relatively more olein than that of the ox or of the sheep.

$\$ 420$. When we come to consider the question, By what processes does the fat make its appearance in the fat-cell? we are brought face to face with much the same kind of problem as that which occupied us in dealing with glycogen. On the one hand we may suppose that the fat is brought to the fat-cell as fat and is in some way taken up by the cell and deposited in the cell substance with little or no change. On the other hand, we may suppose that the fat is manufactured by the fat-cell, in some such way as mucin or pepsin is manufactured by a mucous or a gastric cell, out of and by means of its cell substance, and that the process of fattening, or of producing fat in fat-cells, consists essentially in feeding and so building up the cell substance which subsequently breaks down into fat, and does not consist merely in bringing fat within reach of the cell. Which of these views is the true one, or how far are both these operations carried on in the animal body?

In support of the latter view it may be urged that, not only the more complex living substance, but, as we have nore than once urged, the simpler proteid constituent of living substance, obviously contains what we may call a fatty radicle, so that we night expect fat to be formed out of its metah- 
olism. And as a matter of fact not only in adipose tissue, but in every part of the body, living substance is continuously giving rise to and temporarily depositing in itself some amount of fat, and in what is known as fatty degeneration there seems to be evidence of the formation of fat out of proteid material.

On the other hand, we have traced the fats taken as food, and found that they pass with comparatively little change from the alimentary canal, chiefly through the intermediate passage of the lacteals, into the blood, from which they rapidly disappear after a meal. We might infer from this that an excess of fat thus entering the blood would naturally be disposed of by being simply stored up in the available adipose tissue without any further change; we can imagine that the fat not immediately wanted by the economy passes in some way from the blood to the convective tissue (the white blood-corpuscles which appear loaded with fat after a meal possibly acting as intermediaries), and that the connective-tissue corpuscles swallow the fat brought to them after the fashion of an amoba, not digesting it but simply keeping it in store until it is wanted elsewhere.

What do experiments teach on this matter?

In the first place, it is evident that in an animal fattened on ordinary fattening food, only a small fraction of the fat stored up in the body can possibly come direct from the fat of the food. Long ago in opposition to the views of Dumas and his school, who taught that all construction of organic material, that all actual manufacture of living substance or even of its organic constituents, was confined to vegetables and unknown in animals, Liebig showed that the butter present in the milk of a cow was much greater than could be accounted for by the scanty fat present in the grass or other fodder she consumed. He also urged as an argument in the same direction, that the wax produced by bees, which though having a different composition from fat may be used as an analogy, is out of all proportion to the wax or allied bodies contained in their food, consisting as this does chiefly of sugar. And it has since been shown in many ways that, in fattening animals, the fat accumulated in the body cannot be accounted for by the fat which has been taken in the food. It has been proved by direct analysis. Thus of two young pigs, as much alike as possible, of the same litter, one was killed and analyzed, the amount of fat in the body being among other things determined. The other was fattened for a certain length of time on food whose composition was known, and then killed and analyzed. It was found that for every 100 parts of fat in the food 472 parts of fat were stored up in the body during the fattening period. It is clear that fat may be formed in the body out of something which is not fat.

$\$ 421$. There are two possible sources of this manufactured fat. The carbohydrates of the food form one source. In treating of digestion ( $\$ 243)$, we referred to the possibility of carbohydrates during digestion in the alimentary canal becoming by fermentation converted into butyric acid; and we suggested that higher and more complex members of the same fatty acid series might be obtained out of carbohydrates by somewhat analogous changes, carried on, however, not in the alimentary canal by means of foreign organized ferments, but in the tissues through the activity of the tissues themselves. IVe cannot as yet trace out the steps nor can we definitely point to any particular tissues other than the fat-cells themselves as the seats of any such changes. But there can be no doubt that the carbohydrate material does in some way or other give rise to fat. A carbohydrate diet is the kind of diet most efficacious in producing an accumulation of fat in the body ; sugar or starch, in some form or other, is always a large constituent of ordinary fattening foods. 
Another source of fat is to be found in the proteids. We have seen that the urea of the urine practically represents the whole of the nitrogen which passes through the body. Now in any given quantity of urea, the amount of carbon is far less than that found in the quantity of proteid containing the same amount of nitrogen. Thus the percentage composition of the two being respectively,

\begin{tabular}{|c|c|c|c|c|c|c|}
\hline & & $\begin{array}{c}\text { Carbon. } \\
20.00\end{array}$ & $\begin{array}{c}\text { Hydrogen. } \\
6.66\end{array}$ & $\begin{array}{c}\text { Oxygen. } \\
26.67\end{array}$ & $\begin{array}{c}\text { Nitrogen. } \\
46.67\end{array}$ & Sulphur. \\
\hline & . . & .53 & 7.30 & 23.04 & 15.53 & 1.13 \\
\hline
\end{tabular}

100 grms. of urea contain about as much nitrogen as 300 grms. of proteid; but the 300 grms. of proteid contain 139 grms. $(159-20)$ more carbon than do the 100 grms. urea. Hence the 300 grms. of proteid in passing through the body and giving rise to 100 grms. of urea, would leave behind 139 grms. of carbon, in some combination or other; and this surplus of carbon, if the needs of the economy did not demand that it should be immediately converted into carbonic acid and thrown off from the body, might be deposited somewhere in the form of fat. It has been calculated that in this way 100 grms. of proteid food might furnish $24 \mathrm{grms}$. of fat. We have already seen, in treating of the action of the pancreatic juice ( $\$ 218)$, that there is evidence of a fatty element (viz., leucin, which is amido-caproic acid, and so belongs to the fatty acid series) being thrown off from the complex proteid compound in the very process of digestion ; and though, as we have said, we have no proof that this action of pancreatic juice takes place largely in the normal body, its value as an example is none the less important.

Some observers have pushed this view of the production of fat out of proteids so far as to insist that all the fat formed in the body arises in this way out of proteid material, and that when carbohydrate food gives rise to the formation of fat it does so by shielding from oxidation the carbon moiety of the proteid food taken at the same time, thus permitting it to be stored up as fat. The carbohydrate itself, they argue, never becomes fat but its presence allows fat to be formed out of proteid material. This view has obviously a very important economical bearing, since, if it be true, it is useless to increase the carbohydrate material of food for the purpose of fattening, unless a sufficient proportion of proteid material be given at the same time.

The view, however, has been proved to be untenable by several investigations carried out on different animals. It has been shown than an animal rapidly fattened on a diet consisting of proteids with much carbohydrate will store up far more fat than can possibly be accounted for by the proteids of the diet. Thus a dog, the fat in whose body had been reduced to a minimum by starvation, was fed for a period on measured quantities of proteids and carbohydrates, and killed. The amount of fat found after death in his body, making full allowance for the fat which remained after the starvation and for the fat accompanying the proteids in the meat given as food, was found to be far more than could be supplied by the carbon in the proteids of the food, even supposing that every jot of those proteids which did not go to make up the increase of the proteid "flesh" of the body taking place during the fattening was used for the purpose of forming fat. Similar experiments on geese and pigs have led to similar results; and if fat be formed in this way in the bodies of carnivora and omnivora, we may be sure that the same holds good for the bodies of herbivora. We may therefore conclude that fat can be constructed in the body on the one hand out of proteid material and on the other hand by some direct conversion of carbohydrates. 
$\S 422$. It is clear then that a construction of fat does occur in the body somewhere. What limits can we place on the degree to which this construction is carried? When the foor contains sufficient actual fat to account for the fat stored up in the body, does any construction of fat take place? In the first place we find that when the food contains abnormal fats such as are not present in the body, spermaceti for instance, or erucin (from rape-seed oil), these fats are not to be found, or are found in very small quantity, in the fat which is stored up in the body as a consequence of a large supply of that food. In the second place we may call to mind the statement previously made, that the composition of fat varies in different animals. The fat of a man differs from the fat of a dog, even if both feed on exactly the same food, fatty or otherwise. Were the fat which is taken as food stored up as adipose tissue directly and without change, recourse being had to other sources of food for the construction of fat only in cases where the fat in the food was deficient, we should expect to find that the nature of the fat of the body would vary greatly with the food. So far from this being the case, direct experiments show that the fat of the dog is, as far as composition is concerned, very largely independent of the food, that the normal constituents of fat make their appearance very much as usual, and in very much their appropriate proportion, though their proportion in the food may largely vary, and though some of them may be wholly absent. Thus in one experiment the fat of the body contained considerable quantities of stearin after a diet free from stearin, and in another preserved the normal amount of olein after a diet free from olein.

Of course it is quite possible that in such cases as these, though the stearin, or the olein, when absent from the food, was in some way or other constructed anew, yet at the same time those constituents which were present were simply stored up; and the small quantity of erucin present in the fat of the body after feeding on erucin must have been directly stored up. So also, when an animal is rapidly fattened on a diet consisting of a small quantity of proteid and a large quantity of fat, the amount of fat stored up may be too great to have come from the proteids of the diet, in which case we may infer that it was the actual fat of the food simply deposited in the fat-cells of the body. But even in this case, as more distinctly in the others, it is also open for us to suppose that all the fat taken as food was in some way or other disposed of, and that all the new fat which made its appearance was constructed anew. And the latter view is more perhaps in harmony with the histological facts previously mentioned, as well as supported by other considerations.

At the present, however, we may be content with the following conclusions : 1. Fat is actually formed in the animal body, and the fat present at any moment in the body is not exclusively, if at all, fat merely stored up from the fat of the food. 2. The carbon elements of the newly-formed fat may be supplied either from carbohydrate food, or from the carbon surplus of proteid food, or from fats taken as food which are not the natural constituents of the body-fat. 3. The fat stored up appears as fat granules or drops deposited in the cell-substance of certain cells, and the increase of the fat in the cells is accompanied first by a growth, and subsequently by a consumption of the cell substance; but, as in the analogous case of glycogen, there is no complete evidence to show whether the fat granules which appear are simply deposited by the cell substance in a more or less mechanical manner, without their forming an integral portion of that cell substance, the chief stages of the manufacture of the fat having been gone through elsewhere, or whether they arise from a breaking up, a functional metabolism of the cell substance of the fat-cell itself; the latter view is on the whole, however, the more probable. 


\section{The Mammary Gland.}

$\S 423$. Since milk is a secretion, and indeed an excretion, the mammary gland ought not to be classed as a metabolic tissue, in the limited meaning we are now attaching to those words. Yet the metabolic phenomena giving rise to the secretion of milk are so marked and distinct, have so many analogies with the purely metabolic events which take place in adipose tissue, and so strikingly illustrate metabolic events in general, that it will be more convenient to consider the matter here, rather than in any other connection.

The mammary gland, formed like a sweat gland, of which it may be considered an extreme development, by an ingrowth of the Malpighian layer of the epidermis, is a compound racemose gland, constructed after the general plan of such a gland and thus composed of branching ducts, ending in secreting alveoli.

$\$ 424$. The appearances presented by the alveoli differ widely according as the gland is one which is being used for suckling, or is one in a resting or dormant condition, that is to say, before any pregnancy at all has taken place or in the interval between two suckling periods. In the suckling gland each alveolus consists of a basement membrane, presenting the usual characters, lined with a single layer of cells leaving a wide lumen; but the appearances presented by the cells differ from time to time according to circumstances and are not the same in all the alveoli at the same time. We may, however, distinguish two conditions which, since they seem to correspond to the loaded and discharged conditions of an ordinary gland, we may call the loaded and the discharged phase respectively, conditions intermediate between the two being met with.

In the discharged phase the alveolus is lined by a layer of low cubical or even flattened cells, so that the relatively large area of the alveolus is almost wholly occupied by the lumen in which some of the constituents of the milk may still be retained. Each cell consists of granular cell substance in which is placed a rounded or oval nucleus. Sometimes the free edge of the cell is jagged and uneven as if a portion of the free border had been torn away.

In a fully loaded phase the appearances are very different. The alveolus is now lined with a layer of tall columnar cells projecting unevenly into the lumen, the outline of which is correspondingly irregular and the area of which is much reduced. While the broader base of each cell rests on the basement membrane, the other end, conical or irregular, stretches toward the centre of the lumen. Instead of one nucleus, two or even more are now present, one well formed and normal being placed nearer the base, and the others, often showing signs of breaking or degeneration, nearer the free end. Sometimes constrictions are seen whereby the free peripheral portion of the cell, including one or more of the nuclei, is apparently being separated from the basal portion in which the remaining nucleus is lodged; and occasionally portions or fragments of cells, nucleated or nucleusless, may be seen lying in the cavity of the alveolus. In the cell substance, especially toward the free border of the cell, are numerous oil globules of various sizes as well as granules or particles of other nature : some of the larger oil globules may be seen projecting from the surface as if about to be extruded from the cell ; and in the cavity of the alveolus oil globules with a thinner or thicker coating of cell substance are frequently present.

Between such a fully loaded phase and a completely discharged phase various intermediate conditions may be observed, the cells being of greater or less height, containing one nucleus only or more than one, the cell sub- 
stance occupied with a few or with many oil globules and other granules, and the free border more or less jagged.

$\S 425$. The dormant resting mammary gland, that for instance of an animal which has never been pregnant, is much smaller than a suckling gland, owing to the alveoli being both smaller and less numerous. Each alveolus, moreover, is not a cavity lined with a single layer of epithelium, but a solid cylinder or mass of comparatively small, rounded or polyhedral cells. So long as pregnancy does not occur the growth of these is exceedingly slow, and the products of such metabolism as goes on in them are carried away by the blood, so that under normal circumstances no secretion takes place.

When pregnaney occurs rapid growth of the mamma takes place, numerous new alveoli being formed by budding, but all for a time remaining solid cylinders of cells. At the approach of the birth of the offspring, the central cells undergo metabolic changes, especially a fatty transformation, and either before or after birth are cast off, leaving a single layer to line the alveoli and to carry on the work of secretion as described above. It is generally supposed that these shed cells supply the so-called "colostrum corpuscles" characteristic of the first milk, of which we shall speak presently. At the end of lactation an absorption of some of the alveoli takes place, and in old age still further absorption goes on with great diminution of the lumina.

$\$ 426$. The connective tissue, joining together the lobules of various sizes, surrounding the lobules and running in between the projecting blind ends of the alveoli within the lobules, is rich in bloodvessels, which form capillary networks round the alveoli; it also carries a considerable number of lymphatic vessels which arise in lymph-spaces around the alveoli and elsewhere. Leucocytes are numerous in the spaces of this connective tissue, and some of them may make their way through the basement membrane and between the secreting cells into the cavities of the alveoli and so appear in the milk.

$\$ 427$. The nature of milk. Human milk has a specific gravity of from 1028 to 1034 , and when quite fresh possesses a slightly alkaline reaction. It speedily becomes acid; and cow's milk, even when quite fresh, is sometimes slightly acid, the change of reaction taking place during the stagnation of the milk in the mammary ducts.

The constituents of milk are:

1. Proteids, viz., casein, and an albumin, agreeing in its general features with ordinary serum-albumin, but which, since it is said to differ somewhat in its solubilities and rotary power from serum-albumin, has been called lactalbumin. The casein, as we have seen ( $\$ 193)$, undergoes through the action of rennin a change whereby insoluble casein (tyrein) makes its appearance and the milk is curdled. Casein may, however, be precipitated in an unchanged form by saturating milk with neutral salts, or by the careful addition of acetic acid to diluted milk, or by first adding to the diluted milk a slight quantity of acetic acid and then passing through it a stream of carbonic acid. In the filtrate the presence of the lactalbumin, which occurs in small and variable quantities, may be shown by coagulation with heat, or by precipitation with potassium ferrocyanide, etc. In the process of curdling the casein, as stated in $\$ 193$, appears to be not simply changed into tyrein, but to be split up into tyrein and into another proteid, which unlike the lactalbumin is not coagulated by heat and which appears to be allied to peptone or albumose. This or a similar peptone-like body has also been found in small quantities even in milk which has not curdled; it has been called lactoprotein. The lactalbumin, though coagulated by heat when 
isolated, is not so coagulated as it exists in the natural milk, the alkalinity of the milk, which is increased by boiling, preventing this. Sinilarly casein, though coagulited by heat when simply suspended in water after being precipitated, is not coagulated by heat when it exists in a natural condition in milk; in these respects casein behaves like alkali-albumin, which it resembles in other features also. Hence nilk when boiled does not coagulate as a whole, though in the superficial layers exposed to the air changes take place by which a film or skin, derived chiefly from the albumin but partly from the casein, appear's on the surface; if this be removed a fresh portion undergoes the same change.

2. Fats. 'These are, in the main, palmitin, stearin, and olein; but other fats, supplied by butyric and other fatty acids in combination with glycerin, accompany the above in small quantities. In this respect the fat of milk resembles that of adipose tissue. Lecithin and cholesterin are also present in very small quantity, as well as a yellow coloring matter. The fat present in milk differs in different animals as to the relative proportion of olein, palmitin, and stearin, and as to the kinds and relative amount of the other scantier fats.

'The mixture of these fats, fluid at ordinary temperatures, is present in natural milk in the form of globules of various sizes but for the most part exceedingly small (in man from $2 \mu$ to $5 \mu$ ). Milk is in fact a typical emulsion, and it is the presence of the casein in the milk which brings about the emulsion.

On standing a great deal of the fat collects on the top of the milk in the form of cream, but in this, as in the butter which is formed from it, the globules are still discrete, so long at least as the butter is "fresh." By the use of a centrifugal machine nearly the whole of the fat may be separated from the plasma.

3. Milk sugar or lactose. This is very apt to undergo fermentation into lactic acid, through the agency of an organized ferment; the milk thus becomes sour, and the casein is precipitated in a flocculent form when the acid is produced in sufficient quantity. Since the change will take place even when every care is taken to exclude germs from the atmosphere having access to the milk, the organized ferments must be present in the milk in the ducts of the gland.

4. Salts. Though traces of urea and kreatinin have been noted by some observers, the extractives of milk, beyond the lecithin and cholesterin already mentioned, are insignificant. 'The salts are of more importance; these are chiefly calcic phosphate, of whose function in the process of curdling we spoke in $\S 193$, and potassic and sodic chlorides, with a small quantity of magnesic phosphate. Sulphates appear to be absent. A small quantity of an iron salt is present, and traces of sulphocyanide have been observed. Besides the phosphorus in the actual form of phosphates, milk contains a further considerable quantity of phosphorus in the proteids and in the nuclein, as well as some sulphur in the former. The inorganic constituents of milk may, broadly speaking, be said to differ distinctly from those of blood, and to much more nearly resemble those of the entire body.

The composition of milk in the same animal varies widely from time to time, and besides undergoes marked changes during the period of lactation. The relative general composition of human milk and that of the cow, the mare, and the bitch may perhaps be shown by the following table; but it is difficult to draw an average, since the individual analyses given differ so much ; the figures given for casein and fat in the milk of the bitch may be unusually high. 


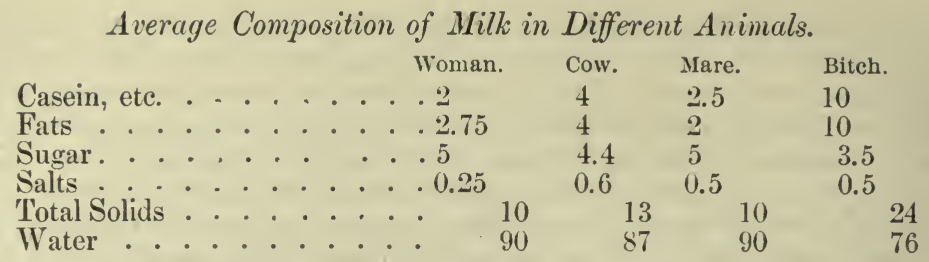

The quantity of milk secreted by a woman in twenty hours at the height of lactation has been calculated at 700 to 800 c.c. A good milch cow will yield about 10 litrés of milk per diem.

$\$ 428$. Colostrum. This is the name given to the milk secreted at the beginning of a period of lactation, just before and for some days after parturition. This milk differs from the subsequent milk in microscopical characters and in chemical composition.

When ordinary milk is examined under the microseope hardly anything is seen besides the fat globules except a very few imperfect cells or portions of cells, consisting of cell substance more or less loaded with fat and containing sometimes a more or less altered nucleus. A few minute granules, thought by some to be particles of suspended casein or nuclein, are, however, also visible.

Colostrum, on the other hand, contains a large number of cells or corpuscles, which have been called "colostrum corpuscles." Some of these closely resemble leucocytes, others are either cells of about the same size, round or irregular, and possessing a nucleus, often misshapen, or are merely portions of cell substance without a nucleus. In all of them the cell subtance may be loaded with fat globules or may be fairly free from fat. Some of these cells appear to be undergoing disintegration; some may at a favorable temperature exhibit slow amoboid movements, and must then at least be regarded as living.

Colostrum also differs from ordinary milk in containing not only a large quantity of albumin (lactalbumin), but also a decided amount of globulin. In consequence of this, colostrum differs from milk, inasmuch as it is distinctly coagulated by heat.

As stated above, during the rapid growth by which the gland is enlarged preparatory to lactation, the alveoli are at first solid masses of cells with little or no lumen, and a lumen is established subsequently by the discharge of the central cells. It is usually supposed that the cells so discharged, some undergoing much, others comparatively little, change, supply the colostrum corpuscles just spoken of, and at the same time furnish the globulin and excess of albumin also characteristic of colostrum. But this is not certain. The alveoli at this time contain peculiar cells resembling colostrum corpuscles except that they are free from fat; and it is suggested that these being discharged and taking up fat in amoboid fashion become colostrum corpuscles. Some regard the colostrum corpuscles as simply leucocytes which have similarly taken up fat.

$\$ 429$. The mammary gland is present both in the female and the male child at birth ; and in both sexes at and for a few days after birth is thrown, in common with all the other secreting glands, into secretory activity, and a small quantity of milk, the "witches' milk," so called by the Germans, is discharged from the nipple. The milk resembles in all essential features the milk of lactation. In both sexes this initial activity soon passes off, the gland in the female further developing at puberty, but in the male remaining, save in exceptional cases, in its infantile condition or somewhat retrograding. 
$\$ 430$. The secretion of milk. From what has been already said, it is obvious that the secretion of milk, while resembling the secretion of the other secreting glands which we have studied in being essentially an activity of the epithelial cells lining the alveoli, nevertheless presents certain interesting features special to itself. If the account given in $\S 424$ be a true one, morphological changes in the cells are more prominent than in the case of other glands; and we may interpret the appearances there related somewhat as follows: When the discharged gland with its low epithelium begins the work of loading, the cells distinctly "grow." Their cell substance increases in bulk and, elongating, projects into the lumen of the alveolus. At the same time the nucleus divides as if the cell were about to give birth to new cells; but at first, at all events, no division of the cell substance takes place, and the new nuclei lie imbedded in a common cell body. The cell substance meanwhile puts on secretory activity ; it deposits in itself material to form milk. The deposit of fat is conspicuous and easily recognized, but we may fairly infer that the other less easily distinguished proteid and carbohydrate materials are deposited in the cell substance in a similar fashion. 'Then follows the ejection of the prepared material, and this may take place in one of two ways. The oil globules of fat may be extruded from the cell substance much in the same way that an amœba extrudes its excrement, and possibly other constituents of milk may be ejected by a similar method. But, besides this, the deferred cell division now takes place in a somewhat imperfect fashion, so that portions of the old cell carrying nuclei with them come asunder from the rest of the cell in which a nucleus is left, and lie loose in the lumen of the alveolus; portions of cell substance free from nuclei appear also to be cast off. Here, in the lumen of the alveolus, they rapidly undergo change ; the cell substance is altered and dissolved, and its load of prepared material, probably undergoing in the act some further change, is set free, the nuclei also undergoing change and becoming ultimately broken up. Hence the constituents of milk are provided for, not only as in other glands by the material with which the cell loads itself and subsequently discharges into the lumen of the alveolus, but also by the actual substance of part of the cell itself. The characteristic nuclein of the milk has thus its origin in all probability in the shed nuclei of the secreting cells, and we may perhaps infer that the still more characteristic casein exists in milk in the form of casein and not of some other proteid in consequence of this intervention of the actual cell substance in the formation of the milk.

$\S 431$. The secretion of milk then would appear to illustrate, even more fully and clearly than do other glands, the truth on which we have so often insisted, that a secretion is eminently the result of the metabolic activity of the secreting cell. The blood is the ultimate source of milk, but it becomes milk only through the activity of the cell, and that activity consists largely in a metabolic manufacture by the cell, and in the cell, of the common things brought by the blood into the special things present in the milk. Experimental results tell the same tale. Thus the quantity of fat present in milk is largely and directly increased by proteid, but not increased-on the contrary diminished-by fatty food. This effect on the mammary gland in particular is in accordance with what we shall presently learn to be the general effect on the body of proteid in contrast to that of fatty food; proteid food seems to increase the general metabolic activity of the body, while fatty food tends to lessen it. Moreover the proteid food seems actually to furnish the fat; and we have already suggested a manner in which proteids may give rise to fat. That the fat of the milk need not necessarily come from the fat of the food is shown by the following experiment: A bitch 
fed on meat for a given period gave off more fat in her milk than she could possibly have taken in her food; and this moreover took place while she was gaining in weight and "laying on fat," so that she could not have supplied the mammary gland with fat by simply transferring fat from the store previously existing in the adipose tissue of her body ; she apparently obtained the fat ultimately from the proteids of her food. And the histological facts given above favor the view that the formation of fat out of proteids in such cases takes place in the cells of the alveoli. The experimental then as well as the histological evidence goes to show that the fat of milk is formed in the cell and by the cell, and is not sinuply gathered out of the blood.

The casein in a similar way seems to be formed by the action of the cell. It cannot be gathered out of the blood, since the blood contains no real casein ; it must be formed in the gland. Some observers have maintained that when milk is kept at $35^{\circ}$, the casein is increased through some ferment action taking place in the milk itself; but this seems not to be the case, and the formation of casein must be regarded as the result of the action of the cell. Even the albumin present appears to be not the ordinary serumalbumin simply passed from the blood through the cell into the lumen of the alveolus, but the slightly different lactalbumin. We may perhaps regard the albumin as less difficult to manufacture than the casein; and we may explain the fact that relatively to the albumin the casein is less at the very beginning and especially toward the end of lactation, by supposing that the cell has in the first case not got into full working order, and in the second case is waning in power. The peptone-like body in milk, though small in quantity, is a further indication of the proteid metabolism taking place in the cell.

That the milk sugar, lactose, also is formed in and by the cell, is indicated by the facts that it is found in no other part of the body, and that its presence in milk is not dependent on carbohydrate food, for it is maintained in abundance in the milk of carnivora when these are fed exclusively on meat, as free as possible from any kind of sugar or glycogen. A glycogen-like body has moreover been described as existing in the cells, and it is suggested that this body is the antecedent of the lactose.

We thus have evidence in the mammary gland of the formation, by the metabolic activity of the secreting cell, of the representatives of the three great classes of food-stuffs, proteids, fats, and carbohydrates.

$\S 432$. That both the secretion and ejection of milk are under the control of the nervous system is shown by common experience, but the exact nervous mechanism has not yet been fully worked out. While the erection of the nipple ceases when the spinal nerves which supply the breast are divided, the secretion continues, and is not arrested even when the sympathetic as well as the spinal nerves are cut.

\section{CHAPTER V.}

\section{NUTRITION.}

\section{The Statistics of Nutrition.}

$\S 433$. THE preceding chapter has shown us how wholly impossible it is at present to master the metabolic phenomena of the body, by attempting to trace out, forward or backward, the several changes undergone by the individual constituents of the food, the body, or the waste products. Another 
method is, however, open to us-the statistical method. We may ascertain the total income and the total expenditure of the body during a given period, and by comparing the two may be able to draw conclusions concerning the changes which must have taken place in the body while the income was being converted into the output. Many researches have been carried out by this method; but valuable as are the results which have been therebv gained, they must be received with caution, since in this method of inquiry a small error in the data may, in the process of calculation and inference, lead to wrong conclusions. The great use of such inquiries is to suggest ideas, but the views to which they give rise need to be verified in other ways before they can acquire real worth.

Composition of the animal body. The first datum we require is a knowledge of the composition of the body, as far as the relative proportion of the various tissues is concerned. In the human body the proportions by weight of the chief tissues, in the fresh state, are probably somewhat as follows:

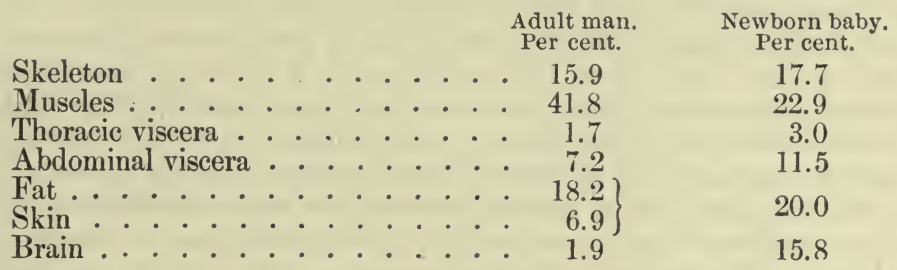

An analysis of a cat has given the following result:

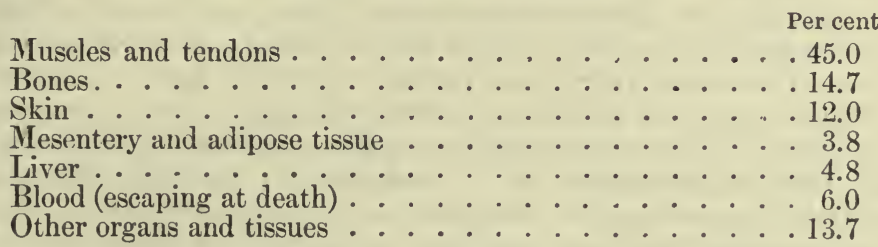

One point of importance to be noticed in these analyses is that the skeletal muscles form nearly half the body; we have already seen ( $\$ 38)$ that about a quarter of the total blood in the body is contained in them, and have already ( $\$ 398)$ insisted that a large part of the metabolism of the body is carried on in the muscles. Next to the muscles we must place the liver, for though far less in bulk than them, it is subject to a very active metabolism; this is suggested by the fact that it alone may hold about a quarter of the whole blood, and is also indicated by the numerous facts brought before us in the preceding chapter.

$\$ 434$. The starving body. Before attempting to study the influence of fond, it will be useful to ascertain what changes occur in the body when all food is withheld. A cat of known weight was starved for thirteen days. At the beginning of the period the body was presumed to have the composition above given; at the close of the period a direct analysis of the body was made. From this it appeared that during the hunger period the cat hand lost 734 grammes of solid material, of which 248.8 were fat and 118.2 muscle, the remainder being derived from the other tissues. The percentages of dry solid matter lost by the more important tissues during the period were as follows: 
Adipose tissue . . . . . . . . . . . . . 97.0

Spleen. . . . . . . . . . . . . . . . . 63.1

Liver . . . . . . . . . . . . . . . . 56.6

Muscles . . . . . . . . . . . . . . . . . . . . 30.2

Blood . . . . . . . . . . . . . . 17.6

Brain and spinal cord . . . . . . . . . . . . 0.0

Thus, the loss during starvation fell most heavily on the fat, indeed nearly the whole of this disappeared. Next to the fat, the glandular organs, the tissues which we have seen to be eminently metabolic, suffered most. Then come the muscles, that is to say, the skeletal muscles, for the loss in the heart was very trifling; obviously this organ, on account of its importance in carrying on the work of the economy, was spared as much as possible; it was, in fact, fed on the rest of the body. The same remark applies to the brain and spinal cord; in order that life might be prolonged as much as possible, these important organs were nourished by material drawn from less. noble organs and tissues. The blood suffered proportionately to the general body-waste, becoming gradually less in bulk, but retaining the same specific gravity; of the total dry proteid constituents of the body, 17.3 per cent. was lost, which agrees very closely with the 17.6 per cent. dry material (almost wholly proteid) lost by the blood. It is worthy of remark that the tissues in general become more watery than in health. Similar observations on other animals have led to sinilar results, the chief discordance being that in some cases the bones have suffered considerable loss, in others comparatively little. We might be inclined to infer from these data the conclusions that metabolism is most active in the adipose tissue, next in such metabolic tissues as the hepatic cells and spleen-pulp, then in the muscles, and so on; but we have no warrant for these conclusions. Because the loss of cardiac and nervous tissue was so small, we must not therefore infer that their metabolism was feeble; they may have undergone rapid metabolism, and yet have been preserved from loss of substance by their drawing upon other tissues for their material. The great loss of adipose tissue is obviously to be explained by the fact that that tissue is essentially a storehouse of material, and the similarly great, though less, loss in the spleen and liver indicates, as, indeed, the facts recorded in the previous chapter suggest, that these organs, too, serve in part as storehouses.

During the starvation period, the urine contained, in the form of urea (and that practically represents all the nitrogen of the urine), 27.7 grammes of nitrogen. Now, the amount of muscle which was lost during the period contained about 15.2 of nitrogen. Thus, more than half the nitrogen of the output during the starvation period must have come ultimately from the metabolism of muscular tissue. This fact we have already used in discussing the history of urea, and shall have occasion to make further use of it hereafter. The amount of urea excreted per diem has been observed, in some cases, to fall very rapidly during the first day or two of starvation, and then to diminish gradually, though often showing considerable irregularities. In other cases no such large initial fall has been observed. It is most marked in animals which have been well fed before the beginning of the starvation, especially in those which have had a rich nitrogenous diet; and the discharge, in these cases, of an extra quantity of urea in the first day or two is obviously connected with that immediate effect of food on the excretion of urea, to which we have already ( $\$ 401)$ referred, and to which we shall have to return in speaking of what is known as "luxusconsumption." 


\section{Comparison of Income and Output of Material.}

$\$ 435$. Method. We have now to inquire how the elements of food are distributed in the excreta, in order that, from the manner of the distribution, we may infer the nature of the intermediate stages which take place within the body. By comparing the ingesta with the excreta, we shall learn what elements have been retained in the body, and what elements appear in the excreta which were not present in the food; from these we may infer the changes which the body has undergone through the influence of the food.

In the first place, the real income must be distinguished from the apparent one by the subtraction of the feces. We have seen that by far the greater part of the feces is undigested matter, $i$. $e$., food which, though placed in the alimentary canal, has not really entered into the body. The share in the feces taken up by matter which has been excreted from the blood into the alimentary canal, is so small that it may be negleeted; certainly, with regard to nitrogen, the whole quantity of this element, which is present in the feces, may be regarded as indicating simply undigested nitrogenous matter.

The income, thus corrected, will consist of so much nitrogen, carbon, hydrogen, oxygen, sulphur, phosphorus, saline matters, and water, contained in the proteids, fats, carbohydrates, salts, and water of the food, together with the oxygen absorbed by the lungs, skin, and alimentary canal. The output may be regarded as consisting of (1) the respiratory products of the lungs, skin, and alimentary canal, consisting chiefly of carbonic acid and water, with small quantities of hydrogen and carburetted hydrogen, these two latter coming exclusively from the alimentary canal ; (2) of perspiration, consisting chiefly of water and salts, for the dubious excretion (see $\$ 366$ ) of urea by the skin may be neglected, and the other organic constituents of sweat amount to very little; and (3) of the urine, which is assumed to contain all the nitrogen really excreted by the body, besides a large quantity of saline matters, and of water. Where great accuracy is required, the total nitrogen of the urine ought to be determined; it is maintained, however, that no errors of serious importance arise when the urea alone, as determined by Liebig's method (which was largely used in the researches forming the basis of the present discussion), is taken as the measure of the total quantity of nitrogen in the urine, since, in this method, other nitrogenous bodies besides urea are precipitated, and so contribute to the quantitative result. It has been, and, indeed, still is, debated whether the body may not suffer loss of nitrogen by other channels than by the urine and feces, whether nitrogen may not leave the body by the skin, or, indeed, in a gaseous state, by the lungs. The balance of the conflicting evidence seems, however, in favor of the view that no such loss takes place. It would appear that though nitrogen, the pivot, so to speak, of the chemical changes of living beings, forms so large a portion of the atmosphere, and, moreover, is physically diffused through the bodies of both plants and animals, free nitrogen is of no chemical use to either of them. It enters into and remains in their bodies as an inert substance, and the nitrogen which leaves a plant or animal, in a gaseous state, is simply a part of the same inert supply, and does not come from the breaking up of the nitrogenous substances of the body or of the food.

Of these elements of the income and output, the nitrogen, the carbon, and the free oxygen of respiration are by far the most important. Since water is of use to the body for merely mechanical purposes, and not solely as food in the strict sense of the word, the hydrogen element becomes a 
dubious one; the sulphur of the proteids and the phosphorus of the fats are insignificant in amount; while the saline matters stand on a wholly different footing from the other parts of food, inasmuch as they are not sources of energy, and pass through the body with comparatively little change. The body-weight must, of course, be carefully ascertained at the beginning and at the end of the period, correction being made where possible for the feces.

It will be seen that the labor of such inquiries is considerable. The urine, which must be carefully kept separate from the feces, requires daily measurement and analysis. Any loss by the skin, either in the form of sweat, or, in the case of woolly animals, of hair, must be estimated or accounted for. The food of the period must be, as far as possible, uniform in character, in order that the analyses of specimens may serve faithfully for calculations involving the whole quantity of food taken; and this is especially the case when the diet is a meat one, since portions of meat differ so much from each other. But the greatest difficulty of all lies in the estimation of the carbonic acid produced and the oxygen consumed. In some of the earlier researches this factor was neglected, and the variations occurring were simply guessed at, through which very serious errors were introduced. No comparison of income and output can be considered satisfactory unless at least the carbonic acid produced be directly measured by means of a respiration chamber. And in order that the comparison should be really complete, the water given off by the skin and lungs must be directly measured also; but this seems to be more difficult than the determination of the carbonic acid.

In the plan originally adopted by Regnault and Reiset, and followed by some other observers, the animal experimented on is allowed to breathe a limited and measured atmosphere. The carbonic acid, as fast as it is formed, is fixed and removed by a strong solution of caustic potash, and the normal percentage of oxygen in the atmosphere is maintained by a supply of this gas from a gas-holder. In this way both the oxygen consumed and the carbonic acid produced are directly determined, while the continual supply of fresh oxygen prevents any evil effects due to breathing a confined portion of air. In order, however, to avoid all possible errors arising from a too restricted atmosphere, a different method has been adopted by Pettenkofer and Voit. Their apparatus consists essentially of a large chamber, capable of holding a man comfortably. By means of a steam-engine a current of pure air, measured by a gasometer, is drawn through the chamber. Neasured portions of the outgoing air are from time to time withdrawn and analyzed; and from the data afforded by these analyses the amounts of carbonic acid (and other gases) and of water given off by the occupant of the chamber during a given time are determined. The oxygen consumed is not determined directly; but if the total amounts of carbonic acid and of water given out by the lungs and skin are ascertained, and the amount of urine and feces known, the quantity of oxygen consumed may be arrived at by a simple calculation. For evidently the difference between the terminal weight plus all the egesta and the initial weight plus all the ingesta can be nothing else than the weight of the oxygen absorbed during the period. This method in turn, however, is also open to objections, since minute errors in the analyses of the small samples of air employed for the determinations attain considerable dimensions when these are multiplied so as to give the changes in the whole mass of air passed through the apparatus. It seems, moreorer, undesirable to leave the quantity used of so important an element as oxygen to be determined by indirect calculations.

Let us imagine, then, an experiment of this kind to have been completely carried out; that the animal's initial and terminal weights have been accurately determined; the composition of the food satisfactorily known to consist of so much proteid, fat, carbohydrates, salts, and water, and to contain 
so much nitrogen and carbon; the weight of the feces and the nitrogen they contain ascertained; the nitrogen of the urine determined; the carbonic acid and water given off by the whole body carefully measured, and the amount of oxygen absorbed calculated-what interpretation can be placed on the results?

Let us suppose that the animal has gained $w$ in weight during the period. Of what does $w$ consist? Is it fat or proteid material which has been laid on, or simply water which has been retained, or some of one and some of the other? Let us further suppose that the nitrogen of the urine passed during the period is less, say by $x$ grammes, than the nitrogen in the food taken, after deduction, of course, of the nitrogen in the feces. This means that $x$ grammes of nitrogen have been retained in the body; and we may with reason infer that they have been retained in the form of proteid material. We may even go further, and say that they are retained in the form of flesh, i. e., of muscle. In this inference we are going somewhat beyond our tether, for the nitrogen might be stored up as some proteid constituent of the hepatic cells or of some other tissue; indeed, it might be for the while retained in the form of some nitrogenous crystalline body. But this last event is unlikely; and if we used the word "flesh" to mean nitrogen-holding living substance (proteid) of any kind, we may without fear of any great error reckon the deficiency of $x$ grammes nitrogen as the storing up of $a$ grammes flesh. There still remain $w-a$ grammes of increase to be accounted for. Let us suppose that the total carbon of the egesta has been found to be $y$ grammes less than that of the ingesta ; in other words, that $y$ grammes of carbon have been stored up. Some carbon has been stored up in the flesh with the nitrogen just considered; this we must deduct from $y$, and we shall then have $y^{\prime}$ grammes of carbon to account for. Now there are only two principal forms in which carbon can be stored up in the body-as glycogen or as fat. The former is, even in most favorable cases, inconsiderable, and we therefore cannot err greatly if we consider the retention of $y^{\prime}$ grammes carbon as indicating the laying on of $b$ grammes fat. If $a+b$ are found equal to $w$, then the whole change in the economy is known; if $w-(a+b)$ leaves a residue $c$, we infer that in addition to the laying on of flesh and fat some water has been retained in the system. If $w-(a+b)$ gives a negative quantity, then water must have been given off at the same time that flesh and fat were laid on. In a similar way the nature of a loss of weight can be ascertained, whether of flesh or fat or of water, and to what extent of each. The careful comparison, the debtor and creditor account of income and output, enables us, with the cautions rendered necessary by the assumptions just now mentioned, to infer the nature and extent of the bodily changes. The results thus gained ought, of course, if an account is kept of the water taken in and given out, to agree with the amount of oxygen consumed, and also to tally with the conclusions arrived at concerning the retention or the reverse of water.

Having thus studied the method, and seen its weaknesses as well as its strength, we may briefly review the results which have been obtained by its means.

$\$ 436$. Nitrogenous metabolism. When a meal of lean meat, as free as possible from fat, is given to a dog which has previously been deprived of food for some time, and whose body therefore, is greatly deficient in flesh, it might be expected that the larger part of the food would be at once stored up to supply pressing deficiencies, and that only the smaller part would be immediately worked off as urea corresponding to the nitrogenous metabolism going on in the body at the time, increased somewhat by the labor thrown on the economy by the very presence of the food. This, however, is not the 
case as far as the nitrogen of the meal is concerned; the larger portion passes off as urea at once, and only a comparatively small quantity is retained. If the diet be continued, and we are supposing the meals given to be large ones, the proportion of the nitrogen which is given off in the form of urea goes on increasing until at last a condition is established in which the nitrogen of the egesta exactly equals that of the ingesta. This condition, which is spoken of as "nitrogenous equilibrium," is attained in dogs with an exclusively meat diet only when large quantities of food are given, and it is not easily maintained for any length of time. The exact quantity of meat required to attain nitrogenous equilibrium varies with the previous condition of the dog; equilibrium is frequently attained when 1500 or 1800 grammes of meat are given daily.

Thus the most striking effect of a purely nitrogenous diet is largely to increase the nitrogenous metabolism of the body; and we shall see later on that it increases the metabolism not only of the nitrogenous but also of the other constituents of the body.

The establishment of nitrogenous equilibrium does not mean that a bodyequilibrium is established, that the body-weight neither increases nor diminishes. On the contrary, when the meal necessary to balance the nitrogen is a large one, the body though it is neither gaining nor losing nitrogen may gain in total weight; and the increase is proved by calculation from the income and output, and indeed by actual examintion of the body, to be due to the laying on of fat. 'The amount so stored up may be far greater than can possibly be accounted for by any fat still adhering to the meat given as food. We are therefore driven to the conclusion that the proteid food is split into a urea moiety and a fatty moiety, that the urea moiety is at once discharged, and that such of the fatty moiety as is not made use of directly by the body is stored up as adipose tissue. And this disruption of the proteid, as we have already ( $\$ 401)$ suggested, explains at the same time why the meat diet so largely and immediately increases the urea of the egesta.

The characteristic effect of proteid food to increase the metabolism of the body is shown on other animals besides the dog, and not only by means of calculations of what is supposed to take place in the body, but also by direct analysis. Thus the analysis of the body of a pig, which had been fed on a known diet, compared with the analysis of that of another pig of the same litter, killed at the time when the first was put on the fixed diet, gave as a result that of the dry nitrogenous material of the food only about 7 per cent. was laid up as dry proteid material during the fattening period, though the amount of proteid food was low. This contrasts strongly with the amount of fat stored up during the same period (see $\$ 420$ ). Similar observations carried out on sheep showed that in these animals the storing up of nitrogenous material was even less, only about 4 per cent. of that given in the food.

Every quantity of proteid material taken into the alimentary canal thus appears to affect proteid metabolism in two ways. On the one hand, it excites a rapid proteid metabolism giving rise to an immediate, and generally large, increase of urea; on the other hand, it serves to maintain the more regular normal proteid metabolism continually taking place in the body, and so contributes to the normal regular discharge of urea. It seems very natural to suppose that the proteid which plays the first of these two parts is not really built up into the tissues, does not become actual living substance, but undergoes the changes which give rise to urea outside the actual living substance in the blood or elsewhere ; and we have seen that under the influence of the pancreatic juice some of the proteid food may undergo the greater part of such a change while it is as yet within the alimentary canal. Hence 
has arisen the very natural distinction to which we have already alluded between "tissue proteids" or " morphotic proteids" which are actually built up into the living substance of the tissues and give rise to urea through the metabolism of living substance, and " circulating proteids" or "floating proteids" which do not at any period of their career within the body become an integral part of the living substance and by their metabolism set free energy not in the way of vital manifestations, but in the form of heat only. We shall latter on consider what is the exact meaning which we ought to attach to the words "becoming part of the living substance;" and hence shall defer until then any discussion of the appropriateness of these phrases and of the validity of the distinction which they formulate.

It was once thought, as we shall presently see, erroneously, that the exclusive purpose of proteid food was to supply the proteid tissues, and that all the energy set free in the body in vital manifestations, such as movement and the like as distinguished from heat, had its origin in proteid metabolism, the metabolism of fats and carbohydrates giving rise to heat only. Hence when it first became known that a certain proportion of proteid food apparently underwent a metabolism giving rise to heat only, without becoming part of the tissues, this seemed to be a wasteful expenditure of precious material ; and the metabolism of this portion of proteid food was accordingly spoken of as a "luxus-consumption," a wasteful consumption.

Before leaving this subject we may call attention to a possible analogy between the history of proteids and that of fats and carbohydrates. The uniform composition of the blood, which the body seems ever striving to maintain, probably applies to its proteids as well as to its other constituents. We have seen that a surplus of non-nitrogenous materials in the blood is withdrawn from the circulation and stored up as fat or glycogen, and it is possible that an excess of proteids might similarly be stored up in some tissue or tissues, in the hepatic cells for instance, though from the facts previously mentioned it is obvious that the power of storage is far less than in the case of fats and carbohydrates. Such a store of proteid matter would represent a sort of circulating proteid, but nevertheless for its final metabolism might have to form an integral part of some living tissue unit.

$\$ 437$. The effects of fatty and of carbohydrate food. Unlike those of proteid food, the effects of fats and carbohydrates cannot be studied alone. When an animal is fed simply on non-nitrogenous food, death soon takes place; the food rapidly ceases to be digested, and starvation ensues. We can therefore only study the nutritive effects of these substances when they are taken together with proteid material.

When a small quantity of fat is taken, in company with a fixed moderate quantity of proteid material, the whole of the carbon of the food reappears in the egesta. No fat is stored up; some even of the previously existing fat of the body may be consumed. As the fat of the meal is increased, a point is soon reached at which carbon is retained in the body as fat. So also with starch or sugar; when the quantity of this is small, there is no retention of carbon; as soon, however, as it is increased beyond a certain limit, carbon is stored up in the form of fat or, to a smaller extent, as glycogen. Fats and carbohydrates, therefore, differ markedly from proteid food in that they are not so distinctly provocative of metabolism. 'This is exceedingly well shown in the results obtained on the pig previously mentioned. It was found that 472 units of fat were laid on for every 100 units of fat taken as such in the food (which consisting of barley-meal, etc. contained a very small amount of actual fat), while for every 100 units of the total dry non-nitrogenous food including fat, starch, cellulose, etc., no less than 21 units were retained in the body in the form of fat. No clearer proof than this could be afforded 
that fat is formed in the body out of something which is not fat. In $\S 421$ we have already discussed this formation of fat out of carbohydrates.

As one might imagine, the presence of fat or carbohydrates in the food is found to decrease the amount of proteid material necessary to establish nitrogenous equilibrium. For instance, with a diet of $800 \mathrm{grms}$. meat and 150 grms. fat, the nitrogen in the egesta became equal to that in the ingesta in a dog, in whose case 1800 grms. meat had to be given to produce the same result in the absence of fat or carbohydrates.

On the other hand, it was found that, with a fixed quantity of fatty or carbohydrate food, an increase of the accompanying proteid led not to a storing up of the surplus carbon contained in the extra quantity of proteid, but to an increase in the consumption of carbon. Proteid food increases not only proteid but also non-nitrogenous metabolism. This explains how an excess of proteid food may, by the increase of general metabolism, actually reduce the fat of the body.

We have at present no exact information concerning the nutritive differences between fats and carbohydrates, beyond the fact that in the final combustion of the two, while carbohydrates require sufficient oxygen to combine with their carbon only, there being already sufficient oxygen in the carbohydrate itself to form water with the hydrogen present, fats require in addition oxygen to combine with some of their hydrogen. Hence in herbivora, living largely on carbohydrates, a larger portion of the oxygen consumed reappears in the carbonic acid of the egesta than in carnivora, in which animals, living chiefly on proteids and fats, more of it leaves the body combined with hydrogen to form water. This relation of the oxygen to the carbonic acid is often expressed as the quotient of the volume of the carbonic acid expired divided by the volume of the oxygen consumed, the "respiratory quotient," $\frac{\mathrm{CO}_{2}}{\mathrm{O}_{2}}$, which is in herbivora about 0.9 and in carnivora about 0.6 or 0.7 . When an herbivorous animal starves, it feeds on its own fat, and under these circumstances the respiratory quotient falls to the carnivorous standard; and indeed many circumstances affect this respiratory quotient. The carbohydrates are notably more digestible than the fats, but on the other hand the fats contain more potential energy in a given weight. As to the nutritive difference between starch and sugar, we know nothing very definite; it has been thought, however, that cane-sugar is rather more fattening than starch.

$\$ 438$. The effects of gelatin as food. It is a matter of common experience that gelatin will not supply the place of proteids as a constituent of food. Animals fed on gelatin together with fat or carbohydrates die very much in the same way as when they are fed on non-nitrogenous material alone. Nevertheless it would appear, as might be expected, that the presence of gelatin in food is not without effect. This nitrogenous equilibrium is established at a lower level of real proteid food when gelatin is added. In a dog, morenver, fed on a diet of gelatin and fat, the excess of nitrogen in the excreta over that in the ingesta is less than when the same dog is fed on a diet of fat alone; that is to say, the gelatin has sheltered from metabolism some proteid constituents of the body; and the consumption of fat seems also to be lessened by the presence of gelatin. These facts become intelligible if we suppose that gelatin is rapidly split up into a urea and a fat moiety in the same way that we have seen a certain quantity of proteid material to be. It is this direct destructive metabolism of proteid matter which gelatin can take up; it seems, however, unable to imitate the other function of proteid matter, and to take part in the formation of living substance; or in the phraseology of a preceding paragraph ( $\$ 436)$, it can take 
the place of circulating but not of tissue proteid. What is the cause of this difference we cannot at present say.

$\$$ 439. Peptone as food. Since proteids are at least largely, as we have seen $(\$ 262)$, converted into and absorbed as peptone, and since, as we have also seen, the peptone appears during the very act of absorption to be reconverted into some other form of proteid matter, possibly serum-albumin, it might seem natural to suppose that peptone given as food would, as far as metabolism is concerned, play the same part as other proteids. Nevertheless, some observers have maintained with regard to both peptones and the allied albumoses that, like gelatin, these bodies "can take the place of circulating but not of tissue proteid." On the whole, however, the evidence goes to show that animals can "lay on flesh" when the proteid in their food consists entirely of peptone or albumose. A difficulty appertaining to digestion prevents any large substitution of peptone for ordinary proteids, since as might be expected diarrhœa is apt to be set up.

$\S 440$. The effects of salts as food. All food contains, besides the substances possessing potential energy, which we have just studied, certain saline matters, organic and inorganic, having in themselves little or no such potential energy, but yet either absolutely necessary or highly beneficial to the body. These must have important functions in directing the metabolism of the body ; the striking distribution of them in the tissues, the preponderance of sodium and chlorides in blood-serum and of potassium and phosphates in the red corpuscles, for instance, must have some meaning; but at present we are in the dark concerning it. The element phosphorus seems no less important, from a biological point of view, than carbon or nitrogen; it is as absolutely essential for the growth of a lowly being like penicillium as for man himself. We find it probably playing an important part as the conspicuous constituent of lecithin and other complex fats belonging to the nervous system; we find it prominent in the peculiar body nuclein; we find it peculiarly associated with the proteids, but we cannot explain its rôle. The element sulphur, again, is only second to phosphorus, and we find it as a constituent of nearly all proteids; but we cannot foretell the exact changes which would take place in the economy if all the sulphur of the food were withdrawn. In the kreatin of the epidermis and its appendages, hairs, etc., sulphur is probably undergoing excretion, though its presence in kreatin may have to do with the peculiar physical characters of corneous epithelium.

We know that the various saline matters are essential to health; that when they are not present in proper proportions nutrition is affected. Dogs fed on food freed as much as possible from all saline matters, but otherwise abundant, with a proper proportion of the food-stuffs, soon exhibit symptoms showing that the metabolism of their tissues, especially of their central nervous system, is going wrong; they suffer from weakness, soon amounting to paralysis, and are often carried off by convulsions. And more or less similar derangements of nutrition follow the absence or a deficiency of individual salts. During starvation these various salts continue to be discharged from the body; in some way or other they are carried along in the metabolic stream, and their presence is in some way essential to the various metabolic processes; hence, they need to be always present in daily food. In what way it is that they thus direct metabolism we do not know; we are aware that the properties and reactions of various proteid substances are closely dependent on the presence of certain salts, but beyond this we know very little. The inorganic salts are those the nutritive value of which has been chiefly studied by experiment, but we have reason to believe that the organic salts, or extractives, which are present in greater or less quantity in all food of both vegetable and animal origin, are no less essential to the proper meta- 
bolic activities of the body. The undoubted connection of scurvy with the lack of fresh vegetable food, other conditions helping, may perhaps turn in part on this, for the evidence that the disease is due to the deficiency of potash alone is not conclusive.

Lastly, water has an effect on metabolism, as shown, among other things, by the fact that when the water of a diet is increased, the urea is increased to an extent beyond that which can be explained by the increase of fluid increasing the facilities of mere excretion.

\section{The Energy of the Body.}

\section{The Income of Energy.}

$\S 441$. Broadly speaking, the animal body is a machine for converting potential into actual energy. The potential energy is supplied by food; this the metabolism of the body converts into the actual energy of heat and mechanical labor. We have in the present section to study what is known of the laws of this conversion, and of the distribution of the energy set free.

Neglecting all subsidiary and unimportant sources of energy, we may say that the income of animal energy consists in the oxidation of food into its waste products - viz., the oxidation of proteids, fats, and carbohydrates into urea, carbonic acid, and water. A principle laid down by the chemist teaches that the potential energy of any body, considered in relation to any chemical change which it may undergo, is the same when the final result is the same, whether that result be gained at one leap or by a series of steps; that, for instance, the energy set free by the oxidation of $1 \mathrm{grm}$. of fat into carbonic acid and water is the same, whatever the changes forward or backward which the fat undergoes before it finally reaches the stage of carbonic acid and water; and similarly, that the energy available for the body in 1 grm. of dry proteid is the energy given out by the complete combustion of that 1 grm., less the energy given out by the complete combustion of that quantity of urea to which the 1 grm. of proteid gives rise in the body. Taking this as our guide, we can readily calculate the amount of potential energy contained in an average twenty-four hours' diet, and thus obtain the average daily income of energy. For the potential energy of most of the substances used as food has been determined by direct calorimetric observations; and the several determinations, though they vary somewhat, agree sufficiently closely to serve as data for the calculations in question.

The total combustion of the following substances has given for one gramme of each substance the following results expressed in calories-that is, in gramme-degree units of heat:

Meat, free from fat, 5103 and 5324. Fibrin, 5511. Egg-albumin, 5579. Thus, taking round numbers, we may say that $1 \mathrm{grm}$. of proteid material contains 5000 or 5500 calories of potential energy, according as we use the lower or higher determinations.

Fat beef or mutton, 9069, 9365, 9423. Butter, 7267 or 9192 . Again, in round numbers, we may say that 1 grm. of fat contains about 9000 calories.

Arrowroot (nearly pure starch), 3912. Starch, 4123. Cellulose, 4146. Dextrose, 3692. Cane sugar, 3866. Here again, taking round numbers, we shall not be far wrong in saying that the potential energy of $1 \mathrm{grm}$. of carbohydrate material is about 4000 calories.

The combustion of $1 \mathrm{grm}$. of urea sets free an amount of energy which has been determined by one observer as 2206 , by another as 2465 calories. 
We have seen ( $\S 421)$ that 1 grm. of proteid gives rise in the body to $\frac{1}{3}$ grm. urea. Hence, to obtain the energy of $1 \mathrm{grm}$. proteid material available for the economy, we must deduct from its potential energy one-third the potential energy of $1 \mathrm{grm}$. urea-that is, in round numbers, 700 or 800 calories. This will give us $5000-700$, or $5500-800$, that is, 4300 or 4700 calories, according as we take the lower or higher data; or we may take as a mean 4500 calories. The data, then, so far, are as follows:

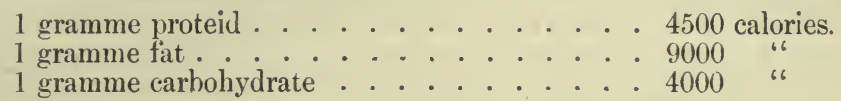

The average diet of an average man-that is, the average amount of each food-stuff respectively taken daily-may be determined experimentally or statistically. Thus, a man may determine by a series of trials the diet on which, while neither losing or gaining weight and maintaining " nitrogenous equilibrium" ( $(436)$, he enjoys good health. Or an average may be struck of a large number of diets used by various people. We shall have something to say of this latter statistical method when we come to speak of diet. For the present purpose we may use one arrived at experimentally, which we will speak of as Ranke's diet, since it was determined by a physiologist of that name from observations on himself. It was composed of 1000 grms. proteid, 100 grms. fat, 240 grms. carbohydrate. Such a diet would give

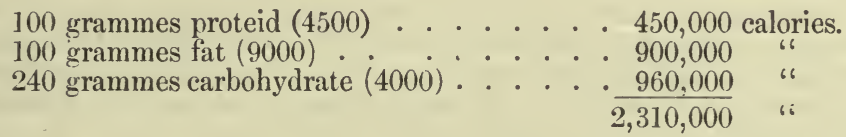

If we translate the units of heat into units of work, the 2,310,000 grammedegree, or 2310 kilogramme-degree calories will give us about 980,000 , or, in round numbers, somewhere about one million kilogramme-metres.

We may, in passing, call attention to the fact that the proteids supply a relatively small part of the total energy, and that the share contributed by the large mass of carbohydrates is not much greater than that belonging to the much smaller quantity of fat. In the average diet obtained by the statistical method, in which the data are largely drawn from public institutions, the (cheaper) carbohydrates are still further increased at the expense of the (dearer) fats, a change which may tend to reduce somewhat the total energy ; but this does not materially affect the broad results just given.

\section{The Expenditure.}

$\S 442$. There are two ways only in which energy is set free from the body: mechanical labor and heat. The body loses energy in producing muscular work, as in locomotion and in other kinds of labor, in the movements of the air in respiration and speech, and, though to a hardly recognizable extent, in the movements of the air or contiguous bodies by the pulsations of the vascular system. The body loses energy in the form of heat by conduction and radiation, by respiration and perspiration, and by the warming of the urine and feces. All the internal work of the body, all the mechanical labor of the internal muscular mechanisms with their accompanying friction, all the molecular labor of the nervous and other tissues, is converted into heat before it leaves the body. The most intense mental 
action, unaccompanied by any muscular manifestations, the most energetic action of the heart or of the bowels, with the slight exceptions mentioned above, the busiest activity of the secreting or metabolic tissues, all these end simply in augmenting the expenditure in the form of heat.

A normal daily expenditure in the way of mechanical labor can be easily determined by observation. Whether the work take on the form of walking, or of driving a machine, or of any kind of muscular toil, a good day's work may be put down at about 150,000 kilogramme-metres.

The normal daily expenditure in the way of heat cannot be so readily determined. Direct calorimetric observations on the whole body are attended with so many difficulties, except in the case of small animals, that their value is uncertain ; and observations made by placing a part only of the body, an arm or leg for example, in the calorimeter, and from the data thus gained calculating the heat produced by the whole body, are subject to many additional sources of error.

The calorimeters usually employed in chemical operations, in measuring, for instance, the heat given out in chemical changes, are unsuitable for experiments on living animals. Such are the mercury calorimeter, in which the chemical action to be studied is made to take place in the midst of a mass of mercury, from the consequent expansion of which through the heat taken up the amount of heat given out is calculated, or the ice calorimeter in which in a similar way the heat given out is calculated by the amount of ice melted. The latter has been used for physiological purposes, but an animal surrounded by ice is under such abnormal conditions that the results are of little value. The methods usually adopted by physiologists are as follows:

In one method, the water calorimeter, the animal is placed in a metal chamber surrounded by a jacket filled with water. The heat given out by the animal warms the water in the jacket, and the amount given out is calculated upon the increase of the temperature of the water. By supplying the animal with air through a long spiral tube passing through the water-jacket, the heat given out in the expired air is prevented from being lost.

This method may be employed in a simpler form, when the heat given out by a part of the body, the arm or leg for instance, is all that has to be determined. The part is then merely placed in a bath of water, from the changes of temperature of which the amount given out is calculated. And this modification of the method may with due precautions be employed for the whole body.

In Rosenthal's calorimeter the chamber in which the body or part of the body is placed is surrounded by, not a water-jacket, but an air-jacket, which thus serves as an air calorimeter. The instrument consists essentially of three concentric copper cylinders; the inner one contains the animal (or other source of heat); the other one serves merely as a casing to protect those inside from changes of temperature due to currents of air and the like; and the middle one encloses an air-space between itself and the inner one. There are special arrangements for closing the cylinders after the introduction of the animal. and for supplying the animal with air for breathing purposes. With the air-jacket, or space between the inner or middle cylinders, are connected a manometer and a thermometer. When an animal (or other source of heat) is placed in the inner cylinder, the temperature and the pressure of the air in the air-jacket are increased; and from the amounts of increase measured by the thernometer and the manometer the amount of heat given out from the animal is calculated.

The calorimeters of D'Arsonval and Rubner are constructed on very similar principles.

Various attempts have been made to acertain the amount of heat given out by the body in an indirect manner, as for instance by calculating the heat given out by the oxidation of the food. As trustworthy as any is the plan of simply subtracting the normal daily mechanical expenditure from the normal daily income. Thus 150,000 kilogramme-metres subtracted from one million kilogramme-metres gives 850,000 kilogramme-metres as the daily 
expenditure in the form of heat; $i . e$., between one-fifth and one-sixth of the total income is expended as mechanical labor, the remaining four-fifths or five-sixths leaving the body in the form of heat. The results given by direct calorimetric observations and by other calculations give somewhat higher figures than these; and indeed these may probably be taken as under rather than over the true amount. In any case they are to be regarded as furnishing nothing more than a rough average, the exact amount varying according to the size, the weight, and the condition of the individual, as well as according to variations in circumstances.

$\S 443$. The energy of mechanical work. We have already in treating of muscle and elsewhere partly discussed this subject, but may here say the rest that has to be said.

The older writers, even after it had been proved that the animal body was constructive, as far as the formation of fat was concerned, still held to the distinction between nitrogenous or plastic and non-nitrogenous or respiratory food. Put broadly, this view was that all the nitrogenous food went to build up the proteid tissues, the muscular flesh and the like, and that the nitrogenous egesta arose solely from the functional metabolism of these tissues, while the non-nitrogenous food was used with equal exclusiveness for respiratory or calorific purposes, being either directly oxidized in the blood, or, if present in excess, stored up as fatty tissue. According to this view the two classes of income corresponded exactly to the two forms of expenditure. We have already urged several objections against this view. We have seen that in the blond itself very little oxidation takes place; that it is the active tissue, and not the passive blood-plasma, which is the seat of oxidation. We have further seen that proteid food may undoubtedly be, in the above sense, respiratory and incidentally give rise to the storing up of fat. One division of the view is thereby overthrown. We have now to inquire whether the other division holds good, whether muscle and the other proteid tissues are fed exclusively on the proteid material of fond, and whether muscular energy comes exclusively from the metabolism of the proteid constituents of muscle. We have already seen (\$63) that when the muscle itself is examined, we find no proof of nitrogenous waste, but, on the other hand, clear evidence of the production of non-nitrogenous bodies, such as carbonic acid. And when we ask the question, Does muscular exercise proportionately increase the urea given off by the body as a whole? for this according to the theory in question it certainly ought to do, the evidence we can obtain, though somewhat varying, gives on the whole a decidedly negative answer.

In the majority of observations no marked change at all in the amount was met with; indeed, in some cases there was a distinct decrease, followed by an increase on the following days. Some observers, however, found a very marked increase, and this was especially the case when the subject under observation took a large amount of food and performed very severe labor. On the whole, the various results obtained by different observers justify the conclusion that exercise by itself, even when severe, does not necessarily increase the amount of urea excreted, but that conditions may obtain in which such an increase undeniably occurs. We may draw the further conclusion that experiments of this kind do not supply the right methor for determining the point at issue. It must be remembered that it is not the muscles alone which feel the influence of the labor; the circulation and indeed the whole body are affected by it. If we suppose a large part or even only some part of the urea to come from other than muscular metabolism, from changes in the hepatic cells for instance, we should expect that these changes, and with them the amount of urea discharged, would be influenced by labor, especially by severe labor. 
In no case has a direct relation between the amount of labor and amount of urea been observed. More than this, the following experience lands us in an absurdity, if we suppose the whole energy of muscular work to arise from proteid metabolism. Two observers performed a certain amount of work (an ascent of a mountain) on a non-nitrogenous diet, and estimated the amount of urea passed during the period. Assuming the urea to represent the oxidation of so much proteid matter, which oxidation represented in turn so much energy set free, they found that, whereas the actual work done amounted to 129.026 and 148.656 kilogramme-kilometres for each observer respectively, the total energy available from proteid metabolism during the period was in the case of the first 68.69 , and of the second 68.376 kilogramme-kilometres. That is to say, the energy set free by the proteid metabolism of the muscles engaged in the work was far less than the amount necessary to accomplish the work actually done, to say nothing of its having to provide as well for the movements of respiration and circulation. Their muscular energy, therefore, must have had other sources than proteid metabolism.

That, on the contrary, the production of carbonic acid is at once and largely increased by muscular exercise is beyond all doubt. One hour's hard labor will increase fivefold the quantity of carbonic acid given off within the hour. And in an experiment directed to this point it was found that a man in twenty-four hours consumed 954 grammes oxygen and produced 1284 grammes carbonic acid when doing work, as against 708 grammes oxygen consumed and 911 grammes carbonic acid produced when remaining at rest, the quantity of urea secreted being in the first case 37 grammes, in the second 37.2 grammes.

It is evident that the conclusions arrived at by the statistical method entirely corroborate those gained by an examination of muscle itself, viz., that during muscular contraction the explosive decomposition which takes place bears chiefly, if not exclusively, on the non-nitrogenous constituents of the muscle, and that it is the non-nitrogenous products which alone escape from the muscle and from the body, any nitrogenous products which result being retained within the muscle, or at least within the body. IVe must, therefore, reject the second as well as the first division of the views under discussion; not only is the muscle not fed exclusively on proteid material, but also its energy does not arise from an exclusively proteid metabolism.

\section{Animal Heat.}

\$444. The sources and distribution of heat. We have already seen that the conception of the non-nitrogenous portions of food being solely calorifacient or respiratory proves to be unfounded when we attempt to trace the history of the food on its way through the body. The same view is still more strikingly shown to be inadequate when we study the manner in which the heat of the body is produced. We may, indeed, at once affirm that the heat of the body is generated by the chemical changes, which we may speak of generally as thuse of oxidation, undergone not by any particular substances, but by the tissues at large. Wherever metabolism is going on, or, to be more exact, wherever destructive metabolism, katabolism, is going on, heat is being set free. In growth and in repair, in the deposition of new material, in the transformation of lifeless pabulum into living tissue, in the constructive metabolism, the anabolism of the body, and in the smaller synthetic processes of which we spoke in dealing with urea ( $\$ 403)$, heat is undoubtedly to a certain extent being absorbed and ren- 
dered latent; the energy of the construction may be, in part at least, supplied by the heat present. But all this, and more than this, viz., the heat present in a potential form in the substances themselves so built up into the tissue, is lost to the tissue during its destructive metabolism; so that the whole metabolism, the whole cycle of changes from the lifeless pabulum through the living tissue back to the lifeless products of vital action, is eminently a source of heat.

Of all the tissues of the body the muscles, not only from their bulk, forming as they do so large a portion of the whole frame, but also from the characters of their metabolism, must be regarded as the chief sources of heut.

In treating ( $\$ 65)$ of the thermal changes in muscle we have seen that in the total energy expended in a muscular contraction, the ratio of that which appears as heat to that which appears as external work is variable. If we take a proportion which is somewhat higher than the mean of the range there given (one-fifth to one-twenty-fifth), and assume that the energy involved in the work done in a muscular contraction is about one-tenth of the total energy expended, the rest going out as heat, then, upon the calculation that the total external work of the body is about one-fifth of the total energy set free in the body, it is clear that the heat given out by the muscles, even if we consider only the heat given out when they are contracting, must form a very large part of the total heat given out by the body. And even if, as recent researches indicate, the muscular machine works more economically than we have hitherto supposed, the amount of heat given out by the skeletal muscles must still remain very large. Moreover, to the skeletal muscle we must add the heart which, never resting, does in the twenty-four hours, as we have seen ( $\$ 127)$, no inconsiderable amount of work, and must give rise to no inconsiderable amount of heat. But the skeletal muscles, though frequently, are not continually contracting; they have periods, at times long periods, of rest; and during these periods of rest, metabolism, of a subdued kind it is true, but still a metabolism involving an expenditure of energy is going on. This quiescent metabolism must also give rise to a certain amount of heat; and if we add this amount, which in the present state of our knowledge we cannot exactly gauge, to that given out during the movements of the body, it is very clear, even in the absence of exact data, that the metabolism of the muscles must supply a very large proportion of the total heat of the body. They are par excellence the thermogenic tissues.

Next to the muscles in importance come the various secreting glands. In these the secreting elements, at the periods of secretion at all events, are in a state of metabolic activity, which activity as elsewhere must give rise to heat. In the case of the salivary gland of the dog the temperature of the saliva secreted during stimulation of the chorda has been found to be as much as $1^{\circ}$ or $1.5^{\circ}$ higher than that of the blood in the carotid artery at the same time, and in all probability the investigation of other secreting glands would lead to similar results. Of all these various glands the liver deserves special attention on account of its size and large supply of blood, and because it appears to be continually at work. If there be any truth in the views urged in the preceding chapter touching the large and varied metabolic work of the liver, we must conclude that a very large amount of heat is set free in this organ; and that holds good even if we make a large allowance for the various synthetic anabolic processes which may take place and by which heat would be absorbed and made latent. We find, indeed, that the blood in the hepatic vein is the warmest in the body. Thus in the $\operatorname{dog}$ a temperature of $40.73^{\circ} \mathrm{C}$. has been observed in the hepatic vein, 
while that of the vena cava inferior was $38.35^{\circ}$ to $39.58^{\circ}$, and that of the right heart $37.7^{\circ}$. The fact that the hlood of the hepatic vein is warmer than that of either the portal vein or the aorta, shows that the increased temperature is not due simply to the liver being far removed from the surface of the body.

The brain, too, may be regarded as a source of heat, since its temperature is higher than that of the arterial blood with which it is supplied; though from the smaller quantity of blood passing through its vessels, as well as from the changes in it being less massive, it cannot, in this respect, compare with either the liver or the muscles as a source of heat to the body.

The blood itself cannot be regarded as a source of any considerable amount of heat, since, as we have so frequently urged, the oxidations or other metabolic changes taking place in it are comparatively slight. The heat evolved by the indifferent tissues, such as bone, cartilage, and connective tissue, may be passed over as insignificant ; and we cannot even regard the adipose tissue as a seat of the production of heat, since the fat of the fat-cells is in all probability not oxidized in situ, but simply carried away from its place of storage to the tissue which stands in need of it, and it is in the tissue that it undergoes the metabolism by which its latent energy is set free. Some amount of heat is also produced by the changes which the food undergoes in the alimentary canal before it really enters the body.

Hence, taking a survey of the whole body, we may conclude that since metabolism is going on to a greater or less extent everywhere, heat is everywhere being generated; but that, looked at from a quantitative point of view, the muscles and the glandular organs must be regarded as the main sources of the heat of the body, the muscles being, in all probability, the more important of the two.

$\S 445$. But heat, while being thus continually produced, is as continually being lost, by the skin, the lungs, the urine, and the feces. The blood passing from one part of the body to the other, and carrying warmth from the tissues where heat is being rapidly generated, to the tissues or organs where heat is being lost by radiation, conduction, or evaporation, tends to equalize the temperature of the various parts, and thus maintains a "constant bodily temperature."

When the production of heat is not great as compared with the loss there is no great accumulation of heat within the body, the temperature of which consequently is but slightly raised above that of surrounding objects. Thus the temperature of the frog, for instance, is rarely more than $0.04^{\circ}$ to $0.05^{\circ}$ above that of the atmosphere, though in the breeding season the difference may amount to $1^{\circ}$. Such animals, and they comprise all classes except birds and mammals, are spoken of as cold-blooded; they have been also called poikilothermic, that is, of varied temperature. Exceptions among them are not uncommon. Some fish, such as the tunny, are warmer than the water in which they live, and in a species of python ( $P$. bivittatus) a difference of as much as $12^{\circ}$ has been observed. In a beehive the temperature may rise at times as much as to $40^{\circ}$. In the so-called warm-blooded animals, birds and manimals, the loss and production of heat are so balanced that the temperature of the bolly remains constant at, in round numbers, $35^{\circ}$ or $40^{\circ}$, whatever be the temperature of the air ; hence these have been called homoiothermic, of constant temperature. The temperature of man is about $37^{\circ}$; in some birds it is as high as $44^{\circ}$ (Hirundo), and in the wolf it is said to be as low as $35.24^{\circ}$.

This temperature is with slight variations maintained throughout life. After death the generation of heat rapidly diminishes, and the body 
speedily becomes cold; but for some short time immediately following upon systemic death, a rise of temperature may be observed, due to the fact that, while the metabolism of the tissue is still going on, the loss of heat is somewhat checked by the cessation of the circulation. The onset of pronounced rigor mortis causes a marked accession of heat, and when occurring after certain diseases may give rise to a very considerable elevation of temperature.

This mean bodily temperature of warm-blooded animals is, during health, maintained, with slight variations of which we shall presently speak, within a very narrow margin, a rise, or indeed a fall of much more than a degree above or below the limit given above being indicative of some failure in the organism, or of some unusual influence being at work. It is evident, therefore, that the mechanisms which coördinate the loss with the production of heat must be exceedingly sensitive. It is obvious, moreover, that the mechanisms may act when the bodily temperature is tending to rise, by either checking the production or by augmenting the loss of heat; conversely when the bodily temperature is tending to fall, they may act by either increasing the production or by diminishing the loss of heat. As the regulation of temperature by variations in the loss of heat is better known than regulation by variations in production, it will be best to consider the former first.

$\$ 446$. Regulation by variations in loss. Heat is lost to the body by the warming of the feces and of the urine, by the warming of the expired air, by the evaporation of the water of respiration, by conduction and radiation from the skin, and by the evaporation of the water of perspiration. It has been calculated that the relative amounts of the loss by these several channels are as follows: In warming the feces and urine about 3 , or, according to others, 6 per cent. By respiration about 20, or, according to others, about only 9 per cent., leaving 77 , or alternately 85 , per cent. for conduction and radiation and evaporation by the skin.

The two chief means of loss, then, which are at all susceptible of any great amount of variation, and which can be used to regulate the temperature of the body, are the skin and the lungs.

The more air passes in and out of the lungs in a given time, the greater will be the loss in warming the expired air, and in evaporating the water of respiration. In such animals as the $\mathrm{dog}$, which do not perspire freely by the skin, respiration is a most important means of regulating the temperature; and in the dog a very close connection may be observed between the production of heat and respiratory activity. 'The changes which give rise to this loss take place before the inspired air reaches the pulmonary alveoli ; both the warming and the evaporation are effected in the nasal and pharyngeal, and to some extent in the bronchial passages. Some observers have maintained that the left side of the heart is warmer than the right, and hence have argued that chemical changes leading to a considerable development of heat take place in the pulmonary capillaries. It would appear, however, that the right ventricle, owing to its lying nearer to the liver, the high temperature of which has already been mentioned, is, in reality, rather hotter than the left. And, indeed, we have no satisfactory evidence of any large amount of heat being produced by any pulmonary metabolism.

The great regulator, however, is undoubtedly the skin; and this has a more or less double action. In the first place, it regulates the loss of heat by means of the vasomotor mechanism. The more blood passes through the skin the greater will be the loss of heat by conduction, radiation, and evaporation. Hence any action of the vasomotor mechanism which, by causing dilatation of the cutaneous vascular areas, leads to a large flow of blood through the skin, will tend to cool the body ; and, conversely, any 
vasomotor action which, by constricting the cutaneous vascular areas, or by dilating the splanchnic vascular areas, causes a smaller flow through the skin, and a larger flow of blood through the abdominal viscera, will tend to heat the body. In the second place, besides this, the special nerves of perspiration will act directly as regulators of temperature, increasing the loss of heat when they promote, and lessening the loss when they cease to promote, the secretion of the skin. 'The working of this heat-regulating mechanism is well seen in the case of exercise. Since every muscular contraction gives rise to heat, exercise must increase for the time being the production of heat; yet the bodily temperature rarely rises so much as a degree centigrade, if at all. By exercise the respiration is quickened, and the loss of heat by the lungs increased. The circulation of blood is also quickened, and the cutaneous vascular areas becoming dilated, a larger amount of blood passes through the skin. Added to this, the skin perspires freely. Thus a large amount of heat is lost to the body, sufficient to neutralize the addition caused by the muscular contraction, the increase which the more rapid flow of blood through the abdominal organs might tend to bring about being more than sufficiently counteracted by their smaller supply for the time. The sense of warmth which is felt during exercise in consequence of the flushing of the skin, is, in itself, a token that a regulative cooling is being carried on. In a similar way the application of external cold or heat defeats its own ends, either partially or completely. Under the influence of external cold, the cutaneous vessels are constricted, and the splanchnic vascular areas. dilated, so that the blood is withdrawn from the colder and cooler regions to the hotter and heat-producing organs. This vascular change may be used to explain the fact that stripping naked in a cold atmosphere often gives rise to a distiuct increase in the mean temperature of the blood, as indicated by a thermometer placed in the mouth, though possibly the effect may be partly due to an actual increase of the production of heat. Under the influence of external warmth, on the other hand, the cutaneous vessels are dilated, a rapid discharge of heat takes place; and if the circumstances be such that the body can perspire freely, and the perspiration be readily evaporated, the temperature of the body may remain very near to the normal, even in an excessively hot atmosphere. Thus, more than a century ago, two observers were able to remain with impunity in a chamber heated even to $127^{\circ} \mathrm{C}$. $\left(260^{\circ} \mathrm{Fahr}\right.$.), and with ease in one so hot that it became painful for them to touch the metal buttons of their clothing. It is unnecessary to give any more examples of this regulation of temperature by variations in the loss of heat; they all readily explain themselves.

$\S 447$. The production of heat, its variations and regulation. As we have already said, the exact determination of the amount of heat produced in the living body is attended with great difficulties; still, certain conclusions have been arrived at based partly on direct calorimetric observations, the more recent ones with improved calorimeters being especially valuable, and partly on what seem to be trustworthy deductions from observed chemical changes.

The rate of production of heat in a living body is determined by a variety of circumstances. In the first place, what may be called the general rate of metabolism, and so of the production of heat, varies in different kinds of animals. Of two animals of the same bulk and weight placed under the same circumstances, one "living faster" than the other, metabolizes its living substance more rapidly, and so produces heat more rapidly. Thus direct calorimetric observations, as far as they at present go, show that a man, on the average, produces more heat, per kilo, per hour, than does a dog, and a dog more than a rabbit. Probably every species has what may 
be called its specific coefficient, and every individual his personal coefficient of heat-production, the coefficient being the expression of the inborn qualities proper to the living substance of the species and of the individual.

A larger living body will naturally produce more heat than a smaller living body of the same nature, since the larger body possesses, so to speak, a greater number of heat-producing units. But this is neutralized by an opposing tendency. The smaller body, having relatively to its bulk a larger amount of surface, loses heat at a more rapid rate than does the larger body; and, therefore, to maintain the balance between loss and production, so as to secure the same constant bodily temperature (and, as we have just seen, the bodily temperature of warm-blooded animals is remarkably uniform), it must produce heat, per unit of its body, at a more rapid rate. As a rule, the greater loss of heat owing to the relatively greater surface is so marked that of two animals having the same constant bodily temperature, of two species of mammals, or of two individuals of the same race, we should expect the smaller one to produce a relatively larger amount of heat. And direct calorimetric observations show that this is so. The struggle for existence has raised what we have just called the specific or personal coefficient of the smaller animal.

From what we have seen concerning the immediate effects of a meal, we should be inclined to expect that food would temporarily increase the production of heat; and not only is this view confirmed by common experience and by our own sensations, but direct calorimetric observations afford experimental proof of its truth. In the dog it has been found that the rate of production increases after a meal, reaching its maximum from the sixth to the ninth hour, and then declining to a level which may be regarded as that secured by the general metabolism of the body, and which appears to be maintained with remarkable constancy until after long starvation the economy begins to break down.

Labor, muscular work, has a powerful influence in increasing the production of heat. As we have seen, of the total heat produced in the body, a certain portion must always be attributed to muscular contractions, which even in the most quiet body are always going on; in an ordinary active body a considerable quantity of heat must be thus generated. Hence, the more active the body the greater the production of heat. As we stated before $(\$ 85)$, in a contraction the proportion of the energy set free to do work to that set free as heat appears to vary under different circumstances; and the increase of heat due to labor probably varies in a corresponding way. The details of this relation have yet to be worked out, but we may at least conclude that, when a man pushes his daily labor beyond the 150,000 kilogramme-metres, the additional energy thus leaving his body as work done is not taken out of the 850,000 kilogramme-metres given in $\S 442$ as the average daily output of heat, but the total setting free of energy and the total production of heat is at the same time increased. And it need hardly be said that the figures in question give only an average estimate for a man of average build and weight, taking an average amount of average food, and doing an average amount of work.

$\S 448$. The production of heat thus determined by these several influences, some of which are themselves regulated by the nervous system, is further regulated in a remarkable manner. For it is not solely by variations in the loss of heat that the constant temperature of the warm-blooded animal is maintained. Variations in the amount of heat actually generated in the body constitute an important factor, not only in the maintenance of the normal temperature, but also in the production of the abnormally high or low temperatures of various diseases. Many considerations have long led 
physiologists to suspect the existence of a nervous mechanism, by which afferent impulses arising in the skin or elsewhere might, through the central nervous system, originate efferent impulses, whose effect would be to increase or to diminish the metabolism of the muscles or other organs, and thus to increase or diminish the amount of heat generated for the time being in the body. The existence, in fact, of a metabolic or thermogenic nervous mechanism, comparable in many respects to the vasomotor mechanism or to the various secreting nervous mechanisms, seems in itself à priori probable. And we have experimental evidence that such a mechanism does really exist.

The warm-blooded animal is distinguished from the cold-blooded animal by the fact that when it is exposed to cold or heat it does not, like the latter, become colder or hotter, as the case may be, but, within certain limits, maintains its normal temperature. If the maintenance of the temperature of the warm-blooded animal during exposure to cold is assisted by an increased production of heat, and is not due simply to a diminished loss, there should be evidence of an increased metabolism during that exposure. We ought to find, under these circumstances, an increased production of carbonic acid and an increased consumption of oxygen, since it is to these products, rather than to the nitrogenous factors, on the peculiarities of which as uncertain signs of metabolism we have already insisted, we must look for indications of the rise or fall of metabolic activity.

Taking the consumption of oxygen, and, though with less confidence, the production of carbonic acid, as a measure of metabolic activity and so of heat-production, it has been shown that a marked contrast in this respect exists between cold-blooded and warm-blooded animals exposed to changes of temperature. In the cold-blooded animal, cold diminishes and heat increases the metabolic activity of the body; as the temperature to which the animal is subjected rises or falls, so the consumption of oxygen and production of carbonic acid is increased or lessened. The body of a cold-blooded animal behaves in this respect like a mixture of dead substances in a chemist's retort; heat promotes and cold retards chemical action in both cases. Very different is the behavior of a warm-blooded animal. In this case, within a lower and a higher limit, cold increases and heat diminishes the bodily metabolism, as shown by the increased or diminished consumption of oxygen and production of carbonic acid as the temperature falls or rises. In these animals there is obviously a mechanism of some kind, counteracting, and indeed overcoming, the more direct effects which alone obtain in coldblooded animals. And that this mechanism is of a nervous nature is indicated by the following facts:

When a warm-blooded animal is poisoned by urari, the temperature falls and the metabolism, measured by the consumption of oxygen and the production of carbonic acid, sinks also; and that the latter is the cause, not the effect, of the former is shown by the fact that the metabolism continues to fall though loss of heat be prevented by surrounding the animals with wrappings of cotton-wool. In such a urarized animal, exposure to higher temperatures augments and exposure to lower temperatures diminishes metabolism; the urarized warm-blooded animal, in fact, behaves like a cold-blooded animal. Similar, but perhaps not such striking or so constant results, are gained by division of the medulla oblongata. After this operation the temperature of the body sinks, and the fall, though partly due to increased loss of heat by the skin, caused by the dilated condition of the cutaneous vessels, is also accompanied by diminished metabolism, and is, therefore, in part due to diminished production of heat. And when an animal is in this condition, exposure to higher temperatures increases and exposure to lower tempera- 
tures diminishes the bodily metabolism. We can best explain these results by supposing that, under normal conditions, the muscles, which as we have seen contribute so largely to the total heat of the body, are placed, by means of their motor nerves and the central nervous system, in some special connection with the skin, so that a lowering of the temperature of the skin leads to an increase, while a heightening of the temperature of the skin leads to a decrease of the muscular metabolism. Further, the centre of this thermotaxic reflex mechanism appears to be placed somewhere in the nervous system above the spinal cord. When urari is given, the reflex chain is broken at its muscular end; when the spinal cord is divided, the break is nearer the centre.

We may add that the muscular metabolism which thus helps to regulate temperature need not involve visible muscular contractions. At the same time, the heat given out by the muscles will be temporarily increased at every contraction which may occur. Thus, the shivering which follows exposure to cold distinctly helps to warm the body; indeed, some observers have been led to think that, in man, this visible effect of cold plays a more important part in his heat regulation than the invisible actions which we have just described. We may also add that the regulative nervous mechanism may apparently be overborne by an exposure to too great heat or cold. When, for instance, the cold to which the animal is exposed becomes excessive, the reaction of the thermotaxic nervous system is powerless against the direct action on the tissues of the depressing influences, and the metabolism, together with the temperature, sinks.

$\$ 449$. In a number of experiments it has been shown that injuries to, such as those caused by puncture or galvanic cautery, or electrical stimulation of, limited portions of the more central portions of the brain may give rise to a great increase of the temperature of the body without producing any other marked symptom. The increase is shown by the increase of metabolism, increased production of carbonic acid, and increased consumption of oxygen, as well as by direct calorimetric observations, to be due to an increased production of heat. This naturally suggests that the portions of the brain in question contain the hypothetical heat centre just mentioned, the lesion on stimulation exciting the centre to activity by direct action on it, instead of in the usual reflex manner. The matter has not, however, as yet been clearly worked out; and indeed observers are not agreed as to the exact parts of the brain injury to which, or stimulation of which, produces the effect.

$\S 450$. By regulative mechanisms of the kind just discussed the temperature of the warm-blooded animal is maintained within very narrow limits. In ordinary health the temperature of a man varies between $36^{\circ}$ and $38^{\circ}$, the narrower limits being $36.25^{\circ}$ and $37.5^{\circ}$, when the thermometer is placed in the axilla. In the mouth the reading of the thermometer is somewhat $\left(0.25^{\circ}\right.$ to $\left.1.5^{\circ}\right)$ higher; in the rectum it is still higher (about $0.9^{\circ}$ ) than in the mouth. The temperature of infants and children is slightly higher and much more susceptible of variation than that of adults, and after forty years of age the average maximum temperature (of health) is somewhat lower than before that epoch. A diurual variation, independent of food or other circumstances, has been observed, the maximum ranging from 9 A.M. to 6 P.M. and the minimum from 11 P.M. to 3 A.M. Meals cause sometimes a slight elevation, sometimes a slight depression, the direction of the influence depending on the nature of the food-alcohol seems always to produce a fall. Exercise and variations of external temperature, within ordinary limits, cause a very slight change, on account of the compensating influences which have been discussed above. The rise from even active 
exercise does not amount to $1^{\circ}$; when labor is carried to exhaustion a depression of temperature may be observed. In travelling from very cold to very hot regions a variation of less than a degree occurs, and the temperature of inhabitants of the tropics is practically the same as of those dwelling in aretic regions.

$\$ 451$. Many of the maladies of the body are characterized by an increase of the bodily temperature known as "fever" or "pyrexia," the thermometer very frequently rising to $39^{\circ}$ or $40^{\circ}$, not unfrequently to $41^{\circ}$, and at times reaching $43^{\circ}$ or even $44^{\circ}$; but these higher temperatures cannot long be borne without the organism failing. And, as we have said, any increase in man of the bodily temperature beyond $38^{\circ}$, or even beyond $37.5^{\circ}$, indicates some disturbance. In most cases the rise of temperature has a definite objective cause, some local inflammation or suppuration, or, as in specific fevers, the presence in the economy of some "materies morbi," of the nature of an organized germ or of some other nature. We cannot here discuss the connection between local inflammation or the specific poison and the high temperature, but we have increasing evidence that the high temperature of fever is due, not merely to a diminution of the loss of heat, though this may be a factor, but also, and indeed chiefly, to an increased production of heat. In fever the production of carbonic acid and the consumption of oxygen, that is to say, the metabolic changes of the tissues, are increased. The urea also is increased, and that in such a way as to confirm the view already expressed that much of the heat comes from such a metabolism of the skeletal muscles as, unlike an ordinary contraction, directly involves the nitrogenous elements. The inordinate metabolism of the body at large thus characteristic of fever is shown by the wasting which it entails. Calorimetric observations also show in a direct manner that the production of heat is increased. Of course, mere increased production alone would be insufficient to raise the temperature of the body, for it might be met, up to a very high limit, by a compensating increase of loss of heat; but in fever this compensation is wanting, and it is perhaps this absence of due regulation which is most characteristic of the febrile condition.

In some maladies the bodily temperature falls distinctly below the normal average, reaching for instance $35^{\circ}$, or even lower. In such cases there can be little doubt that the condition is due to diminished metabolism and diminished heat production.

One of the most marked phenomena of starvation is the fall of temperature, which becomes very rapid during the last days of life. The lowered metabolism diminishes the production of heat, and the lowered temperature in turn still further diminishes the metabolism. Indeed, the low temperature is a powerful factor in bringing about death, for life may be much prolonged by wrapping a starving animal in some bad conductor, so as to economize the bodily heat.

$\$ 452$. Effects of great heat. As we said above, the regulative heat mechanism is unable to withstand the strain of too great an external heat or too prolonged an exposure to a great but less degree of heat. The temperature of the body then rises above the normal; and it has been observed that the temperature is more easily raised by warmth than depressed by cold, at least when neither is very intense. When either in this way by external warmth or through pyrexia the temperature of the body is raised some $6^{\circ}$ or $7^{\circ}$ above the normal, to $45^{\circ}$ or thereabouts, death speedily ensues. The chain of events thus leading to death has not been as yet clearly made out, and most likely the events do not take exactly the same course in all cases; but we shall probably not go far wrong in attributing death to the fact that the high temperature hurries on the metabolism of the several tissues, of 
some more than others, at such a spendthrift rate that their capital is soon exhausted. We have seen ( $\S 314$ ) that too warm blood produces dyspnœa and soon exhausts the metabolic capital of the respiratory centre. Too warm blood similarly hurries on the beats of the heart; an explosion of the contractile substance is each time prematurely brought on before a sufficient quantity of explosive substance is accumulated, each stroke becomes more and more feeble as the rate is quickened, the beats become irregular and finally cease. Either of these two events alone and certainly both together are enough to bring the working of the bodily mechanism to an end; but other tissues beside the heart and the respiratory centre are suffering in the same way, notably the rest of the central nervous system. This, too, is being hurried on unduly in its inner changes, so that not only consciousness is lost and other objective manifestations of nervous action go wrong or fail, but the regulative grasp of the central nervous system on the tissues of the body at large is loosened, and tumult takes the place of order. Whether this or that sign of disorder comes to the front, whether, for instance, convulsions take place, would appear to depend upon the exact turn taken by the abnormal events. In heat-stroke, more commonly known as sun-stroke, the essential condition of which seems to be a rapid rise of the temperature of the body, owing to a sudden failure of the thermotaxic mechanism, the symptoms vary. Sometimes the heart suddenly gives way, at other times the respiratory centre seems to be more directly affected; sometimes convulsions make their appearance, but more commonly death takes place through a comatose condition of the brain, an initial phase of excitement of the central nervous system being not unfrequently witnessed.

Mammalian muscle, it will be remembered ( $\$ 82)$, becomes rigid at about $50^{\circ}$; but death probably always occurs before that higher temperature is reached by the blood, so that a sudden rigor mortis from heat (rigor caloris) cannot be regarded as a factor in death from exposure to too great heat.

$\$ 453$. Effects of great cold. The effects of a too great lowering of the temperature of the body, which is, generally, the result of too great external cold, and rarely, if ever, arises from internal causes lowering the metabolism and thus the production of heat, are, in their origin, the reverse of those of a too high temperature. The metabolism of the tissues is lowered; and not only are the katabolic changes, which lead to the setting free of energy thus affected, but the anabolic changes also share in the depression. Thus "living substance" falls to pieces less readily, but is also made up less readily; and could this slackening of metabolism be carried on in the several tissues at a rate proportionate to the rate at which each tissue lives, life might thus be brought to a peaceful end by gradual arrest of the life of each part of the whole body. And, indeed, in some cases, where the lowering of the temperature takes place gradually, something like this does occur even in warm-blooded animals. The diminished metabolism tells first and chiefly on the central nervous system, especially on the brain, and more particularly on those parts of that organ which are concerned in consciousness. The intrinsic lowering of the cerebral metabolism is further assisted by a slowing of the heart-beat and of the breathing; drowsiness is succeeded by a condition very like to, if not identical with, that known as sleep, which we shall study later on, but by a sleep which insensibly passes into the sleep of death. In some cases, however, especially those in which the lowering of the temperature is sudden and rapid, disorders of the nervous system intervene and convulsions like those of asphyxia are produced: 


\section{On Nutrition in General.}

$\S 454$. It may now be profitable to take a brief survey of the various conclusions at which we have arrived concerning the problems of nutrition.

We have seen that the several tissues, using lymph as a medium, live upon the blood, taking up from the blood the materials for, and returning to the blood the products of, their metabolism. The blood itself we have also seen to be replenished with food from the alimentary canal and with oxygen from the lungs, and to be freed from waste products by means of the excretory organs. In this double action the raw material of the food, on the one hand, undergoes between its being placed in the mouth and its taking part in the metabolism of the tissue which ultimately uses it, many intermediate changes carried on in various parts of the body, and the waste products similarly undergo internediate changes between leaving the tissue and appearing in the urine, the sweat, or the expired air.

We have further seen reason to think that the metabolic events of the body take place, in the main, in the tissues, not in the blood stream on its way between the heart and the tissues. Changes proper to the blood itself take place in the blood; the corpuscles, red and white, with the plasma undergo, like the rest of the body, their proper metabolic cycles, and in this sense blood may be called a tissue, if there is any advantage in using the phrase; but, apart from these intrinsic blood changes, as far as we can see at present the metabolism undergone, during their transit along the blood channels, by the substances which are merely carried in the blood from place to place, is an insignificant part of the total metabolism of the body.

By metabolism of a tissue we understand the total chemical changes taking place in the tissue; and we divide these changes into those which either directly or indirectly are concerned in the building up (anabolic), and those which are, in like manner, concerned in the breaking down (katabolic) of the living substance. We shall explain presently what we mean by the words "directly" and "indirectly," used in this connection. And we may here repeat the caution $(\$ 30)$ that though, for convenience sake, we use the phrase "living substance," what is really meant by the words is not a thing or body of a particular chemical composition, but matter undergoing a series of changes.

$\S 455$. Since the several tissues originate through a differentiation of the simpler primordial protoplasm, we may infer that we have a right to speak of a general plan of metabolism common to all the tissues, modified in various particulars in various tissues. It is more reasonable, for instance, to suppose that there is such a general plan common to both muscle and gland, than to suppose that the metabolism of the one differs wholly from or only accidentally resembles that of the other. And we may profitably take the nutrition of muscle as exemplifying, in the midst of the features special to the muscle, the general plan of vital metabolism. The muscle, in a normal state of things, lives ultimately on the proteids, fats, carbohydrates, salts, and water of the food, and on the oxygen of the inspired air, but lives directly on the blood which brings these things to it. Taking the proteids first, we may ask the question, How does the blood supply the muscle with proteids?

The blood contains three classes of proteids: 1 , serum-albumin ; 2, paraglobulin, and 3 , fibrinogen. With regard to the function of these three kinds of proteids in the nutrition of muscle, our only conclusions at present are indirect onces, based chiefly on the results of experiments as to the rel- 
ative value of these substances in maintaining or restoring the irritability of muscle. It is found that when the washed-out frog's heart ( $\$ 148)$ is fed with defibrinated blood, the restoration is as good as with whole blood; and that while the effects of globulin are uncertain, and while peptone and albumose appear to act in an injurious manner, the restorative effects of serumalbumin are marked. From these results we may provisionally infer that the muscle in its (total) anabolic changes takes up and so lives upon the serum-albumin of the blood. But this conclusion must be regarded as provisional only, and indeed uncertain. For we must remember that the blood supplies not only the food (including oxygen) for the muscle, but also the conditions under which the muscle can live and avail itself of the food offered to it. The complex actions through which a certain quantity of proteid and other material is built up into living muscular substance, need for their execution a favorable medium, need certain physical and chemical conditions; and it may be that the favorable influence of serum-albumin is simply due to its presence in some way assisting the transformation into living substance of raw material still remaining in the muscular fibres and not to its supplying new raw material.

Dextrose is, as we have repeatedly said, always present in the blood in small quantity, and appears to be the only carbohydrate contituent of bloodplasma. Experiments carried out on a large animal, such as the horse or cow, have shown that the venous blood coming from a muscle contains less dextrose than the arterial blood going to the muscle, and that the difference is much increased by throwing the muscle into contraction. From this we may provisionally conclude that dextrose is an essential part of the food of the muscle.

The blood, as we have seen, also contains a certain amount of fat; and if we push the analogy between the whole body and the muscle, we may infer that the muscle takes up fat as food for itself from the blood. But we have no experimental evidence in favor of this. Moreover, we have seen that fat and carbohydrate are, in the animal body, more or less transferable. We have distinct proof that the body can transform carbohydrate into fat; and it is very probable that it can transform fat into carbohydrate. Seeing how much more easily a soluble diffusible carbohydrate like sugar can be carried from place to place by the fluids of the body than can immiscible fats, it seems reasonable to suppose that when the body has to draw upon its store of fat in the cells of adipose tissue, the fat, on leaving the fatcell, is transformed into sugar, its carbon, so to speak, being dealt out to the tissues in the form of dextrose. Indeed, we may, perhaps, dwelling on the fact that a muscle, though itself essentially of proteid build, turns over ( $\$ 85)$ in its daily work so much more carbon than nitrogen, entertain the view that what muscle wants as food is a certain amount of proteid plus an additional quantity of carbon in some form or other, and that dextrose is a convenient form in which the additional carbon can be supplied. And we may hold this view without prejudice to any opinion that the carbon so brought, while being built up into the living substance, may be again arranged as fat, and in the course of the metabolism of the muscle may be later on separated from the living substance and deposited in the fibre as globules of fat. But our knowledge is at present insufficient to decide whether this view is true or not.

The various salts brought to the muscle by the plasma, though they supply no energy, are as essential to the life of muscle as the energy-holding proteid or carbon compound; and experiments made with regard to some of them, calcic salts, for instance, show that their presence or absence materially affects the maintenance or restoration of irritability. Some of these probably play 
the part only of securing by their presence favorable conditions for the due metabolic processes, somewhat after the way in which the presence of calcic phosphate determines the curdling of milk ; but some we probably ought to regard as actually entering into the processes themselves. Of these matters, however, we know very little.

$\$$ 456. The end-products of muscular metabolism are, as we have seen, carbonic acid, lactic acid, and kreatin, or some other nitrogenous bodies, and we have already $(\$ 85)$ said all we have to say concerning the formation of these products. We may, however, briefly consider here the question, What is the relation of these various metabolic processes to the structural elements of the tissue? When we say that the muscular fibre is continually undergoing metabolism do we mean that very jot and tittle of the fibre is undergoing change and that at the same rate? We can hardly suppose this. It seems unlikely, for instance, that the metabolism of the fibrillar substance is identical with that of the interfibrillar substance, whatever be the view we take as to the properties or meaning of the two substances. Further, if we accept the suggestion made in $\S 85$ as to a contractile substance, which, though having peculiar qualities, being peculiarly related to and having peculiar connections with the rest of the fibre, may in a broad way be compared with the glycogen of an hepatic cell, we can conceive that this contractile substance may be manufactured without the whole of it at least having been at any time an integral part of what we may in a stricter sense call the real living substance of the fibre. We should thus be led to regard the metabolic events occurring in muscle as falling into two classes at least: those taking place in the living more permanent framework, and those bearing on the formation and destruction of the contractile substance lodged in that living framework. Further, if we suppose that the metabolism by which the muscles supply so much of the heat of the body, and which, as we have seen, may and does go on independently of contractions, is not a metabolism of the same contractile substance differing from the metabolism of a contraction in being so ordered that all the energy goes out as heat, none being employed to effect a change of form, but is a metabolism of some other "thermogenic" substance, we should have to add a third class to the other two. These, of course, are at present matters of speculation; but on the whole, the evidence we can gather tends, and perhaps increasingly tends, to show that in muscle there does exist such a framework of what we may call more distinctly living substance which rules the histological features of the fibre, and whose metabolism though high in quality does not give rise to massive discharges of energy, and that the interstices, so to speak, of this framework are occupied by various kinds of material related in different degrees to the framework and therefore deserving to be spoken of as more or less living, the chief part of the energy set free by muscle coming directly from the metabolism of some or other of this material. And the same view may be extended to other tissues. Both the framework and the intercalated material undergo metabolism, and have, in different degrees, their anabolic and katabolic changes; both are concerned in the life of the living substance but one more directly than the other, and this is what was meant by the terms "directly" and "indirectly," used in $\$ 454$. Such a mode of expression seems preferable to the more common one, based on the analogy of a firearm, of the muscle fibre firing off the contractile material ; in the firearm there are no such connections between the machine and the charges as obtain in the living mechanism. We may perhaps further be led by this to distinguish between growth as bearing on the framework, and mere temporary nutrition as bearing on the accumulation and expenditure of the lodged material. We may add that since some of the material so lodged in the 
framework will consist of substances which have not yet underyone metabolism, but are either about to be worked up into the framework itself, or are about to be transformed in a more direct way into some product of metabolism, or are substances whose presence is in some way necessary for the carrying on of metabolic processes in which they themselves take no bodily part, we must recognize a continuity without any sharp break between this material which we regard as part of the tissue, and the lymph which simply bathes the tissue and flows through the interstices. Hence such phrases as "tissue proteid" and "floating proteid" ( $\$ 436)$, are undesirable if they are understood to imply a sharp line of demareation between the "tissue" and the blood or lymph, though useful as indicating two different lines or degrees of metabolism.

$\$ 457$. The products of muscular metabolism pass into the lymph bathing the fibre, and so, either by a direct path into the capillaries or by a more circuitous course through the general lymphatic system, into the blood. The fate of the carbonic acid we have fully treated of in dealing with respiration; the little we know concerning the nitrogenous product or products has been stated in dealing with urea; the third recognized product is lactic acid, sarcolactic acid. Did any considerable amount of oxidation take place in the blood stream while the blood is flowing along the larger channels, subject only to the influence of the vascular walls, we might fairly expect that the lactic acid discharge from the muscles would be subjected to oxidizing influences while still within the blood stream of the larger channels. We have, however, no satisfactory evidence of any lactic acid being oxidized in this way. On the contrary, there is a certain amount of experimental and other evidence that lactic acid present in the blood is somehow or other disposed of by the liver; and that if the liver fail to do its duty, lactic acid may appear in the urine. It is tempting to suppose that it might there by a synthetic effort be converted into glycogen, the liver thus utilizing some of the muscular waste product, but the experimental and other evidence is all against this view. In the absence of actual knowledge we infer that it is in the liver oxidized into carbonic acid and water, thus adding its contribution to the supply of heat, or prepared in some way for oxidation elsewhere. Probably such a change is not confined to the liver, but takes place in other organs, such as the spleen. Thus the kind of action on which we dwelt in treating of urea, namely, that the products of the metabolism of one organ are carried to other organs for further elaboration and possible utilization, applies to the non-nitrogenous as well as to the nitrogenous products of muscular metabolism; and if a muscle gives rise to other non-nitrogenous products than carbonic and lactic acid these are probably disposed of in some such way as the lactic acid. In speaking of glycogen in the winter frog $(\$ 380)$ we said that possibly the glycogen so stored up might arise from sugar brought to the liver from other tissues. If that he so, we should further expect that some at least of that sugar, either as such or as some allied substance, would come from the skeletal muscles which form so large a part of the body of the frog; and if so, we must conclude that under the special circumstances obtaining in the winter frog the muscles discharge into the blood a non-nitrogenous product not in the form either of carbonic or lactic acid. It is perhaps, however, more probable that the sugar in question comes from a metabolism of the fat stored up in the "fatty bodies" and elsewhere.

$\$ 458$. As far as we can see at present the plan of nutrition thus briefly sketched out for muscle holds good for the other tissues as well, the chief or at least the most conspicuous differences bearing on the nature and properties of and the changes undergone by the material formed by and held by 
the more distinctly structural work. Thus the mucin of the salivary mucous cell finds its analogue either in the contractile substance itself, or inore probably in some early nitrogenous product of the explosion of the contractile substance, such as may correspond to the myosin of rigid muscle. The metabolism of the hepatic cell seems, as we have seen, to be especially characterized by its returning to the blood a body, viz., sugar, still containing a considerable amount of energy, available for use in other parts of the body. And this suggests the question whether in the normal metabolism of muscular substance a similar something, still holding a considerable quantity of energy, some proteid substance for instance, may not be returned to the blood; so that the metabolism of muscle is imperfectly described in saying that the results are carbonic and lactic acids and an antecedent of urea. If this be so, then muscles may be of other use to the body at large than as mere contractile machines, just as the liver has other uses than the production of bile. And the same considerations may be applied to the other tissues as well.

$\S 459$. Whether the chief product of the metabolism of any tissue be a proteid substance, or a fat, or a carbohydrate, proteid substance is the pivot, so to speak, of the metabolism, and nitrogenous bodies always appear as the products of metabolism. This is strikingly seen in the nutrition of plants where, as far as mere bulk or weight is concerned, the active metabolizing tissue is insignificant compared with the mass of products of metabolism heaped up in the form of starch or cellulose or some allied carbohydrate. The protoplasm of a vegetable cell soon becomes a mere film bearing a heavy burden of heaped-up metabolic products and eventually disappears; and of that film only a part corresponds to what we spoke of above as the living framework of the muscle. Yet that scanty proteid-built framework is more or less directly concerned in the production of the carbohydrate material and the various conversions which that material undergoes. Proteid, nitrogen, changes are entangled with the carbon changes; and since the products of metabolism in the plant are not as in the animal cast out of the organism, but for the most part heaped up within it, we find the plant storing up in parts, where if they serve no useful purpose they at least do no harm, nitrogenous products of metabolism, such as those known as vegetable alkaloids, many of which by their amide nature betray their kinship to the animal nitrogenous product urea.

$\$ 460$. The rate at which in the adult, leaving aside for the present the special nutrition of the young, nutrition is carried on, and the characters of the nutrition, are dependent on a variety of circumstances. Each tissue has of course a line of nutrition of its own which circumstances may favor or hinder, but cannot change in nature; the nutrition of the hepatic cell cannot be altered to that of the muscular fibre. The same tissue, moreover, has in different races and different individuals specific and individual characters of nutrition; the flesh of a dog is not the same as that of a man, the muscle of one man lives differently from that of another, the metabolism per unit of body weight is, as we have seen, greater in the smaller organism, and so on.

Within the limits and subject to the conditions, however, thus fixed by race and personality, general influences produce general variations in nutrition. The rate of nutrition of a tissue, for instance, is dependent on the food, on the amount and nature of the food material brought to the tissue by the blood. We have seen that proteid food, in contrast to carbon food, markedly increases the metabolism of the body. Since this increase tells not only on the nitrogenous but also on the carbon metabolism ( $\$ 437$ ), it cannot be the result of a mere luxus consumption of the proteid food itself; and unless we suppose that the presence of the excess of proteid material either in the ali- 
mentary canal, or while passing through the capillaries of some organ, such as the liver, acts as a stimulus to some reflex nervous machinery through whose action the metabolism of certain or of all the tissues is hurried on, we must conclude that it is the direct access of proteid material to the tissues themselves which stirs them up to increased metabolic activity. That proteid food should do this, and not carbohydrate or fat, seems to be connected with the fact just dwelt on that proteid material is the pivot of metabolism.

$\$ 461$. In the preceding chapters of this work we have had abundant evidence that the metabolism of the tissues is subject to the government of the central nervous system; the contraction of a muscle, the secretory activity of a gland, the increased or diminished production of heat, all afford instances of nervous impulses affecting metabolism. In most of these instances the changes induced fall within the downward, katabolic phase and have a downward character; thus, when a muscle contracts, the result is a conversion of more complex bodies into simpler bodies; and the same, as far as we can see, is true of most other cases. But it is open for us to suppose that nervous impulses might affect the upward, anabolic phase and have a constructive influence. There are no reasons for regarding such an action as impossible; and indeed some phenomena, such as those of inhibitory nerves and the antagonism between these and augmentor nerves, pointedly suggest some such view. Thus, we may suppose that an inhibitory impulse produces such changes in the cardiac muscular substance that the upward constructive processes are assisted and the down ward disruptive processes checked, whereby the setting free of energy is checked, and so the beats hindered or stopped, the inhibitory effect being followed by a period of rebound in which the savings of the inhibited period are spent in increased action. Conversely we may suppose that an augmentor impulse hinders the anabolic and assists the katabolic changes, and conversely also, when it has done its work, leaves the tissue with diminished capital manifested by feebler beats or by the absence of the power to beat. And similarly in the case of the respiratory centre and other tissues. When we have to study the origination of visual impulses in the retina we shall come upon a riew that a wave of light may affect what we shall call a visual substance either by promoting anabolic constructive changes or by increasing katabolic destructive changes according to its wave length. There is then evidence, to a certain extent, for the view on which we are dwelling; but, without discussing the matter any further, we may say that the conception, though suggestive, has not yet been demonstrated, and so far can only be spoken of as probable.

$\$ 462$. One value perhaps of such a view lies in the fact that it warns us against assuming that a nervous impulse can only produce disruptive katabolic changes such as are seen in muscular contraction or in secretion. The effects of stimulating a nerve going to a muscle or a salivary gland are striking and obvious, and the behavior of a muscle or a gland as far as contraction and secretion are concerned is, within certain limits, under experimental control. But there are certain phenomena, seen chiefly in the course of disease, and lying, to a very small extent only, within the control of experiment, which seem to show that the central nervous system governs the metabolic changes, the nutrition, not only of muscle and gland, but of various other tissues, in a deeper and more general way than that of simply promoting (or hindering) contraction or secretion. Thus, as we have seen ( $\$ 81)$, when the connection between a muscle and the central nervous system is severed, the muscle eventually wastes and loses its vitality; when all the nerves going to the submaxillary gland are severed, the gland, instead of being, as in the normal condition, intermittingly active and quiescent, pours forth a continuous "paralytic" secretion and eventually degenerates and wastes. When in 
a rabbit the fifth nerve is divided in the skull the loss of sensation in those parts of the face of which it is the sensory nerve is followed by nutritive changes. Very soon, within twenty-four hours, the cornea becomes cloudy : and this is the precursor of an inflammation which may involve the whole eye and end in its total disorganization. At the same time the nasal chambers of the side operated on are inflamed, and very frequently ulcers make their appearance on the lips and gums. And similar results have been seen in other animals, including man. If the operation be conducted in a young animal, which subsequently lives to maturity, the head may become bilaterally unsymmetrical, as shown especially by the skull. Again, division of both vagus nerves is very apt to be followed by inflammation of both lungs, loy fatty degeneration of the heart, and so by death.

In several of these instances the effect is a mixed one, and the problem complicated. Thus, in the case of division of the fifth nerve, seeing how delicate a structure the eye is, and how carefully it is protected by the mechanisms of the eyelids and tears, it seems reasonable to suppose that the inflammation in question might simply be the result of the irritation caused by dust and contact with foreign bodies, to which the eye, no longer guided and protected by sensations, these being destroyed by the section of the nerve, became subject. In the same way the ulcers on the lips and gums might be explained as injuries inflicted by the teeth on those structures in their insensitive condition. And some observers maintain that the inflammation of the eye may be greatly lessened or altogether prevented if the organ be carefully covered up, and in all possible ways protected from the irritating influences of foreign bodies. Other observers, however, have failed to prevent the inflammation in spite of every care. So, also the inflammation of the lungs following upon division of both vagus nerves seems to be due, not to any direct nutritive action of the pulmonary branches of the vagus on the pulmonary tissue, but to food accumulating in the pharynx, owing to the paralysis of the cesophagus and larynx, and then passing into the air passages, and so setting up inflammation. Death in these cases is, moreover, often the simple result of inanition caused by the paralysis of the cosophagus allowing no food to reach the stomach. The phenomena of the paralytic secretion of saliva are also of a complicated nature.

But even without insisting on such instances as the above, various other phenomena of disease seem to indicate such an influence of the nervous system on nutrition as we are discussing. As examples we might mention the rapid and peculiar degeneration of and loss of contractility in the skeletal muscles in certain affections of the spinal cord, the changes in the muscles being more rapid and profound than in the nerves; the phenomena of bedsores, especially the so-called acute bedsores of cerebral apoplexy; some at least of the cases of vesical affections attendant on spinal cerebral diseases or injuries; the more rapid atrophy and loss of contractility in muscles which follow upon contusions of nerves as compared with the effects of simple section of nerves; the occurrence of certain eruptions, such as lichen, zona, ecthyma, etc., in various spinal or cerebral diseases, and indeed the general phenomena, and especially the topography of the eruption, of a large number of cutaneous diseases. Lastly, but not least, we might quote the general process of inflammation. These are examples of disordered nutrition. To them we might add as instances of altered but yet orderly nutrition the remarkable connections observed between changes in the form of the fingers and growth of the nails and hairs, and certain internal maladies, such, for instance, as the "clubbed fingers" of phthisical and other patients, and the like. We might also call attention to the influence of light on the nutrition of animals. The experience of blind people and blind animals indicates 
some special connection between visual sensations and the nutrition of the skin; and this can hardly be other than a nervous connection. The effects of prolonged darkness on nutrition in general and the experimental results which show that the total metabolism of the body is influenced by light, also suggest some nervous action. The influence of cold, again, in determining the growth of hair points in the same direction.

Making every allowance for the intervention in the production of the phenomena quoted above of such factors as common actions of the nervous system already well known to us, such as vasomotor changes, making every allowance for the consequences of the failure or bluntness of sensation and the absence of those beneficial after-results of muscular activity which we pointed out in $\$ 86$, recognizing, moreover, that changes in one organ may affect the condition of other distant organs by changes induced in the composition or qualities of the blood, there still remains a residue which seems distinctly to point to the conclusion that the influence of the nervous system is not limited to such changes of the muscles as belong to the production of contractions or the generation of heat, but bears on the whole nutrition of the muscle. Similar considerations lead us also to conclude that the influence of the nervous system bears on the whole nutrition of the glands, of the bloodvessels, of the skin, and of the connective tissue in general, in fact of nearly the whole body.

\section{ON Diet.}

$\S 463$. An ordinary man living an ordinary life will need for the maintenance of vigorous health a certain amount of food of a certain kind; this we may take as a normal diet.

Presuming that the experience of man has led him to adopt what is good for him, we may ascertain approximately the normal diet by means of the statistical method, by examining the nature and amount of the daily food of a very large number of individuals. The most valuable data for this purpose are those gained by inquiries among persons who choose their own food; the results gained from the diets used in prisons or other institutions, or among bodies of men such as the army, though more readily arrived at, are open to the objection that the diets in question are determined in part by the theoretical opinions of those whose duty it is to fix the diet. Putting together the various statistical results thus obtained, and selecting the quantities which seem to be most commonly used rather than attempting to strike a strict average or take a strict mean, we find that in an ordinary diet for the twentyfour hours the several food-stuffs are:

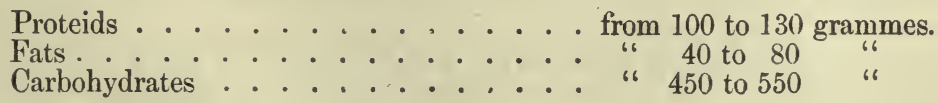

to these we must add

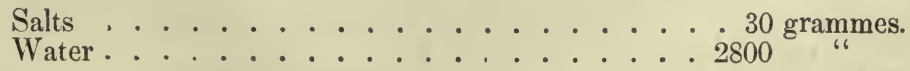

The total (available) potential energy of the lower estimate is 2610 , of the higher 3505 (kilogramme-degree) calories, calculated, in round numbers, on the data of $\S 441$. With such a statistical diet we may compare an experimental diet, that is to say a diet arrived at through a series of trials on an individual man whose body might be taken to be an average one, that diet being considered a normal one in which the body, maintaining vigorous 
health, neither gained nor lost in weight, and remained, moreover, in nitrogenous equilibrium with the nitrogen of the egesta equal to that of the ingesta. To make sure that under such a diet the body was remaining of the same composition, there ought to be evidence of a carbon equilibrium also, otherwise during the period of the experiment fat might be replaced by water (see $\$ 435$ ); but this is unlikely, and we may therefore accept the method as a fair one. It has given in the hands of two different observers the following somewhat different results, the diet A being that already quoted in $\$ 441$ :

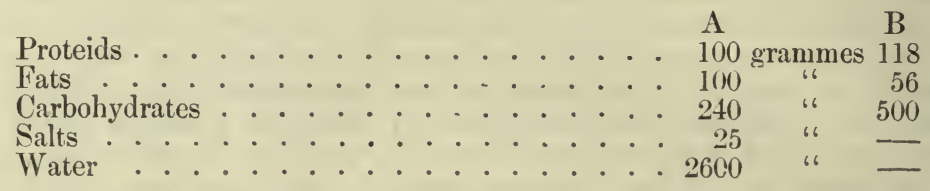

The total (available) potential energy is respectively 2310 and 3035 calories.

On the whole, the diets gained by the two methods agree very largely. To put down a single column of figures as "the normal diet" would be to affect a vain and delusive accuracy. If we desire, for theoretical purposes, to select some one set of figures rather than others, we might be influenced by the considerations that the lower amount of proteids in the experimental diet was nearer the mark than the higher amount of some of the statistical diets, and further that, where cost is not of moment, the substitution of fat for an excess of carbohydrates is desirable. We should be thus led to take the experimental diet A as on the whole the best or most "normal" one, and that is the one which we employed in the calculations of $\$ 441$. It will be observed that the potential energy of this diet is less than that of any of the others, and, as we said while then speaking of it, may be considered low; but there was no evidence that it was insufficient. Still it must be remembered that neither it nor any of the others is to be regarded as distinctly proved to be the real normal diet. Against the experimental diet we may urge that the number of experiments have been few, and conducted on a few individuals only at most, and that a larger number of experiments, with a variety of combinations of different amounts of the several food-stuffs, might lead to a different result; that, for instance, with certain amounts of fats and carbohydrates, the amount of proteid needed to maintain healthy bodily equilibrium, including nitrogenous equilibrium, might be reduced much below the 100 grammes, especially if particular kinds of proteids, fat, or carbohydrates were used, and especial attention ( see $\$ 440$ ) were paid to the salts. And, indeed, a considerable number of observations have been made tending to show that a man of average size and weight may continue in nitrogenous equilibrium and in good health with a daily ration of much less than 100 grammes proteid, with as little as 40 grammes for example. To this we shall have to refer in speaking of a vegetable diet. Against the statistical diet, on the other hand, we may urge that instinct is not an unerring guide, and that the choice of a diet is determined by many other circumstances than the physiological value of the food.

$\S 464$. Taking, however, some such diet as the above to be the approximately true normal diet, we may call attention to the fact that the normal diet is made up of each of the three great food-stuffs, carbohydrates being in excess. We may here remark incidentally that the diets of both the carnivora and herbivora agree with that of omnivora in containing all three food-stuffs ; they differ from each other as to the relative proportions only. As we have seen, the body may be maintained in equilibrium on proteid food 
alone; but an exclusively proteid diet is not only bought dearly in the market, but also paid for dearly within the economy; we are, of course, now speaking of man. To obtain the necessary carbon out of the carbon moiety of proteid unnecessary labor is thrown on the economy, and the system tends to become blocked with the amides and other nitrogenous waste arising out of the nitrogen moiety simply thrown off to secure the carbon.

Fats and carbohydrates are much more akin to each other than is either to proteid; and if, on the one hand, as ( $\$ 455)$ seems possible or even probable, the fat of the food and of the body is converted into sugar either on its way to become built up into the tissue, or in the course of the changes taking place outside the real living framework of the tissue by which it is reduced to carbonic acid, and that, on the other hand, carbohydrates can furnish the fat whose presence in the body is necessary, we might expect that carbohydrate alone without fat might with proteid form a normal diet. But on this point experience is probably to be trusted; and we may infer that in every normal diet some fat at least must be added to the starches and the sugars.

The advantage of this mixture is probably felt while the food is as yet within the alimentary canal. What we have learned concerning digestion leads us to regard it as a complicated process, and we cannot readily imagine that the proteolytic, amylolytic, and adipolytic changes run their several courses, especially in the small and large intestine, apart from and irrespective of each other. We are rather led to suppose that the accompaniment of one set of changes, in some indirect manner, favors the others; and it is for that reason probably that we take our food-stuffs not separately, but mixed in the same meal, often on the same plate, and even in the same mouthful. But apart from this the two food-stuffs, fats and carbohydrates, must play different parts in the economy, so that the one cannot be wholly substituted for the other; and though, beyond the fact that the one seems to be a source of energy and the other not, we do not as yet know the true physiological function of the hydrogen of the fat as compared with that of the differently disposed hydrogen of the carbohydrate, we may perhaps infer that the difference of use within the body of the two kinds of food-stuffs bears not so much on their ultimate consumption to supply energy, as on the various complicated processes which they undergo and arrangements in which they take part before the end of their work is reached. We have had a hint that the carbohydrate more rapidly supplies the heat-giving metabolism than does the fat; and this suggests an advantage to the economy in receiving daily a certain portion of the more tardy material, while at the same time it may be taken to mean that the fat before it is used to give rise to energy has first to be converted into sugar, and so takes more time in its work.

The main carbohydrate of every diet is starch, and as far as we can learn at present, the starch which is so large a part of the cereals and vegetables consumed by man is the same body in all of them; for the use of such bodies as inulin is so insignificant that it may be neglected. Man, however, consumes no inconsiderable quantity of sugar, chiefly cane sugar. Since the starch of a meal does not become available for the economy until it has been converted into sugar, we might be inclined to infer that it was a matter of indifference whether the carbohydrate of a diet were supplied as starch or as sugar. But besides the fact that any large deficit of starch in a diet might seriously interfere with the general course of digestion, especially if as urged above the several digestive processes are more or less dependent on each other, it must be remembered that the sugar into which starch is changed by digestion is maltose, while cane sugar appears to be either 
absorbed as cane sugar or at most only inverted. Moreover, if our laboratory experiments truly represent the digestion taking place in the living body, only part of the starch $(\S 194)$ is changed into maltose, while part becomes some variety of dextrine or of starch. Our knowledge of sugars and of their fate in the economy is too imperfect for us to be able to state the effects on the body of digested starch as compared with those of cane sugar or milk sugar; but that these are or may be different is shown by the experience of medical practice. In many cases the total effect on the body of a diet from which cane sugar is as much as possible eliminated, though starch be allowed, is very different from that of one of which cane sugar forms an appreciable part.

Concerning cellulose, which in herbivora appears certainly to serve as a source of energy and to be a real food-stuff, our knowledge will not allow us to decide whether it has any special uses of its own, or whether the body is simply led to utilize and make the best of what is a necessary accompaniment of the starch of vegetable food.

Concerning the salts present in a diet, we need only repeat what was said in $\$ 440$, that these, though affording of themselves little or no energy, are as essential a part of a diet as the energy-giving food-stuffs, inasmuch as they in some way or other direct metabolism and the distribution of energy. And this is true not only of the inorganic salines, such as chlorides and phosphates, but also of the so-called extractives. As we have seen, the presence of these bodies, both the simpler inorganic and the more complex organic salts, in the blood or in the extra-vascular juices or lymph of the tissues is essential to or directs or modifies the metabolic activity of the several tissues. The beneficial effects, as components of special diets, of such things as beef-tea and meat extract, which consist chiefly of salts and extractives, with a very small quantity of albumose or other forms of proteid, and the effects either beneficial or deleterious of drugs, both turn in common upon their taking a part of some kind or other in, it may be upon their interference with, metabolic processes. The salts and extractives of a diet may be looked upon as necessary daily medicines, and a medicine as a more or less extraordinary variation in these elements of a diet.

Alcohol, to the use of which as a component of an ordinary diet special interest for various reasons attaches, comes in this class. For though observations show that the greater part of a moderate dose of alcohol is oxidized within the body, and so serves as a source of energy, man has recourse to alcohol not for the minute quantity of energy which is supplied by itself, but for its powerful influence on the distribution of the energy furnished by other things. That influence is a very complex one and cannot be fully discussed here. It is stated that moderate or small doses of alcohol diminish the consumption of oxygen and production of carbonic acid, that is to say, diminish the total result of the metabolism of the body, while larger but still not intoxicating doses have a contrary effect and increase the total metabolism. But such a statement affords no sound basis for any conclusion as to the general physiological effect of alcohol, or as to its usefulness as part of an ordinary diet; it does not justify such a conclusion, for example, as that alcoholic drinks, taken in moderation, by diminishing metabolism economize the resources of the body. The prominent physiological problem of dietetics is not either to increase or diminish the metabolism of the body, but to direct that metabolism into proper channels; and whether in each particular case a given dose of alcohol gives a right or a wrong turn to the physiological processes of the body depends on the particular circumstances of the case. For the action of all these bodies of which we are now speaking, in contrast with the actions of the food-stuffs proper, 
is not only complex but variable; so complex and variable that simple experience is at present a more trustworthy guide than speculative physiology. We may add that the physiological action of alcoholic drinks is still further complicated by the fact that most such drinks contain, beside ethylic alcohol, various other allied substances, whose action is even more potent than that of the ethylic alcohol itself, and whose presence very markedly determines the total effect of the drink. Such articles of diet as tea and coffee stand upon very much the same footing as alcohol.

The quantity of fluid which a man drinks or should drink daily, or more correctly the quantity of water which he should daily add to the dry solids of his diet, must vary widely according to circumstances. It will differ according as he is perspiring freely or not, according to the nature of the dry solids of the diet, whether largely carbohydrate or not, and so on. A lower limit, below which excretion is impeded, and a higher limit, above which digestion and metabolism are injuriously affected, probably exist; but we have as yet no adequate data which will enable us to fix either of them.

$\S 465$. In the selection of articles of food to supply the food-stuffs and other constituents of a normal diet, regard must, of course, be had in the first place to the amount of potential energy present in the material. The articles chosen for the daily fare must contain between them so much proteid, fat, and carbohydrate, representing so much available energy. But it is no less important that the potential energy in the material should be really available for the economy. The material must have such qualities that it is digested within the alimentary canal, and further that its digestion and absorption do not give rise to trouble either in the alimentary canal or in that secondary digestion carried on by means of the various metabolic events which we have discussed in preceding sections. A really nutritious substance is one which not only contains in itself an adequate supply of energy, but is of such a nature that its energy can be appropriated by the economy with ease, or at least with as little trouble as possible. We have approximate data for determining how far an estimate of the relative usefulness of various articles of food must be corrected, by allowing for the proportion of each which after an ordinary meal merely passes through the alimentary canal, and the energy of which is not in any way available for the body's use. Thus, a number of observations carried out on healthy individuals gave, in the case of the following articles of food, the following figures as the percentage, reckoned in each case on dry material, which could be recovered from the feces, and was, therefore, not digested and not used by the body : Meat, 5 per cent.; eggs, 5 per cent.; milk, 9 per cent.; bread (white), 4 per cent.; black bread, 15 per cent.; rice, 4 per cent.; maccaroni, 4 per cent.; maize, 7 per cent.; peas, 9 per cent.; potatoes, 11 per cent. It must, however, be remembered that the actual correction to be made in any case will depend on the mode of cooking of the material, on the character of the meal of which it forms part, and on the individual capabilities of the consumer, the latter, too, varying under different circumstances.

The above refers to what may be called rough digestibility, but besides this there are other circumstances to be considered. The same food-stuff in two articles of food, though actually digested, that is to say taken up by the alimentary canal, may, even while still within the alimentary canal, undergo changes in the one case differing from those in the other. A proteid may for instance in one case tend to be entirely converted into peptone, or to break up into leucin, etc., or in other cases to undergo other changes; and a carbohydrate may in one case be absorbed as maltose, and in another 
give rise to lactic acid. Indeed, when we speak of the digestibility or the indigestibility of this or that article of food, we do not in many cases so much mean the relative amount of the substance taken up in some way or other by the alimentary canal as the characters advantageous or otherwise of the changes which it undergoes in being so taken up.

Hence the purely chemical statement of the amount of potential energy present in an article of foor is no safe guide of the physiological value of the substance. A chunk of cheese stands very high on, generally at the top of, a table of the nutritive value of articles of food drawn up on exclusively chemical principles-according to the units of energy present in a unit of the material-but it is very low down in a corresponding physiological table. And similarly a dish of old peas has a very different physiological function from a plate of fresh meat, even when both contain the same amount of nitrogen.

In thus correcting for digestion the nutritive value of a diet it must also be borne in mind that the alimentary canal, while chiefly a receptive organ, is also to some extent $(\$ 245)$ an excretory organ; a free passage through the canal is needed not only for carrying off undigested matter but also for getting rid of excreted matter; and the presence of the former, up to certain limits, assists the discharge of the latter. Were it possible to prepare a diet every jot and tittle of which could be digested and absorbed, the use of such a diet would probably bring about disorder in the economy, through the absence of a sufficiently rapid discharge of the matters excreted into the alimentary canal. Hence cellulose and like substances, even when unutilized through absorption, are not without their use, and experience shows that digestion may be promoted by eating undigestible things.

$\$ 466$. The several foorl-stuffs of a diet may be drawn from the animal or from the vegetable kingdom. Vegetable proteids appear to undergo the same changes in the alimentary canal as do animal proteids, and the main effects on the body of proteids from the two sources seem to be the same. Our knowledge at present, however, is too imperfect to enable us to decide whether the functions of the two are exactly the same, whether the body behaves exactly the same upon a diet in which the proteids are exclusively of vegetable origin, as upon a diet in which, otherwise the same, the proteids are partly of animal origin also. Nor have we much better knowledge of the relative nutritive value of vegetable and animal fats. And as we have already said, we possess little or no exact knowledge as to the part played by those extractives in respect to the amount and nature of which animal food strikingly differs from vegetable food. In attempting, therefore, a judgment from a purely physiological point of view as to the value of an exclusively vegetarian diet compared with a diet of both animal and vegetable origin, we can do little more at present than inquire whether the former supplies the several food-stuffs in adequate quantity, in proper proportion, and in such a form as to be economically utilized by the body.

The careful examination during three separate periods of several days each of the ingesta and egesta of a man, 28 years old, weighing 57 kilos, who had for three years lived on an exclusively vegetable diet, viz., bread, fruit, and oil, gave the following results:

The daily diet consisted on the average of 719 grms. solid matter and 1084 grms. water. It contained

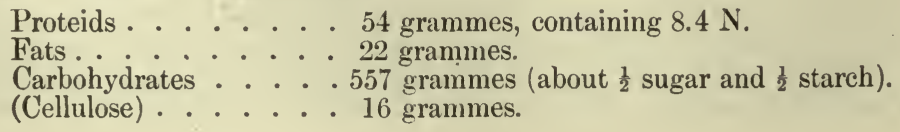


The daily feces weighed, when fresh, 333 grms. containing 75 grms. solid matter, and were therefore both bulky and watery. There were present in the feces, fat 7 grms., starch 17 grms., cellulose 9 grms., showing that 30 per cent. of the fat, 6 per cent. of the starch, and 56 per cent. of the cellulose had not been utilized by the body. The subject had really lived on fat, 15 grms., carbohydrates 540 grms. (and cellulose 7 grms.). The feces contained no less than 3.46 nitrogen. If we reckon the whole of this as proteid, this would give 22 grms. of undigested proteid, so that there has been a waste of 41 per cent. of the proteids, leaving only 32 grms. available for real use in the body; and, indeed, a very small portion only of this nitrogen can be regarded as really discharged from the body itself. The total solids of the feces must be reckoned as partly excreta but chiefly undigested food. If we regard the 75 grms. of solid feces as entirely undigested food, the whole solid food available for the body must be reduced from 719 grms. to 644 grms.

The urine of the day contained 5.33 grms. nitrogen; this added to the 3.46 grms. nitrogen in the feces gives 8.79 grms. nitrogen in the total egesta as compared with the 8.4 grms. nitrogen of the food, indicating a slight loss of nitrogenous material from the body ; but if we suppose that all the nitrogen in the feces was not in the form of undigested food we may neglect this; and indeed the subject of the observation was in apparently good health and stationary weight.

Compared with either of the normal diets given in $\$ 463$, the above diet is striking for the low amount of proteids and of fats and the relative excess of carbohydrates. But though such a diet may be taken as perhaps fairly typical of the daily food of a rigid vegetarian, a much more richly proteid diet may be obtained from sources still strictly vegetable. Thus the diet, entirely vegetable in nature, of an average Japanese laborer of about the same weight as the individual whose data we have just given has been estimated to consist of proteids 102 grms., fat 17 grms., carbohydrates 578 grms. And the diet of a Roumanian peasant, living chiefly on beans and maize with the addition of fat of some kind, has been calculated to furnish no less than proteids 182 grms., fat 93 grms., carbohydrates 968 grms. ; but the real nutritive value of such a diet must need very large correction indeed. ( $C f . \$ 465$.)

The examination of the diet of an individual living with a fair nitrogenous equilibrium and apparently good health on a modified vegetable diet-that is to say, one which included milk and eggs-gave the following: Proteids, 74 grms. ; fat, 58 grms.; carbohydrates, 490 grms., a diet which differs from the normal diet almost solely in the lesser amount of proteids, one-third of which, by the by, was supplied by the animal material, eggs and milk. In another instance, nitrogenous equilibrium and fairly good health were secured, for some weeks at all events, on a vegetable diet yielding proteids, about $100 \mathrm{grms}$. ; fat, $70 \mathrm{grms}$.; carbohydrates, 400 grms.; but in this nearly the whole of the fat was furnished by the animal product butter, and Liebig's extract was freely used.

Confining ourselves, however, to the more strictly vegetarian diet, we may conclude in the first place that, unless the daily food be very large in amount, the proteid element of such a diet falls considerably below the 100 or more grms. given in the normal diet. But we cannot authoritatively say that such a reduction is necessarily an evil ; for, as we stated above ( $\$ 463)$, our knowledge will not, at present, permit us to make an authoritative exact statement as to the extent to which the proteid may be reduced without disadvantage to the body when accompanied by adequate provision of the other elements of food; and this statement holds good whether the body 
be undertaking a small or large amount of labor. A second feature of such a diet is the marked reduction of the fat and its replacement by carbohydrates. Although here again we cannot make a distinctly authoritative statement, the evidence which we possess bears clearly in the direction that such a reduction is a marked disadvantage. A third and very characteristic feature of the strictly vegetarian diet is the relatively large amount of undigested food lost to the body and discharged as feces. Even when the diet is scanty, so that the proteid element is low, the amount of feces relatively to the total food is high; and when a more normal proteid contribution is secured by ample meals the feces becomes exceedingly voluminous. Indeed when, leaving man, we compare the herbivorous with the carnivorous mammal, we find that the former is almost as clearly distinguished from the latter by its frequent and abundant feces as by the anatomical features of its organization. We have already urged that, since the feces serve as a means of excretion of the real waste products of metabolism, a certain amount of vehicle to carry these away is of advantage or even necessary; but there are no facts at present known to us which show that the larger intestinal current of the purely vegetable diet effects any such good as can compensate for the obvious waste of labor incurred in the transport and management, to say nothing of the opportunities of mischief offered by a mass of material more subject to the dominion of foreign organisms than even to that of the body itself, though these opportunities are less than with a corresponding mass of animal origin. With respect to these three features, then, the strictly vegetarian diet seems, on physiological grounds, inferior to one of a mixed nature. There are, as we said, other aspects, still of a physiological kind, to be considered, such as the relative digestibility of vegetable articles of food, the relative metabolic value of the food-stuffs of vegetable origin, and the influence of animal extractives ; but any fuller discussion of these points would be out of place here.

$\S 467$. We have treated the diet discussed above as a normal diet, suitable for man under ordinary or general circumstances. Ought such a diet to be modified for the various exigencies of life; such as labor, age, climate, and the like? work.

We shall discuss the influence of age in the concluding portions of this

We may be inclined, at first sight, to assume that the total amount of the diet should vary with the weight, that is, the size, of the individual; and, indeed, in discussions on nutrition, statements concerning metabolism and amounts of food are often given in terms of per kilo of body weight. In a broad sense, it may be true that a small man needs less food than a large one; but it must be remembered that, as we saw in speaking of animal heat, the smaller organism, having the relatively larger surface, carries on a more rapid metabolism per unit of body weight, and so needs relatively more food. And, moreover, the influence of size is probably far less than the influence exerted by the inborn individual characters of the organism, giving rise to what we may call the personal equation of metabolism. The smaller metabolism of woman, leading to the use of scantier diet, as compared with that of man, is to be regarded in this light rather than with reference to the average lesser weight of woman. The relative metabolism of the two sexes may be illustrated by the case of an active man and his wife, both of about the same age and weight, the man being rather the hearier and the woman rather the older, who in carrying out together an experiment on the relative values of vegetable and animal food, both lived for some time on the same kind of diet, and found that nutritive equilibrium was, in the one case and in the other, maintained when 


\begin{tabular}{|c|c|c|c|}
\hline The man consumed daily about & $\begin{array}{l}\text { Proteids. } \\
.100)\end{array}$ & $\begin{array}{c}\text { Fats. } \\
70\end{array}$ & $\begin{array}{c}\text { Carbohydrates. } \\
400\end{array}$ \\
\hline The wife & . 60 & 67 & 340 \\
\hline
\end{tabular}

The most striking difference is in the proteids.

$\S 468$. With regard to climate, the chief considerations attach to temperature. When the body is exposed to a low temperature the general metabolism of the body is increased owing to a regulative action of the nervous system ( $\$ 448)$. We might infer from this that more food is necessary in cold climates; and, since the increase in the metabolism appears to manifest itself chiefly in a greater discharge of carbonic acid, and therefore to be especially a carbon metabolism, we might infer that the carbon elements of food should be especially increased. When the body is exposed to high temperatures, the same reflex mechanism tends to lower the metabolism; but the effects in this direction are much less clear than those of cold, and soon reach their limits; the bodily temperature is maintained constant under the influence of surrounding warmth not so much by diminished production as by increased loss. We may infer from this that in warm climates not less, but, if anything rather more, food than in temperate climates is necessary in order to supply the perspiration needed for the greater evaporation and discharge of heat by the skin.

In both cold and warni climates, however, man trusts much more to variations in his clothing and immediate surroundings to protect him against cold or to guard him from heat than to any marked variations in his normal diet. In the former he may, perhaps, be expected to eat somewhat more, since, in spite of wrappings, the skin still feels in part the cold, and thus the nervous mechanism for the increase of metabolism is, to a certain extent, set to work. And since the metabolism thus increased appears to affect especially the carbon of the body, he may further be expected to increase the fats rather than the carbohydrates of his food, seeing that the former supply him with the most energy for their weight. But it is very doubtful whether what he might thus be expected to gain over a corresponding increase in carbohydrates is not more than counterbalanced by the increased labor of digestion; and the habits of the dwellers in arctic climates cannot safely be taken as guides in this matter, for their reputed love of fat is probably the result of that being their most available form of carbon. Indeed, the evidence that the increase of metabolism provoked by cold bears exclusively on carbon constituents is so uncertain that it may be doubted whether any change in the normal diet, beyond some increase in the whole, should be made to meet a cold climate. Sinilar reasons would lead one to infer that man in the warmer climate would maintain, on the whole, the same normal diet, the only change being, perhaps, to increase it slightly, possibly throwing the increase chiefly on the carbohydrates with the special view of furthering perspiration.

$\$ 469$. A special diet for the purpose of fattening-that is to say, for the accumulation of adipose tissue out of proportion to the rest of the body-is not needed in the case of man. The power to store up fat in adipose tissue is much more dependent on certain inborn qualities of the organism which we cannot at present define than on the kind of food; of two bodies living on the same diet, and under the same circumstances, one will become fat while the other will remain lean; and it is an object of the agriculturist to develop by breeding and selection a "constitution" which will store up the most fat on the cheapest diet. In fattening animals the chief care, when the selection of the kind of animal has been made, is to provide adequate carbohydrate food, which, as we have seen, is the chief fattener; and the object of the farmer in rearing stock for the butcher is mainly to convert cheap vege- 
table carbohydrate into dear animal fat. Further aids in fattening may be found in providing repose for the body of such a kind that, while sufficient energy is expended to secure adequate digestion and absorption of food, all causes leading to an increase of metabolism by which energy is set free and leaves the body are avoided as much as possible.

To avoid fat rather than to increase it is often an object of human care. This may be effected by diminishing fats and carbohydrates, but also, in a very marked manner, by relatively increasing the proteids. Proteid food, as we have seen, augments the whole metabolism of the body, hurrying on the destruction not only of proteid but of carbon food; and a tendency to corpulency may be counteracted by a diet in which fats and carbohydrates are much restricted, and proteids are largely increased. When, as in what is known as the Banting method, the diet is almost exclusively proteid, the nitrogenous overwork entails dangers on organisms which do not possess the power of ridding themselves freely of the large amount of nitrogenous waste which such a diet produces. A less severe method in which the fats and carbohydrates are diminished only, not entirely done away with, and the proteids only moderately increased, is less open to objection; and such a diet, assisted by other hygienic conditions, has proved successful.

An increase of daily food, largely proteid in nature, given under circumstances such as a large amount of passive exercise and skin stimulation, known as "massage," which will not only favor digestion but also promote metabolism in general, may be given with favorable results. In this way an enormous metabolism may be excited, and yet so carried on that the body gains both in flesh and in fat. Thus, in one case, the patient with an initial weight of $45 \mathrm{kilos}$, and a daily nitrogenous metabolism calculated as 28 grms. proteid, reached in the course of about fifty days a weight of 60 kilos, the daily nitrogenous metabolism being raised on one occasion to 182 grms. proteid, with an average on the whole period of 150 grms. During the treatment no less than 8420 grms. of proteid were taken as food.

$\S 470$. With regard to labor, since, as we have seen, the energy expended as work done is not taken out of and away from the amount set free as heat, the two forms of energy being so related that an increase of work done is accompanied by a greater or less increase of heat set free, it is obvious that a man who is doing a hard day's muscular work needs a larger income of energy for the day than does an idle man. What we have learnt concerning muscular metabolism further shows us that the additional energy needed is not necessarily to be supplied by an increase in the proteid components of the diet; the energy of muscular contraction does not come, as was once thought, from proteid metabolism $(\S 443)$. The fact that it is the carbon metabolism which is augmented in muscular work may suggest that the extra food for extra work should be exclusively carbon compounds; and if, as seems probable, the carbohydrates are more readily and directly available for the functional metabolism of muscle than are the fats, we might be further led to recommend an increase in carbohydrates to form a diet especially suited for labor. But several considerations should make us hesitate before we come to such a conclusion. A muscle is not a machine within the body which can be loaded and fired off irrespective of the rest of the body. In the performance of muscular labor, the condition of the muscle, the amount of energy available in the muscle itself, is of course of prime importance; but, and this perhaps especially holds good in severe labor, of great importance also, we might almost say of no less importance, is, as we have urged ( $\$ 333)$, the power of the body as a whole to avail itself of the energy latent in the muscle. The power of doing work hangs not on the muscle alone, but on the heart, the lungs, the nervous system, and, indeed, on the whole body. It is 
very doubtful whether we ever, even in supreme efforts, draw upon more than a portion of the capital of energy lodged in the muscle itself; fatigue is far more a nervous than a muscular condition, and even the distinctly muscular fatigue is as we have seen $(\$ 86)$, partly at least the result of the accumulation of products and not alone the using up of available energy. In choosing a diet for muscular labor we must have in view not the muscle itself but the whole organism. And though it is possible that future research may suggest minor changes in the various components of a normal diet such as would lessen the strain during labor on this or that part of the body, on the muscles as well as on other organs, our present knowledge would rather lead us to conclude that what is good for the organism in comparative rest is good also for the organism in arduous work, that the diet, normal for the former condition, would need for the latter a limited total increase but no striking change in its composition. In preparing the body for some coming arduous labor, in "training," as it is called, an increase of proteid food, for the purpose of hurrying on the general metabolism of the body, and thus of making "new flesh" and renovating the body, so to speak, in view of the strain to be put upon it, may perhaps suggest itself; but even this is doubtful.

The principles of such a conclusion with regard to muscular work may be applied with still greater confidence to nervous or mental work. The actual expenditure of energy in nervous work is relatively small, but the indirect influence on the economy is very great. The closeness and intricacies of the ties which bind all parts of the body together are very clearly shown by the well-known tendencies of so-called brain work to derange the digestive and metabolic activities of the body; and if there be any diet especially suited for intellectual labor it is one directed not in any way toward the brain, but entirely toward lightening the labors of and smoothing the way for such parts of the body as the stomach and the liver. 



\section{BOOK III.}

\section{THE CENTRAL NERVOUS SYSTEM AND ITS INSTRUMENTS.}

\section{CHAPTER I.}

THE SPINAL CORD.

\section{On Some Features of the Spinal Nerves.}

$\S 471$. WE have called the muscular and nervous tissues the master tissues of the body; but a special part of the nervous system, that which we know as the central nervous system, the brain and spinal cord, is supreme among the nervous tissues and is master of the skeletal muscles as well as of the rest of the body. We have already (Book I., Chapter III.) touched on some of the general features of the nervous system, and have now to study in detail the working of the brain and spinal cord. We have to inquire what we know concerning the laws which regulate the discharge of efferent impulses from the brain or from the cord, and to learn how that discharge is determined, on the one hand, by intrinsic changes originating, apparently, in the substance of the brain or of the cord, and, on the other hand, by the nature and amount of the afferent impulses which reach them along afferent nerves.

As we shall see, the study of the spinal cord cannot be wholly separated from that of the brain, the two being very closely related. Nevertheless, it will be of advantage to deal with the spinal cord by itself as far as we can. The medulla oblongata or spinal bulb ${ }^{1}$ we shall consider as part of the brain. But before we speak of the spinal cord itself, it will be desirable to say a few words concerning the spinal nerves, that is to say, the nerves which issue from the spinal cord.

We have already seen ( $\$ 92)$ that each of the spinal nerves arises by two roots, an anterior root attached to the ventral or anterior surface, and a posterior root attached to the dorsal or posterior surface of the cord. We have further seen that the latter bears a ganglion, a "ganglion of the posterior root" or "spinal ganglion," as we have (\$93) studied the structure of this ganglion.

We stated at the same time that while the trunk of a spinal nerve contained both efferent and afferent fibres, the efferent fibres were gathered up into the anterior root and the afferent fibres into the posterior root; but we gave no proof of this statement.

$\S 472$. Before we proceed to do so, it will be as well to say a few words on the terms "efferent" and "afferent." By efferent nerve-fibres we mean nerve-fibres which in the body usually carry impulses from the central ner-

1 The term medulla oblongata is not only long, but presents difficulties, since the word medulla is now rarely used to denote the whole spinal cord (medulla spinalis) but is generally used to denote the peculiar coat of a nerve-fibre, the white substance of Schwann. In using instead the word bulb or, if necessary, spinal bulb there is little fear of confusion with any other kind of bulb. The adjective is in not uncommon use, in such phrases as "bulbar paralysis." 
vous system to peripheral organs. Most efferent nerve-fibres carry impulses to muscles, striated or plain, and the impulses passing along them give rise to movements; hence they are frequently spoken of as "motor" fibres. But all efferent fibres do not end in or carry impulses to muscular fibres; we have seen for instance that some efferent fibres are secretory. Moreover, all the nerve-fibres going to muscular fibres do not serve to produce movement; some of them, as in the case of certain vagus fibres going to the heart, are inhibitory and may serve to stop movement.

By "afferent" nerve-fibres we mean nerve fibres which in the body usually carry impulses from peripheral organs to the central nervous system. A very common effect of the arrival at the central nervous system of impulses passing along afferent fibres is that change in consciousness which we call a "sensation;" hence afferent fibres or impulses are often called "sensory" fibres or impulses. But as we have already in part seen, and as we shall shortly see in greater detail, the central nervous system may be affected by afferent impulses, and that in several ways, quite apart from the development of any such change of consciousness as may be fairly called a sensation. We shall see reason for thinking that afferent impulses reaching the spinal cord, and, indeed, other parts of the central nervous system, may modify reflex or automatic or other activity without necessarily giving rise to a "sensation." Hence it is advisable to reserve the terms "efferent" and " afferent" as more general modes of expression than " motor" or " sensory."

We have seen in treating of muscle and nerve, that the changes produced in the muscle serve as our best guide for determining the changes taking place in a motor nerve; when a motor nerve is separated from its muscle $(\$ 70)$ the only change which we can appreciate in it is an electrical change. Similarly in the case of an afferent nerve, the central system is our chief teacher; in a bundle of afferent fibres isolated from the central nervous system, in a posterior root of a spinal nerve for instance, the only change which we can appreciate is an electrical change. To learn the characters of afferent impulses we must employ the central nervous system. But in this we meet with difficulties. In studying the phenomena of motor nerves we are greatly assisted by two facts. First, the muscular contraction by which we judge of what is going on in the nerve is a comparatively simple thing, one contraction differing from another only by such features as extent or amount, duration, frequency of repetition and the like, and all such differences are capable of exact measurement. Secondly, when we apply a stimulus directly to the nerve itself, the effects differ in degree only from those which result when the nerve is set in action by natural stimuli, such as the will. When we come, on the other hand, to investigate the phenomena of afferent nerves, our labors are for the time rendered heavier, but in the end more fruitful, by the following circumstances: First, when we judge of what is going on in an afferent nerve by the effects which stimulation of the nerve produces in some central nervous organ, in the way of exciting or modifying reflex action, or modifying automatic action, or affecting consciousness, we are met on the very threshold of every inquiry by the difficulty of clearly distinguishing the events which belong exclusively to the afferent nerve from those which belong to the central organ. Secondly, the effects of applying a stimulus to the peripheral end-organ of an afferent nerve are very different from those of applying the same stimulus directly to the nerve trunk. This may be shown by the simple experience of comparing the sensation caused by bringing any sharp body into contact with a nerve laid bare in a wound with that caused by contact of an intact skin with the same body. These and like differences reveal to us a complexity of impulses, of which the phenomena of motor nerves gave us hardly a hint. 
We shall further see in detail later on that our consciousness may be affected in many different ways by afferent impulses; we must distinguish not only sensory from other afferent impulses, but also different kinds of sensory impulses from each other. Certain afferent nerves are spoken of as nerves of special sense, and the nature of the afferent impulses passing along these special nerves, together with the modifications of consciousness caused by arrival of these impulses at the central nervous system, constitute by themselves a complex and difficult branch of study. "In some of the problems connected with the central nervous system we shall have to appeal to the results of a study of these special senses; but, on the other hand, a knowledge of the central nervous system is necessary to a proper understanding of the special senses; and on the whole it will be more convenient to study the former before the latter.

$\S 473$. 'The proof that the afferent and efferent fibres which are both present in the trunk of a spinal nerve are parted at the roots, the efferent fibres running exclusively in the ventral or anterior root, and the afferent fibres exclusively in the dorsal or posterior root, is as follows:

When the anterior root is divided the muscles supplied by the nerve cease to be thrown into contractions, either by the will or by reflex action, while the structures to which the nerve is distributed retain their sensibility. During the section of the root, or when the proximal stump connected with the spinal cord is stimulated, no sensory effects are produced. When the distal stump is stimulated the muscles supplied by the nerve are thrown into contractions. When the posterior root is divided the muscles supplied by the nerve continue to be thrown into action by an exercise of the will, or as part of a reflex accion, but the structures to which the nerve is distributed lose the sensibility which they previously possessed. During the section of the root, and when the approximal stump is stimulated, the sensory effects are produced. When the distal stump is stimulated no movements are called forth. These facts demonstrate that sensory impulses pass exclusively by the posterior root from the peripheral to the central organs, and that motor impulses pass exclusively by the anterior root from the central to the peripheral organs; and as far as our knowledge goes the same holds good not only for sensory and motor but also for afferent and efferent impulses.

An exception must be made to the above general statement, an account of the so-called " recurrent sensibility" which is witnessed in conscious mammals under certain circumstances. It sometimes happens that when the distal stump of the divided anterior root is stimulated, signs of pain are witnessed. These are not caused by the concurrent muscular contractions or cramp which the stimulation occasions, for they persist after the whole trunk of the nerve has been divided some little way below the union of the roots above the origins of the muscular branches, so that no contractions take place. They disappear when the posterior root is subsequently divided, and they are not seen if the mixed nerve-trunk be divided close to the union of the roots. The phenomena are probably due to the fact that bundles of sensory fibres of the posterior root, after running a short distance down the mixed trunk, turn back and run upward in the anterior root (being distributed probably to the pia mater) and by this recurrent course give rise to the recurrent sensibility:

$\$ 474$. Concerning the ganglion on the posterior root, we may say definitely that we have no evidence that it can act as a centre of reflex action, nor have we any evidence that it can spontaneously give origin to efferent impulses and thus act as an automatic centre, as can the central nervous system itself. The bodies of the nerve-cells behave somewhat differently from the axis-cylinders at some distance from the cells, though, as we have 
seen, these are in reality processes of the nerve-cells; thus the nerve-cells in the ganglion appear to be more sensitive to certain poisons than are the nerve-fibres of the nerve-trunk. But beyond this, our knowledge concerning the function of the ganglion is almost limited to the fact that it is in some way intimately connected with the nutrition of the nerve. As we have already (\$81) said, when a mixed nerve-trunk is divided the peripheral portion degenerates from the point of section downward toward the periphery. The central portion does not so degenerate, and if the length of nerve removed be not too great, the central portion may grow downward along the course of the degenerating peripheral portion, and thus regenerate the nerve. This degeneration is observed when the mixed trunk is divided in any part of its course from the periphery to close up to the ganglion. When the posterior root is divided between the ganglion and the spinal cord, the portion attached to the spinal cord degenerates, but that attached to the ganglion remains intact. When the anterior root is divided, the proximal portion in connection with the spinal cord remains intact, but the distal portion between the section and the junction with the other root degenerates; and in the mixed nerve-trunk many degenerated fibres are seen, which, if they be carefully traced out, are found to be motor (efferent) fibres. If the posterior root be divided carefully between the ganglion and the junction with the anterior root, the small portion of the posterior root left attached to the peripheral side of the ganglion above the section remains intact, as does also the rest of the root from the ganglion to the spinal cord, but in the mixed nerve-trunk are seen numerous degenerated fibres, which when examined are found to have the distribution of sensory (afferent) fibres. Lastly, if the posterior ganglion be excised, the whole posterior root degenerates, as do also the sensory (afferent) fibres of the mixed nerve-trunk. Putting all these facts together, it would seem that the growth of the efferent and afferent fibres takes places in opposition directions, and starts from different nutritive or "trophic" centres. The afferent fibres grow away from the ganglion either toward the periphery or toward the spinal cord. The efferent fibres grow outward from the spinal cord toward the periphery. This difference in their mode of nutrition is frequently of great help in investigating the relative distribution of efferent and afferent fibres. When a posterior root is cut beyond the ganglion, or the ganglion excised, all the afferent nerves degenerate, and in the mixed nerve-branches these afferent fibres, by their altered condition, can readily be traced. Conversely, when the anterior roots are cut, the efferent fibres alone degenerate, and can be similarly recognized in a mixed nerve-tract. When the anterior is divided some few fibres in it do not, like the rest, degenerate, and when the posterior root is divided, a few fibres in the anterior root are seen to degenerate like those of the posterior root; these appear to be the fibres which give to the anterior root its " recurrent sensibility." In the case of certain spinal nerves at all events, it has also been ascertained that when the posterior root is divided, while most of the fibres in the part of the root thus cut off from the ganglion but left attached to the cord degenerate, some few do not. These few appear to have their trophic centre not in the ganglion, but in some part of the spinal cord itself; we shall refer to these later on.

This method of distinguishing nerve-fibres by the features of their degeneration, called the "degeneration method," or sometimes, from the name of the physiologist who introduced it, the "Wallerian method," has proved of great utility. Thus in the vagus nerve, which is composed not only of fibres which spring from the real vagus root, but also of fibres proceeding from the spinal accessory roots, the two may be distinguished by section of the vagus and spinal accessory roots respectively. We shall presently see 
that this method may be applied to the differentiation of tracts of fibres in the brain and spinal cord.

\section{The Structure of the Spinal Cord.}

$\S 475$. Lying within the vertebral canal the spinal cord is protected by its "membranes," the dura mater, the arachnoid membrane and the pia mater. The consideration of the arrangement of these membranes and of the structure of the dura mater and arachnoid we will leave until we come to speak of the vascular and lymphatic supplies of the central nervous system; the histology of the pia mater may more fitly come with that of the spinal cord itself.

Along its whole length from its junction with the bulb at its termination in the filum terminale the spinal cord, while possessing certain general features, is continually changing as to special features. It will be convenient to study first the general structure of some particular part, for instance the middle of the thoracic (dorsal) ${ }^{1}$ region, and afterward to point out the special features which obtain in the several regions.

A transverse vertical section of either a fresh or a hardened and prepared spinal cord at the thoracic region possesses an outline which is, roughly speaking, circular. In the middle of the anterior or ventral surface is a vertical fissure, the ventral or anterior fissure (Fig. 119, $A . F$.), running some way across the thickness of the cord from the ventral toward the dorsal surface. Opposite to it on the posterior or dorsal surface is a corresponding, deeper but narrower, dorsal or posterior fissure (Fig. 119, P. F.) which, however, as we shall see, differs materially in nature from the anterior fissure, and ought to be called a septum rather than a fissure. Between the two fissures the substance of the cord is reduced to a narrow isthmus uniting the two lateral halves, which in a normal cord are like each other in every respect. In the middle of the isthmus lies the section of a small canal, the central canal (Fig. 119,c.c.), which is all that remains of the relatively wide neural canal of the embryo.

Each lateral half consists of an outer zone of white matter surrounding, except at the isthmus, an inner more or less crescentic, or comma-shaped mass of gray matter. The convexity of each crescent is turned toward the median line of the cord, the two crescents being placed back and back and joined together by the isthmus just spoken of. The somewhat broader anterior extremity of the crescent, or head of the comma, is called the anterior cornu or horn; and the narrower posterior extremity of the erescent, or tail of the comma, is called the posterior cornu or horn. The part by which each horn is joined on to the middle part of the crescent is called the cervix, anterior and posterior respectively. The isthmus joining the backs of the two crescents, like the crescents themselves, consists, for the most part, of gray matter, the band running posterior or dorsal to the central canal being called the posterior gray commissure (Fig. 119,p.g.c.), and the band running anterior or ventral to the canal being called the anterior gray commissure (Fig. $119, a . g . c$.$) . The posterior fissure touches the posterior gray commissure,$

"It is very desirable to use the terms "dorsal" and "ventral" for the parts of the cerebro-spinal axis which lie respectively near the dorsal or back part, and the ventral or belly part of the body, instead of the terms posterior and anterior; but if this is done the use of the word dorsal to denote the region of the cord between the lumbar and cervical regions is apt to lead to confusion; hence the introduction of the word thoracic. If this use of dorsal and ventral be adhered to, before and behind, above and below, may conveniently be used to denote nearer the head and nearer the tail (or coccyx) respectively; anterior and posterior may also be used in the same sense except in the case of anterior and posterior fissure and horn, which terms seem too much honored by time to be thrown aside. 
but the anterior gray commissure is separated from the bottom of the anterior fissure by a band of white matter, called the anterior white commissure,

FIG. 119.

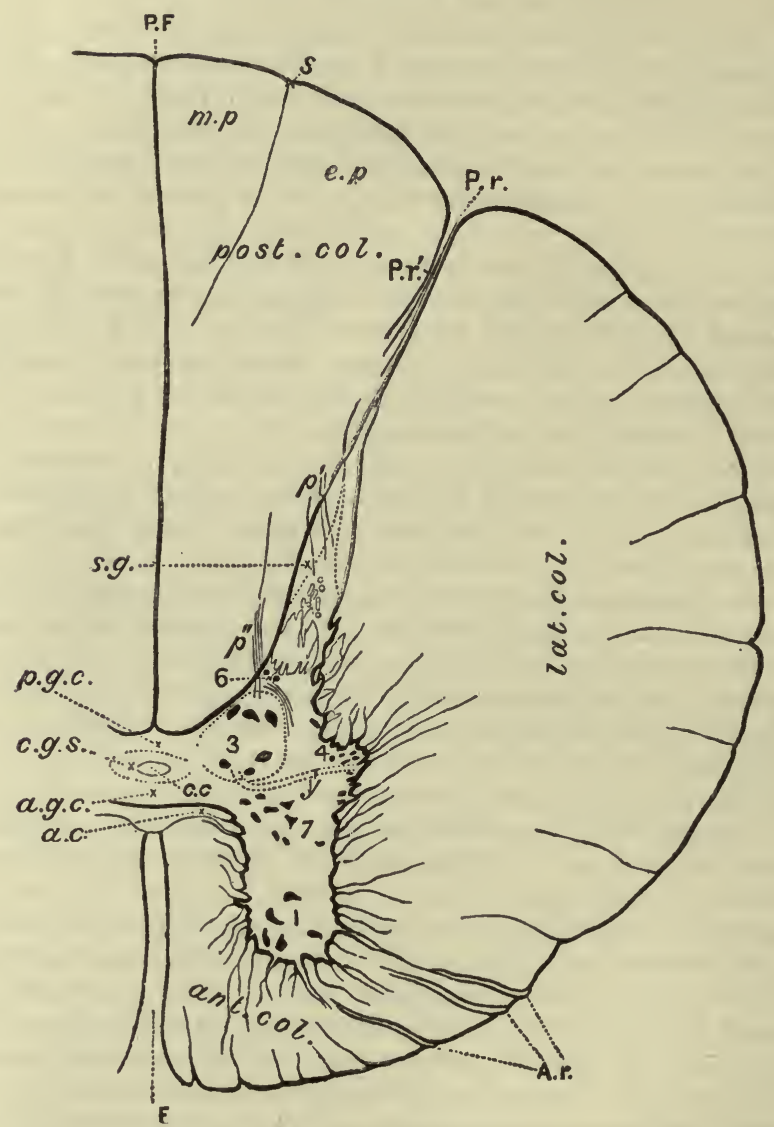

A Transverse Dorso-ventral Section of the Spinal Cord (Human) at the Level of the Sixth Thoracic (Dorsal) Nerve. (Sherrington.) $)^{1}$ Magnified 15 times. One lateral half only is shown. The large conspicuous nerve-cells (drawn from actual specimens) are shaded black to render their relative size, shape and position more obvious; the outline of the gray matter has been made thick and dark in order to render it conspicuous. $A$. $F$., antetror fissure; $P$. $F$., posterior fissure; $c . c$., central canal; $c . g . s$., central gelatinous substance; $A . r$, anterior root : $P$. $r$., lateral (or intermediate) bundle: $P . r^{\prime}$., median bundle or posterior root of spinal nerve; $p^{\prime} p^{\prime \prime}$ fibres of posterior root passing, $p^{\prime}$ indirectly through the substance of Rolando, $p^{\prime \prime}$ directly into gray matter; $a . g . c$. anterior gray commissure; $p . g . c$. , posterior gray commissure; $a_{.} . c$. , anterior white commissure; ant. col., anterior column ; lat. col., lateral column; post. col., posterior column; s. g., the substance of Rolando; s., septum marking out the external posterior column or columu of Burdach, $c . p$., from the median posterior column, or column of Goll, $m . p$. 1, cells of the anterior horn ; 3, posterior column, or vesicular cylinder, or column of Clarke, the area of the cylinder defined by a dotted line; 4 , cells of the intermedio-lateral tract or lateral tract or lateral horn; 6 , cells of the posterior horn; 7 , cells of the anterior cervix; $y$, a tract of fibres passing from the vesicular cylinder to the lateral column.

1 For this and many succeeding figures $I$ am deeply indebted to my friend and former pupil, Dr. Sherrington, who has kindly prepared the figures for me from his original drawings. 
or more simply, the white commissure, or sometimes the anterior commissure (Fig. 119, a. c.).

If the section be taken at the level of the origin of a pair of spinal nerves, it will be seen that the anterior or ventral root, piercing the white matter opposite the head of the comma in several distinct bundles (Fig. $119, A . r$.$) , plunges into the anterior cornu, while the posterior or dorsal$ root (Fig. 119, $P . r ., P . r^{\prime}$ ), having the appearance of a single undivided bundle, passes, in part at least, into the posterior horn. Both roots are dispersed lengthways along the cord, the hinder roots of one nerve being close to the foremost roots of the nerve below, but it is only the anterior roots which are dispersed sideways. The compact bundle of the posterior root divides, with tolerable sharpness, the white matter in each lateral half of the cord into (1) a posterior portion lying between the posterior fissure and the posterior root, which portion since, as we shall see, runs in the form of a column along the length of the cord, is called the posterior column, and (2) into a portion lying to the outside of the posterior root between it and the anterior fissure, called the antero-lateral column. 'This latter may be considered as further divided, by the entrance of the anterior roots into a lateral column (Fig. 119, lat. col.) between the posterior root and the most external bundle of the anterior root, and into an anterior column (Fig. 119, ant. col.), between the anterior fissure and the most external bundle of the anterior root. The part traversed by the bundles of the anterior root, as they make for the anterior horn, accordingly belongs to the anterior column; but some writers speak of the anterior column as lying between the anterior fissure and the nearest bundle of the anterior root, thus making the region of the anterior root belong to neither anterior nor lateral column. And indeed the distinction between the anterior and the lateral column is, to a great extent, artificial.

$\S 476$. The "white matter" consists exclusively of medullated fibres supported partly by connective tissue and partly by a peculiar tissue known as neuroglia, of which we shall presently speak. The fibres are of various sizes, but many of them are large, and in all of them the medulla is conspicuous. They run for the most part longitudinally, so that in transverse sections of the cord nearly the whole of the white matter appears under the microscope to be composed of minute circles, the tranverse sections of the longitudinally-disposed fibres, imbedded in the supporting structures. The "gray matter" also contains medullated fibres, but these are for the most part exceedingly fine fibres possessing a medulla which appears to differ from that of an ordinary nerve-fibre, since it does not stain readily with osmic acid, but is rendered visible by special modes of preparation such as that known as Weigert's. Hence these fine fibres are not apparent in ordinary carmine or other specimens, and indeed their presence was for a long time overlooked. Besides these fine medullated fibres, if we may call them such, the gray matter contains what the white matter does not, nerve-cells with branching processes, naked axis-cylinders, and delicate filaments arising from the division of axis-cylinders or from the branching of nerve-cells, all these various structures being imbedded in neuroglia. Owing to the relative abundance of the white refractive medulla, the white matter possesses in fresh specimens a characteristic, opaque white color; hence the name. The gray matter from the relative scantiness of medulla has no such opaque whiteness, is much more translucent, and in fresh specimens has a gray or rather pinkish-gray color, the reddish tint being due to the presence partly of pigment and partly of blood, for the bloodvessels are much more abundant in the gray matter than in the white.

The pia mater which closely invests the cord all around consists of con- 
nective tissue fairly rich in elastic elements and abundantly supplied with bloodvessels; it is indeed essentially a vascular membrane and furnishes the nervous elements of the cord with their chief supply of blood. It sends in at intervals partitions or septa of the same nature as itself radiating toward the central gray matter. The narrow posterior fissure is completely filled up by a large septum of this kind, indeed, as we have said, is in reality not a fissure but a large septum; but the anterior fissure is too wide for such an arrangement; the whole membrane dips down into this fissure, following the surface of the cord and being reflected at the bottom. From these primary septa, secondary finer septa still composed of ordinary fibrillated connective tissue, carrying bloodvessels, branch off; but these are soon merged into the peculiar supporting tissue, called, as we have said, neuroglia. This consists in the first place of small branching cells, lying in various planes. The branching is excessive, so that the body of the cell is reduced to very small dimensions, indeed at times almost obliterated, the nucleus disappearing while the numerous branches are continued as long, fine filaments or fibres pursuing a devious but for the most part a longitudinal course. In the second place these cells and fibres or filaments are imbedded in a homogeneous ground substance. Relatively to the fibres and ground substance the bodies of the cells (which are called Deiter's cells), especially bodies such as bear obvious nuclei, are very scanty; hence in sections, especially in transverse sections, of the cord the neuroglia has often a dotted or punctated appearance, the dots being the transverse sections of the fine longitudinallydisposed fibres imbedded in the ground substance. Examined chemically, the neuroglia is found to be composed not like comnective tissue of gelatin, but of a substance which appears to be closely allied to keratin, the chief constituent of horny epidermis, hairs and the like, and which has therefore been called neurokeratin (see also $\$ 68$ ). And indeed this neuroglia, though like connective tissue a supporting structure, is not, like connective tissue, of mesoblastic, but of epiblastic origin. The walls of the neural canal of the embryo which are transformed into the spinal cord of the adult consist at first of epithelial, epiblastic cells ; and while some of these cells become nervous elements, others become neuroglia. The epithelial cells which are destined to form neuroglia become exceedingly branched, while their originally protoplasmic cell substance becomes transformed to a large extent into neurokeratin.

The neuroglia fills up the spaces between the radiating larger septal prolongations of the pia mater and the finer branched septa which starting from the larger ones carry minute bloodvessels into the interior of the white matter. In these spaces it is so arranged as to form delicate tubular canals, of very variable size, running for the most part in a longitudinal direction. Each of these tubular canals is occupied by and wholly filled up with medullated nerve-fibre of corresponding size. A medullated nerve-fibre of the white matter of the spinal cord resembles a medullated nerve-fibre of a nerve ( $\$ 68)$ in being composed of an axis-cylinder and a medulla; but it possesses no primitive sheath or neurilemma. This is absent and indeed is not wanted; the tubular sheath of neuroglia affords in the spinal cord (and, as we shall see, in the central nervous system generally) the support which in a nerve is afforded by the neurilemma. Nodes are, according to most authors, absent, but some say they are present.

The white matter of the cord consists then of a more or less solid mass of neuroglia, having the structure just described, which is permeated by minute canals, some exceedingly fine and carrying very fine $2 \mu$ fibres, others larger and carrying fibres up to the size of $15 \mu$. This mass is further broken up into areas by the smaller and larger vascular connective-tissue septa with the 
edges and endings of which the neuroglia is continuous. Most of the nervefibres, as we have said, run longitudinally and in a transverse section of the cord are cut transversely; but, as we shall see, fibres are continually passing into and out of the white matter, and in so doing take a more or less transverse course; these, however, are few compared with those which run in a longitudinal direction. On the outside of the cord below the pia mater the neuroglia is developed into a layer of some thickness from which nerve-fibres are absent; this is often spoken of as an inner layer of the pia mater; but being neuroglia and not connective tissue is of a different nature from the pia mater proper. A layer of this superficial neuroglia also accompanies the larger septa, and a considerable quantity is present in the large septum called the posterior fissure.

The pia mater carries not only bloodvessels but also lymphatics; of these, however, we shall speak when we come to deal with the vascular arrangements of the whole of the central nervous system.

$\S 477$. In the gray matter we may distinguish the larger, more conspicuous nerve-cells and the rest of the gray matter in which these cells lie. We have already ( $\$ 95)$ described the general features of these larger nerve-cells, and shall have presently to speak of their special characters and grouping. Meanwhile the most important point to remember about them besides the fact that they vary largely in form and size is that while one process may or does become an axis-cylinder of a nerve-fibre, the others rapidly branch, and breaking up into fine nerve-filaments are lost to view in the rest of the gray matter.

These larger nerve-cells form, however, a part only, and in most regions of the cord the smaller part, of the whole gray matter. In a transverse section from the thoracic region (Fig. 119) a few only of these larger nerve-cells are seen in the whole section, and though they appear more numerous in sections from the cervical and especially from the lumbar regions (Figs. 121, 122 ), yet in all cases they occupy the smaller part of the area of the gray matter. The larger part of the gray matter consists, besides a neuroglia supporting the nervous elements, of nerve-filaments running in various directions and forming, not a plexus properly so called, but an interlacement of extreme complexity. These filaments are, on the one hand, the fine medullated fibres spoken of above as being recognized with difficulty, and, on the other hand, non-medullated filaments ranging from fairly wide and conspicuous naked axis-cylinders down to fibrils of extreme tenuity, the latter arising apparently either from the division of axis-cylinders and nerve-fibres passing into or out of the gray matter or from the continued branching of processes of nerve-cells. By the modes of preparation now available it has been shown that the fine medullated fibres, so far from being rare, are in certain parts of the gray matter so abundant as even to preponderate over the non-medullated fibres or fibrils. Lastly, besides the conspicuous nervecells spoken of above, which, though of various sizes, may all perhaps be spoken of as large, a very large number of other cells of small size, some of which at all events must be regarded as true nerve-cells, are present in the gray matter.

The neuroglia in which all these structures, nerve-cells, fine medullated nerve-fibres, naked axis-cylinders, and fine filaments are imbedded, is identical in its general characters with that of the white matter, but, as naturally follows from the nature of the nervous elements which it supports, is differently arranged. Instead of forming a system of tubular channels it takes on the form of a sponge-work with large spaces for the larger nerve-cells and fine j)assages for the nervous filaments. At the junction of the gray matter with the white matter, the neuroglia of the one is continuous with that of the 
other, and the connective-tissue septa of the latter run right into the former ; the outline of the gray matter is not smooth and even, but broken by toothlike processes due to the septa. Since, as we have just said, some of the true nerve-cells are very small, and since the nerve-filaments like the neuroglia fibres are very fine and take like them an irregular course, it often becomes very difficult in a section to determine exactly which is neuroglia and which are nervous elements. The neuroglic cells may, however, be distinguished perhaps from the smaller nerve-cells by their nuclei not being so conspicuous or so relatively large as in a nerve-cell, and by their staining differently.

The gray matter, then, may be broadly described as a bed of neurnglia, containing a certain number of branching nerve-cells, for the most part though not exclusively large and conspicuous, but chiefly occupied by what is not so much a plexus as an intricate interweaving of nerve-filaments running apparently in all directions. Some of these filaments are fairly conspicuous naked axis cylinders, and a few are easily recognized medullated fibres of ordinary size; but by far the greater number are either exceedingly fine medullated fibres, whose medulla is only made evident by special modes of preparation, or delicate fibrils devoid of medulla. With the nervous web formed by these filaments the branching processes of the nerve-cells, on the one hand, and the divisions of nerve-fibres passing into or out of the gray matter on the other hand, appear to be continuous. It may be added that the gray matter is well supplied with bloodvessels, these being in it, as stated above, relatively much more numerous than in the white matter.

$\S 478$. The central canal is lined by a single layer of columnar epithelial cells, which are generally described as bearing cilia; but it is not certain that the processes which may be seen projecting from the surfaces of the cells are really cilia. These epithelial cells rest not on a distinct basement membrane, but on a bed of neuroglia, free apparently, or nearly so, from nervous elements which surrounds the central canal and is sometimes spoken of as the substantia gelatinosa centralis (Fig. 119, c. g. s.). The attached basis of the epithelial cells are branched or taper to a filament, and become continuous with the branched cells or fibres of the neuroglia below. As we said above, the neuroglia elements are transformed epithelial cells; and the continuity of the cells, which retaining the characters of epithelial cells form a lining to the canal, with the cells which have become branched and lost their epithelial characters, indicates the epithelial origin of the latter.

The central canal with the surrounding area of neuroglia forms the central part of the isthmus uniting the two lateral halves of the cord. Posterior (dorsal) to this central mass lies the posterior gray commissure (Figs. $119,121,122, p . g$. c.) composed chiefly of fine filaments running transversely, and anterior (ventral) to it lies first the thinner anterior gray commissure (Figs. 119, 121, 122 a.g.c.) of a similar nature, and then the relatively thick white commissure (Figs. 119, 121, 122, a. c.) which is formed by medullated fibres crossing over from one side of the cord to the other, and thus constitutes a decussation of fibres along the whole length of the cord. On each side the central mass of neuroglia of which we are speaking gradually merges into the central gray matter of the corresponding lateral half.

The end or head (caput), as it is frequently called, of the posterior horn is occupied not by ordinary gray matter, but by a peculiar tissue, the substantia gelatinosa of Rolando, which forms a sort of cap to the more ordinary gray matter, but differs in size and shape in different regions of the cord. (Cf. Figs. 119, 120, 121, s. g.) In carmine and some other modes of preparation it is frequently stained more deeply than is the ordinary gray matter, and in such preparations is very conspicuous. It may be described as con- 
sisting of a somewhat peculiar neuroglia traversed by fibres of the posterior root, and containing a large number of cells which, for the most part small, the cell-bodies being. small relatively to the nuclei, are not all alike, some being probably nervous and others not. It takes origin from the cells forming the immediate walls of the embryonic medullary canal. In the embryo this canal is relatively wide, though compressed from side to side, and in transverse sections of the medullary tube appears at a certain stage as a narrow oval slit placed vertically and reaching almost from the dorsal to the ventral surface. The dorsal part of this long slit is later on closed up by the coming together of the walls and the obliteration of the greater part of the cavity, leaving the ventral part to form a circular canal, which by the development of the anterior columns assumes the central position. During this closure of the dorsal part of the canal a mass of the cells lining the canal is cut from the rest on each side, and during the subsequent growth takes up a position at the end of the posterior horn. Hence, though it never apparently contains any cavity, the substance of Rolando may be regarded as an isolated portion of the walls of the medullary canal, which has undergone a development somewhat different from that of the portion which remains as the lining of the central canal. Traces of this origin may be seen even in the adult. Thus, in the lower end of the cord, in what we shall speak of presently as the conus medullaris, the central canal widens out dorsally, and in section (Fig. 120, A) presents on each side a bay $x$ stretching

FIG. 120.

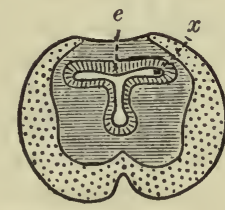

A

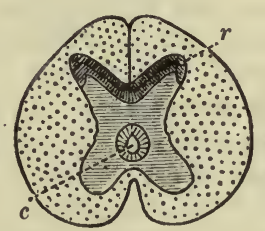

B

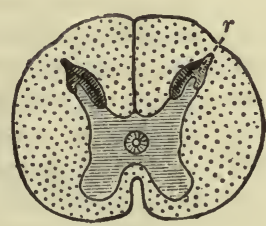

C

Diagram to illustrate the Nature of the Substance of Rolando. The figures are purely diagrammatic and are not drawn to the same scale. In all three figures the gray matter is shaded with fine lines and the white matter with dots. $A$, transverse section of the lower end of the conus medullaris in man; $e$, epithelium lining the medullary canal; $x$, lateral expansion of the canal; $B$, transverse section of the spinal cord of the calf in the lower thoracic region: $r$, sub. stance of Rolando: $c$, central canal ; $C$, transverse section through mid-thoracic region of cord in man.

out toward the position of the posterior horn. At this region of the cord, though both white and gray matter are developed on the ventral surface, the posterior columns do not meet on the dorsal surface, but leave the central canal covered only by tissue which perhaps may be called neuroglia, but is of peculiar nature and origin. In the calf, in a part of the dorsal region the substance of Rolando is not confined to the tip of the posterior horn, but is continued to meet its fellow in the middle line. (Fig. 120, B.) If we imagine the dorsal portion of the canal of $A$ to be cut off from the ventral portion, its cavity to be obliterated, and the lining epithelium with some of the surrounding elements to undergo a special development, the condition in $B$ is reached by the growth of the posterior columns. From $B$ the transition to the normal state of things as in Fig. 120, $c$, is a very slight one. The extreme dorsal tip of the horn, being of a more open texture than the substance of Rolando, is sometimes called the zona spongiosa.

$\$ 479$. The grouping of the nerve-cells. The nerve-cells, at all events the cells which are large enough to to be easily and without doubt recognized to 
be nerve-cells, form, as we have seen, only a part of the gray matter, and in some parts of the cord, in the thoracic region for instance, are so sparse that in a section of the spinal cord in this region thin enough to show its histological features satisfactorily, the bodies of a few only of such cells are visible (Fig. 119); the greater part of the gray matter consists not of the bodies of conspicuous nerve-cells, but of a mass of fibres and fibrils passing apparently in all directions. In the cervical (Fig. 121), and especially in the lumbar (Fig. 122) regions, the nerve-cells are both absolutely and relatively more abundant; but even in a section taken from the lumbar region the nerve-célls, all put together, form the smaller part of the whole area of gray matter. Moreover, in respect of the number of cells, all the sections of even the same region of the cord are not alike. Seeing that the cord may be considered as growing out of the fusion of a series of paired ganglia, each ganglion corresponding to a nerve ( $c f . \S 92)$, we may fairly expect to find the fusion not complete, so that the nerve-cells would appear more numerous opposite a nerve than in the middle between two nerves. In some of the lower animals this arrangement is most obvious, and there are some reasons for thinking that even in man the nerve-cells are metamerically increased at the level of each nerve.

Even when casually observed, it is obvious that the nerve-cells are not scattered in a wholly irregular manner throughout the gray matter, being, for instance, much more conspicuous in the anterior horn than elsewhere; and more careful observation allows us to arrange them to a certain extent in groups.

The cells of the anterior horn are for the most part large and conspicuous, $67 \mu$ to $135 \mu$ in diameter, branch out in various directions, and present an irregular outline in sections taken in different planes. We have reason to think that every one of them possesses an axis-cylinder process, which, in the case at all events of most of the cells, passing out of the gray matter becomes a fibre of the adjacent anterior root. They are obvious and conspicuous in all regions of the cord, though much more numerous and individually larger in the cervical and lumbar enlargements than in the thoracic region. We may further, with greater or less success, divide them into separate groups.

In the cervical and lumbar regions a fairly distinct group of cells is seen lying on the median side of the gray matter close to the anterior column (Figs. 121, 122, 1). This may be called the median group. It appears also in the thoracic region (Fig. 119, 1); indeed, the question arises whether all the cells of the anterior horn in this region do not belong to this group. The other cells so conspicuous in the lumbar and cervical enlargements, and therefore probably in some way associated with the limbs, may be spoken of as forming altogether a lateral group; but we may, though with some uncertainty, subdivide them into two or three groups. Thus in the lumbar region a group of cells (Fig. 122, $2 \gamma$ ) lying near the lateral margin of the more dorsal part or base of the horn may be distinguished, as a lateral sub-group, from the cells occupying the ventral lateral corner of the horn and forming a ventral or anterior sub-group (Fig. 122, $2 \propto$ ); and the same distinction, though with less success, may be made in the cervical region (Fig. 121). Further, we may perhaps in both regions distinguish a group of cells placed more in the very middle of the horn as a central sub.group (Figs. 121, 122, $2 \beta$ ). But, in all cases, the separation of these cells, which we have spoken of as a whole as lateral cells, into minor groups, is far less distinct than the separation of the median group from these lateral cells, especially if we admit that in the thoracic region the median group is alone clearly represented. 


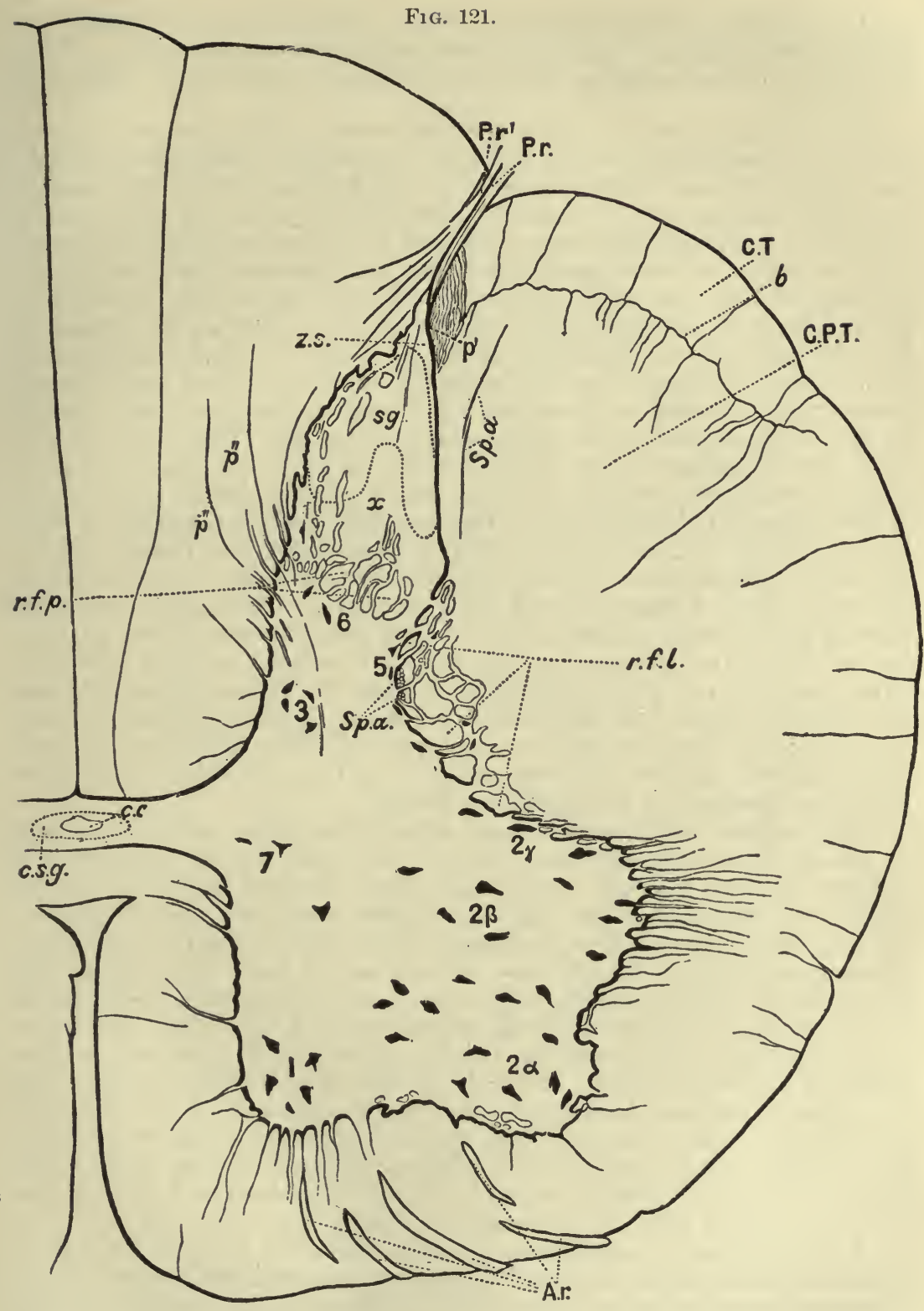

Transverse Dorso-ventral Section of Spinal Cord (Human) at the Level of the Sixth Cervical Nerve. (Sherrington.) This is drawn on the same scale as Fig. 119, that is, magnified fifteen times $r . f . l$., lateral reticular formation; $r . f . p$. posterior reticular formation; $p^{\prime}$, fine fibres of lateral bundle of the posterior root; $p^{\prime \prime}, p^{\prime \prime \prime}$, fibres of median bundle of posterior root entering gray matter from external posterior column ; $x$, gray matter of posterior horn; $S p$. $a$., bundles of fibres belonging to the spinal accessory nerve: in the lateral reticular formation they are seen cut transversely; $b$, is the natural septum of connective tissue marking out the cerebellar tract $C: T$. from the crossed pyramidal tract $C . P . T . ; z .8$., zona spongiosa; $2 \alpha, \beta, \gamma$, lateral cells of the anterior horn; 5 , cells in the region of the lateral reticular formation. The other letters of reference are the same as in Fig. 119. 
In the thoracic region a group of rather smaller cells is seen at the base of the anterior horn, near to the junction with the isthmus (Fig. 119, 7). In the cervical and lumbar region these cells are very scanty (Figs. 121, $122,7)$.

The cells of the posterior horn contrast strongly with those of the anterior horn in being few, and for the most part small. They are branched; and though we have reason to believe that, like the cells of the anterior horn, they possess each an axis-cylinder process, this is not easily determined by actual observation ; the processes do not run out to join the posterior root, as do the corresponding processes in the anterior horn, and therefore are not so readily seen. These cells occur in all regions of the cord, and appear to be arranged in two or more groups. The lateral margin of the posterior horn, at about the middle or neck of the horn, is along the whole length of the cord, but especially in the cervical region, much broken up by bundles of fibres passing in various directions and forming an open network, called the lateral reticular formation (Figs. 121, 122, r. $f$. lat.). In all regions of the cord a number of cells are found associated with this reticular formation, forming the group of the lateral reticular formation (Figs. 121, 122, 5). In all regions of the cord, also a group of cells (Figs. 119, 121, 122, 6) is found in that part of the horn where, a little ventral to the substance of Rolando, the uniform field of gray matter is broken up into a kind of network by a number of bundles of white fibres running in various directions. The network has also been called a reticular formation, and has received the name of posterior reticular formation (Figs. 121, 122, r.f. p.) to distinguish it from the lateral reticular formation just mentioned; the two, however, in some regions (see Fig. 119) join each other, and thus cut off a ventral portion of the posterior horn containing nerve-cells from a dorsal portion, $x$ in Figs. 121, 122, in which no obvious or conspicuous nerve-cells are present.

The groups of cells just mentioned, with the restrictions and modifications spoken of, occur along the whole length of the cord; but the group of cells to which we must now call attention is almost confined to a special region of the cord, or at least is but feebly represented elsewhere. In the thoracic region, especially in the lower thoracic region (we shall return to the limits of the group later on), at the base of the posterior horn (Fig. 119, 3), just ventral to the curve formed by the posterior gray commissure as this bends dorsally to join the posterior horn, is seen on each side of the cord a conspicuous group of cells known as Clarke's column, or the posterior vesicular column or vesicular cylinder. The cells composing this group, though varying in size at different levels, are rather large cells, and are for the most part fusiform, with their long axis placed lengthways along the cord, so that in transverse sections they often appear to have a rather small round body. They are surrounded by, and as it were imbedded in, a mass of fine fibres, the area of which is indicated by a dotted line in Fig. 118.

Also conspicuous in the thoracic region is another group of cells lying on the outer side of the middle of the gray matter at about the junction of the anterior and posterior horns. This is known as the intermedio-lateral tract, and is sometimes called the lateral horn (Fig. 119, 4). The cells composing it are somewhat small spindle-shaped cells with their long axis placed transversely. The group is conspicuous, as we have said, in the thoracic region; it may be recognized in the lumbar region (Fig. 121, 4), but in the cervical region becomes confused with the most dorsally placed or lateral sub-group of the anterior horn. We shall, however, have to return to these groups of cells when we come to speak of the differences between the spinal regions of the cord. 
$\S 480$. The tracts of white matter. At first sight the white matter of the cord appears to be of uniform nature. We can use the nerve-roots to delimitate the anterior, posterior, and lateral columns, but we appear to

Fig. 122 .

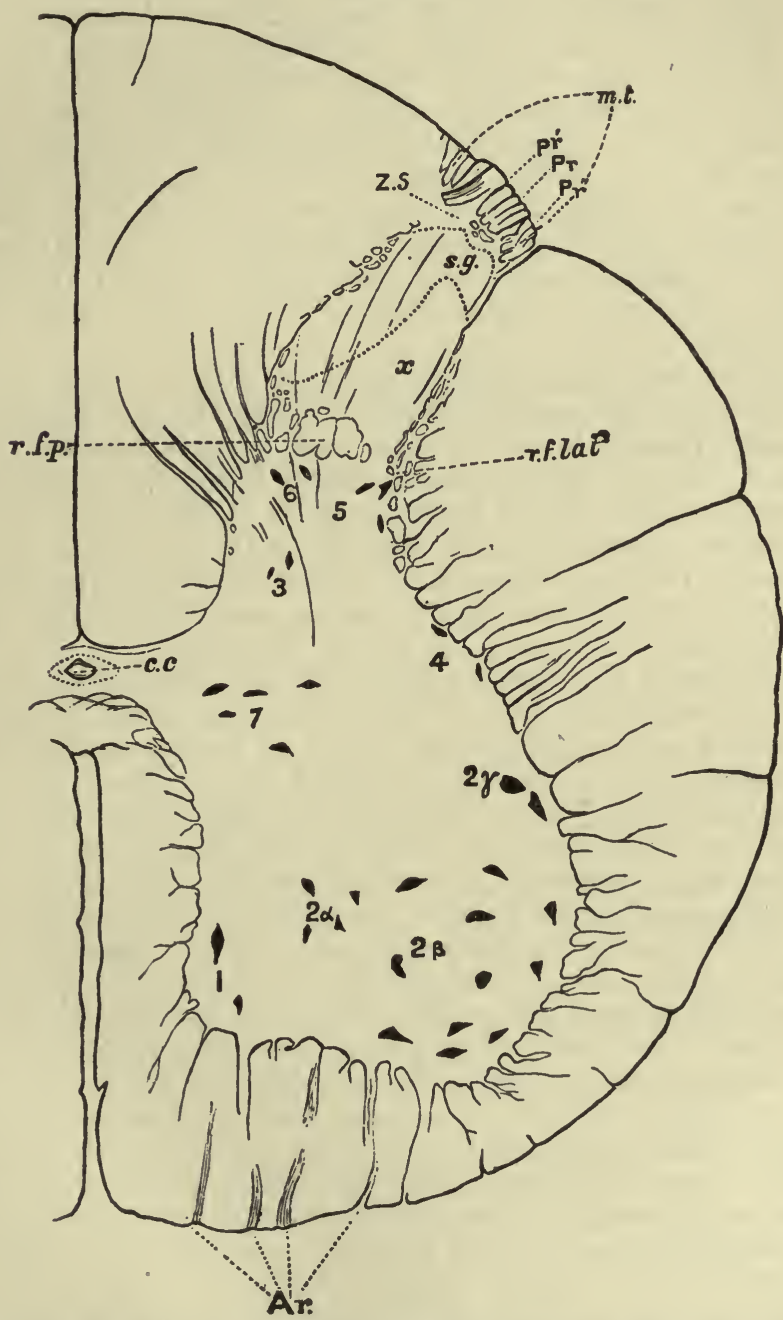

Transverse Dorso-ventral Section of the Spinal Cord (Human) at the Level of the Third Lumbar Nerve. (Sherrington.) This is drawn to the same scale as Figs. 119, 120, and in the same way, except that the outline of the gray matter is not exaggerated, $P r^{\prime}$. median; Pr. intermediate; $P r^{\prime \prime}$. lateral bundles of posterior roots. The region comprised under $m . t$. is the marginal zone of Lissauer's zone. The other letters of reference are the same as in Figs. 119, 121. The three figures, $119,121,122$, are intended to illustrate the main differential features of the cervical, thoracic, and lumbar cord.

have no criteria to distinguish parts in each column. In the cervical and upper thoracic regions of the cord, a septum (Fig. 119, s.) in the posterior column, somewhat more conspicuous than the other septa, has enabled anatomists to distinguish an inner median portion, the median posterior 
column, commonly called the postero-median column or column of Goll (Fig. 119. m. p.), from an outer lateral portion, the external posterior column, commonly called the postero-external column or column of Burdach (Fig. 119, e.p.), the lateral part of which, nearer the gray matter, has, for reasons which we shall see later on, been called the posterior root-zone. But beyond this neither the irregular septa nor other features will enable us to distinguish one part of the white matter as different in nature from another. Nor have we better success when with the scalpel we attempt to dissect out the white matter into separate strands. Nevertheless we have convincing evidence that the white matter is arranged in strands, or tracts, or columns, which have different connections at their respective ends, which behave differently under different circumstances, which we have every reason to believe carry out different functions, but which cannot be separated by the scalpel, because each of them is more or less mixed with fibres of a different nature and origin. The evidence for the existence of these tracts is twofold.

One kind of evidence is embryological in nature. When a nerve-fibre is being formed in the embryo, either in the spinal cord or elsewhere, the essential axis-cylinder is formed first and the less essential medulla is formed later. Now when the developmental history of the spinal cord is studied it is found that, in the several regions of the cord, all the fibres of the white matter do not put on the medulla at the same time. On the contrary, in certain tracts, the medulla of the fibres makes its appearance early, in others later. By this method it becomes possible to distinguish certain tracts from others.

Another kind of evidence is supplied by facts relating to the degeneration of the fibres of the white matter. We have seen $(\$ 474)$ that the degeneration of a nerve-fibre is the result of the separation of the fibre from its trophic centre, and that while the trophic centre of the afferent fibres is in the ganglion on the posterior root, that of the efferent fibres is in some part of the spinal cord. In the case of the efferent fibres the degeneration might be spoken of as descending from the spinal cord to the muscles or other peripheral organs. In the case of the afferent fibres of the trunk of the nerve, the degeneration is also one descending from the ganglion down to the skin or other peripheral organ. When, however, the section is carried through the posterior root of a spinal nerve, the degeneration takes place in the part of the nerve between the section and the spinal cord; it runs up from the section to and into the spinal cord, and may, therefore, be called an ascending degeneration. Thus we may say that when a nerve-trunk or when a nerve-root is cut completely across, all the fibres, which are thereby separated from their trophic centres, degenerate. When the nerve-trunk is divided, all the fibres below the section undergo descending degeneration. If the anterior root be cut across, all the fibres of the root below the section undergo descending degeneration. If the posterior root be cut across, all the fibres of the root above the section undergo ascending degeneration with the exception of certain fibres which do not degenerate at all, and of which we shall speak later on.

When the spinal cord is cut across, for instance in the dorsal region, all the fibres of the white matter do not degenerate either in the part of the cord above the section or in the part below. Some fibres, and indeed some tracts of fibres degenerate, and some do not. Further, some tracts degenerate in the cord above the section, and thus undergo what has been called an ascending degeneration; other tracts degenerate in the cord below the section, and thus undergo what has been called a descending degeneration. These terms must, however, be used with caution. When a nerve-trunk is 
cut across, the degeneration actually descends, in the sense that the progress of the degenerative changes may be traced downward; they begin at the section and travel downward at a rate sufficiently slow to permit a difference being observed between the progress of degeneration at a spot near the section and that of one further off. After section of or injury to the spinal cord, however, it is not possible to trace any such progress either upward or downward; in the tracts both above and below the section or injury, degeneration either begins simultaneously along the whole length of the degenerating tract, or progresses along the tract so rapidly that no differences can be observed, as far as the stage of degeneration is concerned, between parts near to and those far from the section and injury. When, for instance, the cord is divided in the cervical region, subsequent examination of the tracts of the so-called descending degeneration shows that the degeneration is as far advanced in the lumbar region far away from the section as in the cervical region just below the section. Applied to the spinal cord, therefore, the term descending degeneration means simply degeneration below the seat of injury or disease, ascending degeneration means simply degeneration above the seat of injury or disease. We may add that the histological features of the degeneration of fibres in the spinal cord are not wholly identical with those of the degeneration of fibres in a nerve-trunk. Thus, the neurilemma with its nuclei being absent from the fibres of the cord, no proliferation of nuclei takes place; the axis-cylinder and medulla simply break up, are absorbed, and disappear.

Similar degenerations, ascending or descending, or both, are seen when the section is not carried right through the whole cord, but particular parts of the cord are cut through or deeply injured. And similar degenerations occur as the consequences of disease set up in parts of the cord.

In this way the results of sections of or of other injuries to or of diseases of the spinal cord, have enabled us to mark out certain tracts of the white matter as undergoing degeneration and others as not, and, moreover, certain tracts as undergoing descending and others as undergoing ascending degeneration. Further, the delimitation of tracts of white matter by the process of degeneration agrees so well with the results of the embryological method as to leave no doubt that the white matter does consist of tracts which differ from each other in nature and in function.

The several tracts thus indicated vary in different regions of the cord. They may be broadly described as follows:

I. Descending tracts, that is to say, tracts which undergo a descending degeneration in the sense noted above.

The most important and conspicuous is a large tract (Fig. 123, cr.P.) occupying the posterior part of the lateral column, coming close upon the outer margin of the posterior horn, and for the most part not reaching the surface of the cord. We shall have to return to this tract more than once, and may here simply say that it is most distinctly marked out by both the embryological and the degeneration methods, that it may be traced along the whole length of the cord from the top of the cervical region to the end of the sacral region, and that it enters the cord from the brain through the structures called the pyramids of the bulb, which we shall study later on. These pyramids cross over or decussate as they are about to pass into the cord, forming what is known as the decussation of the pyramids, and the tract of fibres in question shares in this decussation. Hence this tract is called the crossed pyramidal tract or more simply the pyramidal tract.

A smaller, less conspicuous descending tract occupies the median portion of the anterior column (Fig. 123, d.P.). This is not only much smaller but also much more variable than the crossed pyramidal tract, is not present in 
the lower animals, being found in man and the monkey only and being better developed in man than in the monkey, and reaches a certain way only down the spinal cord, generally coming to an end in the thoracic region. It, too, comes down from the pyramid, and is a continuation of that part of the pyramid which, unlike the rest, does not decussate in the bulb; thus the tract which coming down from the left side of the brain runs in the left pyramid

FIG. 123.

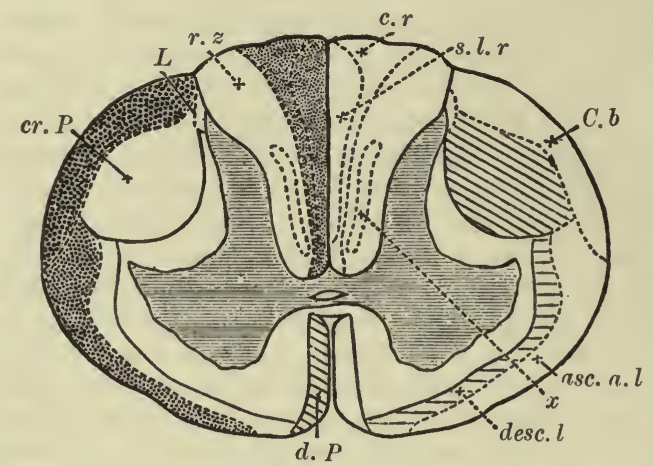

Diagram to illustrate the General Arrangement of the Several Tracts of White Matter in the Spinal Cord. (Sherrington.) The section is taken at the level of the fifth cervical nerve. The relations of the tracts in different regions of the cord are shown in Fig.127. The ascending tracts, tracts of ascending degeneration, are shaded with dots, the descending tracts, tracts of descending degeneration, are shaded with lines; the shading in each case put on one side of the cord, only the reference letters being placed on the other side. cr.P. crossed pyramidal tract, or more shortly, pyramidal tract; d.P. direct pyramidal tract shaded on the side opposite to that on which $c r . P$. is shaded, in order to indicate the difference of the two as to crossing; C.b. cerebellar tract; s.lr. and c.r. together indicate the median posterior tract, or tract of fibres of the posterior roots, $c r$. representing, as is explained more fully in the text, the cervical and s.lr. the sacral, lumbar, anã dorsal roots; asc.a.l. the antero-lateral ascending tract; desc.l. the antero-lateral descending tract. The area, not shaded, marked $x$, is the small descending tract or rather patch mentioned in the text as observed in certain regions of the cord, in the external posterior column r.z. The small area at the tip of the posterior horn, marked $L$, is the posterior marginal zone of Lissauer's zone.

in the bulb, passes down into the left anterior column of the cord. Hence this smaller tract is called the direct pyramidal tract.

These two are the most conspicuous and important descending tracts, but names have been given to two other descending tracts. One, known as the antero-lateral descending tract, is a large tract placed in the antero-lateral column, and seen in section (Fig. 123, desc. l.) as an elongated area stretching fiom the pyramidal tract toward the anterior column and reaching at times as far as the anterior fissure. The area is large, however, because the tract is very diffuse, that is to say, the fibres with descending degeneration, or fibres which degenerate below the section or injury, are very largely mixed up with fibres which do not degenerate; in this respect this tract contrasts with the pyramidal tract, which is to a much greater extent composed of fibres with descending degeneration, though even in it there are a considerable number of fibres which do not degenerate. Indeed, this antero-lateral descending tract is so diffuse that it hardly deserves to be called a tract.

The other is a small, narrow, comma-shaped tract (Fig. 123, x), situated in the middle of the external posterior column which has been observed in the cervical and upper thoracic regions, and has been called the "descending" comma tract. But the degeneration reaches a short way only below 
the section or injury, and the group of fibres thus degenerating can hardly be considered as forming a tract comparable to the other tracts. The area probably represents fibres of the posterior root which take a descending course soon after their entrance into the cord.

II. Ascending tracts, that is to say, tracts in which the degeneration takes place above the section or injury.

A conspicuous ascending tract of a curved shape (Fig. 123, C.b.) occupies the outer dorsal part of the lateral column lying to the outside of the crossed pyramidal tract, between it and the surface of the cord. It appears to begin in the upper lumbar region, being said to be absent from the lower lumbar and sacral cord, and may be traced upward increasing in size through the thoracic and cervical cord to the bulb. In the bulb it may be traced into the restiform body or inferior peduncle of the cerebellum, and so to the cerebellum; for the restiform body serves, as we shall see, in each lateral half of the brain, as the main connection of the cerebellum with the bulb and spinal cord. Hence this tract is called the cerebellar tract.

A second important ascending tract occupies the median portion of the posterior columns (Fig. 123, cr., s.lr.), and so far coincides with what we described above as the median posterior column, in the upper regions of the cord, that it may be called the median posterior tract; it extends along the whole length of the spinal cord, varying at different levels in a manner which we shall presently study, and ending above in the bulb.

A third ascending tract, called the ascending antero-lateral tract, or tract of Gowers, occupies (Fig. 123, asc. a. l.) the outer ventral part of the lateral column. It has somewhat the form of a comma, with the head filling up the angle left between projecting portions of the cerebellar and pyramidal tracts, and the tail stretching away ventrally along the outer margin of the lateral column outside the antero-lateral descending column, the end of the tail often reaching to the anterior roots. It may be traced along the whole length of the cord, but it is not so distinct and compact a tract as the two ascending tracts just mentioned; the fibres with ascending degeneration, that is to say, the fibres degenerating above the section or seat of injury, are very largely mixed with fibres of a different nature and origin.

We may further remark that these several tracts differ from each other, in some cases markedly, as to the diameter of their constituent fibres. Thus the cerebellar tract is composed almost exclusively of remarkably coarse fibres. The mediau posterior tract, on the contrary, is made up of fine fibres of very equable size, while the fibres of the antero-lateral ascending tract are of a size intermediate between the other two. The pyramidal tract, on the other hand, is made up of fibres of almost all sizes mixed together.

The tracts then which are thus marked out are, as descending tracts, the crossed and the direct pyramidal tracts, with the less distinct or important antero-lateral descending tract; and, as ascending tracts, the cerebellar tract, the median posterior tract, and the less distinct antero-lateral ascending tract. If we suppose all these tracts taken away there is still left a considelable area of white matter, namely, nearly the whole of the external posterior column, the exterual anterior column, including the region traversed by the bundles of the anterior roots, and that part of the lateral column which lies between the antero-lateral descending tract and the crossed pyramidal tract on the outside and the gray matter on the inside. From this area of white matter we may put on one side at present the external posterior column, because, as we shall see, this column is largely composed of the fibres of the posterior root which pass through this column, especially through the lateral part of it near the gray matter, on their way to their ultimate destination; hence the alternative name of posterior root-zone. We may simi- 
larly leave for the present the small zone of white matter composed of very fine fibres known as the posterior marginal zone or Lissauer's zone (Fig. 123, $L$.), lying dorsal to the tip of the posterior horn and in the lower regions reaching to the outside of the cord; for this, too, belongs to the fibres of the posterior root. Leaving these parts out of consideration we may say as regards the rest of the white matter that the present state of our knowledge will not allow us to divide it into special tracts. All this area is largely composed of fibres which do not undergo either ascending or descending degeneration as the result of section, injury, or disease. It has been suggested that these fibres either have no trophic centre at all or have double ones, one above and one below, on either of which they can in case of need lean; so that when the fibre is divided at any level, the upper portion is still nourished from some centre above, and the lower from some centre below. At all events, whether this be the true explanation or not, the fibres in this part of the white matter cannot be differentiated into tracts by a study of their degeneration. Fibres of this kind, which we can speak of neither as ascending nor as descending, also occur in the external posterior column mingled with the fibres of the posterior root. And we may repeat the caution, that even in the several ascending and descending tracts just described, especially in those which we spoke of as less distinct or as more diffuse, many fibres are present which undergo neither ascending nor descending degeneration.

$\S 481$. It may be as well perhaps to insist here once more, that when these several tracts or the fibres running in the tracts are spoken of as ascending or descending, what is meant is that the degeneration takes place above the section or seat of injury or disease in the one case, and takes place below in the other. It has been supposed by many that the nervous impulses which these fibres severally carry, travel in the same direction as that taken by the degeneration, that the ascending tracts carry impulses from below upward, that is to say, carry impulses which arising from peripheral organs pass to various part of the spinal cord or of the brain, that they are, in other words, channels of afferent impulses, and that conversely the descending tracts carry efferent impulses. To this view is often added as a corollary, that the tracts which do not degenerate at all carry impulses both ways, and hence cannot be considered as either afferent or efferent channels, but simply as communicating channels. Upon this it may be remarked that impulses do not necessarily travel in the same direction as the degeneration; when a spinal nerve-trunk is divided the afferent fibres as well as the efferent fibres both degenerate in a descending direction toward the periphery, though the former carry impulses in the other direction. Hence the direction of degeneration is no proof of the direction in which impulses travel; moreover, as we have seen, degeneration does not actually travel along the fibres of the spinal cord in the same way that it does along the fibres of a nerve-trunk. It may be that the descending tracts do carry impulses in a descending direction, that is, efferent impulses, and that the ascending tracts serve to carry afferent impulses; but the proof that they do thus respectively act must be supplied from other facts than those of degeneration. Moreover, we shall have to return to these ascending and descending tracts and to study their behavior along the length of the cord before we can use the facts concerning them as a basis for any discussion as to their functions.

$\$ 482$. The connections of the nerve-roots. If we regard the spinal cord, and apparently we have a right to do so, as resulting from the fusion of a series of segments or metameres, each segment, represented by a pair of spinal nerves, being a ganglionic mass, that is to say, a mass containing nerve-cells with which nerve-fibres are connected, we should expect to find that the 
fibres of a spinal nerve soon after entering in or before issuing from the spinal cord are connected with nerve-cells lying in the neighborhood of the attachment of the nerve to the cord. We should, we say, expect to find this; but owing to the difficulty of tracing individual nerve-fibres through the tangled mass of the substance of the cord, our actual knowledge of the termination of the fibres of the posterior root and origin of the fibres of the anterior root is at present far from complete.

With regard to the anterior root, there can be no doubt that a very large proportion of the fibres in the root are continuations of the axis-cylinders of cells in the anterior horn. The fibres which can thus be traced are of large diameter and appear to be chiefly if not exclusively motor fibres for the skeletal muscles. In the frog a laborious enumeration, on the one hand, of the number of fibres in the anterior roots, and, on the other hand, of the number of cells of the anterior horn in the areas corresponding to the nerve-roots has, it is true, shown a very remarkable agreement in the number between the two. We might be inclined from this to conclude that all the fibres of an anterior root start directly from cells in the anterior horn, and that all the cells in the anterior horn end in fibres of the nearest anterior root. But several considerations prevent us from trusting too much to this observation especially in the case of the higher animals. The anterior root contains other fibres than motor fibres for the skeletal muscles, vasomotor fibres for instance, secretory fibres and others; and it is $\grave{a}$ priori unlikely that these should have origin from the same cells as the motor fibres of the skeletal muscles. Moreover, as a matter of fact, some of the fibres have been traced through the anterior horn, on the one hand, toward the posterior horn, and, on the other hand, toward the lateral column; others again are found to pass through the anterior horn of their own side to the bottom of the anterior fissure where, crossing over to the other side and thus forming part of the anterior white commissure, they appear to ascend to the anterior horn of the other side. We cannot at present make any positive statement as to the real origin and exact nature of these fibres which thus upon entering the cord pass by the cells in the anterior horn without joining them, though those which cross by the anterior white commissure are supposed to take origin in the cells of the anterior horn of the other side; it is sufficient for our present purposes to remember that while a large number of the fibres of the anterior root, presumably those supplying the skeletal muscles, take origin in the cells of the anterior horn, shortly before they issue from the cord, others have some other origin. . And similarly we have reason to think that all the cells in the anterior horn do not send out axis-cylinder processes to join the anterior roots of the same side. We may, however, regard a large number at all events of the cells of the anterior horn, at the level of as well as a little below and a little above the level of the exit of any particular anterior root, as constituting a sort of nucleus of origin for the larger number of the fibres, and those most probably the skeletal motor fibres, of that anterior root.

The posterior root enters the cord not in several bundles laterally scattered as does the anterior root, but in a more compact mass. This mass, however, consists of at least two distinct bundles, which upon their entrance into the cord, take different courses. One bundle, the larger one, lying to the inner or median side of the other, consisting of relatively coarse fibres, and called the median bundle (Fig. 121, $P^{\prime}$ ), passes obliquely into the lateral part of the external posterior column, which, as we have said, is in consequence often spoken of as the posterior root-zone. Here the fibres changing their direction run longitudinally for some distance upward (some, however, certainly in the upper cervical region, and probably in other regions, run a 
short distance downward), but eventually either go, as we shall see, to form the median posterior tract or make their way back into the gray matter at the base of the posterior horn and thus join the vesicular cylinder, though some are said to be continued on through the gray matter into the anterior horn. The other smaller bundle placed to the outside of the former, and called the lateral bundle (Fig. 121, $\mathrm{Pr}$ ), may be again divided into an intermediate bundle (Fig. 122, Pr) lying next to the median bundle, and into a still more lateral bundle (Fig. 122, $\operatorname{Pr}^{\prime \prime}$ ). The former, consisting also of coarse fibres, plunges directly through the substance of Rolando at the extremity of, and so into the gray matter of the horn, where the fibres changing their direction run in part at least longitudinally in the gray matter in bundles known as "the longitudinal bundles of the posterior horn" (Figs. $121,122, r . f . p$.$) , some of which appear to pass on to the anterior horn.$ The small, nost external or lateral portion of the lateral bundle, consisting of fine fibres and sometimes spoken of as the lateral bundle, on entering the cord at once ascends for some distance, and thus forms the thin layer of fine fibres, the posterior marginal zone or Lissauer's zone, indicated in Fig. 122 by m. $t$., which lies between the actual extremity of the horn and the surface of the cord, and in the upper regions of the cord (cf. Fig. 121, $p^{\prime}$ ) runs some way upward on the lateral margin of the horn between the gray matter and the crossed pyramidal tract. As it ascends this layer continually gives off fibres to the gray matter of the posterior horn in the cells of which they appear to end.

Thus, while part of the median bundle does not join the gray matter at all but goes to form the median posterior tract, the rest of that bundle and all the other fibres of the root, sooner or later, join the gray matter either of the posterior horn or of some other part.

$\$ 483$. The special features of the several regions of the spinal cord. The cord begins below in the slender filament called the filum terminale, which lying in the vertebral canal, in the midst of the mass of nerve-roots called the cauda equina, rapidly enlarges at about the level of the first lumbar vertebra into the conus medullaris. This may be regarded as the beginning of the lower portion of a fusiform enlargement of the cord known as the lumbar swelling, which reaches as high as about the attachment of the roots of the twelfth or eleventh thoracic nerve at the level of the eighth thoracic vertebra, the broadest part of the swelling being about opposite the third lumbar nerve. Above the lumbar swelling, through the thoracic region the somewhat narrowed cord retains about the same diameter until it reaches the level of the first or second thoracic nerve opposite the seventh cervical vertebra where a second fusiform enlargement, the cervical swelling, broader and longer than the lumbar swelling, begins. The broadest part of the cervical swelling is about opposite to the fifth or sixth cervical nerve; from thence the dianieter of the cord becomes gradually somewhat less until it begins to expand into the bulb, but even in the highest part is greater than in the thoracic region. The sectional area of the cord increases therefore from below upward, but not regularly, the irregularity being due to the lumbar and cervical swellings. The extremity of the filum terminale is said to consist entirely of neuroglia closely invested by the membranes, even the central canal being absent. A little higher up the central canal begins, and nervecells with nerve-fibres make their appearance in the neuroglia; thus a kind of gray matter covered by a thin superficial layer of white matter is established. We have already referred to the peculiar features of the lower end of the conus ( $\$ 477$ ); but higher up the canal becomes central and small, the posterior columns are developed, and the gray matter contains more nervous elements and relatively less neuroglia, becomes in fact ordinary gray 
matter. From thence onward to very near the junction with the bulb, where transitional features begin to come in, the spinal cord may be said to have the general structure previously described.

The sectional area of the white matter increases in absolute size and on the whole in a steady manner from below upward. In other words, in a section at any level, the number of longitudinal fibres forming the white matter is greater than the number at a lower level, and less than the number at a higher level; for any difference which may exist in the diameter of the individual fibres is insufficient to explain the differences in the total sectional

FIG. 124.

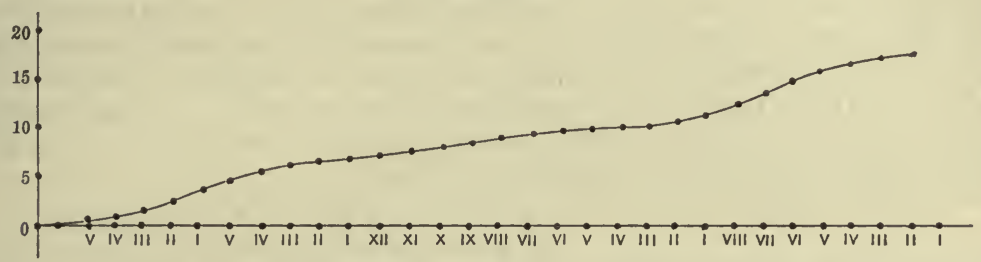

Diagram showing the United Sectional Areas of the Spinal Nerves, proceeding from Below Upward. In this, as in the succeeding figures, $125,126,127,128,129$, all of which refer to man, the left-hand side represents the bottom of the cord and the right-hand the top of the cord, the numerals indicating successively the sacral, lumbar, thoracic, and cervical nerves. The several figures are not drawn to the same scale.

area of the white matter. If we were to measure in man the sectional area of each of the spinal nerves as it joins the cord, and to add them together, passing along the cord from below upward, the results put in the form of a curve would give us some such figure as that shown in Fig. 124; the area gained by adding together the sectional areas of the nerves increases in a fairly steady manner from below upward. The curve of the sectional area of the white matter of the cord taken from below upward would be very similar, but if anything more regular. It must be understood, however,

Fig. 125.

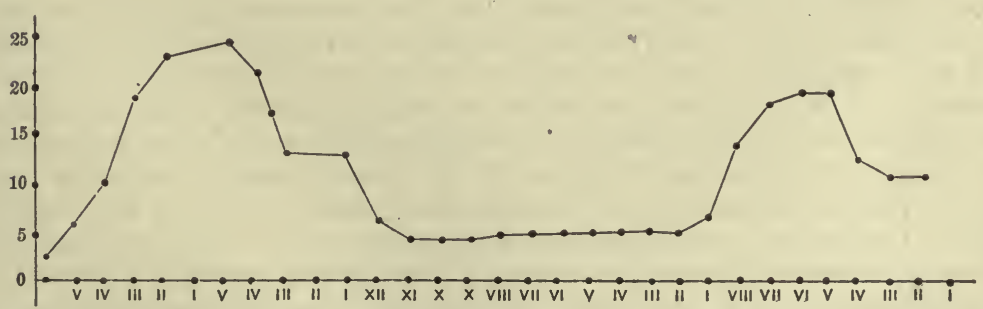

Diagram showing the Variations in the Sectional Area of the Gray Matter of the Spinal Cord along its Length.

that the dimensions of the areas would not be the same in the two cases. The sectional area of the white matter at the top of the cervical region, though greater than anywhere lower down, is far less than the united sectional area of all the nerves below that level. The white matter is not formed by all the fibres from the nerves which join the spinal cord continuing to run along the cord up to the brain; as we have seen, some at least of the fibres end in the gray matter. Nevertheless, the white matter in passing up the cord ap- 
pears to receive a permanent addition at the entrance of each nerve. We may infer that each nerve has a representative of itself starting from the level of its entrance and running up to some part of the brain. Whether the fibres thus representative of the nerve are continuations of the very fibres of the nerve itself, or are new fibres starting from some relay of gray matter, with which the fibres of the nerve are also connected, is another question.

$\$ 484$. The gray matter in contrast to the white matter shows great variations in area along the length of the cord (Fig. 125). From the entrance of the coccygeal nerve upward the area increases very rapidly, reaching a maximum at about the level of the fifth lumbar nerve. It then rapidly decreases to about the level of the eleventh thoracic nerve, maintains about the same dimensions all through the thoracic region, and begins to increase again at about the level of the second thoracic nerve. Its second maximum is reached at about the level of the fifth or sixth cervical nerve, after which the area again becomes smaller, remaining, however, at the upper cervical region much larger than in the thoracic region.

FIG. 126.

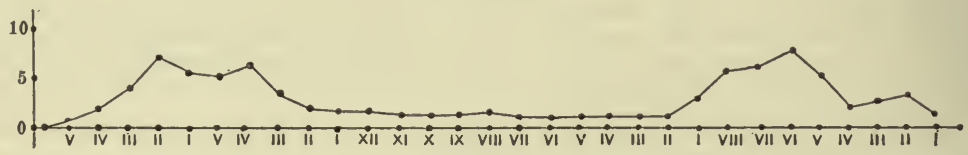

Diagram showing the Relative Sectional Areas of the Spinal Nerves as they Join the Spinal Cord.

The meaning of these variations becomes clear when we turn to Fig. 126, which shows in a similar diagrammatic manner the sectional areas of the several spinal nerves. It will be observed that the increase and decrease of the sectional area of the gray matter follow very closely the increase and decrease of the quantity of nerve, that is to say, neglecting differences in the diameter of the fibres, in the number of nerve-fibres passing into the cord. The sectional areas of the first and second sacral, fourth and fifth lumbar nerves are very large, and opposite to these the sectional area of the gray matter of the cord is very large also; the enlargement of gray matter which is the essential cause of the lumbar swelling is correlated to the large number of fibres which enter and leave the cord at this region to supply chiefly the lower limbs. Similarly the enlargement of gray matter which is the essential cause of the cervical swelling is correlated to the large number of fibres which enter and leave this region of the cord to supply chiefly the upper limbs. In the thoracic region, where the number of fibres entering and leaving the cord is relatively less, the sectional area of the gray matter is also less. Since the attachments of the several spinal nerves are not exactly equidistant from each other along the length of the cord, the sectional area is not an exact measure of bulk; the total bulk of gray matter, for instance, belonging to two nerves which enter the cord close together is less than that of two nerves giving rise to the same sectional area of gray matter as the former two but entering the cord far apart from each other. Still the error which may be introduced by taking sectional area to mean bulk is, for pres. ent purposes at all events, so small that we may permit ourselves to say that in the successive regions of the spinal cord the bulk of gray matter in any segment is greater or less according to the size of the nerve (or pair of nerves, right and left) belonging to that segment.

From this anatomical fact we appear justified in drawing the conclusion 
that at all events a great deal of the gray matter of the spinal cord may be considered as furnishing a nervous mechanism, with which the efferent fibres of each spinal nerve just before they leave the cord, and the afferent fibres soon after they join the cord, are more immediately connected. It may be that the whole of the gray matter is thus directly connected with and thus rises and falls with the fibres of the nerves; or it may be that there is a sort of cord of gray matter, which maintains a uniform bulk along the whole length of the cord and serves as a basis which is here more and there less swollen by the addition of the gray matter more immediately connected with the fibres of the nerves. This question the method which we are now using cannot settle.

$\$ 485$. Owing to these different rates of increase of the gray and white matter respectively along the length of the cord, we find that in sections of the cord taken at different levels the appearances presented vary in a very distinct manner. This is strikingly shown by comparing Figs. 119, 121 and 122. At the level of the third lumbar nerve (Fig. 122) the gray matter is very large, reaching, as we have seen, its maximal sectional area at about this point, so that although the area of white matter is not very great the whole area of the cord is considerable.

At the level of the sixth thoracic nerve (Fig. 119), in spite of the white matter having very decidedly increased, the gray matter has shrunk to such very small dimensions that the total sectional area of the cord has markedly diminished.

At the level of the sixth cervical (Fig. 121) the gray matter has again increased, reaching here, as we have seen, its second maximum; the white matter has also further increased, and that indeed very considerably, so that the total area of the cord is much greater than in any of the lower regions.

Further details of the varying size of the white matter and of the gray matter at different levels are also shown in the series given in Fig. 127. In these, combined with the three figures just referred to, it will be observed that the serial increase and decrease of the gray matter does not affect all parts of the gray matter alike, so that the outline of the gray matter changes very markedly in passing from below upward. In the coccygeal region each lateral half is a somewhat irregular oval, and in the sacral region (Fig. 127, Sac.) the differentiation into anterior and posterior horns is still very indistinct. In the lumbar region the two horns are sharply marked out, though both the posterior and anterior horns are broad and more or less quadrate. In the thoracic region the decrease of gray matter has affected both horns, so that both are pointed and slender, while the junction between them has not undergone so much diminution, so that what has been called the lateral horn is relatively conspicuous. In the cervical region the returning increase bears much more on the anterior horn, which again becomes large and broad, than on the posterior horn, which still remains slender and pointed. Taking the form of the gray matter in the thoracic. region as the more typical form of the gray matter we may say that while the increase in the lumbar swelling bears equally on the anterior and posterior horns, that in the cervical region bears chiefly on the anterior horns.

Now we have no reason to suppose that either afferent impulses reach the lumbar spinal cord in greater numbers from the lower limbs, or along any of the nerves joining this part of the cord, or that those which do reach it are of a more complex nature than is the case with the afferent impulses reaching the cervical cord along the nerves of the upper limbs. The increase of gray matter in the posterior horns is therefore not correlated to any increase in the number or complexity of the afferent impulses reaching the cord; and we may provisionally conclude that at least a large part of the 
gray matter in the posterior horn is not specially concerned in any elaboration or transformation of afferent impulses immediately upon their arrival at the cord. Indeed, we have seen that while there is ample evidence to connect the nerve-cells, and therefore presumably the gray matter in general of the anterior horn with the efferent motor fibres of the anterior root, there is no corresponding evidence as to any large immediate connection of the afferent fibres of the posterior root with the nerve-cells, or indeed any other part of the gray matter of the posterior horn. We may add that, as we shall point out later on, so essential is the concurrence of appropriate afferent impulses to the due carrying out of complex coördinate motor or efferent impulses, that we can scarcely expect to find any increase in the nervous mechanisms devoted to the purely motor function of carrying out motor impulses without a corresponding increase in the nervous mechanisms belonging to the afferent impulses, by means of which these motor impulses

FIG. 127.
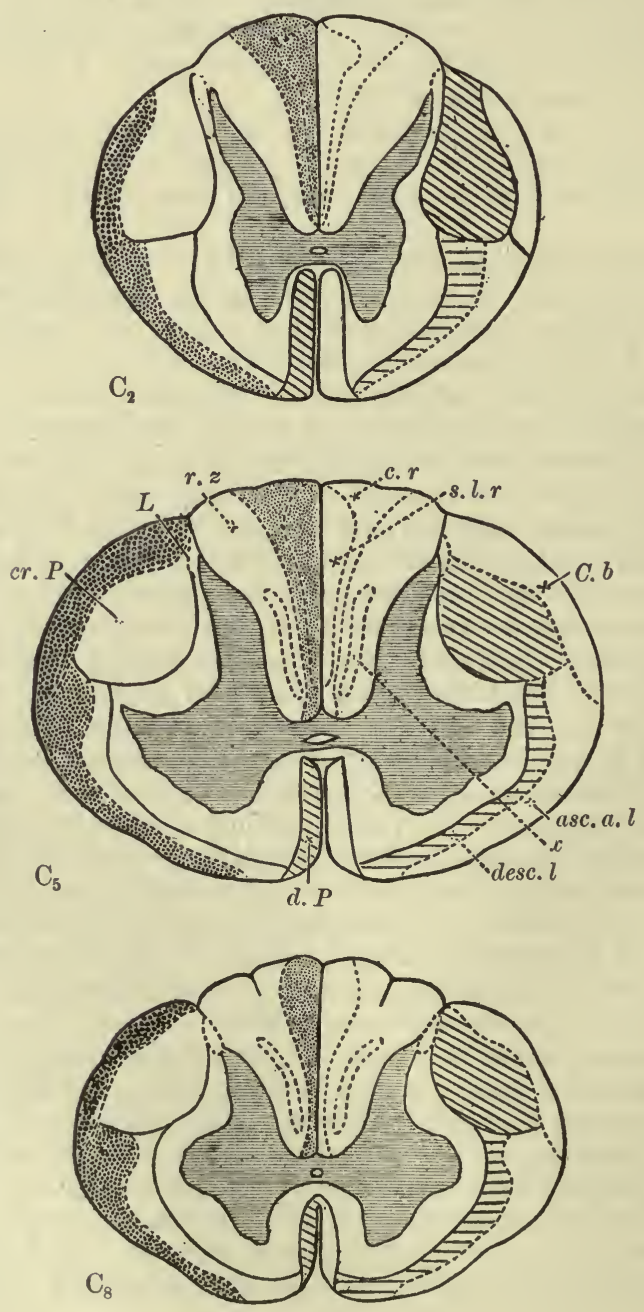

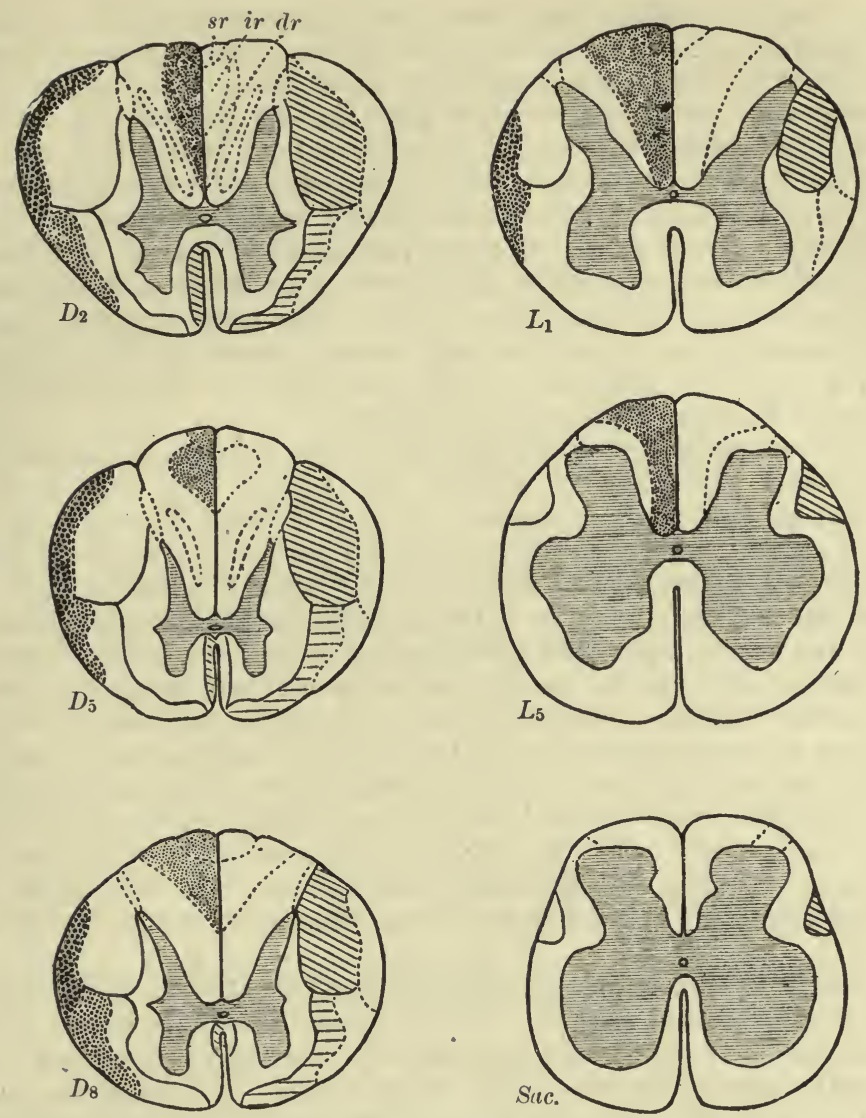

Diagram illustrating some of the Features of the Spinal Cord at Different Levels. (Sherrington.) All the figures are drawn to seale, and represent the cord magnified four times. They show the difference at different levels in the shape and size of the cord, in the outline of the gray matter, and in the relative position of the anterior and posterior fissures, and also show the variations at different levels of the several "tracts" of the white matter.

$C_{2}$ at the level of the second cervical nerve, $C_{5}$ of the fifth cervical, $C_{8}$ of the eighth cervical. $D_{2}$ of the second thoracic, $D_{5}$ of the fifth thoracic, $L_{1}$ of the first lumbar, $L_{5}$ of the fifth lumbar and Sac. of the seeond sacral nerve.

The shading of the tracts is the same as in Fig. 123; but in the median posterior column of $D_{2}$ the areas of fibres coming from the sacral nerves s.r. and lumbar nerves $l . r$ are distinguished from the area $d . r$. of fibres belonging to the thoracic nerves. In $C_{8}$ no distinction is made between any of these sets of fibres; in $L_{5}$ only fibres of saeral nerves are represented; in $L_{1} D_{8} D_{5}$ the more dorsal small portion corresponds in sacral fibres and the next tolumbar, or lumbar thoracic nerves.

are guided and coördinated. Hence, were the nervous mechanisms restricted to the posterior horns, we should expect to find a greater parallelism than does actually exist between them and the anterior horns.

$\$ 486$. The changes in the area of gray matter illustrated by the statements and diagrams given above refer to the gray matter as a whole-that is, not only to nerve-cells, but also to strands and networks of nerve-fibres and nerve-fibrils, and indeed include to a certain extent neuroglia. We have seen $(\S 479)$ that we are able to distinguish certain large and conspicuous nerve-cells in the gray matter, and to arrange these into groups. 
The gray matter contains many other small nerve-cells, which we are not able at present to name or arrange, but whose existence must always be borne in mind. Confining ourselves now, however, to the groups of larger, more conspicuous nerve-cells, we find that, broadly speaking, the chief differences which can be observed in the cells of the anterior horn along the length of the cord are that in the thoracic region the nerve-cells of the anterior horn are few and relatively small, while in the cervical and lumbar region, especially in the latter, they are numerous and large. It is not easy, even if possible, to distinguish in the thoracic region the several groups of cells marked in Figs. 121 and 122 as $2 \propto, \beta, \gamma$; the median group (Figs. 121, $122,1)$, indeed, seems to be the only group present in the mid-thoracic region (Fig. 119, 1). The group of the posterior horn (Figs. 119, 121, 122, 6 ) appears to be about the same in all regions.

With two other groups of nerve-cells striking differences are seen in different regions. The vesicular cylinder, for instance (Fig. 119, 3), is most conspicuous in the thoracic region. It may be said to reach from the seventh or eighth cervical nerve to the third lumbar nerve, being perhaps most developed in the lower thoracic and upper lumbar region. It is absent in the cervical region above the seventh or eighth cervical nerve, and in the lumbar region below the third lumbar nerve; but a similar group of cells is present opposite the second and third cervical nerves; a group of more doubtful likeness is seen in the sacral region below, and the column is said to have a representative in the bulb above the spinal cord proper. It seems natural to infer that the cells forming this vesicular cylinder are connected neither with the ordinary somatic motor fibres governing the skeletal muscles, nor with the ordinary afferent sensory somatic fibres coming from the skin and elsewhere, but in some way with some special sets of fibres; on this point, however, no authoritative statement can as yet be made.

The lateral horn or intermedio-lateral tract (Fig. 119, 4) is also most conspicuous in the thoracic region. In the lumbar region it is lost or traced with great difficulty, and in the cervical region seems to be merged into the most dorsally placed division of the lateral group of cells of the anterior horn. It is possible that this group represents in the limbless thoracic region the cells which are developed into the great lateral group of the anterior horn in the regions of the limbs.

$\$ 487$. The white matter, as we have seen, increases in sectional area with considerable regularity from below upward. If; instead of a diagram of the increase of the whole white matter we construct in a similar way diagrams of the anterior, posterior, and lateral columns respectively, we find that while the sectional area of the lateral column (Fig. 128) increases with some considerable regularity from below upward, though not so regularly as does the whole area of white, matter, both the anterior (Fig. 129) and the posterior (Fig. 130) columns agree to a certain extent with the gray matter in showing a decided increase in both the lumbar and the cervical swellings. We may, provisionally at least, infer from this that, while considerable portions of both the anterior and the posterior columns are, like the adjoining gray matter, in some way or other concerned in the exit and entrance of efferent and afferent fibres, the larger portion of the lateral column is concerned in the transmission of impulses to and fro, between the local mechanisms below, immediately connected with the several spinal nerves, and the brain above. This conclusion seems incidentally confirmed (though these diagrams must not be strained to carry detailed inferences) by the suilden increase of the lateral column above the lumbar swelling, as if the large mass of nervous mechanism for the lower limbs concentrated in 
this region demanded a sudden increase in the number of fibres connecting it with the brain above.

This more or less continuous increase of the lateral column partly explains the change of form in the general outline of the transverse section of the cord which is observed in passing upward from the lower to the higher regions. In the coccygeal, sacral, and lumbar regions the outline, though varying somewhat, chiefly owing to the disposition of the gray matter, is on the whole circular. In the thoracic region, especially in the upper part, the increase of the lateral columns increases the side-to-side

FIG. 128.

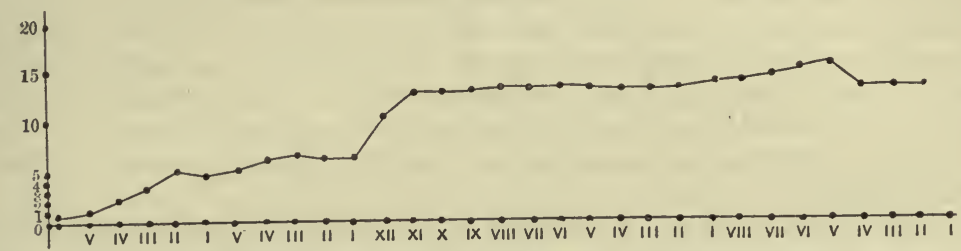

Diagram sbowing the Variations in the Sectional Area of the Lateral Columns of the Spinal Cord along its Length.

FIG. 129.

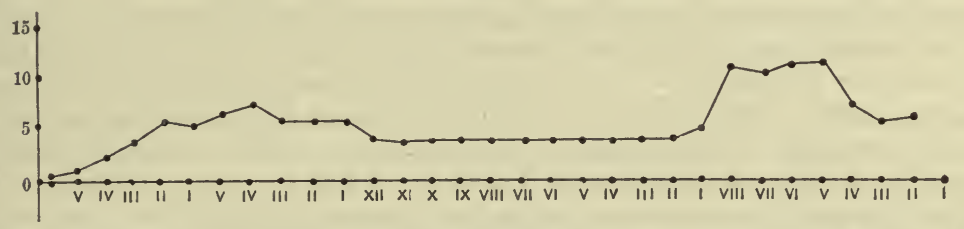

Diagram showing the Variations in the Sectional Area of the Anterior Columus of the Spinal Cord along its Length.

Frg. 130 .

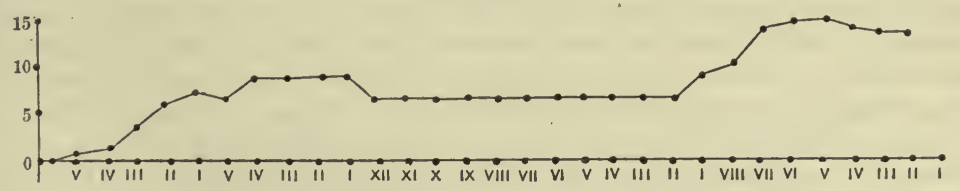

Diagram showing the Variations in the Sectional Area of the Posterior Columns of the Spinal Cord along its Length.

diameter so much that the section becomes oval, and in the cervical region this increase of the side-to-side diameter out of proportion to the dorso-ventral diameter is very marked. The actual outline of the whole transverse section is, however, determined also to a certain extent by the changes of form of the gray matter.

The cord, moreover, undergoes along its length a change which is not very clearly indicated in the diagrams (Figs. 129, 130). By comparing the series of transverse sections given in Fig. 127, it will be seen that the relative position of the central canal shifts along the length of the cord. In the sacral and lumbar regions the central canal is nearly at the centre of the circle of outline, and the posterior and anterior fissures are nearly of equal depth. Even in the upper lumbar region, and still more in the thoracic region, the position of the central canal is shifted nearer to the ventral surface, so that the posterior fissure becomes relatively longer, deeper than the 
anterior. This shifting goes on through the cervical region up to about the level of the second cervical nerve, where it is arrested by the beginning of the changes through which the spinal cord is transformed into the far more complicated bulb.

This lengthening of the posterior fissure indicates an increase in the dorso-ventral diameter of the posterior columns, and this, not being accompanied by a compensating diminution of the side-to-side diameter, shows in turn that the posterior columns undergo an increase in passing upward. From this we may add to the provisional conclusion just arrived at with regard to the lateral columns, the further conclusion that some part of the posterior columns also is concerned in transmitting impulses, in a more or less direct manner, between the various regions of the cord below and the brain above. The anterior columns do not increase in the same marked manner, though over and above the increase due to the lumbar and cervical swellings a continued increase may be observed, especially in the upper cervical region; it is in this upper region that the direct pyramidal tract is best developed.

$\S 488$. The provisional conclusions at which we have arrived are further, to a certain extent at least, confirmed and extended by a study of the behavior at the several regions of the cord of the special tracts of white matter described in $\$ 479$.

The pyramidal tract, that is to say, the crossed pyramidal tract entering the spinal cord above from the pyramid, is very large in the cervical region, having the form and situation shown in Fig. 127, $C_{2} C_{5} C_{8}$. From thence downward it diminishes in size, the diminution being especially rapid in the lumbar swelling (Fig. 127, $L_{1}$ ), where the tract, being no longer covered in by the cerebellar tract, comes to the surface of the cord; but it may be traced by the degeneration method down as far as the coccygeal region, and indeed appears to be coexistent with the entrance of spinal nerves into the cord. Diminution of the tract means a lessening of the number of fibres; and since we cannot suppose that any of the fibres come suddenly to an end in the tract itself, we are led to infer that along the cord, from above downward, fibres are successively leaving the tract and passing to some other part of the cord. We seem further justified in concluding that the fibres which thus successively leave the tract go to join the series of local nervous mechanisms with which the spinal nerves communicate, as we have seen reason to believe, upon their entrance into the cord. Indeed, as we shall see later on, we have reason to think that the nervous mechanisms which the fibres in question join are those belonging to the motor fibres of the anterior roots. This pyramidal tract does not begin 'in the pyramid, but may be traced through the lower parts of the brain right up to special areas in the cortex or surface of the cerebral hemispheres; and very strong reasons may be brought forward in support of the view that the fibres of this tract are fibres which carry impulses from the cortex to successive portions of the spinal cord and there give rise to efferent impulses which pass to appropriate skeletal muscles. The tract, therefore, is not only a descending tract by virtue of the mode of degeneration, but may be spoken of in a broad sense as a tract of efferent impulses descending from the cerebral cortex ; and, indeed, it is maintained that it is the channel of the particular kind of efferent impulses which we shall speak of as voluntary or volitional impulses. We may add that as the tract passes along a path, which we shall subsequently describe, from the cerebral cortex through the lower parts of the brain to the pyramid, it gives off fibres to mechanisms connected with several of the cranial nerves, much in the same way that it gives off fibres to the spinal nerves. 
We may, therefore, picture to ourselves this pyramidal tract as starting in the form of a broad sheaf of fibres from a certain district on the surface of one of the cerebral hemispheres. Putting aside for the present any possible increase of the number of fibres by division of fibres (though we have reason to think that this does, to a certain extent, occur), we may regard the tract as being at its maximum at its beginning in the cortex. As it descends to the decussation of the pyramids in the bulb it loses a certain number of fibres, which pass off to the cranial nerves. Having crossed and entered into the lateral column of the cord it continues to give off fibres to the spinal nerves, probably to the anterior root of each in succession, and so goes on its way down the cord continually diminishing until the last remaining fibres are given off to the last coccygeal nerve.

When degeneration is set up along this tract, as may be done by injuries to particular areas of the cerebral cortex, the main mass of degenerated fibres, after crossing over from one side of the cerebro-spinal axis to the other in the decussation of the pyramids at the lower end of the bulb, during its further progress down the spinal cord, keeps to the side to which it has crossed right down to the end. Hence, as we have said, it is called the crossed pyramidal tract. The main mass of fibres, the degeneration of which has been started by injury to the left side of the brain, crosses over to the right side of the spinal cord and runs down the lateral column of the right side to the end of the cord. Nevertheless, some fibres appear to cross over again in the spinal cord and then to run along the same side as the side of the brain injured-along the left side in the case just mentioned. Such fibres are spoken of as " recrossed fibres."

The direct pyramidal tract (Fig. 127, $d P$ ), except that it does not cross at the decussation of the pyramids, is otherwise similar to the crossed pyramidal tract, and, indeed, is a part of the same strand to which the crossed tract belongs. When degeneration in this tract is started by injury to particular areas of the cerebral cortex, say on the left half of the brain, the degeneration may be traced through the left anterior pyramid, and so to the left median anterior column of the spinal cord. The direct tract is never so extensive or marked as the crossed tract, does not reach so far down, is much more variable both in length and in sectional area, and, as we have said, is almost confined to man. Diminishing as it descends it may be said to cease in the middle thoracic region (Fig. $127, D_{5} D_{8}$ ). Taking an average, we may say that, of the whole strand running in the pyramids above the decussation, about three-fourths of the fibres go to form the crossed and about onefourth to form the direct tract. We shall see later on that the impulses coming down along the united tract in the brain may, broadly speaking, be said to cross over wholly from one side to the other before they reach the skeletal muscles, so that the impulses passing along the fibres in, say, the left pyramid, reach the muscles of the right limbs and right side of the body, whether the fibres cross over at the decussation to form the crossed or remain on the same side to form the direct pyramidal tract. We are, therefore, led to infer that the fibres in the direct tract, as they pass down the cord, cross over in the cord itself before they make connections with the fibres of the anterior roots. Probably the crossing is effected by means of some of the decussating fibres which form the anterior white commissure. A part only, indeed a small part, of the commissure can serve this purpose; most of the fibres of the commissure, and in the lower regions of the cord, where the direct tract no longer exists, all the fibres, must have some other functions. Some of the fibres of this great pyramidal tract leave the tract, as we have said, to join some of the cranial nerves before the pyramids of the bulb are reached; and the impulses passing along these fibres also cross 
over to the opposite side before they issue along the cranial nerves. Hence we infer that these fibres decussate above the decussation of the pyramids just as those of the direct tract decussate below it. So that of the whole strand as it leaves the cerebral cortex, while the main mass of fibres crosses over at the decussation of the pyramids, the rest of the fibres cross the middle line in succession from the level of the third cranial nerve to the level of the lower limit of the direct tract; below the decussation of the pyramids the crossing takes place by means of the anterior commissure of the cord, above the decussation by means of what we shall later on learn to speak of as the raphé of the bulb, or by structures corresponding to this higher up.

$\S 489$. 'The cerebellar tract (Fig. 127, C.b.) is, as we have seen, a tract of ascending degeneration; the degeneration in it makes its appearance above the section or seat of other injury of the cord. It begins somewhat suddenly at the level of the second lumbar nerve region, being absent at least as a distinct tract below ; injury of the cord at the level of the middle and lower lumbar nerves leads to no marked tract of degeneration (though possibly scattered single fibres may degenerate), while injury higher up does. The tract lies, as we have said, close to the surface of the cord in the posterior part of the lateral column just outside the crossed pyramidal tract, and while varying somewhat in the shape of its section from level to level, remains throughout a somewhat narrow crescentic patch. At the top of the spinal cord, it passes, as we have said, from the lateral columns into the restiform bodies of the bulb, and so to certain parts of the cerebellum.

When the section or lesion is limited to one side of the cord, the degeneration is similarly limited to the same side, and that along its whole course up to the cerebellum; there is no evidence of any of the fibres decussating in the cord.

The area of the tract increases from below upward. This has been determined by the embryological method, by noting the appearance of the medulla in the fibres, as well as by comparing the extent of the degeneration following upon a section high up in the cord with that following upon a section lower down. From this we infer that the fibres composing the tract must start successively from other parts of the cord along its length-that is to say, the tract must be fed by fibres coming from other structures in the cord. On the other hand, it is found that the degenerated area following upon a section or injury diminishes as it is traced upward; when, for instance, a section is made in the mid-thoracic region, the area of degeneration in the tract is greater immediately above the section than it is higher up, say in the cervical region. From this we are led to infer that though the tract is successively fed along its course by fibres coming from other parts of the cord, some of the fibres entering the tract, though like their companions undergoing an ascending degeneration, do not like them continue in the tract right up to the cerebellum, but pass off to other parts of the cord on their way upward. This, however, is equivalent to saying that the tract is not a pure or homogeneous one, but consists of at least two sets of fibres, only one of which is continued on to the cerebellum and strictly deserves the name of "cerebellar." It may, perhaps, here be mentioned that while the fibres composing the tract are, as a whole, conspicuously coarse, large fibres, with these there are mingled, especially in the thoracic region, a number of much finer fibres; but these apparently undergo a descending, not an ascending, degeneration, and do not, therefore, really belong to the tract; they may be fibres which have strayed from the pyramidal tract. 
We have as yet no very clear evidence as to the origin of the fibres which compose the tract. Unlike the case of the median posterior tract of which we have next to speak, no degeneration, at least in the lumbar and thoracic regions, appears in the tract after section merely of the roots of the nerves; to produce the degeneration the cord itself must be injured. From this we may infer that the tract is not fed directly by the fibres of the posterior roots. Some observers maintain that the tract is fed by fibres coming from the vesicular cylinder and point out that both the tract and the column begin at the same level somewhat suddenly; but the want of parallelism between the course of the tract and that of the cylinder along the length of the cord, the latter being as we said conspicuous in the thoracic region while the tract steadily increases upward, is distinctly opposed to such a view. From the fact that the degeneration taking place in it is an ascending one, it is supposed that the tract is the channel for ascending, that is to say, in a broad sense, afferent impulses. And considerable interest attaches to the fact that these impulses should be carried, not to the cerebrum but to the cerebellum. Our knowledge on this point, however, is very imperfect, and what can be said in the matter had better be said later on.

$\S 490$. The median posterior tract is the other conspicuous tract of ascending degeneration; it also is supposed to be a channel for ascending afferent impulses; and this view is rendered almost certain by the intimate relations of the tract to the fibres of the posterior roots.

In dealing so far with the tracts of degeneration in the spinal cord we have always spoken of the degeneration as being the result of lesions of the spinal cord itself. Experiments on animals, however, and clinical experience have shown that division or injury of the fibres of the posterior roots is followed by tracts of degeneration in the spinal cord, though no damage whatever may have been done to the substance of the cord itself. These tracts make their appearance in the median posterior columns, the exact path and limits of the degeneration differing with the different spinal nerves. The results of the division of different groups of nerves are so instructive that we may dwell upon them in detail.

If the posterior roots of two or three lumbar nerves (on one side) be divided, an examination of the cord, after an interval long enough to allow degeneration to be well established, will bring to light the following features: The divided roots will be found to have degenerated right up to their entrance into the cord. A section of the cord opposite the entrance of the lowest divided root will show no degeneration of the cord beyond that of the bundles of fibres passing in. A little higher up degeneration will be observed in the external posterior column close to the posterior horn; and as we ascend we find that this degeneration first spreads over a large portion of the external posterior column, and then invades the median posterior column; the degeneration does not affect the whole of the median posterior column but leaves intact a small dorsal portion, roughly triangular in shape, at the angle between the fissure and the dorsal surface of the cord, as well as some portion of the more ventral part of the column nearest the gray commissure. Still a little higher up we should find that degenerated fibres had disappeared from the external portion of the external posterior column close to the gray matter, though still existing in the more median part of that column as well as in the median posterior column to the extent just indicated. Still a little higher up the whole of the degeneration would have disappeared from the external posterior column, but the tract of degeneration in the median posterior column would remain, the extent of degeneration being dependent on the number of roots which had been divided. Lastly, by carrying the sections still higher up the cord we should be able to trace this tract in the 
median posterior column right up to the bulb, where it would come to an end.

If we divided some of the thoracic nerves instead of the lumbar we should obtain very similar results : a degeneration of the external posterior columns a little above the entrance of the roots, spreading across the column toward the median line, and wholly disappearing at a certain height above, accompanied by a degeneration of a part of the median posterior column, reaching from a little distance above the entrance of the divided nerve-roots right up to the bulb. This latter tract of degeneration would, however, not occupy the same position as that consequent upon division of the lumbar nerves; its position would be more ventral, nearer the gray commissure, and rather more lateral. Compare Fig. $127, D_{2}$, where $l r$. indicates the degeneration due to section of the lumbar nerves, and $d r$. that of the thoracic nerves. If we divided some of the cervical posterior roots we should get similar results, with the difference that the tract of degeneration in the median posterior columns would occupy a position still more ventral and still more lateral (Fig. 127, $C_{5}$ c. r.), while if we divided the sacral nerves the tract of degeneration would be dorsal and median to the tract belonging to the lumbar nerves, and would occupy more or less of the triangle left below that tract (Fig. 127, $D_{2} s \cdot r$.). The degeneration, it will be understood, is in all cases confined to the same side of the cord as that of the divided roots. We may add, in order to complete the story of the effects of division of the posterior roots, that the section leads to degeneration of the marginal zone (Lissauer's tract), but this degeneration reaches for a certain distance only up the cord and then disappears. It will be remembered that this zone is fed by fibres (of fine calibre) belonging to the external or lateral bundle of the posterior roots.

These results may be interpreted as follows: The (great majority of the) fibres of the posterior root, cut off from their ganglion by the division, degenerate centripetally toward the spinal cord. We have previously seen that many of the fibres of the root pass into the external posterior column and run up in that column for some distance. The degeneration observed in this column for some distance above the entrance of the divided roots shows that the fibres run lengthways for some distance is this column, while the disappearance of the degeneration a little higher up similarly shows that the fibres eventually leave the column. The appearance of degeneration in the median posterior column shows that some of these fibres have passed into that column from the external posterior column, and the continuation of that degeneration right up to the bulb indicates that these fibres pursue an unbroken course in that column along the whole length of the cord. The area of degeneration, or more exactly the number of degenerated fibres in the continued tract of degeneration in the median posterior column is much less than that in the temporary or short tract of degeneration in the external posterior column. This shows that some only of the fibres passing into the external posterior column go on to join the median posterior column and so reach the bulb; the rest obviously take another path, and we have already seen reason to think that many of these end in the gray matter of the cord. Hence of all the fibres joining the cord in a posterior root, while some, and these we may add are chiefly fine fibres, entering the gray matter directly or passing into the posterior marginal zone, soon make such connections that the degeneration due to the section of the roots spreads no further, a large number, and these chiefly coarse fibres, before they make any such connection pass into and occupy for some length of the cord the external posterior column. We may here remark that though these fibres are spread over the greater part of this column, they do not form the whole of the 
column; they are mixed up with fibres of a different nature and origin. Of these fibres of the posterior root which thus run in the external posterior column while still dependent for their nutritive activity on the ganglion of the root, some, indeed the greater part, leave the tract and make such connections in the gray matter that their degeneration ceases; others, forming the smaller part, pass into the median posterior column, and taking up a definite position in that column pursue an unbroken course to the bulb.

All the fibres, therefore, of the posterior roots do not end in the gray matter soon after their entrance into the cord. A representative of each root is carried right up to the bulb by means of the median posterior column; of the axis-cylinders which leave the ganglion on the root, a certain relatively small number pursue an unbroken course for some little distance through the external posterior column, and for the rest of their way through the median posterior column, along the whole length of the cord above the entrance of the root until they find an ending in the gray matter of the bulb. Further, each spinal nerve has this representative of its posterior root placed in a definite position in the posterior median column, the arrangement being such, as shown in Fig. 127, that the lower (sacral) nerves find their place in the more dorsal and median part of the column, while the nerves above are successively placed in positions more and more ventral and external.

As far as our knowledge goes at present we are led to believe that this median posterior tract is very largely made up of fibres having this origin. It affords a channel by which afferent impulses are carried straight up the cord from the nerve-trunk without making connections on the way. We may repeat that the path is confined to the same side of the cord along its whole length; there is no crossing over to the other side.

In the above description we have spoken only of the result following section of the posterior roots outside the cord; but it will be understood that similar results follow upon section or of injury to or disease of the cord itself affecting the posterior columns or the bundles of the roots as they euter the cord. When such a lesion occurs there may be observed in the region of the cord above the lesion a degeneration of the external posterior column, reaching some little distance up, and a more limited degeneration of a part of the median posterior column stretching right up to the bulb. The position and form of the tract of the degeneration in the median posterior column will depend on the level of the lesion along the length of the cord, according as it interrupts the ascending representatives of the sacral nerves only, or of the lumbar and sacral nerves, or of the dorsal and cervical nerves as well. A complete section or hemi-section of the cord will produce results corresponding to the division on both sides or on one side of all the nerves below the section.

We may add that while, according to some observers, the strand of fibres belonging to a particular root or group of roots having once taken up its position in the median posterior column remains unchanged until it reaches the bulb, according to others it diminishes in area, some of its fibres making connections in the cord itself:

$\S 491$. The antero-lateral ascending tract (Fig. 127, asc. a. l.) is less well known than either of the two preceding; it is also more diffuse, that is to say, the fibres undergoing degeneration are more largely mixed with fibres of a different nature and origin. It appears to extend down the cord to a lower level than the cerebellar tract, but its lower limit has not yet been accurately determined. Since the degeneration taking place in it is an ascending one, it has been inferred that it serves as the path for afferent and indeed for sensory impulses. Degeneration in it is seen only after section or injury of the substance of the cord itself, not after division of the 
posterior roots. If, then, it is to be regarded as a channel of afferent impulses passing into it from the posterior roots, those impulses must pass into it along those fibres of the posterior root which find secondary trophic centres in some part of the gray matter; in this respect this tract resembles the cerebellar träct, and differs from the median posterior tract. The latter is the direct continuation up the cord to the bulb of such fibres as are still trusting for their nutritive activity to the cells of the ganglion on the posterior root; the fibres of both the former trust for their nutritive activity to some part of the gray matter of the cord, and presumably to the nervecells of that gray matter. A further resemblance between the antero-lateral ascending and cerebellar tracts must be admitted, if future researches confirm the opinion of those who hold that the former like the latter, at the top of the cord, pass along the restiform body to the cerebellum. Indeed, under such a view it would appear probable that the antero-lateral tract is simply a more diffuse and outlying part of the cerebellar tract.

$\S 492$. We may now briefly pass in review, somewhat as follows, the chief facts which we have learned concerning the structure of the spinal cord, always keeping in view their physiological meaning.

The important feature of the spinal cord is the presence of what we have called "gray matter," and all our knowledge goes to show that the important powers of the spinal cord, by which it differs from a thick multiple nerve, and by virtue of which we speak of it as a nervous centre or series of centres, are in some way or other associated with this gray matter.

With this gray matter the fibres of the spinal nerves are connected. The greater part of the fibres of the anterior root certainly end in or rather take origin from the gray matter close to the attachment of the root, and the rest most probably join the gray matter at no great distance. The fibres of the posterior root run, as we have seen, for some little distance in the white matter, but if we except the special bundle which runs in the median posterior tract right up the cord to the bulb,without joining the spinal gray matter at all, we may say that the fibres of the posterior root also join the gray matter not far from the attachment of the root.

Morphological reasons lead us, as we have seen, to regard the spinal cord as a series of segments, each segment corresponding to a pair of nerves; and even in the spinal cord of man we may recognize a segmental groundwork, obscured though this is by fusion and overlaid by the several commissural tracts. Each segment of this groundwork we may conceive of as a central mass of gray matter, connected on each side with an anterior and a posterior root, thus constituting a segmental nervous mechanism capable of carrying out certain functions.

Such a segment has been compared to a ganglion, but it differs strikingly from a ganglion, whether of the posterior root or of the splanchnic system, both in structure and in function. A ganglion and the gray matter of a spinal segment both contain nerve-cells, and so far resemble each other; but there the resemblance for the most part ends. In a ganglion the constituent nerve-cell is a development of the axis-cylinder of a fibre into a nucleated cell-body which lies on the course of the fibre, and may, as in a splanchnic ganglion, be placed just where one fibre divides into two or more. We have clear evidence that the cell, that is to say, the nucleus with the adjacent cell substance, exercises an important influence on the nutrition, and so on the functional activity of the nerve-fibre, it acts, as we have seen, as a "trophic centre." There are also reasons for thinking that the cell substance is more sensitive, more readily responsive to changes in its circumstances than is the axis-cylinder at some distance from the cell. But we have no satisfactory evidence that the cell can automatically originate nervous impulses in itself 
as the outeome of its own intrinsic changes. Nor have we any evidence that the cell can exert any marked transforming power over the impulses passing along the fibre; the impulses which travel away from the cell do not appear to differ markedly from those which travel toward it. The several instances in which there seemed to be evidence that splanchnic ganglia acted as centres either of reflex or of automatic action, have, as we have seen, broken down; and it is not even suggested that the ganglia of the posterior roots possess any such powers. The gray matter of the spinal cord, on the other hand, as we have already seen, and as we shall see more in detail, is especially characterized by the possession of reflex and automatic as well as of other powers.

In structure, moreover, such a spinal segment differs strikingly from a ganglion and exhibits features unknown in ganglia. In a ganglion the nerve-fibres may divide, and in a small peripheral ganglion the division may give rise to very delicate fibrils; but the fibres or fibrils resulting from the division leave the ganglion to follow their appropriate courses; the division serves for dispersion only. In the spinal cord, on the other hand, both efferent and afferent fibres divide in such a way that their divisions are lost to view in the gray matter; division here seems to serve the purpose of union. The efferent fibres of the anterior root may be traced back as a process of a cell in the anterior horn. That cell gives off other processes, but no one of these processes is continued as an axis-cylinder process stretching across the gray matter until it becomes a fibre of the posterior root, or as anything like such an axis-cylinder process. On the contrary, all the processes, except the axis-cylinder process, divide into branches, and appear to end in nervous fibrils lost to view in the gray matter. Conversely, though our knowledge of the junction of the posterior fibres with the gray matter is much more imperfect than that of the junction of the anterior fibres, what we do know leads us to believe that the fibres of the posterior root, either by the mediation of cells, or by direct division of the axis-cylinder without the mediation of cells, similarly break up into fibrils and are similarly lost in the gray matter. All the evidence goes to show that the anterior and posterior roots are functionally continuous; this functional continuity is, however, effected not by a gross continuity of axis-cylinders, but in a peculiar manner through the division of branches of nerve-cells or of axis-cylinders into the nervous tangle which forms such a special feature of the gray matter of the cord. We may, perhaps, venture to regard the gray matter of the segmental groundwork, of which we are now alone speaking, as constituting a nervous network or web, formed certainly in part by the rapidly dividing branches of nerve-cells, and probably in part by the divisions of directly dividing nerve-fibres.

In any ordinary section of the spinal cord the gray matter presents to view much more than this nervous groundwork. To say nothing of the indubitable neuroglia and the obscure structures, including small cells, which are claimed now to be neuroglia, now to be nervous in nature, the gray matter in every section shows numerous distinct nerve-fibres crossing it in various directions; of these fibres a few are ordinary medullated fibres, some are non-medullated fibres, that is to say, are naked axis-cylinders, while others, and these the more numerous, are the peculiar medullated fibres of small diameter spoken of in $\S 476$. A large number of these fibres, indeed all the larger ones, though they go to make up what we call gray matter, are not continuous with, and do not belong to, the groundwork or nervous web, at all events do not form part of the groundwork seen in the same section as themselves. They are simply fibres traversing the groundwork, in spaces of the neuroglia bed, on their way up or down the cord, or across the 
cord from one part to another. It may be that some of the finer medullated fibres do really enter into the groundwork, and so contribute to the nervous web; but our knowledge is too imperfect to afford a clear decision on this point. Our inability to define its exact limits need not, however, prevent our recognizing the existence of the groundwork.

The prominence in this groundwork of the larger nerve-cells has led to the conception that the powers of the spinal segment are exercised by these nerve-cells to the exclusion of the other elements of the nervous web. But such a view has not been adequately proved. What we do know is that the nuclei and cell-bodies of the cells of the anterior horn exercise an important influence on the nutrition of the fibres of the anterior root which proceed from them, and possibly also influence the nutrition of the other branches of the cells forming part of the groundwork; and these cells are probably so conspicuous a feature of every section of the spinal cord because of the important task intrusted to them of maintaining in due order the nutrition of the long stretch of motor fibres reaching from them to the muscular fibres or other peripheral organs. The fibres of the posterior root are not so obviously connected with the conspicuous cells of the gray matter; indeed, as we have said, it may be doubted, though the view is maintained by some, whether any cell intervenes to secure the continuity of a posterior fibre with the groundwork, a division of the axis-cylinder serving this purpose; and this becomes intelligible when we bear in mind that the posterior fibres are governed as far as their nutrition is concerned by the nerve-cells of the ganglion on the posterior root, which ought probably to be considered as much a part of the spinal cord as the cells of the anterior horn. The nerve-cell of the ganglion is adequate to secure the due nutrition of the nerve-fibre until it joins the groundwork, and probably helps to maintain the nutrition of the groundwork itself.

Hence we may perhaps, until fresh evidence to the contrary is brought forward, incline to the view that the powers of the gray matter do not depend on the conspicuous cells alone or even chiefly, but on the peculiar molecular constitution and nature of the whole groundwork. The nuclei of the cells of the anterior horn with the cell substance adjacent to each and the cells of the ganglia on the posterior root probably govern the nutrition, and so the functional activity of the groundwork as well as of the issuing and entering fibres; but there appears to be as yet no convincing evidence of any other peculiar powers confined to the cells and absent from other parts of the groundwork. We may add that, in accordance with this view, the other cells of the gray matter, such as those of the vesicular cylinder, are to be regarded as of importance for governing the nutrition of fibres, commissural and others, starting from the spinal segment, and of the part of the groundwork from which by their mediation the fibres start, rather than for determining the functions of the groundwork of the segment or of the fibres receiving impulses from it.

$\$ 493$. The segmental ground work of gray matter belonging to each pair of spinal nerves is so fused with that of all the other pairs as to form along the whole length of the cord a mass of gray matter which appears, under certain circumstances at all events, to be continuous in the sense that impulses may pass in all directions along it. But each spinal segment is in addition connected by means of tracts of white matter with parts more or less distant. The crossed pyramidal tract is such a longitudinal commissural tract, connecting apparently each spinal segment in succession with a certain part of the cortex of the cerebrun. We have reason to think, as we shall see later on, that impulses descending this or that fibre or group of fibres of this tract give rise to the issue of motor impulses along this or that fibre or group of 
fibres of an anterior root. We do not at present know what is the exact manner by which the fibre in the pyramidal tract is connected with the fibre of the anterior root. It seems certain, however, that the connection is not in the form of a fibre isolated from the rest of the gray matter, containing, so to speak, the pyramidal fibre into a cell of the anterior horn whence the fibre of the anterior root issues. Most probably the pyramidal fibre makes connections with the segmental groundwork spoken of above, whether with or without the intervention of a cell we cannot at present tell. The direct pyramidal tract is a like tract of less extent downward, and the less known antero-lateral descending tract is probably of a similar nature.

The cerebellar and antero-lateral ascending tracts are in like manner to be regarded as longitudinal commissures between the successive spinal segment below and some part of the brain above. We have reason to think that these tracts convey upward impulses of a nature which may be called afferent, and are, therefore, in some way probably connected with the posterior roots. We do not know as yet the exact nature of the connection; but probably in those cases also the commissural fibres are united not directly to the posterior fibres, but indirectly by means of the segmental groundwork. And since these tracts do not degenerate after section of the posterior roots, but only after section or other lesion of the cord itself, we may infer that their junction with the groundwork is effected by means of trophic cells, by means of some or other of the cells spoken of a little while before.

The median posterior tract seems to be a commissural tract of a nature different from any of the above. Through it a certain part of each posterior root is brought into connection, not with its own spinal segment, but with the bulb above, and so with the brain, which thus receives direct representatives of each afferent spinal nerve. If, however, as some maintain, the bundle in this tract starting from a spinal nerve below diminishes as it proceeds upward, throwing off fibres to pass elsewhere, though always carrying some fibres right up to the bulb, we must add to the above the further view that this tract connects also each posterior root, not with its own segment, but with other more or less distant segments.

$\S 494$. All the evidence which we possess goes to show that each strand of each of these tracts runs isolated, that is to say, makes no connections with adjoining structures at any part of its course, from its beginning or end in the brain and its end or beginning in its appropriate spinal segment, or in the case of the median posterior tract from its beginning in the ganglion of a posterior root and its end in the bulb or in some distant spinal segment. In the crossed pyramidal tract, for instance, we have reason to think that one or more fibres run a quite unbroken and isolated course from the cortex of the cerebrum through various parts of the brain, along the whole length of the cord until they reach the lowermost spinal segmental mechanism. These tracts serve in no way to connect one segmental mechanisn with another. The segmental mechanisms are, however, connected together; and the connections between them seem to be of two kinds. In the first place, as we have already suggested, the segmental pieces of gray matter are so fused together as to form what appears to be a continuity of gray matter from one end of the cord to the other. Though we cannot actually track our way histologically through, and are still less aware of the physiological nature of the labyrinth of nerve-cells, fibres, and fibrils which make up what we have called the groundwork, we may with considerable probability assume that the passage of nervous impulses along it is determined as much by the condition of the material as by its anatomical disposition; that, for instance, the restrictions to the flow of an impulse are brought about much more frequently by the refusal of the molecules of nervous matter to take up the 
molecular disturbance which is the essence of the impulse; that is to say, by molecular resistance than by actual breaks of continuity in the nervous matter. Indeed, we have some reasons for thinking that actual structural continuity of nervous material is not essential to functional continuity ; that a nerve-fibril, for instance, may produce its due effect on another nerve-fibril or on a nerve-cell, if sufficiently in contact with it, though the microscope fails to demonstrate actual continuity.

But besides the gray matter there are areas of white matter which do not belong either to the nerve-roots as these are making their way into the gray matter, or to any of the tracts which we have mentioned. These comprise the strands of fibres which do not undergo either ascending or descending degeneration when parts of the spinal cord are injured or diseased. The area of white matter left when all the various tracts of ascending and descending degeneration detailed above are taken out, seems at all events in the higher parts of the cord (Fig. 127), relatively small, and future observations may continue to still further reduce it; but it inust be remembered that none of the above mentioned tracts are "pure;" they are all more or less mixed up, and some largely mixed up, with fibres which do not degenerate. Our knowledge is at present too scanty to allow us to make any statement with confidence concerning the function either of the fibres forming the white matter not yet marked out into tracts, or of the fibres scattered among the acknowledged tracts. But we may, at all events provisionally, assume that these fibres serve in the main as commissures connecting the successive segmental mechanisms with each other; we may conclude that changes taking place in one segmental mechanism can by means of these fibres produce correlated changes in some other distant segmental mechanism, without calling into action any of the gray matter of the intervening segmental mechanisms.

The commissures which we may suppose to be thus furnished by white matter are longitudinal commissures connecting the segmental mechanisms of the same lateral half of the spinal cord with each other. A transverse connection between the two lateral halves is afforded in some measure by the anterior white commissure. We shall see, however, later on, reasons for thinking that many impulses besides those passing along the anterior commissure cross from one side of the cord to the other; and these, whether they pass along distinct fibres or along the general groundwork, must travel by the gray matter of the isthmus forming the anterior and posterior gray commissures.

Thus, as far as we can see at present, the spinal cord consists of a series of segmental mechanisms with their respective afferent and efferent roots (the gray matter of the several segments being continuous along the cord), of encephalic ties of white matter between the several segments and the brain, of longitudinal commissural tracts connecting together the several segmental mechanisms, and of transverse commissures running largely in the gray matter.

\section{The Reflex Actions of the Spinal Cord.}

$\S 495$. In the preceding portions of this work we have repeatedly seen that though we can learn much concerning the working of an organ or tissue or part of the body by studying its behavior when isolated from the rest of the body, all the conclusions thus gained have to be checked by a study of the behavior of the sanie organ or part while it is still an integral part of the intact body. All the several organs and tissues are so bound together by various ties that the actions of each depend on the actions of the rest; and to 
say that the life of each part is a function of the life of the whole, is no less true than to say that the life of the whole is a function of the life of each part. This is especially borne in upon us when we come to study the actions of the central nervous system. We may, on anatomical grounds, separate the spinal cord from the brain; but when we come to consider the respective functions of the two, we are brought face to face with the fact that in actual life a large part of the work of the brain is carried out by means of the spinal cord, and conversely the spinal cord does its work habitually under the influence of, if not at the direct bidding of, the brain. We may gain certain conclusions by studying the behavior of the spinal cord isolated from the brain, or of parts of the spinal cord isolated from each other; but we must be even more cautious than when we were dealing with other parts of the body, and must greatly hesitate to take it for granted that the work which we can make the spinal cord or a part of the spinal cord do, when isolated from the brain, is the work which is actually done in the intact body when the brain and spinal cord form an unbroken whole. Moreover, this caution becomes increasingly necessary when in our studies we pass from the simpler nervous system of one animal to the more complex nervous system of another; for it is by the complexity of their central nervous systems, more than by anything else, that the "highest" animals are differentiated from those "below" them. When we compare a rabbit, a dog, a monkey, and a man, the differences in the vascular, digestive, and respiratory systems of the four, striking as they may appear, sink into insignificance compared with the differences exhibited by their respective central nervous systems. We need caution when from the results of experiments on dogs or rabbits we draw conclusions as to the digestion or circulation of man, but we need far greater caution when from the behavior of the isolated spinal cord of one of these animals we infer the behavior of the intact spinal cord of man.

A further difficulty meets us when an experimental investigation entails operative interference with the central nervous system. Removal or section of, or other injury to parts of the brain or spinal cord is very apt to give rise in varying degree to what is known as "shock." The cutting or tearing or other lesion of any considerable mass of nervous substance affects the activity, not only of the structures immediately injured, but of other, it may be far distant structures. The nature of "shock" is not as yet thoroughly understood, but may perhaps, in part at all events, be explained by regarding the lesion as a very powerful stimulus, which, partly by way of inhibition but still more by way of exhaustion, depresses or suspends for a while normal functions, and thus gives rise to temporary diminution or loss of consciousness, of volition, of reflex movements, and other nervous actions. Thus a section through the spinal cord, even when made with the sharpest instrument and with the utmost skill, so as to avoid all bruising as much as possible, may for a while suspend all reflex activity of the cord, or indeed all the obvious activities of the whole central nervous system. We may add that such a "shock" of the central nervous system may also be produced by sudden lesions not bearing directly on the central nervous system, as, for instance, by extensive injury to a limb.

Moreover, in many cases in which the effects of experimental interference have been watched for some considerable time, days, months, or years after the operation, it has been observed, on the one hand, that phenomena which are conspicuous in the early period may eventually disappear, and, on the other hand, that activities which are at first absent may later on make their appearance; movements, for instance, which are at first frequent after a while die away, and conversely, movements which at first seemed impossible are later on easily achieved. We have to distinguish or to attempt to distin- 
guish between the temporary and the lasting effects of the operation, including among the former not only those of urdinary "shock," but others of slower development or longer duration. In many instances where a part of the central nervous system is by section or otherwise suddenly separated from the rest, the phenomena suggest that the separated part is at first profoundly influenced as to its activities by the withdrawal of various influences which previously were being exerted upon it by the rest of the system, but later on accommodates itself to the new conditions, and learns, so to speak, to act without the help of those influences. And indeed it is possible that some of the effects of even immediate "shock" may be due, not as suggested above, to the action of an inhibitory or exhausting stimulus, but to the sudden cessation of habitual influences.

Still, in spite of all these difficulties, it is possible not only to ascertain the working of an isolated portion of the central nervous system, but even to infer from the results some conclusions as to the share taken by that portion in the working of the entire and intact system. There can be no doubt, for instance, that the spinal cord can, quite apart from the brain, carry out various reflex actions, and, moreover, it does carry out actions of this kind when in the intact organism it is working in contact with the brain. Indeed, the carrying out of various reflex actions seems to be one of the most important functions of the spinal cord, so much so that though the brain or, at least, parts of the brain can also and do develop reflex actions, the spinal cord offers the best field for the study of these actions. We have already ( $\$ 97)$ touched on the general features of reflex actions, and elsewhere have incidentally dwelt on particular instances; we may therefore confine ourselves now to certain points of special interest.

$\$ 496$. Reflex movements are perhaps best studied in the frog and other cold-blooded animals, since in these the actions of the cord are less dependent on, and hence less obscured by the working of, the other parts of the central nervous system. They obtain, however, in the warm-blooded mammal also, but in these special preparations are necessary to secure their full development. In the frog the shock, which, as we have said, follows upon division of the spinal cord and for awhile suspends reflex activity, soon passes away; within a very short time after the bulb, for instance, has been divided the most complicated reflex movements can be carried on by the frog's spinal cord when the appropriate stimuli are applied. With the mammal the case is very different. For days even after division of the spinal cord the parts of the body supplied by nerves springing from the cord below the section may exhibit very feeble reactions only. In the dog, for instance, after division of the spinal cord in the lower dorsal region, the hind limbs hang flaccid and motionless, and pinching the hind foot evokes as a response either slight irregular movements or none at all. Indeed, were our observations limited to this period we might infer that the reflex actions of the spinal cord in the mammal were but feeble and insignificant. If, however, the animal be kept alive for a longer period, for weeks, or better still for months, though no union or regeneration of the spinal cord takes place, reflex movements of a powerful, varied, and complex character manifest themselves in the hind limbs and hinder parts of the body; a very feeble stimulus applied to the skin of these regions promptly gives rise to extensive and yet coördinate movements. Indeed, the more the matter is studied, the stronger is the evidence that the reflex movements carried out by isolated portions of the spinal cord of the mammal are hardly less definite, complete, and purposeful than those witnessed in the frog. It is worthy of attention, as bearing out the remarks made above on the great differentiation of the central nervous system in the higher animals, that the reflex phenomena in mammals 
vary very much not only in different species but also in different individuals and in the same individual under different circumstances. Race, age, and previous training seem to have a marked effect in determining the extent and character of the reflex actions which the spinal cord is capable of carrying out; and these seem also to be largely influenced by passing circumstances, such as whether food has been recently taken or not. It has been asserted that the isolated spinal cord of the rabbit, which has been the subject of so many experiments, is, as compared with that of the dog and many other mammals, singularly deficient in the power of carrying out complex reflex movements.

In studying reflex actions in man we are met with the difficulty that we never have to deal with a portion of the spinal cord separated from the rest of the central nervous system under the favorable circumstances of experimental investigation. In man, we must be content to examine reflex actions either while the whole nervous system is intact, or when a portion of the cord has been wholly or partially separated by some more or less diffuse disease or by some accident involving more or less crushing of the nervous structures. Hence, the caution already given, as to drawing inferences concerning man from the results of experiments on animals, acquires still greater force.

$\$ 497$. Confining ourselves at first to the results of experiments on animals we may say that in both cold-blooded and warm-blooded animals the salient feature of ordinary reflex actions is their purposeful character, though every variety of movement may be witnessed, from a simple spasm to a most complex manœuvre. And in all reflex movements, both simple and complex, we can recognize certain determining influences which more or less directly contribute to the shaping of this purposeful character.

Thus the features of any movement taking place as part of a reflex action are in part determined by the characters of the afferent impulses. Simple nervous impulses generated by the direct stimulation of afferent nerve-fibres generally evoke as reflex movements merely irregular spasms in a few muscles; whereas the more complicated differentiated sensory impulses generated by the application of the stimulus to the skin, readily give rise to large and purposeful movements. It is easier to produce a complex reflex action by a slight pressure on or other stimulation of the skin than by even strong induction-shocks applied directly to a nerve-trunk. If in a brainless frog, the area of skin supplied by one of the dorsal cutaneous nerves be separated by section from the rest of the skin of the back, the nerve being left attached to the piece of skin and carefully protected from injury, it will be found that slight stimuli applied to the surface of the piece of skin easily evoke reflex actions, whereas the trunk of the nerve may be stimulated with even strong currents without producing anything more than irregular movements. In ordinary mechanical and chemical stimulation of the skin it is not a single impulse but a series of impulses which passes upward along the sensory nerve, the changes in which may be compared to the changes in a motor nerve during tetanus. In every reflex action, in fact, the central mechanism may be looked upon as being thrown into activity through a summation of the afferent impulses reaching it. Hence while a reflex action is readily called forth by even feeble induction-shocks applied to the skin if they be repeated sufficiently rapidly, a solitary induction-shock is ineffectual unless it be strong enough to cause in the skin or nerves changes of an electrolytic nature sufficient to give rise of themselves to a series of impulses.

$\$ 498$. When a muscle is thrown into contraction in a reflex action, the pitch of the sound which it gives forth does not vary with the stimulus, but 
is constant, being the same as that given forth by a muscle thrown into contraction by the will. From which we infer, even bearing in mind the discussion in $\$ 78$ concerning the nature of the muscular sound, that in a reflex action the afferent impulses do not simply pass through the centre in the same way that they pass along afferent nerves, but are profoundly modified. And in accordance with this we find, as we shall see, that a reflex action takes up an amount of time, the greater part of which is spent in the carrying out of the central changes, and which though variable is always much longer, and may be very much longer, than that taken up by the mere passage of a nervous impulse along a corresponding length of nerve-fibre. The term reflex action is therefore an unsuitable one. The afferent impulse is not simply reflected or turned aside into an efferent channel; on its arrival at the centre, it starts changes of a different nature from and more complex than its own; and the issue of efferent impulse is the result of those more complex changes, not the mere continuation of the simpler afferent impulse. In other words, the interval between the advent at the central organ of afferent, and the exit from it of efferent impulses, is a busy time for the nervous substance of that organ; during it many processes, of which we have at present very little exact knowledge, are being carried on.

$\S 499$. The character of the movement forming part of a reflex action is also influenced by the intensity of the stimulus. A slight stimulus, such as gentle contact of the skin with some body, will produce one kind of movement; and a strong stimulus, such as a sharp prick applied to the same spot of skin, will call forth quite a different movement. When a decapitated snake or newt is suspended and the skin of the tail slightly touched with the finger, the tail bends toward the finger; when the skin is pricked or burnt, the tail is turned away from the offending object. And so in many other instances. It must be remembered, of course, that a difference in the intensity of the stimulus entails a difference in the characters of the efferent impulses; gentle contact gives rise to what we call a sensation of touch, while a sharp prick gives rise to pain, consciousness being differently affected in the two cases because the afferent impulses are different. Hence the instances in question are in reality fuller illustrations of the dependence, to which we called attention above, of the characters of a reflex movement on the characters of the afferent impulses.

Further, as we have already pointed out ( $\$ 97)$, while the motor impulses started by a weak stimulus applied to an afferent nerve are transmitted along a few, those started by a strong stimulus may spread to many efferent nerves. Granting that any particular afferent nerve is more especially associated with certain efferent nerves than with any others, so that the reflex impulses generated by afferent impulses entering the cord by the former pass with the least resistance down the latter, we must evidently admit further that other efferent nerves are also, though less directly, connected with the same afferent nerve, the passage into the second efferent nerve meeting with a greater but not an insuperable resistance. When a frog is poisoned with strychnine, a slight touch on any part of the skin may cause convulsions of the whole body ; that is to say, the afferent impulses passing along any single afferent nerve may give rise to the discharge of efferent impulses along any or all of the efferent nerves. This proves that a physiological, if not an anatomical, continuity obtains between all parts of the spinal cord which are concerned in reflex action, that the nervous network intervening between the afferent and efferent fibres forms along the whole length of the cord a functionally continuous field. This continuous network, however, we must suppose to be marked out into tracts presenting greater or less resistance to the progress of the impulses into which afferent impulses, coming along this 
or that afferent nerve, are transformed on their advent at the network; and accordingly the path of any series of impulses in the network will be determined largely by the energy of the afferent impulses. And the action of strychnine may be, in part, explained by supposing that it reduces and equalizes the normal resistance of this network, so that even weak impulses travel over all its tracts with great ease.

$\S 500$. Further, the movement, forming part of a reflex action, varies in character according to the particular part of the body to which the stimulus is applied. The reflex actions developed by stimulation of the internal viscera are different from those excited by stimulation of the skin. We have reason to think that the contraction of, or other changes in a skeletal muscle may produce, by reflex action, contractions of other muscles; and such reflex actions also differ from those started by stimulation of the skin. In reflex actions started by applying a stimulus to the skin the movements vary largely, according to the particular area of the skin which is affected. Thus, pinching the folds of skin surrounding the anus of the frog produces different effects from those witnessed when the flank or toe is pinched; and, speaking generally, the stimulation of a particular spot calls forth particular movements. In the case of the simple reflex movements, it appears to be a general rule that a movement started by the stimulation of a sensory surface or region on one side of the body is developed on the same side of the body, and if it spreads to the other side, still remains most intense on the same side; the movement on the other side, moreover, is symmetrical with that on the same side. It has been maintained that "crossed" or diagonal reflex movements, as where stimulation of one fore-foot leads to movements of the opposite hind limb, do not occur unless some portion of the bulb be left attached to the spinal cord. Seeing that locomotion of four-footed animals is largely effected by diagonal movements of the limbs, one would rather have expected to find the spinal cord itself provided with mechanisms to assist in carrying them out; and, indeed, it is affirmed that in the case of cold-blooded animals and of many young mammals, after a division of the spinal cord below the bulb, a gentle stimulation will provoke a diagonal movement, slight pressure on one fore foot, for example, giving rise to movements in the opposite hind leg; a strong stimulus, however, will produce an ordinary one-sided movement. Again, when in a dog the cord has been divided in the lower thoracic region so that the hind limbs depend on the lumbar cord alone, a rhythmically repeated drawing up and letting down of the hind limbs is witnessed when these are allowed to hang down; and these movements, which appear to be of a reflex nature excited by the pendent position of the limbs, are often seen to alternate regularly in the two limbs, the right leg being extended while the left leg is being drawn up and vice versa. It may further be observed that if the foot of one pendent limb be pinched while the other limb is passively flexed the flexion of the limb which is pinched is accompanied by an extension of the other limb. In these respects, however, different animals, as already urged, differ from ('ach other.

$\S 501$. From these and similar phenomena we may infer that the nervous network spoken of above is, so to speak, mapped out into nervous mechanisms by the establishment of lines of greater or less resistance, so that the disturbances in it generated by certain afferent impulses are directed into certain efferent channels. It may be added that though conspicuously purposeful movements seem to need the concurrent action of several segments of the cord, and as a rule, the greater the length of the cord involved the more complex and the more distinctly purposeful the movement, still the movements evoked by even a segment of the cord may be purposeful in character; 
hence we must conclude that every segment of the nervous network is mapped out into mechanisms. But the arrangement of these mechanisnis, especially of the more complex ones, is not a fixed and rigid one. We cannot always predict exactly the nature of the movement which will result from the stimulation of any particular spot, because the result will vary according to the condition of the spinal cord, especially in relation to the strength and character of the stimulus. Moreover, under a change of circumstances a movement quite different from the normal one may make its appearance. Thus when a drop of acid is placed on the right flank of a brainless frog, the right foot is almost invariably used to rub off the acid; in this there appears nothing more than a mere "mechanical" reflex action. If, however, the right leg be cut off, or the right foot be otherwise hindered from rubbing off the acid, the left foot is, under the exceptional circumstances, used for the purpose. This at first sight looks like an intelligent choice. A choice it evidently is ; and were there many instances of choice, and were there any evidence of a variable automatism, like that which we call "volition," being manifested by the spinal cord of the frog, we should be justified in supposing that the choice was determined by an intelligence. But, as we shall have occasion later on to point out, a frog, deprived of its brain so that the spinal cord only is left, makes no spontaneous movements at all. Such an entire absence of spontaneity is wholly inconsistent with the possession of intelligence. Then again the above experiment, if not the only instance, is, at all events, by far the most striking instance of choice on the part of a brainless frog. We are, therefore, led to conclude that the phenomena must be explained in some other way than by being referred to the working of an intelligence. Moreover, this conclusion is supported by the behavior of other animals. Thus similar vicarious reflex movements may be witnessed in mammals, though not perhaps to such a striking extent as in frogs. In dogs, in which partial removal of the cerebral hemispheres has apparently heightened the reflex excitability of the spinal cord, the remarkable scratching movements of the hind leg which are called forth by stimulating a particular spot on the loins or side of the body, are executed by the leg of the opposite side, if the leg of the same side be gently held. In this case the vicarious movements are effectual, the leg not being, as in the case of the frog, crossed over so as to bear on the spot stimulated, and cannot be considered as betokening intelligence. Again, the "mechanical" nature of reflex actions is well illustrated by the behavior of a decapitated snake. When the body of the animal in this condition is brought into contact at several places at once with an arm or a stick, complex reflex movements are excited, the obvious purpose as well as effect of which is to twine the body round the object. A decapitated snake will, however, with equal and fatal readiness twine itself round a red-hot bar of iron, which is made to touch its skin in several places at the same time.

$\S 502$. In considering the nature of the events in the spinal cord which determine the behavior of the frog in the instance just mentioned we must bear in mind that the movements in question are "coördinated;" that is to say, not only are many distinct muscles brought into play, but certain relations are maintained between the amount, duration, and exact time of occurrence of the contraction of each muscle and those of the contractions of its fellow muscles sharing in the movement. In the absence of such coördination the movement would become irregular and ineffectual. We shall have occasion later on in dealing with voluntary movements to point out that the coördination and hence the due accomplishment of a voluntary movement is dependent on certain afferent impulses passing up from the con- 
tracting muscles to the central nervous system, and guiding the discharge of the efferent impulses which call forth the contractions. When these afferent impulses affect consciousness we speak of them as constituting a "muscular sense;" it is, as we shall see, by the "muscular sense" that we become aware of and can appreciate the condition of our muscles. But we have reason to think that the afferent impulses which constitute the basis of the muscular sense, whatever be their exact nature, in order to play their part in bringing about the coördination of a voluntary movement need not pass right up to the brain and develop a distinct muscular "sense," but may produce their effect by working on the nervous mechanisms of the spinal cord with which the motor fibres carrying out the movement are connected. In other words, the coördination of a voluntary movement takes place in the part of the spinal cord which carries out the movement, and not in the brain, though the latter may be conscious of the whole movement including its coördination.

But if the spinal cord possesses mechanisms for carrying out coördinated movements, which in the case of voluntary movements are discharged by nervous impulses descending from the brain, we may infer that in reflex actions the same mechanisms are brought into action though they are discharged by afferent impulses coming along afferent nerves instead of by impulses descending from the brain. The movements of reflex origin, in all their features except their exciting cause, appear identical with voluntary movements; the two can only be distinguished from each other by a knowledge of the exciting cause. And it seems unreasonable to suppose that the spinal cord should possess two sets of mechanisms in all respects identical, save that the one is discharged by volitional impulses from the brain and the other by afferent impulses from afferent nerves.

We are led therefore to the conclusion that in a reflex action two kinds of afferent impulses are concerned: the ordinary afferent impulses which discharge the nervous mechanism within the cord and so provoke the movement, and the afferent impulses which connect that nervous mechanism with the muscles about to be called into play, and which take part in the coördination of the movement provoked. The nature of these latter afferent impulses is at present obscure; but if we admit, as we seem compelled to do, that the character of a reflex action is determined by them as well as by the afferent impulses which actually discharge the mechanism, it seems possible that a fuller knowledge of these coördinating afferent impulses may afford an adequate explanation of the fact that when, as in the case of the frog in question, the usual set of muscles cannot be employed by the nervous mechanism, recourse is had to another.

We have avoided the introduction of the word "consciousness" as unnecessarily complicating the question; and it would be out of place to discuss psychological problems here. We may remark, however, that since we have no objective proofs of consciousness outside ourselves, and only infer by analogy that such and such an act is an outcome of consciousness on account of its likeness to acts which are the outcome of our own consciousness, we conclude that the brainless frog possesses no active consciousness like our own, because absence of spontaneous movements seems to be irreconcilable with the existence of an active consciousness whose very essence is a series of changes. Consciousness, as we recognize it, seems to be necessarily operating as, or to be indissolubly associated with the presence of, an incessantly repeated internal stimulus; and we cannot conceive of that stimulus failing to excite mechanisms of movement which, as in the case of the brainless frog, are confessedly present. We may, however, distingnish between an active continuous consciousness, such as we usually understand by the term, 
and a passing and momentary condition, which we may speak of as consciousness, but which is wholly discontinuous from an antecedent or from a subsequent similar momentary condition; and indeed we may suppose that the complete consciousness of ourselves, and the similarly coniplete consciousness which we infer to exist in many animals, has been gradually evolved out of such a rudimentary consciousness. We may, on this view, suppose that every nervous action of a certain intensity or character is accompanied by some amount of consciousness, which we may, in a way, compare to the light emitted when a combustion, previously giving rise to invisible heat, waxes fiercer. We may thus infer that when the brainless frog is stirred by some stimulus to a reflex act, the spinal cord is lit up by a momentary flash of consciousness coming out of darkness and dying away into darkness again; and we may perhaps further infer that such a passing consciousness is the better developed the larger the portion of the cord involved in the reflex act and the more complex the movement. But such a momentary flash, even if we admit its existence, is something very different from consciousness as ordinarily understood, is far removed from intelligence, and cannot be appealed to as explaining the "choice" spoken of above.

$\$ 503$. Lastly, the characters of a reflex movement are, as we need hardly say, dependent on the intrinsic condition of the cord. The action of strychnine just alluded to is an instance of an apparent augmentation of reflex action best explained by supposing that the resistances in the cord are lessened. There are probably, however, cases in which the explosive energy of the nervous substance is positively increased above the normal. Conversely, by various influences of a depressing character, as by various anæsthetics or other poisons, reflex action may be lessened or prevented; and this again may arise either from an increase of resistance or from a diminution in the actual discharge of energy. So also various diseases may so affect the spinal cord as to produce, on the one hand, increased reflex excitability, so that a mere touch may produce a violent movement, and, on the other hand, diminished reflex excitability, so that it becomes difficult or impossible to call forth reflex action.

$\$ 504$. When we come to study the reflex actions of man we should at first perhaps be inclined to infer that, since in him the spinal cord is so largely used as the instrument of the brain, the independent reflex actions of the cord, at least such as affect skeletal muscles, are in him of much less importance than they appear to be in animals, and experience seems to support this view. But it must be remembered that in his case, as we have already stated ( $\$ 496)$, we lack the guidance of experimental results; we are obliged to trust to the entangled phenomena of disease or to a study of the behavior of the cord while it is still a part of an intact nervous system; and each of these methods presents difficulties of its own. The movements, which in the intact human body we can recognize as indubitable reflex actions, are, as a rule, simple and unimportant. They are, in by far the greater number of instances, occasioned by stimulation of the skin or of the mucous membrane, for the most part involve a few muscles only, and rarely indicate any very complex coördination. The flexion, followed by extension, of the leg, which is called forth by tickling the sole of the foot, or the winking of the eye when the cornea or conjunctiva is touched, may perhaps be regarded as the type of these movements. A very common form of reflex action is that in which a muscle or group of muscles is thrown into contraction by stimulation of the overlying or neighboring skin, as when the abdominal muscles contract upon stroking the skin of the abdomen or the testicle is retracted upon stroking the inside of the 
thigh. A reflex movement may occur as the result of stimulation of an organ of special sense, parts of the central nervous system other than the spinal cord serving as the centre. A sound or a flash of light readily produces a start, a bright light makes the eye wink and may cause the person to sneeze (the greater coördination manifest in this act being due to the fact that the complex respiratory mechanism is brought into play ( $\$ 334$ ), and reflex movement may result from a taste or smell. A special form of reflex action, or at least an action resembling a reflex action, is called forth by sharply striking certain tendons; for instance, striking the tendon below the patella gives rise to a sudden extension of the leg, known as the "kneejerk"; but it will be best to discuss these "tendon reflexes," or "muscle reflexes," as they are called, later on in another connection.

On the whole the reflex movements carried out by the intact nervous system of man are, we repeat, scanty and comparatively simple; but we are not justified in inferring from this that the human spinal cord, left to itself, is incapable of doing more; that owing to the predominant activity of the brain it has lost the powers possessed by the spinal cord in the lower animals. For it may be that the cord, when joined to the brain, is through various influences proceeding from the latter in a different condition from that in which it is when separated from the brain; indeed, we have reason to think that this is so; and we may here remark that in the lower animals, as in man, the development of reflex movements is difficult and uncertain in the presence of the brain.

When we turn to the teaching of disease, however, we again find that reflex movements carried out by the cord or by parts of the cord are, on the whole, scanty and simple.

In some stages of certain diseases of the spinal cord extensive reflex movements are witnessed; but these are not purposeful, coördinated movements, such as have been described above as occurring in frogs and mammals after experimental interference, but rather mere exaggerations of the simpler reflex movements witnessed when the nervous system is intact. In cases of paraplegia (such being the term generally used when disease or injury has cut off the cord, generally the lower part of the cord, from the brain, so that the will cannot bring about movements in, and the mind derives no sensation from, the parts below the lesion, the legs for instance), it sometimes happens that contact with the bedclothes or other external objects sets up from time to time rhythmically repeated movements, the legs being alternately drawn up and thrust out again. And an exaggeration of the "knee-jerk" or other " tendon reflexes" is a very common symptom in certain spinal diseases. It is rarely, if ever, that reflex movements of a really complicated character are observed. Moreover, clinical experience shows that in man, when a portion of the cord is isolated, reflex actions carried out by means of that portion, so far from being exaggerated, are much more commonly exceedingly feeble or absent altogether. In the cases in which the physiological continuity of the lower with the upper part of the cord has been broken by disease, by some growth invading the nervous structures, or by some changes of the nervous structures themselves, we may attempt to explain the absence from the lower part or coördinate reflex activity, such as is seen in the lower animal, as due to the disease not only affecting the powers of the actually diseased part, but influencing the whole cord below, and either by inhibition, of which we shall speak presently, or in some other way depressing its functions. But the same absence of complex reflex movements is also often observed in cases in which the cord has been severed by accident, and, indeed, though accidental injuries to the human cord generally produce more profound and extensive mischief 
than that which results in animals from experimental interference, clinical experience tends, on the whole, to support the view that in man the more complete subordination of the spinal cord to the brain has led to the dying out of the complex reflex actions which are so conspicuous in the lower animals. This, however, cannot be regarded as distinctly proved.

When we come to study voluntary movements, we shall see reason to think that in man, as in the lower animals, the will in carrying out these movements makes use of complex nervous mechanisms situated in the spinal cord-nervous mechanisms into the working of which, as urged above, afferent impulses enter largely; and it seems improbable that these spinal mechanisms should be capable of being thrown into action by the will only. In the act of walking, for instance, it is highly probable that the movements of the legs are the direct results of the action of nervous mechanisms in the lumbar cord brought into play by the will, being thus, in an indirect manner only, the products of volitional impulses; and even in man, though clinical experience only affords us instances of this machinery working apart from the brain in a damaged condition and under unfavorable circumstances, so that the resemblance of the movements observed to the complete act of walking is but feeble, still it seems similarly probable that under more favorable circumstances the lumbar cord separated from the brain might as part of a reflex act carry out the movements in a more complete and coördinate manner.

$\$ 505$. We have dwelt above chiefly on reflex actions, in which the efferent impulses cause contractions of skeletal muscles, since these are undoubtedly the most common and the most prominent forms of reflex action; but it must not be forgotten that the efferent impulses of reflex origin may produce contractions of other muscles, as well as other effects, such as secretion, for instance. On several of these we have dwelt, from time to time in previous parts of this work, and it will be unnecessary to repeat them here. But it may be worth while to point out that the spinal cord, by serving as a reflex centre for innumerable ties which correlate the nutritive or metabolic activities of the several tissues to events taking place in other parts of the body, plays a conspicuous part in securing the welfare of the whole body. In dealing ( $\$ 462)$ with the general problems of nutrition, we stated that an orderly nutrition appears to be in some way dependent on nervous influences. Many of these nervous influences appear to issue from the spinal cord, either as parts of a reflex act or as the outcome of some automatic processes. When in a dog the lumbar cord is wholly separated from the rest of the cord by section, the nutrition of the hind limbs and the general health of the animal may, with care, be maintained in a very satisfactory condition; but if that small separated piece of the cord be destroyed, death inevitably ensues before long, in spite of every care and precaution, being brought about apparently by the disordered nutrition of the hind limbs and other parts supplied by nerves coming from the lumbar cord. In man extensive injuries to the spinal cord are followed by bedsores and other results of impaired nutrition; and, indeed, death is generally brought about in this way in cases of paraplegia caused by accidental crushing or severance of the cord.

$\$ 506$. Inhibition of reflex action. The reflex actions of the spinal cord, like other nervous actions, may be totally or partially inhibited, that is to say, may be arrested or hindered in their development by impulses reaching the centre while it is already in action. Thus, if the body of a decapitated snake be allowed to hang down, slow rhythmic pendulous movements, which appear to be reflex in nature, soon make their appearance; all these may be for a while arrested by slight stimulation, as by gently stroking the tail. 
We have already seen that the action of such nervous centres as the respiratory and vasomotor centres, which frequently, at all events, is of a reflex nature, may be either inhibited or augmented by afferent impulses. The micturition centre in the nuammal, which is also largely a reflex centre, may be easily inhibited by impulses passing downward to the lumbar cord from the brain, or upward along the sciatic nerves. In the case of dogs, whose spinal cord has been divided in the thoracic region, micturition set up as a reflex act by simple pressure on the abdomen or by sponging the anus is at once stopped by sharply pinching the skin of the leg. And it is a matter of common experience that in man micturition may be suddenly checked by an emotion or other cerebral event. The erection centre in the lumbar cord, also in large measure a reflex centre, is similarly susceptible of being inhibited by impulses reaching it from various sources. And, indeed, many similar instances of the inhibition of reflex movements might readily be quoted.

Several apparent instances of the inhibition of reflex acts are not really such; in these cases all the nervous processes of the act may take place in their entirety and yet fail to produce their effect on account of a failure in the muscular part of the act. Thus, when we ourselves by an effort of the will stop the reflex movements which otherwise would be produced by tickling the soles of the feet, we achieve this to a large extent by throwing voluntarily into action certain muscles, the contractions of which antagonize the action of the muscles engaged in carrying out the reflex movements. But it may be doubted, even in these cases, whether inhibition is always or wholly to be explained in this way; and certainly in very niany instances of reflex inhibition no such muscular antagonism is present, and the reflex act is checked at its nervous centre.

When the brain of a frog is removed, and the effects of shock have passed away, reflex actions are developed much more readily and to a much greater degree than in the entire animal, and in mammals also reflex excitability has been observed to be increased by removal of the cerebral hemispheres. This suggests the idea that in the intact nervous system the brain is habitually exerting some influence on the spinal cord, tending to prevent the normal development of the spinal reflex actions. And we learn by experiment that stimulation of certain parts of the brain has a remarkable effect on reflex action. If a frog, from which the cerebral hemispheres have been removed (the optic lubes, bulb, and spinal cord being left intact), be suspended by the jaw, and the toes of the pendent leg be from time to time dipped into very dilute sulphuric acid, a certain average time will be found to elapse between the dipping of the toe and the resulting withdrawal of the foot. If, however, the optic lobes or optic thalami be stimulated, as by putting a crystal of sodium chloride on them, it will be found on repeating the experiment, while these structures are still under the influence of the stimulation, that the time intervening between the action of the acid on the toe and the withdrawal of the foot is very much prolonged. That is to say, the stimulation of the optic lobes has caused impulses to descend to the cord, which have there so interfered with the nervous processes engaged in carrying.out reflex actions as greatly to retard the generation of efferent impulses, or, in other words, has inhibited the reflex action of the cord. And similar results may be obtained in mammals by stimulating certain parts of the corpora quadrigemina, which bodies are homologous to the optic lobes of frogs. From this it has been inferred that there is present in this part of the brain a special mechanism for inhibiting the reflex actions of the spinal cord, the impulses descending from this mechanism to the various centres of reflex action being of a specific inhibitory nature. But, as we have already 
seen, impulses of an ordinary kind, passing along ordinary sensory nerves, may inhibit reflex action. We have quoted instances where a slight stimulus, as in the pendulous movements of the snake, and where a stronger stimulus, as in the case of the micturition of the dog, may produce an inhibitory result; we may add that in the frog adequately strong stimuli applied to an afferent nerve will inhibit, $i$. e., will retard or even wholly prevent, reflex action. If the toes of one foot are dipped into dilute sulphuric acid at a time when the sciatic of the other leg is being powerfully stimulated with an interrupted current, the period of incubation of the reflex act will be found to be much prolonged, and in some cases the reflex withdrawal of the foot will not take place at all. And this holds good, not only in the complete absence of the optic lobes and bulb, but also when only a portion of the spinal cord, sufficient to carry out the reflex action in the usual way, is left. There can be no question here of any specific inhibitory centres, such as have been supposed to exist in the optic lobes. But if it is clear that inhibition of reflex action may be brought about by impulses which are not in themselves of a specific inhibitory nature, we may hesitate to accept the view that a special inhibitory mechanism in the sense of one giving rise to nothing but inhibitory inıpulses is present in the optic lobes of frogs, and after removal of the brain that the exaltation of reflex actions which is manifest is due to the withdrawal of such a specific inhibitory mechanisn.

The presence of the brain does obviously produce an effect which may be broadly spoken of as inhibitory, and a specific action of the brain, in an effort of the will, may stop or inhibit a specific reflex action; but we must not in these matters be led too much away by the analogy of the special and limited cardiac inhibitory mechanism. There we have apparently to deal with fibres, whose exclusive duty it is to convey inhibitory impulses from the bulb to the cardiac muscle, and inhibition of the heart, at least through nervous influences, is exclusively carried out by them. But already, in studying the nervous mechanism of respiration, we have seen reason to think that afferent impulses passing along the same nerves and probably along the same fibres may, according to circumstances, now inhibit, now augment the respiratory centre, and have thus been led to speak of inhibitory impulses, that is, impulses producing an inhibitory effect, apart from specific inhibitory fibres. In the complex working of the central nervous system we may still more expect to come across similar instances of the same channels serving as the path either of inhibition or of augmentation. In all probability actions or processes which we may speak of as inhibitory do play, as indeed we shall see, an important part in the whole work of the central nervous system; in all probability many of the phenomena of nervous life are the outcome of a contest between what we may call inhibitory and exciting or augmenting forces; but in all probability, also, we ought rather to seek for the explanation of how vagus impulses inhibit the beat of the heart by reference to the inhibitory phenomena of the central nervous system, than to attempt to explain the latter by the little we know of the former. At present, however, we must be content with the fact that experiments on animals show that the brain not only by some action or other may inhibit particular spinal reflex movements, but also habitually exercises a restraining influence on the reflex activity of the whole cord, though we are unable to state clearly how this inhibition is carried out.

We say "experiments on animals," because though we know, as stated above, by an appeal to our own consciousness, that an action of the brain, an effort of the will, may stop a particular reflex act, we have no evidence 
that in man separation of the cord from the brain leads, as in animals, to heightened reflex activity. Is diseases, or injuries to the cord, reflex actions are, as we have said, sometimes exaggerated, but it is possible, and indeed probable, that the increase is due to the morbid processes producing a greater irritability of the cord itself, and not to the withdrawal of any inhibitory influences. In many cases, in perhaps the greater number, no exaggeration but a diminution or even absence of reflex activity is observed ; so much so that could we trust explicitly to clinical experience, we should be inclined to conclude that the scantiness of spinal reflex action in man was due not to any preoccupation of the cord by influences proceeding from a dominant brain, but to an inherent paucity of spinal reflex mechanisms. But we have already said all we have at present to say on this point.

$\$ 507$. The time required for reflex actions. When one eyelid is stimulated with a sharp electrical shock, both eyelids blink. Hence, if the length of time intervening between the stimulation of the right eyelid and the movement of the left eyelid be measured, this will give the total time required for the various processes which make up a reflex action. It has been found to be from 0.0662 to 0.0578 second. Deducting from these figures the time required for the passage of afferent and efferent impulses along the fifth and facial nerves to and from the bulb, and for the latent period of the contraction of the orbicularis muscle, there would remain 0.0555 to 0.0471 second for the time consumed in the central operations of the reflex act. The calculations, however, necessary for this reduction, it need not be said, are open to sources of error; moreover, the reflex act in question is carried out by the bulb and not by the spinal cord proper. Blinking thus produced is a reflex act of the very simplest kind; but, as we have seen in the preceding pages, reflex acts differ very widely in nature and character; and we accordingly find, as indeed we have incidentally mentioned, that the time taken up by a reflex movement varies very largely. This, indeed, is seen in blinking itself. When the blinking is caused not by an electric shock applied to the eyelid, but by a flash of light falling on the retina, in which case complex visual processes are involved, the time is distinctly prolonged; moreover, the results in different experiments in which light serves as the stimulus are not nearly so uniform as when the blinking is caused by stimulation of the eyelid.

In general it may be said that the time required for any reflex act varies very considerably with the strength of the stimulus employed, being less for the stronger stimuli; this we should expect, seeing that the efferent impulses of the reflex act are not simply afferent impulses transmitted through the central organ, but result from internal changes in the central organ started by the afferent impulse or impulses; and these internal changes will naturally be more intense and more rapidly effected when the afferent impulses are strong. It is stated that when the movement induced is on the same side of the body as the surface stimulation of which starts the act, the time taken up is less than when the movement is on the other side of the body, allowance being made for the length of central nervous matter involved in the two cases; that is to say, the central operations of a reflex act are propagated more rapidly along the cord than across the cord. The rapidity of the act varies, of course, with the condition of the spinal cord, the act being greatly prolonged when the cord becomes exhausted; and a similar delay has been observed in cases of disease. The time thus occupier by purely reflex actions must not be confounded with the interval required when the changes taking place in the central nervous system are of a more complicated nature, and more or less distinctly involve mental operations; of the latter we shall speak later on. 


\section{The Automatic Actions of the Spinal Cord.}

$\S 508$. We speak of an action of an organ or of a living body as being spontaneous or automatic when it appears to be not immediately due to any changes in the circumstances in which the organ or body is placed, but tu be the result of changes arising in the organ or body itself and determined by causes other than the influences of the circumstances of the moment. Some automatic actions are of a continued character; others, like the beat of the heart, are repeated in regular rhythm; but the most striking automatic actions of the living body, those which we attribute to the working of the will and which we call voluntary or volitional, are characterized by their apparent irregularity and variableness. Such variable automatic actions form the most striking features of an intact nervous system, but are conspicuously absent from a spinal cord when the brain has been removed.

A brainless frog placed in a condition of complete equilibrium in which no stimulus is brought to bear on it, protected, for instance, from sudden passing changes in temperature, from a too rapid evaporation by the skin and the like, remains perfectly motionless until it dies. Such apparently spontaneous movenents as are occasionally witnessed are so few and seldom, that we can hardly do otherwise than attribute them to some stimulus, internal or external, which has escaped observation. In the mammal (dog) after division of the spinal cord in the dorsal region regular and apparently spontaneous movements may be observed in the parts governed by the lumbar cord. When the animal has thoroughly recovered from the operation the hind limbs rarely remain quiet for any long period; they move restlessly in various ways; and when the animal is suspended by the upper part of the body, the pendent hind limbs are continually being drawn up and let down again with a monotonous rhythmic regularity, suggestive of automatic rhythmic discharges from the central mechanisms of the cord. In the newly born mammal too, after removal of the brain, movements apparently spontaneous in nature are frequently observed. But all these movements, even when most highly developed, are very different from the movements, irregular and variable in their occurrence though orderly and purposeful in their character, which we recognize as distinctly voluntary. Even admitting that some of the movements of the brainless mammal may resemble voluntary movements in so far as they are due to changes taking place in the spinal cord itself independent of the immediate influence of any stimulus, we are not thereby justified in speaking of the spinal cord as developing a will in the sense that we attribute a will to the brain.

$\$ 509$. In the case of the beat of the heart, the automatic rhythmic discharge of energy appears to be exclusively the outcome of the nolecular nutritive changes taking place in the cardiac substance. The beat may be modified, as we have seen, by nervous impulses reaching the cardiac substance along certain nerves; but the actual existence of the beat is wholly independent of these extraneous influences; the rhythmic discharge continues when they are entirely absent. The automatic rhythmic discharge of respiratory impulses from the respiratory centre is alsu dependent on the intrinsic molecular changes of the centre, these being, as we have seen, largely determined by the character of the blood streaming through it ; but in this case extrinsic nervous impulses, reaching the centre along the vagus and other nerves, play a much more important part than do similar impulses in the case of the heart. They act so continually on the centre and enter so largely into its working, that we are compelled to regard the activity of the centre as fed, if we nay use the word, not only by the intrinsic molecular nutritive processes of the centre itself, but also by the extrinsic nervous in- 
fluences which flow into the centre from without. The autornatism of the spinal cord as a whole resembles, in this respect, that of the respiratory centre rather than that of the heart. It has for its basis doubtless the intrinsic molecular changes of the gray matter, on whose remarkable constitution we dwelt in a previous section; the metabolic events of this substance are so ordered as to give rise to discharges of energy; but the discharge appears to be also intimately dependent on the inflow into the gray matter of afferent impulses and influences. The normal discharges of efferent impulses from the cord undoubtedly take place under the influences of these incoming impulses; and it may be doubted whether the gray matter of the cord would be able in the absence of all afferent impulses, to generate any sustained series of discharges out of its merely nutritive intrinsic changes. The automatic activity of the cord is fed not only by intrinsic nutritive events, but also by extrinsic influences.

In this feature we may, however, find perhaps the reason why the automatic activity of the spinal cord is so limited as compared with that of the brain. In spite of certain striking but superficial characters of which we shall speak later on, the gray matter of the brain presents no histological features so different from those of the gray matter of the cord as to justify us in concluding that the one is capable and the other incapable of developing the impulses which we call volitional out of the molecular nutritive changes of its substance. We are, therefore, led to the conclusion that the fuller automatic activity of the brain is due to the intrinsic changes of its substance being so much more largely assisted by the influx of various afferent impulses and influences, notably those of the special senses. To this question, however, we shall have to return later on.

$\$ 510$. In treating of the vascular system we saw that the central nervous system exercised through the vasomotor nerves such an influence on the muscular coats of the bloodvessels as to maintain what we spoke of as "tone," section of vaso-constrictor fibres leading to "loss of tone." We saw further that arterial tone, though normally dependent on the general vasomotor centre in the bulb, could be kept up by the cord itself, that, for instance, a tone of the bloodvessels of the hind limbs could be maintained by the isolated dorso-lumbar cord. This maintenance of arterial tone may be spoken of as one of the "automatic" functions of the spinal cord. We have also seen that plain muscular fibres, other than those of the arteries, notably the fibres forming sphincters, such as the cardiac and pyloric sphincters of the stomach, the sphincter of the bladder, and especially the sphincter of the anus, also possess tone, and that the tone of these sphincters is also dependent on the spinal cord, or on some part of the central nervous system. We need not repeat the discussions concerning these mechanisms and other instances of the spinal cord exercising an automatic influence over various viscera; we have referred to them here, since they serve as an introduction to a question which been much debated, and which has many collateral and important bearings, namely, the question whether the spinal cord exercises an automatic function in maintaining a tone of the skeletal muscles.

The question is not one which can be settled off-hand by a simple experiment. Most observers agree that the section of a motor nerve does not produce any clearly recognizable immediate lengthening of a muscle supplied by the nerve, in the same way that section of a vaso-constrictor nerve undoubtedly gives rise to a relaxation of the muscular fibres in the arteries governed by it; and it has been inferred from this that skeletal tone does not exist. But there are several facts to be taken into consideration before we can come to a just decision. 
The skeletal muscles have been described as being placed " on the stretch" in the living body. If a muscle be cut away from its attachments at each end, it shortens ; if it be cut across, it gapes. In other words, the muscle in the living body possesses a latent tendency to shorten, which is continually being counteracted by its disposition and attachments. In studying muscular contraction we saw ( $\$ 85$ ) that the shortening of a contraction is followed by a relaxation or return to the former length, both the contraction and relaxation being the result of molecular changes in the living muscular substance. We have now to extend our view and to recognize that, apart from the occurrence of ordinary contractions, molecular changes are by means of nutritive processes continually going on in the muscle in such a way that the muscle, though continually on the stretch, does not permanently lengthen, but retains the power to shorten upon removal or lessening of the stretch, and conversely though possessing this power of shortening permits itself to lengthen when the stretch is increased. In this way the muscle is able to accommodate itself to variations in the amount of stretch to which it is from time to time subjected. When a flexor muscle, for instance, contracts, the antagonistic extensor muscle is put on an increased stretch and is correspondingly lengthened; when the contraction of the flexor passes off the extensor returns to its previous length; and so in other instances. Thus by virtue of certain changes within itself a muscle maintains what may be called its natural length in the body, always returning to that natural length both after being shortened and after being stretched. In this the muscle does no more than do the other tissues of the body which, within limits, retain their natural form under the varied stress and strain of life; but the property is conspicuous in the muscle; and its effects in skeletal muscles correspond so closely to those of arterial tone, that we may venture to speak of it as a skeletal tone. Indeed, the molecular changes at the bottom of both are probably the same.

These changes are an expression of the life of the muscle; they disappear when the muscle dies and enters into rigor mortis; and, moreover, during life they vary in intensity so that the "tone" varies in amount according to the nutritive changes going on. We have seen reason to believe that the nutrition of a muscle, as of other tissues, is governed in some way by the central nervous system. We saw, in treating of muscle and nerve ( $\$ 81)$, that the irritability of a muscle is markedly affected by the section of its nerve, $i$.e., by severance from the central nervous system; and again ( $\$ 462)$, in speaking of the so-called trophic action of the nervous system, we referred to changes in the nutrition of muscles occasioned by diseases of the nervous system. And experience, especially clinical experience, shows that the nutritive changes which determine tone are very closely dependent on a due action of the central nervous system. When we handle the limb of a healthy man, we find that it offers a certain amount of resistance to passive movements. This resistance, which is quite independent of, that is to say; which may be clearly recognized in the absence of all distinct muscular contractions of volitional or other origin, is an expression of muscular tone, of the effort of the various muscles to maintain their "natural" length. In many cases of disease this resistance is felt to be obviously less than normal; the limb is spoken of as "limp" or "flabby," or as having "a want of tone." In other cases of disease, on the other hand, this resistance is markedly increased; the limb is felt to be stiff or rigid; more or less force is needed to change it from a flexed to an extended, or from an extended to a flexed condition; and in the range of disease we may meet with very varying amounts of increased resistance, from a condition which is only slightly above the normal to one of extreme rigidity. In some cases the condition of the muscle is such as at 
first sight seems much more comparable to a permanent ordinary contraction than to a mere exaggeration of normal tone; but all intermediate stages are met with, and indeed these extreme cases may be taken as indicating that the molecular processes which maintain what we are now calling tone are at bottom of the same nature as those which carry out a contraction; they serve to show the fundamental identity of the skeletal tone with the more obvious arterial tone.

Clinical experience then shows that the central nervous system does exert on the skeletal muscles such an influence as to give rise to what we may speak of as skeletal tone-changes in the central nervous system, leading in some cases to diminution or loss of tone, in other cases to exaggeration of tone, manifested often as conspicuous rigidity. The question why the changes take one direction in one case and another in another is one of great difficulty (the occurrence of extreme rigidity being especially obscure), and cannot be discussed here. We have called attention to the facts simply because they show the existence of skeletal tone and its dependence on the central nervous system. This conclusion is confirmed by experiments on animals, and these also afford proof that in animals the spinal cord can by itself, apart from the brain, maintain the existence of such a tone. In a frog, after division of the cord below the brain, the limbs during the period of shock are flabby and toneless ; but after a while, as the shock passes off, tone returns to the muscles, and the limbs offer when handled a resistance like that of the limbs of an entire frog. When the animal is suspended the hind limbs do not hang perfectly limp and helpless, but assume a definite position; and that this position is due to some influence proceeding from the spinal cord is shown by dividing the sciatic nerve on one side; the hind limb on that side now hangs quite helpless. This more pendent position shows that some of the flexors have lengthened in consequence of the section of the nerve, and this result may be taken as refuting the argument, quoted above against the existence of tone, which is based on the statement that a muscle cannot be observed to lengthen after section of its nerve. It may be here remarked that if the brainless frog, whose hind limbs are more or less pendent when the body is suspended, be placed on its belly, the hind limbs are brought into a flexed position under the body by means of obvious muscular contraction; and from this it might be inferred that the maintenance of the position of the pendent limb was also the result of a feeble contraction. But no obvious contractions can be observed in the latter case, as in the former; and when in the former the limb has once been brought into the flexed position, that position, like the pendent position, is maintained without obvious contractions. As we said above, "tone" may pass into something which appears to be identical with a contraction, but where no obvious contractions are observed it seems preferable to speak of the state of the muscle as one of tone.

In the dog, after division of the cord in the thoracic region, the hind limbs during the period of shock are limp and toneless. In the warmblooded animal, as we have said, the effects of shock are much more lasting than in the cold-blooded animal; and in the dog the tone of the skeletal muscle returns much more slowly than in the frog. Indeed, when the division of the cord has taken place low down the skeletal tone returns very slowly, and may be manifested very feebly, or even be absent altogether. But under favorable circumstances, when a sufficient length of cord has been left, a fairly normal tone is reëstablished. In man, in accordance with the facts previously mentioned ( $\$ 504)$, skeletal tone, which has been lost through the continuity of the cord being broken by disease or accident, appears rarely if ever to return fully in the regions below the lesion. 
We may therefore on the whole of the evidence conclude that the maintenance of skeletal tone is one of the functions of the cord; but we may here repeat that the condition of the cord, on which depends the issue from the cord along the efferent cords of the influences, whatever their nature, which produce tone in the muscle, may be, and indeed is, in its turn dependent on afferent impulses. In the case of the frog quoted above the tone of the pendent limbs disappears or is greatly lessened when the posterior roots of the sciatic nerves are divided, though the anterior roots be left intact. In the absence of the usual stream of afferent impulses passing into it, the cord ceases to send forth the influences which maintain the tone. Hence the maintenance of tone presents many analogies with a reflex action, especially when we remember that, as stated above, tone passes insensibly into contraction; and it may be a mere matter of words whether we speak of the maintenance of tone as an automatic or as a reflex action of the cord. We may, however, distinguish the part played by the afferent impulses in assisting the cord to a condition in which it is capable of maintaining tone from the part played by an afferent impulse in causing a reflex action; in the former the action of the afferent impulses seems analogous to that of a supply of arterial blond in maintaining an adequate irritability of the nervous substance, in the latter the afferent impulses lead directly to a discharge of energy. And it is convenient to distinguish the two things by different names.

$\S 511$. The close connection between tone and reflex action is illustrated by the so-called "tendon phenomena," which, on the one hand, are considered as cases of ordinary reflex action, and, on the other hand, have been regarded as exemplifying a special influence of the spinal cord on the irritability of the muscle. It is well known that when the leg is placed in an easy position, resting for instance on the other leg, a sharp blow on the patellar tendon will cause a sudden jerk forward of the leg brought about by a contraction of the quadriceps femoris; it is necessary or at least desirable for a good development of the jerk, that the tendon (and muscle) should be somewhat on the stretch. Similarly the muscles of the calf may be thrown into action by tapping the tendo Achillis, put somewhat on the stretch by flexion of the foot; and in some cases the same muscles may be made to execute a series of regular rhythmatic contractions, called "clonic" contractions, by suddenly pressing back the sole of the foot, so as to put them on the stretch. These, and other instances of a like kind, at first sight appear to be, and indeed are by many observers maintained to be, cases of reflex action, due to afferent impulses started in the tendon; hence they have been frequently spoken of as "tendon-reflex." Other observers maintain that they are not reflex, but due to direct stimulation of the muscles, the vibrations set up in the more or less tense tendon being transmitted to the muscles, and so throwing the latter into contractions. The chief arguments against their being reflex are that the interval between the lap and the contraction is very short ( 0.03 or $0 .(14$ second), shorter than the ordinary interval of a reflex action ( $\$ 507)$, and that the movement persists after section of the nerves of the tendon. The first argument is perhaps not a very strong one, and the second may be met by supposing that in such a case at least, if not always, the reflex act really begins in the muscle, being started in it by the vibrations transmitted to it along the tendon.

But even if we admit that the movements are purely muscular, started and carried out in the muscle without the help of the usual reflex chain of afferent impulses, spinal centre, and efferent impulses, we must at the same time admit that they are closely dependent on the integrity of the spinal cord and of the connections between the cord and the muscle. In the case 
of animals they disappear when the spinal cord is destroyed, or the nerves going to the muscles are severed, or even when the posterior roots only are divided. The measure of their development both in animals and in man is also closely dependent on the condition of the spinal cord and of the central nervous system generally. They may be incieased or diminished, augmented or inhibited by a coincident voluntary effort directed toward some other end, or by the coincident development of a sufficiently distinct sensation. In general it may be said that whatever favors the activity of the spinal cord tends to increase them, and whatever depresses the activity of the spinal cord tends to diminish them. They are diminished or wanting in certain diseases of the spinal card (e.g., locomotor ataxia) and exaggerated in others ; so much so indeed that they have become of practical clinical importance as a means of diagnosis. Whether we regard them as instances of ordinary reflex action, or consider that they are carried out by the muscle itself and that the cord intervenes only so far as to increase, maintain, or diminish the irritability of the muscular substance, it remains good that they are prominent whenever the conditions increase the reflex or other excitability of the cord, and diminish or disappear when the conditions lower or abolish that excitability.

$\$$ 512. Disease in man reveals other actions of the spinal cord which bear features different from those of an ordinary reflex movement, and yet have been described as reflex in nature. For instance, certain affections of the cord are characterized by the legs becoming rigid in extreme extension, the rigidity of the straightened limbs being often so great that when a bystander lifts up one leg from the bed the other leg is raised at the same time. The rigidity is due to the extensor muscles being thrown into a state of contraction, which is so uniform and long continued that it may be spoken of as a "tonic" contraction; such a tonic rigidity mav, however, be replaced by a series of rhythmic, "clonic" contractions. It has sometimes been observed that the limbs when flexed are supple and free from rigidity, but that rigidity sets in so soon as they are brought into the position of extension, the leg becoming suddeuly fixed and straight somewhat in the way that a clasp-knife springs back when opened. It seems clear that the peculiar contraction is carried out by means of the spinal cord, but the whole action, though it is often spoken of as a "muscle-reflex," is very unlike an ordinary reflex movement. In an ordinary movement an extensor is brought into action when a limb is flexed-not when it is already extended; and if in a reflex act the condition of the muscle about to be thrown into action determines in any way the discharge of impulses from the reflex centre, we should expect that the stretching of an extensor muscle by flexion-not its relaxation by extension-would determine the discharge of extensor impulses. In the case of the diseases in question just the opposite seems to take place; the position which appears to determine the development of the remarkable contraction is precisely that in which the strain upon the extensors is at its minimum. It may be doubted, therefore, whether the word reflex should be used to denote such phenomena; but the phenomena themselves deserve attention, especially, perhaps, as showing how in the disorders of the gray matter of the cord due to disease impulses or influences which are latent only in health become actual and effective.

It remains for us to speak of the part played by the spinal cord, as the instrument of the brain, in the execution of voluntary morements and in the development of conscious sensations; but it will be best to consider these matters in connection with the brain itself, to the study of which we must now turn. 


\title{
CH A PTER II.
}

\author{
THE BRAIN.
}

\section{On Some General Features of the Structure of the Brain.}

$\S 513$. IT would be out of place to attempt to give here a complete description of the structure of the brain; but certain features must be kept fresh in the mind as a basis for physiological discussion; and to these we must now turn our attention, a general acquaintance with the topographical anatomy of the brain being presupposed. ${ }^{1}$

Like the spinal cord, the brain consists of "white matter," in which the nervous elements are almost exclusively medullated fibres, and of "gray matter," in which nerve-cells and other nervous elements are also present; but the gray matter of the brain is much more variable in structure than that of the spinal cord, and possesses features peculiar to itself; these we shall study later on.

For physiological purposes the brain may be conveniently divided into parts corresponding to the divisions which appear in it in the embryo. At an early stage in the life of the embryo, that part of the medullary tube which is about to become the brain differs from that which is about to become the spinal cord, in that the central canal, which in the latter is of fairly uniform bore along its whole length, is in the former alternately widened and narrowed, so that the tube forms a series of vesicles, the cerebral vesicles, succeeding each other lengthways. At first these vesicles are three in number, called respectively fore-brain, mid-brain, and hind-brain ; but the fore-brain, after having developed on each side a lateral vesicle, the optic vesicle, subsequently transformed into the retina and optic nerve, gives rise in front of itself to a pair of vesicles placed side by side, or rather to a single vesicle with a deep median furrow, the vesicle of the cerebrum, containing a cavity divided by a median partition into two cavities, lying side by side, which open into the cavity of the original fore-brain by a $\mathrm{Y}$-shaped opening. This embryonic chain of vesicles is developed into the aduit brain by unequal growth of the walls and unequal expansion of the cavities, certain features being also impressed upon it by the bend on the longitudinal axis, which takes place in the region of the mid-brain and is known as the cranial flexure.

$\$ 514$. In the hind part of the hinder vesicle or hind-brain, the ventral, basal portion or floor is thickened to form the bulb, while the greater part of the dorsal portion or roof does not thicken at all, is not transformed into nervous elements, but remains as a single layer of epithelium, adherent to the pia mater overlying it, and so forms a thin covering to the lozengeshaped cavity of the vesicle, now known as the fourth ventricle.

In the front part of the same hind-brain, on the contrary, the roof and sides are enormously developed into the conspicuous cerebellum overhanging the front part of the fourth ventricle, while the floor is also thickened into the pons Varolii.

This thickening of the pons is largely made up, on the one hand, of horizontal nerve-fibres, which run transversely from each side of the cerebellum into the pons, or from one side of the cerebellum to the other, and, on the other hand, of longitudinal fibres, which run forward from the bulb and are

1 Figs. 131 and 146 , which will be found in succeeding sections, may with advantage be consulted in reading this section, though not specially referred to in the text. 
wrapped round by and interlaced with the others. At the front margin of the pons these longitudinal fibres, augmented in number, appear as two thick strands, the crura cerebri, forming the floor of the mid-brain, the roof of which is thickened into the corpora quadrigemina, and the cavity of which is reduced to a narrow tubular passage, the rqqueduct of Sylvius, or iter a tertio ad quartum ventriculum.

At the level of the fore-brain the crura cerebri, diverging rapidly from each other as they pass forward, leave the median portion of the floor of the vesicle now known as the third ventricle very thin, but form, especially behind and ventrally, thick lateral walls, which are further increased in thickness by the development on each side of a mass largely composed of gray matter, known as the optic thalamus. The roof of the third ventricle, like that of the fourth ventricle, is not developed into nervous elements, but remains extremely thin, and consists of nothing more than a single layer of epithelium.

$\S 515$. In front of the third ventricle each diverging crus cerebri spreads out in a small radial fashion into the corresponding half of the paired vesicle of the cerebellum now developed into the preponderant cerebral hemispheres, the two cavities of which are now known as the lateral ventricles. The growth of the cerebral hemispheres is not only much greater than that of the rest of the brain, but also takes place in a special manner. At their first appearance the cerebral hemispheres lie wholly in front of the fore-brain or vesicle of the third ventricle, but in their subsequent growth, while expanding in nearly all directions, they extend especially backward. Thus, in the adult brain, on the dorsal surface they not only completely cover up the third ventricle but also overlap the mid-brain, reaching so far back as to cover the front border of the cerebellum, while on the ventricle surface, though in the middle line they leave exposed the floor or ventral portions of the walls of the third ventricle, at the sides they are seen to reach as far backward as on the dorsal surface. The median furrow on the dorsal surface which separates each hemisphere from its fellow is at first shallow, but rapidly deepens, so that as the hemispheres grow they become separated from each other by a narrow, deep longitudinal fissure, into which, as we shall see, a fold of the dura mater dips. This fissure is not only deep vertically-i. e., from the dorsal surface ventrally-but at the front of the brain runs backward in the middle line almost as far as the level of the third ventricle, so as completely to separate from each other the anterior parts of each hemisphere, known as the anterior lobes; at the back of the brain also it similarly runs forward in the middle line for a considerable distance, so as to separate from each other the posterior lobes. Hence the two great masses of the cerebral hemisphere are united with each other, not along their whole length, but for about a third of that length, the isthmus or bridge thus connecting them lying at some depth below the dorsal surfice at the bottom of the longitudinal fissure, in about the middle third of its length.

At its first appearance each lateral ventricle is of a more or less oval form, its walls are of uniform thickness, and it lies in front of the third ventricle. During the growth of the hemispheres it acquires a peculiar shape and becomes divided into an anterior cornu or horn stretching into the anterior portion, a posterior horn stretching into the posterior portion, and a descending horn, which curves laterally and ventrally into the middle portion of the hemisphere; owing to the great backward extension of the hemispheres the lateral ventricles come to lie not only in front of but also at the side of, and indeed, to a certain extent, above or dorsal to the third ventricle; and during the growth of the parts the originally wide $\mathrm{Y}$-shaped opening which 
placed the hind ends of the two lateral ventricles in communication with the front of the third ventricle becomes narrowed into a slit-like passage of similar form, the foramen of Monro, which still opening into the front of the third ventricle, now leads on each side from a point rather in front of the middle of the lateral ventricle.

As the hemisphere enlarges, the growth of the walls of the vesicle is not uniform in all parts. At an early period there may be observed in the ventral wall or floor of the vesicle a thickening, which assuming a special, more or less semilunar, form and projecting into the cavity becomes the body known as the corpus striatum. As development proceeds the corpus striatum on each side becomes attached to the optic thalamus, lying behind and to the median side of itself, the radiating fibres of the crus cerebri passing between the two, and also as we shall see dividing the corpus striatum into two bodies, called the nucleus caudatus and nucleus lenticularis. A notable result of this growth and change of position of the hemispheres and of the coalescence of the corpus striatum with the optic thalamus is that the latter body, though really belonging to the third ventricle, comes to project somewhat into the lateral ventricle; a strip of the upper surface of the optic thalamus, along its outer, lateral edge, forms a portion of the floor of the lateral ventricle in the median region on each side of the third ventricle. Besides this special development of the corpus striatum, the walls of each vesicle, with the exception of the median part by which the two vesicles coalesce with each other, become (we are now speaking of the higher mammals) thickened much in the same way all over, the surface being folded so as to give rise to convolutions or gyri separated by furrows or sulci; and the thickening taking place in such a way as to give the ventricle its peculiar shape. The median coalesced part undergoes a different and peculiar change. This part, which at first lies in front of the third ventricle, through the changes brought about by the growth of the hemispheres so shifts its position as to lie immediately over, dorsal to third ventricle, very much as if this part of the cerebral vesicles had been folded back over the fore-brain. In the junction itself we may distinguish a dorsal and a ventral portion. The dorsal portion is developed into a system of transverse commissural fibres passing across from one hemisphere to the other. In the median region these fibres form a thick compact band, called the corpus callosum, which may be exposed to view at the bottom of the longitudinal fissure, while on each side they spread away in all directions to nearly all parts of the surface of the hemispheres, passing over and helping to form the roof of the lateral ventricles. The band is not flat but curved ventralward; hence in a longitudinal vertical section of the brain taken in the middle line it presents a curved form with the concavity directed ventralward. While this dorsal portion of the junction is developed at the sides as well as in the middle line, the ventral portion is developed in the median region only, and that in a special way, so that it forms below, ventral to, the corpus callosum an arched plate, in the shape of a triangle with the apex directed forward, called the fornix, which lies immediately above the thin epithelial roof of the third ventricle. In front, the narrower apical portion of the fornix lies at some little distance below, ventral to, the corpus callosum, and here the junction between the two vesicles is reduced to a thin sheet, the septum lucidum; but behind, the broader basal portion of the fornix is arched up so as to lie immediately under and touch the corpus callosum. Hence the septum lucidum has the form of a more or less triangular vertical sheet, broad in front and narrowing behind, separating the two lateral ventricles. The sheet may be conceived of as being double and formed by the apposition of two layers, one belonging to each ventricle; between these two layers is developed a narrow 
closed cavity containing fluid, called the fifth ventricle. But while the lateral ventricles open by the foramen of Monro into the third ventricle, and the third ventricle is continuous by means of the aqueduct with the fourth ventricle, which again passes into the central canal of the spinal cord, the whole series being develuped out of the same embryonic neural canal, the fifth ventricle communicates with none of them; it is a cavity of different origin.

The corpus callosum or dorsal portion of the junction between the vesicles spreads out, as we have said, laterally along its whole length, and thus forms a broad band joining the two hemispheres together; the middle portion spreads out in a more or less straight direction, though curving over the ventricle upward and downward to reach various parts of the hemisphere, while the front and hind ends bend round on each side forward and backward to reach the anterior and posterior parts. Thus through the corpus callosum the thick wall of one ventricle is made continuous with that of the other. The disposition of the fornix or ventral portion of the junction is very different. At its apex in front the fornix bifurcates into two bands, known as the pillars of the fornix, which on each side become continuous with, and take a peculiar course in the walls of the third ventricle. In like manner behind, the angles of the base of the fornix are continuous with the walls of the lateral ventricles, that is to say, with the thick mass of the hemispheres, being also prolonged as two special strands of fibres called the crura of the fornix. But along each side of the triangle, between the attachments in front and behind, the substance of the fornix is not continued into the substance of the corresponding hemisphere; the edge of the fornix appears on each side to lie loose on the dorsal surface of the optic thalamus, which here forms the median portion of the floor of the lateral ventricle; between the optic thalamus below and the fornix above there seems to be a narrow slit by which the cavity of the lateral ventricle communicates with parts outside itself. In reality, however, there is no actual breach of continuity though there is a breach of nervous substance. The slit is bridged over by a layer of epithelium, by means of which the edge of the fornix is made continuous with the upper surface of the optic thalamus, and the median wall of the lateral ventricle made complete. But this layer of epithelium has the following peculiar relations to the pia mater covering the brain :

We have said that the roof of the third ventricle, like that of the fourth ventricle, consists only of a layer of epithelium devoid of nervous elements. We have further seen that the fornix and the hind part of the corpus callosum with which it is continuous overlie the third ventricle, the free base of the fornix with the rounded hind end of the corpus callosum above forming together the hind border of the junction or bridge between the two hemispheres. The pia matter covering the dorsal surface of the brain, passing forward under this curved border, spreads over the top of the third ventricle, becoming adherent to the layer of epithelium just referred to, and thus forms a vascular sheet called the velum interpositum, which serves as the actual roof of the third ventricle, immediately below, ventral to, the fornix; it cannot be seen without previously removing the fornix. At the lateral edge of the fornix, on each side, this same vascular sheet of pia mater projects from beneath the fornix into the lateral ventricle, carrying with it the layer of epithelium which, as we said, made the edge of the fornix actually continuous with the rest of the walls of the lateral ventricle; the part of the pia mater thus seen projecting beyond the edge of the fornix when the lateral ventricle is laid open is called the choroid plexus. To this peculiar intrusion of the pia mater, by which the nutrition of the brain is assisted, we shall return when we come to speak of the vascular arrangements of the 
brain. Meanwhile we may point out, that while this vascular ingrowth seems to make the cavity of the third ventricle continuous with that of the lateral ventricle on each side, and all three with the exterior of the brain, it really does not do so. The cavity of the third ventricle is made complete by the layer of epithelium forming its roof, and the cavity of the lateral ventricle is made complete by the layer of epithelium passing from the lateral edge of the fornix over the choroid plexus to the other parts of the wall of the ventricle. To pass along this line from the actual cavity of the lateral into that of the third ventricle one must first pierce the epithelium covering the choroid plexus, thus gaining access to.the pia mater of the plexus and of the velum, and then again pierce the epithelium coating the under surface of the vellum and forming the roof of the third ventricle It is only by the foramen of Munro that a real communication exists between the cavity of the lateral and that of the third ventricle.

Thus by the large growth and backward extension of the cerebral hemispheres, the third ventricle comes to form, as it were the front end of the cerebro-spinal axis, the crura cerebri expanding on each side of the third ventricle into the cerebral hemispheres which cover up the ventricle on the dorsal surface, but leave its wall exposed on the ventral surface. Attached to the dorsal surface of the third ventricle at its hind end, ventral to and somewhat projecting beyond the base of the fornix, lies the pineal gland with its attachments, the remnants of a once-important median organ; and attached to the ventral surface of the ventricle, at the apex of a funnelshaped projection, the infundibulum, lies the pituitary body, also a remnant of important ancestral structures.

$\$ 516$. We may then divide the whole brain into a series of parts corresponding to the main divisions of the embryonic brain. At the front lie the cerebral hemispheres, with the lateral ventricles, developed out of the cerebral vesicles; and with these are associated the corpora striata, the term cerebral hemisphere being sometimes used so as to include these bodies, and sometimes so as to exclude them. Next come, corresponding to the original fore-brain, the parts forming the walls of the third ventricle, conspicuous among which are the optic thalami; for these bodies, though they appear to intrude into the lateral ventricles, belong properly to the third ventricle. In the mid-brain which follows, the cavity, now the tubular passage of the aqueduct, is roofed in by the two pairs, anterior and posterior, or corpora quadrigemina, the dimensions of which are not very great; but a thick floor is furnished by the crura cerebri. In each crus we must distinguish hetween a dorsal portion called the tegmentum, in which a large quantity of gray matter is present, and in which a great complexity in the arrangement of fibres exists, and a ventral portion, the pes or crusta, which is a much more uniform mass of longitudinally disposed fibres. As the crura passing forward diverge into the cerebral hemisphere on each side, the tegmentum ceases at the hinder end and ventral parts of the optic thalamus; it is the pes which supplies the mass of fibres radiating into each cerebral hemisphere. In a view of the ventral surface of the brain, the base of the brain as it is frequently called, the crura may be seen emerging from the anterior border of the pons. This we have spoken of as the thickened floor of the front part of the hind-brain, but in reality it encroaches a little on the mid-brain, the hind part of the corpora quadrigemina being in the same dorso-ventral plane as the front part of the pons. (See Fig. 131.) In the main, however, the pons belongs to the fore part of the hind-brain, the roof and sides of which are developed, as we have said, into the cerebellum. This superficially resembles the cerebral hemispheres in its large size, and in the special development of its surface, which is formed of gray matter 
folded in a remarkable manner and often spoken of as cortex. The cerebellum, though the lateral portions, called the hemispheres, project above the median portion, called the vermis, is, unlike the cerebrum, a single mass; each lateral half, however, sends down ventrally a mass of fibres which, running transversely, partly end in the pons and partly are continued across the pons into the other lateral half; this mass of fibres, thus constituting, as we have said, a considerable part of the pons, forms on each side, just as it leaves the cerebellum to enter the pons, a thick strand, called the middle peduncle of the cerebellım. From the cerebellum there also proceeds backward into the bulb on each side a thick strand of fibres, the inferior peduncle of the cerebellum or restiform body; and a third strand, the superior peduncle of the cerebellum, passes forward on each side into the region of the corpora quadrigemina. As the cerebellar peduncles converge behind the corpora quadrigemina the angle between them is filled up by a thin sheet of nervous matter, the vaive of Vieussens, which thus for a little distance backward forms a ronf for the front part of the fourth ventricle, just where the lozenge-shaped cavity is narrowing to become the aqueduct. Behind the cerebellum and pons comes the bulb, which, as we have said, is the thickener floor of the hind part of the hindbrain, the roof of the cavity being here practically wanting.

Of these several divisions the first division, that of the cerebral hemispheres, including the corpora striata, stands apart from the rest by reason both of its origin and the character of its development. As we shall seee, this anatomical distinction corresponds to a physiological difference.

Of the other parts of the brain the crura cerebri deserve special attention. We may regard these as starting in the cord, but largely augmented in the bulb; they traverse the pons, where they are still further increased, and passing beneath the corpora quadrigemina, with which as well as with the cerebellum they make connections, end partly in the region of the optic thalami and walls of the third ventricle, but to a great extent in the cerebral hemispheres. We may, in a certain sense, consider the rest of the brain as built upon and attached to these fundamental basal or ventral strands.

$\$ 517$. Connected with the brain are a series of paired nerves, the cranial nerves. The first and second pair, the olfactory nerves and the optic nerves, differ in their origin and mode of development from all the rest so fundamentally as to cause regret that they are included in the same category. We shall consider these by themselves in due course. The remaining pairs, from the third pair to the twelfth, forming a much more homogeneous category, we shall also consider in their proper place. We must now turn to study in greater detail some of the structural features of the brain, and we may with advantage begin with the bulb.

\section{The Bulb.}

$\S 518$. The spiual cord, as it ascends to the brain, becomes changed into the more complex bulb, partly by a shifting of the course of the tracts of white fibres, partly by an opening up of the narrow central canal into the wide and superficial fourth ventricle, but chiefly by the development of new gray matter.

When the anterior ventral aspect of the bulb is examined (Fig. 131, C.), it will be seen that the anterior columns of the cord are interrupted for some distance in the median line by bundles of fibres (Py. dec.) which, appearing to rise up from deeper parts, cross over from side to side and so confuse the line of the anterior fissure. This is the decussation of the pyra- 


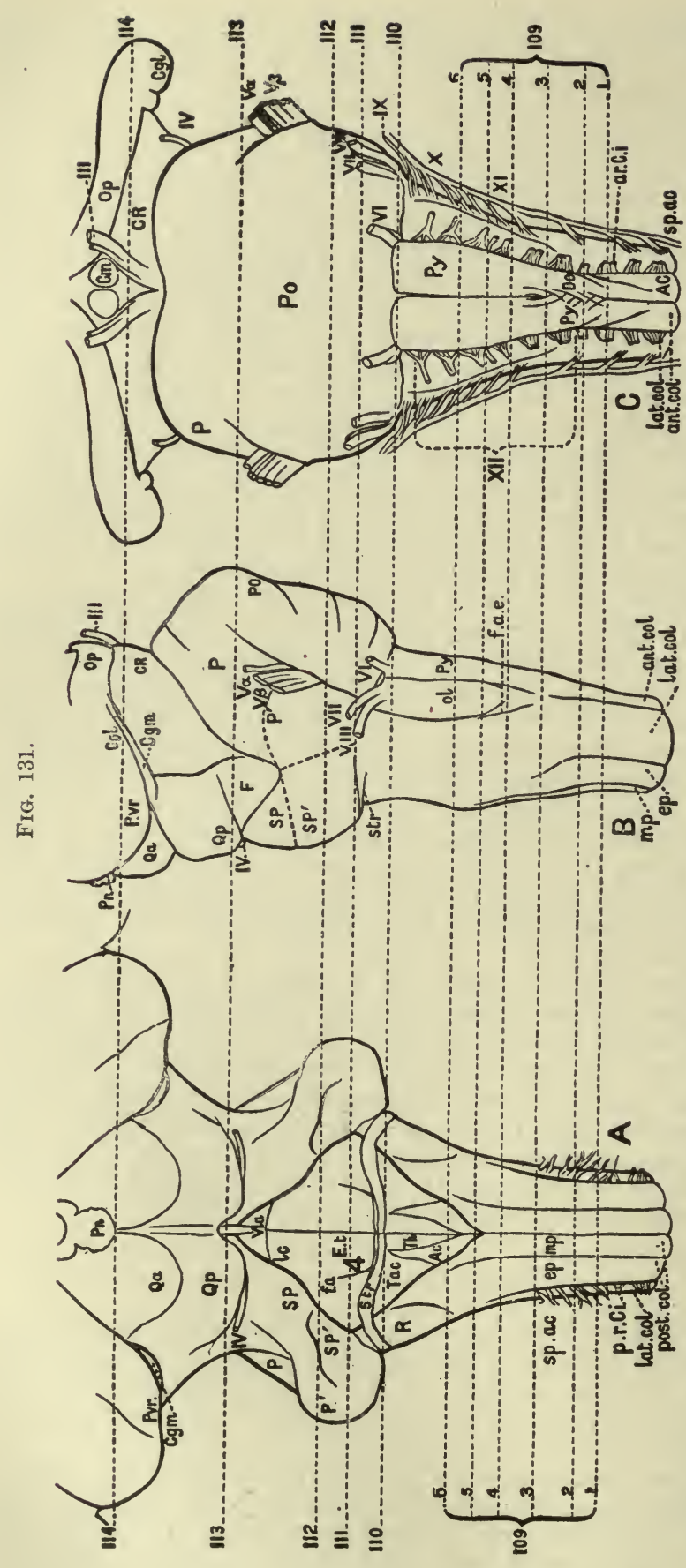

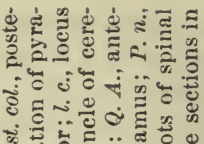

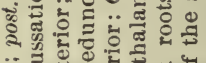

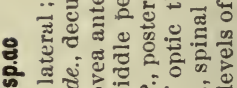
क्ष

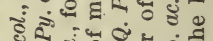
चैं 的嵒

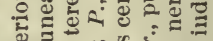

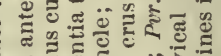
ชैं

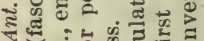

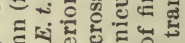
제웡

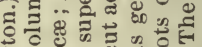

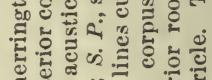

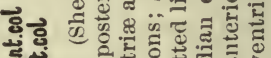
ब. कौ जु क्षे

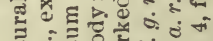
हैं 乙 $\therefore$ 远

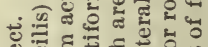

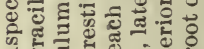
यद 50 .

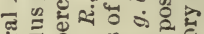
मे ब 5 ह o क्ष 政

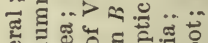

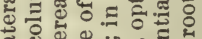

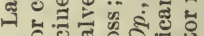
ه

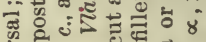
क्ष संखु

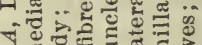
4.0 .

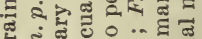

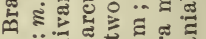

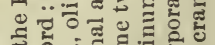

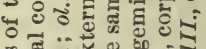
क है ह

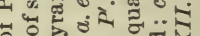
षै हैंत हैं ज्ञ

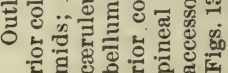


mids, above which the place of the anterior columns of the spinal cord is taken by two larger, more prominent columns, the pyramids of the bulb $(P y$.$) , which are continued forward to the hind margins of the pons. On$ the other side of, lateral to, each pyramid lies a projecting oval mass, the olivary body or inferior olive (ol.) separating the pyramid from a column of white matter, the restiform body $(R)$, which, occupying the lateral region of the bulb, when traced backward appears to continue the line of the lateral column of the cord, and when traced forward is seen to run up to the cerebellum as the inferior peduncle of that organ. On the posterior dorsal aspect no such decussation is seen. The two posterior columns of the cord diverge from each other, leaving between them a triangular space, the calamus scriptorius, which is the hind part of the lozenge-shaped shallow cavity of the fourth ventricle. As the cord passes into the bulb, the posterior column, as a whole, grows broader, and the division into a median posterior and an external posterior column becomes very obvious and distinct by the appearance of a conspicuous furrow separating the two. At some distance, however, in front of the point of divergence of the columns or apex of the calamus scriptorius, the furrow becomes less marked, and it eventually fades away. In its course the furrow takes such a line that the median posterior column, forming the immediate lateral boundary of the fourth ventricle, has the appearance of a strand broad behind but thinning away in front, while the external posterior column, also broadening as it advances forward, seems to be wedged in between the median posterior column on its median edge and the restiform body on its lateral edge; hence the former is here called the fasciculus (or funiculus) gracilis (m.p.), and the latter the fasciculus (or funiculus) cuneatus (e.p.). Further forward both columns seem to merge with each other and with fibres which curve round to form part of the restiform body; the relations, however, of these two columns to each other and to the other parts of the bulb, as well as the nature of the other several changes by which the cord is transformed into the bulb, are disclosed by transverse vertical (dorso-ventral) sections, to the study of which we must now turn.

A section (Fig. 132, 1) taken at the hind margin of the decussation, at which level the first cervical nerve takes origin, when compared with a section of the cord at the level of the second cervical nerve (cf. Fig. 127, $\mathrm{C}_{2}$ ), shows that certain changes are already taking place in the gray matter. The anterior horns are not much altered, but the posterior horns are, as it were, pushed out laterally and dorsally so that the posterior columns, which as yet retain their previous great depth, become very much broader than they are lower down, encroaching, so to speak, on the lateral columns. At the same time the substance of Rolando (s.g.), forming the head or caput of the horn, has enlarged into a more or less globular form, and lies near the surface of the cord though separated from it by a compact tract of longitudinal fibres ( $V . a$.), which, as we shall see, belongs to the fifth cranial nerve. A considerable development of the reticular formation $(f$. ret.) at the side of the gray matter ventral to the posterior horn has also taken place, and this with the shifting of the position of the posterior horn has driven the lateral horn $(l . h$.) nearer to the anterior horn. From this lateral horn a root of the eleventh spinal accessory cranial nerve (XI.) may be seen taking origin. Further, a great increase of gray matter round the central canal may also be observed.

These changes, however, are of degree only; what seems to be an absolutely new feature is the presence of bundles of fibres $(P y$. dec.) which starting from the anterior column of one side cross over to and are apparently lost in the gray matter of the neck of the anterior horn of the other side; in 
so crossing the fibres push aside the bottom of the anterior fissure. When the course of these fibres is investigated, either by simple microscopic observation, or still better by the method of degeneration, it is found that they may be traced from the anterior column of one side, across the anterior commissure, through the neck of the anterior horn to the lateral column of the opposite side, and to that part of the lateral column which we have previously described as the crossed pyramidal tract.

In a section a little higher up (Fig. 132, 2), these decussating fibres form on each side a large strand which starts from a part of the anterior column, now becoming distinctly marked off as the pyramid (Py.), and is apparently lost in the reticular formation, but in reality passes on to the crossed pyramidal tract of the lateral column. This strand, as it crosses over, completely cuts off the head of the anterior horn from the more central gray matter, and forms with its fellow a large area of decussating fibres between the bottom of the anterior fissure and the central gray matter. When a surface view of the bulb is examined the decussation is seen to be effected by alternate bundles, passing now from right to left, now from left to right; and in transverse sections we find correspondingly that the anterior fissure appears bent now to the left and now to the right, according as the section cuts through a bundle passing from left to right or from right to left.

In sections still higher up (Fig. 132,3 and 4) this conspicuous strand of fibres crossing obliquely from side to side will be no longer seen; decussating fibres are seen dorsal to the anterior fissure, but these, of which we shall speak presently, are of different nature and origin. The fibres which in sections below were seen in the act of crossing are now gathered into masses of longitudinal fibres, the pyramids $(P y$.) one on each side of the anterior fissure, each with a sectional area of a rounded triangular form clearly marked out from the surrounding structures; the section is taken above the decussation of the pyramids. Or, tracing the changes from below upward, we may say that the decussation is now complete; on each side the whole of the crossed pyramidal tract of the spinal cord has, in the region of the bulb below the level of the present sections, crossed over to the other side, and joining with the direct pyramidal tract of the anterior column of the cord of the same side has become the pyramid of the bulb. In other words, the decussation of the pyramids is, as we have already hinted, the passing off from each pyramid and the crossing over to the opposite side of the cord of those fibres which are destined to become the crossed pyramidal tract of the spinal cord of the opposite side, while the rest of the pyramid pursues its. course on the same side as the direct pyramidal tract.

$\S 519$. In the spinal cord the bottom of the anterior fissure is separated from the central canal by nothing more than the anterior white commissure and a narrow band of gray matter, composed of the anterior gray commissure and of part of the central gelatinous substance. During the decussation of the pyramids, the decussating fibres push, as it were, the central canal with its surrounding gray matter to some distance from the bottom of the anterior fissure. In sections above the decussation the bottom of the fissure does not again approach the central canal, but continues to be removed to some distance from it, and, as we pass upward, to an increasing distance, by the interposition of tissue which consists largely of decussating fibres. These, however, though they seem to continue on the decussation of the pyramids, are shown by the embryological and degeneration methods to have no connection with the pyramids, but belong to another system of decussation. As we have seen $(\$ 477)$ the anterior commissure along the whole length of the cord contains decussating fibres. Some of these in the upper part of the cord are fibres crossing from the direct pyramidal tract of one side to the gray 
1

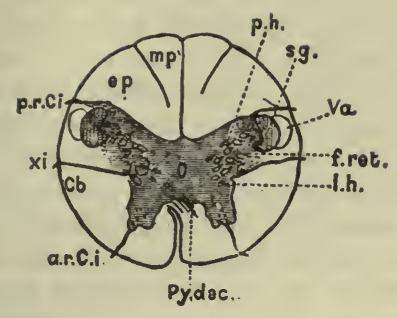

2

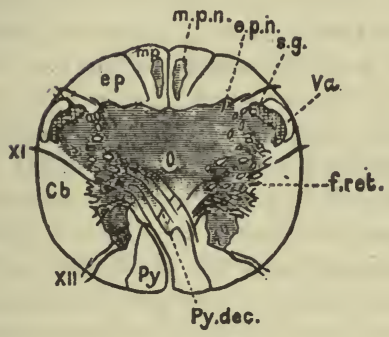

3

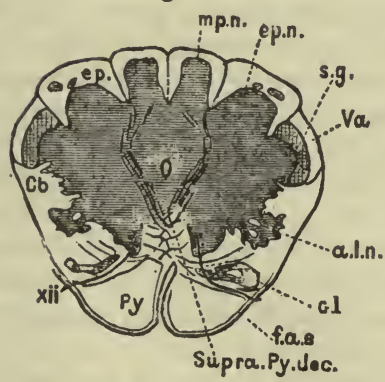

FIG. 132.

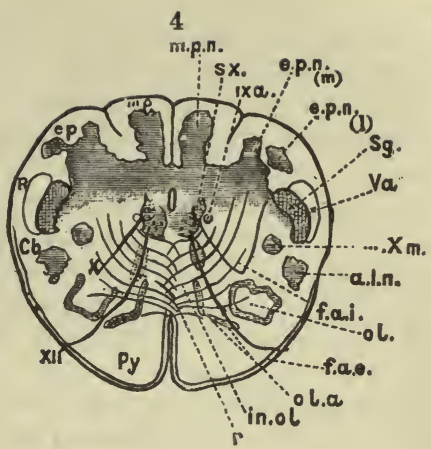

5

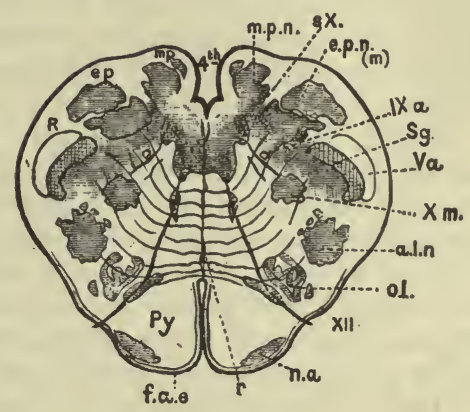

6

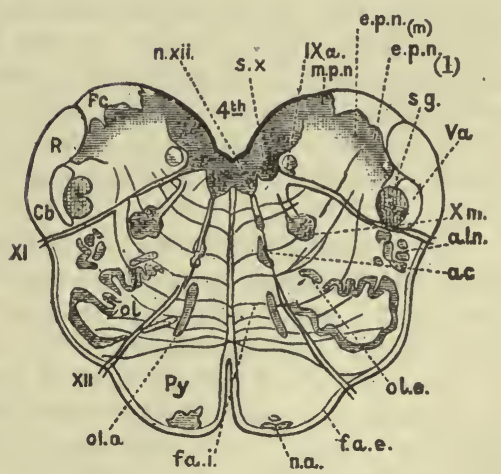

Transverse Dorso-ventral Sections of the Bulb (Man) at Different Levels. (Sherrington.) Fig. 132 and Figs. 133-137 form a series of transverse dorso-ventral sections of the brain taken at different levels from the hind end of the bulb to the front of the third ventricle; the several levels are shown by the lines drawn in Fig. 131. They are all magnified twice. The details are shown, for the sake of simplicity, in diagrammatic fashion; the white matter is left unshaded, the course of the fibres being indicated in a few important instances only; the gray matter is shaded formally, the nerve-cells being indicated in the case only of the nuclei of the cranial nerves. The want of complete bilateral symmetry which is often met with in such sections is indicated in several of the figures.

1. At the hiud limit of the decussation of the pyramids; 2 . In the middle of the decussation; 3. At the upper end of the decussation; 4. Just below the point of the calamus scriptorius; 5 . Just above the point; 6 . Through the middle of the ala cinerea.

Py. Pyramids; Py. dec. decussation of the pyramids; Supra Py.dec. superior decussation; $f$. a. $i$. internal arcuate fibres; $f, a$. $e$. external arcuate fibres; $C b$. position of cerebellar tract; $R$. resti- 
matter of the other side, and so may be regarded as part of the whole pyramidal tract; but others are of different origin; and even in the region of the actual decussation of the pyramids some of the fibres which cross over do not belong to the pyramidal tract. This system of decussating fibres becomes increasingly prominent above the decussation of the pyramids, and through it the ventral area of the bulb between the central canal and the anterior fissure is much increased. The fibres as they cross form a middle line of partition, the raphe (Fig. 132, 4, 5, r) which increases in depth in the upper parts of the bulb, and on each side of the raphe help to break up the gray matter (which previously formed the anterior horns) into what is called the reticular formation. We shall return to this presently, but may here call attention to a special development of these decussating fibres which is seen just above the decussation of the pyramids. In a section at this level (Fig. 132, 3) a strand of fibres (Supra Py. dec.) may be seen to start chiefly from the gracile nucleus but also to some extent from the cuneate nucleus, to sweep round the central gray matter, and to decussate ventral to this between it and the bottom of the anterior fissure. This is called the superior decussation, or, for reasons which we shall see later on, the sensory decussation.

$\$ 520$. We must now turn to the posterior fissure and its relations to the fourth ventricle. We saw that at the beginning of the pyramidal decussation, the posterior horns had been thrown backward and outward so as to increase the posterior columns. The posterior fissure is still of great depth, so that by the increase of depth and maintenance of depth the posterior column, the lateral limit of which is still sharply marked out by the swollen hear of the posterior horn as well as by the highest posterior rootlets of the first cervical nerve, acquires at this level its maximum of bulk.

From this point forward the depth of the posterior fissure and the dorsoventral diameter of the posterior columns diminishes. The head of the horn (Fig. 132, 2) is thrown still further outward into the lateral regions; developments of gray matter at the base and to some extent at the neck of the horn (of these we shall speak presently) encroach (Fig. 132, 3) dorsally on the white matter of the columns; and the central gray matter appears to rise dorsally at the expense of the posterior fissure, in coincidence with the development described above as taking place on the ventral side of the canal.

Still a little further forward, in a section, for instance (Fig. 132, 4), a little way behind the apex of the calamus scriptorius, the central gray matter, which still forms a rounded mass around the central canal, is brought yet nearer to the posterior fissure.

In a section yet a little further forward (Fig. 132, 5) carried through the hinder narrower part of the fourth ventricle itself, it is seen that the central canal has opened out on the dorsal surface, and that the gray matter, which

form body or inferior peduncle of the cerebellum; e. p. external posterior column, fasciculus cuneatus; $m . p$. median posterior column, fasciculus gracilis; $r$. raphe; $l . h$. lateral horn; $m . p . n$. nucleus of the median posterior column or gracile nucleus; $e . p$. $n$. nucleus of the external posterior column or cuneate nucleus ; e. p. n. $(m)$ median division and $e . p . n$. (l) lateral division of the same; ol. olivary body; ol. a. median accessory, and ol.e. lateral accessory olive; in.ol. interolivary layer: $a . l . n$. lateral (antero-lateral) nucleus; $n . a$. arcuate nucleus; $a . c$. remnant of anterior horn; f. ret. reticular formation; s.g. substance of Rolando; $a . r . c .1$. anterior root, and $p . r$. c. I. posterior root of first cervical nerve; XI. root of spinal accessory nerve: XII. twelfth or hypoglossal nerve; $n$. XII. nucleus of the same in $6:$ the nucleus may be traced, however, through $2,3,4,5$, in connection with the fibres of the nerve; $s$. X. sensory or main part of the glossopharyngeal-vago-accessory nucleus: X. $m$. motor nucleus of the vagus, or nucleus ambiguus; IX. a. ascending root of the glosso-pharyngeal nucleus; V. a. ascending root of the fifth nerve; 4 th. fourth ventricle; the ependyma or lining is indicated by a thick dark line; and in 5 and 6 , the tooth-like section of the projecting obex is shown. 
in previous sections surrounded it, is now exposed to the surface on the floor of the ventricle, the median posterior columns being thrust aside. In a still more forward section (Fig. 132, 6) this gray matter in correspondence with the increasing width of the ventricle occupies a still wider area, thrusting still further aside the narrowing upper ends of the two posterior columns.

During these successive changes the large, wide posterior (both external posterior and median posterior) columns of the cervical spinal cord and beginning bulb, are reduced to small dimensions and in the end disappear; but before we speak of the course and fate of the tracts of fibres constituting these columns we must turn to the important changes of the gray matter.

$\$ 521$. A transverse section through the lower end of the decussation (Fig. 132, 1) shows, as we have said, few differences as regards the gray matter from one taken at the level of the second cervical nerve. The changes noticeable are mainly the changes in position of the posterior horns, the increase of central gray matter around the central canal, the approach of the lateral horn, from which spring the roots of the spinal accessory nerve, to the anterior horn, and an increase of the reticular formation in the bay ventral to the posterior horn.

In the middle of the decussation (Fig. 132, 2) the decussating fibres are cutting the head of the anterior horn away from the base of the horn and the central gray substance, and the isolated head is diminished in size, being separated from the surface of the cord by an increasing thickness of white matter. The lateral horn and origin of the spinal accessory root do not share in this isolation, but are driven back again dorsally toward the posterior root to join the reticular formation which is increasing in area, while the lateral column of white matter is diminishing in bulk by the withdrawal of the pyramidal tract.

Still a little further forward, the anterior horn seems at first sight to have wholly disappeared (Fig. 132, 3 and 4), but its disappearance is coincident with an increase of the reticular formation in the position of the lateral columns, as well as with the growth of tissue mentioned above between the anterior fissure and the central gray matter. In fact, between the anterior pyramids on the ventral side and the largely increased and laterally expanded gray matter on the dorsal side, a large area of peculiar tissue now extends on each side for a considerable distance from the middle line of the raphe, encroaching on what was the lateral column of white matter; and a corresponding area of similar tissue may be traced from this level through the higher parts of the bulb up into the pons and crura cerebri. The tissue consists of nerve-fibres running transversely, longitudinally, and in other directions, so as to form a network, the bars of which are often curved; and with these fibres are found branched nerve-cells in considerable number, some of them small, both fibres and cells being as elsewhere imbedded in neuroglia. Though differing from the ordinary gray matter of the cord by the more open character of its network, it may be considered as a form of gray matter. We may consider it as being in reality the gray matter of the apparently lost anterior horn broken up and dispersed by the passage of a large number of fibres and bundles of fibres, especially of the decussating fibres spoken of in $\$ 518$, which since they curve through this area from the middle line laterally are called arcuate or arciform fibres, internal arcuate fibres (Fig. 132, 6, f. a. i.) to distinguish them from the external arcuate fibres (f. a.e.) of which we shall speak presently, Fragments of more compact gray matter also belonging probably to the anterior horn are seen at intervals in this area (Fig. 132,6,ac.) and elsewhere. We have seen that 
nearly all the way along the cord the gray matter of the neck of the posterior horn is similarly broken up by bundles of fibres into what we there called the reticular formation (Figs. 121, 122, r.f. p. and $r . f . l$. ) ; and this area in the bulb though it possesses characters of its own is also called the reticular. formation. In the more lateral portion of this formation, the network is more open and irregular, the fibres are finer, and the nerve-cells are more abundant than in the median portion where the nerve-cells, except in the immediate neighborhood of the raphe, are less numerous or even absent, and the fibres are coarser. These two parts are sometimes distinguished as the outer or lateral and the inner or median formation. In the middle line the fibres distinctly interlace and decussate in an oblique manner, some rumning nearly vertically in the dorso-ventral plane, thus constituting, as we have said, a thick raphe, which, however, at its edges gradually mergesin to the more open network.

$\$ 522$. Within the area, bounded by the pyramids ventrally, the expanded gray matter dorsally, the raphe in the middle line, and the white matter laterally, certain distinct compact masses of gray matter make their appearance, as we pass upward toward the pons.

One of the most important of these gives rise to the olivary body, or inferior olive, which, as we have seen, projects as an oval mass (Fig. 131, ol.) on each side of the pyramids, reaching from a level which is somewhat higher up than the lower limit of the pyramids, almost but not quite to the pons. The olivary body, as a whole, consists partly of white matter, that is, of fibres, and partly of gray matter, sometimes called the olivary nucleus. 'This latter is disposed in the form of a hollow flask or curved bowl, with deeply folded or plaited walls, having a wide open mouth directed inward toward the middle line, and forward toward the pons (Fig. 132, 4, 5, 6, ol.). The flask is filled within by white matter, and covered up on its outside with white matter as well as traversed by fibres. The gray matter thus forming this flask-shaped nucleus consists of small rounded nerve-cells, lying in a bed of tissue which is partly ordinary neuroglia, and partly a fine nervous network.

Lying to the median side of the olivary body, immediately dorsal to the anterior pyramid is another small mass of gray matter, in the form of a disc, appearing in transverse sections as a thick bent rod, in some sections consisting of two parts (Fig. 132, 4, ol. a.). This is the accessory olivary nucleus. A very similar body lies dorsal to the olivary nucleus, in the lateral reticular formation ; this is also called an accessory olivary nucleus, being distinguished (Fig. 132, 6, ol.e.) by the name outer accessory nucleus from the above-mentioned inner accessory nucleus. It will be observed in these transverse sections that the inner accessory nucleus is separated from the olivary nucleus by a bundle of white fibres (Fig. 132, 4, 5, 6, XII.) which, running ventrally from the gray matter in the dorsal region, comes to the surface between the anterior pyramids and the olivary body. This is the hypoglossal or twelfth cranial nerve.

On the surface of the anterior pyramid itself is seen on each side a small mass of gray matter (Fig. 132, 5, 6, n. a.), which siuce it appears to be connected with a system of superficial transverse fibres, which we shall describe directly as the external arcuate fibres (Fig. 132, 3, 4, 5, 6, f. a. e.), is called the arcuate nucleus. It seems to belong to the same group as the accessory olives.

Lastly, a small somewhat diffuse collection of gray matter is seen in sections as a rounded mass of irregular form placed laterally to the reticular formation (Fig. 132, 4, 5, 6, a.l.n.). This, which at its first appearance seems to be budded off from the general mass of gray matter (Fig. 132, 3, 
a.l.n.) and which is probably a detached portion of the base of the anterior horn or of the lateral region of the gray matter, is called the lateral or antero-lateral nucleus.

Hence, besides the diffuse reticular formation, this ventral part of the bulb contains more sharply defined collections of gray matter in the olivary nucleus, and the other bodies just mentioned.

$\S 523$. We must now turn to the dorsal part of the bulb. Here in the first place we must distinguish between the portions of gray matter which are more immediately connected with the cranial nerves taking origin from this part of the bulb, and the portions which have no such obvious connection. In the spinal cord, the anterior horns supply, as we have seen, the origins of the successive anterior motor nerves; but in the transformation of the cord into the bulb the anterior horns have been broken up or displaced; and the parts of the anterior horns serving as the nuclei of origin for motor nerves have been translated from the ventral to the more dorsal regions. Hence, it is in the more dorsal part of the gray matter that we have to seek for the nuclei of origin not only of afferent but also of motor cranial nerves. It will be convenient to consider all these nuclei of origin of cranial nerves by themselves, and we may here confine ourselves to the gray matter of other nature. We may, however, say that these nuclei from that of the third nerve backward are more or less closely associated with the gray matter immediately surrounding the central canal. This central gray matter, in the narrow sense of the term, is marked out somewhat low down (Fig. 132, 3) by the fibres of the sensory decussation which sweep round it; it appears in sections higher up as a fairly distinct region (Fig. 132, 4); and it is this part of the gray matter which is exposed on the floor of the fourth ventricle when the central canal (Fig. 132, 5, 6) opens out into that space. We say exposed; but in reality the true gray matter is covered by a superficial layer of tissue of a peculiar nature (indicated in Fig. 132, 5, 6, by a thick black line) similar to that which is found at the hind end of the conus medullaris in the spinal cord.

We saw that at the level of the first cervical nerve coincident with the horizontal flattening out of the posterior horns the posterior columns assumed very large dimensions. In this region (Fig. 132, 1) they consist entirely of white matter-that is, of longitudinal fibres.

At a little higher level, however, at the level of the middle of the decussation for example, an islet of gray matter (Fig. 132, 2, m. p. n.) makes its appearance in the median posterior column. A little further forward, at the level of the established pyramids, it will be seen (Fig. 132, 3) that this islet is the hind end of an invasion from the more centrally placed gray matter, and that at the same time there has taken place a similar inroad of gray matter into the external posterior column (Fig. 132, 3, e. p. n.); indeed, a slight extension of gray matter into the external posterior column may be seen even before this (Fig. 132, 2, e.p.n.). It will further be observed that these gray masses have so largely encroached on the white matter that both the median posterior or fasciculus gracilis and the external posterior column or fasciculus cuneatus, instead of being simply tracts of white fibres, as they were in the hinder part of the bulb and in the cord, have now become columns of gray matter covered by a relatively thin layer of white fibres. These columns of gray matter are now called respectively the median posterior nucleus or nucleus fasciculi gracilis, or, more shortly, the gracile nucleus; and the external posterior nucleus, or nucleus fasciculi cuneati, or the cuneate nucleus. From the ventral aspect of these nuclei a larger number of fibres pass ventrally, with a more or less curved course, to form, as we have seen ( $(518)$, the superior decussation and to pursue certain paths through the 
reticular formation, of which we shall speak later on. It is at this level and for some little distance above (Fig. 131, 4,5) that these nuclei acquire their greatest development. Further forward (Fig. 132, 6), when the fourth ventricle has opened out and the nuclei of the cranial nerves are becoming conspicuous, and the posterior columns have been thrust aside laterally, both these nuclei have diminished in size; still further forward they become still smaller, and toward the pons they gradually disappear.

The mass of gelatinous substance, forming at the level of the first cervical nerve the swollen caput of the horn close to the surface but separated from it by a band of fibres $(V a$. $)$ of fine calibre, to which we have already referred as belonging to the fifth cranial nerve, increases in bulk at a somewhat higher level (Fig. 132, 2, 3, s. g.) and forms on the surface a slight projection, called the tubercle of Rolando. It soon, however, becomes thrust ventrally by the divergence of the posterior columis, and more and more covered up by the fibres which are going to form the increasing restiform body (Fig. $132,4,5,6, R)$. Retaining this position the islet of gelatinous substance diminishes in size further forward (Fig. 133, s.g.), and eventually disappears.

$\$ 524$. The fibres of the bulb. It is obvious, from what has already been said, that the arrangement into posterior, lateral, and anterior columns, so clear and definite in the spinal cord, becomes broken up in the bulb; indeed it will be best, in treating of the bulb, not to attempt to trace out these

FIG. 133.

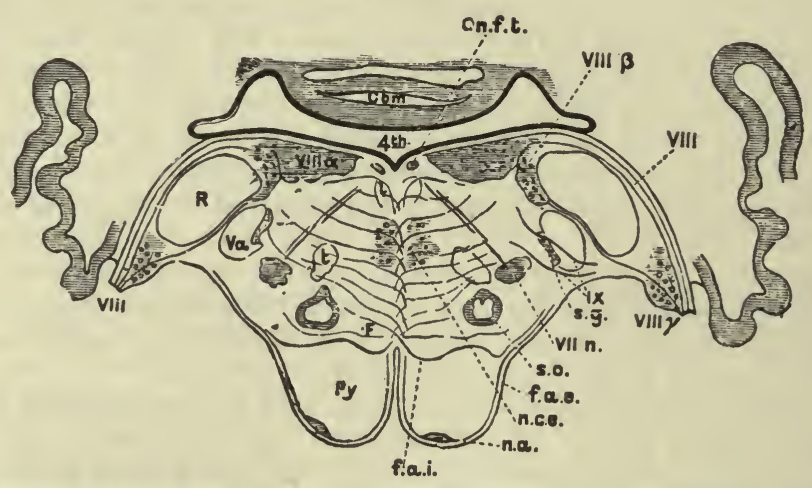

Througln the Bulb just Behind the Pons. (sherrington.) Taken in the line 110, Fig. 131. Py. pyramids; $R$. restiform body ; Cbm. cerebellum; $F$. fillet; $f$. $a$. e. external, $f . a$. $i$. internal arcuate fibres; $t$. bundle of fibres from olive to the lenticular nucleus; $l$. posterior longitudinal bundles; $n$. f.t. nucleus of the fasciculus teres; s. o. superior olive; $n$. c. e. nucleus centralis (the marks within it are sections of bundles of fibres by which it is traversed); $8 . g$. substance of Rolando; V. $a$. ascending root of fifth nerve; VII. $a$. nucleus of the seventh nerve; VIII. auditory nerve, chiefly the dorsal or cochlear root; VIII. $\infty$ median nucleus; VIII. $\beta$. lateral nucleus; VIII. $\gamma$. accessory nucleus of auditory nerve; IX. fibres of root of ninth nerve passing through ascending root of fifth nerve.

columns, but to speak of the course of the several tracts into which these columns may be divided.

The direct and cross pyramidal tracts of the cord unite to form, as we have seen, the pyramid of the bulb, and so pass on to the pons. We need say nothing more at present concerning this important pyramidal strand, except that, as we trace it down from the pons to the spinal cord, it gives off to the bulb itself fibres which make connections with the motor fibres of the cranial nerves proceeding from this region.

Concerning the course taken by the other less conspicuous "descending" 
tract, the antero-lateral descending tract, our knowledge is very imperfect; nothing definite can be said at present.

The cerebellar tract, occupying near to the surface a position which in the series of sections (Fig. 132, Cb.) appears now rather more ventral, now more dorsal, eventually passes into the restiform body, of which it forms a large part, and thus reaches the cerebellum. The antero-lateral ascending tract possibly also takes the same course, but this is not as yet certain.

The median posterior tract or columu, becoming the fasciculus gracilis, ends in the gracile nucleus; and in a similar manner the external posterior column, or fasciculus cuneatus, ends in the median and lateral masses of the cuneate nucleus. As we have seen, the white matter of these columns diminishes as the nuclei increase; and the nuclei after absorbing, so to speak, the white matter diminish in turn; the ascending degeneration observed in these columns stops at these nuclei. It is a suggestive fact that as these nuclei diminish forward the restiform body increases in bulk.

The remaining fibres of the cord, belonging partly to the anterior column and partly to the lateral column, not gathered into any of the above-mentioned tracts, appear to end, chiefly at all events, in the reticular formation of the bulb itself, though some are carried on to the higher parts of the brain.

$\S 525$. Thus of the various tracts or strands of the spinal cord two only are known definitely and certainly to pass as conspicuous unbroken strands through the bulb to or from higher parts; namely, the pyramidal tract to the cerebrum and the cerebellar tract to the cerebellum. All, or nearly all, the rest of the longitudinal fibres of the cord reaching the bulb end, as far as we know at present, in some part or other of the bulb; and we may infer that some or other nerve-cells of the bulb serve as relays to connect these fibres of the cord with other parts of the brain.

The gracile and cuneate nuclei stand out conspicuously as relays of this kind, and through them the posterior columns of the cord make secondary connections on the one hand with the cerebellum, and on the other hand with various regions of the cerebrum. We have said $(\$ 519)$ that fibres passing ventrally from the gracile and cuneate nuclei sweep in a curved course through the reticular formation as the internal arcuate fibres (Fig. $132, f . a . i$.). The hindmost of these form the superior decussation already referred to, as seen in sections of the fore part of and in front of the pyramidal decussation (Fig. 132, 3, Supra Py. dec.). After decussating ventral to the central canal, these fibres form an area called the inter-olivary layer (Fig. 132, 4, in. ol.), lying dorsal to the pyramids between two of the olivary nuclei. 'This layer may be regarded as the hind end or beginning on each side of a remarkable longitudinal strand called the fillet (Figs. 131, B. F., $133, F$.), of the connections of which in the front part of the brain we shall speak hereafter. Thus these two nuclei are the source of fibres which cross to the other side of the bulb, and reaching the inter-olivary layer dorsal to the pyramids run up to higher parts of the brain by the fillet. We may add that the formation of the fillet is also probably assisted by fibres from a tract which lies just dorsal to the inter-olivary layer, and is derived from the anterior columus of the cord. Besides its fibres of descending degeneration the anterior column contains fibres of ascending degeneration, and these rise dorsally in the bulb to form the tract in question. Though the whole tract is of some length, the component fibres appear to be short.

The gracile and cuneate nuclei give rise also to other fibres which, though also sweeping ventrally and crossing to the other side, do not, when they reach the inter-olivary region, assume a longitudinal direction, as do the fibres forming the fillet, but as external arcuate fibres (Fig. 132, f. a. e.) 
pursue a course which is at first ventral along the side of the anterior fissure, and then lateral over the ventral surface of the pyramid and olivary nucleus, by which path they reach the lateral surface of the bulb, and so the restiform body and cerebellum. In this way the two nuclei in question contribute to the restiform body of the opposite side of the bulb. These external arcuate fibres, which as they sweep round the ventral surface of the pyramid traverse the arcuate nucleus, though they vary much in individual brains, form a considerable portion of the white matter seen on the ventral and lateral surfaces of the bulb; it is by them that the olivary nucleus is covered up.

The cuneate and gracile nuclei, besides this crossed and somewhat roundabout connection with the restiform body of the opposite side, are believed to have more direct connection with the restiform body of the same side by means of fibres which pass by a more or less direct lateral path from them to it. Accepting this view, we may say that the two nuclei are comnected with the opposite side of the cerebellum by external arcuate fibres, and with the same side of the cerebellum by the other fibres just mentioned. In any case, the connection between the two nuclei and the cerebellum is large and important.

Thus the important strand of fibres which is called in the bulb the restiform body, and higher up the inferior peduncle of the cerebellum, is connected with the spinal cord in two chief ways; directly by means of the cerebellar tract and indirectly by means of the cuneate and gracile nuclei, which, as we have said, diminish in bulk forward as the restiform body increases. By the relay of the gracile nucleus it is brought into connection with the median posterior column along the whole length of the cord, and so with that division of the posterior roots which $(\$ 490)$ in each of the several spinal nerves goes to form that column. By the relay of the cuneate nucleus it is brought into connection with such parts of the external posterior column as end in that nucleus, and thus probably with other fibres of the posterior roots of the upper spinal nerves. And if we admit that the cerebellar tract is connected, by the relay of the vesicular cylinder or by other nerve-cells, with the rest of the posterior roots of the spinal nerves, we may conclude that the restiform body is, by means of these relays, a continuation of the spinal posterior roots.

The restiform body and so the cerebellum is also specially connected with the olivary body of the opposite side; for when in young animals one side of the cerebellum is removed, the olivary body of the opposite side atrophies. The course of the fibres maintaining this connection is not as yet accurately known, but they probably pass from the olivary nucleus of one side through the inter-olivary layer, and so laterally through the reticular formation of the other side. Lastly, we may add that a tract which is sometimes included in the restiform body as its median or inner division has quite a different origin from any of the above; the fibres which compose it come, as we shall see, from the auditory nerve.

The further connections of the bulb with the cerebrum it will be best to leave until we come to deal with the structural arrangement of the rest of the brain.

Meanwhile enough has been said to show that the bulb differs very materially in structure from the spinal cord. The gray matter of the bulb is far more complex in its nature than is that of any part of the cord; and the arrangement of the several strands and tracts of fibres is far more intricate. The structural features on the whole perhaps suggest that the main functions of the bulb are twofold: on the one hand, it seems fitted to serve as a headcentre governing the spinal cord, the various reins of which, with the exceptions noted, it holds, as it were, in its hands; on the other hand, it ap- 
pears no less adapted to act as a middleman between parts of the spinal cord below and various regions of the brain above. As we shall see, experiment and observation give support to these suggestions.

\section{The Disposition and Connections of the Gray and White Matter of the Brain.}

THE GRAY MATTER.

$\S 526$. As we pass up from the bulb to the higher parts of the brain, the differentiation of the gray matter into more or less separate masses, which we have seen begin in the bulb, becomes still more striking. We have to distinguish a large number of areas or collections of gray matter, more or less regular in form and more or less sharply defined, from the surrounding white matter; to such collections the several terms corpus, locus, nucleus, and the like, have from time to time been given. These areas or collections vary greatly in size, in form, and in histological characters; they differ from each other in the form, size, features, and arrangement of the nerve-cells, in the characters of the nervous network of which the nerve-cells form a part, and especially perhaps in the extent to which the more distinctly gray matter is traversed and broken up by bundles of white fibres. Guided by the analogy of the spinal cord, as well as by the results of experiments and observations directed to the brain itself, we are led to believe that the complex functions of the brain are intimately associated with this gray matter; and a full knowledge of the working of the brain will carry with it a knowledge of the nature and meaning of the intricate arrangement of the cerebral gray matter. At present, however, our ignorance as to these things is great; and, though various theoretical classifications of the several collections of gray matter have been proposed, it will perhaps be wisest to content ourselves here with a very broad and simple arrangement. We will divide the whole gray matter of the brain into four categories only: 1 . The central gray matter lining the neural canal; and this we may consider the nuclei of the cranial nerves, some of which are closely associated with it. 2. The superficial gray matter of the roof of some of the main divisions of the brain, such as that of the cerebral hemispheres, and of the cerebellum. 3. The intermediate gray matter more or less closely connected with the crura cerebri. 4. Other collections and areas of gray matter. We will, moreover, confine ourselves at present for the most part to their general features and topography, reserving what we have to say concerning their histological characters for another occasion.

\section{The Central Gray Matter and the Nuclei of the Cranial Nerves.}

$\$ 527$. The ventricles of the brain, like the central canal of the spinal cord, of which they are a continuation, are lined by an epithelium which is in general a single layer of columnar cells said to be ciliated throughout, though it is often difficult to demonstrate the cilia. Beneath this epithelium lies a layer of somewhat peculiar neuroglia, forming with the epithelium, as we have said ( $\$ 523)$, the ependyma, which, well developed in the floor of the fourth ventricle and in the walls of the third ventricle and of the aqueduct, is thin and scanty in the lateral ventricles. Beneath, and more or less connected with the ependyma in the sides and floor of the third ventricle, is a fairly conspicuous layer of gray matter, which is well developed in the parts of the floor exposed on the ventral surface of the brain, and known as the lamina terminalis, the anterior and posterior perforated spaces, the tuber 
cinereum, etc. This layer is not continued forward into the lateral ventricles of the cerebral hemispheres, but it is well developed backward along the aqueduct (Figs. 136, 137), and in the floor of the fourth ventricle, and through the bulb becomes, as we have seen ( $\$ 523$ ), continuous with the central gray matter of the cord. The nerve-cells of this gray matter are on the whole small and in many places scant.

$\$ 528$. The several roots of the cranial nerves from the third nerve backward may be traced within the brain substance to special collections of gray matter, called the nuclei of the cranial nerves, some of which lie close upon the central gray matter, while others are placed at some distance from it. The optic nerve and what is sometimes called the olfactory nerve, namely, the olfactory bulb and tract, may advantageously be dealt with apart, since these two nerves are not, like the other cranial nerves, simple outgrowths from the walls of the original neural canal, but are in reality elongated vesicles, budded off from the neural canal, the cavities of which have been obliterated. We may add that part of the retina, and of the gray matter of the olfactory tract, may perhaps be considered as corresponding to the nuclei of which we are speaking, the retinal and proper olfactory fibres being connected with them very much as the fibres of the remaining cranial nerves are connected with their respective nuclei. In the brain the segmental regularity of the nerve-roots so conspicuous in the spinal cord is very greatly obscured. We shall have something to say on this point later on ; but at present we may be content to treat the several nerves in a simple topographical manner. They may be seen in a ventral view of the brain (Fig. 131, C.), leaving the brain at various levels by what is called their "superficial origin ;" the third nerve issuing in front of the pons, and the last or hypoglossal stretching back to the hind end of the bulb. Part, indeed, of the eleventh nerve, the spinal accessory nerve properly so-called, makes connections with the spinal cord below the bulb as far back as the sixth or seventh cervical nerve, or even lower; but this part may by these connections be distinguished from the remaining part of the nerve, as well as from all other cranial nerves. The nuclei to which the nerve-roots may be traced within the brain substance, sometimes spoken of as the "deep origin," range in position from the hinder part of the bulb to the hind end of the third ventricle. The nucleus of the hypoglossal nerve begins in the bulb just above the decussation of the pyramids, the nucleus of the third nerve ends beneath the hind end of the floor of the third ventricle; and all the rest of the nuclei may be broadly described as placed between these limits in various parts of the floor of the central canal or in adjoining structures, though part of one nucleus, namely, that of the fifth nerve, can be traced, as we shall see, back into the spinal cord as far as the second cervical nerve, and probably extends still further. Fig. 138 is a diagram showing in a roughly approximate manner the nuclei of the several nerves as they would appear in a bird'seye view of the floor of the aqueduct and fourth ventricle looked at on the dorsal aspect.

$\$ 529$. The twelfth or hypoglossal nerve. The nucleus of this nerve, which it will be convenient to take first (Fig. 138, XII.), is a long column of gray matter lying in the bulb parallel to, and very close to, the median line. It reaches from the hinder part of the fourth ventricle at about the level of the hind end of the auditory nucleus, as far back as beyond the hind end of the olivary body. At its extreme hind end or beginning (Fig. 132, 2) it occupies a ventral position and is a part of the anterior horn; thence it gradually rises dorsally (Fig. $132,3,4,5$ ), but so long as the central canal remains closed, continues to occupy a distinctly ventral position in reference to the central canal; in its front part it is, by the opening up 
of the fourth ventricle, brought into an apparently more dorsal position (Fig. 132, 6).

The nucleus consists mainly of large nerve-cells with distinct axis-cylinder processes, which though pursuing a somewhat irregular course may be traced into the fibres of the nerve. These, starting from the ventral surface of the nucleus along its length, run ventrally through the reticular formation, and making their way in a series of bundles, between the olivary nucleus on the lateral side and the pyramid and median accessory olive on the median side, gain the surface along the groove which separates the pyramid from the olivary body.

$\S 530$. The ninth or glosso-pharyngeal, tenth or vagus, and eleventh or spinal accessory nerves. It will be advantageous to consider these three nerves together.

In the spinal accessory nerves we must distinguish, as we have said, two parts: the "spinal accessory" proper, formed by the roots which come off from the cervical spinal cord, reaching as far down as the sixth or seventh cervical nerve, and the "bulbar accessory," whose roots come off from the bulb just below the vagus.

The spinal accessory proper takes origin in the group of cells lying in the extreme lateral margin of the anterior horn, from whence the fibres proceed directly outward through the lateral column, and issue from the cord along a line immediate between the anterior and posterior roots; the upper roots undergo, with the portion of the lateral horn from which they spring, the shifting spoken of in $\$ 518$.

The bulbar accessory starts from an elongated nucleus in the bulb which is common to it, to the vagus, and to the glosso-pharyngeal; hence we have taken these three nerves together. This (Fig. 138) stretches further forward than the hypoglossal nucleus, reaching the level of the transverse fibres called striæ acusticæ (str.), but does not extend so far behind.

In transverse sections of the bulb, which pass a little below and a little above the point of the calamus scriptorius (Fig. 132, 4, 5), two nuclei or collections of cells are seen in the gray matter round the central canal. The more ventral one is the hypoglossal nucleus, the more dorsal one the beginning or hind part of the combined accessory-vago-glosso-pharyngeal nucleus.

When a little further forward the central canal opens out into the fourth ventricle (by which change the hypoglossal nucleus (Fig. 132, 6 n. xii.) is brought nearer to the dorsal surface in the floor of the fourth ventricle) this combined nucleus, increasing in breadth, is thrown to the side and assumes a more lateral position, lying now on the side of, but still somewhat dorsal to, the hypoglossal nucleus, between it and the now diminishing gracile nucleus. In this position the nucleus appears to consist of two parts, a median and lateral, the median part having conspicuous nerve-cells of moderate size, the lateral part having but few cells and those of small size. From this level the nucleus runs forward, maintaining nearly the same position in the floor of the fourth ventricle, but gradually becoming thinner, and ends, as we have said, at about the level of the striæ acusticæ on the dorsal surface corresponding on the ventral surface to a level a little behind the hind margin of the pons.

From this combined nucleus, but chiefly from the median part, fibres sweep in a ventral and lateral direction through the dorsal part of the reticular formation, pass ventral to, or in some cases through, the gelatinous substance and the strand of fibres connected with the fifth nerve (Fig. 132, $V$. a), and reach the surface of the bulb on its lateral aspect in a line between the olivary and restiform bodies (Fig. 131, C.). Along this line may be seen 
(Fig. 131, C.), a series of roots ; of these the lowest, the accessory roots, spring from the hind part, the highest, the glosso-pharyngeal roots, from the front part (and it is these especially which pierce the gelatinous substance-Fig. $133, I X . a)$, and the intermediate, the vagus roots, from the middle part of the combined nucleus. Hence we may speak of the hind part of the whole nucleus as being the accessory nucleus, the middle part as the vagus nucleus, and the front part as the glosso-pharyngeal nucleus.

All the fibres, however, of the roots of these three nerves do not take origin from the nucleus in question; some of the fibres start in a different way. In sections of the bulb above the decussation of the pyramid a patch of gray matter is seen lying in the lateral part of the reticular formation (Fig. 132, X. m.), about midway between ventral and dorsal surfaces. What is thus disclosed by sections is a column of gray matter, the "nucleus ambiguus" (Fig. 138, $n$ a.), stretching about as far forward and backward as the combined accessory-vago-glosso-pharyngeal nucleus, but placed distinctly more ventrally and somewhat more laterally. (In Fig. 138, it and the combined nucleus are represented on different sides of the diagram to avoid confusion through the overlapping of the shading.) From it fibres curve round (Fig. 132, X. m.) to join the accessoryvago-glosso-pharyngeal roots, but especially the vagus roots. It may, therefore, be considered as a second nucleus of the vagus (and possibly of the other) roots.

But there is yet a third source of some of the fibres of the nerves of which we are speaking. In sections through the bulb there may be seen just ventral to and a little lateral to the combined nucleus (Fig. 132, 4, 5, 6, IX. a.) the circular section of a longitudinal bundle of fibres. In the hinder sections (Fig. 132, 4) the bundle is a very thin one, and still further back it is lost to view, though there are reasons for thinking that some of the fibres are continued back into the cervical cord, as far as the origin of the fourth cervical nerve or even beyond; in the more forward sections (Fig. 132, 5 and 6 ) it increases in diameter and may be traced forward to the front end of the combined nucleus into which it merges. It is a bundle of fibres which, starting successively in the lateral gray matter of the cervical cord and higher up in the reticular formation of the bulb, run longitudinally forward; the bundle at first increases in size by the addition of fresh fibres at each step; but further forward the fibres leave the bundle to pass into the roots of the nerves of which we are speaking, especially of the glosso-pharyngeal, and the bundle eventually ends in front by passing into the glosso-pharyngeal roots. The gray matter from which these fibres take origin does not form a defined compact area, is not, therefore, a nucleus in the sense in which we are now using the term, but is diffused among the rest of the gray matter along a considerable length. The fibres are, nevertheless, fibres of nerve-roots, and the bundle is called the ascending root of the glosso-pharyngeal, the term ascending being used since it is customary to trace such structures from below upward, that is, from behind forward; though since the fibres in question are probably afferent fibres carrying impulses backward from the nerves to the gray matter, "descending" would be the more appropriate word. The bundle has also been called the fasciculus solitarius; and, since its position has been supposed to correspond to that of the area marked out experimentally as the respiratory centre ( $\$ 304)$, it has been spoken of as the respiratory bundle.

The roots of these three nerves, then, the bulbar accessory, the vagus, and the glosso-pharyngeal-all leaving the surface of the brain along the line between the olive and the restiform body, and all so far alike that it is impossible upon mere inspection to say where in the series the fibres of 
the middle nerve, the vagus, begin and end-spring from three sources, the combined nucleus, the nucleus ambiguus, and the ascending root.

$\$ 531$. The eighth or auditory nerve. This nerve differs from the other nerves which we are now considering, in being a nerve of special sense; its arrangements are complicated. In a view of the base of the brain. (Fig. $131, C$ ) the nerve is seen to leave the surface of the brain from the ventral surface of the fore part of the restiform body at the hind margin of the pons as two strands or roots, one of which winds round the restiform body so as to reach its dorsal surface, while the other appears to sink into the substance of the bulb to the median side of the restiform body; and in a transverse section of the bulb (Fig. 133), just behind the pons, the two ruots may be seen embracing the restiform body, one passing on its dorsal and the other on its ventral side. The former is called the dorsal root (Fig. 133), or sometimes the lateral root, or, since it reaches back or lower down than the other, the posterior or inferior root; the latter is called the ventral root (Fig. 135), or sometimes the median root, or, since it reaches further forward or higher up than the other, the anterior or superior root. When we come to study the ear we shall find that one division of the auditory nerve is distributed to the cochlea alone, and is called the nervus cochlearis, the rest of the nerve being distributed to the utricle, saccule and semicircular canals as the nervus vestibularis. As we shall see, there are reasons for thinking that the vestibular nerve carries up to the brain from the semicircular canals impulses other than those, or besides those, which give rise to sensations of sound, whereas, the cochlear nerve appears to be exclusively concerned in hearing; and in some structural details these two divisions of the auditory nerve differ from each other. Hence it is important to note that according to careful investigations the cochlear nerve is the continuation of the dorsal root and the vestibular nerve the continuation of the ventral root. With these roots of the auditory nerve proper also issues, a little in front of the ventral root, the small nerve called the portio intermedia Wrisbergi, which goes to join the facial nerve.

The auditory nucleus, as a whole, is a broad mass, having in transverse sections of the bulb a somewhat triangular form, lying in the lateral parts of the floor of the fourth ventricle, reaching in front somewhat beyond the level of the striæ acusticæ, and overlapping behind the front parts of the nucleus ambiguus and the combined accessory-vago-glosso-pharyngeal nucleus: it extends laterally some distance outside the former nucleus.

The nucleus, however, consists of two distinct parts, a median or inner nucleus (Fig. 138, VIII., m.), characterized by the presence of small cells, and a lateral or outer nucleus (Fig. 138, VIII., l.), the cells of which are much larger, some of them being very large. The lateral nucleus is placed somewhat deeper than, ventral to, the median nucleus; it also extends farther forward (Figs. 133 and 134, VIII., $\beta$ ), so that the front end of the whole nucleus is furnished by the lateral nucleus alone, which at its front end occupies a more dorsal position than at its hind end.

Moreover, this auditory nucleus thus placed in the floor of the fourth ventricle is not the whole of the nucleus of the auditory nerve. At the convergence of the dorsal and ventral roots on the ventral surface of the restiform body is placed a group of cells, forming a swelling which, in its general appearance and in the characters of its cells is not unlike a ganglion on the posterior root of a spinal nerve. This is called the accessory nucleus.

When we trace the fibres of the nerve centralward into the brain, we find that a large number, at least, of the fibres of the dorsal root, cochlear nerve (Fig. 133), end, according to most observers, in the cells of the accessory 
nucleus or in nerve-cells lying dorsal to the accessory nucleus, and especially in a group of cells giving rise to the tuberculum acusticum, which, small in man, is conspicuous in some animals. Hence, the farther part of this dorsal root, as it winds round the lateral and dorsal surface of the restiform body, consists largely, if not wholly, of fibres which are derived not directly from the trunk of the nerve, but indirectly through the relay of the accessory nucleus or of other cells. Reaching the dorsal surface of the restiform body, these fibres appear on the floor of the fourth ventricle as the strice acustica (Fig. 131, str.), and end partly in the median nucleus, partly in other regions of the bulb. The exact determination, however, of the endings of this root

FIG. 134.

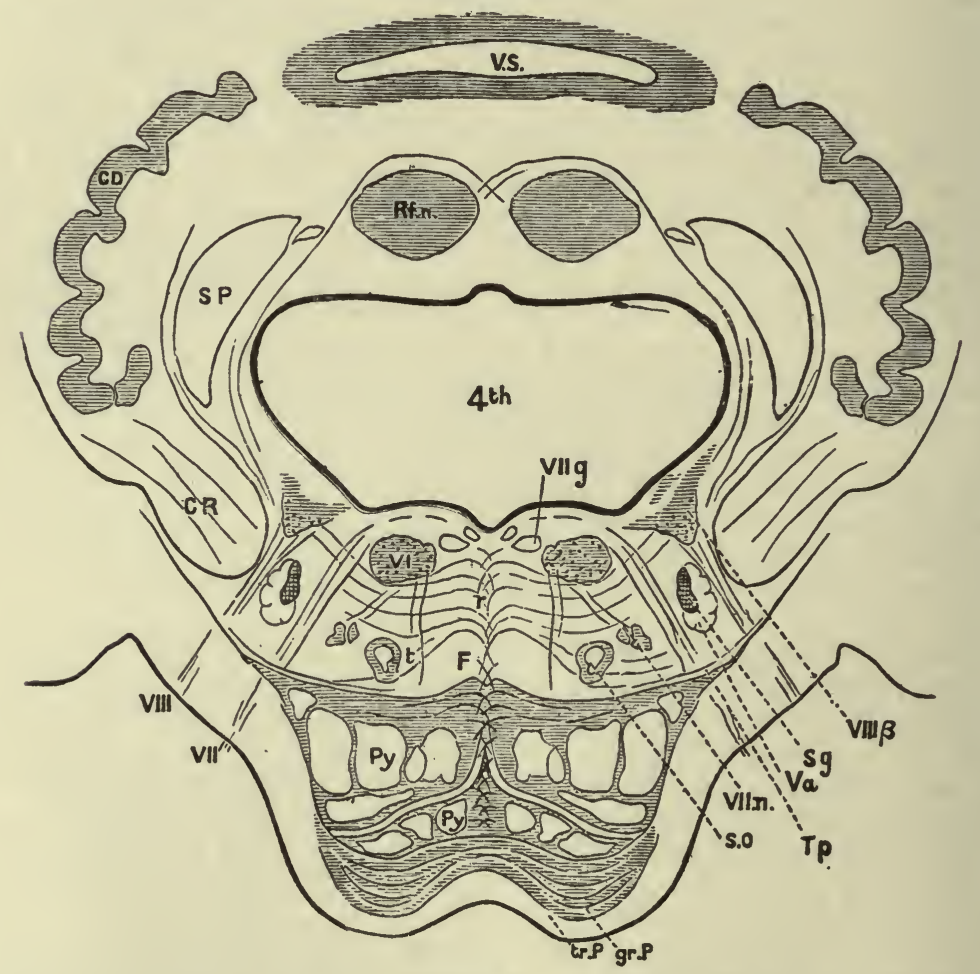

Through the widest part of the Fourth Ventricle. (Sherrington.) Taken in the line 111, Fig. 131. $P y$., pyramidal fibres cut transversely ; $t r . P$., the superficial (ventral) transverse fibres of the pons. The shaded part of the pons ( $g r . P$.) indicates gray matter mingled with the deeper transverse fibres. $F$, the fillet: $T p$., the trapezium: $C . R$., the restiform body or inferior peduncle of the cerebellum, cut across obliquely : $S$. $P$., the superior peduncles of the cerebelluin; $r$., raphe ; s. o., superior olive; $C$. D., corpus dentatum of the cerebellum; $R f . n$., the nucleus of the roof; s. $g$., tubercle of Rolando; V.S., section through sulcus in the vermis superior of the cerebellum , $t$., bundle from the olive to the lenticular nueleus: VIII., the eighth or auditory nerve, its ventral or vestibular root proceeding from $V I I I . \beta$. the front part of the lateral auditory nucleus; VII. $n$., the nueleus of the seventh or facial nerve; VI., the nucleus of the sixth nerve; VII. $g$., fibres of the seventh nerve cut across as they sweep round the nucleus of the sixth before issuing from the pons at VII.; 4th, the fourth ventricle, here roofed in by the cerebellum; the shading of the central gray matter immediately surrounding the ventricle is, for the sake of simplicity omitted.

is a matter of considerable difficulty; some observers regard the accessory nucleus as homologous, not with the Gasserian and with the spinal ganglia, 
but with the other, true, cranial nuclei ; and in any case we must probably consider the median division of the auditory nucleus, not as a nucleus in the sense in which we are now using it, but rather as a secondary connection within the bulb.

When we trace the ventral root, vestibular nerve (Fig. 134), inward, we find that it makes, according to most observers, no connections at all with the accessory nucleus; and that it passes (Fig. 134, VIII.) to the median side of the restiform body, between it and the ascending root of the fifth nerve, and so reaches the lateral division of the nucleus, in the large cells of which most at least of its fibres are said to end, and which, therefore, may be regarded as the nucleus of the ventral root. On this point, however, all authors are not agreed. The lateral auditory nucleus, with the fibres proceeding to and from it, lying as they do to the median or inner side of the restiform body proper, are sometimes spoken of as the median or inner division of the restiform body; and from the nucleus a considerable number of fibres pass up with the restiform body into the cerebellum as a continuation of this "median division of the restiform body." Some authors maintain that these fibres are continued straight on from the nerve to the cerebellum; but the more recent investigations seem to show that they all make connections with the nerve-cells of the lateral nucleus on their way. These fibres constitute a connection between the auditory (vestibular) nerve and the cerebellum, the physiological significance of which we shall see later on ; we may, perhaps, compare it to the connection between the posterior roots of the spinal nerves and the cerebellum through (the vesicular cylinder and) the cerebellar tract.

The other central connections of the lateral nucleus are, like those of the accessory and of the median nucleus, complicated and obscure. But we may call attention to a set of fibres which, starting apparently in the accessory nucleus, run directly transverse in the ventral region of the tegmentum just dorsal to the transverse fibres of the pons, forming what is called the trapezium (Fig. 134, Tp.).

Lastly, we may add that the fibres of the peculiar portio intermedia appear to take origin from the accessory nucleus.

$\$$ 532. The seventh or facial nerve. The nucleus (Fig. 138, VII., and Figs. 133, 134, VII. n.) of this nerve (it being borne in mind that the motor fibres for the orbital region (the orbicular muscle, etc.), though they run in the trunk of this nerve, really belong to the third nerve and take origin from the hind part of the nucleus of the third nerve) is narrower in front than behind, reaches from the level of the striæ acusticæ some distance into the region of the pons, and occupies in the midst of the reticular formation, a little dorsal of the patch of gray matter called the upper olive, a position corresponding closely to that of the nucleus ambiguus. The cells of the nucleus are large, and possess well-marked axis-cylinder processes, which are gathered up at the dorsal surface of the nucleus to form the root. This, rising up dorsally, describes a loop (Fig. 134, VII. g.) round the nucleus of the sixth or abducens nerve, running forward for some little distance dorsal to that nucleus, and then descends again ventrally, passing to the lateral side of its own nucleus, between it and the ascending root of the fifth (V.a.); it thus gains the surface of the brain at the hinder margin of the pons, lateral to the abducens, opposite the front end of the groove between the olivary body and the restiform body. As it thus encircles the nucleus of the abducens, it looks as if it were receiving fibres from that body; but the evidence goes to show that these fibres simply pass through the nucleus, and do not take origin from any of its cells.

$\S 533$. The sixth or abducens nerve. This nerve starts from a compact oval 
nucleus (Fig. 138, VI.), lying at the level of the hinder part of the pons, and therefore of the front part of the fourth ventricle, in the central gray matter of the floor of the ventricle, or rather just between it and the reticular formation, a little on one side of the median line (Fig. 134, VI.). A slight swelling of the floor of the fourth ventricle, eminentia teres, marks its position (Fig. 138, e.t.). The nucleus contains fairly large nerve-cells, with distinct axis-cylinder processes. These are gathered at the median side of the nucleus to form the thin root, which, passing ventrally and laterally, at some little distance from the median raphe, through the reticular formation, runs backward above the pyramidal bundles of the pons, and finally comes to the surface at the hinder edge of the pons, opposite the front end of the pyramid (Fig. 131, C.).

FiG. 135.

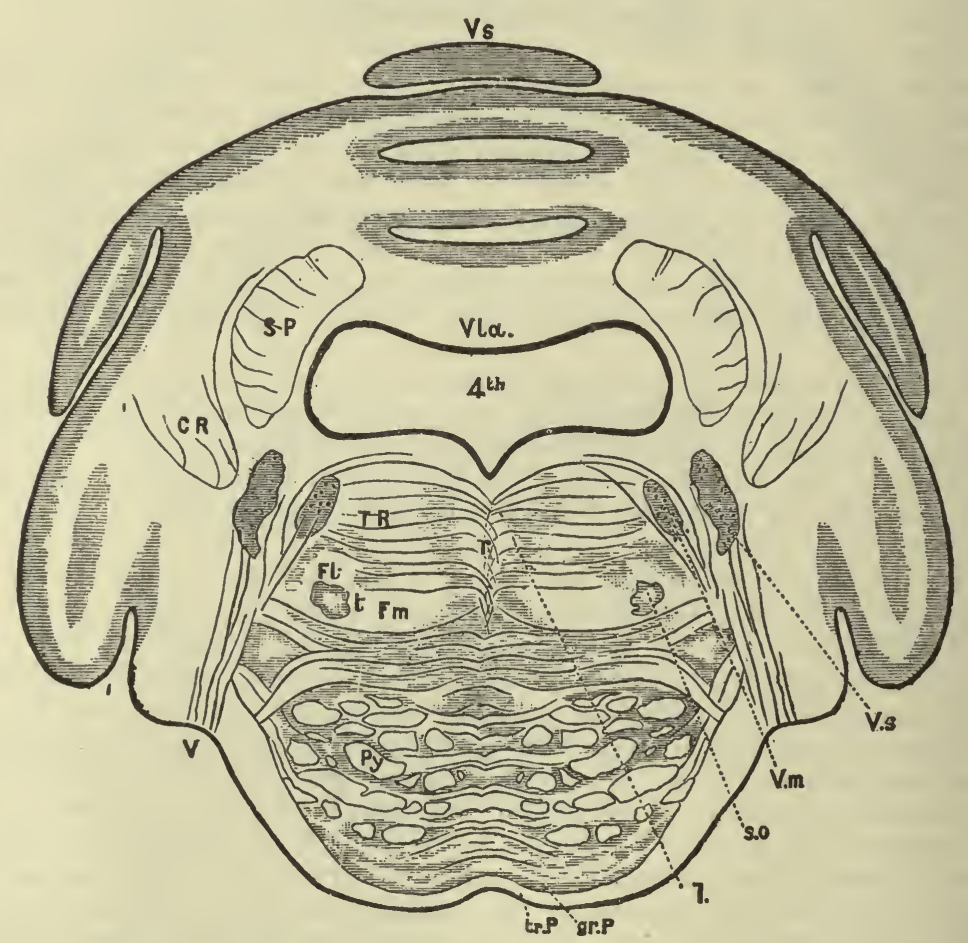

Through the Pons at the Exit of the Fifth Nerve. (Sherrington.) In the line 112, Fig. 131. $C$. $R$. remains of restiform body; S. P. superior peduncle of the cerebellum; $F . m$. median; $F . l$. lateral fillet; T. R., tegmental reticular formation; tr. $P$. superficial transverse fibres of the pons ; $l$. posterior longitudinal bundles; V.s. superior vermis (sections of three folia are shown. one being detached; between them the intervening sulci laid open by the section are seen); I'la. valve of Vieussens or anterior velum: $r$. raphe; $P y$. pyramidal fibres; $g r$. $P$. gray matter of the pons; s. o., superior olive; $t$., placed on the left side indicates the position of a bundle of longitudinal fibres which may be traced forward into the subthalamic regions; $V . m$. motor nucleus $V$. s., sensory nucleus; and $V$. roots of the fifth nerve. 4 th, fourth ventricle; shading of central gray matter omitted as in Fig. 134.

$\S 534$. The fifth or trigeminal nerve. This nerve, as it comes to the surface on the ventral aspect of the pons (Fig. 131, C.), near the front edge, at some distance from the median line, consists of two parts, a smaller motor 
root and a larger sensory root, the latter bearing the large ganglion of Gasser; and the origin of the nerve is in many ways complex. Both roots may be traced in an oblique direction (Fig. 135, V.), inward and toward the dorsal surface, through the pons to the reticular formation beneath the floor of the front part of the fourth ventricle, the smaller motor root taking up a position median to the larger sensory root.

Here the motor root comes into connection with a collection of nervecells (Figs. 138 and 135, V.m.), which may be regarded as its nucleus; but this is not the whole nucleus of the motor root. From the level of the nucleus there stretches forward as far as the level of the anterior corpora quadrigemina a bundle of longitudinal fibres which, since it is usually traced from the front backward until it passes into the root of the nerve, is spoken of as the descending root of the fifth nerve.

This descending root begins as a few scattered bundles of fibres at the level of the anterior corpora quadrigemina, in the peripheral lateral part of the central gray matter surrounding the aqueduct, dorsal, and lateral (Fig. 137, V.d.), to the nucleus of the third nerve (Fig. 137, III. n.). From thence the fibres pass backward, augmenting in number, and soon form a compact bundle, semilunar in transverse section, lying lateral to the fourth nerve as this is rising dorsally (Fig. 136, V.d.) ; still increasing in

\section{FIG. 136.}

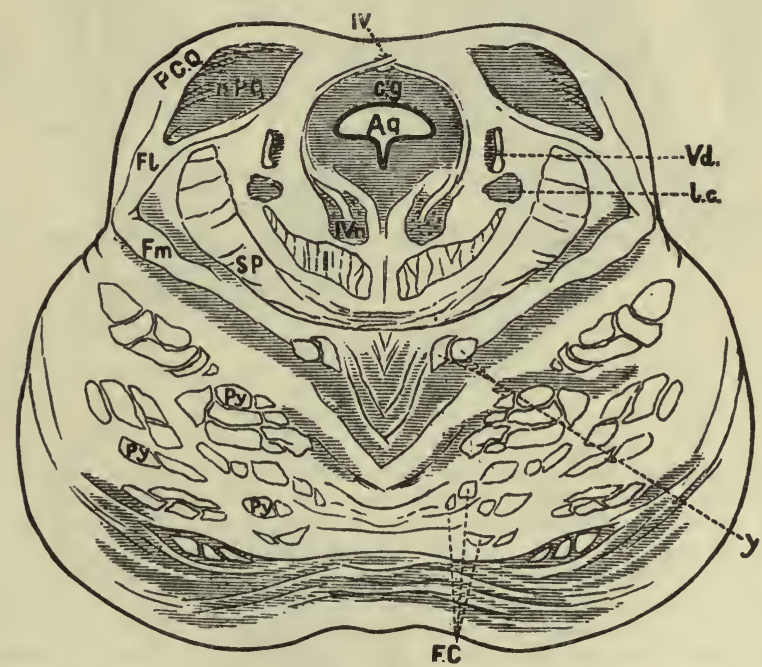

Through the Fore Part of the Pons. (Sherrington.) In the line 113, Fig. 131. Py. pyramidal fibres; $F$. C. fibres from the frontal cortex; S. P. superior peduncle of the cerebellum; Fm. median portion; $F l$. lateral portion of the fillet; $l$. posterior longitudinal bundles; $P . C$. Q. posterior corpora quadrigemina; $y$. fibres which become detached from the fillet, and further forward form (the innermost) part of the pes of the crus; $l$. $c$. locus cæruleus; $n . P$. Q. nucleus of the posterior corpora quadrigemina-the outline is made toosharp: $I V$. bundles of the fourth nerve decussating, $I V . n$. its nucleus; V. $d$. descending root of the fifth nerve; $A q$. the aqueduct; $c . g$. the region of central gray matter.

number in their course backward, they gradually assume a more ventral position as the aqueduct opens into the fourth ventricle. All along its course this descending root has attached to it large $(70 \mu$ or more in diameter), sparse spheroidal nerve-cells of striking appearance; these, however, seem too few to give origin to all of the fibres, and there are some reasons 
for connecting this root with the collection of gray matter called "locus cæruleus." (Fig. 136, l. c.).

We may probably regard this descending root as belonging to the motor division of the nerve ; but it is stated that many of the fibres of this root pass into the sensory root, eventually finding their way, according to some observers, into the ophthalmic branch.

The sensory root may be similarly traced into a nucleus, the sensory nucleus (Figs. 138 and 135, V. s.) lying lateral to the motor nucleus, and connected with this is the striking tract of fibres, to which already we have so frequently alluded, and which is called the ascending root of the fifth nerve.

This ascending root begins as a bundle or bundles of few fibres which may be traced backward as far, at least, as the level of the second cervical nerve, and is soon conspicuous in transverse sections (Figs. 132 et seq., V.a.) as a semilunar patch of white matter forming a sort of cap on the outside of the swollen caput of the posterior horn, between this structure and the longitudinal fibres which are beginning to form the restiform body on the

FIG. 137.

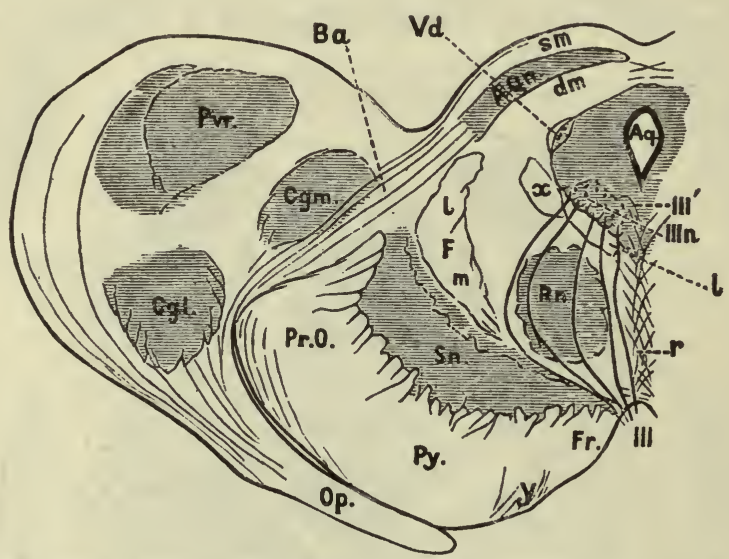

Through the Crus and Anterior Corpora Quadrigemina. (Sherrington). One-half only is shown in the line 114, Fig. 131. Py. the pyramidal portion of the pes: Fr. the region of the pes occupied by fibres from the frontal portion of the cortex; $\mathrm{Pr}$. $O$. the region occupied by fibres coming from the occipital portion of the cortex; $y$. fibres coming from the fillet; Op. the optic tract; $F$. the fillet, $l$. the lateral portion, $m$. the median portion; $l$. the posterior longitudinal bundle; $B . a$. the brachium of the anterior corpus quadrigeminum; $x$. fibres from the posterior commissure of the cerebrum; $r$. raphe; S. $n$. substantia nigra; $R$. $n$. red nucleus; $C . g$. l. lateral, and $C . g . m$. median corpus geniculatum; Pvr. pulvinar of optic thalamus; $A . Q . n$. nucleus or gray matter of anterior corpus quadrigeminum ; III. $n$. nucleus of III. third nerve; III.' rootlets from the dorsal part of III. $n$. the nucleus of the third nerve, which cross the median line to emerge with rootlets derived from the nucleus of the opposite side; s. m. superficial layer of fibres of the ant. corp. quad.; $d$. $m$. deep layer: $A q$. aqueduct surrounded by cerebral gray matter.

surface. Passing upward, and continually augmenting in bulk, the root clings, as it were, to the gelatinous substance of the caput of the posterior horn, and sinks with it inwardly and ventrally as this becomes covered up first by the restiform body and subsequently by the issuing trunk of the great eighth or auditory nerve (Figs. 133, 134). Passing still forward, beyond the disappearing gelatinous substance, the root, still growing larger and divided into several distinct bundles, runs into the reticular formation of the pons and, reaching the level of the sensory nucleus, suddenly bends rounds and joins the sensory root. 
Fig. 138.

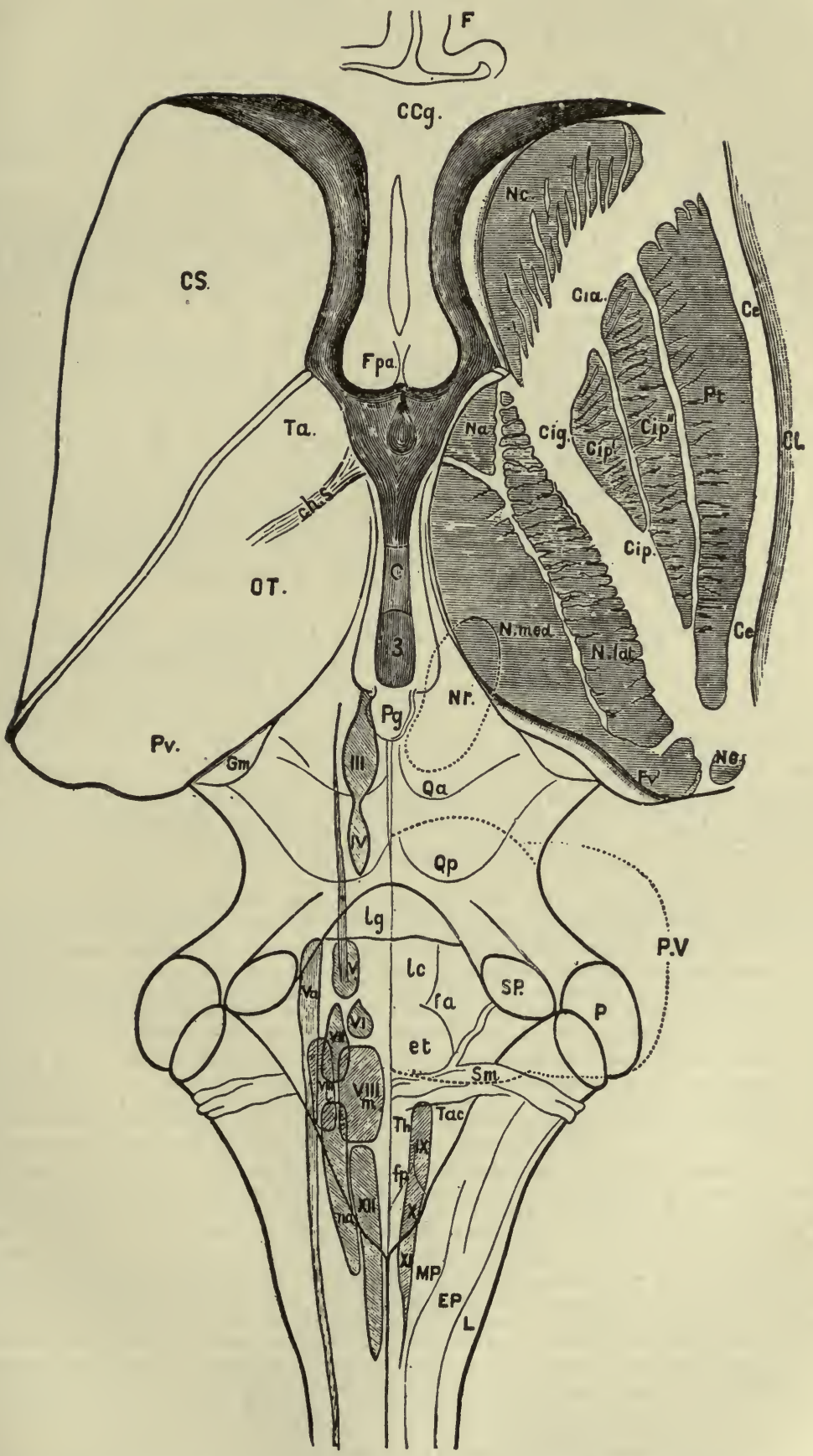

Diagram to illustrate the Position of the Nuclei of the Cranial Nerres. (Sherrington.) 'The brain is supposed to be viewed from the dorsal aspect, the cerebral hemispheres and cerebellum 
This ascending root differs from the descending root in not having conspicuously attached to it any collection of nerve-cells; in this respect it resembles the ascending root of the glosso-pharyngeal, and we may add part of the posterior ront of an ordinary spinal nerve, the fibres of which, as we have seen, pass into the gray matter without being obviously connected with nerve-cells. In its lower part at least it consists of extremely fine fibres, and indeed looks very much like a continuation of the bulb of the marginal (Lissauer's) zone of the spinal cord.

$\$ 535$. The fourth or trochlear nerve. The nucleus of this nerve (Fig. $138, I V$.$) is a column of somewhat large multipolar cells on each side of the$ median line below the aqueduct (Fig. 136, $I V . n$.), reaching from the level of the junction of the anterior and posterior corpora quadrigemina to the hinder level of the latter body.

The root, starting from the lateral surface of the nucleus, does not take at first a ventral direction, but sweeps laterally and dorsally in the outer layers of the central gray matter (Fig. 136), and so curving round to the dorsal surfaces reach the valve of Vieussens, where in the median line it decussates with its fellow in the substance of the valve; such a decussation at a distance from the nucleus of origin is exceptional in the cranial nerves. Leaving the surface of the brain in the valve, it takes a superficial course, curving (Fig. 131, B) laterally and ventrally, and makes its appearance in a ventral view of the brain at the front edge of the pons, on the lateral edge of the crus (Fig. 131, C.).

$\S 536$. The third or oculo-motor nerve. The nucleus of this nerve (Fig. $138, I I I ., 137, I I I . n$.) is a column of, for the most part, fairly large multipolar cells lying on each side close to the median line, in the gray matter of the central canal, just dorsal to a bundle of fibres which we shall speak of as the longitudinal posterior bundle; it reaches from the level of the posterior commissure in the third ventricle to the level of the junction of the anterior and posterior corpora quadrigemina. In a section taken through its middle (Fig. 137) the nucleus is seen to give off fibres which run vertically toward the ventral surface, traversing the tegmentum and a body $(R n$.)

having been cut away. The nuclei are represented as if seen through transparent material. On the right side, the corpus striatum and optic thalamus have been cut away horizontally to some little depth in order to show their internal structure. $L$. lateral, $E$. $P$. external posterior, and $M$. $P$. median posterior column of the cord ; $I$. $P$. inferior peduncle, S. $P$. superior peduncle, and $P$. middle peduncle of the cerebellum, all cut across. The dotted curved lines, upper and lower, on the right half of the figure to which the dotted line $P$. $V$. outside the figure points, mark the upper and lower boundaries of the pons on the ventral aspect. The outline of the fourth ventricle is shown by a bold thick line. In the floor of the ventricle are shown, on the right half: $f p$. fovea posterior; $T h$. trigonum hypoglossi ; $T$. ac. trigoumm acusticum; e.t. eminentia teres: $s . m$. striæ medullares on acusticæ; $f . c$. fovea anterior; $l$. $c$. locus cæruleus; $l . g$. valve of Vieussens; $Q p$. posterior, and Qa. anterior corpus quadrigeminum; $P g$. pineal gland; $N r$. the outline of the red nucleus; 3 , the third ventricle, in which $C$. indicates the middle or soft commissure; $F$. p. a. the pillars of the fornix, behind which is indicated in the cavity of the third ventricle the hollow of the infundibulum; C. C. g. the genu of the corpus callosum, between which and the fornix the cavity uften called the fifth ventricle is indicated; $F$. portion of convolution of frontal hemisphere cut across. On the left side are shown: C.S. corpus striatum; O. T. optic thalamus; $P v$. pulvinar; $T$. a. tuberculum anterius; $c h$. s. choroidal sulcus marking the place of reflection of the choroidal plexus. On the right side are exposed: $N$. C. head of, Nc. end of tail of nucleus caudatus; Cip'., Cip". the two parts of the globus pallidus, and Pt. putamen of the nucleus lenticularis; $N$. $a$. anterior nucleus: $N$. med. medium nucleus, $N$. lat. lateral nucleus, and $P v^{\prime}$. pulvinar of the optic thalamus; Cia. front limb, Cig. knee or genu, Cip. hind limb of internal capsule; $\mathrm{Ce}$. external capsule ; $C l$. claustrum. The numcrals III. to XII. indicate the nuclei of the respective cranial nerves, all shown on the left side with the exception of the accessory vago-glosso-pharyngeal IX.. X., IX., which to avoid confusion is placed on the right side. $V$. is the motor nucleus of the fifth nerve with the descending root, $V . a$. the sensory nucleus of the same with the long ascending root VIII. $m$. median nucleus, VIII. $l$. lateral nucleus of the auditory nerve: $n . a . n u-$ cleus ambiguus. The ascending root of the ninth nerve is seen at the hind end of the combined nucleus of IX.,X., XI. 
which we shall presently speak of as the "red nucleus," but apparently making no connections with these structures, and pierce the median edge of the pes, emerging (Fig. 131, C.) on the surface to the median side of each crus. As we shall see later on, this nerve is now exclusively efferent, whatever it may have been in more primitive beings. We shall also see later on that impulses starting from the cerebrum of one side pass to the nerve of the other side, that is to say, decussate; and this is also the case with the other efferent cranial nerves. 'The fibres which appear to take origin from the nerve-cells of the nucleus do not cross over after emerging from the nucleus, but keep to the same side; there is no distant decussation as in the case just noted of the fourth nerve. There are, however, fibres (Fig. 137, $I I I^{\prime}$.) which leaving the nucleus cross the median raphe from one side to the other, and these possibly are the paths for the decussation of the impulses; but they may be fibres passing from the crus across the raphe to the nucleus. This nerve has special relations with the optic tract, but of these we shall speak when we come to deal with the functions of the nerves.

$\$ 537$. In attempting to understand the nature and relations of these cranial nerves, it must be borne in mind that, while morphological studies lead us to believe that, as the vertebrate body has been developed out of an invertebrate ancestry, so the briin of the vertebrate has arisen by a series of modifications from the nervous structures placed at the head and around the mouth of an invertebrate, the same studies teach us that such an evolution has been accomplished by means of profound changes. We have, for instance, reason to think that the mouth of the vertebrate does not correspond to the mouth of the invertebrate, but is a new structure, whose appearance has been accompanied by a considerable dislocation of parts. We must accordingly expect to find the indications of a segmental arrangement greatly obscured on the one hand by transposition, and on the other by fusion.

The twelfth or hypoglossal nerve is one whose nature seems fairly simple. It is in function exclusively an efferent nerve. The large cells, with conspicuous axis-cylinder processes, which characterize its nucleus, are exactly like those of the anterior horn of the spinal cord which give origin to the fibres of an anterior root. The nucleus, moreover, in its position corresponds to part of the anterior horn of the spinal cord, if we take into account the shifting involved in the decussation of the pyramids, and in the new developments of the bulb. If we compare Fig. 132 with any section of the cord, we see that the hypoglossal nerve corresponds to an anterior root of the spinal cord, but that the fibres, after leaving the cells from which they take their origin, traverse in the former a large tract and in the latter case a small tract of tissue. Whether the whole nerve corresponds to the fibres of several segments fused together or to those of one segment spread out longitudinally is, for our present purposes, of secondary importance.

Recognizing the hypoglossal nerve as the homologue of a spinal anterior root, we may go on to claim the nuclei of the third and fourth nerves as similar groups of cells of the anterior horn, giving rise to anterior roots. The position of the nuclei, the character of the cells, the function of the fibres, all support this view. The case is perhaps not so clear as that of the hypoglossal nerve, since there are reasons for thinking that these nerves have undergone in the course of evolution greater changes than has the hypoglossal nerve; still these reasons do not oppose the above conclusion.

The nucleus of the exclusively motor sixth nerve does not exactly correspond to those of the third and fourth in position; but we may probably place it in the same series with them. Thus we have in succession the third, fourth, sixth, and twelfth nerves, with their respective nuclei, as the anterior roots of nerves of their several segments. 
In the fifth nerve the dislocation and fusion spoken of above has introduced difficulties. The motor nucleus, with the fibres of the motor root to which it gives origin, has by some been considered as homologous to the series just described; but it is at once obvious that we cannot look upon this great fifth nerve as corresponding to one spinal nerve, with its anterior and posterior root, great as the superficial resemblance seems to be. The features of the remarkable ascending root forbid this. The fibres of this root may be traced back, as we have said, to the very beginning of the bulb, and, indeed, into the spinal cord beyond; as far as can be ascertained, they are not in an obvious and direct manner connected with nerve-cells along their course; but the bundle of fibres clings, as we have seen, to the gelatinous substance of the posterior horn of the spinal cord and to the continuation of this along the bulb, and the fibres are lost in this structure. The root, therefore, as we have said, corresponds very closely to part at least of the posterior root of a spinal nerve, and though the matter has not yet been experimentally proved, we may infer that the trophic centres of these fibres are to be found in the cells of the Gasserian ganglion.

But if the ascending root be of the nature of a posterior root (and we may incidentally remark that the term ascending has been unhappily chosen, since, if it be an afferent root, the direction of the impulses which it carries will be a descending one, namely, from the entrance in the pons toward the hinder parts), we can hardly suppose that it belongs to a single segment, or is the complement of the motor root alone; in it, most probably, the posterior fibres of several segments are blended together. Further, we may, perhaps, infer that the other fibres of the sensory root which end directly in what we have called the sensory nucleus, are in nature quite distinct from the fibres of the ascending root; and, if so, difficulties arise as to the nature and homologies of the nucleus in question. These, however, we must not discuss here, nor can we enter into the question of the nature of the descending root, concerning the fibres of which, as we have said, authorities differ as to whether they pass into the motor or sensory root. We have said enough to show that this fifth nerve is extremely complex, and that its apparent conformity to a simple spinal nerve is in reality misleading.

The fibres of the vagus, glosso-pharyngeal, and bulbar accessory, taken together, are partly efferent, partly afferent. The combined nucleus of these three nerves, the cells of which are small and devoid of conspicuous axiscylinder processes, is usually regarded as a sensory nucleus, and in the diagram (Fig. 138) is shaded accordingly. It may, perhaps, be compared to the sensory nucleus of the fifth. Thus, the ascending root, or fasciculus solitarius, presents many analogies with the ascending root of the fifth, and we are led to regard this as, like it, a gathering of certain afferent fibres of the posterior roots of several segments; in its case also the term ascending is misleading. But there are many difficulties in connection with this nucleus, as with the fifth. We must not enter into a detailed discussion concerning them, but may remark that we have here perhaps to deal with complexities due to the fact that certainly many vagus and glosso-pharyngeal fibres, and probably some of those of the fifth, are splanchnic in function.

The nucleus ambiguus contains large conspicuous cells and we may probably regard it as a motor nucleus, especially of the vagus fibres. We may also, perhaps, place it and the nucleus of the seventh nerve in the same category, and further class with them the motor nucleus of the fifth, looking upon all three as so many detached portions of gray matter, corresponding to some part of the anterior horn of the spinal cord. Whether they are exactly homologous to the hypoglossal nucleus, and their fibres to simple anterior roots, is not so clear. 
Lastly, the auditory nerve, both from its character as a nerve of special sense and from the remarkable features of its nuclei, is even more difficult. Most probably it results from the fision of more roots than one; but it is impossible at present to obtain a clear conception of the nature of the whole nerve.

\section{The Superficial Gray Matter.}

$\S 538$. The whole of the surface of each cerebral hemisphere for some little depth inward consists of gray matter, possessing special characters; this is called the cortical gray matter, or the cortex cerebri, or shortly and simply the cortex. As we shall see, by its histological and still more by its physiological features, it stands apart from all other kinds of gray matter.

The whole of the surface of the cerebellum is also covered with gray matter, which, while possessing features of its own, so far resemble the cerebral cortex, in its histological characters that it too has been spoken of as cortex, as the cortex cerebelli. By its functional manifestations, however, it differs widely from the cerebral cortex ; and since there are many advantages in being able to use the word cortex in connection with the cerebrum only, it is desirable not to speak of a cerebellar cortex but to employ the term "superficial gray matter of the cerebellum."

The third ventricle and the hinder part of the fourth ventricle are not roofed in by nervous material, and possess no superficial gray matter at all. In the corpora quadrigemina, which form the roof of the aqueduct or cavity of the mid-brain, gray matter is present and possesses, in the case of the anterior corpora quadrigemina at least, characters to a certain extent analogous to those of the cortex and to the cerebellar superficial gray matter; but it will be best to consider the gray matter of these bodies as belonging to another category.

\section{The Intermediate Gray Matter of the Crural System.}

$\S 539$. We have seen ( $\$ 516)$ that the crura cerebri form the prominent part of a system of longitudinal fibres stretching from each cerebral hemisphere to the bulb and to the spinal cord. This system of fibres, upon which we may consider the various parts of the brain to be, as it were, founded, we may speak of as the crural system. It is, it is true, not one continuous strand, but a number of different strands, having different beginnings and endings; but these all contribute to the crura and are so far alike as to justify us in considering them as a system. The cortical gray matter of each hemisphere is, as we shall see, connected with various parts of this system, and in one sense we may regard this system as beginning in the cortex of each hemisphere, and ending in the spinal cord. But certain masses of gray matter in the hemisphere not strictly cortical, and several important masses and areas of gray matter lying between the hemisphere and the cord, are connected with the system; and these we may speak of as the "intermediate gray matter of the crural system."

Corpus striatum and optic thalamus. Of all these several collections of gray matter, the largest, most conspicuous, and perhaps the most important are the two masses in the front part of the system known as the corpus striatum and optic thalamus. The former is, as we have seen ( $\$ 515)$, a development of the wall of the cerebral vesicle, the latter a development of the wall of the vesicle of the third ventricle. They are, therefore, of different origin; although in the course of the growth of the brain they become closely attached to each other, they are at the outset quite separate and distinct. Moreover, as we shall see, they differ from each other so essentially, in their nature and relations, that they cannot be considered as homologous bodies; and the term 
"basal ganglia" often applied to them is, therefore, unfortunate. Nevertheless, it will render the description of their topographical relations easier, if for a little while we consider them together.

When the lateral ventricle is laid open from above, part of the corpus striatum is seen projecting into the cavity of the ventricle. In front the projecting part is broad, forming the lateral wall and part of the floor of the ventricle, and to its median side lies the cavity of the ventricle, separated from its fellow by the septum lucidum. Further back the projecting part, becoming gradually narrower, assumes a more lateral position and passes into the descending horn. In this part of its course there lies on its median side, separated from it by a narrow band called the tænia semicircularis or stria terminalis, the optic thalamus, a narrow strip of the surface of which is seen projecting outside the edge of the choroid plexus. If now, both lateral ventricles be laid open by removal of the corpus callosum, and the fornix with the velum interpositum and choroid plexus be taken away, so as fully to expose the third ventricle, and also, in order to obtain a better view, the whole of the hinder part of the cerebrum containing the posterior horns of the lateral ventricle, be completely cut away, it is seen (Fig. 138) that the two optic thalami (O.T.) present themselves as two large oval bodies, placed obliquely athwart the diverging crura cerebri and converging in front to form the immediate walls of the third ventricle. In front and to the sides of the optic thalami are seen the corpora striata (C.S.) forming anteriorly the lateral walls of the two lateral ventricles, and diverging behind to allow of the interposition of the optic thalami. On each side of the brain, then, these two bodies, the corpus striatum and optic thalamus, appear as two masses of gray matter placed on the crus cerebri as this, diverging firom its fellow, begin to spread out into the cerebral hemisphere, the corpus striatum being placed somewhat in front of the optic thalamus. The relations of the two bodies, moreover, are such that while the optic thalamus alone forms the wall of the third ventricle to which it properly belongs, and the corpus striatum forms part of the wall of the lateral ventricle to which it in turn properly belongs, the optic thalamus also projects into and seems to form part of the wall of the lateral ventricle, though at its origin it had nothing to do with the cerebral vesicle.

We spoke just now of these bodies as being placed on the crura cerebri, but though their dorsal surfaces thus project from the dorsal surface of the diverging crura, a large portion of each body is, so to speak, imbedded in the substance of the diverging crus, and what is seen in the above surface view is only a part of each body, and, indeed, in the case of the corpus striatum, only a small part. In order to understand the nature and relations of these two important bodies we must study sections taken through a cerebral hemisphere in various planes (Figs. 139-146).

Each crus is made up, as we have seen, of a dorsal portion or tegmentum consisting largely of gray matter, and a rentral portion or pes consisting exclusively of longitudinally disposed fibres. The tegmentum ends partly in structures lying ventral to the thalamus, partly in the thalamus itself; and we may for the present leave this part of the crus out of consideration. The fibres of the pes, while continuing their oblique course forward and outward, soon rise dorsally by the side of the thalamus and hence, in a transverse dorso-ventral section at the level of the hind part of the thalamus (Fig. 139), are seen leaving their previous position ventral to the substantia nigra $\left(S_{n}^{\prime}\right)$ and passing ( Cip) by the side of the thalamus on their way to the central white matter of the hemisphere. In this part of their course they form a thick strand separating the thalamus (In.) from a large mass of gray matter which, roughly triangular in section, is divided by 
partitions of white matter into three parts $\left(G p^{\prime}, G p^{\prime \prime}, P t\right)$, and of which we shall speak directly as the nucleus lenticularis.

FIG. 139.

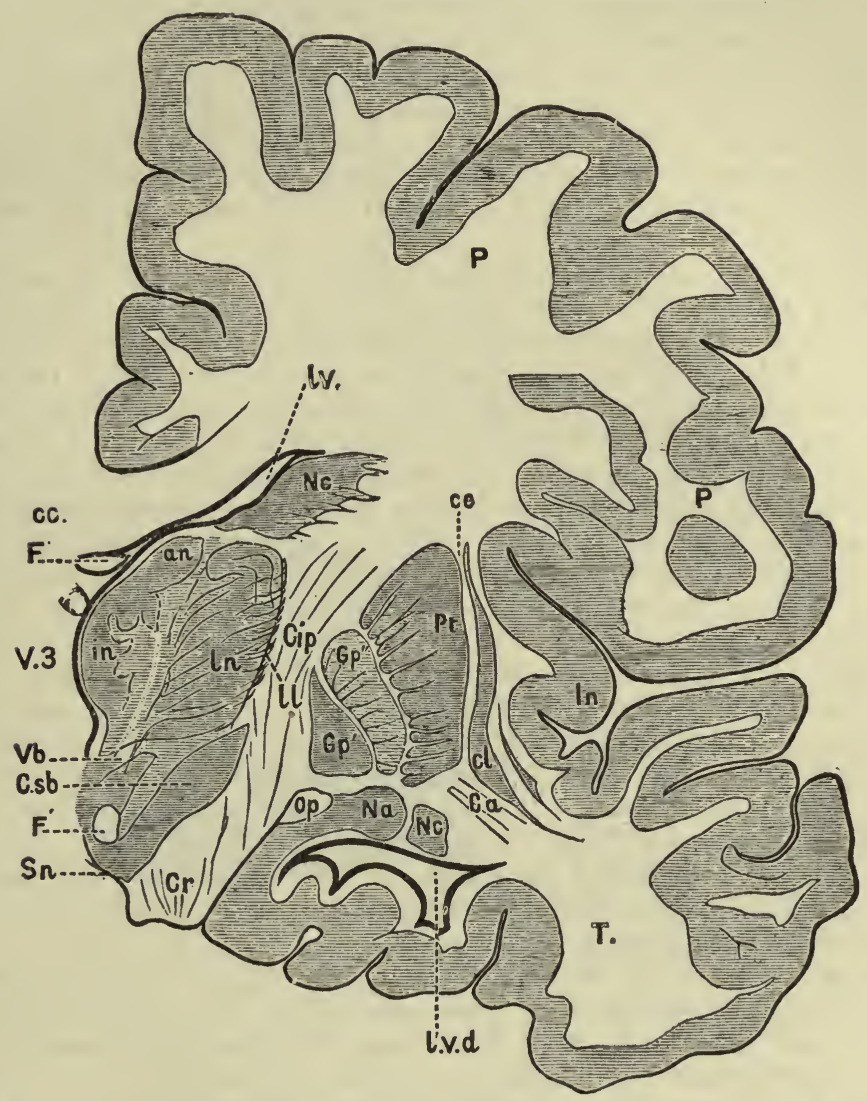

Diagrammatic Outline of a Transverse Dorso-ventral Section through the Right Hemisphere (Man), at Level Posterior to the Knee of the Internal Capsule. (Sherrington.) Natural size. Nc, nucleus caudatus; in the upper part of the figure the section of the nucleus is through the narrower portion which succeeds the wider front end or head; in the lower part of the figure the section passes through the tail of the nucleus near its end, and this portion of it has for the sake of clearness been sundered from the gray matter at $\mathrm{Na}$, nucleus amygdalæ, more distinctly than in reality is the case. $G p^{\prime}, G p^{\prime \prime}$, globus pallidus, seen here in two segments, and $P t$, putamen of nucleus lenticularis; an, the anterior; $i n$, the inner ; and $l n$, the lateral nucleus of the optic thalamus ; at $l$ is seen the "latticed layer" lying next to Cip, the posterior limb of the internal capsule aud containing many strands of fibres which mingle with it. In the thalamus between the anterior and internal nuclei on the one hand and the lateral nucleus on the other is a layer shaded less deeply in the fignre, representing the internal medullary lamina of the thalamus, consisting largely of white matter. Other collections of white matter within the thalamus are $V b$, the bun. dle of Vieq. d'Azyr, and $F$, the lower end of the anterior pillar of the fornix; $F$, the upper end of the anterior pillar of the fornix, below $c c$ the corpus callosum; C.sb, corpus subthalamicum; forming a fairly continuous mass with the thalamus; $S n$, substantia nigra; $c l$, claustrum; $c e$, external capsule; $\mathrm{Ca}$, terminal portion of anterior commissure; In, the insula or island of Reil, $l v$, lateral ventricle; l.v.d.. descending horn of lateral ventricle; $V .3$, in the position of the third ventricle; the outlines of the cavities are made diagrammatically distinct by thick black lines; Op optic tract: $P P$. parietal lobe; $T$, temporal lobe.

\section{If instead of taking a transverse we take a longitudinal dorso-ventral}


(or, as it is called, sagittal) section (Fig. 145) we find that the fibres forming the strand in question do not continue parallel to each other as they rise dorsally but diverge in a radiating manner, forming the so-called corona radiata. If again we take horizontal sections at proper levels (Figs. 138, $144)$, we find that this strand or rather thick band of dorsally directed radiating fibres not only stretches ( $\mathrm{Cip}$ ) between the thalamus and the gray mass just spoken of, but reaching further forward passes ( $\mathrm{Cia}$ ) between the same gray mass on the lateral side and another gray mass $(N c)$ on the median side, the latter from its position being evidently the part of the corpus striatum which projects into the lateral ventricle. The same horizontal sections further teach us that the front part of the band ( $\mathrm{Cia}$ ) is bent at an angle upon the hind part ( $\left.C_{i p}\right)$.

It appears, then, from these sections that the fibres of the pes as they rise up dorsally into the hemisphere spread out in the form of a fan bent upon itself. This fan-like expansion of the pes is called the internal capsule, the angle formed by the bend being called its genu or knee ( $\mathrm{Cig}$ ), the part in front of the knee the front limb, and the part behind the knee the hind limb. And horizontal sections at levels more dorsal than those given in Figs. 138144 would show that the fibres composing this fan-like internal capsule, as they rose dorsally, curved away in various directions to reach nearly all parts of the surface of the hemisphere. We may add that though the internal capsule is mainly composed of fibres which thus stretch all the way from the cerebral cortex to the pes of the crus, it also contains other fibres of which we shall speak later on.

$\$ 540$. The gray mass separated from the thalamus by the hind limb of the internal capsule is called as a whole the nucleus lenticularis, since in horizontal section it presents a certain though distant resemblance to a lens. Of the three divisions into which it is split up by the partitions of white matter, the two median ones $G p^{\prime}, G p^{\prime \prime}$, are spoken of together as the globus pallidus, the name being given to them on account of their paler color. The third, lateral division $P t$, is called the putamen. The use of these two names for the two different parts of the one body, appears to be justified by the different connections and features of the two parts.

The gray mass which in a horizontal section (Fig. 138, $\mathrm{Nc}$ ) is separated from the nucleus lenticularis by the front limb of the external capsule, and which projects into the lateral ventricle, is called the nucleus caudatus. The nucleus caudatus and the nucleus lenticularis form together the corpus striatum; the former, since it projects into the lateral ventricle, being the part of the corpus striatum seen when the lateral ventricle is laid open, is sometimes spoken of as the intra-ventricular portion of the whole body, while the nucleus lenticularis, which is wholly hidden in the hemisphere and in no part projects into the lateral ventricle, is called the extra-ventricular portion.

But only a part, indeed only a relatively snall part, of the nucleus caudatus is disclosed in such a horizontal section ; to learn the somewhat peculiar form and relations of the whole nucleus a number of sections of a hemisphere taken in different planes must be studied; and these will at the same time explain why the nucleus is called "caudatus." These teach us that the nucleus has somewhat the form of a comma (Fig. 142). The thick, rounded head forms the lateral wall of the front part of the lateral ventricle; thence the body passes backward, narrowing rapidly and diverging somewhat laterally; in its course it arches over the nucleus lenticularis, curving so much that the end of the tail, sweeping round the hinder border of that body and changing its direction, runs eventually ventral to it. In a horizontal section taken at a certain depth, such as that represented in Fig. 138, only a portion 
of the head or body $(\mathrm{Nc})$ in the front part of the figure, and a transverse section of the end of the tail $(N c)$ in the hind part of the figure are seen; all the intervening portion of the nucleus lies above the plane of the section. In a transverse, dorso-ventral section taken somewhat anteriorly through the front limb of the capsule (Fig. 140), the head or body of the nucleus cauda-

FIG. 140.

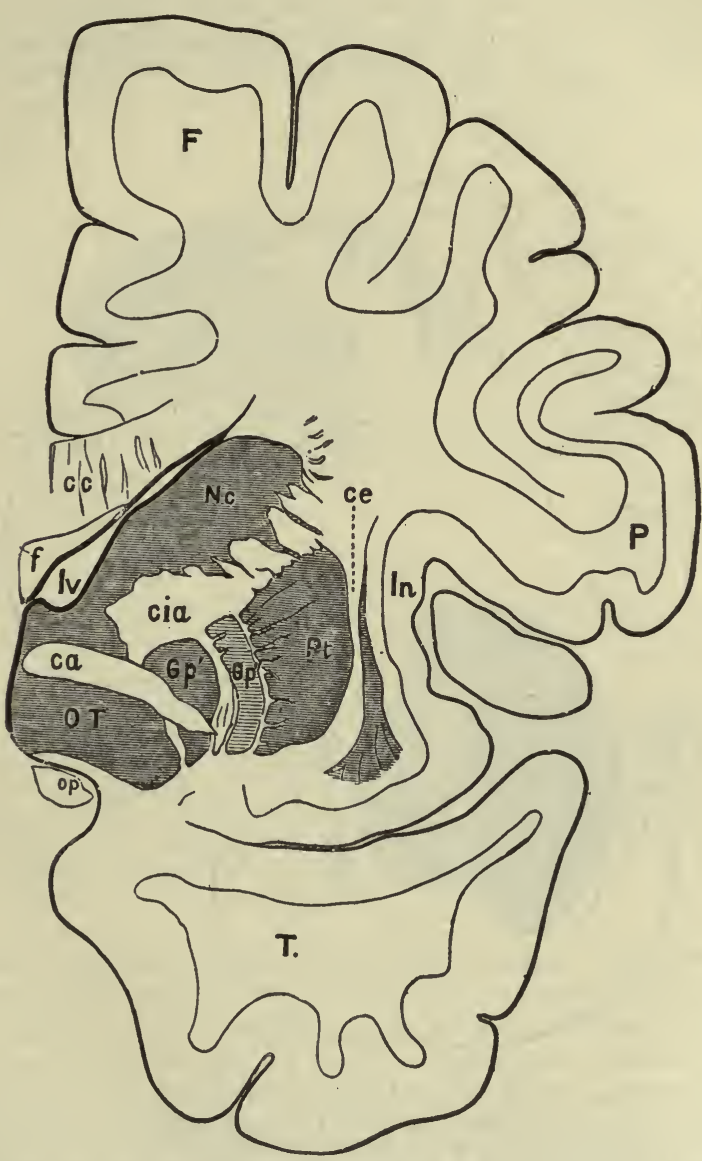

Diagrammatic Outline of a Transverse Dorso-ventral Section through the Right Hemisphere (Man) at a Level Anterior to Fig. 139. (Sherrington.) Natural size. Nc, nucleus caudatus; $G p^{\prime}$, $G p^{\prime \prime}$, globus pallidus, seen here in two segments, and $P t$, putamen of nucleus lenticularis; $O T$, optic thalamus, with $c a$, anterior commissure, in close relation to cia, anterior limb of internal capsule; $c e$, external capsule ; op, optic tract; $c c$, corpus callosum; $f$, fornix; $l v$, a space that in its upper part belongs to the lateral ventricle, in its lower was filled by the fold of subarachnoid tissue and pia mater, the side fringe of which, covered with epithelium, forms the choroid plexus; this fold was detached in the making of the section and was removed; $I n$, the insula; $F$, frontal lobe; $P$, parietal lobe; $T$, temporal lobe. For greater clearness the cortical gray matter, which is shaded in Fig. 139, is in this figure left unshaded.

tus $(N c)$, which has not yet reached its greatest dimensions, is seen lying dorsal to the nucleus lenticularis, separated from it by the white mass of the front limb (cia) of the capsule, though this is somewhat broken up by strands of gray matter passing from one nucleus to the other. In a transverse dorso- 
ventral section, taken still more anteriorly through the frontal lobe (Fig. 141 ), the head of the nucleus caudatus is seen at about it greatest size, and the diminishing uucleus lenticularis $(N l)$, represented by the putamen alone, is becoming fused with it, the two nuclei being separated by a small quantity of white matter of the internal capsule, and that largely broken up by bundles of gray matter, giving rise to a striated appearance. In a similar section still further forward, the nucleus lenticularis would be absent, the head of the nucleus caudatus appearing by itself. Returning to the hinder part of the hemisphere, we find in a dorso-ventral section taken through the hind limb of the capsule (Fig. 139) that while the nucleus lenticularis is

Fig. 141.

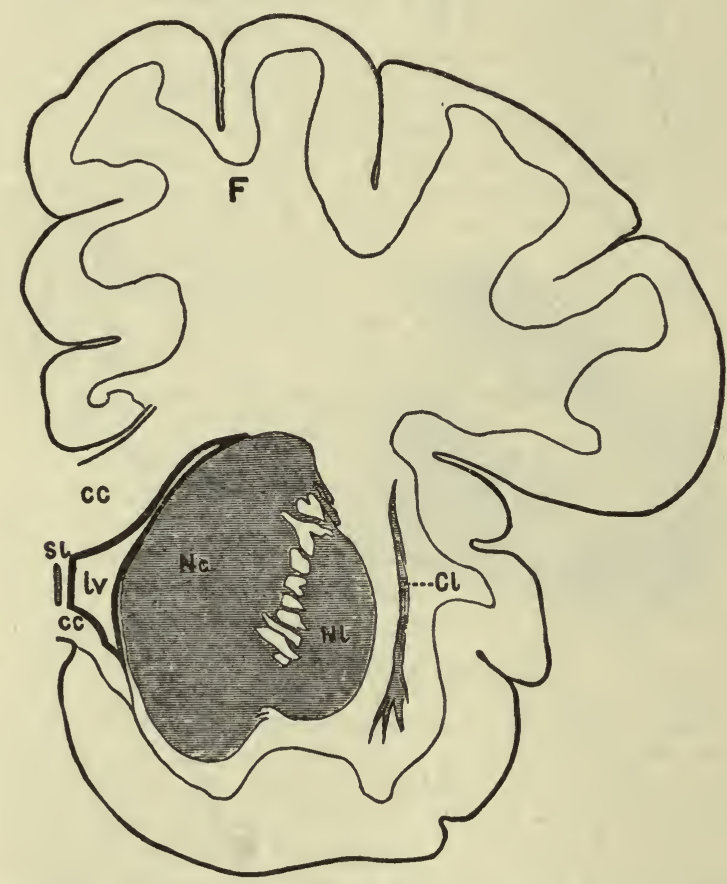

Diagrammatic Outline of a Transverse Dorso-ventral Section of Right Hemisphere (Man) through the Frontal Lobe. (Sherrington.) Natural size. $\mathrm{Nc}$, head of nucleus caudatus, and $\mathrm{Nl}$, the front end of the putamen of the nucleus lenticularis becoming fused with it; $c c$, corpus callosum, cut through at its front bend or rostrum, so that both dorsal and ventral portions are shown; between these is seen the fifth ventricle or cavity in the septum lucidum $S l$; $l v$, lateral ventricle; $C l$, claustrum ; $F$, frontal lobe. Cortical gray matter, as in Fig. 140, left unshaded.

here at its greatest size, the head of the nucleus caudatus $(\mathrm{Nc})$, lying dorsal to the nucleus lenticularis and separated from it by a considerable thickness of internal capsule, has much diminished; the same section, moreover, shows ventral to the nucleus lenticularis and clinging to the descending horn of the lateral ventricle (l.v.d.), the extreme tip of the tail of the nucleus caudatus $(\mathrm{Nc})$ soon about to fuse with the small mass of gray matter called the nucleus amygdalce $(N a)$. A sagittal (longitudinal dorso-ventral) section taken at some distance from the median line (Fig. 142) shows the curved course of the larger portion of the nucleus caudatus, the extreme head as well as the latter part of the tail lying out of the plane of the section; and 
a similar section taken nearer the middle line (Fig. 145) shows how the nucleus in the middle portion is broken up by bands of fibres of the internal capsule traversing it, and thus contributing to the striated appearance; the same section also shows that the globus pallidus, as well as the putamen, becomes continuous with the nucleus caudatus.

FIG. 142.

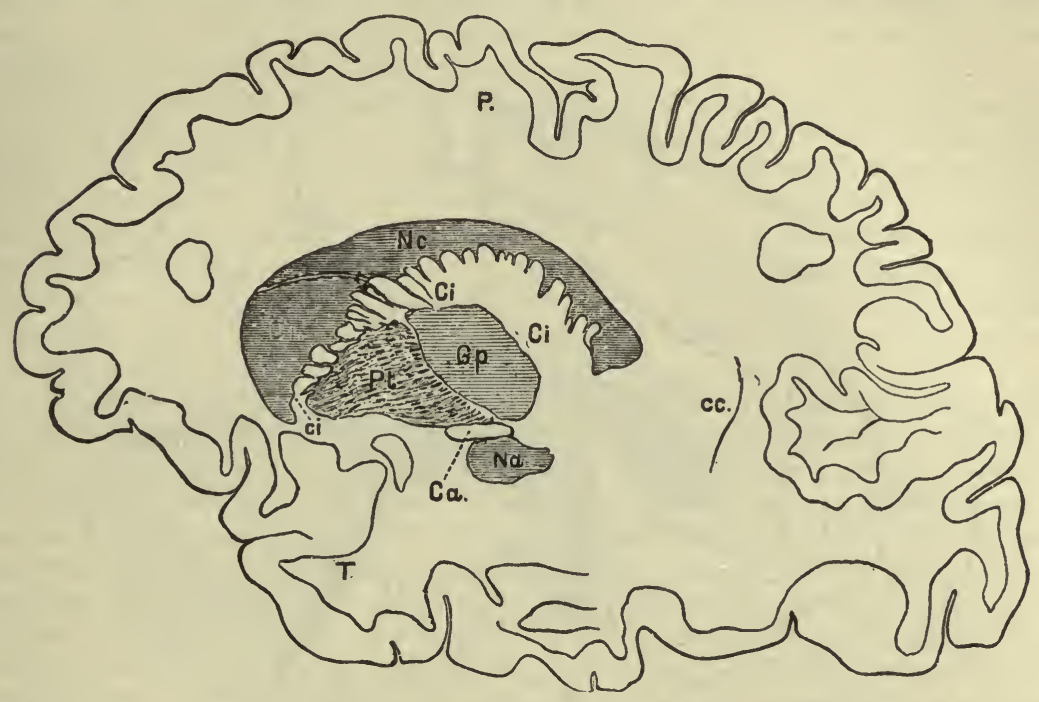

Diagrammatic Outline of a Sagittal Section taken through the Right Hemisphere (Man), Seen from the Mesial Surface. (Sherrington.) Half natural size. The plane of the section is not truly sagittal, but slightly inclined. Nc, the caudate nucleus exposed to the left of the letters $N c$ in nearly its entire anterior extent, to right of the letters in a considerable part of its posterior extent. It forms an arch of gray matter over the gray matter of $P t$, the putamen, and $G p$, the globus pallidus of the lenticular nucleus; $\mathrm{Na}$, the amygdaloid nucleus; $\mathrm{Ci}, \mathrm{Ci}, \mathrm{Ci}$, the internal capsule; $C a$, the anterior commissure; $c c$, the hinder limit of fibres of the splenium corporis callosi, $P$, the parietal lobe; $T$, the temporal.

Thus, when we speak of the corpus striatum as a whole we mean a large mass of gray matter lying lateral to the optic thalamus, reaching nearly as far back as that body and stretching much further forward, as far forward in fact as does the lateral ventricle; but it is important to remember that it is divided into two masses or nuclei, which are fused together, and that imperfectly at the very front only. These two nuclei are, the one the commashaped nucleus caudatus, the bulk of which is placed forward projecting into the lateral ventricle, and which on the whole is the more dorsal portion of the whole body, the other the irregularly shaped nucleus lenticularis, the bulk of which is placed further back than the lateral ventricle, by the side of the optic thalamus, and which on the whole is the more ventral portion of the whole body. It is no less important to remember that the radiating fibres, which we call the internal capsule, pass in the hinder region of the whole body between the thalamus and the nucleus lenticularis, forming the hind limb of the capsule, and in the front region between the nucleus caudatus and the nucleus lenticularis, forming the front limb of the capsule, the front and hind limbs being bent on each other so as to form an angle, the so-called knee.

$\S 541$. The optic thalamus as a whole is a somewhat oval mass of gray 
matter lying, as we have said, athwart the diverging crus, in which it is partly imbedded. Its curved median side covered with a thin layer of central gray matter forms the lateral wall of the third ventricle (Figs. 138, 139, 144 ), and in a longitudinal vertical section of the brain taken in the line of the middle of the third ventricle (Fig. 143, O.T.) is seen occupying the space between the fornix and hind end (splenium) of the corpus callosum above and the diverging crus below. Its more or less straight lateral border abuts

FIG. 143.

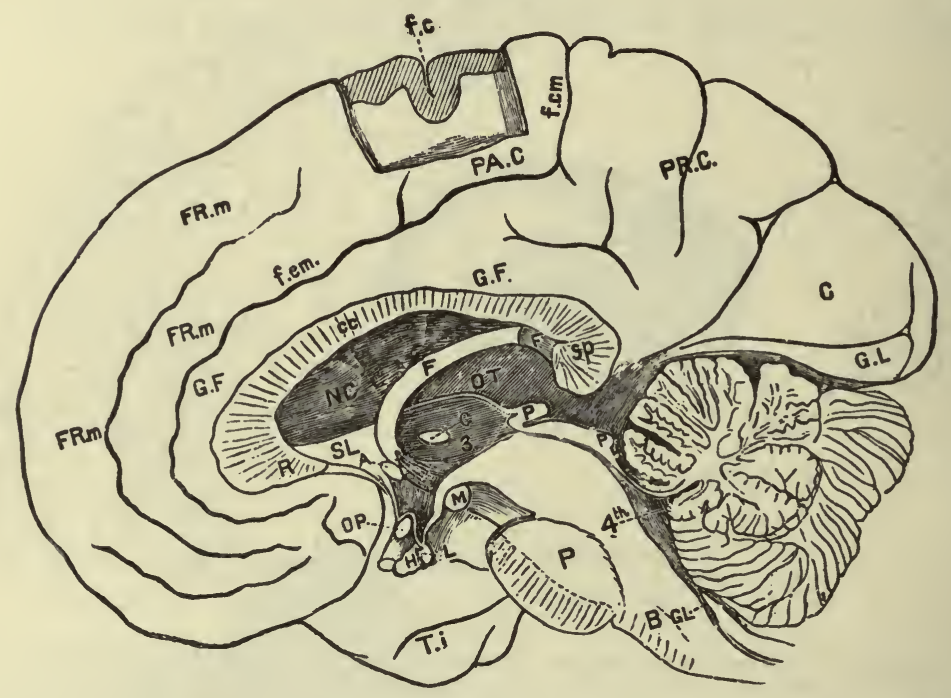

View of Right Half of Brain of Man as Disclosed by a Longitudinal Section in the Median Line through the Longitudinal Fissure.' (Sherrington.) Half natural size. The bulb, seen in longitudinal section at $B$, passes into the pons, $P$, and into the crus cerebri, which last is cut obliquely across as it diverges into the hemisphere and passes ont of the section. A part of the ventral surface of the crus is shown in the shaded part marked $C R$. At $G L$ the central canal of the spinal cord is seen opening out into the fourth ventricle (4th), overhung by the cerebellum (bisected in the middle line), and passing on by the aqueduct beneath the posterior, $Q P$, and anterior, $Q A$, corpora quadrigemina into the third ventricle (3). The posterior corpus quadrigeminum is continuous behind with the valve of Vieussens, attached to the superior peduncle of the cerebellum, and seen in a longitudinal section overhanging the front part of the fourth ventricle. The corpora quadrigemina appear relatively small because the section passes in the median line in the depression between the right and left bodies of the two pairs; and immediately in front of them is the section of the mesially placed pineal gland $P$, which overhangs the opening of the aqueduct into the third ventricle, and the right arm of which, running in the lateral wall of the third ventricle, is shown by an unshaded tract. The roof of the third ventricle is seen to be furnished by the arch of the fornix $F$, shown unshaded in longitudinal section. Posteriorly the body of the fornix passes into the diverging right posterior pillar, where $F$ is shaded, and is lost to view under the overhanging rounded hind end or splenium $(S p)$ of the corpus callosum. In front the body of the fornix is seen passing just behind the transverse section of the anterior commissure $A$ into the diverging right anterior pillar $f$, which is lost to view as it stretches in the lateral wall of the ventricle toward the corpus mammillare or albicans $\boldsymbol{M}$. The small white cross immediately behind $f$ indicates the position of the foramen of Monro. The bulging median surface of the optic thalamus OT, is seen forming the lateral wall of the hinder (and owing to the cranial flexure, the more dorsal) part of the third ventricle, and on this, below the area of the pineal gland, is seen, unshaded, the section of the soft or middle commissure $C$. Between the pineal gland $(P)$ and the splenium $(S p)$ is seen the hind end or pulvinar of the thalamus projecting into the so-called transverse fissure of the brain, shown shaded in the figtire, by which the pia mater, passing on beneath the posterior part of the cerebrum and above the cerebellum, gains access to the third ventricle, the position of the velum being shown by the thin black line stretching from the splenium to the fornix. The front (and more ventral) part of the third ventricle is seen to end in the infundibulum, attached to which is the pituitary body $H$, seen in section at $L$. In front of the infundibulum is seen the 
on the internal capsule (Figs. 138, 139, 144). Its dorsal surface, as we have already seen, also forms part of the wall of the third ventricle and is free; but there lies close above it the prolongation of the pia mater, forming the velum interpositum with its choroid plexus ( $\$ 515)$, which creeps in over it beneath the projecting hind end of the corpus callosum and the fornix (Fig. 143). Its ventral surface is fused with the crus; indeed the tegmental or dorsal portion of the crus may be said to end in it and in certain structures lying ventral to the thalamus, in what is called the "subthalamic region" (Fig. 139), while the fibres of the pes pass first ventral and then lateral to it to form the internal capsule.

The gray matter of the whole body is more or less distinctly divided by sheets of white matter, as seen both in horizontal and in vertical sections (Figs. 136, 139, 144), into three parts which have received the name of nuclei, namely, the median or inner nucleus (Fig. $139 \mathrm{in}$ ), which with the thin layer of central gray matter forms the side wall of the third ventricle; the larger lateral nucleus $(l n)$, which abuts upon the internal capsule; and the smaller anterior nucleus (an), which lies on the dorsal surface of the front part of the body, and which thus at its front end appears to project into the lateral ventricle.

These three nuclei form, however, not the whole of the optic thalamus, but only the larger front portion; behind them lies the important portion called the pulvinar, into which the hind part of the median nucleus merges; this is partly imbedded in the crus ventrally, and in the hemisphere laterally, and is partly free, coming to the surface beneath the hind end of the corpus callosum. In a median longitudinal section of the brain (Fig. 143) it is the pulvinar which forms the cushion-like (hence the name) end of the thalamus beneath the overhanging splenium of the corpus callosum, by the side of the pineal gland; and in the horizontal view (Fig. 138, Pvr), in which the hemispheres are supposed to have been removed, the same pulvinar is seen projecting over the crus by the side of the anterior corpus quadrigeminum. The buried portion of the pulvinar is exposed in a transverse section taken through the anterior corpus quadrigeminum (Fig. 137); the extreme end of this part of the pulvinar $(P v r)$ is here seen lying dorsal and lateral to the pes of the crus, immediately above two masses of gray matter, the corpora geniculata $(\mathrm{Cgl}$. Cgm.), of which we shall speak later on. One of these, the lateral corpus geniculatum $(C . g . l$.$) , is especially connected with the$ optic tract $(o p)$, and, as we shall see hereafter, the pulvinar itself is also connected with the optic tract, and is an important part of the central apparatus of vision.

\$542. The substantia nigra, the red nucleus, and other gray matter of the tegmentum. Nerve-cells and groups of nerve-cells, or areas of gray matter, too siriall to deserve special names, are scattered throughout the tegmentum

optic nerve cut across at the optic decussation $O P$, stretching from which to the anterior commissure is the lamina terminalis. Stretching between the corpus callosum $c c$ (seen in longitudinal section with a striated appearance, and ending in front at the rostrum $R$ and behind at the splenium $S p$ ) dorsally and the fornix ventrally is seen (unshaded) the septum lucidum $S L$, but the greater part of this has been cut away in order to disclose the right lateral ventricle, in the wall of which is seen the bulging nucleus caudatus $N C$. Above the corpus callosum is seen the mesial surface of the right hemisphere forming the right lateral wall of the longitudinal fissure. On this mesial surface appears immediately above the corpus callosum the arched gyrus fornicatus $G F$, defined above by the calloso-marginal fissure $f . \mathrm{cm}$. The whole of the surface seen in the frontal region in front of the calloso-marginal fissure, though divided by fissures, is called the marginal convolution. In the middle parletal region a block of the cerebral substance has been removed in order to show the position of the central fissure or fissure of Rolando, $f . c$, and immediately below this is seen a part of $P A . C_{.}$, the paracentral lobule. In the occipital region, $P R . C_{.}$, is the precuneus or quadrate lobule, and $C$, the cuneus, while at $G . L$. is seen a part of the lingual lobule. T. $i$. is a part of the inferior temporo-occipital convolution, the greater part of which is hidden from view by the pons and crus. 
along its course. But, besides these and the nuclei of the third and fourth cranial nerves, of which we have already spoken, certain larger collections of gray matter deserve attention. A conspicuous mass of gray matter, circular in transverse section, placed in the midst of the tegmentum on each side but somewhat near the middle line, and stretching from the hinder margin of the third ventricle beneath the anterior corpus quadrigeminum (Figs. 137, 138), is, from the red tint it possesses, called the red nucleus, nucleus, or locus ruber. It is traversed by fibres of the third nerve as these make their way ventrally from the nucleus to the surface.

IVe must consider also as belonging to the tegmentum a large area of gray matter, somewhat lens-shaped in section (Fig. 137, Sn), which lies between the pes and tegmentum, sharply marking off the one from the other. From its dark appearance, due to the abundance of black pigment, it is called the substantia nigra or locus niger. It acquires its largest dimensions at about the middle of the length of the crus, coming to an end in front (Fig. 139, Sn) and fading away behind (Fig. 136), as the crus passes beneath the posterior corpora quadrigemina. These two, the red nucleus and the substantia nigra, are perhaps the most important collections of gray matter in the tegmentum, but we may add that at the front of the crus as the substantia nigra comes to an end there is seen in a somewhat similar position ventral to the hind part of the optic thalamus a collection of gray matter called the corpus subthalamicum (Fig. 139, G. sb).

At the hinder part of the crus, as it is about to plunge into the pons, while the pes, now decreasing relatively in size, still continues to be ordinary white matter composed of longitudinal bundles of medullated fibres, the tegmentum takes on more and more the structure which in speaking of the bulb we called reticular formation, and which, as we saw, deserves to be considered as a kind of gray matter.

The gray matter of the pons. When the conjoined crura as we trace them backward plunge beneath the pons, the longitudinal fibres of the pes of each crus are, as we have said, soon split up into bundles and scattered anong the transverse fibres belonging to the pons itself. Dorsal to this system of transverse and longitudinal fibres forming the pons proper, between it on the rentral surface and the central gray matter with the posterior corpora quadrigemina on the dorsal surface, is a region which may be called tegmental, since it is a continuation of the tegmentum of the crus. In the front part of the pons (Fig. 136), where the posterior corpora quadrigemina still form the dorsal roof of the section, this tegmental area, which is much broken up by certain strands of longitudinal fibres, of which we shall speak later on, contains scattered nerve-cells, and is largely composed of reticular formation. In this is placed on each side a group of nerve-cells, the locus corruleus (Fig. $136, l$. c.), to which we have already referred (\$534) as probably serving in part as the origin of the descending root of the fifth nerve $(V . d$.$) , just ven-$ tral to which it lies. This acquires larger dimensions further back, in the front part of the fourth ventricle (Fig. 138, l.c.) between the levels represented in Figs. 135 and 136, and is a collection of large spindle-shaped nerve-cells; it has a bluish tint when its black pigment is seen shining through the surrounding more or less transparent nuaterial, hence the name.

In the hinder parts of the pons (Figs. 134,135), where the cerebellum is seen overhanging the open fourth ventricle, the reticular formation of the tegmental area is still more conspicuous. The only special collection of gray matter in this region to which we need call attention is one which, consisting, like the olivary body of the bulb (or inferior olive), of a wall of gray matter surrounding and surrounded by white matter, is called the upper olive (Figs. 134, 135̄, s. o.). 
The ventral part of the pons, or the pons proper, unlike the pes of the crus, contains, mixed with the fibres, a very considerable quantity of gray matter. This is fairly abundant in the front part of the pons (Fig. 136) below the corpora quadrigemina, but increases even more behind this (Figs. 125, 135). Hence, though the pons proper is largely built up of transverse and longitudinal fibres, and though it contains no compact aggregations of gray matter receiving special names, it does contain scattered throughout it a very large quantity of gray matter, far more, indeed, than is present in the tegmental portion; the gray matter of the pons-that is, of the pons propermust be regarded as forming a very important part of the gray matter of the crural system, and of no little physiological significance.

Behind the pons the crural system is continued into the bulb, with whose structure we have already dealt.

\section{Other Collections of Gray Matter.}

$\S 543$. Of these, three deserve chief attention, and may be classed together, though they differ in nature.

The gray matter of the corpora quadrigemina. On each side of and somewhat dorsal to the central gray matter of the aqueduct, which, as we have seen, is well developed, especially on the ventral side, collections of gray matter form the chief part of the corpora quadrigemina, both anterior and posterior.

'The gray matter of the anterior corpora quadrigemina (Fig. 137, A. Q.n.) is more distinctly marked off from, and separated by a wider tract of white matter from, the central gray matter of the aqueduct than is that of the posterior corpora quadrigemina (Fig. 137, $n P Q$ ); it is, moreover, of a different nature. Indeed the two pairs of bodies have quiet different relations, are of different nature, and perform different functions.

Corpora geniculata. The two optic nerves, as we shall see in detail later on, give rise, through the optic decussation, to the two optic tracts. Each optic tract (Figs. 131, 137, $O p$ ) winds round the crus cerebri on its ventral surface to reach the substance of the hemisphere in the region below the optic thalamus, and as it does so is described as dividing into a lateral and median portion. The lateral portion just as it sweeps round the far edge, that is the outer or lateral edge, of the crus bears a rounded swelling (Fig. 131, B and C, Cgl.), the lateral or outer corpus geniculatum, the interior of which consists largely of gray matter (Fig. 137, Cgl). The median portion similarly bears another like swelling occupying a more median position, the median or inner corpus geniculatum (Fig. 131, A and B, Cgm), the interior of which (Fig. 137, Cgm) also consists of gray matter. It is to be regretted that these two bodies should bear the same name, for they are different in their origin, in their connections, and in their functions. The lateral body is said to be derived from the fore-brain, that is from the vesicle of the third ventricle, has definite connections with the retinal optic fibres, and is distinctly concerned in vision; the median body is derived from the mid-brain, is not definitely connected with the retinal fibres, and appears to be in no way concerned in vision. We shall, however, return later on to the connections and probable functions of these bodies.

Corpus dentatum of the cerebellum. In the midst of the mass of white matter which is formed in the interior of the cerebellum by the confluence of the three peduncles is found (Fig. 134, C D) an area of gray matter arranged, like the olivary body of the bulb, as a sharply folded or plaited band in the shape of a flask or bowl. As in the similar olivary body the gray matter of the flask is covered up by and its interior filled up with white 
matter; the mouth of the flask is, on each side, directed toward the median. line; the fibres pass chiefly to the superior peduncle.

FIG. 144.

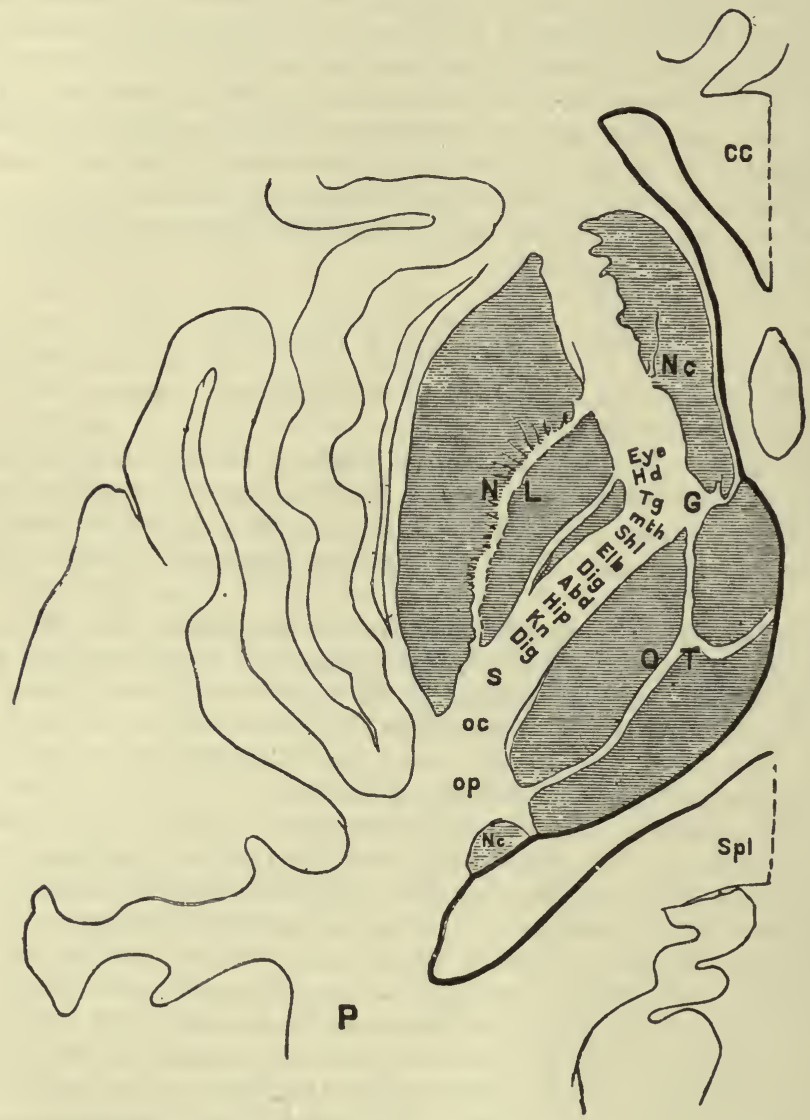

Outline of Horizontal Section of Brain, to show the Internal Capsule. Natural size. The section is taken at a level more ventral than shown in Fig. 138. The gray matter of the cortex and claustrum is left unshaded, but that of the corpus striatum and optic thalamus is shaded; OT, optic thalamus, showing the median, lateral, and anterior nuclei ; $N L$, nucleus lenticularis, showing the putamen large, and the inner division of the globus pallidus very small; $N C$, nucleus caudatus, the large head in front of, and the diminishing tail behind, the thalamus; $G$, the knee of the internal capsule. From "Eye" to "Dig," marks the position of the pyramidal tract as a whole, and the several letters indicate broadly the relative positions of the several constituents of the tract, named according to the movements with which they are concerned: thus Eye, movements of the eyes; $H d$, of the head; $T g$, of the tongue; $m t h$, of the mouth; Shl, of the shoulder; $E l h$, of the elbow: Dig, of the hand; $A b d$, of the abdomen; Hip, of the hip; $K n$, of the knee; Dig, of the foot; $S$, the temporo-occipital tract; oc, fibres to the occipital lobe; op, optic radiation. At this level the fibres of the frontal tract, in the fore limb of the capsule in front of the pyramidal tract, run almost horizontally, parallel with the plane of the section. (Cf. Fig. 146, Fron). $c c$, the rostrum of the corpus callosum, $\mathrm{Spl}$, the splenium of the same, both cut across horizontally. The thick dark line indicates the boundary of the cavities of the anterior and descending horns of the lateral ventricle and of the third ventricle, the two ventricles being laid open into one by the removal of the velum and choroid plexus, etc. The oval outline in the fore part of this cavity indicates the fornix. Lateral to the nucleus lenticularis is seen in outline the claustrum, the cortex of the island of Reil and the operculum or convolution overlapping the island of Reil. $P$ is inserted to show which is the hind part of the section. 
There are also other collections of gray matter in the central white matter of the cerebellum, one of which, called the "nucleus of the roof," is connected with the two inferior peduncles.

\section{THE ARRANGEMENT QF THE FIBRES OF THE BRAIN.}

$\S 544$. The systems, tracts, and bundles of fibres in which the white matter of the brain is arranged, may be distinguished from each other, partly through mere mechanical separation by means of the scalpel, partly by being traced out with the help of the microscope, but, as in the spinal cord, much more fully and completely by differences of development, and by the method of degeneration.

FIG. 145.

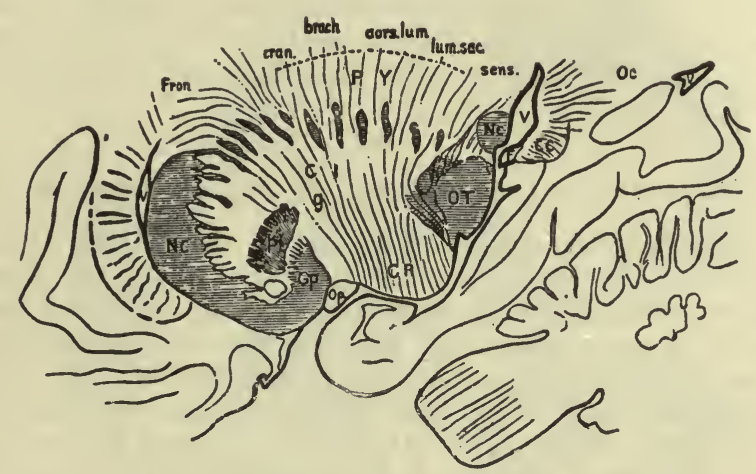

Outline of a Sagittal Section through the Hemisphere-Man. (Sherrington.) The section is taken not far to the right of the median plane and is one-half linear or natural size. The gray matter of the corpus striatum and thalamus is shaded. $N c, N c$, the caudate nucleus; $P t$, the putamen, and $G p$, the globus pallidus of the lenticular nucleus; $O T$, the optic thalamus; $C I$, the internal capsule with a streaked appearance revealing approximately the direction taken by fibrebundles passing into it from the portion of corona radiata over it. In these sets of bundles may be broadly distinguished a frontal system, fron, a pyramidal system, $P Y$ (subdivisible into cranial (cran.), brachial (brach.), dorso-lumbar (dors. lum.), and lumbo-sacral (lum. sac.) parts, and a temporo-occipital system, sens.; the situation of the genu of the internal capsule is indicated by $g$. $C R$, the crus cerebri; $O c$, the so-called optic radiations passing into the occipital lobe; $c c$, the splenial end of the corpus callosum; $v, v, v$. the lateral ventricle cut across in three different places ; $F$, the fornix in cross-section; $O p$, the optic tract in cross-section. Part of the cerebellum is seen in outline to the right.

We have seen that a marked feature of the brain is presented by the two crura cerebri which, running forward from the hind parts of the brain, spread out into each cerebral hemisphere. We have also seen that the crus in the wide sense of the word consists of two parts, a dorsal part, the tegmentum, and a ventral part, the pes or crusta, and that these two parts differ very strikingly from each other in structure and in relations. The pes consists exclusively of bundles of longitudiual fibres, and we may trace these from the cerebral hemispheres into the pons and some of them beyond the pons into the bulb and spinal cord. The tegmentum is more complex in structure; it consists of gray matter, and of fibres and bundles of fibres having various relations both with the collections of gray matter lying within itself and with surrounding structures. It too has connections with the parts lying in front of it, and with the parts lying behind it; we may trace it, too, backward through the pons into the bulb and forward to the optic thalamus. If we allow ourselves to conceive of the optic thalamus as 
constituting the front ending of the tegmentum, we may arrange a large part of the brain into two main regions-into a tegmental region stretching from the optic thalamus through the dorsal portion of the pons to the dorsal portion of the bulb, and into a region, which we may call the pedal region, stretching from the internal capsule through the ventral portion of the pons to the ventral portion of the bulb.

The fibres of the brain, as a whole, may be broadly classified into longitudinal tracts conuecting parts of the brain with succeeding parts and into transverse or commissural tracts between one lateral half and the other, and into tracts connected with the several cranial nerves. Taking the longitudinal fibres first, we may in accordance with the division just explained into a pedal and a tegmental region, consider these as forming, on the one hand, a peilal and, on the other hand, a tegmental system.

Both systems begin, as we shall see, in the cortex of the cerebral hemispheres. We shall have to deal with the topography of the cortex later on, but may here say that the first broad division of the whole surface of a hemisphere is into four main regions : frontal, parietal, occipital, and temporal (Figs. 139, 140, 144).

\section{LONGITUDINAL FIBRES OF THE PEDAL SYSTEM.}

$\S 545$. The pyramidal tract. We have already (\$ 488) said that the pyramidal tract of the spinal cord may be traced to a particular region of the cerebral cortex. We shall study the details of this region, which is often spoken of as the "motor area "later on, but may here say that broadly speaking it is parietal in position and corresponds to the parts of the cortex gathered round the fissure of Rolando. Fibres passing from the gray matter of the cortex of this region to the white matter below, and so contributing their share to the central white matter of the hemisphere, converge (Figs. 145,146 ) to form part of the internal capsule, namely, that part which in a horizontal section (Fig. 144, Eye to Dig) occupies the knee and stretches for more than half, or nearly twn-thirds, along the hind limb of the capsule, between the optic thalamus on the inside and the nucleus lenticularis on the outside. From the knee and hind limb of the capsule they pass by the side of and ventral to the optic thalamus (Fig. 139, 146), and so contribute to form the beginning of the crus cerebri. In thus converging to take up their position in the capsule and in their further passage to the crus the fibres follow a course of somewhat complicated curvature. As we trace the capsule from more dorsal to more ventral levels, we find it continually changing in form ; the exact shape of the capsule shown in Fig. 144 only holds good for the level at which the section was taken; it differs somewhat from that shown in Fig. 138 taken at a slightly different level, and sections still more dorsal or still more ventral would present still greater differences. When we examine a series of horizontal sections, taken in succession from the dorsal to the ventral regions, we find that the knee shifts its position and changes in the width of its angle, that the two limbs vary in direction, in size, and in shape, and that at last the bent, flattened capsule passes into the more or less rounded crus by the rapid disappearance of the fore limb, and the consequent extinction of the angle; so that in one sense it is the hind limb which becomes the crus, and the fibres of the fore limb may be said to pass into the crus through the ventral portion of the hind limb. Hence it is obvious that the fibres of the pyramidal tract, like the other fibres of the capsule, are continually changing their direction as they pass through the capsule. Moreover, while the fibres from the different parts of the "motor area" assume definite positions in relation to each other as they pass into 
the capsule, their relative positions are not constant, but vary somewhat. To this point, however, we shall return when we come to speak of the function of this tract.

FIG. 146.

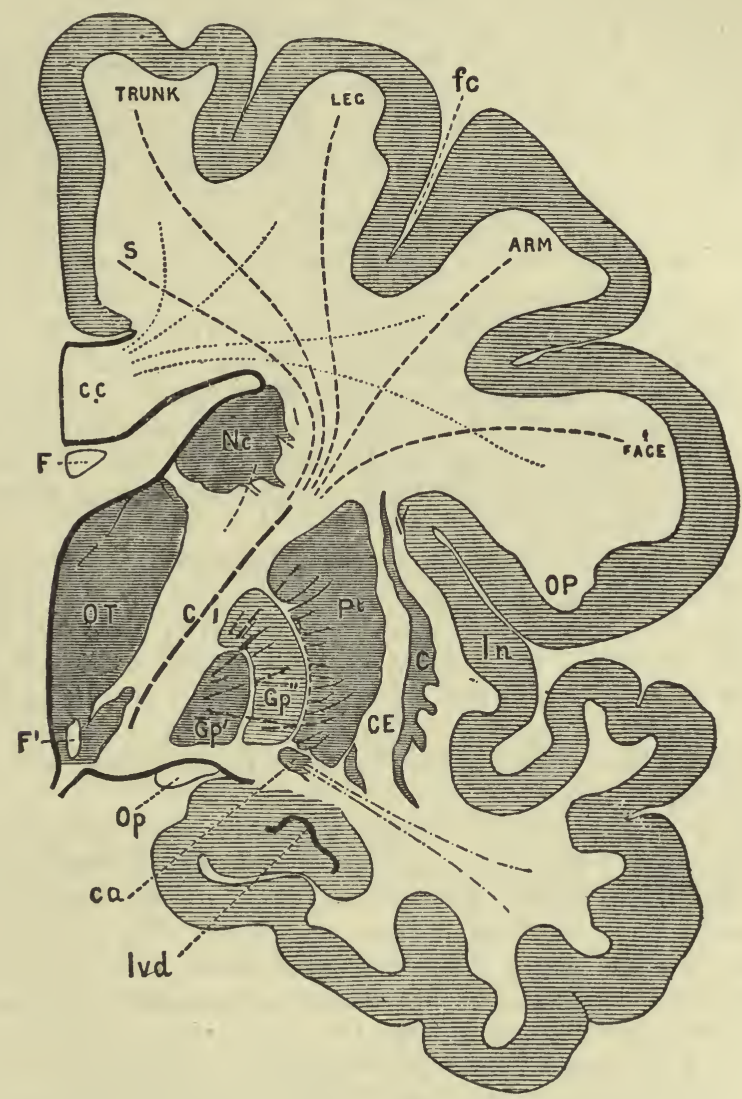

Outline of a Transverse Dorso-ventral Section of the Right Half of the Brain. Natural size. (Sherrington.) The section, which is taken at the level of the knee of the capsule, and is therefore intermediate between those shown in Figs. 139 and 140, is introduced to illustrate the course of the constituents of the pyramidal tract. OT, optic thalamus: $N c$, nucleus caudatus-the head only appears in this section: $P t$, putamen; $G p^{\prime \prime}, G p,^{\prime}$ the two parts of the globus pallidus of the nucleus lenticularis; $C$, the claustrum ; $C E$, the external capsule ; $I n$, the island of Reil ; $c a$, the anterior commissure, shaded to render it distinct and the fibres from the temporo-sphenoidal lobe which pass into it being indicated by broken lines; $O p$, the optic tract; $l v d$, the end of the descending horn of the lateral ventricle; $F$, the fornix; $F^{v}$, the end of the anterior pillar of the fornix in the base of the thalamus; $c c$, corpus callosum; $O P$, anterior part of the occipital lobe. $f c$ is the central fissure, or fissure of Rolando. The course of the fibres of the pyramidal tract connected respectively with the trunk, leg, and arm and hence with spinal nerves, and of those connected with the face and hence with cranial nerves, is shown by broken lines. These are all seen converging into the internal capsule, $C I$. This figure should in respect to the course of these fibres be compared with the horizontal section shown in Fig. 144, and the sagittal figure shown in Fig. 145. S indicates the course of the most anterior and dorsal part of the temporo-occipital tract. The fine dotted lines converging to the corpus callosum, $c c$, indicate the course of the callosal fibres.

In the crus these fibres run exclusively in the pes and form a compact strand (Fig. 137, $P y$ ) occupying the central and larger portion of the pes between a small median portion on the inside and a lateral portion on the 
outside. Maintaining this position along the crus they enter the pons, but here the previously compact strand is split up by the interlacing transverse fibres of the pons into a number of scattered bundles, which, however, as a whole, still keep their central position. They form the greater part of, but not all, the bundles seen cut transversely in transverse sections of the pons (Figs. 135, 136). Further backward they become the pyramid of the bulb, and so give rise in the spinal cord to the direct and crossed pyramidal tracts. These fibres from the motor area of the cortex of the cerebrum are thus the source of the pyramidal tracts of the spinal cord, and hence the whole strand of fibres from the cortex downward has been called the pyramidal tract. We have said ( $\$ 488$ ) that we have reasons for thinking that the pyramidal tract in the spinal cord makes connections through the gray matter of the anterior horn with the anterior roots of all the spinal nerves in succession; and similarly we have reason to think that along its course in the crus, in the pons, and in the bulb, before it reaches the cord, the tract also makes connections with the nuclei of those cranial nerves which are motor in function. During the passage of the tract through the internal capsule the fibres destined for cranial nuclei occupy the knee, while those belonging to the spinal cord run in the hind limb. Some authors limit the term pyramidal tract to the spinal moiety, since this alone forms the pyramid; but this is undesirable.

This tract is well marked out by the degeneration method, and the degeneration in it is a descending one, the trophic centres of the fibres being cells in the gray matter of the cortex. Removal of or injury to the cortex of the whole motor area gives rise to a degeneration along the whole tract, and removal of or injury to part of the area gives rise to degeneration of some of the strands. The tract is also well marked out by the embryological method; the fibres belonging to it acquire their medulla at times different from those of other fibres.

Anterior or frontal cortical. Fibres from the gray matter of the cortex in front of the motor area also pass to the internal capsule, but occupy the fore limb (Fig. 145, fron). Thence they pass to the crus, of which they form the small inner, median portion of the pes (Fig. 137, Fr.), and from the crus pass into the pons; in transverse sections of the pons they are seen as scattered bundles (Fig. 136, F. C.) to the median side of the pyramidal fibres. But here they seem to end ; the degeneration of the tract is a descending one, and ceases here. Most probably the fibres end in the nervecells of the gray matter, which, as we have seen, is abundant in the pons. It is also probable that through these nerve-cells the fibres of this tract are connected with transverse fibres passing along the middle cerebellar peduncle into the cerebellum of the opposite side; but this has not been definitely proved.

Posterior or temporo-occipital cortical. Fibres from the gray matter of parts of the cortex behind the motor area also converge to the internal capsule, forming the hinder end of the hind limb behind the pyramidal tract (Fig. 144, S). These fibres also contribute to form the crus cerebri, passing into the pes, of which they occupy the outer lateral portion (Fig. 137, Pr. O.). From the crus they pass into the pons, where, like the fibres of the preceding tract, they appear to end, and probably in a like manner. This tract has been described as one of ascending degeneration, but in all probability like the preceding is one of descending degeneration.

The above three tracts of fibres may, therefore, all be regarded as starting from or having their trophic centres in the cortical gray matter of the hemispheres, as all helping to form, first, the internal capsule and then the pes of the crus cerebri. But while the pyramidal tract passes, in part, to the 
spinal cord, the other two cease at the pons, and probably through the gray matter of the pons make comnections with the cerebellum. Further, while the pyramidal tract coming from the middle region of the cortex occupies a middle position in the capsule and a middle position in the crus, the system from the front part of the cortex occupies a front position in the capsule and an inner or median position in the crus, and the system from the hind part of the cortex, a hind position in the capsule and an outer or lateral position in the crus. As the three systems pass from the cortex through the capsule to form the pes of the crus, their positions in relation to each other are shifted from one plane into another. As the fibres spread out from the pes through the capsule to all parts of the cortex, or, put in another way, as they converge from the cortex through the capsule to the pes, they form a fan, the corona radiata, which is not only curved, but the constituent parts of which cross each other.

Besides these three systems all passing from varions regions of the cortex to the crus, there is yet a fourth strand contributed to the pes by the cerebral hemisphere, though not starting in the cortex. From the nucleus caudatus fibres pass down to the crus, and take up a position in the pes dorsal to the tract just mentioned, occupying a lens-shaped area immediately ventral to the substantia nigra, and probably passing into the substantia nigra itself. These cannot be traced further down than the pons, where they appear to end, though possibly some terminate higher up in the substantia nigra. This tract has a descending degeneration, and may be regarded as a tract analogous to the front and hind cortical tracts, though it begins not in the cortex but in the nucleus caudatus; it is not, however, a very pure tract, many fibres of the pyramidal tract passing into it in the pes.

These are the main tracts of the pedal system. For, though the nucleus lenticularis gives off fibres to the internal capsule, our knowledge of the further course of these is at present imperfect, and though there seem to be longitudinal fibres connecting the bulb, the pons, and the pes at various levels, these are not numerous, and at all events do not form conspicuous strands.

\section{LONGITUDINAL FIBRES OF THE TEGMENTAL SYSTEM.}

$\$$ 546. Cortical fibres. Although the fibres of the pedal system form, as we have seen, the greater part, they do not form the whole, of the internal capsule. Fibres coming from all or nearly all-parts of the cortex, though they help to form the internal capsule, do not go on to form the pes, but pass to the optic thalamus (Fig. 139, l.l.) and appear to end in the gray matter of that body. In their passage through the capsule the fibres of this nature from the frontal and parietal regions of the cortex, occupy the extreme end of the anterior limb in front of the strand of the fibres of the pedal system (Fig. 144, Th.). The fibres from the occipital and temporal regions-those from the occipital regions being the most numerous, and indeed being very conspicuous-occupy the extreme hind end of the hind limb of the capsule, behind the temporo-occipital division of the pedal system (Fig. 144, Op.). Since, as we shull see, we have reason to associate the occipital region of the cortex with vision, the fibres thus radiating to (or from) the thalamus through the extreme hind limb of the capsule from (or to) the occipital cortex have been called the optic radiation.

All the above tracts of fibres, though joining the thalamus and not passing on to the pes, take part in the formation of the internal capsule. But a considerable number of fibres coming from the temporo-occipital region, 
and especially from the temporal region, pass to the thalamus without joining the capsule; they pass ventral to and behind the pes as this plunges into the hemisphere to become the capsule, and so reach the thalamus.

We may here perhaps diverge for a moment to point out the contrast between the optic thalamus and the corpus striatum, or at least the nucleus caudatus. The former does not contribute to the pedal system, the latter supplies a marked contribution. The former receives fibres from all parts of the cortex; there are no such special contributions from the cortex to the latter. And this difference accords with the experience that when parts of the cortex are removed, or are congenitally absent, no degeneration or want of development is observed in the corpus striatum, while degeneration or want of development is observed in the optic thalamus as well as in parts of the pedal and tegmental systems. Hence, while we may regard the optic thalamus as an intermediate mass of gray matter receiving fibres from the cortex, and connecting the cortex with lower parts of the tegmental region, the corpus striatum appears rather to be analogous to the cortex itself, to be a special modification of the cortex, sending fibres down into the pedal system, but itself receiving no special tracts of fibres from the cortex. Indeed we may probably regard the corpus striatum as the oldest part of the superficial gray matter of the hemisphere, the more ordinary cortex being a later development.

The tegmentum proper, lying ventral to the hind end of and behind the thalamus, in which region, as we have seen, the conspicuous red nucleus is situated, is thus, by reason of its connection with the thalamus, indirectly connected with the cortex. But besides this, it has direct connections of its own with the cortex. Some of the fibres of the optic radiation, as well as fibres from the temporal and occipital regions described above as sweeping round the base of the internal capsule, are said to pass not to the thalamus, but to the tegmentum. Other fibres from the frontal and parietal regions traversing the lenticular nueleus in the sheets of white matter splitting the nucleus into parts are also said to reach the tegmentum either by piercing through or by sweeping round the internal capsule. On their path these fibres are, according to some observers, joined by fibres coming from the lenticular nucleus itself, and possibly from the caudate nucleus, which fibres, on the view that these nuclei are modified cortex, may also be considered as cortical. Thus the fore part of the tegmental region is brought into ample connection with the cerebral hemisphere partly by fibres joining the thalamus, partly by fibres passing directly to the tegmentum proper.

The mode of degeneration of these cortical fibres of the tegmental system is at present a matter of dispute. Nor is the general nature of the fibres conclusively determined, though it is generally supposed that they carry impulses from the thalamus and parts of the tegmentum to the cortex.

$\$ 547$. In the tegmentum from the subthalamic region to the bulb the reticular formation is, as we have seen, more or less abundant; this, and the occurrence of various bundles of fibres, gives the region great complexity; and we must confine ourselves here to touching on one or two important longitudinal strands which traverse it.

The superior peduncle of the cerebellum is one of the most important of these. This, on each side consists of a bundle of fibres which, taking origin chiefly from the gray matter of the nucleus dentatus and the smaller neighboring collection of gray matter, and also in part from the superficial gray matter, leaves the cerebellum in front of and to the median side of the restiform body and passes forward toward the corpora quadrigemina to converge with its fellow. At first the two peduncles are superficial and dorsal in position (Figs. 134, 135, S. P.) and the space between them is bridged over by the 
valve of Vieussens (Fig. 135, Vla); but, still converging, they som sink ventrally beneath the posterior corpora quadrigemina and at the level of the junction between the anterior and posterior corpora quadrigemina meet and decussate ventral to those bodies in the ventral region of the tegmentum (Fig. 136, S. P.). Beyond the decussation they are continued forward in the tegmentum ventral to the anterior corpora quadrigemina as two strands, one on each side, which appear to end in the red nuclei.

In this way the peduncles connect certain parts of the gray matter of the cerebellum with the tegmental region, and more particularly with the red nucleus, and thus indirectly with the structures with which that region is itself connected.

The fillet. This, as we have seen ( $\$ 525)$, takes origin in the bulb, in the inter-olivary layer between the inferior olives, from fibres which are derived through the supra-pyramidal or sensory decussation from the gracile and cuneate nuclei. From this origin it passes forward on each side as a flat band into the tegmental region of the pons, receiving accessions from the superior olive and other collections of gray matter, and dividing there into two strands, the median (Figs. 135, 136, Fm) and lateral (Figs. 135, 136, Fl, and Fig. 131, $B F$ ) fillet. The lateral division ends partly in the gray matter of the posterior corpus quadrigeminum, and partly in the white matter underlying (Fig. 137, $\mathrm{dm}$ ) the anterior corpus quadrigeminum; the median division. passing further forward appears partly to end in the gray matter of the anterior corpus quadrigeminum, but partly to be continued on to the subthalamic region of the tegmentum ventral to the thalamus, thence to the thalamus, and so to the cortex.

The longitudinal posterior bundles. In a transverse section through the fore part of the pons at the level of the posterior corpora quadrigemina a rather conspicuous bundle of longitudinal fibres (called the longitudinal posterior bundle) is seen on each side, cut transversely, in the dorsal region of the tegmentum just ventral to the nucleus of the fourth nerve (Fig. 136, l). Traced backward from the aqueduct beneath the fourth ventricle, it becomes less conspicuous (Fig. 135, l), though maintaining its position dorsal to the reticular formation, and at the hind end of the bulb appears to be a continuation forward of those fibres, "ground fibres," of the anterior column of the cord which probably serve as successive short longitudinal commissures between the segments of the cord. While the somewhat analogous fillet runs ventral to the reticular formation, this posterior longitudinal bundle runs always dorsal to that structure. It may be traced forward as far as the nucleus of the third nerve, as is seen in transverse sections lying immediately ventral to that group of cells (Fig. 137, l), but its further connections forward have not as yet been determined. It is relatively more prominent in the lower than in the higher animals, and its fibres acquire their medulla relatively early. It is supposed to be coinnected with the nuclei of the nerves governing the muscles of the eye, and so to be concerned in the movements of that organ.

Tracts from the corpora quadrigemina. From each corpus quadrigeminum there passes obliquely forward and downward on each side a band of fibres, connected with the gray matter of the corpus and known as the brachium. The anterior brachium (Fig. 137, Ba), as we shall see in dealing with the optic nerve, joins the lateral corpus geniculatum and helps to form the optic tract, but some of its deeper-lying fibres proceed to the occipital cortex forming part of the fibres which we have ( $\$ 545)$ described as passing from the occipital cortex to and past the thalamus. The posterior brachium passes to the median corpus geniculatum; having received fibres from and probably given fibres up to that body, it is continued on to the tegmentum, and, ac- 
cording to some authors, through the tegmentum by the hind part of the hind limb of the internal capsule to the temporal region of the cortex, mingling in its course with fibres from the thalamus.

\section{Transverse or So-Called Commissural Fibres.}

$\S 548$. The two chief masses are those, on the one hand, belonging to the cerebrum, and those, on the other hand, belonging to the cerebellum.

In the cerebrum the most imposing mass of transverse fibres form the corpus callosum. Starting from the cortex in nearly all parts of the hemisphere, the fibres converge toward the thick body of the corpus callosum placed in the middle line, and thence diverge to nearly all parts of the cortex of the hemisphere on the other side, interlacing in their course with the cortical fibres of the pedal and tegmental systems. It is supposed that by means of these fibres, each part of the cortex of one hemisphere is brought into connection with the corresponding part of the other hemisphere.

Besides these callosal fibres from one hemisphere to another, the white matter of each hemisphere contains fibres called " association fibres," passing from one convolution to another of the same hemisphere.

The small anterior white commissure though it is placed in the front part of the third ventricle (Fig. 143, $A$ ) and, in part of its course, lies along the thalamus (Fig. 140, Ca), is really a commissure of particular parts of the cerebral hemispheres. A portion, very small in man, belongs to the olfactory tract; the rest takes origin on each side in a limited portion of the cortex (Fig. 139, Ca), which we shall later on speak of as the temporo-sphenoidal convolution, and in which callosal fibres are deficient, whence it arches forward through the globus pallidus, past the thalamus (Figs. 146, $c a, 140, C a$ ) to the front part of the third ventricle. It may be remarked that this commissure is still found in those lower animals which do not possess an obvious corpus callosum.

The small posterior commissure may be regarded as simply a commissure between the two thalami, but it also helps to unite the tegmentum of the two sides, and some fibres are said to pass on each side into the hemisphere. The middle or soft commissure of the third ventricle (Fig. 138, c), though it contains transverse fibres, is, in the main, a collection of gray matter, indeed, a part of the central gray matter.

The fornix, together with, at all events, part of the septum lucidum which joins it with the corpus callosum, must also be regarded as a commissural structure. But its relations are peculiar; for while, behind, the diverging posterior pillars begin in the cerebral hemispheres, namely, in the walls of the descending horn of the lateral ventricle on each side, in front the anterior pillars or columns, leaving the cerebral hemispheres, pass along the lateral walls of the third ventricle (Fig. 143, $f$ ), and apparently end in the gray matter of the corpora albicantia. Whether the band of fibres, known as Vicq d'Azyr's bundle (Fig. 139, Vb), which running in the lateral wall of the third ventricle leads dorsally from each corpus albicans up to the anterior nucleus of the thalamus, is really to be considered as a continuation of the fornix is disputed; it may more probably be regarder as a part of the system spoken of above as connecting the cortex with the thalamus.

In the cerebellum true commissural fibres are supplied by the middle peduncles; but by no means all the fibres of these peduncles are of this nature. The fibres of the middle peduncle, in contrast to those of the superior peduncle which starts chiefly from the nucleus dentatus, or other 
internal gray matter, and to those of the inferior peduncle which start chiefly from the superficial gray matter of the vermis, appear to start from the superficial gray matter of the whole surface, from that of the median vermis as well as from that of the lateral hemispheres; they thus form the greater part of the central white matter. Sweeping down into the pons, they form the transverse fibres of that body, interlacing with the longitudinal fibres of the crural system and intermingling with the abundant gray matter.

Of these transverse fibres of the pons, a certain number are truly commissural; they make no connections with cells in the pons, but continue their way unbroken across it ; they start in the superficial gray matter of one side of the cerebellum and end in the superficial gray matter of the other side, the parts of the gray matter thus united being probably corresponding parts. The most ventrally placed transverse fibres of the pons which form a superficial layer of white matter, free from gray matter (Fig. 134, $t r . P$.) are probably of this nature, as are also the transverse fibres placed most dorsally, just ventral to the tegmental region.

A large number of the transverse fibres are not of this nature. They cross from one side of the cerebellum to the opposite side of the pons, but end in the pons apparently in the nerve-cells of the gray matter; and it is supposed that by these nerve-cells they are brought into connection with the longitudinal fibres of the pedal system and thus with the cerebrum. They are transverse appendages of the pedal system, not true commissural fibres, though they do cross the median line.

It is further supposed that other fibres of the middle peduncle reaching the pons do not cross the median line, but keeping to the same side and changing their direction, take a longitudinal upward course either with or without the intervention of nerve-cells, and so make their way to the tegmentum. But this is not certain.

We must also consider as commissural structures the numerous fibres crossing or serving to form the median raphe in the bulb. This raphe, with similar commissural fibres, is present in the tegmental portion of the pons, and, indeed, in the tegmentum itself.

Fibres also cross from one side to the other in connection with the cranial nerves, but these, as well as all the tracts specially connected with the cranial nerves, including the olfactory and optic nerves, had better be considered by themselves.

\section{Summary.}

$\S 549$. It may perhaps appear from the foregoing that the brain consists of a number of isolated masses of gray matter, and some large, some small, connected together by a multitude of ties of white matter arranged in perplexing intricacy ; and the addition of numerous collections of gray matter and strands of white matter, of which we have made no mention, would still further increase the perplexity. Nevertheless, a systematic arrangement may be recognized, at least, to a certain extent.

The least conspicuous, but perhaps in point of origin the oldest part of the brain, seems to be what we have called the central gray matter. This seems to serve chiefly as a bed for the development of the nuclei of the cranial nerves.

Next to the central gray matter and more or less associated with it comes what is called the tegmental region, of which the reticular formation, coming into prominence in the bulb and continued on to the subthalamic region, forms, as it were, the core. Belonging to the tegniental system are numerous 
masses of gray matter from the conspicuous optic thalamus and the red nucleus in front to the several nuclei of the bulb behind. This complex tegmental system, which may, perhaps, be regarded as a more or less continuous column of gray matter, comparable to the gray matter of the spinal cord, serves as a sort of backbone to the rest of the central nervous system. With the spinal cord it is connected by various ties, besides being, as it were a continuation of the spinal gray matter, and around it are built up the great mass of the cerebrum, and the smaller but still larger mass of the cerebellum; the less important corpora quadrigemina we may for simplicity's sake neglect.

At the hind end we find various parts of the spinal cord becoming connected with this tegmental system, either passing into it and becoming, as far as our present knowledge goes, lost in it, or supplying strands or fibres which passing into it become through it connected with other parts. Thus the anterior column of the cord exclusive of the direct pyramidal tract, the lateral column exclusive of the crossed pyramidal and cerebellar tracts (and possibly the antero-lateral ascending tract), together with part of the posterior column, appear to join the tegmental system, while part of the posterior column, after the relay of the gracile and cuneate nuclei, passes through the system as the fillet destined for various structures.

At the front end we find all parts of the cerebral cortex (though some regions, namely, the temporo-occipital, to a greater extent than others), connected with the thalamus and other parts of the tegmental system; and the corpus striatum may possibly possess like connections.

The relations of the cerebellum to this system are notable. On the one hand the cerebellum is directly connected with the system, partly by fibres which pass from the bulb to join the restiform body or inferior peduncle, partly by the superior peduncles which are in a measure lost in the tegmentum, and partly probably by fibres of the middle peduncles also making connections with the tegmentum. On the other hand, the cerebellum forms around the tegmental system a great junction between the spinal cord and the cerebrum. To the spinal cord it is joined in a direct manner by the cerebellar tract and possibly by the antero-lateral ascending tract, and in an indirect manner by the relay of the gracile and cuneate nuclei. To all parts of the cerebral cortex it appears to be joined by those conspicuous strands of the pedal system which end in the pons, and there make conriections with the fibres of the middle peduncle. And we may here perhaps remark that while this connection between the cerebrum and cerebellum is wholly a crossed one, each cerebral hemisphere being joined with the opposite half of the cerebellum, the connections between the spinal cord and the cerebellum are largely uncrossed ones, that by the cerebellar tract being wholly uncrossed, and that with the posterior column by the relay of the gracile and cuneate nuclei being in part uncrossed.

Thus the cerebral cortex has a double hold, so to speak, on the rest of the central nervous system, first through the tegmental system, and secondly through the cerebellar junction. But in addition to this there is another tie between the cerebral cortex and the whole length of the cerebro-spinal. axis, or at least between it and the whole series of motor mechanisms in succession from the nucleus of the third nerve to the nucleus, if we may so call it, of the anterior root of the coccygeal nerve, namely, the great pyramidal tract, which thus appears as a something superadded to all the rest of the central nervous system.

When the cerebral hemispheres are removed this pyramidal tract falls away, as does also the pedal system leading from the cerebrum to the pons, but there still remains the tegmental system with its cerebellar and other 
adjuncts, and this, as we shall see, constitutes a nervous machinery capable of carrying out exceedingly complicated acts.

\section{On the Phenomena Exhibited by an Animal Deprived of its Cerebral Hemispheres.}

$\S 550$. The cerebral hemispheres, as we have more than once insisted, seem to stand apart from the rest of the brain. In the case of some animals it is possible to remove the cerebral hemispheres and to keep the animal not only alive, but in good health for a long time-days, weeks, or even months after the operation. In such case we are able to study the behavior of an animal possessing no cerebral hemispheres, and to compare it with that of an intact animal. Such an experiment is best carried out on a frog. In this animal it is comparatively easy to remove the cerebral hemispheres, including the parts corresponding to the corpora striata, leaving behind intact and uninjured the optic thalami with the optic lobes (or representatives of the corpora quadrigemina), the small cerebellum, and the bulb. If the animal be carefully fed and attended to, it may be kept alive for a very long time-for more than a year, for instance.

The salient fact about a frog lacking the cerebral hemispheres is that, as in the case of a frog deprived of its whole brain, the signs of the working of an intelligent volition are either wholly absent or extremely rare. The presence of the bulb and the middle parts of the brain (for so we may conveniently call the cerebral structures lying between the cerebral hemispheres and the bulb) insures the healthy action of the vascular, respiratory, and other nutritive systems; food placed in the mouth is readily and easily swallowed; the animal when stimulated executes various movements; but if it be left entirely to itself, and care be taken to shield it from adventitious stimuli, either it remains perfectly and permanently quiescent, or the apparently spontaneous movements which it carries out are so few and so limited as to make it very doubtful whether they can fairly be called volitional. Such a frog, for instance, after being kept alive for some time and made to exhibit the phenomena of which we are about to speak, has been placed on a table with a line drawn in chalk around the area covered by its body, and left to itself has subsequently been found dead without having stirred outside the chalked circle.

We must here, however, repeat the caution laid down in $\$ 495$, as to the ultimate effects of an operation on the central nervous system. The longer the frog is kept alive and in good health after the removal of the cerebral hemispheres, the greater is the tendency for apparently spontaneous movements to show themselves. For days, or even weeks, after the operation there may be no signs whatever of the working of any volition ; but after the lapse of months, movements, previously absent, of such a character as to suggest that they ought to be called voluntary, may make their appearance. To this point we shall return, but may, in the meanwhile, state that even in their most complete development such movements do not negative the view that the frog in the absence of the cerebral hemispheres is wanting in what we ordinarily call a "will."

$\$ 551$. We have seen that a frog from which the whole hrain has been removed, and the spinal cord only left, appears similarly devoid of a "will;" but the phenomena presented by a frog possessing the middle portions of the brain differ widely from those presented by a frog possessing a spinal cord only. We may; perhaps, broadly describe the behavior of a frog from which the cerebral heinispheres only have been removed by saying that such an 
animal, though exhibiting no spontaneous movements, can by the application of appropriate stimuli be induced to perform all, or nearly all, the movements which an entire frog is capable of executing. It can be made to swim, to leap, and to crawl. Left to itself, it assumes what may be called the natural posture of a frog, with the fore limbs erect and the hind limbs flexed, so that the line of the body makes an angle with the surface on which it is resting. When placed on its back it immediately regains this natural posture. When placed on a board it does not fall from the board when the latter is tilted up so as to displace the animal's centre of gravity; it crawls up the board until it gains a new position in which its centre of gravity is restored to its proper place. Its movements are exactly those of an entire frog, except that they need an external stimulus to call them forth. They differ, moreover, fundamentally from those of an entire frog in the following important feature: they inevitably follow when the stimulus is applied; they come to an end when the stimulus ceases to act. By continually varying the inclination of a board on which it is placed, the frog may be made to continue crawling almost indefinitely; but directly the board is made to assume such a position that the body of the frog is in equilibrium, the crawling ceases; and if the position be not disturbed the animal will remain impassive and quiet for an almost indefinite time. When thrown into water the creature begins at once to swim about in the most regular mauner, and will continue to swim until it is exhausted, if there be nothing present on which it can come to rest. If a small piece of wood be placed on the water the frog will, when it comes in contact with the wood, crawl upon it and so come to rest. If disturbed from its natural posture, as by being placed on its back, it immediately struggles to regain that posture; only by the application of continued force can it be kept lying on its back. Such a frog, if its flanks be gently stroked, will croak; and the croaks follow so regularly and surely upon the strokes that the animal may almost be played upon like a musical, or, at least, an acoustic, instrument. Moreover, provided that the optic nerves and their arrangements have not been injured by the operation, the movements of the animal appear to be influenced by light; if it be urged to move in any particular direction, it seems in its progress to avoid obstacles, at least such as cast a strong shadow; it turns its course to the right or left, or sometimes leaps over the obstacle. In fact, even to a careful observer, the differences between such a frog and an entire frog which was simply very stupid or very inert would appear slight and unimportant, except in this, that the animal without its cerebral hemispheres is obedient to every stimulus, and that each stimulus evokes an appropriate movement; whereas, with the entire animal it is impossible to predict whether any result at all, and, if so, what result, will follow the application of this or that stimulus. Both may be regarded as machines; but the one is a machine and nothing more; the other is a machine governed and checked by a dominant volition.

Now, such movements as crawling, leaping, swimming, and, indeed, as we have already urged, to a greater or less extent all bodily movements are carried out by means of coördinate nervous motor impulses, influenced, arranged, and governed by coincident sensory or afferent impulses. Muscular movements are determined by afferent influences proceeding from the muscles and constituting the foundation of the muscular sense; they are also directed by means of afferent impulses passing centripetally along the sensory nerves of the skin, the eye, the ear, and other organs. Independently of the particular afferent impulses, which, acting as a stimulus, call forth the movement, very many other afferent impulses are concerned in the generation and coördination of the resultant motor impulses. Every bodily movement, such as those of which we are speaking, is the work of a more or less 
complicated nervous mechanism, in which there are not only central and efferent but also afferent factors. And, putting aside the question of consciousness, with which we have here no occasion to deal, it is evident that in the frog deprived of its cerebral hemispheres all these factors are present, the afferent no less than the central and the efferent. The machinery for all the necessary and usual bodily movements is present in all its completeness. We may regard the share, therefore, which the cerebral hemispheres take in executing the movements of which the entire auimal is capable, as that of putting this machinery into action or of limiting its previous activity. The relation which the higher nervous changes concerned in volition bear to this machinery may be compared to that of a stimulus, always bearing in mind that the effect of a stimulus on a nervous centre may be either to start activity or to increase $o^{\circ}$ to curb or to stop activity already present. We might almost speak of the will as an intrinsic stimulus. Its operations are limited by the machinery at its command. We may infer that in the frog the action of the cerebral hemispheres in giving shape to a bodily movement is that of throwing into activity particular parts of the nervous machinery situated in the lower parts of the brain and in the spinal cord; precisely the same movement may be initiated in the absence of the cerebral hemispheres by applying such stimuli as shall throw precisely the same parts of that machinery into the same activity.

Very marked is the contrast between the behavior of such a frog which, though deprived of its cerebral hemispheres, still retains the other parts of the brain, and that of a frog which possesses a spinal cord only. The latter when placed on its back makes no attempt to regain its normal posture; in fact, it may be said to have completely lost its normal posture, for even when placed on its belly it does not stand with its fore feet erect, as does the other animal, but lies flat on the ground. When thrown into water, instead of swimming it sinks like a lump of lead. When pinched or otherwise stimulated it does not crawl or leap forward; it simply throws out its limbs in various ways. When its flanks are stroked it does not croak; and when a board on which it is placed is inclined sufficiently to displace its centre of gravity it makes no effort to regain its balance, but falls off the board like a lifeless mass. Though, as we have seen, the various parts of the spinal cord of the frog contain a large amount of coördinating machinery, so that the brainless frog may, by appropriate stimuli, be made to execute various purposeful coördinate movements, yet these are very limited compared with those which can be similarly carried out by a frog possessing the middle and lower parts of the brain in addition to the spinal cord. It is evident that a great deal of the more complex machinery of this kind, especially all that which has to deal with the body as a whole, and all that which is concerned with equilibrium and is specially governed by the higher senses, is seated not in the spinal cord but in the brain. We do not wish now to discuss the details of this machinery; all we desire to insist upon at present is that in the frog the nervous machinery required for the execution, as distinguished from the origination, of bodily movements even of the most complicated kind, is present after complete removal of the cerebral hemispheres, though these movements are such as to require the coöperation of highly differentiated afferent impulses.

$\$ 552$. In warm-blooded animals the removal of the cerebral hemispheres is attended with much greater difficulties than in the case of the frog. Nevertheless, in the bird the operation may be carried out with approximate success. Pigeons, for instance, have been kept alive for five or six weeks after complete removal of the cerebral hemispheres, with the exception of portions of the crura and corpora striata immediately sur- 
rounding the optic thalami; these parts were left in order to ensure the intact condition of the latter bodies.

When the immediate effects of the operation have passed off, and for some time afterward, the appearance and behavior of the bird are strikingly similar to those of a bird exceedingly sleepy and stupid. It is able to maintain what appears to be a completely normal posture, and can balance itself on one leg, after the fashion of a bird which has in a natural way gone to sleep. Left alone in perfect quiet, it will remain impassive and motionless for a long time. When stirred it moves, shifts its position; and then, on being left alone, returns to a natural, easy posture. Placed on its side or its back it will regain its feet; thrown into the air, it flies with considerable precision for some distance before it returns to rest. It frequently tucks its head under its wings, and at times may be seen to clean its feathers; when its beak is plunged into corn, it eats. It may be induced to move not only by ordinary stimuli applied to the skin, but also by sudden loud sounds, or by flashes of light; in its flight it will, though imperfectly, avoid obstacles, and its various movements appear to be to a certain extent guided not only by touch but also by visual impressions.

In a certain number of cases this sleepy, drowsy condition passes off and is succeeded by a phase in which the bird, apparently spontaneously, without the intervention of any obvious stimulus, moves rapidly about. It does not fly, that is to say, it does not raise itself from the ground in flight, but walks about incessantly for a long while at a time, the periods of activity alternating with periods of repose. It seems, from time to time, to wake up and move about, and then to go to sleep again; and it has been observed that during the night it appears to be always asleep. It is obvious, therefore, that the sleepy, quiescent condition is not due simply to the absence of the cerebral hemispheres, but is a temporary effect of the operation, and that spontaneous movements, that is to say, movements not started by any obvious stimulus, may occur after removal of the cerebral hemispheres. But the movements so witnessed differ from those of an intact bird. They are, it is true, varied; and the variations are in part dependent on external circumstances, the bird being guided by tactile, and as we have said, visual sensations, or, to be more exact, by impressions made upon the sensory nerves of the skin and on the retina; but they do not show the wide variations of voluntary movements. The bird never flies up from the ground, never spontaneously picks up corn, and its aimless, monotonous, restless walks, resembling the continuous swimning of the frog thrown into the water after being deprived of its cerebral hemispheres, forcibly suggest that the activity is the outcome of some intrinsic impulse generated in the nervous machinery in some way or other, but not by the working of a conscious intelligence as in the impulse which we call the will.

Still we must not shut our eyes to the fact that spontaneous movements, whatever their exact nature, are manifested by a bird in the absence of the cerebral hemispheres, and become the more striking the more complete the recovery from the passing effects of the mere operation. Could such birds be kept alive for any considerable time, possibly further developments might be witnessed, and indeed cases are on record where birds have been kept alive for months after the operation, and have shown spontaneous movements of a still more varied character than those just described; but in such cases the removal of the hemispheres has not been complete, portions of the ventral regions being left behind; and, though a mere remnant left around the optic thalami can hardly be regarded as a sufficient cause for the spontaneity of which we are speaking, a larger mass, still more or less retaining its normal structure, might have a marked effect. And we 
may here perhaps remark that all these facts seem to point to the conclusion that what may be called mechanical spontaneity, sometimes spoken of as "autonatism," differs from the spontaneity of the "will" in degree rather than in kind. Looking at the matter from a purely physiological point of view (the only one which has a right to be employed in these pages), the real difference between an automatic act and a voluntary act is that the chain of physiological events between the act and its physiological cause is in the one case short and simple, in the other long and complex. Werhave seen that a frog lacking its cerebral hemispheres, viewed from one standpoint, appears in the light of a mechanical apparatus, on which each change of circumstances produces a direct, unvarying, inevitable effect. And yet it is on record that such a frog, if kept alive long enough for the most complete disappearance of the direct effects of the operation, will bury itself in the earth at the approach of winter, and is able to catch and swallow flies and other food coming in its neighborhood, although in other respects it shows no signs of an intelligent volition, and answers with unerring mechanical certainty to the play of stimuli. IVe may add that in some fishes the removal of their cerebral hemispheres, which in these animals form a relatively small part of the whole brain, produces exceedingly little change in their general behavior.

These, however, are not the considerations on which we wish here to dwell; we have quoted the behavior of the bird deprived of its cerebral hemispheres mainly to show that in this warm-blooded animal, as in the more lowly cold-blooded frog, the parts of the brain below or behind the cerebral hemispheres constitute a nervous machinery by which all the ordinary bodily movements may be carried out. The bird, like the frog, suffers no paralysis when the cerebral hemispheres are removed; on the contrary, though its movements have not been studied so closely as those of the frog, the bird without its cerebral hemispheres seems capable of executing at all events all the ordinary bodily movements of a bird. And in the bird as in the frog, the afferent impulses passing into the central nervous system, whether they give rise to consciousness or not, play an important part not only in originating but in guiding and coördinating the efferent impulses which stir the muscles to contract, the coördination being effected partly in the spinal cord, but largely and indeed chiefly in the parts of the brain lying behind the cerebral hemispheres. It is further worthy of notice that spontaneity of movement of the kind which we have described is much more prominent in the more highly developed bird than in the more lowly frog. The cerebral hemispheres are not the only part of the central nervous system which has undergone a greater development in the bird; the other parts of the brain have also acquired a far greater complexity than in the frog.

$\$ 553$. In the mammal the removal of the cerebral hemispheres is still more difficult than in the bird; the animal cannot be kept alive for more than a few hours; but in some mammals it is possible to observe during those few hours phenomena kindred to those witnessed in the bird and in the frog. The rabbit or rat, from which the whole of both hemispheres has been removed with the exception of the parts immediately surrounding the optic thalami, can stand, run, and leap. Placed on its side or back it at once regains its feet. Left alone it generally remains as motionless and impassive as a statue, save now and then when a passing impulse seems to stir it to a sudden but brief movement; but sometimes it seems subject to a more continued impulse to move, in which case death usually follows very speedily. Such a rabbit will remain for minutes together utterly heedless of a carrot or cabbage-leaf placed just before its nose, though 
if a morsel be placed within its mouth it at once begins to eat. When stirred it will with ease and steadiness run or leap forward; and obstacles in its course are very frequently, with more or less success, avoided. In some cases the animal (rat) has been described as following by movements of the head a bright light held in front of it (provided that the optic nerves and tracts have not been injured during the operation), as starting when a shrill and loud noise is made near it, and as crying when pinched, often with a long and seemingly plaintive scream. So plaintive is the ery which it thus gives forth as to suggest to the olsserver the existence of passion; this, however, is probably a wrong interpretation of a rocal action; the cry appears plaintive simply because, in consequence of the completeness of the reflex nervous machinery and the absence of the usual restraints, it is prolonged.

Without insisting too much on such results as these, and allowing full weight to the objection which may be urged, that in some of these cases parts of the cerebral hemispheres surrounding the optic thalami were left, there still remains adequate evidence to show that a mammal such as a rabbit, in the same way as a frog and a bird, may in the complete or all but complete absence of the cerebral hemispheres maintain a natural posture, free from all signs of disturbance of equilibrium, and is able to carry out with success, at all events all the usual and common bodily movements. And as in the bird and frog, the evidence also shows that these movements not only may be started by, but in their carrying out are guided by and coördinated by afferent impulses along afferent nerves, including those of the special senses. But in the case of the rabbit it is even still clearer than in the case of the bird that the effects of these afferent impulses are different from those which result when the impulses gain access to an intact brain. The movements of the animal scem guided by impressions made on its retina, as well as on other sensory nerves; we may perhaps speak of the animal as the subject of sensations; but there is no satisfactory evidence that it possesses either visual or other perceptions, or that the sensations which it experiences give rise to ideas. Its avoidance of objects depends not so much on the form of these as on their interference with light. No image, whether pleasant or terrible, whether of food or of an enemy, produces an effect on it, other than that of an object reflecting more or less light. And we may infer that it lacks the possession of an intelligent will. But it must always be remembered that some of the phenomena are due to the operation producing other results than the mere absence of the part removed. We must bear in mind that in all the above experiments, while the positive phenomena, the things which the animal continues able to do, are of great value, the negative phenomena, the things which the animal can no longer do, are of much less, indeed of doubtful value. The more carefully and successfully the experiments are carried out, the narrower become what we may call the "deficiency phenomena," the phenomena which are alone and directly due to something having been taken away. Were it possible to keep the rabbit alive long enough for the mere effects of the operation to pass completely away, we should not only probably witness, as in the case of the bird, a greater scope of movement and more frequent spontaneity, but possibly find a difficulty in describing the exact condition of the animal.

$\S 554^{\circ}$. Hitherto attempts to witness similar phenomena in more highly organized. mammals, such as the dog, have failed; these animals do not recover from the operation of removing the whole of both their hemispheres sufficiently to enable us to judge whether they, like the frog, the bird, and the rabbit, can carry out coördinate bodily movements in the absence of the hemispheres, or whether in them this part of the brain, so largely developed, has usurped functions which in the lower animals belong to other parts. 
Our knowledge is largely confined to the experience that when in a dog the cerebral convolutions are removed piecemeal at several operations, the animal may be kept alive and in good health for a long tine, many months at least, even after these parts of the brain have been reduced to very small dimensions, and that under these circumstances the animal is not only able to carry out with some limitations his ordinary bodily movements, but also exhibits a spontaneity obviously betokening the possession not merely of a conscious volition but of a certain amount of intelligence. Unless we are willing to believe that a mere fragment, so to speak, of the hemispheres can take on most extended powers, such an experience seems to show that in the $\log$ as in the rabbit and in the bird, the development of so-called higher functions is not limited to the cerebral hemispheres, that the middle and lower portions of the brain in the higher animals as compared with the lower do not increase in bulk merely as the instruments of the hemispheres, but like the hemispheres acquire more and more complex functions. IVe may perhaps go so far as to ask the question whether the volition and intelligence which such a dog exhibits is not as much the product of the parts lying behind the hemispheres as of the stump left in the front.

If we can thus say little about the condition of a dog without the cerebral hemispheres, we can say still less about the monkey, which in all matters touching the cerebral nervous system serves as our best, indeed our only guide for drawing inferences concerning man; but in all probability the monkey in this respect bears somewhat the same relation to the dog that the dog bears to the bird.

In short, the more we study the phenomena exhibited by animals possessing a part only of their brain, the closer we are pushed to the conclusion that no sharp line can be drawn between volition and the lack of volition, or between the possession and absence of intelligence. Between the musclenerve preparation at the one limit and our conscious willing selves at the other there is a continuous gradation without a break; we cannot fix on any linear barrier in the brain or in the general nervous system, and say, "Beyond this there is volition and intelligence, but up to this there is none."

This, however, is not the question with which we are now dealing. What we want to point out is that in the higher animals, including at least some inammals, as in the frog, after the removal of the cerebral hemispheres, even though conscious volition and intelligence appear to be largely, if not entirely lost, the body is still capable of executing all the ordinary movements which the animal in its natural life is wont to perform, in spite of these movements necessitating the coöperation of various afferent impulses; and that therefore the nervous machinery for the execution of these movements lies in some part of the brain other than the cerebral hemispheres. We have reason for thinking that it is situated in the structures forming the middle- and hind-brain; as we shall see, interference with these parts produces at once remarkable disorders of movement.

\section{The Machinery of Coördinated Movements.}

$\S 555$. We may now direct our attention for a while to some considerations concerning the nature of this complex nervous machinery for the coördination of bodily movements, and especially concerning the part played by afferent impulses. Most of our knowledge on this point has been gained by a study of animals not deprived of, but still possessing, their cerebral hemispheres, or by deductions from the data of our own experience; but it is possible in most cases to eliminate from the total results the phenomena which are due to the working of a conscious intelligence. Some of the most 
striking facts bearing on this matter have been gained by studying the effects of operative interference with certain parts of the internal ear, known as the semicircular canals.

When in a pigeon the horizontal membranous semicircular canal is cut through, the bird is observed to be continually moving its head from side to side. If one of the vertical canals be cut through, the movements are up and down. The peculiar movements may not be witnessed when the bird is perfectly quiet, but they make their appearance whenever it is disturbed or attempts in any way to stir. When the injury is confined to one canal only, or even to the canals of one side of the head only, the condition after a while passes away; when the canals of both sides have been divided, it becomes much exaggerated, lasts much longer, and in some cases is said to remain permanently. After such injuries it is found that these peculiar movements of the head are associated with what appears to be a great want of coördination of bodily movements. If the bird be thrown into the air, it flutters and falls down in a helpless and confused manner; it appears to have lost the power of orderly flight. If placed in a balanced pusition, it may remain for some time quiet, generally with its head in a peculiar posture: but directly it is disturbed, the movements which it attempts to execute are irregular and fall short of their purpose. It has great difficulty in picking up food and in drinking; and in general its behavior very much resembles that of a person who is exceedingly dizzy.

It can hear perfectly well, and therefore the symptoms cannot be regarded as the result of any abnormal auditory sensations, such as a "roaring" in the ears. Besides any such stimulation of the auditory nerve as the result of the section would speedily die away, whereas these phenomena may last for a very considerable time.

The movements are not occasioned by any partial paralysis, by any want of power in particular muscles or group of muscles; though removal of the canals of one side has been described as leading to diminished muscular force on the same side of the body, the mere diminution of force is insufficient to explain the phenomena. Nor, on the other hand, are the movements due to any uncontrollable impulse; a very gentle pressure of the hand suffices to stop the movements of the head, and the hand in doing so experiences no strain. The assistance of a very slight support enables movements otherwise impossible or most difficult to be easily executed. Thus, though when left alone the bird has great difficulty in drinking or picking up corn, it will continue to eat with ease if its beak be plunged into water or into a heap of barley; the slight support of the water or the grain seems sufficient to steady its movements. In the same way it can, even without assistance, clean its feathers and scratch its head, its beak and foot being in these opeations guided by contact with its own body.

The amount of disorder thus induced differs in different birds, and some movements are more affected than others. As a general rule, it may be sair that the more complex and intricate a movement, the fuller and more delicate the coördination needed to carry it out successfully, the more markedly is it disordered by the operation; thus, after injury of the canals, while a pigeon cannot fly, a goose is still able to swim.

In mammals (rabbits) section of the canals also produces a certain amount of loss of coördination, but much less than that witnessed in birds; and the movements of the head are not so marked, peculiar oscillating movements of the eyeballs, differing in direction and character according to the canal or canals operated upon, becoming, however, prominent. In the frog no deviations of the head are seen, but there is some loss of coördination in the movements of the body. In fishes no effect at all is produced. 
Injury to the bony canals alone is insufficient to produce the symptoms ; the membranous canals themselves must be divided or injured. The characteristic movements of the head may, however, be brought about in a bird without opening the bony canal, by suddenly heating or cooling a canal, especially its ampullar terminations, or by the making or breaking of a constant current directed through the canal.

There can be no doubt that these characteristic movements of the head are the result of afferent impulses started in the nervous endings of the auditory nerve over the ampulla of the canal and conveyed to the brain along that nerve. And that injury to or other stimulation of each of the three canals should produce in each case a different movement of the head, the direction of the movement being different according to the plane in which the canal lies, shows that these impulses are of a peculiar nature. This is further illustrated by the following experiment: If the horizontal canal be carefully laid bare, and the membranous canal opened so as to expose the endolymph, blowing gently over the opened canal with a fine glass canula will produce a definite movernent of the head, which is turned to the one side or to the other, according as the current of air drives the endolymph toward or away from the ampulla. From this it is inferred that a movement of the endolymph over, or an increased pressure of the endolymph on, the nervous endings in the ampulla gives rise to afferent impulses which in some way determine the issue of efferent impulses leading to the movement of the head. It is further suggested that since the planes of the three canals lie in the three axes of space, any change in the position of the head nust lead to changes in the pressure of the endolymph on the walls of the ampullæ or to movements of endolymph over those walls, and so must give rise to impulses passing up the auditory nerve; and that since every change of position will affect the three canals differently (whereas, the changes of pressure of the endolymph involved in a "wave of sound" will affect all three ampullæ equally), those impulses will differ according to the direction of the change. A still further extension of this view supposes that since in any one position of the head the pressure of the endolymph will differ in the three ampullæ, mere position of the head, as distinguished from change of position, is adequate to generate afferent impulses differing in the different positions.

Let us now for a while turn aside to ourselves and examine the coördination of the movements of our own bodies. When we appeal to our own consciousness we find that our movements are governed and guided by what we may call a sense of equilibrium, by an appreciation of the position of our body and its relations to space. When this sense of equilibrium is disturbed we say we are dizzy, and we then stagger and reel, being no longer able to coördinate the movements of our bodies or to adapt them to the position of things around us. What is the origin of this sense of equilibrium? By what means are we able to appreciate the position of our body? There can be no doubt that this appreciation is in large measure the product of visual and tactile sensations; we recognize the relations of our body to the things around us in great measure by sight and touch; we also learn much by our muscular sense. But there is something besides these. Neither sight nor touch nor muscular sense can help us when, placed perfectly flat and at rest on a horizontal rotating table, with the eyes shut and not a muscle stirring, we attempt to determine whether or not the table and we with it are being moved, or to ascertain how much it and we are turned to the right or to the left. Yet under such circumstances we are conscious of a change in our position, and some observers have been even able to pass a tolerably successful judgment as to the angle through which 
they have been moved. There can be no doubt that such a judgment is based upon the interpretation by consciousness of afferent impulses which are dependent on the position of the body, but which are not afferent impulses belonging to sensations of touch or sight, or taking part in the muscular sense. And it is urged with great plausibility that the afferent impulses in question are those which we have just referred to as started in the semicircular canals.

If we admit the existence of such ampullar impulses, if we may venture so to call them, and recognize them as contributing largely not only to our direct perception of the position of the head and thus of the body, but also in a more indirect way to what we have called the sense of equilibrium, we should expect to find that when they are abnormal the sense of equilibrium is disturbed, and that in consequence a failure of coördination in our movements results. And the loss of coördination which we described above as resulting from injury to the semicircular canals has accordingly been attributed to a deficiency or disorder of normal ampullar impulses.

But we must here distinguish between two things. It seems clear that when the membranous canals are injured or otherwise stimulated, afferent impulses are generated which, on the one hand, may produce peculiar movements of the head, and, on the other hand, seem able when the injury is large to cause a loss of coördination of bodily movements. But it does not necessarily follow' from this that in a normal condition of things afferent impulses are continually passing up to the brain from the semicircular canals, and that the loss of coördination which follows upon injury to the canals is due to these normal impulses being deficient or altered. It may be that such normal impulses do not exist, and that the loss of coördination is the result of the central machinery for coördination being interfered with by quite new impulses generated by the injury to the canal with the consequent loss of endolymph acting as a stimulus to the endings of the nerve. For the experience quoted above, though it proves that afferent impulses other than those of sight, touch, and the muscular sense do reach the brain and afford a basis for a judgment as to the position of the body, does not by itself prove that those impulses come from the semicircular canals; the arrangement of the canals is undoubtedly suggestive; but it is quite possible that the afferent impulses in question may be generated by one or other of various changes, vasomotor and others, of the tissues of the body which are involved in a change of position. And if it be true, as affirmed by some observers, that both auditory nerves may be completely and permanently severed without any effect on the coördination of movements, it is obvious that the incoördination which follows upon section of the semicircular canals is due to some special irritation set up by the operation, and not to the mere absence of any normal ampullar impulses. On the other hand, if the effects are those of irritation, it is difficult to understand how they can, as according to certain observers they certainly do, become permanent. It has, however, been strongly urged that in such cases of permanent incoördination, the operation has set up secondary mischief in the brain, in the cerebellum for instance, with which, as we have seen ( $\$ 531)$, the vestibular auditory nerve makes special connections, and that the permanent effects are really due to the disease going on here; and we have reason, as we shall see, to think that the cerebellum is concerned in the coördination of movements. It cannot, therefore, be regarded as settled that the canals are the source of normal impulses, or that our conscious appreciation of the position of the head and so of the body in space is based on such impulses. But such a view is not disproved; and in any case it remains true that injury to the canals does in some way or other, either by generating new impulses or by altering pre- 
existing ones, so modify the flow of afferent impulses into the machinery of coördination as to throw that machinery out of gear.

$\S 556$. We have dwelt on these phenomena of the semicircular canals because they illustrate in a striking manner the important part played by afferent impulses in the coördination of movements. IVe saw reason to think ( $\$ 502)$ that even in an ordinary reflex movement carried out by the spinal cord or by a portion of the cord, afferent impulses, other than those which excite the movement are at work, determining such coördination as is present. In such a case the coördinating afferent impulses are relatively simple in character and start chiefly at all events in the muscles concerned. In an animal possessing the lower parts of the brain, though deprived of the cerebral hemispheres, the coördinating afferent impulses, in accordance with the greater diversity and complexity of the movements which the animal is able to execute, are far more potent and varied. Besides afferent impulses from the muscles, forming the basis of what we have called the muscular sense, afferent impulses from the skin, forming the basis of the sense of touch in the wide meaning of that word, other afferent impulses of obscure character from the viscera and various tissues, and the peculiar afferent ampullar impulses of which we have just spoken, important special afferent impulses borne along the nerves of sight and hearing come into play. The frog, the bird, and even the mammal, deprived of the cerebral hemispheres, though it may show little signs or none at all of having a distinct volition, is, as we have urged, indubitably affected by visual and auditory impressions, and whether we admit or not that such an animal can rightly be spoken of as being conscious, we cannot resist the conclusion that afferent impulses started in its retina or internal ear produce in its central nervous system changes similar to those which in a conscious animal form the basis of visual and auditory sensations, and we must either call these changes sensations or find for them some new word. Whatever we call them, and whether consciousness is distinctly involved in them or not, they obviously play an important part as factors of the coördination of movements. Indeed, when we appeal to the experience of ourselves in possession of consciousness, we find that though various sensations clearly enter into the coördination of our movements, we carry out movements thus coördinated without being distinctly aware of these coördinating factors. In every movement which we make the coördination of the movement is dependent on the impulses or influences which form the basis of the muscular sense, yet we are not distinctly conscious of these impulses; it is only, as we shall see, by special analysis that we come to the conclusion that we do possess what we shall call a muscular sense. So again, taking the matter fiom a somewhat different point of view, many of our movements, markedly, as we shall see, those of the eyeballs, are coördinated by visual sensations, and when we sing or when we dance to music our movements are coördinated by the help of sensations of sound. In these cases distinct sensations in the ordinary sense of the word intervene; if we cannot see or cannot hear, the movement fails or is imperfect; yet even in these cases we are not directly conscious of the sensations as coördinating factors; it neerls careful analysis to prove that the success of the movement is really dependent on the sound or on the sight. These and other facts suggest the view that the point at which the various afferent impulses which form the basis of the sensations of a conscious individual enter into the coördination mechanism is or may be some way short of the stage at which the complete conversion of the impulse into a perfect sensation takes place. The events which constitute what we may call visual impulses, as these leave the retina to sweep along the optic nerve are, we must admit, very different from those which in the appropriate parts of the brain con- 
stitute what we may call conscious vision; and probably between the beginning and the end there are progressive changes. It is probable, we say, that these visual events may affect the coördinating mechanism at some stage of their progress before they reach their final and perfect form. If this be so we may further conclude that though, when the whole nervous machinery is present in its entirety, the afferent impulses which take part in coördination must inevitably at the same time give rise to conscious sensations, they might still effect their coördinating work when, owing to their imperfection or lack of the terminal part of the nervous machinery, the impulses failed to receive their final transformation, and conscious sensations were absent. In other words, the coördinating influences of sensory or afferent impulses are not essentially dependent on the existence of a distinct consciousness.

$\S 557$. We have raised this point partly for the sake of illustrating the working of the coördination machinery in the absence of the cerebral hemispheres, but also in order to aid in the interpretation of the subjective condition which we speak of as giddiness or dizziness or vertigo. The condition of the pigeon after an injury to the semicircular canals is comparable to that of a person who is giddy or dizzy, and, indeed, vertigo is the subjective expression of a disarrangement of the coördination machinery, especially of that concerned in the maintenance of bodily equilibrium. It may be brought about in many ways. When a constant current of adequate strength is sent through the head from ear to ear, we experienced a sense of vertigo; our movements then appear to a bystander to fail in coördination, in fact to resemble those of a pigeon whose semicircular canals have been injured; and, indeed, the effects are probably produced in the same way in the two cases. In what is called Ménière's disease attacks of vertigo seem to be associated with disease in the ear, being attributed by many to disorder of the semicircular canals, and cases have been recorded of giddiness as well as deafness resulting from disease of the auditory nerve. Visual sensations are very potent in producing vertigo. Many persons feel giddy when they look at a waterfall; and this is a case in which both the sense of giddiness and the disarrangement of coördination is the result of the action of a pure sensation and nothing else. In the well-known intense vertigo which is caused by rapid rotation of the body visual sensation plays a part when the rotation is carried on with the eyes open, but only a part ; for vertigo may be induced, though not so readily, by rotation with the eyes completely shut. In the latter case it has been suggested that the vertigo is caused by abnormal ampullar impulses, but these can only contribute to the result, which is in the main caused by direct disturbance of the brain. When the rotation is carried out with the eyes open, the vertigo which is felt when the rotation ceases is partly caused by the visual sensations, on account of the behavior of the eyeballs, ceasing to be in harmony with the rest of the sensations and afferent impulses which help to make up the coördination. The rotation sets up peculiar oscillating movements of the eyeballs, which continue for some time after the rotation has ceased; owing to these movements of the eyeballs the visual sensations excited are such as would be excited if external objects were rapidly moving, whereas all the other sensations and impulses which are affecting the central nervous system are such as are excited by objects at rest. In a normal state of things the visual and the other sensations and impulses which go to make up the coördinating machinery are in accord with each other in reference to the events in the external world which are giving rise to them; after rotation they are for a time in disaccord, and the coördinating machinery is in consequence disarranged.

When we interrogate our own consciousness, we find that we are not dis- 
tinctly conscious of this disaccord; the visual sensations are so prepotent in consciousness that we really think the external world is rapidly whirling round; all that we are further conscious of is the feeling of giddiness and our inability to make our bodily movements harmonize with our visual sensations. So that even in the cases where the loss of coördination is brought about by distinct sensations, what we really appreciate by means of our consciousness is the disarrangement of the coördinating machinery. It is the appreciation of this disorder which constitutes the feeling of vertig(); both the feeling of giddiness and the disordered movements are the outcome, one subjective and the other objective, of the same thing. It is not because we feel giddy that we stagger and reel; our movements are wrong because the machinery is at fault, and it is the faulty action of the machinery which also makes us feel giddy.

We may here perhaps remark that it is an actually disordered condition of the coördinating mechanism which gives rise to the affection of consciousness which we call giddiness, not a mere curtailing of the mechanism or any failure on its part to make itself effective. Complete blindness limits the range of activity of the machinery but leaves the remainder intact, and no giddiness is felt. So again in certain diseases of the nervous system the muscular sense is interfered with over considerable regions of the body, and in these regions coördination fails or is imperfect, but the central machinery is not thereby affected, though its area of usefulness is limited, and no giddiness is experienced; and so in other instances.

$\$ 558$. Forced movements. So far we have dwelt on disorders of the coördinating machinery brought about by the action of various afferent impulses. We have now to call attention to some peculiar phenomena which result from operative interference with parts of the brain, and which in some instances at least may be taken to illustrate how this complex machinery works when some of its inner wheels are broken.

All investigators who have performed experiments on the brain have observed, as the result of injury to various parts of it, remarkable movements which have the appearance of being irresistible, compulsory, forced. They vary much in the extent to which they are developed; some are so slight as hardly to deserve the name, while others are strikingly intense. One of the most common forms is that in which the animal rolls incessantly round the longitudinal axis of its own body. This is especially common after section of one of the crura cerebri, or of the middle and inferior peduncles of the cerebellum, or after unilateral section of the pons, but has also been witnessed after injury to the bulb and corpora quadrigemina. Sometimes the animal rotates toward and sometimes away from the side operated on. Another form is that in which the animal executes "circus movements," $i$. e., continually moves round and round in a circle of longer or shorter radius, sometimes toward and sometimes away from the injured side. This may be seen after several of the above-mentioned operations, and in one form or another is not uncommon after various unilateral injuries to the brain. There is a variety of the circus movement, the "clock-hand movement," said to occur frequently after lesions of the posterior corpora quadrigemina, in which the animal moves in a circle, with the longitudinal axis of its body as a radius and the end of its tail for a centre. And this form again may easily pass into a simple rolling movement. In yet another form the animal rotates over the transverse axis of its body, tumbles head over heels in a series of somersaults; or it may run incessantly in a straight line backward or forward until it is stopped by some obstacle. These latter forms of forced movements are sometimes seen after injury to the corpus striatum even when a very limited portion of the gray matter is affected. And many of these 
forced movements may result from injuries which appear to be confined to the cerebral cortex.

When the phenomena are well developed, every effort of the animal brings on a movement of this forced character. Left to itself and at rest the animal may present nothing abnormal, its posture and attitude may be quite natural; but when it is excited to move or when it attempts of itself to move, it executes not a natural movement, but a forced one, turning round or rolling over as the cuse may be. In severe cases the movement is continued until the animal is exhausted; when the exhaustion passes off the animal may remain for some little time quiet, but some stimulus, intrinsic or extrinsic, soon inaugurates a fresh outbreak, to be again followed by exhaustion.

In some of the milder forms, that for instance of the circus morement with a long radius, the curved character of the progression appears simply due to the fact that in the effort of locomotion volitional impulses do not gain such ready access to one side of the body as to the other, the injury having caused some obstacle or other. Hence the contractions of the muscles of one side (the left, for instance) of the body are more powerful than the other, and in consequence the body is continually thrust toward the other (the right) side. As is well known, we ourselves, when our walk is not guided by visual sensations, tend to describe a circle of somewhat wide radius, the deviation being due to a want of bilateral symmetry in our limbs; and the above circus movement is only an exaggeration of this.

But the other more intense forms of forced movements are more complicated in their nature. No mere blocking of volitional impulses will explain why an animal whenever it attempts to move rolls rapidly over or rushes irresistibly forward or backward. It is not possible with our present knowledge to explain how each particular kind of movement is brought about; and, indeed, the several kinds are probably brought about in different ways, for they differ so greatly from each other that we only class them together because it is difficult to know where to draw the line between then. But we may regard the more intense forms as illustrating the complex nature of what we have called the coördinating machinery, the capabilities of which are, so to speak, disclosed by its being damaged. Such gross injuries as are involved in dividing cerebral structures or in injecting corrosive substances into this or that part of the brain, must, of necessity, partly by blocking the way to the impulses which in a normal state of things are continually passing from one part of the brain to another, partly by generating new unusual impulses, seriously affect the due working of the general coördinating machinery. The fact that an animal can, at any moment, by an effort of its own will, rotate on an axis or run straight forward, shows that the nervous mechanism for the execution of those movements is ready at hand in the brain, waiting only to be discharged; and it is easy to conceive how such a discharge might be affected either by the substitution for the will of some potent intrinsic afferent impulse or by some misdirection of volitional impulses. Persons who have experienced similar forced movenients as the result of disease report that they are frequently accompanied, and seem to be caused, by disturbed visual or other sensations; thus they attribute their suddenly falling forward to the occurrence of the sensation that the ground in front of them is suddenly sinking away beneath their feet. Without trusting too closely to the interpretations the subjects of these disorders give of their own feelings, and remembering what was said above concerning vertigo, we may at least conclude that the unusual movements are in many cases due to a disorder of the coördinating mechanism, brought about by strange or disordered sensory impulses. And this view is supported by the fact that many of these forced movements are accompanied by a peculiar 
and wholly abnormal position of the eyes, which alone might perhaps explain many of the phenomena.

$\$ 559$. The phenomena presented by animals deprived of their cerebral hemispheres show that this machinery of coördination is supplied by cerebral structures lying between the cerebral hemisphere above and the top of the spinal cord below. But when we ask the further question, How is this machinery related to the various elements which go to make up this part of the brain? the only answers which we receive are of the most imperfect kind.

In the case of the fiog we can, after removal of the cerebral hemispheres, make an experimental distinction in the parts left between the optic thalami with the optic nerves and tracts, the optic lobes, and the bulb with the rudimentary cerebellum. When the optic thalami are removed, as might be expected, the evidence of visual impressions modifying the movements of the animal disappears; and it is stated that apparently spontaneous movements are much more rare than when the thalami are intact. When the optic Iobes as well as the cerebral hemispheres are removed, the power of balancing is lost; when such a frog is thrown off its balance by inclining the plane on which it is placed, it slips back or falls down; the special coordinating mechanism for balancing must, therefore, in this animal have a special connection with the optic lobes. But after removal of these organs the animal is still capable of a great variety of coördinate movements; unlike a frog retaining its spinal cord only, it can swim and leap, it maintains a normal posture, and when placed on its back immediately regains the normal posture. The cerebellum of the frog is so small, and in removing it injury is so likely to be done to the underlying parts, that it becomes difficult to say how much of the coördination apparent in a frog possessing cerebellum and bulb is to be attributed to the former or to the latter; probably, however, the part played by the former is small.

In the case neither of the bird nor of the mammal have we any exact information as to the behavior of the animal after removal of the parts behind the hemispheres, in addition to the hemispheres themselves. Our knowledge is confined to the results of the ablation or of the stimulation of parts, the cerebellum for instance, in animals in which the rest of the brain has been left intact. Observations of this kind have disclosed many interesting facts, besides the forced movements just referred to, but they have not led to, and indeed could hardly be expected to lead to, any clear views as to. the point which we are now discussing. It does not follow that every part, injury or stimulation of which interferes with coördinated movements, or gives rise to definite, forced, or other movements, is to be considered as part of the machinery under consideration. The corpora striata and cerebral hemispheres form, as we have seen, no part of the machinery, yet injury to them may disorder the machinery; and the fact that removal of or injury to the cerebellum disorders the machinery is no proof by itself that the cerebellum is an essential part of the machinery.

If we may trust to deductions from structural arrangements, we might be inclined to infer that the anatomical relations of the tegmental region from the bulb upward point to its serving as the foundation of the machinery in question. Behind it has full connections with various parts of the cord, while in front by means of the optic thalami and anterior corpora quadrigemina, if not by other ways as well, it is so far associated with the optic nerves that the path seems open for visual impulses to gain access to it. To this foundation, however, we must add the cerebellum, on account of its relations to it, to the cord, and to the bulb through the restiform bodies, including its ties with the auditory nerve. And if we add the cerebellum 
we must also probably add the pons. We may exclude the pes of the crus, since this is composed exclusively of fibres,bringing the cerebral hemispheres, including the corpora striata, into connection with the pons, bulb, and cord, and so with the coördinating machinery itself, as well as with other parts of the nervous system. And observation, as far as its goes, supports this deduction from anatomical relationships. We will, however, defer what else we have to say on this point until after we have discussed the carrying out of voluntary movements.

\section{On Some Histological Features of the Brain.}

$\S 560$. The white matter of the brain, as we have already said, like that of the spinal cord, consists of medullated fibres of various sizes imbedded in neuroglia and supported by septa of connective tissue derived from the pia mater. Save that cells, or even groups or rows of cells, for the most part small cells, about many of which it may be debated whether they are nervecells or neuroglia cells, are frequently seen between the fibres and bundles of fibres, the white matter of the brain seems essentially identical with that of the spinal cord.

The gray matter of the brain in general also corresponds to the gray matter of the cord in consisting of branching nerve-cells, fine medullated fibres of peculiar nature, non-medullated fibres and fibrils, with a few ordinary medullated fibres, all supporterl in neuroglia.

The "central" gray matter is extremely like that of the cord except that the nervous elements are imbedded in a relatively larger quantity of neuroglia. Immediately underneath the epithelium lining the several ventricles and the aqueduct, the neuroglia is especially developed, forming a distinct layer which may be regarded as a continuation of the central gelatinous substance of the spinal cord, and which, with the epithelium overlying it, forms what is known as the ependyma. The "nuclei" of the cranial nerves are, as we have seen, comparable to the groups of nerve-cells in the spinal cord.

A great deal of gray matter of the brain may be spoken of as more "diffuse" or "scattered," more broken up by bundles of fibres than is the case in the spinal cord. The "reticular formation" of the bulb and of the tegmental region is an extreme form of this diffuse gray matter. And even in such collections of indubitable gray matter as the corpus striatum, optic thalamus, and the like, the pure gray matter, if we may use the term, is much more interrupted and broken up by conspicuous bundles of white fibres than is the case in any region of the spinal cord. In the corpora quadrigemina the gray matter is broken up by sheets or bundles of white matter.

The nerve-cells of the several collections of gray matter are not all alike ; they present in different regions differences in size, form, and in other characters. The cells of the nucleus caudatus, for instance, are rather small and often round or spindle-shaped, while those of the optic thalamus are large, branched, and rich in pigment. The cells of the substantia nigra are spindleshaped, of moderate size, and so loaded with black pigment (in man) as to justify the name; those of the locus cæruleus are very large and spherical, with just so much pigment as to give a bluish tint. But our knowledge of the finer histological details of the various masses of gray matter is at present too imperfect to afford any basis whatever for physiological deductions ; and it will be hardly profitable to dwell upon them. Two regions of gray matter alone call for special description, the cortex cerebri and the superficial gray matter of the cerebellum. 


\section{The Superficial Gray Matter of the Cerebellum.}

$\$ 561$. The surface of the cerebellum is increased by being folded or plaited into leaf-like folds, and each of these primary folds is similarly folded into a number of secondary, also leaf-like, folds or lamellæ. Each of these lamellæ consists of a central core of white matter, the fibres of which pass inward to and contribute to form the central white matter of the cerebellum, and of a superficial layer of gray matter. A section through a lamella perpendicular to the surface shows that the gray matter consists essentially of two layers: a layer lying next to the white nuatter formed by densely crowded small cells, called the nuclear layer, and between this and the superficial pia mater a much thicker layer of peculiar nature, called the molecular layer. Between these two layers, and connected, as we shall see, with both of them, lies a row of very large and remarkable cells, called the cells of Purkinje, the bodies of which abut on the nuclear layer, and the long branches of which traverse the molecular layer; these cells so placed may be said to constitute a third layer. Before proceeding further, we may here remark that a section of the lamellæ, that is, one of the secondary, not one of the primary, folds, while still remaining a vertical section (that is, perpendicular to the surface), may be carried through the lamella in different planes, and that, of these several planes, the sections taken in two of them are especially instructive, namely, the one taken in what we may call the longitudinal plane, passing from the top of the lamella to its base, and the one taken at right angles to the former, in what we may call the transverse plane. The nuclear layer and the molecular layer present the same broad features in both longitudinal and transverse sections, but the long-branched processes of the cells of Purkinjé since they run in the transverse plane are adequately seen in transverse sections only; longitudinal sections show only their profiles.

The molecular layer is of a peculiar nature. In many modes of preparation and in many sections it appears chiefly composed of a granular or dotted ground substance; hence the name molecular, as if it were an aggregation of molecules. The dots, however, are sections of fine fibrils, some of which are neuroglia fibrils but others are undoubtedly nervous. The layer consists in fact partly of nervous elements, and here perhaps even more than elsewhere it is extremely difficult to say with regard to many of the elements whether they are neuroglial or nervous in nature. A considerable portion of the whole area of the molecular layer is taken up by the conspicuous branched processes of the cells of Purkinjé ; and scattered about lie numerous small cells, some of which are neuroglia cells, but some of which are undoubtedly nerve-cells. The most conspicious feature of the layer, however, is the presence in large numbers of the fine fibrils; but before we speak of these it will be desirable to turn to the cells of Purkinjé and the nuclear layer.

The cell of Purkinjé possesses a large $(40 \mu$ by $30 \mu)$ flask-shaped body, surrounding a large, conspicuous, clear, rounded nucleus; it has much the appearance of a large ganglion cell. The base of the flask rests on the nuclear layer, and from it there proceeds a single axis-cylinder process which, passing through the nuclear layer somewhat obliquely, and in its passage acquiring a medulla, joins the central white substance as a medullated fibre. The cells, as we have said, form a single layer only, but since this covers the nuclear layer over the whole of the lamella, a considerable number of the fibres of the white central matter, though only a very small fraction of the whole, are thus derived from these cells of Purkinjé. The narrowed neck of the flask running outward in the molecular layer divides in an arborescent 
fashion into a large number of branches which, spreading out laterally in the transverse plane and stretching as far as the surface, ramify through the molecular layer, and are eventually lost to view as exceedingly fine fibrils. Some observers maintain that some of the fine processes are continuous with processes of the small nerve-cells of the molecular layer, but this is not admitted by all. In any case the fibrillar terminations of these cells of Purkinjé contribute to the fine fibrils of the molecular layer.

The nuclear layer in ordinary stained specimens has the appearance of a mass of nuclei closely crowded together in a bed of reticular nature; and since the nuclei usually stain deeply, the layer stands out in strong contrast to the much less deeply stained molecular layer. Careful examination with special modes of preparation shows, however, that while some of the nuclei are nuclei belonging to neuroglia and bloodvessels, the majority belong to small nerve-cells of a peculiar nature. In these cells the nucleus is surrounded by cell substance, which, forming a thin layer immediately around the nucleus, is chiefly disposed as thin spreading branches, some of which end in a peculiar arborescence not unlike a muscle end-plate; these processes contribute with the neuroglia to form the reticular-looking bed spoken of above. No process can be traced inward to the central white matter; but one of the processes gives off a branch, which passing vertically outward takes on the appearance of a delicate axis-cylinder process and runs, without dividing, into the molecular layer for a variable distance, sometimes reaching close to the surface, but at last divides at right angles into two fibrils, which run in the longitudinal plane in opposite directions for a considerable distance, and are ultimately lost to view. Since these cells in the nuclear layer are very numerous and each gives rise in the above manner to longitudinal fibrils, the molecular layer is traversed by a multitude of fibrils, visible as such in longitudinal sections, but appearing as dots in transverse sections, in which the cells of Purkinjé are best displayed.

Besides these longitudinal fibrils proceeding from the cells of the nuclear layer, special modes of preparation similarly disclose numerous transverse as well as more or less oblique fibrils. Many of these appear to result from the branching of the small nerve-cells of the molecular layer, and some of those so arising descend to the layer of the cells of Purkinjé and end around the bodies of those cells in remarkable nests of fibrils, without, however, actually making connections with them.

The medullated fibres of the central white matter of a lamella pass on all sides into the nuclear layer; or, put in another way, medullated fibres passing out of the nuclear layer at all points converge to form the central white matter. Some of these fibres, as we have seen, begin or end in the cells of Purkinjé. None of them appear to join the cells of the nuclear layer, and we have no evidence that any of them end or begin in any way in the nuclear layer. A certain number, however, may be seen to pass through the nuclear layer and between the cells of Purkinje into the molecular layer, where losing their medulla they divide and apparently contribute to the numerous fibrils of the molecular layer. The presumption, therefore, is that all the fibres of the white matter begin or end either in the cells of Purkinjé or the fibrils of the molecular layer.

The superficial gray matter of the cerebellum then resembles the gray matter of the spinal cord in so far as it consists of branching nerve-cells, nerve-fibres, and nerve-fibrils imbedded in neuroglia; but the disposition and features of the several factors are peculiar. We may take, perhaps, as the key of the structure the fibrils of the molecular layer; this layer is relatively very thick, about $400 \mu$, much thicker than the nuclear which, however, varies in thickness, being generally thickest at the top of the fold; 
hence the number of fibrils in it may be spoken of as enormous. These fibrils seem certainly to be connected on the one hand with the cells of the nuclear layer, and on the other hand with the scattered small cells of their own layer; but we have no evidence that these two sets of fibrils are continuous with each other; on the contrary, it seems more probable that the two sets of cells represent two independent systems. We can hardly doubt that these fibrils are in functional connection with the medullated fibres of the central white matter; but we have no clear evidence that the system of scattered cells is continuous either with the cells of Purkinjé, and so with the medullated fibres belonging to those cells, or with the medullated fibres which end independently in the molecular layer; and we have no evidence at all that the system of the cells of the nuclear layer is connected with either. We can hardly think otherwise than that the molecular changes which sweep to and fro along the tangle of these fibrils (whose nutrition is probably governed and hence whose functional activity is probably regulated by the nuclear and scattered cells respectively) are influenced by or originate the nervous impulses passing along the medullated fibres of the white matter; and hence we must conclude that either a continuity exists which has as yet escaped detection, or, what is quite possible if not probable, that one fibril can act upon another by simple contact or even at a distance. Further, while the cell of Purkinjé, with its large cell-body and nucleus, its conspicuous axis-cylinder process and its other branched processes presents many analogies with motor cells, such as those of the anterior horn of the spinal cord, and raises the presumption that the impulses which move along its axis-cylinder process proceed outward from the cell as motor or at least as efferent impulses, we have no direct proof that this is so. And though it is tempting to suppose that the other medullated fibres, which like the fibres of a posterior root are lost in the gray matter, without the intervention of a conspicuous cell, carry efferent impulses, we have as yet no proof of this. All we can say is that the gray matter is connected in two different ways with at least two sets of fibres, which probably therefore, have different functions.

We may here add the remark that the large body of the cell of Purkinjé lies, as indeed do the other nervous elements, in an appropriate space in the bed of neuroglia. Between the surface of the cell and the wall of neuroglia is a space, generally so narrow as to be potential rather than actual, but which may sometimes be considerable. Whether small or large it contains lymph, and the cavity in which the cell lies is in connection with the lymphatics of the brain. Each cell then lies in a lymph-space; but we merely mention the fact now; we shall have to return to the matter when we come to deal with the lymphatic and vascular arrangements of the brain and spinal cord.

\section{The Cerebral Cortex.}

$\S 562$. While the superficial gray matter of the cerebellum does not differ strikingly as to its histological features in different regions, very considerable differences are observed in different regions of the cerebral cortex. A general plan of structure may perhaps be recognized, but as we pass from one part of the cerebral surface to another we find modifications continually taking place. IVe must content ourselves here with attempting a description of the general plan, followed by an indication of the more striking characteristics of certain regions.

The cortical gray matter, having an average thickness of about $3 \mathrm{~mm}$., but varying considerably in different regions from $1.8 \mathrm{~mm}$. in some parts of 
the occipital lobe to $4.2 \mathrm{~mm}$. at the dorsal summit of the precentral convolution, is, like other gray matter, composed of nerve-cells, and of nerve-fibres and fibrils supported by neuroglia. The nerve-cells, at least the conspicuous and easily recognized nerve-cells, are scattered, and appear, in sections, to be imbedded in and separated from each other by a not inconsiderable but variable quantity of somewhat peculiar ground substance, not unlike that which forms so large a part of the molecular layer of the cerebellum. Part of this ground substance, which apparently is not confined to any particular layer, but stretches throughout the thickness of the cortex, is undoubtedly neuroglial in nature, but part, and probably the greater part, is nervous in nature ; it is largely composed of fine fibrils traversing it in various directions, the transverse sections of the fibrils giving it a characteristic dotted or "molecular" appearance; and the majority of these fine fibrils are probably the continuations of branching nerve-cells or dividing nerve-fibres, the remainder being neuroglial fibrils. In this respect it resembles the molecular layer of the cerebellum, but it is, to a much greater extent than is that layer, traversed by medullated nerve-fibres, especially by fine medullated fibres like those seen in the gray matter of the spinal cord ( $\$ 476$ ).

The nerve-cells imbedded in this ground substance in more or less distinct layers are of various kinds. The most conspicuous, abundant and characteristic nerve-cells found in the cortex of all regions of the cerebellum are those which from their shape are called pyramidal cells. These vary very much in size and have been distinguished as "small pyramidal" cells averaging $12 \mu$ in length by $8 \mu$ in breadth, and "large pyramidal" cells, sometimes called "ganglionic cells," of which the medium size is about $40 \mu$ in length by $20 \mu$ in breadth. Some of the latter, occurring in special regions, are of very large size, $120 \mu$ by $50 \mu$, and have been called "giant cells."

The features of a "large pyramidal" cell are very characteristic. Such a cell appears in a well-prepared vertical section of the cortex as an elongated isosceles triangle placed vertically, with the base looking toward the underlying white substance and the tapering apex pointing to the surface. The cell substance is finely granulated or fibrillated, the fibrillæ sweeping round in various directions; it not unfrequently contains pigment. In the midst of this cell substance rather near the base lies a large, clear, conspicuous round or oval nucleolated nucleus. At the base the cell substance is prolonged into a number of processes. One of these, generally starting from about the middle of the base, runs for some distance without dividing, and soon acquiring a medulia may be recognized as an axis-cylinder process; the fibre to which it gives origin sweeps with a more or less curved course into the subjacent white matter. In some instances the axis-cylinder process, by a T-division like that seen in a ganglion of a posterior root ( $\$ 93)$, gives rise to two fibres, one of which may take a horizontal direction; in some regions of the cortex, the occipital for instance, the axis-cylinder process is said to give rise by division to several fibres. The other processes from the base, especially those from the angles of the triangle, rapidly branch into fine fibrils which are soon lost to view in the ground substance. The apex of the triangle is also prolonged into a process, which, giving off fine lateral branches, makes, as it were, straight for the surface, but ultimately branching into fine fibrils is lost to view at some distance from the body of the cell. The cell lies in a cavity of the ground substance, which it appears normally to fill, but from the walls of which it sometimes shrinks, developing between itself and the wall of the cavity a space which may contain not only lymph, but occasionally leucocytes. In prepared specimens the retraction within its cavity of the artificially shrunken cell may be often observed. 
The "small pyramidal" cells have much the same features; that is to say, the cells are characterized by their pyramidal form, though this is naturally not so distinct, by their vertical position, and by the possession of branching processes which are lost in the molecular ground substance; the presence, however, of a midbasal axis-cylinder process has not been clearly demonstrated.

Other nerve-cells are more like the ordinary nerve-cells of the spinal cord and of the internal cerebral gray matter; they are branched cells, of irregular, not of pyramidal, form, and for the most part small, $18 \mu$ by $10 \mu$. They may be characterized by the relatively large size, $7 \mu$, of the nucleus, and do not possess an axis-cylinder process; at least, such a process has not yet been demonstrated. They are frequently spoken of as "angular" cells.

Another kind of cell, the "fusiform cell," which is found in all regions of the cortex, has a characteristic spindle-shape, the cell substance being prolonged at the opposite poles into tapering, ultimately branched processes. The long axis of the cell is generally placed horizontally, following the curvature of the cortex, and being thus at the sides of the sulci vertical to the surface of the brain; it is, however, at times inclined at various angles.

Still another kind of cell, the "granule cell," or " nuclear cell," is one in which the nucleus is surrounded by a relatively small quantity of cell substance, $9 \mu$ by $7 \mu$, more or less spherical in form in ordinary preparations, but probably breaking up into delicate branched processes. Cells of this kind are sparsely scattered throughout the cortex generally, but in particular regions-e.g., the occipital-are crowded together into a layer, which in many respects resembles the nuclear layer of the cerebellum, and has been called the "granular" or "nuclear" layer.

I astly, throughout the cortex are found, besides indubitable nerve-cells and indubitable neuroglial cells, numerous small, somewhat irregular cells, concerning which it may be debated whether they are really nervous or simply neuroglial in nature. Moreover, in using the names given above for the various kinds of nerve-cells, it must be remembered that many transitional forms are observed; cells, for instance, may be seen intermediate in form between pyramidal cells and "fusiform" or "angular" cells.

The medullated nerve-fibres which take part in the cortex may be considered provisionally as forming two categories. In the first place, fibres sweep up vertically into the cortex from the subjacent "central white matter," taking at first a curved course as they enter into the gray matter, and then appearing to run straight toward the surface. These are arranged in the deeper levels in bundles, leaving vertical columns of the gray matter between them; but at more superficial levels the bundles spread out and are gradually lost to view. Besides these distinct vertical fibres and bundles of fibres of the ordinary medullated kind, which we have reason to think are the ends (or beginnings) on the one hand of fibres of the pedal and tegmental systems, and on the other hand of fibres of the corpus callosum, or the other commissural fibres spoken of as "association" fibres ( $\$ 548)$, an exceedingly large number of fibres of the peculiar fine medullated kind run in various directions, forming a dense network in the ground substance of the gray matter between the cells. We may add that this system of fine medullated fibres is of late growth, and is not fully developed in man until two or three years after birth. Many of the medullated fibres, coarse as well as fine, take a horizontal direction parallel to the surface, and in certain regions are specially developed into a layer or into two layers so.as to form a horizontal streak or streaks.

The vascular pia mater invests closely, as we have said, the whole surface of the cortex, dipping down into the sulci; and from it, as in the case of the 
spinal cord, processes carrying bloodvessels and bearing lymph-spaces pass inward to supply the gray matter with blood. But while, as we shall see later on, the supply of bloodvessels to the gray matter is considerable, the truly connective-tissue elements of the pia mater processes are soon merged into neuroglia. Immediately beneath the pia mater forming the immediate surface of the cortex is a thin layer consisting of neuroglia enly.

$\S 563$. The nerve-cells of the above several kinds are arranged more or less distinctly in layers parallel to the surface, so that the whole thickness of the cortex may by means of them be, more or less successfully, divided into a series of zones, one above the other; and we may, as we have said, recognize on the one hand a general arrangement common to the whole surface, and on the other hand modifications existing in the several regions. The general arrangement may be said to be one of five layers or zones, usually counted from the surface inward.

The fifth layer, lyiug next to the central white matter, fairly uniform in characters and thickness (about $1 \mathrm{~mm}$.) over the greater part of the brain, is characterized by the presence of somewhat sparsely scattered "fusiform" cells, though other branched cells are present. It is broken up into vertical columns by the bundles of vertical fibres, and its demarcation from the white matter below is somewhat indistinct, owing to the fact that in the brain the white matter, especially that lying beneath the cortex, contains cells and small groups of cells lying between the bundles of flbres to a much greater extent than does the white matter of the spinal cord.

The fourth layer, lying above the preceding, varies much more both in thickness $(0.35 \mathrm{~mm}$. to $0.15 \mathrm{~mm}$.) and in its characters. The constituent cells are on the one hand large pyramidal cells, and on the other hand "granule" or "nuclear" cells. In some regions it may be subdivided into two layers, the small "nuclear" cells being so abundant as to form in the upper part of the layer a separate layer called the "granule" or "nuclear" layer. This fourth layer, like the preceding fifth layer beneath it, is split up into vertical columns by the bundles of vertical fibres, but to a less degree. It is marked in its lower part by a horizontal streak due to numerous, mostly fine, medullated fibres running horizontally. In the cortex of the island of Reil this horizontal layer is developed into a conspicuous sheet of medullated fibres, separating the fourth and fifth layers by a distinct interval of obvious white matter. This fifth layer of fusiform cells, thus detached from the rest of the cortex, is what is called the claustrum (Figs. 138, 139, cl.).

In the third layer, the constituent cells are the characteristic pyramidal cells. These are for the most part large, though diminishing in size from below upward, and the layer has been called the "layer of large pyramidal cells," though in certain regions the largest pyramidal cells, and notably the giant-cells, are found in the preceding, fourth, layer. The cells are, on the whole, scattered somewhat sparsely, though frequently gathered into small groups, and among them occur small "nuclear" and other cells. The bundles of vertical fibres spread out rapidly in this layer, so that the columnar arrangement becomes lost, and many of the fibres undoubtedly become axis-cylinder processes of the pyramidal cells. Though the layer varies in thickness ( $1 \mathrm{~mm}$. to $0.4 \mathrm{~mm}$.), and in some of its features in different regions, the characteristic pyramidal cells are present over the whole surface of the hemisphere. In the lower part of the layer a second horizontal streak of closely interwoven horizontal fibres frequently makes its appearance.

The second layer, generally a thin one, though varying from $0.25 \mathrm{~mm}$. to $0.75 \mathrm{~mm}$. in thickness, is also formed by pyramidal cells, but is distinguished from the layer below by the absence of large and medium-sized cells and by the presence of numerous small cells closely packed together; it has been 
called "the layer of small pyramidal cells." As we have said, these smaller pyramidal cells differ somewhat from the larger cells; and the cells in this layer are sometimes described as " angular."

The first and most superficial layer is characterized by the predominance of the molecular ground substance, the cells being few, far between, small, and irregular. The ground substance itself seems to be more largely neuroglial in nature than in the other layers, and, as we said above, its extreme surface appears to be furnished by neuroglia alone. The layer is generally spoken of as the "peripheral " or "superficial layer," or sometimes as the "molecular" layer. The tapering vertical processes of the pyramidal cells may be traced into this layer, which indeed varies in thickness according to the abundance of pyramidal cells in the subjacent layers; numerous somewhat fine medullated fibres also traverse it in a horizontal direction.

$\$ 564$. The general arrangement just described varies, as we have said, in different regions of the cerebral surface. We must content ourselves here with pointing out the characteristics of two or three important regions.

The region which we have ( $\$ 545)$ called the " motor area " or " region" is characterized on the one hand by the great thickness $(1 \mathrm{~mm}$.) of the third layer, that of large pyramidal cells, as well as by the number and size of the cells contained in it, and on the other hand, and especially, by the prominence in the fourth layer of remarkable clusters of very large pyramidal cells, of the kind which are referred to above ( $\$ 562)$ as being frequently called "ganglionic;" it is in this region that "giant cells" are found in the fourth layer, namely, in the upper part of the precentral and at the summit of the postcentral convolution, and in the paracentral lobule, acquiring their greatest size at the top of the precentral convolution.

The occipital region is characterized by the prominence of the "granule" or "nuclear" cells. These not only form a distinct division of the fourth layer, but are also conspicuous in other layers, their arrangements being such that some authors have been led to divide the cortex of this region into seven or even eight layers. In the present state of our knowledge we may be content with insisting that the great mark of this occipital region is the abundance of these small "nuclear" cells, together with other small "angular" cells, whereby the pyramidal cells seem to be made less conspicuous. It is worthy of notice, however, that in the third, but more especially in the fourth layer, a few cells of very large size are met with, which by their large branched cell substance and conspicuous axis-cylinder process resemble the large cells in the motor region; but it should be noted that while these large cells occur (at least in man and in the monkey, though not in some of the lower animals, as the rabbit) in very definite clusters in the motor region, they occur singly in the occipital region. In this occipital region the layer of horizontal fibres in the fourth layer is very conspicuous, and owing to the number of ordinary medullated fibres present, forms a white streak visible even to the naked eye.

In the frontal region, in front of the motor region, the arrangement is more in accordance with what we have described as the general plan. The two pyramidal layers are well marked, as is also the fourth layer; but the layer of large pyramidal cells is much thinner than in the motor region, as is also, though to a less extent, the fourth layer, while the fifth layer, that of fusiform cells, is thicker than elsewhere. Small "nuclear" cells are perhaps more abundant in this region throughout all layers than in the motor region, but are far less conspicuous than in the occipital region.

We may here remark that the transition in structure from one region to 
another is very gradual, not sharp and distinct, and is perhaps especially gradual in passing from the motor region backward to the occipital region. It is not possible to recognize histologically the limit, for instance, of the motor region as determined experimentally.

In special regions of the brain, for instance in the olfactory bulb, of which we shall speak later on, very great modifications of the general plan may be observed in the cortex. We cannot enter upon these, but may just refer to the cornu ammonis or hippocampus. At the ventral end of the temporal lobe the gyrus hippocampi, the structure of whose cortex follows the general plan, is thrust inward so as to project into the cavity of the descending horn of the lateral ventricle, forming the ridge-like prominence known by the above name. The substance of the cornu ammonis is therefore cortical substance covered on the side of the ventricle by a thin prolongation of the central white matter, which is in turn covered by the ependyma lining the ventricle. A vertical section of this substance shows that while the fifth and fourth layers are reduced to small dimensions, the third layer, that of large pyramidal cells, is well developed, though narrow. The cells are large and remarkably long, and the tapering processes are arranged so regularly as to give rise, especially in stained preparations, to a marked radiate appearance. At the level of the second layer there occurs a large development of capillary bloodvessels and a scarceness of cells, giving rise to a "lacunar" appearance; and the first or molecular layer is of some considerable thickness. From the prominence of the pyramidal cells in this region, the third layer in the general plan of the cortex has sometimes been spoken of as the "formation of the cornu ammonis."

$\S 565$. In the present state of knowledge it is impossible to come to any satisfactory conclusion concerning the meaning of the variety and arrangement of the cells and other constituents of the cortex. The cells with their branches, the nerve-fibres and the nerve-fibrils form a network of gray matter which we may compare with the gray matter of the spinal cord ( $\S 492)$, but which is obviously, as we might expect, far more complex than that is. We may conclude, and experimental observation confirms the conclusion, that the large pyramidal cells with recognizable axis-cylinder processes serve as trophic centres for the fibres which appear to start from them. And we may, though with less confidence, explain the large size of these cells in the motor region, by the fact that they give rise to fibres of the pyramidal tract stretching a long way from their origin in the cell, and therefore demanding great nutritive activity on the part of the cell. We may perhaps also conclude that these fibres are efferent, motor fibres, destined to carry impulses from the cortex to the peripheral, or at least distant parts. And we may further, with distinctly less confidence, however, assume that the size of the cell is correlated to the energy which has to be expended in the discharge of efferent, motor impulses. If we accept these conclusions we inust also bear in mind that such cells, with axis-cylinder processes continued on as fibres, are not limited to, though most abundant in the motor region, but are found in all regions of the cortex; and we must hence conclude that impulses, which we must call efferent, proceed from all parts of the cortex.

It is obvious, however, that the connection of the cortical network of gray matter with the fibres of the white matter is effected in part only, and that a small part, by the method of axis-cylinder processes definitely prolonged from the cell substance of cells. A part, and probably a greater part, of the fibres sweeping up from the subjacent white matter, whether they be fibres of the pedal and tegmental systems or callosal or "association" fibres, end in the gray matter in some other way than by bodily being continued 
in the cell substance of cells; they plunge into and break up within the network, of which fibrils no less than cells form a conspicuous part; and we may here repeat the remark which we made in speaking of the cerebellum concerning the actual continuity of the elements of the network. Moreover, besides the vertical fibres obviously coming from the subjacent white matter, we have in this gray matter to deal with the fibres of horizontal and other directions, which may come from white matter not far off, but which may come from some neighboring gray matter; our present knowledge will not enable us to settle this point.

In the spinal cord we were able to divide all the fibres into afferent and efferent respectively; though even here we met with some difficulty. Dealing with the cerebral cortex, which, as we have already seen, is certainly especially concerned in voluntary movements and in the development of full sensations, we may be tempted to consider the fibres connected with the gray matter as similarly divisible into motor and sensory; and we may go on to suppose that the fibres joining the cortex as axis-cylinder processes of recognizable cells are motor fibres, and that all the other fibres joining the gray matter in some other way are sensory fibres. But in doing so we are going beyond our tether; in all probability the nervous processes going on in the cortex are far too complex to permit; such a simple classification of the functions of fibres as that into motor and sensory; and any attempt to arrange either fibres or regions of the cortex as simply motor or sensory is probably misleading. But we shall have to return to these matters when we deal with the functions of the cortex.

\section{On Voluntary Movements.}

$\S 566$. When we examine ourselves we recognize certain of our movements as "voluntary ;" we say that we carry them out by an effort of the "will." And when we witness the movements of other people or of animals we regard as also voluntary such of those movements as by their characters and by the circumstances of their occurrence seem to be carried out in the same way as our own voluntary movements. Even in the case of some of our own movements we are not always clear whether they are really voluntary or no; and in the case of other people and of animals it is still more difficult to decide the question. It would be out of place to attempt to discuss here how voluntary movements really differ from involuntary movements, or, in other words, what is the nature of the will; we must be content to take a somewhat rough use of the words "voluntary," "volitional," and "will" as a basis for physiological discussion. We may, however, remark that as far as the muscular side of the act, if we may use such an expression, is concerned, a voluntary movement does not differ in kind from an involuntary movement. It is perfectly true that a skilled man may by practice learn to execute muscular manœuvres which he would not have learned to execute had not intelligent volition been operative within him; but our own experience teaches us that many more or less intricate movements which have undoubtedly been learned by help of the will may be carried out under circumstances of such a kind that we feel compelled to regard them as, at the time, involuntary ; and it may at least be debated whether every movement which we can carry out by an effort of the will may not appear, under appropriate circumstances, as part of an involuntary act. In the case of the lower animals, in the frog deprived of its cerebral hemispheres, for instance, we have seen that voluntary differ from involuntary movements, not by their essential nature, but by the relation which their occurrence bears to circumstances. We have, therefore, to seek for the distinction between 
voluntary and involuntary, not in the coördination of the muscular and nervous components of a movement, but in the nature of the process which starts the whole act.

The histories related in a preceding section, of various animals deprived of their cerebral hemispheres, while they have further shown the difficulty of drawing a sharp line between the presence and absence of volition, such as when we appeal to our own consciousness we seem able to draw, have taught us that in a broad sense the presence of volition is, in the higher vertebrata, dependent on the possession of the cerebral hemispheres; and we have now to inquire what we know concerning the way in which the cerebral cortexfor this, as we have seen, is the important part of the cerebral hemisphereby the help of other parts of the nervous system carries out a voluntary movement.

$\S 567$. With this view we may at once turn to the results of experimental interference with the cortex. When the surface of the brain is laid bare by removal of the skull and dura mater, mechanical stimulation of the cortex produces little or no effect, thus affording a contrast with the results of mechanically stimulating other portions of the brain, or other nervous structures. And for a long time the cortex was spoken of as insensible to stimulation. When, however, the electric current is employed, either the make and break of the constant current or the more manageable interrupted current, very marked results follow. It is found that certain movements follow upon electric stimulation of certain regions or areas. The results, moreover, differ in different animals. It will be convenient to begin with the dog, on which animal the observations of this kind were first conducted.

When the surface of the dog's brain is viewed from the dorsal surface a short but deep sulcus it seen toward the front, running outward almost at right angles from the great longitudinal fissure; this is called the crucial sulcus (Fig. 147), the gyrus or convolution in front and behind it, and sweeping around its end, being called the sigmoid gyrus. It will hardly be profitable to discuss here either the homology of this sulcus or the names of the other sulci and convolutions of the dog's brain. We mention this sulcus because it is found that stimulation of the cortex in a region which may be broadly described as that of the neighborhood of this crucial sulcus gives rise to movements of various parts of the body, whereas no such movements result from stimulation of the extreme frontal region in front of the area around the crucial sulcus, or from stimulation of the occipital region behind this area. Certain exceptions may be made to this broad statement, but these it will be best to discuss in reference to the more highly developed monkey.

The region of the cortex in the neighborhood of the crucial sulcus may then be termed an "excitable" or " motor" "region, inasmuch as stimulation of this region leads to movements carried out by skeletal muscles, while stimulation of other regions does not. Further, stimulation of particular districts or areas of the region leads to particular movements carried out by particular muscles. For instance, stimulation of the more median parts of the gyrus behind the crucial sulcus (Fig. 147, $C$ ) leads to movements of the hind limb, whereas stimulation of the lateral part or outer end of the same gyrus leads to movements of the fore limb, and we may here distinguish between an area, stimulation of which (Fig. 147, $E$ ) leads to flexion of the fore limb, and an area (Fig. 147, A), stimulation of which leads to extension of the same limb. In a similar way stimulation of other areas within the "motor" region leads to movements of this kind or of that kind of the tail, of the eyes, of the mouth, of other parts of the face, of the 
tongue, and so on. Obviously in the dog this region of the cortex has connections with the skeletal muscles which do not obtain between other regions of the cortex and those muscles; and further, the region in question is topographically differentiated, so that certain areas or districts of this region are specially connected with certain skeletal muscles or groups of muscles. We may speak of a "localization of function" in this region as compared with

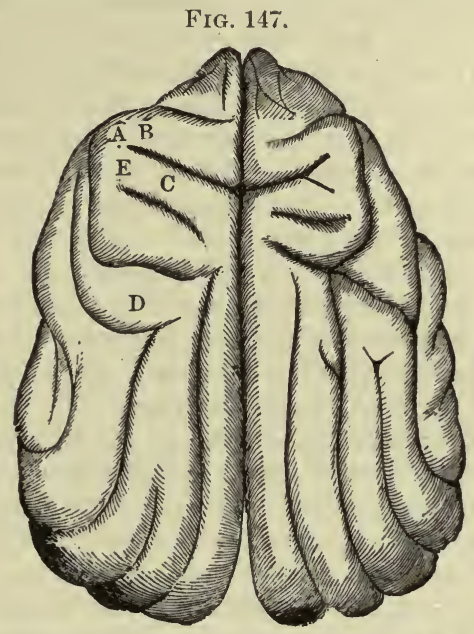

The Areas of the Cerebral Convolutions of the Dog, according to Hitzig and Fritsch. (1) $B$, The area for the muscles of the neck. (2) $A$, The area for the extension and adduction of the fore limb. (4) $E$, The area for the flexion and rotation of the fore limb. (1) $C$, The area for the hind limb. Running transversely toward and separating 1 and 2 from 3 and 4 is seen the crucial sulcus. (5) $D$, The facial area.

other regions of the cortex, and in the several areas within the region as compared with each other.

The muscles which are thus thrown into contraction are the muscles of the opposite side of the body. When "the fore-limb area," as we may call it, of the right hemisphere is stimulated, it is the left fore limb which is moved; and so with the other areas; it is only in exceptional cases, as in certain movements of the eyes, that the effect is bilateral; a movement confined to the same side as that stimulated is never witnessed.

The results are most clear when the current employed as a stimulus is not stronger than is just sufficient to produce the appropriate movement (roughly speaking, a current just perceptible to the tongue of the operator is in ordinary cases a useful one), and when the cortex is in good nutritive condition. In any experiment the results obtained by the earlier stimulations, soon after the cortex has been exposed, are the best; after repeated stimulations the surface is apt to become hyperæmic, and it is then frequently observed that the movements resulting from the stimulation of a particular area are not confined to the appropriate muscles, but spread to the corresponding muscles of the opposite side, then to muscles connected with other cortical areas, and at last to the muscles of the body generally; at the same time the movements lose their distinctive purposeful character and the animal is thrown into convulsions of an epileptiform kind. It not unfrequently happens that an experiment has to be stopped in consequence of the onset of these epileptiform convulsions. The response of movement to stimulation may be observed while the animal is under the moderate influence of an anæsthetic, but a too profound anæsthesia lessens or annuls the effects. 
In order to carry out a closer analysis of the phenomena it is desirable to watch or record the contraction of a particular group of muscles, or perhaps better still a particular muscle, $e . g$., the area for extension of the hind limb may be studied by help of the extensor digitorum communis of the limb. When this is done, the following important facts may be observed: The area of cortex having been found which gives the best movements, and the stimulus being no stronger than is necessary, isolation of the area from its lateral surrourdings by a circular incision carried to some little depth will not prevent the development of contractions in the muscle; but these do cease, even without the circular incision, if by a horizontal section the gray cortex is separated from the subjacent white matter. After removal of the cortex, stimulation of the white matter underlying the area produces the appropriate contraction; not only, however, is a stronger stimulus necessary, but also the latent period, that is the time intervening between the beginning of the application of the stimulating current and the beginning of the muscular contraction, is appreciably shortened. The appropriate contractions not only appear when the white matter immediately below the cortex is stimulated, but by making successive horizontal sections and stimulating each in turu, the effect may, so to speak, be traced through the central white matter of the hemisphere down to the internal capsule. We may conclude from these results, that when the current is applied to the surface of the cortex, certain parts of certain structures in the gray matter are stimulated, the process having a marked latent period, and that as the outcome of the changes induced in the gray matter, impulses pass along the fibres leading down from the gray matter to the internal capsule and so by the pedal system of fibres to the spinal cord and motor' spinal roots. 'The anatomical considerations advanced in a previous section lead us to suppose that the fibres in question belong to the great pyramidal tract, on which we have so much insisted; and, as we shall see, all our knowledge confirms this view.

It must not, however, be supposed that the several areas stimulation of which produces each its distinctive movement, are in the dog sharply defined from each other; when the term area for extension of the hind limb is used, it must not be supposed that the area can be defined by an outline within which stimulation produces nothing but extension of the hind limb, and outside which stimulation never produces extension of the hind limb. All that is meant is that extension of the hind limb is the salient and striking result of stimulating the area. When we study the various movements, and especially perhaps when we study, by help of a graphic record, the contractions of various individual muscles resulting from the stimulation of various parts of the motor region, we find not only that the areas for particular movements or particular muscles are very diffuse, but that the several areas largely overlap each other. If, for instance, we were to map out on the same diagram the several areas belonging to four or five muscles of different parts of the body, such as the extensors of the digits of the fore and of the hind limb, the flexors of the same, and the orbicular muscle of the eyelid, that is to say, the several areas within which in turn stimulation of the cortex produced contraction of the particular muscle, the overlapping would be so great that the whole figure would appear highly confused. In a similar way the excitable motor region as a whole would gradually merge into, be broken up into, the unexcitable frontal, occipital, and temporal regions, in front, behind, and below. In other words, the localization in the cortex of the dog is to a marked degree imperfect.

In this respect the dog, corresponding to its position in the animal hierarchy, is intermediate between such animals as the rabbit, the bird, and the 
frog, on the one hand, and the more highly developed monkey on the other ; and that is one reason why we have taken the dog first and dwelt so long upon it. In the rabbit, a similar localization may be observed, but far less definite, far more diffuse; it becomes still less in the bird, and is hardly recognizable in the frog. It will not be profitable to dwell on the details of the phenomena of these lower animals; but the phenomena of the monkey, leading up as they do to those of man, call for special notice.

$\$ 568$. When in a monkey, in an individual, for instance, belonging to the genus Macacus, the surface of the cerebrum is explored with reference to the effects of electric stimulation, it is found that when the current is applied to the precentral or ascending frontal and the post-central or ascending parietal convolutions which lie respectively in front of and behind the important central fissure or fissure of Rolando (cf. Fig. 148), movements of the fore limb follow. The "motor area for the fore limb" thus discovered is more circumscribed and definite than is the corresponding area in the dog. Its outline (Fig. 149) is roughly that of a truncated triangle bisected by the central fissure, with the broad base at some distance from the mesial line, and the truncated apex reaching on the lateral surface of the hemisphere to a well-marked hend in the lower part of the central fissure. Behind, it reaches as far as the intra-parietal fissure which somewhat sharply defines its hind border, and in front it ceases no less definitely at some little distance behind the precentral fissure. Further examination shows that the whole area is divided into areas corresponding to movements of particular parts of the forearm, and that these are arranged in a definite relation to each other. In the more dorsal part of the area, at the base of the triangle, stimulation produces movements of the shoulder (Fig. 149); if the electrodes be shifted ventrally, movements of the elbow make their appearance; if still more ventrally, movements of the wrist come in, and these are in turn succeeded ventrally by movements of the digits generally, of the forefinger, and lastly of the thumb. A very striking experiment may be made by applying a current of suitable strength, first at the lower ventral border of the area, and then gradually advancing upward toward the mesial line; the thumb is moved first, then the forefinger, then the rest of the digits, then the wrist, next the elbow, and lastly the shoulder. Further, in certain parts of the area the resulting movement is flexion of the appropriate segment of the limb, in other parts extension, in certain parts abduction, in other parts adduction, and so on.

Similar exploration shows that the "area for the hind limb" lies on the median side of the area for the fore limb, stretching besides on to the mesial surface along the marginal convolution which forms the dorsal portion of the wall of the great longitudinal fissure ; it reaches as far back as the intraparietal sulcus, and is succeeded in front by the "area for the trunk" (Fig. 150). Within this general area for the hind limb we may similarly distinguish special areas for the hip (Figs. 149, 150) in the front portion, for the knee and ankle behind this, and for the digits still further backward, the area for the great toe being, however, in front of the area for the other digits.

In front of the areas for the limbs and trunk, on the median dorsal surface, dipping down into the mesial surface along the marginal convolution (Fig. 150) and reaching laterally on the lateral dorsal surface to the dorsal extremity of the precentral sulcus (Fig. 149), is the "area for the head," that is to say, for the movements of the head brought about by contractions of the muscles of the neck.

Ventral to this again, in front of the precentral sulcus, is the "area for the eyes," that is to say, for contractions of the ocular muscles; and behind 
the precentral suleus, ventral to the arm area lies a snall area for movements of the eyelids, brought about by contractions of the orbicularis muscle. Ventral to this again is the "area for the face," in which we may distinguish an area for the mouth, that is an area stimulation of which produces changes in the buccal orifice, opening, shutting, drawing to one side, etc., and an area for movements of the tongue. These two areas reach downward to the fissure of Sylvius and backward to the line of the intra-parietal sulcus. In front of them, occupying all the ventral part of the precentral convolution and reaching forward as far as the precentral sulcus, where it

FIG. 148.
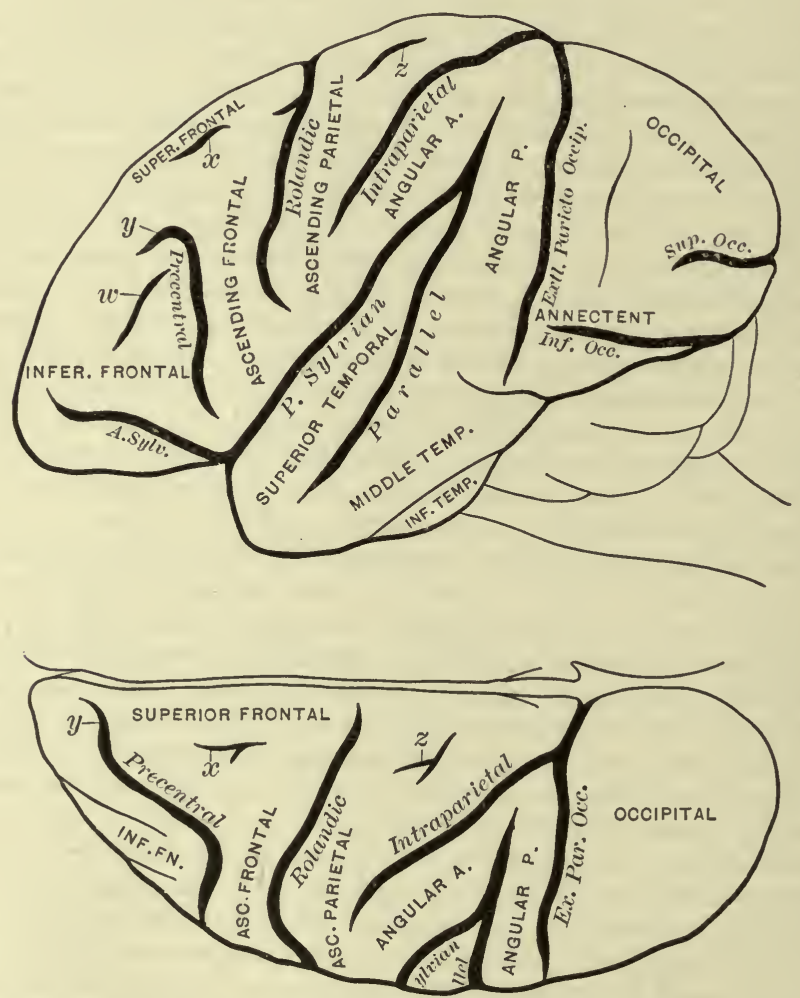

Outline of Brain of Monkey (Macacus) to show Principal Sulci (Fissures) and Gyri (Convolutions). (Sherringtnn, after Horsley and Schafer.) Natural size. The brain figured is the same as that in Fig. 149, and the two figures should be consulted together. Over each sulcus, purposely printed very thick, the name is written in SMALL CAPITALS, over each gyrus in italice. $x$ indicates the small depression, hardly to be called a sulcus, which is supposed to be homologous with the superior frontal sulcus of man: and $w, y, z$, similarly indicate sulci whose homologies are not certain. For some synonyms see Figs. 152, 154.

meets the area for the eyes, lies an area stimulation of which produces movements of the pharynx or larynx, as well as of the mouth or face, and which may be divided into areas for mastication, for swallowing, and for the production of the voice.

We might speak of these several areas in another way by referring to the nerves concerned in carrying out the several movements, though in doing 
so we must remember that there is not an exact correspondence between the relative position of a muscle along the axis of the body or along the axis of a limb and the relative position along the cerebro-spinal axis of the nerve or nerves governing the muscle. We may, however, adopting this method, note that the sacral and lumbar nerves are represented by the most mesial portion of the whole motor area and by the hind division of this mesial portion; that the lumbar and thoracic nerves are represented by

FIG. 149.
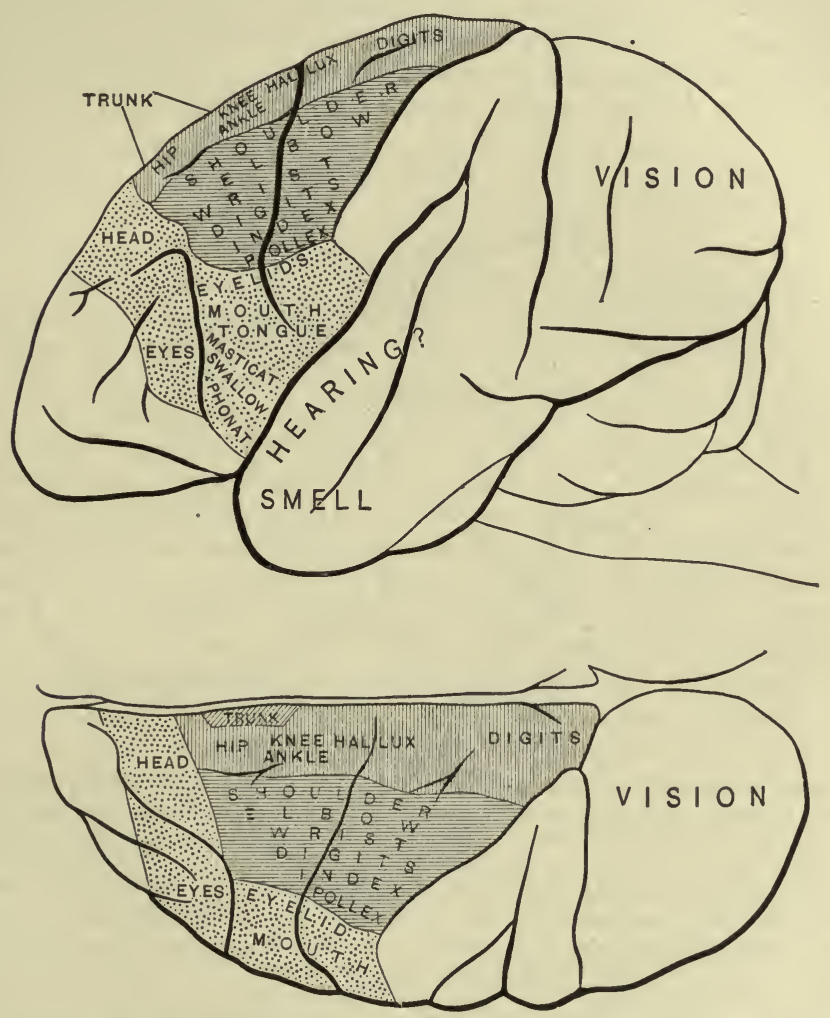

Left Hemisphere of the Cerebrum of Monkey (Macacus), Viewed from its Left Side and from Above. (Sherrington, after Horsley and Beevor.) Natural size. The figure shows the positions of the portions of the cortex concerned with movement of various parts, and with the senses of sight, smell, and hearing, The cortical area conmected with the movements of the leg is shaded vertically across, that with the movements of the arm horizontally, and that with the movements of the trunk in a slanting direction; the area connected with the movements of the head (neck), face, and eyes is dotted. The course of the chief fissures is indicated by single limes.

the front division of the same mesial portion; that the upper thoracic with the lower cervical nerves belong to a region lying lateral to, and the upper cervical nerves to one lying in front of, the preceding area; and, lastly, that the remaining lateral and ventral portions of the whole motor region appertain to the cranial nerves. But the topographical differentiation does not come out so clearly by this method as by that of taking for our guide distinctive movements of the several parts of the body.

It will be observed that all these areas taken together, represented by 
the portion of Figs. 149, 150 shaded in one way or another, occupy chiefly the parietal region of the cerebral surface, though they also reach into the frontal region. Stimulation of the frontal region in front of this motor area or of the occipital region behind, whether on the lateral or on the mesial surface, or of the temporal region, whether also on the latter or on the mesial surface, or of the gyrus fornicatus (Fig. 150) connecting the frontal and occipital regions on the mesial surface, and running ventral to the marginal gyrus, does not give rise to movements ; or, to be more exact, does not give rise to movements comparable to those just described as resulting from stimulation of various parts of the motor region. Movements do take place when certain parts of the occipital or of the temporal region are stimulated, but these are not only feeble and experimentally uncertain, but appear to be of a different nature from those resulting from stimulation of

FIG. 150.

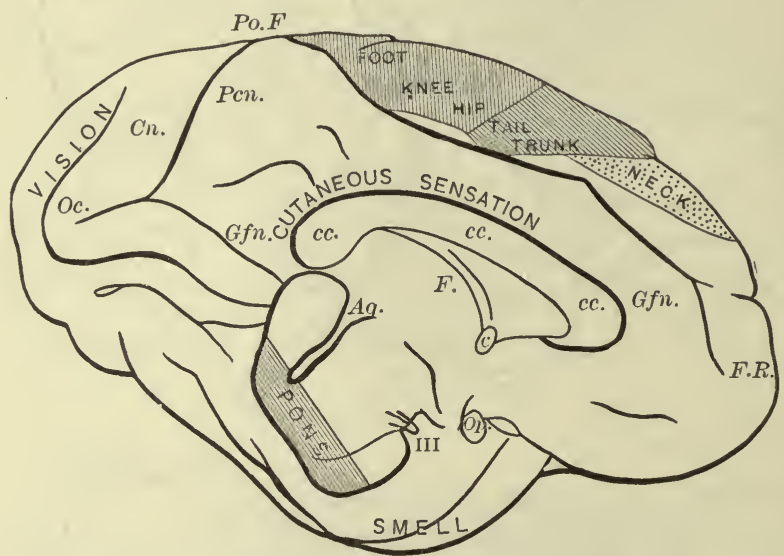

Mesial Aspect of the Ieft Half of the Brain of Mucacus, displayed by Section in the Median Sagittal Plane and Removal of the Cerebelium. (Sherrington, after Horsley and Beevor.) Natural size. The batehed and stippled parts of the surface show the regions of the cortex connected with movements of the foot, knee, hip, tail, trunk, and neck respectively. The several positions of the areas of cortex connected with vision and smell and with cutaneous sensation are indicated by the appropriate words. The plane of section has passed through the corpus callosum, $c c ., c c ., c c$., and through the anterior commissure, $c$., sparing the left pillar of the fornix, $F$; behind it has bisected the anterior part of the pons, laying open the aqueduct, Aq. (iter a tertio ad quartum ventrieulum); Pons, the left half of the pons in frontal section; Op., the optic commissure cut across; III, the root of the third cranial nerve; $F R$., the frontal pole; Oc., the occipital pole; $C n$., the cuneus; Pcn, the precuneus; $G f n, G f n, G f n$, the gyrus fornicatus; the unlettered fissure seen to form the upper boundary of this gyrus in its supra-callosal part is the calloso-marginal; Po $f$, the parieto-occipital fissure.

the motor region; it will be convenient to speak of the nature and meaning of this kind of movement when we come to discuss the development of sensations.

$\$ 569$. It is obvious from the foregoing that the mechanism for the development of these movements of cerebral origin are far more highly differentiated in the monkey than in the dog. But even in the monkey (Macacus and allied forms) the differentiation is still very incomplete. If we explore, for instance, the area for the wrist, we find that its limits are ill-defined. In some parts of the area we obtain movements of the wrist only, but in other parts of the area stimuiation produces not only movements of the wrist, but also of the shoulder or of the digits, or of the neck; and so with the other areas. 
If, however, not a Macacus or other ordinary monkey, but the more highly developed orang-outang be taken as the subject of experiments, the differentiation is found to be distinctly advanced; the several areas are more sharply defined, and what is important to note, the respective areas tend to be separated from each other by portions of cortex stimulation of which gives rise to no movement at all.

The opportunities of stimulating the cortex of man himself have been few and far between, and have, for the most part, been conducted under unfavorable circumstances; but as far as the results so obtained go, they show that the topographical distribution of areas for the several movements is carried out on the same plan as in the monkey (we are purposely confining ourselves now to the results of artificial stimulation); and, moreover, justify the conclusion, which à priori reasons would lead us to adopt, that in man the differentiation is advanced still further than in the monkey.

Thus, when we survey a series of brains in succession, from the more lowly frog, through the bird, the rabbit, the dog, and other lower mammals up to the monkey, the anthropoid ape, and so to man himself, we find an increasing differentiation of the cerebral cortex, by which certain areas of the cortex are brought into special connection with certain skeletal or other muscles in such a way that stimulation of a particular portion of the gray matter gives rise to a particular movement, and to that alone.

$\S 570$. In treating of the structure of the brain we spoke ( $\$ 545)$ of the pyramidal tract as starting from the motor region of the cortex; and it is obvious that the fibres of this tract must be concerned in the development of the movements which we have just described. When the movements are brought about by stimulation of the fibres in some part of their course, in the internal capsule, for instance, there can be no doubt that the stimulation starts impulses which travelling down the tract to the origin of certain cranial or spinal nerves, in some way give rise to coördinate motor impulses along the motor fibres of the nerves; and we may with reason speak of the impulses then passing along the tract as motor or efferent in nature. When the stimulus is applied direct to the cortex, we may assume that processes, started in the gray matter, eventuate in similarly efferent impulses along the fibres of the tract. All the evidence leads us to regard this tract as an efferent tract.

When the spinal cord is divided in the lower dorsal region and the electrodes of an electrometer are brought into connection with the transverse cut surface and with some point of the longitudinal surface above, the electrometer gives evidence of currents of action (manifested as negative variations of a demarcation current or current of rest ( $\$ 67)$ whenever the motor area of the hind limb is stimulated, but not when other parts of the cortex are stimulated. We have already said that stimulation of any part of the motor region may, under abnormal conditions, give rise to general epileptiform convulsions; when these occur during such an experiment as the above, currents of action manifest themselves in the lower dorsal cord, whether the stimulation giving rise to the convulsions be applied to the area for the hind limb or to any part of the motor region. It has been further observed that the currents of action developed within the spinal cord tally in a very exact manner with the muscular movements. The convulsions begin with a sustained "tonic" contraction of the muscles, and the electrometer shows a similar sustained current of action; this is followed by rhythmic movements of the muscles, accompanied by corresponding rhythmic movements of the mercury of the electrometer. Without insisting too much on the exact interpretation of these results, we may take them as at least showing that, when the notor region of the cortex is excited, nervous 
impulses accompanied by "currents of action" pass downward along the fibres of the pyramidal tract.

The results of stimulating the fibres of the tract in their course through the corona radiata and the internal capsule, and the results obtained by studying the degenerations following upon injury to or removal of the several parts of the cortical motor region, agree in marking out the paths taken by the several constituents of the tract through the central white nintter of the hemisphere, the corona radiata, and the capsule. Comparing Figs. 149, 150, with Figs. 144, 145, and 146, it will be seen that the portions of the tract destined for the cranial nerves, and so for the movements of the eyes, the mouth, face, tongue, pharynx, and larynx, starting from the ventral parts of the more frontal district of the motor region, take up their position at the knee of the internal capsule; and the portion destined for those upper cervical nerves which carry out movements of the head through the muscles of the neck, starting from the extreme frontal and dorsal parts of the area, is also apparently directed to the knee of the capsule. The rest of the tract, starting from the part of the area lying at once behind and mesial to the above, occupies in the capsule a position posterior to them in the hind limb of the capsule; and it will be observed that the tract for the fore limb which begins on the lateral surface of the tracts for the trunk and hind limb, shifts its course in relation to theirs, so that in the capsule it is front of them, not lateral to them. It may further be observed that while in the tracts for the trunk and hind limb the same fore-and-aft order which obtains on the surface is reproduced in the capsule, even apparently to the strange precedence of the ankle over the knee, the order of the several elements in the fore-limb tract which is lateral on the surface becomes regularly fore and aft in the capsule. In the capsule the several elements are arranged in a linear order, corresponding broadly to that of the distribution of the muscles along the longitudinal axis of the body; on the cortex they are disposed in an order the cause of which is at present not very clear, but which is probably determined by the respective relations of the several parts of the motor region to the functional activity of the other parts of the cortex. In the shifting from the one order to the other, the several constituent fibres, as we have said, describe a somewhat peculiar course; and when we remember, as stated in $§ 545$, that the order shown in Fig. 144 is only the order obtaining at one particular level of the capsule, and that from the dorsal beginnings of the capsule in the corona radiata to its ventral end in the pes the capsule is continually changing in form, and its fibres therefore continually shifting their relations to each other, the whole course of the several fibres of the tract from their origin in the cortex until they are gathered up into the central portion of the pes (Fig. 137, Py.) must be a very complicated one.

When the area of one hemisphere is stimulated, the movement which results is in most cases seen on the other side of the body, and on that other side alone. Thus when the area for the fore limb on the left hemisphere is stimulated it is the right fore limb which is moved. This is in accordance with what we have learned of the pyramidal tract and its ultimate entire decussation before it reaches the motor nerves, the decussation either occurring massively as in the case of the crossed pyramidal tract, or in a more scattered manner aloug the upper part of the spinal cord in the case of the direct pyramidal tract; and, as we have seen, there is a similar decussation for such part of the pyramidal tract as is connected with the cranial nerves above the decussation of the pyramids. Except in the case of certain areas for movements naturally bilateral, of which we shall speak presently, the movement is normally on the crossed side, and on the crossed side only. Under abnormal conditions, however, the limb on the other side-that is, of 
the same side as the hemisphere stimulated-may move also. But such an abnormal movement of the same side has not the same characters as the proper movement of the crossed limb. Instead of being an orderly coördinate movement, it is a more simple, either tetanic or perhaps tonic, or rhythmic, clonic, contraction of the muscles. Obviously its mechanism is of a different nature from that by which the proper movement of the crossed limb is effected; but it is important to bear in mind that a movement of the uncrossed limb may take place; and further, that the abnormal conditions continuing, sinilar movements of an uncoördinated character may spread to the hind limb and other parts of the crossed side, though the stimulation be still confined to the arm area, then to other parts of the uncrossed side, until, as we have said, the whole body is thrown into epileptiform couvulsions. This feature must not be forgotten. In fact, it may be fairly insisted upon that while we may speak of a particular coördinate movement as being the normal outcome of an ordinary careful stimulation of a particular area in a normal condition, it is no less true that diffuse uncoördinated movements, culminating in general epileptiform convulsions, are the natural outcome of the stimulation of any area in an abnormal condition. And in attempting to form any opinion of the nature of the first act we must bear the second in mind.

As we said above, the movements resulting from cortical stimulation are most conveniently described in terms of parts of the body-of the arm, of the thumb, of the tongue, etc. The movements of the same part may be further distinguished by means of the nomenclature usually adopted in speaking of muscular movements, such as flexion, extension, abduction, adduction, etc.; so that, within the area bearing the name of some particular part-such as the wrist, for instance-we have to distinguish an area for the flexion and another for the extension of that joint; and in like manner in reference to other parts. But it will be readily understood that it is easier to map out the area for a particular part than to distinguish the areas corresponding to the several movements of that part. Hence the nomenclature usually adopted in speaking of the motor region is one based on the parts of the body moved, rather thau on the character of the movements. The more closely, however, the movements in question are studied, the more probable it appears that the localization which obtains in the cortex is essentially a localization corresponding not to parts of the body or to nerves or to muscles, but to movements. In considering this point it must be remembered how rude and barbarous a method of stimulation is that of applying electrodes to the surface of the gray matter compared with the natural stimulation which takes place during cerebral action; the one probably is about as much like the other as is striking the keys of a piano at a distance with a broomstick to the execution of a skilled musician. Were it in our power to stimulate the cortex in any way at all approaching the natural method, we should, in all probability, arrive at two results : on the one hand, we should be able to produce at will a variety of movements of different degrees of complexity, some very simple, others very complex, and for these we should have to use names suggested by the characters and purpose of each movement, and by these alone; on the other hand, we should find very decided limits to the number and kind of movements which we could evoke, limits fixed in the case of each subject partly by inherited organization, partly by the training of the individual.

Some such results of refined experimentation are, indeed, already foreshadowed by the rude results of our present rough methods. The movements which usually follow stimulation of the motor region, and which we have described as flexion, etc., are, so to speak, the elementary factors of 
ordinary bodily movements, the detached and imperfect chords of a musical piece; and in the following facts relating to their production we can recognize the influences of organization and habit. As we have said, stimulation of the motor area of one hemisphere produces movements, as a rule, which are limited to one side of the body, and that the opposite side. Now both in ourselves and in the higher animals a large number of bodily movements, especially of the limbs, are habitually unilateral; and, putting aside the question why there should be two halves of the brain, and why the one half of the brain should be associated with the cross half of the body, we may recognize in the unilateral crossed movement resulting from stimulation of the cortex an accordance with natural habits. But some movements of the body are ordinarily bilateral; the two eyes, for iustance, are ordinarily moved together, and the two sides of the trunk move together very much more frequently than do the two fore limbs or the two hind limbs. And in accordance with this we find that stimulation of the motor area for the eyes on either hemisphere produces movements of both eyes, and stimulation of the trunk area of one hemisphere is also very apt to produce bilateral action of the trunk muscles; in such instances the movements on both sides are quite normal movements. We may incidentally remark that removal of the trunk area leads to a good deal of bilateral degeneration, that is, to degeneration of strands in the pyramidal tracts of both sides, whereas such a bilateral degeneration is comparatively scanty after removal of the leg or arm area.

That it is the movement and not the part moved which is, so to speak, represented on the cortex is further shown by the relative magnitude of the several cortical areas when they are mapped out according to parts of the body. The area for the arm, for instance (cf. Figs. 149, 150), is, so to speak, enormous compared to that of the trunk when the relative bulks of these two parts of the body are considered; and within the arm area itself the space occupied by the thumb and forefinger and digits is, bulk for bulk, out of proportion to the space allotted to the shoulder; so also the area for the eyes, or for the mouth is out of proportion to the size of those organs. But these relative sizes of the respective areas become intelligible when we bear in mind relative mobility, nimbleness, and delicacy of execution; in these respects the shoulder is far behind the thumb, while the eyes and mouth surpass most other parts of the body.

We are brought yet a step further when we compare, in respect of the cortical motor region, animals of different grades of organization ; and the results thus obtained lead us to the conclusion that the motor region is correlated not to movements in general, but to movements of a particular kind. Taking in series the rabbit, the dog, the monkey, and man, we find in passing from one to the other, an increase in prominence and in differentiation of the motor region accompanied by an increase in the bulk of the pyramidal tract; among the many striking differences between the brains of these several animals, these two features, the increasing complexity of the motor region and the increasing size of the pyramidal tract, are among the most striking. The size of the pyramidal tract is itself correlated to the complexity of the motor region, and, being the more easily determined, may be used as indicating both; the difference in the size of the pyramidal tract in these animals is seen all along the whole length of the cord (Fig. 151). Now as regards mere quantity of movement, if we may use such an expression, the differences between these animals are of no great moment. If we were to take the amount of energy expended as movement in twenty-four hours per gramme of muscle present in the body in each of the four cases. 
we should certainly not find any correspondence between that aud the size of the pyramidal tract. If, however, we take a particular kind of movement, what we may perhaps call skilled movement, that is, movement carried out by means of intricate changes in the central nervous system, we do find a remarkable parallelism in the above cases between the amount of such skilled movement entering into the daily life of the individual and the size of the pyramidal tract. In these two respects man is much above the monkey, and the monkey far above the dog. We may conclude then that the cortical

FIG. 151.
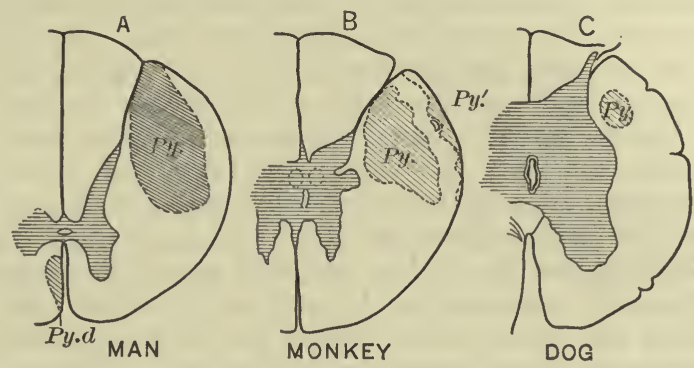

Diagram to illustrate the Relative Size of the Pyramidal Tract in the Dog, Monkey, and Man. (Sherrington.) The figure shows in outline the lateral half of the cord, at the level of the fifth thoracic nerve, in $A$, man ; $B$, monkey ; $C$, dog. $A$ is a reproduction of $D^{5}$ in Fig. $127 ; B$ and $C$ are drawn of the same size as $A$. Py., shaded obliquely, the pyramidal tract; the depth of shading indicates that the tract is more crowded with true pyramidal fibres as well as larger in $A$ than in $B$, and in $B$ than in $C$. In $B, P y^{\prime}$ is an outlying portion of the pyramidal tract separated from the rest by the cerebellar tract; $P y . d$., the direct pyramidal tract, present in man only. The gray matter seems relatively large in $C$ because the section was taken from a very young puppy.

motor region is in some way especially concerned with the kind of movement which we have called "skilled."

$\$$ 571. These skilled movements are, to a large extent, though not exclusively, voluntary movements. We have, in a previous section, seen reason to believe that the cerebral cortex is in some way especially associated with the development of voluntary movements. Putting together this conclusion and the conclusions just arrived at, we are naturally led to the further conclusion that the cortical motor region, with the pyramidal tract belonging to it, plays an important part in carrying out voluntary movements. Do other facts support this view; and if so, what light do they throw on the question as to what part and what kind of part the motor region thus plays?

In this connection we naturally desire to know what are the results of removing from an otherwise intact animal the whole motor region and more especially this or that particular portion of it. Before proceeding fiurther, however, we may once more call attention to the caution given in $\$ 495$ and repeated in $\$ 553$; indeed, when we consider the high organization and complex functions which obviously belong to the cortex, when we bear in mind that it appears to govern, and must therefore be bound by close ties to almost all the rest of the central nervous system, we must be prepared to find after removing a portion of cortex that the pure "deficiency" phenomena, those which result from the mere absence of a piece of the cortex, are largely obscured by the other effects of the uperation.

In the rabbit the results have been almost purely negative. When in this animal the part of the cortex which may be considered as the motor region 
is removed, nothing remarkable is observed in the movements of the animal We can hardly suppose that the operations of the central nervous system are the same in an injured as in an intact animal, and the differences induced ought to be betrayed by the movements of the body; but at present they have escaped observation.

In the dog the removal of an area is followed by a loss or diminution of voluntary movement in the corresponding part of the body. When, for instance, the area for the fore limb is removed from the left hemisphere, the right fore limb is completely or partially "paralyzed." In carrying out its ordinary movements the operated animal makes little or no use of its right fore limb. But this state of things is temporary only. After a while the animal regains power over the limb, and in successful cases recovery is so complete that it is impossible to point out in the limb any appreciable deviation from the normal use. And careful examination after death has shown not only that the area had been wholly removed, but also that there was no regeneration of the lost parts; the removal of the cortex lears in such cases, as usual, to degeneration of the corresponding strand in the pyramidal tract right away from the cerebral surface to the endings of the strand in the cervical and dorsal spinal cord. Nor can it be urged in such cases that diffused remnants of the arm area had been left in the remaining parts of the motor region; for the whole motor region has been removed, and yet the animal has recovered to such an extent that a casual observer could detect no differences between the movements of the two sides of the body. Closer examination did disclose certain imperfections of movement; but the operation had involved injury to or produced changes in structures other than the motor region, and the imperfections might have been due to the additional damage. Nor can it be urged that, in such a case, where one side is removed, the remaining hemisphere takes on double functions; for the greater part of the motor areas have been removed on both sides, and yet the animal's movements have been so far apparently complete that a casual observer would see nothing strange in them. Again, the whole motor region has been removed from one hemisphere in a young puppy, and some time later when the movements seemed to have recovered their normal condition, the removal of the motor region of the other hemisphere has produced merely a paralysis of the crossed side of the body, and that as before only of a temporary character.

Two things have to be noted here. In the first place, the removal of an area does affect the movements which are brought about by stimulating that area, it leads to their disappearance or at least to great diminution of them; and this affords an additional argument that the connection between the area and the movement is a real and important one. In the second place, the physiological effect is temporary only, though the anatomical results of the operation are permanent, for the cortex is never renewed, and the pyramidal tract degenerates along its whole length, never to be restored; this shows that we have to deal here with events of a very complex character. When a particular movement results from stimulation of the appropriate cortical area, we may be sure that whatever takes place in the cortex and along the pyramidal tract, motor impulses, duly coördinated, pass along certain anterior roots to certain muscles; and we know that if we removed a sufficient length of each of those anterior ronts that particular movement would be lost for the rest of the life of the individual. We may, therefore, infer that the events which, whatever be their exact nature, taking place in the cortex and along the pyramidal tract lead ultimately to the issue of motor impulses along the anterior roots, differ essentially from the events attending the transmission of ordinary motor impulses. 
In the case of the monkey, the results of removing parts of the cortical motor region have not been so accordant as in the case of the dog. The two animals agree perfectly in so far that the removal of a particular area leads, as an immediate result, to the loss of the corresponding movement; but while in some instances recovery of the movement has in the monkey as in the dog after a while taken place, in other instances the "paralysis" has appeared to be permanent. As a rule the paralysis caused by a large lesion is not only more extensive, but also of longer duration than that caused by a small one; and natural bilateral movements, as of the eyes, reappear earlier than unilateral movements. The facts, however, within our knowledge relating to the permanence of the effect are neither numerous nor exact enough to justıfy at present a definite conclusion. On the one hand, the positive cases where recovery has taken place are of more value than the negative ones, since in the latter the recovery may have been hindered by concomitant events of a nature which we may call accidental; and it is at least à priori most unlikely that the pyramidal tract mechanism, if we may use the expression, though it may differ in the monkey and the dog in degree of development, differs so essentially in kind that damage of it leads in the one case to permanent, and in the other to mere temporary, loss of function. We may add that we should further expect to meet in the monkey with more prominent and more lasting complications due to the subsidiary effects of the operation, and it may be doubted whether in any of the recorded experiments the animal has been allowed to live a sufficient time for these subsidiary events to have cleared away, leaving only what we have called the "deficiency" phenomena, due to the loss of the cortical area alone. On the other hand, it must be remembered that the movements of the monkey are more intricate in origin, more "skilled," than those of the dog; and it may be that differences in the characters of movements determine the possibility of their recovery. In illustration of this we may quote the experience that, after the removal of the arm area in the monkey, a certain awkwardness in the movements of the thumb is one of the last effects of the operation.

$\S 572$. Before we proceed, however, any further in the discussion, it will be of advantage to turn aside to what is known concerning the cortical motor region in man. As we have already said, theoretical considerations lead us to believe that the cortical motor region in man is disposed in accordance with the plan of the anthropoid ape as ascertained experimentally, but with the differentiation carried still further; and the few cases of experimental stimulation of the human cortex support this view. Our chief knowledge in this matter is derived from the study of disease; and in this the advantages of dealing with one of ourselves are largely counterbalanced by the disadvantages due to disease being so often anatomically diffuse and physiologically changeful and progressive.

We said above that during experiments on animals stimulation of any part of the motor region may under abnormal conditions lead to general epileptiform convulsions. Now clinical study has shown that in man certain kinds of epileptic attacks are of similar cortical origin. In these cases it has been observed that the attack begins in a particular movement, by contractions of particular muscles or of the muscles of a particular region of the body, of the hand, foot, toe, thumb, etc., and then spreads in a definite order or " march" over the muscles of other regions until the whole body is involved. When in an experiment on an animal epileptiform convulsions supervene, they similarly start from the region of the body, the motor area of which is beneath the electrodes at the time, and similarly spread by a definite "march" over the whole body. Hence, in the human epileptiform 
attacks of which we are speaking, it has been inferred that the immediate exciting cause of the attack is to be sought in events taking place in that part of the cortex which serves as the area for the movement which ushers in the attack. Further inquiry has not only confirmed this view, but has also shown that the topography of the cortical areas in man, as thus determined, very closely follows that of the monkey.

Other diseases of the cortex have been marked, among other symptoms, by loss or impairment of particular movements. In most of such cases the cortical lesion has been of such an extent as to involve a number of special areas at the same time, and so to lead to loss or impairment of movement over relatively considerable regions of the body, such as the whole of one arm; and in general the teaching of these cases of disease, while confirming the deductions from the monkey, and giving us some general idea of the topography of the human motor cortical region, has at present given us approximate results only. Figs. 154 and 155 show in broad diagrammatic manner the position and relative extent of the motor areas for the leg, arm, and face in man as far as has yet been ascertained. To assist the reader we give at the same time diagrams (Figs. 152 and 153) illustrating the nomenclature of the surface of the human brain.

One area is of special and instructive interest. Speech is an eminently "skilled" movement. We have seen that in the monkey the area for the mouth and tongue lies at the ventral end of the central fissure or fissure of Rolando, ventral to the arm area, and that the extreme ventral and front part of the motor region just above the fissure of Sylvius supplies an area which we marked as that of phonation (Fig. 149). In the monkey the area of phonation is determined by experimental stimulation; in man, in a similar position, on the third or lowest frontal convolution, sometimes called Broca's convolution, ventral to and in front of, and probably overlapping backward, the area which in Fig. 154 is marked "face," and which includes the mouth and tongue, clinical study has disclosed the existence of an area which may be spoken of as the area of "speech." Lesions of the cortex in this area cause a loss of or interference with speech, the condition being known as aphasia; to this we shall presently return. In Fig. 154 this area is shown in an approximate manner.

The movements of speech are essentially bilateral movements. In the dog and monkey various bilateral movements may be excited by stimulation of the appropriate area in either hemisphere; and analogy would lead us to suppose that in man the movements of speech would be connected with the speech area in both one and the other hemisphere. The results of lesions, however, show that it is in most cases especially the left hemisphere which is connected with speech; it is a lesion in the third frontal convolution of the left hemisphere, often associated with other lesions of the same hemisphere leading to paralysis of the right side of the body and face, which causes aphasia, it being only in exceptional cases that the condition results from a lesion of the corresponding area of cortex on the right hemisphere.

In man, then, clinical study corroborates the conclusions deduced from the experimental investigation of the dog and of the monkey, but still leaves us in uncertainty as to the question what and what alone are the absolutely permanent effects of the loss of a cortical area and nothing else. On the one hand, in the cases in which recovery of a movement follows upon its loss or impairment, it is open for us to suppose that the lesion itself was temporary, and that with the cure of the malady the cortical area regained its normal condition. On the other hand, where the disease continues, the permanency of the loss of any movement may be attributed to the disease doing more than merely suspend the function of the cortical area. Aphasia, 
especially in young persons, has been followed by recovery, but in such cases it has been supposed that the dormant area on the right side has been awakened to activity by the loss of the left area; and in support of this

FIG. 152.

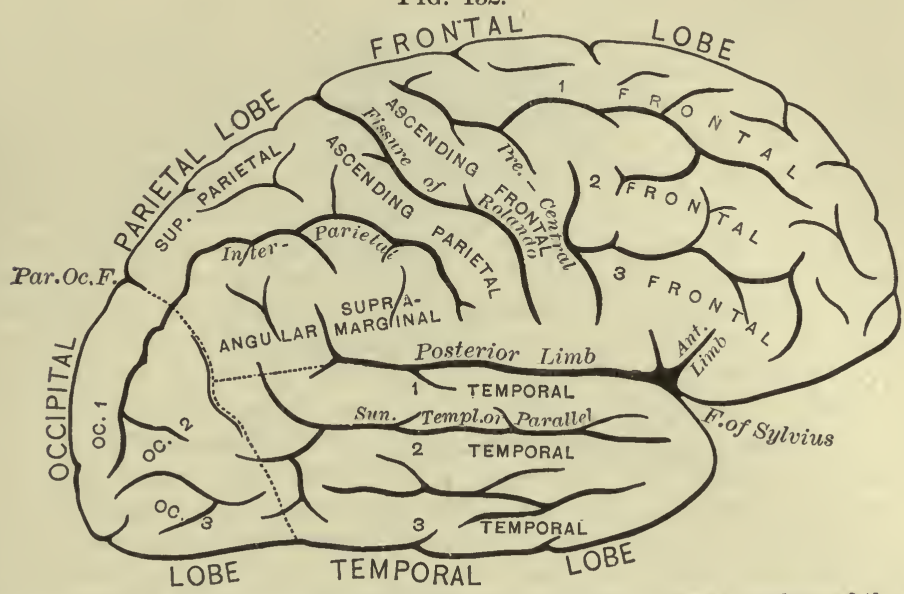

Diagram of the Gyri (convolutions) and Sulci (fissures) on the Lateral Surface of the Right Hemisphere of Man. (Gowers.)

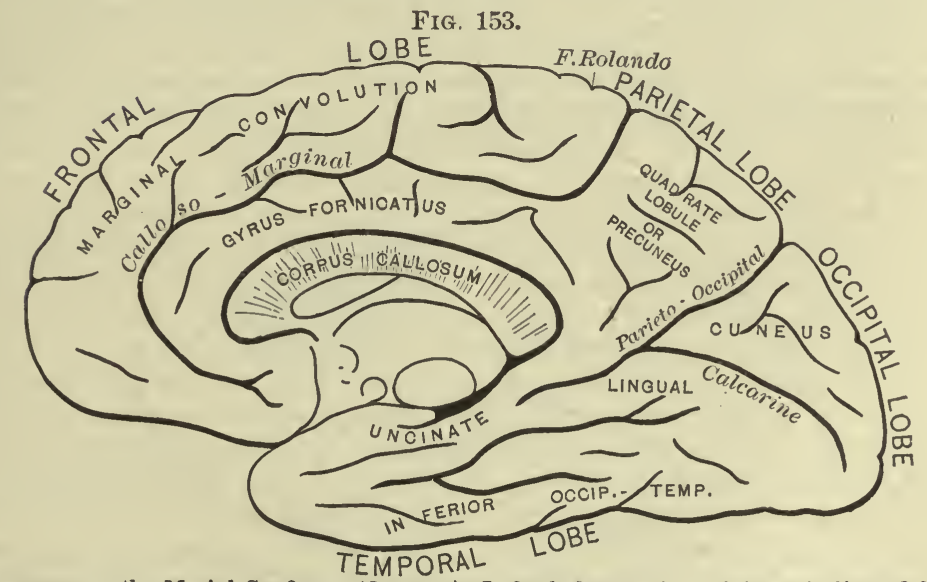

The same on the Mesial Surface. (Gowers.) In both figures the sulci are indicated by italic and the convolutions by roman type. The following list of some synonyms may perhaps be of use in connection with these figures and those of the brain of the monkey (Figs. 149 and 150).

Gyri or convolutions. Precentral or anterior central = ascending frontal. Postcentral or posterior central $=$ ascending parietal. Superior temporal $=$ infra-marginal $=$ first temporal. Triangular lobule $=$ cuneus. Central lobe $=$ island of Reil. Paracentral lobule $=$ the mesial face of the superior frontal within the marginal gyrus. Cingulum = the part of the gyrus fornicatus which adjoins the corpus callosum. Gyrus hippocampi = uncinate gyrus, though the latter name is sometimes restricted to the front part of the hippocampal gyrus; the two may be considered as a continuation of the gyrus fornicatus, and the three together, forming a series, have been called "the great limbic lobe."

Sulci or fissuers. Central $=$ Rolandic, or of Rolando. Perpendicular ptari eto-occipital. Parietal $=$ intra-parietal or sometimes inter-parietal. Tempor $=0$-sphenoidal lobe $=m$ poral lobe.

view cases have been recorded in which a first aphasia, due to a lesion on the left side has been followed by a second aphasia due to a subsequent lesion 
occurring on the right side. On the whole, perhaps, the evidence of clinical study tends to show that in man the loss of movement due to the destruction

Fig. 154 .

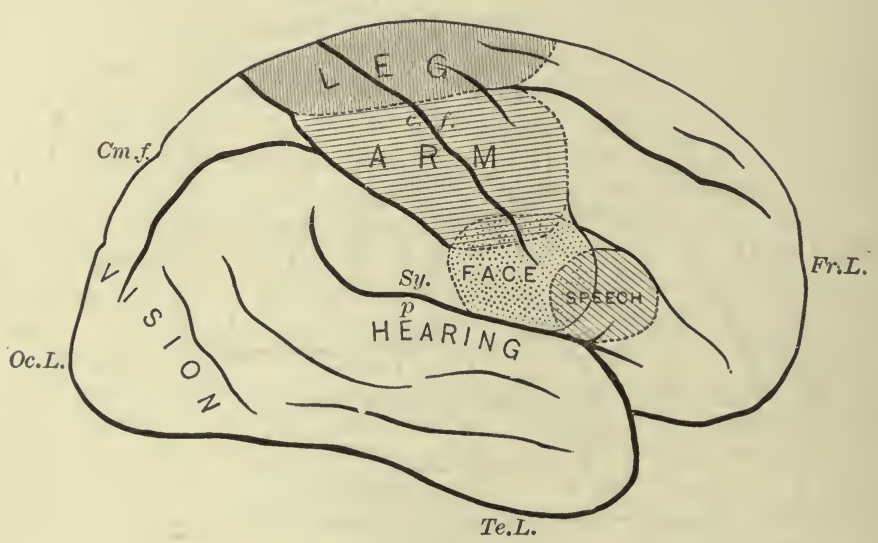

The Lateral Surface of the Right Cerebral Hemisphere of Man in Outline, to illustrate the Cortical Areas. (Reduced from nature.) The position of the areas of the cortex concerned with movements of the face, arm, and leg, and with the senses of sight and hearing are approximately shown. The position of the area connected with speech (Broca's centre) is also shown for the sake of comparison of it with the position of the otherareas; the representation of speech in the cortex cerebri lies, however, in the left hemisphere chiefly. Oc. $L$, occipital lobe ; Fr. $L$, frontal lobe ; $T$ e. $L$, temporal lobe; $S y$. $f$, the fissure of Sylvius; C.f, the central fissure (Rolandic); Cm.f, indicates the position of the posterior end of the calloso-marginal fissure.

Fig. 155.

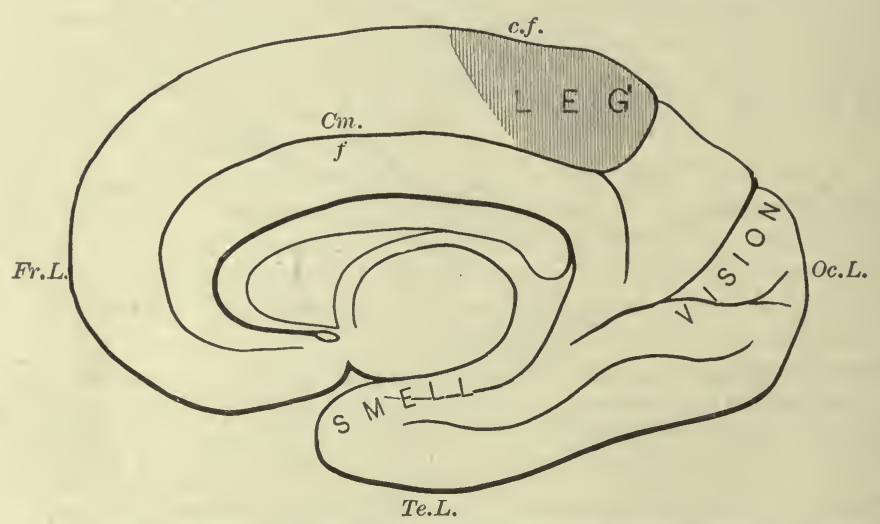

The Mesial Surface of the Right Cerebral Hemisphere of Man in Outline, to illustrate the Cortical Areas. The areas shown are those connected with the movements of the leg and with the senses of sight and smell. Fr. $L$, the frontal lobe of the hemisphere: Oc. $L$, the occipital lobe; $T e . L$, the temporal lobe; $C m . f$, the calloso-marginal fissure separating the marginal gyrus above from the gyrus fornicatus below; $C f$, marks the situation of the central fissure, the fissure itself not being apparent on the mesial aspect of the hemisphere. The corpus callosum and the anterior commissure are seen in cross section.

by disease of an area is a permanent one, though actual demonstration of this is wanting. 
$\S 573$. We may now return to the discussion of the question, What is the part played by a motor area, and by the contribution from that area to the pyramidal tract in carrying out the movements with which the area is associated?

We may premise that the evidence points very distinctly to the conclusion that whatever be the nature of the whole chain of events of which the cortical area seems to be a sort of centre, the fibres of the pyramidal tract serve as the channel of processes which we must regard as efferent in nature. It is perfectly true that in many cases at least the removal of a cortical area has led to diminished sensibility of the part in which movements are excited by stimulation of the area; and there are many facts, of which we shall presently quote a very striking one, which go to show that the cortex of the motor region is largely influenced by sensory impulses from various parts of the body; but we cannot suppose that the pyramidal tract is the channel by which such sensory impulses reach the cortex. As we have previously ( $\$ 481)$ urged, the fact that the degeneration of the fibres in the tract is a descending one, and cannot be trusted by itself to prove that the direction in which the fibres carry impulses is only that from the cortex downward; but this added to the fact that when the fibres of the tract are stimulated at any part of their course, movements, the signs of the occurrence of efferent centrifugal impulses, are produced, leaves no doubt that the tract is one of efferent fibres. Hence we may infer that whatever be the nature of the events taking place in a motor area during the carrying out of a movement, the part played by the fibres of the pyramidal tract is that of carrying efferent impulses from the area to the muscles concerned.

Let us consider first the movements of speech in man, the evidence touching the connection of which with an area on the third frontal convolution appears so very clear. Speech is eminently a "skilled" movement; it involves the most delicate coördination of several muscular contractions, and we may certainly say of it that it has to be "learned." The whole chain of coördinated events by which the utterance of a sentence, a word, or any vocal sign is accomplished consists of many links, the breaking of any of which will lead to failure of one kind or another in the act.- Something may go wrong in the glossal or other muscles, in the nerve-endings in those muscles, or in the fibres of the nerves, hypoglossal and others, between the central nervous system and the muscles, or something may go wrong in that part of the central nervous system, the bulb to wit, in which a certain amount of coördination is carried out just previous to the issue of the motor impulses. Damage done to any of these parts of the mechanism may lead to dumbness or to imperfect speech. In the latter case the imperfections have a certain character ; if we are at all able to gather the wish of the speaker, we recognize that he is attempting to utter the right words in the right sequence, but that his efforts are frustrated by imperfect coördination or imperfect muscular action; his speech is "thick," the syllables are blurred and the like. Disease of the bulb at times leads to imperfect speech of this kind in which the imperfection may be recognized as due to the lack of proper coördination of motor impulses. The affection of speech known as "aphasia," which is caused by lesions of the cortex, is of a different character, and the forms of imperfect speech caused by bulbar disease have justly been distinguished from true aphasia by the use of other terms. Cases of complete aphasia in which all power of speech is lost, do little more than help us to ascertain the topographical position in the cortex of the "speech" area, but cases of partial aphasia are especially instructive. IVithout attempting to go into the details of the subject and into the many considerations which 
have to be had in mind in dealing with it, for there are different kinds of aphasia, we may venture to say that the striking feature of partial aphasia is the failure to say certain words or syllables, and the tendency to substitute some wrong word or syllable for the right one. The words or syllables which are uttered are rightly pronounced without defect of articulation; and in many cases, though the right word cannot be produced as a direct effort of the will, it may be uttered under the influence of an emotion, or, indeed, sometimes as the result of some physical processes more complex than those involved in the mere volitional effort to say the word. An instructive case is recorded of a man suffering from slight aphasia, who, after several failures to say the word "no" by itself, at last said, "I can't say no, sir."

From the phenomena of partial aphasia we may draw the deduction that the cortical speech area does not carry ou't the whole of the coördination of the impulses involved in articulation. That coördination is exceedingly complex, and we ought, perhaps, to recognize in it more than one degree or kind of coördination. The failure of articulation in disease of the bulb shows that a certain amount of coördination takes place there ; for the affections of speech due to bulbar disease are not the same as those resulting from the mere loss of this or that muscle or nerve. We must, of course, admit that some, possibly a great deal, of coördination of a certain kind takes place in the cortex, for the bulb cannot by itself be made to speak; exactly how much, the knowledge at present at our disposal leaves a matter of great uncertainty; but it is sufficient for our present purpose to recognize that whatever may be the nature of the events taking place in the cortical area during the act of speech, those events make use of the machinery already provided in the bulb. The word spoken does not start, so to speak, ready made in the cortex; it is not that a group of impulses start from the cortex with their coördination fully achieved, and pass along certain nerve-fibres to certain muscles, making their way without change through the tangle of the bulb, as if this were merely a bundle of lines offering paths for, but exercising no influence over the impulses. We must rather suppose that something takes place in the cortex of the third frontal convolution, as the result of which efferent impulses pass along the appropriate fibres of the pyramidal tract to the bulb, and there start a series of events leading to the issue of the coördinated impulses by which the word is spoken.

$\S 574$. We have no reason whatever to think that the cortical area for speech differs in its fundamental characters from other divisions of the motor region, and are justified in carrying on to other areas the deduction we have just drawn in connection with the speech area. With that end in view we may now turn back to the experimental results obtained on the dog, and it will make our discussion simpler if we take as an illustration some large area such as the fore-limb area.

We have seen that stimulation of this area produces what we may, to start with, speak of simply as movements of the fore limb; and guided by the analogy of speech in man we may confidently conclude that when the dog voluntarily moves the fore limb, the act is carried out by means of events taking place in the fore-limb cortical area. The simplicity of the electrical phenomena resulting from cortical stimulation, which we described in $\$ 658$, might at first sight lead us to conclude that the whole matter was fairly simple; and, indeed, some writers appear to entertain the conception that in a voluntary movement such as that of the fore limb, all that takes place is that the "will" stimulates certain cells in the cortical area, causing the discharge of motor impulses along the pyramidal fibres connected with 
those cells, and that these motor impulses travel straight down the pyramidal tract to the motor fibres of the appropriate nerves, undergoing possibly some change at the place in the cord where the pyramidal fibre makes junction with the fibre of the anterior root, but deriving their chief if not their whole coördination from the cortex itself, that is to say, being coördinated at their very starting-point. That such a view is untenable and that the simplicity of the electrical phenomena is misleading is shown by the following two considerations among others: On the one hand, as was shown in a previous section, the coördination of movements mav be carried out apart from the cortex, namely, in the absence of the hemispheres; and we can hardly suppose that there should be two quite distinct systems of coördination to carry out the same movement, one employed when volition was the moving cause, and the other when something else led to the movement. On the other hand, the analogy of speech justifies us in concluding that the cortical processes do take advantage of coördination effected by the action of other parts of the nervous system.

Bearing this in mind, we may recall attention to the remarkable effects which result from the removal of the area. These are twofold. In the first place, there is more or less complete paralysis of the limb; all the movements of the limb are for a time ineffective. It is not that purely voluntary movements are alone, so to speak, cut out; the reflex and other movements are also impaired or temporarily abolished, and, as we have already said, in many cases at least the sensations of the limb are interfered with. These troubles are, of course, in part the effects of the mere operative interference belonging to what we spoke of in $\S 495$, as being of the nature of shock. But, even giving full weight to this consideration, there remains the fact that the cortical area is associated with the various coördinating and other nervous mechanisms belonging to the limb by such close ties that these are thrown into disorder when it is injured. And side by side with this we may put the remarkable fact previously stated, that during an abnormal condition of the cortical area stimulation of the area, instead of producing the appropriate movements confined to the limb, may give rise to movements of other parts culminating in epileptiform convulsions.

In the second place, this paralysis is temporary only ; the voluntary movements are after a while regained, and that in spite of the fore-limb moiety of the pyramidal tract permanently degenerating along its whole length. neither it nor the cortical area ever being regenerated. This shows that whatever be the chain of events in the intact animal, it is possible for the "will " of the animal to get at the muscles and motor mechanisms of the fore limb by some other path than that provided by the appropriate cortical area and corresponding path of the pyramidal tract ; and the facts previously recorded $(\$ 571)$ show that that other part is not the corresponding part of the pyramidal system belonging to the other half of the hemisphere, and, indeed, is not any part at all of the whole pyramidal system. 'The "will," whatever be the processes by which it takes origin, and wherever be the place where they are carried on, is able in the absence of the pyramidal system to produce its effect on the motor fibres of the brachial nerves by working on other parts of the central nervous system.

Hence while admitting, as we must do, that in the intact animal the cortical area and pyramidal tract play their part in carrying out voluntary movements, their action is not of that simple character supposed by the view referred to above. On the contrary, we are driven to regard them rather as links, important links, it is true, but still links in a complex chain. As we have already urged, we may probably speak of the changes taking place in the pyramidal fibres as being on the whole of the nature of efferent impulses; 
but we should be going beyond the evidence if we concluded that they were identical with the ordinary efferent impulses of motor nerves. And above all it must not be left unnoticed that the cortical area has close, if not direct, connections of a sensory nature with the part in whose movenients it is concerned. 'This is shown by the following remarkable results which may make their appearance when stimulation of the curtex is carried on while the animal $(\mathrm{dog})$ is in a particular stage of the influence of morphine. If a subminimal stimulus be found, that is, a current of such intensity that applied to a motor area it will produce no movement, but if increased ever so slightly will give a feeble constriction of the appropriate muscle, it may be observed that a slight stimulus, such as gently stroking the skin over the muscles in question, will render the previous subminimal stimulus effective, and so call forth a movement. Thus, if the area experimented on be that connected with the lifting of the forepaw, and the subminimal stimulus be applied to the area at intervals, after several applications followed by no movements, a gentle stroke or two over the skin of the paw will lead to the paw being lifted the next time the stimulus is applied to the area. A similar result, but less sure and striking, may follow upon the stimulation of parts of the body other than the part corresponding to the area stimulated. Then, again, it has been observed that in certain other stages of the influence of morphine, the cortex and the rest of the nervous system are in such a condition that the application of even a momentary stimulus to an area leads not to a simple movement, but to a long-continued tonic contraction of the appropriate muscles. Under these circumstances a gentle stimulus, such as stroking the skin or blowing on the face, applied immediately after the application of the electric stimulus to the area, suddenly cuts short the contraction, and brings the muscles at once to rest and normal flaccidity.

These experiments show that the development of the processes in the cortex leading to the issue of what we have agreed to call efferent impulses along the pyramidal fibres is markedly affected by sensory impulses, and especially by sensory impulses started in the skin overlying and corresponding to the muscles put into movement. How these sensory impulses reach the cortex we do not exactly know; but we have no evidence to show that afferent, centripetal impulses can travel backward, so to speak, along the pyramidal fibres; and it is more reasonable to suppose that the sensory impulses in question reach the cortex by the ordinary paths of sensory impulses, which we shall presently discuss. We may therefore take the results of the experiments as showing how close is the connection of the motor area with the sensory mechanisms of the spinal cord and lower parts of the brain, and as illustrating the complexity of the chain of events by which the motor area brings about voluntary movements.

$\$ 575$. We have above used the general phrase "movements of the limb," since in the dog it is not easy to pick out certain movements as being particularly skilled movements. In the monkey such a distinction is easier. In this animal, as we have said, recovery of voluntary movement also takes place after removal of a cortical area, or at least has done so in many cases; and while the phenomena immediately following removal on the whole resemble those witnessed in the dog, a certain order of recovery may be observed; the more skilled movements are the last to return. When, for instance, the arm area is removed, the delicate movements of the hand, of the thumb and finger, are the last to be reëstablished; and a condition of things may be met with in which the animal after removal, say of the arm area in the left hemisphere, uses by preference the left hand at a time when, if prevented from using that hand, he is able to use the right; that is to say, the recovery in the right limb after the removal of the area on the left side 
is nearly but not quite complete; the "will" can gain access to the right hand, but not so easily as to the left hand, and this latter is used, though under ordinary circumstances it would not be used.

When we turn to man, in whom the great development of the pyramidal system and differentiation of the cortical area is paralleled by the prominence of skilled and trained movements, the analogy of the phenomena of speech, if it be true, as clinical histories seem to show, that destruction by disease of the speech area of both sides causes permanent aphasia, would lead us to conclude that at least highly skilled voluntary movements are carried out by the pyramidal system and by that alone. But in reference to this it must be remembered that such a permanent aphasia may be due, not to mere loss of the pyramidal channel, not to the will being merely unable to gain access to lower coördinating mechanisms, but to the absence of the differentiated cortical gray matter, by reason of which absence the will cannot initiate the first processes of the act of speech; it may be that were it able to do so, the processes so started might, in the absence of the pyramidal tract, find some other way to the bulbar mechanism, as in the case of the unskilled movements of the dog. This point, however, clinical histories have not definitely settled. Moreover, in dealing with the phenomena of the nervous system of man, as revealed by disease, we meet in reference to the cerebral cortex the same difficulty that we dwelt upon in dealing with the spinal cord ( $\$ 504)$. Lesions of the pyramidal system, of the internal capsule for instance, lead to the loss not only of skilled, but of all voluntary movements ; according to the character and position of the lesion this or that part of the body is wholly withdrawn from the influence of the will. And it is possible to maintain the thesis that man has become so developed as to his nervous system and the motor cortex, so accustomed to make use exclusively of the pyramidal system, that the will has lost the power, still possessed by lower animals, to gain access by some path other than the pyramidal one to the immediate nervous mechanisms of movement. The data for forming a satisfactory conclusion as to this point are so few and uncertain that it would be unprofitable to discuss the question here; but we may venture to point out that, great as is the development of the cerebral cortex and the pyramidal system in man, that development is accompanied by a hardly less striking expansion of other parts of the brain not directly connected with the pyramidal system which we have previously seen reason to associate with the coördination of movements, for example the cerebellum. And, indeed, it is clear that, admitting the pyramidal tract to be the ordinary channel by which volitional impulses pass to, or by which the will gains access to, the motor mechanisms immediately associated with the anterior roots of this or that spinal nerve, we must also admit that those volitional impulses passing along the pyramidal tract, or at least some of the processes constituting the will, are in connection with, and thus are influenced by the condition of, other parts of the brain. When, for instance, a gymnast executes a skilled voluntary movement in which all his four limbs and other parts as well perhaps of his body are involved, it is probably the case that changes of the nature of efferent impulses sweep down his pyramidal tract, and that these impulses, starting in a definite order from his cortex, that is to say, having undergone a certain amount of initial coördination at their very origin, meet with further coördination in the spinal gray matter, which serves as a set of nuclei of origin for the motor nerves concerned in the movement before they issue as ordinary motor impulses along the anterior roots. But this is not all. Should the gymnast's semicircular canals happen to be injured and his cerebellum thereby be troubled, or mischief fall on some other part of the brain which like this has no direct connection with either the pyramidal 
tract or the motor cortex, the movement fails through lack of coördination, though both the cortex, the pyramidal tract, and the spinal motor mechanisms remain as they were before. Obviously the carrying out of a voluntary movement is a very complex proceeding, and the motor cortex with the pyramidal tract is only one part of the whole mechanism; so far from the whole business being confined to these, it is perhaps no exaggeration to say that in each movement of the kind most parts of the whole brain have a greater or less share.

The exact nature of the part played by the cortex and the pyramidal tract in voluntary movements our present knowledge is inadequate to define. When we pass in review a series of brains from the lower to the higher and see how the pyramidal system is, so to speak, grafted on to the rest of the brain, when we observe how the increasing differentiation of the motor cortex runs parallel to the increasing possession of skilled educated movements, we may perhaps suppose that a "short cut" from the cortex to the origins of the several motor nerves, such as is afforded by the pyramidal fibres, from the advantages it offers to the more primitive path from segment to segment along the cerebr()-spinal axis has by natural selection been developed into being in man the chief and most important instrument for carrying out voluntary movements ; but, we repeat, it remains even in its highest development a link in a chain, and a knowledge of how the whole chain works is at present hidden from us.

We must not here wander into psychological problems, but may repeat that in the above discussion we have used the word "will" in a general sense only. A man may be brought into a condition, for instance in certain hypnotic phases, in which he can carry out all the various skilled movements which he has inherited or which he has learned; and yet, according to some definitions of the word "will," those movements could not be said to be initiated by his will. It can hardly be doubted that in such cases the motor cortex and pyramidal tract play their usual part. But we may pass from such cases as these through others, until we come to cases where a skilled movement which has been learned and practised by the working of an intelligent will, may continue to be carried out under circumstances which seem to preclude the intervention of any conscious will at all; and the transition from one case to another is so gradual, that it is impossible to suppose that there has been any shifting of the machinery employed for carrying out the movement. So that a volitional origin is not an essential feature of these so-called voluntary movements, and the machinery of the motor cortex and pyramidal tract is available for other things than pure volitional impulses.

$\S 576$. The preceding discussion will enable us to be very brief concerning a question which has from time to time been much discussed, and which has acquired perhaps fictitious importance, viz., the question as to how volitional impulses leading to voluntary movements travel along the spinal cord. The conclusions at which we have arrived, namely, that in the normal carrying out of voluntary movements the chief part is played by efferent impulses passing along the pyramidal tract, carries with it the answer that volitional impulses travel in the spinal cord along the pyramidal tract.

In the dog, in which the whole pyramidal tract crosses at the decussation of the pyramids, we should expect to find that a break in the pyramidal tract of one side of the cord at any point along its length caused loss of voluntary movement on the same side below the level of the break. And experiments as far as they go support this view. No one it is true has attempted to divide or otherwise cause a break in the pyramidal tract alone, leaving the rest of the cord intact; and, indeed, even if an injury were 
limited to the area marked out as the pyramidal tract, fibres other than pyramidal fibres would be injured at the same time, since the tract is never a "pure" one. But it has been found that a section of a lateral half of the cord, a lateral hemisection, or a section limited to the lateral column of one side, has for one of its principal effects loss of voluntary movement on the same side in the parts supplied by motor nerves leaving the cord below the level of the section. We say "one of its principal effects" because, besides the concomitant interference with sensations concerning which we shall speak presently, the loss of voluntary movement is not absolutely confined to the same side; there is some loss of power on the crossed side, at least in a large number of cases. IVe must not lay stress on this crossed paralysis because it is probably one of the effects of the mere operation, not a pure "deficiency" phenomenon, and, indeed, appears soon to pass away. But taking into consideration what was said above concerning the effects of removing cortical areas, it is important to note that in the experience of many experimenters the loss of voluntary power on the operated side diminishes after a while, and that the animal if kept alive and in good health long enough appears to regain almost full voluntary power over the affected parts. In such cases, as in other operations on the central nervous system, there is no regeneration of nervous tissue; the two surfaces of the section unite by connective not nervous tissue, and the tracts which as the result of the section degenerate downward or upward are permanently lost. Hence, even if we admit that in the intact animal a voluntary movement is chiefly carried out by means of efferent impulses passing along the pyramidal tract right down to the motor mechanisms of the cord immediately connected with the motor nerves, we must also admit that the "will" under changed circumstances can find other channels for gaining access to the same mechanisms.

It has been further observed that if in the dog a hemisection be made at one level, for instance in the lower thoracic region of the cord, and then, after waiting until the voluntary power over the hind limb of that side has returned, a second hemisection, this time on the other side, be made at a higher level, this second operation is followed by results similar to those of the first; there is loss of voluntary power on the side operated on, with some loss of power on the crossed side, and as in the first case this loss of power not only on the same but also on the crossed side may eventually disappear. This shows among other things that the recovery after the first operation was not due to the remaining pyramidal tract doing the work of both. Further, the hemisection may be repeated a third time, the third hemisection being on the same side as the first, with at least a very considerable return of power over both limbs. That is to say, under such abnormal circumstances voluntary impulses may, so to speak, thread their way in a zigzag manner from side to side along the mutilated cord until they reach the appropriate spinal motor mechanisms. Such an abnormal state of things does not, however, really militate against the view that under normal circumstances volitional impulses normally travel along the pyramidal tract; but it does show what, indeed, has already been shown by the phenomena of strychnine poisoning ( $\$ 499$ ) that in the central nervous system the passage of nervous impulses (using these words in the general sense of changes propagated along nervous material) is not rigidly and unalterably fixed by the anatomical distribution of tracts of fibres; in all such discussions as those in which we are engaged we must bear in mind that physiological conditions as well as anatomical continuity are potent in determining the passage of these impulses.

$\S 577$. When we reflect on the great prominence of the pyramidal tract in the spinal cord of man as compared with that of the dog, we may justly infer not only that the pyramidal tract is under normal circumstances more 
exclusively the channel of volitional impulses in man than in such lower animals, but also, bearing in mind the discussion in a previous chapter ( $\$ 504$ ) concerning the activities of the spinal cord of man, that the potential alternatives presented by the spinal cord of the dog are greatly reduced in that of man. And such clinical histories of disease or accidental injury in man as we possess support this conclusion. Lesions confined to one-half of the cord, or even lesions confined to the lateral column of one-half, appear to lead to loss of voluntary power on the same side, and the same side only, in the parts below the level of the lesion; and the same symptoms have been observed to accompany disease limited apparently to the pyramidal tract of one side. Moreover, though cases of recovery of power have been recorded, we have not such satisfactory evidence as in animals of the volitional impulses ultimately making their way along an alternative route; but here the same doubts may be entertained as were expressed in discussing the reflex acts of the cord in man.

When we say that the loss of voluntary power is seen on the side of the lesion only, we should add that this statement appears to apply chiefly to the thoracic and lower parts of the cord. We have seen that in man, in the upper regions of the cord, the pyramidal tract is only partly crossed; a variable but not inconsiderable number of the pyramidal fibres do not cross at the decussation of pyramids, but running straight down as the direct pyramidal tract effect their crossing lower down in the cervical and upper thoracic regions. Hence, we should infer that a hemisection of, or a lesion confined to one side of, the cervical cord would affect the voluntary movements of the crossed side as well as of the same side, though not to the same extent. But we have no exact information as to this point. And, indeed, the purpose of the direct tract is not clear; there is no adequate evidence for the view which has been held that these direct fibres are destined for the upper limbs and upper part of the body ; since they are the last to cross we should, à priori, be inclined to suppose that they were distributed to lower rather than higher parts.

$\S 578$. We may now briefly summarize what we know conceruing voluntary movements. And it will be convenient to trace the events in order backward.

Certain muscles are thrown into a contraction which even in the briefest movements is probably of the nature of a tetanus. In almost every movement more than one muscle, as defined by the anatomists, is engaged, and in many movements a part of several muscles is employed, and not the whole of each. It is perhaps partly owing to the latter fact that a muscle which has become tired in one kind of movement, may show little or no fatigue when employed for another movement, though we must bear in mind that in a voluntary movement fatigue is much more of nervous than of muscular origin.

Besides the active muscles, if we may so call them, which directly carry out the movement, the metabolism of which supplies the energy given out as work done, other muscles, some of which are antagonistic to the active muscles and some of which may be spoken of as adjuvant, enter into the whole act. In flexion, for instance, of the forearm on the arm it is not the flexor muscles only but the extensors also which are engaged. According to the immediately preceding position and use of the arm, and according to the kind and amount of flexion which is to be carried out, the extensors will be either relaxed, that is to say, inhibited, or thrown into a certain amount of contraction. And in some of the more complicated voluntary movements the part played by adjuvant muscles is considerable. Hence, in a voluntary movement the will has to gain access not only to the active muscles, but also 
to the antagonistic and adjuvant muscles; and every voluntary movement, even one of the simplest kind, is a more or less complex act.

The impulses which lead to the contraction of the active muscles reach the muscles along the fibres of the anterior roots (we may for the sake of simplicity take spinal nerves alone, neglecting the peculiar cranial nerves), and such evidence as we possess goes to show that the impulses governing the antagonistic and adjuvant muscles travel by the anterior roots also; the question whether the inhibition of the antagonistic muscles when it takes place is carried out by inhibitory impulses passing as such along the fibres, or simply by central inhibition of previously existing motor impulses, need not be considered now. These anterior roots are connected, as we have seen, with the gray matter of the cord, and in each hypothetical segment of the cord we may recognize the existence of an area of gray matter which, though we cannot define its limits, we may, led by the analogy of the cranial nerves, call the nucleus of the nerve belonging to the segment; and we may further recognize in such a nucleus what we may call its efferent and its afferent side.

Every voluntary movement, even the simplest, is, as we have repeatedly insisted, a coördinated movement, and in its coördination afferent impulses play an important part. The study of reflex actions ( $\$ 502)$ has led us to suppose that each spinal segment presents a nervous mechanism in which a certain amount of coördination is already present, in which efferent impulses are adjusted to afferent impulses. But the results obtained by stimulating separate anterior nerve-roots show that, in the case of most muscles at all events, the especially active muscles of the limbs for instance, each muscle is supplied by fibres coming from more than one nerve-root, that is to say, the spinal nucleus, or at least the spinal motor mechanism for any one muscle, extends over two or three segments. Hence à fortiori in a voluntary movement, involving as this does in most cases more than one muscle, the spinal mechanism engaged in the act spreads over at least two or three segments, thus allowing of increased coördination. In that coördination the impulses serving as the foundation of muscular sense play an important part, but other afferent impulses, such as those from the adjoining skin, also have their share in the matter; and it is worthy of notice that not only is the skin overlying a muscle served, broadly speaking, by nerve-roots of the same segment as the muscle itself, afferent in one case, efferent in the other, but in the parts of the body where coördination is especially complex, in the fingers for instance, not only is each muscle supplied from more than one segment, but also each piece of skin is supplied in the same way by the posterior roots of more than one nerve.

In the case of the frog it is clear that in reflex movements a large amount of coördination is carried out by these various spinal mechanisms; and as we have urged, we may safely infer that in the voluntary movements of the frog, the will makes use of this already existing coördination, whatever be the exact path by which in this animal the will gains access to the spinal mechanisms. In the dog we may conclude that in voluntary movements the spinal mechanisms, with coördinating functions, are also set in action, in this case by impulses passing straight fiom the cortex to the mechanisms by the pyramidal tract, though apparently, in the absence of the pyramidal tract, the will can work upon the mechanisms by changes travelling through other parts of the cerebro-spinal axis. And in the monkey and man, subject to the doubts already expressed as to the potentialities of the human spinal cord, we may probably also infer that in each voluntary movement some, perhaps we may say much, of the coördination is carried out by the spinal mechanism set into action through impulses along the pyramidal tract. We 
may probably further infer that a careful adjustment obtains between the beginnings of the pyramidal tract in the cortex and its endings in the cord, so that the topography of "areas" or "foci" in the cortex above is an image or projection of the spinal mechanisms below.

The complex character, on which we insisted just now, of almost every voluntary movement necessitates that in every such movement a large area of spinal mechanism is involved. But this is not all. The movements of any part of the legs, for instance, are not deternimed, nor is the coördination of the movements affected, simply by what is going on in the legs and the part of the spinal cord belonging to them. The discussion in a previous section has shown that much of the coördination of the body is carried out by the middle portions of the brain, and on these the motor area must have its hold as on the spinal mechanisms.

The details of the nature of that hold are at present unknown to us; but it must be remembered that not all the fibres passing down from the motor region, not all those even proceeding from the densest and most clearly defined motor areas, are pyramidal fibres. With the pyramidal fibres are mingled fibres having other destinations, and some of these probably pass to the thalamus and so join the great tegmental region. Moreover, the motor region must have close ties with other regions of the cortex whence, as we have seen ( $\$ 545)$ fibres pass to the pons to make connections with the cerebellum. On the other hand, as we have seen ( $\$ 525)$ the cerebellum is especially comnected with what we may fairly consider the afferent side of the spinal cord and bulb. These facts must merely be taken as indicating the possibilities by which the motor region is kept in touch with the great coördinating mechanism; it would be venturesome at present to say much more.

In an ordinary voluntary movement an intelligent consciousness is an essential element. But many skilled movements initiated and repeated by help of an intelligent conscious volition may, when the nervous machinery for carrying them out has acquired a certain facility (and in all the higher processes of the brain we must recognize that, in nervous material at all events, action determines structure, meaning by structure molecular arrangement and disposition), be carried out under appropriate circumstances with so little intervention of distinct consciousness that the movements are then often spoken of as involuntary. All the arguments which go to show that the distinctly conscious voluntary skilled movement is carried out by help of the appropriate motor area go to show that the motor area must play its part in these involuntary skilled movements also. So that distinct consciousness is not a necessary adjunct to the activity of a motor area. And it is worthy of notice that some of these, in their origin, purely voluntary skilled movements, which by long-continued training have become almost as purely involuntary, are hampered rather than assisted by being " thought about."

The word "training" suggests the reflection that the physiological interpretation of becoming easy by practice is that new paths are made, or the material of old paths made more mobile by effort and use. We have already urged ( $\$ 494)$ that the gray matter of the spinal cord is a network, in which the passage of impulses is determined by physiological conditions rather than anatomical continuity, and the same considerations may with still greater force be applied to the brain. We must suppose that training promotes the growth and molecular mobility of the motor area and of all its connections. There are doubtless limits to the changes which can be effected, but within these limits the will, blundering at first in the maze of the nervous network, gradually establishes easy paths; though even to the end it blunders-in trying to carry out one movement it often accomplishes another. 
Lastly, without attempting to enter into psychological questions, we may at least say that the birthplace of what we call the "will," is not conterminous with the motor area ; the will arises from a complex series of events, some of which take place in other regions of the cortex, and probably in other parts of the brain as well. With these parts the motor area has ties concerned not in the carrying out of volition, but in the generation of the will. So that, looking round on all sides, it is obvious, as we have said, that the motor area is a mere link in a complex chain. It is, moreover, a link of such a kind, that while the changes which the breaking of it makes in the daily life of a lowly animal, such as the dog, in whom the experience of the individual adds relatively little to the nervous and psychical storehouse transmitted from his ancestors, can hardly be appreciated by a bystander, those which the breaking of it makes in the daily life of a man, whose brain at any moment is not only a machine fitted for present and future work but a closely packed record of his past life, are obvious not only to the individual himself, but to his fellows.

\section{On the Develophent within the Central Nervous System of}

Visual aNd OF SOME OTHER Sensations.

\section{Visual Sensations.}

$\S 579$. In the chain of events through which some influence brought to bear on the periphery of a sensory nerve gives rise a sensation, we are able, with more or less success, to distinguish between those events which are determined by the changes at the periphery and those which are the expression of changes induced in the central nervous system. Thus when certain rays of light proceeding from an object and falling upon the eye give rise to visual perception of the object, two sets of events happen : the rays of light, by help of the mechanisms of the eye, partly dioptric, partly nervous, give rise to certain changes in the fibres of the optic nerve, which we may call visual impulses; and these visual impulses reaching the brain along the optic nerve give rise to visual sensations and so to visual perception of the object. We shall later on, under the heading of "the senses," deal chiefly with the peripheral events, and have now to consider some points connected with the central events, to learn what we know concerning how the various sensory impulses travelling along the several kinds of sensory nerves behave within the central nervous system. In doing so we shall have from time to time to refer to peripheral events, but only occasionally, and never in any great detail. It will be convenient to begin with the special sense of sight, and we must first briefly call attention to a few points which we shall have to study in fuller detail hereafter.

The eye is so constructed that images of external objects are brought to a focus on the retina, the stimulation of which by light starts the visual impulses along the fibres of the optic nerve; and the distinctness with which, by means of the visual sensations arising out of these visual impulses, we perceive external objects is dependent on the sharpness of the retinal images. The eye is further so constructed that, in any position of the eye, the rays of light proceeding from a portion only of the external world fall upon the retina; or in other words, in any one position of the eye only a portion of the external world is visible at the same time. The portion so seen is spoken of as the visual field for that position.

The image thrown on the retina is an inverted one, so that the top of an actual object is represented by the lower, and the bottom by the upper, part 
of the retinal image; similarly the actual left-hand side of the retinal image corresponds to the right-hand side of the actual object, and the right-hand side to the left-hand side. Hence the right-hand half of the visual field corresponds to the left-hand side of the retina, and the left-hand half to the right-hand side.

The eye can be moved in various directions, and since in the visual field the portion of external nature which can be seen at the same time differs with each different position, a large range of vision is thus secured; and this can be further increased by movements of the head. Moreover, we normally make use of two eyes; our normal vision is binocular, and the visual field of the right eye differs from that of the left eye. There is one striking difference which must always be borne in mind. A section carried through the eye in a vertical and front-to-back plane, through what we shall learn to call the optic axis (Fig. 156,o.x.) (the exact details of the plane may be left for the present), will divide the retina into two lateral halves, and in each retina one-half will be on the nasal side next to the nose, and the other half will be on the malar or temporal side, next to the cheek or temple. It must be remembered that the nasal halves and temporal halves of the two retinas do not occupy corresponding positions in space. The temporal half of the left retina is on the left side of its own eye, whereas the temporal half of the right retina is not on the left, but on the right, side of its eye; and so with the nasal halves. Now, in the right eye, the right-hand side of the visual field corresponds to the nasal half of the retina, and the left-hand side of the visual field to the temporal half of the retina, whereas in the left eye the right-hand side of the visual field corresponds to the temporal half of the retina, and the left-hand side to the nasal half. 'This is shown in Fig. 156, where the left-hand visual field and the retinal area concerned are shown shaded in each eye.

When we look at an object with the two eyes, though two retinal images are produced, one in one eye and one in the other, we perceive one object only, not two. This is the essential fact of binocular vision; when certain parts of each retina are stimulated at the same time we are conscious of one sensation only, not two ; and the parts of the two retinas which, stimulated at the same time, give rise to one sensation are spoken of as "corresponding parts." From the structure and relations of the two eyes it follows that the temporal side of the right, and the nasal side of the left, eye are such corresponding parts, while the nasal side of the right eye corresponds to the temporal side of the left eye. But the whole of each retina is not employed in binocular vision. Owing to the position of the two eyes in relation to the nose, it comes about that an object held very much on one side, to the lefthand side for instance, while it is capable of producing an image on the extreme nasal side of the left eye, and can be seen, therefore, by that eye, cannot produce an image on the temporal side of the right eye; the nose blocks the way. It is, therefore, not seen by the right eye, and the vision of it is monocular by the left eye only. In Fig. 156 it may be seen that the left visual field of the left eye $(L . F . L$.) extends more to the left, and is larger than the left visual field of the right eye (I.F.R.), and that the right retinal area, corresponding to the left visual field, extends further along the nasal side of the left side $\left(a^{\prime}\right)$ than it does along the temporal side of the right eye $\left(a^{\prime}\right)$, the difference being due to the presence of the nose $\left(F^{\prime}\right)$. And similar conditions obtain with regard to the extreme right-hand side of the risual field.

$\S 580$. After these preliminary statements, we may now turn to consider some anatomical facts concerning the ending of the optic nerve in the brain. 
Fig. 156

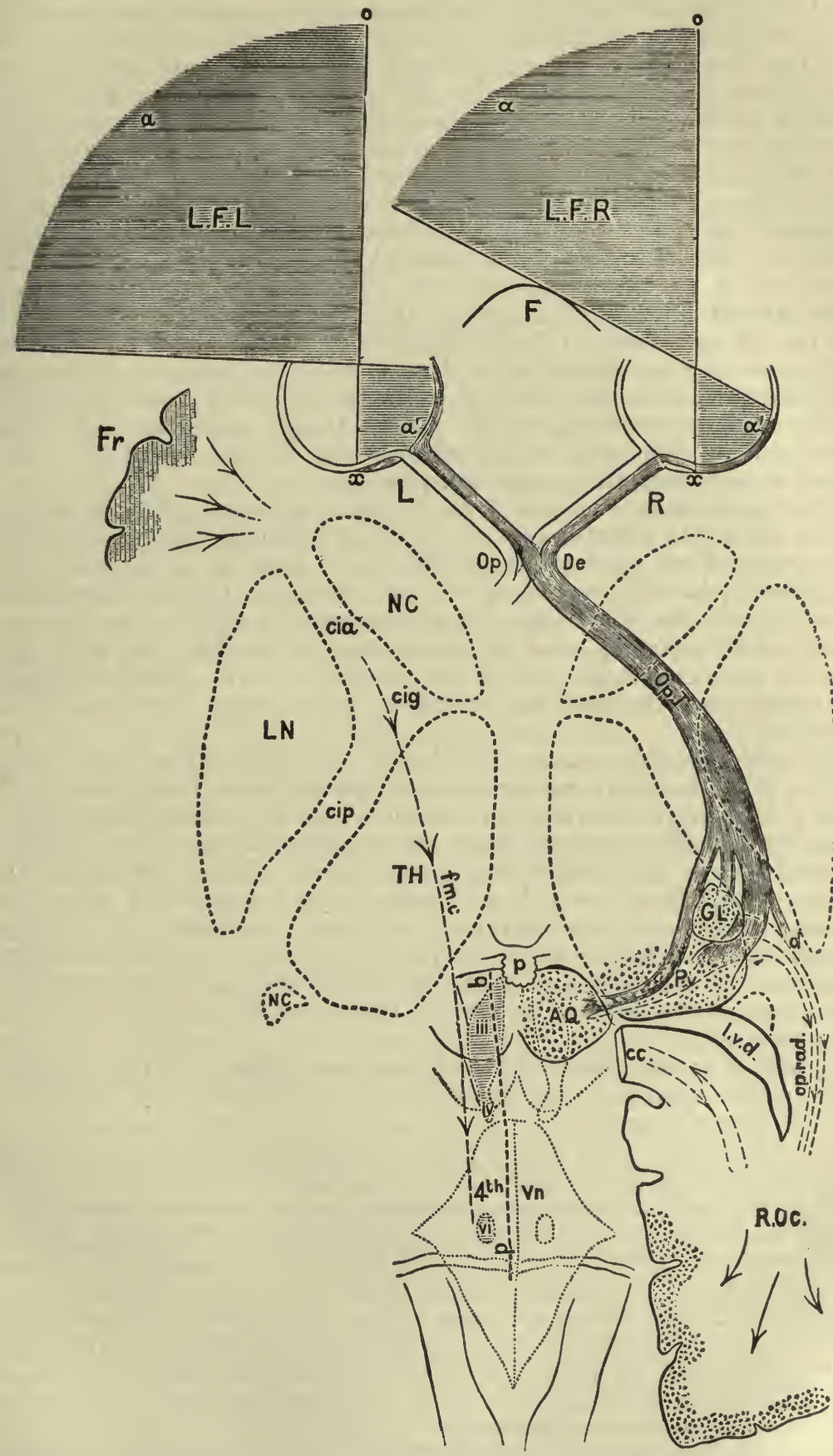

Diagram to illustrate the Nervous Apparatus of Vision in Man. (Sherrington.) $L$, the left eye $R$, the right eye; $0, x$., the optic axis; $F$, the outline of the face between the eyes; $O p T$, the right 
The optic nerve of each eye consists of nerve-fibres coming from all parts of the retina of that eye; but the two optic nerves meet. ventral to the floor of the third ventricle, cross each other at the optic chiasma (Fig. $156, O p . D e$.$) , and are thence continued on under the name not of optic$ nerves, but of optic tracts ( $O_{p} . T$.). The decussation of fibres which takes place in the chiasma has peculiar characters. At their decussation (we are speaking now of man) the fibres in the optic nerve belonging to the temporal half of the eye in which the nerve ends pass into one optic tract, namely, the optic tract of the same side, while the fibres belonging to the nasal half pass into another optic tract, namely the optic tract of the opposite side. Thus the fibres of the temporal half of the right eye and of the nasal half of the left eye pass into the right optic tract, and the fibres of the nasal half of the right eye and of the temporal half of the left eye pass into the left optic tract. Compare Fig. 156, in which the fibres forming the right optic tract are shaded, while those forming the left optic tract are left unshaded. Now the nasal half of one retina and the temporal half of the other retina are "corresponding" parts. Hence, while each optic tract contains fibres belonging to half of each eye, the two halves thus represented in each tract are corresponding halves.

The amount and character of the decussation taking place in the optic chiasma differs in different animal types, the difference having relation to the amount of binocular vision, which in turn depends on the position of the eyes in the head, that is, on the prominence of the face between the eyes. In the fish, for instance, with laterally placed eyes, no binocular vision at all is possible, and the decussation is complete; the whole optic nerve of each eye crosses over to the other optic tract. Between this and the arrangement in man, just described, various stages obtain in various animals.

The chiasma also contains at its hinder part fibres which have no connection with the optic nerves or the eyes, but are simply commissural tracts passing from one side of the brain, namely, from the median corpus geniculatum ( $(543)$ along one optic tract, through the chiasma to the other optic tract, and so to the median corpus geniculatum of the other side of the brain. These fibres are spoken of as the inferior or posterior (optic) commissure or arcuate commissure, or Gudden's commissure. It was once thought that in a similar way fibres passed from one retina along one optic nerve through the front part of the chiasma to the other optic nerve, and to the other retina, forming an anterior (optic) commissure; but this seems to be an error.

$\S 581$. The optic vesicle is, as we have seen, budded off from the forebrain or forerunner of the third ventricle, and the optic chiasma is

optic tract (shaded) supplying, through $O p D e$, the optic decussation, the temporal side of the retina of the right eye and the nasal side of the retina of the left eye; L.F.L and L.F.R, the left visual fields of the left and right eye respectively; the two fields and the parts of the two retinas whose excitation produces vision over the fields are shaded, the object $a$ in the field of the right side giving rise to an image at $a^{\prime}$, and $a$ on the left side an image at $a^{\prime}$. The right optic tract is represented as ending in $G L$, the lateral corpus geniculatum; in $P v$, the pulvinar; and in $A Q$, the anterior corpus quadrigeminum, all three stippled; op rad, the optic radiation from these bodies to R.Oc, the right occipital lobes whose stippled cortex indicates the "visual area;" $d$, the "direct" tract to the cortex; $c c$, corpus callosum, cut across at the splenium; $l . v . d$, descending horn of the lateral ventricle; the left side has been utilized to indicate at $F$, shaded with lines, the cortical motor area for the eyes; $f m$. $c$. indicates the path from it to iii, iv, vi, the nuclei of the third, fourth, and sixth nerves; $p . b$, the posterior longitudinal bundle, shown as a broken line; $N C$, the nucleus caudatus; $L N$, the nucleus lenticularis; and $T H$, optic thalamus shown in outline; cia, the front limb; cig, the knee, and cip, the hind limb of the internal capsule. The outlines of the fourth ventricle, 4 th $V n$, and of the posterior corpora quadrigemina are shown by dotted lines, that of the bulb is shown by a fine line; $p$, the pineal gland. 
attached to and forms part of the floor or ventral wall of that ventricle. In a view of the basal or ventral surface of the brain the diverging optic tracts are seen to separate the anterior perforated space and lamina cinerea in front from the posterior perforated space, tuber cinereum with the infundibulum, and corpora albicantia behind, all these being parts of the floor of the third ventricle. From the gray matter in this floor, fibres forming what is sometimes spoken of as Meynert's commissure, belonging neither to the optic nerves nor to the inferior commissure, join the optic tracts, eventually leaving them to pass to the pes. Hence the whole of the optic tract is by no means derived from the optic nerve; the fibres just mentioned and the inferior commissure form parts of the optic tract not connected with the retina.

Each optic tract crosses obliquely, being in crossing firmly attached to the ventral surface of the crus cerebri of the same side (Fig. 131, C), and is soon lost to view, being covered up by the temporo-sphenoidal lobe of the hemisphere. When this is removed the tract is seen to sweep dorsally round the crus toward the dorsal aspect, and as we have already (\$543) said, to become connected on the further side of the crus with the two corpora geniculata, lateral and median. We may say at once that the median corpus geniculatum has no connection with that part of the tract which is derived from the optic nerve, and is not concerned in vision, but is connected with that part of the tract, sometimes called the median part, which goes to form the inferior commissure. We may confine our attention to that part of the tract which consists exclusively of fibres coming from the retinas of the two eyes, for it is this part, and this part only, which is concerned in vision.

$\$ 582$. This ends in three main ways, as shown diagrammatically in Fig. 156. In the first place, part of the tract ends in the lateral corpus geniculatum $(G L$.$) , formed of alternating layers of white and gray matter,$ the gray matter containing in some parts large nerve-cells, and in others small nerve-cells. In these cells of one kind or another, many of the fibres appear to end. In the second place, a very large number of fibres passing the corpus geniculatum on its ventral and lateral surfaces spread out into the pulvinar $(P V$.$) . In the third place, others in considerable number,$ taking a more median direction, reach the anterior corpus quadrigeminum $(A Q$.$) . These two sets also, like the first, end apparently in the nerve-cells$ of the respective bodies. Thus, the really optic fibres of the optic tract end in one of three collections of gray matter, the lateral corpus geniculatum, the pulvinar, or the anterior corpus quadrigeminum. Further, we have reasons for thinking that a considerable part at all events of the gray matter of these three bodies is associated with and, in a certain sense, dependent on the fibres of the optic nerves; the reasons are as follows: We know that when a nerve-fibre is cut away from its trophic centre it degenerates; but the division and the loss of the peripheral degenerating portion has no obvious effect on the trophic centre; when a spinal nerve, for instance, is divided below the spinal ganglion, though the nerve below the section degenerates, the ganglion and the piece of nerve in connection with it remain very much as before; we have it, however, in our power to bring about changes of a deeper and wider character, a cessation of growth amounting to atrophy, by operative interference with nervous structures before they are fully developed. Thus, in an adult animal, a section of an optic nerve or removal of the eye leads to degeneration in the optic nerve and optic tract; the optic fibres have their trophic centre in certain cells of the retina, of which we shall speak in treating of vision, and cut away from that centre they degenerate; by this means the nature of the optic decussa- 
tion in animals, and, indeed, in man, has been ascertained. But if the eyes be removed (removal of both eyes being desirable on account of the characters of the optic decussation) in a newborn animal, not only do both the optic nerves and the greater part of both optic tracts cease only to be further developed and degenerate, but the bodies mentioned above, the two lateral corpora geniculata, the pulvinar on each side, and the two anterior corpora quadrigemina, do not fully develop; certain parts of them undergo atrophy. The development of these nervous structures seems therefore to be largely dependent on their functional connection with the eyes by means of the optic tracts and nerves.

The same method confirms the view expressed above, that the median corpus geniculatum has no connection with vision. When the eyes of newborn animals are extirpated, neither the median corpora geniculata nor the posterior corpora quadrigemina show any signs of atrophy, and the part of the optic tract which does not degenerate is the inferior commissure connecting the two median corpora geniculata. Obviously these parts are associated with functions of the brain other than those of sight. The lateral corpora geniculata, the pulvinar, and the anterior corpora quadrigemina, are, we may repeat, alone to be regarded as the chief central parts in which the optic nerve ends. We may also repeat that owing to the peculiarity of the optic decussation each optic nerve thus finds its endings in both sides of the brain.

While the optic chiasma is, as we have seen, helping to form the floor of the third ventricle, it gives off fibres to the posterior perforated spot. Some of these have been supposed to pass directly in the wall of the ventricle to the nucleus of the third (oculo-motor) nerve, and to serve as a channel for afferent impulses, causing constriction of the pupil; but to this we shall return in dealing hereafter with the movements of the pupils.

$\$ 583$. Though the above three bodies are undoubtedly the chief endings of the optic nerve, three primary visual centres, if we may so call them, it is also believed that some fibres of the optic tract, making connections with neither of these three bodies, pass by the crus cerebri straight to certain parts of the cerebral hemisphere (Fig. $156, d$ ); but this fourth ending is by no means so clearly established as are the other three.

And undoubtedly the main connection of the cerebral hemisphere with the optic tract is not a direct one, but an indirect one, through the three bodies in question. A number of fibres proceeding from the occipital cortex and reaching the thalamus through the hind limb of the internal capsule formed what was called the "optic radiation." These fibres beginning (or ending) in the cortex of the occipital region, end (or begin) (Fig. 156, op. rad.) to a large extent in the pulvinar and in the lateral corpus geniculatum, but also in the anterior corpus quadrigeminum, reaching it by the anterior brachium ( $\$ 547)$. When even in a grown animal the occipital cortex is destroyed, not only these fibres, but also parts of the pulvinar and external corpus geniculatum, undergo degeneration, and there is some change in the anterior corpus quadrigeminum. When the same cortex is destroyed in a newborn animal the sane parts atrophy; and in such cases the optic tract and nerve, which are but little affected by the operation in the adult animal, are also involved in the atrophy. We may add that removal of both eyes in the newborn animal is said to lead, besides the atrophy of the three bodies in question, to diminisher occipital lobe due to lack of white matter. We may therefore conclude that in the complex act of vision two orders of central apparatus are involved; we may speak of two kinds of centres for vision-the primary or lower visual centres supplied by the three bodies of which we are speaking, and a secondary or higher visual centre supplied by 
the cortex in the occipital region of the cerebrum. And experimental results accord with this view.

Before we proceed to discuss those results, one or two preliminary observations may prove of use.

In the first place, as we have previously urged, the interpretation of the results of an experiment, in which we have to judge of sensory effects, are far more uncertain than when we have to judge of motor effects, that is, of course, when the experiment is conducted on an animal. We can estimate the motor effect quantitatively, we can measure and record the contraction of the muscle, but in estimating a sensory effect we have to depend on signs, our interpretation of which is based on analogies which may or may not be misleading. We are on safer ground when we can appeal to man himself in the experiments instituted by disease; but the many advantages thus secured are often more than counterbalanced by the diffuse characters or the complex concomitants of the lesion. In dealing with sensory effects we must expect and be content for the present with conclusions less definite and more uncertain even than those gained by the study of motor effects.

In the second place, in dealing with vision, it will be desirable to know the meaning which we are attaching to the words which we employ. By blindness, that is, "complete" or "total " blindness, we mean that the movements and other actions of the body are in no way at all influenced by the amount of light falling on the retina. Of partial or incomplete or imperfect vision, using the word vision in its widest sense, there are many varieties; and we may illustrate some of the defects of the visual machinery, regarded as a whole, with its central as well as its peripheral parts, by referring to certain defects of vision due to changes in the eye itself. The eye may fall into such a condition that the mind can only appreciate, and that to a varying degree, the difference between light and darkness; the mind is aware that the retina (or it may be part of the retina) is being stimulated to a less or greater degree, but cannot perceive that one part of the retina is being stinulated in a different way from another part; a sensation of light is excited, but not a set of visual sensations corresponding to the sets of pencils of luminous rays, which, reflected or emanating from external objects in a definite order, are falling upon the eye. The eye again may fall into another condition, in which such sets of visual sensations are excited, but on account of dioptric imperfections or for other reasons the several sensations are not adequately distinct; the mind is aware through the eye of the existence of "things," but cannot adequately recognize the character of those things; the visual images are blurred and indistinct. And a large number of gradations are possible between the extreme condition in which only those objects which present the strongest contrast with their surroundings are visible to a condition which only just falls short of normal vision. Imperfections of this kind, of varying degree, may result from failure, not in the peripheral apparatus, not in the retina or optic nerve or other parts of the eye, but in the central apparatus; the retinal image may be sharp, the retina and the optic fibres may be duly responsive, but from something wrong in some part or other of the brain the visual sensations excited by the visual impulses may fail in distinctness, and that in varying degree; imperfections of vision, whether of central or peripheral origin, in which visual sensations fail in distinctness, are generally spoken of under the not wholly unexceptionable name of amblyopia.

If one optic nerve be divided, total blindness of one eye will result; but if one optic tract be divided, it follows from what has been said above that half-blindness in the corresponding halves of both eyes will result. If, for instance, the right optic tract (Fig. 154, Op. T.) be divided, the left, visual 
fields of both eyes will be blotted out. The same condition will be brought about by failure in the optic tract at its central ending, provided of course the mischief be confined to the ending of the one tract. Such a half-blindness or half-vision is spoken of as hemianopsia or hemianopia or hemiopia; the words left and right are generally used in reference to the visual field; thus, left hemianopsia is the blotting out of both left visual fields through failure of the right optic tract.

If, instead of the whole optic nerve being divided, certain bundles only were cut across, partial blindness would be the result, a portion of the visual field would be blotted out, and mischief limited to a few bundles of one optic tract would lead to corresponding blots in the corresponding halves of the visual fields of both eyes.

Further, an affection of half the retina or of a limited area in the retina might occur of such a character as to lead, not to complete, but to partial blindness, to a hemi-amblyopia or to a partial amblyopia. The part of the retina so affected might be central, or peripheral, or a quadrant, or any patch of any size, form, and relative position. And we may further imagine it at least possible that mischief in the brain might be so limited as to produce any of the above partial effects, though the retina, optic nerve, and optic tracts all remained intact.

The above visual imperfections we have illustrated by changes in the peripheral apparatus, but there is a kind of imperfection which we nay still call a visual imperfection, though it is of purely central origin. In a normal state of things a visual sensation excited in the brain is or may be linked on to a chain of physical events; we often, then, speak of it as a visual idea. When we see a dog the visual sensation, or rather the group of sensations making up the visual perception of the dog, does not exist by itself, apart from all the other events of the brain; it joins and affects them, and among the events which it so affects may be and often are psychical events; the visual perception "enters into our thoughts" and modifies them. Between the visual impulse, as it travels along the optic nerve or tract, and its ultimate psychical effect, a whole series of events intervene; and we may take it for granted that the chain may be broken or spoiled at any of its links, at the later as well as at the earlier ones. We may therefore consider it possible that the break or damage may occur at the links by which the fully developed visual sensation joins on to psychical operations. We may suppose that an object is seen and yet does not affect the mind at all, or affects it in an abnormal way.

These foregoing considerations emphasize the difficulty and uncertainty of interpreting the visual condition of an animal which has been experimented upon. When, for instance, after an operation, an animal ceases to be influenced in its previous normal manner by the visual effects of external objects, a most careful psychical analysis is often necessary to enable us to judge whether the newly introduced disregard of this or that object is due to the mere visual sensation being blurred or blunted, or to some failure in the psychical appreciation of the sensations; and in most cases such an analysis is beyond our reach. The greatest caution is needful in drawing conclusions from experiments of this kind, especially from such as appear to have been hastily carried out or hastily observed; and we must be content here to dwell on some of the broader features only of the subject.

$\$ 584$. Since we have in this matter to trust so much to analogies with our own experience, we may turn at once to the monkey as being more instructive than any of the lower animals. We have already said that electrical excitation of the occipital cortex behind the motor region may produce movements, but that these movements are in character different from those 
caused by stimulation of the motor region itself. In the monkey stimulation of parts of the occipital region, the occipital lobe, and the angular gyrus, for instance, may give rise to movements of the eyes, of the eyelids, and of the head, that is of the neck, all the movements so produced being such as are ordinarily connected with vision. It will not be profitable to enter here into the details concerning the exact topography of the excitable parts or of the special characters of the movements so called forth. But it is important to note that these movements are unlike the movements excited by stimulation of the appropriate motor area, inasmuch as their occurrence is far less certain, they need a stronger stimulus to bring them ont; when evoked they are feeble, being easily antagonized by appropriate stimulation of the motor area, and they have a much longer latent period. They are not due to any indirect stimulation of the motor area, through "association" fibres connecting the spot stimulated with the motor area, or otherwise, since they persist after removal of the motor area. Movements of this kind may also be witnesserl in the dog. They are obviously the result of impulses transmitted in some direct manner from the cortex to some parts below, and may be taken as an indication that the parts of the cortex in question are in some way connected with vision. The exact manner, however, in which they are brought about is at present obscure. The explanation of their genesis which is frequently offered, namely, that the stimulation so affects the cortical gray matter as to give rise to visual sensations, and that the movements express these sensations, does not seem satisfactory. For, if it be possible that the gross changes which the electric current sets going in the cortical gray matter can reproduce the psychical events which take place in that gray matter in the normal action of the brain, we should expect stimulation of any and every part of the cortex to call forth some movement or other, since it cannot be doubted that every part of the cortex is in some way or other engaged in psychical operations, and that every psychical phase tends to express itself in movement: whereas, outside the motor region, with the exceptions we are now discussing, the cortex is, as we have seen, "inexcitable," and even within the motor region itself the excitable substance is scattered, with increasing segregation as we advance along the animal scale, among inexcitable substance. When we speak of the region or substance as inexcitable, we do not mean that the electric current produces no effect; we only mean that the effect is not manifested by movement; the real difference between the excitable motor region and the inexcitable rest of the cortex is probably that in the several motor areas the current, playing upon the beginnings of the pyramidal fibres, is able to inaugurate simple motor impulses or something like them, whereas elsewhere the molecular changes induced by the current are too confused to reach their normal expression. There can be no doubt, of course, that molecular changes in this or that part of the brain, set going by processes other than actual visual impulses along the optic nerves, may give rise to visual sensations; and, as we shall see in dealing with the senses, the subject of such "subjective" sensations is unable to distinguish them from sensations of "ohjective" origin; but it is at least unlikely that the coarse disturbances started by a tetanizing current should take such a definite form. Moreover, the view in question is disproved by the experimental result that the same movements are brought about when the cortex is pared away and the electrodes are applied to the subjacent white matter. This result suggests the existence of efferent tracts or bundles of a special kind, differing from those of the pyramidal kind, though like them making connections with the ocular and other muscles; we have, however, as yet no other evidence of such tracts existing.

$\S 585$. The results of removal of the cortex support the same general 
conclusion, though there is much discordance among the various observers, both as to the particular results and especially as to their interpretation. One broad fact comes out in all the observations, namely, that the removal of or injury to the hind region of the cortex always produces some disturbance of vision, and produces disturbance of vision more surely and to a greater extent than does injury to or removal of any other region of the cortex; but beyond this broad fact there is much dispute, and we must be content here with a very brief statement.

In the monkey, some observers have found that removal of the occipital lobe on one side (the region marked "vision" in Figs. 149 and 150) caused hemiopia, the effect on the visual fields being a crossed one; when the right lobe was removed there was blindness in the left visual fields, that is, in the right halves of the retinas of both eyes; in other words, the visual impulses passing along the right optic tract failed to produce their usual effect, so that the animal disregarded objects on its left-hand side. We may remark that the decussation of the optic nerves in the monkey is very similar to that in man. When both occipital lobes were removed, total blindness resulted. But, and this is most important, not only was the hemiopia caused by the removal of one lobe transient, but also according to some observers, the lost vision returned after the total removal of both lobes, though some impairment might be noticed long afterward, so long in fact as the animal was kept alive.

In the hands of other observers destruction of the angular gyrus of one side (Fig. 148) has led to hemiopia, failure in the left (or right) visual field, indicating failure in the central endings of the right (or left) optic tract, being caused by removal of the right (or left) gyrus, and destruction of both angular gyri has led to total blindness, not only the hemiopia, but the total blindness being, however, apparently transitory. And cases have been observed in which the transient blindness due to removal of the occipital lobes has been succeeded by permanent hemiopia upon the subsequent removal of the angular gyrus. Indeed, the general, but not uniform, tendency of the many experiments which have been made is to connect, in the monkey, both the occipital lobe and the angular gyrus with vision.

In the dog, removal of portions of the occipital cortex have also led to partial and transient blindness, or, according to some, to permanent blindness; but the difficulties of judging the visual condition of a dog are very considerable, and his vision is so different from that of a man, so much less binocular, for instance, than his, that it would not be profitable to relate at length the results obtained in the $\operatorname{dog}$ or to discuss the conclusions which have been derived from them. We will only say that some observers have been led to think that the lateral part of the retina is connected with the lateral part of the visual occipital area, the front part with the front part, and so on, the retina being, as it were, projected on to the occipital cortex ; but the facts are not clear enough to make it worth while to dwell upon them here.

In man, clinical histories so far conform to the results of experiments on the monkey as to associate the occipital cortex, and more particularly the cuneus (see Figs. 152 and 153), with vision. They have, however, raised a point on which we have not yet touched. In the experiments on the monkey quoted above, the result (putting aside transient effects due probably to "shock") of interference with one side of the brain was hemiopia; and this is what we might expect from the anatomical relations; the optic tract goes straight to the tegmental masses of its own side, and the optic radiation passes from those masses to the occipital cortex of the same side; there is no decussation, save of the fibres of the optic nerve, as they pass into the 
optic tract at the chiasma. Clinical histories teach the same lessons as these experiments on animals; lesions limited to the occipital lobe have for a symptom hemiopia; and this is said to be especially the result of mischief limited to the apex of the occipital lobe, that is, to the cuneus. But experiments on monkeys have been made in which destruction of one angular gyrus has produced, not hemiopia, but crossed blindness or crossed amblyopia, that is to say, has affected the whole of the retina of one eye, and that the crossed eye, the eye of the same side not being, or being supposed not to be, at all affected; similar results have also been stated to follow upon removal of one occipital lobe. And a few clinical cases have been recorded in which disease, especially of the angular gyrus, seemed to affect the vision of the whole of the crossed eye. (It must be remembered that the angular gyrus of man corresponds to a part only of the whole angular gyrus of the monkey. ( $C f$. Fig. 148 with Fig. 152.) Some authors have, in accordance with this, put forward the theory that the occipital lobe serves as a cortical centre for the optic tract of its own side only, and so for one-half of each retina, while in front of this on the angular gyrus is a centre in which both optic tracts are represented. But the clinical histories bearing on this point cannot be regarded as wholly satisfactory ; and with reference to the experimental results we may once more insist, and the warning applies perhaps with particular force to these experiments on vision, on the danger of confounding those immediate effects of operative interference, which are of the nature of "shock" in the wide sense of that word, with those pure "deficiency" phenomena which are alone the outcome of the loss of the part removed. It is difficult to resist the conclusion that much of the transitory blindness which is observed in these experiments belongs to the former category, that the effect is transient because it is of the nature of shock, and not because the loss of faculty is supplied by some other cortical area being subsequently substituted for the one removed. In the dog, injury to the frontal region of the cortex unaccompanied by any secondary mischief in the occipital region has led to impaired vision; and this was probably an instance of "shock," for we have no other reason to connect the frontal region of the cortex with vision. We must be very careful in drawing the conclusion that, because an operation produces transient blindness, the part operated on has a direct share in vision; and we may well hesitate to accept the view that the whole retina is represented in the crossed hemisphere.

In conclusion we may say that, when all the many results which have been arrived at by experiment or by clinical observation are duly weighed, it will be felt that while the evidence for the occipital lobe, especially the cuneus, being concerned in the matter is convincing, we cannot in the present state of our knowledge dogmatically exclude the angular gyrus, and that hence the only clear and consistent statement which can be made with any confidence is the broad and simple one that the hind region of the cortex is in some way intimately concerned in vision.

$\S 586$. Such an attitude becomes all the more necessary when we ask ourselves the question, What is it which actually takes place in the cortex during vision? Are we to conceive of it as if a visual impulse set going along the fibres of the optic tract underwent no essential change until it reached the cortex, as if it there suddenly developed into a "visual sensation?" We can hardly suppose this. Between the cortex and the optic tract, the lower visual centres, the tegmental masses, intervene; and we can hardly suppose that interference with these bodies produces the same effect on vision as simple section of the optic tract. We have seen in a previous section that the frog and the bird certainly, and according to some observers 
also the rabbit, are in the absence of the cerebral hemispheres not totally blind, their movements being guided by retinal impressions; and cases are recorded of the dog being obviously still guided in some measure by retinal impressions after the occipital lobes had been wholly or almost wholly removed. And, though this is a matter at present outside exact knowledge, and though it is perhaps possible for simple afferent impulses to determine even complex movements without the intervention of "consciousness," we are probably justified in assuming that the simple visual impulses, travelling along the fibres of the optic tract, undergo important transformations in the tegmental masses, and that the changes which are propagated along the fibres of the optic radiation constitute something quite different from the impulses along the optic tract or nerve.

Judging from the analogy of the motor region we may probably assume that in vision the cortical events are psychical in nature, and that the function of the optic radiation is to furnish what we may call crude visual sensations for further psychical elaboration.

Nor need this view compel us to suppose that injury to or removal of the cortex must produce only psychical blindness or psychical impairment of vision, though this point has probably not been sufficiently held in view during the various experiments, sufficient care not having been taken to determine how far the blindness was purely psychical. Bearing in mind the degeneration following upon lesions of the occipital cortex, and the farreaching effects of any operation on the brain, we may suppose that injury to the cortex affects the lower centres as well; and some of the transient impairment of vision, on which we have just dwelt, may, perhaps, be explained as the effect of the cortical injury on the lower centres.

Although the matter is thus in many of its details at present outside our exact knowledge, we may probably conclude that in the complex act of complete vision, while part, especially the more psychical part, is carried out in the cortex, more particularly of the occipital region, part is accomplished in the lower centres, the tegmental masses. As to the several functions of the three masses, we know almost absolutely nothing. Electric stimulation, and, it is said, mechanical stimulation also, of the anterior corpora quadrigemina in mammals, or the optic lobes in lower animals, calls forth movements of the eyes, and of various parts of the body; and removal of them causes blindness and in some cases loss of coördination of movements. Our knowledge on these points is not very exact; but from the above facts as well as from the connections of the anterior corpora quadrigemina with the parts of the brain behind we may suppose that these bodies are more especially concerned with the part visual impulses play in determining the coördination of movements. We must remember, however, that all three masses are connected with the cortex, and probably all three play a part in vision even of the highest psychical kind.

\section{Sensations of Smell.}

$\$ 587$. In many animals in whom the sense of smell is acute, a portion of the cortex, known as the "pyriform lobe" or "hippocampal lobule," and which is anatomically continuous with the front end of the hippocampal gyrus (the part to which the name uncinate gyrus is often restricted), acquires relatively large dimensions. This and the anatomical relations just mentioned would lead us to suppose that a part of the cortex which is continuous with the front end of the hippocampal gyrus is in some way connected with smell. The argument from comparative anatomy, however, is one which must be used with caution; since, besides the great difficulty of 
determining the homologies of parts of the brain in different animals, relative increase in the part in question might be correlated to other things than the power of smell, and might be determined by circumstances having no relation to smell.

The experimental evidence, though, on the whole, it gives support to the view, is conflicting; and when the difficulty of determining whether a "dumb animal" can or cannot smell is borne in mind, this will not be wondered at. The observation that electrical stimulation of the region in question gives rise to movements of the nostrils, which have been interpreted as sniffing in response to subjective olfactory sensations, cannot have much weight; and while some observers have found that the removal of this part of the brain destroys the sense of smell, others have obtained negative results.

The few clinical histories which bear upon the matter are, perhaps, more trustworthy. These seem to show that a lesion involving the cortex of this region, but leaving the olfactory bulb and tract, as well as other parts of the brain, intact, may destroy or greatly impair smell. And we may, perhaps, give particular weight to the cases in which epileptiform attacks, preceded by an "aura" in the form of a peculiar smell, have been associated with disease limited to this region; for the phenomena of "aura" seem to be connected with cortical processes.

Though the evidence on the whole goes to show that the cortex at the front end of the hippocampal gyrus is especially connected with smell, and we have so marked it (in Fig. 155), yet the whole matter stands on a somewhat different footing from the sense of sight. In man the relations of smell to the other operations of the brain (though, as we shall see in dealing with the senses, somewhat peculiar) are far more limited than are those of vision, and the psychical development of simple olfactory sensations is extremely scanty.

\section{Sensations of Taste.}

$\S 588$. This special sense, though so closely associated with smell, stands, together with the special sense of hearing, on a different footing from the two preceding special senses, since the nerves concerned belong to the category of ordinary cranial nerves, and we lack, in reference to them, the anatomical leading which is offered to us in the case of the optic and olfactory nerves.

We shall see in dealing with the senses that the fifth nerve and the glosso-pharyngeal nerve have been considered as nerves of taste, but that the matter is one subject to controversy; the gustatory function of the fifth is attributed to the peculiar chorda tympani nerve, and other questions have been raised. Whatever view we take, however, the nerves of taste are ordinary cranial nerves, and we have no anatomical guidance as to the fibres of either of the above two nerves making special connections with any part of the cortex. Though sensations of taste enter largely into the life of animals, and, indeed, of man himself, we have no satisfactory indications which will enable us to connect this special sense with any part of the cortex; the view, indeed, has been put forward that some part of the cortex in the lower portion of the temporal lobe, not far from the centre for smell, serves as a centre for taste; but the arguments in favor of this view are not, as yet at least, convincing.

\section{Sensations of Hearing.}

$\$ 589$. The cochlear division of the eighth or auditory nerve may be assumed to be a nerve of the special sense of hearing, and of that alone; 
the vestibular division serves, as we have seen, for other functions than those of hearing ( $\$ 555)$, but, as we shall urge in dealing with the senses, is not to be regarder as wholly useless for the purposes of that sense. The cochlear division we have traced ( $\$ 531)$ into the bulb, and the vestibular division into the lateral auditory nucleus (which, perhaps, may be regarded as a continuation or segmental repetition forward of the cuneate nucleus or of part of that nucleus), and into the cerebellum, the cerebellar continuation being probably the part of the nerve which serves for coördinating functions. The connections of the auditory nerve with the cerebral hemisphere belong to the same category as those of other afferent cranial, and we may add spinal, nerves; we have no very clear anatomical guide toward any particular part of the cortex.

When we turn to the empirical results furnished by experiment and clinical observations, we find that these, though even less definite and less accordant than in the case of the senses of sight and smell, point to part of the first or superior temporal (temporo-sphenoidal) convolution (Figs. 149, 152 , and 154) lying in the temporal lobe just ventral to the Sylvian fissure, as being especially concerned in hearing in some such way as the occipital lobe is concerned in vision.

Electrical stimulation of this region of the cortex gives rise to " pricking of the ears," and other movements such as are frequently connected with auditory sensations; but such phenomena are in this instance, perhaps, to be depended upon even less than in other similar instances. While some observers maintain that this convolution, the operation including other portions of the temporal lobe as well, may be removed from a monkey without producing any certain signs of deafness, other observers have found that removal of it on one side affected the hearing of the ear on the opposite side, and removal on both sides brought the animal into a condition in which, without being, perhaps, absolutely deaf, it reacted toward sound in a very imperfect manner indeed, very different from its normal behavior. The scanty clinical histories bearing on this matter are not very decisive; for though deafness has been observed in connection with disease affecting the superior temporal convolution, the lesion has usually invaded other parts as well, and the deafness has been associated with other symptoms, notably aphasia. An auditory "aura" has, however, at times been observed in connection with disease of this region, as also a peculiar psychical failure, known as "word-deafness," in which, though sounds are heard-that is to say, auditory sensations are felt, it may be even as usual-the perception or psychical appreciation of the sounds is lacking, and a spoken word is not recognized.

Lastly, we may add that though, as we said, the anatomical leading is not definite, observers have found that in newborn animals, on the one hand, destruction of the part of the cortex probably corresponding to the region mentioned above leads to atrophy of the median corpus geniculatum, and to some extent of the posterior corpus quadrigeminum; and, on the other hand, destruction of the interual ear leads to an atrophy of part of the lateral fillet of the opposite crossed side, which may be traced to the posterior corpus quadrigeminum; and thence to the median corpus geniculatum; and section of the lateral fillet on one side leads, among other results, to atrophy of the striæ acusticæ and tuberculum acusticum ( $\$ 531$ ) of the crossed side. This suggests that the path of auditory impulses is along the cochlear nerve to the lateral fillet of the crossed side, and so by the posterior corpus quadrigeminum and median corpus geniculatum to the cortex of the temporal lobe of that crossed side, the two latter bodies bearing toward hearing a relation somewhat like that borne toward sight by the anterior 
corpus quadrigeminum and lateral corpus geniculatum. But the matter needs further investigation.

There remains the special sense of touch, but this we had better consider in connection with sensations in general.

\section{On the Development of Cutaneous and some other Sensations.}

$\S 590$. The sensations with which we have just dealt arise through impulses passing along special nerves or parts of special nerves, the optic nerve, the olfactory nerve, etc. We have now to deal with sensations arising through impulses along the nerves of the body generally. These are of several kinds. In the first place, there are sensations which we may speak of as "cutaneous sensations," the impulses giving rise to which are started in the skin covering the body, or in the so-called mucous membrane lining certain passages. These sensations, which, as we shall see in dealing with the senses, are dependent on the existence of special terminal organs in or near the skin, are sensations of "touch," in the narrow meaning of that word, by which we appreciate contact with and pressure on the skin, and the sensations of "temperature," which again we may, as we shall see, divide into sensations of "heat" and sensations of "cold." These sensations may be excited in varying degree by impulses passing along any nerve branches of which are supplied to the skin. Then there are the sensations constituting the "muscular sense," to which we have already referred, and these again may be excited in any nerve having connections with the skeletal muscles.

As we shall see in dealing with the senses, when a nerve is laid bare and its fibres are stimulated directly either by pressure, such as pinching, or by heat, or by cold, or in other ways, the sensations which are caused do not enable us to appreciate whether the stimulation is one of contact or pressure, or of temperature, or of some other kind ; we only experience a "feeling," which at all events when it reaches a certain intensity we speak of as "pain." And we have reason to think that at least from time to time impulses along various nerves gives rise to sensations which have been spoken of as those of "general sensibility," by which in addition to other sensations, such as those of touch and of the muscular sense, we become aware of changes in the condition and circumstances of our body. When the stimulation of the skin exceeds a certain limit of intensity, the sense of touch or temperature is lost in, that is to say, is not appreciated as separate from, the sense of pain; and under abnormal circumstances acute sensations of pain are started by changes in parts, for example tendons, the condition of which under normal circumstances we are not conscious of appreciating through any distinct sensation, though it may be that these parts do normally give rise to feeble impulses contributing to "general sensibility." It may therefore be debated whether "pain" is a phase of all sensations, or of general sensibility alone, or a sensation sui generis. We shall have something further to say on this matter when we treat of the senses; meanwhile it will be convenient for present purposes if we consider that the sensations we have to deal with just now are the sensations of touch and of temperature, those of the muscular sense, and those of general sensibility, including those of pain.

$\$ 591$. The fairly convincing evidence that the occipital cortex has special relations with vision, and the less clear evidence that other regions have special relations with smell and hearing, suggest that special parts of the cortex have special relations with the sensations now under consideration. But in the cases of the senses of sight and smell we had a distinct anatomical leading; and we have seen how uncertain is the evidence where such an 
anatomical leading fails, as in hearing and taste. In the case of sensations of the body at large, the auatomical leading similarly fails. Moreover, any attempt to push the aualogy of sight raises the following question: If there were two optic nerves on each side of the head, would there be two cortical areas, one for each nerve, in each hemisphere, or one visual area only? And again, if the optic nerve were the instrument for some sense in addition to that of sight, would there be two cortical areas, one for each sensation, or one area only serving as the cortical station, so to speak, of the whole nerve? If we push the analogy of sight it is open for us, since we cannot give a definite answer to the above question, to suppose either that there is one area for touch, another area for temperature, and so on, each for the whole body, or that there is an area for sensations of all kinds for each afferent nerve, or, that there is an intricate arrangement which supplies all the combinations of the two which are required for the life of the individual. Of the three hypotheses the latter is the more probable; but if so, it is by its very nature almost insusceptible of experimental proof, especially when we bear in mind what we have already said touching the difficulty of judging the sensations of animals. If the judgment of visual sensations is difficult, how much more difficult must be the judgment of sensations of touch and temperature? Indeed, sensations of pain are the only sensations of which we can form a quantitative judgment in animals; and our method of judging even these, namely, by studying the movements or other effects indirectly produced, is a most imperfect one.

We can learn, therefore, almost absolutely nothing in this matter from experimental stimulation of the cortex in animals. As we have previously ( $(584)$ urged, the absence of movements when parts of the cortex other than the motor regions are stimulated is no evidence that the stimulation does not give rise to psychical events into which sensations enter; and movements follow stimulation of the motor area, not because that area is wholly given up to motor events, but because from the histological arrangement the stimulus gets ready access to relatively simple motor mechanisms. That the motor region has close connections with sensory factors is not only almost certaiu on theoretical grounds, but is shown in many ways, for example by the experiment, described in $\S 574$, of exalting the sensitiveness of a motor area by generating peripheral sensory impulses.

Nor can the effects on sensation of removal of parts of the cortex be interpreted with clearness and certainty. In the monkey removal or destruction of the gyrus fornicatus (Figs. 148 and 150) on the mesial surface of the brain, ventral to the calloso-marginal sulcus which forms on the mesial surface the ventral limit of the motor region (an operation of very great difficulty), has brought the whole of the opposite side of the body to a condition which has been described as an anæsthesia, that is, a loss of all cutaneous tactile sensations, and an analgesia, that is, a loss of sensations of pain, the condition being accompanied by little or no impairment of voluntary movements and, though apparently diminishing as time went on, lasting until the death of the animal some weeks afterward. Again, removal of the continuation of the gyrus fornicatus into the gryus hippocampi has, in other instances, led to a more transient anæsthesia also of the whole or greater part of one side of the body. And it is asserted that removal of no other region of the cortex interferes with cutaneous and painful sensations in so striking and lasting a manner as does the removal of parts or of the whole of this mesial region.

These results, however, do not accord with clinical experience which, though scanty, seems, as far as it goes, to show that in man, when mischief apparently limited to the cortex produces loss of sensation, it is the parietal 
lobe corresponding to the motor region which is affected; but there appears to be no record of any case of a cortical lesion affecting sensation without affecting movement. We have previously called attention to the fact that the temporary loss or impairment of movement which follows removal of an area is frequently, if not always, accompanied by an impairment of cutaneous sensations in the limb or part "paralyzed;" and side by side with this we may put the experience that in the human epileptiform attacks of cortical origin, the seizure is at times ushered in by peculiar sensations, called the "aura," in the part movements of which inaugurate the march of convulsive movements. But these things do not show that the cortical area is the "seat of sensations;" they rather illustrate what we said concerning the complexity of the chain of which the events in the cortical area are links, and the close tie between sensory factors and the characteristic elements of the motor region.

In the dog, while removal of almost any considerable portion of the cortex affects sensation, removal of parts of the frontal region producing, perhaps, less effect than removal of parts in other regions, the loss or impairment of sensation appears to be transient, though having a duration broadly proportionate to the extent of cortex removed; and when a very large portion of the cortex is removed, some imperfection appears to remain to the end. We have already referred to the case of a dog from which the greater part of both cerebral hemispheres had been removed, but which remained capable of carrying out most of the ordinary bodily movements, and that apparently in a voluntary manner; in this case the "blunting" of cutaneous sensations was perhaps more striking than the imperfection of movement. It will be worth while to consider the condition of this dog a little closely, on account of the light which it throws on the problem which we are now discussing.

Clinical experience shows that in man the integrity of the cerebral hemispheres, and of the connection of the hemispheres with the rest of the central nervous system, is essential to the full development of sensatious ; and that in this respect, each hemisphere is related to the crossed side of the body. A very common form of paralysis or "stroke" is that due to a lesion of some part of one hemisphere (the exact position of the lesion need not concern us now), frequently caused by rupture of a bloodvessel, in which the patient loses all power of voluntary movement and all sensations on the crossed side of his body (including the face); he is said to be suffering from hemiplegia, "one-sided stroke." Not only do voluntary impulses fail to reach the muscles of the affected side, but sensory impulses, such as those which started, for instance, in the skin, would, under normal conditions, lead to sensations of touch, of heat or cold, or of pain, fail to affect consciousness, when they originate on the affected side; the patient cannot on that side feel a rough surface, or a hot body, or the prick of a pin. For the sake of clearness we suppose the loss of movement and sensation to be complete, but it might, of course, be partial. Such a case shows, we repeat, that the integrity of the cerebral hemisphere, and of the connection of that hemisphere, we may say of the cortex of that hemisphere, with the other parts of the nervous system, is essential to the development of the sensations; but it does not prove that the cortex of the hemisphere is the "seat" of the sensations, it does not prove that the afferent and sensory impulses started in the skin undergo no material change until they reach the cortex and are then suddenly converted into sensations ; it only proves that in the complex chain of events by which sensory impulses give rise to full conscious sensations the events in the cortex furnish an indispensable link. And the phenomena of the dog in question, on the one hand, illustrate how complex the chain is, and, on the other hand, 
sugrgest that the completeness of the loss of sensation in the hemiplegic man is not a pure "deficiency" phenomenon, but is due to the lesion affecting the chain of events in some way or other besides merely removing the link furnished by means of the cortex. For, as we previously urged, the dog in question, however curtailed its psychical life may have been, seemed to a casual observer to feel and move much as usual. Neglecting visual and auditory sensations with which we are not now dealing, it needed careful observation to ascertain that some of the animal's movements fell short, the failure being apparently due to the lack of adequately energetic coördinating sensory impulses; a stronger stimulus than usual had to be applied to the skin in order to call forth the usual movements and other tokens that the stimulus was "felt." As we have before urged, it is impossible to suppose that the mere stump of cerebrum left in this case could have taken on all the functions of the lost hemispheres; and naking, as we have previously done, full allowance for the differentiation between man and dog, we must conclude that in the more general sensations with which we are now dealing, as with the more special visual sensations, the full development of a complete sensation is a complex act of more stages than one between the afferent impulse along the afferent nerve and the affection of consciousness which we subjectively recognize as "the sensation ;" the cortical events are only some among several. It follows that any analogy between the cortical events which play their part in a sensation and the cortical events which immediately precede the issue of impulses from the motor region along the fibres of the pyramidal tract is misleading; the highly differentiated motor localization does not justify us in concluding that there exists a similar topographical distribution of sensation.

$\S 592$. We may now attack the problem in a different way, and instead of beginning with the cortex begin with afferent impulses started along afferent nerves from their peripheral endings, and attempt to trace them centralward. And first we may call to mind what anatomical guidance we possess $(\S 482)$.

The fibres of posterior roots, the channels of afferent impulses, end in the spinal cord in at least two main ways. One set is continued on, not broken by any relays, as the median posterior tract, and by this tract representatives of all the spinal nerves are connected with the gracile nucleus in which ( $\$ 523)$ the median posterior column ends. The other fibres of a posterior root appear to end in the gray matter not far from their entrance; but from the gray matter there starts the cerebellar tract, which, though not conclusively proved to be, may be assumed to be, an efferent tract. We may therefore probably suppose that afferent impulses along certain of the fibres of the postcrior root make their way upward along the cerebellar tract, and there are some reasons for regarding the vesicular cylinder and the cells which represent this where it is not conspicuous in the regions of the cord, as a relay between the two systems of fibres. There are also the more scattered fibres of the ascending antero-lateral tract ( $\$ 480)$, which probably is also an afferent tract, and therefore probably also connected with the posterior roots; but, as we have seen, our knowledge of this tract is imperfect, though, if, as some urge, it ends in the restiform body, we may perhaps consider it as similar at least to the cerebellar tract, and treat the two as one.

Thus there seem to be at least two main recognized paths, in the form of tracts of fibres, for afferent impulses along the cord; une along the median posterior column, the other along the lateral column in the cerebellar tract. The latter passes straight up to the cerebellum by the restiform body, travelling along the same side of the cord, and any crossing of impulses passing along this tract must take place before they enter the tract; we have, how- 
ever, no anatomical guidance for such a crossing. The other path, along the median posterior tract, comes to end in the gracile nucleus; it has, indeed, been urged that the gracile nucleus is thus connected chiefly with the lower limbs and lower part of the body, and that the analogous posterior root fibres from the upper limbs and neck pass similarly into the cuneate nucleus, or at least into the median division of that nucleus, but this cannot be considered as proved. Moreover, both the posterior columns, median and external, bring to these nuclei fibres which have started from some relay in the gray matter lower down, and which are not fibres coming straight without any relay from the posterior roots; these, however, we cannot distinguish from each other in their course beyond the nuclei. From the gracile and cuneate nuclei the path onward is a double one, one broad, one narrow. The broad path, the one having most fibres and presumably carrying most impulses, leads to the cerebellum by the restiform body; and here the path, previously continued exclusively along the same side of the cord, becomes partly crossed though remaining partly uncrossed, the sensory decussation in the bulb being the crossed and the other fibres passing from the nuclei straight to the restiform body being the uncrossed one $(\$ 525)$; the uncrossed one we may, perhaps, look upon as really an upper part of the cerebellar tract. The narrow path is the fillet ( $\$ 547)$, by which some of the fibres from the nuclei are continued on toward the cerebrum. This path is a crossed one, the crossing taking place in the sensory decussation, and it carries relatively few impulses, the chief increase in the size of the fillet as it passes onward being due to fibres coming from structures other than the gracile and cuneate nuclei.

Hence of the sensory impulses travelling along continuous tracts in the spinal cord, these tracts apparently keeping always to the same side, the great majority pass to the cerebellum; and of these again the greater number, all those along the cerebellar tract, and some of those passing through the gracile and cuneate nuclei, remain uncrossed to the end. The only path by which all these impulses thus passing to the cerebellum can gain access to the cortex of the cerebrum, is by some or other of the ties between the cerebellum and the cerebral cortex. The relatively few impulses which pass along the fillet are for the most part carried to the middle parts of the brain, for only a small portion of the fillet passes to the cortex ( $\$ 547)$, and it is not clear that this part of the fillet comes from the gracile and cuneate nuclei, so that most of these impulses can gain access to the cortex only by the relays of these middle parts of the brain.

Very striking, indeed, are these constant relays along the path of sensory impulses; in this respect the sensory impulses offer a strong contrast to the motor impulses. But a still more complex system of relays has to be mentioned; for yet a third path is open for seusory afferent impulses along the cord. We must admit the possibility of afferent impulses travelling along the network of the gray matter, their path being either absolutely confined to the gray matter, or leaving the gray matter at intervals, and joining it again by means of those longer or shorter commissural or internuncial fibres which unite the longitudinal segments of gray matter, and form no inconsiderable portion of the whole white matter of the cord. We have seen ( $\$ 499)$ that under abnormal circumstances impulses pass freely in all directions along the gray matter, and we may conclude that under normal circumstances they can pass along it under restrictions and along lines determined by physiological conditions. The fibres in the white matter which do not show either descending or ascending degeneration are probably, as we have said ( $\$ 494)$, internuncial fibres, connecting segments of gray matter in a longitudinal direction; and, though we have no exact knowlenge touch- 
ing this matter, we may suppose that some of these convey impulses upward, and others downward.

If, as some maintain, the fibres of the ascending antero-lateral tract end not in the cerebellum, but in the gray matter of the bulb, or higher up, we have a fourth path for sensory impulses, which, after the primary relay in the segmental gray matter, pass straight up to the bulb.

$\S 593$. How do experimental results and clinical histories accord with such an anatomical programme?

We may first call attention to an experiment, which, though somewhat old, carried out on rabbits, and confiner to one region only of the cord, the lower thoracic, has nevertheless a certain value on account of its affording more or less distinctly quantitative and measurable results. We have seen ( $\$ 161)$ that afferent impulses started in afferent fibres, in those, for instance, of the sciatic nerve, so affect the vasomotor centre in the bulb as to cause a rise of blood-pressure, at least in an animal under urari. Those afferent impulses must pass by some patl or other from the roots which supply the sciatic nerves with afferent fibres along the thoracic and cervical cord to the bulb. If the path be blocked, the stimulation of the sciatic nerve will fail to produce the usual rise of blood-pressure. Now in a rabbit the amount of rise of blood-pressure following upon the stimulation of one sciatic nerve with a certain strength of current having been ascertained, it is found that a much less rise of blood-pressure or none at all follows the same stimulation after division of certain parts of the cord in the mid or upper thoracic region; that is to say, the section of the cord has partially or completely blocked the path of the afferent impulses. Further, the block is conspicuous when the lateral column is divided, and is not increased by other parts of the cord being divided at the same time; when both lateral columns are divided, the block is almost complete. And further, supposing one sciatic, say the right, is the one which is stimulated, a block occurs both when the lateral column of the same, right, side and when that of the crossed, left, side is divided, but is greater when the division is on the crossed than when it is on the same side. We may infer that the impulses, which reach the lumbar cord by the roots of the sciatic nerve, travel up the cord, or give rise within the lumbar cord to events which we may compare to nervous impulses, and which travel up the cord in such a manner that in the lower thoracic region they pass almost exclusively along the fibres of the lateral column, some having kept to the same side of the cord, but more having crossed over to the opposite side before reaching the thoracic region.

This result was obtained in rabbits, and the experiment was carried out in the lower thoracic region only; the conclusions to be drawn from it hold good for that animal only, and for that part only of its cord. Moreover, the experiment only tests the path of such impulses as reach and affect the vasomotor centre in the bulb. It is, however, exceedingly probable that the impulses which, generated in sensory nerves, affect the vasomotor centre are impulses which, in the conscious animal, give rise to sensations of pain ; in an intact animal changes in the vasomotor centre occasioned by the stimulation of sensory nerves are accompanied by signs of more or less pain. And indeed this is confirmed by the fact that similar results were obtained when, the experiment being conducted in a similar way, signs of pain instead of variations in blood-pressure were taken as the tokens of the blocking of impulses. Hence, assuming this, we may regard the experiment as indicating that the impulses which form the basis of painful sensations pass by the lateral columns in the lower thoracic region of the cord of the rabbit, and therefore, though this is a further assumption, by the same columns along the whole length of the cord. We further may infer that while some of the 
impulses keep to the same side of the cord, others, and indeed the greater number, cross to the opposite side.

These conclusions entail assumptions, but the main interpretation of the whole experiment entails a still greater assumption. The testing of the influence of the sciatic stimulation was carried out soon after the section of the cord, and yet we have assumed that the block of the impulses was due to a pure deficiency phenomenon, the absence of a usual path. But we have no right to do this. It is possible that the section produced, in some way or other, a depressing or inhibitory effect lower down in the cord, affecting struetures other than the lateral columns; all our experience, indeed, of the effects of 'operations on the cord would lead us to expect this. It is further possible that a section of the lateral column might produce this depressing effect, while sections of other parts did not, or might produce more effect than the former. It is possible, for instance, that the section of the thoracic lateral eolumn inhibited, for the period during which the experiment was carried out, the gray matter of the lumbar cord, and that the block really took place in this gray matter. Until the uncertainties thus attending the interpretation are removed the experiment is not valid as a proof that the lateral columns are the paths of afferent impulses; it would, however, still serve to indicate that the afferent impulses reaching the cord along the sciatic nerve crossed over to a large extent before they came under the influence of the inhibition, since we have no evidence to show that such an inhibitory action of the section would be exerted chiefly on the crossed side.

Again, we have seen that the afferent impulses affecting the vasomotor centre gain access to that centre without the help of the parts of the brain above the bulb; the existence of the vasomotor centre was made out ( $\$ 162)$, by combining stimulation of a sciatic nerve with a series of operations consisting in making successive transverse sections of the bulb from above downward; and it was not until the sections reached the vasomotor centre that the blood-pressure effects of the sciatic stimulation were modified. Hence, if the experiment be taken as showing that not only afferent impulses affecting the vasomotor centre, but other afferent impulses also travel by the lateral columns, it would also seem to show that these other impulses pass in like manner to the bulb, and gain access to the cortex through the bulb. This increases a difficulty which presents itself even when the afferent impulses affecting the vasomotor centre are alone considered. If the experiment means anything, it means that the impulses having in some way or other reached the lateral column, travel up that column by some continuous path, and indeed is generally taken as having that meaning. But if we put aside the very doubtful view that the ascending antero-lateral tract ends in the bulb, there is no continuous afferent tract in the lateral column ending in the bulb; the only definite continuous afferent traet in the lateral column of which we have any clear knowledge, namely, the cerebellar tract, ends not in the bulb but in the cerebellum. And if we attempt to get out of the difficulty by supposing that those impulses at least which affeet the vasomotor centre, after travelling for some distance in the cerebellar traet, leave that tract for some path leading to the bulb (and the cerebellar tract does probably give off as well as receive fibres along its course), we practically admit that the experiment does not prove the existence of a continuous path.

A further difficulty is raised by the fact that, according to the interpretation which we are discussing, the section of the lateral column breaks the paths of what we may consider two kinds of impulses: those, the larger number, which have already crossed from one side of the cord to the other, 
and those which have remained on the same side. For, as we have already said, we have evidence, in man at least and some other animals, that afferent impulses cross completely over somewhere or other on their path before they are developed into full sensations; and we have also evidence, though less strong, that they cross not long after their entrance into the cord. But, if we suppose this to be the case in the rabbit also, it follows that in the experiment in question the impulses which were blocked on their passage along the lateral column of the same side, whatever the way by which they reached that lateral column, were pursuing a path which would eventually have led them to the other side of the cord. Hence, the section of the lateral column, in breaking their path, broke not a continuous path keeping to the lateral column up the length of the cord, but a path which soon left the lateral column to pass elsewhere. The experiment, therefore, as far as the impulses passing up the same side are concerned, does not prove that they pursue a continuous path along the lateral column; and if so, what becomes of the validity of the experiment as regards the impulses crossing over from the other side? for the experiment in itself makes no distinction between the two.

We may add, however, that though the point has not been specially investigated, it is possible that in the rabbit, in whose hind limbs bilateral movements are so predominant, there is associated with the movements a bilateral arrangement for sensations, and that those impulses which remain along the same side of the cord as the nerve in which they originate, are carried up to the brain without any crossing at all.

$\S 594$. The results of this vasomotor experiment then, though they are frequently quoted, do not when closely considered afford adequate proof that afferent impulses pursue a continuous path along the lateral columns of the cord, and, moreover, the facts brought to light by the experiment show but little accord with the anatomical programme. We have dwelt on it so long because it is more or less illustrative of the many difficulties attending the interpretation of experiments of this kind; and it is in this respect all the more valuable because the actual experimental results are sharp and clear. IVe may pass over more rapidly the numerous experiments on the lower mammals, such as rabbits and dogs, in which other indications of sensation have been made use of, chiefly those which are the signs of painful sensations; these have been carried out in various regions of the cord, but chiefly in the thoracic region, and in them a like uncertainty of interpretation is further increased by the want of exactness and agreement in the results.

If we content ourselves with making no distinction between the different kinds of afferent impulses, and in the case of these animals it would hardly be profitable to attempt to make a distinction, we may say that the several experiments so far agree that they point to the lateral columus as being the chief paths of afferent, sensory impulses, or to speak more exactly, to the passage of these impulses being especially blocked by section of the lateral columns. Some observers find that in the dog and other lower mammals a section of the lateral column on one side, or at least a hemisection of the cord, produces "loss of sensation" on the opposite side greater than on the same side, or confined to the opposite side and even accompanied by an exaltation of sensation, a hyperesthesia, on the same side. Other observers again, and these certainly competent observers, find that, in the dog, section of one side affects sensations on both sides, and, indeed, chiefly on the same side. We may perhaps once more repeat the warning of the difficulty attending the quantitative and qualitative determination of sensations in such an animal as the dog; and may remark that in all these cases of unilateral section the increased blood-supply due to failure of the normal vaso-con- 
strictor tone must influence the peripheral development of sensory impulses.

In these experiments, as in those on voluntary movements, it is most important to distinguish between immediate or temporary and more lasting effects; and observers have found that the loss of sensation following a hemisection of the cord, like the loss of voluntary movement, is temporary only, and eventually disappears, though the recovery is slower and less complete than is the case with movements. As with voluntary movement ( $\$ 576)$ so with sensation, recovery, though less complete than that of movement, is possible when a hemisection on one side has been at a later date followed by a hemisection on the other side. We may, therefore, repeat in reference to sensations the remarks which we made in reference to movement. There is, however, an important difference between the two cases : in respect to movement we have evidence that under normal conditions the pyramidal tract plays an important part and that any other path for volitional impulses is more or less an alternative one, whereas in respect to sensation we have no anatomical or other distinct proof of any such normal path.

The experiments on monkeys are in like manner neither accordant nor decisive; and even in these animals with their more varied signs of sensations, the interpretation of these signs is beset with fallacies. Some observers have found that a hemisection (in the thoracic region) produced loss of sensation on the crossed side, accompanied by little or no loss on the same side; other observers again have failed to obtain after a hemisection satisfactory proof of any such marked loss on the crossed side. Further, large portions of the lateral column, the more internal parts adjacent to the gray matter being left, have been removed without any very obvious and certainly without any lasting defects of sensation on the one side or on the other.

$\$ 595$. The clinical histories of diseases of the spinal cord in man bring to light in a fairly clear manner a fact of some importance, namely, that the several impulses which form the bases of the several kinds of sensations, of touch, heat, cold, and pain, and of the muscular sense, are transmitted along the cord in different ways and presumably by different structures, for disease may impair one of these sensations and leave the others intact. Thus cases of spinal disease are recorded, in which on one side of the body or in one limb ordinary tactile sensations seemed to be little impaired, and yet sensations of pain were absent; when a needle was thrust into the skin no pain was felt, though the patient was aware that the needle had been pressed upon the skin at a particular spot; and conversely, in other cases pain has been felt upon the insertion of a needle, though mere contact with or pressure on the skin could not be appreciated. Again, cases are recorded in which the skin was sensitive to touch or pain, but not to variations of temperature; it is further stated that cases have been met with in which cold could be appreciated, but not heat, and vice versa; and there are some facts which point to sensations of pain being more closely associated with those of heat, and tactile sensations with those of cold, than those of pain with those of touch or those of heat with those of cold. Cases of spinal disease are also recorded in which the muscular sense appeared to be affected apart from other sensations. We shall return to these matters later on in dealing with the senses; we refer to them now simply as showing that disease, limited as far as can be ascertained to the spinal cord, may affect the several sensations separately, and therefore as suggesting that the several kinds of impulses, forming the bases of the several kinds of sensations, are transmitted in different ways and follow different "paths" along the spinal cord. 
Clinical histories, moreover, agree, at least to a large extent, in showing that when the lesion is confined to one-half of the cord, the sensations affected in the parts below the level of the lesion are chiefly or even exclusively those of the crossed side. But there is not entire accordance, especially as to the crossing being complete. And with regard to the muscular sense there is a distinct conflict of opinion; the majority of cases seem to show that in unilateral disease or injury to the cord, the muscular sense in company with the voluntary movements fails on the sanne side; but cases have been recorded in which the muscular sense in company with other sensations seemed to be affected on the crossed side; it must be remembered, however, that it is very difficult to appreciate a deficiency of muscular sense mingled with deficiencies in other sensations, and we should à priori expect the muscular sense to run parallel with motor impulses.

When, however, we appeal to clinical histories or indications as to the several paths within the spinal cord taken by these several impulses, the answer is a most uncertain one, as indeed might be expected from the too often diffuse character of the lesions of disease; and it is perhaps not too much to say that no satisfactory deductions at all can be made.

$\S 596$. Whether, then, we turn to experiments on animals or to the study of disease, the teachings with regard to sensation, in contrast to those with legard to voluntary movement, are in the highest degree uncertain and obscure. A few general reflections will perhaps help us to appreciate the value of such facts as we possess.

We have seen reason to think that in every movement, whether voluntary and of cortical origin, or involuntary and started either as a simple spinal reflex or through the working of some part or other of the brain, the motor impulses, which sweep down the motor fibres to the muscles, issue marshalled and coördinated from the gray matter of the cord (for the sake of clearness we may omit the cranial nerves), from what we have called the motor mechanisms of the cord. Analogy would lead us to suppose that the afferent impulses, forming the bases of the sereral kinds of sensations, similarly left the afferent fibres to join the gray matter of the cord in what we may call the sensory mechanism. And such anatomical leading as we possess seems to support this view; with the exception of the median posterior tract, to which we will return immediately, all the fibres of a posterior root seem to end in the gray matter not very far from the entrance of the root. We have seen that a coördinate reflex movement may be carried out by at least a few segments of the cord; that a reflex movement may be started by stimuli of various kinds and therefore presumably by afferent impulses of various kinds; and that impulses forming the basis of the muscular sense are essential to the coördination of the movement. All our knowledge goes to show that in reflex movement carried out by a few segments of the cord, the whole chain of events between the arrival of the afferent impulses along the posterior root and the issue of efferent impulses along the anterior root may be carried out by gray matter, and gray matter alone. We may further infer that, while on the one hand the same procedure might obtain not through a few segments only but along the whole length of the cord, there would be an advantage, especially in respect to the rapidity of transmission, in employing internuncial tracts of fibres between the several segments, the advantage being greater the more distant the segments which have to work together.

We might further suppose that it would be of advantage to possess some direct path between the cerebral cortex and the spinal sensory mechanism immediately connected with the posterior root, such as is afforded by the pyramidal tract between the cortex and the spinal motor mechanism 
immediately connected with the anterior root. But no anatomical evidence of such a tract is fortheoming; and, as we have before remarked, along all the tracts which seem to be sensory in nature, in contrast to what takes place in the motor tracts, relays of gray matter are continually being interpolated.

The median posterior tract, since it gathers up representatives of successive nerves, presents itself as the nearest approach to such a sensory homologue of the pyramidal tract, though it ends in the bulb and is not continued directly on to the cortex. And possibly it does play a somewhat analogous part, in so far as it serves as a special connection between the brain and the whole series of spinal nerves. But we are wholly ignorant as to what it really does; and whatever be the exact nature of the part which it plays, it probably has relations not to one kind of sensation only, but to all the different kinds of sensation. It has indeed been supposed by some to be especially a tract for the impulses of the muscular sense; but neither experiment nor clinical study affords adequate proof of this view. The condition known as locomotor ataxia, the salient feature of which is loss or impairment of muscular sense, is associated with disease of the posterior root and of its entrance into the cord, not with disease confined exclusively to the median posterior column. Moreover, the tract cannot carry all the impulses of muscular sense, since some of them must pass at once into the gray matter to take part in the coördination of reflex movements, and must therefore travel by fibres which do not form this tract. Similarly there is no adequate proof of the tract being an exclusive channel for tactile or for painful sensations.

We may also, perhaps, urge similar considerations with regard to the cerebellar tract, which, though starting from a relay of gray matter, is thence onward to the cerebellum a continuous tract. This tract also has been supposed to carry impulses of a particular kind, and more particularly those of muscular sense. There is less à priori objection to this view, since the tract starts from the gray matter, where the impulses of muscular sense may have already done their, so to speak, local work, and ends in the cerebellum, which, as we have seen, seems especially connected with the coördination of movements. But with respect to this tract also neither experiment nor clinical study affords any clear and decisive proof that it is solely or even especially concerned with the muscular sense.

With regard to the antero-lateral ascending tract our knowledge is too imperfect to justify us in supposing that it is the special or exclusive channel for any one kind of sensation, or indeed in drawing any conclusions at all concerning it.

But when we subtract from the white matter of the cord these continuous tracts of ascending degeneration of presumably sensory or afferent function, and the continuous tracts of descending degeneration, which we may confidently speak of as motor or at least efferent, there are left only the fibres which we have $(\$ 494)$ supposed to be longitudinal commissural or internuncial fibres between successive segments. We are thus driven back to our former conclusion, that sensory impulses pass either by the gray matter alone, or by a series of steps as it were, by relays of gray matter connected by internuncial tracts of fibres, whose length we cannot ascertain, but which may be short. That such internuncial tracts intervene is rendered probable on the one hand by the fact that section of the white matter, leaving the gray matter untouched, does affect sensations, and on the other hand by the fact that the several kinds of sensation appear to travel along the cord by separate paths, or at least may be separately blocked. It is of course, as we have already urged, possible that the effect of a section of a tract of fibres 
may not be the mere block due to loss of continuity, but some action on the gray matter with which the fibres are connected, whereby that gray matter fails of its usual functions and ceases to carry onward the sensory impulses reaching it from below ; it is also possible that this or that lesion of disease may, directly or indirectly, affect particular parts of the gray matter or affect the gray matter in a particular way, so that a certain kind of sensory impulse and none other is blocked. On the other hand, we have reason to think that the rate at which impulses travel along the gray matter is very slow compared with that along nerve-fibres; and in the struggle for life rapidity of transmission of nervous impulses is of great importance. Hence the view that the internuncial fibres intervene has more to commend it ; it is, moreover, to a certain extent supported by clinical histories. But, if we accept this view, we must at the same time admit that, in aninals at least, the lines provided by the internuncial tracts are not rigid - that within limits and under circumstances alternative routes are possible.

$\$ 597$. We may here, perhaps, raise once more, and this time more pointedly than before, the doubt whether we are justified in assuming, as we generally do assume, that the events which take place in the fibres connecting relays of gray matter within the central nervous system are exactly the same as those which take place in the fibres of nerves outside the central system during the passage of what we call a nervous impulse. Most of our knowledge of a nervous impulse has been gained by the study of the motor nerve of a muscle-nerve preparation. Our knowledge of the processes in afferent nerves is much more imperfect ; but there are many facts which at least suggest that the molecular events constituting an afferent impulse along an afferent nerve are different from, and probably more complicated than, those constituting an efferent impulse along an efferent nerve. And, with regard to the processes taking place in fibres within the central nervous system, we have hardly any exact experimental knowlege at all. It has been maintained by many observers that not only the gray matter, but also the tracts of white matter in the spinal cord, while they are capable of conveying impulses in one direction or the other, are incapable of being so excited by artificial stimuli as to generate new impulses. These observers maintain that when movements or signs of sensation follow the direct stimulation of various parts of the cord, the effects are due to issuing motor fibres or entering sensory fibres having been stimulated, and not to a stimulation of the intrinsic substance of the parts themselves; they propose accordingly to call these parts "kinesodic" and "xsthesodic" respectively, that is to say, serving as paths for motor or sensory impulses without being themselves either motor or sensory. The evidence on the whole goes to show that this view is a mistaken one; that the various tracts of the spinal cord, like the pyramidal tract, and indeed other parts of the brain, are excitable toward artificial stimuli. The question cannot, however, be considered as definitely closed; and the very fact that it has been raised illustrates the point on which we are now dwelling. We may further quote, in similar illustration of the same point, the following remarkable fact which was observed in the series of experiments referred to in $\$ 576$ on the effects of repeated hemisection of the spinal cord in dogs: The animal had partially recovered voluntary movements in his hind limbs after a third hemisection of the thoracic cord, and yet when, at his death, a strong tetanizing current was directed through the bulb and cervical cord, no movements of the hind limbs followed; the impulses started by artificial stimulation could not pass the bridge which sufficed for volitional impulses of natural origin. It is not too much to say that our experimental knowledge as to the events which accompany the activity of the structures within the central nervous system is almost entirely 
limited to the recognition of the "currents of action" referred to in $\$ 570$. WV are already going beyond our tether when we assume on the strength of this that the processes started in the fibres of the pyramidal tract by artificial stimulation are in all respects identical with those started in the fibres of a motor nerve. We are going still more beyond our tether when we assume that the processes starter in the same pyramidal fibres as the outcome of natural events in the motor cortex are of the same kind. But these assumptions are trifles compared with the assumption that the events taking place in the fibres of the optic radiation passing from the pulvinar to the occipital cortex are identical with the events taking place in the fibres of the optic tract on the way to the pulvinar, or that the events travelling along the spinal cord to the brain as the result of a prick of the little finger are identical with those which the prick has started in the fibres of the ulnar nerve. Of the lalter events we know a little; of the former events we know next to nothing. And we may here ask the question, What is the meaning of these continual relays of gray matter along the sensory tract, unless it be that at each relay some transformation, some further elaboration of the impulses take place, until what were the relatively, but only relatively, simple impulses along the fibres of the peripheral nerve are by successive steps changed in the complex events which we call a conscious sensation? This is what we had in mind when we gave $(\$ 565)$ a note of warning concerning the danger of considering all the events in the central nervous system as either motor or sensory in nature. It is perhaps not an exaggeration to represent the views of some observers as if they supposed that afferent impulses, say tactile impulses, that is, impulses eventually giving rise to tactile sensations, travelled unchanged from the skin to the cortex, and there suddenly blossomed into sensations. If such a view were true, undoubtedly the chief task of physiology, almost the only one, would be to ascertain the tract along which these impulses passed. But if, on the other hand, the views just now urged have any real foundation, the question of tracts or paths sinks into insignificance compared with the almost untouched problems as to what are the several changes by which simple impulses are developed into full sensations, and when and how the changes are effected.

$\S 598$. Seeing how unsatisfactory is our present knowledge with regard to the tracts or paths of sensations in the relatively simple spinal cord, it would be useless to attempt any discussion as to their paths in the much more complex brain. If it be probable that the passage is effected by relays of gray matter in the former, the same method is much more probable in the latter; and if neither experiment nor clinical study throws much light on the path up to the bulb, these cannot be expected to give much help in the maze of gray matter and fibres by which the bulb is joined to the cortex. The several defined areas or collections of gray matter, and the several strands and tracts of fibres which we briefly described in a previous section, must have of course a meaning; but it may be doubted whether we have even so much as a correct glimpse of that meaning in any one case, if we except those which are in immerliate connection with the cranial nerves and their nuclei. Seeing that the thalamus appears, on the one hand, to be connected with all or nearly all parts of the cortex, and, on the other hand, to serve as the front of the tegmental system, it is tempting to suppose that it plays an important part in sensations pertaining to the body generally, as part of it, the pulvinar, certainly does with reference to the special sense of sight; but we have no decisive indications as to what part it plays. And the part which it plays, whatever that may be, is not an exclusively sensory one, since both experimental and morbid lesions of the thalamus are apt to produce disorders of movement as well as other efferent effects. WV ought 
perhaps to say the parts which it plays, for it is a complex body, having many ties and probably performing many duties.

'The conspicuous fillet again, seeming as it does to be a special internuncial tract connecting what appear to be more particularly afferent or sensory parts of the bulb, such as the gracile and cuneate nuclei, with various parts of the middle brain and probably with the cortex, presents itself as a probable path of sensations of one kind or another from the body at large, the "narrow path" of the anatomical programme ( $\$ 592)$; but in reference to this too, beyond its probable connection with the auditory sensations ( $\$ 589)$, we lack evidence.

A conspicuous part of the brain, namely, the cerebellum, naturally arrests our attention on account of its large connections with what appear to be afferent structures; in the anatomical programme we called it " the broad path." By the cerebellar tract it has an uncrossed grip upon what is practically the whole length of the spinal cord; by the other constituents of the inferior peduncle it has a like uncrossed grip upon what appear to be afferent structures in the bulb, the gracile and cuneate nuclei, as well as on the eighth (vestibular) nerve and probably representatives of other afferent cranial nerves; it has further a crossed grip through the gracile and cuneate nuclei on the afferent posterior columns of the whole cord. It is, of course, possible that the cerebellar tract, though in itself uncrossed, lays its hand, by means of the vesicular cylinder for instance, on impulses which have already crossed from the posterior roots of the other side; for as we have seen the evidence as a whole shows that sensory impulses do cross over; but neither has the crossing of the impulses been definitely proved, nor has the path of the crossing been clearly demonstrated; while, on the contrary, the fibres of the auditory nerve which pass to the cerebellum, and which, as we have suggested ( $\$ 531$ ), may be compared to an outlying part of the cerebellar tract, certainly continue uncrossed into the peduncle of the same side. We may conclude, therefore, that the ties of the cerebellum with the posterior roots are both crossed and uncrossed. And we may regard this double grip of the cerebellum on the cord, this grip on both sides of it; as an additional evidence that the ties of the cerebellum with the spinal cord are not merely for the purpose of serving as the channel for the impulses of muscular sense, but are the means by which the cerebellum transforms or elaborates sensory impulses, not of muscular sense alone or chiefly, but probably of all kinds, in order that they may take part in cerebral operations, of which the coördination of bodily movements may be one, but probably is only one, of several or even of many.

\section{Some Other Aspects of the Functions of the Brain.}

$\S 599$. It is difficult to say anything definite concerning the transmission of sensory impulses and the development of sensations; it is still more difficult to say anything definite, beyond what has been already incidentally said, concerning the parts played in the work of the brain by the various aggregations of gray matter and tracts of fibres forming the middle part of the brain. Neither experiment nor elinical study has as yet afforded any clear or sure leading.

To what has already been said about the cerebellum, we may add the following;

Electrical stimulation of the surface of the cerebellum, in the monkey and in other animals, has led to movements of the eyes, and of other parts of the head; but we cannot from such results draw any satisfactory inferences. 
The removal of various parts of the cerebellum, especially of the medium parts, has led to a want of coördination in bodily movements; and an unsteady gait, due to a like want of adequate coördination, is a fiequent symptom of cerebellar disease. But the incoördination which makes its appearance immediately after removal of, or injury to, the cerebellum may eventually disappear, even though large portions have been removed; and many cases of cerebellar disease have been recorded in which the most perfect coördination of movements was retained. Hence, the results of experimental and clinical study, while on the whole supporting the conclusion that the cerebellum has in some way'to do with coördination, throw little or no light on the exact nature of the part which the organ plays in the complex process, but perhaps rather show that we are at present wholly ignorant of how coördination is brought about.

Many hypotheses have been put forward as to the work carried out by the cerebellum, but none of these can be said to have an adequate basis. And, indeed, if there be any value in the reflections we have repeatedly made in previous pages, the physiologist ought not to use the words "functions of the cerebellum." From a physiological point of view it is, so to speak, a matter of accident that various structures, the seats of various physiological processes, have, from morphological causes, been gathered together into the body which anatomists call the cerebellum. The task of the physiologist is to unravel the ties binding these various cerebellar structures with other parts of the central nervous system, and so with various parts of the body at large.

We must content ourselves here with calling attention to two or three broad and suggestive facts concerning its structure and connections.

In the first place, one striking fact about the cerebellum is the very large development of commissural fibres connecting together the superficial gray matter of the two hemispheres for the greater part of their extent, and passing, not only through the pons $(\$ 548)$ as part of the middle peduncle, but also through the median vermis. This great commissure is second only to the great callosal commissure of the cerebrum; and from the fact that median lesions of the cerebellum, those which do most damage to this commissure, are the most effective in causing incoördination and forced movements, we may infer that it in some way plays an important part in coordination.

A second striking fact is one on which we have already just dwelt, the connection, chiefly an uncrossed one, through the inferior peduncle, with the afferent structures of the bulb and spinal cord. We may now add that the fibres of this peduncle passing into the centre of the white matter of the cerebellar hemisphere of the same side enclose the gray matter of the nucleus dentatus and appear largely to end in that body, though some pass on to the vermis.

A third striking fact is the connection, this being, as far as we know, wholly a crossed one, through the pons and pes, with the cerebral cortex both of the extreme frontal region and of the temporo-occipital region and possibly or even probably with more scattered cortical elements of the parietal (motor) region. This connection is one between cortex and cortex, or, at least, between cerebral cortex and cerebellar superficial gray matter, for the fibres of the middle peduncle passing from the gray matter of the pons which serves as a relay end in the surface of the lateral hemisphere of the cerebellum. The frontal cortical fibres passing to the pes have a descending degeneration, that is, from the cortex to the pons, and we may probably assume that the similar temporo-occipital fibres similarly degenerate downward to the pons ( $\$ 545)$. From this it has been inferred that 
this cerebro-cerebellar connection carries impulses from the cebral cortex to the cerebellum; and it has been further inferred that these impulses are of the nature of motor impulses. As we have more than once urged, the character of degeneration, that is, whether "ascending" or "descending," is not a satisfactory proof of the direction taken by impulses; but it is, perhaps, of more importance to remember that, as we have also urged, we have no right to assume that the impulses passing along such a tract as the one in question must be either sensory or motor, or indeed that such a tract serves as an instrument for producing effects in one direction only.

That during life the fibres of which we are speaking serve as an important chain by which cerebral cortex and cerebellum affect each other, there can be but little doubt; but we are wholly in the daik as to what really takes place along the fibres. We have seen $(\$ 506)$ reason to think that the brain may and does exert an inhibitory influence over the spinal cord; and the mechanical certainty with which an animal deprived of its cerebral hemispheres responds to stimuli, in contrast to the uncertainty attending the result of stimuli applied to an intact animal, as well as all the experience of our own daily life, shows that the cerebral cortex can work in an inhibitory manner on other parts of the brain; the remarkable "forced movements" on which we dwelt in a previous section seem, in some instances, to be the result of the abrupt snap of some inhibitory boud. Conversely all the experience of our daily life, many of the phenomena of the condition known as hypnotism and of allied conditions, as well as various experimental results such as that quoted in $\$ 574$, where a sensory impulse seems to inhibit the activity of a notor area, show that the cortex may itself in turn be inhibited by other parts of the central nervous system. But we have at present no satisfactory indications as to the paths of inhibitory impulses or as to how inhibition is brought about; nor have we any proof that the cerebro-cerebellar tract is an inhibitory one in either direction.

We may add that some of the fibres of the middle peduncle appear to be neither commissural nor connected with the cortical fibres in the pes, but to end in other ways; and tracts have been described as continuing onward, some of the cerebellar fibres of the middlc peduncle on the one hand upward toward the cerebrum, and, on the other hand, downward toward the spinal cord. It has been further urged that these tracts are efferent in function.

Lastly, we may call attention to the superior peduncles. These, which, as we have seen, appear to come largely from the gray matter of the nucleus dentatus and to end in the tegmentum, largely in the red nucleus, may be regarded as constituting through the relay of the front part of the tegmentum another tie, presumbly of a different nature from the foregoing, between the cerebellum and the cortex; indeed, it used to be called processus a cerebello ad cerebrum. It is an obviously crossed tract (Fig. 136, SP); it connects one nucleus dentatus, and so presumably by that relay the fibres of the inferior peduncle ending in that body, and perhaps other fibres proceeding from the superficial gray matter of one side of the cerebellum, with the red nucleus and other parts of the tegmentum of the crossed side, and thus with the cortex of the crossed side. It has been supposed that the direction of impulses passing along it is from the cerebrum to the cerebellum, but we have no clear proof of this; indeed, as to what it does, we have no satisfactory evidence either experimental or clinical.

We may here incidentally remark that, in consequence of afferent tracts being traced to or toward the tegmentum, and of the sharp contrast presented between the tegmentum and the conspicuously motor pyramidal tract 
in the pes, the view has gained ground that the tegmentum is essentially a sensory structure. But there does not appear to be adequate evidence either clinical or experimental for such a conclusion. The thalamus, which we have regarded as the front so to speak of the tegmentum, cannot, as we have al ready urged ( $\$ 598)$, be considered exclusively or especially sensory. And many of the ties of the tegmentum, such as the fibres from the corpora striata ending in the substantia nigra, for this may be considered as properly belonging to the tegmentum, are of the kind which we may suppose to be efferent or motor. Indeed, we may probably regard the whole tegmentum as being broadly the analogue in the forward segments of the cerebro-spinal axis of both the anterior and posterior gray matter of the spinal segments behind.

Though we are thus in the dark concerning what goes on in the cerebellum, it may be worth while to call attention once more to the remarkable characters of the superficial gray matter ( $\$ 561)$. The many points of resemblance between it and the cerebral cortex cannot but suggest that the processes taking place in it have some analogies with cortical cvents. And it is, at least, a fact of some significance that congenital deficiency, or atrophy of the cerebral hemisphere of one side, is frequently accompanied by a corresponding deficiency of the crossed cerebellar hemisphere.

$\S 600$. Both the anterior and posterior corpora quadrigemina are complex in structure; not only do they differ from each other, but also in each the gray matter differs in different parts, both as to its nature and appearance and as to its connections with tracts of fibres. If we have little right to speak of the "functions of the cerebellum," we have even less right to speak of the "functions of the corpora quadrigemina" or of either pair of them. The anterior pair, as we have seen, has to do in some way with vision; but we have reason to think that a part only of the whole body is thus concerned; and there is some foundation for the view that of this part one portion belongs, so to speak, to the optic tract, and another portion to the cortical fibres of the optic radiation. Possibly still another part is concerned in bringing, as we have $(\$ 586)$ suggested, visual impulses to bear on the coördination of movements.

Stimulation of the surface of the posterior pair, besides giving rise to movements of various parts of the body, has in monkeys and some other animals the singular effect of producing a vocal utterance in the form of a cry or bark. But we cannot make much use of these results for the purpose of drawing conclusions as to what share these bodies take in the whole work of the brain. In the frog, the optic lobes correspond to the two pair of corpora quadrigemina together ; and the cry just mentioned may, perhaps, be put side by side with the fact that in the frog the optic lobes seem to furnish a mechanism for croaking; when the optic lobes are destroyed, the reflex croaking mentioned in $\$ 551$ is done away with. The probable connection of the posterior corpora quadrigemina with hearing is also interesting in this connection; but we have no satisfactory evidence of any special ties between the bodies in question and either the cortical area for phonation or the vocal mechanism in general ; the occurrence of the cry remains so far an isolated fact.

In frogs, in which the cerebellum is very small, the optic lobes seem to be particularly concerned in the coördination of movements. When the brain is removed by means of a section behind the optic lobes the animal loses the power of balancing itself $(\$ 551)$, which it possesses when the section passes in front of the optic lubes; and injury to the optic lobes produces incoördination of movement and often "forcerl movements." It has been maintained that the loss of coördination is in these cases due to re- 
moval of or injury to the central gray matter in the walls of the third ventricle, and not to mere removal of or injury to the optic lobes; but the whole evidence goes to show that in the frog and in the bird the optic lobes do play a part in the coördination of movement, though lesions of the central gray matter around the third ventricle, or, indeed, of the thalamus or other parts of the tegmentum, may give rise to loss of coördination or to "forced movements."

In the mammal removal of or injury to the posterior corpora quadrigemina does not cause blindness, but may, like a lesion of the anterior pair, give rise to loss of coördination or to forced movements; the effect, however, is, in most instances, very temporary. The connection of the anterier. pair with vision suggests a clew as to how this pair takes part in coördination; but as to how the posterior pair could intervene in the matter we have hardly so much as a hint; for, even if we admit a connection between them and the sense of hearing, and, remembering that a loud sound would often cause a person to reel, further admit that purely auditory impulses, as distinct from what we have called ampullar impulses, may take part in the general coördination of bodily movements and in the maintenance of equilibrium, as they certainly do in the special coördination of laryngeal movements, still we are not much nearer an understanding of the matter. We may add that section of the lateral fillet, which appears as a conspicuous tie between the posterior corpora quadrigemina and the parts of the nervous system behind them, does not appear to have any marked effect in producing incoördination.

In fine, beyond the broad facts on which we dwelt in a previous section, namely, that we maintain our equilibrium and carry out complex movements involving often several parts of our body, through what we call coördiuation, that afferent impulses supply important factors of this coördination, and that the cerebellum, through the vestibular nerves in part at all events, together with other portions of the middle brain, are in some way its chief instruments, we as yet know very little. We have certainly no adequate knowledge as to how either pair of corpora quadrigemina exactly iutervene in the matter, or, indeed, as to what other parts they play in the general work of the brain.

With regard to other tracts of fibres or areas of gray matter we have nothing to say, except as regards those which are more or less immediately connected with certain of the cranial nerves, such for instance as the nerves for movements of the eyes, and these it will be best to consider when we have to deal with the nerves themselves.

$\$ 601$. Besides the somatic functions which in previous discussions we have chiefly had in view, the brain as a whole undoubtedly carries out splanchnic functions; concerning these, however, we must be very brief.

Of the respiratory and vasomotor functions of the bulb we have already treated in their appropriate places, and we have referred $(\S 449)$ to the experimental evidence that a lesion of the corpus striatum or of the front part of the optic thalamus has a remarkable influence on the development. of heat in the body. We have further seen that the higher parts of the brain, acting through the bulb, exercise powerful influences on respiration, on the vasomotor system, and on the beat of the heart. Daily experience affords abundant instances of actions such as these, as well as of the influence of the brain on other organic functions. We can bring our will to bear on the mechanism of micturition ( $\$ 364$ ) which is almost wholly, and on the mechanism of defecation ( $\$ 236$ ) which is largely, splanchnic in nature. These movements, however, are not skilled movements; and as we explained in dealing with them, the action of the brain as regards them seemed limited 
to augmenting or inhibiting the activity of spinal centres. We should, therefore, hardly expect them to be specially represented in the cortical motor region. But emotions have a much wider and more powerful influence over the splanchnic functions than has the will, and have the power of affecting the work of certain organs, for instance the heart and secreting glands, which the will is unable to touch. And since we have every reason to believe that the cortex is closely associated with the emotions, we may naturally infer that elements of the cortex supply a link in the chain through which an emotion influences this or that splanchnic activity; we may, accordingly, expect to find that stimulation of some part or other of the cortex produces splanchnic effects. The results of experimental investigation, however, are both scanty and discordant; but the greater weight should perhaps be attached to the positive results. Thus, some observers find that stimulation of the cortex, the locality being in the dog some part of the sigmoid gyrus, produces movements of the bladder; and they trace the path of this influence through the front part of the thalamus and the tegmentum to the bulb and so to the cord, excluding the cerebellum, which other observers believed to be concerned in the matter. Some observers again find that stimulation of the cortex produces a flow of "chorda saliva," while others maintain that the secretion, when it does occur, is an indirect and not a direct effect of the cortical stimulation; and it may be remarked that the cortical area, which is claimed to be a "salivation area," lying in the dog on the convolutions dorsal to and in front of the Sylvian fissure, is not either the area connected with the facial nerve, or that allotted to taste or smell.

Similarly, stimulation of parts of the cortex has in the hands of various observers led to movements or to arrest of movements of the intestines, to changes in the beat of the heart, and to various vasomotor and other effects ; but it will not be profitable to enter into any further details. We may, however, add the remark that when the cortical motor area for a limb is removed, or suffers a lesion, the temporary paralysis which is thereby caused is accompanied by a rise of temperature in the limb; this may be at times very great indeed-in the monkey, for instance, the hand or foot on the paralyzed side may be as much as $10^{\circ} \mathrm{C}$. higher than that of the other side. The effect is partly due to vasomotor paralysis, but, especially considering that the muscles of the limb are relatively quiescent and so producing less heat than usual, cannot be due to that alone. The remarkable result may be taken as still further illustrating the complexity of the processes connected with the cortical motor area; the area is in some way associated with the vascular arrangements and nutrition of the muscles with whose movements it is concerned.

$\S 602$. There remain yet a few words to be said about the cortex. We regard, and justly so, the spontaneous intrinsic activity of the brain as the most striking feature of its life. The nearest approach to it which we find elsewhere in the body is perhaps the rhythmic beat of the heart. The analogy between the "regular automatism" of the one, and the "irregular automatism" of the other is a striking one; and indeed our knowledge of the relatively simple spontaneity of the heart has probably influenced to a large extent our conceptions of the complex spontaneity of the brain. In the heart the rhythmic discharge of energy is chiefly determined by intrinsic chemical changes, by the metabolism of the cardiac substance; the influence of external circumstances, apart from those which provide an adequate supply of proper blood, is wholly subsidiary and serves only to raise or to lower the intrinsic changes from time to time, as occasion may demand. And the analogy of the heart has perhaps led us to exaggerate the part played in the brain by the like intrinsic chemical metabolism. 
(We are here, of course, viewing the action of the brain from the only standpoint admissible in these pages, the purely physiological one; but such a mode of treatment does not prejudge other points of view.) Some writers. use expressions which seem to imply the conception that the nervous changes forming the basis of the psychical and other processes of the brain are chiefly the direct outcome of the chemical metabolism of the gray matter and especially of the nerve-cells. They speak of "the discharge of energy" from these cells in the same way that we can speak of the discharge of energy from a cardiac fibre. But, to say nothing of the low rate of nervous metabolism as measured in terms of chemical energy, we have no experimental or other evidence of nervous substance in any part of the body being, like the cardiac substance, the seat of an important metabolism carried on irrespective of influences other than purely nutritive ones. In the case of nerve-cells interpolated along nerves composed of fibres of the same kind, as in the sporadic ganglia, all the instances where the nerve-cells were supposed to initiate active processes have, on examination, broken down; as we lave seen, the ganglia of the heart do not supply the moving cause of the heart-beat. It is only in the central nervous system, where nerve-cells, as part of gray matter, are found at the meeting of nerve-fibres of different kinds, that we have any evidence of "discharge of energy" from the cells.

As we pointed out $(\$ 510)$ in speaking of the spinal cord, the discharge of efferent impulses from the central nervous system, though it undoubtedly must have a certain chemical basis, namely, the metabolism of the nervous substance, is, in the first place, dependent on the advent of afferent impulses. But this, if true of the spinal cord, is still more true of the brain, which receives or may receive not only all the impulses which reach it through the cord, but especially potent and varied impulses directly through the cranial nerves. All life long, the never-ceasing changes of the external world continually break as waves on the peripheral endings of the afferent nerves; all life long, nervous impulses, now more now fewer, are continually sweeping inward toward the centre; and the nervous metabolism, which is the basis of nervous action, must be at least as largely dependent on these influences from without, as on the mere chemical supply furnished by the blood.

We have developed this point because of the influence it must have on our conceptions of the physiological processes taking place in the cortex. If we accept the view just laid down, we must regard the supereminent activity of the cortex and the characters of the processes taking place in it as due not so much to the intrinsic chemical nature of the nervous substance which is built up into the cortical gray matter as to the fact that impulses are continually streaming into it from all parts of the body, that almost all influences brought to bear on the body make themselves felt by it. To put the matter in a bald way we may ask the question, What would happen in the cortex if, its ordinary nutritive supply remaining as before, it were cut adrift from afferent impulses of all kinds? We can hardly doubt but that volitional and other physical processes would soon come to a standstill and consciousness vanish. This is, indeed, roughly indicated by the remarkable case of a patient whose almost only communication with the external world was by means of one eye, he being blind of the other eye, deaf of both ears, and suffering from general anæsthesia. Whenever the sound eye was closed, he went to sleep. It is further indirectly illustrated by the following experimental result. We have seen $(\$ 567)$ that a vertical incision carried through the depth of the gray matter around an area does not prevent stimulation of the surface of the area producing the usual movements. 
But after such an incision the animal suffers a paralysis of the movements connected with the area, like that resulting from the removal of the gray matter of the area; and the operation is said to be followed by degenerative changes in the area, and degeneration of the pyramidal fibres starting from it. Some of this effect may be due to nutritive changes brought about by injury to the pia mater and division of bloodvessels; but it cannot be wholly accounted for in this way; it appears as if the life of the area is curtailed when its nervous ties are broken.

IVe may conclude then that we are not justified in speaking of consciousness of volition, or other psychical processes, even admitting that these fail when the cortex is removed, as being functions of the cortex in the same way that we speak of the functions of other organs; they are rather functions of the connections of the cortex with the other parts of the central nervous system.

We should add that they are also functions of the connections of the several parts of the cortex with each other. All our knowledge goes to show that psychical processes are dependent on, or are in some way associated with the cortex; but whatever classification of psychical functions we adopt, we are wholly unable to make out any localization of functions, such as we can make out for movements, visual sensations and the like. Even taking the broad and elementary division into "the emotions" and "the intellect," we cannot satisfactorily allot either division to any particular part of the hemisphere. In dogs, removal of particular parts of the hemispheres has indeed been observed to change the character of the animal, converting for instance a vicious, morose dog into a mild and inoffensive one; and removal of the front part of the hemisphere seems to have frequently a marked effect in rendering the animal more impressionable and excitable; he becomes much more demonstrative and "gushing" in his behavior than before. But these are mere hints, and the clinical histories of disease in man do not enable us to say much more. Such knowledge as we do possess rather tends to show that the psychical processes in proportion as they become more complex involve a greater number of nervous factors, and, therefore, have for their material basis a greater width of nervous area, or in other words their localization becomes less definite. Thus, while we may localize the beginning of a psychical process, a visual sensation for instance, and one of its terminal acts, such as the issue of impulses along the pyramidal tract, we cannot put our finger on the seat of the intermediate transactions. These even in the simplest process must be complex, and must involve many factors. Our simplest conceptions of the external world are based on a combination of visual sensations and tactile sensations. It being granted that the visual sensation, in one phase of its development, is connected with certain changes in some spot of the occipital cortex, there nust be some tie between this and the corresponding nervous seat of the tactile sensation wherever that may be, and further ties between these and other parts of the cortex. Hence, as we said, the psychical process is a function of connections.

Many of these ties are most probably furnished by the association fibres passing from one part of the cortex to a neighboring part. We must also probably admit that impulses, or, to use a more general word, processes, may travel laterally along the tangle of the cortical gray matter, for this, like the gray matter of the spinal cord, seems to form a physiological continuity, no more broken by the fissures than is the cord by its segmental arrangement; and we know nothing as to the limits which must be placed on the distance to which such processes may travel from their focus of origin. Further, seeing how completely in the dark we are as to the reason why we 
possess two hemispheres, and especially seeing that, as shown by speech, the whole of each hemisphere is not identical in action with the whole of the other, we may perhaps suppose that the fibres of the corpus callosum, which form so large a part of the central white matter of the hemisphere, have other duties than that of merely keeping the points of one hemisphere in touch with the corresponding points of the other hemisphere. But, when we have made every allowance for all these direct intercortical connections, we are driven to the conclusion that the indirect ties between one part of the cortex and another through the lower parts of the brain are of no less, perhaps of greater importance. This, indeed, is shown by the relations of the motor region. We have already urged, that even as regards the mere carrying out of a skilled movement (and we may add, whether that be voluntary or involuntary in the ordinary common use of the words) the motor region must have other ties with the part moved than merely the efferent tie of the pyramidal fibres; it must have sensory afferent ties, and the course of these, including perhaps even those which belong to the muscular sense, we may regard as an indirect one along the spinal cord and middle parts of the brain, though the details are as yet unknown to us. It must, moreover, as we have also seen, have ties, at least in many cases, with parts other than the part moved, for instance with the general coördinating machinery. And the ease with which some not very obvious change will permit the stimulation of a limited motor area to start epileptiform convulsions shows how many and close are the ties in another direction. Further, when we go beyond the final phases of the process in the motor cortex to those which precede the issue of the efferent impulses, we find the ties multiplying. For instance, since our movements are so largely guided by visual sensations, there must be ties between the motor cortex and the central visual apparatus, it may be of the occipital cortex, but it may also be of the lower visual centres. As we insisted, the motor area is only a link in a complex chain; and what we can see, dimly though it be, in reference to the cortical motor processes, probably holds good for those other cortical processes as well, of whose nervous genesis we know at present nothing. Hence even the higher psychical events cannot truly be spoken of as functions of the cortex, meaning that they are simply the outcome of molecular changes in the cortical gray matter; they are rather to be regarded as the outcome of complex processes in which the parts of the brain below the cortex play a part no less important than that of the cortex itself. If so, the fibres passing down from the cortex to the middle brain have functions by which they take part even in our psychical life, functions for which neither the words motor nor sensory are fitting.

\section{On the Time taken up by Cerebral Operations.}

$\S 603$. We have already seen ( $\$ 507$ ) that a considerable time is taken up in a purely reflex act, such as that of winking, though this is perhaps the most rapid form of reflex movement. When the movement which is executed in response to a stimulus involves cerebral operations a still longer time is needed; and the interval between the application of the stimulus and the commencement of the muscular contraction varies according to the nature of the mental labor involved.

The simplest case is that in which a person makes a signal immediately that he perceives a stimulus-ex. gr., closes or opens a galvanic circuit the moment that he feels an induction-shock applied to the skin, or sees a flash of light, or hears a sound. By arrangements similar to those employed in measuring the velocity of nervous impulses, the moment of the application 
of the stimulus and the moment of the making of the signal are both recorded on the same travelling surface, and the interval between them is carefully measured. This interval, which has been called the "reaction period" or "reaction time," may be divided into three stages: 1 . The time during which afferent impulses are generated in the peripheral sense organs and transmitted along the afferent nerves to the central nervous system; this may be called the "afferent stage." 2. The time during which, through the operations of the central nervous system, the afferent impulses are transformed into efferent impulses; this may be called the "central stage." 3. The time taken up by the passage of the efferent impulses along the efferent nerves and the transformation of the nervous impulses into muscular contractions ; this may be called the "efferent stage." In the efferent stage the events are comparatively simple, and though not absolutely constant, do not vary largely; we are able to form a fairly satisfactorily estimate of its duration, and so of the share in the whole reaction period which may be allotted to it. The events of the afferent stage are much more complex, and the estimates of its duration, being arrived at in an indirect manner and chiefly based upon calculations of the whole reaction time, are very uncertain. Hence all attempts to estimate the length of the "central " stage, the "reduced reaction period," as it is sometimes called, by subtracting the efferent and afferent stages, must be subject to much error. But a good deal may be learned by studying the variations under different circumstances of the reaction period as a whole.

Taking first of all the cases in which the events of. the central stage are simple, such as those where the subject has merely to make a signal upon feeling a sensation, we find that the length of the reaction period is dependent on the intensity of the stimulus, being shorter with the stronger stimulus. But variations in the strength of the stimulus, especially in the case of minimal stimuli, have a much more striking effect in determining the certainty of the reaction than in affecting the length of the period. Thus, when the signal is made in response to some visual sensation, upon seeing an electric spark, for instance, if the spark be a very weak one the subject of the experiment often fails to make a signal at all, though he may rarely fail if the spark be a strong one.

Some of the most marked variations in the length of the reaction period are determined by the individuality of the subject. Thus, with the same stimulus applied under the same circumstances, the reaction period of one person will be found very different from that of another.

The length of the reaction period varies also according to the nature and disposition of the peripheral organs stimulated. In general, it may be said that cutaneous sensations produced by the stimulus of an electric shock applied to the skin (the signal, for instance, being made by the right hand when the shock is felt by the left hand) are followed by a shorter reaction period than are auditory sensations, while the period of these is in turn shorter than that of visual sensations produced by luminous objects; on the other hand, the shortest period of all is said to be that of visual sensations produced by direct electrical stimulation of the retina. Roughly speaking, we may say that the reaction period is for cutaneous sensations one-seventh, for hearing one-sixth, and for sight one-fifth of a second.

Practice materially shortens the reaction period ; indeed, after long practice, making the signal, at first a distinct effort of the will, takes on the characters of a reflex act, with a correspondingly shortened interval. Iastly, we may add that in the same individual and with the same stimulus, the length of the period will vary according to circumstances, such as the time of year, the weather, and the like, as well as according to the condition of the 
individual, whether fresh or fatigued, fasting or replete, having taken more or less alcohol, and the like.

The reaction period of vision has long been known to astronomers. It was early found that when two observers were watching the appearance of the same star a considerable discrepancy existed between their respective reaction periods, and that the difference, forming the basis of the so-called "personal equation," varied from time to time, according to the personal condition of the observers.

$\S 604$. The events taking place in the central stage are, of course, complex, and this stage may be subdivided into several stages. Without attempting to enter into psychological questions, we may at least recognize certain elementary distinctions. The afferent impulses started by the stimulus, whatever be their nature, when they reach the central nervous system undergo changes, and as we have seen, probably complex changes, before they become sensations; and further changes, now of a more distinctly psychical character, are necessary before the mind can duly appreciate the characters of these sensations and act accordingly. Then come the psychical processes through which these appreciated sensations, or perceptions, or apperceptions, as they are sometimes called, determine an act of volition. Lastly, there are the executive processes of volition, the processes which, psychical to begin with, end in the issue of coördinate motor impulses, or, in other words, start the distinctly physiological processes of the efferent stage. We may thus speak of the time required for the perception of the stimulation, of the time required for the action of the will, and of the time required for the complex psychical processes which link these two together. Accepting this elementary analysis, it is obvious that the total length of the central stage may be varied by differences in the length of each of these parts; and a more complete analysis would, of course, open the way for further distinctions. Hence, by studying the variations of the whole reaction time under varying forms of psychical activity, we may form an estimate of time taken up by various psychical processes.

We may take as an instance the case in which the subject of the experiment has to exercise discrimination. The mode of making the signal being the same, and the stimulus being of the same order in each trial-that is to say, visual, or cutaneous, or auditory, etc- - and general circumstances remaining the same, two different stimuli are employed, and the subject is required to make a signal in response to the one stimulus, but not to the other; the subject has to discriminate between the psychical effects of the two stimuli. Suppose, for example, the stimulus is the sound of a spoken or sung vowel, and the subject is required to make a signal when $a$ is spoken or sung, but not when $o$ is spoken or sung. If the subject's whole reaction period be determined (1) in the usual way, with either $a$ or $o$ spoken (and the result will be found not to differ materially whether $a$ or $o$ be used), the subject knowing that only $a$ or only $o$ will be spoken, and then be determined again (2) when he has to discriminate in order that he may make the signal when $a$ is spoken, but not when $o$ is spoken, he not knowing which is about to be spoken, the whole reaction period will be found to be distinctly longer in the second case. The experiment may be varied by making use of all the vowel sounds taken irregularly as the stimulus, the subject responding by a signal to one only, as arranged beforehand. And, of course, other orders of stimulus may be used, either visual, the signal being made when a red light is shown but not when other colors are shown, or tactile, the signal being made when one part of the body is touched but not when other parts are touched, and the like.

In such experiments, where the subject has to distinguish, to discrimi- 
nate, between two or more events, the prolongation of the reaction period is o!)viously due to the longer time required for the psychical processes taking place during what we have called the central stage. In the two cases, one without and the other with discrimination, not only are the afferent and efferent stages the same in both, but we have no reason to suppose that in the central stage there is any difference between the two cases as to the time taken up by the transformation of simple sensory impulses into perceptions, or as to that taken up by the will in gaining access to the motor apparatus and so starting the processes of the efferent stage; the delay takes place in the psychical processes intervening between these two parts, and the amount of delay is the measure of the time needed for the processes involved in the discrimination. This "discrimination period" has been found to differ in the same individual according to the sensation employed, visual, auditory, etc., and according to the kind of difference in the sensation which has to be discriminated, for instance in visual sensations between colors or between objects in different parts of the field of vision. In a series of observations made in this way, the discrimination period, $i$. e., the prolongation of the simple reaction period due to having to discriminate, was found to range from 0.011 second to 0.062 second.

Another series of observations may be made in the following way: The signal being one made with the hand, the simple reaction period for a stimulus is determined with the signal given by the right hand. Two kinds of stimuli are then employed, both of the same order, two vowel sounds for instance, and the subject is directed to respond to one vowel with the right hand and to the other with the left hand. It is found, the subject being right-handed, that the reaction period is greater when the signal is made with the left hand. In this case the delay takes place not in the recognition of the effects of the stimulus, nor in the processes through which the will is formed upon that recognition; these are the same in the two cases; it takes place in the processes by which the will is brought to bear on the nervous motor apparatus for making the signal, on the cortical origin, for example, of the pyramidal tract; these processes take a longer time in the case of the unaccustomed left hand than in the case of the usual right hand. In this way we obtain a measure, so to speak, of the volitional side of psychical processes.

In a somewhat similar way we may obtain a measure of the time required for perception. A strong sensation following too closely upon a weak one will prevent the psychical recognition of the weaker one. If, for instance, two or three letters in white on a black background be presented to the eye, and a large white surface be presented afterward at an interval which is made successively shorter and shorter, it is found that when the interval is made very brief indeed the letters cannot be perceived at all. In proportion as the interval is prolonged, the recognition of the letters increases, until at an interval of about 0.05 second they are fully and clearly recognized. That is to say, the time required for perception is in such a case of about that length.

The duration of all these psychical processes, as of the simple reaction period itself, varies of course under different circumstances, and the discrimination period may be conveniently used for measurements of the varying effects of circumstances. Practice shortens the discrimination period as it does the simple reaction perior. One of the most powerful influences is that of attention. And it is stater that the shortening of the period is greater when the attention is concentrated on the making of the signal than when it is more especially directed to recognition of the stimulus; in other words, the volitional processes are more amenable than are the perceptive 
processes to the psychical action which we call attention. On the other hand, the period is distinctly prolonged if the observer be distracted by concomitant sensations. For example, the period for discriminating between two visual sensations is prolonged if powerful auditory sensations be excited at the same time.

The same niethod of measurement may be used in other ways and under other circumstances with reference to psychical processes. It must be remembered, however, that all such observations are open to many fallacies and need particular caution. It not unfrequently happens that false results are obtained ; for instance, the subject, expecting the stimulus to be brought to bear upon him and straining his attention, makes the signal before the stimulus actually comes off. And the interpretation of the results obtained are in many cases very difficult; but it would be out of place to dwell upon these matters any further here.

\section{The Lymphatic Arrangements of the Brain and Spinal Cord.}

$\$ 605$. The membranes of the brain and spinal cord. The cerebro-spinal canal is lined by a tough lamellated nembrane. composed of connective tissue with a small amount of elastic network, called the dura mater, which, somewhat closely adherent to the walls of the cranial cavity, is separated from those of the vertebral canal by a considerable space, containing bloodvessels, especially large venous sinuses, and some fat. It may be considered as a development of the periosteum lining the cerebro-spinal cavity. It sends tubular sheaths for some distance along the several cranial and spinal nerves; and forms between the cerebral hemispheres, in the longitudinal fissure, a conspicuous sickle-shaped vertical fold, the falx cerebri, as well as a smaller horizontal or oblique fold between the cerebellum and cerebrum known as the teutorium.

The vascular pia mater is closely attached to the surface of the brain and spinal cord, dipping down, as we have seen, into the ventral or anterior fissure of the cord as well as into the fissures of the brain. Sheath-like investments of pia mater are continued along the several nerves as they leave the cerebrospinal cavity; and in the vertebral canal an imperfect partition half-way between the dorsal and ventral surfaces of the cord is furnished by a membrane of connective tissue which, continuous along its whole length with the pia mater, is attached to and fused with the dura mater at intervals only, namely, between the successive nerve-roots. Since its outer edge has thus a toothed appearance, this membrane is called the ligamentum denticulatum. Between the pia mater next to the brain and cord and the dura mater next to the bony walls is a cavity, which is divided into two by a thin membrane, the arachnoid, composed of interwoven bundles of connective tissue. The space between the arachnoid and the dura mater is called the subdural space, and the space between the arachnoid and the pia mater is called the subarachnoid space. When the brain is exposed by removing the roof of the skull and slitting open the dura mater, the subdural space is laid bare, and the arachnoid is seen stretching over the pia mater; in the vertebral canal the arachnoid lies close to the dura mater, so that usually, when the dura mater is slit open and turned back, the arachnoid is carried with it and the cavity exposed is that of the subarachnoid space. The arachnoid, like the dura mater and the pia mater, is continued for some distance over the nerves as they leave the cerebro-spinal cavity; so that each nerve at its exit is surrounded by a tubular prolongation of the subdural space, and within this a similar tubular prolongation of the subarachnoid space.

The subdural space is broken up to a slight extent only by bridles carry- 
ing nerves and bloodvessels, especially venous sinuses, between the pia mater and dura mater, and over the surface of the brain by villus-like projections of the arachnoid called Pacchionian glands, some of which pierce the venous sinuses of the dura mater. It is lined throughout, both on its dural and on its arachnoid wall, by an epithelium of flat epithelioid cells, and may be compared to a serous cavity, such as that of the peritoneum. Like the serous cavities it contains normally a small quantity only of fluid, and its size is potential rather than actual.

The subarachnoid space on the other hand is, especially in certain regions, such as the dorsal portions of the vertebral canal and the base of the brain, much broken up by bridles of comnective tissue passing from it to the pia mater, as well as by a network of sponge-like arrangement of bundles of connective tissue lying immediately beneath itself, and giving it, when viewed from below, a honeycomb or fenestrated appearance. The under surface of the membrane itself, as well as all the trabeculæo of the sponge-work and the bridles, are covered with an epithelium of flat epithelioid cells, which is continued also over the pia mater and the ligamentum denticulatum, and lines the tubular sheath-like prolongations of the space along the issuing nerveroots. The subarachuoid space, therefore, like the subdural space, may be regarded as a serous or large lymphatic space, but it is an actual not a mere potential space; it always contains an appreciable quantity of fluid, which, however, is not ordinary lymph, but is furnished in a particular way and deserves special study. To understand the nature and origin of this cerebrospinal fluid, as it is called, we must turn to some special arrangements of the pia mater.

$\$ 606$. The pia mater proper, consisting of interwoven bundles of connective tissue with some elastic fibres and a considerable number of connective-tissue corpuscles, serves, as we have said, as the bearer of bloodvessels to the nervous structures which it invests. The small arteries, as they pass into the nervous substance by way of the septa, are surrounded by perivascular lymphatic canals, with which spaces in the neuroglial groundwork, both of the brain and spinal cord, especially spaces surrounding the larger nerve-cells, are continuous. As is the case with other tissues, so with the central nervous system, the several elements of the tissue are bathed with lymph derived from the blood; and this, oozing through the spaces into the perivascular canals and the other lymphatic vessels of the pia mater, makes its way into the subarachnoid space; but the fluid in the subarachnoid space has other sources besides.

The roof of the fourth ventricle is, as we have said ( $\$ 514)$, reduced to a single layer of non-nervous columnar epithelium, which appears as a mere lining to the pia mater overlying it. In the hinder part of the ventricle this roof is perforated by a distinct narrow oval orifice, the foramen of Majendie. By this orifice, which passes right through both the pia mater and the underlying layer of epithelium, the cavity of the fourth ventricle, and so the whole series of cavities derived from the original medullary canal, the lateral and third ventricles, the aqueduct, and the central canal of the spinal cord, are marle continuous with the subarachnoid space. There are also other less conspicuous communications between the subarachnoid space and the fourth ventricle. Hence the cerebro-spinal fluid is made common to all these cavities, and is furnished not only by the pia mater investing the outside of the brain and spinal cord, but also, and indeed probably to a larger extent, by the epithelium lining the several cavities of the cerebro-spinal axis, especially perhaps by those portions of that epithelium which coat the processes of pia mater projecting into those cavities at certain places.

IVe saw previously ( $\$ 515$ ) that a large fold of the pia mater, carrying in 
with it the thin non-nervous epithelium which alone represents at the place the original wall of the medullary canal, is thrust inward at the transverse fissure of the brain, beneath the fornix, to form the velum interpositum, thus supplying a roof to the third ventricle, and that it thence projects into each lateral ventricle as the choroid plexus of each side, reaching firom the foramen of Monro in front along the edge of the fornix to the tip of the descending horn. The velum being a fold of the pia mater consists theoretically of two layers, and between the upper dorsal layer and the lower ventral layer lies a thin bed of connective tissue carrying arteries forward from the hind edge of the corpus callosum, and similarly carrying veins backward; these vessels supply the choroid plexus with an abundant supply of blood. In the choroid plexus the folded pia nater is developed into a number of villuslike processes, the primary processes bearing secondary ones. Each process consists, like a villus, of a basis of connective tissue, in which the bloodvessels end in close-set eapillary loops, covered with an epithelium. The epithelium, though continuous with the rest of the epithelium lining the lateral ventricle, and thus, as we have said, shutting off the lateral from the third ventricle (except at the foramen of Monro), and though like it derived from the wall of the original medullary canal, is different in structure. Over the ventricle generally the epithelium consists of ordinary short columnar, apparently ciliated cells, with more or less transparent cell substance; the cells orer the choroid plexus are cubical, often irregular in form, and their cell substance is loaded with granules, some of which are pigmentary. They have very much the appearance of " active" secreting cells; and indeed a branched process of the plexus may be compared to an everted alveolus of a secreting gland, with the epithelium outside and the bloodvessels within. It cannot be doubted that these cells play an important part in secreting into the cavity of the ventricle fluid which, passing thence by the foramen of Monro into the third and so into the fourth ventricle, finds its way by the foramen of Majendie into the subarachnoid space.

As the velum overhangs the third ventricle it sends down vertically two longitudinal linear fringes, which, resembling in structure the choroid plexuses of the lateral ventricle, are called the choroid plexuses of the third ventricle. From the roof of the fourth ventricle there hangs down on each side a similar linear fringe, the choroid plexus of the fourth ventricle, which is especially developed at its front end beneath the overhanging cerebellum. These subsidiary choroid processes doubtless assist in furnishing cerebrospinal fluid, but their share is small compared with that of the main choroid plexuses of the lateral ventricle.

$\$ 607$. The cerebro-spinal fluid. The specimens of cerebro-spinal fluid which have been examined as to their composition are not quite comparable with each other, since while some (such as those obtained from cases where a fracture of the base of the skull has placed the subarachnoid space at the base of the brain, where it is largely developed, in communication with the external meat!ıs, and the fluid escapes by the ear) may be regarded as normal, others (such as those obtained from cases of hydrocephalus where the ventricles contain an unusual quantity of fluid, or from cases of spinal malformations) must be considered as abnormal. In most of the more complete analyses, the fluid examined has belonged to the latter class; and the following statements apply, strictly speaking, to them alone.

With this caution, we may say that cerebro-spinal fluid is a transparent colorless or very slightly yellowish fluid, of faint alkaline reaction, free from histological elements. The specific gravity is about 1010 or less, the amount of solids being on an average 1 per cent. Of these by far the greater part, 0.8 or 0.9 per cent., is supplied by salts, the total quantity of which as well 
as the relative amount of the several constituents being about the same as obtain in blood and lymph. The comparative deficiency of solids is due to the scantiness of the proteids, which rarely exceed 0.1 per cent. These are chiefly globulin and a form of albumose, or even peptone; albumin is said to be generally absent. The fluid, save apparently in exceptional cases, does not clot, and contains neither fibrogenous factors nor fibrin ferment. It very frequently contains a substance which like dextrose reduces Fehling's solution but which is not sugar; it appears to be pyrocatechin or a closely allied body.

Seeing that a fluid of such a composition is of a different nature from ordinary lymph, furnished entirely in the ordinary way, we might be inclined to infer that probably a very large part of the whole mass of the fluid is furnished by the secreting epithelium of the choroid plexus. But it must be borne in mind, that the foregoing analyses refer chiefly to fluid appearing under abnormal circumstances, and it would be hazardous to draw any wide inference from them. We have little or no exact experimental evidence as to how much fluid is actually secreted by the choroid plexuses; and if the fluids which have been analyzed do represent a mixture of ordinary lymph supplied through the pia mater with the peculiar secretion of the choroid plexus and cerebro-spinal canal, some further change beyond the mere mingling of the two fluids is needed to explain the remarkable absence of albumin which has been so strongly insisted upon by various authors.

$\$ 608$. We may fairly suppose that during life the fluid is continually being supplied, from the one source or the other; but we have no very exact knowledge as to the rate at which it is furnished. In the dog, the fluid has been observed to escape at a rate varying very largely under different circumstances, and ranging from 1 c.c. in forty minutes to as much as 1 c.c. in six minutes, the total quantity discharged in twenty-four hours varying from 36 c.c. to 240 c.c. In the cases of fracture of the base of the skull mentioned above, a very considerable flow has been frequently observed; but it may be doubted whether the abnormal circumstances of such cases have not raised the secretion above normal. The rate of flow was found in the dog to be much increased by the injection of substances (normal saline solution) into the blood, but to be relatively little influenced by artificial heightening of arterial pressure. This has been put forward as indicating that the fluid is chiefly furnished as a secretion and not as an ordinary transudation of lymph ; but it cannot be regarded as affording a valid argument. The pressure under which the fluid exists is also very variable; it is closely dependent on the vascular arrangements of which we shall have to speak presently. In the dog the average pressure has been estimated at about $10 \mathrm{~mm}$. of mercury.

If the fluid is thus continually formed it must always find a means of escape. This is probably supplied by the tubular prolongations of the subarachmoid space along the nerve-roots; these are continuous with the lymphatic vessels of the nerves, and so with the lymphatics of the body generally ; and in the skull, the passages of this kind along the cranial nerves, especially along the two nptic nerves into the orbits, afford a ready means of escape. It is also urged that some of the fluid escapes through the Pacchionian glands directly into the blood of the venous sinuses. In a dead body fluid introduced into the subarachnoid space through an opening over the bulb, disappears at even a very low pressure with great rapidity. The circumstances then are, however, not the sane as in life; and the few experiments which have been made seem to show that, during life, a somewhat high pressure is required to secure the escape of fluid introduced in addition to that naturally secreted. Thus it is stated that when in a dog normal saline 
solution is introduced into the subarachnoid cavity at the lower end of the spinal cord very little resorption takes place so long as the pressure remains as low as about 10 c.c. of mercury ; as the pressure is increased beyond this resorption quickly increases. But it may be doubted whether the resorption of added fluid is a fair test of the escape of fluid naturally present; and the experiment is of value rather as showing simply that there are means of escape than as affording a measure of the rate of escape. Besides, the immediate effects of applying pressure at the caudal end of the spinal cord are not the same as those of applying the pressure within the skull.

The rate of possible escape is not without importance as regards the mechanical importance of the cerebro-spinal fluid. 'Thus it has been urged that when an extra quantity of blood is driven into the skull, any injurious intercranial compression is prevented, not only by the transference of a corresponding quantity of cerebro-spinal fluid through the foramen of Majendie from the cranium into the spinal canal, the walls of which are less rigidly complete, but also by the direct escape of the fluid from the cavity of the skull along the cranial nerves in the manner described. It has also been urged that the fluid at the base of the skull, in the large subarachnoid spaces of which it gathers in larger quantity than elsewhere, acts as a sort of protective water cushion to the delicate cerebral substance, and that, in general, the presence of the fluid is mechanically useful to the welfare of the brain, removal of the fluid by aspiration being said to lead to hemorrhage from the pia mater and to various nervous disorders. But our knowledge as to the part which the fluid plays is at present very imperfect; and its very peculiar chemical characters suggest that it has some chemical functions.

\section{The Vascular Arrangements of the Brain and Spinal Cord.}

$\S 609$. The bloodvessels reach the nervous structures by means of the pia mater. In the spinal cord arteries coming from the vertebral, intercostal, and other arteries, and travelling along the nerve-roots join the pia mater, and then through the fissures and septa reach all parts of the cord; but, as we have previously remarked, the capillary network is much denser, and, therefore, the blood-supply much greater in the gray than in the white matter. The veins, also gathered up along the septa and fissures into the pia mater, those coming from the gray matter forming, before they reach the external pia mater, a conspicuous longitudinal vein on each side of the posterior gray commissure, pass from the pia mater to the large venous sinuses of the dura mater and so to adjoining veins.

In the brain two important features of the distribution of the arteries deserve special attention. In the first place, the quadruple supply by the right and left vertebral and internal carotid arteries is made one by remarkable anastomoses forming the circle of Willis. The right and left vertebral arteries entering the vertebral canal at the level of the sixth cervical vertebra and running forward toward the brain, join beneath the ventral surface of the bulb to form the single median basilar artery. This, after giving off branches to the bulb, cerebellum, and pons, divides into the right and left posterior cerebral arteries. Each internal carotid entering the skull reaches the base of the brain in the region of the floor of the third ventricle, and, passing ventral to and athwart the optic tract, gives off the large and important middle cerebral artery along the fissure of Sylvius, and, then, turning forward and toward the median line, passes dorsal to the optic nerve to end in the anterior cerebral artery. Just, however, as it gives off the middle artery, it sends backward, inclining to the middle line, a relatively large 
branch, the posterior communicating artery, which joins the posterior cerebral near the origin of this from the basilar artery. Moreover, the two anterior cerebral arteries, soon after they have crossed the optic nerves, just as they are about to run straight forward along the frontal lobes, are joined together by a short, wide branch, the anterior communicating artery. In this way the vertebral arteries through the basilar artery join with the carotid arteries to form around the optic chiasma beneath the floor of the third ventricle an arterial circle, the circle of Willis.

Blood can pass along this circle in various ways-from the basilar artery along the right posterior communicating artery to the right internal carotid, and so by the right anterior cerebral artery and anterior communicating artery to the left side of the circle, and similarly from the basilar artery along the left side to the right, or from the right or from the left carotid through the circle, to the right hand or to the left hand in each case. Since the channel of the circle is a fairly wide one, the passage in various directions is an easy one; all the vessels radiating from the circle, including the basilar artery and its branches, can be supplied by the carotids alone, or by the vertebrals alone, or even loy one carotid or one vertebral alone. In this way an ample supply of blood to the brain is secured in the face of any hindrance to the flow of blood along any one of the four channels.

In what may, perhaps, be considered the usual arrangement, the calibre of the posterior communicating arteries is rather smaller than the other parts of the circle, so that, other things being equal, most of the vertebral blood will pass by the posterior cerebral arteries, while the carotid blood passes to the middle and anterior cerebral arteries; but many variations are met with. We may also here, perhaps, call to mind the fact that the left carotid coming off from the top of the aorta offers a shorter path for the blood than does the right carotid which comes off from the innominate artery.

Another special feature of the arterial supply to the brain is that the three large cerebral arteries-posterior, middle, and anterior-are distributed almost exclusively to the cortex and to the subjacent white matter, while the deeper parts of the hemisphere, the nucleus caudatus, thalamus, and the like, with the capsule and other adjoining white matter, are supplied by smaller arteries coming direct from the circle of Willis, or from the very beginnings of the three cerebral arteries. It is stated that these two systems make no anastomoses with each other; but this appears to vary much in different individuals. We may add that the anterior cerebral artery supplies the cortex of the dorsal aspect of the frontal lobe as well as the front and middle portions of the whole mesial surface of the liemisphere; while the middle cerebral, always large, is distributed to the side of the brain, that is, the parietal lobe, with the ventral part of the frontal lobe and the dorsal part of the temporal lobe; the posterior cerebral supplying the rest of the cortex, that is to say, the occipital lobe, including the hind part of the mesial surface of the hemisphere, together with the ventral part of the temporal lobe. The distribution of these arteries, therefore, does not correspond to functional divisions, for while the middle cerebral supplies a large part of the motor region, it does not supply the whole of it, and does supply parts outside of it. Though the small arteries as they run in the pia mater on the surface of the cortex anastomose freely, there is very little anastomosis between the small arteries which, leaving the pia mater, dip down into the substance of the brain; lience, when these latter arteries are blocked, the nutrition of the part of the cortex supplied by them is apt to be impaired.

$\S 610$. The venous arrangements of the brain have very special characters. 
Along the upper convex border of the sickle-shaped fold of dura mater, the falx cerebri, is developed a large venous sinus, the superior longitudinal sinus. This, triangular in section, increasing in calibre from before backward, is a sinus, not a vein ; its walls are formed of nothing but connective tissue lined with epithelium, muscular elements being entirely absent. Though its channel is broken by bridles of connective tissue passing across it, it possesses no valves, and, indeed, these are absent from all the sinuses and veins of the brain. Most of the blood returning from the cortex and subjacent white matter is carried into this sinus by veins, the mouths of which are for the most part directed forward, that is to say, against the direction of the blood stream. Along the lower concave border of the falx is a similar sinus, the inferior longitudinal sinus, which, however, is small, and into which relatively few veins open.

From the deeper parts of the brain, and especially from the choroid plexus, blood is conveyed by the veins of Galen along the velum interpositum to the transverse fissure, where the veins of Galen join the inferior longitudinal sinus to form the straiglit sinus. This, running along the line formed by the intersection of the vertical falx with the (more or less) horizontal tentorium, joins the end of the superior longitudinal sinus to form the reservoir or cellar, called the torcular Herophili, from which the lateral sinus, passing on each side along the convex border of the tentorium and gathering veins from the cerebellum and hind regions, as well as from the base of the brain, delivers the blood into the internal jugular vein.

It should be added that veins from the nose and, through the oplithalmic veins, from the face join the veins and sinuses of the brain, and that the socalled emissary veins pass through the cranium from the scalp to the superior longitudinal and lateral sinuses.

The channels for the venous blood of the brain are therefore not veins, but sinuses; not so much tubes for maintaining a uniform current, as longitudinal reservoirs, which. while affording an easy onward path, can also be easily filled and easily emptied, and in which the blood can move to and fro without the restrictions of valves. This arrangement is correlated to the peculiar surroundings of the brain, which is not, like other organs, protected merely by skin or other extensible or elastic tissue, but is encased by a fairly complete inextensible envelope, the skull. As a consequence of this, when at any time an extra quantity of blood is sent from the heart to the brain room must be made for it by the increased exit of the fluids already present. For any pressure on the brain-substance beyond a certain limit is injurious to its welfare and activity, as is seen in certain maladies, where blood passing by rupture of the bloodvessels out of its normal channels remains effused on the surface of the brain or elsewhere, and thus taking up the room of the proper brain-substance leads, by "compression," as it is called, to paralysis, loss of consciousness, or death. Some room may, as we have seen ( $\$ 608)$, be provided by the escape of cerebro-spinal fluid from the skull. But, within the limits of the normal cerebral circulation, the characteristic venous sinuses especially serve to regulate the internal pressure ; they form temporary reservoirs from which a comparatively large quantity of blood can be rapidly discharged from the cranium, the flow from the sinuses being greatly assisted by the low or negative pressure obtaining in the veins of the neck at each inspiratory movement of the chest.

$\S 611$. The supply of blood to the brain seems at first sight not to correspond to the importance of this the chief organ of the body. In the rabbit it would appear that hardly more than one per cent. of the total quantity of the blood of the body is present at any one time in the brain, a quantity but little more than half that which is found in the kidneys; and while the 
weight of blood, in the brain at any one time amounts to about five per cent. of the total weight of the organ, being about the same as in the nuscles, in the kidney.it amounts to nearly twelve per cent, and in the liver to as much as nearly thirty per cent. Making every allowance for the relative small size and functional importance of the rabbit's brain, the blood-supply of even the human brain must still be small; and making every allowance for rapidity of current, the interchange between the blood and the nervous elements must also be small. In other words, the netabolism of the brain-substance is of importance not so much on account of its quantity as of its special qualities.

The circulation in the brain may be studied by help of various methods. A manometer may be connected with the peripheral end of the divided internal carotid artery, a second manometer being attached in the usual way to the central portion. Since the peripheral manometer records the blood-pressure in the circle of IVillis transmitted along the peripheral portion of the carotid artery, variations of pressure in the circle of Willis may thus be studied; and a comparison of the peripheral with the central manometer will indicate what general changes are taking place in the circulation through the brain. Thus a fall of pressure in the peripheral manometer unaccompanied by any corresponding fall in the central manometer would show that the "peripheral resistance" in the brain was being lowered, in other words, that the vessels were being dilated.

In another method, in the dog, the outflow of venous blood from the lateral sinus through the posterior facial vein has been measured. The freedom with which blood passes along the sinuses justifies the assumption that the outflow through the open vein gives an approximate measure of the rate of flow under natural conditions; still the results are only approximate, and besides, the continued loss of blood introduces error.

A third method is a plethysmographic one. The skull is made.to serve as the box of the plethysmograph or oncometer $(\$ 346)$; a small piece of the roof having been removed by the trephine, a membrane is fitted to the hole, and the movements of the membrane are recorded by help of a piston and lever or directly by a lever. In young subjects, the fontanelle, or portion of the cranium not yet ossified, may be utilized as a natural membrane, and its movements recorded in a similar manner. When the instrument is fitted to the hole in a water-tight manner, this method records variations in interual pressure; and we may take it for granted, unless otherwise indicated, that greater or less pressure is due to more or less blood passing to the brain. But the amount of pressure brought to bear on the recording instrument will also depend on the readiness with which the cerebro-spinal fluid escapes from the cavity of the skull; if there be a hindrance to the escape, or, on the other hand, an increased facility of escape, the same increase of supply of blood will produce in one case a less, in the other a greater movement of the lever. If the membrane be attached loosely to the hole so as to allow free escape of the cerebro-spinal fluid, the lever practically resting on the surface of the cerebral hemisphere, the method records variations in the dorso-ventral diameter of the hemisphere, and these may be taken as measuring variations in the volume of the brain and so in the blood-supply. In neither form, however, does the method by itself give us all the information which we want. An increase of blood in the brain, and therefore an expansion of the brain, and so a movement of the recording instrument, may result either from a fuller arterial supply or from hindrance to the venous outflow; the former condition is, at least in most cases, favorable to, the latter always and distinctly injurious to, the activity of the nervous structures; hence the teachings of the lever must 
be corrected by a simultaneous observation of the general arterial pressure and of the blood-pressure in the veins of the neck. Moreover, the argument which we used ( $\$ 353)$ in reference to the kidney may be applied here and probably with equal force, namely, that the value of the blood stream for the nutrition of the tissue is dependent not alone on the amount of bloodpressure but also and especially on the rapidity of the flow; indeed, this second factor is of particular importance in view of the need of supplying the nervous elements with an adequate interchange of gases. Now of the rapidity of flow the plethysmographic method can give us indirect information only.

$\$ 612$. By one or other or all of these methods certain important facts have been made out. The volume of the brain as determined by the amount of blood present in it, is continuously undergoing changes brought about by various causes. Each heart-beat makes itself visible on the cerebral as on the renal plethysmographic tracing, and as we have seen in speaking of respiration, the diminution of pressure in the great veins of the neck during inspiration leads to a shrinking, and the reverse cliange during expiration to a swelling of the brain. The plethysmograph also shows variations, larger and slower than the respiratory undulations, and brought about by various causes, such as the position of the head in relation to the trunk, movements of the limbs, modifications of the respiratory movements, and apparently phases of activity of the brain itself, as in waking and sleeping; undulations corresponding to the Traube-Hering variations ( $\$ 330)$ of blood-pressure may not unfrequently be observed.

All the variuus methods show that the flow through the brain is largely determined by a vasomotor action of some kind or another. And this we might indeed infer from ordinary experience. When the head is suddenly shifted from the erect to a hanging position, there must be a tendency for the blood to accumulate in the cranial cavity, and conversely when the head is suddenly shifted from a hanging to an erect position, there must be a tendency for the supply of blood within the cranium to be for a while less than normal. Either change of position, and especially perhaps the latter, would lead to cerebral disturbances, which in turn would in ourselves be revealed by affections of our consciousness. That a perfectly healthy and especially young organism, whose vasomotor mechanisms are at once effective and delicately responsive, can pass swiftly from one position of the head to the other without inconvenience, whereas those in whom the vasomotor mechanisms have by age or otherwise become imperfect are giddy when they attempt such rapid changes, is in itself adequate evidence of the importance of the vasomotor arrangements affecting the circulation through the brain. The several methods agree in showing that increased general arterial pressure, such as that, for instance, induced by stimulation of a sensory nerve, leads to a greater flow of blood to the brain; the volume of the brain is increased and the venous outflow by the lateral sinus is quickened. Conversely, a lowering of arterial pressure leads to a lessened flow of blood to the brain.

Seeing that the cerebral arteries have well-developed muscular coats, the basilar artery in fact being conspicuous in this respect, one would be led to suppose that the brain possessed special vasomotor nerves of its own; and recognizing the importance of blood-supply to rapid functional activity one would perhaps anticipate that by special vasomotor action, the supply of blood to this or that particular part of the brain might be regulated apart from changes in the general supply. The various observations, however, which have hitherto been made have failed to demonstrate with certainty any such special vasomotor nerves or fibres directly governing cerebral 
vessels. It would be hazardous to insist tor much on this negative result, especially since the observations have been chiefly directed to the nerves of the neck, the experimental difficulties of investigating the presence of vasomotor fibres in the cranial nerves being very great. Still it may be urged and indeed has been urged that the flow of blood through the brain is so delicately responsive to the working of the general vasomotor mechanism just because it has no vasomotor nerves of its own. In such an organ as the kidney, an increase of general blood-pressure, as we have more than once insisted, may or may not lead to a greater flow through the kidney according as the vessels of the kidney itself, through the action of the renal vasomotor nerves, are dilated or constricted; and as we have seen, a constriction of the renal vessels may be one of the contributors to the increased general pressure. In the brain, on the other hand, an increase of general arterial pressure seems always to lead to increase of flow. Thus in the Traube-Hering undulations just mentioned, the expansions of the brain are coincident with the rises of the general pressure, whereas in the normal kirlney and in other organs the local Traube-Hering undulation reverses the general one, the shrinkings are synchronous with the rises of pressure, the local constriction being one of the factors of the general rise. It is argued that in the absence of vasomotor nerves of their own, the cerebral vessels are wholly, so to speak, in the hands of the general vasomotor system, so that when the blood-pressure is high owing to a large vaso-constriction in the abdominal viscera, more blood must necessarily pass to the brain, and when again the blood-pressure falls through the opening of the splanchnic flood-gates ( $\$ 159)$ less blood necessarily flows along the cerebral vessels. And indeed one may recognize here a sort of self-regulating action; for diminishing the supply of blood to the vasomotor centre in the bulb acts, as we know, as a powerful stimulus in producing vaso-constriction, and so leads to a rise of blood-pressure; but this very rise of blood-pressure drives more blood to the brain, including the bulb, and thus the injurious effects to the brain threatened by an anæmic condition are warded off by the very beginning of the anæmia itself. All these advantages are, however, quite compatible with the coexistence of special vasomotor mechanisms.

$\$ 613$. Moreover, the flow of blood to, and consequent change in the bulk of, the brain, and indeed the flow of blood through the brain, as measured by the venous outflow, may be modified independently of changes in the general blood-pressure. For instance, stimulation of the motor region of the cortex quickens the venous outflow, without producing any marked change in the general blood-pressure; this feature becomes very striking at the onset of epileptiform convulsions when these make their appearance. It is difficult not to connect such a result of functional activity with some special vasomotor nervous arrangement comparable to that so obvious in the case of a secreting gland. Again, it has been observed that certain drugs have an effect on the volume of the brain, quite incommensurate with their effect on the vasomotor system; thus in particular the injection into the general blood-stream of a weak acid produces a large and immediate expansion of the brain, while the introduction of a weak alkali similarly gives rise to similar considerable shrinking. It is suggested that these effects are produced by the acid or alkali acting directly on the muscular coats of the minute arteries and so leading to relaxation or contraction respectively. In treating of the chemistry of nervous substance $(\$ 70)$ we stated that "the gray matter of the central nervous system is said to be slightly acid during life and to become more acid after death." Recent observations go to show that the gray matter of the cortex is faintly alkaline during life and under normal conditions, but becomes acid after death or when its blood-supply is 
interfered with; and it has been urged that nervous gray matter like muscular substance develops acidity during activity as well as upon death, the acidity being probably due in each case to some form of lactic acid. And just as it has been suggested that the dilatation of the minute arteries of a skeletal muscle, accompanying or following the contraction of the muscle, is brought about by the acid generated during the contraction causing a relaxation of the muscular coats of the minute arteries, so it has been suggested that a similar acidity, the product of nervous activity, similarly leads in nervous tissue to a dilatation of the vessels of the part. The existence of special vasomotor mechanisms would, however, afford a more satisfactory explanation of these and other phenomena; in spite of the negative results so far obtained, the matter is obviously one needing further investigation. Meanwhile we have abundant evidence that, however brought about, the flow of blood through the brain, and probably through particular parts of the brain, is varied in accordance with the needs of the brain itself and the events taking place elsewhere in the body.

\section{CH A P T E R I I I.}

\section{SIGHT.}

$\$ 614$. A RAY of light falling on the retina gives rise to what we call a sensation of light; but in order that distinct vision of any object may be gained, an image of the object must be formed on the retina, and the better defined the image the more distinct will be the vision. Hence, in studying the physiology of vision, our first duty is to examine into the arrangements by which the formation of a satisfactory image on the retina is effected; these we may call briefly the dioptric mechanisms. We shall then have to inquire into the laws according to which rays of light impinging on the retina give rise to sensory impulses, and those according to which the impulses thus generated give rise in turn to sensations. Here we shall come upon the difficulty of distinguishing between the unconscious or physical and the conscious or psychical factors. And we shall find our difficulties increased by the fact, that in appealing to our own consciousness we are apt to fall into error by confounding primary and direct sensations with states of consciousness which are produced by the weaving of these primary sensations with other operations of the central nervous system, or, in familiar language, by confounding what we see with what we think we see. These two things we will briefly distinguish as visual sensations and visual judgments; and we shall find that both in vision with one eye, but more especially in binocular vision, visual judgments form a very large part of what we frequently speak of as our sight.

$\$ 615$. [The eyeball is of as pheroidal shape. It consists of two segments of different-sized spheres. The larger segment is situated posteriorly, and constitutes about five-sixths of the walls of the eyeball. From its free margin projects the smaller segment, which is that of a smaller sphere. The posterior segment is composed of a whitish, opaque, firm wall, consisting of three coats or tunics-the sclerotic, the choroid, and retina. The anterior segment is continuous with the sclerotic coat. (Fig. 157.) It is a transparent, elastic, convex organ, called the cornea. The cornea consists of three layers-an anterior and posterior elastic lamina, having between them a 
layer which is the proper tissue of the organ. This middle layer is composed of about sixty superimposed lamina of fusiform fibrous cells. In the interstices between the laminæ are found tubular spaces, which contain a transparent fluid. 'The anterior and posterior elastic laminæ are structureless and highly elastic. When separated from the proper corneal tissue they have a great tendency to curl up, suggesting that they are active agents in the retention of a proper curvature of the cornea. The cornea is covered on its anterior surface by the conjunctival mucous membrane, which consists of three or four layers of pavement epithelial cells; the deeper layers of cells are oblong, and placed perpendicularly. The conjunctiva at this point has no perceptible basement membrane. The posterior surface of the cornea is covered by a transparent serous membrane, which consists of a simple layer of polygonal pavement epithelial cells resting on an elastic mem-

FIG. 157.

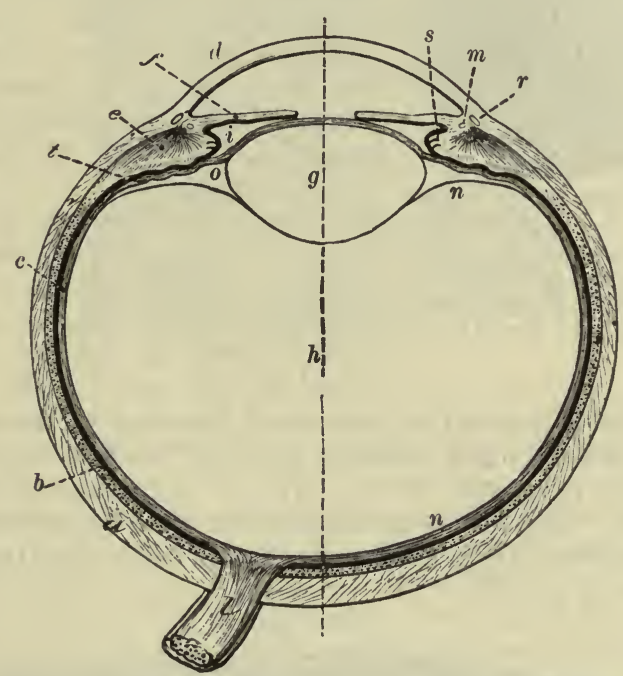

Diagram of a Horizontal Section of the Eyeball. $a$, outer or sclerotic coat ; $d$, the cornea; $b$, middle or choroidal coat ; $m$, ciliary ligament ; $s$, ciliary process ; $e$, ciliary muscle, and $f$, iris ; $c$. inner coat of retina, continuous with the optic nerve behind, with a dark layer outside it; $g$, lens; $t$, suspensory ligament of the lens; $h$, vitreous body $\cdot n$, hyaloid membrane $i$, posterior chamber; $o$, canal of Petit; $r$, sinus circularis iridis; $l$, optic nerve. The dotted line through the centre is the longitudinal axis of the ball.

brane. This is called the membrane of Demours. The cornea has no bloodvessels, and therefore derives its nutriment by diffusion.

The sclerotic coat is so named on account of the firmness of its texture and of its hardness. It forms the outer tunic of the posterior segment. It is whitish, opaque, smooth, excepting at the points of attachment of the muscles of the eyeball. It is composed of white fibrous tissue, arranged more or less in bundles, which interlace each other in various directions. Anteriorly the interlacements are in a generally transverse direction; posteriorly the direction is longitudinal. This coat also contains yellow elastic fibres and fusiform nucleated cells. It is continuous anteriorly with the cornea, and posteriorly with the perineurium of the optic nerve. At the internal border of the junction with the cornea is a venous sinus called the sinus circularis iridis or canal of Schlemm. The optic nerve pierces it about $2.6 \mathrm{~mm}$. internal to the antero-posterior axis of the eyeball. At this point the coat is perfo- 
rated by minute openings for the passage of the nerve-filaments. One of these openings, which is relatively large, gives passage to the arteria centralis retince. Surrounding this point of entrance of the optic nerve are many small openings for the passage of the ciliary nerves and vessels. The in-

FiG. 158.

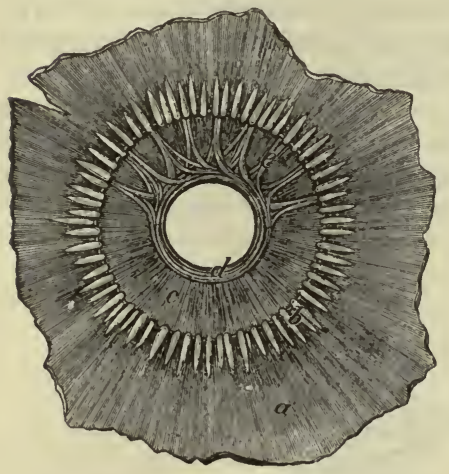

Inner View of the Front of the Choroid Coat with its Ciliary Processes, and the Back of the Iris. $a$, anterior piece of the choroid coat ; $b$, ciliary processes; $c$, iris ; $d$, sphincter of the pupil ; $e$, bundles of fibres of the dilator of the pupil.

or internal extremities to the suspensory ligament of the lens.

The ciliary muscle arises from the point of junction of the sclerotic coat and the cornea. It consists of two portions-a radiating or meridional and a circular layer. The radiating fasciculi are situated externally and have a meridional direction. (Fig. 159.) From this layer numerous fasciculi inter-

FIG. 159 .

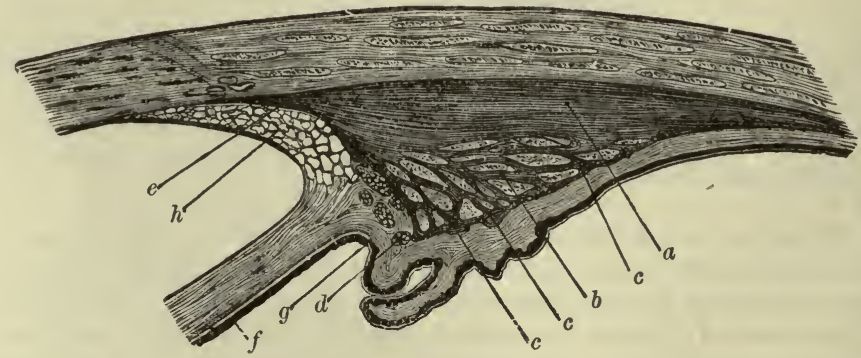

Section of the Ciliary Region of the Eye in Man. a, meridional muscular fasciculi of the musculus ciliaris ; $b$, deeper-seated radiating fasciculi; $c, c, c$, annular plexus; $d$, annular muscle of Müller; $f$, muscular lamina on the posterior surface of the iris; $g$, muscular plexus at the ciliary border of the iris; $e$, annular tendon of the musculus ciliaris; $h$, ligamentum pectinatum.

lace between the fasciculi of the circular layer, which occupies an internal position to the radiating layer. The ciliary muscle is inserted into the exterual surface of the anterior portion of the choroid coat, the fibres extending somewhat posterior to the anterior margin of the retina. This muscle is a very important factor in the mechanism of accommodation. 
The iris is a fibro-muscular curtain which is suspended between the cornea and the crystalline lens. It is attached by its circumference to the internal wall of the sinus c. iridis. In its centre is a round perforation called the pupil, which is susceptible of considerable variations in size. This membrane is composed of a fibro-connective tissue having a general radiating direction from the pupillary border. Within this tissue are found pigmentcells and unstriated muscular tissue. The muscular tissue element consists of radiating and circular fasciculi. (Fig. 160.) The circular fasciculi form

FIG. 160.

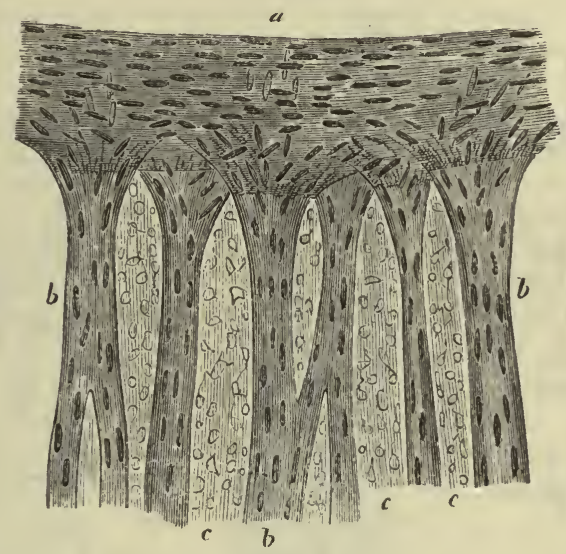

Muscular Structure of the Iris of a White Rabbit. $a$, sphincter of the pupil; $b, b$, radiating fasciculi of dilator muscle; $c, c$, connecting tissue with its corpuscles.

a sphincter at the pupillary margin; the radiating fasciculi radiate from the sphincter to the circumference. At the circumference of the iris the membrane lining the anterior chamber forms fibrous processes, which are termed the ligamentum iridis pectinatum. The posterior surface is covered with a pigmentary layer, which is a continuation of the pigment layer of the retina.

The retina or third coat consists of two portions: the pigmentary membrane and terminal elements of the optic nerve. The pigmentary membrane or external layer, which has been called the system of the uvea, covers the whole of the internal surface of the ciliary processes, the iris, and the choroid. It consists of a single layer of hexagonal nucleated pigment-cells of a dark-brown color. From the internal surface of this membrane delicate fibres are continued between the cellular elements of the nervous layer. It is frequently dissected with the choroid coat, and spoken of as one of its laminæ. The color of the iris in different individuals is dependent upon the density of the fibro-connective tissue anterior to the $u v e a$, and to the amount of pigment-granules in it. In persons with dark eyes the pigment in this tissue is relatively more abundant.

The internal or nervous layer of the retina is composed essentially of the terminal nerve elements of the optic nerve. Externally it is covered with the pigmentary layer ; internally it is lined by a homogeneous transparent structure called the hyaloid membrane. The structure of the retina is one of great complexity. It consists of nine distinct layers, seven of which are layers of nerve elements. All of these layers are bound together and supported by a connective tissue which contains bloodvessels. This layer ex- 
tends from the entrance of the optic nerve to a point where the aunular fasciculi of the ciliary muscle are found; at this point the nervous elements cease to exist, and the layer has an irregular dentated margin called the ora serrata. Beyond this the nervous layer is continued as a mere fibrous extenuation.

The optic nerve pierces the sclerotic and the choroid coats, and the pigmentary membrane of the retina, when it rapidly divides into vast numbers of fibres, which consist alone of the axis-cylinders or their ultimate fibrillæ.

FIG. 161.

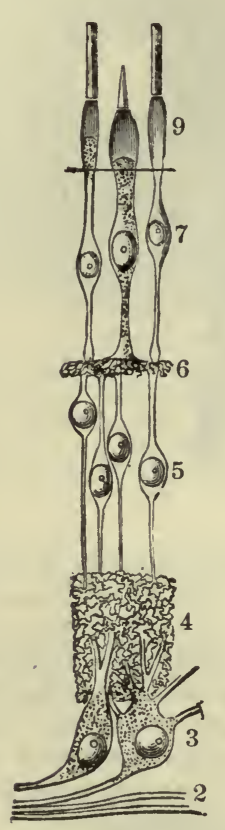

Fig. 162.

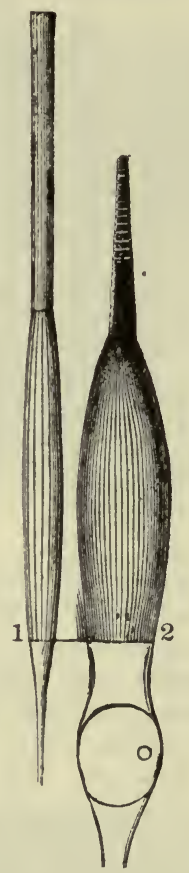

FIG. 163.

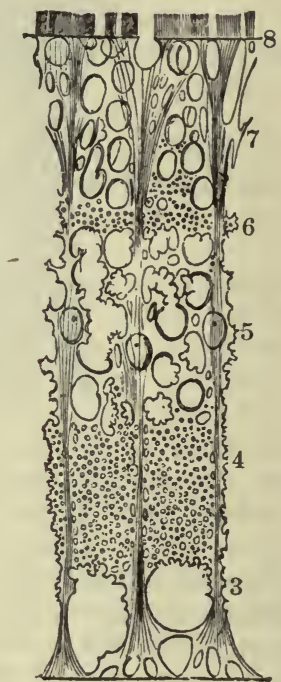

Fig. 161.-Diagrammatic Representation of the Connections of the Nerve-fibres in the Retina. 1, membrana limitans interna; 2 , optic nerve-fibre layer; 3 , layer of ganglion cells; 4 , internal granulated or molecular layer; 5, internal granule layer; 6 , external granulated or molecular layer; 7, external granule layer; 8 , membrana limitans exterior; 9, bacillary layer, or layer of rods and cones.

Fig. 162.-Rod and Cone from the Retina of Man, preserved in a two per cent. solution of perosmic acid to show the fine fibres of the surface, and the different lengths of the internal segment. The outer segment of the cone is broken up into disks, which, however, are still adherent to one another ; at the base of the cone are seen a few fine hairs. (+1000 diameters.)

FIG. 163.-Diagrammatic Representation of the Connective Tissue and Radial Fibres of Muller of the Retina as seen near the Ora Serrata. The numbers correspond to those of the several layers of the retina shown in Fig. 161.

This layer of fibres is continuous over nearly the whole of the internal surface, and is called the second or optic nerve-fibre layer. On its internal surface, between it and the hyaloid nembrane, is a delicate structure called the first layer, or membrana limitans interna. The third or ganglion layer is composed of multipolar ganglion cells, similar to those found in the cerebral substance. In the posterior portion of the retina these ganglion cells are in several layers; at the macula lutea there are as many as eight, and at the anterior portion of the retina there is but a single layer. From each of these 
cells fibres are continued to the fifth or internal granule layer, which consists of granular cells with nuclei. Between the third and fifth layers is a layer of vesicular matter containing nerve-fibrils of extreme minuteness. This layer is the fourth or internal granulated or molecular layer. The sixth or external granulated or molecular layer consists of parallel interlaced fibres, containing nuclei and smooth cells. The seventh or external granule layer is very similar to the fifth. The cighth laver consists of a delicate membrane of connective tissue, called the membrana limitans externa. The ninth or bacillary layer, or layer of rods and cones, or Jucob's membrane, is composed of two elements, the rods and cones. These layers are supported by connective tissue and a peculiar neuroglial structure known as the radial fibres of Müller. (Fig. 163.) The rods are cylindrical bodies, each ending externally in a truncated, flattened extremity, and internally as an attenuated fibre, which probably communicates with the deeper layer of ganglion cells. The cones, as their name indicates, are conical-shaped bodies. Each consists of two portions, a conical body having projecting from its apex a rod-like segment, which appears in all respects like the rods. This segment is called the cone rod. The terminal extremities of the cone rods do not extend as far externally as the extremities of the rods. The rods and cones have been demonstrated to consist of two segments or limbs, which are composed of filaments, granular matter, and nuclei. The outer limb of the rods contains a pinkish pigment known as "visual purple," which is extremely sensitive to light.

The optic nerve, where it pierces the coats of the eye, projects somewhat beyond the surface of the retina, as a papilla; here the essential nerve elements of the retina are absent, and luminous rays are unperceived; hence, it is called the blind spot. About $2.6 \mathrm{~mm}$. external to the point of entrance of the optic nerve, and in the exact centre of the retinal surface corresponding to the antero-posterior axis of the eye, is the "yellow spot of Sömmerring," or macula lutea. (Fig. 164.) It is an elliptical-shaped spot, having its long diameter transverse. In the centre of the macula lutea is a depression called the fovea centralis. At this point the nervous layer of the retina is very much modified in the composition of the different layers. The nervous layer is much thicker than at any other part of the membrane. The ganglion (third) and external granulated (sixth) layers are even more thickened. The ganglion layer consists of six or eight laminæ of cells. The rods of the ninth layer are absent, and are replacerl by cones. In the fovea centralis the internal gran-

FIG. 164.

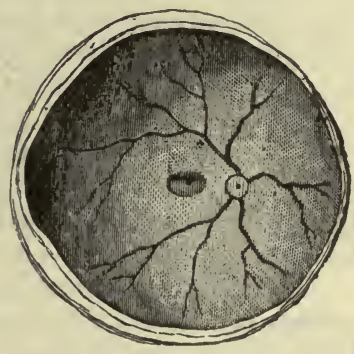

Objects on the Inner Surface of the Retina. In the centre of the ball is the yellow limbus luteus, here represented by shading, and in its middle the dark spot. To the inner side is the nerve, with its accompanying artery. (After Sömmerring.) ulated (fourth), the internal granule (fifth), and the optic nerve-fibre (second) layers are wanting. The ganglion cell (third), the external granulated (sixth), and the external granule (seventh) layers are increased in thickness. The ganglion layer of cells in the fovea consists of three laminæ. In all portions of the nervous layer the rods greatly predominate in number over the cones, excepting in the macula lutea, where they are entirely absent. The retina is much thicker posteriorly, becoming thinner as it extends forward, the nervous layer gradually disappearing in the anterior portion of the membrane.

The interior of the eyeball is divided into two portions by the crystalline 
lens and its suspensory ligament. The anterior portion contains the aqueous humor, the posterior contains the vitreous body.

The crystalline lens measures about $7 \mathrm{~mm}$. in transverse diameter, and about $4 \mathrm{~mm}$. antero-posterior diameter. It is a transparent biconvex body, somewhat flattened anteriorly. It consists of a number of segments which radiate from the centre, similar to the segments of an orange. These segments are composed of superimposed laminæ of varying density. The most superficial are soft and gelatinous; the deeper are relatively hard, so that they form a kernel or nucleus. The laminæ are made up of parallel fibres, with an undulating course, the convexities and concavities of the adjoining fibres fitting accurately into each other. The lens is covered with a capsule consisting of a transparent, elastic, fragile membrane, which has a tendency to curl up, with its external surface innermost.

The suspensory ligament of the lens is formed by a continuation of the hyaloid membrane which lines the vitreous body. The hyaloid membrane is a delicate transparent structure situated between the vitreous body and membrana limitans interna of the retina. It is continued in front of the ora serrata, where it divides into two layers. The posterior is attached to the posterior portion of the capsule of the lens; the anterior portion gradually becomes thicker as it extends forward behind the ciliary processes, and is attached to the anterior surface of the capsule. This thickened portion of the membrane, which is corrugated where it has attached the ciliary processes, is called the zone of Zinn. These two layers constitute the suspensory ligament. Between them is a triangular canal, with its base corresponding to the crystalline lens. This is called the canal of Petit.

The vitreous body is contained within the cavity formed by the hyaloid membrane and the posterior surface of the lens. It consists of a clear, colorless, albuminous fluid, having an extremely delicate interlacement of fibres extending in all directions through it. These fibres are not discernible in the adult, but can readily be seen in the frotus.

The aqueous humor is contained within the space formed by the posterior surface of the cornea and the anterior surface of the lens. The space, which is divided into two chambers by the iris, is filled with a clear, colorless, limpid fluid containing saline and proteid substances in solution. This fluid constitutes the aqueous humor.

The anterior external portion of the eyeball, comprising the surface of the cornea and about 6 or $8 \mathrm{~mm}$. of the sclerotic coat, is covered by the conjunctival mucous membrane.]

\section{Dioptric Mechanisus.}

\section{The Formation of the Image.}

$\S 616$. The eye is a camera, consisting of a series of surfaces and media arranged in a dark chamber, the iris serving as a diaphragm; and the object of the apparatus is to form on the retina a distinct image of external objects. That a distinct image is formed on the retina may be ascertained by removing the sclerotic from the back of an eye, and looking at the hinder surface of the transparent retina while rays of light proceeding from any external object are allowed to fall on the cornea.

$\$ 617$. A dioptric apparatus in its simplest form consists of two media separated by a (splierical) surface; and the optical properties of such an apparatus depend upon (1) the curvature of the surface, (2) the relative refractive power of the media. The eye consists of several media, bounded by surfaces which are approximately spherical, but of different curvature. The 
surfaces are all centred on a line called the optic axis, which meets the retina at a point somewhat above and to the inner (nasal) side of the fovea centralis. In passing from the outer surface of the cornea to the retina the rays of light traverse in succession the cornea, the aqueous humor, the lens, and the vitreous humor. Refraction takes place at all the surfaces bounding these several media, but particularly at the anterior surface of the cornea, and at both the anterior and posterior surfaces of the lens. Since the anterior and posterior surfaces of the cornea are parallel, or very nearly so, the rays of light would suffer little or no change of direction in passing through the cornea, if it were bounded on both sides by the same medium. The direction of the rays of light in the aqueous humor would, therefore, remain the same if the cornea were made exceedingly thin; if, in fact, its two surfaces were made into one, forming a single anterior surface to the aqueous humor; or, which comes to the same thing in the end, since the refractive power of the substance of the cornea is almost exactly the same as that of the aqueous humor, the refraction at the posterior surface of the cornea may be neglected altogether. Thus the two surfaces of the cornea are practically reduced to one. The lens varies in density in different parts, the refractive power of the central portions being greater than that of the external layers; but the refractive power of the whole may, without any serious error, be assumed to be uniform. The refractive power of the vitreous humor is almost exactly the same as that of the aqueous humor.

$\$ 618$. Thus the apparently complicated natural eye may be simplified into a "diagrammatic eye," in which the refracting surfaces are reduced to three, viz.: (1) the anterior surface of the cornea, (2) the anterior surface of the lens separating the lens from the aqueous humor, and (3) the posterior surface of the lens separating the lens from the vitreous humor. The media will similarly be reduced to two: the substance of the lens and the aqueous or vitreous humor. This "diagrammatic eye" is of great use in the various calculations which become necessary in studying physiological optics; for the magnitudes which are derived by calculation from it represent the corresponding magnitudes in an average natural eye with sufficient accuracy to serve for all practical purposes. The values adopted by Listing for the constants of this "diagrammatic eye," and to him we are indebted for the introduction of it, are as follows:

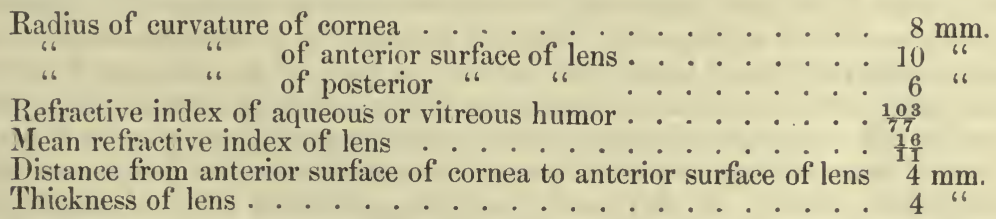

The calculated position of the principal posterior focus, $i$. e., the point at which all rays falling on the cornea parallel to the optic axis are brought to a focus, is in the diagrammatic eye $14.6470 \mathrm{~mm}$. behind the posterior surface of the lens, or $22.6470 \mathrm{~mm}$. behind the anterior surface of the cornea. That is to say, the fovea centralis must occupy this position in order that a distinct inage of a distant object may be formed upon it. It must be understood that these values refer to the eye when at rest, $i$. $e$, when it is not undergoing any strain of accommodation.

\section{Accommodation.}

$\S 619$. When an object, a lens, and a screen to receive the image are so arranged in reference to each other that the image falls upon the screen in 
exact focus, the rays of light proceeding from each luminous point of the object are brought into focus on the screen in a point of the image corresponding to the point of the object. If the object be then removed further away from the lens, the rays proceeding in a pencil from each luminous point will be brought to a focus at a point in fiont of the screen, and, subsequently diverging, will fall upon the screen as a circular patch composed of a series of circles, the so-called diffusion circles, arranged concentrically round the principal ray of the pencil. If the object be removed, not further from, but nearer to the lens, the pencil of rays will meet the screen before they have been brought to focus in a point, and consequently will in this case also give rise to diffusion circles. When an object is placed before the eye, so that the image falls into exact focus on the retina, and the pencils of rays proceeding from each luminous point of the object are brought into focus in points on the retina, the sensation called forth is that of a distinct image. When, on the contrary, the object is too far away, so that the focus lies in front of the retina, or too near, so that the focus lies behind the retina, and the pencils fall on the retina not as points, but as systems of diffusion circles, the sensation produced is that of an indistinct and blurred image. In order that objects both near and distant may be seen with equal distinctness by the same dioptric apparatus, the focal arrangements of the apparatus must be accommodated to the distance of the object, either by changing the refractive power of the lens or by altering the distance between the lens and the screen.

$\S 620$. That the eye does possess such a power of accommodation is shown by every-day experience. If two needles be fixed upright some two feet or so apart into a long piece of wood, and the wood be held before the eye so that the needles are nearly in a line, it will be found that if attention be directed to the far needle, the near one appears blurred and indistinct, and that conversely, when the near one is distinct, the far one appears blurred. $\mathrm{By}$ an effort of the will we can at pleasure make either the far one or the near one distinct; but not both at the same time. When the eve is arranged so that the far needle appears distinct, the image of that needle falls exactly on the retina, and each pencil from each luminous point of the needle unites in a point upon the retina; but when this is the case the focus of the near needle lies behind the retina, and each pencil from each luminous point of this needle falls upon the retina in a series of diffusion circles. Similarly, when the eye is arranged so that the near needle is distinct, the image of that needle falls upon the retina in such a way, that each pencil of rays from each luminous point of the needle unites in a point on the retina, while each pencil from each luminous point of the far needle unites at a point in front of the retina, and then diverging again falls on the retina in a series of diffusion circles. If the near needle be gradually brought nearer and nearer to the eye, it will be found that greater and greater effort is required to see it distinctly, and at last a point is reached at which no effort can make the image of the needle appear anything but blurred. The distance of this pcint from the eye marks the limit of accommodation for near objects. Similarly, if the person be short-sighted, the far needle may be moved away from the eye, until a point is reached at which it ceases to be seen distinctly, and appears blurred. In the one case the eye, with all its power, is unable to bring the image of the needle sufficiently forward to fall on the retina; the focus lies permanently behind the retina. In the other the eye cannot bring the image sufficiently backward to fall on the retina; the focus lies permanently in front of the retina. In both cases the pencils of rays from the needles strike the retina in diffusion circles.

$\$ 621$. The same phenomena may be shown with greater nicety by 
what is called Scheiner's Experiment. If two smooth holes be pricked in a card, at a distance from each other less than the diameter of the pupil, and the card be held up before one eye, with the holes horizontal, and a needle placed vertically be looked at through the holes, the following facts may be observed: When attention is directed to the needle itself, the image of the needle appears single. Whenever the gaze is directed to a more distant object, so that the eye is no longer accommodated for the needle, the image appears double and at the same time blurred. It also appears double and blurred when the eye is accommodated for a distance nearer than that of the needle. When only one needle is seen, and the eye therefore is properly accommodated for the distance of the needle, no effect is produced by blocking up one hole of the card, except that the whole field of vision seems dimmer. When, however, the image is double on account of the eye being accommodated for a distance greater than that of the needle, blocking the left-hand hole causes a disappearance of the right-hand or opposite image, and blocking the right-hand hole causes the left-hand image to disappear. When the eye is accommodated for a distance nearer than that of the needle, blocking either hole causes the image on the same side to vanish. The diagram will explain how these results are brought about.

Let $a$ (Fig. 165) be a luminous point in the needle, and $a e, a f$ the extreme right-hand and left-hand rays of the pencil of rays proceeding from it, and passing respectively through the right-hand $e$, and left-hand $f$, holes in the card. (The figure is supposed to be a horizontal section of the eye.) When the eye is accommodated for $a$, the rays $e$ and $f$ meet together in the point $c$, the retina occupying the position of the plane $n n$; the luminous point appears as one point, and

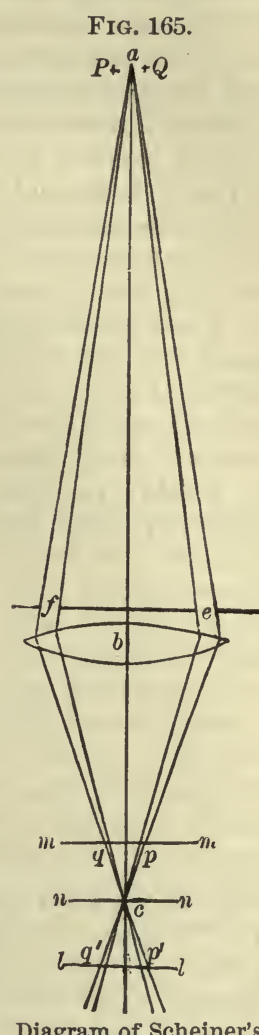

Diagram of Scheiner's Experiment. the needle will appear as one needle. When the eye is accommodated for a distance beyond $a$, the retina may be considered to lie ${ }^{1}$ no longer at $n n$, but nearer the lens, at $m m$ for example; the rays $a e$ will cut this plane at $p$, and the rays $a f$ at $q$; hence the luminous point will no longer appear single, but will be seen at two points, or rather as two systems of diffusion circles, and the single needle will appear as two blurred needles. The rays passing through the right-hand hole $e$, will cut the retina at $p-i . e$., on the right-hand side of the optic axis; but, as we shall see in speaking of the judgments pertaining to vision, the image on the right-hand side of the retina is referred by the mind to an object on the lefthand side of the person; hence the affection of the retina at $p$, produced by the rays $a e$ falling on it there, gives rise to the image of the spot $a$ at $P$, and similarly the left-hand spot $q$ corresponds to the right-hand $Q$. Blocking the left-hand hole, therefore, causes a disappearance of the right-hand image, and vice versa. Similarly, when the eye is accommodated for a distance nearer than the needle, the retina may be supposed to be removed to $l l$, and the right-hand $a e$ and left-hand $a f$ rays, after uniting at $c$, will diverge

1 Of course in the actual eye, as we shall see, accommodation is effected by a change in the lens, and not by an alteration in the position of the retina; but for convenience sake, we may here suppose the retina to be moved. 
again, and strike the retina at $p^{\prime}$ and $q^{\prime}$. The blocking of the hole $e$ will now cause the disappearance of the image $q^{\prime}$ on the left-hand side of the retina, and this will be referred by the mind to the right-hand side, so that $Q$ will seem to vanish.

If the needle be brought gradually nearer and nearer to the eye, a point will be reached within which the image is always double. This point marks with considerable exactitude the near limit of accommodation. With shortsighted persons, if the needle be removed further and further away, a point is reached beyond which the image is always double; this marks the far limit of accommodation.

The experiment may also be performed with the needle placed horizontally, in which case the holes in the card should be vertical.

The adjustment of the eye for near or far distances may be assisted by using two needles, one near and one far. In this case, one needle should be vertical and the other horizontal, and the card turned round so that the holes lie horizontally or vertically according to whether the vertical or horizontal needle is being made to appear double.

$\$ 622$. In what may be regarded as the normal eye, the so-called emmetropic eye [Fig. 166], near the limit of accommodation is about 10 or $12 \mathrm{~cm}$.

[FIG. 166.

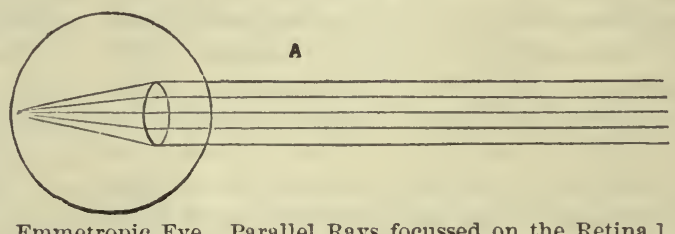

Emmetropic Eye. Parallel Rays focussed on the Retina.]

and the far limit may be put for practical purposes at an infinite distance. The "range of distinct vision," therefore, for the emmetropic eye is very great. In the myopic, or short-sighted eye [Fig. 167] the near limit is brought much closer ( 5 or $6 \mathrm{~cm}$.) to the cornea; and the far limit is at a variable, but not very great distance, so that the rays of light proceeding from an object not many feet away are brought to a focus, not on the retina,

[FIG. 167.

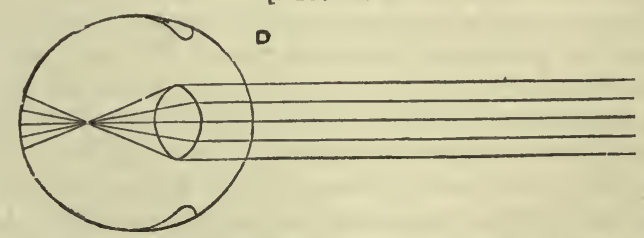

Myopic Eye. Rays coming from a distance focussed too soon.]

but in the vitreous humor. The range of distinct vision is therefore, in the myopic eye very limited. In the hypermetropic [Fig. 168], or long-sighted eye, the rays of light coming from even an infinite distance are, in the passive state of the eye, brought to a focus beyond the retina. The near limit of accommodation is at some distance off, and a far limit of accommodation does not exist. The presbyopic eye, or the long sight of old people, resembles the hypermetropic eye in the distance of the near point of accommodation, but differs from it inasmuch as the former is an essentially defective condition of the accommodation mechanism, whereas in the latter the power of 
accommodation may be good, and yet, from the internal arrangements of the eye, be unable to bring the image of a near object on to the retina. When a normal eye becomes presbyopic, the far limit may remain the same, but since the power of accommodating for near objects is weakened or lost, the change is distinctly a reduction of the range of distinct vision. In the normal emmetropic eye, when no effort of accommodation is made, the principal focus of the eye lies on the retina, in the myopic eye in front of it, and in the hypermetropic eye behind it.

[FIG. 168.

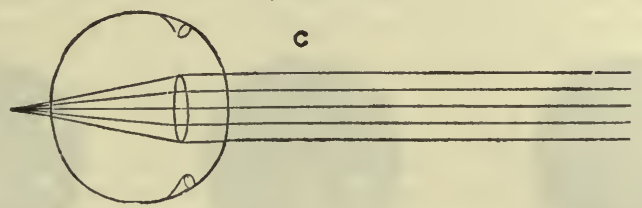

Hypermetropic Eye. Rays coming from a distance focussed too late.]

$\S 623$. Mechanism of accommodation. In directing our attention from a far to a very near object, we are conscious of a distinct effort, and feel that some change has taken place in the eye; when we turn from a very near to a far object, if we are conscious of any change in the eye, it is one of a different kind. The former is the sense of an active accommodation for near objects ; the latter, when it is felt, is the sense of relaxation after exertion.

Since the far limit of an emmetropic eye is at an infinite distance; no such thing as active accommodation for far distances need exist. The only change that will take place in the eye in turning from near to far objects will be a mere passive undoing of the accommodation previously made for the near object. And that no such active accommodation for far distance takes place is shown by the facts-that the eye, when opened after being closed for some time, is found not in medium state, but adjusted for distance ; that when the accommodation mechanism of the eye is paralyzed by atropine or nervous disease, the accommodation for distant objects is unaffected; and that we are conscious of no effort in turning from moderately distant to far distant objects. The sense of effort often spoken of by myopic persons as being felt when they attempt to see things at or beyond the far limit of their range seems to arise from a movement of the eyelids, and not from any internal changes taking place in the eye.

$\$ 624$. What, then, are the changes which take place in the eye when we accommodate for near objects? It might be thought, and, indeed, once was thought, that the curvature of the cornea was changed, becoming more convex, with a shorter radius of curvature, for near objects. Young, however, showed that accommodation took place as usual when the eye (and head) is immersed in water. Since the refractive powers of aqueous humor and water are very nearly alike, the cornea with its parallel surfaces, placed between these two fluids, can have little or no effect on the direction of the rays passing through it when the eye is immersed in water. And accurate measurements of the dimensions of an image on the cornea have shown that these undergo no change during accommodation, and that, therefore, the curvature of the cornea is not altered. Nor is there any change in the form of the bulb; for any variation in this would necessarily produce an alteration in the curvature of the cornea, and pressure on the bulb would act injuriously by rendering the retina anæmic and so less sensitive. In fact, there are only two changes of importance which can be ascertained to take place in the eye during accommodation for near objects. 
One is that the pupil contracts. When we look at near objects, the pupil becomes small; when we turn to distant objects, it dilates. This, however, cannot have more than an indirect influence on the formation of the image ; the chief use of the contraction of the pupil in accommodation for near objects is to cut off the more divergent circumferential rays of light.

$\$ 625$. The other and really efficient change is that the anterior surface of the lens becomes more convex. If a light be held before the eye, three reflected images may, with care and under proper precautions, be seen by a bystander:

[FIG. 169.
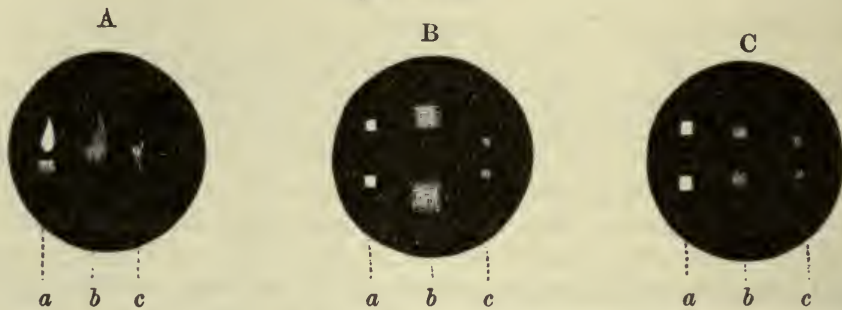

Diagram of Images reflected from the Eye.]

one a very bright one caused by the anterior surface of the cornea $(a)$, a second less bright, by the anterior surface of the lens $(b)$, and a third very dim, by the posterior surface of the lens $(c)$; when the images are those of an object, such as a candle, in which a top and bottom can be recognized, the two former images are seen to be erect, but the third inverted. When the eye is accommodated for near objects, no change is observed in either the first or the third of these images; but the second, that from the anterior surface of the lens, is seen to become distinctly smaller, showing that the surface has become more convex. When, on the contrary, vision is directed from near to far objects, the image from the anterior surface of the lens grows larger, indicating that the convexity of the surface has diminished, while no change takes place in the curvature either of the cornea or of the posterior surface of the lens. And accurate measurements of the size of the image from the anterior surface of the lens have shown that the variations in curvature which do take place are sufficient to account for the power of accommodation which the eye possesses.

The observation of these reflected images is facilitated by the simple instrument introduced by Helmholtz and called a phakoscope. It consists of a small, dark chamber, with apertures for the observed and observing eyes; a needle is fixed at a short distance in front of the former, to serve as a near object, for which accommodation has to be made; and a lamp or candle is so disposed as to throw an image on each of the three surfaces of the observed eve. Since the distance between two images is more readily appreciated than is a simple change of size of a single image. two prisms are employed so as to throw a double image of the lamp on each of the three surfaces [Fig. 169, B. C ]. When the anterior surface of the lens becomes more convex the two images reflected from that surface approach each other (C), when it becomes less convex they retire from each other (B).

These observations leave no doubt that the essential change by which accommodation is effected is an alteration of the convexity of the anterior surface of the lens. And that the lens is the agent of accommodation is further shown by the fact that after removal of the lens, as in the operation for cataract, the power of accommodation is lost. In the cases which have been recorded, where eyes from which the lens had been removed seemed 
still to possess some accommodation, we must suppose that no real accommodation took place, but that the pupil contracted when a near object was looked at, and so assisted in making vision more distinct.

$\$ 626$. This increase of the convexity of the lens has been supposed to be due to a compression of the circumference of the lens by a contraction of the iris; but this is disproved by the fact that accommodation may take place in eyes from which the iris is congenitally absent. It has also been attributed to vasomotor changes, to increased fulness of the vessels of the iris or ciliary processes surrounding the lens; but this also is disproved by the fact that acconmodation may be effected after death, in an eye which is practically bloodless, by stimulating the ciliary ganglion or ciliary nerves with an interrupted current or by other means. The real nature of the mechanism seems to be as follows.

$\$ 627$. The lens when examined after removed from the eye is found to be a budy of considerable elasticity. IV hen the curvature of the anterior surface of the lens is determined, as may be done by appropriate means, in its natural position in the eye at rest, aid then again determined after the lens has been removed from the eye, the anterior surface is found to be more convex in the latter than in the former case. There seems to be in the eye in its natural condition, some agency at work keeping the anterior surface of the lens somewhat flattened. The suspensory ligament, attached to the choroid and ciliary processes behind, and passing over the front of the lens, is just such a structure as would produce this effect. In the natural position of the choroid this ligament is tense, and tends to flatten the front of the lens. When the choroid is pulled forward, the ligament becomes slack and the lens bulges out forward. Further, the ciliary muscle attached on the one hand to a fairly fixed region, the juuction of the sclerotic and cornea, and on the other to the looser and more movable choroid, would naturally, when thrown into contraction, pull forward the choroid and so slacken the suspensory ligament, and hence permit the elastic lens to bulge out forward. And we have experimental evidence, carried out on lower animals, that stimulation of the ciliary ganglion or of its so-called radix brevis does lead, on the one hand, to a contraction of the ciliary muscle and pulling forward of the choroid, and, on the other hand, to an increased curvature of the anterior surface of the lens. Hence we may conclude that accommodation for near objects consists essentially in a contraction of the ciliary muscle, which, by pulling forward the choroid coat and the ciliary process, slackens the suspensory ligament, and allows the lens to bulge forward by virtue of its elasticity, and so to increase the convexity of its anterior surface [Figs. 170 and 171].

Accommodation is in most cases a voluntary act; since, however, the change in the lens is always accompanied by movements in the iris, it will be convenient to consider the latter before we discuss the nervous mechanism of the whole act.

$\S 628$. Movements of the pupil. Though by making the efforts required for accommodation we can at pleasure contract or dilate the pupil, it is not in our power to bring the will to act directly on the iris by itself. This fact alone indicates that the nervous mechanism of the pupil is of a peculiar character, and such indeed we find it to be. The pupil is contracted (1) when the retina (or optic nerve) is stimulated, and when light falls on the retina, the brighter the light the greater being the contraction, (2) when we accommodate for near objects. The pupil is also contracted when the eyeball is turned inward, when the aqueous humor is deficient, in the early stages of poisoning by chloroform, alcohol, etc.; in nearly all stages of poisoning by morphine, physostigmine, and some other drugs; and in deep 
slumber. The pupil is dilated (1) when stimulation of the retina (or optic nerve) is diminished or arrested as in passing from a bright into a dim light or into darkness, (2) when the eye is adjusted for far objects. Dilatation also occurs when there is an excess of aqueous humor, during dyspnoea, during violent niuscular efiorts, as the result of a stimulation of sensory nerves, as an effect of emotions, in the later stages of poisoning by chloroform, etc., and in all stages of poisoning by atropine and some other drugs, and in pathological conditions of the nervous system.

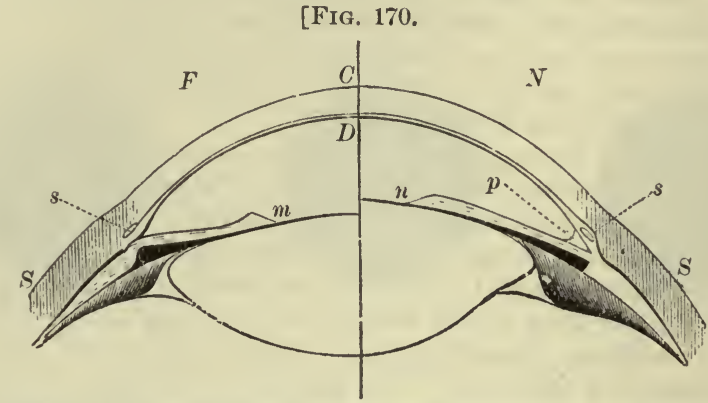

Diagram to illustrate Accommodation. $C$, cornea; $S$, sclerotic ; $F, C, N$, vertical plane of the cornea ; $B, C, D$, axis of the eye ; $s, s$, canal of Schlemm; , angle formed by the iris and cornea, or margin of anterior chamber ; $m$, position of iris and curvature of lens in an eye converged for parallel rays, distant vision, or negative accommodation; $n$, position of iris and curvature of lens required for neur objects or for positive accommodation.]

[FIG. 171.

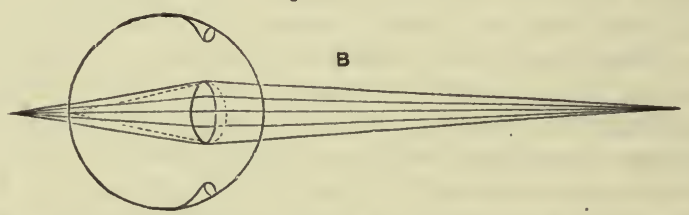

Emmetropic Eye. The dotted lines show how accommodation for the diverging rays of near objects is effected by the bulging of the lens. The dotted lines indicate the change in the lens and the effect on the light rays.]

$\$ 629$. Contraction of the pupil is caused by contraction of the circular fibres or sphincter of the iris. Dilatation is caused by contraction of the radial fibres of the iris; for though the existence of radial fibres has been denied by many observers, the preponderance of evidence is clearly in favor of their being really present.

Considering how vascular the iris is, it does not seem unreasonable to interpret some of the variations in the condition of the pupil as the results of simple vascular turgescence or of depletion brought about by vasomotor action or otherwise, the small or contracted pupil corresponding to the dilated and filled and the large or dilated pupil to the constricted and emptied condition of the bloodvessels. Thus slight oscillations of the pupil may be observed synchronous with the heart-beat and others synchronous with the respiratory movements. But the variations in the pupil seem too marked to be merely the effects of vascular changes, and indeed that constriction of the pupil cannot be wholly the result of turgescence, nor dilatation wholly the result of depletion of the vessels of the iris, is shown by the facts that both these events may be witnessed in a perfectly bloodless eve, and that the movements of the pupil when brought about by agents which also affect the 
bloodvessels begin some time before the changes in the calibre of the bloodvessels, and indeed may be over before these have arrived at their maximum. Moreover, the fibres of the sympathetic, which, as we shall see, are concerned in causing dilatation of the pupil, run a somewhat different course from those which govern the bloodvessels of the eye. We may, therefore, adhere to the view that the main changes of the pupil in the direction of narrowing and widening are brought about by contractions of the plain muscular fibres in the iris.

$\$ 630$. Muscular contractions leading to changes of the pupil may be observed in the eye removed from the body, and indeed in the extirpated iris. The plain muscular fibres of the iris, like other plain muscular fibres, are remarkably sensitive to variations in temperature. Besides this there seems to be, in certain animals at least, a connection within the eye between the iris and retina of such a kind that light falling into an extirpated eye will lead to a narrowing of the pupil. Putting aside, however, such exceptional events we may lay down the broad principle that contraction of the pupil, brought about by light falling on the retina, is a reflex act, of which the optic is the afferent nerve, the third or oculo-motor the efferent nerve, and the centre some portion of the brain lying below the corpora quadrigemina in the front part of the floor of the aqueduct of Sylvius. This is proved by the following facts: When the optic nerve is divided, the falling of light on the retina no longer causes a contraction of the pupil; when the third nerve is divided, stimulation of the retina or of the optic nerve no longer causes contraction; but direct stimulation of the peripheral portion of the divided third nerve causes extreme contraction of the pupil. If the region of the brain spoken of above as a centre be carefully stinulated contraction of the pupil will take place even in the absence of light and after division of the optic nerve. After removal of the same centre stimulation of the retina is ineffectual in narrowing the pupil. But if the centre and its connections with the optic nerve and third nerve be left intact and in thoroughly sound condition, contraction of the pupil will occur as a result of light falling on the retina, though all other nervous parts be removed.

\$ 631. The nervous centre is not a double centre with two completely independent halves, one for each eye ; there is a certain amount of functional communion between the two sides, so that when one retina is stimulated both pupils contract. It might be imagined that this cerebral centre acted as a tonic centre, whose action was simply increased, not originated, by the stimulation of the retina; but this is disproved by the fact that if the optic nerve be divided subsequent section of the third nerve produces no further dilatation.

In considering the movements of the pupil, however, we have to deal not only with a narrowing of the pupil thus brought about in a reflex way by contraction of the circular sphincter fibres, and with the absence of such a narrowing, but also with active dilatation due to a contraction of the radial dilator fibres, and this renders the whole matter much more complex than might be supposed to be the case from the simple statement just made.

$\$ 632$. The iris is supplied, in common with the ciliary muscle and choroid, by the short ciliary nerves (Fig. 172, s. c.) coming from the ophthalmic or lenticular (ciliary) ganglion (l. c.) which is connected by its roots with the third nerve $(r . b$.$) , the cervical sympathetic nerve (sym.), and$ with the nasal branch of the ophthalmic division of the fifth nerve $(\imath \cdot l$.$) .$ The short ciliary nerves are, moreover, accompanied by the long ciliary nerves (l.c.) coming from the same nasal branch of the ophthalmic division of the fifth nerve. What are the uses of these several nerves in relation to the pupil?

$\$ 633$. If the cervical sympathetic in the neck be divided, all other por- 
tions of the nervous mechanism being intact, a contraction of the pupil (not always very well marked) takes place, and if the peripheral portion (i.e., the

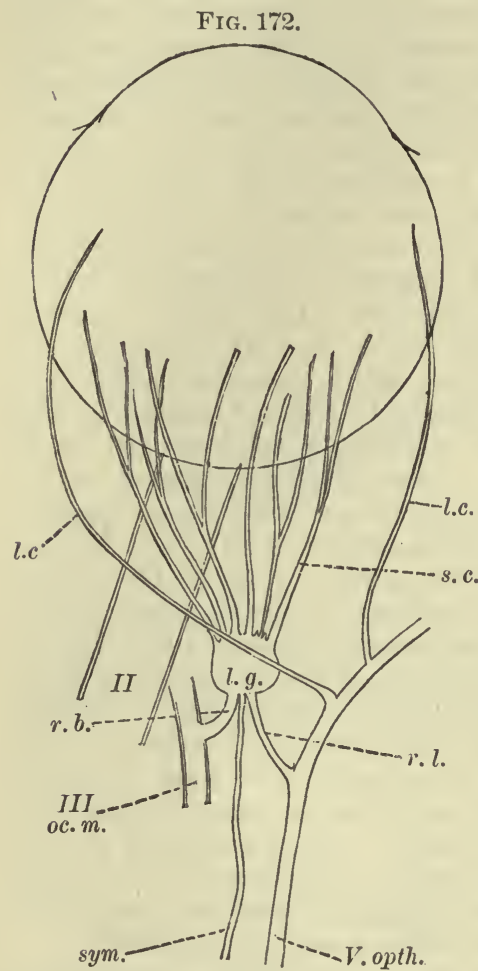

Diagrammatic Representation of the Nerves Governing the Pupil. II. optic nerve; $l$. $g$. lenticular gauglion; $r . b$. its short root from $I I I . ; o c . m$. third or oculomotor nerve ; sym. its sympathetic root; $r . l$. its long root from $V$. ophthm. the nasal branch of the ophthalmic division of the fifth nerve s. c. the short ciliary nerves from the lenticular ganglion; $l$.c. the long ciliary nerve from the nasal branch of the ophthalmic division of the fifth nerve.

after division of the medulla oblongata and consequent severance of the efferent paths from the centre in the aqueduct of Sylvius, dilatation of the pupil may still be brought about, in some animals at least, by dyspnœa or by adequate stimulation of sensory nerves. A question is raised here in fact somewhat similar to that raised in connection with the medullary respiratory centre (p. 370) ; and here as there we may probably conclude that the independent action of such a spinal centre is of subordinate importance.

$\S 635$. The pupil then seems to be under the dominion of two antagonistic mechanisms : one a contracting mechanism, reflex in nature, the third nerve serving as the efferent and the optic as the afferent tract; the other a dilating mechanism, apparently tonic in nature, but subject to augmentation from various causes, and of this the cervical sympathetic is the efferent channel. Hence, when the third or optic nerve is divided, not only does 
contraction of the pupil cease to be manifest, but active dilatation occurs, on account of the tonic dilating influence of the sympathetic being left free to work. When, on the other hand, the sympathetic is divided, this tonic dilating influence falls away, and contraction results. When the optic or third nerve is stimulated, the dilating, effect of the sympathetic is overcome, and contraction results; and when the sympathetic is stimulated, any contracting influence of the third nerve which may be present is overcome, and dilatation ensues.

$\$ 636$. But there are considerations which show that the matter is still more complex than this. A small quantity of atropine introduced into the eye or into the system causes a dilatation of the pupil. This might be attributed to a paralysis of the third nerve, and, indeed, it is found that after atropine has produced its effects the falling of light on the retina no longer causes contraction of the pupil. A difficulty, however, is introduced by the fact that when the third nerve is divided, and when, therefore, the contracting effects of stimulation of the retina are placed entirely on one side, and there is nothing to prevent the sympathetic producing its dilating effects to the utmost, dilatation is still further increased by atropine. When physostigmine is introduced into the eye or system, contraction of the pupil is caused, whether the third nerve be divided or not; and when the dose is sufficiently strong the contraction is so great that it cannot be overcome by stimulation of the sympathetic. The dilatation which is caused by a sufficient dose of atropine may be greater than that which can ordinarily be produced by stimulation of the sympathetic, and the contraction caused by a sufficient dose of physostigmine may be greater than that which is ordinarily produced in a reflex manner by stimulation of the optic nerve, or even than that produced by direct stimulation of the third nerve. Evidently these drugs act either directly on the plain muscular fibres of the iris or on some local mechanism, the one in such a way as to cause dilatation, the other in such a way as to cause contraction. Such a local mechanism cannot, however, lie in the ophthalmic ganglion, for both drugs continue to produce these effects in a most marked degree after the ganglion has been excised. We must suppose, therefore, that the mechanism if it exists is situated in the iris itself or in the choroid, where, indeed, ganglionic nervecells are abundant. The movements of the iris in the extirpated eye, spoken of just now, may perhaps be attributed to the same local mechanism. Further it is stated that with stimulation of the sympathetic, the latent period, $i$. e., the period intervening between the beginning of stimulation and the beginning of the movement of the iris, is much greater than with stimulation of the third nerve, indicating that the former acts through a local mechanism but the latter more directly on the muscular fibres. The whole question, however, of this local mechanism, and of the exact mode of action of the various drugs and of the changes in the body which lead to contraction or dilatation respectively of the pupil, needs fuller discussion than we can afford to give to it here. We may add that the local action of atropine in contrast to any action on the cerebral centre is well illustrated by applying atropine to one eye locally. The pupil of that eye dilates widely; in consequence more light falls on the retina, and this so affects the cerebral centre, which as we have seen is not strictly unilateral but in communion with its fellow, that increased constricting impulses pass from both centres, and these, though ineffectual in the atropinized eye, lead in the untouched eye to an increased narrowing of the pupil.

$\$ 637$. The share of the fifth nerve in the work of the iris seems to be in part a sensory one; the iris is sensitive, and the sensory impulses which are generated in it pass from it along the fibres of the fifth nerve. 
We may sum up the nervous mechanism of the pupil then somewhat as follows: The salient and most frequently repeated event, the contraction of the pupil upon exposure to light, is a reflex act, the centre of which is placed in the brain; and the correlative widening of the pupil upon diminution of light is due to the tonic action of the sympathetic making itself felt upon the waning of its antagonist. The contraction of the pupil in the earlier stages of the action of alcohol and chloroform and in slumber is probably due to an increased action of the contracting centre, but the narrow pupil caused by such drugs as morphine and physostigmine is due, chiefly at least, to a local action. 'The dilating effects of such drugs as atropine are also largely due to a local action, but in the widened pupil of the later stages of alcohol poisoning and of dyspnœa we can probably trace the effects of an exhaustion of the cerebral contracting centre, assisted possibly by an increased activity of the dilating centre.

$\$ 638$. There remains a word to be said concerning the contraction of the pupil which takes place when the eye is accommodated for near objects, and when the pupil is turned inward (the two being closely allied, since the eyes converge to see near objects), and the return to the more dilated condition when the eye returns to rest and regains the accommodation for far objects. These are instances of what are called "associated movernents." Two movements are thus spoken of as "associated" when the special central nervous mechanism employed in carrying out the one act is so connected by nervous ties of some kind or other with that employed in carrying out the other, that when we set the one mechanism in action we unintentionally set the other in action also. The ciliary muscles which bring about acconmodation are governed in this action by fibres that may be traced, through the ciliary nerves and lenticular ganglion, along the third or oculo-motor nerve, to a centre which lies (in dogs) in the hind part of the floor of the third ventricle, and which is especially connected with the most anterior bundles of the roots of the third nerve. This centre is under the command of our will; when we wish to accommodate for near objects we throw it into action, and, when in action, it calls also into action by "association" the centre for the contraction of the pupil; when the action of the accommodation centre ceases and the eye falls back to the condition of rest, in which it is accommodated for far objects, the action of the pupil-contracting centre ceases also, and the pupil therefore widens.

$\$ 639$. The mechanism of accommodation may also be affected in a local manner. And the drugs which have a special action on the pupil, such as atropine and Calabar bean, also affect the mechanism of accommodation. Atropine paralyzes it, so that the eye remains adjusted for far objects; and physostigmine throws the eye into a condition of forced accommodation for near objects. This double action has been explained by the supposition that while atropine paralyzes, physostigmine throws into tonic or tetanic contraction, on the one hand, the circular muscles of the iris and, on the other, the ciliary muscles; but the phenomena, on inquiry, appear too complicated to be explained in so simple a manner.

We can accomodate at will; but few persons can effect the necessary change in the eye unless they direct their attention to some near or far object, as the case may be, and thus assist their will by visual sensations. By practice, however, the aid of external objects may be dispensed with; and it is when this is achieved that the pupil may be made to dilate or contract at pleasure, accommodation being effected without the eye being turned to any particular object. 


\section{Imperfections in the Dioptric Apparatus.}

$\$ 640$. The enmetropic eye may be taken as the normal eye. The myopic and hypermetropic eyes may be considered as imperfect eyes, though the former possesses certain advantages over the normal eye. An eye might be myopic from too great a convexity of the cornea or of the anterior surface of the lens, or from permanent spasm of the accommodation-mechanism, or from too great a length of the long axis of the eyeball. The last appears to be the usual cause. Similarly, most hypermetropic eyes possess too short a bulb. Moreover, in the strongly-marked myopic eye there is frequently hypertrophy of the longitudinal (meridional) fibres of the ciliary muscle, often spoken of exclusively as the ciliary muscle, and atrophy or absence of the circular fibres; in the hypermetropic eye, on the other hand, the circular fibres are well developed and the meridional fibres scanty. The presbyopic eye is, as we have seen, an eye normally constituted in which the power of accommodation has been lost or is failing through increasing weakness of the ciliary muscle or a loss of elasticity in the lens, or through the parts becoming rigid.

\$641. Spherical aberration. In a spherical lens the rays which impinge on the circumference are brought to a focus sooner than those which pass nearer the centre, and the rays proceeding from a luminous point are no longer brought to a single focus at one point, but form a number of foci at different distances. Hence when rays are allowed to fall on the whole of the lens, the image formed on a screen placed in the focus of the more central rays is blurred by the diffusion-circles caused by the circumferential rays which have been brought to a premature focus. In an ordinary optical instrument spherical aberration is obviated by a diaphragm which shuts off the more circumferential rays. In the eye the iris is an adjustable diaphragm ; and when the pupil contracts in near vision the more divergent rays proceeding from a near object which tend to fall on the circumferential parts of the lens are cut off. As, however, the refractive power of the lens does not increase regularly and progressively from the centre to the circumference, but varies most irregularly, the purpose of the narrowing of the pupil cannot be simply to obviate spherical aberration; and indeed the other optical inperfections of the eye are so great that such spherical aberrations as are caused by the lens produce no obvious effect on vision.

$\$ 642$. Astigmatism. We have hitherto treated the eye as if its dioptric surfaces were all parts of perfect spherical surfaces. In reality this is rarely the case, either with the lens or with the cornea. Slight deviations do not produce any marked effect, but there is one deviation, known as regular astigmatism, which, present to a certain extent in most eyes, very largely developed in some, frequently leads to very imperfect vision. This defect is due to the dioptric surface being not spherical but more convex along one meridian than another, more convex, for instance, along the vertical than along the horizontal meridian. When this is the case the rays proceeding from a luminous point are not brought to a single focus at a point, but possess two linear foci, one nearer than the normal and corresponding to the more convex surface, the other further than the normal focus and corresponding to the less convex surface. If the vertical meridians of the surface be more convex than the horizontal, then the nearer linear focus will be horizontal and the further linear focus will be vertical, and vice versa. (This can be shown much more effectually on a model than in a diagram in which we are limited to two dimensions.) Now, in order to see a vertical line distinctly, it is much more important that the rays which diverge from the line in a series of horizontal planes should be brought to a focus prop- 
erly than those which diverge in the vertical plane of the line itself; and sinilarly, in order to see a horizontal line distinctly, it is much more important that the rays which diverge from the line in a series of vertical planes should be brought to a focus properly than those which diverge in the horizontal plane of the line itself. Hence a horizontal line held before an astigmatic dioptric surface, most convex in the vertical meridians, will give rise to the image of a horizontal line at the nearer focus, the vertical rays diverging from the line being here brought to a linear horizontal focus. Similarly, a vertical line held before the same surface will give rise to an image of a vertical line at the further focus, the horizontal rays diverging from the vertical line being , here brought to a linear vertical focus. In other words, with a dioptric surface most convex in the vertical meridians horizontal lines are brought to a focus sooner than are vertical lines.

Most eyes are thus more or less astigmatic, and generally with a greater convexity along the vertical meridians. If a set of horizontal or vertical lines be looked at, or if the near point of accommodation be determined by Scheiner's experiment (p. 747), for the needle placed first horizontally and then vertically, the horizontal lines or needle will be distinctly visible at a shorter distance from the eye than the vertical lines or needle. Similarly, the vertical line must be further from the eye than a horizontal one if both are to be seen distinctly at the same time. The cause of astigmatism is, in the great majority of cases, the unequal curvature of the cornea ; but sometimes the fault lies in the lens, as was the case with Young.

When the curvature of the cornea or lens differs not in two meridians only but in several, irregular astigmatism is the result. A certain amount of irregular astigmatism exists in most lenses, thus causing the image of a bright point, such as a star, to be not a circle but a radiate figure.

$\$ 643$. Chromatic aberration. The different rays of the spectrum are of different refrangibility, those toward the violet end of the spectrum being brought to a focus sooner than those near the red end. This in optical instruments is obviated by using compound lenses made up of various kinds of glass. In the eye we have no evidence that the lens is so constituted as to correct this fault ; still the total dispersive power of the instrument is so small that such amount of chromatic aberration as does exist attracts little

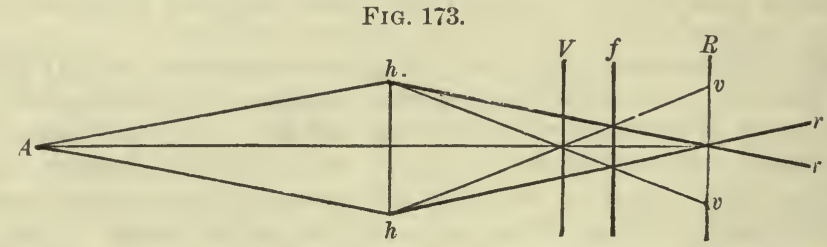

Diagram illustrating Chromatic Aberration. $h h$ is the dioptric surface; $h v$ represents the blue and $h r$ the red rays; $V$ is the focal plane of the blue, $R$ of the red rays.

notice. Nevertheless, some slight aberration may be detected by careful observation. When the spectrum is observed at some distance the violet end will not be seen in focus at the same time as the red. If a luminous point be looked at through a narrow orifice covered by a piece of violet glass, which while shutting out the yellow and green allows the red and blue rays to pass through, there will be seen alternately an image having a blue centre with a red fringe, or a red centre with a blue fringe, according as the image of the point looked at is thrown on one side or other of the true focus. Thus supposing $f$ (Fig. 173) to be the plane of the mean focus of $A$, the violet rays will be brought to a focus in the plane $V$, and the red 
rays in the plane $R$. If the rays be supposed to fall on the retina between $V$ and $f$, the diverging or blue rays will form a centre surrounded by the still converging red rays; whereas if the rays fall on the retina between $f$ and $R$, the converging red rays will form a centre with the still diverging blue rays forming a fringe around them. If the rays fall on the retina at $f$, the two kinds of rays will be mixed together; as will be seen from the figure, the circumferential converging red ray $h r$ as it cuts the plane of the retina is, in ordinary vision, accompanied by the diverging violet ray $h v$, and thus by a sort of compensation, we see together even the rays which differ most in refraction.

$\S 644$. Entoptic phenomena. The various media of the eye are not uniformly transparent; the rays of light in passing through them undergo local absorption and refraction, and thus various shadows are thrown on the retina, of which we become conscious as imperfections in the field of vision, especially when the eye is directed to a uniformly illuminated surface. These are spoken of as entoptic phenomena, and are very varied, many forms having been described.

The most common are those caused by the presence of floating bodies in the vitreous humor, the so-called muscce volitantes. These are readily seen when the eye is turned toward a uniform surface, and are frequently very troublesome in looking through a microscope. They are especially obvious when divergent rays fall upon the eye. They assume the form of rows and groups of beads, of single beads, of streaks, patches, and granules, and may be recognized by their almost continual movement, especially when the head or eye is moved up and down. When an attempt is made to fix the vision upon them, they immediately float away. Tears on the cornea, temporary unevenness on the anterior surface of the cornea after the eyelid has been pressed on it, and imperfections in the lens or its capsule, also give rise to visual images. Not unfrequentily a radiate figure corresponding to the arrangement of the fibres of the lens makes its appearance.

Imperfections in the margin of the pupil appear in the shadow of the iris which bounds the field of vision; and the movements of the iris in one eye may be rendered visible by looking at a bright point or luminous surface through a pin-hole in a card placed close in front of the eye, in the anterior focus in fact, and then alternately closing and opening the other eye; the field of the first may be observed to contract when light enters and to expand when the light is shut off from the second. The media of the eye are fluorescent: a condition which favors the perception of the ultra-violet rays. If a white sheet or white cloud be looked at in daylight through a Nicol's prism, a somewhat bright double cone or double tuft, with the apices touching, of a faint blue color, is seen in the centre of the field of vision, crossed by a similar double cone of a somewhat darker yellow color. These are spoken of as Haidinger's brushes; they rotate as the prism is rotated, and are supposed to be due to the unequal absorption of the polarized light in the yellow spot. The prism must be frequently rotaterl, as when the prism remains at rest the phenomena fade. Lastly, the optical arrangements have a further imperfection in that the dioptric surfaces are not truly centred on the optic axis.

\section{Visual Sensations.}

$\$ 645$. Light falling on the retina excites sensory impulses, and these passing up the optic nerve to certain parts of the brain, produce changes in certain cerebral structures, and thus give rise to what we call a sensation. In a sensation we ought to be able to distinguish between the events through 
which the impact of the rays of light on the retina is enabled to generate sensory impulses, and the events, or rather series of events, through which these sensory impulses (for, judging by the analogy of motor nerves, we have no reason to think that they undergo any fundamental changes in passing along the optic nerve), by the agency of the cerebral arrangements, develop into a sensation. Such an analysis, however, is, at present at least, in most particulars, quite beyond our power ; and we must therefore treat of the sensations as a whole, distinguishing between the peripheral and central phenomena, on the rare occasions when we are able to do so.

\section{The Origin of Visual Impulses.}

$\$ 646$. Of primary importance to the understanding of the way in which luminous undulations give rise to those nervous changes which pass along the optic nerve as visual impulses, is the fact that the rays of light produce their effect by acting not on the optic nerve itself but on its terminal organs. They pass through the anterior layers of the retina apparently without inducing any effect; it is not till they have reached the region of the rods and cones that they set up the changes concerned in the generation of visual impulses; and the impulses here generated travel back to the layer of fibres in the anterior surface of the retina and thence pass along the optic nerve. That the optic fibres are themselves insensible to light and that visual impulses begin in the region of rods and cones is shown by the phenomena of the blind spot and of Purkinje's figures respectively.

$\S 647$. Blind spot. There is one part of the retina on which rays of light falling give rise to no sensations; this is the entrance of the optic nerve, and the corresponding area in the field of vision is called the blind spot. If the visual axis of one eye, the right for instance, the other being closed, be fixed on a black spot in a white sheet of paper, and a small black object, such as the point of a quill pen dipped in ink, be moved gradually sideways over the paper away to the outside of the field of vision, at a certain distance the black point of the quill will disappear from view. On continuing the movement still further outward the point will again come into view and continue in sight until it is lost in the periphery of the field of vision. If the pen be used to make a mark on the paper at the moment when it is lost to view, and at the moment when it comes into sight again, and if similar marks be made along the other meridians as well as the horizontal, an irregular outline will be drawn circumscribing an area of the field of vision within which rays of light produce no visual sensation. This is the blind spot. The dimensions of the figure drawn vary, of course, with the distance of the paper from the eye. If this distance be known, the size as well as the position of the area of the retina corresponding to the blind spot may be calculated from the diagrammatic eye (p. 745). The position exactly coincides with the entrance of the optic nerve, and the dimensions (about $1.5 \mathrm{~mm}$. diameter) also correspond. While drawing the outline as above directed the indications of the large branches of the retinal vessels as they diverge from the entrance of the nerve can frequently be recognized. The existence of the blind spot is also shown by the fact that an image of light, sufficiently small, thrown upon the optic nerve by means of the ophthalmoscope, gives rise to no sensations.

The existence of the blind spot proves that the optic fibres themselves are insensible to light; it is only through the agency of the retinal expansion that these can be stimulated by luminous vibrations.

$\$ 648$. Purkinjé's figures. If one enters a dark room with a candle, and while looking at a plain (not parti-colored) wall, moves the candle up and 
down, holding it on a level with the eyes by the side of the head, there will appear in the field of vision of the eye of the same side, projected on the wall, an image of the retinal vessels, quite similar to that seen on looking into an eye with the ophthalmoscope. The field of vision is illuminated with a glare, and on this the branched retinal vessels appear as shadows. In this mode of experimenting the light enters the eye through the cornea, and an image of the candle is formed on the nasal side of the retiria; and it is the light emanating from this image which throws shadows of the retinal vessels on to the rest of the retina. A far better method is for a second person to concentrate the rays of light, with a lens of low power, on to the outside of the sclerotic just behind the cornea; the light in this case emanates from the illuminated spot on the sclerotic and passing straight through the vitreous humor throws a direct shadow of the vessels on to the retina. Thus the rays passing through the sclerotic at $b$, Fig. 174, in the direction $b \nu$, will throw a shadow of the vessel $\nu$ on to the retina at $\beta$; this will appear as a dark line at $B$ in the glare of the field of vision. This proves that the structures in which visual impulses originate must lie behind the retinal vessels, otherwise the shadows of these could not be perceived.

If the light be moved from $b$ to $a$, the shadow on the retina will move from $\beta$ to $\alpha$, and the dark line in the field of vision will move from $B$ to $A$. If the distance $B A$ be measured when the whole image is projected at a

FIG. 174.

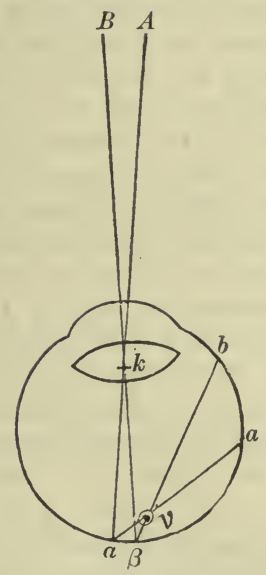

Diagram illustrating the Formation of Purkinje's Figures when the Illumination is Directed through the Sclerotic.
Fig. 175 .

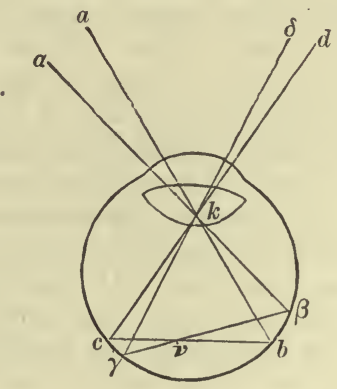

Diagram illustrating the Formation of Purkinje's Figures when the Illumination is Directed through the Cornea.

known distance, $k B$ from the eye, $k$ being the optical centre, ${ }^{1}$ then, knowing the distance $k \beta$ in the diagrammatic eye, the distance $\beta \alpha$ can be calculated. But if the distance $\beta \alpha$ be thus estimated, and the distance $b a$ be directly measured, the distances $\beta \nu, \alpha \nu, b \nu, a \nu$ can be calculated, and if the appearance in the field of vision is really caused by the shadow of $\nu$ falling on $\beta$, these distances ought to correspond to the distances of the retinal vessels $\nu$ from the

\footnotetext{
1 For the properties of the optical centre we must refer the reader to the various treatises on optics. The optical centre of a lens is the point through which all the prineipal rays, of the various pencils of rays falling on the lens, pass. The diagrammatic eye of Listing (p. 745) has two optical centres, but these may, without serious error, be further reduced for practical purposes to one lying in the lens near its posterior surface, at about $15 \mathrm{~mm}$. distance from the retina.
} 
sclerotic $b$ on the one hand, and from that part of the retina $\beta$ where visual impressions begin, on the other. H. Müller found that the distance $\beta$, thus calculated corresponded to the distance of the retinal vessels from the layer of rods and cones. Thus Purkinjé's figures prove in the first place that the sensory impulses which form the commencement of visual sensations originate in some part of the retina behind the retinal vessels, $i$. $e$, somewhere between them and the choroid coat; and H. Müller's calculations go far to show that they originate at the most posterior or external part of the retina, viz., the layer of rods and cones. It must be adnitted, however, that $H$. Müller's results were not sufficiently exact to allow any great stress to be placed on this argument.

In the second method of experimenting the image always moves in the same direction as the light, as it obviously must do. In the first method, where the light enters through the cornea, the image moves in the same direction as the light when the light is moved from right to left, provided the movement does not extend beyond the middle of the cornea, but in the opposite direction to the light when the latter is moved up and down. In Fig. 175, which represents a horizontal section of an eye, if $a$ be moved to $\alpha$, $b$ will move to $\beta$, the shadow on the retina $c$ to $\gamma$, and the image $d$ to $\delta$. If, on the other hand, $a$ be supposed to move above the plane of the paper, $b$ will move below, in consequence $c$ will move above, and $d$ will appear to move below, $i$. $e$, $d$ will sink as $a$ rises.

It is desirable in these cases to move the light to and fro, especially in the first method, as the retina soon becomes tired, and the image fades away. Some observers can recognize in the axis of vision a faint shadow corresponding to the edge of the depression of the fovea centralis.

$\S 649$. The retinal vessels may also be rendered visible by looking through a small orifice, such as a pin-hole in a card placed close to the eye, at a bright field such as the sky, and moving the orifice very rapidly from side to side or up and down. If the movement be from side to side the vessels which run vertically will be seen; if up and down, the horizontal vessels. The fine capillary vessels are seen more easily in this way than by Purkinjés method. The same appearances may also be produced by looking through a microscope from which the objective has been removed and the ere-piece only left (or in which, at least, there is no object distinctly in focus in the field), and moving the head rapidly from side to side or backward and forward. Or the microscope itself may be moved; a circular movement of the field will then bring both the vertically and horizontally directed vessels into view at the same time.

$\$ 650$. The photo-chemistry of the retina. In seeking to understand how it is that rays of light falling upon the region of the rods and cones can give rise to sensory, visual impulses in the optic nerve, we may adopt one or other of two views. On the one hand, we may suppose that the vibrations of the ether are able, through the means of the retinal apparatus of the rods and cones for example, to give rise in some way or other to molecular vibrations which are the beginning of the nervous impulses in the optic nerve. No satisfactory explanation of how such a change can be brought about has been offered, and indeed the difficulties of such a conception are very great. On the other hand, we may more naturally turn to a chemical explanation. We are familiar with the fact that rays of light are able to bring about the decomposition of very many chemical substances, and we accordingly speak of these substances as being sensitive to light. All the facts dwelt on in this book illustrate the great complexity and corresponding instability of the composition of protoplasm. And we might reasonably suppose that protoplasm itself would be sensitive to light; that is to say, that rays of light 
falling on even undifferentiated protoplasm might set up a decomposition of that protoplasm, and so inaugurate a molecular disturbance; in other words, that light might act as a direct stimulus to protoplasm. As a matter of fact, however, such evidence as we at present possess goes to show that native undifferentiated protoplasm is, as a rule, not sensitive to light (that is, to those particular waves which, when they fall on our retina give rise in us to the sensation of light), though in the case of some lowly organisms, whose protoplasm exhibits very little differentiation and in particular contains no pigment, a sensitiveness to light has been observed. Nor can we be surprised at this indifference of protoplasm when we reflect that what we may call pure protoplasm is remarkable for its transparency, that is to say, the rays of light pass through it with the slightest possible absorption. But in order that light may produce chemical effects, it must be absorbed; it must be spent in doing the chemical work. Accordingly, the first step toward the formation of an organ of vision is the differentiation of a portion of protoplasm into a pigment at once capable of absorbing light and sensitive to light, i. e., undergoing decomposition upon exposure to light. An organism, a portion of whose protoplasm had thus become differentiated into such a pigment, would be able to react toward light. The light falling on the organism would be in part absorbed by the pigment, and the rays thus absorbed would produce a chemical action and set free chemical substances which before were not present. We have only to suppose that the chemical substances are of such a nature as to act as a stimulus to the protoplasm of other parts of the organism (and we have manifold evidence of the exquisite sensitiveness of protoplasm in general to chemical stimuli), in order to see how rays of light falling on the organism might excite movements in it, or modify movements which were being carried on, or might otherwise affect the organism in whole or in part.

Such considerations as the foregoing may be applied to even the complex organ of vision of the higher aninials. If we suppose that the actual terminations of the optic nerve are surrounded by substances sensitive to light, then it becomes easy to imagine how light falling on these sensitive substances should set free chemical bodies possessed of the property of acting as stimuli to the actual nerve-endings, and thus give rise to visual impulses in the optic fibres. We say "easy to imagine," but we are at present far from being able to give definite proofs that such an explanation of the origin of visual impulses is the true one, probable and enticing as it may appear.

$\S 651$. One of the most striking features in the structure of the retina is the abundance of black pigment in the retinal, or as it is sometimes called choroidal, epithelium. It is difficult to suppose that the sole function of this pigment is to absorb the superfluous rays of light, and that the rays thus absorbed are put to no use, but simply wasted. And indeed it has been shown that the pigment is sensitive to light; but the changes in it induced by light are excessively slow. Moreover, its presence cannot be of fundamental importance, since vision is not only possible but fairly distinct with albinos, in which this pigment is absent.

Then again, in the vast majority of vertebrate animals, the outer limbs of the rods are suffused with a purplish-red pigment, the so-called visual purple, which is so eminently sensitive to light that images of external objects may by appropriate means be photographed in it on the retina. When the eye of a frog or of a rabbit is examined in an ordinary way, with full exposure to light, the retina appears colorless. But if the eye be kept in the dark for some time before it is examined, the retina, if removed rapidly, will be found to be of a beautiful purplish-red color. Upon exposure to light the 
color changes to yellow and then fades away, leaving, however, the retina not only white, but more opaque than it was before. Upon examination with the microscope it is found that the purple color is confined exclusively to the rods and to the outer limbs of the rods, the inner limbs being wholly devoid of it.

$\S 652$. The color of the rods is due to the presence of a distinct pigment, the "visual purple," diffused through the substance of the outer limbs; and this may be extracted from the rods by dissolving these in an aqueous solution of bile-salts. A clear purple solution is thus obtained, which is capable of being bleached by the action of light, and in its general features and behavior is similar to the pigment as it naturally exists in the retina.

Visual purple is found, as we have said, exclusively in the outer limbs of the rods; it has never yet been found in the cones, and it is accordingly absent from the retinas (such as those of snakes), which are composed of cones only, and from the macula lutea and fovea centralis of the retinas of man and the ape. The intensity of the coloration varies in different animals, and the retinas even of some animals possessing rods (bat, dove, hen) seem to be wholly devoid of the visual purple; it is generally well marked in retinas in which the outer limbs of the rods are well developed. Its absence or presence is not dependent on nocturnal habits, since the intense color of the retina of the owl is in strong contrast to the absence of color in the bat It has been found in the retina of the embryo.

$\S 653$. The visual purple is bleached not only by white, but also by monochromatic, light. Of the various prismatic rays the most active are thi greenish-yellow rays, those to the blue side of these coming next, the leas active being the red. Now it is precisely the greenish-yellow rays which ar most readily absorbed by the color itself. A natural colored retina or a solu. tion of visual purple gives a diffuse spectrum without any defined absorption bands, and according to the amount of coloring material through which tho light passes, absorption is seen either to be limited to the greenish-yellow part of the spectrum or to spread thence toward the blue, and, to a much. less extent, toward the red. Thus the various prismatic rays produce photo-chemical effect on the visual purple in proportion as they are absorbew by it. Under the action of light the visual purple, whether in solution 0 . in its natural condition in the rods, passes through a purplish-orange to i yellow, and finally becomes colorless; and we appear to be justified in speaking of a "visual yellow" and "visual white" as products of the photo-chemical changes undergone by the visual purple.

For the restoration of the visual purple, after it has been destroyed by light, the maintenance of the circulation of the blood through the tissues of the eye is not essential. The choroidal epithelium has by itself, provided that it still retains its tissue life, the power of regenerating the purple. If a portion of the retina of an excised eye be raised from its epithelial bed, bleached, and then carefully restored to its natural position, the purple will return if the eye be kept in the dark. The choroidal epithelium may, in fact, be spoken of as a "purpurogenous" membrane.

$\S 654$. If the image of some bright object, such as a lamp or a window, be thrown on to the retina, either of an eye in its natural position or of one recently excised, care having been taken to keep the retina for some time previous away from any rays of light, the portion of the retina on which the rays have fallen will be found to be bleached, the rest of the retina remaining purple. In fact, an "optngram" of external objects may thus be obtained; and if the retina be removed and treated with a four per cent. solution of potash alum before the choroidal epithelium has had time to obliterate the bleaching effects, the retina may remain permanently in that 
condition; the photo-chemical effect may, as the photographers say, be "fixed."

It seemed very tempting, especially upon the first discovery of it, to suppose that this visual purple is directly concerned in vision. If we suppose that visual purple itself is inert toward the endings of the optic nerve, but that either visual yellow or visual white, $i$. e., some product of the action of light on visual purple, may act as a stimulus to those endings, the way seems opened to understanding how rays of light can give rise to sensory impulses in the optic nerve. Unfortunately visual purple is absent from the cones, and from the fovea centralis, which, as we shall see, is the region of distinct vision; it is further entirely wanting in some animals which undoubtedly see very well; and, lastly, animals, such as frogs, naturally possessing the pigment, continue to see very well, and even apparently to see colors, when their visual purple has been absolutely bleached, as it may be by prolonged exposure of the eyes to strong light. We cannot therefore, at present at least, explain the origin of visual impulses by the help of visual purple. At the same time its history suggests that some substances, sensitive like it to light, but unlike it, colorless and therefore escaping observation, may exist, and by photo-chemical changes be the means of exciting the optic nerves. And, as we shall see later on, one theory of color vision is based on the assumption that vision is carried on in some way or other by changes in what may be called the visual substances present in the retina, these substances being used up and regenerated as vision is going on.

But even admitting as probable the existence of these sensitive visual substances, the changes in which lead to stimulation of the real endings of the retinal nervous mechanism, we cannot at present state anything definite concerning those nerve-endings or the manner of their stimulation. It may be that even the outer limbs of the rods and cones, in spite of the apparent break of continuity between the outer and inner limbs, are really nervous in nature. It may be, on the other hand, that the outer limbs are either purely dioptric in function, or are associated with the sensitive visual substances in such a way that the purely nervous structures must be considered as extending no further at least than the inner limbs. We cannot as yet make any definite statement in the one direction or the other.

$\S 655$. In connection with the origin of visual impulses, we may perhaps call attention to the remarkable changes which the cells of the retinal pigment epithelium undergo under the influence of light. When an eye has been shut off from all light for some little time the pigment is concentrated in the bodies of the cells, and the remarkable filamentous processes of the cells, with the pigment granules or crystals which they carry, extend a slight distance only between the limbs of the rods and cones (about one-third down the length of the outer limbs of the rods). Under the influence of light these processes, loaded with pigment, thrust themselves a much longer way down toward the external limiting membrane; in consequence a considerable quantity of pigment is found massed between the outer and even the inner limbs of the rods and cones; indeed, the outer limbs of the rods swelling at the same time become jammed, as it were, between the masses of pigment, causing the epithelial layer to adhere very closely to the layer of rods and cones.

$\S 656$. The retina and optic nerve, like other nervous structures, develop electric currents, which may be spoken of as currents of rest and currents of action. They may be shown by placing one electrode on the retina of a bisected eye, or on the cornea of a whole one, and the other on the optic nerve, or hind part of the eyeball, or even on some distant part of the body. They are also manifested by the isolated retina itself. The phenomena 
appear somewhat complicated by the appearance now of positive, now of negative, variations; but this fact comes out clearly, that the incidence of light on the irritable retina develops an electric change, the magnitude of which is to a certain extent proportionate to the intensity of the light acting as a stimulus. The changes accordingly diminish and cease to appear as the retina gradually loses its irritability after death. IVe may add that these electric phenomena appear to be quite independent of the condition of the visual purple.

\section{Simple Sensations.}

$\S 657$. Relations of the sensation to the stimulus. If we put aside for the present all questions of color, we may say that light, viewed as a stimulus affecting the retina, varies in intensity, that is, in the energy of the luminous vibrations as manifested by their amplitude, and in duration, that is, in the length of time a succession of waves continues to fall upon the retina. The effect of the light will also depend on the extent of retinal surface exposed to the luminous vibrations at the same time. Taking a luminous point, in order to eliminate the latter circumstance, we may make the following statements :

The sensation has a duration much greater than that of the stimulus, and in this respect is comparable to a muscular contraction caused by such a stimulus as a single induction-shock. The sensation of a flash of light, for instance, lasts for a much longer time than that during which luminous vibrations are falling on the retina. Hence, when two stimuli, such as two flashes of light, follow each other at a sufficiently short interval, the two sensations are fused into one; and a luminous point moving rapidly round in a circle gives rise to the sensation of a continuous circle of light. This again is quite comparable to muscular tetanus. The interval at which fusion takes place, that is, the interval between successive stimuli which must be exceeded in order that successive distinct sensations may be produced, varies according to the intensity of the light, being shorter with the stronger light; with a faint light it is about $\frac{1}{10}$ second, with a strong light $\frac{1}{30}$ or $\frac{1}{50}$ second. This may be shown by rotating rapidly before the eye a disc arranged with alternate black and white sectors of equal width. With a faint illumination the flickering, indicative of the successive sensations from the white sectors not being completely fused, ceases when the rotation becomes so rapid that each pair of black and white sectors takes only $\frac{1}{10}$ second in passing before the eye. When a brighter illumination is used the rapidity must be increased before the flickering disappears. That part of the sensation which is recognized as lasting after the cessation of the stimulus is frequently spoken of as the "after-image."

Though the duration of the after-image is longer with the stronger light (that caused by looking even momentarily at the sun lasting for some time), the commencement of the decline of the sensation begins relatively earlier, hence the greater difficulty in the complete fusion of successive sensations with the stronger light. The interval at which fusion takes place differs with different colors, being shortest with yellow, intermediate with red, and longest with blue.

The duration of a stimulus necessary to call forth a sensation is exceedingly short; thus the shortest possible flash, such as that of an electric spark, gives rise to a sensation of light.

Objects in motion when illuminated by a single electric spark appear motionless, the stimulus of the light reflected from them ceasing before they can make an appreciable change in their position. When a moving body is 
illuminated by several rapid flashes in succession, several distinct images corresponding to the positions of the body during the several flashes are generated; the images of the body corresponding to the several flashes fall on different parts of the retina.

$\$ 658$. The intensity of the sensation varies with the luminous intensity of the object; a wax candle appears brighter than a rushlight. The ratio, however, of the sensation to the stimulus is not a simple one. If the luminosity of an object be gradually increased from a very feeble stage to a very bright one, it will be found that though the corresponding sensations likewise gradually increase, the increments of the sensations due to increments of the luminosity gradually diminish; and at last an increase of the luminosity produces no appreciable increase of sensation; a light, when it reaches a certain brightness, appears so bright that we cannot tell when it becomes any brighter. Hence it is much easier to distinguish a slight difference of brightness between two feeble lights than the same difference between two bright lights; we can easily tell the difference between a rushlight and a wax candle; but two suns, or even two bright lamps, one of which differed from the other merely by just the number of luminous rays which a wax candle emits in addition to those sent forth by a rushlight, would appear to us to have exactly the same brightness. In a darkened room an object placed before a candle will throw what we consider a deep shadow on a sheet of paper or any white surface. If, however, sunlight be allowed to fall on the paper at the same time from the opposite side the shadow is no longer visible. The difference between the total light reflected from that part of the paper where the shadow was, and which is illuminated by the sun aloue, and that reflected from the rest of the paper which is illuminated by the candle as well as by the sun, remaius the same; yet we can no longer appreciate that difference.

On the other hand, if using two rushlights we throw two shadows on a white surface and move one rushlight away until the shadow caused by it ceases to be visible, and, having noted the distance to which it had to be moved, repeat the same experiment with two wax candles, we shall find that the wax candle has to be moved just as far as the rushlight. In fact, it is found by careful observation that, within tolerably wide limits, the smallest difference of light which we can appreciate by visual sensations is a constant fraction (about $\frac{1}{10}$ th) of the total luminosity employed. The same law holds good with regard to the other seuses as well. The smallest difference in length we can detect between two lines, one an inch long and the other a little less than an inch, is the same fraction of an inch that the smallest difference in length we can detect between a line a foot long and one a little less than a foot, is of a foot. Put in a more general form, then, the law, which is often called Weber's law, is as follows: When a stimulus is continually increased, the increase of stimulus necessary to call forth the smallest appreciable increase of sensation always bears the same proportion to the whole stimulus.

$\$ 659$. Distinction and fusion of sensations. When light falls on a large portion of the retina the total sensation produced is greater in amount than when a small portion only of the retina is affected; a large piece of white paper produces a greater total effect on our consciousness than a small one, though, if the surfaces be uniformly and equally illuminated, the intensity of the sensation is in each case the same; the small piece of paper appears as bright or as "white" as the large one. If the images of two luminous objects fall on the retina at sufficient distances apart, the consequent sensations

1 From which Fechner, by an assumption, obtained a mathematical expression or formula, which is sometimes incorrectly spoken of as Fechner's law. 
are distinct, and the intensity of each sensation will depend solely upon the luminosity of the corresponding object. If, however, the two objects are made to approach each other, a point will be reached at which the two sensations are fused into one. When this occurs the intensity of the total sensation produced will be greater than that of either of the sensations caused by the single objects. A number of luminous points scattered over a wide surface would appear each to have a certain brightness; each would give rise to a sensation of a certain intensity. If they were all gathered into one spot, that spot would appear far brighter than any of the previous points; the intensity of the sensation would be greater. We may therefore suppose the retina to be divided into areas corresponding to sensational units. If the images from two luminous objects fall on separate visual areas, if we may so call them, two distinct sensations will be produced; if, on the contrary, they both fall on the same visual area, one sensation only will be produced. Where the sensations are separate, the intensity of the one (with exceptions hereafter to be mentioned) is not affected by the presence of the other; but where they become fused the intensity of the united sensations is greater than either of, though not equal to the sum of, the single sensations. The existence of these sensational units is the basis of distinct vision. When we speak of the smallest size visible or distinguishable, we are referring to the dimensions of the retinal areas corresponding to these sensational units. The retinal area must be carefully distinguished from the sensational unit, for the sensation is, as we have seen, a process whose arena stretches from the retina to certain parts of the brain, and the circumscription of the sensational unit, though it must begin as a retinal area, must also be continued as a cerebral area in the brain, the latter corresponding to, and being as it were the projection of, the former. With most people two stars appear as a single star when the distance between them subtends an angle of less than 60 seconds; and the best eyes generally fail to distinguish two parallel white streaks when the distance between the two, measured from the middle of each, subtends an angle of less than 73 seconds. Some, however, can distinguish objects 50 seconds distant from each other. An angle of 73 seconds in an object corresponds in the diagrammatic eye (see p. 745 ) to the length of $5.36 \mu$ in the retinal image, ${ }^{1}$ and one of 50 seconds to $3.65 \mu$.

$\$ 660$. In the human eye 50 cones may be counted along a line of $200 \mu$ in length drawn through the centre of the yellow spot; this would give $4 \mu$ for the distance between the centres of two adjoining cones in the yellow spot, the average diameter of a cone at its widest part being $3 \mu$, and there being slight intervals between neighboring cones. Hence, if we take the centre of a cone as the centre of an anatomical retinal area, these anatomical areas correspond very fairly to the physiological visual areas as determined above. That is to say, if two points of the retinal image are less than $4 \mu$ apart, they may both lie within the area of a single cone; and it is just when they are less than about $4 \mu$ apart that they cease to give rise to two distinct sensations. It must be remembered, however, that the fusion or distinction of the sensations is ultimately determined by the brain and not by the retina. Two points of the retinal image less than $4 \mu$ apart might lie both within the area of a single cone; but the reason why, under such circumstances, they give rise to one sensation only is not because one conefibre only is stimulated. Two points of a retinal image might lie, one on the area of one cone and another on the area of an adjoining cone, and still be less than $4 \%$ apart; in such a case two cone-fibres would be stimulated,

${ }^{1} \mathrm{By} \mu$ is meant the micromillimeter, one one-thousandth of a millimeter. 
and yet only one sensation would be produced. So also in the less sensitive peripheral parts of the retina two points of the retinal image might stimulate two cones a considerable distance apart, and yet give rise to one sensation only.

In the case where the two points lie entirely within the area of a single cone, it is exceedingly probable that, even if the adjacent cones or conefibres in the retina are not at the same time stimulated, impulses radiate from the cerebral ending of the excited cone into the neighboring cerebral endings of the neighboring cones; in other words, the sensation-area in the brain does not exactly correspond to and is not sharply defined like the retinal area, but gradually fades away into neighboring sensation-areas. We may imagine two points of the retinal image so far apart that even the extreme margins of their respective cerebral sensation-areas do not touch each other in the least; in such a case there can be no doubt about the two points giving rise to two sensations. We might, however, imagine a second case where two points were just so far apart that their respective sensationareas should coalesce at their margins, and yet that, in passing from the centre of one sensation-area to the centre of the other, we should find on examination a considerable fall of sensation at the junction of the two areas ; and in a third case we might imagine the two centres to be so close to each other that in passing from one to the other no appreciable diminution of sensation could be discovered. In the last case there would be but one sensation, in the second there might still be two sensations if the marginal fall were great enough, even though the areas partially coalesced. Thus, though the mosaic of rods and cones is the basis of distinct vision, the distinction or fusion of two vision impulses is ultimately determined by the disposition and condition of the cerebral centres. Hence the possibility of increasing by exercise the faculty of distinguishing two sensations, since by use the cerebral sensation-areas become more and more differentiated. This, however, is even more strikingly shown in touch than in sight.

\section{Color Sensations.}

$\S 661$. When we allow sunlight reflected from a cloud or sheet of paper to fall into the eye, we have a sensation which we call a sensation of white light. When we look at the same light through a prism, and allow different parts of the spectrum to fall in succession into the eye, we have sensations which we call respectively sensations of red, orange, yellow, green, blue, violet, etc., light. In other words, rays of light falling on the retina give rise to different sensations, according to the wave-lengths of the rays. Though we speak of the spectrum as consisting of a few colors, such as red, orange, etc., there are an almost infinite number of intermediate tints in the spectrum itself ; and we perceive in external nature a large number of colors, such as purple, brown, gray, etc., which do not correspond to any of the color sensations gained by regarding the successive parts of the spectrum. We find, however, on examination, that certain distinct color sensations, not corresponding to any of the colors of the spectrum may be obtained by the fusion of the sensations caused by two or more of the prismatic colors. Thus purple, which is not present in the spectrum, may be at once produced by fusing the sensations of blue and red in proper proportions. Moreover, many of the various tints and shades of nature may be imitated by fusing a particular color sensation with the sensation of white, or by allowing a certain quantity of light of a particular color to fall sparsely over the area of the retina, which is at the same time protected from the access of any other light, $i$. e., as we say, by mixing the color with black. Thus the browns 
of nature result from various admixtures of yellow, red, white, and black ; and a small quantity of white light, scattered over a large area of the retina, $i$. e., white largely mixed with black, forms a gray. In fact, the qualities of a color depend (1) on the nature of the prismatic color or colors, i.e., on the wave-lengths of the constituent rays, falling on a given area of the retina; (2) on the amount of this colored light which falls on the area of the retina in a given time; and (3) on the amount of white light falling on the same area at the same time. When rays corresponding to a prismatic color fall upon the retina unaccompanied by any white light, the color is said to be "saturated;" and a color is spoken of as more or less saturated according as it is mixed with less or more white light. When we are led to describe a color as being of such a tint or hue, we are guided by the first of the above conditions. But we have no common phrases by which we distinguish the second of the above conditions from the third. The word "pale," it is true is most frequently used to express a color very slightly saturated; but the words " rich" or "deep" are used sumetimes as meaning highly saturated, sometimes as meaning simply that a large quantity of light of the particular hue is passing into the eye. So also with the phrase "bright;" this we often use when a large amount of colored and white light fall at the same time on the same retinal area, but we sometimes also use it to express the mere intensity of the sensation.

The best method of fusing color sensations is that adopted by Maxwell, of allowing two different parts of the spectrum to fall on the same part of the retina at the same time. The use of the pure prismatic colors eliminates errors which arise when pigments, the colors of which are not pure, but mixed, are employed. And where pigments are used, it is the sensations to which the pigments give rise which must be mixed and not the pigments themselves. Thus while the sensations gained by looking at gamboge yellow and indigo respectively when fused give rise to a sensation of white, gamboge and indigo themselves when mixed appear green. The color of the mixed pigment is due to the fact that the rays which reach the eye from the mixture are those which are least absorbed by the two pigments. The gamboge absorbs the blue rays very largely, but the green to a much less extent; while the indigo absorbs the red and yellow rays very largely, but also absorbs very little of the green. Hence green is the predominant hue of the mixture. When pure pigments, $i$. e., pigments corresponding as closely as possible to the prismatic colors, are used, satisfactory results may be gained, either by using the reflected image of one pigment, and arranging so that it falls on the retina at the same spot as the direct image of the other pigment, or by allowing the image of one pigment to fall on the retina before the sensation produced by the other has passed away. The first result is easily reached by Helmholtz's simple method of placing two pieces of colored paper a little distance apart on a table, one on each side of a glass plate inclined at an angle. By looking with one eye down on the glass plate the reflected image of the one paper may be made to coincide with the direct image of the other, the angle which the glass plate makes with the table being adjusted to the distance between the two pieces of paper. In the second method, the "color top "is used; sectors of the colors to be investigated are placed on a disc made to rotate very rapidly, and the image of one color is thus brought to bear on the retina so soon after the image of another that the two sensations are fused into one.

$\S 662$. When the sensation corresponding to the several prismatic colors are fused together in various combinations, the following remarkable results are brought about :

1. When red and yellow in certain proportions are mixed together the result is a sensation of orange, quite indistinguishable from the orange of the spectrum itself. Now the latter is produced by rays of certain wave-lengths, whereas the rays of red and of yellow are respectively of quite different wave-lengths. The orange of the spectrum cannot be made up by any mix- 
ture of the red and the yellow of the spectrum in the sense that the red and yellow rays can unite together to form rays of the same wave-lengths as the orange rays; the three things are absolutely different. It is simply the mixed sensation of the red and yellow which is so like the sensation of orange; the mixture is entirely and absolutely a physiological one. In the same way we may by appropriate mixtures produce the sensations corresponding to other parts of the spectrum. Now we must suppose that rays of different wave-lengths give rise to different sensory impulses; that, for instance, the sensory impulses generated by orange rays are different from those generated by red and by vellow rays. Hence we are led by the fact of mixed sensations being identical with other apparently simple sensations to infer that the sensory impulses which any ray originates are either themselves of a complex character, or in becoming converted into sensations give rise to complex or mixed sensations; that, for instance, the impulse or sensation which a ray in the middle of the orange gives rise to, is not a simple impulse or sensation answering exclusively to the color of that ray, but that the ray gives rise either to a complex impulse which becomes converted into a complex sensation, or to a simple impulse which eventually develops into a mixed or complex sensation, into the composition of which in each case other orange tints and shades of red and yellow enter.

$\S 663$. 2. When certain colors are mixed together in pairs in certain definite proportions, the result is white. These colors are:

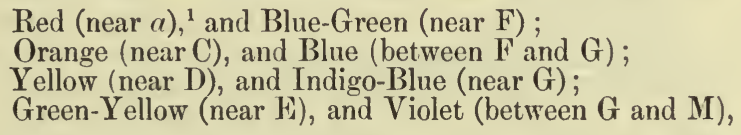

and are said to be "complementary" to each other. To these might be added the peculiar non-prismatic color purple, which with green also gives white.

3 . If we select arbitrarily any three colors corresponding to any three parts of the spectrum sufficiently far apart-say, for instance, red, green, and blue-we can, by a proper adjustment of the proportions of each, produce white. Further, these three colors can be taken in such proportions as with a proper addition, if necessary, of white to produce the sensations of all other colors. ${ }^{2}$ That is to say, given three standard sensations, all the other sensations may be gained by the proper mixture of these.

$\$ 664$. It is obvious from the foregoing that our real color sensations are much fewer in number than those which we appear to have when we look on the colors of the spectrum or of nature ; that rays of light awake in us certain simple sensations, which mixed in various proportions reproduce all our sensations. And the question arises, What is the nature or what are the characters of these simple sensations?

When we examine our own sensations of light we find that certain of these seem to be quite distinct in nature from each other, so that each is something sui generis, whereas we easily recognize all other sensations as various mixtures of these. Thus red and yellow are to us quite distinct; we do not recognize anything common to the two; but orange is obviously a mixture of red and yellow. The sensations caused by different kinds of

1 These letters refer to Frauenhofer's lines.

${ }^{2}$ A few highly saturated colors cannot be so reproduced, but a mixture of any one of them with white can. We may, perhaps, therefore speak of these saturated colors as being reproduced by a proper combination of the three arbitrarily selected colors, with the subtraction of white. 
light, which thus appear to us distinct, and which we may speak of as "fundamental sensations," are white, black, red, yellow, green, blue. Each of these seems to us to have nothing in common with any of the others, whereas in all other colors we can recognize a mixture of two or more of these.

This result of common experience suggests the idea that these fundamental sensations are the primary or simple sensations, spoken of above as those out of which all other sensations may be supposed to be compounded. And a theory has been proposed to reconcile the various facts of color vision with the supposition that we possess these six fundamental sensations. This theory, known as that of Hering, is somewhat as follows: The six sensations readily fall into three pairs, the members of each pair having analogous relations to each other. White and black naturally go together, the one being the antagonistic or correlative of the other. There is a similar connection between red and green, the one being the complementary of the other, and between yellow and blue, which are similarly complementary. We saw reason, a short time back (p. 765), for believing that vision originates in the changes taking place in certain visual substances (or a visual substance) in the retina. And the theory of which we are speaking supposes that there exist in the retina, or at least somewhere in the visual apparatus, three distinct visual substances which are continually undergoing a double metabolism, one constructive, of assimilation or building up, and the other destructive, of dissimilation or breaking down. One of these substances is further of such a nature that when dissimilation is in excess of assimilation we have a sensation of white, and when assimilation is in excess a sensation of black. With a second substance excess of dissimilation provokes red, of assimilation green; and with the third substance, yellow and blue respectively. When in the latter two substances dissimilation and assimilation are exactly equal, no effect is produced; but with the first substance this condition produces in us the effect of gray. Further, these substances are of such a kind that while the first or white-black substance is influenced by rays along the whole range of the spectrum, the two other substances are differently influenced by rays of different wave-length. Thus in the part of the spectrum which we call red, the rays promote a rapid dissimilation of the red-green substance with comparatively slight effect in either direction on the yellow-blue substance; hence our sensation of red. In that part of the spectrum which we call yellow the rays effect a marked dissimilation of the yellow-blue substance, but their action on the red-green substance is equal in the direction of both assimilation and dissimilation ; hence our sensation of yellow. The green rays, again, promote assimilation of the redgreen substance, leaving the assimilation of the yellow-blue substance equal to the dissimilation, and similarly blue rays cause assimilation of the yellowblue substance, and leave the red-green substance neutral. Finally, at the extreme blue end of the spectrum the rays once more provoke dissimilation of the red-green substance. When orange rays fall on the retina, there is an excess of dissimilation of both the red-green and the yellowblue substance; when greenish-blue rays are perceived there is an excess of assimilation of both these substances; and other intermediate tints correspond to variable amounts of dissimilation or assimilation of two or more of these substances.

$\$ 665$. When all the rays together fall on the retina, the red-green and yellow-blue substances remain in equilibrium, but the white-black substance is violently dissimilated; and we say the light is white.

Another theory (known as the Young-Helmholtz theory, because it was introduced by Young and more fully elaborated by Helmholtz) strives to 
reduce the matter to still further simplicity. Starting from the fact mentioned a short time since, that all color sensations, including the sensation of white, may be obtained by the appropriate mixture of three standard sensations, this theory teaches that our visual apparatus is so constituted as, when excited, to give rise to three primary sensations, and that these primary sensations are called forth in different degrees by different rays of light, so that each ray gives rise to a different mixture of the three. Several sets of three such primary sensations might be chosen, which would satisfy the conditions of giving rise, by appropriate mixture, to all sensations of color, including white; lut for reasons into which we cannot enter fully here, the sensations which may thus be taken as primary sensations appear to correspond to our sensations of red, green, and blue or violet. Such a view of three primary color sensations is represented in the diagram (Fig. 176). Thus the red

Fig. 176.

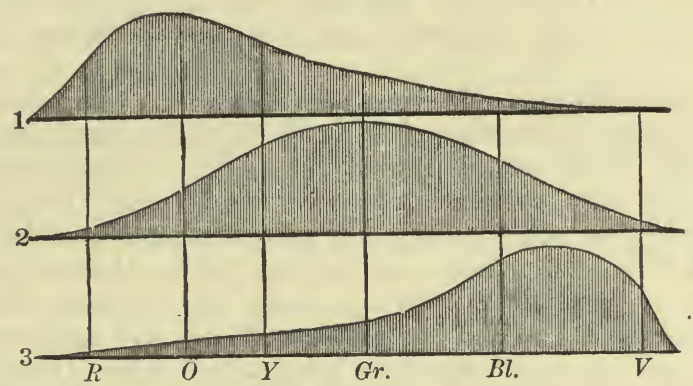

Diagram of Three Primary Color Sensations. 1, is the so-called "red ;" 2, "green," and 3, "violet" primary color sensation. $R, O, Y$, etc., represent the red, orange, yellow, etc., color of the spectrum, and the diagram shows by the height of the curve in each case, to what extent the several primary color sensations are respectively excited by vibrations of different wavelengths.

primary sensation, excited to a certain extent by the rays at the extreme red end, is most powerfully affected by the rays at a little distance from the end, the rays from this point onward toward the blue end producing less and less effect. The curve of the green primary sensation begins later, and reaches its maximum in the green of the spectrum, while the blue or violet primary sensation is still later, and only reaches its maximum toward the blue end of the spectrum. Each ray calls forth each sensation, but to a different degree, and the total result of each ray, or of each group of rays, is determined by the proportionate amount of the three sensations. Thus the sensation of orange ( $O$ in the figure) is brought about by a mixture of a great deal of the primary red with much less of the primary green, and hardly any of the primary blue; the orange sensation is converted into a yellow - sensation by diminishing the primary red and largely increasing the primary green, the primary blue undergoing also some slight increase. And similarly with all the other sensations. When each of the primary sensations is excited to a maximum, as when ordinary light falls on the retina, the result is a sensation of white. According to this theory, black is simply the absence of sensation from the visual apparatus.

In the view, as originally put forward by Young, the three primary sensations were supposed to be represented by three sets of fibres, each set of fibres being differently affected by different rays of light, and the impulses passing to the brain along each set awakening a distinct sensation. No such distinction of fibres can be found in the retina; but an anatomical basis of this kind is not necessary for the theory; we can easily conceive of the same 
fibre transmitting three distinct kinds of impulses ; or we may suppose that the visual substances are three in number instead of six, the changes in each substance provoking a primary sensation.

$\$ 666$. Such are the two main theories of color vision ; and much may be said in favor of both of them; at the same time both of them present many difficulties. To discuss them fully is a task beyond the limits of this book, and to discuss them in any but a full manner would be unsatisfactory. We must be satisfied, therefore, with the foregoing simple statement of the two views. Independently of any theory, however, we may remember (1) that all the sensations which we experience under the action of light of whatever kind may be reduced to six-white, black, red, yellow, green, and blue; and (that these may be all reproduced by various mixtures of three standard sensations, if black be allowed to indicate the absence of all sensation. These are matters of fact; what is at present debated is whether the six fundamental sensations are the outcome of three primary sensations or whether they represent six distinct conditions of the visual apparatus.

$\$ 667$. Color-blindness. Persons vary much in their power of appreciating and discriminating color, $i$. e., in the intensity and accuracy of their color sensations. Some people regard as similar, colors which to most people are glaringly distinct; the former are said to be "color-blind." The most common form of color-blindness is that of persons unable to distinguish green and red from each other. As in the case of Dalton, they tell a red gown lying on a green grass plot, or a red cherry among the green leaves, by its form, and not by its color. They confound not only red, green, and certain forms of brown, but also rose, purple, and blue. Such persons are often spoken of as " red blind." On the Hering theory they lack the redgreen visual substance; hence, all the color sensations they possess must be those of yellow and blue free from all mixture of red or green; and such accounts as have been given of their sensations by those persons who are "red blind" in one eye, but possess normal vision with the other, accord with this conclusion. On the Young-Helmholtz theory, such persons lack the primary red sensation; and hence the sensations which they have must be inixtures of green and blue alone, our yellow appearing to them a bright green, and our green-blue a kind of gray.

All such red-blind people ought, on either theory, to be less affected than are persons with normal eyes, by the red end of the spectrum; this ought with them to be shortened and obscure. In a certain number of persons who confound red and green, this is the case; but in some instances no such lack of appreciation of the red end of the spectrum can be ascertained. Such cases have been supposed to be green blind, that is, lacking the primary sensation of green. According to the Hering theory green blindness apart from red blindness is impossible, the only two possible color defects being red-green and blue-yellow blindness. And the existence of distinct green blindness has been held to contradict that theory. On the other hand, the Hering theory admits the possibility of total color-blindness, $i . e$, the inability to see anything but white and black; and this on the YoungHelmholtz theory, is impossible, since for vision to exist at all, one of the three primary sensations must be present; a man to see at all must see things in various shades of either red, or of green, or of violet, though he may confound this single-colored vision with the normal vision of white of different intensities. But, indeed, a full examination of color-blindness rather increases than diminishes the difficulties of deciding between the two rival theories.

$\S 668$. Influence of the pigment of the yellow spot. In the macula lutea, 
which part of the retina we use chiefly for vision, images falling on other parts of the retina being said to give rise to "indirect vision," the yellow pigment absorbs some of the greenish-blue rays. Hence, the sensation which we receive from objects which we are in the habit of calling white is that which, if this pigment were absent, we should receive from objects more or less yellow. We may use this feature of the yellow spot for the purpose of making the spot, so to speak, visible to ourselves, by an experiment suggested by Maxwell. A solution of chrome alum, which only transmits red and greenish-blue rays, is held up between the eye and a white cloud. The greenish-blue rays are absorbed by the yellow spot, and here the light gives rise to a sensation of red; whereas in the rest of the field of vision the sensation is that ordinarily produced by the purplish solution. The yellow spot is consequently marked out as a rosy patch. 'This very soon, however, dies away.

In speaking of sensation as a function of the stimulus (p. 766), we referred to white light only; but the different colors are unequal in the relations borne by the intensity of the stimulus to the amount of sensation produced. Thus the more refrangible blue rays produce a sensation more readily than the yellow or red rays. Hence, in dim lights, as those of evening and moonlight, the blues preponderate, and the reds and yellows are less obvious. So also when a landscape is viewed through a yellow glass, the yellow hue suggests to the mind bright sunlight and summer weather, although the actual illumination which reaches the eye is diminished by the glass. Conversely, when the same landscape is viewed through a blue glass the idea of moonlight or winter is suggested.

The theory of three primary color sensations may be used to explain why any colored light, if made sufficiently intense, appears white. Thus a violet light of moderate intensity appears violet because it excites the primary sensation of violet much more than those of green and red. If the stinjulus be increased the maximum of violet stimulation will be reached, while the stimulation of green will continue to be increased and even that of red to a slight degree. The result will be that the light appears violet mixed with green, that is blue. If the stimulus be still further increased while the green and violet are both excited to the maximum, the red stimulation may be increased until the result is violet, green, and red in the proportions which make white light. And so with light of other colors.

$\$ 669$. After-images. We have already seen that in vision the sensation lasts much longer than the stimulus. Under certain circumstances, such as particular conditions of the eye, an intense stimulus, etc., the sensation is so prolonged that it is spoken of as an after-image. 'Thus, if the eye be directed to the sun, the image of that body is present for a long while after; and, if, on early waking, the eye be directed to the window for an instant and then closed, an image of the window with its bright panes and darker sashes, the various parts being of the same color as the object, will remain for an appreciable time. These images, which are simply continuations of the sensation, are spoken of as positive after-images. They are best seen after a momentary exposure of the eye to the stimulus.

When, however, the eye has been for some time subject to a stimulus, the sensation which follows the withdrawal of the stimulus is of a different kind; what is called a negative after-image, or negative image, is produced. If, after looking steadfastly at a white patch on a black ground, the eye be turned to a white ground, a gray patch is seen for some little time. A black patch on a white ground similarly gives rise on a gray ground to a negative image in the form of a white patch. This may be explained as the result of exhaustion. When the white patch has been looked at steadily for some time, that 
part of the retina on which the image of the patch fell becomes tired; hence, the white light, coming from the white ground subsequently looked at, which falls on this part of the retina, does not produce so much sensation as in other parts of the retina; and the image, consequently, appears gray. And so, in the other instance, the whole of the retina is tired, except at the patch ; here the retina is for a while most sensitive, and hence the white negative image.

When a red patch is looked at, the negative image is a green-blue, that is, the color of the negative image is complementary to that of the object. Thus, also, orange produces a blue, green a pink, yellow an indigo-blue, negative image, and so on. This, too, can be explained as a result of exhaustion on either hypothesis of color vision. When the colored patch is looked at, one of the three primary color sensations is much exhausted, and the other two less so, in varying proportions, according to the exact nature of the color of the patch; and the less exhausted sensations become prominent in the after-image. Thus, the red patch exhausts the red sensation, and the negative image is made up chiefly of green and blue sensations, that is, appears to be greenish-blue, or bluish-green, according to the tint of the red. On the other hypothesis, we may suppose that, owing to the continued effect of looking at the red patch, dissimilation of the red-green substance becomes less and less, leading to a prominence and, indeed, to an actual increase of the process of assimilation of the same substance; hence, the sensation of green dominating in the negative image.

Similarly, when the eye, after looking at a colored patch, is turned to a colored ground, the effects may easily be explained by reference to the comparative exhaustion of the color sensations excited by the patch and the ground respectively; if a yellow ground be chosen after looking at a green object, the negative image will appear of a reddish-yellow, and so on.

$\$ 670$. The theory of three primary sensations does not so readily explain why negative images should make their appearance without any subsequent stimulation of the retina. When the eyes are shut and all access of light, even through the eyelids, carefully avoided, the field of vision is not absolutely dark; there is still a sensation of light, the so-called "proper light" of the retina. If a white patch on a black ground be looked at for some time, and the eyes then shut, a negative (black) image of the spot will be seen on the ground of the "proper light" of the retina, having in its immediate neighborhood a specially bright corona. So, also, if a window be looked at and the eyes then closed, the positive after-image with bright panes and dark sashes gives rise to a negative after-image with bright sashes and dark panes; and similar effects appear with colors. These and similar facts have been largely used in support of the Hering theory. When the eye has been looking at red, and so has caused dissimilation of the redgreen substance, mere rest, as on shutting the eyes, favors assimilation of the same substance and thus leads to a sensation of green. And the rhythmic oscillations from one color to its correlative and back again, frequently observed under these conditions and which point to assimilation and dissimilation alternately gaining the upper hand, are not without analogies in other common instances of protoplasmic metabolism.

\section{Visual Perceptions.}

$\$ 671$. Hitherto we have studied sensations only, and have considered an external object, such as a tree, as simply a source of so many distinct sensations, differing from each other in intensity and kind (color). In the mind these sensations are coördinated into a perception. We are not only 
conscious of a number of sensations of bright and dim lights, of green, brown, black, etc., but these sensations are so related to each other and by virtue of cerebral processes so fashioned into a whole, that we "see a tree." We sometimes, in illustration of such an effect, speak of an image or picture in the mind corresponding to the physical image on the retina.

When we look upon the external world, a variety of images are formed at the same time on the retina, and give rise to a number of contemporaneous visual sensations. The sum of these sensations constitutes " the field of vision," which varies, of course, with every movement of the eye. This field of vision, being in reality an aggregate of sensations, is, of course, a subjective matter; but we are in the habit of using the same phrase to denote the sum of external objects which give rise to the aggregate of visual sensations; in common language the field of vision is "all that we can see" in any position of the eye, and we have a field of vision for each eye separately and for the two eyes combined.

$\$ 672$. Using for the present the words in their subjective sense, we may remark that we are able to assign to each constituent sensation its place among the aggregate of sensations constituting the field of vision; we can, as we say, localize the sensation. We can say whether it belongs to (what we regard as) the right hand or left hand, the upper or the lower part of the field of vision. We are able to distinguish the relative positions of any two distinct sensations; and the relative positions, together with the relative intensities and qualities (color) of the sensations arising from any object determine our perception of the object. It need hardly be remarked that this localization is purely subjective. We simply determine the position of the sensation in the field of vision (which is itself a wholly subjective matter); we do not determine the position of the object. The connection between the position of the object in the external world and the position of the sensation in the field of vision cannot be determined by visual observation alone. All the information which can be gained by the eye is limited to the field of vision, and provided that the relative position of the sensations in the field of vision remained the same, the actual position of external objects might, as far as vision is concerned, be changed without our being aware of it.

As a matter of fact the field of vision in one important particular does not correspond to the field of external objects. The image on the retina is inverted; the rays of light proceeding from an object which by touch we know to be on what we call our right hand, fall on the left-hand side of the retina. If, therefore, the field of vision correspond to the retinal image, the object would be seen on the left hand. We, however, see it on the right hand, because we invariably associate right-hand tactile localization with left-hand visual localization ; that is to say, our field of vision, when interpreted by touch, is a re-inversion of the retinal image.

The dimensions of the field of vision of a single eye are about 145 degrees for the horizontal and 100 degrees for the vertical meridian, the former being distinctly greater than the latter. The horizontal dimension of the field of vision for the two eyes is about 180 degrees. By movements of the eyes, however, even apart from those of the head, the extent may be considerably increased.

The satisfactory perception of external objects requires distinct vision; and of this, as we have already said, the formation of a distinct image on the retina is an essential condition. We can receive visual sensations of all kinds with the most imperfect dioptric apparatus, but our perception of an object is precise in proportion to the clearness of the image on the retina.

$\S 673$. Region of distinct vision. If we take two points, such as two black 
dots, only just so far apart that they can be seen distinctly as two when placed near the axis of vision, and then, keeping the axis fixed, move the two points out into the circumferential parts of the field of vision, it will be found that the two soon appear as one. The two sensations become fused, as they would do if brought nearer to each other in the centre of the field. The further away from the centre of the field, the further apart must two points be in order that they may be seen as two. In other words, vision is much more distinct in the centre of the field than toward the circumference. Practically the region of distinct vision may be said to be limited to the macula lutea, or even to the fovea centralis; by continual movements of the eye we are constantly bringing any object which we wish to see in such a position that its image falls on this region of the retina.

The diminution of distinctness does not take place equally from the centre to the circumference along all meridians. The outline described by a line uniting the points where two spots cease to be seen as two when moved along different radii from the centre is a very irregular figure.

The sensations of color are much more distinct in the centre of the retina than toward the circumference. If the visual axis be fixed and a piece of colored paper be mover toward the outside of the field of vision, the color undergoes changes and is eventually lost, red disappearing first, and blue last, the object remaining visible, though with very indistinct outlines, when its color can no longer be recognized. A purple color becomes blue, and a rose color a bluish white. In fact, there seems to be a certain amount of red-blindness in the peripheral parts of all retinas.

\section{Modified Perceptions.}

$\S 674$. Since our perception of exterual objects is based on the distinctness of the sensations which go to form the perception, it might be expected that when an image of an object is formed on the retina the sensory impulses would correspond to the retinal image, the sensations correspond to the sensory impulses and the perception correspond to the sensations, and that, therefore, the mental condition resulting from our looking at any object or view would correspond exactly to the retinal image. We find, however, that this is not the case. The sensations and probably even the simple sensory impulses produced by an image react upon each other, and these reactions modify our perceptions, independently of the physical conditions of the retinal image. There arise certain discrepancies between the retinal image and the perception, some having their source in the retina, some in the brain, and others being of such a nature that it is difficult to say where the irrelevancy is introduced.

$\$ 675$. Irradiation. A white patch on a dark ground appears larger, and a dark patch on a white ground smaller, than it really is (Fig. 177). This is especially so when the object is somewhat out of focus, and may in this case be partly explained by the diffusion circles which, in each case, encroach from the white upon the dark. But over and beyond this, any sensation coming from a given retinal area occupies a larger share of the field of vision, when the rest of the retina and central visual apparatus are at rest, than when they are simultaneously excited. It is as if the neighboring, either retinal or cerebral, structures were sympathetically thrown into action at the same time.

$\$ 676$. Contrast. If a white strip be placed between two black strips, the edges of the white strip near the black will appear whiter, than its median portion; and if a white cross be placed on a black background, the centre of the cross will appear sometimes so dim, compared with the parts 
close to the black, as to seem shaded. This occurs even when the object is well in focus; the increased sensation of light which causes the apparent greater whiteness of the borders of the cross is the result of the "contrast" with the black placed adjoining it. Still more curious results are seen with colored objects. If a small piece of gray paper be placed on a sheet of green paper, and both covered with a sheet of thin tissue paper, the gray paper will appear of a pink color, the complementary of the green.

FIG. 177.

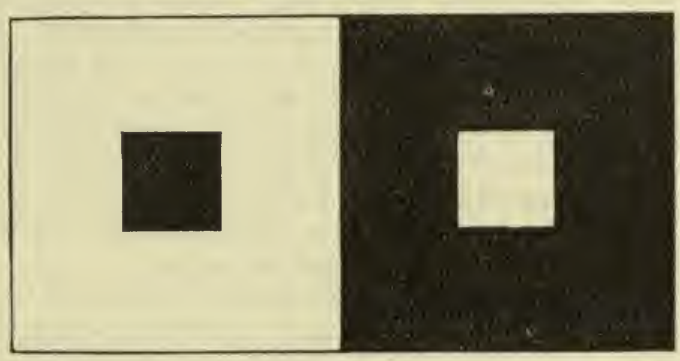

This effect of contrast is far less striking, or even wholly absent, when the small piece of paper is white instead of gray, and generally disappears when the thin covering of tissue paper is removed. It also vanishes if a bold, broad black line be drawn round the small piece of paper, so as to isolate it from the ground color. If a book or pencil be placed vertically on a sheet of white paper, and illuminated on one side by the sun and the other by a candle, two shadows will be produced, one from the sun, which will be illuminated by the yellowish light of the candle, and the other from the candle, which will in turn be illuminated by the white light of the sun. The former naturally appears yellow; the latter, however, appears not white but blue; it assumes, by contrast, a color complementary to that of the candle-light which surrounds it. If the candle be removed, or its light shut off by a screen, the blue tint disappears, but returns when the candle is again allowed to produce its shadow. If, before the candle is brought back, and vision be directed through a narrow blackened tube at some part falling entirely within the area of what will be the candle's shadow, the area, which in the absence of the candle appears white, will continue to appear white when the candle is made to cast its shadow, and it is not until the direction of the tube is changed so as to cover part of the ground outside the shadow, as well as part of the shadow, that the latter assumes its blue tint.

$\$ 677$. Filling up the blind spot. Though, as we have seen, that part of the retina which corresponds to the entrance of the optic nerve is quite insensible to light, we are conscious of no blank in the field of vision. When in looking at a page of print we fix the visual axis so that some of the print must fall on a blind spot, no gap is perceived. We could not expect to see a black patch, because what we call black is the absence of the sensation of light from structures which are sensitive to light; we must have visual organs to see black. But there are no visual organs in the blind spot, and consequently we are in no way at all affected by the rays of light which fall on it. There is in our subjective field of vision no gap corresponding to the gap in the retinal image. We refer the sensations coming from two points of the retina lying on opposite margins of the blind spot to two points lying close together, since we have no indication of the space which separates them. Concerning the effects which are produced when an object in the field of view passes into the region of the blind spot there has been much 
discussion. In ordinary vision, of course, the existence of the blind spot is of little moment since it is outside the region used for distinct vision, and besides, the image of an object does not fall on the blind spots of both eyes at the same time. [See Fig. 179.]

$\$ 678$. Ocular spectra. So far from our perceptions exactly corresponding to the arrangements of the luminous rays which fall on the retina, we may have visual sensations and perceptions in the entire absence of light. Any stimulation of the retina or of the optic nerve sufficiently intense will give rise to a visual sensation. Gradual pressure on the eyeball causes a sensation of rings of colored light, the so-called phosphenes; a sudden blow on the eye causes a sensation of flashes of light, and the seeming identity of the visual sensations so brought about with visual sensations produced by light is well illustrated by the statement once gravely made in a German court of law by a witness who asserted that on a pitch-dark night he recognized an assailant by help of the flash of light caused by the assailant's hand coming in violent contact with his eye. Electrical stimulation of the eye or optic nerve will also give rise to visual sensations.

The sensations which may arise without any light falling on the retina need not necessarily be undefined; on the contrary they may be most clearly defined. Complex and coherent visual images or perceptions may arise in the brain without any corresponding objective luminous cause. These socalled ocular spectra or phantoms, which are the result of an intrinsic stimulation of some (probably cerebral) part of the visual apparatus, have a distinctuess which gives them an apparent objective reality quite as striking as that of ordinary visual perceptions. They may occasionally be seen with the eyes open (and therefore while ordinary visual perceptions are being generated) as well as when the eyes are closed. They sometimes become so frequent and obtrusive as to be distressing, and form an important element in some kinds of delirium, such as delirium tremens.

$\$ 679$. Appreciation of apparent size. By the eye alone we can only estimate the apparent size of an object, we can only tell what space it takes in the field of vision, we can only perceive the dimensions of the retinal image, and therefore have a right only to speak of the angle which the diameter of the object subtends. The real size of an object must be determined by other means. But our perception of even the apparent size of an object is so modified by concurrent circumstances that in many cases it cannot be relied on. The apparent size of the moon must be the same to every eye, and yet while some persons will be found ready to compare the moon in the midheavens with a three-penny piece, others will liken it to a cart-wheel; that

FIG. 178.

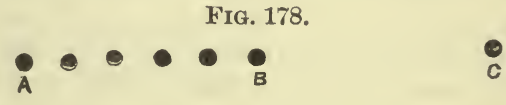

is to say, the angle subtended by the moon seems to the one to be about equal to that subtended by a three-penny piece held at the distance from the eye at which it is most commonly looked at, and to the other about equal to that subtended by a cart-wheel similarly viewed at the distance at which it is most commonly looked at. If a line such as $A C$, Fig. 178, be divided into two equal parts, $A B, B C$, and $A B$ be divided by distinct marks into several parts, as is shown in the figure, while $B C$ is left entire, the distance $A B$ will always appear greater than $C B$. So also, if two equal squares be marked, one with horizontal and the other with vertical alternate dark and light bands, the former will appear higher, and the latter broader, than it really is. Hence short persons affect dresses horizontally striped in order to 
increase their apparent height, and very stout persons avoid longitudinal stripes. Two perfectly parallel lines or bands, each of which is crossed by slanting parallel short lines, will appear not parallel, but diverging or converging according to the direction of the cross-lines.

Again, when a short person is placed side by side with a tall person, the former appears shorter and the latter taller than each really is. The moon on the horizon appears larger than when at the zenith, because in the first position it can be most easily compared with terrestrial objects. The absence of comparison may, however, contribute to an opposite effect, as when a person looks larger in a fog; being seen indistinctly, he is judged to be further off than he really is, and so appears larger than he naturally would do at the distance at which he is supposed to be. So, conversely, distant mountäins, when seen distinctly in a clear atmosphere appear small, because, on account of their distinctness, they are judged to be nearer than they really are. Indeed, our daily life is full of instances in which our direct perception is modified by circumstances. Among those circumstances previous experience is one of the most potent, and thus simple perceptions become mingled with what are in reality judgments, though frequently made unconsciously. But this intrusion of past experience into present perceptions and sensations is most obvious in binocular vision, to which we now turn.

\section{Binocular Vision.}

\section{Corresponding or Identical Points.}

$\$ 680$. Though we have two eyes, and must therefore receive from every object two sets of sensations, our perception of an object is under ordinary circumstances a single one; we see one object, not two. But putting either eye into an unusual position, as by squinting, we can render the perception double; we see two objects where one only exists. From which it is evident that singleness of perception depends on the image of the object falling on certain parts of each retina at the same time, these parts being so related to each other that the sensations from each are blended into one perception; and it is also evident that the movements of the eyeballs are adapted to bring the image of the object to fall on these "corresponding" or "identical" parts, as they are called, of each retina.

When we look at an object with one eye the visual axis of that eye is directed to the object, and when we use two eyes the visual axes of the two eyes converge at the object, the eyeballs moving accordingly. The corresponding points of the two retinas are those on which the two images of the object fall when the visual axes converge at the object. Thus in Fig. 179, if $x x, x x^{\prime}$ be the two visual axes, $x x^{\prime}$ being the centres of the fover centrales of the two eyes, then, the object $y, x, z$ being seen single, the point $y$ on the one retina will " correspond" to or be "identical" with the point $y^{\prime}$ on the other, and the point $x$ in the one to the point $x^{\prime}$ in the other. Hence a point lying anywhere on the right side of one retina has its corresponding point on the right side of the other retina, and the points on the left of one correspond with those on the left of the other. Thus, while the upper half of the retina of the left eye corresponds to the upper half of the retina of the right eye and the lower to the lower, the nasal side of the left eye corresponds with the malar side of the right, and the malar of the left with the nasal side of the right.

The blending of the two sensations into one only occurs when the two images of an object fall on these corresponding points of the two retinas. Hence it is obvious that in single vision with two eyes the ordinary move- 
ments of the eyeballs must be such as to bring the visual axes to converge at the object so that the two images may fall on corresponding points. When the visual axes do not so converge, and when, therefore, the images do not fall on corresponding points, the two sensations are not blended into one perception and vision becomes double.

FIG. 179.
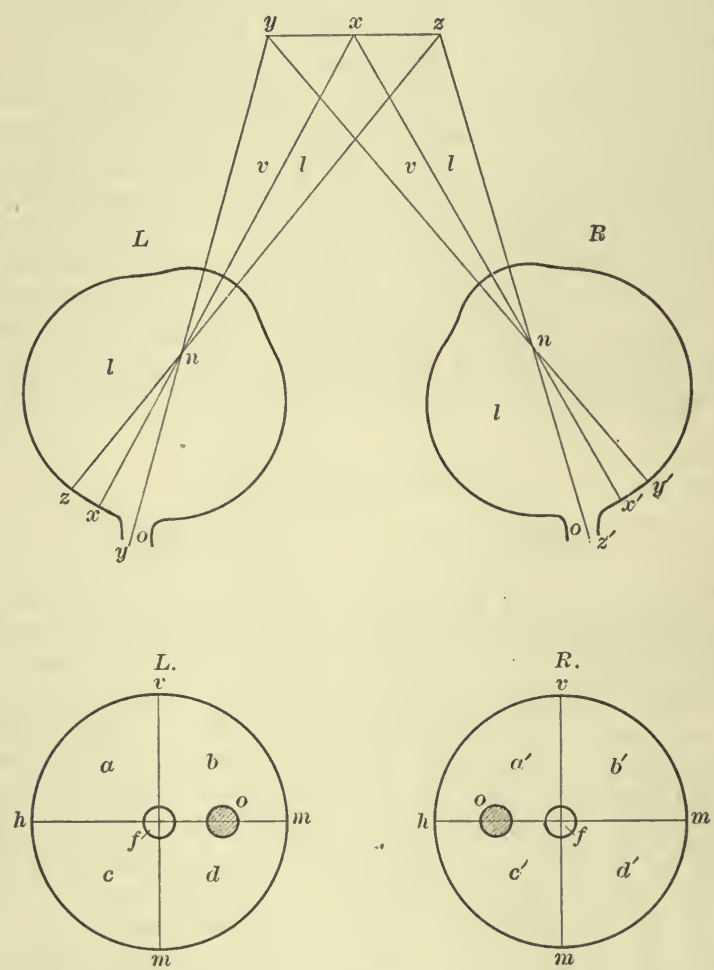

Diagram illustrating Corresponding or Identical Points: $L$, the left, $R$, the right eye ; $n$, nodal point; $o$, optic nerve (blind spot); $x$, forea. $x^{\prime} y^{\prime} z^{\prime}$ in the right eye are corresponding points to $x y$ $z$ in the left eye. $v l$, visual axis. The two figures below are projections of $L$ the left and $R$ the right retina. $f$, fovea; $o$, blind spot. $a$ and $c$ on the temporal side of $L$ correspond to $a^{\prime}$ and $c^{\prime}$ on the nasal side of $R$. $v m h m$, lines separating quadrants.

\section{Movements of the Eyeballs.}

$\S 681$. The eye is virtually a ball placed in a socket, the bulb and the orbit forming a ball-and-socket joint. In its socket-joint the optic ball is capable of a variety of movements, but it cannot by any voluntary effort be moved out of its socket. It is stated that by a very forcible opening of the eyelids the eyeball may be slightly protruded; but this trifling locomotion may be neglected. By disease, however, the position of the eyeball in the socket may be materially changed.

Each eyeball is capable of rotating round an immobile centre of rotation, which has been found to be placed a little $(1.77 \mathrm{~mm}$.) behind the centre of the eye ; but the movements of the eye round the centre are limited in a pecu- 
liar way. The shoulder-joint is also a ball-and-socket joint; and we know that we can not only move the arm up and down round a horizontal axis passing through the centre of rotation of the head of the humerus, and from side to side round a vertical axis, but we can also rotate it round its own longitudinal axis. When, however, we come to examine closely the movements of the eyeball we find that though we can move it up and down round a horizontal axis, as when with fixed head we direct our vision to the heavens or to the ground, and from side to side, as when we look to left or right, and though by combining these two movements we can give the eyeball a variety of inclinations. we cannot, by a voluntary effort, rotate the eyeball round its longitudinal visual axis. 'The arrangement of the muscles of the eyeball will permit of such a movement, but we cannot by any direct effort of will bring it about by itself. In certain movements of the eye rotation of the eyeball does take place and by bringing about these movements we can indirectly cause rotation; but we cannot rotate the eyeball except thus indirectly as a part of these movements.

If, when vision is directed to any object the head be moved from side to side, the eyes do not move with it ; they appear to remain stationary, very

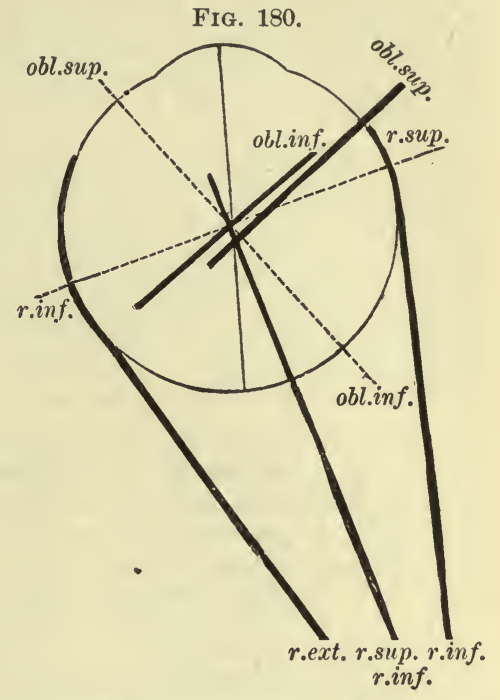

Diagram of the Attachments of the Muscles of the Eye and of their Axes of Rotation, the latter being represented by dotted lines.

The axis of rotation of the rectus externus and internus, being perpendicular to the plane of the paper, cannot be shown. (After Fick.)

much as the needle of a ship's compass remains stationary when the head of the ship is turned. The change in the position of the visual axes to which the movement of the head would naturally give rise is met by compensating movements of the eyeballs; were it not so, steadiness of vision would be impossible.

$\$ 682$. There is one position of the eyes which has been called the primary position. It corresponds to that which may be attained by looking at the distant horizon with the head vertical and the body upright; but its exact determination requires special precautions. The visual axes are then parallel to each other and to the median plane of the head. All other positions of the eyes are called secondary positions.

$\$ 683$. Muscles of the eyeball. The eyeball is moved by six muscles, the recti inferior, superior, internus, and externus, and the obliqui inferior and superior. It is found by calculation from the attachments and directions of the muscles, and confirmed by actual observation, that the six muscles may be considered as three pairs, each pair rotating the eye round a particular axis. The relative attachments and the axes of rotation are diagrammatically shown in Fig. 180. The rectus superior and the rectus inferior rotate the eye round a horizontal axis, which is directed from the upper end of the nose to the temple; the obliquus superior and obliquus inferior round a horizontal axis directed from the centre of the eyeball to the occiput; and the rectus internus and rectus externus round a vertical axis (which, being at right angles to the plane of the paper, cannot be shown in the diagram), 
passing through the centre of rotation of the eyeball parallel to the median plane of the head when the head is vertical. Thus the latter pair acting alone would turn the eye from side to side, the other straight pair acting alone would move the eye up and down, while the oblique muscles acting alone would give the eye an oblique movement. The rectus externus acting alone would turn the eye to the malar side, the internus to the nasal side, the rectus superior upward, the rectus inferior downward, the oblique superior downward and outward, and the inferior upward and outward. The recti superior and inferior in moving the eye up and down also turn it somewhat inward and at the same time give it a slight amount of rotation; but this is corrected if the oblique muscles act at the same time; and it is found that the rectus superior acting with the obliquus inferior moves the eye upward, and the rectus inferior with the obliquus superior downward in a vertical direction. In oblique movements also, the obliqui are always associated with the recti. Hence the various movements of the eyeball may be arranged as follows:

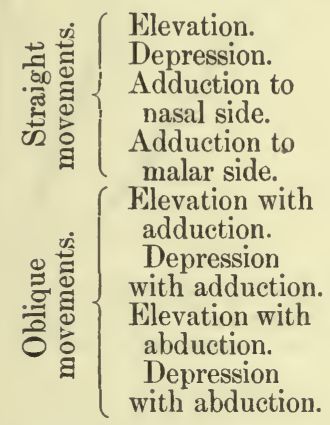

Rectus superior and obliquus inferior.

Rectus inferior and obliquus superior.

Rectus internus.

Rectus externus.

Rectus superior and internus with obliquus inferior.

Rectus inferior and internus with obliquus superior.

Rectus superior and externus with obliquus inferior.

Rectus inferior and externus with obliquus superior.

\$ 684. Coördination of visual movements. Thus even in the movements of a single eye a considerable amount of coördination takes place. When the eye is moved in any other than the vertical and horizontal meridians, impulses must descend to at least three muscles, and in such relative energy to each of the three as to produce the required inclination of the visual axis. But the coördination observed in binocular vision is more striking still. If the movements of any person's eyes be watched it will be seen that the two eyes move alike. If the right eye moves to the right, so does also the left; and, if the object looked at be a distant one, exactly to the same extent; if the right eye looks up, the left eye looks up also, and so in every other direction. Very few persons are able by a direct effort of the will to move one eye independently of the other; though some, and among them one distinguished both as a physiologist and an oculist, have acquired this power. In fact, the movements of the two eyes are so arranged that in the various movements the images of any object should fall on the corresponding points of the two retinas, and that thus single vision should result. We cannot by any direct effort of our will place our eyes in such a position that the rays of light proceeding from any object shall be brought to a focus on parts of the two retinas which do not correspond, and thus give rise to two distinct visual images. We can bring the visual axes of the two eyes from a condition of parallelism to one of great convergence, but we cannot, without special assistance, bring them from a condition of parallelism to one of divergence. The stereoscope will enable us to create a divergence. If in a stereoscopic picture the distance between the pictures be increased very gradually so as carefully to maintain the impression of a single object, the 
visual axes may be brought to diverge. Similarly if a distant object be looked at with a prism before one eye, and the image of the object be kept carefully single, while the prism is turned very slowly up or down, then on suddenly removing the prism a double image is for a moment seen; showing that the eye before which the prism was placed has moved in disaccordance with the other. The double image, however, in a few seconds after the removal of the prism becomes single, on account of the eyes coming into accordance.

It is only when loss of coördination occurs, as in various diseases and in alcoholic or other poisoning, that the movements of the two eyes cease to agree with each other. It is evident, then, that when we look at an object to the right, since we thereby abduct the right eye and adduct the left, we throw into action the rectus externus of the right eye and the rectus internus of the left, and similarly when we look to the left we use the rectus externus of the left and the rectus internus of the right eye. On the other hand, when we look at a near object, and therefore converge the visual axes, we use the recti interni of both eyes; and when we look at a distant object, and bring the axes from converging toward parallelism, we use the recti externi of both eyes. In the various movements of the eye there is therefore, so to speak, the most delicate picking and choosing of the muscular instruments. Bearing this in mind, it cannot be wondered at that the various movements of the eye are dependent for their causation on visual sensations. In order to move our eyes we must either look at or for an object; when we wish to converge our axes we look at some near object, real or imagiuary, and the convergence of the axes is usually accompanied by all the conditions of near vision, such as increased accommodation and contraction of the pupil. And so with other movements. The close association of the movements of the eye may be illustrated by the following case: Suppose the eyes, to start with, accommodated for the far distance, and that it is desired to direct attention to a nearer point lying in the visual line of the right eye. In this case no movement of the right eye is required; all that is necessary is for the left eye to be turned to the right, that is, for the rectus internus of the left eye to be thrown into action. But in ordinary movements the contraction of this muscle is always associated with either the rectus externus of the right eye, as when both eyes are turned to the right, or the rectus internus of that eye, as in convergence; the muscle is quite unaccustomed to act alone. This would lead us to suppose that in the case in question the contraction of the rectus internus of the left eye is accompanied by a contraction of both recti externus and internus of the right eye, keeping that eye in lateral equilibrium. And the peculiar oscillating movements seen in the right eye, as well as the sense of efforts in the right eye which is felt by the person, show this to be the case.

$\S 685$. Such a complex coördination requires for its carrying out a distinct nervous machinery, and we have reasons for thinking that such a machinery exists in certain parts of the corpora quadrigemina or in the underlying structures. In the nates there appears to be a common centre for both eyes, stimulation of the right side producing movements of both eyes to the left, of the left side movements to the right; while stimulation in the middle line behind causes a downward movement of both eyes with convergence of the axes and in the front an upward movement with return to parallelism, both accompanied by the naturally associated movements of the pupil. Stimulation of various parts of the nates causes various movements, depending on the position of the spot stimulated. After an incision in the middle line, stimulation of the nervous centre on one side produces movements in the eye of the same side only. 
The Horopter.

$\S 686$. When we look at any object we direct to it the visual axis, so that when the object is small, the "corresponding" parts of the two retinas, on which the two images of the object fall, lie in their respective fovex centrales. But while we are looking at the particular object, the images of other

FIG. 181.

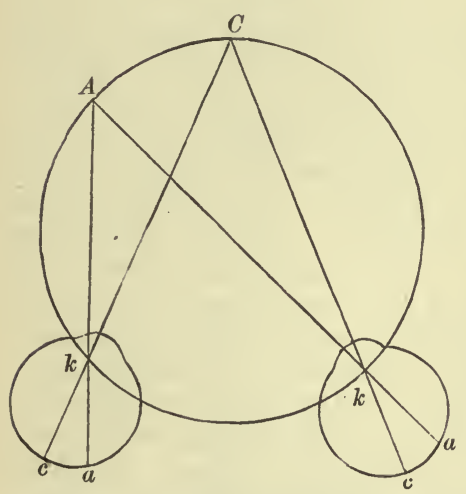

Diagram illustrating a Simple Horopter. When the visual axes converge at $C$, the inages $a, a$ of any point $A$ on the circle drawn through $C$ and the optical centres $k, k$ will fall on corresponding points. objects surrounding it fall on the retina surrounding the fovea, and thus go to form what is called indirect vision. And it is obviously of advantage that these images also should fall on "corresponding " parts in the two eyes. Now for any given position of the eyes there exists in the field of vision a certain line or surface of such a kind that the images of the points in it all fall on corresponding points of the retina. A line or surface having this property is called a horopter. The horopter is, in fact, the aggregate of all those points in space which are projected on to corresponding points of the retina; hence its determination in any particular case is simply a matter of geometrical calculation. In some instances it becomes a very complicated figure. The case whose features are most easily grasped is a circle drawn in the plane of the two visual axes through the point of the convergence of the axes and the optic centres of the two eyes. It is obvious from geometrical relations that in Fig. 181 the images of any point in the circle will fall on corresponding points of the two retinas. When we stand upright and look at the distant horizon, the horopter is (approximately, for normal emmetropic eyes) a plane drawn through our feet, that is to say, is the ground on which we stand; the advantage of this is obvious.

\section{VisUal Judgments.}

$\$ 687$. Binocular vision is of use to us, inasmuch as the one eye is able to fill up the gaps and imperfections of the other. For example, over and above the monocular filling up of the blind spot, of which we spoke on page 779 , since the two blind spots of the two eyes, being each on the nasal side, are not " corresponding" parts, the one eye supplies that part of the field of vision which is lacking in the other. And other imperfections are similarly made good. But the great use of binocular vision is to afford us means of forming visual judgments concerning the form, size, and distance of objects.

$\$ 688$. Judgment of distance and size. The perceptions which we gain simply and solely by our field of vision concern two dimensions only. We can become aware of the apparent size of any part of the field corresponding to any particular object, and of its topographical relations to the rest of the field, but no more. Had we nothing more to depend on, our sight would be almost valueless as far as any exact information of the external world was concerned. By the association of visual sensations with sensations of touch, and with sensations derived from the movements of the eyeballs required to make any such part of the field as corresponds to a particular 
object distinct, we are led to form judgments, $i$. e., to draw conclusions concerning the external world by means of an interpretation of our visual perceptions. Looking before us, we say we see a certain object of a certain color nearly in front of us or much on our right hand or much on our left; that is to say, we judge such an object to be in such a position because from the constitution of our brain, strengthened by all our experience, we associate such a part of our field of vision with such an object. The subjective visual complex sensation or perception is to us a symbol of the external object.

Even with one eye we can, to a certain extent, form a judgment, not only as to the position of the object in a plane at right angles to our visual axis, but also as to its distance from us along the visual axis. If the object is near to us, we have to accommodate for near vision; if far from us, to relax our accommodation mechanism so that the eye becomes adjusted for distance. The muscular sense (of which we shall speak presently) of this effort enables us to form a judgment whether the object is far or near. Seeing the narrow range of our accommodation, and the slight muscular effort which it entails, all molecular judgments of distance must be subject to much error. Everyone who has tried to thread a needle without using both eyes knows how great these errors may be. When, on the other hand, we use two eyes, we have still the variations in accommodation, and, in addition, have all the assistance which arises from the muscular effort of so directing the two eyes on the object that single vision will result. When the object is near, we converge our visual axes; when distant, we bring them back toward parallelism. This necessary contraction of the ocular muscles affords a muscular sense, by the help of which we form a judgment as to the distance of the object. Hence, when by any means the convergence which is necessary to bring the object into single vision is lessened, the object seems to become more distinct, when increased, to move toward us-as may be seen in the stereoscope.

$\S 689$. The judgment of size is closely connected with that of distance. Our perceptions, gained exclusively from the field of vision, go no further than the apparent size of the image, $i$. e., of the angle subtended by the object. The real size of the object can only be gathered from the apparent size of the image when the distance of the object from the eye is known. Thus perceiving directly the apparent size of the image, we judge the distance of the object giving the image, and upon that come to a conclusion as to its size. And, conversely, when we see an object, of whose real size we are otherwise aware, or are led to think we are aware, our judgment of its distance is influenced by its apparent size. Thus when in our field of vision there appears the image of a man, knowing otherwise the ordinary size of a man, we infer, if the image be very small, that the man is far off. The reason of the image being small may be because the man is far off, in which case our judgment is correct; it may be, however, because the image has been lessened by artificial dioptric means, as when the man is looked at through an inverted telescope, in which case our judgment becomes a delusion. So also an image on a screen when gradually enlarged seems to come forward, when gradually diminished seems to recede. In these cases the influence on our judgment of the muscular sense of binocular adjustment, or monocular accommodation, is thwarted by the more direct influence of the association between size and distance.

$\$ 690$. Judgment of solidity. When we look at a small circle, all parts of the circle are at the same distance from us, all parts are equally distinct at the same time, whether we look at it with one eye or with two eyes. When, on the other hand, we look at a sphere, the various parts of which are 
at different distances from us, a sense of the accommodation, but much more a sense of the binocular adjustment, of the convergence or the opposite of the two eyes, required to make the various parts successively distinct, makes us aware that the various parts of the sphere are unequally distant; and from that we form a judgment of its solidity. As with distance of objects, so with solidity, which is at bottom a matter of distance of the parts of an object, we can form a judgment with one eye alone; but our ideas become much more exact and trustworthy when two eyes are used. And we are much assisted by the effects produced by the reflection of light from the various surfaces of a solid object; so much so, that raised surfaces may be made to appear depressed, or vice versa, and flat surfaces either raised or depressed, by appropriate arrangements of shadings and shadow.

FIG. 182.

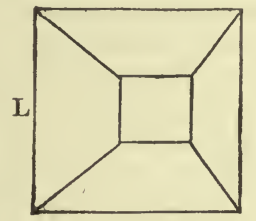

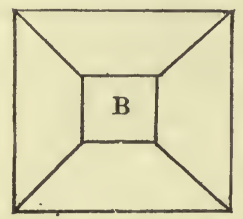

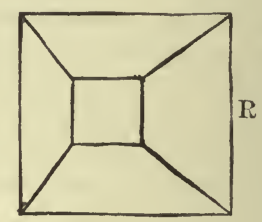

$\S 691$. Binocular vision, moreover, affords us a means of judging of the solidity of objects, inasmuch as the image of any solid object which falls on the right eye cannot be exactly like that which falls on the left, though both are combined in a single perception of the two eyes. Thus, when we look at a truncated pyramid placed in the middle line before us, the image which falls on the right eye is of the kind represented in Fig. 182, R, while that which falls on the left eye has the form of Fig. 182, L; yet the perception gained from the two images together corresponds to the form of which Fig. 182, B, is the projection. Whenever we thus combine in one perception two dissimilar images, one of the one and the other of the other eye, we judge that the object giving rise to the images is solid.

This is the simple principle of the stereoscope, in which two slightly dissimilar pictures, such as would correspond to the vision of each eye separately, are, by means of reflecting mirrors, as in Wheatstone's original instrument, or by prisms, as in the form introduced by Brewster, made to cast images on corresponding parts of the two retinas, so as to produce a single perception. Though each picture is a surface of two dimensions only, the resulting perception is the same as if a single object, or group of objects, of three dimensions had been looked at.

It might be supposed that the judgment of solidity which arises when two dissimilar images are thus combined. in one perception was due to the fact that all parts of the two images cannot fall on corresponding parts of the two retinas at the same time, and that therefore the combination of the two needs some movement of the eyes. Thus, if we superimpose R on L (Fig. 182), it is evident that when the bases coincide the truncated apices will not, and vice versa; hence, when the bases fall on corresponding parts, the apices will not be combined in one image, and vice versa; in order that both may be combined, there must be a slight, rapid movement of the eyes from the one to the other. That, however, no such movement is necessary for each particular case is shown by the fact that solid objects appear as such when illuminated by an electric spark, the duration of which is too short to permit of any movement of the eyes. If the flash occurred at the moment that the eyes were binocularly adjusted for the bases of the pyramids, the two 
apices not falling on exactly corresponding parts would give rise to two perceptions, and the whole object ought to appear confused. That it does not, but, on the contrary, appears a single solid, must be the result of cerebral operations, resulting in what we have called a judgment.

$\$ 692$. Struggle of the two fields of vision. If the images of two surfaces, one black and the other white, are made to fall on corresponding parts of the eye, so as to be united into a single perception, the result is not always a nixture of the two impressions, that is, a gray, but, in many cases, a sensation similar to that produced when a polished surface, such as plumbago, is looked at: the surface appears brilliant. The reason, probably, is because when we look at a polished surface, the amount of reflected light which falls upon the retina is generally different in the two eyes; and hence we associate an unequal stimulation of the two retinas with the idea of a polished surface. So, also, when the impressions of two colors are united in binocular vision, the result is, in most cases, not a mixture of the two colors, as when the same two impressions are brought to bear together at the same time on a single retina, but a struggle between the two colors, now one and now the other, becoming prominent, intermediate tints, however, being frequently passed through. This may arise from the difficulty of accommodating at the same time for the two different colors (see p. 758); if two eyes, one of. which is looking at red and the other at blue, be both accommodated for red rays, the red sensation will overpower the blue, and vice versa. It may be, however, that the tendency to rhythmic action, so manifest in other simpler manifestations of protoplasmic activity, makes its appearance also in the higher cerebral labors of binocular vision.

\section{The Protected Mechanisms of the Eye.}

$\S 693$. The eyeball is protected by the eyelids, which are capable of movements called respectively opening and shutting the eye. The eye is shut by the contraction of the orbicularis muscle, carried out either as a reflex or voluntary act by means of the facial nerve. The eye is opened chiefly by the raising of the upper eyelid through the contraction of the levator palpebræ carried out by means of the third nerve. The upper eyelid is also raised and the lower depressed, the eye being thus opened, by means of plain muscular fibres existing in the two eyelids and governed by the cervical sympathetic. The shutting of the eye, as in winking, is in general effected more rapidly than the opening.

The eye is kept continually moist partly by the secretion of the glands in the conjunctiva, and of the Meibomian glands, but chiefly by the secretion of the lachrymal gland. Under ordinary circumstances the fluid thus formed is carried away by the lachrymal canals into the nasal sac and thus into the cavity of the nose. When the secretion becomes too abundant to escape in this way it overflows on to the cheeks in the form of tears.

If a quantity of tears be collected, they are found to form a clear, faintly alkaline fluid, in many respects like saliva, containing about 1 per cent. of sulids, of which a small part is proteid in nature. Among the salts present sodium chloride is conspicuous.

$\S 694$. The nervous mechanism of the secretion of tears, in many respects, resembles that of the secretion of saliva. A flow is usually brought about either in a reflex manner by stimuli applied to the conjunctiva, the nasal mucous membrane, tongue, optic nerve, etc., or more directly by emotions. Venous congestion of the head is also said to cause a flow. The efferent nerves belong either to the cerebro-spinal system (the lachrymal and 
orbital branches of the fifth nerve) or arise from the cervical sympathetic, the afferent nerves varying according to the exciting cause.

The act of blinking undoubtedly favors the passage of tears through the lachrymal canals into the nasal sac, and hence when the orbicularis is paralyzed tears do not pass so readily as usual into the nose; but the exact mechanism by which this is effected has been much disputed. According to some authors, the contraction of the orbicularis presses the fluid onward out of the canals, which upon the relaxation of the orbicularis dilate and receive a fresh quantity. Others maintain that a special arrangement of muscular fibres keeps the canals open even during the closing of the lids, so that the pressure of the contraction of the orbicularis is able to have full effect in driving the tears through the canals.

\section{CH A P TER I V.}

\section{HEARING, SMELL, AND TASTE.}

$\S 695$. As in the eye, so in the ear, we have to deal first with a nerve of special sense, the stimulation of which gives rise to a special sensation; secondly, with terminal organs through which the physical changes proper to the special sense are enabled to act on the nerve; and thirdly, with subsidiary apparatus, by which the usefulness of the sense is increased. The central connections of the auditory nerve are such that whenever the auditory fibres are stimulated, whether by means of the terminal organs in the usual way or by the direct application of stimuli, electrical, mechanical, etc., the result is always a sensation of sound. Just as stimulation of the optic fibres produces no other sensation than that of light, so stimulation of the auditory fibres produces no other sensation than that of sound. ${ }^{1}$ The terminal organs of the auditory nerve are of two kinds : the complicated organ of Corti in the cochlea, and the epithelial arrangements of the maculæ and cristæ acusticæ in other parts of the labyrinth. Waves of sound falling on the auditory nerve itself produce no effect whatever; it is only when by the medium of the endolymph they are brought to bear on the delicate and peculiar epithelium cells which constitute the peripheral terminations of the nerve, that sensations of sound arise. Such delicate structures are for the sake of protection naturally withdrawn from the surface of the body where they would be subject to injury. Hence, the necessity of an acoustic apparatus, forming the middle and external ear, by which the waves of sound are most advantageously conveyed to the terminal organs.

\section{Hearing.}

$\S 696$. [The ear, or organ of hearing, is composed of three parts, called the external, middle, and internal ear.

The external ear consists of an outer projecting portion, called the pinna, and the auditory canal, or meatus auditorius externus. (Fig. 183.) The pinna is a somewhat oblong funnel-shaped organ, the smaller portion of the funnel being attached to the skull by ligamentous tissue, the larger portion serving to collect and convey the sonorous undulations to the meatus. It is composed of cartilage covered by integument. Its surface is irregularly

\footnotetext{
${ }^{1}$ It will be seen later on that there are reasons for thinking that impulses passing along the auditory nerve may give rise to other effects than auditory sensations.
} 
curved and depressed. The outer projecting rim is the helix; anterior to the helix is a second elevation, called the antihelix, which describes a curve partially around a deep depression which leads to the meatus, called the

FIG. 183.

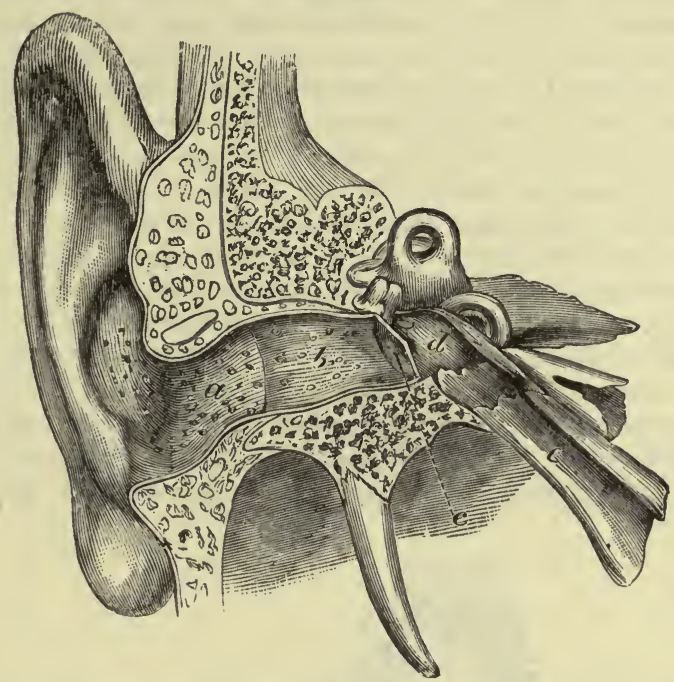

Vertical Section of the Meatus Auditorius and Tympanum. (Scarpa.) a, cartilaginous part of the meatus; $b$, osseous portion; $c$, membrana tympani; $d$, cavity of the tympanum; $e$, Eustachian tube.

FIG. 185.

FIG. 184.

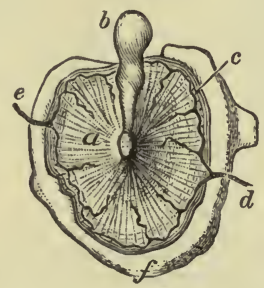

Fig. 184.-Inner view of the Membrana Tympani in the Fœtus, with the Malleus attached. $a$, membrane or drum of the tympanum; $b$, malleus; $c$, band of circular fibres at the circumference; $d$, inferior, and $e$, superior tympanic artery; $f$, tympanic bone.

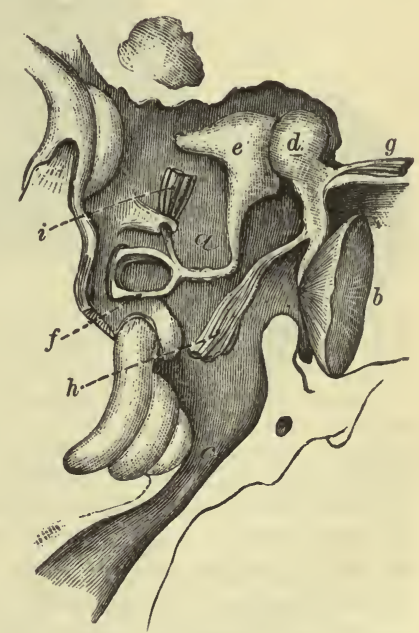

Fig. 185.-Plan of the Ossicles in Position in the Tympanum, with their Muscles. $a$, cavity of the tympanum; $b$, membrana tympani ; $c$, Eustachian tube; $d$, malleus; $e$, incus; $f$, stapes ; $g$, laxator tympani muscle; $h$, tensor tympani; $i$, stapedius.

concha. Between the helix and antihelix is the fossa of the helix. The antihelix bifurcates at its superior portion, and encloses the fossa of the antihelix. Projecting posteriorly from the anterior portion of the concha is a 
papillary prominence called the tragus; posterior to this, separated by a fissure, is the antitragus, which is a continuation of the helix. On the inferior portion of the pinna is a soft pendulous portion, termed the lobule. The meatus leads from the concha to the middle ear, from which it is separated by the tympanic membrane. Its direction is forward, inward, and slightly upward; its lower surface being longer than the upper, on account of the obliquity of the position of the tympanic membrane. The canal consists of an external membrano-cartilaginous portion, which is continuous with the pinna, and an internal osseous portion formed by the mastoid bone. In the external portion of the canal are found numerous hairs and sehaceous glands; in the internal portion are found the ceruminous glands, which secrete a peculiar substance commonly known as the earwax.

$\$ 697$. The middle ear or tympanum is an irregular flattened cavity, situated in the petrous portion of the temporal bone, and lined with a mucous membrane. It is separated from the meatus by a membranous diaphragm, which is the tympanic membrane; and from the internal ear by an osseo-membranous partition, which forms a common wall for both. Through the Eustachian tube it communicates with the pharynx. On its posterior wall are seen orifices of the mastoid cells. The tympanic membrane is a semi-transparent oval membrane, concave on its external and convex on its
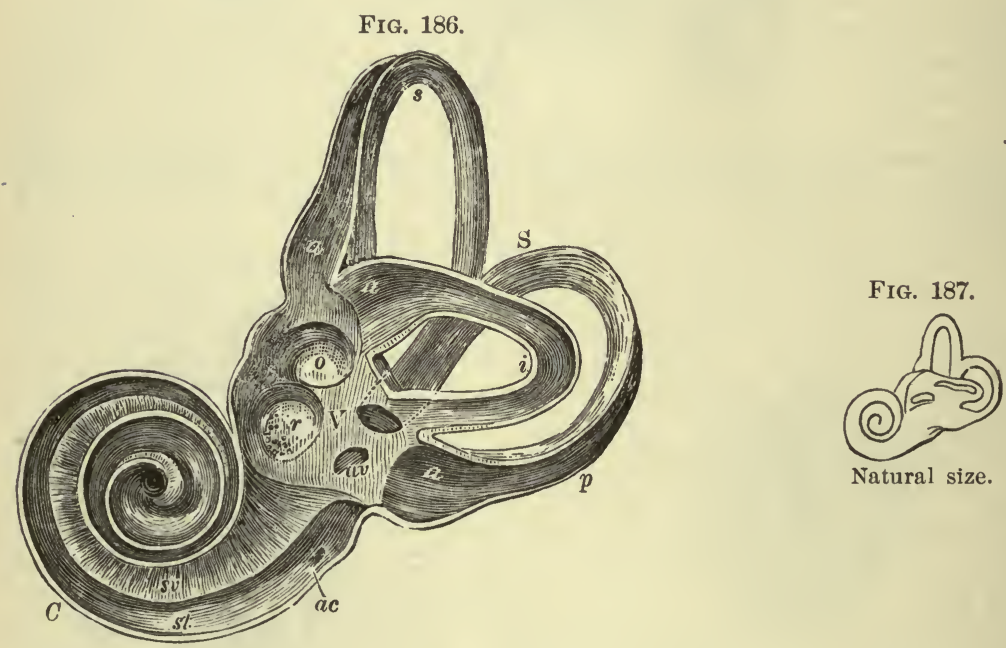

Natural size.

Interior of the Osseous Labyrinth. (After Sömmerring.) $V$, vestibule; av, aqueduct of the vestibule; $o$, fovea hemielliptica; $r$, fovea hemispherica; $S$, semicircular canals; $s$, superior; $p$, posterior; $i$, horizontal ; $a, a, a$, the ampullar extremity of each; $C$, cochlea; $a c$, aqueduct of the cochlea ; $s v$, osseous zone of the lamina spiralis, above which is the scala vestibuli, communicating with the vestibule; st, scala tympani below the spiral lamina.

internal surface, where it has attached the long process of the malleus, one of the ossicles. It is placed in an oblique position, sloping downward, forward, and inward at an angle of about $45^{\circ}$. Its circumference is attached to a groove in the temporal bone. In the fotus this portion of the bone exists as a separate piece, called the tympanic bone (Fig. 184), but it afterward becomes ossified to the temporal. The tympanic membrane consists of three layers-the external, middle, and internal. The external is a continuation of the integument covering the meatus; the internal is a continuation of the mucous membrane lining the tympanum; the middle layer, 
which is the most important, is tense, strong, and fibrous, made up of circular and radiating fibres, with a small amount of elastic tissue intermixed.

$\$ 698$. In the internal wall of the tympanum are two small openingsthe fenestra ovalis and fenestra rotunda-which communicate with the labyrinth. The fenestra rotunda is closed by a membrane. Extending between the tympanic membrane and the fenestra ovalis are the ossicles, consisting of three small bones, which form a system of levers. These ossicles are termed, from their resemblance to particular objects, the malleus, incus, and stapes. (Fig. 185.) The malleus consists of a head, neck, long and short process, and handle. The head articulates with the roof of the tympanum and in a depression of the incus; the handle is directed downward and attached by its whole length to the tympanic membrane; the long process (processus gracilis) is directed forward and has attached the insertion of the laxator tympani muscle; the short process, which is at the base of the long process, has attached the insertion of the tensor tympani muscle. The incus consists of a body, a long and short process. The hody of the incus has a depression in which articulates the liead of the malleus; the short process is attached to the posterior wall of the tympanum; the long process (lenticular process) is placed almost vertically, and at its end is a rounded

FIG. 188.

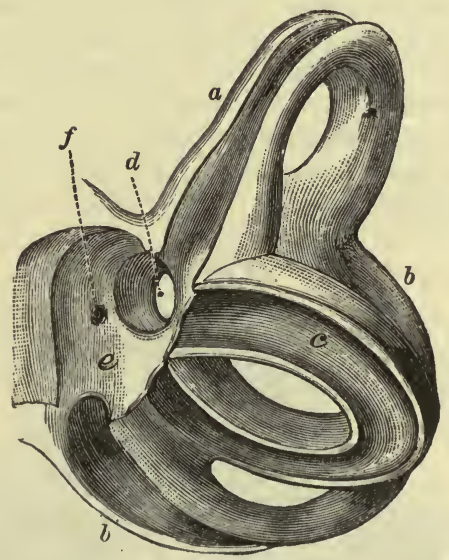

Representation of the Semicircular Canals Enlarged. (From a model in University College Museum.) $a$, superior vertical ; $b$, posterior or inferior vertical ; and $c$, horizontal canal ; $d$, common opening of the two vertical canals; $e$, part of the vestibular cavity; $f$, opening of the aqueduct of the vestibule.

process (the os orbiculare), which articulates with the head of the stapes. The stapes consists of a head, neck, two crura, and a base. The head articulates with the long process of the incus; the neck serves as a point of insertion of the stapedius muscle; the crura diverge from the neck and unite with the oval base at its greatest diameter. The base is fixed in the fenestra ovalis by attachments formed by the lining membranes of both the tympanum and internal ear. These ossicles are connected with each other and to the walls of the tympanum by ligaments, and at their articulations they are furnished with cartilages and synovial membranes. They are enveloped by prolongations of the mucous membrane lining the tympanum.

$\$ 699$. The internal ear or labyrinth is the most essential portion of the auditory apparatus. It consists of three portions-the vestibule, semicircular 
canals, and cochlea-and is situated within the petrous portion of the temporal bone. Within the osseous labyrinth is a membranous labyrinth to which the auditory nerve is distributed. The vestibule is an irregular chamber which serves as a common means of communication between the tympanum and the semicircular canals and cochlea. On its external wall is the fenestra ovalis, closed by the base of the stapes. On its internal wall is a depression called the fovea hemispherica, which is perforated by minute openings for the passage of auditory nerve-filaments. Above and posterior to this depression is another, the fovea hemielliptica. Posterior to the fovea hemispherica is the orifice of the aqueductus vestibuli. In the posterior wall are five openings leading to the semicircular canals. Anteriorly, it communicates with the cochlea by the aperturce scaloe vestibuli cochleoe. The semicircular canals are three in number-superior, posterior or inferior, and horizontal. They form the greater portion of a circle, and communicate with the vestibule by five openings, one of which is common to the superior and horizontal canals. The superior canal is situated vertically and at right angles with the posterior surface of the petrous bone; the posterior canal is also vertical and parallel with the posterior surface of the petrous bone; the inferior canal is placed horizontally and at right angles to the others. At the commencement of each of these canals is a dilated portion, called the ampulla.

$\$ 700$. The cochlea occupies the anterior portion of the labyrinth. Its base, which corresponds to the internal auditory meatus, is perforated by many minute orifices for the passage of filaments of the cochlear branch of the auditory nerve. The cochlea consists of a central axis, or modiolus, which has a spiral canal wound around it. This canal makes two and a half complete turns, and terminates in the apex of the cochlea in an expansion termed the infundibulum. (Fig. 189.) The modiolus is somewhat

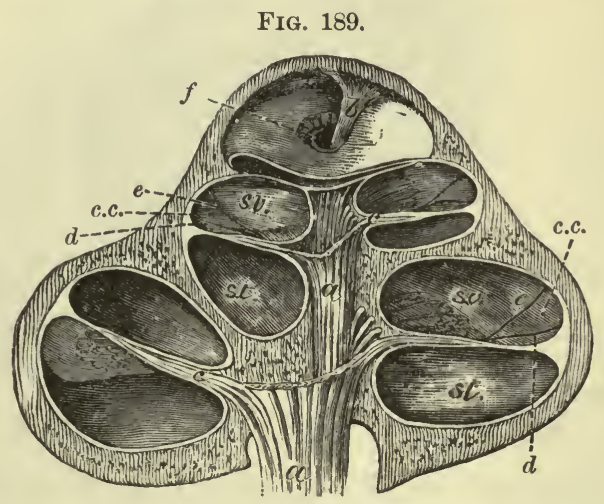

Section through the Cochlea. (Breschet.) $a$, axis with its canals; $b$, infundibulum or enlarged upper end of the axis; $c$, septum of the cochlea; $d$, membrane of Corti ; $e$, membrane of Reissner; $f$, hiatus or hellicotrema; st, scala tympani ; $s v$, scala vestibuli.

cone-shaped, and forms the internal wall of the canal, being perforated in its centre and sides by apertures for the passage of the filaments of the auditory nerve. The canal is divider into two passages or scalce by a septum called the lamina spiralis, which is partly osseous and partly membranous. The osseous portion projects from the modiolus, midway across the canal; it consists of two laminæ, between which the nerve-filaments run. The membranous portion extends from the external margin of the osseous lamina 
to the external wall of the canal. It consists of two layers; the superior is the membrane of Corti, or membrana tectoria; the inferior the membrana basilaris, which is attached externally to the planum semilunare. These membranes are placed parallel with each other and contain between them the organ of Corti, which rests on the basilary membrane. (Fig. 190.)

$\S 701$. The scala vestibuli ${ }^{1}$ communicates below with the vestibule by the aperturce scalce vestibuli cochlece; the lower passage, or scala tympani, communicates with the tympanum by the fenestra rotunda. These scalæ communicate at the apex of the cochlea by an opening termed the hiatus or helicotrema, which exists in consequence of a deficiency of the last half turn of the lamina spiralis.

The osseous portion of the lamina spiralis has on its superior external portion a denticulated cartilaginous substance called the lamina denticulata. From the superior surface of the spiral lamina, and internal to the lamina denticulata, is a delicate membrane extending upward and outward at an angle of about 45 degrees to the external wall of the scala. This is called the membrane of Reissner. It divides the scala into two passages, the lower of which is the ductus cochlearis. This duct ends in the apex of the cochlea in a coca, and communicates at the base with the saccule by the ductus reuniens; it contains the essential portion of the auditory apparatus of the cochlea, and is a part of the membranous labyrinth.

FIG. 190.

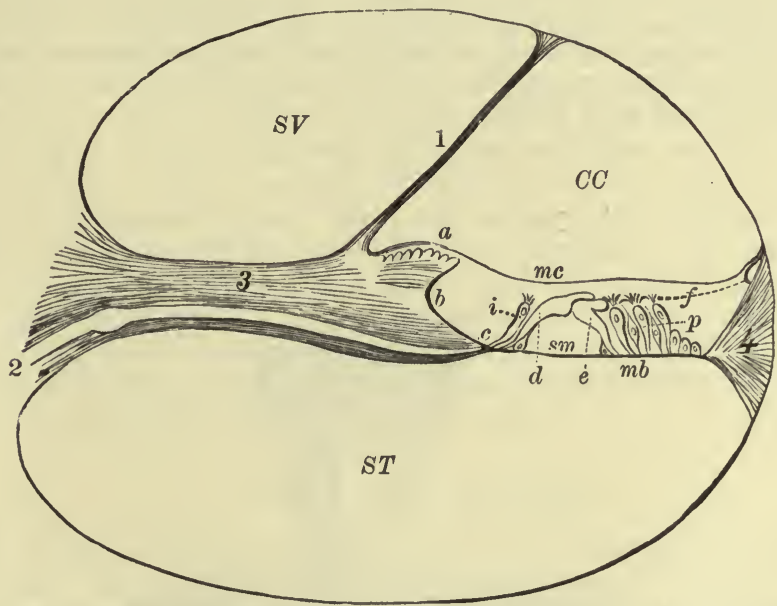

A Diagram of a Section of the Tube of the Cochlea, enlarged. (Modified from Henle.): $S V$, scala vestibuli; $S T$, scala tympani; $C C$, canal of the cochlea; 1 , membrane of Reissner; 2 , cochlear branch of the auditory nerve; 3 , lamina spiralis ossea; 4 , planum semilunare; $a$, lamina denticulata ; $b$, sulcus spiralis; $c$, tympanic lip of the sulcus spiralis; $d$, inner rods of Corti ; $e$, outer rods of Corti ; $f$, lamina reticularis; $i$, inner hair-cells ; $m b$, membrana basilaris; $m c$, membrane of Corti ; $p$, outer hair-cells; $8 m$, central space between the rods.

$\$ 702$. The organ of Corti rests upon the basilary membrane. It consists of the inner and outer hair-cells, and two rows of elongated cells, placed parallel with each other, having an inclining position so that their free extremities rest against each other and thus form the arch of Corti, which covers the central space. (Fig. 190.) These rows are called the inner and outer rods or pillars of Corti. From the superior extremity of both the inner and

1 The upper scala is divided into two parts by a membranous partition, the upper of which is called the scala vestibuli ; the other, ductus cochlearis (Fig. 190). 
outer rods, finger-like processes project externally. At their bases corresponding to the central space are single rows of nucleated cells. On the

FIG. 191.

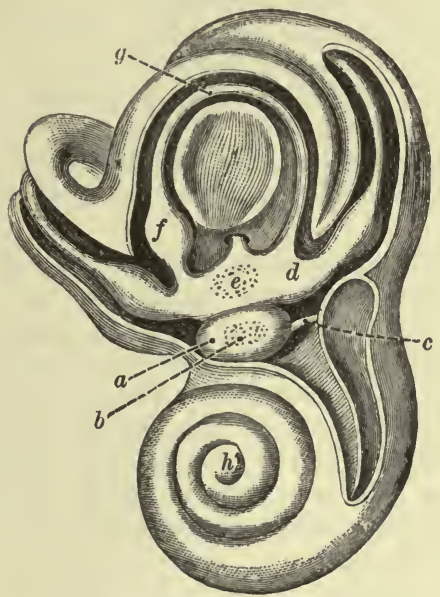

FIG. 192.

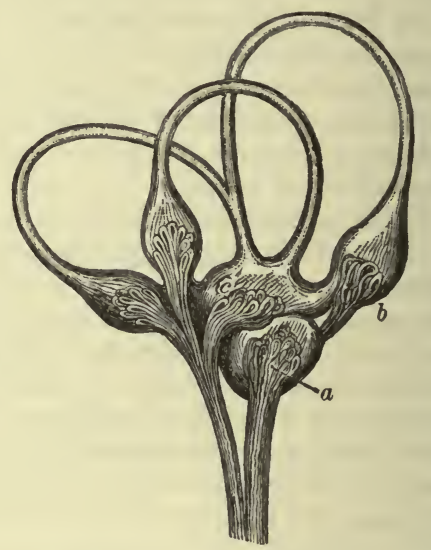

Fig. 191.- Petrous Bone partly Removed to Show the Membranous Labyrinth in Place. (Breschet.) $a$, small sac; $b$, its otolith ; $c$, ductus reuniens ; $d$, large sac or utricle ; $e$, its otolith; $f$, ampullary enlargements on a semicircular tube ; $g$, semicircular tube.

Fig. 192.-Distribution of Nerves to the Membranous Labyrinth. (Breschet.) $a$, nerve to the saccule; $b$, nerve entering the ampullary enlargement on a semicircular tube; $c$, branch of the nerve entering the large sac or utricle.

Frg. 193.

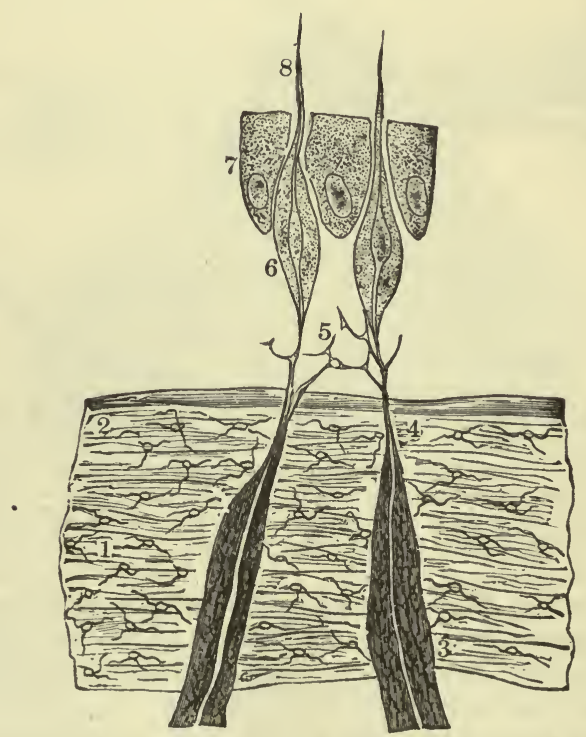

Diagram of the mode of Termination of the Auditory Nerve in the Ampullæ and Sacculi. 1, wall of the ampulla; 2 , structureless basement-membrane; 3 , doubly contoured nerve-fibres; 4 , axis-cylinder traversing the basement-membrane; 5 , plexiform union of fine nerve-fibres with interspersed nuclei ; 6 , fusiform cells, with nucleus and dark fibre in their interior; 7 , supportiug cells ; 8 , auditory hairs. 
internal side of the inner rod is a single row, and on the external side of the outer rods are three rows of elongated ciliated cells. Extending across the top of the organ of Corti, from the inner hair-cells to the external wall of the canal, is a very delicate structure called the reticular membrane. The auditory nerve-filaments probably terminate in the ciliated cells, being intimately connected with the cilia.

$\$ 703$. The osseous labyrinth is lined by a fibro-serous membrane which secretes a watery fluid called the perilymph. The perilymph fills the scalæ of the cochlea, and surrounds the ductus cochlearis and the membranous portions of the labyrinth, which are situated in the vestibule and semicircular canal.

$\S 704$. The membranoue labyrinth is a closed sac consisting of the semicircular canals, a vestibular portion, and the ductus cochlearis of the cochlea. The semicircular canals are of the same form as the osseous canals, and are contained within them. The vestibular portion consists of an expanded body, the utricle, and a smaller body, the saccule. The utricle is situated at the fovea hemielliptica ; the semicircular canals open on its internal surface. The saccule lies at the fovea hemispherica; it is connected with the ductus cochlearis by the ductus reuniens. In the walls of the saccule and utricle are two calcareous bodies called the otoliths. The walls of the ampulla, according to Bowman, also contain some grains of a similar substance. The walls of the membranous labyrinth consist of a fibrous tissue, lined by pavement nucleated epithelial cells, having a structureless basement-membrane. These epithelial cells are much modified at the place of entrance of the fibres of the auditory nerve. The vestibular branches of the auditory nerve are distributed to the ampullæ, utricle, and saccule. (Fig. 192.) In the utricle and the saccule the fibres terminate in oval plates, called the maculce acustica, which are more or less colored by the deposition of yellow pigment. In the ampullæ the fibres terminate in elevations called the cristce acustica. After the nerve-filament pierces the membranous wall at these points, the axis-cylinder alone penetrates the basement-membrane; it then forms a plexus of delicate nerve-fibres with nuclei, and finally terminates in fusiform epithelial cells which have terminal cilia called the auditory hairs. (Fig. 193.) These ciliated cells are supported by columnar epithelium.

The membranous labyrinth is lined by polygonal nucleated epithelium, which secretes the endolymph which fills the sac.]

\section{The Acoustic Apparatus.}

$\S$ 705. Waves of sound can and do reach the endolymph of the labyrinth by direct conduction through the skull. Since, however, sonorous vibrations are transmitted with great difficulty from the air to solids and liquids, and most sounds come to us through the air, some special apparatus is required to transfer the aërial vibrations to the liquids of the internal ear. This apparatus is supplied by the tympanum and its appendages.

$\S 706$. The concha. The use of this, as far as hearing is concerned, is to collect the waves of sound coming in various directions, and to direct them on to the membrana tympani. In ourselves of moderate service only, in many animals it is of great importance.

$\$$ 707. The membrana tympani. It is a characteristic property of stretched membranes that they are readily thrown into vibration by aërial waves of sound. The membrana tympani, from its peculiar conformation, being funnel-shaped with a depressed centre surrounded by sides gently convex outward, is peculiarly susceptible to sonorous vibrations, and is most readily thrown into corresponding movements when waves of sound reach it 
by the meatus. It has, moreover, this useful feature, that unlike other stretched membranes, it has no marked note of its own. It is not thrown into vibrations by waves of a particular length more readily than by others. It answers equally well within a considerable range to vibrations, of very different wave-lengths. Had it a fundamental tone of its own, we should be distracted by the prominence of this note in most of the sounds we hear. When sounds impinge on the solids of the head, as when a watch is held between the teeth, the membrana tympani is still functional. Vibrations are conveyed from the temporal bone to it and hence pass in the usual way, in addition to those transmitted directly from the bone to the perilymph.

$\$ 708$. The auditory ossicles. The malleus, the handle of which descending forward and inward, is attached to the membrana tympani, and the incus, whose long process is connected by means of its os orbiculare or lenticular process and the stapes to the fenestra ovalis, form together a body which rotates round an axis, passing through the short process of the incus, the bodies of the incus and malleus, and the processus gracilis of the malleus. [Fig. 194.] When the malleus is carried inward, the incus moves inward

[FIG. 194.

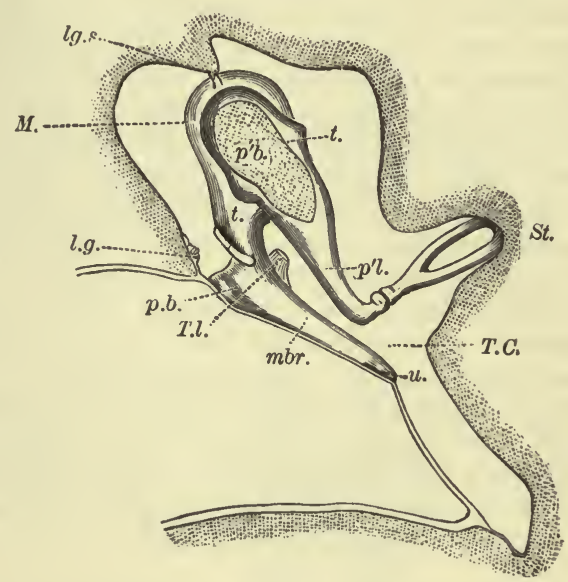

The Ossicles in Position. Magnified four times. (After Hensen.) The figure represents a section through tympanum in the line of the long axis of the malleus and incus; the short process of the incus, $p^{\prime} b^{\prime}$, has been cut through.

$T . C$, the tympanic cavity ; $m b r$, handle of malleus; $u$, umbo; $p . b$, short process of the malleus shown in dotted outline as pushing ontward the membrana flaccida; $T . l$, the attachment of the tendon of the tensor tympani; $l g$, the attachment of the external ligament of the malleus; $l g . s$, the superior ligament of the malleus; $t . t$, the teeth of the incus; $p^{\prime} l$, the long process shaft of the incus; St, the stapes.]

too, and when the malleus returns to its position, the incus returns with it, the peculiar saddle-shaped joint with its catch-teeth permitting this movement readily, but preventing the stapes being pulled back when the membrana tympani with the malleus is for any reason pushed outward more than usual; the joint then gapes, so as to permit the malleus to be moved alone. Various ligaments, the superior or suspensory, anterior, and external, also serve to keep the malleus in place. The whole series of ossicles may be regarded as a single-armed lever, moving on the ligamental attachment of the short process of the incus to the posterior wall of the tympanum, the weight being brought to bear at the end of the long process of the incus, and the power at the end of the handle of the malleus. The long, malleal 
arm of this lever is about $9 \frac{1}{2} \mathrm{~mm}$., the short, staperial, $6 \frac{1}{3} \mathrm{~mm}$. in length ; hence, the movements of the stapes are less than those of the tympanum; but the loss in amplitude is made up by a gain of force, which is in itself an obvious advantage.

Thus every movement of the tympanic membrane is transmitted through this chain of ossicles to the membrane of the fenestra ovalis, and so to the perilymph of the labyrinth; the vibrations of the tympanic membrane are conveyed with increased intensity, though with diminished amplitude, to the latter. That the bones thus move en masse has been proved hy recording their movements in the usual graphic method. A very light style attached to the incus or stapes is made to write on a travelling surface; when the membrana tympani is thrown in to vibrations by a sound the curves described by the style indicate that the chain of bones moves with every vibration of the tympanum. On the other hand, the comparatively loose attachments of the several bones is an obstacle to the molecular transmission of sonorous vibrations through them. Moreover, sonorous vibrations can only be transmitted to or pass along such bodies as either are very long compared to the length of the sound-waves, or, as in the case of membranes and strings, have one dimension very much smaller than the others. Now the bones in question are not especially thin in any one dimension, but are in all their dimensions exceedingly small compared with the length of the vibrations of even the shrillest sounds we are capable of hearing; hence, they must be useless for the molecular propagation of vibrations.

[FIG. 195.

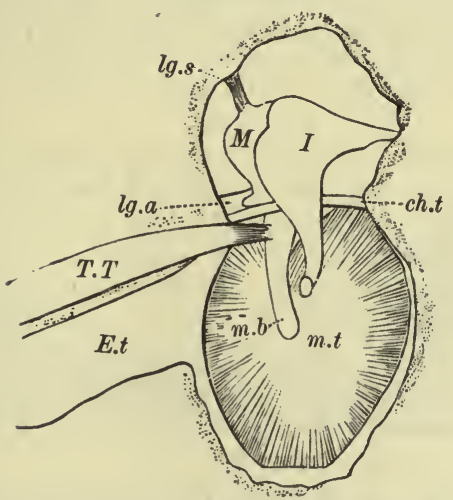

[FIG. 196.

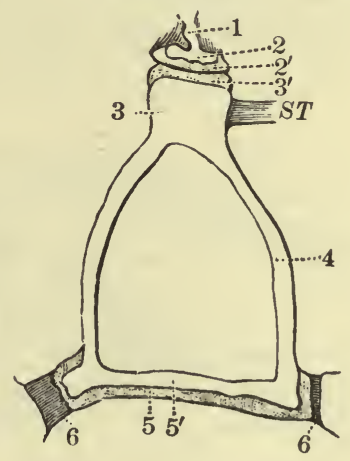

FIG. 195.-Diagram of the Outer Wall of the Tympanum (Right Ear) as Seen from the Mesial Side, showing Insertion of Tensor Tympani. Magnified twice. (After Schwalbe.) m.t, membrana tympani; $m . b$, bandle of $M$, the malleus; $I$, the incus; E.t, Eustachian tube; $T . T$, tensor tympani, the tendon of which is attached to the bandle of the malleus; lg.a, the anterior, and lg.s, the superior, ligament of the malleus; ch.t, the chorda tympani nerve passing through the tympanic cavity.

FIG. 196.-The Stapes in Position. Much magnified. (Schwalbe.) 1, the end of the shaft of the incus ; 2 , its expansion or os orbiculare; $2^{\prime}$, the articular cartilage of the same ; 3 , the capitulum of the stapes ; $3^{\prime}$, its articular cartilage ; 4 , the hoops of the stapes; 5 , the foot-plate of the stapes ; $5^{\prime}$, its articular cartilage; 6 , the membrane of the fenestra ovalis; $S T$, the tendon of the stapedius muscle attached to the capitulum of the stapes.]

$\S$ 709. The tensor tympani muscle even in a quiescent state is of use in preventing the membrana tympani being pushed out far. (Fig. 195.) When it contracts it renders the membrana tympani more tense, and hence has been supposed to act as a damper, lessening the amount of vibration of the membrane in the case of too powerful sounds; it is said to be readily thrown 
into contraction at the commencement of a suund or noise, but to return to rest during the continuance of a musical note. Efferent impulses reach it through fibres of the fifth nerve, and its activity is regulated by a reflex action. In some persons the muscle seems to be partly under the dominion of the will, since a peculiar crackling noise which these persons can produce at pleasure appears to be caused by a contraction of the tensor tympani.

The so-called laxator tympani is considered to be not a muscle at all, but a part of the ligamentous supports of the malleus.

$\$ 710$. The stapedius muscle by pulling upon the head of the bone (Fig. 196 ) is supposed to regulate the movements of the stapes, and especially to prevent its base being driven too far into the fenestra ovalis during large or sudden movements of the membrana tympani. It is governed by fibres from the facial nerve.

$\$ 711$. The Eustachian tube. This serves to maintain an equilibrium of pressure betwen the external air and that within the tympanum, and to serve as an exit for the secretions of that cavity. Were the tympanum permanently closed the vibrations of the membrana tympani would be injuriously affected by variations of pressure occurring either inside or outside. The Eustachian tube is undoubtedly open during swallowing, but it is still disputed whether it remains permanently open or is opened only at intervals; probably it is, at most times, neither widely open nor closely shut.

\section{Auditory Sensations.}

$\S 712$. Each vibration communicated by the stapes to the perilymph travels as a wave over the vestibule, the semicircular canals, and other parts of the labyrinth; and from the perilymph is transmitted through the mem-

FIg. 197 .

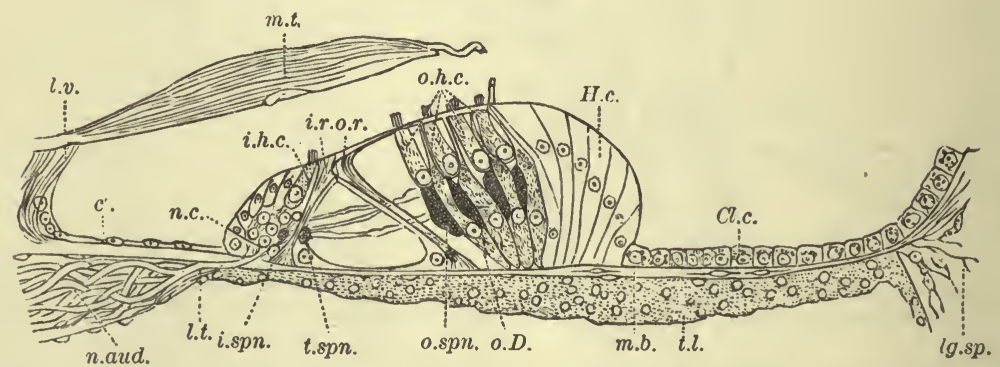

Diagram of the Organ of Corti. (After Retzius.) i.r, inner rod of Corti ; o.r, outer rod of Corti. i.h.c, inner hair-cells; n.c, the group of nuclei beneath it; o.h.c, outer hair-cell, or cell of Corti, of the first row ; c.D, its twin cell of Deiters-four rows of these twin cells are shown.

$n . a u d$, the auditory nerve perforating the tympanic lip, $l . t$, and lost to view among the nuclei beneath the inner hair-cell; $i . s p . n$, the inner spiral strand of nerve-fibrillæ; $t . s p . n$, the spiral strand of the tunnel; $0.8 p . n$, the outer spiral strand belonging to the first row of outer hair-cells; the three succeeding spiral strands belonging to the three other rows are also shown. Nervefibrillæ are shown stretching radially across the tumnel.

H.c, Hensen's cells; Cl.c, Claudius's cells; m.b, basilar membrane; $t . l$, lymphatic epithelioid lining of the basilar membrane on the side toward the scala tympani; lg.sp, spiral ligament; $c^{\prime}$, cells lining the spiral groove, overhung by $l . v$, the vestibular lip; m.t, the tectorial membrane-a fragment of it is seen torn from the rest and adherent to the organ of Corti just outside the outermost row of outer hair-cells.

branous wall to the endolymph. From the vestibule it passes on into the scala restibuli of the cochlea, and descending the scala tympani, ends as an impulse against the membrane of the fenestra rotunda. In the regions of the maculæ and cristæ the vibrations of the endolymph are supposed to throw 
into corresponding vibrations the so-called auditory hairs. In the cochlea the vibrations of the perilymph are supposed to throw into vibrations the basilar membrane with the superimposed organ of Corti, consisting of the rods of Corti with the inner and outer hair-cells. (Fig. 197.) The vibrations thus transmitted to these structures give rise to nervous impulses in the terminations of the auditory nerves, and these impulses reaching certain parts of the brain produce what we call auditory sensations. We are accustomed to divide our auditory sensations into those caused by noises and those caused by musical sounds. It is the characteristic of the latter that the vibrations which constitute them are periodical; they occur and recur at regular intervals. When no marked periodicity is present in the vibrations, when the repetition of the several vibrations is irregular, or the period so complex as not to be readily appreciated, the sensation produced is that of a noise. There is, however, no abrupt line between the two. Between a pure and simple musical sound produced by a series of vibrations, each of which has exactly the same wave-length, and a harsh noise in which no consecutive vibrations may be alike, there are numerous intermediate stages.

$\$ 713$. In both noises and musical sounds we recognize a character which we call loudness. This is determined by the amplitude of the vibrations; the greater the disturbance of the air (or other medium) the louder the sound. In a musical sound we recognize also a character which we call pitch. This is determined by the wave-length of the vibrations; the shorter the wave-length, the larger the number of consecutive vibrations which fall upon the ear in a second, the higher the pitch. We are able to speak of a whole series of tones or musical sounds of different pitch, from the lowest to the highest audible tone. And even in many noises we can, to a certain extent, recognize a pitch, indicating that among the multifarious vibrations there is a periodicity of certain groups of vibrations.

$\S$ 714. Lastly, we distinguish musical sounds by their quality; the same note sounded on a piano and on a violin produce very different sensations, even when a series of vibrations having in each case the same period of repetition is set going. This arises from the fact that the musical sounds generated by most musical instruments are not smple but compound vibrations. When the note $\mathrm{C}$ in the treble, for instance, is struck on the piano, and we analyze the total sound, we find that it can be resolved partly into a series of vibrations with a period characteristic of the pure tone of the treble $\mathrm{C}$, and partly into other series of vibrations with periods characteristic of the $\mathrm{C}$ in the octave above, of the $\mathrm{G}$ above that, of the $\mathrm{C}$ in the next octave, and of the $\mathrm{E}$ above that. And the sensation which we associate with the sound of the treble $\mathrm{C}$ on the piano is determined by the characters of the complex vibration arising out of these several constituent simple vibrations. Almost all musical sounds are thus composed of what is called a "fundamental tone" accompanied by a number of "overtones." And the overtones varying in number and relative prominence in different instruments, give rise to a difference in the sensation caused by the whole tone. So that while the fundamental tone determines the pitch of the sound, the quality of the sound is determined by the number and relative prominence of the overtones. In a somewhat similar way we distinguish the quality of noises, such as a banging, crackling, or rustling noise, by an appreciation of sudden or irregular changes in the amplitude and period of the constituent vibrations.

$\S 715$. Since we have a very considerable appreciation, capable by exercise of astonishing enlargement, of the loudness, pitch, and quality of a wide range of noises and musical sounds, it is clear that, within the 
limits of hearing, each vibration or series of vibrations must produce its effect on the auditory nerves, according to the measure of its intensity and period. Out of those effects, out of the sensory impulses to which the several vibrations thus give rise, are generated our sensations of the noise or of the sound.

The vibrations of a musical sound (and since noises are so imperfectly understood, we may, with benefit, chiefly confine ourselves to musical sounds), as they pass through the air (or other medium) are not discrete; the vibrations corresponding to the fundamental tone and overtones do not travel as so many separate waves; they all together form one complex disturbance of the medium; and it is as one composite wave that the sound falls on the membrana tympani, and passing through the auditory apparatus, breaks on the terminations of the auditory nerve. And when two or more musical sounds are heard at the same time, the same fusion of the waves occurs. Since we can distinguish several tones reaching our ear at the same time, it is clear that we must possess in our minds or in our ears some means of analyzing these composite waves of sound which fall on our acoustic organs, and of sorting out their constituent vibrations.

$\$ 716$. There is at hand a simple and easy physical method of analyzing composite sounds. If a person standing before an open piano sings out any note, it will be observed that a number of the strings of the piano will be thrown into vibration, and on examination it will be found that those strings which are thus set going correspond in pitch to the fundamental tone and to the several overtones of the note sung. The note sung reaches the strings as a complex wave, but these strings are able to analyze the wave into its constituent vibrations, each string taking up those vibrations and those vibrations only which belong to the tone given forth by itself when struck. If we suppose that each terminal fibril of the auditory nerve is connected with an organ so far like a piano-string that it will readily vibrate in response to a series of vibrating impulses of a given period and to none other, and that we possess a number of such terminal organs sufficient for the analysis of all the sounds which we can analyze, and that each terminal organ so affected by particular vibrations gives rise to a sensory impulse and thus to a sensation of a distinct character-if we suppose these urgans to exist, our appreciation of sounds is in a large measure explained. In the organ of Corti we find structures, the arrangement of which irresistibly suggests to us that these are the organs we are seeking. We have only to suppose that of the long series of rods of Corti, varying regularly as these do from the bottom to the top of the spiral, in length and in the span of their arch, each pair will vibrate in response to a particular tone, and the whole matter seems explained. But the more the subject is inquired into, the more complex and difficult it appears; and we are obliged to conclude that the part played by the rods of Corti is only a subordinate part of the function of the whole organ of Corti.

In the first place, it is difficult to see how the rods of Corti, even ii" they are thrown into vibration, can originate sensory impulses, for the fibrils of the auditory nerve terminate in the inner and outer hair-cells, and it is in these cells, and not along the course of these fibrils as they pass under and between the rods of Corti, that the sensory impulses must begin. In the second place, the variation in length of the fibres along the series is insufficient for the work assigned to them. Moreover, they appear not to be clastic. Lastly, they are wholly absent in birds, who very clearly can appreciate musical sounds. This last fact proves indubitably that the rods in question are not absolutely essential for the recognition of tones. In the face of these 
difficulties it has been suggested that the basilar membrane, which is present in birds as well as in mammals, and which, being tense radially but loose longitudinally, $i$. e., along the spiral of the cochlea, may be considered as consisting of a number of parallel radial strings, each capable of independent vibrations, is the sought-for organ of analysis; for it may be shown mathematically that a membrane so stretched in one direction only is capable of vibrating in such a manner. And the radial dimensions of the basilar membrane give a much greater range of difference than do the rods of Corti, diminishing in man downward from $0.495 \mathrm{~mm}$. at the top to $0.04125 \mathrm{~mm}$. near the bottom of the spiral, whereas the difference in length of the latter is simply that between 0.048 and $0.085 \mathrm{~mm}$. for the inner and between 0.019 and $0.085 \mathrm{~mm}$. for the outer fibres. According to this view, a particular simple vibration reaching the scala tympani of the cochlea throws into sympathetic vibrations a small portion of the basilar membrane, the vibrations of which in turn so affect the structures overlying it that sensory impulses are generated. The sensory impulses reaching the brain give rise to a corresponding sensation of a particular tone.

The remarkable reticular membrane which has such peculiar relations with the hair-cells, and through them with the basilar membrane, must, one might imagine, have some special function; but it is impossible at present to assign to it any satisfactory duty. The structural arrangements seem, if anything, to indicate, that when a segment of the basilar membrane is thrown into vibrations, the overlying hair-cells, reticular membrane, and rods of Corti vibrate en masse with it. But this renders the whole matter still more difficult. Indeed the whole subject is in the highest degree obscure, and the most we can say is that the organ of Corti as a whole seems to be in some way connected with the appreciation of tones, but that at present it is very hazardous to attempt to explain how it acts, or to assign particular functions to particular parts. The distinction between the inner and outer hair-cells seems to be very parallel to that between the rods and cones of the retina; but even this analogy may be a fallacious one.

It has been observed that among the auditory hairs of the crustacea, some will vibrate to particular notes; but the auditory hairs of the mammal are far too much of the same length to permit the supposition that they can act as organs of analysis.

If the organ of Corti is the means by which we appreciate tones, it is evident that by it also we must be able to estimate loudness, for the quality of a musical sound is dependent on the relative intensity, as well as on the nature, of the overtones. And since noise is at best but confused music, the cochlea must be a means of appreciating noises as well as sounds. But this would leave nothing whatever for the rest of the labyrinth to do in respect to the appreciation of sound save so far as the difference in structure between the hair-cells of Corti, with their short, thick rods, and the hair-bearing structures in the maculæ and cristæ, with their thin, delicate hairs, may possibly indicate a difference of function, the latter being more susceptible to the irregular vibrations of noises. That the vestibule and semicircular canals are, however, concerned in hearing is shown by its being the only auditory organ in the ichthyopsida, unless we suppose that in the higher vertebrates its function has been wholly transferred to the cochlea. That the semicircular canals may have duties apart from hearing we shall show later on.

$\$ 717$. Concerning the function of the other parts of the internal ear we know very little. The otoliths have been supposed to intensify the vibrations of the endolymph; but since apparently they are lodged in a quantity of mucus it is probable that they really act as dampers. A similar damping 
action has been suggested for the membrane of Corti (membrana tectoria) overhanging the fibres and hair-cells; and some writers have supposed that muscular fibres present in the planum semilunare may by tightening the basilar membrane serve as a sort of acconımodation mechanism.

It must, however, be borne in mind that even making the fullest allowance for the assistance afforded us by the organ of Corti, the appreciation of any sound is ultimately a mental act. The analysis of the vibrations by the fibres of Corti or the basilar membrane is simply preliminary to a synthesis of the sensory impulses so generated into a complex sensation. We do not receive a distinct series of specific auditory impulses resulting in a specific sensation for every possible variation in the wave-length of sonorous vibrations any more than we receive a distinct series of specific visual impulses for every possible wave-length of luminous vibrations. In each case we have probably a number of primary sensations, from the various mingling of which, in different proportions, our varied complex sensations arise; the difference between the eye and the ear being that whereas in the former the number of primary sensations appears to be limited to three or at most to six, in the latter, thanks to the organ of Corti, the number is very large; what the exact number is we cannot at present tell. Our appreciation for a sound is at bottom an appreciation of the combined effect produced by the relative intensities to which the primary auditory sensations are, with the help of the organ of Corti, excited by the sound.

$\$ 718$. Whatever be the explanation of the manner in which our distinct auditory sensations arise, the range and precision of our appreciation of musical sounds is very great. Vibrations with a recurrence below 30 a sec. ond $^{1}$ are unable to produce a sensation of sound; if the waves are powerful enough we may feel them, but we do not hear them if the vibrations are simple, and such as would give rise to a pure tone; if the fundamental tone is accompanied by overtones we may hear these, and are thus apt to say we hear the former when in reality we only hear the latter. The note of the 16 -feet organ pipe, 33 vibrations a second, gives us the sensation of a droning sound. A tone of forty vibrations is, however, quite distinct. In the other direction it is possible to hear a note caused by 38,000 vibrations a second, though the limit for most persons is far lower-about 16,000. Some persons hear grave sounds more easily than high ones and vice versa. This may be so pronounced as to justify the subjects being spoken of as deaf to grave or high tones respectively. The range in different animals is very different.

The power of distinguishing one note from another varies, as is well known, in different individuals, according as they have or have not a "musical ear." A well-trained ear can distinguish the difference of a single or even of a half vibration a second, and that through a long range of notes. The range of an ordinary appreciation of tones lies between 40 and 4000 vibrations a second, $i$. e. , between the lowest bass $\mathrm{C}\left(\mathrm{C}_{1} 32\right.$ vibrations) and the highest treble $\mathrm{C}\left(\mathrm{C}^{5} 4224\right.$ vibrations) of the piano; tones above and below these, even when audible, being distinguished from each other with great difficulty.

$\S 719$. When two consecutive sounds follow each other at a sufficiently short interval the sensations are fused into one. In this respect auditory sensations are of shorter duration than ocular sensations. When ocular sensations are repeated ten times in a second they become fused (p. 766), whereas the ticks of a pendulum beating 100 in a second are readily audible as distinct sounds. - When two tuning-forks not quite in tune are struck together the interference of the vibrations gives rise to an alternating rise and

1 By some authors the limit is placed as low as 24 or even 15 a second. 
fall of the sound, known as "beats." When the beats follow each other as rapidly as 132 in a second they cease to be recognized, that is to way, the sensations which they cause become fused. Before they disappear they give a peculiar disagreeable roughness to the sound. The pleasure given by musical sounds depends largely on the absence of this incomplete fusion of sensations.

$\$ 720$. Corresponding to entoptic phenomena there are various entotic phenomena, sensations or modifications of sensations originating in the tympanum or in the labyrinth; moreover, sensations of sound may rise in the auditory nerve or in the brain itself, without any vibration whatever falling on the labyrinth.

\section{Auditory Judgments.}

$\S 721$. In seeking for the cause of our visual sensations we invariably refer to the external world. The sensation caused by direct stimulation of the optic nerve or retina by a blow or a galvanic current, we identify with that caused by a flash of light. A sensation arising from any stimulation of the left side of our retina we regard as caused by some object on the right-hand side of our external visible world. In a similar way, but to a less extent, we project our auditory sensations into the world outside us, and when the auditory nerve is affected we seek the cause in vibrations starting at a greater or less distance from us. We do not think of the sound as originating in the ear itself.

This mental projection of the sound is much more complete when the ear is stimulated by vibrations reaching it through the membrana tympani than when the vibrations are conducted by the solids of the head directly to the perilymph of the labyrinth. When the meatus externus is filled with fluid and the vibrations of the membrana tympani are in consequence interfered with the apparent outwardness of sounds is to a very large extent lost; sounds, however caused, seem under these circumstances to arise in the ear. Hence it would seem that the vibrations of the membrana tympani, or possibly the action of the muscles attached to the ossicula, give rise to obscure sensations of which, by themselves, we are not distinctly conscious, but which nevertheless lead us to judge that the sounds heard by means of the tympanum come from ontside the ear.

$\S 722$. Our judgment of the distance of sounds is very limited. A sound whose characters we know appears to us near when it is loud and far off when it is faint. A blindfold person will be unable to distinguish between the difference of intensity produced on the one hand by a tuning-fork being held before him, first with the broad edge of the fork toward him and then with the narrow edge, and the difference on the other hand caused by the removal of the tuning-fork to a distance. We can, on the whole, better appreciate the distance of noises than of musical sounds.

$\$ 723$. Our judgment of the direction of sounds is also very limited. Our chief aid in this is the position in which we have to place the head in order that we may hear the sound to the best advantage. If a tuning-fork be held in the median vertical plane over the head, though it is easy to recognize it as being in the median plane, it becomes very difficult when the eyes are shut to say what is its position in that plane, $i . e_{\text {., }}$ whether it is more toward the front or back of the head. In this respect, too, our appreciation is more accurate in the case of noises than of musical sounds, with the exception of those given out by the human voice, the direction of which can be judged better than even that of a noise. 


\section{SMELL.}

$\$ 724$. [The nasal fossæ are two irregular cavities which communicate anteriorly with the air through the anterior nares and posteriorly with the pharynx through the posterior nares. The fossæ are partially divided into upper, middle, and lower air-passages or chambers by the superior, middle, and inferior turbinated bones. They are lined by the Schneiderian or pituitary mucous membrane, which is continuous anteriorly with the integument and posteriorly with the mucous membrane of the pharynx ; and with the membrane lining the ducts and sinuses connected with the fossæ. At the position of the distribution of the olfactory nerve-filaments it is much thicker, more vascular, pignented, and lined by columnar nucleated epithelial cells; the remaining portion of the membrane covering the fossæ, excepting near the anterior nares, is lined by columnar ciliated epithelium. This membrane contains racemose mucous glands, which secrete mucus for the purpose of keeping the membrane constantly moist, which is a condition essential to perfect olfaction.

$\S 725$. The olfactory tract is a prolongation of the cerebrum, which terminates anteriorly in a bulbous expansion, the olfactory ganglion. It consists principally of gray matter. This ganglion rests upon the cribriform plate

FrG. 198.

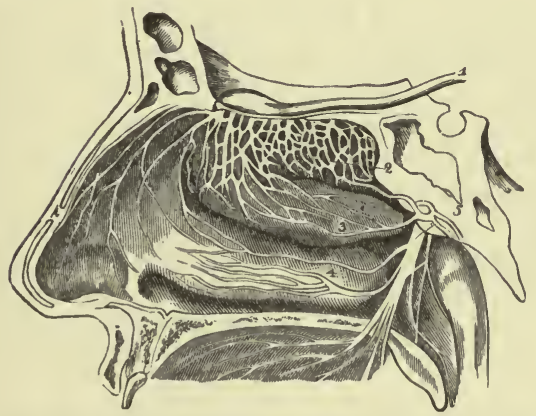

Fig. 198.-Vertical Section of Right Nasal Fossa, showing Outer Side of Fossa. 1, olfactory tract; 2, olfactory nerves; 3 , middle turbinated bone; 4 , lower turbinated bone; 5 , branches from the fifth nerve. Branches of the fifth are also shown in the anterior portion. (After Arnold.)

Fig. 199-Cells of the Olfactory Mucous Nembrane. hart Clarke.)

of the ethmoid bone, and in this position sends about twenty filaments, which consist of gray matter alone, through the cribriform plate to be distributed to the pituitary membrane of the upper third of the septum nasi, the upper portion of the root of the nose, the superior, and a portion of the middle turbinated bones. (Fig. 198.) The whole surface corresponding to the distribution of the olfactory nerve is colored brownish by the pigment in the epithelial cells of the mucous glands and membrane. This pigmented region is called the regio olfactoria, and is the essential portion of the nasal fossæ concerned in olfaction.

$\$ 726$. According to Schultze, the epithelium of the regio olfartoria is of two kinds: The first (Fig. 199, a) consists of yellow nucleated proto- 
plasmic cells, which have a cylindrical body terminating at its free extremity as a squared truncated surface ; the other extremity of the body is stretched out as a filamentous prolongation, which expands into a triangular plate as it approaches the submucous tissue. From the base of this plate a number of filaments are given off, which are prolonged into the subnucous tissue. The second variety of epithelial cells (c) is found at the borders of the regio olfactoria. They are similar to those just described, excepting that their free surface is covered with cilia. Between the epithelial cells the olfactory nerves terminate. These terminal filaments $(b, f)$ are long, delicate structures, which have a number of fusiform expansions along their course; in the largest expansion is found an oval nucleus. The terminal filaments are called the olfactory cells. As yet no connection between the subepithelial and interepithelial nerve-filaments has been demonstrated. The epithelial cells ( $d$ and $e$ ) in the above figure are shown connected with the subepithelial tissue. The fifth nerve supplies the fossæ with sensory filaments.]

$\$ 727$. Odorous particles present in the inspired air passing through the lower nasal chambers diffuse into the upper nasal chambers, and falling on the olfactory epithelium produce sensory impulses which, ascending to the brain, give rise to sensations of smell. We may presume that the sensory impulses are originated by the contact of the odorous particles with the peculiar rod-shaped olfactory cells described by Max Schultze; but we are as much in the dark about this matter as about the development of visual sensory impulses in the rods and cones or of auditory sensory impulses in the organ of Corti.

The susidiary apparatus of smell is exceedingly meagre. By the forced nasal inspiration, called sniffing, we draw air so forcibly through the nostrils that currents pass up into the upper as well as the lower nasal chambers; and thus a more complete contact of the odorous particles with the olfactory membrane than that supplied by mere diffusion is provided for.

We have every reason to think that any stimulus applied to the olfactory nerve will produce the sensation of smell; but the proof of this is not so clear as in the case of the optic and auditory nerves. We are, however, subject to sensations of smell not caused by objective odors. 'The olfactory membrane is the only part of the body in which odors as such can give rise to any sensations; and the sensations to which they give rise are always those of smell. The mucous membrane of the nose is, however, also an instrument for the development of afferent impulses other than the specific olfactory ones. Chemical stimulation of the olfactory membrane by pungent substances such as ammonia gives rise to a sensation distinct from that of smell, a sensation which affords us no information concerning the chemical nature of the stimulus, and which is indistinguishable from the sensations produced by chemical stimulation of other parts of the nasal membrane as well as of other surfaces equally sensitive to chemical action. It is probable that these two kinds of sensations thus arising in the olfactory membrane are conveyed by different nerves, the former by the olfactory, the latter by the fifth nerve.

$\$ 728$. For the development of smell it appears necessary that the odorous particles should be conveyed to the nasal membrane in a gaseous medium, or, at least, that the surface of the membrane should not be exposed at the same time to the action of fluids. Thus, when the nostril is filled with rosewater, the odor of roses is not perceived; and simply filling the nostrils with distilled water suspends for a time all smell, the sense returning gradually after the water has been removed; the water apparently acts injuriously on the delicate olfactory cells. 
Each substance that we smell causes a specific sensation, and we are not only able to recognize a multitude of distinct odors, but also to distinguish individual odors in a mixed smell.

As in the previous senses, we project our sensation into the external world; the smell appears to be not in our nose, but somewhere outside us. We can judge of the position of the odor, however, even less definitely that we can of that of a sound.

The sensation takes some time to develop after the contact of the stimulus with the olfactory membrane, and may last very long. When the stimulus is repeated the sensation very soon dies out; the sensory terminal organs speedily become exhausted. Mental associations cluster more strongly around sensations of smell than around any other impressions we receive from without. And reflex effects are very frequent, many people fainting in consequence of the contact of a few odorous particles with their olfactory cells.

Apparently the larger the surface the more intense the sensation; animals with acute scent having a proportionately large area of olfactory membrane. The quantity of material required to produce an olfactory sensation may be, as in the case of musk, almost immeasurably small.

When two different odors are presented to the two nostrils, an oscillation of sensation similar to that spoken of in binocular vision (p. 788) takes place.

$\S 729$. The assertion that the olfactory nerve is the nerve of smell has been disputed. Cases have been recorded of persons who appeared to have possessed the sense of smell, and yet in whom the olfactory lobes were found after death to be absent. Direct experiments on animals, however, show that loss of the olfactory lobes entails loss of smell. On the other hand, it is stated that section or injury of the fifth nerve causes a loss of smell though the olfactory nerve remains intact; but in these cases it has not been shown that the olfactory membrane remains intact, and it is quite possible that, as in the case of the eye, changes may take place in the nasal membrane as the result of the injury to the fifth nerve, sufficient to prevent its performing its usual functions.

\section{TASTE.}

$\$ 730$. [The peripheral organs concerned in the sense of taste are localized in the mucous membrane covering the dorsum of the tongue, the fauces, soft palate, and uvula, and possibly a portion of the upper part of the pharynx. This membrane is analogous in structure to other membranes of its type, except on the dorsum of the tongue, where its structure is similar to that of the integument. At this position it consists of a corium, with a papillary and a superficial epithelial layer.

The structure of the corium is similar to that of the skin, but is thinner and less compact. It serves as a point of insertion of the muscular fibres of the tongue.

\$731. The papilloe are thickly distributed over the whole dorsal surface, but more particularly marked in the anterior two-thirds. They project as minute prominences, which give the tongue a roughened, characteristic appearance. The papillæ are of two kinds, the simple and compound. The simple papillæ are similar to those found in the skin; they are found scattered over the whole dorsal surface between the compound papillæ. They are most numerous in the posterior portion of the organ. The compound papillæ are of three varieties: the papillæ maximæ or circumvallatæ, the papillæ mediæ or fungiformes, and the papillæ minimæ or filiformes. 
The papilloe circumvallatoe (Fig. 200), which are the largest, are about eight or ten in number and form a V-shaped row at the junction of the middle and posterior two-thirds of the tongue. They consist of a central, broad papilla, surrounded by an annular ring or wall of about the same elevation and separated from the central papilla by a circular fissure. The central papilla, as well as the surrounding wall, is covered by simple papillæ. Each of them receives one or more capillary loops and nerve-filaments.

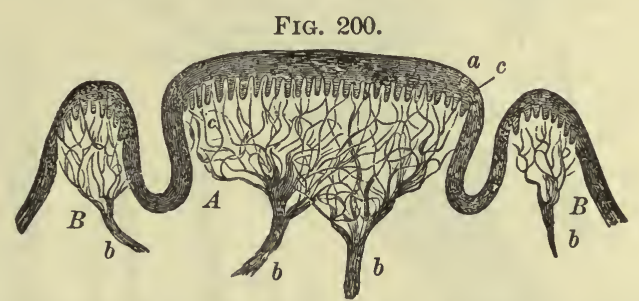

Vertical Section of the Circumvallate Papillæ. (From Kölliker.) $A$, the papillæ; $B$, the surrounding wall; $a$, the epithelial covering; $b$, the nerves of the papilla and wall spreading toward the surface; $c$, the secondary papillæ. $10 \% 1$.

The papillo fungiformes (Fig. 201) are found principally on the tip and sides of the tongue, although scattered sparsely over the whole of the anterior two-thirds. These are so named from their fungiform shape, being expanded at their free extremity and projecting on a short, thick pedicle. They are covered by simple papillæ, and contain plexuses of vessels and nerves.

Frg. 201.
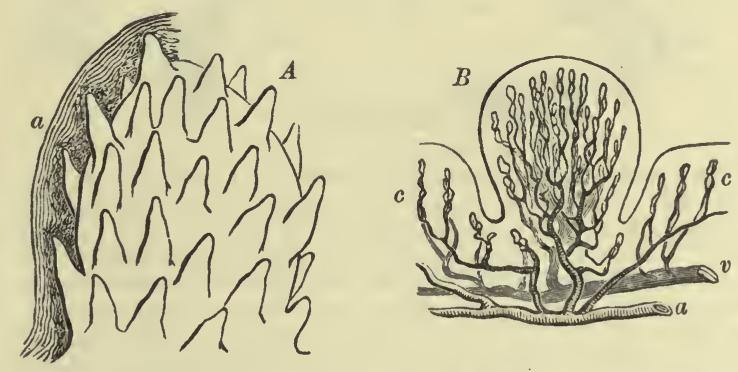

Surface and Section of the Fungifurm Papillæ. (From Kölliker, and after Todd and Bowman.) $A$, the surface of a fungiform papilla, partially denuded of its epithelium, $35 / 1 ; a$, epithelium. $B$, section of a fungiform papilla with the bloodvessels injected; $a$, artery; $v$, vein ; $c$, capillary loops of simple papillæ in the neighboring structure of the tongue.

The papilloe filiformes (Fig. 202) are by far the most numerous, and are found thickly distributed over the entire surface of the anterior two-thirds of the tongue. They are minute, conical in shape, and generally arranged in bipenniform rows, which are more or less parallel with the two rows of papillæ circumvallatæ. Their free surface is covered with simple papillæ. The epithelium covering them is greatly modified and appears in the form of hair-like processes. (Fig. 202.) These processes are bathed in mucus, are movable, and have a general inclination pointing backward. The existence of these hair-like processes on the filiform papillæ suggests that this variety of papillæ is intimately connected with the tactile sensibility of the tongue, and not with gustation. In carnivora and herbivora these processes are of a horny structure, and perform an active function in the attrition and prehension of food. In man their special function appears, through 
their intimate connection with the tactile sense, to guide the tongue in its variable and complicated movements.

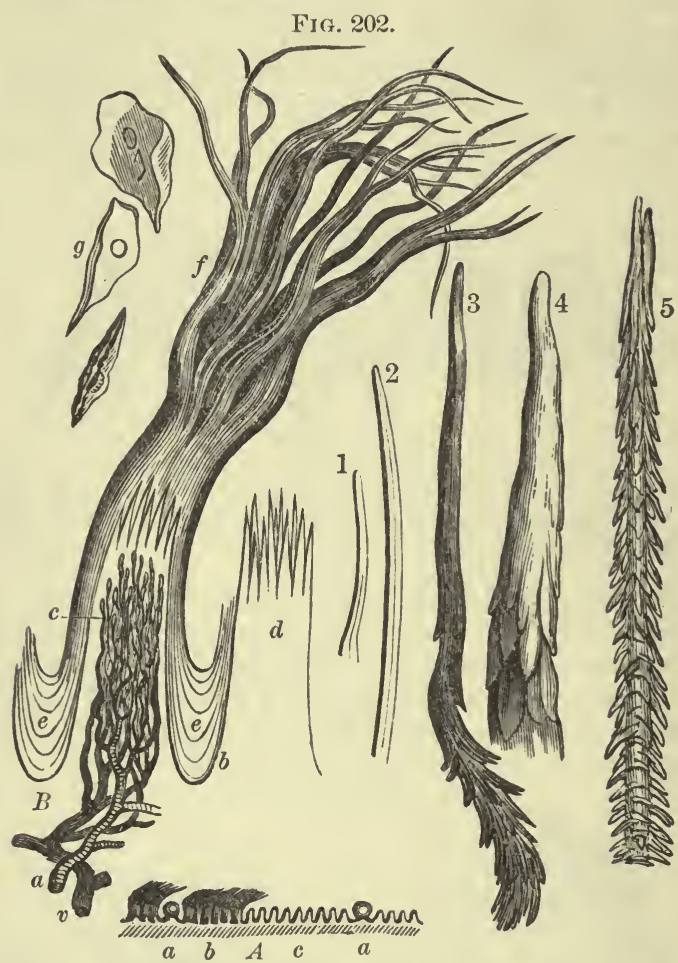

$A$, vertical section near the middle of the dorsal surface of the tongue ; $a, a$, fungiform papillæ ; $b$, filiform papillæ, with their hair-like processes; $c$, similar ones deprived of their epithelium, magnified 2 diameters; $B$, filiform compound papillæ; $a$, artery; $v$, vein ; $c$, capillary loops of the secondary papillæ; $b$, line of basement-membrane; $d$, secondary papillæ, deprived of $e, e$, the epithelium; $f$, hair-like processes of epithelium capping the simple papillæ, magnified 25 diameters; $g$, separated nucleated particles of epithelium, magnified 300 diameters. 1, 2, hairs found on the surface of the tongue; $3,4,5$, ends of hair-iike epithelial processes, showing varieties in the imbricated arrangement of the particles, but in all a coalescence of the particles toward the point; 5 encloses a soft hair, magnified 160 diameters. (After Todd and Bowman.)

FIG. 203.

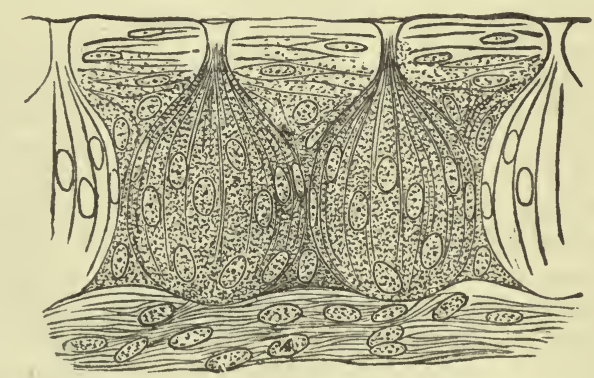

Gustatory Bulbs from the Lateral Gustatory Organ of the Rabbit. (Magnified 450 diameters.)

$\$ 732$. The ultimate terminations of the gustatory nerves are yet enveloped in obscurity. According to Engelmann, the glosso-pharyngeal nerves 
terminate in flask-shaped organs which are termed the gustatory bulbs or taste buds. (Fig. 203.) These bulbs are found principally in the papillary surface of the wall of the circumvallate papillæ. They are also found in the fungiform papillæ, but are less numerous. They consist of a flask-shaped fundus, which rests upon the subepithelial tissue, and a mouth which opens upon the surface of the mucous membrane. The mouth is known as the gustatory pore. 'The fundus of the flask is composed of two varieties of cells ; the outer or investing cells are fusiform, nucleated, and granular, placed parallel and arranged concentrically in a direction from the base to the neck; they thus form a wall which encloses elongated nucleated cells with filamentous processes, which extend through the gustatory pore and project as very finely pointed or truncated extremities. These inner cells are called the gustatory cells, and are supposed to be the essential terminal elements concerned in gustation. Their relation to the gustatory nerves has not as yet been clearly demonstrated, but they are evidently connected with the ganglionic plexuses of nerve-fibres at the papillary bases. The gustatory nerves are also supposed to terminate in the epithelium of the papillæ.]

$\S 733$. The word taste is frequently used when the word smell ought to be employed. We speak of "tasting" odoriferous substances, such as an onion, wines, etc., when in reality we only smell them as we hold them in our mouth; this is proved by the fact that the so-called taste of these things is lost when the nose is held, or the nasal membrane rendered inert by a catarrh.

The terminal organs of the sense of taste thus more strictly defined are the endings of the glosso-pharyngeal and lingual nerves in the mucous membrane of the tongue and palate, those nerves serving as the special nerves of taste. Whether the so-called gustatory buds can be regarded as specific organs of taste appears doubtful. The subsidiary apparatus is confined to the tongue and lips, which by their movements assist in bringing the sapid substances into contact with the mucous membrane of the mouth.

Though we can hardly be said to project our sensation of taste into the external world, we assign to it no subjective localization. When we place quinine in our mouth, the resulting sensation of taste gives us no information as to where the quinine is, though we may learn that by conconitant general sensations arising in the buccal mucous membrane.

$\S 734$. We recognize a multitude of distinct tastes, which may be broadly classified into acid, saline, bitter, and sweet tastes. Sapid substances have the power of producing these sensations by virtue of their chemical nature. But other stimuli will also give rise to sensations of taste. When the tongue is tapped, a taste is felt; and when a constant current is passed through the mouth, an alkaline or, in some persons, a bitter metallic taste is developed when the anode, and an acid taste when the kathode, is placed on the tongue. It is probable that in these cases the terminal organs are indirectly affected by the current. When hot or pungent substances are introduced into the mouth, sensations of general feeling are excited, which obscure any strictly gustatory sensations which may be present at the same time.

Though analogy would lead us to suppose that a stimulus applied to any part of the course of the real gustatory fibres of either the glosso-pharyngeal or lingual nerves would give rise to a sensation of taste and nothing else, the proof is not forthcoming, since both these nerves are mixed nerves containing other afferent fibres as well as those of taste.

When the constant current is used as a means of exciting taste, gustatory sensations are found to be developed in the back, edges, and tip of the tongue, the soft palate, the anterior pillar of the fauces, and a small tract of the posterior part of the hard palate. They are absent from the anterior 
and middle dorsal and under surface of the tongue, the front portion of the hard palate, the posterior pillars of the fauces, the gums, and the lips. Sapid substances are unsuitable as a test for this purpose, on account of their rapid diffusion. Bitter substances produce most effect when placed on the back, and sweet substances when placed on the tip, of the tongue; but the tasting power of the tip of the tongue varies very much in different individuals, and in many seems almost entirely absent. It is said that acids are best appreciated by the edge of the tongue.

$\$ 735$. It is essential for the development of taste that the substance to be tasted should be dissolved, and the effect is increased by friction. The larger the surface the more intense the sensation. The sensation takes some time to develop, and endures for a long time, though this may be in part due to the stimulus remaining in contact with the terminal organs. A temperature of about $40^{\circ}$ is the one most favorable for the production of the sensation. At temperatures much above or below this, taste is much impaired. The nerves of taste are, as we have said, the glosso-pharyngeal and the lingual or gustatory. The former supplies the back of the tongue, and section of it destroys taste in that region. The latter is distributed to the front of the tongue, and section of it similarly deprives the tip of the tongue of taste. There is no reason for doubting that the gustatory fibres in the glosso-pharyngeal are proper fibres of that nerve; but it has been urged by many that the gustatory fibres of the lingual are derived from the chorda tympani, and that those fibres of the lingual which come from the fifth are employed exclusively in the sensations of touch and feeling; the evidence in favor of this view is, however, inconclusive.

\section{CHAPTER V.}

\section{FEELING AND TOUCH.}

\section{General Sensibility and Tactile Perceptions.}

$\$ 736$. WE have taken the foregoing senses first in the order of discussion on account of their being eminently specific. The eye gives us only visual sensations, the ear only auditory snsations. The sensations are produced in each case by specific stimuli; the eye is only affected by light and the ear only by sound. Moreover, the information they afford us is confined to the exterual world; they tell us nothing about ourselves. The various visual sensations which arise in our retina are referred by us not to the retina itself, but to some real or imaginary object in the world without (including as part of the external world such portions of our own bodies as are visible to ourselves). Such also, with diminishing precision, is the information gained by hearing, taste, and smell.

All the other afferent nerves of the body, centripetal impulses along which are able to affect our consciousness, are the means of conveying to us information conerning ourselves. The sensations, arising in them from the action of various stimuli, are referred by us to appropriate parts of our own body. When any body comes in contact with our finger, we know that it is our finger which has been touched; from the resultant sensations we not only learn the existence of certain qualities in the object touched, but we also are 
led to connect the cognizance of these qualities with a particular part of our own body.

$\$ 737$. Like the more specific senses previously studied, the sensations of which we are now speaking, and which may be referred to under the name of touch, using that word for the present in a wide meaning, require for their production terminal organs; and the chief but not exclusive organ of touch is to be found in the epidermis of the skin and certain underlying nervous structures. For the development of specific tactile sensations these terminal organs are as essential as are the terminal organs of the eye for sight or of the ear for hearing. Contact of the skin with a hard or with a hot body gives rise to a distinct sensation, whereby we recognize that we have touched a hard or a hot body. But the application of either body or of any other stimulus to a nerve-trunk gives rise to a sensation of general feeling only, corresponding to the simple sensation of light which is produced by direct stimulation of the optic nerve. We have no more tactile perception of a body which is in contact with a nerve-trunk than we could have visual perception of any luminous object, the rays proceeding from which were strong enough to excite sensory impulses when directed on to the optic nerve instead of on to the retina, supposing such a thing to be possible. It is further characteristic of these ordinary nerves of general feeling, that the sensations caused by any stimulation of them beyond a certain degree develop that state of consciousness which we are in the habit of speaking of as "pain." Putting aside the general feeling which many parts of the eye possess, a very strong luminous stimulation of the retina is required to produce a sensation of pain, if indeed it can be at all brought about; whereas a very moderate stimulation of the skin, and almost every stimulation of an ordinary nerve-trunk, is said by us to be painful.

Though the skin is the chief organ of touch, the mucous membrane lining the various passages of the body also serves as an instrument for the same sense, but only for a short distance from the respective orifices. We can recognize hard or hot bodies with our lips or mouth, but a hot liquid, when it has reached the oesophagus or stomach, simply gives rise to a sensation of pain; we cannot distinguish the sensation caused by it from the sensation caused by a draught of a too acid fluid.

From parts and tissues of the body other than the skin and the portions of mucous membrane just mentioned we have obscure sensations of general feeling, by which we are made vaguely aware of the general condition of our body, though our judgments in this matter are chiefly influenced by what we shall have to speak of directly as a muscular sense. In all parts of the body, however, on occasions all too frequent, this general feeling may become prominent as pain.

$\S 738$. The stimuli which, when applied to the skin, give rise to tactile perceptions are of two kinds only: (1) mechanical, that is, the contact of bodies exerting varying degrees of pressure; and (2) thermal, $i . e_{\text {. }}$, the raising or lowering of the temperature of the skin by the approach or contact of hot or cold bodies. We can judge of the weight and of the temperature of a body, because we can, through touch, perceive how much it presses when allowed to rest on our skin or how hot it is. But we can through touch derive no other perceptions and form no other judgments. An electric shock sent through the skin will give rise to a sensation, but the sensation is an indefinite one, because the electric current acts not on the terminal organs of touch, but on the fine nerve-branches of the skin. We cannot distinguish the sensation so caused from a mechanical prick of similar intensity; we cannot perceive that the sensation is caused by an electric current. Similarly, certain chemical substances, such as a strong acid, will give rise to a 
sensation, but we cannot perceive the acid, we can form no judgment of its nature such as we could if we tasted it; and if the acid does not permeate the skin so as to act directly and chemically on the fine nerve-fibres, we cannot distinguish the acid from any other liquid giving rise to the same simple contact impressions. The terminal organs of the skin are such as are only affected by pressure or by temperature. Conversely, pressure or a variation in temperature brought to bear on a nerve-trunk, instead of on the terminal organs, produces no specific tactile sensations of pressure or temperature, but merely general sensations of feeling rapidly rising into pain.

\section{Tactile Sensations.}

Sensations of Pressure.

$\S 739$. As with visual, so with tactile, and, indeed, with all other sensations, the intensity of the sensation maintains that general relation to the intensity of the stimulus which we spoke of at p. 767 as being formulated under IVeber's law. We can distinguish the difference of pressure between one and two grammes as readily as we can that between ten and twenty or one hundred and two hundred.

When two sensations follow each other in the same spot at a sufficiently short interval, they are fused into one; thus, if the finger be brought to bear lightly on a rotating card having a series of holes in it, the holes cease to be felt as such when they follow each other at a rapidity of about 1500 in a second. The vibrations of a cord cease to be appreciable by touch when they reach the same rapidity. When sensations are generated at points of the skin too close together they become fused into one; but to this point we shall return presently.

$\S 740$. The sensation caused by pressure is at its maximum soon after its beginning, and thenceforward diminishes. The more suddenly the pressure is increased, the greater the sensation; and if the increase be sufficiently gradual, even very great pressure may be applied without giving rise to any sensation. A sensation in any spot is increased by contrast when the surrounding areas are not subject to pressure. Thus, if the finger be dipped into mercury, the pressure will be felt most at the surface of the fluid; and if the finger be drawn up and down, the sensation caused will be that of a ring moving along the finger.

All parts of the skin are not equally sensitive to pressure; small differences of simple pressure are more readily appreciated when brought to bear on the palmar surface of the finger, or on the forehead, than on the arm or on the sole of the foot. In making these determinations, all muscular movements should be avoided in order to eliminate the muscular sense, of which we shall speak presently; and the area stimulated should be as small and the surfaces in contact as uniform as possible. In a similar manner, small, consecutive variations of pressure, as in counting a pulse, are more readily appreciated by certain parts of the skin than by others; and the minimum of pressure which can be felt differs in different parts. In all cases, variations of pressure are more easily distinguished when they are successive than when they are simultaneous.

\section{Sensations of Temperature.}

$\S 741$. When the temperature of the skin is raised or lowered in any spot, we receive sensations of heat and cold respectively; and by these sensations of the temperature of our skin, we form judgments of the temper- 
ature of bodies in contact with it. Bodies of exactly the same temperature as the region of the skin to which they are applied produce no such thermal sensations, though we can, from the very absence of sensation, form a judgment as to their temperature; and good conductors of heat appear respectively hotter and colder than bad conductors raised to the same temperature.

$\$ 742$. We may consider the skin as having at any given time and in any given spot a normal temperature at which the sensation of temperature is at zero; for under ordinary circumstances we are not directly conscious of the temperature of our skin; it is only when the normal temperature at the spot is raised or lowered that we have a sensation of heat or cold respectively. This normal temperature may be at the same time different in different parts of the body; thus, at a time when neither the forehead nor the hand are giving rise to any sensation of temperature, we may, by putting the hand to the forehead, frequently feel the former hot or cold because the normal temperatures of the two parts differ. The normal temperature in any spot may also vary from time to time. Thus, when the hand is placed in a warm medium for some time, the sensation of warmth ceases; a new normal temperature is established with the zero of sensation at a higher level, a depression or elevation of this new temperature giving rise, however, as before, to sensations of heat and cold respectively. That it is the changed condition, and not the change itself, of which we are conscious, is shown by the fact that when a portion of the skin is cooled, by brief contact with a cold metal, for instance, we are still conscious of the spot being cold after the cooling agent has been removed-that is, at a time when a cooled spot is in reality being heated by the surrounding warmer tissues.

$\$ 743$. The change in temperature of the skin necessary to produce a sensation must have a certain rapidity; and the more gradual the change the less intense the sensation. The repeated dipping of the hand into hot water produces a greater sensation than when the hand is allowed to remain all the time in the water, though in the latter case the temperature of the skin is most affected. The effects of contrast are also seen in these sensations as in those of pressure.

We can with some accuracy distinguish variations of temperature, especially those lying near the normal temperature of the skin. 'These sensations, in fact, follow Weber's law, though apparently sensations of slight cold are more vivid than those of slight heat, the range of most accurate sensation seeming to lie between $27^{\circ}$ and $33^{\circ}$.

The regions of the skin most sensitive to variations in temperature are not identical with those most sensitive to variations in pressure. Thus the cheeks, eyelids, temples, and lips are more sensitive than the hands. The least sensitive parts are the legs, and front and back of the trunk.

$\S 744$. The simplest view which can be taken with regard to the distinction between pressure sensations and temperature sensations, and which is suggested by the facts just mentioned, is to suppose that two distinet kinds of terminal organs exist in the skin, one of which is affected only by pressure, and the other only by variations in temperature; and that the two kinds of peripheral organs are connected with different parts of the central sensory organs by separate nerve-fibres. Certain pathological cases have been quoted as showing not only that this is the case, but that the two sets of fibres pursue different courses in the spinal cord. Thus in certain diseases or injuries to the brain or spinal cord, hyperæsthesia as regards temperature has been observed unaccompanied by an augmentation of sensitiveness to pressure; and, conversely, instances have been seen where the patient could tell when he was touched, but could not distinguish between hot and cold. On the other hand, there are facts which show a close dependence between 
the sensations of pressure and temperature. When each stimulus is brought to bear on a very limited area, the two sensations are frequently confounded, especially in those regions of the body where sensations are not acute. So, also, a penny cooled down nearly to zero, and placed on the forehead will be judged by most people to be as heavy or even heavier than two pennies of the temperature of the forehead itself; and, conversely, a body warmer than the skin will often appear heavier than a body of the same weight, but of the same temperature as the skin. Moreover, cases have been recorded where a hot body, such as a heated spoon, was felt, though the application of the same spoon at the temperature of the body produced no sensations, and yet the heated spoon was not recognized as a hot body, but appeared to be simply something touching the skin. It may be argued that these instances show nothing more than the changes in the skin, whatever they be, which give rise to sensations of pressure, are modified by the temperature of the skin for the time being, whereby the judgment as to the pressure which is being exerted is rendered faulty; but they may also be taken to indicate that variations in pressure and temperature affect the same terminal organs, and the same nerve-fibres, though affecting them in a different way, and generating nervous impulses so far different that they give rise to different sensations. And we may here note that we certainly cannot speak of nerves of warmth in the same sense in which we speak of nerves of sight or of hearing. A stimulus (of whatever kind) applied to an optic or auditory nerve, if adequate, gives rise, as we have seen, to a sensation of light or of sound; a stinulus, on the other hand, applied to the trunk of a cutaneous nerve, gives rise only to general feeling or pain; though the nerve certainly contains fibres by which sensations of pressure and of temperature reach the brain, the general feeling which stimulation of the trunk causes is akin neither to sensations of pressure nor to those of warmth.

$\$ 745$. The rapidity with which hot or cold bodies brought into contact with the skin give rise to sensations of temperature, suggests that the terminal apparatus for generating these sensations, whatever be its nature, is placed in the epidermis, and indeed as near as possible to the surface. Pressure, on the other hand, can be readily transmitted through even a thick layer of skin. And those who maintain the existence of different terminal organs for pressure and temperature, regard the nerve-endings in the epidermis as the latter, and the corpuscular tactus, end-bulbs, and allied organs as the former. But the evidence we possess concerning this matter is at present inconclusive.

\section{Tactile Perceptions and Judgments.}

$\S$ 746. When a body presses on any part of our skin, or when the temperature of the skin at that point is raised, we are not only conscious of pressure or of heat, but perceive that a particular part of our body has been touched or heated. We refer the sensations to their place of origin, and we thus by touch perceive the relations to ourselves of the body which gives rise to the tactile sensations, in the same way as in our visual perception of external objects we refer to external nature the sensations originating in certain parts of the retina. When we are touched on the finger and on the back we refer the sensations to the finger and to the back respectively, and when we are touched at two places on the same finger at the same time we refer the sensations to two points of the finger. In this way we can localize our sensations, and are thus assisted in perceiving the space relations of objects with which we come in contact.

$\S 747$. This power of localizing pressure sensations varies in different 
parts of the body. The following table, from Weber, gives the distance at which two points of a pair of compasses must be held apart, so that when the two points are in contact with the skin the two consequent sensations can be localized with sufficient accuracy to be referred to two points of the body, and not confounded as one:

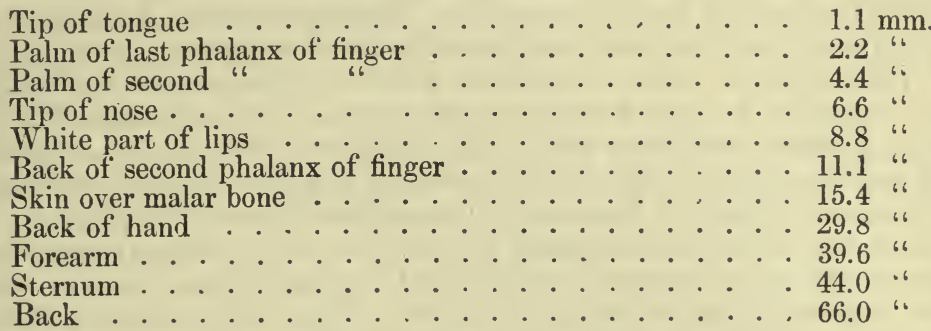

And an analogous distribution has been observed in reference to the localization of sensations of temperature. As a general rule, it may be said that the more mobile parts are those by which we can thus discriminate sensations most readily. The lighter the pressure used to give rise to the sensations, the more easily are two sensations distinguished; thus two points which, when touching the skin lightly, appear as two, may, when firmly pressed, give rise to one sensation only. The distinction between the sensations is obscured by neighboring sensations arising at the same time. Thus, two points brought to bear within a ring of heavy metal pressing on the skin, are readily confused into one. And it need hardly be said that these tactile perceptions, like all other perceptions, are immensely increased by exercise.

$\S 748$. Our "field of touch," if we may be allowed the expression, is composed of tactile areas or units, in the same way that our field of vision is composed of visual areas or units. The tactile sensation is, like the visual sensation, a symbol to us of some external event, and we refer the sensation to its appropriate place in the field of touch. All that has been said (p. 768) concerning the subjective nature of the limits of visual areas, applies equally well, mutatis mutandis, to tactile areas. When two points of the compasses are felt as two distinct sensations, it is not necessary that two, and only two, nerve-fibres should be stimulated; all that is necessary is that the two cerebral sensation-areas should not be too completely fused together. The improvement by exercise of the sense of touch must be explained not by an increased development of the terminal organs, not by a growth of new nervefibres in the skin, but by a more exact limitation of the sensational areas in the brain, by the development of a resistance which limits the radiation taking place from the centres of the several areas.

$\S 749$. By a multitude of simultaneous and consecutive tactile sensations thus converted into perceptions we are able to make ourselves acquainted with the form of external objects. We can tell by variations of pressure whether a surface is rough or smooth, plane or curved, what variations of surface a body presents, and how far it is heavy or light; and from the information thus gained we build up judgments as to the form and nature of objects, judgments, however, which are most intimately bound up with visual judgments, the knowledge derived by one sense correcting and completing that obtained by the other. As in other senses, so in this, our sensations may mislead us and cause us to form erroneous judgments. This is well illustrated by the so-called experiment of Aristotle. It is impossible in an ordinary position of the fingers to bring the radial side of the middle finger 
and the ulnar side of the ring finger to bear at the same time on a small object, such as a marble. Hence, when with the eyes shut we cross one finger over the other, and place a marble between them so that it touches the radial side of the one and the ulnar side of the other, we recognize that the object is such as could not under ordinary conditions be touched at the same time by these two portions of our skin, and therefore judge that we are touching not one but two marbles. Upon repetition, however, we are able to correct our judgment, and the illusion disappears.

$\$ 750$. Distinct tactile sensations are, as we have seen, produced only when a stimulus is applied to a terminal organ. When sensations or affections of general sensibility other than the distinct tactile sensations are developed in the termination of a nerve, we are still able, though with less exactitude, to refer the sensation to a particular part of the body. Thus, when we are pricked or burned, we can feel where the prick or burn is. When a sensory nerve-trunk is stimulated, the sensation is always referred to the peripheral terminations of the nerve. Thus a blow on the ulnar nerve at the elbow is felt as a tingling in the little and ring fingers corresponding to the distribution of the nerve, and sensations started in the stump of an amputated limb are referred to the absent member. When cold is applied to the elbow it is felt as cold in the skin of the elbow; but a cooling of the ulnar nerve at this spot, since stimulation of a nerve-trunk gives rise to general sensations only, simply gives rise to pain which is referred to the ulnar side of the hand and arm.

\section{The Muscular Sense.}

$\$ 751$. When we come into contact with external bodies we are conscious not only of the pressure exerted by the object on our skin, but also of the pressure which we exert on the object. If we place the hand and arm flat on a table, we can estimate the pressure exerted by bodies resting on the palm of the hand, and so come to a conclusion as to their weights; in this case we are conscious only of the pressure exerted by the body on our skin. If, however, we hold the body in the hand, we not only feel the pressure of the body, but we are also aware of the muscular exertion required to support and lift it. We possess a muscular sense; and we find by experience that when we trust to this muscular sense as well as to sensations of pressure, we can form much more accurate judgments concerning the weight of bodies than when we rely on sensations of pressure alone. When we want to tell how heary a body is, we are not in the habit of allowing it simply to press on the hand laid flat on a table; we hold it in our hand and lift it up and down. We appeal to our muscular sense to inform us of the amount of exertion necessary to move it, and by help of that, judge of its weight. And in all the movements of our body we are guided, even to an astonishing degree of accuracy, as is well seen in the discussions concerning vision, by an appreciation, more or less distinctly conscious, of the amount of the contraction to which we are putting our muscles. In some way or other we are made aware of what particular muscles or groups of muscles are being thrown into action, and to what extent that action is being carried. We are also conscious of the varying condition of our muscles, even when they are at rest; the tired and especially the paralyzed limb is said to "feel" heavy. In this way the state of our muscles largely determines our general feeling of health and vigor, of weariness, ill health and feebleness.

It has been suggested that since muscle possesses little or no general sensibility, comparatively little pain being felt for instance when muscles are cut, our muscular sense is chiefly derived from the traction of the contract- 
ing muscle on its attachments ; and undoubtedly in many instances of cramp, the pain is chiefly felt at the joints; and, as we know, Pacinian bodies are abundant around the joints. Afferent nerves, however, having a different disposition from the ordinary motor nerves which terminate in end-plates, have been described as present in muscle; and analogy would lead us to suppose that these afferent fibres, though possessing a low general sensibility, might be easily excited in a specific manner by a muscular contraction; but further investigations are necessary before these can be accepted as the true nerves of the muscular sense.

$\$ 752$. In favor of the view that the muscular sense is peripheral and not central in origin may be urged the fact that the sense is felt when the muscles are thrown into contraction by direct galvanic stimulation instead of by the agency of the will. Many authors, while admitting the existence of a muscular sense of peripheral origin, contend that we also possess and are very largely guided in our movements by what might be called a "neural" sense of central origin. That is to say, the changes in the central nervous system involved in initiating and carrying out a movement of the body so affect our consciousness that we have a sense of the effort itself.

It has been observed that when the posterior roots are divided, movements become less orderly, as if they lacked the guidance of a muscular sense; and although the impairment of the movements may be due in part to the coincident loss of tactile sensations, it is probable that it is increased by the loss of the muscular sense. There is a malady or rather a condition attending various diseased states of the central nervous system called locomotor ataxia, the characteristic feature of which is that, though there is no loss of direct power over the muscles, the various bodily movements are effected imperfectly and with difficulty, from want of proper coördination. In such diseases the pathological mischief is frequently found in the posterior columns of the spinal cord and the posterior roots of the spinal nerves, that is, in distinctly afferent structures; and the phenomena seem in certain cases at least to be due to inefficient coördination caused by the loss both of the muscular sense and of ordinary tactile sensations. The patients walk with difficulty, because they have imperfect sensations both of the condition of their muscles and of the contact of their feet with the ground. In many of their movements they have to depend largely on visual sensations; hence when their eyes are shut, they become singularly helpless. In other cases again ataxia may be present without any impairment of touch; but a discussion of the varied phenomena of this class of maladies cannot be entered into here.

\section{CHAPTER VI.}

\section{SPECIAL MUSCULAR MECHANISMS.}

\section{The Vorce.}

$\S$ 753. [THE larynx is a membrano-cartilaginous chamber, broader above than below, and situated in the anterior median portion of the neck. It consists of a number of cartilages, which are articulate with each other, connected by ligaments, moved by a number of muscles, and lined by a mucous membrane. 
The principal cartilages are the thyroid, cricoid, the two arytenoid, and the epiglottis.

The thyroid cartilage is the largest and consists of two quadrilateral plates or alæ, which are continuous with each other in front, where they form the prominence called the pomum Adami. The posterior borders of the thyroid cartilage serve as a point of attachment of the stylo-pharyngeus and palato-pharyngeus muscles. The upper part of each of these borders terminates in a superior cornu, which articulates with the hyoid bone; the lower portion terminates in the inferior cornu, which articulates with the cricoid cartilage. The upper border between the cornua is connected with

FIG. 204.

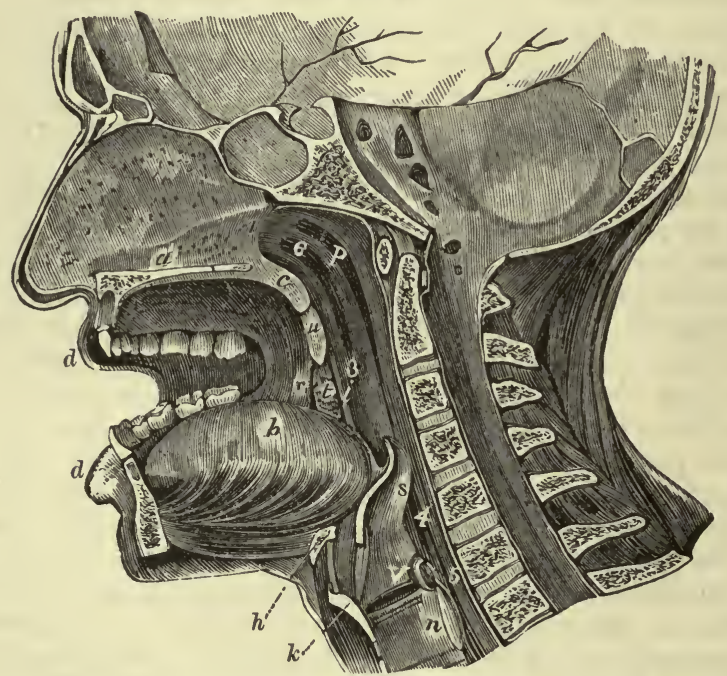

Median Section of Mouth, Nose, Pharynx, and Larynx. a, septum of nose, below it section of hard palate; $b$, tongue; $c$, section of velum pendulum palati; $d, d$, lips; $u$, uvula; $r$, anterior arch or pillar of fauces : $i$, posterior arch ; $t$, tonsil ; $\mathrm{P}$, pharynx : $h$, hyoid bone ; $k$, thyroid cartilage; $n$, cricoid cartilage ; $s$, epiglottis ; $v$, glottis ; 1 , posterior opening of the nares; 3 , isthmus faucium ; 4, superior opening of larynx ; 5, passage into œesophagus ; 6 , mouth of right Eustachian tube.

the hyoid bone by the thyro-hyoid membrane. The lower border is connected with the cricoid cartilage by the thyro-cricoid membrane at the median line, and at the sides by the crico-thyroid muscles.

The cricoid cartilage is situated below the thyroid cartilage with its broad portion posteriorly. At the upper part of its broad portion are two smooth surfaces on which the arytenoid cartilages articulate.

The arytenoid cartilages are pyramidal in form and articulate on the upper surface of the cricoid. Each cartilage has an external, posterior, and internal (median) surface, an apex and a base. The apex is pointed backward and inward, and is surmounted by a small cartilaginous tubercle, called the cartilage of Santorini (Fig. 207). The base, which articulates with the cricoid cartilage, presents at its external internal angle a projection called the processus vocalis. At the posterior internal angle is a second projection, called the processus muscularis.

$\S 754$. The superior opening of the larynx is formed anteriorly by the epiglottis, posteriorly by the apices of the arytenoid cartilages, and laterally by the aryteno-epiglottidean folds stretching between these points. The in- 
ferior opening corresponds to the inferior border of the cricoid cartilage. Between these points is the cavity of the larynx, which has stretching across its sides the vocal cords. The vocal cords consist of two pairs ; the superior or false vocal cords are membrano-ligamentous bands, which extend from the receding angle of the thyroid to the exterual surfaces of the arytenoid cartilages ; the inferior or true vocal cords (chordee vocales) are membrano-ligamentous bands which stretch across the cavity of the larynx from the receding angle of the thyroid to the processus vocales of the arytenoid cartilages. Between the borders of the true and false vocal cords is an elliptical opening, the ventricle, which leads to a space running upward and behind the false vocal cords, called the sacculus laryngis. The mucous

FIG. 205.

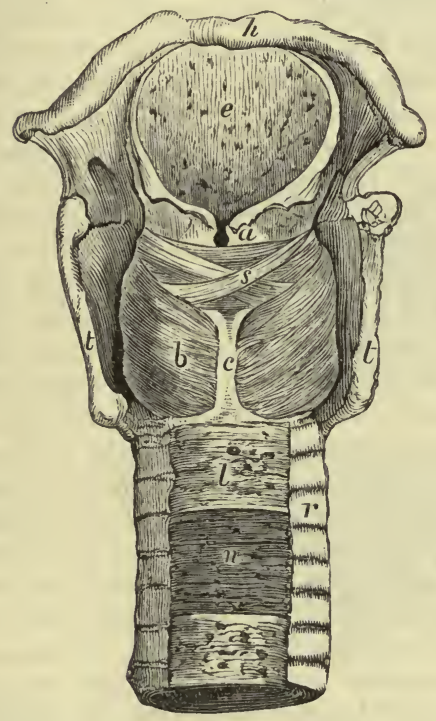

FIG. 206.

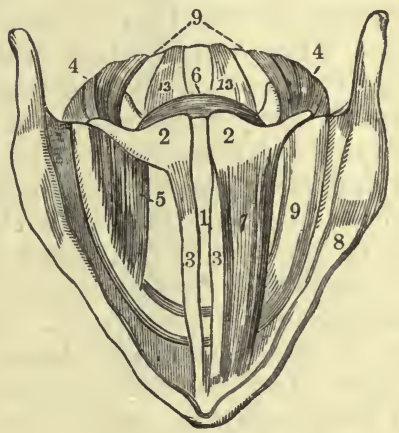

FIG. 207.

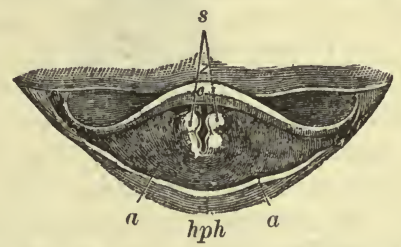

Fig. 205.-View of the Larynx and part of the Trachea from Behind with the Muscles Dissected $h$, the body of the hyoid bone; $e$, epiglottis ; $t$, the posterior borders of the thy roid cartilage; $c$, the median ridge of the cricoid; $a$, upper part of the arytenoid; $s$, placed on one of the oblique fasciculi of the arytenoid muscle ; $b$, left posterior crico-arytenoid muscle ; ends of the incomplete cartilaginous rings of the trachea $;$, fibrous membrane crossing the back of the trachea; $n$, muscular fibres exposed in a part. (From Quain's Anatomy.)

Fig. 206.-View of the Larynx from Above. 1, aperture of glottis; 2 , arytenoid cartilages ; 3 , vocal cords ; 4 , posterior crico-arytenoid muscles; 5 , lateral crico-arytenoid muscle of right side, that of left side removed; 6 , arytenoid muscle ; 7 , thyro-arytenoid muscle of left side, that of right side removed ; 8 , thy roid cartilage $; 9$, cricoid cartilage ; 13 , posterior crico-ary tenoid ligament. With the exception of the arytenoid muscle, this diagram is a copy from Mr. Willis's figure.

Fig. 207.-View of the Upper Part of the Larynx as seen by means of the Laryngoscope during the Utterance of a Grave Note. $c$, epiglottis; $s$, cartilages of Santorini ; $a$, arytenoid cartilages; $z$, base of the tongue; $p h$, posterior wall of the pharynx.

membrane lining this sac contains a great number of follicular glands which discharge a mucous secretion for the purpose of lubricating the true vocal cords.

Between the true vocal cords is an opening which is called the rima glottidis. The form of the glottis varies very much both in the inspiratory and expiratory acts, and in the act of phonation.

$\$ 755$. The muscles of the larynx are divided anatomically into the intrinsic and extrinsic. The former are nine in number, four of them being 
in pairs. They are the essential muscles concerned in the movements of the arytenoid cartilages and chordæ vocales. The extrinsic muscles connect the larynx with adjacent parts, and are for the most part concerned in the elevation and depression of the organ.

The larynx is lined with a mucous membrane which is continuous above with that lining the pharynx and mouth, and below with that lining the trachea. Above the chorda vocales it is lined with pavement epithelium, excepting at the lower anterior portion, where it is ciliated; below the chordæ vocales the epithelium is of a ciliated columnar variety. The mucous membrane contains many mucous glands, which are pretty uniformly distributed; they are, however, very abundant in the part of the membrane lining the sacculus laryngis.]

$\$ 756$. A blast of air, driven by a more or less prolonged expiratory movement, throws into vibrations two elastic membranes-the chordoe vocales. These impart their vibrations to the column of air above them, and so give rise to the sound which we call the voice. Since the sound is generated in the vocal cords, we may speak of them and of those parts of the larynx which decidedly affect their condition as constituting the essential vocal apparatus; while the chamber above the vocal cords, comprising the ventricles of the larynx with the false vocal cords, the pharynx and the cavity of the mouth, the latter varying much in form, constitutes a subsidiary apparatus of the nature of a resonance tube, modifying the sound originating in the vocal cords. In the voice, as in other sounds, we distinguish : 1, Loudness. This depends on the strength of the expiratory blast. 2 , Pitch. This depends on the length and tension of the vocal cords. Their length may be regarded as constant, or varying only with age. It consequently determines the range only of the voice, and not the particular note given out at any one time. The shrill voice of the child is determined by the shortness of the cords in infancy, and the voices of a soprano, tenor, and baritone are all dependent on the respective length of their vocal cords. 'Their tension is, on the contrary, variable; and the chief problems connected with the voice refer to variations in the tension of the vocal cords. 3, Quality. This depends on the number and character of the overtones accompanying any fundamental note sounded, and is determined by a variety of circumstances, chief among which is the physical quality of the cords.

$\$ 757$. The vocal cords, attached in front to the thyroid cartilage, end behind in the processus vocales of the arytenoid cartilages. Hence a distinction has been drawn between the rima vocalis, $i . e$, the opening bounded laterally by the vocal cords, and the rima respiratoria, or space between the arytenoid cartilages behind the processus vocales; these names, however, are not free from objections. In quiet breathing (Fig. 208, $B$ ) the two form together a V-shaped space, which, as we have seen (p. 343), in deep inspiration is widened into a rhomboidal opening by the divergence of the processus vocales (Fig. 208, $C$ ). When a note is about to be uttered, the vocal cords are by the approximation of the processus vocales brought into a position parallel to each other, and the whole rima is narrowed (Fig. $208, A)$. By their parallelism and by the narrowness of the interval between them the cords are rendered more susceptible of being thrown into vibration by a moderate blast of air. 'The problems we have to consider are, first, by what means are the cords brought near to each other or drawn asunder as occasion demands; and, secondly, by what means is the tension of the cords made to vary. We may speak of these two actions as narrowing or widening of the glottis, and tightening or relaxation of the vocal cords. 
\$ 758. Narrowing of the glottis. The change of form of the glottis is best understood when it is borne in mind that each arytenoid cartilage is, when seen in horizontal section (Fig. 208), somewhat of the form of a triangle, with an internal or median, an external, and a posterior side, the processus vocalis being placed in the anterior angle at the junction of the median and external sides. When the cartilages are so placed that the processus vocales are approximated to each other, and the internal surfaces of

FIG. 208.
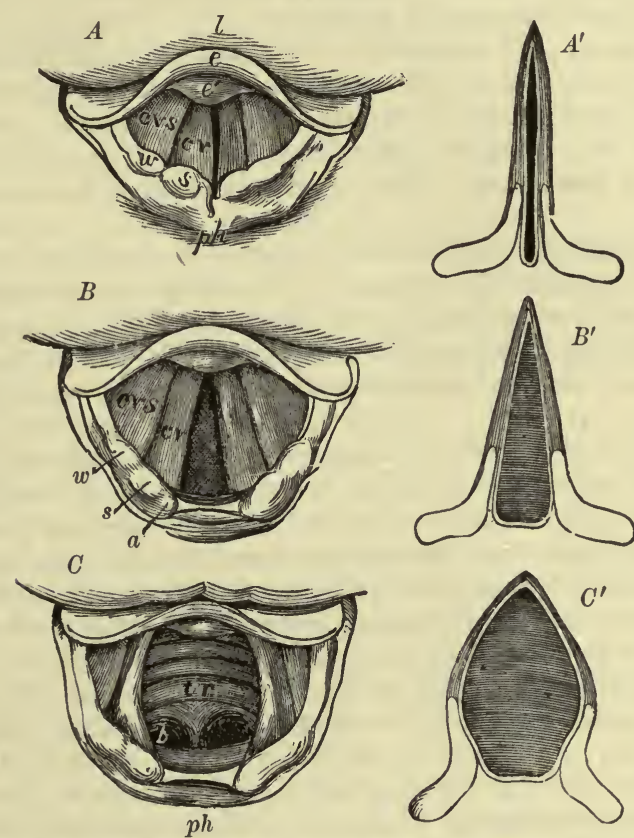

The Larynx as Seen by Means of the Laryngoscope in Different Conditions of the Glottis. (From Quain's Anatomy, after Czermak.) $A$, while singing a high note; $B$, in quiet breathing; $C$, during a deep inspiration. The corresponding diagrammatic figures $A^{\prime}, B^{\prime}, C^{\prime}$, illustrate the changes in position of the arytenoid cartilages and the form of the rima vocalis and rima respiratoria in the above three conditions. $l$, the base of the tongue; $e$, the upper free part of the epiglottis; $e^{\prime}$, the tubercle or cushion of the epiglottis; $p h$, part of the anterior wall of the

- pharynx behind the larynx; $w$, swelling in the aryteno-epiglottidean fold caused by the cartilage of Wrisberg; s, swelling caused by the cartilage of Santorini ; $a$, the summit of the arytenoid cartilage; $c v$, the true vocal cords; $c v s$, the false vocal cords; $t r$, the trachea with its rings; $b$, the two bronchi at their commencement.

the cartilages nearly parallel, the glottis is narrowed. When on the contrary the cartilages are wheeled round on the pivots of their articulations, so that the processus vocales diverge, and the internal surfaces of the cartilages form an angle with each other, the glottis is widened.

\$ 759. There are several muscles forming together a group which has been called by Henle the sphincter of the larynx. These are : 1, the thyroary-epiglotticus, proceeding from the inner surface of the thyroid cartilage and from the arytenoid epiglottidean ligament, and sweeping round the outer ridge of the arytenoid cartilage of its own side to be inserted into the processus muscularis of the arytenoid cartilage of the other side; 2 , the thyroarytenoideus externus, passing from the reëntrant angle of the thyroid cartilage to be inserted into the outer edge of the arytenoid cartilage of the same 
side ; 3 , the thyro-arytenoideus internus, passing from the angle of the thyroid cartilage to the processus vocalis and outer side of the arytenoid cartilage; 4 , the arytenoideus ( posticus), passing transversely from one arytenoid cartilage to another. All these muscles, when they act together, grasp round the glottis and tend to close it up; and each of them, acting alone, has, with the exception of the last named (arytenoideus), the same effect. In addition to these, the crico-arytenoideus lateralis, which passes from the lateral border of the cricoid cartilage upward and backward to the outer angle of the arytenoid, by pulling this outer angle forward throws the processus vocalis inward, and so also narrows the glottis.

$\$ 760$. Widening of the glottis. The crico-arytenoideus posticus, passing from the posterior surface of the cricoid cartilage to the outer angle of the arvtenoid cartilage behind the attachment of the lateral crico-arytenoideus, pulls back this outer angle, and so causing the processus vocalis to move outward, widens the glottis. The arytenoideus posticus, acting alone, has a similar effect.

$\$ 761$. Tightening of the vocal cords. The crico-thyroideus pulls the thyroid downward and forward, and so increases the distance between that cartilage and the arytenoids when the latter are fixed. Supposing, then, the arytenoideus and crico-arytenoideus posticus to fix the arytenoids, the effect of the contraction of the crico-thyroideus would be to tighten the rocal cords.

$\$ 762$. Slackening of the vocal cords. This is effected by the whole sphincter group just mentioned, but more especially by the thyro-arytenoidei externus and internus; these, acting alowe, supposing the arytenoid cartilages to be fixed, would pull the thyroid cartilage upward and backward, and so shorten the distance between the processus vocalis and that body.

$\$ 763$. Thus almost every movement of the larynx is effected not by one muscle only, but by several, or at least by more than one, acting in concert. The movements which give rise to the voice are preëminently combined and coördinate movements. When we remember how a very slight variation in the tension of the vocal cords must give rise to a marked difference in the pitch of the note uttered, and yet what a multitude of fine differences of pitch are at the command of a singer of even moderate ability, it appears exceedingly probable that the various muscular combinations required to produce the possible variations in pitch are of such a kind that frequently a part only, possibly a few fibres only, of a particular muscle may be thrown into contraction, while all the rest of the muscle remains quiet. Taking into view, moreover, the great range of pitch possessed by even common voices, as compared with the possible variations of tension of which the vocal cords in their natural length are capable, it has been suggested that some of the fibres of the thyro-arytenoideus internus, which, passing either from the thyroid or from the arytenoid, appear to end in the vocal cords themselves, may, by fixing particular points of the cords, so to speak, "stop" them; and by thus artificially shortening the length actually thrown into vibration, produce higher notes than the cords in their natural length are capable of producing. It has also been suggested that the processus vocales may overlap each other, and thereby shorten the length of cord available for vibration.

$\S 764$. These various muscles are supplied by the vagus nerve, or rather by spinal accessory fibres running in the vagus trunk. The superior laryngeal is the afferent nerve supplying the mucous membrane, but it also contains the motor fibres distributed to the crico-thyroid muscle; hence, when this nerve is divided on one side the corresponding vocal cord is relaxed and high notes become impossible. It is worthy of notice that this, the chief 
tensor, and therefore the most important, muscle of the larynx, has a separate and distinct nervous supply. According to some authors the arytenoideus posticus also receives its nervous supply from this nerve; but this is denied by others.

The inferior laryngeal or recurrent branch supplies all the other muscles. When this nerve is divided the voice is lost, since the approximation and parallelism of the vocal cords can no longer be effected. When in a living animal both recurrent nerves are divided, the glottis is seen to become immobile and partially dilated, the vocal cords assuming the position in which they are found in the body after death, and which may be considered as the condition of equilibrium between the dilating and constricting muscles. During forcible inspiration the glottis passes from this condition in the direction of more complete dilatation ; during forcible expiration, the change is one of constriction. When the peripheral portion of one recurrent nerve is stimulated, the vocal cord of the same side is approximated to the middle line; when both nerves are stimulated, the vocal cords are brought together and the glottis is narrowed. Though the nerve is distributed to both dilating and constricting muscles, the latter overcome the former when the nerve is artificially stimulated. In the complete closure of the glottis, which is so important a part of the act of coughing (p. 403), the group of muscles which we have spoken of as constituting a sphincter is thrown into forcible contractions by the recurrent laryngeal nerve.

$\S 765$. Though fundamentally a voluntary act, the utterance of a given note is not affected by the direct passage of simple volitional impulses down to the laryngeal muscles. So complex and coördinate a movement as that of sounding even a simple and natural note requires a coördinating nervous mechanism in which, as in other complex muscular actions, afferent impulses play an important part. Auditory sensations, if not as important for an accurate management of the voice as are visual sensations for the movements of the eye, are yet of prime importance. This is recognized when we say that such and such a one whose power over his laryngeal muscles is imperfect "has no ear."

A person may speak or sing in two kinds of voice. In the one the sounds are full and strong, and the resonance chamber which is supplied by the trachea, bronchi, and indeed by the whole chest, is thrown into powerful and palpable vibrations; hence this voice is spoken of as the chestvoice. The other kind of voice, called the falsetto, is thin and poor, deals chiefly with high notes, and is not accompanied by the same conspicuous vibrations of the chest. Much controversy has taken place as to the exact manner in which these two voices are respectively produced. The prevailing opinion teaches that in the chest-voice the vocal cords are somewhat thick, their substance being thrust inward toward the median line by the contraction of the thyro-arytenoidei externi muscles, and the opening between them, sometimes so narrow as to be almost linear, extends along their whole length. In the falsetto voice, on the other hand, the vocal cords are said to be thin and membranous, and the note to be given forth by a vibration, not of the whole width of the cords, as in the chest-voice, but of the extreme edges only, the lateral parts, though not absolutely at rest, vibrating with a different rhythm. Though the whole larynx in the falsetto voice is stretched in the antero-posterior direction, and the vocal cords correspondingly elongated, the rima vocalis does not extend along their whole length; at their posterior part the cords are in contact, and indeed, according to some authors, the high falsetto notes are produced by a sort of "stopping" of the cords. The sense of effort which accompanies the falsetto voice indicates that the changes in the larynx which bring it about are effected by some special muscular 
manœuvres, as is also suggested by the fact that the ease with which falsetto notes can be uttered is readily increased by practice. The change from the chest to the falsetto voice is an abrupt one, and the combined range may be very extensive, as in the case of persons who can carry on a duet, singing alternately, for instance, in a tenor (chest) and a soprano (falsetto) voice.

$\$ 766$. The ventricles of Morgagni are apparently of use in giving the vocal cords sufficient room for their vibrations, and perhaps supply a secretion by which the vocal cords are kept adequately moist. The purpose of the false vocal cords is not exactly known. Some authors think that in the falsetto voice they are brought down into contact with, and thus serve to stop, the true vocal cords.

At the age of puberty a rapid development of the larynx takes place, leading to a change in the range of the voice. The peculiar harshness of the voice when it is thus "breaking" seems to be due to a temporary congested and swollen condition of the mucous membrane of the vocal cords accompanying the active growth of the whole larynx. The change in the mucous membrane may come on quite suddenly, the voice "breaking," for instance, in the course of a night.

\section{SPEECH.}

Vowels.

$\S 767$. Every sound, every note (for all vocal sounds when considered by themselves are musical sounds), caused by the vibrations of the vocal cords, besides its loudness due to the force of the expiratory blast, and its pitch due to the tension of the cords, has a quality of its own, due to the number and relative prominence of the overtones which accompany the fundamental tone. Some of these features which make up the quality are imposed on the note by the nature of the vocal cords, but still more arise from various modifications which the relative intensities of the overtones undergo through the resonance of the cavity of the mouth and throat. Whenever we hear a note sounded by the larynx we are able to recognize in it features which enable us to state that one or other of the "vowels" is being uttered. Vowel sounds are in fact only extreme cases of quality, extreme prominence of certain overtones brought about by the shape assumed by the buccal and pharyngeal passages and orifices, as the vibrations pass through them. Each vowel has its appropriate and causative disposition of these parts. When $i$ (ee in feet) is sounded, the sounding-tube of the upper air-passages is made as short as possible, the larynx is raised and the lips are retracted, the whole cavity of the mouth taking on the form of a broad flask with a narrow neck. During the giving out of $e$ (a in fat) the shape of the mouth is similar, but somewhat longer. For the production of $a$ (as in father) the mouth is widely open, so that the buccal cavity is of the shape of a funnel with the apex at the pharynx. With 0 , the buccal cavity is again flask-shaped, with the mouth more closed than in $a$, but the lips, instead of being retracted as in $i$ and $e$, are somewhat protruded, so that the sounding-tube is prolonged. The greatest length of the tube is reached in $u(00)$, in which the larynx is depressed and the lips protruded as much as possible. While the two latter vowels are being uttered, the general form of the buccal cavity is that of a flask with a short neck and a small opening, the orifice being smaller for $u$ than for 0 .

$\S 768$. Each of these various "vowel" forms of the mouth possesses a note of its own, one toward which it acts as a resonance chamber. Thus, if several tuning-forks of various pitch be held while sounding before a mouth which has assumed the particular form necessary for sounding $U$, it will be 
fourd that the resonance will be particularly great with the fork having the pitch of the bass $b$-flat. Similarly, other and higher notes will be intensified when the mouth is moulded to utter the other vowels. And it is the experience of singers that each vowel is sung with peculiar ease on a note having a prominent overtone corresponding to the tone proper to the mouth when moulded to utter the vowel. The precise nature of the vowel sounds is, however, still disputed.

As the vibrations are travelling through the pharyngeal and buccal cavities, the posterior nares are closed by the soft palate; and it may be shown, by holding a flame before the nostril, that no current of air issues from the nose when a vowel is properly said or sung. When the posterior nares are not effectually closed the sound acquires a nasal character. The same happens when the anterior nares are closed, as when the nose is held between the fingers, the nasal chamber then forming a cavity of resonance.

\section{Consonants.}

$\S 769$. Vowels are, as their name implies, the only real vocal sounds; it is only on a vowel that a note can be said or sung. Our speech, however, is

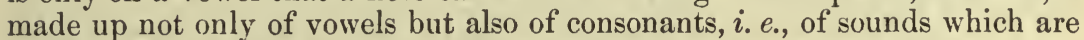
produced not by the vibrations of the vocal cords but by the expiratory blast being in various ways interrupted or otherwise modified in its course through the throat and mouth.

The distinction between the two is, however, not an absolute one, since, as we have seen, the characters of the several vowels depend on the form of the mouth, and in the production of some consonants ( B, D, M, N, etc.) vibrations of the vocal cords form a necessary though adjuvant factor.

Consonants have been classified according to the place at which the characteristic interruption or modification takes place. Thus it may occur:

1. At the lips, by the movement or position of the lips in reference to each other or to the teeth, giving rise to labial consonants.

2. At the teeth, by the movement or position of the front part of the tongue in reference to the teeth or the hard palate, giving rise to dental consonants.

3. In the throat, by the movement or position of the root of the tongue in reference to the soft palate or pharynx, giving rise to guttural consonants.

Among the dentals again may be distinguished the dentals commonly so called, such as $\mathrm{T}$, the sibilants such as $\mathrm{S}$, and the lingual L, all differing in the relative position of the tongue, teeth, and palate.

Consonants may also be classified according to the character of the movements which give rise to them. Thus they may be either explosive or continuous.

1. Explosives. In these the characters are given to the sound by the sudden establishment or removal of the appropriate interruption. Thus, in uttering the labial $\mathrm{P}$, the lips are first closed, then an expiratory current of air is driven against them, and upon their being suddenly opened, the sound is generated. Similarly, the dental $\mathrm{T}$ is generated by the sudden removal of the interruption caused by the approximation of the tip of the tongue to the front of the hard palate, and the guttural $K$ by the sudden removal of the interruption caused by the approximation of the root of the tongue to the soft palate.

The labial $\mathrm{B}$ differs from $\mathrm{P}$, inasmuch as it is accompanied by vibrations of the vocal cords (that is, a vowel sound is uttered at the same time), and these vibrations continue after the removal of the interruption. Hence, $B$ is 
often spoken of as being uttered with voice and $\mathrm{P}$ without voice; and $\mathrm{D}$ and $\mathrm{G}$ (hard) with voice bear the same relation to $\mathrm{T}$ and $\mathrm{K}$ without voice.

The continuous consonants may further be divided into-

2. Aspirates. In these the sound is generated by a rush of air through a constriction formed by the partial closure of the lips, or by the raising of the tongue against the hard or soft palate, etc. Thus, $F$ is sounded when the lips are brought into partial, and not as in $\mathrm{P}$ and $\mathrm{B}$ into complete approximation, and a current of air is driven through the narrowed opening. $F$ is uttered without any accompanying vibration of the vocal cords, $i$. e., without voice. With voice it becomes $\mathrm{V}$.

The sibilant $\mathrm{S}$ is formed by a rush of air past an obstruction caused by the partial closure of the teeth, the front of the tongue being depressed at the same time; and $\mathrm{S}$ accompanied with vibrations of the vocal cords becomes Z.

In Sh the dorsal surface of the tongue is raised so as to narrow the passage between that organ and the palate for a considerable portion of its length.

Th is formed by placing the tongue between the two partially open rows of teeth; and the hard and soft Th bear to each other the same relation as do $\mathrm{P}$ and $\mathrm{B}$.

$\mathrm{L}$ is produced when the passage is closed in the middle by pressing the tip of the tongue against the hard palate and the air is allowed to escape at the sides of the tongue.

When the constriction in an aspirate is formed by the approximation of the root of the tongue to the soft palate, we have the guttural $\mathrm{CH}$ (as in loch) without voice and $\mathrm{GH}$ (as in lough) with voice.

3. Resonants or nasals. In these, all of which must have vibrations of the vocal cords as a basis, the usual passage through the mouth is closed either in a labial, dental, or guttural fashion, and the peculiar character is given to the sound by the nasal chambers acting as a resonance cavity. Thus in M, the passage is closed by the approximation of the lips, in $\mathrm{N}$ by the approximation of the tongue to the hard palate, and in NG by the approximation of the root of the tongue to the soft palate.

4. The various forms of $\mathrm{R}$ are often spoken of vibratory, the characteristic sounds being caused by the vibration of some or other of the parts forming a constriction in the vocal passage. Thus the ordinary $R$ is produced by vibrations of the point of the tongue elevated against the hard palate, the guttural $R$ by the vibrations of the uvula or other parts of the walls of the pharynx; and in some languages there seems to be an $R$ produced by the vibrations of the lips.

$\mathrm{H}$ is caused by the rush of air through the widely opened glottis. When, in sounding a vowel, the sound coincides with a sudden change in the position of the vocal cords from one of divergence to one of approximation, the vowel is pronounced with the spiritus asper. When the vocal cords are brought together before the blast of air begins, the vowel is pronounced with the spiritus lenis. The Arabic $\mathrm{H}$ is produced by closing the rima vocalis, the epiglottis and false vocal cords being depressed, and sending a blast of air through the rima respiratoria.

On many of the above points, however, there are great differences of opinion, the discussion of which as well as of other more rare consonantal sounds would lead us too far away from the purpose of this book. The following tabulated statement must, therefore, be regarded as introduced for convenience only. 
Explosives. Labials, without voice . . . . P.

with voice ..... B.

Dentals, without voice. . . . T.

with voice ..... D

Gutturals, without voice . . . . K (hard C).

with voice..... G (hard).

AsPirates. Labials, without voice..... F.

with voice ..... V.

Dentals, without voice ..... I, S (soft C), Sh, Th (hard).

with voice ..... Z, Zh (in azure, the French $\mathrm{j}$ ), Th (soft).

Gutturals, without voice . . . . CH (as in loch).

with voice . . . . GH as in lough).

Resonants. Labial, ........... M.

Dental, . . . . . . N

Guttural, . . . . . . NG.

Vibratory. Labial, not known in European speech.

Dental, R (common).

Guttural, R (guttural).

$\S 770$. Whispering is speech without any employment of the vocal cords, and is effected chiefly by the lips and tongue. Hence, in whispering the distinction between consonants needing and those not needing voice, such as $\mathrm{B}$ and $\mathrm{P}$, becomes for the most part lost.

\section{Locomotor Mechanisms.}

$\S 771$. The skeletal muscles are for the most part arranged to act on the bones and cartilages as on levers, examples of the first kind of lever being rare, and those of the third kind, where the power is applied nearer to the fulcrum than is the weight, being more common than the second. This arises from the fact that the movements of the body are chiefly directed to moving comparatively light weights through a great distance, or through a certain distance with great precision, rather than to moving heavy weights through a short distance. The fulcrum is generally supplied by a (perfect or imperfect) joint, and one end of the acting muscle is made fast by being attached either to a fixed point, or to some point rendered fixed for the time being by the contraction of other muscles. There are few movements of the body in which one muscle only is required; in the majority of cases several muscles act together in concert; nearly all our movements are coördinate movements. Where gravity or the elastic reaction of the parts acted on does not afford a sufficient antagonism to the contraction of a muscle or group of muscles, the return to the condition of equilibrium is provided for by the action either elastic or contractile of a set of antagonistic muscles; this is seen in the case of the face.

$\$ 772$. The erect posture, in which the weight of the body is borne by the plantar arches, is the result of a series of contractions of the muscles of the trunk and legs, having for their object the keeping the body in such a position that the line of gravity falls within the area of the feet. That this does require muscular action is shown by the facts, that a person when standing perfectly at rest in a completely balanced position falls when he becomes unconscious, and that a dead body cannot be set on its feet. The line of gravity of the head falls in front of the occipital articulation, as is shown by the nodding of the head in sleep. The centre of gravity of the combined head and trunk lies at about the level of the ensiform cartilage, in front of the tenth dorsal vertebra, and the line of gravity drawn from it passes behind a line joining the centres of the two hip-joints, so that the erect body 
would fall backward were it not for the action of the muscles passing from the thighs to the pelvis, assisted by the anterior ligaments of the hip-joints. The line of gravity of the combined head, trunk and thighs falls, moreover, a little behind the knee-joints, so that some, though little, muscular exertion is required to prevent the knees from being bent. Lastly, the line of gravity of the whole body passes in front of the line drawn between the two anklejoints, the centre of gravity of the whole body being placed at the end of the sacrum; hence some exertion of the muscles of the calves is required to prevent the body falling forward.

$\S 773$. In walking, there is in each step a moment at which the body rests vertically on the foot of one, say the right, leg, while the other, the left leg, is inclined obliquely behind with the heel raised and the toe resting on the ground. The left leg, slightly flexed to avoid contact with the ground, is then swung forward like a pendulum, the length of the swing or step being determined by the length of the leg; and the left toe ${ }^{1}$ is brought to the ground. On this left toe as a fulcrum, the body is moved forward, the centre of gravity of the body describing a curve the convexity of which is upward and the left leg necessarily becoming straight and rigid. As the body moves forward, a point will be reached sinilar to that with which we supposed the step to be started, the body resting vertically on the left foot, and the right leg being directed behind in an oblique position. The movement on the left foot, however, carries the body beyond this point, and in doing so swings the right leg forward until it is the length of a step in advance of its previous position, and its toe in turn forms a fulcrum on which the body, and with it the left leg, is again swung forward. Hence in successive steps the centre of gravity, and with it the top of the head, describes a series of consecutive curves, with their convexities upward, very similar to the line of flight of many birds.

Since in standing on both feet the line of gravity falls between the two feet, a lateral displacement of the centre of gravity is necessary in order to balance the body on one foot. Hence in walking the centre of gravity describes not only a series of vertical, but also a series of horizontal curves, inasmuch as at each step the line of gravity is made to fall alternately on each standing foot. While the left leg is swinging, the line of gravity falls within the area of the right foot, and the centre of gravity is on the right side of the pelvis. As the left foot becomes the standing foot, the centre of gravity is shifted to the left side of the pelvis. The actual curve described by the centre of gravity is, therefore, a somewhat complicated one, being composed of vertical and horizontal factors. The natural step is the one which is determined by the length of the swinging leg, since this acts as a pendulum; and hence the step of a long-legged person is naturally longer than that of a person with short legs. The length of the step, however, may be diminished or increased by a direct muscular effort, as when a line of soldiers keep step in spite of their having legs of different lengths. Such a mode of marching must obviously be fatiguing, inasmuch as it involves an unnecessary expenditure of energy.

In slow walking there is an appreciable time, during which, while one foot is already in position to serve as a fulcrum, the other, swinging, foot has not yet left the ground. In fast walking this period is so much reduced that one foot leaves the ground the moment the other touches it; hence there is practically no period during which both feet are on the ground together.

When the body is swung forward on the one foot acting as a fulcrum with such energy that this foot leaves the ground before the other, swinging,

1 This indicates perhaps what should be done rather than the actual practice; most people put the heel to the ground first, the contact with the toe coming later. 
foot has reached the ground, there being an interval during which neither foot is on the ground, the person is said to be running, not walking.

In jumping, this propulsion of the body takes place on both feet at the same time; in hopping, it is effected on one foot only.

$\$ 774$. The locomotion of four-footed animals is necessarily more complicated than that of man. The simple walk, such as that of the horse, is executed in four times, with a diagonal succession; thus, right fore-leg, left hind-leg, left fore-leg, right hind-leg. In the amble, such as that of the camel, the two feet of the same side are put down at one and the same time, this movement being followed by a similar movement of the other two legs; it corresponds, therefore, very closely to human walking. In the trot, which corresponds to human running, the two diagonally opposite feet are brought to the ground at the same time, and the body is propelled forward on them. Concerning this, however, as well as concerning the still more complicated gallop and canter, observers are not agreed, and much discussion has arisen.

The other problems connected with the action of the various skeletal muscles of the body are too special to be considered here. 



\section{BOOK IV.}

\section{THE TISSUES AND MECHANISUS OF REPRODUCTION.}

\section{CHA P TER I.}

\section{ORGANS OF REPRODUCTION.}

$\$ 775$. MANY of the individual constituent parts of the body are capable of reproduction-i. e., they can give rise to parts like themselves; or they are capable of regeneration- $i$. $e$., their places can be taken by new parts more or less closely resembling themselves. The elementary tissues undergo during life a very large amount of regeneration. Thus, the old epithelial scales which fall away from the surface of the body are succeeded by new scales from the underlying layers of the epidermis; old blood-corpuscles give place to new ones; worn-out muscles, or those which have failed from disease, are renewed by the accession of fresh fibres; divided nerves grow again; broken bones are united; connective tissue seems to disappear and reappear almost without limit; new secreting cells take the place of the old ones which are cast off; in fact, with the exception of some cases, such as cartilage, and these doubtful exceptions, all those fundamental tissues of the body, which do not form part of highly differentiated organs, are, within limits fixed more by bulk than by anything else, capable of regeneration. That regeneration by substitution of molecules, which is the basis of all life, is accompanied by a regeneration by substitution of mass.

In the higher animals regeneration of whole organs and members, even of those whose continued functional activity is not essential to the wellbeing of the body, is never witnessed, though it may be seen in the lower animals ; the digits of a newt may be restored by growth, but not those of a man. And the repair which follows even partial destruction of highly differentiated organs, such as the retina, is in the higher animals very imperfect.

In the higher animals the reproduction of the whole individual can be effected in no other way than by the process of sexual generation, through which the female representative element or ovum is, under the influence of the male representative or spermatozoon, developed into an adult individual.

We do not purpose to enter here into any of the morphological problems connected with the series of changes through which the ovum becomes the adult being; or into the obscure biological inquiry as to how the simple, all-but-structureless ovum contains within itself, in potentiality, all its future developments, and as to what is the essential nature of the male action. These problems and questions are fully discussed elsewhere; they do not 
properly enter into a work on physiology, except under the view that all biological problems are, when pushed far enough, physiological problems. We shall limit ourselves to a brief survey of the more important physiological phenomena attendant on the impregnation of the ovum and on the nutrition and birth of the embryo.

$\$ 776$. [The female organs of generation are anatomically divided into the internal and external organs. The latter comprise the labia majora and minora, the clitoris, the hymen, the meatus urinarius, the vulvo-vaginal glands, and the mucous and sebaceous glands which are distributed in the mucous membrane covering the parts. The external organs play a very subsidiary part in the function of reproduction, and they will be passed by with this brief notice.

The internal organs comprise the vagina, uterus, Fallopian tubes, and ovaries.

The vagina is a musculo-membranous canal, about four to six inches long, directed obliquely upward and backward, and extending from the hymen to the cervix uteri, where it is attached at a point a short distance

Fig. 209.

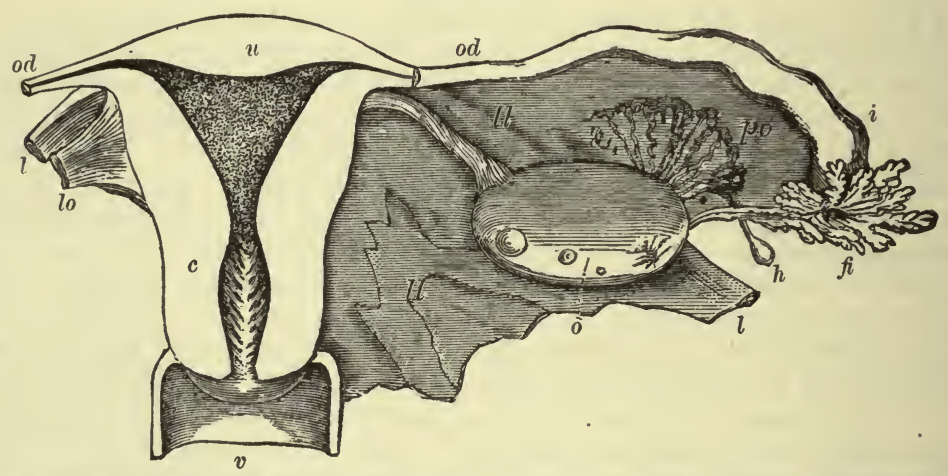

Diagrammatic View of the Uterus and its Appendages, as seen from Behind. (From Quain.) Half natural size. The uterus and upper part of the vagina have been laid open by removing the posterior wall; the Fallopian tube, round ligament, and ovarian ligament have been cut short, and the broad ligament removed on the left side : $u$, the upper part of the uterus; $c$, the cervix opposite the os internum; the triangular shape of the uterine cavity is shown, and the dilatation of the cervical cavity with the rugæ, termed arbor vitæ; $v$, upper part of the vagina; od, Fallopian tube or oviduct; the narrow communication of its cavity with that of the cornu of the uterus on each side is seen; $l$, round ligament; $l o$, ligament of the ovary ; 0 , ovary ; $i$, wide outer part of the right Fallopian tube ; $f$, its fimbriated extremity ; $p o$, parovarium; $h$, one of the hydatids frequently found connected with the broad ligament.

above the os uteri. Its walls consist of an external coat of longitudinal muscular fibres, a middle erectile coat, and an internal mucous coat. The mucous membrane is continuous below with that covering the external genitals, and above with the mucous membrane lining the uterus. The anterior and posterior surfaces are marked by longitudinal folds or raphe, from which a number of transverse folds are given off. This membrane is provided with mucous glands, and is thickly covered with sensitive papillæ.

The uterus is a flattened, pyriform, muscular organ (Fig. 209). Anatomically it is divided into the fundus, neck, and cervix. The neck indicates the point of division between the lower constricted portion, which is the cervix, and the upper expanded portion, the fundus. The cervix, which extends from the neck to the end of the organ, projects into the vagina, at which point it is marked by a transverse fissure, called the os uteri. 
The cavity of the uterus is somewhat triangular in shape, and very much flattened antero-posteriorly. The inferior angle of the cavity is continuous with the canal running through the cervix to the vagina. The superior angles are called the cornua; at the bottom of each is an orifice of a Fallopian tube. The uterus is composed of three coats; a serous (formed by the peritoneum), a muscular, and a mucous coat. The mucous coat is continuous with that lining the Fallopian tubes and vagina. It is covered with columnar ciliated epithelium, and, if examined with a lens, the openings of the mucous follicles will be seen to be very profusely distributed over the surface. If a vertical section is made, as in Fig. 210, the tubules will be seen to be arranged perpendicularly to the surface, having a wavy course. In the impregnated uterus they become much swollen and enlarged. The mucous membrane lining the cervix, on account of its peculiar appearance, is called the arbor vitce uterinus.

The Fallopian tubes (Fig. 209) are about four inches in length, and extend from the cornua of the uterus to the ovaries, where they end in enlarged expanded extremities, the margins of which are covered by long, slender processes, one of them being connected to the ovary. This portion of the tube is called the fimbriated extremity. The tubes are composed of a serous, muscular, and mucons layer. The mucous membrane is covered with ciliated columnar epithelium.

The ovaries (Fig. 209) are flattened, ovoidal bodies, which are situated one on each side of the uterus, and enclosed in the folds of the broad ligaments. They are each connected with the uterus by a ligament, and with the Fallopian tube by one of its fimbriæ. Thev each consist of a fibrous coat

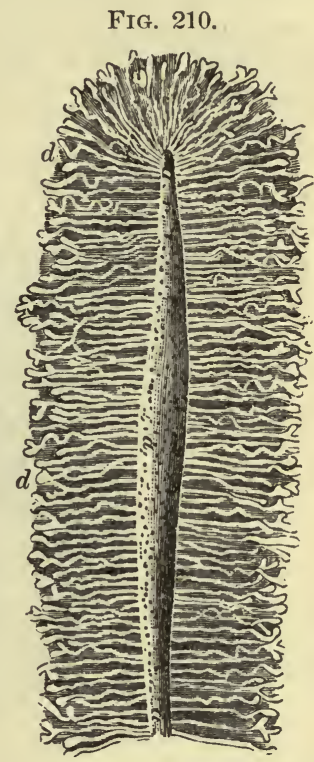

Section of the Lining Membrane of a $\mathrm{Human}$ Uterus at the Period of Commencing Pregnancy, showing the arrangements and other peculiarities of the glands, $d, d, d$, with their orifices, $a, a, a$, on the internal surface of the organ. Twice the natural size.

(tunica albuginea) which encloses the stroma of the organ. (Fig. 211.) The stroma is composed of a soft, vascular fibrous tissue, having imbedded in it a number of small bodies, called Graafian vesicles, which are in divers stages of development. These vesicles commence their development in the deeper portions of the ovary, and as they approach maturity gradually make their way to the surface, where they project as prominences, and, their capsule finally rupturing, discharge their contents into the Fallopian tube. Each vesicle consists of an external coat formed by the ovary, an internal coat or capsule, and within this a layer of cells, which constitutes the membrana granulosa. The interior of the vesicle consists of an albuminous fluid, in which is suspended the ovule.

$\$ 777$. The male generative organs. The same physiological interest is not centred in the male organs of generation as in those of the female, the principal interest being concentrated upon the organs which secrete the male fluid by which the ovule is impregnated. Our remarks will, therefore, be almost entirely confined to the organs concerned in the secretion of this fluid.

The male organs comprise the penis, or organ of copulation, the prostate and Cowper's glands, the testicles, and vasa deferentia and vesiculæ seminales. 
The prostate gland surrounds the neck of the bladder and commencement of the urethra (Fig. 211). It secretes a milky fluid, which is conveyed by the prostatic ducts to the floor of the urethra. Cowper's glands are two small glands which are situated between the layers of the deep perineal fascia at the anterior part of the membranous urethra. They secrete a viscid fluid, which is conveyed by ducts to the floor of the urethra.

The testes or testicles are two small, flattened, ovoidal glands, which are situated in a musculo-membranous pouch, called the scrotum, and suspended by the spermatic cords. Each testicle consists of two parts: the gland proper and the epididymis. The gland (Fig. 213) is composed of an outer

FIG. 211.

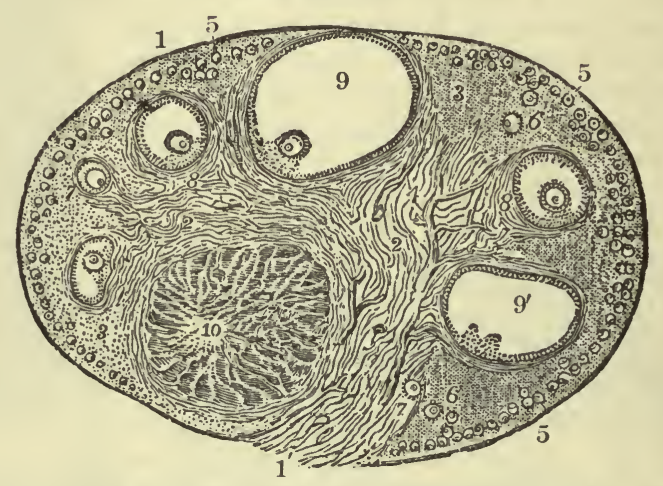

View of a Section of Prepared Ovary of the Cat. (After Schron.) $\times 6$. 1, outer covering and free border of the ovary : $1^{\prime}$, attached border; 2 , the ovarian stroma, presenting a fibrous and vascular structure; 3 , granular substance lying external to the fibrous stroma; 4 , bloodvessels : 5 , ovigerms in their earliest stages occupying a part of the granular layer near the surface; 6 , ovigerms which have begun to enlarge and to pass more deeply into the ovary; 7 , ovigerms round which the Graafian follicle and tunica granulosa are now formed, and which have passed somewhat deeper into the ovary and are surrounded by the fibrous stroma; 8 , more advanced Graafian follicle with the ovum imbedded in the layer of cells constituting the proligerous disc; 9 , the most advanced follicle containing the ovum; 9', a follicle from which the ovum has accidentally escaped; 10 , corpus luteum.

fibrous coat, the tunica albuginea, this being covered by a serous membrane, the tunica vaginalis. The substance of the gland consists of a number of pyramidal lobular divisions, which are situated with their bases toward the surface. Each lobule is composed of several convoluted tubuli seminiferi, and are separated from adjoining lobules by prolongation of fibrous tissue from the tunica albuginea. The tubules are composed of a homogeneous basement membrane, which is lined by granular nucleated epithelium. In the apices of the lobules they have a straight course, and form the vasa recta. They then enter the fibrous tissue of the mediastinum (Fig. 213), and form a plexus of tubes called the rete testis, which end in the upper part of the mediastinum as the vasa efferentia, and these becoming very much convoluted from the globus major or head of the epididymis. 'The tubules of the globus major unite to form a single tube, which is very much convoluted, and constitutes the body and globus minor of the epididymis, and is then continued from the globus minor to the base of the bladder as the excretory duct or vas deferens.

The vas deferens, commencing at the globus minor, ascends in the posterior part of the spermatic cord through the spermatic canal into the pelvis, where it runs to the base of the bladder and becomes enlarged, sacculated, 
and narrowed, and joins with the duct of the vesicula seminalis to form a common ejaculatory duct. The walls of the vas deferens are composed of fibrous and muscular tissue, which is lined by a mucous membrane with columnar epithelium.

The vesiculae seminales are two elongated sacculated bodies, placed ex-

FIG. 212.

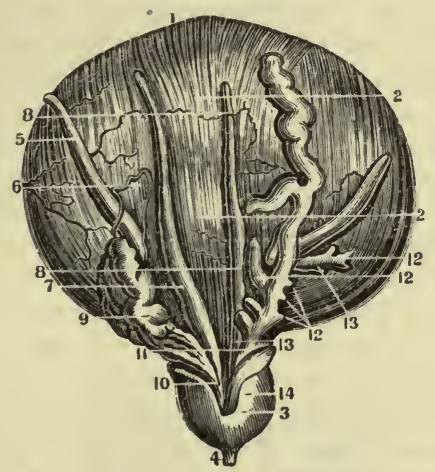

FIG. 213.

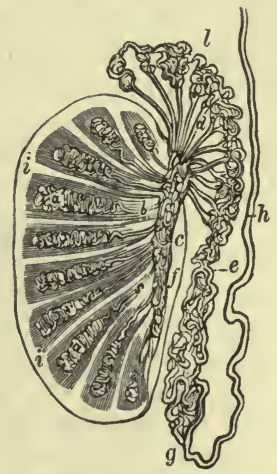

FIg. 212.-The Base of the Male Bladder, with the Vesiculæ Seminales and Prostat iland. (After Haller.) 1, the urinary bladder; 2, the longitudinal layer of muscular fibres; 3 , . ase prostate gland; 4 , membranous portion of the urethra; 5 , the ureters ; 6 , bloodvessels ; 7 , lef, 3 , right vas deferens; 9 , left seminal vesicle in its natural position; 10 , ductus ejaculatorius of the left side traversing the prostate gland; 11, right seminal vesicle injected and unravelled; 12,13 , blind pouches of vesiculæ; 14 , right ductus ejaculatorius traversing the prostate.

FIG. 213. $-a$, lobules ; $b$, vasa recta ; $c$, mediastinum; $d$, vasa efferentia ; $e$, body of epi (idymis : $f$, rete testes; $g$, globus minor; $h$, vas deferens ; $i$, tunica albuginea and its interlobu'ar reflections ; $l$, globus major.

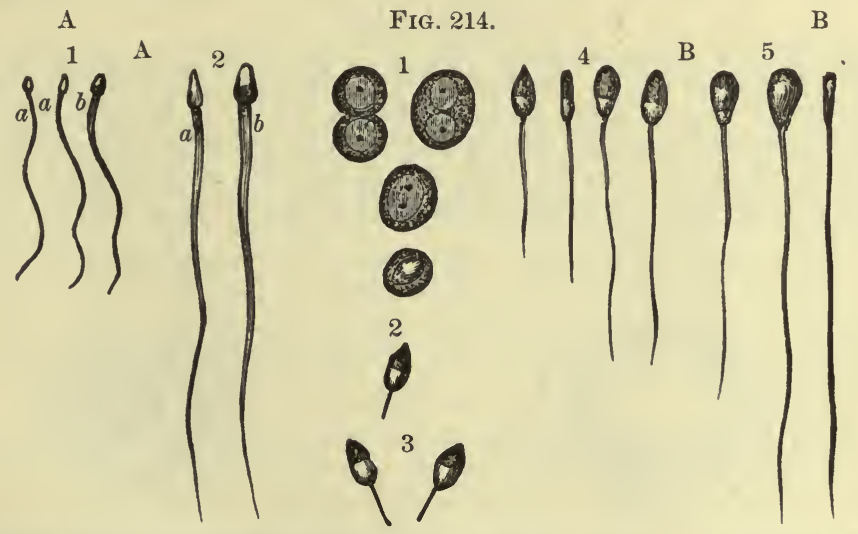

$A$, Spermatozoa from the Human Vas Deferens. (After Kölliker.) 1, magnified 350 diameters ; 2 , magnified 800 diameters; $a$, from the side ; $b$, from above. $B$, Spermatic Cells and Spermatozoa of the Bull undergoing Development. (After Kölliker.) $450 ; 1$. 1, spermatic cells, with one or two nuclei, one of them clear; 2,3 , free nuclei, with spermatic filaments forming; 4 , the filaments enlongated and the body widened; 5 , filaments nearly developed.

ternal to the vasa deferentia. The structure of the seminal vesicles is similar to that of the vasa deferentia, consisting of a fibro-muscular wall lined with a mucous membrane, which is covered by granular, nucleated, polygonal epithelium cells. These organs serve as receptacles for the seminal fluid secreted by the testes, and at the same time produce a secretion of their own 
which is added to it. The ejaculatory ducts, which are formed by the union of the ducts of the vasa deferentia and vesiculæ seminales, open into the prostatic portion of the urethra. Their coats are thinner, but have essentially the same structure as the vasa deferentia, with which they are continuous.

$\S 778$. The seminal fluid is a complex secretion, being composed of the anatomical elements of spermatozoa, which are formed in the testes, and of the secretions of the vasa deferentia, vesiculæ seminales, the prostrate and Cowper's glands, and the mucous glands of the urethra. The seminal fluid is of a thick, whitish striated appearance, and, if examined microscopically, is seen to contain innumerable bodies which are in active motion. These are the spermatozoids, and are the essential male elements concerned in the fecundation of the ovule. Each of these bodies (Fig. 214) consists of a flattened, ovoidal head, having at its base a tapering caudate appendage in active vibratile motion. These anatomical elements were at first considered animalcula, but they are now looked upon as free mases of protoplasm with ciliary appendages, which endow them with the power of migration.

The spermatozoa are developed from the nuclei of vesicles which are formed in the tubules of the testes. The nuclei are metamorphosed into the heads of the spermatozoa, the ciliary appendages being afterward developed as a sort of outgrowth. Different stages of the development and other interesting features are shown in the above figure.]

\section{CH A P'TER I I.}

\section{MENSTRUATION.}

\$779. From puberty, which occurs at from 13 to 17 years of age, to the climacteric, which arrives at from 45 to 50 years of age, the human female is subject to a monthly discharge of ova from the ovaries, accompanied by special changes, not only in those organs but also in the Fallopian tubes and uterus, as well as by general changes in the body at large, the whole

[FIG. 215.

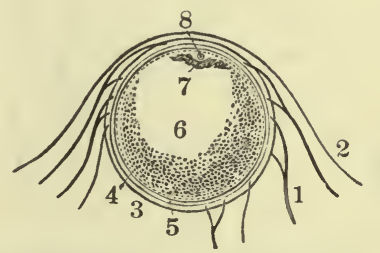

[FIG. 216.

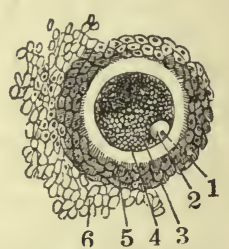

FIG. 215.-Section of Graafian Follicle of a Mammal. (After Von Barr.) 1, stroma of the ovary with bloodvessels; 2 , peritoneum ; 3 and 4 , layers of the external coat of the Graafian follicle ; 5 , membrana granulosa : 6, fluid of the Graafian follicle; 7, granular zone, or discus proligerus, containing the ovule (8).]

Fig. 216.-Ovula of the Sow. (After Barry.) 1, germinal spot; 2, germinal vesicle; 3 , yolk ; 4 , zona pellucida; 5 , discus proligerus; 6 , adherent granules or cells.]

constituting "menstruation." The essential event in menstruation is the escape of an ovum from its Graatian follicle (Fig. 215). The whole ovary at this time becomes congested, and the ripe follicle bulges from its surface. The most projecting portion of the wall of the follicle, which has previously 
become excessively thin, is now ruptured, and the ovum, which having left its earlier position, is lying close under the projecting surface of the follicle, escapes, together with the cells of the discus proligerus (Fig. 216), into the Fallopian tube. How the entrance of the ovum into the Fallopian tube is secured is not exactly known. Some maintain that the ovary is grasped by the trumpet-shaped fimbriated mouth of the Fallopian tube, itself turgid and congested, the movement necessary to bring this about being effected by the plain muscular fibres present in the mouth of the tube. Others, rejecting this view, and asserting that the turgescence of the tube does not occur until after the ovum has become safely lodged in the tube, suggest

FIG. 217.

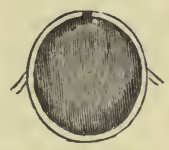

11

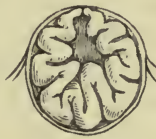

$e$

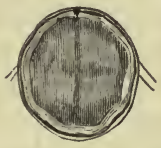

$b$

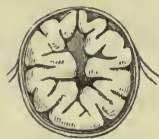

$f$

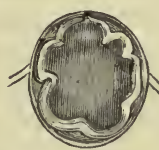

,

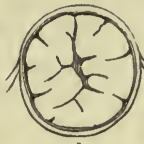

9

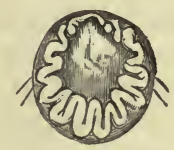

d.

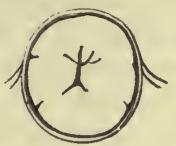

h

Successive Stages of the Formation of the Corpus Luteum in the Graafian Follicle of the Sow (as seen in Vertical Section). At $a$ is shown the state of the follicle immediately after the expulsion of the ovule, its cavity being filled with blood, and no ostensible increase of its epithelial lining having yet taken place; at $b$ a thickening of this lining has become apparent; af $c$ it begins to present folds, which are deepened at $d$, and the clot of blood is absorbed pari passu, and at the same time decolorized; a continuance of the same process, as shown at $e, f, g, h$, forms the corpus luteum, with its delicate cicatrix.

that the ovum is carried in the proper direction by currents in the peritoneal cavity set up by the action of the ciliated epithelium lining the tube, currents whose direction and strength seem, as shown by experiment, to be adequate to carry into the uterus particles present in the peritoneal fluid. Arrived in the tube, the ovum travels downward, very slowly, by the action probably of the cilia lining the tube, though possibly its progress may occasionally be assisted by the peristaltic contractions of the muscular walls. The stay of the ovum in the Fallopian tube may extend to several days. There is an effusion of blood into the ruptured follicle, which is subsequently followed by histological changes in the coats of the follicle resulting in a corpus luteum ${ }^{1}$ (Fig. 217). The discharge of the ovum is accompanied not

1 [The following tabular statement by Dalton expresses the principal differences between the corpus luteum of the non-pregnant and pregnant female:

Corpus Luteum of Menstruation.

At the end of three Three-quarters of an inch in diamweeks.

One Month.

Two Months.

Six Months.

Nine Months. eter: central clot reddish; convoluted wall pale.

Smaller; convoluted wall bright yellow; clot still reddish.

Reduced to the condition of an insigniticant cicatrix.

Absent.

Absent.
Corpus Luteum of Pregnancy.
Larger: convoluted with bright yellow ; clot still reddish.

Seven-eighths of an inch in diameter ; convoluted wall bright yellow; clot perfectly decolorized.

Still as large as at end of second month ; clot fibrinous; convoluted wall paler.

One-half an inch in diameter; central clot converted into radiating cicatrix : the external wall tolerably thick and convoluted, but without any bright yellow color.] 
only by a congestion or erection of the ovary and Fallopian tube, but also by marked changes in the uterus, especially in the uterine mucous membrane. While the whole organ becomes congested and enlarged, the mucous membrane, and especially the uterine glands, are distinctly hypertrophied. The swollen internal surface is thrown into folds which almost obliterate the cavity; and a hemorrhagic discharge, often considerable in extent, constituting the menstrual or catamenial flow, takes place from the greater part of its surface. The blood as it passes through the vagina becomes somewhat altered by the acid secretions of that passage, and when scanty coagulates but slightly; when the flow, however, is considerable, distinct clots may make their appearance. The swollen and hypertrophied mucous membrane then undergoes a rapid degeneration, and is shed, passing a way sometimes in distinct masses, forming the latter part of the menstrual flow. The loss of the mucous membrane is so complete, that the bases only of the uterine glands are left, and from the epithelial cells lining these the regeneration of the new membrane is said to take place. It is not certain that menstruation, in the human subject at all events, is always accompanied by a discharge of an ovum; indeed cases have been recorded in which menstruation continued after what appeared to be complete removal of both ovaries. And it seems probable also that under certain circumstances, $e$. g., coitus, a discharge of an ovum may take place at other times than at the menstrual period. Since, however, the time during which both the ovum and the spermatozoon may remain in the female passages alive and functionally capable is considerable, probably extending to some days, coitus effected either some time after or some time before the menstrual escape of an ovum might lead to impregnation and subsequent development of an embryo; hence the fact that impregnation may follow upon coitus at some time after or before menstruation is no very cogent argument in favor of the view that such a coitus has caused an independent escape of an ovum. The escape of the ovum is said to precede, rather than coincide with or follow, the catamenial flow. If no spermatozoa come in contact with the ovum it dies, the uterine membrane returns to its normal condition, and no trace of the discharge of an ovum is left, except the corpus luteum in the ovary.

$\$ 780$. It is obvious that in these phenomena of menstruation we have to deal with complicated reflex actions affecting not only the vascular supply, but, apparently in a direct manner, the nutritive changes of the organs concerned. Our studies on the nervous action of secretion render it easy for us to conceive in a general way how the several events are brought about. It is no more difficult to suppose that the stimulus of the enlargement of a Graafian follicle causes nutritive as well as vascular changes in the uterine mucous membrane, than it is to suppose that the stimulus of food in the alimentary canal causes those nutritive changes in the salivary glands or pancreas which constitute secretion. In the latter case we can to some extent trace out the chain of events; in the former case we hardly know more than that the maintenance of the lumbar cord is sufficient, as far as the central nervous system is concerned, for the carrying on of the work. In the case of a dog in which the spinal cord had been completely divided in the dorsal region while the animal was as yet a mere puppy, "heat" or menstruation took place as usual. 


\section{CH A PTER III.}

\section{IMPREGNATION.}

$\$ 781$. Is coitus the discharge of the semen containing the spermatozoa is most probably effected by means of the peristaltic contractions of the vesiculæ seminales and vasa deferentia, assisted by rhythmical contractions of the bulbo-cavernosus muscle, the whole being a reflex act, the centre of which appears to be in the lumbar spinal cord. In the dog emissions of semen can be brought about by stimulation of the glans penis after complete division of the spinal cord in the dorsal region. The emission of semen is preceded by an erection of the penis. This we have already seen (p. 274) is in part at least due to an increased vascular supply brought about by means of the nervi erigentes; it is probable, however, that the condition is further secured by a compression of the efferent veins of the corpora cavernosa by means of smooth muscular fibres present in those bodies. The semen being received into the female organs, which are at the time in a state of turgescence resembling the erection of the penis, but less marked, the spermatozoa find their way into the Fallopian tubes, and here (probably in its upper part) come into contact with the ovum. In the case of some animals impregnation may take place at the ovary itself. The passage of the spermatozoa is most probably effected mainly by their own vibratile activity; but in some animals a retrograde peristaltic movement travelling from the uterus along the Fallopian tubes has been observed; this might assist in bringing the semen to the ovum, but inasmuch as these movements are probably parts of the act of coitus, and impregnaton may be deferred till some time after that event, no great stress can be laid upon them.

As the result of the action of the spermatozoa on the ovum, the latter, instead of dying as when impregnation fails, awakes to great nutritive activity accompanied by remarkable morphological changes; it enlarges and develops into an embryo.

$\$ 782$. [Preceding the time of the occurrence of the entrance of the spermatozoon into the egg, certain histological changes have been observed to occur, and in order thoroughly to understand these, as well as the changes which follow in the ovum, it will first be necessary to review the histology of the egg.

The ovule is a minute cell, the wall being formed by a structureless, transparent membrane, called the zona pellucida, or vitelline membrane. Within this is the yolk, or vitellus, which consists of a granular semi-fluid mass, having suspended in it a nucleus or germinal vesicle which contains a nucleolus or germinal spot. The germinal vesicle consists of a very delicate, transparent, homogeneous membrane which encloses a fluid with granules, suspended in which is an eccentric nucleolus of a graular and fibrillated structure.

$\$ 783$. Previous to the occurrence of the impregnation of the ovule a very interesting series of changes have been observed to take place. According to Balfour, the first interesting point to be noticed is the migration of the germinal vesicle toward the cell wall. The vesicular wall then becomes wavy and gradually disappears, while at the same time the nucleolus or germinal spot has undergone metamorphosis, so that what remains of these structures is a spindle-shaped mass. One extremity of this mass gradually projects through the cell wall and is thrown off as a polar vesicle. From the other remaining portion a second polar vesicle is formed, the part of the 
mass then remaining in the ovule being permanent, and is called the female pronucleus. The next change observed is the appearance of a zone, of radial striæ around the pronucleus and its migration to the centre of the egg. The spermatozoon then penetrates the wall of the ovule, probably at the point of the formation of the polar vesicles. The tail of the spermatozoon becomes absorbed, and the head is metamorphosed into the male pronucleus. From the male pronucleus a number of radiating striæ are given off in all directions, and it then migrates toward the female pronucleus, and afterward fuses with it, forming a single or cleavage nucleus.

$\$ 784$. Cleavage or segmentation of the vitellus then begins (Fig. 218), by which process the nucleus thus formed divides into two parts, each taking

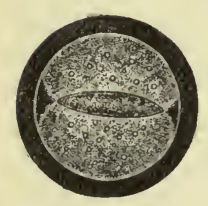

$b$

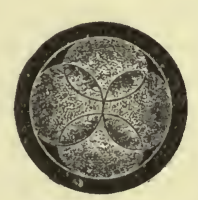

FIG. 218.

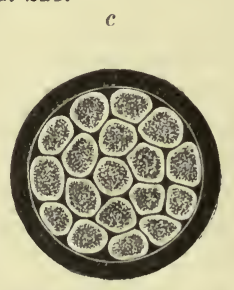

$d$

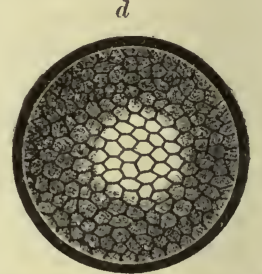

Diagrams of the Various Stages of Cleavage of the Yolk. (After Dalton.)

with it half of the vitelline mass. These two divide into four, and these four into eight, and so on indefinitely until an agglomerate mass of nucleated cells results, each of which contains a part of the cleavage nucleus. This

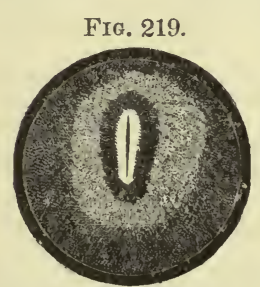

Impregnated Egg, with Commeneement of Formation of Embryo. (After Dalton.) Showing the area germinativa or embryonic spot, the area pellucida, and the primitive groove or trace. mass of cells is called the mulberry mass, and the cells constituting it arrange themselves about the interior of the zona pellucida and form the blastodermic vesicle or membrane. This membrane then splits up into two layers, the external and internal, a third or middle layer being afterward formed between them.

Immediately after the formation of the two layers of blastoderm, an opaque rounded collection of small cells occurs, called the area germinativa or embryonic spot. (Fig. 219.) This spot then becomes elongated, and in its longitudinal axis the first trace of the embryo appears as a faint line, termed the primitive trace, this being in the midst of a clear elongated mass of cells, the area pellucida, which is itself surrounded by a more opaque zone.

$\$ 785$. In front of the primitive trace two folds are formed from which a groove is prolonged backward in a line with the primitive trace. These folds gradually extend along the entire length of the groove, and from the lamince dorsales, which, by growing, project more and more above the groove, and, gradually approaching each other, coalesce and enclose the neural canal, which will afterward contain the cerebro-spinal axis. At about the same period, corresponding to the development of the dorsal lamina similar lamina are given off from the under surface of the blastoderm. These are the lamince ventrales, which, by gradually enlarging and finally coalescing, enclose the abdominal cavity. Beneath the floor of the groove above described a delicate, whitish collection of cells appears. This is the chorda dorsalis or notochord, around which are afterward developed. the bodies and processes of the vertebra. 
During this period other changes have also taken place. The cephalic and caudal extremities have become fixed and form the cephalic and caudal flexures; and the embryo also being curved upon itself laterally, the vitelline mass appears separated from it by a constriction. This constriction gradually increasing, finally separates the vitelline mass as a vesicular body, it being connected with the body of the embryo by the vitelline duct. (Fig. 220.) The vesicular body thus formed is called the umbilical vesicle. This at first communicates with the intestinal cavity, but as development proceeds the duct of communication becomes closed, and the vesicle is merely attached by a pedicle, and finally disappears altogether. At the time of the development of the bloodvessels, vessels appear on the surface of the umbilical vesicle, constituting the vascular area, the chief vessels being the omphalomesenteric arteries and veins. The vessels of the vascular area absorb the nutritive material contained within the vesicle and convey it to the embryo for its sustenance.

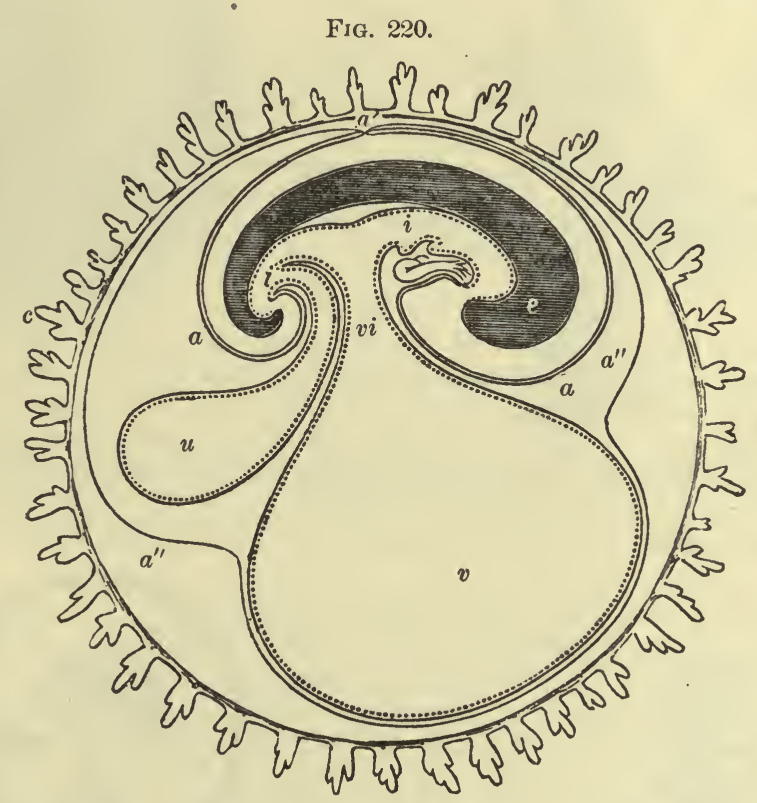

Diagrammatic Section showing the Relation in a Mammal and in a Man between the Primitive Alimentary Canal and the Membranes of the Ovum. The stage represented in this diagram corresponds to that of the fifteenth or seventeenth day in the human embryo, previous to the expansion of the allantois ; $c$, the villous chorion; $a$, the amnion; $a^{\prime}$, the place of convergence of the amnion and reflection of the false amnion, $a^{\prime \prime} a^{\prime \prime}$, or outer or corneous layer; $e$, the head and trunk of the embryo, comprising the primitive vertebræ and cerebro-spinal axis; $i, i$, the simple alimentary canal in its upper and lower portions; the yolk-sac or umbilical vesicle; $v i$, the vitelline duct : $u$, the allantois connected by a pedicle with the anal portion of the alimentary canal.

$\S 786$. Shortly after the occurrence of the commencement of the formation of the umbilical vesicle, double folds, formed of the external layer of the blastoderm, are given off from the cephalic and caudal extremities, and laterally, which curve around over the dorsal surface of the embryo, where they meet and coalesce, and, their point of junction becoming absorbed, form the amniotic cavity. (Figs. 220, 221, 222, and 223.) The outer layer of the fold, or false amnion, gradually expands and covers the whole of the internal surface of the vitelline membrane, which it ultimately replaces; the 
inner layer, or true amnion, is continuous with the skin of the embryo at the umbilicus, and closely envelops it. The amniotic cavity or sac thus formed becomes filled with the liquor amnii, which gradually increases in quantity as pregnancy advances, up to about the fifth or sixth month, when the quantity gradually decreases up to the time of labor.

FIG. 221.

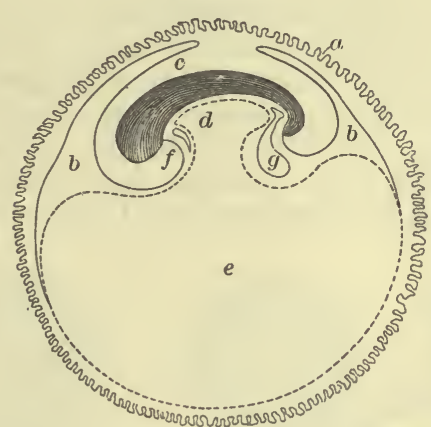

Fig. 222.

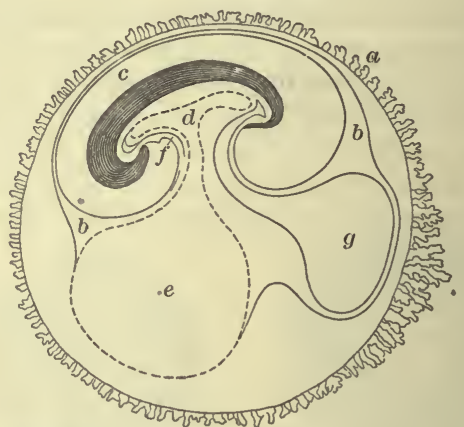

$a$, chorion with villi. The villi are shown to be best developed in the part of the chorion to which the allantois is extending; this portion ultimately becomes the placenta. $b$, space between the two layers of the amnion; $c$, amniotic cavity; $d$, situation of the intestine, showing its connection with the umbilical vesicle; $e$, umbilical vesicle; $f$, situation of heart and vessels; $g$, allantois. (After Todd and Bowman.)

$\$ 787$. At about the time of the commencement of the development of the amnion a new organ, the allantois, appears as a pyriform mass of cells at a point immediately posterior to the vitelline duct and projecting through the same opening. (Fig. 223.) This mass of cells undergoes rapid growth, spreading itself between the true and false amniotic folds, finally completely

FIG. 223.

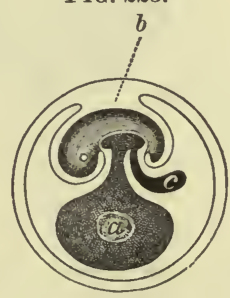

Fig. 224.

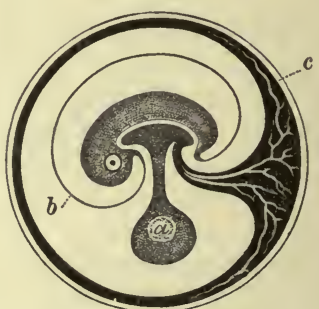

Fig. 223.-Diagram of Fecundated Egg. (After Dalton.). $a$, umbilical vesicle; $b$, amniotic cavity ; $c$, allantois.

Fig. 224.-Fecundated Egg with Allantois nearly Complete. $a$, inner layer of amniotic fold; $b$, outer layer of ditto; $c$, point where the amniotic folds come in contact. The allantois is seen penetrating between the outer and inner layers of the amniotic folds. This figure, which represents only the amniotic folds and the parts within them, should be compared with Figs. 221 and 222 , in which will be found the structures external to these folds.

enclosing the embryo and amnion (Fig. 224), becoming at the same time adjoined to the false amnion, when it is developed into the true chorion. During the process of the development of the allantois, it has become very vascular; at first there are two arteries and two veins, afterward one of the veins disappears. These vessels constitute the umbilical vessels, forming part of the umbilical cord which connects the allantois with the embryo. During 
the development of the allantois it presents three distinct anatomical portions : a portion which becomes constricted off, as it were, from the rest and forms the urinary bladder ; the outer portion forms the chorion, the intermediate portion forming the umbilical cord.

FIG. 225.

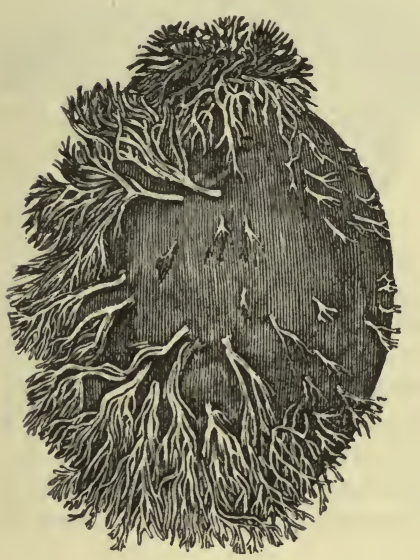

FIG. 226.

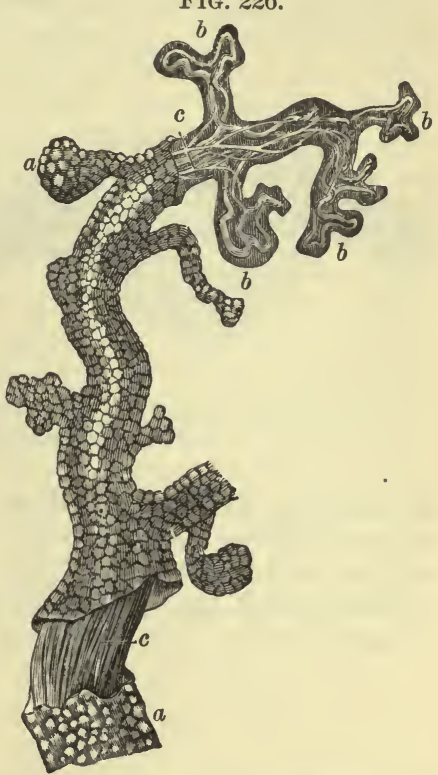

Fig. 225.-Entire Human Ovum of Eighth Week; sixteen lines in length (not reckoning the tufts); the surface of the chorion partly smooth and partly rendered shaggy by the growth of tufts.

Fig. 226.-Portion of One of the Fœtal Villi, about to form part of the Placenta; highly magnified. $a, a$, its cellular covering; $b, b, b$, its looped vessels; $c, c$, its basis of connective tissue.

During the development of the embryo up to this time, the first chorion was formed by villosities formed on the vitelline membrane; and following that by villosities developed upon the false amnion. The allantois then becoming developed, completely covers the internal surface of the false amnion, which then gradually disappears as a distinct structure. The true chorion is then formed by the allantois, which becomes covered by a growth of a multitude of vascular shaggy tufts or villi (Fig. 225). These villi, at first, are distributed over the entire surface of the organ, but they soon commence disappearing, except at a small area corresponding to the attachment of the pedicle which connects the allantois with the embryo. At this point they become greatly increased in number, and also in size and vascularity. These villi are composed of a fibro-granular matrix, in which are numerous capillary loops, and are covered with a layer of epithelial cells (Fig. 226). This portion of the chorion forms the foetal portion of the placenta.

No sooner, however, have these changes begun in the ovum than correlative changes, brought about probably by reflex action, but at present most obscure in their causation, take place in the uterus. The mucous membrane of this organ, whether the coitus resulting in impregnation be coincident with a menstrual period or not, becomes congested, and a rapid growth takes place, characterized by a rapid proliferation of the epithelial and subepithelial tissues. Unlike the case of menstruation, however, this new growth does not give way to immediate decay and hemorrhage, but remains, and may be 
distinguished as a new temporary lining to the uterus, the so-called decidua. Into this decidua the ovum, on its descent from the Fallopian tube, in which it has undergone developmental changes, extending most probably as far, at least, as the formation of the blastoderm, if not further, is received; and in this it becomes imbedded, the new growth closing in over it. (Figs. 227, 228.) Meanwhile the rest of the uterine structures, especially the muscular tissue,

FIG. 227.

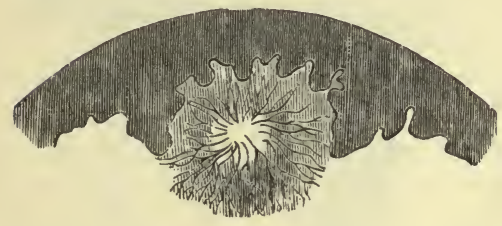

FIG. 228.

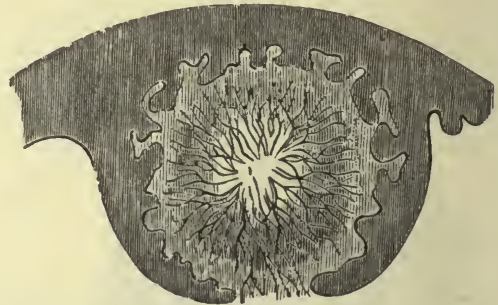

FIG. 227.-First Stage of the Formation of the Decidua Reflexa around the Ovum. Fig. 228.-More Advanced Stage of Decidua Reflexa.

become also much enlarged; as pregnancy advances a large number of new muscular fibres are formed. As the ovum continues to increase in size, it bulges into the cavity of the uterus, carrying with it the portion of the decidua which has closed over it. Henceforward, accordingly, a distinction is made in the now well-developed decidua between the decidua reflexa, or

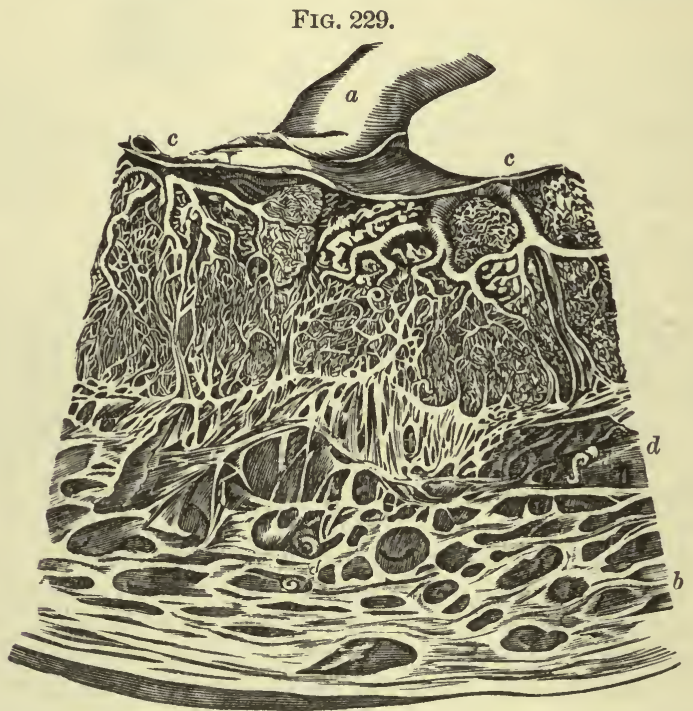

Section of a Portion of a Fully Formed Placenta, with the part of the Uterus to which it is attached. $a$, umbilical cord ; $b, b$, section of uterus, showing the venous sinuses; $c, c, c$, branches, of the umbilical vessels ; $d, d$, curling arteries of the uterus.

that part of the membrane which covers the projecting ovum, and the decidua vera, or the rest of the membrane lining the cavity of the uterus, the two being continuous around the base of the projecting ovum. That part of the decidua which intervenes between the ovum and the nearest uterine 
wall is frequently spoken of as the decidua serotina. As the ovum develops into the fotus with its membranes, the decidua reflexa becomes pushed against the decidua vera; about the end of the third month, in the human subject, the two come into complete contact all over, and ultimately the distinction between them is lost. In the region of the decidua serotina, the allantoic vessels of the fœetus develop a placenta.

$\S 788$. In the earliest stages of the development of the placenta, the delicate villous processes of the chorion insinuate themselves into the hypertrophied follicles of the decidua serotina. The villi then undergo a rapid increase in size and vascularity, becoming branched into secondary and tertiary ramifications; while, at the same time, corresponding changes are taking place in the follicles, by which they become greatly increased in size and vascularity, and at the same time forming diverticula in which are imbedded the ramifications of the villi. The villi and follicles thus grow simultaneously, and finally become blended with each other and are no longer separate structures. The follicular bloodvessels first form capillary plexuses; these vessels, however, become enlarged, forming frequent anastomoses, and finally coalescing to form venous sinuses (Fig. 229), in which are bathed the foetal villi. There is no continuity established between the maternal and fœetal blood; the interchange of nutritive material necessary for the growth and development of the f'etus takes place through the delicate walls of the villi.]

For further account of the various changes by which these events are brought about, as well as of the history of the embryo itself, we must refer the reader to anatomical treatises.

\section{CHAPTER IV.}

\section{THE NUTRITION OF THE EMBRYO.}

\$ 789. During the development of the chick within the hen's egg the nutritive material needed for the growth first of the blastoderm, and subsequently of the embryo, is supplied by the yolk, while the oxygen of the air, passing freely through the porous shell, gains access to all the tissues both of the embryo and the yolk, either directly or by the intervention of the allantoic vessels. The mammalian embryo, during the period which precedes the extension of the allantoic vessels into the cavities of the uterine wall to form the placenta, must be nourished by direct diffusion, first from the contents of the Fallopian tube and subsequently from the decidua; and its supply of oxygen must come from the same sources. All analogy would lead us to suppose that, from the very first, oxidation is going on in the blastodermic and embryonic structures; but the amount of oxygen actually withdrawn from without is probably exceedingly small in the early stages, seeing that nearly the whole energy of the metabolism going on is directed to the building up of structures, the expenditure of energy in the form of either heat or external work being extremely small. The marked increase of bulk which takes place during the conversion of the mulberry mass into the blastodermic vesicle shows that at this epoch a, relatively speaking, large quantity of water at least, and probably of nutritive matter, must pass from without into the ovum; and subsequently, though the blastoderm and em- 
bryo may for some time draw the material for their continued construction at first hand from the yolk-sac or umbilical vesicle, both this and they continue probably until the allantois is formed to receive fresh material from the mother by direct diffusion.

$\$ 790$. As the thin-walled allantoic vessels come into closer and fuller connection with the materual uterine sinuses, until at last in the fully formed placenta the former are freely bathed in the blood streaming through the latter, the nutrition of the embryo becomes more and more confined to this special channel. The blood of the foetus flowing along the umbilical arteries effects exchanges with the venous blood of the mother, and leaves the placenta by the umbilical vein richer in oxygen and nutritive material and poorer in carbonic acid and excretory products than when it issued from the fotus.

As far as the gain of oxygen and the loss of carbonic acid are concerned these are the results of simple diffusion. Venous blood, as we have already seen, always contains a quantity of oxy-hæmoglobin, and the quantity of this substance present in the blood of the uterine veins is sufficient to supply all the oxygen that the embryo needs; the blood of the foetus, containing less oxygen than even the venous blood of the mother, will take up a certain though small quantity. The foetal blood travelling in the umbilical artery must, in proportion to the extent of the nutritive changes going on in the embryo, possess a higher carbonic tension than that in the umbilical vein or uterine sinus; and by diffusion gets rid of this surplus during its stay in the placenta. The blood in the umbilical arteries and veins is, therefore, relatively speaking, venous and arterial respectively, though the small excess of oxy-hæmoglobin in the blood of the umbilical vein is insufficient to give it a distinctly arterial color, or to distinguish it as sharply from the more venous blood of the umbilical artery, as in ordinary arterial from ordinary venous blood. Thus, the foetus breathes by means of the maternal blood, in the same way that a fish breathes by means of the water in which it dwells.

The blood of the foetus is very poor in hæmoglobin, corresponding to its low oxygen consumption. When the mother is asphyxiated the foetus is asphyxiated too, the oxygen of the latter passing back again into the blood of the former; and the asphyxia thus produced in the foetus is much more rapid than that which results when the oxygen is used up by the tissues of the fœtus alone, as when the umbilicus is ligatured and the fœetus not allowed to breathe.

If oxygen and carbonic acid thus pass by diffusion to and from the mother and the foetus, one might fairly expect that diffusible salts, proteids, and carbohydrates would be conveyed to the latter, and diffusible excretions carried away to the former, in the same way; and if fats can pass directly into the portal blood during ordinary digestion, there can be no reason for doubting that this class of food-stuffs also would find its way to the foetus through the placental structures. We do know from experiment that diffusible substances will pass both from the mother to the fotus, and from the foetus to the mother; but we have no definite knowledge as to the exact form and manner in which, during normal intra-uterine life, nutritive materials are conveyed to or excretions conveyed from the growing young. The placenta is remarkable for the great development of cellular structures, apparently of an epithelial nature, on the border-land between maternal and fotal elements; and it has been suggested that these form a temporary digestive and secretory (excretory) organ. But we have no exact knowledge of what actually does take place in these structures. From the cotyledons of ruminants may be obtained a white creamy-looking fluid, which from 
many features of its chemical composition might almost be spoken of as a "uterine milk."

Speaking broadly, the foetus lives on the blood of its mother, very much in the same way as all the tissues of any animal live on the blood of the body of which they are the parts.

$\$ 791$. For a long time all the embryonic tissues are "protoplasmic" in character; that is, the gradually differentiating elements of the several tissues remaiu still imbedded, so to speak, in undifferentiated protoplasm; and during this period there must be a general similarity in the metabolism going on in various parts of the body. As differentiation becomes more and more marked, it obviously would be an economical advantage for partially elaborated material to be stored up in various foetal tissues, so as to be ready for immediate use when a demand arose for it, rather than for a special call to be made at each occasion upon the mother for comparatively raw material needing subsequent preparatory changes. Accordingly, we find the tissues of the foetus at a very early period loaded with glycogen. The muscles are especially rich in this substance, but it occurs in other tissues as well. The abundance of it in the former may be explained partly by the fact that they form a very large proportion of the total mass of the foetal body, and partly by the fact that, while during the presence of the glycogen they contain much undifferentiated protoplasm, they are exactly the organs which will ultimately undergo a large amount of differentiation, and, therefore, need a large amount of material for the metabolism which the differentiation entails. It is not until the later stages of intra-uterine life, at about the fifth month, when it is largely disappearing from the muscles, that the glycogen begins to be deposited in the liver. By this time histological differentiation has advanced largely, and the use of the glycogen to the economy has become that to which it is put in the ordinary life of the animal; hence we find it depositerl in the usual place. Besides being present in the fotal, glycogen is found also in the placental, structures; but here probably it is of use, not for the foetus, but for the nutrition and growth of the placental structures themselves. We do not know how much carbohydrate material finds it way into the umbilical vein; and we cannot, therefore, state what is the source of the fotal glycogen; but it is at least possible, not to say probable, that it arises, in part at all events, from a splitting up of proteid material.

$\$ 792$. Concerning the rise and development of the functional activities of the embryo our knowledge is almost a blank. We know scarcely anything about the various steps by which the primary fundamental qualities of the protoplasm of the ovum are differentiated into the complex phenomena which we have attempted in this book to expound. We can hardly state more than that while muscular contractility becomes early developed, and the heart probably, as in the chick, beats even before the blood-corpuscles are formed, movements of the foetus do not, in the human subject, become pronounced until after the fifth month ; from that time forward they increase and subsequently become very marked. They are often spoken of as reflex in character; but only a preconceived bias would prevent them from being regarded as largely automatic. The digestive functions are naturally, in the absence of all food from the alimentary canal, in abeyance. Though pepsin may be found in the gastric membrane at about the fourth month, it is doubtful whether a truly peptic gastric juice is secreted during intra-uterine life; trypsin appears in the pancreas somewhat later, but an amylolytic ferment cannot be obtained from that organ till after birth. The date, however, at which these several ferments make their appearance in the embryo appears to differ in different animals. The excretory functions of 
the liver are developed early, and about the third month bile pigment and bile salts find their way into the intestines. The quantity of bile secreted during intra-uterine life accumulates in the intestine, and especially in the rectum, forming, together with the smaller secretion of the rest of the canal, and some desquamated epithelium, the so-called meconium. Bile salts, both unaltered and variously changed, the usual bile pigments, and cholesterin, are all present in the meconium. The distinct formation of bile is an indication that the products of fotal metabolism are no longer wholly carried off by the maternal circulation; and to the excretory function of the liver there are now added those of the skin and kidney. The substances escaping by these organs find their way into the allantois or into the amnion, accord-

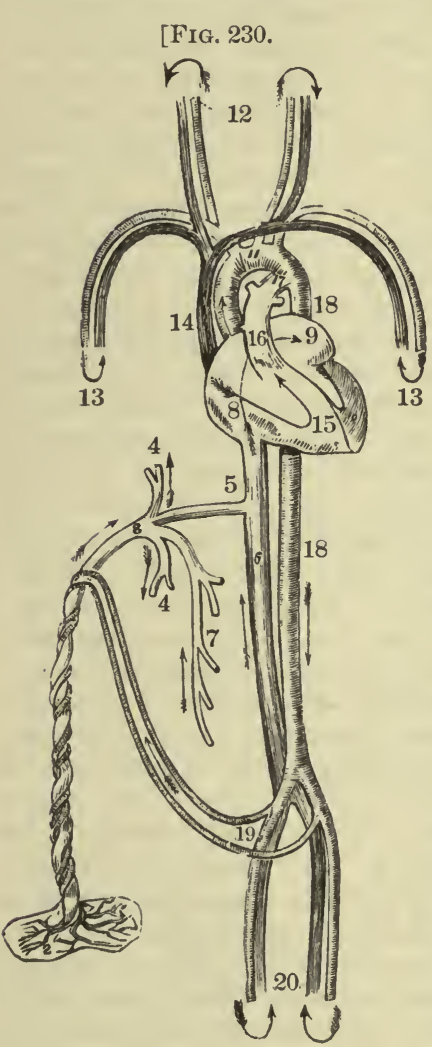
ing to the arrangement of the foetal membranes in different classes of animals; in both these fluids urea or allied bodies have been found as well as the ordinary saline constituents; the latter may or may not have been actually secreted. From the allantoic fluid of ruminants the body allantoin has been obtained, and human and other amniotic fluids have been found to

Diagram of the Fœtal Circulation. 1, the umbilical cord, consisting of the umbilical veiu and two umbilical arteries, proceeding from the placenta (2); 3 , the umbilical vein dividing into three branches, two $(4,4)$ to be distributed to the liver, and one (5), the ductus venosus, which enters the inferior vena cava $(6) ; 7$, the portal vein, returning the blood from the intestines, and uniting with the right hepatic branch; 8 , the right auricle: the course of the blood is denoted by the arrow proceeding from 8 to 9 , the left auricle; 10 , the left ventricle; the blood following the arrow to the arch of the aorta (11), to be distributed through the branches given off by the arch to the head and upper extremities; the arrows 12 and 13 represent the return of the blood from the head and upper extremities through the jugular and subclavian veins to the superior rena cava (14), to the right auricle (8), and in the course of the arrow through the right ventricle $(15)$, to the puimonary artery $(16) ; 17$, the ductus arteriosus, which appears to be a proper continuation of the pulmonary artery : the offsets at each side are the right and left pulmonary arteries cut off. The ductus arteriosus joins the descending aorta $(18,18)$, which divides into the common iliacs, and these into the internal iliacs, which become the umbilical arteries (19), and return the blood along the umbilical cord to the placenta, and the external iliacs.(20), which are continued into the lower extremities. The arrows at the termination of these vessels mark the return of the venous blood by the veins to the inferior vena cava.]

contain urea. It is maintained by some, however, that the fluid in the amnion is secreted by the mother, and that hence the substances present in it are of maternal origin.

$\S 793$. About the middle of intra-uterine life, when the foetal circulation [Fig. 230] is in full development, the blood flowing along the umbilical vein is carried chiefly by the ductus venosus into the inferior vena cava and so into the right auricle. Thence it is directed by the valve of Eustachius through the foramen ovale into the left auricle, passing from which into the left ventricle it is driven into the aorta. Part of the umbilical blood, however, instead of passing directly to the inferior cava, enters by the portal vein into the hepatic circulation, from which it returns to the inferior cava 
by the hepatic veins. The inferior cava also contains blood coming from the lower limbs and lower trunk. Hence the blood which passing from the right auricle into the left auricle through the foramen ovale is distributed by the left veutricle through the aortic arch, though chiefly blond coming direct from the placenta, is also blood which on its way from the placenta has passed through the liver, and blood derived from the tissues of the lower part of the body of the foetus. The blood descending as foetal venous blood from the head and limbs by the superior vena cava does not mingle with that of the inferior vena cava, but falls into the right ventricle, from which it is discharged through the ductus arteriosus (Botalli) into the aorta, below the arch, whence it flows partly to the lower trunk and limbs, but chiefly by the umbilical arteries to the placenta. A small quantity only of the contents of the right ventricle finds its way into the lungs. Now the blood which comes from the placenta by the umbilical vein direct into the right auricle is, as far as the foetus is concerned, arterial blood; and the portion of umbilical blood which traverses the liver probably loses at this epoch very little oxygen during its transit through that gland, the liver being at this period a simple excretory rather than an actively metabolic organ. Hence the blood of the inferior vena cava, though mixed, is on the whole arterial blood; and it is this blood which is sent by the left ventricle through the arch of the aorta into the carotid and subclavian arteries. Thus the head of the foetus is provided with blood comparatively rich in oxygen. The blood descending from the head and upper limbs by the superior vena cava is distinctly venous; and this passing from the right ventricle by the ductus arteriosus is driven along the descending aorta, and together with some of the blood passing from the left ventricle around the aortic arch falls into the umbilical arteries and so reaches the placenta. The foetal circulation then is so arranged that while the most distinctly venous blood is driven by the right ventricle back to the placenta to be oxygenated, the most distinctly arterial (but still mixed) blood is driven by the left ventricle to the cerebral structures, which have more need of oxygen than have the other tissues. Contrary to what takes place afterward, the work of the right ventricle is in the foetus greater than that of the left; and, accordingly, that greater thickness of the left ventricular walls, so characteristic of the adult, does not become marked until close upon birth.

In the later stages of pregnancy the mixture of the various kinds of blood in the right auricle increases preparatory to the changes taking place at birth. But during the whole time of intra-uterine life the amount of oxygen in the blood passing from the aortic arch to the medulla oblongata is sufficient to prevent any inspiratory impulses being originated in the medullary respiratory centre. This, during the whole period elapsing between the date of its structural establishment, or rather the consequent full development of its irritability, and the epoch of birth, remains dormant; the oxygen-supply to the protoplasm of its nerve-cells is never brought so low as to set going the respiratory molecular explosions. As soon, however, as the intercourse between the maternal and umbilical blood is interrupted by separation of the placenta or by ligature of the umbilical cord, or when, as by the death of the mother, the umbilical blood ceases to be replenished with oxygen by the maternal blood, or when in any other way blood of sufficiently arterial quality ceases to find its way by the left ventricle to the medulla oblongata, the supply of oxygen in the respiratory centre sinks, and when the fall has reached a certain point an impulse of inspiration is generated and the foetus for the first time breathes. This action of the respiratory centre may be assisted by adjuvant impulses reaching the centre along various afferent nerves, such as those started by exposure of the body 
to the air, or to cold; but these are subordinate, not essential. A retarded first breath may be hurried on by dashing water on the face of the newborn infant; but, on the other hand, the foetus, upon the cessation of the placental circulation, will make its first respiratory movements while it is still invested with the intact menbranes and thus sheltered from the air and indeed from all external stimuli.

$\$ 794$. Before this first breath is taken the pulmonary alveoli contain no air, and the lungs when thrown into water sink at once; they are then said to be "atelectatic." After the first breath, the alveoli contain air and the lungs float when thrown into water. A striking difference, however, exists between the lungs of a newborn infant and those of an older person. When the pleural cavity of the former is opened, the lungs do not collapse, no air is driven out by the trachea ; that partial distention of the lungs, and negative thoracic pressure, appears not to be established immediately upon birth. That portion of the residual air in the lungs of the adult which, remaining after the most forcible expiration, is still driven from the lungs upon the pleural cavity being laid open, and which might be called " collapse air," is wanting in the newborn infant. When the change from one condition to the other is effected is not at present known; it may possibly arise from the growth of the chest outstripping that of the lungs.

When the first breath is taken, as under normal circumstances it is, with free access to the atmosphere, the lungs become filled with air, the scanty supply of blood which at the moment was passing from the right ventricle along the pulmonary artery returns to the left auricle brighter and richer in oxygen than ever was the fotal blood before. With the diminution of resistance in the pulmonary circulation caused by the expansion of the thorax, a larger supply of blood passes into the pulmonary artery instead of into the ductus arteriosus, and this derivation of the contents of the right ventricle increasing with the continued respiratory movements, the current through the latter canal at last ceases altogether, and its channel shortly after birth becomes obliterated. Corresponding to the greater flow into the pulmonary artery, a larger and larger quantity of blood returns from the pulmonary veins into the left auricle. At the same time the current through the ductus venosus from the umbilical vein having ceased, the flow from the inferior cava has diminished; and the blood of the right auricle finding little resistance in the direction of the ventricle, which now readily discharges its contents into the pulmonary artery, but finding in the left auricle, which is continually being filled from the lungs, an obstacle to its passage through the foramen ovale, ceases to take that course. Any return of blood from the now vigorous and active left auricle into the right auricle is prevented by the valve which, during the later stages of intrauterine life, has been growing up in the left auricle over the foramen ovale. At birth the edge of this valve is to a certain extent free, so that, in case of an emergency, as when the pulmonary circulation is obstructed, a direct escape of blood into the left auricle from. the over-burdened right auricle can take place. Eventually, in the course of the first year, adhesion takes place, and the separation of the two auricles becomes complete. With its larger supply of blood and greater work the left ventricle acquires the greater thickness characteristic of it during life. Thus the foetal circulation, in consequence of the respiratory movements to which its interruption gives rise, changes its course into that characteristic of the adult. 


\section{H A P T E R V.}

\section{PARTURITION.}

$\$ 795$. In spite of the increasing distention of its cavity, the uterus remains quiescent, as far as any marked muscular contractions are concerned, until a certain time has been run. In the human subject the period of gestation generally lasts from 275 to 280 days, $i$. e., about 40 weeks, the general custom being to expect parturition at about 280 days from the last menstruation. Seeing that in many cases it is uncertain whether the ovum which develops into the embryo left the ovary at the menstruation preceding or succeeding coitus, or, as some have urged, independent of menstruation, by reason of the coitus itself, an exact determination of the duration of pregnancy is impossible.

In the cow the period of gestation is about 280 days, in the mare about 350 , sheep about 150 days, dog about 60 days, rabbit about 30 days.

$\S 796$. The extrusion of the fotus is brought about partly by rhythmical contractions of the uterus itself and partly by a pressure exerted by the contraction of the abdominal muscles, similar to that described in defecation. The contractions of the uterus are the first to appear, and their first effect is to bring about a dilatation of the os uteri; it is not until the later stages of labor, while the foetus is passing into the vagina, that the abdominal muscles are brought into play.

$\$ 797$. The whole process of parturition may be broadly considered a reflex act, the nervous centre being placed in the lumbar cord. In a dog, whose dorsal cord had been completely severed, parturition took place as usual ; and the fact that, in the human subject, labor will progress quite naturally while the patient is unconscious from the administration of chloroform, shows that in woman also the whole matter is an involuntary action, however much it may be assisted by direct volitional efforts. That the uterus is capable of being thrown into contractions through reflex action, excited by stimuli applied to various afferent nerves, is well known. The contraction of the uterus, which is so necessary for the prevention of hemorrhage after delivery, may frequently be brought about by exerting pressure or by dashing cold water on the abdomen, by the introduction of foreign bodies into the vagina, and especially by putting the child to the nipple. And we learn from experiments on animals that rhythmic contractions of the uterus resembling at least those of parturition, may be brought about in a reflex manner by stimulating various afferent nerves. Similar movements may be induced by direct stimulation of the spinal cord along its whole length, as well as of various parts of the brain; but there are reasons for thinking that in these cases the impulses started in the brain and upper part of the spinal cord produce their effects by working upon what may be called a " parturition" centre in the upper lumbar regions of the cord. And it would appear that the uterine contractions which are induced by such drugs as ergot, as well as those caused by asphyxia, are, at all events in part, brought about by the agency of the same lumbar centre. From this centre the paths for the efferent impulses appear (in the dog) to be two-fold; one along sympathetic tracts, by nerves passing from the inferior mesenteric ganglion to the hypogastric plexus, and the other along spinal tracts by branches of the sacral nerves to the same plexus. It is stated that the characters of the 
movements induced by stimulating these two tracts are somewhat different, and moreover that the sympathetic tract is vaso-constrictor and the spinal tract vaso-motor in nature; but the matter has not yet been fully worked out.

$\S 798$. We are, however, hardly justified in considering the rhythmical contractions of the uterus during parturition as simple reflex acts excited by the presence of the foetus. We are utterly in the dark as to why the uterus, after remaining apparently perfectly quiescent (or with contractions so slight as to be with difficulty appreciated) for months, is suddenly thrown into action, and within, it may be, a few hours or even less get rid of the burden it has borne with such tolerance for so long a time; none of the various hypotheses which have been put forward can be considered as satisfactory. And until we know what starts the active phase, we shall remain in ignorance of the exact manner in which the activity is brought about. The peculiar rhythmic character of the contractions, each "pain" beginning feebly, rising to a maximum, then declining, and finally dying away altogether, to be succeeded after a pause by a similar pain just like itself, pain following pain like the tardy long-drawn beats of a slowly beating heart, suggests that the cause of the rhythmic contraction is seated, like that of the rhythmic beat of the heart, in the organ itself. And this view is supported by the fact that contractions of the uterus similar to those of parturition have been observed in animals even after complete destruction of the spinal cord; and the movements induced by asphyxia seem in part, and those caused by some drugs such as ammonia seem to be wholly, due to an intrinsic action of the uterus itself. Nevertheless, general evidence supports the conclusion that, in a normal state of things at all events, the contractions of the uterus, like those of the lymph-hearts, are largely dependent on the spinal cord.

The occurrence of contractions in conseqence of an asphyxiated condition of the blood explains why, when pregnant animals are asphyxiated, an extrusion of the foetus frequently takes plaee. There is no evidence, however, that the onset of labor is caused by a gradual diminution of oxygen in the blood, reaching at last to a climax. Nor are there sufficient facts to connect parturition with any condition of the ovary resembling that of menstruation.

The action of the abdominal muscles in parturition is, on the other hand, obviously a reflex act carried out by means of the spinal cord, the necessary stimulus being supplied by the pressure of the fotus in the vagina or by the contraction of the uterus. Hence the whole act of parturition may with reason be considered as a reflex one.

Whether it be wholly a reflex or partly an automatic one, the act can readily be inhibited by the action of the central nervous system. Thus emotions are a very frequent cause of the progress of parturition being suddenly stopped; as is well known, the entrance into the bedroom of a stranger often causes for a time the sudden and absolute cessation of "labor" pains, which previously may have been even violent. Judging from the analogy of micturition, between which and parturition there are many points of resemblance, we may suppose that this inhibition of uterine contractions is brought about by an inhibition of the centre in the lumbar cord.

$\$ 799$. After the expulsion of the fotus, the foetal placenta separates from the uterine walls, and is, together with the remnants of the membrahes, expelled after it. 'The uterus then falls into a firm tonic contraction similar to that of the emptied bladder, by which means hemorrhage from the vessels torn by the separation of the placenta is avoided. The lining membrane of the uterus is gradually restored, the muscular elements are reduced by a rapid fatty degeneration. and in a short time the whole organ has returned to its normal condition. 


\section{CHA PTER VI.}

THE PHASES OF LIFE.

$\S 800$. The child has at birth, on an average, rather less than one-third the maximum length, and about one-twentieth the maximum weight, to which in future years it will attain.

The composition of the body of the newborn babe, as compared with that of the adult, will be seen from the following table, in which the details are more full than those given on p. 482 .

\begin{tabular}{|c|c|c|c|}
\hline \multirow[b]{2}{*}{ Eye. . } & \multicolumn{2}{|c|}{$\begin{array}{l}\text { Weight of organ in percentage } \\
\text { of body-weight. }\end{array}$} & \multirow{2}{*}{$\begin{array}{l}\text { Weight of organ in } \\
\text { adult, as compared } \\
\text { with that of newborn } \\
\text { babe taken as } 1 . \\
1.7\end{array}$} \\
\hline & $\begin{array}{l}\text { Newborn babe. } \\
\text {. } 0.28\end{array}$ & $\begin{array}{l}\text { Adult. } \\
0.028\end{array}$ & \\
\hline Brain ...... & .14 .34 & 2.37 & 3.7 \\
\hline Kidneys. . & . 0.88 & 0.48 & 12 \\
\hline sin... & .11 .3 & 6.3 & 12 \\
\hline Liver & $\begin{array}{l}.39 \\
\end{array}$ & 2.77 & 13.6 \\
\hline Heart... . . & . 0.89 & 0.52 & 15 \\
\hline Stomach and intestin & e 2.53 & 2.34 & 20 \\
\hline Lungs....... & . 2.16 & 2.01 & 20 \\
\hline Skeleton... . . & .16 .7 & 15.35 & 26 \\
\hline Muscles, etc... & .23 .4 & 43.1 & 28 \\
\hline Testicle . . . . & . 0.037 & 0.8 & 60 \\
\hline
\end{tabular}

It will be observed that the brain and eyes are, relatively to the whole body-weight, very much larger in the babe than in the adult, as is also, though to a less extent, the liver. This disproportion is a very marked embryonic feature, and, as far as the brain and eye are concerned at least, has a morphological or phylogenic, as well as a physiological or teleological, significance. Inasmuch as the smaller body has relatively the larger surface, the skin is naturally proportionately greater in the babe. It is chiefly by the accumulation of muscle or flesh, properly so called, that the child acquires the bulk and weight of man, the skeletal framework, in spite of its being specifically lighter in its earlier cartilaginous condition, maintaining throughout life about the same relative weight.

$\S 801$. The increase in stature is very rapid in early infancy, proceeding, however, by decreasing increments. During or shortly before puberty, there is again a somewhat sudden rise, with a subsequent more steady but diminishing increase up to about the twenty-fifth year. From thence to about fifty years of age the height remains stationary, after which there may be a decrease, especially in extreme old age.

$\$ 802$. The increase in weight is also very rapid at first, and proceeding, like the height, with diminishing increments, may continue till about the fortieth year. After the sixtieth year a decline of variable extent is generally witnesserl. It is a remarkable fact, however, that in the first few days of life, so far from there being an increase, there is an actual decrease of weight, so that, even on the seventh day the weight still continues to be less than at birth.

$\$ 803$. The saliva of the babe is active on starch, and its gastric juice, unlike that of many newhorn animals, has good peptic powers, from which we may infer that its digestive processes in general are identical with those of the adult; but the feces of the infant contain, besides considerable quan- 
tity of undigested food (fat, casein, etc.), unaltered bile-pigment, and undecomposed bile-salts.

804. The heart of the babe (see Table, p. 855) is, relatively to its body-weight, larger than the adult, and the frequency of the heart-beat much greater, viz., about 130 or 140 per minute, falling to about 110 in the second year, and about 90 in the tenth year. Corresponding to the smaller bulk of the body, the whole circuit of the blood system is traversed in a shorter time than in the adult (12 seconds as against 22); and, consequently, the renewal of the blood in the tissues is exceedingly rapid. The respiration of the babe is quicker than that of the adult, being at first about 35 per minute, falling to 28 in the second year, to 26 in the fifth year, and so onward. The respiratory work, while it increases absolutely as the body grows, is, relatively to the body-weight, greatest in the earlier years. It is worthy of notice that the absorption of oxygen is said to be relatively more active than the production of carbonic acid; that is to say, there is a continued accumulation of capital in the form of a store of oxygenholding explosive compounds. This, indeed, is the striking feature of infant metabolism. It is a metabolism directed largely to constructive ends. The food taken represents, undoubtedly, so much potential energy; but before that energy can assume a vital mode, the food must be converted into tissue; and, in such a conversion, morphological and molecular, a large amount of energy must be expended. The metabolic activities of the infant are more pronounced than those of the adult, for the sake, not so much of energies which are spent on the world without, as of energies which are for a while buried in the rapidly increasing mass of flesh. Thus, the infant requires, over and above the wants of the man, not only an income of energy corresponding to the energy of the flesh actually laid on, but also an income corresponding to the energy used up in making that living sculptured flesh out of the dead amorphous proteids, fats, carbohydrates, and salts, which serve as food. Over and above this, the infant needs a more rapid metabolism to keep up the normal bodily temperature. This, which is no less, indeed, slightly $\left(0.3^{\circ}\right)$ higher, than that of the adult, requires a greater expenditure, inasmuch as the infant with its relatively far larger surface, and its extremely vascular skin, loses heat to a proportionately much greater degree than does the grown-up man. It is a matter of common experience that children are more affected by cold than are adults.

This rapid metabolism is, however, not manifest immediately upon birth. During the first few days, corresponding to the loss of weight mentioned above, the respiratory activities of the tissues are feeble; the embryonic habits seem as yet not to have been completely thrown off, and as was stated on p. 305, newborn animals bear with impunity a deprivation of oxygen which would be fatal to them later on in life.

$\S 805$. The quantity of urine passed, though scanty in the first two days, rises rapidly at the end of the first week, and in youth the quantity of urine passed is, relatively to the body-weight, larger than in adult life. This may be, at least in quite early life, partly due to the more liquid nature of the food, but is also in part the result of the more active metabolism. For not only is the quantity of urine passed, but also the amount of urea and some other urinary constituents secreted, relatively to the body-weight, greater in the child than in the adult. The presence of uric, of oxalic, and, according to some, of hippuric acids in unusual quantities is a frequent characteristic of the urine of children. It is stated that calcic phosphates, and indeed the phosphates generally, are deficient, being retained in the body for the building up of the osseous skeletın.

$\S 806$. Associated probably with these constructive labors of the growing 
frame is the prominence of the lymphatic system. Not only are the lymphatic glands largely developed and more active (as is probably shown by their tendency to disease in youth), but the quantity of lymph circulation is greater than in later years. Characteristic of youth is the size of the thymus body, which increases up to the second year, and may then remain for a while stationary, but generally before puberty, has suffered a retrogressive metamorphosis, and frequently hardly a vestige of it remains behind. The thyroid body is also relatively greater in the babe than in the adult; the spleen, on the other haud, which grows rapidly in early infancy, is not only absolutely, but also relatively, greater in the adult. It need hardly be said that the recuperative power of infancy and early youth is very marked.

$\$ 807$. It would be beyond the scope of this work to enter into the psychical condition' of the babe or the child, and our knowledge of the details of the working of the nervous system in infancy is too meagre to permit of any profitable discussion. It is hardly of use to say that in the young the whole nervous system is more irritable or more excitable than in later years; by which we probably to a great extent mean that it is less rigid, less marked out into what, in preceding portions of this work, we have spoken of as nervous mechanisms. It may be mentioned that stimulation of the various cerebral areas, in newborn animals, does not give rise to the usual localized movements. The sense of touch, both as regards pressure and temperature, appears well developed in the infant, as does also the sense of taste, and, possibly, though this is disputed, that of smell. The pupil (larger in the infant than in the man) acts fully, and Donders observed normal binocular movements of the eyes in an infant less than an hour old. The eye is (in man) from the outset fully sensitive to light, though of course visual perceptions are imperfect. As regards hearing, on the other hand, very little reaction follows upon sounds-i.e., auditory sensations seem to be dull during the first few days of life; this may be partly, at least, due to absence of air from the tympanum and a tumid condition of the tympanic mucous membrane. As the child grows up his senses rapidly culminate, and in his early years he possesses a general acuteness of sight, hearing, and touch which frequently becomes blunted as his psychical life becomes fuller. Children, however, are said to be less apt at distinguishing colors than in sighting objects; but it does not appear whether this arises from a want of perceptive discrimination or from their being actually less sensitive to variations in hue. A characteristic of the nervous system in childhood, the result, probably, of the more active metabolism of the body, is the necessity for long or frequent and deep slumber.

$\$ 808$. Dentition marks the first epoch of the new life. At about seven months the two central incisors of the lower jaw make their way through the gum, followed immediately by the corresponding teeth in the upper jaw. The lateral incisors, first of the lower and then of the upper jaw, appear at about the ninth month, the first molars at about the twelfth month, the canines at about a year and a half, and the temporary dentition is completed by the appearance of the second molars usually before the end of the second year.

$\S 809$. Ahout the sixth year the permanent dentition commences by the appearance of the first permanent molar beyoud the second temporary molar; in the seventh year the central permanent incisors replace their temporary representatives, followed in the next year by the lateral incisors. In the ninth year the temporary first molars are replaced by the first bicuspids, and in the tenth year the second temporary molars are similarly replaced by the second bicuspids. The canines are exchanged about the eleventh or 
twelfth year, and the second permanent molars are cut about the twelfth or thirteenth year. There is then a long pause, the third or wisdom tooth not making its appearance till the seventeenth, or even twenty-fifth year, or in some cases not appearing at all.

$\S 810$. Shortly after the conclusion of the permanent dentition (the wisdom teeth excepted) the occurrence of puberty marks the beginning of a new phase of life; and the difference between the sexes, hitherto merely potential, now becomes functional. In both sexes the maturation of the generative organs is accompanied by the well-known changes in the body at large; but the events are much more characteristic in the typical female than in the aberrant male. Though in the boy, the breaking of the voice and the rapid growth of the beard which accompany the appearance of active spermatozoa, are striking features, yet they are, after all, superficial. The curves of his increasing weight and height, and of the other events of his economy, pursue for a while longer an unchanged course; the boy does not become a man till some years after puberty; and the decline of his functional manhood is so gradual that frequently it ceases only when disease puts an end to a ripe old age. With the occurrence of menstruation, on the other hand, at from thirteen to seventeen years of age, the girl almost at once becomes a woman, and her functional womanhood ceases suddenly at the climacteric in the fifth decennium. During the whole of the childbearing period her organism is in a comparatively stationary condition. While before the age of puberty, up to about the eleventh or twelfth year, the girl is lighter and shorter than the boy of the same age, in the next few years her rate of growth exceeds his; but she has then nearly reached her maximum, while he continues to grow. Her curve of weight from the nineteenth year onward to the climacteric remains stationary, being followed subsequently by a late increase, so that while the man reaches his maximum of weight at about forty, the woman is at her greatest weight about fifty.

$\$ 811$. Of the statical differences of sex, some, such as the formation of the pelvis and the costal mechanism of respiration, are directly connected with the act of childbearing, while others have only an indirect relation to that duty; and indications, at least, of nearly all the characteristic differences are seen at birth. The baby boy is heavier and taller than the baby girl, and the maiden of five breathes with her ribs in the same way as does the matron of forty. The woman is lighter and shorter than the man, the limits in the case of the former being from 1.444 to 1.740 metres of height, and from 39.8 to 93.8 kilos of weight, in the latter from 1.467 to 1.890 of height, and from 49.1 to 98.5 kilos of weight. The muscular system and skeleton are both absolutely and relatively less in wonian, and her brain is lighter and smaller than that of man, being about 1272 grammes to 1424 . Her metabolism, as measured by the respiratory and urinary excreta, is also not only absolutely but relatively to the body-weight less, and her blood is not only less in quantity, but also of lighter specific gravity, and contains a smaller proportion of red corpuscles. Her strength is to that of man as about 5 to 9 , and the relative length of her step as 1000 to 1157 .

$\S 812$. From birth onward (and indeed from early intra-uterine life) the increment of growth progressively diminishes. At last a point is reached at which the curve cuts the abscissa line, and the increment becomes a decrement. After the culmination of manhood at forty and of womanhood at the climacteric, the prime of life declines into old age. The metabolic activity of the body, which at first was sufficient not only to cover the daily waste, but to add new material, later on is able only to meet the daily wants, and at last is too imperfect even to sustain in its entirety the existing frame. Neither as regards vigor and functional capacity, nor as regards weight and 
bulk, do the turning-points of the several tissues and organs coincide either with each other or with that of the body at large. We have already seen that the life of such an organ as the thymus is far shorter than that of its possessor. The eye is in its dioptric prime in childhood, when its media are clearest and its muscular mechanisms most mobile, and then it for the most part serves as a toy ; in later years, when it could be of the greatest service to a still active brain, it has already fallen into a clouded and rigid old age. The skeleton reaches its limit very nearly at the same time as the whole frame reaches its maximum of height, the coalescence of the various epiphyses being pretty well completed by about the twenty-fifth year. Similarly the muscular system in its increase tallies with the weight of the whole body. The brain, in spite of the increasing complexity of structure and function to which it continues to attain even in middle life, early reaches its limit of bulk and weight. , At about seven years of age it attains what may be considered as its first limit, for though it may increase somewhat up to twenty, thirty, or even later years, its progress is much more slow after than before seven. The vascular and digestive organs as a whole may continue to increase even to a very late period. From these facts it is obvious that though the phenomena of old age are, at bottom, the result of the individual decline of the several tissues, they owe many of their features to the disarrangement of the whole organism produced by the premature decay or disappearance of one or other of the constituent bodily factors. Thus, for instance, it is clear that were there no natural intrinsic limit to the life of the muscular and nervous systems, they would nevertheless come to an end in consequence of the nutritive disturbances caused by the loss of the teeth. And what is true of the teeth is probably true of many other organs, with the addition that these cannot, like the teeth, be replaced by mechanical contrivances. Thus the term of life which is allotted to a muscle by virtue of its molecular constitution, and which it could not exceed were it always placed under the most favorable nutritive conditions, is, in the organism, determined by the similar life-terms of other tissues; the future decline of the brain is probably involved in the early decay of the thymus.

$\S 813$. Two changes characteristic of old age are the so-called calcareous and fatty degenerations. These are seen in a completely typical form in cartilage, as for instance, in the ribs; here the protoplasm of the cartilage-corpuscle becomes hardly more than an envelope of fat-globules, and the supple matrix is rendered rigid with amorphous deposits of calcic phosphates and carbonates, which are at the same time the signs of past and the cause of future nutritive decline. And what is obvious in the case of cartilage is more or less evident in other tissues. Everywhere we see a disposition on the part of protoplasm to fall bask upon the easier task of forming fat rather than to carry on the more arduous duty of manufacturing new material like itself; everywhere almost we see a tendency to the replacement of a structured matrix by a deposit of amorphous material. In no part of the system is this more evident than in the arteries; one common feature of old age is the conversion by such a change of the supple elastic tubes into rigid channels, whereby the supply to the various tissues of nutritive material is rendered increasingly more difficult, and their intrinsic decay proportionately hurried.

$\$$ 814. Of the various tissues of the body the muscular and nervous are, however, those in which the functional decline, if not structural decay, becomes soonest apparent. The dynamic coefficient of the skeletal muscles diminishes rapidly after thirty or forty years of life, and a similar want of power comes over the plain muscular fibres also; the heart, though it may not diminish, or even may still increase in weight, possesses less and less 
force, and the movements of the intestine, bladder, and other organs, diminish in vigor. In the nervous system, the lines of resistance, which, as we have seen, help to map out the central organs into mechanisms, and so to produce its multifarious actions, become at last hindrances to the passage of nervous impulses in any direction, while at the same time the molecular energy of the impulses themselves becomes less. The eye becomes feeble, not only from cloudiness of the media and presbyopic inuscular inability, but also from the very bluntness of the retina; the sensory and motor impulses pass with increasing slowness to and from the central nervous system, and the brain becomes a more and more rigid mass of protoplasm, the molecular lines of which rather mark the history of past actions than serve as indications of present potency. The epithelial glandular elements seem to be those whose powers are the longest preserved; and hence the man who in the prime of his manhood was a "martyr to dyspepsia" by reason of the sensitiveness of gastric nerves and the reflex inhibitory and other results of their irritation, in his later years, when his nerves are blunted, and when, therefore, his peptic cells are able to pursue their chemical work undisturbed by extrinsic nervous worries, eats and drinks with the courage and success of a boy.

$\$ 815$. Within the range of a lifetime are comprised many periods of a more or less frequent recurrence. In spite of the aids of a conplex civilization, all tending to render the conditions of his life more and more equable, man still shows in his economy the effects of the seasons. Some of these are the direct results of varying temperature, but some probably, such as the gain of weight in winter and the loss in summer, are habits acquired by descent. Within the year, an approximately monthly period is manifested in the female by menstruation, though there is no exact evidence of even a latent similar cycle in the male. The phenomena of recurrent diseases, and the marked critical days of many other maladies, may be regarded as pointing to cycles of smaller duration than that of the moon's revolution, unless we admit the view urged by some authors that in these cases the recurrence is to be attributed rather to periodical phases in the disease-producing germ itself, than to variations in the medium of the disease.

$\$ 816$. Prominent among all other cyclical events is the fact that most animals possessing a well-developed nervous system, nust, night after night, or day after day, or at least time after time, lay them down to sleep. The salient feature of sleep is the cessation of the automatic activity of the brain; it is the diastole of the cerebral beat. But the condition is not confined to the cerebral hemispheres; all parts of the body either directly or indirectly take share in it. The phenomena of sleep are perhaps seen in their simplest form in the winter sleep of hibernation, to which especially cold-blooded animals, but also to some extent warm-blooded animals, are subject. In these cases the cold of winter slackens the vibrations and lessens the explosions of the protoplasm, not only of nervous but also of muscular and glandular structures; indeed the activity of the whole body is lowered, in some respects almost to actual arrest. At the same time that the labor of the cerebral molecules becomes insufficient to develop consciousness, the respiratory centre is either wholly quiescent or discharges feeble impulses at rare intervals, and the heart beats with a slow, infrequent stroke, not by reason of any inhibitory restraint, but because its very substance in its slow molecular travail can gather head for explosions only after long pauses of rest. And such few and distant beats as do occur are amply sufficient to meet the needs of the feeble metabolism of the several tissues. The sleep of every day differs from the sleep of winter cold chiefly because the slackening of molecular activities is due in the former not to extrinsic but to intrinsic causes, 
not to changes in the medium, but to exhaustion of the subject, and because the phenomena are largely confined to the cerebral hemispheres. It is true that the whole body shares in the condition. The pulse and breathing are slower, the intestine and other intermal muscular mechanisms are more or less at rest, the secreting organs are less active, some apparently being wholly quiescent, and the sleeper on waking rubs his eyes to bring back to his conjunctiva its needed moisture. Indeed the whole metabolism and the dependent temperature of the body are lowered; but we cannot say at present how far these are the indirect results of the condition of the nervous system, or how far they indicate a partial slumbering of the several tissues.

$\$ 817$. Thoracic respiration is said to become more prominent than diaphragmatic respiration during sleep, and the Cheyne-Stokes rhythm of respiration (see p. 385) is frequently observed. During sleep the pupil is contracted, during, deep sleep exceedingly so ; and dilatation, often unaccompanied by any visible movements of the limbs or body, takes place when any sensitive surface is stimulated; on awaking also the pupils dilate. The eyeballs have been generally described as being during sleep directed upward and converging or, according to some authors, diverging; but others maintain that in true sleep the visual axes are parallel and directed to the far distance. The eyes of children have been described as continally executing during sleep movements, often irregular and unsymmetrical and unaccompanied by changes in the pupils.

$\S 818$. We are not at present in a position to trace out the events which culminate in this inactivity of the cerebral structures. It has been urged that during sleep the brain is anæmic ; but even if this anæmia is a constant accompaniment of sleep, it must, like the vascular condition of a gland or any other active organ, be regarded as an effect, or at least as a subsidiary event, rather than as a primary cause. Nor can the view which regards sleep as the result of a shifting of the mechanical arrangements of the cranial circulation be considered as satisfactory. The explanation of the condition is rather to be sought in purely molecular changes; and the analogy between the systole and the diastole of the heart, and the waking and sleeping of the brain, may be profitably pushed to a very considerable extent. The sleeping brain in many respects closely resembles a quiescent but still living ventricle. Both are, as far as outward manifestations are concerned, at rest, but both may be awakened to activity by an adequately powerful stimulus. Both, though quiescent, are irritable, in both the quiescence will ultimately give place to activity, and in both an appropriate stimulus applied at the right time will determine the change from rest to action. Just as a single prick will under certain circumstances awaken a ventricle, which for some seconds has been motionless, into a rhythmic activity of many beats, so a loud noise will start a man from sleep into a long day's wakefulness. And just as in the heart the cardiac irritability is lowest at the beginning of the diastole and increases onward till a beat bursts out, so is sleep deepest at its commencement after the day's labor; thence onward slighter and slighter stimuli are needed to wake the sleeper. For, judging of the depth of ordinary nocturnal sleep by the intensity of the noise required to wake the sleeper, it may be concluded that, increasing very rapidly at first, it reaches its maximum within the first hour; from thence it diminishes, at first rapidly, but afterwards more slowly.

$\$ 819$. We cannot, however, at present make any definite statements concerning the nature of the molecular changes which determine this rhythmic rise and fall of cerebral irritability. The fact that the products of protoplasmic activity when they accumulate within the protoplasm appear to become in the end an obstruction to that activity, has suggested the idea that 
the presence in the cerebral tissue of an excess of the products of nervous metabolism is the cause of sleep. Indeed lactic acid, the increase of which was supposed to be the cause of the acid reaction of muscular and nervous tissues after exercise, has been especially pointed to in this connection; but, as we have seen, the acid reaction in question appears not to be due to any increased production of lactic acid. Besides, if the accumulation of metabolic products of any kind were the cause of sleep, it is not clear why we should ever have any hope of waking. Mifore may be said in favor of the conception that during the waking hours the expenditure of oxygen exceeds the income, and that the quiescence, which we call sleep, comes from the exhaustion of the body's store of oxygen, more especially of that "intramolecular" oxygen of which we spoke in dealing with the respiration of the tissues. But to this view must be added some hypothesis, such as the byplay of some inhibitory mechanism, whereby the respiratory centre is not roused to increased activity by this lack of oxygen, for, as we have seen, the breathing shares in the slumber of the body, though continuing to play with an amount of energy which permits a gradual restoration of the lost store of oxygen and so finally brings on the awakening which ends the sleep. And the necessity for such a complication indicates that the explanation is, at present at least, inadequate.

The phenomena of sleep show very clearly to how large an extent an apparent automatism is the ultimate outcome of the effects of antecedent stimulation. When we wish to go to sleep we withdraw our automatic brain as much as possibie from the influence of all extrinsic stimuli; and an interesting case is recorded of a lad whose connection with the external world was, from a complicated anæsthesia, limited to that afforded by a single eye and a single ear, and who could be sent to sleep at will by closing the eye and stopping the ear.

$\$ 820$. The cycle of the day is, however, manifested in many other ways than by the alternation of sleeping and waking, with all the indirect effects of these two conditions. There is a diurnal curve of temperature (see p. 537), apparently independent of all immediate circumstances, the hereditary impress of a long and ancient sequence of days and nights. Even the pulse, so sensitive to all bodily changes, shows, running through all the immediate effects of the changes of the minute and the hour, the working of a diurnal influence which cannot be accounted for by waking and sleeping, by working and resting, by meals and abstinence between meals. And the same may be said concerning the rhythm of respiration, and the products of pulmonary, cutaneous, and urinary excretion. There seems to be a daily curve of bodily metabolism, which is not the product of the day's events. Within the day we have the narrower rhythm of the respiratory centre with the accompanying rise and fall of activity in the vasomotor centres. And lastly, there stands out the fundamental fact of all bodily periodicity, that alternation of the heart's systole and diastole which ceases only at death. Though, as we have seen, the intermittent flow in the arteries is toned down in the capillaries to an apparently continuous flow, still the constantly repeated cycle of the cardiac shuttle must leave its mark throughout the whole web of the body's life. 


\section{CHAPTER VII.}

\section{DEATH.}

$\$ 821$. When the animal kingdom is surveyed from a broad standpoint, it becomes obvious that the ovum, or its correlative the spermatozon, is the goal of an individual existence; that life is a cycle beginning in an ovum and coming round to an ovum again. The greater part of the actions which, looking from a near point of view at the higher animals alone, we are apt to consider as eminently the purposes for which animals come into existence, when viewed from the distant outlook whence the whole living world is surveyed, fade away into the likeness of the mere byplay of ovumbearing organisms. The animal body is in reality a vehicle for ova; and after the life of the parent has become potentially renewed in the offspring, the body remains as a cast-off envelope whose future is but to die.

Were the animal frame not the complicated machine we have seen it to be, death might come as a simple and gradual dissolution, the "sans everything" being the last stage of the successive loss of fundamental powers. As it is, however, death is always more or less violent; the machine comes to an end by reason of the disorder caused by the breaking down of one of its parts. Life ceases not because the molecular powers of the whole body slacken and are lost, but because a weakness in one or other part of the machinery throws its whole working out of gear.

$\$ 822$. We have seen that the central factor of life is the circulation of the blood, but we have also seen that blond is not only useless, but injurious, unless it is duly oxygenated: and we have further seen that in the higher animals the oxygenation of the blood can only be duly effected by means of the respiratory muscular mechanism, presided over by the medulla oblongata. Thus the life of a complex animal is, when reduced to a simple form, composed of three factors: the maintenance of the circulation, the access of air to the hæmoglobin of the blood, and the functional activity of the respiratory centre ; and death may come from the arrest of either of these. As Bichat put it, death takes place by the heart, or by the lungs, or by the brain. In reality, however, when we push the analysis further, the central fact of death is the stoppage of the heart, and the consequent arrest of the circulation; the tissues then all die, because they lose their internal medium. The failure of the heart may arise in itself, on account of some failure in its nervous or muscular elements, or by reason of some mischief affecting its mechanical working. Or its stoppage may be due to some fault in its internal medium, such for instance as a want of oxygenation of the blood, which in turn may be caused by either a change in the blood itself, as in carbonicoxide poisoning, or by a failure in the mechanical conditions of respiration, or by a cessation of the action of the respiratory centre. The failure of this centre, and indeed that of the heart itself, may be caused by nervous influences proceeding from the brain, or brought into operation by means of the central nervous system; it may, on the other hand, be due to an imperfect state of blood, and this in turn may arise from the imperfect or perverse action of various secretory or other tissues. The modes of death are in reality as numerous as are the possible modifications of the various factors of life; but they all end in a stoppage of the circulation, and the withdrawal from the tissues of their internal medium. Hence we come to consider the death of the body as marked by the cessation of the heart's beat, a cessation from which no recovery is possible; and by this we are enabled 
to fix an exact time at which we say the body is dead. We can, however, fix no such exact time to the death of the individual tissues. They are not mechanisms, and their death is a gradual loss of power. In the case of the contractile tissues, we have apparently in rigor mortis a fixed term by which we can mark the exact time of their death. If we admit that after onset of rigor mortis recovery of irritability is impossible, then a rigid muscle is one permanently dead. In the case of the other tissues we have no such objective sign, since the rigor mortis of simple protoplasm manifests itself chiefly by obscure chemical signs. And in all cases it is obvious that the possibility of recovery, depending as it does on the skill and knowledge of the experimenter, is a wholly artificial sign of death. Yet we can draw no other sharp line between the seemingly dead tissue whose life has flickered down into a smouldering ember which can still be fanned back again into flame, and the handful of dust, the aggregate of chemical substances into which the decomposing tissue finally crumbles.

Moreover, the failure of the heart itself is at bottom loss of irritability, and the possibility of recovery here also rests, as far as is known at present, on the skill and knowledge of those who attempt to recover. So that, after all, the signs of the death of the whole body are as artificial as those of the death of the constituent tissues. 


\section{A P PEN DIX.}

\section{ON THE CHEMICAL BASIS OF THE ANIMAL BODY.}

THE animal body, from a chemical point of view, may be regarded as a mixture of various representatives of three large classes of chemical substances, viz., proteids, carbohydrates, and fats, in association with smaller quantities of various saline and other crystalline bodies. By proteids are meant bodies containing carbon, oxygen, hydrogen, and nitrogen in a certain proportion, varying within narrow limits, and having certain general features; they are frequently spoken of as albuminoids. By carbohydrates are meant starches and sugars and their allies. We have also seen that the animal body may be considered as an assemblage of protoplasm under various modifications and of numerous products of protoplasmic activity. We do not at present know anything definite about the molecular composition of active living protoplasm; but when we submit protoplasm to chemical analysis, in which act it is killed, we always obtain from it a considerable quantity of the material spoken of as proteid. And many authors go so far as to speak of protoplasm as being purely proteid in nature; they regard the living protoplasm as proteid material, which, in passing from death to life, has assumed certain characters and presumably has been changed in construction, but still is proteid matter ; they sometimes speak of protoplasm as "living proteid" or "living albumin." It is worthy of notice, however, that even simple forms of protoplasm, like that constituting the body of a white corpuscle, forms of protoplasm which we may fairly consider as native protoplasm; when they can be obtained in sufficient quantity for chemical analysis, are found to contain some representatives of carbohydrates and fats as well as of proteids. We might, perhaps, even go so far as to say, that in all forms of living protoplasm, the proteid basis is found upon analysis to have some carbohydrates and some kind of fat associated with it. Further, not only does the normal food, which is eventually built up into protoplasm, consist of all three classes, but as we have seen in the sections on nutrition, protoplasm gives rise by metabolism to members of the same three classes; and, as far as we know at present, carbohydrates and fats, when found in the body out of proteid food, are so formed by the agency of living protoplasm, by some living tissue. Hence there is at least some reason for thinking it probable that the molecule of protoplasm, if we may use such a phrase, is far more complex than a molecule of proteid matter, that it contains in itself residues, so to speak, not only of proteid, but also of carbohydrate and fatty material.

Be this as it may, for no dogmatic statement can at present be made, when we examine the various tissues and fluids of the animal body from a chemical point of view we find present in different places, or at different times, several varieties and derivatives of the three chief classes; we find many forms of proteids, and bodies closely allied to proteids, in the forms of mucin, gelatin, ete.; many varieties of fats; and several kinds of carbohydrates.

We find, moreover, many other bodies which we may regard as stages in the constructive or destructive metabolism of both native and differentiated protoplasm, and which are important not so much from the quantity in which they occur in the animal body at any one time as from their throwing light on the nature of animal metabolism; these are such bodies as urea, other organic crystalline bodies, and the extractives in gencral.

In the following pages the chemical features of the more important of these various substances which are known to occur in the animal body will be briefly considered, such characters only being described as possess or promise to possess 
physiological interest. The physiological function of any substance must depend ultimately on its molecular (including its chemical) nature; and though at present our chemical knowledge of the constituents of an animal body gives us but little insight into their physiological properties, it cannot be doubted that such chemical information as is attainable is a necessary preliminary to all physiological study.

\section{PROTEIDS.}

These form the principal solids of the muscular, nervous, and glandular tissues, of the serum of blood, of serous fluids, and of lymph. In a healthy condition, sweat, tears, bile, and urine contain mere traces, if any, of proteids. Their general percentage composition may be taken as

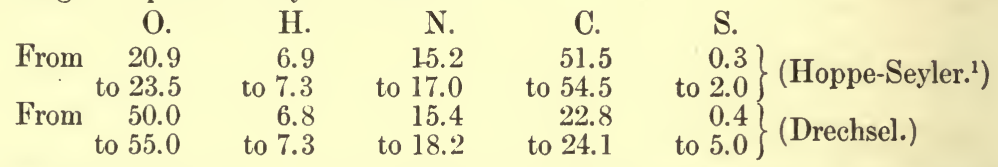

These figures are obtained from a consideration of numerous analyses, slight differences in the various results being immaterial, where the purity of the substance operated upon cannot be definitely determined.

In addition to the above constituents, proteids leave on igmition a variable quantity of ash. In the case of egg-albumin the principal constituents of the ash are chlorides of sodium and potassium, the latter greatly exceeding the former in amount. The remainder consists of sodium and potassium, in combination with phosphoric, sulphuric, and carbonic acids, and very small quantities of calcium, magnesium, and iron, in union with the same acids. There is also a trace of silica. 2 The ash of serum-albumin contains an excess of sodium chloride, but the ash of the proteids of muscle contains an excess of potash salts and phosphates. The nature of the connection of the ash with the proteid is still a matter of obscurity. Globin from hæmoglobin is said to leave no ash on ignition.

Proteids as met with in the animal body are all amorphous; some are soluble, some insoluble in water, and all are, for the most part, insoluble in alcohol and ether; they are all soluble in strong acids and alkalies, but in becoming dissolved mostly undergo decomposition. Their solutions possess a left-handed rotatory action on the plane of polarization, the amount depending on various circumstances, and being, with one exception, viz., peptones, changed by heating.

Crystals into whose composition certain proteid (especially globulin) ${ }^{3}$ elements enter were long since observed in the seeds of many plants; as yet they have not been obtained sufficiently isoluted or in quantities large enough to permit any accurate analysis to be made. A method of isolating in quantity and recrystallizing these substances has, however, ${ }^{4}$ been indicated, and it seems probable that analysis of these may lead to interesting information on the subject of the constitution and combinations of proteids.

The presence of proteids may be determined by the following tests:

1. Heated with strong nitric acid, they or their solutions turn yellow, and this color is, on the addition of ammonia, or caustic soda or potash, changed to a deep orange hue. (Xanthoproteic reaction.)

2. With Millon's reagent they give, when present in sufficient quantity, a precipitate, which turns red on heating. If they are only present in traces, no precipitate is obtained, but merely a red coloration of the solution.

3. If mixed with some concentrated solution of sodic hydrate, and one or two drops of a solution of cupric sulphate, a violet color is obtained, which deepens in tint on boiling.

The above serve to detect the smallest traces of all proteids. The two following tests may be used when there is more than a trace present, but do not hold for every kind of proteid.

4. Render the fluid strongly acid with acetic or other acid, and add a few drops of a solution of ferrocyanide of potassium; a precipitate shows the presence of proteids.

5. Render the fluid, as before, strongly acid with acetic acid, add an equal volume of a concentrated solution of sodic sulphate, and boil. A precipitate is formed if proteids are present.

\footnotetext{
1 Hdb. Phys. Path. Chem. Anal., Ed. iv. (1875), S. 223.

2 See Gmelin, Hdb. Org. Chem., Bd. viii. S. 285.

3 Vines, Journ of Physiol vol, iii. (1880), p. 93.

4 Drechsel, Journ. f. jrakt Chem., N. F., Bd. xix. (1879), S. 331.
} 
This last reaction is useful, not only on account of its exactness, but also because the reagents used produce 110 decomposition of other bodies which may be present; and hence after filtration the same fluid may be further analyzed for other substances. Additional methods of freeing a solution from proteids are: acidulating with acetic acid and boiling, avoiding any excess of the acid; precipitation by excess of alcohol; in the latter case the solution must be neutral or faintly acid. Hoppe-Seyler ${ }^{1}$ recommends the employment of a saturated solution of freshly precipitated ferric hydrate, in acetic acid; this is added to the solution, and on boiling the whole of the proteids are precipitated as well as the ferric salt, the latter as a basic acetate. Brücke's method of removing the last traces of proteids from glycogen solution is also of use (see Glycogen). Precipitation of the last traces of proteids by means of hydrated oxide of lead at a boiling temperature ${ }^{2}$ may be also employed.

[Solutions may entirely be freed from proteids by one of the following methods: (1) Add acetic acid to faint acidity, then tannic acid; (2) render acid with hydrochloric acid, then add iodide of mercury and potassium; (3) render decidedly acid with hydrochloric acid, then precipitate any proteids in solution with phosphotungstic acid.]

Proteids may be conveniently divided into classes.

\section{Class I. Native Albumins.}

Members of this class, as their name implies, occur in a natural condition in animal tissues and fluids. They are soluble in water, are not precipitated by very dilute acids, by carbonates of the alkalies, or by sodium chloride. They are coagulated by heating in solution to a temperature of about $70^{\circ} \mathrm{C}$. If dried at $40^{\circ} \mathrm{C}$. the resulting mass is of a pale yellow color, easily friable, tasteless, inodorous, and soluble.

\section{Egg-albumin.}

Forms in aqueous solution a neutral, transparent, yellowish fluid. From this it is precipitated by excess of strong alcohol. If the alcohol be rapidly removed the precipitate may be readily redissolved in water; if subjected to longer action a coagulation occurs, and the albumin is then no longer thus soluble. Strong acids, especially nitric acid, cause a coagulation similar to that produced by heat or by the prolonged action of alcohol; the albumin becomes profoundly changed by the action of the acid and does not dissolve upon removal of the acid. Mercuric chloride, argentic nitrate, and lead acetate, precipitate the albumin, forming insoluble compounds of variable composition with it: the precipitants may be removed by means of sulphuretted hydrogen and the albumin again obtained, apparently unaltered, in solution.

Strong acetic acid in excess gives no precipitate, but when the solution is concentrated the albumin is transformed into a transparent jelly. A similar jelly is produced when a strong caustic potash is added to a concentrated solution of eggalbumin. In both these cases the substance is profoundly altered, becoming, in the one case, acid; in the other, alkali-albumin.

The specific rotatory power of egg-albumin in aqueous solution is, for yellow light $-35.5^{\circ}$. Hydrochloric acid, added until the reaction is strongly acid, increases this rotation to $-37.7^{\circ}$. The formation of the gelatinous compound with caustic potash is at first accompanied by an increase, but this is followed by a decrease of rotation.

Preparation. White of hen's egg is broken up with scissors into small pieces, diluted with an equal bulk of water, and the mixture shaken strongly in a flask till quite frothy; on standing, the foam rises to the top, and carries all the fibres in whose meshwork the albumin was contained. The fluid from which the foam has been removed, is strained, and treated carefully with dilute acetic acid as long as any precipitate is formed; the precipitate is then filtered off, and the filtrate after neutralization purified by dialysis and then concentrated at $40^{\circ}$ to its original bulk.

\section{Serum-albumin.}

This form of albumin resembles, to a great extent, the one previously described. The following may suffice as distinguishing features:

1. The specific rotation of serum-albumin is $-56^{\circ}$; that of egg-albumin is $35.5^{\circ}$, both measured for yeliow light. 
2. Serum-albumin is not coagulated by being shaken up with ether; egg-albu$\min$ is.

3. Serum-albumin is not very readily precipitated by strong hydrochloric acid, and such precipitate as does occur is readily redissolved on further addition of the acid; the exact reverse of these two features holds good for egg-albumin.

4. Precipitated or coagulated serum-albumin is readily soluble, egg-albumin is with difficulty soluble, in strong nitric acid.

5. Egg-albumin, if injected subcutaneously or into a vein, appears unaltered in the urine ${ }^{1}$ serum-albumin similarly injected does not thus normally pass out by the kidney.

[6. Gautier states that 10 c.c. of the following solution, added to 2 c.c. of the solution to be tested, will precipitate egg-albumin but not serum-albumin: Caustic soda, sp. gr. $0.7,250$ c.c. ; 1 per cent. sulphate of copper, 50 c.c.; glacial acetic acid, 700 c.c.]

Serum-albumin is found not only in blood-serum, but also in lymph, both that contained in the proper lymphatic channels and that diffused in the tissues; in chyle, milk, transudations, and many pathological fluids.

It is this form in which albumin generally appears in the urine.

In addition to the above, Scherer ${ }^{2}$ has described two closely related bodies, to which he gives the names paralbumin and metalbumin. The first he obtained from ovarian cysts; its alkaline solutions are remarkable for being very ropy. It seems doubtful whether this body is a proteid; it differs sensibly in composition from these. Haerlin ${ }^{8}$ gives as its composition, O. 26.8, H. 6.9. N. 12.8 , C. $51.8, \mathrm{~S} .1 .7$ per cent. It seems to be associated with some body like glycogen, capable of being converted into a substance giving the reactions of dextrose. Metalburnin, found in a dropsical fluid, resembles the preceding, but is not precipitated by hydrochloric acid, or by acetic acid and ferrocyanide of potassium; it is precipitated, but not coagulated, by alcohol; its solution is scarcely coagulated on boiling.

Albumins are generally found associated with small but definite amounts of saline matter. A. Schmidt ${ }^{4}$ says that they may be freed from these by dialysis, and that they are then not coagulated on boiling. From this it might be inferred that the albumin and the saline matters were peculiarly related, and that the latter played some special part during the coagulation of the former by heat. Schmidt's observations, however, have not been conclusively corroborated by subsequent observers.

\section{Class II. Derived Albumins (Albuminates).}

\section{Acid-albumin.}

When a native albumin in solution, such as serum-albumin, is treated for some little time with a dilute acid, such as hydrochloric, the properties become entirely changed. The most marked changes are: (1) that the solution is no longer coagulated by heat; (2) that when the solution is carefully neutralized the whole of the proteid is thrown down as a precipitate; in other words, the serum-albumin which was soluble in water, or at least in a neutral fluid containing only a small quantity of neutral salts, has become converted into a substance insoluble in water or in similar neutral fluids. The body into which serum-albumin thus becomes converted by the action of an acid is spoken of as acid-albumin. Its characteristic features are that it is insoluble in distilled water, and in neutral saline solutions, such as those of sodic chloride, that it is readily soluble in dilute acids or dilute alkalies, and that its solutions in acids or alkalies are not coagulated by boiling. When suspended, in the undissolved state, in water, and heated to $70^{\circ} \mathrm{C}$., it becomes coagulated, and is then undistinguishable from coagulated serum-albumin, or indeed from any other form of coagulated proteid. It is evident that the substance when in solution in a dilute acid is in a different condition from that in which it is when precipitated by neutralization. If a quantity of serum- or eggalbumin be treated with dilute hydrochloric acid, it will be found that the conversion of the native albumin into acid-albumin is gradual; a specimen heated to $70^{\circ} \mathrm{C}$. immediately after the addition of the dilute acid, will coagulate almost as usual; and another specimen taken at the same time will give hardly any

IStokvis, Rech. exp. sur les Condit. pathol. de l'Albuminurie, Bruxelles, 1867 ; also Lehmann, Arch. f. Pathol. Anat., Bd. xxx. (1864), S. 593.

${ }^{2}$ Ann der Chem. und Pharm., Bd. Ixxxii., S. 135.

8 Chem. Centralblatt, 1862 , No. 56.

4 Pflüger's Archiv, xi. (1875), S. 1. 
precipitate on neutralization. Some time later, the interval depending on the proportion of the acid to the albumin, on temperature, and on other circumstances, the coagulation will be less, and the neutralization precipitate will be considerable. Still later, the coagulation will be absent, and the whole of the proteid will be thrown down on neutralization.

If finely-chopped muscle, from which the soluble albumins have been removed by repeated washing, be treated for some time with dilute $(0.2$ per cent.) hydrochloric acid, the greater part of the muscle is dissolved. The transparent acid filtrate contains a large quantity of proteid material in a form which, in its general characters at least, agrees with acid-albumin. The acid solution of the proteid is not coagulated by boiling, but the whole of the proteid is precipitated on neutralization; and the precipitate, insoluble in neutral sodic chloride solutions, is readily dissolved by even dilute acids or alkalies. The proteid thus obtained from muscle has been called syntonim, but we have at present no satisfactory test to distinguish the acid-albumin (or syntonin) prepared from muscle from that prepared from egg- or serum-albumin. When coagulated allumin or other coagulated proteid or fibrin is dissolved in strong acids, acid-albumin is formed; and when fibrin or any other proteid is acted upon by gastric juice, acid-albumin is one of the first products; and these acid-albumins cannot be distinguished from acid-albumin prepared from muscle or native albumin. Though hydrochloric acid is perhaps the most convenient acid for forming acid-albumin, other acids may also be used for the purpose of preparing it. Acid-albumin is soluble not only in dilute alkalies, but also in dilute solutions of alkaline carbonates; its solutions in these are not coagulated by boiling.

If sodic phosphate in excess is added to an acid solution of acid-albumin, the acid-albumin is precipitated; this also occurs on adding sodic acetate or phosphate.

As special tests of acid-albumin may be given: 1. Partial coagulation of its solution in lime-water on boiling. 2. Further precipitation of the same solution after boiling, on the addition of calcic chloride, magnesic sulphate, or sodic chloride.

Dissolved in very dilute hydrochloric acid, acid-albumin (syntonin) prepared from muscle possesses a specific lævo-rotatory power of $-72^{\circ}$ for yellow light, this being independent of the concentration. ${ }^{1}$ On heating the solution in a closed vessel in a water-bath the rotatory power rises to $-84.8^{\circ}$.

The body known as parapeptone, which makes its appearance during the peptic digestion of proteids, is closely allied to the substances just described.

\section{Alkali-albumin.}

If serum- or egg-albumin or washed muscle be treated with dilute alkali instead of with dilute acid, the proteid undergoes a change quite similar to that which was brought about by the acid. The alkaline solution, when the change has become complete, is no longer coagulated by heat, the proteid is wholly precipitated on neutralization, and the precipitate, insoluble in water and in neutral sodic chlorine solution, is readily soluble in dilute acids or alkalies. Indeed in a general way it may be said that acid-albumin and alkali-albumin are nothing more than solutions of the same substance in dilute acids and alkalies respectively. When the precipitate obtained by the neutralization of a solution of acid-albumin in dilute acid is dissolved in a dilute alkali, it may be considered to become alkali-albumin; and conversely when the precipitate obtained from an alkali-albumin solution is dissolved in dilute acid, it may be regarded as acid-albumin.

It is stated ${ }^{2}$ as a characteristic reaction of this modified or derived albumin that it is not precipitated when its alkaline solutions are neutralized in the presence of alkaline phosphates; solutions of acid-albumin, on the contrary, are said to be precipitated on neutralization in the presence of alkaline phosphates, and this difference is considered to be a distinguishing feature of the two proteids. But doubt has been cast on this statement. ${ }^{3}$

Alkali-albumin may be prepared by the action not only of dilute alkalies, but also of strong caustic alkalies on native albumins as well as on coagulated albu-

${ }^{1}$ Hoppe-Seyler, Hdb. Phys. Path. Chem. Anal., Ed.iv. (1875), S. 246.

2 Hoppe-Seyler, loc. cit., S. 245.

3 Soyka. Ptlüger's Arch., Bd. xii. (1876), S. 347. 
min and other proteids. The jelly produced by the action of caustic potash on white of egg, spoken of in Class I., 1, is alkali-albumin; the similar jelly produced by strong acetic acid is acid-albumin. One of the most productive methods of obtaining alkali-albumin is that introduced by Lieberkühn, ${ }^{1}$ and consists in adding a strong solution of caustic potash to purified white of egg until the above-mentioned jelly is obtained. This is then cut into small pieces, and dialyzed until quite white. The lumps are then dissolved by heating on the water-bath, and the alkali-albumin precipitated by the careful addition of acetic acid.

Both alkali- and acid-albumin are with difficulty precipitated by alcohol from their alkaline or acid solutions. The neutralization precipitates, however, become coagulated under the prolonged action of alcohol.

The body "protein," described by Mulder, appears, if it exists at all, to be closely connected with this body. All subsequent observers have, however, failed to confirm his views.

The rotatory power of alkali-albumin varies according to its source; thus when prepared by strong caustic potash from serum-albumin, the rotation rises from $-56^{\circ}$ (that of serum-albumin) to $-86^{\circ}$; for yellow light. Similarly prepared from egg-albumin, it rises from $-38.5^{\circ}$ to $-47^{\circ}$; and if from coagulated white of $\mathrm{egg}$, it rises to $-58.8^{\circ}$. Hence the existence of various forms of alkali-albumin is probable.

In addition to the methods given above, alkali-albumin may be also readily obtained by shaking milk with strong caustic soda solution and ether, removing the ethereal solution, precipitating the remaining fluid with acetic acid and washing the precipitate with water, cold alcohol and ether.

The most satisfactory method of regarding acid-and alkali-albumin is to consider them as respectively acid and alkali compounds of the neutralization precipitate. We have reason to think that when the precipitate is dissolved in either an acid or an alkali, it does enter into combination with them. The neutralization precipitate is in itself neither acid-nor alkali-albumin, but may become either, upon solution in the respective reagent.

It is probable that several derived albumins exist, ${ }^{2}$ differing according to the proteid from which they are formed or possibly according to the mode of their preparation, and that each of these may exist in its correlative forms of acid-and alkali-albumin; but the whole subject requires further investigation.

Acid-albumin, prepared by the direct action of dilute acids on native albumin, or on muscle-substance, contains sulphur, as shown by the brown coloration which appears when the precipitate is heated with caustic potash in the presence of basic lead acetate. Alkali-albumin, at all events as prepared by the action of strong caustic potash or soda, does not contain any sulphur; and the acid-albumin, prepared by the solution in an acid of the neutralization precipitate from such an alkali-albumin solution, is similarly free from sulphur.

\section{Casein.}

This is the well-known proteid existing in milk. When freed from fat, and in the moist condition, it is a white, friable, opaque body. In most of its reactions it corresponds closely with alkali-albumin; thus it is readily soluble in dilute acids and alkalies, and is re-precipitated on neutralization; if, however, potassic phosphate is present, as is the case in milk, the solution must be strongly acid before any precipitate is obtained.

Various reactions have at different times been assigned to casein as distinguishing it from the closely allied body alkali-albumin. Later researches have, however, in most cases cast so much doubt on these differences that the identity or non-identity of casein and alkali-albumin must still be left an open question, the discussion of which would be out of place here.

Casein, as occurring in milk, has had several reactions ascribed to it, as characteristic; but these lose their importance on considering that milk contains, in addition to casein, other substances, such as potassic phosphate, and a number of bodies which yield acids by fermentation. The presence of potassic phosphate has an especial influence on the reactions of casein. In the entire absence of this salt, acetic acid in the smallest quantities, as also carbonic anhydride, gires a precipitate; but if this salt is present, carbonic anhydride gives no precipitate, and acetic acid only one when the solution is acid from the presence of free acid, and not from that of acid potassic phosphate. ${ }^{8}$

1 Poggendorff's Annalen, Bd. Ixxxvi., S. 118.

2 Mörner. PHüger's Arch., Bd. xvii. (1878), S. 468.

3 See Kühne, Lehrb. d. Physiol. Chem., 1868, S. 565. 
When prepared from milk by magnesic sulphate (see below), freed by ether from fats, and dissolved in water, casein possesses a specific rotatory power of $-80^{\circ}$ for yellow light; in dilute alkaline solutions, of $-76^{\circ}$; in strong alkaline solutions, of $-91^{\circ}$; in dilute hydrochloric acid, of $-87^{\circ}$.

Casein has been asserted to occur in muscle, in serous fluids, and in blood-serum (serum-casein). In many cases it has probably been confounded with globulins (see Class III.); but blood-serum and muscle-plasma undoubtedly contain an alkali-albumin in addition to whatever globulin may be present, and the usual doubt exists as to the identity of this with true casein. Its presence may be shown by adding dilute acetic acid to blood-serum which has been fieed from globulin by a current of carbonic anhydride; a distinct precipitate is thrown down. A substance similar to casein has also been described as existing in unstriated muscle and in the protoplasm of nerve-cells.

Preparation. Dilute milk with several (10 to 15 ) times its bulk of water, add dilute acetic acid till a precipitate begins to appear, then pass a current of carbonic anhydride, filter, and wash the precipitate with water, alcohol, and ether; the complete removal of the fat carried down with the casein presents some difficulties. Magnesic sulphate added to saturation also precipitates casein from milk; the precipitate as thus formed is readily soluble on the addition of water.

\section{Class III. Globulins.}

Besides the native albumins there are a number of native proteids which differ from the albumins in not being soluble in distilled water; they need for their solution the presence of an appreciable, though it may be a small, quantity of a neutral saline body, such as sodic chloride. Thus they resemble the albuminates in not being soluble in distilled water, but differ from them in being soluble in dilute sodic chloride or other neutral saline solutions. Their general characters may be stated as follows:

They are insoluble in water, soluble in dilute (1 per cent. ) solutions of sodic chloride; they are also soluble in dilute acids and alkalies, bèing changed on solution into acid- and alkali-albumin respectively, unless the acids and alkalies are exceedingly dilute. The saturation with solid sodic chloride of their solutions in dilute sodic chloride, precipitates most members of this class.

\section{Globulin (Crystallin).}

If the crystalline lens be rubbed up with fine sand, extracted with water and filtered, the filtrate will be found to contain at least three proteids. On passing a current of carbonic anhydride a copious precipitate occurs; this is globulin.

The addition of dilute acetic acid to the filtrate from the globulin gives a precipitate of alkali-albumin $;^{1}$ and the filtrate from this, if heated, gives a further precipitate, due to serumalbumin.

In its general reactions globulin corresponds almost exactly with the next members of this class (paraglobulin and fibrinogen), but has no power to form or promote the formation of fibrin in fluids containing the above-mentioned bodies, and possesses the following special features: 1. According to Lehmann, its oxygenated neutral solutions become cloudy on heating to $73^{\circ} \mathrm{C}$, , and are coagulated at $93^{\circ} \mathrm{C}$. 2. It is readily precipitated on the addition of alcohol. According to HoppeSeyler, it is not precipitated on saturation with sodic chloride, resembling vitellin in this respect.

According to Kühne 2 and Eichwald ${ }^{3}$ a globulin with properties identical with those just given may be precipitated from dilute serum by the cautious addition of acetic acid. This body is stated by Weyl 4 to be the same as paraglobulin (fibrinoplastin), the latter differing from it only by a small admixture of fibrin-ferment.

\section{Paraglobulin (Fibrinoplastin).}

Preparation. Blood-serum is diluted tenfold with water, and a brisk current of carbonic anhydride is passed through it. The first-formed cloudiness soon becomes

\footnotetext{
1 But see also Pflüger's Arch., Bd. xiii. (1876), S. 631.

${ }^{2}$ Lehrb. d. Physiol. Chem., 1868, S. 175 .

3 Beitrage zur Chem. d. gewehebild. Subst., Berlin, 1873, H. 1.

${ }^{4}$ Zeitschr. f. Physiol. Chem., Bd. i. (1878), S. 79.
} 
a flocculent precipitate, which is finally quite granular, and may easily be separated by decantation and filtration; it should be washed on the filter with water containing carbonic acid.

It has usually been stated that paraglobulin may be separated from serum by saturation with sodic chloride. But Hammarsten ${ }^{1}$ has shown that this is only in part true, a considerable portion of the globulin remaining unprecipitated. The separation may, however, be completely effected by saturation with magnesic sulphate. When determined by this method the amount of paraglobulin in serum is very considerable, amounting in some cases, according to Hammarsten, to as much as 4.565 per cent. (reckoned on 100 c.c. of serum). The quantity seems to vary in different animals, the precipitation being much more complete in serum from oxblood than in that from the blood of horses.

From its solution in dilute sodic chloride, paraglobulin may be precipitated by a current of carbonic anhydride or the addition of exceedingly dilute (less than 1 pro mille) acetic acid. If the acid is strong, the precipitated proteid becomes immediately changed into acid-albumin (Class II., 1). In pure water, free from oxygen, paraglobulin is insoluble, but on shaking with air or passing a current of oxygen, solution readily takes place; from this it may be reprecipitated by a current of carbonic anhydride. Very dilute alkalies dissolve this body without change; if, however, the strength of the alkali be raised even to 1 per cent. the paraglobulin is changed into alkali-albumin (Class II., 2).

According to Kühne and A. Sehmidt the solutions of this body in water containing oxygen or in very dilute alkalies are not coagulated on heating. The sodic chloride solutions do, however, coagulate when heated to $68^{\circ}-70^{\circ} \mathrm{C} .{ }^{2}$ and if the substance itself be suspended in water and heated to $70^{\circ} \mathrm{C}$. it is coagulated. Although insoluble in alcohol, its solutions are with difficulty precipitated by this reagent.

Paraglobulin occurs not only in blood-serum, but it is also found in white corpuscles, in the stroma of red corpuscles (to some extent at least), in connective tissue, the cornea, aqueous humor, lymph, chyle, and serous fluids.

For the occurrence of globulin in urine see Edlefsen ${ }^{3}$ and Senator. 4

\section{Fibrinogen.}

The general reactions of this body are identical with those of paraglobulin. The most marked difference between the two is the point at which coagulation of their solutions takes place. Hammarsten ${ }^{5}$ has shown that fibrinogen in a 1-5 per cent. solution of sodic chloride coagulates at from $52^{\circ}-55^{\circ} \mathrm{C}$., whereas, as stated above, paraglobulin (fibrinoplastin) coagulates first at from $68^{\circ}-70^{\circ} \mathrm{C}$. This, however, is disputed by A. Schmidt, who holds that the substance coagulating at $52^{\circ}-55^{\circ}$ is not fibrinogen, but a sort of nascent fibrin. There is also a marked difference in the precipitability of the bodies by sodic chloride. (See below.) Other differences between the two may be thus enumerated: In precipitating fibrinogen by a current of carbonic anhydride the containing fluid must be much more strongly diluted, and the gas must pass for a much longer time. The precipitate thus obtained differs from that of paraglobulin in that it forms a viscous deposit, adhering more closely to the sides and bottom of the containing vessel; there is also no flocculent stage previous to the viscous precipitate.

Fibrinogen occurs in blood, chyle, serous fluids, and in various transudations. The relations of fibrinogen and paraglobulin to the formation of fibrin have been discussed in the text, p. 33 .

Preparation. ${ }^{6}$ Salted plasma, obtained by centrifugalizing blood whose coagulation is prevented by the addition of a certain proportion of magnesic sulphate, is mixed with an equal volume of a saturated $\left(35.87 \text { per cent. at } 14^{\circ} \mathrm{C} \text {. }\right)^{7}$ solution of sodic chloride; the fibrinogen is thus precipitated, while the paraglobulin remains in solution. The adhering plasma may be removed by washing with a solution of

1 Pflüger's Archiv, Bd. xvii. (1878), S. 446 ; Bd. xviii. (1878), S. 38.

3 Centralblatt f. med. Wiss., Jahrg. 1870, S. 367. Also Arch. f. klin. Med., Bd. vii. S. 69.

4 Virchow's Archiv, Bd. Ix.S. 476. 5 Upsala Läkareförenings förhandlingar. Bd. xi., 1876.

6 See Hammarsten, Nov. Act. Reg. Scc. Sci.. Upsala, Ser. iii. vol. x. (1875), p. 31. Also Pfiuger's Archiv, Bd. xix. (1879), S. 563, and Bd. xxii. (1880), S. 431.

7 Poggiale, Ann. Chim. Phys. (3), vol. viii. p. 469. 
sodic chloride, and the fibrinogen finally purified by being several times dissolved in and reprecipitated by sodic chloride.

There is no proof that the whole of the substance thrown down by carbonic anhydride from diluted blood-serum is fibrinoplastic; indeed we know that a true globulin devoid of fibrinoplastic properties may be prepared from serum. ${ }^{1}$ Weyle ${ }^{2}$ considers that there is only one globulin in serum, which he characterizes by the name of "serum-globulin," and regards fibrinoplastin as a mixture of this body with a portion of fibrin-ferment. We know for certain (see p. 28) that the whole of the tibrinoplastic precipitate, used to cause the coagulation of a fibrinogenous fluid, does not enter into the composition of the fibrin produced; we also know that such a precipitate may lose its fibrinoplastic powers without any marked change in its general reactions. It would seem advisable, therofore, to speak of the deposit produced by earbonic anhydride in dilute serum, or by saturation with sodic chloride in undiluted serum, as globulin, and to distinguish it as fibrinoplastic globulin when it is able to give rise to fibrin. Fibrinogen similarly might be spoken of as fibrinogenous globulin. The name crystallin, rather than globulin, might then be given to the substance obtained from the crystalline lens.

\section{Myosin.}

This is the substance which forms the chief proteid constituent of dead, rigid musele; its general properties and mode of preparation have been already deseribed at p. 84. In the moist condition it forms a gelatinous, elastic, clotted mass; dried, it is very brittle, slightly transparent, and elastic. From its solution in sodic chloride it is precipitated, either by extreme dilution or by saturation with the solid salt. When precipitated by dilution and submitted to the prolonged action of water, myosin loses its property of being soluble in solutions of sodic chloride. ${ }^{3}$ The sodic chloride solution, if exposed to a rising temperature, becomes milky at $55^{\circ} \mathrm{C}$., and gives a floceulent precipitate at $60^{\circ} \mathrm{C}$. This precipitate is. however, no longer myosin, for it is insoluble in a 10 per cent. sodic chloride solution, and does not, until after many days' digestion, yield syntonin on treatment with hydrochloric acid ( 0.1 per cent.). It is, in fact, coagulated proteid (see Class V.).

Myosin is excessively soluble in dilute acids and alkalies. Advantage may be taken of its solubility in the former to extract it from muscles. ${ }^{4}$ But if the reagents are at all concentrated, myosin undergoes in the act of solution a radical change, becoming in the one case acid-albumin or syntonin, in the other alkalialbumin (Class II.).

Like fibrin, it can in some cases decompose hydrogen dioxide, and oxidize guaiacum with formation of a blue color.

\section{Vitellin.}

As obtained from the yolk of egg, of which it is the chief proteid constituent, vitellin is a white granular body, insoluble in water, but very soluble in dilute sodic chloride solutions; it surpasses myosin in this respect, for the solution may be easily filtered. Its eoagulation temperature is higher than that of myosin, lying, according to Weyl, ${ }^{5}$ between $70^{\circ} \mathrm{C}$. and $80^{\circ} \mathrm{C}$. Saturation with sodic chloride gives no precipitate; in this respect it differs from most other members of this class. In yolk of egg vitelin is always associated with, and probably exists in combination with, the peculiar complex body lecithin.

Jenis, and after him Hoppe-Seyler, have shown that vitellin before the treatment requisite to free it from lecithin possesses properties quite different from other proteids.

A theory has been advanced that vitellin is really a complex body like hæmoglobin, and on treatment with alcohol splits up into coagulated proteid and lecithin. When well purified it contains 0.75 per cent. sulphur, but no phosphorus. Dilute acids or alkalies readily convert it in its uncoagulated form into a member of Class II. 
Fremy and Valenciennes ${ }^{1}$ have described a series of proteids, viz, ichthin, ichthidin, etc. derived from fish and amphibia. They appear to be either identical with, or closely allied to, vitellin.

Preparation. Yolk of egg is treated with successive quantities of ether as long as this extracts any yellow coloring matter; the residue is dissolved in moderately strong (10 per cent.) sodic chloride solution, and filtered. The filtrate on falling into a large excess of water is precipitated. In this state it is mixed with lecithin and nuclein, and in order to free it from these it was usually treated with alcohol. ${ }^{2}$ This, as above stated, entirely changes the vitellin into a coagulated form. It seems probable that the separation of vitellin from the other bodies with which it is mixed in the yolk of egg may be effected by precipitating the sodic chloride solution by the addition of excess of water; the precipitate is then redissolved in 10 per cent. solution of sodic chloride, and the process repeated as rapidly as possible.

\section{Globin.}

Globin, stated by Preyer ${ }^{3}$ to be the proteid residue of the complex body hæmoglobin (see $\mathbf{p}$. 351 ), ought probably to be considered as an outlying member of this class. It is, however, not readily soluble either in dilute acids or sodic chloride solutions. It is said to be absolutely free from ash.

\section{Class IV. Fibrin.}

Insoluble in water and dilute sodic chloride solutions ; soluble, with difficulty, in dilute acids and alkalies, and more concentrated neutral saline solutions.

Fibrin, as ordinarily obtained, exhibits a filamentous structure, the component threads possessing an elasticity much greater than that of any other known solid proteid.

If allowed to form gradually in large masses, the filamentous structure is not so noticeable, and it resembles in this form pure India-rubber. Such lumps of fibrin are capable of being split in any direction, and no definite arrangement of parallel bundles of fibres can be made out.

At ordinary temperature fibrin is insoluble in water, being dissolved only at very high temperatures, and then undergoing a complete change in its characters. In hydrochloric solutions of 1-5 per cent. fibrin swells up and becomes transparent, but is not dissolved. ${ }^{4}$ In this condition the mere removal of the acid by an excess of water, neutralization, or the addition of some salt, causes a return to the original state. If, however, the acid be allowed to act for many days at ordinary temperatures, or for a few hours at $40^{\circ}-60^{\circ} \mathrm{C}$., solution takes place, and the resulting proteid is syntonin. In dilute alkalies and ammonia, fibrin is much more readily soluble, though in this case also the solution is greatly aided by warming; the resulting fluid contains no longer fibrin, but alkali-albumin. This property is not distinctly characteristic of fibrin, although it dissolves perhaps more readily in both dilute acids and alkalies than do coagulated proteids. None of these solutions can be coagulated on heating, which is intelligible when it is remembered that they no longer contain fibrin, but either acid- or alkali-albumin. In addition to the above, fibrin is soluble, though with difficulty and only after a considerable time, in 10 per cent. solutions of sodic chloride, potassic nitrate, or sodic sulphate, the solution being often accompanied by putrefactive changes. These solutions may be coagulated by a temperature of $60^{\circ} \mathrm{C}$., and are precipitated by dilution with water or saturation with solid sodic chloride; in fact, by the action of the neutral saline solutions the fibrin has become converted into a body exceedingly like myosin or globulin. ${ }^{5}$

On ignition of fibrin a residue of inorganic matter is always obtained; it is, however, considered that sulphur is the only one of these elements which enters essentially into its composition. In other respects fibrin corresponds entirely in general composition with the other proteids.

Suspended in water and heated to $70^{\circ} \mathrm{C}$, , it loses its elasticity and becomes opaque ; it is then indistinguishable from other coagulated proteids.

1 Compt. Rend., T. xxxriii. pp. 469, 525.

2 Weyl, op. cit., S. 74.

3 Die Blutkrystalle (1871), S. 166.

${ }^{4}$ Complete solution may, however, take place if the fibrin, as is frequently the case, contains any adherent pepsin.

${ }_{5}$ Gautier, Compt. Rend., T. Ixxix. (1Si4), p. 227. 
A peculiar property of this body remains yet to be mentioned, viz., its power of decomposing hydrogen dioxide. Pieces of fibrin placed in this fluid, though themselves undergoing no change, soon become covered with bubbles of oxygen; and guaiacum is turned blue by fibrin in presence of hydrogen dioxide or ozonized turpentine.

Pieparation. By vigorously stirring blood with a bundle of twigs and then washing with water until it is quite white. If required perfectly pure and colorless it should be prepared from plasma free from corpuscles. If the blood, before stirring, be diluted with an equal bulk of water. the subsequent washing of the fibrin is much facilitated, and it may readily be obtained quite white. Any adherent fats may be removed by ether.

When globulin, myosin, and fibrin are compared each with the other, it will be seen that they form a series in which myosin is intermediate between globulin and fibrin. Globulin is excessively soluble in even the most dilute acids and alkalies; fibrin is almost insoluble in these; while myosin, though more soluble than fibrin, is less soluble than globulin. Globulin again dissolves with the greatest ease in a very dilute solution of 'sodic chloride. Iyosin, on the other hand, dissolves with difficulty; it is much more soluble in a 10 per cent. than in a 1 per cent. solution of sodic chloride; and even in a 10 per cent. solution the myosin can hardly be said to be dissolved. so viscid is the resulting fluid and with such difficulty does it filter. Fibrin again dissolves with great difficulty and very slowly in even a 10 per cent. solution of sodic chloride, and in a 1 per cent. solution it is practically insoluble. When it is remembered that fibrin and myosin are, both of them, the results of coagulation, their similarity is intelligible. Myosin is, in fact, a somewhat more soluble form of fibrin, deposited not in threads or filaments but in clumps and masses.

\section{Class V. Coagulated Proteids.}

These are insoluble in water, dilute acids and alkalies, and neutral saline solutions of all strengths. In fact, they are really soluble only in strong acids and strong alkalies, though prolonged action of even dilute acids and alkalies will effect some solution, especially at high temperatures. During solution in strong acids and alkalies a destructive decomposition takes place, but some amount of acid-or alkalialbumin is always produced.

Very little is known of the chemical characteristics of this class. They are produced by heating to $70^{\circ} \mathrm{C}$. solutions of egg- or serum-albumin, globulins, suspended in water or dissolved in saline solutions; by boiling for a short time fibrin suspended in water or dissolved in saline solutions, or precipitated acid-and alkali-albumin suspended in water. They are readily converted at the temperature of the body into peptones by the action of gastric juice in an acid, or of pancreatic juice in an alkaline medium.

All proteids in solutions are precipitated by an excess of strong alcohol. If the precipitant be rapidly removed they are again soluble in water, but if the precipitated proteids are subjected for some time to the action of the alcohol they are, with the exception of peptones, coagulated and lose their solubility. It appears, however, that the proteids contained in the aleurone-grains of plants are exceedingly resistant to this coagulating action of alcohol. ${ }^{1}$

It seems scarcely necessary to point out the distinction in the use of the word "coagulation" as applied to blood-or muscle-plasma on the one hand and to the action of heat and alcohol upon proteids on the other. The difference is obvious when it is remembered that in the first case the coagulation leads to the formation of fibrin (Class IV.), or myosin (Class III.), and that these bodies may then further be coagulated by heat or alcohol as described above.

\section{Class VI. Peptones. ${ }^{2}$}

Very soluble in water, and not precipitated from their aqueous solutions by the addition of acids or alkalies, or by boiling. Insoluble in alcohol, they are precipitated with difficulty by this reagent, and are unchanged in the process; they differ from all other proteids in not being coagulated by prolonged exposure to alcohol. They are not precipitated by cupric sulphate, ferric chloride, or, except in the instances to be mentioned presently, by potassic ferrocyanide, and acetic acid. In these points they differ from most other proteids. On the other hand, precipita-

1 See Vines, Journ. of Physiol., rol. iii. p. 108.

[ 2 The albumoses are so closely related to peptones that they are commonly considered together with peptones as a class.] 
tion is caused by chlorine, iodine, tannin, mereuric chloride, nitrates of mercury and silver, and both acetates of lead; also by bile-acids in an acid solution. In common with all proteids, these bodies possess a specific lævo-rotatory power over polarized light; but they differ from all other proteids in the fact that boiling produces no change in the amount of rotation.

A solution of peptones, mixed with a strong solution of caustic potash, gires, on the addition of a mere trace of cupric sulphate, a pink color. An excess of the cupric salt gives a violet color, which deepens in tint on boiling, in fact the ordinary proteid reaction. Other proteids simply give the violet color. But the most characteristic feature of peptones is their relatively great diffusibility, a property which they alone, of all.the proteids, may be said to possess, since all other forms of proteids pass through membranes with the greatest difficulty, if at all.

The diffusibility of peptones is, however, absolutely small as compared with that of crystalline bodies such as sodic chloride; in fact, solutions of peptones may be freed from salts by dialysis, a process employed in their preparation.

Notwithstanding their probable formation in large quantities in the stomach and intestine, to judge from the results of artificial digestion, a very small quantity only can be found in the contents of these organs. They are probably absorbed as soon as formed. Another point of interest is their reconversion into other forms of proteids, since this must occur to a great extent in the body. We are, however, as yet ignorant of the manner in which this reverse change is effected.

Preparation. All proteids with the exception of lardacein, yield peptones (and other products) on treatment with acid gastric or alkaline pancreatic juice most readily at the temperature of the human body. Peptones are likewise produced in the absence of pepsin and trypsin, by the action of dilute and moderately strong acids at medium temperatures. also by the action of distilled water at high temperatures under pressure. For various methods of preparing peptones, see Maly, ${ }^{1}$ Adamkiewiez, ${ }^{2}$ Henninger ${ }^{3}$ and Pekelharing. ${ }^{4}$

It appears possible to reobtain ordinary coagulable proteids from peptones by the action of either prolonged heating to $140^{\circ}-170^{\circ} \mathrm{C}$. or of dehydrating agents. ${ }^{5}$

No difference in percentage composition between peptones and the proteid from which they are formed has, at present, been definitely established.

We have used the word "peptones" in the plural number because we have reason to think that more than one kind of peptore exists. Meissner ${ }^{6}$ described three peptones, naming them respectively A-B- and C-peptone. He distinguished them as follows: A-peptone is precipitated from its aqueous solutions by concentrated nitric acid, and also by potassic ferrocyanide in the presence of even weak acetic acid. B-peptone is not precipitated by concentrated nitric acid, nor will potassic ferrocyanide give a precipitate unless a considerable quantity of strong acetic acid be added at the same time. C-peptone is precipitated neither by nitric acid nor by potassic ferrocyanide and acetic acid, whatever be the strength of the acetic acid. In place, however, of speaking of all these as peptones, it is better to consider C-peptone as the only real peptone, and the A- and B-peptones as not peptones at all. Nevertheless we have reason, from the researches of Kühne, to speak of more than one peptone, viz., of a hemipentone which is capable under the action of trypsin of being converted into leucin and tyrosin, and of an antipeptone which resists such a decomposition. The name antipeptone is given to the latter on account of this resistance which it offers toward trypsin; the name hemipeptone, given to the former, signifies that this peptone is the twin or correlative half of antipeptone.

We have seen ( $p .255)$ that when any proteid is digested with pepsin, what we masy preliminarily call a by-product makes its appearance. This by-product which has many resemblances to acid-albumin or syntonin, appearing as a neutralization precipitate soluble in dilute acids and alkalies but insoluble in distilled water, is

1 PHüger's Arch., Bd.ix. (1874), S. 585.

2 Die Natur u. Nähwerth d. Peptons (1877), S. 33.

3 De la Nature et du Role physiolngiques des Peptones, Paris, 1878

4 Pthuger's Arch., Bd. xxii. (1882), S. 185.

'Heuninger, loc. cit.; Hofmeister, Zeitsch. f. physiol. Chem., Bd. 11. (1878), S. 206; Pekelharing, loc. cit.

6 Zeitsch. f. rat. Med., Bde. vii., viii., x., xii. u. xiv 
generally spoken of as parapeptone. According to Finkler ${ }^{1}$ this neutralization precipitate is especially abundant if the pepsin be previously modified by exposure to a temperature of $40^{\circ}$ to $60^{\circ} \mathrm{C}$. The pepsin thus modified is spoken of by Finkler as "isopepsin." Many authors regard parapeptone, syntonin, and acid-albumin as being the same thing. Meissner, however, gave the name parapeptone to a body which need not and probably does not make its appearance during normal natural digestion or during artificial digestion with a thoroughly active pepsin, but which is formed when proteids are subjected to the action of weak hydrochloric acid, either alone or in company with an imperfectly acting pepsin, and which in certain characters is quite distinct from ordinary syntonin or acid-albumin. Its distinguishing feature is that it cannot be changed into peptone by the action of even the most energetic pepsin, though it is really so converted under the influence of trypsin; otherwise it very closely resembles syntonin. We have here an indication that the simple characters by which we have described acid-albumin may be borne by bodies having marked differences from each other. The researches of Kühne ${ }^{2}$ have thrown an important light on these differences. The fundamental notion of Kühne's view is that an ordinary native albumin or fibrin contains within itself two residues, which he calls respectively an anti-residue and a hemi-residue. The result of either peptic or tryptic digestion is to split up the albumin or fibrin, and to produce on the part of the anti-residue antipeptone, and on the part of the hemi-residue hemipeptone, the latter being distinguished from the former by its being susceptible of further change by tryptic digestion into leucin, tyrosin, etc. Antipeptone remains as antipeptone even when placed under the action of the most powerful trypsin, provided putrefactive changes do not intervene.

Before the stage of peptone (whether anti- or hemi-) is reached, there is an intermediate stage corresponding to the formation of syntonin. In both normal peptic and tryptic digestion antipeptone is preceded by an anti-albumose and hemipeptone by a hemi-albumose. Of these the anti-albumose is closely related to syntonin, and has hitherto been regarded as syntonin. The hemi-albumose has not been so frequently observed; it was, however, isolated by Meissner; it is apparently the body called by him A-peptone. It possesses several peculiar features. If its solutions are heated they partially coagulate at about $60^{\circ}-63^{\circ} \mathrm{C}$. : the precipitate is soluble at about $70^{\circ} \mathrm{C}$. and is reprecipitated as the temperature again falls. It also yields a precipitate with nitric acid and potassic ferrocyanide, and this also is soluble at the higher temperature, reprecipitating on cooling. In these respects it closely resembles a proteid body observed by Bence-Jones in the urine of osteomalacia. It approaches myosin in being readily soluble in a 10 per cent. solution of sodic chloride.

If, however, albumin be digested with insufficient or with imperfectly active pepsin, or simply with dilute hydrochloric acid at $40^{\circ} \mathrm{C}$., anti-albumose is not formed, but in its place a body makes its appearance which Kühne calls anti-albumate. $^{3}$ Its characteristic property is that it cannot be converted by peptic digestion into peptone, though it can be so changed by tryptic digestion. It is in fact the parapeptone of Meissner.

It may perhaps be advisable, now that Meissner's parapeptone is cleared up, to reserve the name parapeptone for the initial products of both peptic and tryptic digestion, and to speak of anti-albumose and hemi-albumose as being both parapeptones. But in this sense parapeptone will be an intermediate and not a collateral product of digestion.

Meissner also described a particularly insoluble form of his parapeptone as dyspeptone, and another intermediate product as a metapeptone; but further investigation of both these bodies, as well as his B-peptone, is necessary. Under the influence of dilute hydrochloric acid, anti-albumate becomes changed into a body which Kuihne calls anti-albumid, and which seems identical with the very insoluble proteid described by Schützenberger as "hemiprotein," and probably with Meissner's dyspeptone. The same body is produced at once in company with products belonging to the hemi-group by the action of 3 to 5 per cent. sulphuric acid on native albumin or fibrin. The following tables show the relations and genesis of

1 Pflüger's Archiv, Bd. xiv. (1887), S. 128.

2 Only a short account of these has as yet been published. Verhandl. d. Naturhist.-med. Verein, Heidelberg, Bd. i. Heft 4, 1876.

3 An albumate must not be confounded with an albuminate. 
the bodies we have just described. The several products (antipeptone, etc.) are given in duplicate, on the hypothesis (which, though not proved, is probable) that the changes of digestion are essentially hydrolytic changes, ${ }^{1}$ accompanied by a reduplication; that, just as a molecule of starch splits up into at least two molecules of dextrose, or as a molecule of cane-sugar splits up into a molecule of dextrose and a molecule of levulose, so a molecule of anti-albumose, for instance, splits up into two molecules of antipeptone, and so on. But the whole scheme is, of course, only provisional.

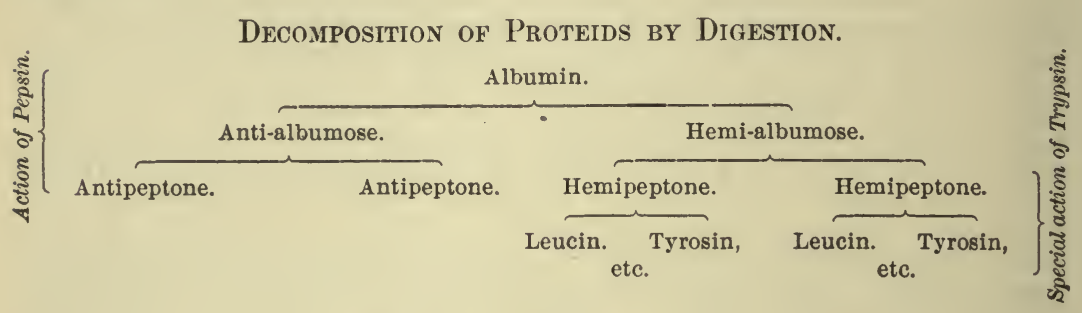

Decomposition by Acids.

1.

By 0.25 per cent. $\mathrm{HCl}$ at $40^{\circ} \mathrm{C}$.

Albumin.

Anti-albumate. $\overbrace{\text { Anti-albumid. } \quad \text { Hemipeptone. }}^{\text {Hemi-albumose. }}$

2.

By $3-5$ per cent. $\mathrm{H}_{2} \mathrm{SO}_{4}$ at $100^{\circ} \mathrm{C}$.

Albumin.

Anti-albumid.

Hemi-albumose.

Leucin, Tyrosin, etc. Hemipeptone.

\section{Class VII. Lardacein, or the so-called Amyloid Substance.}

The substance to which the above name is applied is found as a pathological deposit in the spleen and liver, also in numerous other organs, such as the bloodvessels, kidneys, lungs, etc.

It is insoluble in water, dilute acids and alkalies, and neutral saline solutions.

In centesimal composition it is almost identical with other proteids, ${ }^{2}$ viz. :

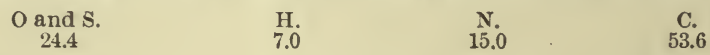

The sulphur in this body exists in the oxidized state, for boiling with caustic potash gives no sulphide of the alkali. The above results of analysis would lead at once to the ranking of lardacein as a proteid, and this is strongly supported by other facts. Strong hydrochloric acid converts it into acid-albumin, and caustic alkalies into alkali-albumin. On the other hand it exhibits the following marked differences from other proteids. It wholly resists the action of ordinary digestive fluids; it is colored red, not yellow, by iodine, and violet or pure blue by the joint action of iodine and sulphuric acid. From these last reactions it has derived one of its names, "amyloid," though this is evidently badly chosen; for not only does it differ from the starch group in composition, but by no means can it be converted

1 Henninger, loc. cit., p. 49.

2 C. Schmidt, Ann. d. Chem. u. Pharm., Bd. cx. S. 250, and Friedreich u. Kekuél, Virchow's Archiv, Bd. xvi. S. 50 . 
into sugar: this latter is one of the crucial tests for a true member of the carbohydrate group. According to Heschl ${ }^{1}$ and Cornil, ${ }^{2}$ anilin-violet (methyl-anilin) colors lardaceous tissue rosy red, but sound tissue blue.

The colors mentioned above as being produced by iodine and sulphuric acid are much clearer and brighter when the reagents are applied to the purified lardacein. When the reagents are applied to the crude substance in its normal position in the tissues, the colors obtained are always dark and dirty looking.

Purified lardacein is readily soluble in moderately dilute ammonia, and can. by evaporation, be obtained from this solution in the form of tough, gelatinous Hakes and lumps; in this form it gives feeble reactions only with iodine. If the excess of ammonia is expelled, the solution becomes neutral, and is precipitated by dilute acids.

Preparation. The gland or other tissue containing this body is cut up into small pieces, and as much as possible of the surrounding tissue remored. The pieces are then extracted several times with water and dilute alcohol, and if not thus rendered colorless are repeatedly boiled with alcohol containing hydrochloric acid. The residue after this operation is digested at $40^{\circ} \mathrm{C}$., with good artificial gastric juice in excess. Everything, except lardacein and small quantities of mucin, nuclein, keratin, together with some portion of the elastic tissue, will thus be dissolved and removed. ${ }^{3}$ From the latter impurities it may be separated by decantation of the finely-powdered substance.

The chief products of the decomposition of proteids are ammonia, carbonic anhydride, leucin, and tyrosin. Several other bodies, for the most part, like leucin, amidated acids, such as aspartic acid, glutamic acid, etc., have also been obtained; also by tryptic digestion, hypoxanthin, and perhaps xanthin. But urea has never yet been derived by direct decomposition from proteid material, the statements to this effect having been based on errors. In spite of numerous researches, we cannot at present state definitely what is the real constitution of a proteid, or in what manner these several residues are contained in the undecomposed substance. It is unnecessary to give here any of the formulæ, nearly all empirical, which have been made to represent a proteid; they all give with equal exactitude the percentage composition, but beyond this they are untrustworthy. Of the various attempts which have been made to assign to proteids some definite molecular structure, none appear, at the present stage of information, sufficiently reliable for general acceptance.

Among the most elaborate labors in this direction may be mentioned those of Hlasiwetz and Haberman. In their first publication,4 starting from the general similarity of the products of decomposition of the proteids and carbohydrates, they tried to establish a definite relation between the two classes of bodies. In this they were not successful, and in their second research 5 they came to the conclusion that the carbohydrates take no part in the formation of the proteids.

Other experiments in the same direction have been made by Schützenberger. ${ }^{6}$ He shows that albumin can be decomposed into carbonic anhydride and ammonia, and that the ratio of these two is the same as though urea had been the body on which he operated. From this he concludes that "the molecule of albumin contains the grouping of urea and represents a complex ureide." In his second publication ${ }^{7}$ he confirms his previous results, stating that the ammonia, carbonic anhydride, and oxalic acid, produced by the decomposition of proteids, are so connected quantitatively as to be capable of derivation from varying proportions of urea and oxamide. He also obtained from the decomposition of proteids a nitrogenous residue which conld be formulated as giving rise to all the amidated acids and other bodies spoken of above. Thus, according to him, albumin built up as a complex ureide, decomposes into ammonia, carbonic, oxalic, and acetic acids, and this nitrogenous body ; this last then gives rise to the other products of decomposition. ${ }^{8}$

It will be noticed that in the general description of the various proteids distinctive reactions for each could not be given, but that varying solubilities were the chief means at our disposal for distinguishing them. They may be arranged according to their solubilities in the following tabular form:

1 Wien. med. Wochenschr, No, $32, \mathrm{~s}, 714$

2 Compt. Rend., T. Ixxx.(1875), p. 1288.

3 Kühne und Rudneff, Virchow's Archiv, Bd. xxxiii. (1865), S. 66.

4 Ann. d. Chem. u. Pharm., Bd. clix. S. 304.

${ }^{6}$ Comptes Rendus, T. lxxx. (1875), p. 232 . Bull. de la Soc. Chim. xxiii., 161, 193, 216, 242, 385,

483 , xxiv. 2 et 145

7 Comptes Rendus, T. Ixxxi. p. 1108. Bull. de la Soc. Chim. xxv. 147.

8 See also Schützenberger, Ann. de Chim, et de Phys., T. xvi. (1879), p. 280 
Soluble in distilled water-

Aqueous solution not coagulated on boiling . . . . Peptones.

Aqueous solution coagulated on boiling . . . . Albumins.

Insoluble in distilled water:

Soluble in $\mathrm{NaCl}$ solution 1 per cent. . . . . . . .

Soluble in $\mathrm{HCl} 0.1$ per cent. in the cold . . . . .

Insoluble in $\mathrm{NaCl}$ solution 1 per vent.

Insoluble in $\mathrm{HCl} 0.1$ per cent. in the cold, but soluble at $60^{\circ} \mathrm{C}$.

Insoluble in $\mathrm{HCl} 0.1$ per cent. at $60^{\circ} \mathrm{C}$; soluble in strong acids.

Soluble in gastric juice ......... Coagulated albumin. Insoluble

Globulins.

Acid-and Alkalialbumin.

Fibrin.

Lardacein.

Such a classification is, however, obviously a wholly artificial one, useful for temporary purposes, but in no way illustrating the natural relations of the several members. Nor is a division into "native" and "derived" proteids much more satisfactory. It is true that we may thus put together serum- and egg-albumin, with vitellin, myosin, and fibrin, on the one hand ; and peptones, coagulated proteids, and acid-with alkali-albumin, on the other. But in what light are we to consider casein, seeing that, though a natural product, it has so many resemblances to alkali-albumin? Moreover, the system of classification must be useless which would place fibrinoplastic globulin and fibrinogen in the same class as fibrin, and yet we can hardly speak of either of the two former bodies as derived proteids. If the view be true that when fibrin is converted into peptone the large molecule of the former is split up, with assumption of water, into two smaller molecules of the latter, one belonging to the "anti" and the other to the "hemi" group, we might speculate on a possible classification of all proteids into hemi-proteids, antiproteids, and holo-proteids. Thus serum- and egg-albumin, myosin, and fibrin would be undoubtedly holo-proteids, peptones either anti- or hemi-proteids, and we should have to distinguish probably in the heterogenenus group of derived albumins both anti-, hemi-, and holo-proteid members. It is possible, moreover, that fibrinoplastic and fibrinogenous globulin and casein may be natural hemi- or anti-proteids, and not holo-proteids. But we have at present no positive knowledge on these points.

\section{[ENZYMes.}

Enzymes are unorganized soluble ferments, such as ptyalin, pepsin, trypsin, amylopsin, rennin, fibrin-ferment, etc., and have been considered in various parts of this work.]

\section{Nitrogenous Non-crystalline Bodies allied to Proteids.}

These resemble the proteids in many general points, but exhibit among themselves much greater differences than do the proteids. As regards their molecular structure nothing satisfactory is known. Their percentage composition approaches that of the proteids, and like these they yield, under hydrolytic treatment, large quantities of leucin and in some cases tyrosin. They are all amorphous.

\section{Mucin. $(\mathrm{O}, 35.75 . \quad \mathrm{H}, 6.81 \text {. N, 8.50. C, 48.94. })^{1}$}

The characteristic component of mucus. Its exact composition is not yet known, the figures given above being merely an approximation.

As occurring in the normal condition it gives to the fluids which contain it the well-known ropy consistency, and can be precipitated from these by acetic acid, alcohol, alum, and mineral acids; the latter, if in excess, redissolve the precipitate, but this is not the ease with acetic acid. In its precipitated form it is insoluble in water, but swells up strongly in it, and this effect is increased by the presence of many alkali salts. Alkalies and alkaline earths dissolve it readily. Its solutions do not dialyse; they give the proteid reactions with Millon's reagent and nitric acid, but not that with sulphate of copper, and are precipitated by basic lead acetate only when neutral or faintly alkaline. According to Eichwald, ${ }^{2}$ 
when heated with dilute nineral acids, mucin yields acid-albumin, and another body which in many of its properties closely resembles a sugar, inasmuch as it reduces solutions of cupric sulphate. Prolonged boiling with sulphuric acid gives leucin and about 7 per cent. of tyrosin.

Preparation. ${ }^{1}$ Ox-gall or an aqueous extract of finely chopped submaxillary gland is acidulated with acetic acid; the precipitated mucin is then washed with water, dissolved in dilute sodic carbonate and finally precipitated with acetic acid. It may also be obtained from snails. ${ }^{2}$

\section{Chondrin. (O, 31.04. H, 6.76. N, 13.87. C, 47.74. S, 0.60 per cent. $)^{3}$}

This is usually regarded as forming the essential part of the matrix of hyaline cartilage, and is contained in the interstices of the fibres in elastic cartilage. A similar substance can be prepared from the cornea. Boiled with water, it dissolves slowly, forming an opalescent solution, which is precipitated by acetic acid, lead acetate, dilute mineral acids, alum, and salts of silver and copper; an excess of the last four reagents redissolves the precipitate. Solutions of this body gelatinize on standing, even if very dilute; the solid mass is insoluble in cold water, readily soluble in hot water, alkalies, and ammonia.

The aqueous and alkaline solutions of chondrin possess a left-handed rotatory power on polarized light of $-213.5^{\circ}$; in presence of excess of alkali this becomes $-552.0^{\circ}$, both measured for yellow light. ${ }^{4}$

It seems, according to the observations of many, that chondrin can, by heating with hydrochloric acid, be converted into a body whose reactions resemble those of syntonin, and another substance, which like the similar product from mucin, so far resembles grape-sugar that it reduces cupric salts in alkaline solution $;^{5}$ it appears, however, to contain nitrogen. The existence of chondrin as a distinct substance has, however, been denied ${ }^{6}$ on the supposition that it is in all cases a mere mixture of other bodies. It is stated that a substance having all the reactions of the socalled chondrin, may at any time be produced by a mixture of mucin, glutin, and inorganic salts. The extreme similarity in the reactions of chondrin and mucin point to a close relationship between the two. The whole subject, however, requires more complete investigation. With alkalies or dilute sulphuric acid chondrin gives leucin, but no tyrosin or glycin. Whether chondrin exists as such in cartilage is uncertain; it seems probable that it does not, since its extraction from eartilage requires an amount of boiling with water much greater than that requisite to dissolve dried chondrin.

Preparation. From cartilage by extracting with water and precipitating with acetic acid.

[Chondrin is not probably a distinct substance, but a mixture of mucin and gelatin.]

\section{Gelatin or Glutin. ${ }^{7}(\mathrm{O}, 23.21 . \quad \mathrm{H}, 7.15 . \quad \mathrm{N}, 18.32 . \quad \mathrm{C}, 50.76 . \quad \mathrm{S}, 0.56$ per cent.)}

This is the substance which is yielded when connective-tissue fibres are heated for several days with very dilute acetic acid, at a temperature of about $15^{\circ} \mathrm{C}$., or by the prolonged action of water in a Papin's digester. The elastic elements of connective tissue are unaffected by the above treatment.

As obtained in this way glutin is when heated a thin fluid, solidifying on cooling to the well-known gelatinous form. When dried it is a colorless, transparent, brittle body, swelling up, but remaining undissolved in cold water; heating, or the addition of traces of acids or alkalies, readily effects its solution. When dissolved in water it possesses a læro-rotatory power of $-130^{\circ}$, at $30^{\circ} \mathrm{C}$.; the addition of strong alkali or acetic acid reduces this to $-112^{\circ}$ or $-114^{\circ}$, both measured for yellow light. ${ }^{\circ}$ Its solutions will not dialyse.

Mercuric chloride and tannic acid are the only two reagents which yield insoluble

1 Eichwald, op. cit. and Chem. Centralb., 1866, No. 14. Staedeler, Ann. d. Chem. u. Pharm., Bd. cxi. S. 14. Landwehr, Zeitschr. f, physiol. Chem., Bd. v. (1881), S. 371.

2 Landwehr, Zeitschr. f. phy siol. Chem., Bd. vi. (1882), S. 75.

3 I. v. Mering, Beitrag zur Chemie des Knorpels, 1873.

4 Hoppe-Seyler, Hab. phys. path. chem. Anal., Aufl. 1875, S. 262.

5 De Bary, Hoppe-Seyler's Untersuch., Hft. 1, S. 71.

6 Morochowetz, Verhand. Naturhist.-med. Ver., Heidelberg, Bd. i. (1876), Hft. 5.

7 Not to be confounded with the vegetable proteid "gluten."

8 Hoppe-Seyler, Hdb. d. phys. path. chem. Anal., 4 Aufl. 1875, S. 222. 
precipitates with this body. Its presence prevents the action of Trommer's sugartest, since it readily dissolves the precipitated cuprous oxide. The proteid reactions of glutin are so feeble that they are probably due merely to impurities. Heated with sulphuric acid it yields ammonia, leucin, and glycin, but no tyrosin.

[When gelatin is digested it undergoes alterations similar to those of fibrin and albumin, forming gelatoses and gelatin-peptones, but the results of recent investigation indicate that while gelatin is valuable as a food-stuff for furnishing energy, it is not of value for the growth of the nitrogenous tissues.

It appears improbable that glutin exists ready-formed in connective-tissue fibres, since these do not swell up in water, and only yield glutin after prolonged treatment with boiling water; to which it may be added that while glutin is acted upon by trypsin, the connective-tissue fibres in their natural condition resist its action (see p. 284). When glutin is submitted for some time to the action of dilute hydrochloric acid at $38^{\circ} \mathrm{C}$, and the change is brought about even more readily by the action of pepsin, it loses its power of gelatinizing and is now diffusible through porous nembranes: the name of gelatin-peptone has been given to the product thus obtained. ${ }^{1}$

\section{Elastin. (O, 20.5. H, 7.4. N, 16.7. C, 55.5 per cent. $)$}

This characteristic component of elastic fibres is left on the removal of all the glutin, mucin, etc., from such tissues as "ligamentum nuchæ," advantage being taken of its not being altered when it is heated with water, even under pressure, with strong acetic acid, or with dilute alkalies. When moist it is yellow and elastic, but on drying becomes brittle. It is soluble in strong alkalies at boiling temperatures, and concentrated sulphuric and nitric acids dissolve it even in the cold; it is also dissolved by the action of papaya juice. It is precipitated from solutions by tannic acid, but not by the addition of ordinary acids. Notwithstanding that it closely approaches the proteids in its percentage composition, and gives distinct although feeble proteid reactions, any very close relationship between the two appears improbable, since elastin when treated with sulphuric acid, yields leucin $(30-40$ per cent. ) only and no tyrosin.

Hilger ${ }^{2}$ has obtained a similar body from the shell membrane of snakes' eggs.

Keratin. $^{3}(\mathrm{O}, 20.7-25.0 . \quad \mathrm{H}, 6.4-7.0 . \quad \mathrm{N}, 16.2-17.7 . \quad \mathrm{C}, 50.3-52.5 . \quad \mathrm{S}$, $0.7-5.0$ per cent.)

This body, though somewhat resembling the proteids in general composition, differs from them and also from the preceding bodies so widely in other properties, that its description is placed here for convenience rather than anything else. Hair, nails, feathers, horn, and epidermic scales consist for the most part of keratin. Heated with water in a digester at $150^{\circ} \mathrm{C}$. keratin is partially dissolved with evolution of sulphuretted hydrogen; the solution then gives with acetic acid and ferrocyanide of potassium a precipitate soluble in excess of the acid. Prolonged boiling with alkalies and acids, even acetic, dissolves keratin; the alkaline solutions evolve sulphuretted hydrogen on treatment with acids. 'The sulphur. in keratin is evidently very loosely united to the substance, and in all its reactions there appears to be a want of similarity between keratin and either proteids, mucin or gelatin. The most common of its products of decomposition are leucin (10 per cent.) and tyrosin (3.6 per cent.), and some aspartic acid; no glycin is formed. What is generally known as keratin is probably a compound body, which has not yet been resolved into its components.

Ewald and Kähne 4 have described a new body to which, since it occurs as a constituent of nervous tissue (both of nerves and of the central nervous system), and is yet closely identical with ordinary horny tissue. they give the name of neuro-keratin. It is prepared in quantity from the brain by extracting this tissue with alcohol and ether, and subjecting the residue to the action of pepsin and trypsin. The final residue is neuro-keratin, and amounts to 15 to 20 per cent. of the original tissue.

Nuclein. $\mathrm{C}_{29} \mathrm{H}_{49} \mathrm{~N}_{9} \mathrm{P}_{3} \mathrm{O}_{22}$.

Discovered by Miescher ${ }^{5}$ in the nuclei of pus corpuscles and in the yellow corpuscles of yolk of egg. Other observers have subsequently obtained it from yeast,

1 Hofmeister, Zeitschr. f. physiol. Chem., Bd. il. (1878), S. 299.

2 Ber. d. Deutsch. Chem. Gesellsch., 1873, S. 166. But see also next reference.

s Lindwall, "Nagra bidrag till kann. om. Ker., Upsalä Läkarefs. förh. xvi. (1881), p. 546.

4 Verhand. Naturhist.-med. Ver., Heidelberg, Bd. i. (1876), Heft 5.

5 Med.-chem. Untersuch., Hoppe-Seyler, Heft 4, 1872, S, 441 u. 502. 
from semen, from the nuclei of the red blood-corpuscles of birds and amphibia, from hepatic cells, and it is probably present in all nuclei.

When newly prepared it is a colorless amorphous body, soluble to a slight extent in water, readily soluble in many alkaline solutions; but its solubilities alter on keeping. If added gradually in sufficient quantity to a solution of caustic alkali it first neutralizes the solution and then renders it acid. It seems to possess an indistinct xantho-proteic reaction, but gives no reaction with Millon's fluid. It yields precipitates with several salts, e.g., zinc chloride, argentic nitrate, and cupric sulphate.

Preparation. ${ }^{1}$ Since nuclein is very resistant to the action of pepsin, it may be obtained from the granular residue consisting chiefly of nuclei, which occurs after digesting pus with pepsin. The most remarkable feature of this body is its large percentage of phosphorus, 9.59 per cent. This phosphorus is readily separated by boiling with strong hydrochloric acid or caustic alkalies; the same occurs when solutions of nuclein are acidulated and allowed to stand.

\section{Chitin. $\mathrm{C}_{15} \mathrm{H}_{26} \mathrm{~N}_{2} \mathrm{O}_{10}^{\prime}{ }^{2}$}

Although not found as a constituent of any mammalian tissue, this substance composes the chief part of the exo-skeleton of many invertebrates. It may probably be regarded as the animal analogue of the cellulose of plants, and from this point of view it possesses considerable morphological interest. Both cellulose and chitin appear to yield some form of sugar when treated with strong acids.

When purified, chitin is a white amorphous body, often retaining the shape of the tissue from which it has been prepared. It is insoluble in all reagents except strong mineral acids, the best solvents being sulphuric or hydrochloric acids. The immediate addition of water to these solutions reprecipitates the chitin in an unaltered form; but the prolonged action of sulphuric acid causes a decomposition resulting, according to some observers, in the formation of an amorphous fermentable carbohydrate; and when hydrochloric acid is used an amidated carbohydrate is obtained to which the name of glycosamin ${ }^{3}\left(\mathrm{C}_{6} \mathrm{H}_{13} \mathrm{NO}_{5}\right)$ has been given.

Preparation. ${ }^{4}$ The cleansed exo-skeleton of a lobster is thoroughly extracted with dilute hydrochloric acid and then with caustic soda. To purify it finally it is submitted to prolonged boiling with a solution of potassic permanganate.

\section{[Nucleo-albumin.}

This term is applied to a class of substances which seem to be composed of nuclein and an albuminous proteid. Casein is probably a nucleo-albumin, for when subjected to peptic digestion a residue of nuclein is obtained.]

\section{CARBOHYDRATES.}

Certain members only of this class occur in the human body; of these the most important and widespread are those known as glycogen and the two sugars, grapesugar or dextrose (glucose), with which diabetic sugar seems to be identical, ${ }^{5}$ and maltose. Next to these comes milk-sugar. Inosit is another body of this class, although it differs in many important points from the preceding two.

Sugars are often considered to be polyatomic alcohol. Several of them stand in peculiar relation to mannit, and may be converted into that substance by the action of sodium amalgam. ${ }^{6}$

[When solutions of sugars are warmed in the presence of phenyl-hydrazin and dilute acetic acid, amorphous or crystalline substances separate, which are termed osazones. 'These differ according to the different sugars.]

\section{Dextrose (grape-sugar). $\quad \mathrm{C}_{6} \mathrm{H}_{12} \mathrm{O}_{6}+\mathrm{H}_{2} \mathrm{O}$.}

Occurs in the contents of the alimentary canal to a variable extent dependent on the nature of the food taken. It is also a normal constituent of blood, chyle, and lymph. Concerning its presence in the liver, see p. 538. The amniotic fluid

1 See Kossel, Zeitschr. f. physiol. Chem., Bd. iii. (1879), S. 284 ; iv. (1880), S. 290 ; vii. (1883), S. 7. "Untersuch. über d. Nuclein u. ihre Spaltungsprod, Strassb., 1881.

2 Ledderhose, Zeitschr. f. physiol. Chem., Bd. ii. (18-8), S. 213.

a Jedderhose, loc. cit., Bd. iv. (1880), S. 139.

4 Bütschli, Arch. f. Anat. u. Physiol., Jahrg., 1874, S. 362.

5 The question, however, whether several varieties of sugar occurring in the animal body have not been confounded together under the common name of dextrose or glucose may be considered at present an open one.

6 Linnemann, Ann. d. Chem. u. Pharm., Bd. cxxiii., S. 136. 
also contains this body. Bile in the normal condition is free from sugar, so also is urine, though this point has given rise to great dispute. ${ }^{1}$ The disease diabetes is characterized by an excess of dextrose in the fluids and tissues of the body (see p. 448).

When pure, dextrose is colorless and crystallizes from its aqueous solution in six-sided tables or prisms, often agglomerated into warty lumps. The crystals will dissolve in their own weight of cold water, requiring, however, some time for the process; they are very readily soluble in hot water. Dextrose is somewhat sparingly soluble in alcohol, and crystallizes from anhydrous alcohol in prisms frce from water of erystallization; it is, moreover, insoluble in ether.

The freshly prepared cold aqueous solution of the crystals possesses a dextro-rotatory power of $+104^{\circ}$ for yellow light. This, quickly on heating, more slowly on standing, falls to $+56^{\circ}$, at which point it remains constant.

Dextrose readily forms compounds with acids and many salts; the latter are very unstable, decomposition rapidly ensuing on heating them. When its metallic compounds are decomposed, the decomposition is, in many cases, accompanied by the precipitation of the metals, e.g., silver, gold, mercury, bismuth. Caustic alkalies readily decompose them, as also does ammonia.

Dextrose is readily and completely precipitated by lead acetate and ammonia.

An important property of this body is its power of undergoing fermentations. Of these the two principal are: 1. Alcoholic. This is produced in aqueous solutions of dextrose, under the influence of yeast. The decomposition is the following: $\mathrm{C}_{6} \mathrm{H}_{12} \mathrm{O}_{6}=2 \mathrm{C}_{2} \mathrm{H}_{6} \mathrm{O}+2 \mathrm{CO}_{2}$, yielding (ethyl) alcohol and carbonic anhydride. Other alcohols of the acetic series are found in traces, as also are glycerin, succinic acid, and probably many other bodies. The fermentation is most active at about $25^{\circ} \mathrm{C}$. Below $5^{\circ} \mathrm{C}$. or above $45^{\circ} \mathrm{C}$. it almost entirely ceases. If the saccharine solution contains more than 15 per cent. sugar it will not all be decomposed, as excess of alcohol stops the reaction. 2. Lactic. This occurs in the presence of decomposing nitrogenous matter, especially of casein, and is probably the result of the action of a specific ferment. ${ }^{2}$ The first stage is the production of lactic acid, $\mathrm{C}_{6} \mathrm{H}_{12} \mathrm{O}_{6}=2 \mathrm{C}_{3} \mathrm{H}_{6} \mathrm{O}_{3}$. In the second butyric acid is formed with evolution of hydrogen and carbonic anhydride: $2 \mathrm{C}_{3} \mathrm{H}_{6} \mathrm{O}_{3}=\mathrm{C}_{4} \mathrm{H}_{8} \mathrm{O}_{2}+2 \mathrm{CO}_{2}+4 \mathrm{H}$. The above changes, the first of which is probably undergone by sugar to a considerable extent in the intestine, are most active at $35^{\circ} \mathrm{C}$.; the presence of alkaline carbonates is also favorable. It is, moreover, essential that the lactic acid should be neutralized as fast as it is formed, otherwise the presence of the free acid stops the process.

The preparation, detection, and estimation of dextrose are so fully given in various books that they need not be detailed here.

\section{Maltose. $\mathrm{C}_{12} \mathrm{H}_{22} \mathrm{O}_{11}+\mathrm{H}_{2} \mathrm{O}$.}

This form of sugar was first described by Dubrunfaut ${ }^{3}$ as a product of the action of malt extract on starch. Its existence was for a long time doubted until O'Sullivan ${ }^{4}$ repeated and confirmed the previous experiments. According to him it crystallizes in fine acicular erystals, possesses a specific rotatory power of $+150^{\circ}$ and a reducing power which is only one-third as great as that of dextrose. It seems probable that this is the chief sugar obtained by the action not only of diastase but of ptyalin and pancreatic ferment upon starch and perhaps also upon glycogen; ${ }^{5}$ although some dextrose may at the same time be formed. Musculus and Gruber ${ }^{6}$ have shown that maltose may also be formed by the action of dilute sulphuric acid on starch, and that it is capable of undergoing alcoholic fermentation.

Preparation. See Musculus and Gruber (loc. cit.).

\section{Milk-sugar. $\mathrm{C}_{12} \mathrm{H}_{22} \mathrm{O}_{11}+\mathrm{H}_{2} \mathrm{O}$.}

Also known as lactose. It is found in milk, and is characteristic of this secretion. It is said, however, to occur abnormally in the urine of lying-in women. ${ }^{7}$

It yields, when pure, hard, colorless crystals, belonging to the rhombic system (four-sided prisms). It is less soluble in water than dextrose, requiring for solu-

1 See Seegen, Der Diabetes Mellitus, 2 ed., S. 196.

2 Lister, Path. Soc. Trans., vol. for 1878, p. 425, also Quart. Journ. of Micros. Sci., vol xviii. (1878), p. 177 .

3 Ann. Chim. Phys. (3) xxi. (1847), p. 178.

4 Journ. Chem. Soc., Ser. 2, vol. x. (1872), p. 579

5 Musculus u. v. Mering, Zeitschr. f. physiol. Chem., Bd. ii. (1878), S. 403.

- Zeitschr. f. physiol. Chem., Bd. ii. (1878), S. 177. ${ }_{7}$ Hofmeister, Ibid., Bd. 1. (1877), S. 101. 
tion six times its weight of cold, but only two parts of boiling, water; it is entirely insoluble. in alcohol and ether. It is fully precipitated from its solutions by the addition of lead acetate and ammonia.

When treshly dissolved, its aqueous solution possesses a specific dextro-rotatory power of $+93.1^{\circ}$ for sodium light; this diminishes slowly on standing, rapidly on boiling, until it finally remains constant at $+52.5^{\circ}$. The amount of rotation is independent of the encentration of the solution.

Lactose unites readily with bases, forming unstable compounds; from its metallic compounds the metal is precipitated in the reduced state on boiling; it reduces copper salts as readily as dextrose, but to a less extent, viz., in the ratio of $70: 100$.

Lactose is generally stated to admit of no direct alcoholic fermentation; this may, however, sometimes be induced by the prolonged action of yeast. By boiling with dilute mineral acids lactose is converted into galactose, which readily undergoes alcholic fermentation and possesses a greater rotatory power than lactose.

It may be remarked here that though isnlated lactose is incapable of direct alcoholic fermentation,milk itself may be fermented. Berthelot was umble in this direct alcoholic fermentation to detect any intermediate change of the lactose into any other fermentable sugar.

Lactose is, however, directly capable of undergoing the lactic and butyric fermentation; the circumstances and products are the same as in the case of dextrose (see above). The action is generally productive of a collateral small quantity of alcohol.

Lactose is thus distinguished from dextrose by its smaller solubility in water, insolubility in alcohol, crystalline form, lower cupric oxide reducing power, and its incapability of undergoing direct alcoholic fermentation.

Preparation. After the removal of the casein and other proteids of the milk, the mother-liquor is evaporated to the crystallizing point; the crystals are purified by repeated crystallization from warm water.

4. Inosit. $\mathrm{C}_{6} \mathrm{H}_{12} \mathrm{O}_{6}+2 \mathrm{H}_{2} \mathrm{O}$.

This substance occurs but sparingly in the human body; it was found originally by Scherer ${ }^{1}$ in the muscles of the heart. Cloetta showed its presence in the lungs, kidneys, spleen, and liver, ${ }^{2}$ and Miuller in the brain. ${ }^{3}$ It oceurs, also, in diabetic urine and in that of "Bright's disease," and is found in abundance in the vegetable kingdom.

Pure inosit forms large efflorescent crystals (rhombic tables); in microscopic preparations it is usually obtained in tufted lumps of fine crystals. Easily soluble in water, it is insoluble in alcohol and ether. It possesses no action on polarized light, and does not reduce solutions of metallic salts.

It admits of no direct alcoholic, but is capable of undergoing the lactic, fermentation; according to Hilger, ${ }^{4}$ the acid formed is sarcolactic. It is unaltered by heating with dilute mineral acids.

Preparation. It may be precipitated from its solutions by the action of basic lead acetate and ammonia; the lead is then removed by sulphuretted hydrogen, and the inosit precipitated with excess of alcohol.

As a special test (Scherer's) may be mentioned the production of a bright violet color by careful evaporation to dryness on platinum foil, with a little ammonia and calcium chloride.

\section{Dextrin. $\mathrm{C}_{6} \mathrm{H}_{10} \mathrm{O}_{5}$.}

By boiling starch-paste with dilute acids, or by the action of ferments, the starch is converted into an isomeric body, to which, from its action on polarized light, the name dextrin has been given. It is soluble in water, but is precipitated by alcohol. It does not undergo alcoholic fermentation until after it has been changed into dextrose, nor can it reduce metallic salts. It yields a reddish port-wine color with iodine, which disappears on warming and does not return on cooling. Further action of acids or of ferments converts dextrin into dextrose. Dextrin is present in the contents of the alimentary canal after a meal containing starch, and has also been found in the blood.

There is not the least doubt that several modifications of dextrin exist, and may be obtained by the action of acids and ferments on starch. Of these two of the 
best known are those described by Brücke ${ }^{1}$ under the name of erythrodextrin and achroödextrin, the former giving a red color with iodine, the latter not yielding any color at all. Erythrodextrin may be readily converted into a sugar by the action of ferments, and thus is not found as a product of the complete action of ptyalin on starch. Achroödextrin, on the other hand, is not thus converted by ferments, and therefore remains in solution, together with the sugar formed by the action of ptyalin on starch. Achroödextrin may be converted into dextrose by boiling with dilute hydrochloric acid.

\section{Glycogen. $\mathrm{C}_{6} \mathrm{H}_{10} \mathrm{O}_{5}$.}

Belongs to the starch division of carbohydrates. Discovered by Bernard in the liver and other organs (see p. 437).

Glycogen is, when pure, an amorphous powder, colorless and tasteless, readily soluble in water, insoluble in alcohol and ether. Its aqueous solution is generally, though not always, strongly opalescent, but contains no particles visible microscopically; the opalescence is much reduced by the presence of free alkalies. The same solution possesses, according to Hoppe-Seyler, a very strong dextro-rotatory power, about three times as great as that of dextrose ${ }^{2}$ it dissolves hydrated cupric oxide; but this is not reduced on boiling.

By the action of dilute mineral acids (except nitric) it is partially converted into a form of sugar very closely resembling, though probably differing somewhat from, true dextrose, and the same conversion is also readily effected by the action of amylolytic ferments. The sugar into which the glycogen of the liver is naturally converted after death (see p. 438) appears to be true dextrose $;^{3}$ so also the sugar of diabetes. The result of the action of diastase, or salivary or pancreatic ferment, upon glycogen is, however, according to Musculus and v. Mering, ${ }^{4}$ a mixture of achroödextrin and maltose; the quantity of dextrose making its appearance at the same time being very small.

Opalescent solutions of glycogen usually become clear on the addition of caustic alkali; Vintschgau and Dietl ${ }^{5}$ have shown that this is accompanied on boiling by a change which converts a portion of the glycogen into a substance to which they gave the name of $\beta$-glycogen-dextrin. (Kühne ${ }^{6}$ had previously described a body to which he gave the name glycogen-dextrin. That described by Vintschgau and Dietl differs slightly from Kuihne's body, hence the name. According to these authors one-fifth of the glycogen is at the same time changed into some other, at present undetermined, substance. Normal lead acetate gives a cloudiness, the basic salt a precipitate, in solutions of glycogen.

As tests for this body may be used the formation of a port-wine color with iodine; this disappears on warming, but returns on cooling. The same color is produced by the action of iodine on dextrin, but this does not reappear on cooling after its disappearance by warming.

Preparation of glycogen. The following is Brïcke's ${ }^{7}$ method: The filtered or simply strained decoction of perfectly fresh liver or other glycogenic tissue is, when cold, treated alternately with dilute hydrochloric acid and a solution of the double iodide of potassium and mercury ${ }^{8}$ as long as any precipitate occurs. In the presence of free hydrochloric acid the double iodide precipitates proteid matters so completely as to render their separation by filtration easy. The proteids being thus got rid of, the glycogen is precipitated from the filtrate by adding alcohol to the extent of between 60 and 70 per cent. Too much alcohol is to be avoided, since other substances as well as glycogen are thereby precipitated. The glycogen is now washed with alcohol, first of 60 and then of 95 per cent., afterward with ether, and finally with absolute alcohol. It is then dried over sulphuric acid.

1 Sitzber. d. Wien. Akad., 1872, iii. Abth. Also, Vorlesungen, 2 Aufl., 1875, Bd. i., S. 224.

2 See Kulz, Pflüger's Arch., Bd. xxjv. (1881), S. 85.

3 Pflüger's Arch., Bd. xix. (1879), S. 106, and xxii. (1880), S. 206. Also, Kulz, Ibid., Bd. xxiv.

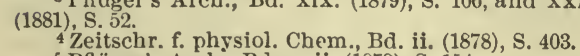

4 Zeitschr. f. physiol. Chem., Bd. ii. (187
5 Pflüger's Arch., Bd. xvii. (1878), S. 154.

6 Lehrb. d. physiol. Chem. (1868). S. 63.

7 Sitzungsber. d. Wiener Akad., Bd. lxiii. (1871), ii. Abth.

8 This may be prepared by precipitating potassic iodide with mercuric chloride and dissolving the washed precipitate in a hot solution of potassic iodide as long as it continues to be taken up. On cooling, some amount of precipitate occurs, which must be filtered off; the filtrate is then ready for use. 


\section{[7. Animal Gum.}

This carbohydrate is found in milk and urine, and is prepared by the action of superheated water on the salivary glands.]

\section{Tunicin. $\left(\mathrm{C}_{6} \mathrm{H}_{10} \mathrm{O}_{5}\right)_{n}$.}

This body is regarded by many observers as identical with the true cellulose of plants, while others have ascribed to it properties differing from those of cellulose sufficiently to justify its receiving.a distinct name. It appears to be more resistant to the action of chemical reagents than plant cellulose.

It constitutes the chief part of the integument of the ascidia or tunicata. As prepared from this source it is, when pure, quite white, and usually retains the shape of the tissue. It is unacted upon by any reagent except strong acids and alkalies, and by the action of the former it yields some form of sugar.

\section{FATS, THEIR DERIVATIVES AND ALLIES.}

\section{The Aceitic Acid Series.}

General formula, $\mathrm{C}_{n} \mathrm{H}_{2 n} \mathrm{O}_{2}$ (monobasic).

This, which is one of the most complete homologous series of organic chemistry, runs parallel to the series of monatomic alcohols. Thus formic acid corresponds to methyl alcohol, acetic acid to ethyl (ordinary) alcohol, and so on. The several acids may be regarded as being derived from their respective alcohols by simple oxidation; thus ethyl alcohol yields by oxidation acetic acid $\mathrm{C}_{2} \mathrm{H}_{6} \mathrm{O}+\mathrm{O}_{2}=\mathrm{C}_{2} \mathrm{H}_{4} \mathrm{O}_{2}+$ $\mathrm{H}_{2} \mathrm{O}$. The various members differ in composition by $\mathrm{CH}_{2}$, and the boiling-points rise successively by about $19^{\circ} \mathrm{C}$. Similar relations hold good with regard to their melting-points and specific gravities. The acid properties are strongest in those where $n$ has the least value. The lowest members of the series are volatile liquids, acting as powerful acids; these successively become less and less fluid, and the highest members are colorless solids, closely resembling the neutral fats in outward appearance. Consecutive acids of the scries present but very small differences of chemical and physical properties, hence the difficulty of separating them; this is further increased in the animal body by the fact that exactly those acids which present the greatest similarities usually occur together.

'The free acids are found only in small and very variable quantities in various parts of the body; their derivatives, on the other hand, form most important constituents of the human frame, and will be considered further on.

\section{Formic Acid. CHO.OH}

When pure is a strongly corrosive, fuming fluid, with powerful irritating odor, solidifying at $0^{\circ} \mathrm{C}$., boiling at $100^{\circ} \mathrm{C}$, and capable of being mixed in all proportions with water and alcohol. It has been obtained from various parts of the body, such as the spleen, thymus, pancreas, muscles. brain, and blood; in the latter its presence may be due to the action of acids on the hæmoglobin. According to some authors, ${ }^{1}$ it occurs only in urine.

Heated with sulphuric acid it yields carbonic oxide and water; with caustic potash it gives hydrogen and oxalic acid.

\section{Acetic Acid. $\mathrm{C}_{2} \mathrm{H}_{3} \mathrm{O} . \mathrm{OH}$.}

Is distinguished by its characteristic odor; its boiling-point is $117^{\circ} \mathrm{C}$.; it solidifies at $5^{\circ}$, and is fluid at all temperatures above $15^{\circ} \mathrm{C}$. It is soluble in all proportions in alcohol and water.

It occurs in the stomach as the result of fermentative changes in the food, and is frequently present in diabetic urine. In other organs and fluids it exists only in minute traces.

With ferric chloride it yields a blood-red solution, decolorized by bydrochloric acid. (It differs in this last reaction from sulphocyanide of iron.) Heated with alcohol and sulphuric acid, the characteristic odor of acetic ether is obtained. It does not reduce silver nitrate.

1 Buliginsky, Hoppe-Seyler's Med. chem. Mittheilung, Heft 2, S. 240. Thudichum, Journ. Chem. Soc., vol. viii. p. 400. 


\section{Propionic Acid. $\mathrm{C}_{3} \mathrm{H}_{5} \mathrm{O} . \mathrm{OH}$.}

This acid closely resembles the preceding one. It possesses a very sour taste and pungent odor; it is soluble in water, boils at $141^{\circ} \mathrm{C}$., and may be separated from its aqueous solution by excess of calcic chloride.

It occurs in small quantities in sweat, in the contents of the stomach, and in diabetic urine when undergoing fermentation. It is similarly produced, mixed, however, with other products, during alcoholic fermentation, or by the decomposition of glycerin. It partially reduces silver nitrate solution on boiling.

\section{Butyric Acid. $\mathrm{C}_{4} \mathrm{H}_{7} \mathrm{O} . \mathrm{OH}$.}

An oily, colorless liquid, with an odor of rancid butter, soluble in water, alcohol, and ether, boiling at $162^{\circ} \mathrm{C}$. Calcic chloride separates it from its aqueous solution.

Found in sweat, the contents of the large intestine, feces, and in urine. It occurs in traces in many other fluids, and is plentifully obtained when diabetic urine is mixed with powdered chalk and kept at a temperature of $35^{\circ} \mathrm{C}$. It exists as a neutral fat in small quantitios in milk.

\section{Valerianic Acid. $\mathrm{C}_{5} \mathrm{H}_{9} \mathrm{O} . \mathrm{OH}$.}

An oily liquid, of penetrating odor and burning taste; soluble in 30 parts of water at $12^{\circ} \mathrm{C}$.; readily soluble in alcohol and ether. Boils at $175^{\circ} \mathrm{C}$.; possesses, in fiee and combined form, a feeble right-handed rotation of the plane of polarization.

It is found in the solid excrements, and is formed readily by the decomposition, through putrefaction, of impure leucin, ammonia being at the same time evolved; hence its occurrence in urine when that fluid contains leucin, as in cases of acute atrophy of the liver.

Caproic Acid.

Caprylic Acid.

Capric (Rutic) Acid. $\mathrm{C}_{10} \mathrm{H}_{19} \mathrm{O} .0 \mathrm{H}$.

These three occur together (as fats) in butter and are contained in varying proportions in the feces from a meat diet. The first is an oily fluid, slightly soluble in water; the others are solids and scarcely soluble in water; they are soluble in all proportions in alcohol and ether. They may be prepared from butter, and separated by the varying solubilities of their barium salts.

Laurostearic Acid. Myristic Acid.

$$
\begin{aligned}
& \mathrm{C}_{12} \mathrm{H}_{23} \mathrm{O} \text {.OH. } \\
& \mathrm{C}_{14} \mathrm{H}_{27} \text { O.OH. }
\end{aligned}
$$

These occur as neutral fat in spermaceti, in butter, and other fats. They present no points of interest.

Palmitic Acid. $\mathrm{C}_{16} \mathrm{H}_{31} \mathrm{O} . \mathrm{OH}$.

Stearic Acid. $\mathrm{C}_{18} \mathrm{H}_{35} \mathrm{O} . \mathrm{OH}$.

These are solid, colorless when pure, tasteless, odorless crystalline bodies, the former melting at $62^{\circ} \mathrm{C}$, the latter at $69.2^{\circ} \mathrm{C}$. In water they are quite insoluble ; palmitic acid is more readily soluble in cold alcohol than stearic; both are readily dissolved by hot alcohol, ether, or chloroform. Glacial acetic acid dissolves them in large quantity, the solution being assisted by warming. - They readily form soaps with the alkalies, also with many other metals. The varying solubilities of their barium salts afford the means of separating them when mixed $;^{1}$ this may also be applied to many others of the higher members of this series.

These acids in combination with glycerin (see below), together with the analogous compound of oleic acid, form the principal constituents of human fat. As salts of calcium they occur in the feces and in "adipocere," and probably in chyle, blood, and serous fluids, as salts of sodium. They are found in the free state in decomposing pus, and in caseous deposits of tuberculosis.

The existence of margaric acid intermediate to the above two is not now admitted, since Heintz ${ }^{2}$ has shown that it is really a mixture of palmitic and stearic acids. Margaric acid possesses the anomalous melting-point of $59.9^{\circ} \mathrm{C}$ A mixture of 60 parts stearic and 40 of palmitic
acid melts at $60.3^{\circ}$ 


\section{Acids of the Oleic (Acrylic) Series. $\mathrm{H}\left(\mathrm{C}_{n} \mathrm{H}_{2 n-3}\right) \mathrm{O}_{2}$ (monobasic).}

Many acids of this series occur as glycerin compounds in various fats. They are very unstable and readily absorb oxygen when exposed to the air. The higher members are decomposed on attempting to distil them. 'Their most peculiar property is that of being converted by traces of $\mathrm{NO}_{2}$ into solid, stable metameric acids capable of being distilled. They bear an interesting relation to the acids of the acetic series, breaking up when heated with caustic potash into acetic acid and some other member of the same series, thus:

$$
\begin{gathered}
\text { Oleic acid. Potassic acetate. Potassic palmitate. } \\
\mathrm{HC}_{18} \mathrm{H}_{33} \mathrm{O}_{2}+2 \mathrm{KHO}=\mathrm{KC}_{2} \mathrm{H}_{3} \mathrm{O}_{2}+\mathrm{KC}_{16} \mathrm{H}_{31} \mathrm{O}_{2}+\mathrm{H}_{2} .
\end{gathered}
$$

\section{Oleic Acid. $\mathrm{C}_{18} \mathrm{H}_{33} \mathrm{O} . \mathrm{OH}$.}

This is the only acid of the series which is physiologically important. It is found united with glycerin in all the fats of the human body.

When pure it is, at ordinary temperatures, a colorless, odorless, tasteless, oily liquid, solidifying at $4^{\circ} \mathrm{C}$. to a crystalline mass. Insoluble in water, it is soluble in alcohol and ether. It cannot be distilled without decomposition. It readily furms soaps with potassium and sodium, which are soluble in water; its compounds with most other bases are insoluble. It may be distinguished from the acids of the acetic series by its reaction with $\mathrm{NO}_{2}$ and by the changes it undergoes when exposed to the air.

\section{The Neutral Fats.}

These may be considered as ethers formed by replacing the exchangeable atoms of hydrogen in the triatomic alcohol glycerin (see below) by the acid radicles of the acetic and oleic series. Since there are three such exchangeable atoms of hydrogen in glycerin. it is possible to form three classes of these ethers; only those, however, which belong to the third class occur as natural constituents of the human body: those of the first and second are of theoretical importance only.

They possess certain general characteristics. Insoluble in water and cold alcohol, they are readily soluble in hot alcohol, ether, chloroform, etc.; they also dissolve one another. They are neutral bodies, colorless and tasteless when pure, are not capable of being distilled without undergoing decomposition. and yield as a result of this decomposition solid and liquid liydrocarbons, water, fatty acids, and a peculiar body, acrolein. (Glycerin contains the elements of one molecule of acrolein and two molecules of water.)

They possess no action on polarized light.

They may readily be decomposed into glycerin and their respective fatty acids by the action of caustic alkalies or of superheated steam.

$$
\text { Palmitin (Tri-palmitin). } \left.\quad\left(\mathrm{C}_{16} \mathrm{H}_{36} \mathrm{O}\right)_{3} \mathrm{H}_{5}\right) \mathrm{O}_{3} \text {. }
$$

The following reaction for the formation of this fat is typical for all the others:

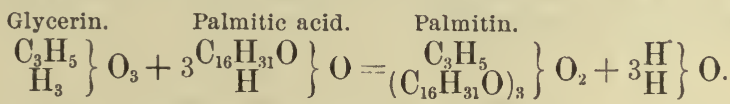

Palmitin is slightly soluble in cold alcohol, readily so in hot alcohol, or in ether; when pure it crystallizes in fine needles; if mixed with stearin, it generally forms shapeless lumps, although the mixture may at times assume a crystalline form, and was then regarded as a distinct body, namely, margarin. It possesses three different melting-points, according to the previous temperatures to which it has been subjected. It solidifies in all cases at $45^{\circ} \mathrm{C}$.

Preparation. From paln oil, by removing the free palmitic acid with alcohol and crystallizing repeatedly from ether.

$$
\text { Stearin (Tri-stearin). } \left.\quad \underset{\mathrm{C}_{3} \mathrm{H}_{5}}{\left(\mathrm{C}_{18} \mathrm{H}_{32} \mathrm{O}\right)_{3}}\right\} \mathrm{O}_{3} .
$$

This is the hardest and least fusible of the ordinary fats of the body, is also the least soluble, and hence is the first to crystallize out from solutions of the mixed 
fats. It crystallizes usually in square tables. It presents peculiarities in its fusingpoints similar to those of palmitin.

Preparation. From mutton suet, its separation from palmitin and olein being effected by repeated crystallization from ether, stearin being the least soluble.

$$
\text { Olein (Tri-olein). } \left.\quad \begin{array}{c}
\left(\mathrm{C}_{18} \mathrm{H}_{33} \mathrm{O}\right)_{3} \\
\mathrm{C}_{3} \mathrm{H}_{5}
\end{array}\right\} \mathrm{O}_{3} .
$$

It is obtained with difficulty in the pure state, and is then fluid at ordinary temperatures. It is more soluble than the two preceding ones. It readily undergoes oxidation when exposed to the air, and is converted by mere traces of $\mathrm{NO}_{2}$ into a solid isomeric fat. Olein yields, on dry distillation, a characteristic acid, the sebacic, and is saponified with much greater difficulty than are palmitin and stearin.

Preparation. From olive oil, either by cooling to $0^{\circ} \mathrm{C}$. and pressing out the olein that remains fluid, or by disśolving in alcohol and cooling, when the olein remains in solution while the other fats crystallize out.

Glycerin. $\left.\begin{array}{c}\mathrm{C}_{3} \mathrm{H}_{5} \\ \mathrm{H}_{3}\end{array}\right\} \mathrm{O}_{3}$.

This principal constituent of the neutral fats may, as above stated, be looked upon as a triatomic alcohol.

When pure, glycerin is a viscid, colorless liquid, of a well-known sweet taste. It is soluble in water and alcohol in all proportions, insoluble in ether. Exposed to very low temperature it becomes almost solid; it may be distilled in close vessels without decomposition, between $275^{\circ}-280^{\circ} \mathrm{C}$.

It dissolves the alkalies and alkaline earths, also many oxides, such as those of lead and copper; many of the fatty acids are also soluble in glycerin.

It possesses no rotatory power on polarized light.

It is easily recognized by its ready solubility in water and alcohol, its insolubility in ether, its sweet taste, and its reaction with bases. The production of acrolein is also characteristic of glycerin.

$$
\mathrm{C}_{3} \mathrm{H}_{8} \mathrm{O}_{3}-2 \mathrm{H}_{2} \mathrm{O}=\mathrm{C}_{3} \mathrm{H}_{4} \mathrm{O} \text { (Acrolein). }
$$

Preparation. By saponification of the various oils and fats. It is also formed in small quantities during the alcoholic fermentation of sugar. ${ }^{1}$

Soaps. These may be formed by the action of caustic alkalies on fats. The process consists in a substitution of the alkali for the radicle of glycerin, the latter combining with the elements of water to form glycerin. Thus:

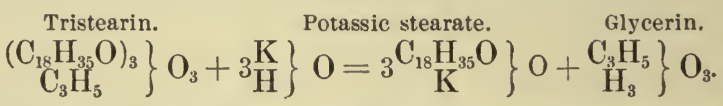

Pancreatic juice can split up fats into glycerin and free fatty acids, and the bile is known to be capable of saponifying these fatty acids. The amount of soaps formed in the alimentary canal is, however, small and unimportant.

\section{Acids of the Glycolic Series.}

Running parallel to the monatomic alcohols $\left(\mathrm{C}_{n} \mathrm{H}_{2 n}+{ }_{2} \mathrm{O}\right)$ is the series of diatomic alcohols or glycols $\left(\mathrm{C}_{n} \mathrm{H}_{n}+{ }_{2} \mathrm{O}_{2}\right)$. Thus corresponding to ethyl alcohol is the diatomic alcohol, ethyl-glycol. As from the monatomic alcohols, so from the glycols, acids may be derived by oxidation; from the latter (glycols), however, two series of acids can be obtained. known respectively as the glyeolic and oxalic series. The first stage of oxidation of the glycol gives a meniber of the glycolic series. Thus:

$$
\begin{aligned}
& \text { Ethyl-glycol. Glycolic acid. } \\
& \mathrm{C}_{2} \mathrm{H}_{6} \mathrm{O}_{2}+\mathrm{O}_{2}=\mathrm{C}_{2} \mathrm{H}_{4} \mathrm{O}_{3}+\mathrm{H}_{2} \mathrm{O} \text {, or more generally } \\
& \mathrm{C}_{n} \mathrm{H}_{2 n+2} \mathrm{O}_{2}+\mathrm{O}_{2}=\mathrm{C}_{n} \mathrm{H}_{2 n} \mathrm{O}_{3}+\mathrm{H}_{2} \mathrm{O} \text {. }
\end{aligned}
$$

1 Pasteur, Ann. d. Chem. u. Pharm., Bd. cvi., S. 338. 
By further oxidation a member of the glycolic series can be converted into a member of the oxalic series. Thus:

$$
\begin{aligned}
& \text { Oxalycolic acid. } \\
& \begin{array}{c}
\mathrm{C}_{2} \mathrm{H}_{4} \mathrm{O}_{3}+\mathrm{O}_{2}=\mathrm{C}_{2} \mathrm{H}_{2} \mathrm{O}_{4}+\mathrm{H}_{2} \mathrm{O} \text {, or more generally } \\
\mathrm{C}_{n} \mathrm{H}_{2 n} \mathrm{O}_{3}+\mathrm{O}_{2}=\mathrm{C}_{n} \mathrm{H}_{2 n-2} \mathrm{O}_{4}+\mathrm{H}_{2} \mathrm{O} \text {. }
\end{array}
\end{aligned}
$$

The acids of the glycolic series are diatomic but monobasic; but those of the oxalic series are diatomic and dibasic.

\begin{tabular}{|c|c|c|c|c|c|}
\hline Radicle. & Alcohol. & Acid. & Glycols. & Acid I. & Acid II. \\
\hline Methyl $\left(\mathrm{CH}_{3}\right)$ & $\mathrm{CH}_{3}(\mathrm{OH})$ & $\begin{array}{l}\text { Formic. } \\
\mathrm{HCHO}_{2}\end{array}$ & & $\begin{array}{l}\text { Carbonic. } \\
\mathrm{H}_{2} \mathrm{CO}_{3}\end{array}$ & \\
\hline Ethyl $\left(\mathrm{C}_{2} \mathrm{H}_{5}\right)$ & $\mathrm{C}_{2} \mathrm{H}_{5}(\mathrm{OH})$ & $\mathrm{HC}_{2} \mathrm{H}_{3} \mathrm{O}_{2}$ & $\mathrm{C}_{2} \mathrm{H}_{4}(\mathrm{OH})_{2}$ & $\mathrm{HC}_{2} \mathrm{H}_{3} \mathrm{O}_{3}$ & $\mathrm{H}_{2} \mathrm{C}_{4} \mathrm{O}_{4}$ \\
\hline Propyl $\left(\mathrm{C}_{3} \mathrm{H}_{7}\right)$ & $\mathrm{C}_{3} \mathrm{H}_{7}(\mathrm{OH})$ & $\mathrm{HC}_{3} \mathrm{H}_{5} \mathrm{O}_{2}$. & $\mathrm{C}_{3} \mathrm{H}_{6}(\mathrm{OH})_{2}$ & $\mathrm{HC}_{3} \mathrm{H}_{5} \mathrm{O}_{3}$ & $\mathrm{H}_{2} \mathrm{C}_{3} \mathrm{H}_{2} \mathrm{O}_{4}$ \\
\hline Butyl $\left(\mathrm{C}_{4} \mathrm{H}_{9}\right)$ & $\mathrm{C}_{t} \mathrm{H}_{9}(\mathrm{OH})$ & $\mathrm{HC}_{4} \mathrm{H}_{7} \mathrm{O}_{2}$ & $\mathrm{C}_{4} \mathrm{H}_{8}(\mathrm{OH})_{2}$ & $\mathrm{CH}_{5} \mathrm{H}_{7} \mathrm{O}_{3}$ & $\mathrm{H}_{2} \mathrm{C}_{4} \mathrm{H}_{4} \mathrm{O}_{4}$ \\
\hline
\end{tabular}

The following table may be given to show the general relationship of alcohols and acids :

\section{Glycolic Acid Series.}

\section{Lactic Acid. $\mathrm{C}_{3} \mathrm{H}_{6} \mathrm{O}_{3}$.}

Next to carbonic acid, the most important member of this series, as far as physiology is concerned, is lactic acid.

Lactic acid exists in four isomeric modifications, but of these only three have been found in the human body. 'These three all form syrupy, colorless fluids, soluble in all proportions in water, alcohol, and ether. They possess an intensely sour taste, and a strong acid reaction. When heated in solution they are partially distilled over in the escaping vapor. 'They form salts with metals, of which those with the alkalies are very soluble and crystallize with difficulty. The calcium and zinc salts are of the greatest importance, as will be seen later on.

1. Ethylidene-lactic acid. This is the ordinary form of the acid, obtained as the characteristic product of the well-known "lactic fermentation." It occurs in the contents of the stomach and intestines. According to Heintz. ${ }^{1}$ it is found also in muscles, and according to Gscheidlen ${ }^{2}$ in the ganglionic cells of the gray substance of the brain. In many diseases it is found in urine, and exists in a large amount in this excretion after poisoning by phosphorus. ${ }^{3}$

It may be prepared by the general methods of slowly oxidizing the corresponding glycol or by acting on monochlorinated propionic acid with moist silver oxide. In obtaining it from the products of lactic fermentation, the crusts of zinc lactate are purified by several crystallizations, and the acid liberated from the compounds by the action of sulphuretted hydrogen.

2. Ethylene-lactic acid. This acid is found accompanying the next to be described, in the watery extract of muscles. ${ }^{4}$ From this it is separated by taking advantage of the different solubilities in alcohol of the zine salts of the two acids. It seems probable, however, that it has not yet been prepared in the pure state by this method.

Wislicenus first obtained this acid by heating hydroxycyanide of ethylene with aqueous solutions of the alkalies.

The same observer found it also in many pathological fluids.

3. Sarcolactic acid. This acid has not yet been procured synthetically. As its name implies, it is that form of the acid which chielly occurs in muscles, and

\footnotetext{
1 Ann. d. Chem. u. Pharm., Bd. clvii., S. 320.

2 Pfiüger's Archiv. Bd. viii. (1873-74), S. 171

3 Schultzen and Riess, Ueber acute Phosphorvergiftung. Chem. Centralb., 1869, S. 681.

4 Ann. d. Chem. u. Pharm., Bd. cxxvili., S. 6.
} 
hence exists in large quantities in Liebig's "extract of meat." It is often found also in pathological fluids. This is the only acid of the series which possesses any power of rotating the plane of polarized light; it is otherwise indistinguishable from the preceding ethylidene-lactic acid, and is generally represented by the same formula. The free acid has dextro-, the anhydride lævo-rotatory action. The specific rotation for the zine salt in solution is $-7.65^{\circ}$ for yellow light.

[FIG. 231.

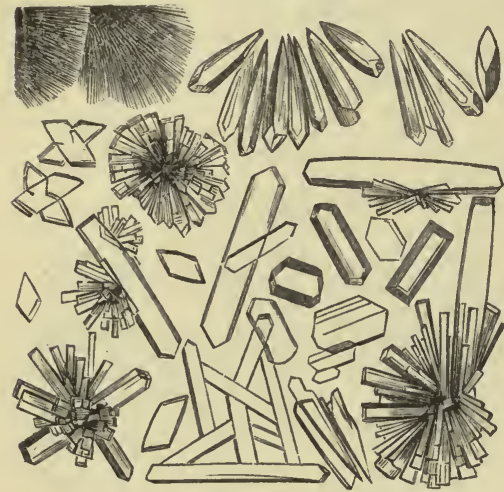

Zinc Sarcolactate. (After Kühne.)]
[FIG. 232.

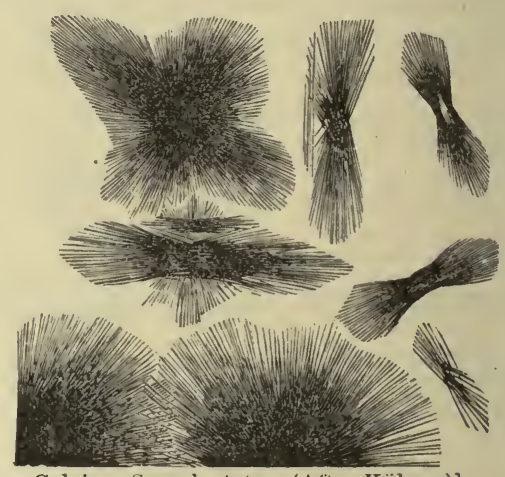

Calcium Sarcolactate. (After Kühne.)]

The zine and calcium salts [Figs. 231, 232] for sarcolactic acid are more soluble, both in water and alcohol, than those of ethylidene-lactic acid, but less so than those of ethylene lactic acid, and the same salts of ethylene-lactic acid contain more water of crystallization than those of the other two.

Heintz ${ }^{1}$ has compared the above acids to the modifications capable of existing in tartaric acid. 2

Hydracrylic acid, the fourth in this series of lactic acids, is distinguished by the nature of its decomposition on heating. It is never found as a constituent of animal bodies.

\section{Oxalic Acid Series.}

Oxalic Acid. $\mathrm{H}_{2} \mathrm{C}_{2} \mathrm{O}_{4}$.

In the free state this acid does not occur in the human body. Calcic oxalate, however, is a not unfrequent constituent of urine, and enters into the composition

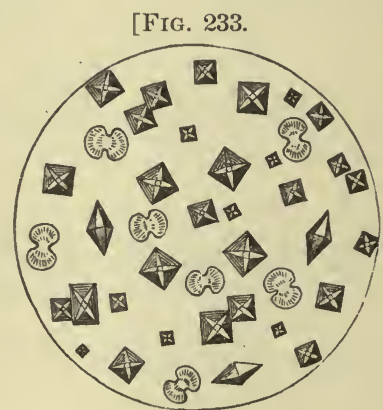

Calcium Oxalate.]

of many urinary calculi, the so-called mulberry calculus consisting almost entirely of it. It may occur in feces, and in the gall-bladder, though this is rarely observed.

1 Op. cit.

2 See, further, Wislicenus, op. cit. Also Ann. d. Chem. u. Pharm., Bd. clxvi., S. 3; Bd. clxvii., S. 302 , and Zeitschr. f. Chem., Bd. xiii., S. 159. 
As ordinarily precipitated from solutions of ealcic salts by ammonic oxalate, calcic oxalate is quite amorphous, but in urinary deposits it assumes a strong characteristic erystalline form, viz., that of rectangular octohedra [Fig. 233]. In some cases it presents the anomalous forms of rounded lumps, dumb-bells, or square columins with pyramidal ends. It is insoluble in water, alcohol, and ether, also in ammonia and a.cetic acid. Mineral acids dissolve this salt readily, as also to a smaller extent do solutions of sodic phosphate or urate. All the above characteristics serve to detect this salt; its microscopical appearance, however, is generally of most use for this purpose.

The pure acid is prepared either by oxidizing sugar with nitric acid, or decomposing ligneous tissue with caustic alkalies.

\section{Succinic Acid. $\mathrm{H}_{2} \mathrm{C}_{4} \mathrm{H}_{4} \mathrm{O}_{4}$.}

This is the third acid of the oxalic series, being separated from oxalic acid by the intermediate malonic acid, $\mathrm{H}_{2} \mathrm{C}_{3} \mathrm{H}_{2} \mathrm{O}_{4}$. It occurs in the spleen, the thymus, and thyroid bodies, hydrocephalic and hydrocele fluids.

According to Meissner and Shepard ${ }^{1}$ it is found as a normal constituent of urine. This is contested by Salkowski, ${ }^{2}$ and also by v. Speyer. It seems probable, however, that since wines and fermented liquors contain succinic acid, and this latter passes unchanged into the urine, that it may thus be occasionally present in this excretion.

Succinic acid crystallizes in large rhombic tables, also at times in the form of large prisms; they are soluble in 5 parts of cold water, and 2.2 of boiling, slightly soluble in alcohol, and almost insoluble in ether. The crystals melt at $180^{\circ} \mathrm{C}$., and boil at $236^{\circ} \mathrm{C}$., being at the same time decomposed into the anhydride and water. The alkali salts of this acid are soluble in water, insoluble in alcohol and ether.

Preparation. Apart from the synthetic methods, it may readily be obtained by the fermentation of calcic malate, acetic acid being produced sinultaneously.

Its presence is recognized by the microscopic examination of its crystals, and its characteristic reaction with normal lead acetate. With this it gives a precipitate, easily soluble in excess of the precipitant, but coming, down again on warming and shaking. ${ }^{3}$

\section{Cholesterin. $\quad\left(\mathrm{C}_{26} \mathrm{H}_{44} \mathrm{O}\right.$. $)$}

This is the only alcohol which occurs in the human body in the free state. (The triatomic alcohol glycerin is almost always found combined as in the fats; and cetyl-alcohol, or æthal, is obtained only from spermaceti.) It is a white crystalline body, crystallizing in fine needles from its solution in ether, chloroform, or benzol; from its hot alcoholic solutions it is deposited on cooling in rhombic tables [Fig. 234]. When dried it melts at $145^{\circ} \mathrm{C}$., and distils in closed vessels at $360^{\circ} \mathrm{C}$. It is quite insoluble in water and cold alcohol : soluble in solutions of bile salts.

Solutions of cholesterin possess a left-handed rotatory action on polarized light, of $-32^{\circ}$ for yellow light, this being independent of concentration and of the nature of the solvent.

Heated with strong sulphuric acid it yields a hydrocarbon; with concentrated nitric it gives cholesteric acid and other products. It is capable of uniting with acids and forming compound ethers.

Cholesterin occurs in small quantities in the blood and many tissues, and is present in abundance in the white matter of the cerebro-spinal axis and in nerves.

It is a constant constituent of bile, forming frequently nearly the whole mass of some gall-stones. It is found in many pathological fluids, hydrocele, the fluid of ovarian cysts, etc.

Preparation. From gall-stones by simple extraction with boiling alcohol, and treatment with alcoholic potash to free from extraneous matter.

As tests for this substance may be given: With concentrated sulphuric acid and a little iodine a violet color is obtained, changing through green to red or blue. This is applicable to the microscopic crystals. After dissolving in chloroform a blood-red solution is formed on the addition of an equal volume of concentrated

1 Untersuch. über d. Entsteh. d. Hippursaủre. Hanover, 1866.

2 Pflüger's Archiv, Bd. ii. (1869), S. 367, and Bd. iv. (1871), S. 95.

For further particulars see Meissner, op. cit., and Meissner and Solly, Zeitschr f. rat. (Med. 3)

B. xxiv., S. 97 . 
sulphuric acid; this solution if exposed to the air in an open dish turns blue, green, and finally yellow; the sulphuric acid under the chloroform has a green

[FIG. 234.

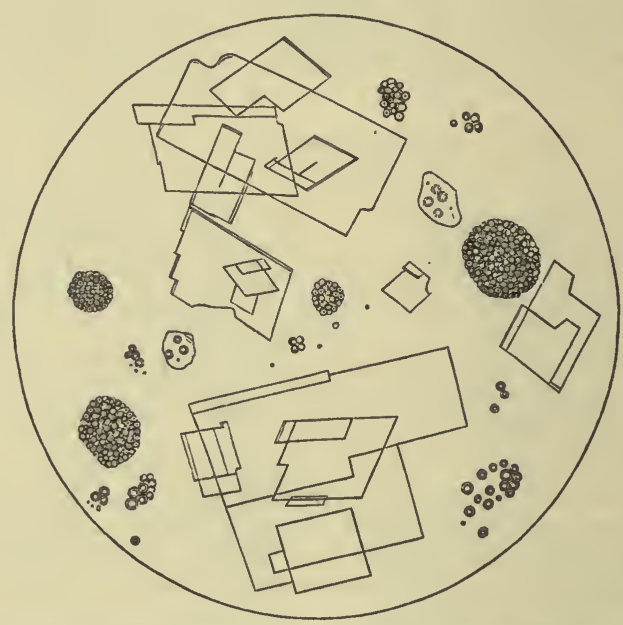

Cholesterin Crystals and Fatty Aggregations and Molecules Spontaneously Deposited in the Urine.]

fluorescence. After evaporation to dryness with nitric acid, the residue turns red on treating with ammonia.

This body is described here rather for the sake of convenience than from its possessing any close relationship to the substances immediately preceding.

\section{Complex Nitrogenous Fats.}

\section{Lecithin. $\mathrm{C}_{44} \mathrm{H}_{90} \mathrm{NPO}_{9}$.}

Occurs widely spread throughout the body. Blood, bile, and serous fluid contain it in small quantities, while it is a conspicuous component of the brain, nerves, yolk of egg, semen, pus, white blood-corpuscles, and the electrical organs of the ray.

When pure it is a colorless, slightly crystalline substance, which can be kneaded, but often crumbles during the process. It is readily soluble in cold, exceedingly so in hot alcohol; ether dissolves it freely though in less quantities, as also do chloroform, fats, benzol, carbon disulphide, etc. It is often obtained from its alcoholic solution by evaporation, in the form of oily drops. It swells up in water and in this state yields a flocculent precipitate with sodium chloride.

Lecithin is easily decomposed; not only does this decomposition set in at $70^{\circ} \mathrm{C}$. but the solutions, if merely allowed to stand at the ordinary temperature, acquire an acid reaction, and the substance is decomposed. Acids and alkalies, of course, effect this much more rapidly. If heated with baryta water it is completely decomposed, the products being neurin, glycerin-phosphoric acid, and baric stearate. This may be thus represented:

$$
\begin{aligned}
& \text { Lecithin. Stearic acid. Glycerin-phosphoric acid. Neurin. } \\
& \mathrm{C}_{44} \mathrm{H}_{90} \mathrm{NPO}_{9}+3 \mathrm{H}_{2} \mathrm{O}=2 \mathrm{C}_{18} \mathrm{H}_{36} \mathrm{O}_{2}+\mathrm{C}_{3} \mathrm{H}_{9} \mathrm{PO}_{6}+\mathrm{C}_{5} \mathrm{H}_{15} \mathrm{NO}_{2} \text {. }
\end{aligned}
$$

When treated in an ethereal solution with dilute sulphuric acid, it is merely split up into neurin and distearyl-glycerin-phosphoric acid. Hence, Diakonow ${ }^{1}$ regards lecithin as the distearyl-glycerin-phosphate of neurin, two atoms of hydrogen in the

1 Hoppe-Seyler's Med.-Chem. Untersuch., Heft ii. (1867), S. 221 ; Heft iii. (1868), S. 405. Centralbl. f. d. med. Wiss. (1868), Nr. 1, 7, u. 28 . 
glycerin-phosphoric acid being replaced by the radicle of stearic acid. It appears also that there probably exist other analogous compounds in which the radicles of oleic and palmitic acids take part.

Preparation. Usually from the yolk of egg, where it occurs in union with vitellin. Its isolation is complicated, and the reader is referred to Hoppe-Seyler. ${ }^{1}$

\section{Glycerin-phosphoric Acid. $\mathrm{C}_{3} \mathrm{H}_{9} \mathrm{PO}_{6}$.}

Occurs as a product of the decomposition of lecithin. and hence is found in those tissues and fluids in which this latter is present; in leukæmia the urine is said to contain this substance. It has not been obtained in the solid form. It has been produced synthetically by heating glycerin and glacial phosphoric acid; it may be regarded as formed by the union of one molecule of glycerin with one of phosphoric acid, with elimination of one molecule of water. It is a dibasic acid; its salts with barium and calcium are insoluble in alcohol, soluble in cold water. Solutions of its salts are precipitated by lead acetate.

\section{Protagon. $\left(\mathrm{C}_{160} \mathrm{H}_{308} \mathrm{~N}_{5} \mathrm{PO}_{35}\right.$ ?)}

A crystalline body containing nitrogen and phosphorus, obtained by Liebreich ${ }^{2}$ from the brain substance and regarded by him as its principal constituent. The researches of Hoppe-Seyler and Diakonow tended to show that protagon was merely a mixture of lecithin and cerebrin. A repetition of Liebreich's experiments has, however, led Gamgee and Blankenhorn ${ }^{3}$ to confirm the truth of his results. Protagon appears to separate out from warm alcohol on gradual cooling in the form of very small needles, often arranged in groups; it is slightly soluble in cold, more soluble in hot alcohol, and ether. It is insoluble in water, but swells up and forms a gelatinous mass. It melts at $200^{\circ} \mathrm{C}$., and forms a brown syrupy fluid.

Preparation. Finely divided brain substance, freed from blood and connective tissue, is digested at $45^{\circ} \mathrm{C}$. with alcohol ( 85 per cent.) as long as the alcohol extracts anything fiom it. The protagon which separates out from the filtrate is well washed with ether to get rid of all cholesterin and other bodies soluble in ether, and finally purified by repeated erystallization from warm alcohol.

\section{Nourin (Cholin). $\quad \mathrm{C}_{5} \mathrm{H}_{15} \mathrm{NO}_{2}$.}

Discovered by Strecker ${ }^{4}$ in pig's gall, then in ox-gall. It does not occur in the free state except as a product of the decomposition of lecithin. It is a colorless fluid, of oily consistence, possesses a strong alkaline reaction, and forms with acids very deliquescent salts. The salts with hydrochloric acid and the chlorides of platinum and gold are the most important.

Neurin is a most unstable body, mere heating of its aqueous solution sufficing to split it up into glycol, trimethylamin and ethylene oxide.

Preparation. From yoke of egg. For this see Diakonow. ${ }^{5}$

Wurtz ${ }^{6}$ has obtained it synthetically, first by the action of glycol hydrochloride on trimethylamin, and then by that of ethylene oxide and water on the same substance. The above, together with the mode of its decomposition, point to the idea that neurin may be regarded as trimethyloxyethyl-ammonium hydrate, $\mathrm{N}\left(\mathrm{CH}_{3}\right)_{3}\left(\mathrm{C}_{2} \mathrm{H}_{5} \mathrm{O}\right) \mathrm{OH}$.

\section{Cerebrin. $\mathrm{C}_{17} \mathrm{H}_{33} \mathrm{NO}_{3}$ (?).}

Is found in the axis-cylinder of nerves, in pus-corpuseles, and largely in the brain. In former times many names were given to the substance when in an impure state, e. g., cerebric acid, cerebrote, etc. W. Müller ${ }^{7}$ first prepared it in the pure form, and constructed the above formula from his analysis; the mean of these is $\mathrm{O}, 15.85 ; \mathrm{H}, 11.2 ; \mathrm{N}, 4.5 ; \mathrm{C}, 68.45$. Great doubts are, however, thrown upon its purity by the researches of later observers. According to Liebreich ${ }^{8}$ and Diakonow, ${ }^{9}$ it is a glucoside. ${ }^{10}$

\footnotetext{
1 Med.-Chem. Untersuch., Heft ii. (1867), S. 215.

2 Ann. d. Chem. u. Pharm., Bd. cxxxiv., S. 29

3 Zeitschr. f. physiol. Chem., Bd. iii. (1879), S. 260, and Journ. of Physiol., vol. ii. (1879), p. 113.

4 Ann. d. Chem. u. Pharm., Bd. cxxiii., S. 353 ; Bd. cxlviii., S. 76.

5 Op. cit. (sub. Lecithin).

7 Aun. d. Chem. u. Pharm., Bd.cv., S. 361

8 Arch.f. pathoi. Anat., Bd. xxxix. (1867).

9 Centrallb. f. d. med. Wiss., 1868, No. 7.

10 See also Geogheghail, Zeitschr. f. physiol. Chem., Bd. iii. (1879), S. 332.
} 
Cerebrin is a light, colorless, exceedingly hygroscopic powder, which swells up strongly in water, slowly in the cold, rapidly on heating. When heated to $80^{\circ} \mathrm{C}$. it turns brown, and at a somewhat higher temperature melts, bubbles up, and finally burns away. It is insoluble in cold alcohol or ether; warm alcohol dissolves it easily. Heated with dilute mineral acids, cerebrin yields a sugar-like body, possessing left-handed rotation, but incapable of fermentation.

Preparation. For this see W. Miiller. ${ }^{1}$

[Charcot's Crystals. 'These crystals [Fig. 235], first discovered by Charcot, have been obtained from the semen, the blood of leukæmies, the expectoration of

[FIG. 235.

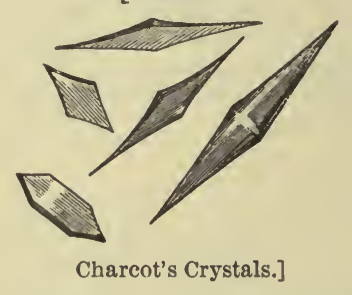

asthmatics, and the various tissues. It is not identical with ethylinimine or piperazine, as was supposed.]

\section{NITROGENOUS METABOLITES.}

\section{The Urea Group, Amides, and Smillar Bodies.}

\section{Urea. $\left(\mathrm{NH}_{2}\right)_{2} \mathrm{CO}$.}

The chief constituent of normal urine in mammalia and some other animals; the urine of birds also contains a small amount. Normal blood, serous fluids, lymph, and the liver all contain the same body in traces. It is not found in the muscles as a normal constituent, but may make its appearance there under certain pathological conditions.

[Fig. 236.

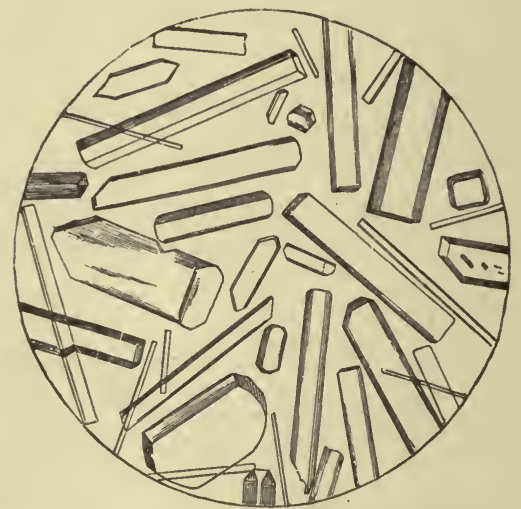

Urea Crystals separated by slow evaporation from Aqueous Solution. (After Funke.)]

When pure it crystallizes from a concentrated solution in the form of long, thin, glittering needles. [Fig. 236.] If deposited slowly from dilute solutions, the form is that of four-sided prisms with pyramidal ends; these are always anhydrous. It possesses a somewhat bitter cooling taste, like saltpetre. It is readily soluble in water and alcohol, the solutions being neutral. In anhydrous ether it is insoluble. 
The crystals may be heated to $120^{\circ} \mathrm{C}$. without being decomposed; at a higher temperature they are first liquefied and then decomposed, leaving no residue. Heated with strong acids or alkalies, decomposition ensues, the final products being carbonic anhydride and ammonia. The same decomposition may also occur as the result of the action of a specific ferment on urea in an aqueous solution. ${ }^{1}$ Nitrous acid at once decomposes it into carbonic anhydride and free nitrogen. It readily forms compounds with acids and bases; of these the following are of importance:

Nitrate of Urea. $\left(\mathrm{NH}_{2}\right)_{2}$ CO. $\mathrm{HNO}_{3}$.

Crystallizes in six-sided or rhombic tables [Fig. 237]. Insoluble in ether and nitric acid, soluble in water, slightly soluble in alcohol.

[FIG. 237.

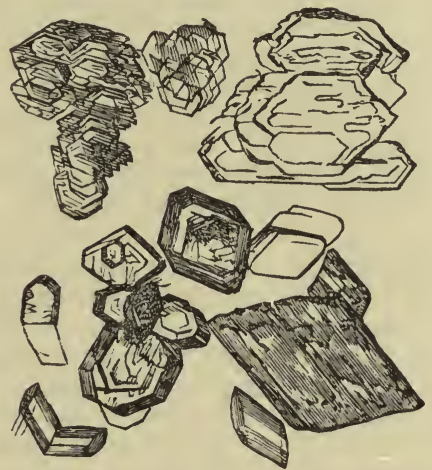

Crystals of Nitrate of Urea. (Krukenberg, after Kühne.)]

Oxalate of Urea. $\left[\left(\mathrm{NH}_{2}\right)_{2} \mathrm{CO}\right]_{2}, \mathrm{H}_{2} \mathrm{C}_{2} \mathrm{O}_{4}+\mathrm{H}_{2} \mathrm{O}$.

Often crystallizes in long thin prisms [Fig. 238], but under the microscope is obtained in a form closely resembling the nitrate; it is slightly soluble in water, less so in alcohol.

「FIG. 238.

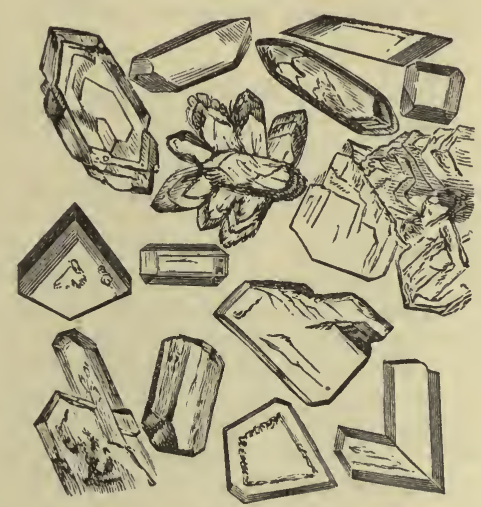

Crystals of Oxalate of Urea. (Krukenberg, after Kübne.)]

With mercuric nitrate urea yields three salts, containing respectively, four, three, and two equivalents of mercuric oxide to one of urea. The first is the pre-

1 Musculus, Pflüger's Archiv, Bd. xii. (1876), S. 214. Jaksch, Zeitsch. f. physiol. Chem., Bd. v. (1881), S. 395 . 
cipitate formed in Liebig's quantitative determination of urea, and may be represented by the formula: $2 \mathrm{~N}_{2} \mathrm{H}_{4} \mathrm{CO} \cdot \mathrm{Hg}\left(\mathrm{NO}_{3}\right)_{2} 3 \mathrm{HgO}$. The exact constitution of these salts has not yet been determined.

Preparation. Ammonic sulphate and potassic cyanate are mixed together in aqueous solution, and the mixture is evaporated to dryness. The residue, when extracted with absolute alcohol, yields urea. From urine, either by evaporating to dryness, having previously precipitated the urine with normal and basic lead acetate in succession and removed the lead by sulphuretted hydrogen, and then extracting with alcohol; or concentrating only to a syrup, and then forming the nitrate of urea; this is washed with pure nitrie acid and decomposed with baric compound.

Detection in solution. In addition to the microscopic appearance of the crystals obtained on evaporation, the nitrate and oxalate should be formed and examined. Another part should give precipitate with mercuric nitrate, in the absence of sodic chloride, but not in the presence of this last salt in excess. A third portion is treated with nitric acid containing nitrous fumes; if urea is present, nitrogen and carbonic anhydride will be obtained. To a fourth part nitric acid in excess and a little mercury are added, and the mixture is warmed. In presence of urea a colorless mixture of gases $\left(\mathrm{N}\right.$ and $\left.\mathrm{CO}_{2}\right)$ is given off. A fifth portion is kept melted for some time, dissolved in water, and cupric sulphate and caustic soda are added; a red or violet color, due to biuret, is developed.

Quantitative determination. For this some special manual must be consulted. ${ }^{1}$ It will suffice here to point out that the determination is made either with a solution of mercuric nitrate of known strength (Liebig); by decomposing the urea by means of sodic hypobromite into nitrogen, carbonic anhydride, and water, and measuring the nitrogen $(\mathrm{Knop})\left[\mathrm{N}_{2} \mathrm{H}_{4} \mathrm{CO}+3 \mathrm{NaBrO}=3 \mathrm{NaBr}+\mathrm{CO}_{2}+2 \mathrm{H}_{2} \mathrm{O}\right.$ $\left.+\mathrm{N}_{2}\right]$, or by heating the urea with caustic baryta in a sealed tube, the urea being determined by the weight of baric carbonate formed (Bunsen).

Urea is generally considered to be an amide of carbonic acid, $i$. $e_{\text {, }}$ carbamide. The amide of an acid is formed when water is removed from the ammonium salt of the acid; if the acid be dibasic and two molecules of water be removed, the result is often spoken of as a diamide. Thus if from ammonic carbonate $\left(\mathrm{NH}_{4}\right)_{2}$ $\mathrm{CO}_{3}$, two molecules of water, $2 \mathrm{H}_{2} \mathrm{O}$, be removed, carbonic acid being a dibasic acid, the result is urea; thus:

$$
\left(\mathrm{NH}_{4}\right)_{2} \mathrm{CO}_{3}-2 \mathrm{H}_{2} \mathrm{O}=\left(\mathrm{NH}_{2}\right)_{2} \mathrm{CO},
$$

which may be written either according to the ammonia type as

$$
\left.\begin{array}{l}
\mathrm{CO} \\
\mathrm{H}_{2}
\end{array}\right\} \mathrm{N}_{2} \quad \text { or as } \quad \mathrm{CO}\left\{\begin{array}{l}
\mathrm{NH}_{2} \\
\mathrm{NH}_{2}
\end{array}\right.
$$

two atoms of amidogen $\left(\mathrm{NH}_{2}\right)$ being substituted for two atoms of hydroxyl (HO).

This connection between carbonic acid and urea is shown by the fact that ammonic carbonate may be formed out of urea by hydration, as when urea is subjected to the specific ferment mentioned above. Regarded, then, as a diamide of carbonic acid, urea may be spoken of as carbamide. But the theoretical derivation of urea from ammonic carbonate by dehydration cannot be realized in practice, whereas urea can readily be formed from ammonic carbamate, and Kolbe is inclined to regard it, not as the diamide of carbonic acid, but as the amide of carbamic acid. Ammonium carbamate, $\mathrm{CO}_{2} \mathrm{~N}_{2} \mathrm{H}_{6}$ minus $\mathrm{H}_{2} \mathrm{O}$, gives urea, $\mathrm{CO}, \mathrm{N}_{2}, \mathrm{H}_{4}$-which, if earbamic acid be written as $\mathrm{CO}, \mathrm{OH}, \mathrm{NH}_{2}$, may be written as $\mathrm{CO}, \mathrm{NH}_{2}, \mathrm{NH}_{2}$, one atom of amidogen being substituted for one atom of hydroxyl, and not two, as when the substance is regarded as derived from carbonic acid. Drechsel's experiments indicate a ready derivation of urea from ammonic carbamate. He has obtained urea by the electrolysis of a solution of this salt with rapidly alternating currents, thus removing the elements of water

1 Neubauer and Vogel, Analyse des Harns, viii. Aufl., 1881, S. 264. 
from the carbamate by such alternating processes of oxidation and reduction as may be supposed to take place in the body. The reaction is expressed as follows :

$$
\begin{aligned}
& \text { 1. } \mathrm{NH} . \mathrm{CO} \mathrm{O} \cdot \mathrm{NH}_{4}+\mathrm{O}=\mathrm{NH}_{2} \cdot \mathrm{CO} \mathrm{O} \mathrm{NH}+\mathrm{NH}_{2} \mathrm{O} \text {. } \\
& \text { 2. } \mathrm{NH}_{2} \cdot \mathrm{CO} \cdot \mathrm{ONH}_{2}+\mathrm{H}_{2}=\mathrm{NH}_{2} \cdot \mathrm{CO} \cdot \mathrm{NH}_{2}+\mathrm{H}_{2} \mathrm{O} \text {. }
\end{aligned}
$$

Wanklyn ${ }^{1}$ and Gamgee, ${ }^{2}$ however, since urea, when heated with a large excess of potassic permanganate, gives off all its nitrogen in a free state, and not in the oxidized form of nitric acid, as do all other amides, conclude that it is not an anide at all, that it is isomeric only and not identical with carbamide.

It is important to remember that urea is also isomeric with ammonic cyanate, C $\left\{\begin{array}{l}\mathrm{N} \\ \mathrm{ONH}_{4}\end{array}\right.$, and, indeed, was first formed artificially by Wöhler (1823) from this body. We thus have three isomeric compounds, ammonium cyanate, urea, and carbamide, related to each other in such a way that urea may be obtained readily either from ammonium cyanate or from ammonic carbamate, and may with the greatest ease be converted into ammonic carbonate. ${ }^{3}$ Now urea is a much more stable body than ammonic cyanate, and in the transformation of the latter into the furmer energy is set free; and it is worthy of notice that, though the presence of sulphocyanides in the saliva probably indicates the presence of cyanic residues in the body, the nitrogenous products of the decomposition of proteids belong chiefly to the class of amides, cyanogen compounds being rare among them. Pfluiger ${ }^{4}$ has called attention to the great molecular energy of the cyanogen compounds, and has suggested that the functional metabolism of protoplasm by which energy is set free may be compared to the conversion of the energetic unstable cyanogen compounds into the less energetic and more stable amides. In other words, ammonium cyanate is a type of living, and urea of dead nitrogen, and the conversion of the former into the latter is an image of the essential change which takes place when a living proteid dies.

Compound ureas. The hydrogen atoms of urea can be replaced by alcohol and acid radicles. The results are compound ureas or ureides when the hydrogen is replaced by an acid radicle. Many of them are called acids, since the hydrogen from the amide group, if not all replaced as above, can be replaced by metal. Thus, the substitution of oxalyl (oxalic acid) gives parabanic acid,

$$
\mathrm{N}_{2}\left\{\begin{array}{l}
\mathrm{CO} \\
\mathrm{H}_{2} \text { or } \mathrm{CO}, \mathrm{NH}_{2}, \mathrm{~N} \cdot \mathrm{C}_{2} \mathrm{O}_{2} ; \\
\mathrm{C}_{2} \mathrm{O}_{2}
\end{array}\right.
$$

of tartronyl (tartronic acid), dialuric acid, $\mathrm{CO}, \mathrm{NH}_{2}, \mathrm{~N}_{2} \mathrm{C}_{3} \mathrm{H}_{2} \mathrm{O}_{3}$; of mesoxalyl (mesoxalic acid), alloxan, $\mathrm{CO}, \mathrm{NH}_{2}, \mathrm{~N}_{2} \mathrm{C}_{3} \mathrm{O}_{3}$. These bodies are interesting as being also obtained by the artificial oxidation of uric acid. (See below.)

Uric Acid. $\mathrm{C}_{5} \mathrm{H}_{4} \mathrm{~N}_{4} \mathrm{O}_{3}$.

The chief constituent of the urine in birds and reptiles; it occurs only sparingly in this excretion in man and most mammalia. It is normally present in the spleen, and traces of it have been found in the lungs, muscles of the heart, pancreas, brain, and liver. Urinary and renal calculi often consist largely of this body or its salts. In gout, accumulations of uric acid salts may occur in various parts of the body, forming the so-called gouty concretions.

It is when pure a colorless, crystalline powder, tasteless, and without odor. The crystalline form is very variable, but usually tends toward that of rhombic tables. ${ }^{5}$ When impure it crystallizes readily, but then possesses a yellowish or brownish color. In water it is very insoluble ( 1 in 14,000 or 15,000 of cold water); ether and alcohol do not dissolve it appreciably. On the other hand, sulphuric acid takes it up without decomposition, and it is also readily soluble in many salts of the alkalies, as in the alkalies themselves. Ammoria, however, scarcely dissolves it.

1 Arch. f. Physiol., 1880, S. 550

2 Journ. Chem. Soc., 2, vol. vi. p. 25

3 The following literature is interesting in connection with the question of the cyanic or amide origin of urea-Drechsel: Ber. d. k. s. Gesell. d. Wiss., Leipzig, Sitz. 25 Juli, 1875 ; Arch. f. Physiol. 1880, S. 505. v. Knieriem : Zt. f. Biol., Bd. x. (1874), S. 263. Munk: Zt. f. physiol. Chem., Bd. ii. (1878), S. 29. E. Salkowski : Centralbl. f. d. med. Wiss., 1875, No. 58; Ber. d. Deutsch. Chem. Gesell., 1875, S. 116. Zeitsch. f. physiol. Chem., Bd. i. (1877), Sn. 1 u. 374 ; Bd. iv. (1880), Sn. 54 u. 103. Schmiedeberg: Arch. f. exp. Pathol., Bd. viii. (1877), S. 1.

4 Pflïger's Archiv., Bd. X. (1875), S. 337.

6 See Ultzmann and K. B. Hoffmann, Atlas der Harnsedimente, Wien, 1872. 
Salts of uric acid. Of these the most important are the acid urates of sodium, potassium and ammonium. The sodium salt crystallizes in many different forns [Fig. 239], these not being characteristic, since they are almost the same for the corresponding compounds of the other two bases. It is very insoluble in cold water ( 1 in 1100 or 1200), more soluble in hot ( 1 in 125). It is the principal constituent of several forms of urinary sediment, and constitutes a large part of many calculi ; the excrement of snakes contains it largely. The potassium resembles the sodium salt very closely, as also does the compound with ammonium; the latter occurs generally in the sediment from alkaline urine. [Fig. 240.]

[FIG. 239.

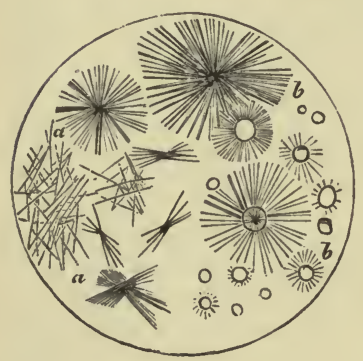

[FIG. 240.

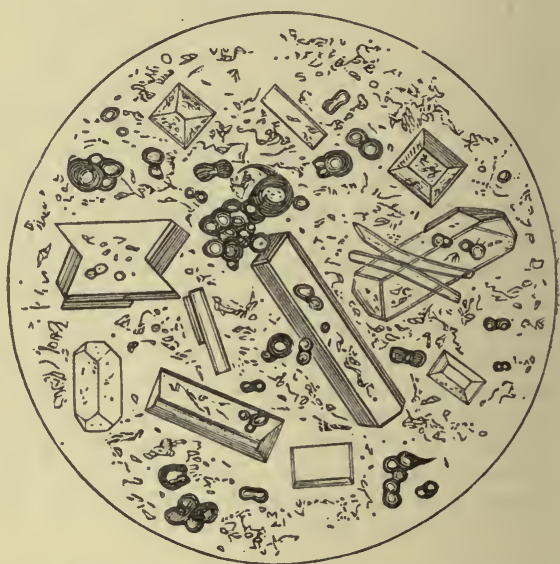

FIG. 239.-Urate of Soda. $a$, from a gouty concretion; $b b$, artificially prepared by adding liq. sodæ to the amorphous urate deposit.]

FIG. 240.-The Normal Deposit from Ammoniacal Urine, showing Crystals of AmmoniacoMagnesian Phosphate, Amorphous Phosphate of Lime, and Spheres of Urate of Ammonia.]

Preparation. . Usually from guano or snake's excrement. From guano by boiling with caustic potash (1 part alkali to 20 of water) as long as ammonia is evolved. In the filtrate a precipitate of acid urate of potassium is formed by passing a current of carbonic anhydride; this salt is then washed, dissolved in a caustic potash, and decomposed by carefully pouring its solution into an excess of hydrochloric acid.

The presence of uric acid is recognized by the following tests: The substance having been examined microscopically, a portion is evaporated carefully to dryness with one or two drops of nitric acid. The residue will, if uric acid is present, be of a red color, which on the addition of ammonia turns to purple. This is the murexide test, and depends on the presence of alloxan and alloxantin in the residue. Schiff $^{\prime}$ has given a delicate reaction for uric acid. The substance is dissolved in sodic carbonate and dropped on paper moistened with a silver salt. If uric acid be present a brown stain is formed. due to the reduction of the carbonate of silver. An alkaline solution of uric acid can, like dextrose, reduce cupric sulphate, with precipitation of the cuprous oxide.

Uric acid resists very largely the action of even strong acids and alkalies, exhibiting in this respect a marked difference from urea. It might therefore perhaps be supposed that urea residues do not pre-exist in uric acid; nevertheless by oxidation uric acid does give rise not only to ordinary urea, but also, and at the same time, to the compound ureas (ureides) spoken of above. Thus, by oxidation with acids,

$$
\begin{aligned}
& \text { Uric acid. } \\
& \mathrm{C}_{5} \mathrm{H}_{4} \mathrm{~N}_{4} \mathrm{O}_{3}
\end{aligned}+\mathrm{H}_{2} \mathrm{O}+\mathrm{O}=\mathrm{C}_{4} \mathrm{~N}_{2} \mathrm{H}_{2} \mathrm{O}_{4}+\mathrm{CN}_{2} \mathrm{H}_{4} \mathrm{O} \text {. }
$$

Now alloxan, as was stated above, is a compound urea, viz., mesoxalyl-urea, and by hydration can be converted into mesoxalic acid and urea, thus :

${ }^{1}$ Ann. d. Chem. u. Pharm., Bd. cix. S. 65. 


$$
\begin{aligned}
& \text { Alloxan. } \\
& \mathrm{C}_{4} \mathrm{~N}_{2} \mathrm{H}_{2} \mathrm{O}_{4}+2 \mathrm{H}_{2} \mathrm{O}=\mathrm{C}_{2} \mathrm{H}_{2} \mathrm{O}_{5}+\mathrm{CN}_{2} \mathrm{H}_{4} \mathrm{O} \text {; }
\end{aligned}
$$

and by the action of chlorine uric acid can be split up directly into a molecule of mesoxalic acid and two molecules of urea:

$$
\begin{aligned}
& \text { Uric acid. } \\
& \mathrm{C}_{5} \mathrm{H}_{4} \mathrm{~N}_{4} \mathrm{O}_{3}+\mathrm{Cl}_{2}+4 \mathrm{H}_{2} \mathrm{O}=\mathrm{C}_{3} \mathrm{H}_{2} \mathrm{O}_{5}+2 \mathrm{CN}_{2} \mathrm{H}_{4} \mathrm{OC}+2 \mathrm{HCl} \text {. }
\end{aligned}
$$
acid,

By oxidation with alkalies, uric acid is converted into allantoin and carbonic

$$
\begin{aligned}
& \text { Uric acid. } \\
& \mathrm{C}_{5} \mathrm{H}_{4} \mathrm{~N}_{4} \mathrm{O}_{3}+\mathrm{H}_{2} \mathrm{O}+\mathrm{O}=\mathrm{C}_{4} \mathrm{H}_{6} \mathrm{~N}_{4} \mathrm{O}_{3}+\mathrm{CO}_{2} \text {; }
\end{aligned}
$$

and allantoin, by hydration, becomes allanturic or lantanuric acid and urea,

$$
\begin{gathered}
\text { Ullantoin. } \\
\mathrm{C}_{4} \mathrm{H}_{6} \mathrm{~N}_{4} \mathrm{O}_{3}
\end{gathered}+\mathrm{H}_{2} \mathrm{O}=\mathrm{CH}_{4} \mathrm{~N}_{2} \mathrm{O}+\mathrm{C}_{3} \mathrm{H}_{4} \mathrm{~N}_{2} \mathrm{O} \text {. }
$$

Now allanturic acid is a compound urea, with a residue of glyoxylic acid. By other oxidations of uric acid, parabanic acid (oxalyl-urea), oxaluric acid (which is hydrated parabanic acid), and dialuric acid (tartronyl-urea) are obtained. In fact, all these decompositions of a molecule of uric acid lead to the production of urea and of a carbon acid of some kind or other. The relation of uric acid to urea, as illustrated by the above reactions, is brought very prominently into view by the synthesis of uric acid which has recently been performed.' It is obtained by simply fusing together glycocine (amido-acetic acid) and urea at a temperature of 200$230^{\circ} \mathrm{C}$. The converse formation of glycocine from uric acid with the simultaneous production of ammonia and carbonic anhydride has been known for some time. Since in this latter reaction the ammonia and carbonic anhydride are in the proportions in which they would be obtained from cyanic or cyanuric acid, uric acid has been regarded as built up from residues of cyanuric acid and glycin, just as hippuric acid is formed from glycin and benzoic acid. It was also at one time supposed that uric acid might be regarded as tartronyl cyanamide.

$$
\mathrm{N}_{2}\left\{\begin{array}{l}
\mathrm{C}_{3} \mathrm{H}_{2} \mathrm{O}_{3} \\
\left(\mathrm{CN}^{2}\right)_{2} \\
\mathrm{H}_{2}
\end{array}\right.
$$

If the existence of some cyanogen residue is thus assumed in the molecule of uric acid, then it must be supposed that before urea can be obtained from it a molecular change takes place by which a portion at least of the nitrogen of the uric acid is converted into the same condition as the rest of the nitrogen, viz., into the amide state.

If this be so, since the metabolism of the animals in which urie acid replaces urea cannot be supposed to be fundamentally different from that of the urea-producing animals, we may infer that the antecedent of both uric acid and urea in the regressive metabolism of proteids is, as we suggested above, a body containing some at least of its nitrogen in the form of cyanogen. ${ }^{2}$

\section{Kreatin. $\mathrm{C}_{4} \mathrm{H}_{9} \mathrm{~N}_{3} \mathrm{O}_{2}$.}

Occurs as a constant constituent of the juices of muscles, though possibly it may be formed during the process of extraction by the hydration of kreatinin. Kreatin is not a normal constituent of urine, but it is said to occur in traces in several fluids of the body. When found in urine its presence is probably due to the conversion of kreatinin, a constant constituent of urine, into kreatin during its extraction, since Dessaignes ${ }^{3}$ has shown that the more rapidly the separation is effected, the less is the quantity of kreatin obtained, and the greater the amount of kreatinin.

In the anhydrous form it is white and opaque, but crystallizes with one molecule of water in colorless, transparent rhombic prisms [Fig. 241, a]. It possesses a some-

1 Horbaczewski; Ber. d. Deutsch. Chem. Gesell., Jahrg. 1882, S. 2678.

2 See v. Knieriem, Zeitschr. f. Biol., Bd. xiii. (1877), S. 36. Schröder, Zeitschr. f. physiol. Chem., Bd. ii. (1878), S. 228 .

3 Jahrb. Pharm. (3), Bd. xxxii. S. 41. 
what bitter taste, is soluble in cold, extremely soluble in hot water, is less soluble in absolute than in dilute alcohol, and is soluble in ether.

It is a very weak base, scarcely neutralizing the weakest acids. It forms crystalline compounds with sulphuric, hydrochloric, and nitric acids.

[FIG. 241.

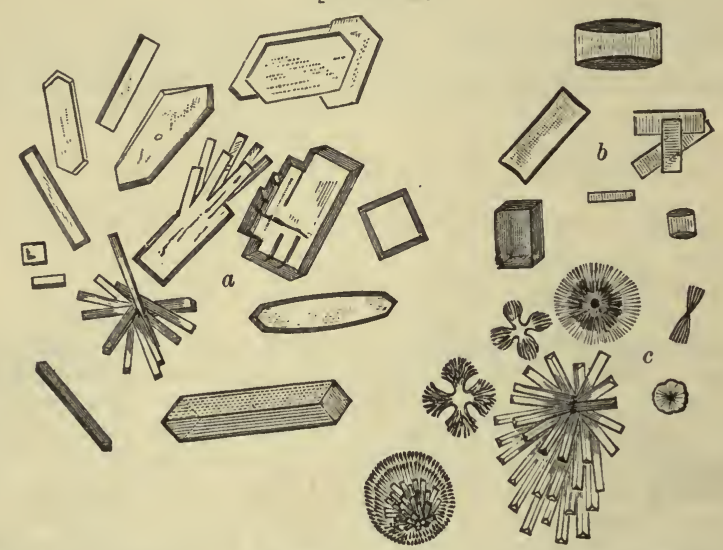

Crystals of Kreatin and Kreatinin. $a$, erystals of kreatin; $b$, crystals of kreatinin; $c$, crystals of chloride of zinc and kreatinin.]

Preparation. From extract of muscle by precipitating completely with basic lead acetate, and crystallizing out the kreatin, mixed with kreatinin. From this latter it is separated by the formation of the zinc-salt of kreatinin, kreatin not readily yielding a similar compound.

Kreatin may be converted into kreatinin under the influence of acids, the transformation being one of simple dehydration.

Kreatin may be decomposed into sarcosin (methyl-glycin) and urea :

$$
\mathrm{C}_{4} \mathrm{H}_{9} \mathrm{~N}_{3} \mathrm{O}_{2}+\mathrm{H}_{2} \mathrm{O}=\mathrm{C}_{3} \mathrm{H}_{7} \mathrm{NO}_{2}+\mathrm{CH}_{4} \mathrm{~N}_{2} \mathrm{O} \text {; }
$$

it may be formed synthetically ${ }^{1}$ by the action of sarcosin and cyanamide:

$$
\mathrm{C}_{3} \mathrm{H}_{7} \mathrm{NO}_{2}+\mathrm{CH}_{2} \mathrm{~N}_{2}=\mathrm{C}_{4} \mathrm{H}_{9} \mathrm{~N}_{3} \mathrm{O} \text {. }
$$

Sarcosin is glycin in which one atom of hydrogen has been replaced by the alcohol radicle methyl, thus:

$$
\text { Glycin } \left.\left.\underset{\mathrm{NH}_{2}}{\mathrm{C}_{2} \mathrm{H}_{3} \mathrm{O}}\right\} \text { O becomes } \underset{\mathrm{NH}_{2}}{\mathrm{C}_{2} \mathrm{H}_{2}\left(\mathrm{CH}_{3}\right) \mathrm{O}}\right\} \mathrm{O} \text {; }
$$

like glycin, sarcosin has not been found in free state in the body.

Kreatinin. $\mathrm{C}_{4} \mathrm{H}_{7} \mathrm{~N}_{3} \mathrm{O}$.

This, which is simply a dehydrated form of kreatin, occurs nornally as a constant constituent of urine and of muscle extract. It crystallizes in colorless shining prisms [Fig. 241, $b$ ], possessing a strong alkaline taste and reaction. It is readily soluble in cold water (1 in 11.5), also in alcohol, but is scarcely soluble in ether. It acts as a powerful base, forming with acids and salts compounds with crystallize well. Of these the most important is the salt with zine chloride $\left(\mathrm{C}_{4} \dot{\mathrm{H}}_{7} \mathrm{~N}_{3} \mathrm{O}\right)_{2}$ $\mathrm{ZnCl}_{2}$. It is formed when a concentrated solution of the chloride is added to a not too dilute solution of kreatinin. Since the compound is very little soluble in alcohol it is better to use alcoholic rather than aqueous solutions. It crystallizes in warty lumps composed of aggregated masses of prisms or fine needles. [Fig. 241, c.]

Preparation. Either by the action of acids on kreatin, or from human urine by concentrating and precipitating with lead acetate; in the filtrate from this a second

\footnotetext{
1 Sitzungsber. d. Bayerisch. Akad., 1868, Heft 3, S. 472.
} 
precipitate is caused by the addition of mercuric chloride, and consists of a compound of this salt with kreatinin. The mercury is removed by sulphuretted hydrogen, and the kreatin purified by the formation of the zinc salt and washing with alcohol.

Kreatinin-zinc chloride may be converted into kreatin, by the action of hydrated oxide of lead on its boiling aqueous solution.

Allantoin. $\mathrm{C}_{4} \mathrm{H}_{6} \mathrm{~N}_{4} \mathrm{O}_{3}$.

The characteristic constituent of the allantoic fluid of the fœetus; it occurs also in the urine of animals for a short period after their birth. . Traces of it are sometimes detected in this excretion at a later date.

It crystallizes in small, shining, colorless prisms [Fig. 242], which are tasteless

[FIG. 242.

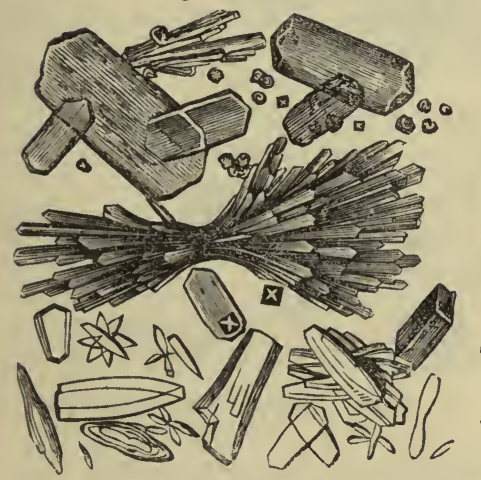

[FIG. 243.

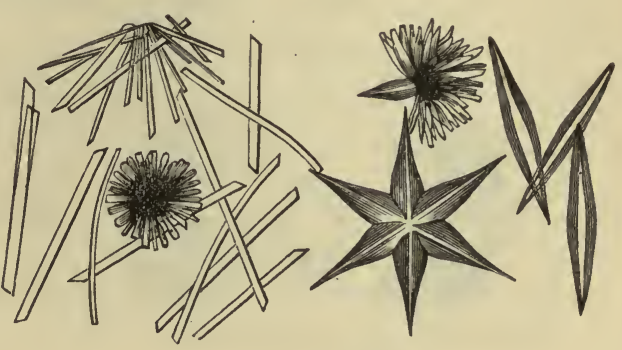

FIG. 242.-Crystals from Concentrated Urine of Calf, showing in Centre a Large Bundle of Prisms of Allantoin. (After Kühne.)]

FIG. 243.-Hypoxanthin-silver-nitrate, $\mathrm{C}_{5} \mathrm{H}_{4} \mathrm{~N}_{4} \mathrm{O} \cdot \mathrm{AgNO}_{3}$. (Krukenberg, after Kühne.)]

and odorless. They are soluble in 160 parts of cold, more soluble in hot water, insoluble in cold alcohol and ether, soluble in hot alcohol. Carbonates of the alkalies dissolve them, and compounds may be formed of allantoin with metals but not with acids.

Allantoin, as already stated, p. 901, is one of the products of the oxidation of uric acid, and by further oxidation gives rise to urea.

Preparation. This is best carried out by the careful oxidation of uric acid, either by means of potassic permanganate or ferrocyanide, or by plumbic oxide.

Hypoxanthin or Sarkin. $\mathrm{C}_{5} \mathrm{H}_{4} \mathrm{~N}_{4} \mathrm{O}$.

Is a normal constituent of muscle, occurring also in the spleen, liver, and medulla of bones. In lukæmia it appears in the blood and urine. It crystallizes in fine

[FIG. 244 .

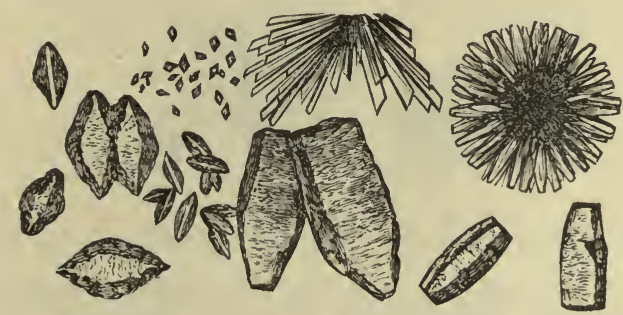

Hypoxanthin-nitrate, $\mathrm{C}_{5} \mathrm{H}_{4} \mathrm{~N}_{4} \mathrm{O} \cdot \mathrm{HNO}_{3}$. (Kühne).]

needles which are soluble in 300 parts of cold, more soluble in hot water, insoluble in alcohol, soluble in acids and alkalies. It forms crystalline compounds with acids 
and bases [Figs. 243, 244, 245]. It is precipitated by basic acetate of lead, the precipitate being soluble in a solution of the normal acetate. Its preparation from

[FIG. 245.

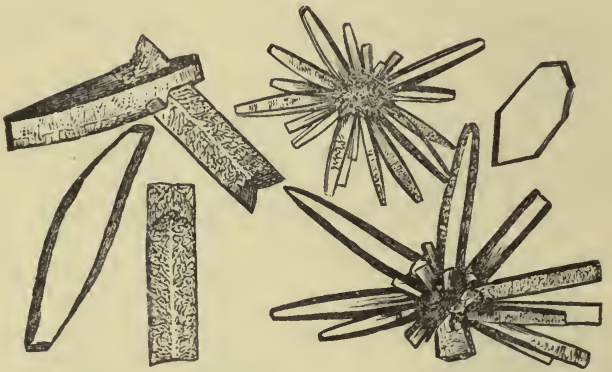

Hypoxanthin-hydrochloride, $\mathrm{C}_{5} \mathrm{H}_{4} \mathrm{~N}_{4} \mathrm{O} . \mathrm{HCl}$. (Kühne.)]

$\cdot$

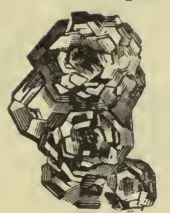

[FIG. 246.

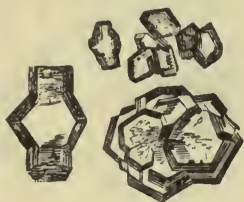

Xanthin-hydrochloride, $\mathrm{C}_{5} \mathrm{H}_{4} \mathrm{~N}_{4} \mathrm{O}_{2} \cdot \mathrm{HCl}$. (Kühne.)]
[FIG. 247.

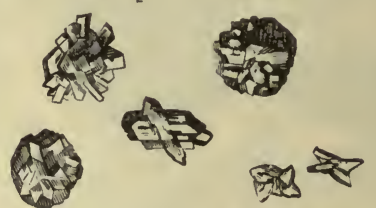

Xanthin-nitrate, $\mathrm{C}_{5} \mathrm{H}_{4} \mathrm{~N}_{4} \mathrm{O}_{2} \cdot \mathrm{HNO}_{3}$. (Kühue.)]

muscle-extract depends on its precipitation first by basic acetate of lead. and then by an ammoniacal solution of silver nitrate after the removal of kreatin.

Both hypoxanthin and the next body, xanthin, can also be obtained fron proteids by the action of putrefactive changes, of water at boiling temperature, of dilute hydrochloric acid $(0.2$ per cent.) at $40^{\circ} \mathrm{C}$, and by the action of gastric and pancreatic ferments. 1 Chittenden has noticed a peculiar difference between fibrin and egg-albumin when submitted to the above processes; he finds that the latter does not yield hypoxanthin when treated with boiling water, with dilute hydrochloric acid, or gastric ferment, while the former does. Egg-albumin, on the other hand, yields hypoxanthin by the action of pancreatic ferment in alkaline solution, but not so readily as fibrin does.

Xanthin. $\mathrm{C}_{5} \mathrm{H}_{4} \mathrm{~N}_{4} \mathrm{O}_{2}$.

First discovered in a urinary calculus, and called xanthic oxide. More recently it has been found as a normal, though scanty, constituent of urine, muscles, and several organs, such as the liver, spleen, thymus, etc.

[FIG. 248.

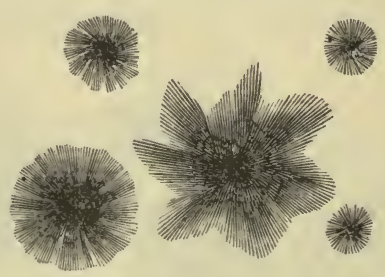

Crystals of Xanthin-silver-nitrate, $\mathrm{C}_{5} \mathrm{H}_{4} \mathrm{~N}_{4} \mathrm{O}_{2} \cdot \mathrm{AgNO}_{3}$. (Krukenberg, after Kühne.)]

When precipitated by cooling from its het, saturated, aqueous solution it falls in white flocks, but if the solution be allowed to precipitate slowly it is obtained in

I Salomon, Zeitschr. f. physiol. Chem., Bd. ii. (1878-1879), S. 60. Krause, Inaug. Diss., Berlin, 1878. Chittenden, Journ. of Physiol., vol, ii. (1879), p. 28. See also Drechsel, Ber, d. Deutsch. Chem. Gesell.. Jahrg. xili. (1880), S. 240. Salomon, Ibid., S. 1160. Kossel, Zeitsch. f. physiol. Chem., Bd. v. (1881), Sn. 152 u. 267. 
small scales. When pure it is a colorless powder, very insoluble in water, requiring 1500 times its bulk for solution at $100^{\circ} \mathrm{C}$. Insoluble in alcohol and ether, it readily dissolves in dilute acids and alkalies, forming crystallizable compounds. [Figs. 246, 247, 248.]

Hypoxanthin by oxidation becomes xanthin. Both these bodies as well as the following, guanin and carnin, are evidently closely allied to uric acid; indeed, uric acid by the action of sodium-amalgam may be converted into a mixture of xanthin and liypoxanthin.

Preparation. It is obtained from urine and the aqueous extract of muscles by a process similar to that for hypoxanthin, and is then scparated from the latter by the action of dilute hydrochloric acid; this separation depends on the different solubilities of the hydrochlorides of the two bodies. For further information see Neubauer and Vogel. ${ }^{1}$

Carnin. $\mathrm{C}_{7} \mathrm{H}_{8} \mathrm{~N}_{4} \mathrm{O}_{3}$.

Discovered by $\mathrm{Weidel}^{2}$ in extract of meat, of which it constitutes about 1 per cent.

It crystallizes in white masses composed of very small, irregular crystals; it is soluble with difficulty in cold, more easily soluble in hot water, insoluble in alcohol and ether. Its aqueous solution is not precipitated by normal lead acetate, but is by the basic acetate of this metal. It unites with acids and salts, forming crystalline compounds.

Preparation. Is found in the precipitate caused in extract of meat by basic acetate of lead. ${ }^{3}$

This body possesses an interesting relation to hypoxanthin, into which it may be converted by the action either of nitric acid or, still better, of bromine.

\section{Guanin. $\mathrm{C}_{5} \mathrm{H}_{5} \mathrm{~N}_{5} \mathrm{O}$.}

First obtained from guano, but recently observed as occurring in small quantities in the pancreas, liver, and muscle extract.

[FIG. 249 .

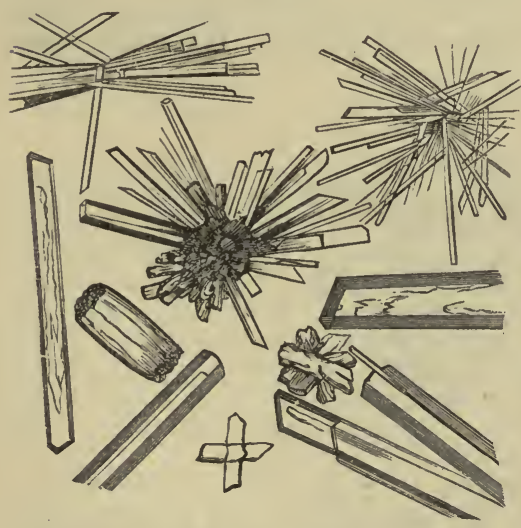

Guanin Hydrochloride. $\mathrm{C}_{5} \mathrm{H}_{5} \mathrm{~N}_{5} \mathrm{O} \cdot \mathrm{HCl}+\mathrm{H}_{2} \mathrm{O}$. (After Kühne.)]
[FIG. 250.

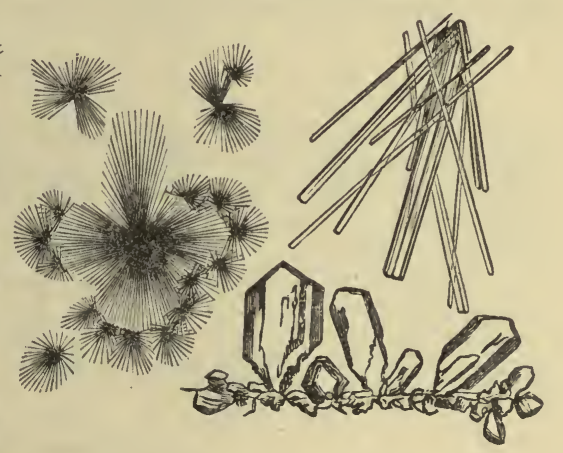

Guanin Nitrate. $\mathrm{C}_{5} \mathrm{H}_{5} \mathrm{~N}_{5} \mathrm{O} \cdot \mathrm{HNO}_{3}+11 / 2 \mathrm{H}_{2} \mathrm{O}$. (After Kühne.)]

It is a white amorphous powder, insoluble in water, alcohol, ether, and ammonia. It unites with acids, alkalies, and salts to form crystallizable compounds. [Figs. 249, 250.]

Preparation. From guano by boiling successively with milk of lime and caustic soda, precipitating with acetic acid, and purifying by solution in hydrochloric acid and precipitation by ammonia.

1 IIarn-Analyse, ed. viii. (1881), S. 26. Also the literature quoted above on hypoxanthin.

2 Ann. d. Chem. u. Pharm., Bd. clviii. S. 365. 
Guanin may, by the action of nitrous acid, be converted into xanthin. By oxidation it can be made to yield principally guanidine and parabanic acid, accompanied, however, by small quantities of urea, xanthin, and oxalic acid. Capranica has given several reactions characteristic of this body. ${ }^{1}$

Its separation from hypoxanthin and xanthin depends on its insolubility in water and behavior with hydrochloric acid.

Kynurenic acid. $\mathrm{C}_{20} \mathrm{H}_{14} \mathrm{~N}_{2} \mathrm{O}_{6}+2 \mathrm{H}_{2} \mathrm{O}$.

Found in the urine of dogs, and first described by Liebig. ${ }^{2}$ When pure it crystallizes in brilliant white needles, insoluble in cold, soluble in hot alcohol. The only salt of this body which erystallizes well is that formed with barium. For preparation and other particulars see Liebig, ${ }^{3}$ Schultzen, and Schmiedeberg. ${ }^{4}$

Glycin. $\mathrm{C}_{2} \mathrm{H}_{2}\left(\mathrm{NH}_{2}\right) \mathrm{O}(\mathrm{OH})$. Also called Glycocol and Glycocin.

Does not occur in a free state in the human body, but enters into the composition of many important substances, $e_{.} g$. , hippuric and bile acids. It crystallizes into large, colorless, hard rhombohedra, which are easily soluble in water, insoluble in cold, slightly soluble in hot alcohol, insoluble in ether. It possesses an acid reaction, but a sweet taste. It has also the property of uniting with both acids and bases to form crystallizable compounds. In this it exhibits its amide nature, and that it is an amide is rendered evident from the methods of its synthetic preparation; thus mono-chloracetic acid and ammonia give glycin and ammonic chloride; $\mathrm{C}_{3} \mathrm{H}_{3} \mathrm{Cl}_{2} \mathrm{O}_{2}+2 \mathrm{NH}_{3}=\mathrm{C}_{2} \mathrm{H}_{2}\left(\mathrm{NH}_{2}\right) \mathrm{O}(\mathrm{OH})+\mathrm{NH}_{4} \mathrm{Cl}$. It is amido-acetic acid. Heated with caustic baryta it yields ammonia and methylamine.

Preparation. From glutin by the action of acids or alkalies; from hippuric acid by decomposing it with hydrochloric acid at a boiling temperature and removing by precipitation the simultaneously formed benzoic acid.

\section{Taurin. $\mathrm{C}_{2} \mathrm{H}_{7} \mathrm{NO}_{3} \mathrm{~S}$.}

In addition to entering into the composition of taurocholic acid taurin is found in traces in the juices of muscle and in the lungs.

It crystallizes in colorless, regular six-sided prisms [Fig. 251]; these are readily soluble in water, less so in alcohol. The solutions are neutral, It is a very stable

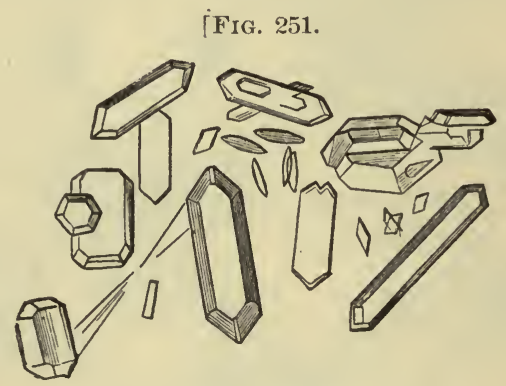

Taurin Crystals.]

compound, resisting temperatures of less than $240^{\circ} \mathrm{C}$.; it is not acted on by dilute alkalies and acids, even when boiled with them. It is not precipitated by metallic salts.

Taurin is amido-isethionic acid; and may be synthetically prepared from isethionic (ethyl-sulphuric) acid by the action of ammonia; thus:

$$
\left.\left.\underset{\mathrm{H}}{\mathrm{C}_{2} \mathrm{H}_{5}}\right\} \mathrm{SO}_{4}+\mathrm{NH}_{3}=\underset{\mathrm{NH}_{2} \mathrm{H}_{5}}{\mathrm{C}_{2}}\right\} \mathrm{SO}_{3}+\mathrm{H}_{2} \mathrm{O} \text {. }
$$

1 Zeitschr. f. phys. Chem., Bd. iv. (1880), S. 240.

2 Ann. d. Chem. u. Pharm., Bd. Ixxxvi. S. 125, and Bd. cviii., S. 354.

3 Op. cit.

4 Anu. d. Chem. u. Pharm., Bd. clxiv. S. 155. 
Preparation. As a product of the decomposition of bile, and is purified by removing any traces of bile acids by means of lead acetate, and then successively crystallizing from water.

Leucin. $\mathrm{C}_{6} \mathrm{H}_{13} \mathrm{NO}_{2}$.

Is one of the principal products of the decomposition of nitrogenous matter, either under the influence of putrefaction or of strong acids and alkalies. It occurs, however, normally in the pancreas, spleen, thymus, thyroid, salivary glands, liver, etc., and is one of the products of the tryptic (pancreatic) digestion of proteids; in acute atrophy of the liver it is present in the urine in large quantity in company with tyrosin.

As usually obtained in an impure form it crystallizes in rounded lumps [Fig. 252], which are often collected together and sometimes exhibit radiating striation. When

[FIG. 252.

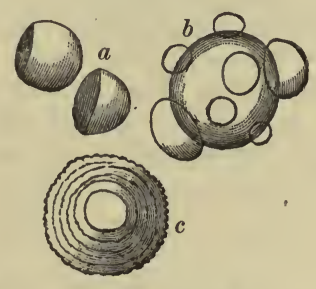

Leucin Crystals.]

pure, it forms very thin, white, glittering, flat crystals. These are easily soluble in hot water, less so in cold water and alcohol, insoluble in ether. They feel oily to to the touch, and are without smell and taste. Acids and alkalies dissolve them readily, and crystallizable compounds are formed.

Carefully heated to $170^{\circ} \mathrm{C}$. it sublimes, but at a higher temperature is decomposed, yielding amylamin, carbonic anhydride, and ammonia. In the presence of putrefying animal matter it splits up into valeric acid and ammonia.

Leucin is amido-caproic acid, and may be represented thus :

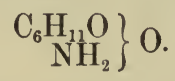

Preparation. From horn shavings by boiling with sulphuric acid, neutralizing with baryta and separating from tyrosin by successive crystallization. See also Kühne, ${ }^{1}$ who prepares it by the action of pancreatic ferment (trypsin) on proteids.

Scherer has given the following test for leucin. The suspected substance is evaporated carefully to dryness with nitric acid; the residue, if it is leucin, will be almost transparent and turn yellow or brown on the addition of caustic soda. If this be again very carefully concentrated with the alkali, an oily drop is obtained, which is quite characteristic of this substance. Leucin, if not too impure, may be easily recognized by its subliming on being heated; a characteristic color of amylamin is at the same time evolved.

\section{Asparagin. $\mathrm{C}_{4} \mathrm{H}_{8} \mathrm{~N}_{2} \mathrm{O}_{3}$}

Is not found as a constituent of the animal body, but appears to be formed by the decomposition of proteids, notably during the germinative changes of the proteids in leguminous seeds. ${ }^{2}$ It is a crystalline body, and when boiled with acids or alkalies is readily converted into aspartic acid.

\section{Aspartic (or asparaginic) Acid. $\mathrm{C}_{4} \mathrm{H}_{7} \mathrm{NC}_{4}$.}

This acid has been obtained in small quantities among the products of the pancreatic digestion of fibrin ${ }^{3}$ and vegetable glutin, ${ }^{4}$ although not occurring as a con-

1 Virchow's Archiv, Bd. xxxix., S. 130.

2 Landwirthski u. Versuchs Stationen, Bd. xvii. 1.

8 Radziejewski u. Salkowski, Ber. d. Deutsch. chem. Gesell., Jahrg. vii. (1874), S. 1050.

1 V. Knieriem, Zeitschr. f. Biol., Bd. xi. (1875), S. 198. 
stituent of any animal tissue or secretion. It is, on the other hand, found normally in plants, notably in beet-sugar molasses. It arises also as a constant product of the action of alkalies and other reagents on both vegetable and animal proteids, and of acids on gelatin. ${ }^{1}$ It thus possesses considerable interest in respect of its relation to the proteids. It crystallizes in rhombic prisms which are but sparingly soluble in cold water or alcohol, readily soluble in boiling water. Its acid solutions are dextro-rotatory, its alkaline lævo-rotatory and reduce Fehling's fluid. It forms a characteristic readily crystallizable compound with copper. Nitrous acid converts it into malic acid.

\section{Glutaminic Acid. $\mathrm{C}_{5} \mathrm{H}_{9} \mathrm{NO}_{4}$.}

The eircumstances and conditions under which this body occurs are in general the same as for the aspartic acid, and hence as a product of proteid decomposition it acquires some importance. It has not, however, as yet been obtained by the action of pancreatic ferments on proteids, and in this it differs from the preceding body.

It crystallizes in rhombic tetrahedra or octahedra ; is not very soluble in cold, but readily soluble in hot water; insoluble in alcohol and ether. Its acid solutions possess a strong dextro-rotatory power, and it reduces Fehling's fluid.

\section{Cystin. $\mathrm{C}_{3} \mathrm{H}_{7} \mathrm{NSO}_{2}$.}

Is the chief constituent of a rarely occurring urinary calculus in men and dogs. It may also occur in renal concretions and in gravel, and is occasionally found in urine.

From calculi it is obtained, by extraction with ammonia, as colorless six-sided tables or rhombohedra [Fig. 253], which are neutral and tasteless. It is insoluble

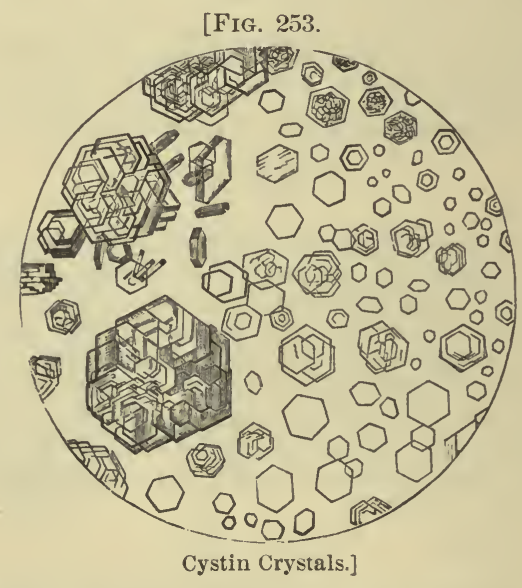

in water, alcohol, and ether, soluble in ammonia and the other alkalies, and also in mineral acids. The fact that this body is one of the few crystalline substances, occurring physiologically, which contain sulphur, renders its detection very easy. Apart from its insolubility in water, etc., it yields, with caustic potash and salts of either silver or lead, a brown coloration due to the presence of the sulphides of these metals.

According to Dewar and Gamgee, ${ }^{2}$ cystin is amido-sulpho-pyruvic acid, and its formula is $\mathrm{C}_{3} \mathrm{H}_{6} \mathrm{NSO}_{2}-$ pyruvic being lactic acid minus two atoms of hydrogen.

1 Horbaczewski, Sitzb. d. k. Akad. d. Wiss., Wien, 1880. 2 Abth., Juni Heft. 2 Journ. of Anat. and Physiol., Nov., 1870, p. 143. 


\section{The Arovatic Series.}

\section{Benzoic Acid. $\mathrm{HC}_{7} \mathrm{H}_{5} \mathrm{O}_{2}$.}

This is not found as a normal constituent of the body, but owes its presence in urine to the fermentative decomposition of hippuric acid, whereby glycin and benzoic acid are formed:

$$
\begin{aligned}
& \text { Hippuric acid. } \\
& \mathrm{C}_{2} \mathrm{H}_{4}\left(\mathrm{C}_{7} \mathrm{H}_{5} \mathrm{O}\right) \mathrm{NO}_{2}+\mathrm{H}_{2} \mathrm{O}=\mathrm{C}_{2} \mathrm{H}_{5} \mathrm{NO}_{2}+\mathrm{C}_{7} \mathrm{H}_{6} \mathrm{O}_{2} \text {. }
\end{aligned}
$$

The sublimed acid is generally crystallized in fine needles, which are light and glistening; any odor they possess is not due to the acid, but to an essential oil, with which they are mixed. When precipitated from solution the crystalline form is always indistinct. This acid is soluble in 200 parts cold or 25 parts of boiling water, but is easily soluble in alcohol or ether. It sublimes readily at $145^{\circ} \mathrm{C}$.; it also passes off in the vapors arising from its heated solutions.

Preparation. Either as above from hippuric acid by fermentation, by boiling the hippuric acid with acids or alkalies, or by sublimation from gum-benzoin.

Tyrosin. $\mathrm{C}_{9} \mathrm{H}_{11} \mathrm{NO}_{3}$.

Generally accompanies leucin, and is perhaps found normally in small quantities in the pancreas and spleen. It is also usually obtained in large quantities by the decomposition of proteid matter, either by putrefaction or the action of acids.

The researches of Radziejewski ${ }^{1}$ render it probable that tyrosin does not occur normally in any part of the human organism, except as a product of pancreatic digestion.

All attempts to synthetize tyrosin were for some time fruitless, although evidence was obtained sufficient to indicate the probable existence in its molecule of some aromatic (phenyl) radicle. ${ }^{2}$ More recently the synthesis has been performed, ${ }^{3}$ and we now have every reason for regarding tyrosin as para-hydroxy-phenyl $a$ alanine. This synthesis, as well as that of uric acid, referred to above, is of considerable importance, since the more definite the knowledge which is possessed of the true molecular structure of the products of proteid decomposition, the more reason is there for expecting that the synthesis of a proteid itself may be realizable in the not very remote future.

[FIG. 254.

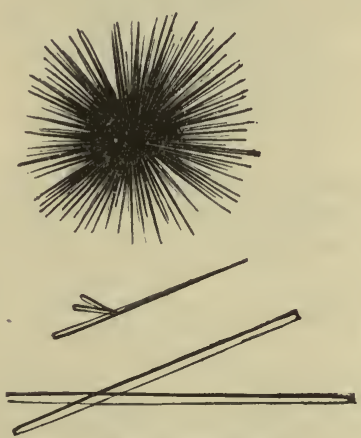

Tyrosin Crystals.]

Tyrosin crystallizes in exceedingly fine needles which are usually collected into feathery masses [Fig. 254]. The crystals are snow-white, tasteless and odorless, almost, insoluble in cold water, readily soluble in hot water, acids, and alkalies,

1 Archiv. f. path. Anat., Bd. xxxvi., S. 1. Zeitschr. f. anal. Chem., Bd. v., S. 466.

2 Barth., Chem. Centralbl., 1865 , S. 1029 ; 1869, S. 761 ; 1872, S. 830 . Hüfner, Ibid., 1869, S. 139. Beilstein u. Kühlberg, Ibid., 1872, S. 830.

3 Erlenmeyer u. Lipp., Ber. d. Deutsch. Chem. Gesell., Jahrg. xv. (1882), S. 1544. 
insoluble in alcohol and ether. If crystallized from an alkaline solution tyrosin often assumes the form of rosettes composed of fine needles arranged radiately.

Tyrosin does not sublime by heating, but is decomposed with an odor of phenol and nitrobenzol. On boiling with Millon's reagent it gives a reaction almost identical with, but much more marked than, that for proteids (Hoffman's test). If tyrosin is treated on a watch-glass with one or two drops of strong sulphuric acid, then diluted with a little water, neutralized with calcic carbonate, and the solution filtered, a characteristic violet color is obtained on the addition of a drop of acid-free ferric chloride (Piria's test).

Preparation. By means similar to those employed for leucin, the separation of the two depending on their widely differing solubilities. According to Kühne's method, ${ }^{1}$ large quantities are easily obtained as the result of pancreatic digestion.

\section{Hippuric Acid. $\mathrm{C}_{9} \mathrm{H}_{9} \mathrm{NO}_{3}$. Or Benzoyl-glycin. $\mathrm{C}_{2} \mathrm{H}_{4}\left(\mathrm{C}_{7} \mathrm{H}_{5} \mathrm{O}\right) \mathrm{NO}_{2}$.}

Is found in considerable quantities in the urine of herbivora, and also, though to a much smaller amount, in the urine of man. It is formed in the body by the union with dehydration of glycin and benzoic acid.

Crystallized from a saturated aqueous solution it assumes the form of fine needles; if from a more dilute solution, white, semitransparent four-sided prisms are obtained [Fig. 255]. These when pure are odorless, with a somewhat bitter

[FIG. 255 .

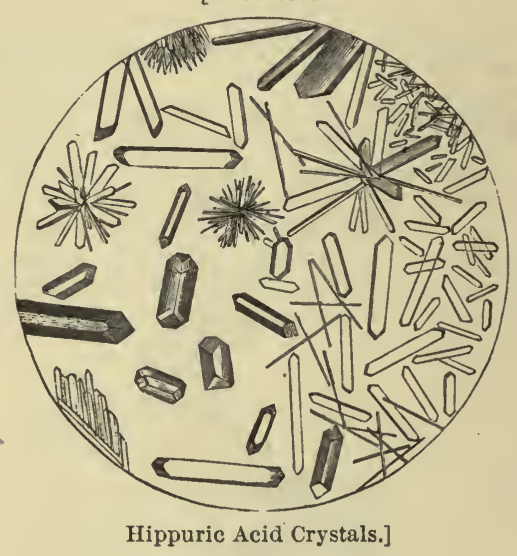

taste. They are soluble in 600 parts of cold water, readily soluble in boiling water, readily soluble in alcohol, less so in ether. All the solutions redden litmus.

Hippuric acid is monobasic, and forms salts which are readily soluble in water (except the iron salts); from these, if in sufficiently concentrated solutions, excess of hydrochloric acid precipitates the acid in fine needles. When heated with concentrated mineral acids it is resolved into benzoic acid and glycin. The same decomposition occurs in presence of putrefying bodies. Strong nitric acid produces an odor of nitrobenzol.

Preparation. Fresh urine of horses or cows is treated with milk of lime in order to form calcic hippurate and thus prevent the decomposition of the hippuric acid, filtered, and the filtrate evaporated to a small bulk; the hippuric acid is then precipitated by adding an excess of hydrochloric acid; the acid is then purified by several crystallizations from boiling water.

When heated in a small tube, hippuric acid gives a sublimate of benzoic acid and ammonic benzoate, accompanied by an odor like that of new hay, while oily, red drops are observed in the tube. This is very characteristic and distinguishes it from benzoic acid. 


\section{Phenylic (carbolic) Acid, or Phenol. $\quad \mathrm{C}_{6} \mathrm{H}_{6} \mathrm{O}$.}

This body is undoubtedly obtained as the result of the putrefactive decomposition of proteids, notably in putrefactive pancreatic digestions. ${ }^{1}$ It may be obtained from the distillate of such digestive mixtures. It is also found in the contents of the alimentary canal under the same conditions which give rise to indol. When so occurring a portion of it may be obtained from the feces, while the rest reappears in the urine. ${ }^{2}$

Buliginsky ${ }^{3}$ says the urine of maly animals, of cows and horses always, contains a substance insoluble in alcohol, and not precipated.by lead acetate and ammonia, which by the action of dilute mineral acids gives carbolic acid. The sume acid applied to the body externally or internally also passes into the urine. ${ }^{4}$ Similarly benzol $\left(\mathrm{C}_{6} \mathrm{H}_{0}\right)$, when taken into the stomach, appears as a carbolic acid in the urine. 5

[F1G. 256.

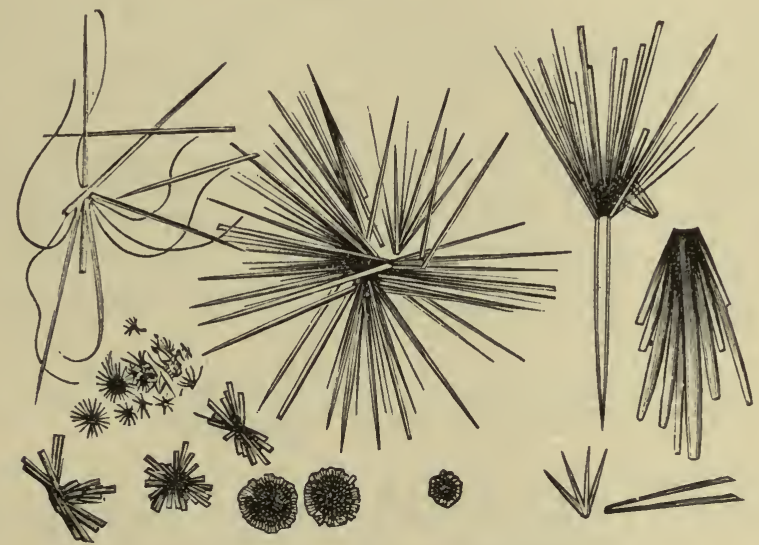

Crystals of Kynurenic Acld. (After Kühne.)]

[FIG. 257.

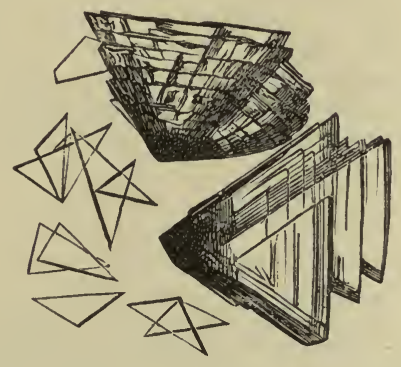

Orystals of Barium Kynurenate. (After Kühne.)]

The pure acid crystallizes in long, colorless prismatic needles; they melt at $35^{\circ} \mathrm{C}$, and boil at $180^{\circ} \mathrm{C}$. It is readily soluble in alcohol and ether, slightly soluble in water (1 part in 20). In most cases it acts as a weak acid, forming crystalline salts with the alkalies. With nitric acid it yields picric acid. Its solutions reduce silver and mercury salts.

1 Baumann, Zeitsch. f. physiol. Chem., Bd. i. (1877), S. 70.

2 Salkowski, Ber, d. Deutsch. Chem, Gesell, Bd. ix (1876) S. 1595 Centralb $f$ med. Wiss. (1876), S. 818. Ber. d. Deutsch. Chem. Gesell., Bd. x. (1877), S. 842. Virchow's Arch., Bd. Ixxii. (1878), S. 409. See also Centralbl. f. d. med. Wiss. (1878), Nos. 30, 31, 34, 42, and Zeitschr. f. physiol. Chem., Bd. ii. (1878), S. 241.

${ }^{3}$ Hoppe-Seyler, Med. chem. Untersuch., Heft ii. (1867), S. 234.

4 Almén, Neues Jahrb. d. Pharm., Bd. xxxiv., S. 111. Salkowski, Pflüger's Archiv, Bd. v. (1871-72), S. 385 .

5 Schultzen u. Naunyn, Reichert u. DuBois Reymond's Archiv (1867), Heft iii., S. 349. 
Preparation. By the dry distillation of salicylic acid, also from the acid products of the distillation of coal. It is obtained in the last portions of the distillate when preparing indol, and is separated by forming a compound with bromine, $\mathrm{C}_{6} \mathrm{HBr}_{3} \mathrm{O}_{3}$.

[KYNURENIC ACID. This acid is obtained from the urine of dogs. It crystallizes in long needles or four-sided prisms [Fig. 256], and with barium forms peculiar triangular crystals [Fig. 257].

\section{The Bile Series.}

\section{Cholalic (or Cholic) Acid. $\mathrm{H} \cdot \mathrm{CH}_{24} \mathrm{H}_{39} \mathrm{O}_{5}+\mathrm{H}_{2} \mathrm{O}$.}

Occurs in traces in the small intestine, in large quantities in the contents of the large intestine, and the feces of men, cows, and dogs. In icterus, the urine often contains traces of this acid. But its principal interest lies in its being the startingpoint for the various bile acids (see below). The pure acid may be amorphous or crystalline, in the latter case crystallizing from hot alcoholic solutions in tetrahedra. These crystals are insoluble in water and ether. In the amorphous form it is somewhat soluble in water and ether. Heated to $200^{\circ} \mathrm{C}$., it is converted into water and dyslysin $\left(\mathrm{C}_{24} \mathrm{H}_{43} \mathrm{O}_{3}\right)$.

This acid possesses, in the anhydrous condition, a specific rotatory power of $+50^{\circ}$ for the yellow light; when it crystallizes with $\mathrm{H}_{2} \mathrm{O}$, the rotation is $+35^{\circ}$. The rotatory power of the alkali salts is always less than the above, and when in solution in alcohol the rotation is independent of the concentration. For the alcoholic solution of the sodium salt the rotation is $+31.4^{\circ}$.

Preparation. By the decomposition of bile acids by means of acids, alkalies, or fermentative changes.

Bayer ${ }^{1}$ has examined the bile acids obtained from human bile, and has prepared from them cholalic acid. To this he assigns the formula $\mathrm{C}_{18} \mathrm{H}_{28} \mathrm{O}_{4}$. If this be so, then cholalic acid of human bile would seem to be a budy entirely different from that obtained from ox-bile, and analyzed by Strecker. Bayer's results, however, require further confirmation.

\section{Pettenkofer's test. ${ }^{2}$}

This well-known test for bile acids depends on the reaction of cholalic acid in presence of sugar and sulphuric acid. If to a solution of the acid a little sugar be added and then sulphuric acid, keeping the temperature below, but not much below, $70^{\circ} \mathrm{C}$., a beautiful reddish purple is obtained. If diluted with alcohol this solution gives a characteristic spectrum with two absorption bands, one between $\mathrm{D}$ and $\mathrm{E}$, nearest to $\mathrm{E}$, the other close to $\mathrm{F}$ on the red side of $\mathrm{F}$.

The reaction is much impeded by the presence of coloring matters; moreover, proteids and other bodies easily decomposed by sulphuric acid, such as amyl-alcohol and oleic, give a similar result; the coloring matter produced from these bodies does not, however, give the absorption bands described above. ${ }^{3}$

Glycocholic Acid. $\mathrm{C}_{26} \mathrm{H}_{43} \mathrm{NO}_{6}$.

This body was first obtained in the crystalline form and described by Gmelin (1826), who gave it the name of "cholic" acid.

To avoid confusion it is now best to use the term "cholic" as a synonym for "cholalic," Demarcay, who first (1838) described the cholalic acid as a product of the decomposition of bile acids, having given it the name of cholic acid. The name cholalic acid is perhaps the best, since it indicates the method by which the bile acids are split up, viz., by treatment with alkali.

This is the principal bile acid of ox-gall : it is also present in the bile of man, but has so far not been observed in that of carnivora. In icterus the urine may contain traces of this acid.

It crystallizes in fine, glistening needles. These are slightly soluble in cold water, readily so in hot water and alcohol, but insoluble in ether. They possess a bitter and yet sweet taste, and a strong acid reaction.

The salts of this acid are readily soluble in water and crystallize well. The salts, as well as the free acid, exert right-handed polarization amounting to $+29.0^{\circ}$ for the acid, and $+25.7^{\circ}$ for the sodium salt, both measured for yellow light.

1 Zeitschr. f. physiol. Chem., Bd. ii. (1878-79), S. 358.

2 Pettenkofer, Annalen d. Chem. u. Pharm., Bd. lii: (1844), S. 90.

8 For further information on this subject see: Bischoff, Zeitschr. f. rat. Med., Ser. 3, Bd. xxi., S. 126. Schulze. Aun. d. Chem. 11. Pharm., Bd. lxxi. (1849), S. 266. Schenk, Anatom.-physiol. Untersuch., Wien, 1872, S. 47. Adamkiewicz, Pflïger's Arch., Bd. ix. (1874), S. 156. 
Glycocholic acid is a compound of glycin and cholalic acid; thus :

$$
\begin{aligned}
& \text { Cholalic acid. Glycin. } \\
& \mathrm{C}_{24} \mathrm{H}_{40} \mathrm{O}_{5}+\mathrm{C}_{2} \mathrm{NH}_{5} \mathrm{O}-\mathrm{H}_{2} \mathrm{O}=\mathrm{C}_{26} \mathrm{H}_{43} \mathrm{NO}_{6} \text {. }
\end{aligned}
$$

Prolonged boiling with dilute mineral acids or caustic alkalies decomposes glycocholic acid into glycin and cholalic acid; if dissolved in concentrated sulphuric acid and then warmed, glycocholic acid by the removal of one molecule of water yields cholonic acid, $\mathrm{C}_{28} \mathrm{H}_{41} \mathrm{NO}_{5}$. The barium salt of this last acid is insoluble in water, which fact is of importance, since cholonic acid possesses nearly the same specific rotatory power as glycocholic acid.

Preparation. From ox-gall by evaporation to a syrup, decolorizing with animal charcoal, extracting with strong alcohol, and precipitating by a large excess of ether. Its separation from taurocholic acid depends on its precipitation by normal lead acetate, taurocholic acid not being precipitated by this reagent.

Taurocholic Acid. $\mathrm{C}_{26} \mathrm{H}_{45} \mathrm{NSO}_{7}$.

Occurs also in ox-gall, but is found especially plentiful in human bile and that of carnivora, notably of the dog.

It crystallizes with difficulty in very fine needles, which are exceedingly deliquescent. When dried it is an amorphous powder, with pure bitter taste, easily soluble in water and alcohol, insoluble in ether. All its salts are soluble in water, and are precipitated by basic lead acetate in the presence of free ammonia. The sodium salt dissolved in alcohol has a specific rotatory power of $+24.5^{\circ}$; if dissolved in water this rotation is less, and in this respect it resembles glycocholic acid.

This acid is far more unstable than the preceding one, being decomposed if boiled with water. The products of decomposition are taurin and cholalic acid.

Taurocholic acid is a compound of taurin and cholalic acid; thus :

$$
\begin{gathered}
\text { Cholalic acid. Taurin. } \\
\mathrm{C}_{24} \mathrm{H}_{40} \mathrm{O}_{5}+\mathrm{C}_{2} \mathrm{H}_{7} \mathrm{NO}_{3} \mathrm{~S}-\mathrm{H}_{2} \mathrm{O}=\mathrm{C}_{26} \mathrm{H}_{45} \mathrm{NO}_{7} \mathrm{~S} \text {. }
\end{gathered}
$$

Preparation. From the bile of dogs by a process similar to that for glycocholic acid. It is separated from traces of this latter and from cholalic acid by precipitation with basic lead acetate and ammonia. ${ }^{1}$

\section{Bile Pigments.}

\section{Bilirubin. $\mathrm{C}_{16} \mathrm{H}_{18} \mathrm{~N}_{2} \mathrm{O}_{3}$.}

It is found chiefly in the fresh bile of man and carnivora, to which it gives the characteristic dark golden-red color. It frequently constitutes a considerable part of some kind of gall-stones, not, however, as free bilirubin, but as a compound with earthy matter, chiefly chalk; the gall-stones of oxen and pigs often contain 40 per cent. of this compound. ${ }^{2}$ These are, therefore, the best material from which to prepare bilirubin.

Preparation. The gall-stones are treated with strong acetic or dilute hydrochloric acid, to separate the earthy matter, and the residue is thoroughly washed with water and alcohol and dried. From this residue the prolonged action of hot chloroform extracts the bilirubin, which may either be obtained in the amorphous form by precipitation with alcohol of its solution in chloroform, or as well-defined crystals by the slow evaporation of the chloroform solution.

The most usual form of the crystals is that of rhombic prisms; they are readily soluble in chloroform and alkaline solutions only.

By treatment with oxidizing agents, such as nitrous acid, bilirubin takes up oxygen and becomes biliverdin, the color at the same time changing to green. The possible oxidation does not end here, and if continued a series of products are obtained, each with a characteristic color, as in the well-known Gmelin's test. ${ }^{3}$ of these only the final product of the oxidation has been obtained in a state of sufficient purity to enable any definite statements to be made of its characteristics. ${ }^{4}$

This is the body known as Choletelin (see below).

1 Parke, Tübing. Med.-chem. Unters., Bd. i., S. 160.

2 Maly, Sitzber. d. Wien. Akad., Bd. Ivii. (1868), ii. Abth., Febr. Hft.

3 Tiedmann u. Gmelin, Die Verdauung, 1826, S. 79.

4 Heynsius u. Campbell, Pflüger's Arch., Bd.iv. (1871), S. 497. 
Biliverdin. $\mathrm{C}_{16} \mathrm{H}_{18} \mathrm{~N}_{2} \mathrm{O}_{4}{ }^{1}$

This product of the oxidation of bilirubin gives the characteristic color to the bile of herbivora and to biliary vomits. It occurs also probably at times in the urine of jaundice and in the pigmentary matter of the placenta. It is found, or occurs in traces only, in gall-stones.

Preparation. An impure product is obtained by precipitating ordinary herbivorous bile with baric chloride, washing the precipitate with water and alcohol, and decomposing it with hydrochloric acid. The biliverdin thus obtained is washed with ether and dissolved in alcohol. From its solution in the latter it is obtained as an amorphous green powder by slow evaporation. Pure biliverdin is best prepared by the slow oxidation in the air of bilirubin, dissolved in dilute caustic soda.

It does not crystallize, and is insoluble in ether or chloroform; readily soluble in alcohol. When oxidized it gives the same play of colors as does bilirubin, with the formation of the same final and intermediate products.

Neither this body nor bilirubin gives any characteristic absorption bands.

There seems now no reason for doubting that the bile pigments are derived ultimately from the coloring matter of the blood.

Virchow has described ${ }^{2}$ the gradual changes in old blood-clots, as of cerebral hemorrhage, which lead to the presence of the so-called hæmatoidin-crystals. Though these have not been obtained in sufficient quantities to enable their composition to be finally fixed by a chemical analysis, ${ }^{3}$ still the identity of their crystalline form with that of bilirubin, and the fact that they both give the same play of colors when oxidized, as in Gmelin's test, justify the assumption that hæmatoidin and bilirubin are identical. ${ }^{4}$ Moreover, the balance of experimental evidence distinctly supports the view that a liberation from the corpuscles of the coloring matter of the blood in the bloodvessels by an injection of chloroform, water, etc., leads generally to the appearance of bile-pigments in the urine. ${ }^{5}$ The occurrence of bilirubin crystals in the urine has frequently been observed after the operation of transfusion of blood in man. The chemical possibility of the conversion of hæmoglobin into biliverdin is readily seen by a comparison of the formulæ of hæmatin (see p. 464) and bilirubin. The former has, according to Hoppe-Seyler, ${ }^{6}$ the composition indicated in the formula $2\left(\mathrm{C}_{34} \mathrm{H}_{35} \mathrm{~N}_{4} \mathrm{FeO}_{5}\right)$, while that of bilirubin is $\mathrm{C}_{16} \mathrm{H}_{18} \mathrm{~N}_{2} \mathrm{O}_{3}$. Although the conversion has not as yet been directly effected, the following facts are significant: If bilirubin is treated with sodium amalgam the substance known as hydrobilirubin (see below) is obtained. If hæmatin is dissolved in caustic soda and treated with sodium amalgam or in hydrochloric acid solution with zinc dust, a substance is obtained which is now recognized as identical with hydrobilirubin. ${ }^{7}$ This is the most direct chemical evidence of the relation of the coloring matters of the blood and bile.

\section{Choletelin. $\left.\mathrm{C}_{16} \mathrm{H}_{18} \mathrm{~N}_{2} \mathrm{O}_{6}(?)\right)^{8}$}

This substance is obtained as the final product of the oxidation of either bilirubin or biliverdin. It is best prepared by acting upon bilirubin with nitrous acid in presence of alcohol; the various colors of Gmelin's reaction are observed and the final reddish-yellow solution, if poured into water, yields a precipitate of choletelin. It is not crystalline and is soluble in alcohol, ether, and chloroform. When freshly prepared it seems to give an uncertain absorption band if examined in an acid solution. On this account some observers ${ }^{9}$ have been led to regard it as identical with hydrobilirubin (urobilin). There is, however, no doubt that they are quite distinct bodies. $^{10}$

\footnotetext{
1 Maly, Sitzb. d. Wien. Akad., Bd. Ixx. (1874), iii. Abth.

2 Arch. f. path. Anat., Bd. i., S. 383.

3 Robin, Ann. d. Chem. u. Pharm., Bd. cxvi., S. 89.

4 But see also Preyer, Die Blutkrystalle, 1871, S. 187.

5 Tarchanoff, Pflüger's Arch., Bd. ix. (1874), S. 53. See also Bd. x. (1875), S. 208.

7 Hoppe-Seyler, Med.-chem. Untersuch., Heft iv., 1871, S. 523. Ber. d. Deutsch. chem. Gesell. vii. (1874), S. 1065 .

8 Maly, Sitzb. d. Wien. Akad., Bd. Ivii. (1868); 2 Abth. Febr. und Bd. lix., 1869; 2 Abth. April. See also Heynsius and Campbeli, loc. cit.

9 Heynsius and Campbell, loc. cit. Stokvis, Centralbl. f. d. med. Wiss., No. 14 (1873), S. 211

10 Maly, Centralbl. f. d. med. Wiss., No. 21 (1875), S. 321. Liebermann, Pflüger's Arch., Bd. xi. (1875), S. 181.
} 
Hydrobilirubin. $\mathrm{C}_{32} \mathrm{H}_{40} \mathrm{~N}_{4} \mathrm{O}_{7}$.

This body was first described by Maly ${ }^{1}$ as resulting from the action of sodium amalgam on an alkaline solution of bilirubin. When the reaction is complete the solution is precipitated with hydrochloric acid, the precipitate dissolved in ammonia, again precipitated by acid, and the substance thus finally obtained is washed with water. It is readily soluble in alcohol, less so in ether. Its alkaline solutions are yellow, and these turn pink on the addition of acid. Both its acid and alkaline solutions, the latter especially on the addition of a few drops of chloride of zinc, give a characteristic absorption band between $\mathrm{b}$ and $\mathrm{F} .{ }^{2}$ In the colors of its alkaline and acid solutions and the greenish fluorescence of its ammoniacal solution on the addition of chloride of zine, and in its absorption spectrum, hydrobilirubin shows its close relation to the urobilin (see below), with which indeed it is now considered to be identical. It is also identical with a body named stercobilin, ${ }^{3}$ which had previously been described as a product of the alteration of the bile-pigments in the alimentary canal occurring in feces. There is no difficulty in seeing how this change (hydrogenation) can be brought about in the intestine since it is known that a considerable quantity of hydrogen may make its appearance by fermentative processes in the intestine, and in its nascent state might readily produce the simple change which is known to occur when bilirubin is converted into hydrobilirubin.

\section{Pigments of Urine.}

Our knowledge of these bodies is at present limited and imperfect. Most probably ${ }^{4}$ they are numerous, but only two appear sufficiently well characterized to deserve mention here.

\section{Urobilin. $\mathrm{C}_{32} \mathrm{H}_{40} \mathrm{~N}_{4} \mathrm{O}_{7}$.}

As stated above, this is now regarded as identical with hydrobilirubin. It was first described by Jaffé ${ }^{5}$ as a well-characterized normal urinary pigment and its identity with hydrobilirubin subsequently determined. ${ }^{6}$

Normal urine contains only small quantities of urobilin, but there is present a substance (chromogen) which under the influence of acids, with absorption of oxygen, yields urobilin. The urine of fever frequently contains a considerable amount of actual urobilin as such.

The properties described above for hydrobilirubin are identical with those of urobilin. Its preparation from urine is somewhat difficult, and for this some special manual must be consulted. ${ }^{7}$

\section{Uroerythrin.}

Is considered to be the substance which gives to the urine of rheumatism its characteristic color. Very little is known of its chemical properties. ${ }^{8}$ It appears to be an amorphous reddish body with an acid reaction, slowly soluble in water, alcohol, and ether. When treated with caustic alkali it turns green. Urine containıng this body takes on a characteristic reddish-yellow color on the addition of concentrated hydrochloric acid.

Thudichum considers that normal urine contains only one pigment, which he calls urochrome. Maly is inclined to regard this as the same as urobilin.10 More recently Thudichum has upheld his former views. ${ }^{11}$

\section{Indican. $\mathrm{C}_{8} \mathrm{H}_{7} \mathrm{NSO}_{4}$.}

\section{The Indigo Series.}

A body was long ago described ${ }^{12}$ as occurring in the urine and sweat of men and other animals which yielded by the action of acids the blue coloring matter

1 Centralb. f. d. med. Wiss., No. 54, 1871. Annal. d. Chem., Bd. clxiii. (1872), S. 77.

2 Vierordt, Zeitschr. f. Biol., Bd. ix. (1873), S. 160 .

3 Vanlair and Masius, Centralbl. f. d. med. Wiss., No. 24, 1871.

- Vierordt, Die quantitativ Spectralanalyse, etc., Tubingen, 1876, S. 81

6 Centralb. f. d. med. Wiss., 1868, S. 243. Virchow's Arch., Bd. xlvii. (1869), S. 405.

6 Maly, Ann. d. Chem. u. Pharm., Bd. clxiii. (1872), S. 77.

7 Vide Neubauer and Vogel, Harnanalyse, ed. viii. (1881), S. 81.

8 Heller's Archiv (2) Bd. iii. (1854), S. 361.

9 Brit. Med Journ., N. S., No. 201, 1864, p. 509

10 Maly, Ann. d. Chem. u. Pharm., loc. cit., 1872, S. 90.

11 Journ. Chem. Soc., Ser. 2, vol. xiii (1875), pp. 397, 401.

12 Schunk, Phil. Mag., vol. x. p. 73; xiv. p. 228; xv. pp. 29, 117, 183. Chem. Centralbl., $1856, \mathrm{~S}$. 50 ; 1857, S. 957 ; 1858, S. 225 . Hoppe-Seyler, Arch. f. path. Anat., Bd. xxvil., S. 388 . Jaffe, Pfluger's Arch., Bd. iil. (1870), S. 448. 
indigo as one of the products of its decomposition. Schunk considered this substance to be identical with the indican known to occur in several plants (Indigofera, Isatis). Hoppe-Seyler, ${ }^{1}$ on the other hand, having regard to the greater ease with which the indican from plants undergoes decomposition, regarded them as most probably different substances. Baumann has shown ${ }^{2}$ that the two are really different, and has confirmed his earlier statements in a more recent publication. ${ }^{3}$ According to him, the indican obtained from urine is not a glucoside (so also Hoppe-Seyler) and yields sulphuric acid by the action of hydrochloric acid. $\mathrm{He}$ assigns to it the formula $\mathrm{C}_{8} \mathrm{H}_{6} \mathrm{~N} . \mathrm{O} . \mathrm{SO}_{2} . \mathrm{OH}$, and regards it as indoxy-sulphuric acid. The acid itself is not yet known in the free state, but it yields stable salts such as that of potassium, $\mathrm{C}_{8} \mathrm{H}_{6} \mathrm{~N}$. SO $\mathrm{SO}_{4} \mathrm{~K}$. It occurs largely in the urine as the result of the presence of indol in the alimentary canal. In this way Baumann and Brieger ${ }^{4}$ were enabled to obtain large quantities by giving indol to a dog. For its preparation their original paper must be consulted.

When treated in aqueous solution with hydrochloric acid in presence of oxygen it yields indigo-blue.

$$
2 \mathrm{C}_{8} \mathrm{H}_{6} \mathrm{NSO}_{4} \mathrm{~K}+\mathrm{O}_{2}=2 \mathrm{C}_{8} \mathrm{H}_{5} \mathrm{NO}+2 \mathrm{KHSO}_{4} \text {. }
$$

It is always estimated in urine by conversion into indigo-blue.

\section{Indigo. $\mathrm{C}_{8} \mathrm{H}_{5} \mathrm{NO}$.}

It is formed, as stated above, from indican, and gives rise to the bluish color sometimes observed in sweat and urine.

It may, by slow formation from indican, be obtained in fine crystals; these are insoluble in water, slightly soluble, with a faint violet color, in alcohol and ether. Chloroform also dissolves then to a slight extent. Indigo is soluble in strong sulphuric acid, forming at the same time two compounds with this acid; these are soluble in water. It possesses a pure blue color; when pressed with a hard body a reddish copper-colored mark is left, and the crystals exhibit the same color if seen in reflected light.

The soluble compounds with sulphuric acid give an absorption band in the spectrum which lies close to the $\mathrm{D}$ line and to the red side of it. This may be used to detect indigo.

Treated with reducing agents, indigo is decolorized, being reduced to indigowhite. The latter contains two atoms more hydrogen than indigo.

Indol. $\mathrm{C}_{8} \mathrm{H}_{7} \mathrm{~N}$.

To this body the specific odor of the feces is partly due. It is obtained as the final product of the reduction of indigo, and also by the distillation of proteid matter with caustic alkalies. ${ }^{5}$

It often occurs among the products of the action of pancreatic ferment on proteids; its presence in such cases appears, however, to be due, not to the action of the trypsin, but to a simultaneous putrefaction under the influence of bacteria, etc. $^{6}$ If the pancreatic digestion be earried on in the presence of salicylic acid, indol does not make its appearance. Indol is a crystalline body, soluble in boiling water, alcohol, and ether. It passes over in the steam when its aqueous solution is boiled. It is characterized by the following reactions: A strip of pine-wood moistened with hydrochloric acid is colored bright crimson when dipped into a solution of indol. Its alcoholic solution turns red when treated with nitrous acid, and its aqueous solution gives a copious red precipitate with the same reagent. It also yields a characteristic crystalline compound with picric acid.

1 Handb. d. path. chem. Anal., ed. iv. (1875), S. 191.

2 PAüger's Arch., Bd. xiii. (1876), S. 301. Zeitschr. f. physiol. Chem., Bd. i. (1877-78), S. 60.

3 Zeitschr. f. physiol. Chem., Bd. iii. (1879), S. 254.

4 Zeitschr. f. physiol. Chem., Bd. iii. (1879), S. 254. See also Ber. d. Deutsch. chem. Gesell., xii. (1879), Sn. 1098, 1192, 2166; and xiii. (1880), S. 408.

5 Kühne, Ber, d. deutsch. chem. Gesell., viii. (1875), S. 206.

6 Kühne, Verhand. Heidelb. naturhist.-med. Ver., N.S., Bd. i., Hft. 3. Ber. d. Deutsch. chem. Gesell. (1875), S. 206. 
Skatol. $\mathrm{C}_{9} \mathrm{H}_{9} \mathrm{~N}(?)$.

Noticed by Brieger ${ }^{1}$ as one of the products of putrefactive changes in the small intestine. Secretan ${ }^{2}$ had previously described a similar substance as arising from the putrefaction of albumin.

Skatol is crystalline and contains nitrogen; it is more soluble in water than indol, and does not give rise to any red coloration with nitrous acid.

Skatol readily passes into the urine when it occurs in the alimentary canal, and then gives a violet-red reaction with strong hydrochloric acid.

$\mathrm{v}$. Nencki ${ }^{3}$ prepares this substance by the putrefaction of a mixture of finely divided pancreas and muscle substance. After the addition of acetic acid the mass is distilled, when the skatol readily passes over. From the distillate it is precipitated by picric acid, and the precipitate when again distilled with ammonia gives off pure skatol, which may be finally purified by crystallization.

\section{[PTOMAINES AND LEUKOMAINES.}

These substances comprise the so-called animal alkalies, and belong to a class of amines. They are called alkaloids because of their close resemblance in toxic properties to the same class of substances obtained from plants. The difference between ptomaines and leukomaines as classes is that the former are products of abnormal metabolism and the latter of normal metabolism. Ptomaines are usually highly toxic, while leukomaines are of feeble toxicity. It seems probable that in specific diseases the disease germs form ptomaines and that the peculiar pathological states are due in a large measure to these substances acting as peculiar poisons.]

1 Ber. d. Deutsch. chem. Gesell., Jahrg. x. (1877), S. 1027.

2 Recherches sur putrefaction de l'albumine. Geneva, 1876.

8 Centralbl. f. d. med. Wiss., 1878, S. 849. 


\section{N D E X.}

AERRATION, chronic, 758

A spherical, 757

Absorption, from alimentary canal, 325 of diffusible substances and water, 331 of fats, 329

Accommodation, 745,751 mechanism of, 749

Acetic acid series, 887

Acid-albumin, 84 formation of, 255

Acid, acetic, 887 aspartic, 907 benzoic, 909 butyric, 888 capric, 888 caproic, 888 caprylic, 888 cholic, 912 ethylene-lactic, 891 ethylidene-lactic, 891 formic, 887 glutaminic, 908 glycerin-phosphoric, 895 glycocholic, 912

hippuric, 910 kynurenic, 906 lactic, 891

laurostearic, 888 myristic, 888 oleic, 889 oxalic, 892 palmitic, 888 phenylic, 911 propionic, 888 sarcolactic, 891 stearic, 888 succinic, 892 taurocholic, 913 uric, 899 salts of, 900 valerianic, 888

Acoustic apparatus, 798

Adipose tissue, 470

Afferent impulses, 526, 706

After-images, 775 negative, 775 positive, 775

Albumin, acid, 868 alkali, 869

derived, 868

egg, 867

native, 867

nucleo-, 884

serum, 865
Albuminous glands, changes in, during secretion, 269

Alkali albumin, 85

Allantoin, 903

Allantois, 842

Alvergniat's pump, 349

Amblyopia, 695

Amides, $896^{\circ}$

Amœba, characteristics of, 17

Amceboid movements, 121

Anelectrotonus, 103

Animal body, chemical basis of, 863

Animal gum, 888 heat, 496

distribution of, 496

production of, 500 regulation by variations in loss, 499

sources of, 496

temperature of body, 498

Aphasia, 676

complete, 679

partial, 679

Apnœea, 384

Aqueous humor, 744

Area for mastication, 666

production of voice, 666

speech, 676

swallowing, 666

A romatic series, 909

Arteries, changes in calibre of, 211

Ascites, 322

Asparagine, 907

Aspartic acid, 907

Asphyxia, 386

Astigmatism, 757

Atropine, action of, on heart, 208 on pupil, 756

A uditory judgments, 805 sensations, 800

Automatic actions, 131

Automatism, irregular, 721

regular, 721

DENZOIC acid, 909

B Bile, 278

acids, 280

formation of, 456

action of, on food, 281

antiseptic qualities of, 281

characters of, 278

composition of, 278

formation of constituents of, 453 
Bile, influence on peptic digestion, 309 pigments of, $279,453,913$ Gmelin's test for, 280 resorption of, 291 salts, 280 tests for, 280 Pettenkofer's, 280

secretion of, 288 series, 912

Bilirubin, 279, 912

Biliverdin, 280, 913

Binocular vision, 781

Bladder, muscles of, 427

Blindness, 695 color, 774

Blind spot, filling up of, 779

Blood, 24 carbonic acid in, 360 changes in quantity of, 236 chemical composition of, 50 clotting of, 26

buffy coat in, 27

causes of, 38

corpuscles in, 37

crassamentum in, 26

effect of neutral salts on, 32

effect of sodium chloride on, 28

effect of temperature on, 31

fibrin in, 28

fibrin ferment in, 34

fibrinogen in, 33

in arteries, 37

in veins, 36

length of time of, 27

paraglobulin in, 30

plasmine in, 32

rapidity of, 27

serum in, 29

serum-albumin in, 30

color of, 37

brightness of, due to reflection of

light through corpuscles, 39

venous and arterial, 356

composition of, 50

gases, 349

methods of obtaining, 346

hæmatin, 359

features of, 359

method of obtaining, 359

hæmin, 360 crystals of, 360

hæmoglobin, 351

characters of, 351

method of obtaining, 352

spectroscopic features of, 353 varieties of crystals. 352

composition of, methæmoglobin, 360

corpuscles, 37

average number of, in human blood, 40

characteristics of, 37

red, structure of, 40 hæmoglobin in, 40

white, 43

effect of deficient aëration on, 382

platelets, 49
Blood plaques, 49

pressure, 137

curves from carotid of rabbit, 140

quantity of, its distribution in body, 54

rate of circulation, 150

relations of oxygen in, 350

respiratory changes in, 346

supply, influence of, on contractile tissues, 113

Brain, 584 of liver, 289

absence of signs of volition and intelligence, 637

and spinal cord, lymphatic arrangements of, 728

membranes of, 728

vascular arrangements of, 732 .

arteries of, distribution and characters 732

blood-supply of, 734

methods of investigating, 735

bulb of, 589

fibres of, 598

central gray matter of, 601

cerebro-spinal fluid, 731

changes in gray matter of, 595

commissural fibres of, 634

connections of gray and white matter of, 601

corpora geniculata, 625

quadrigemina, 625

gray matter of, 625

corpus striatum, 625

cortical fibres, 631

cranial nerves, 589

embryonic, 584

fibres of, 627

functions of, 716

gracile and cuneate nuclei of, 597

gray matter of, 601

histological features of, 653

intermediate gray matter of, 615

longitudinal fibres of, 628

nature and relations of the several nuclei of, 613

olivary nucleus of, 596

optic thalamus, 615

posterior bundles of, 633

cortical of, 630

pyramidal tract of, 628

splanchnic functions of, 720

structure of, 584

superficial gray matter of, 615

superior or sensory decussation, 592

Brain, tracts from corpora quadrigemina of, 633

venous arrangement of, 733

white matter of, 530

Broca's convolution, 676

Burdach, columns of, 540

Butyric acid, 889

CALABAR bean, action of, on pupil, 756

$\cup$ Calorimeters, 494

Capillaries, circulation in, 143, 231 
Capillaries, pressure of blood in, 142 stagnation or stasis, 230 vessels of muscle, 77

Capric acid, 889

Caproic acid, 889

Caprylic acid, 889

Carbohydrates, 88,884

Carbonic acid exhaled, 344, 364

Cardiac contraction, features of, 196 curves, discussion of, 168 impulse, 162 phases, duration of, 173

Carnin, 899

Casein, 259, 868

Cell body, 126

Cells, pyramidal, 656

Central nervous system, vasomotor functions of, 222

Cerebellar tract, course of, 556

Cerebellum, corpus dentatum of, 625 functions of, 716,719

gray matter of, $6 \overline{3} 3$

histology of, 654 superior pedicle of, 632

Cerebral convolutions, areas of, 662 of the dog, 662

cortex, 655

hemispheres, phenomena exhibited by animals deprived of, $63 \bar{i}-643$

operations, time taken up by, 724

Cerebrin, 98, 895

Cerebro-spinal fluid, 730 nerves, 124

Cerebrum, under surface or base of, 123

Changes of living tissue, 46

Charcot's crystals, 896

Chauveau and Lortet's hæmatachometer, 153

Chemical changes, 89

Cheyne-Stokes respiration, 385

Chitin, 883

Cholalic acid, 912

Cholesterin, 97, 279, 893

Choletelin, 915

Chondrin, 882

Chorda saliva, 721 tympani nerve, nature and action of, 264

Chordæ vocales, 821

Choroid coat, 740

Chromatic aberration, 757

Chyle, characters of, 316

Chyme, 307

Ciliary movement, 119

Circles, diffusion, 746

Circulation, causes of irregular heart-beat, 238

circumstances determining character of flow, 144

effects of alcohol on, 241, 242 of food on, 244

fotal, 851

hydraulic principles of, 144

influence of exercise on, 242

Clarke, columns of, 538

Cochlea, 794
Cold, sensations of, 703

Color-blindness, 774 sensations, 769

Colostrum, 480

Composition of bile, 279 of blood, 50

of gastric juice, 252

of milk, 480

of perspiration, 432

of saliva, 246

of starving body, 484

of the animal body, 483 of urine, 409

Conclia, 798

Connective tissue, 134

Constant current, action of, 100

Contractile muscles, $8 j$ tissues, 54, 116

Contraction, breaking, 101

idio-muscular, 112

making, 101

tetanic, 57

wave of, 78,117

Contrast of visual perceptions, 778

Coördinated movements, machinery of, 643652

Cord, spinal, 529

volitional impulses in, 585

Cornea, 738

Cornu ammonis, 660

Corpora geniculata, 625

quadrigemina, functions of, 719

Corpus luteum, 840

Corpuscles, connective tissue, 134 number of, in human blood, 40 of blood, 37

Cortex, cerebral, 655,721

histology of, $655-661$

psychical processes in, 722

Corti, organ of, 795

pillars of, 795

rods of, 795

Cortical areas, removal of, 674

motor region, $662-668$

Cramps, muscular, 118

Cristæ acusticæ, 798

Crystalline lens, 744

Crystals, Charcot's, 896

Currents, constant, 58

electrotonic, 104

induced, 58

of action, 95

of rest, 95

Curves of endocardiac pressure, 168

Cutaneous sensations, 703

Cystin, 908

\section{I) ANIELL'S battery, 57 \\ Death, 863}

Decomposition of proteids by digestion, 879

Defecation, 301

Deglutition, 294

Depressor nerve, 225

Detection in solutions, $\mathrm{S} 08$

Development of embryo, 841 
Development of placenta, 846

Dextrin, 248, 886

Dextrose, 248, 884

Diabetes, 448

Diagrammatic eye, 745

Diaphragm as a respiratory muscle, 339

Dicrotism, 185

Diet, normal, 513 modifications of, 519

Difference in respiration of sexes, 339

Digestion, 245 decomposition of proteids by, 879

Dilatation of arteries, 134

Dioptric apparatus, imperfections in, 757 mechanisms, 744

Discharge of energy, 722

Diuretics, 425

Divisions of ear, 790 of spinal cord, 530

Dubois-Revmond kev, 60

Ducts of mammary glands, 477

Dyspnœa, 339, 380

AAR, 790

cochlea of, 794

division of, 790

Eustaclian tube, 798

external, 790

internal, or labyrinth, 793

meatus auditorius externus, 790

membrane of Reissner, 796

membrani tympani, 792

membranous labyrinth, 798

organ of Corti, 796

osseous labyrinth of, 797

ossicles of, 798

perilymph, 797

physiological anatomy of, 790

stapedius muscle of, 800

tensor tympani muscle of, 799

Efferent impulses, 525

Elastin, 883

Electrical changes, 92 stimuli, 57

Electrodes, non-polarizable, 92

Electrotonic currents, 104

Electrotonus, 101 variations of irritability during, 103

Eleventh nerve, 603

Embryo, nutrition of, 847

Emmetropic eye, 752

Energy, discharge of, 722

expenditure of, 493

income of, 492

of mechanical work, 495

Entoptic phenomena, 759

Enzymes, 880

Epiglottis, 820

Epileptiform convulsions, 671, 675

Epithelium of the ducts, functions of, 276

Ethylene-lactic acid, 892

Ethylidene-lactic acid, 892

Eustachian tube, 800

Eupncea, 380

Exhalation of aqueous vapor, 344
Exhalation of carbonic acid, 344, 364

of organic matters, 346

Exhaustion, causes of, 115

Expiration, 342

Eye, 738

apparent size, 780

ciliary region of, 740

diagrammatic, 745

emmetropic, 752

horizontal section of, 739

hypermetropic, 748

images reflected from, 750

mechanisms of, 789

movements of, 781

muscles of, 781

myopic, 748

physiological anatorny of, 738-744

pigment cells of, 741

presbyopic, 748

TACIAL nerve, 607

course of, 607

Fallopian tubes, 832

Faradization, 63

Fats, complex nitrogenous, 892

formation of, 474

nature of, in adipose tissue, 472

neutral, 890

their derivatives and allies, 888

Feces, 313

Female generative apparatus, 834

Fibres, afferent, 124

efferent, 124

sensory, 124

vaso-constrictor, 216

vaso-dilator, 216

course of, 218

Fibrin, 875

in clotting of blood, 28

Fibrinogen, 872

Fick's spring manometer, 176

Fifth nerve, 608 branches of, 609

Flatulence, 308

Fœtal circulation, 850

Food, action of bile and pancreatic juice in small intestine, 308

changes of, in large intestine, 312

in mouth, $306^{\circ}$

in small intestine, 308

in stomach, 306

effects of fatty and carbohydrate, 489

of gelatin as, 490

salt as, 491

peptone as, 491

-stuff's, 245

classes of, 245

Formic acid, 888

Fourth nerve, 612

Fovea hemispherica, 794

Functional activity, influence of, on contractile tissues, 114

CALEN, veins of, 734

G Gall-stones, 286 
Gases in blood, 50

Gastric and intestinal movements, nervous mechanisms of, 302

digestion, circumstances affecting, 257 acidity, 258

juice, 251 temperature, 258

action of, on milk, 259

on proteids, 252

chemical characters of, 251

formation of free acid of, 276

nature of the action of, 258

secretion of, 266

Gelatin, 134, 882

Generative organs, female, 834 male, 833

Globin, 874 physical anatomy of, 834

Globulins, 871

Glottis, narrowing of, 823 widening of, 824

Glutamidic acid, 908

Glutin, 882

Glycerin, 891 -phosphoric acid, 895

Glycin, 906

Glycogen, $88,437,887$

characters of, 438

conversion of, into sugar by liver, 438

in muscle, 447

in placenta, 448

uses of, in liver, 446

Glycolic acid series, 892

Gmelin's test for bile, 280

Goll, columns of, 540

Graafian follicle, 938

Gray matter, 128

Grove's battery, 57

Guanin, 905

Gudden's commissure, 693

Gum, animal, 888

Gustatory bulbs, 811 mucous membrane, physiological anatomy of, 808 pore, 811

$\mathrm{H}^{\mathrm{s}}$ EMACYTOMETER of Gowers, 42

H Hæmadromometer, Volkmann's, 151

Hæmatachometer, Chauveau and Lortet's, 153

Hæmatin, 359

Hrmin, 360

Hæmoglobin, 351

in red blood-corpuscles, 40

Hearing, sensations of, 701

Heart, 157

-beat, augmentation of, 203

government of, by the nervous system, 199

reflex inhibition of, 203

cardiac cycle of, 158

sound and tambour, 166

change of form of, 160

duration of the several phases of the cardiac cycle, 173
Heart, endocardiac pressure, 164 methods of determining, 165

first sound of, 163

impulse of, 162

influences regulating beat of, 209

main events occurring in ventricle dur.

ing a beat, 171

negative pressure, nature and cause of, 172

normal beat of, 157

analysis of, 193

development of, 192

regulation of beat of, 191

second sound of, 163

sounds of, 162

summary of events constituting a beat, 174

valves of, 158

ventricular systoles, 159

visible movements of, 157

work done by, 175

Heat, sensations of, 703

Helmholtz's arrangement for equalizing the make and break shocks, 63

phakoscope, 750

Hemiamblyopia, 696

Hemianopia, 696

Hemianopsia, 696

Hemiopia, 696

Hippocampal lobule, 700

Hippuric acid, 910 formation of, 422

Histohæmatin, 86

Horopter, 786

Human brain, nomenclature of the surface of, 676 , ovum, 836

Hyaloid membrane of eye, 741

Hydrobilirubin, 915

Hypernœe, 380

Hypnotic phases, 684

Hypoglossal nerve, 602 course of, 602

Hypoxanthin, 903

IMAGE, formation of, 744

Imperfect speech, 679

Impregnation, 841

Impulse, nervous, 98

Indican, 915

Indigo, 916

series, 915

Indol, 284,916

Iuduction coil, 59, 71

Inflammation, phenomena of, 232

Inhibition, features of, 200

of frog's heart by stimulation of vagus nerve, 200

Inhibitory nerves, 132

Inogen, 116

Inosit, 886

Inspiration, 340

Intercostal muscles in respiration, 341

Interfibrillar substance, 80

Intestinal digestion, 308 
Iris, 741

Irradiation, 778

TACOB'S membrane, 743

Jaundice, 456

Juice, gastric, 251

action on proteids, 253

artificial, 253

composition of, 252

pancreatic, 281

action on food-stuffs, 282

\section{K ATELECTROTONUS, 103}

I Keratin, 883

Kidneys, vaso-constrictor nerves of, 415

vaso-dilator nerves of, 416

vasomotor mechanisms of, 411

Kinesodic, 714

Kreatin, 88, 901

Kreatinin, 902

Kymograph, 142

Ludwig's, 142

Kynurenic acid, 906

ICTEALS, 313

1 Lactic acid, 892

Laminæ in a hardened lens, 744

Lardacein, 879

Large intestine, movements of, 300

Larynx, 819

cartilages of, 820

cavity of, 821

muscles of, 821

vocal cords, 821

Latent period, 70

Laurostearic acid, 889

Lecithin, 97, 894

Leclanché battery, 57

Leucin, 907

Leucocythæmia, characterized by increase of white corpuscles, 49

Leukomaines, 917

Life, phases of, 855,862

Ligamentum nuchæ, 134

Limb, movements of, 682

Liver, nerves of, 449

Living body, study of, 17

Load, influence of, 110

Lobes of brain, 585

Ludwig's kymograph, 142 stromuhr, 152

Lungs, 332

atelectatic, 334

capacity of, 336

pressure exerted in breathing, 335

Lymph, characters of, 315

chemical composition of, 315

-hearts, structure and functions, 324

microscopic characters of, 215

movements of, 317

MACULA acusticæ, 798

I Magnetic interrupter, 62
Male generative apparatus, 835

glands, 835

prostate, 836

semen, 838

spermatozoa, 838

testicles, 835

tunica albuginea, 836

vas deferens, 836

Maltose, 248, 885

vesiculæ seminales, 837

Mammary glands, 477

changes occurring in, 478

relation to nervous system, 482

secretion of milk, 481

structure, 477

Manometer, mercury, 139 venous, 146,147

Marey's tambour with cardiac sound, 167

Mastication, 294

Maximun manometer of Goltz and Gaule, 165

Maxwell's method of fusing color sensations, 770,775

Mechanisms, dioptric, 744

of accommodation, 749

locomotor, 829

Membrana tympani, 798

Membrane of Reissner, 795

Menstruation, 836

Mercurial gas pump, Ludwig's, 346

Metabolism, general features of, 506 influence of nerves on, 510

Metabolites, nitrogenous, 896

Metamere, neural, 123

Methæmoglobin, 360

Meynert's commissure, 693

Microörganisms, action of, in alimentary canal, 311

Micturition, 427

involuntary, 429

nervous mechanism of, 428

voluntary, 429

Middle ear, 792

Milk, composition of, 480

constituents of, -478

human, 478

quantity secreted, 480

secretion of, 481

sugar, 885

Millon's reagent, 29

Morse key, 60

Motor area, characteristics of, 659

for leg, arm, and face in man, position and relative extent of, 676

Movements of heart, 157

of limbs, 682

of pupil, 751

skilled, 673

Mucin, 246, 881

Mucous gland, changes in during secretion, 271

Muscæ volitantes, 759

Muscle and nerve, degree of irritability of, 111

experiments with, 60

phenomena of, 55 
Muscle case, 80

chemistry of, 83

contractions, simple, 65

currents, 92

curve, 65

double, 73

from the gastrocnemius of the frog, 65

single induction shock repeated rapidly, $73,74,75$ slowly, 73

dead, 84

during contraction, changes taking place in, 77

energy of, 115

Muscle fibres, microscopic changes, 81

-nerve preparation, 57, 102

plasma, 85

as a machine, 107

sartorius of frog, 78

size and form of, 111

stapedius, 800

under polarized light, 81

Muscular and nervous action, nature of, 115 contraction, single, 57 irritability, 55 simple, 57

irritability, 57

sense, 808

Myoglobulin, 86

Myograph, pendulum, 69 spring, 69

Myosin, 84, 873

Myosinogen, 86

Myristic acid, 889

NASAL fossa, right, 806

Nerve and muscle, electric currents of, 92 cells, grouping of, 535

of spinal cord, 129 variations in, 552

centre, 130

chemistry of, 97

eighth or auditory, 605

eleventh or spinal accessory, 603

fibres in retina, connections of, 742

fifth or trigeminal, 608

fourth or trochlear, 612

ninth or glosso-pharyngeal, 603

roots, connections of, 544

seventh or facial, 607

sixth or abducens, 608

tenth or vagus, 603

tetanization of, 99

third or oculo-motor, 612

twelfth or hypoglossal, 602

Nerves, electric currents in, 99

Nervous impulse, 98

changes in nerve during passage of, 97

measurement of velocity of, 70

system, central, 128

tissues, general features of, 122

Neurin, 98, 895
Neurokeratin, 98

Neutral fats, 890

Nicol prism, 81

Nitrate of urea, 897

Nitrogenous metabolism, 487

metabolites, 896

non-crystalline bodies allied to proteids, 881

Nuclein, 883

Nucleo-albumin, 884

CCIPITAL region, prominence of nuclear cells in, 659

Ocular spectra, 780

CEdema, 322

CEsophagus, movements of, 296

Oleic series, acids of, 890

Olein, 891

Olfactory mucous membrane, cells of, 807 sensations, 700

Oncograph, 413

Oncometer, 412

Optic radiation, 694

thalamus, 615

Organic matter, exhalation of, 345

Organs of Corti, 795

of reproduction, 832

Ovaries, 835

Ovum, 838

Oxalate of urea, 897

Oxalic acid series, 892

DALMITIC acid, 889

Palmitin, 890

Pancreas, changes in, during secretion, 269 of rabbit, 269

Pancreatic juice, 282

action of, on fats and starch, 284 on food-stuffs, 282

characters of, 282

secretion of, 286

Papillæ, circumvallate, 809

filiform, 809

fungiform, 809

Paraglobulin, 871

in the clotting of blood, 30

Paralysis, crossed, 68j

Parapeptone, 256

Parotid gland, nervous mechanism of, 265

Parturition, 853

Pendulum myograph, 68

Pepsin, 258

Peptogenous food, 277

Peptones, 256, 875

Perceptions, general sensibility and tactile, 812

modified, 778

Peristaltic movements, 292 influences bearing on, 305

Perspiration, 431

Pettenkofer's test for bile salts, 280, 912

Phenylic acid, 911

Physostigmine, action of, on pupil, 756

Pituitary body, 468 
Plasmine, in clotting of blood, 32

Pons Varolii, 584 gray matter of, 623

Posterior column of spinal cord, 531

Presbyopic eye, 748

Pressure, arterial, 138

in capillaries, 143

in veins, 138

Products of digestion, course taken by fats, 325

\section{by proteids, $32 \pi$}

by sugar, 326

by water and salts, 326

Proprionic acid, 889

Prostate gland, 834

Protagon, 98, 895

Proteid bodies, characters of the more important, 253

Proteids, 866

classification of, 257

coagulated, 875

decomposition of, by digestion, 878

general composition of, 28

xanthoproteic test for, 28

Protoplasm, 20

Pseudopodia, 121

Psychical processes, duration of, 725

Ptomaines, 313, 345, 896

Pulse, 176

anacrotic, 185,190

katacrotic, 185

methods of recording, 176

venous, 190

Pulse-curve, characters of, 181

Pulse-curves described by sphygmographic levers, 177

Pulse-tracing from an artificial model, 179

from carotid artery of healthy man, 185

Pulse-wave, anacrotic, causes of, 190 changes of, along the arterial tract, 182

diacrotic, 185

cause of, 185

features of, 182

length of, 185

predicrotic, 189

velocity of, 184

Pupil, movements of, 751

nerves governing, 753

Purkinjé, cells of, 653

Purkinjé's figures, 760, 762

Pyramidal cells, 656

Pyrexia, 504

Pyriform lobe, 700

UADRIGEMINAL bodies, 625

Quality of sound, 801

Quantitative determination, 898

Quantity of aqueous vapor exhaled, 344

of blood, 53

mode of estimating, 54

of carbonic acid exhaled, 344

of organic matters exhaled, 345
RAPIDITY of circulation, 150

R. in capillaries, 154

in veins, 154

of clotting of blood, 27

Reaction of urine, 409

Red-blind, 774

blood-corpuscles, 37

counting of, $4^{2}$

apparatus for the, 42

diameter of, 37

disintegration of, 42

number of, 41

shape of, 37

structure of, 37

study of, 37

marrow in bone, a source of red corpuscles, 43

Reflex actions, 130

nature of, 130

of spinal cord, 564

inhibition, 203

Refraction, 745

Regulation of heat, 499

Reissner, membrane of, 795

Rennin, 250

Reproduction, 832

corpus luteum, 840

of menstruation, 840

of pregnancy, 840

development of embryo, 842

$$
\text { allantois, } 844
$$

placenta, 847

segmentation of ovum in, 842

vascular area, 843

Graafian follicle in, 838

impregnation of ovule, 841

menstruation, 838

organs of, 832

Fallopian tubes, 835

male, 835

ovaries, 835

uterus, 834

vagina, 834

ovule in, 841

ovum in, 838

spermatozoa in, 841

Respiration, 332

absorption of oxygen in, 361

apncea in, 384

apparatus for taking tracings, 337

asphyxia in, 336,386

phenomena of, 386

carbonic acid exhaled, amount of, 344

changes of air in, 344

Cheyne-Stokes, 385

complemental air in, 334

dyspnœea in, 336,380

effect of breathing foreign gases, 388

of changes in atmospheric pressure, 389

of muscular exercise on, 383

exhalation of aqueous vapor, 344

of carbonic acid, 344,364

of organic matters, 345

expiration, 342 
Respiration, expired air, impurities of, 345 Sarkin, 903

nature of, 345

temperature of, 344

facial and laryngeal, 343

graphic records of movements of, 336

influence of vagus nerves on, 372

inspiration, 340

labored inspiration, 342

modified movements of, 403

coughing, 403

crying, 404

hiccongh, 403

langhing, 404

sighing, 403

sneezing, 403

sobbing, 403

yawning, 403

movements of diaphragm, 339

of expiration, 342

of inspiration, 339

muscles of, 340

nervous mechanism of, 369

number of, 339

relations of respiratory system to vas-

cular and other systems, 390

residual air in, 334

ribs in, action of, 341

function of, 341

stationary air in, 833

tidal air in, 333

visible movements in, 339

Respiratory centre, 370

changes in blood, 346

in the tissues, 365

undulations, 391

\section{Retina, 741}

connective tissues of, 741

inner surface of, 743

of man, rod and cone from, 742 photo-chemistry of, 762

Ribs, action of, in respiration, 341 function of, in respiration, 340

Rigor mortis, 83

Ritter-Valli law, 112

Rods of Corti, 795

Rolando, substantia gelatinosa of, 534 tubercle of, 598

CACRAL nerves of dog, 125

Saline matters in blood, 51

Saliva, 246

action of, on starch, 247

characters of mixed, 250

of parotid, 251

of sublingual, 251

of submaxillary, 251

chemical characters of, 246

composition of, 246

nature of amylolytic action of, 249

secretion of, by means of chorda tympani nerve, 261

Salts of bile, 280

of uric acid, 900

Santorini, cartilage of, 820

Sarcolactic acid, 892
Scala tympani, 794 vestibuli, 790

Scheiner's experiment, diagram of, 747

Schlemm, canal of, 739

Sclerotic coat, 739

Secretion, general nature of, 273 nature of the act of, 275

Segnentation of ovum, 842

Semicircular canals, 794

Seminal fluid, 838

Seminiferous tubules, 836

Sensation, 759

Sensations, auditory, 800

cutaneous, 703

distinction and fusion of, 766

of cold, 703

of color, 769

primary, 773

of hearing, 701

of heat, 703

of pressure, 814

of smell, 700

of taste, 701

of temperature, 703,814

of touch, 703

relation of, to stimulus, 766

simple, 766

tactile, 814

visual, 689,759

Sense, muscular, 814

Sensory impulses, 759

Serous fluid, chemical character of, 316

Serum-albumin in clotting of blood, 30

Serum in clotting of blood, 29

Seventh nerve, 607

Sexes, difference in respiration, 339

Sight, 738

Sixth nerve, 608

Skatol, 917

Skin, absorption by, 433

cutaneous respiration, 432

perspiration, amount of, 431 composition of, 432

secretion of sweat, mechanism of, 434

Small intestine, contents of, 308 movements of, 292,300

Smell, sensations of, 700

Soaps, 891

Somatic and splanchnic nerves, 123

Sömmerring, yellow spot of, 743

Sound, 801

pitch of, 801

quality of, 801

Sounds of the heart, 162

Specific gravity of urine, 405

Spectra, ocular, 780

Spectroscopic analysis of hæmoglobin, 353

Speech, 826

areas for, 676

imperfect, 679

cansed by bulbar disease, 680

thick, 679

Spermatozoa, 838

Spherical aberration, 757 
Sphygmograph, Dudgeon's, 177 Marey's 177

Spinal accessory nerve, 603 cord, 525

antero-lateral ascending tract of 5 . 9

ascending tracts of, 543

automatic actions of, 578

central canal of, 530,534

cerebellar tract, 556

columns of Burdach, 540 of Clark, 539

of Goll, 540

complexity of reflex movements of, 570,574

cornu of, 530

descending tracts of, 541

features of, 548

fissures of, 529

gelatinous substance of Rolando, 534

gray matter, nature of, 560-563 structure of, 523

longitudinal, commissural tracts of, 563

loss of tone of skeletal muscles in, 579

reflex actions of, 564 inhibitions of, 574

time required for, 577

relative size and form of, 549

reticular formation, 538

rigidity of muscles through action of, 583

structure of, 529

tendon phenomena of, 582

white matter, structure of, 530 tracts of, 540

disease, sensations of pain in, 711 ganglia, $12 \tilde{j}$

nerves, 525

Spleen, chemical constituents of, 453

movements of, 451

red corpuscles in, 43

Spring manometer, Fick's, 176

Stapedius muscle, 800

Starch, 247

Stearic acid, 889

Stearin, 890

Stellate nerve cells, 127

Stimuli, characters of, 107

Stimulus, nature and mode of application of, 107

Stomach, gastric juice in, 266 movements of, 297

Submaxillary gland, 261 nerves of, 261-263

Succinic acid, 893

Succus entericus, 285

nature and action of, 285

Supra-renal bodies, 467

chemical constituents of, 468

functions of, 469

structure of, 468

Sylvius, aqueduct of, 585

Syntonin, 85
T'ACTII.E perceptions and judgments, 816 Tambour, Marey's 167

Taste, 808

buds, 811

circumvallate papillæ, 809

filiform papillæ, 809

fungiform papillæ, 809

gustatory bulbs, 811

sensations of, 701

Taurin, 906

'Taurocholic acid, 913

Temperature, 498

effects of great cold on, 505 of great heat on, 504

influence of food on, 501

on contractile tissues, 113

of muscular action on, 501

of time of day on, 503

of body, 497, 498

of various animals, 497

pyrexia, 504

sensations of, 703

Tensor tympani muscle of ear, 799

'Tenth nerve, 603

'Testicles, $836^{\circ}$

'Tests for bile, 280

Tetanic contractions, 57, 73

Tetanus, 73 contractions in, 109

Thalani optici, 623

Thermal changes, 90

Thermopile, 90

Third nerve, 612

Thoracic duct, 314

'Thymus, functions of, 469

structure of, 470

Thyroid body, 465

functions of, 466

structure of, 465

Tidal air, 333

Tissues, contractile, 54

Tongue, 808

Tonic contraction, 118, 669

Tracing from heart of cat, 161 of respiration, 338

Transudation, phenomena of, 319

Traube-Hering variations, 736

Trigeminal nerve, 608

Trochlear nerve, 612

Trypsin, 274

Trypsinogen, 275

Tunica albuginea, 836

Tunicin, 885

Twelfth nerve, 602

Tyrosin, 909

TMBILICAL vesicle, 843

Urari, poisoning by, 56

rise of blood-pressure in animals

Urea, 896 under, 708

formation of, in liver, 459,460

group, 896

nitrate of, 897

oxalate of, 897 
Urea, relations to cyanogen compounds, 464 Vision, binocular, 781 svnthesis of, 406,462

Uric acid, $405,462,899$

Urine, 404 salts of, 900

abnormal constituents of, 409 albumin, 410 sugar, 410

acidity of, 409

amount of, 408

composition of, 409

ferments in, $40 \mathrm{~S}$

general characters of, 404

hippuric acid in, 406

inorganic salts in, 406

non-nitrogenous constituents of, 407

normal organic constituents of, 405

pigments, 481,915

reaction of, 409

relations of secretion of, to food and drink, 425

secretion of, 410 glomerular, 420

specific gravity of, 405

urea in, 405

uric acids in, 405

Urobilin, 915 amount of, in, 405

Uroerythrin, 915

Uterus, 834

os uteri, 835

structure of, 833

Utricles of ear, 797

VAGINA, 834

Vagus nerve, 603

Valerianic acid, 889

Various forms of stimuli, 58

Vascular mechanism, 133

Vas deferens, 836

Vaso-constrictor fibres, course of, 218 nerves, 124

-dilator fibres, course of, 220

-motor actions, 211 effects of, 220

Veins, blood-pressure in, 137 circulation in, rapidity of, 154 vasomotor nerves of, 231

Velocity of blood in arteries, 150 in capillaries, 154

of the pulse wave, 154

Venous pulse, 190

sinuses, 734

Vertigo, phenomena and causation of, 647

Vesiculere seminales, 837

Vibrating tuning-fork with Despretz signal, 70

Vierordt hæmatachometer, 153

Vision, 759

in man, apparatus of, 691

region of distinct, 777

Visual tield, 689

impulses, origin of, 760

judgments, 786

$$
\begin{aligned}
& \text { of distance, } 786 \\
& \text { of size, } 786 \\
& \text { of solidity, } 787
\end{aligned}
$$

movements, coördination of, 784

perceptions, 776

purple, 764

contrast of, 778

sensations, 689,759

white, 764

vellow, 764

Vitellin, 873

Vitreous body, 744

Vocal cords, 821

movements of, 823

slackening of, 824

tightening of, 824

Volitional impulses in the cord, 684

Volkmann's hæmadromometer, 151

Voluntary movements, 661,679

action of, on the lower animals, 662

Vomiting, 299

W ATERY vapor, exhalation of, 344

Wave, contraction, 79

White blood-corpuscles, 43

chemical examination of, 45

migration of, 48

movements of, 47

nuclei of, 44

number of, 44

origin of, 47

proportion of, to red, 44

size of, 44

structure of, 44

transformation into red, 49 work of, 47

White matter, 97,128

Willis, circle of, 733

Work done by heart, 175

X ANTHIN, 904

X Xanthoproteic test for proteids, 28

VOUNG-HELMHOLTZ theory of color sensations, $772-775$

TINN, zone of, 744

4 Zona pellucida, 841
Zymogen, $27 \bar{j}^{\circ}$

spongiosa, 535 


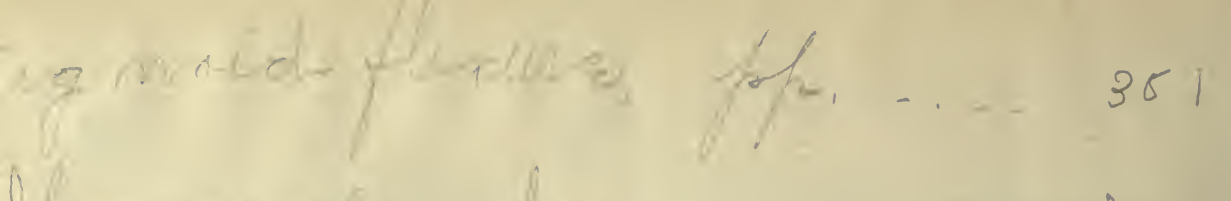

bert caveal zake, " zor 





